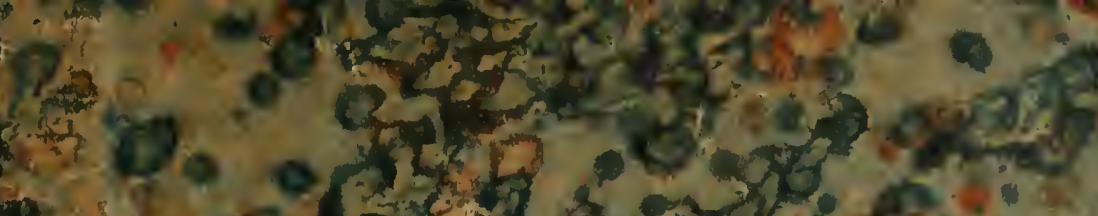

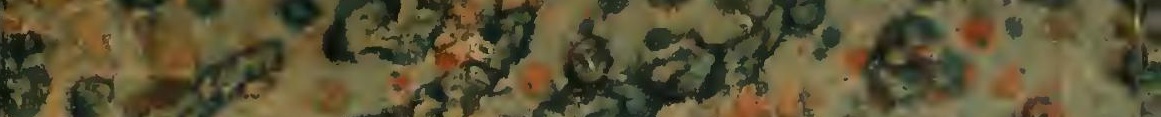

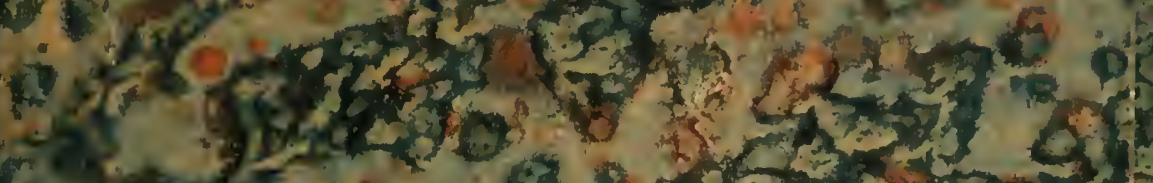

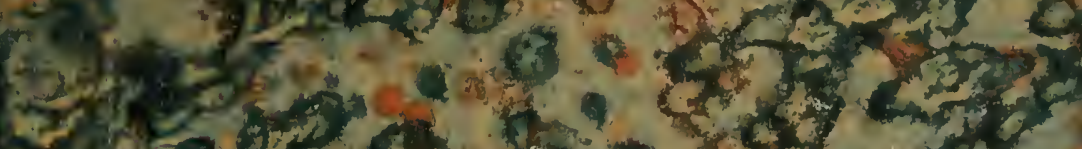

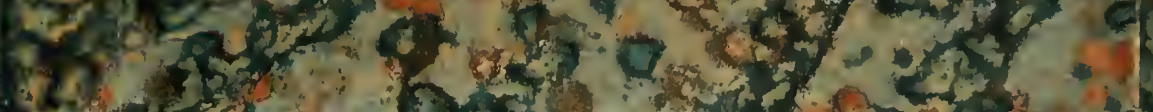
$4: 250$ -

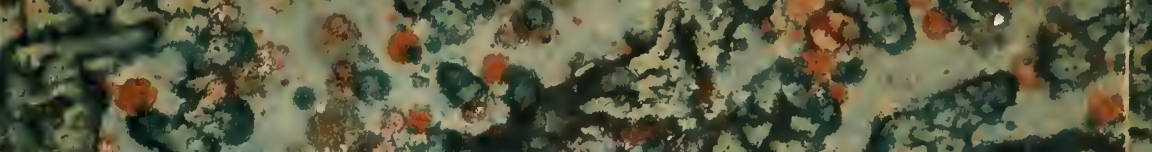

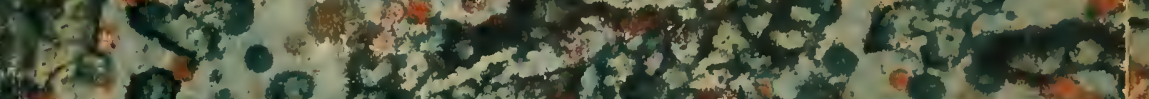

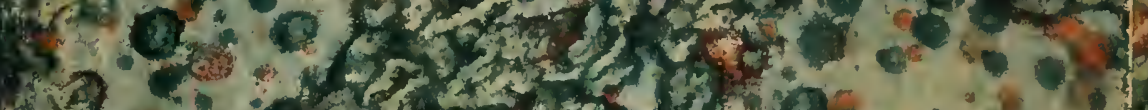

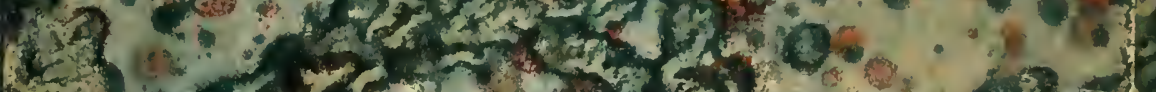
S.t.

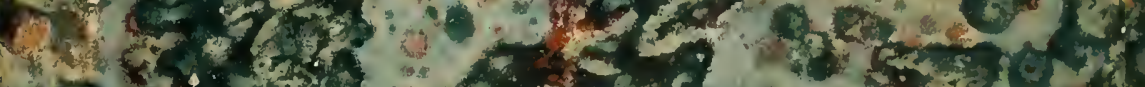

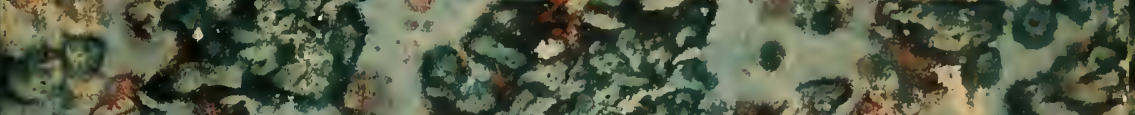

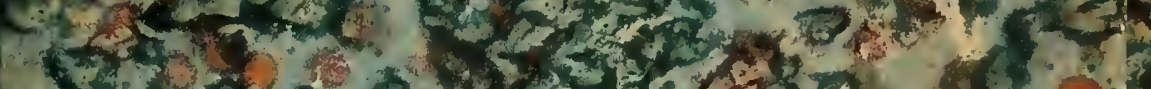

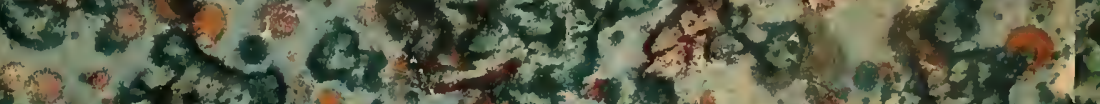

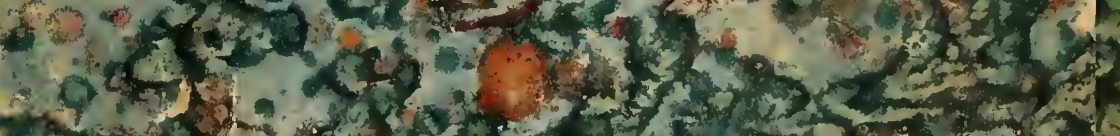

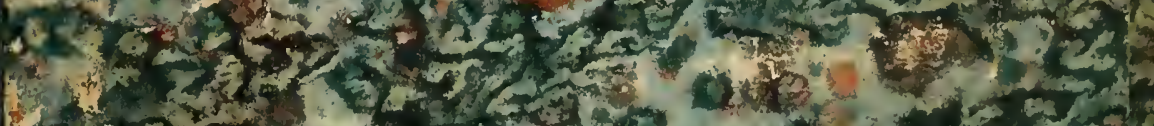

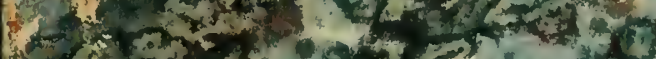

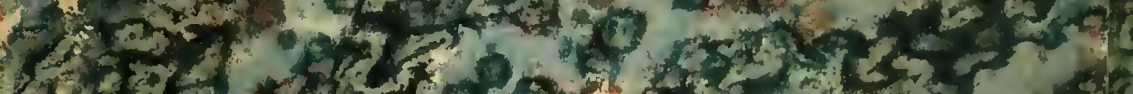

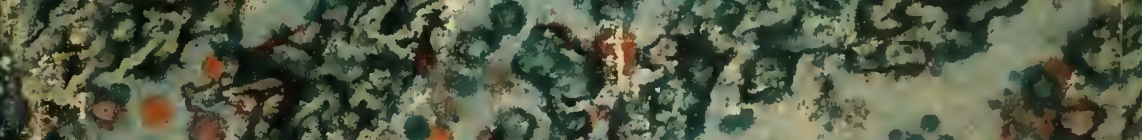

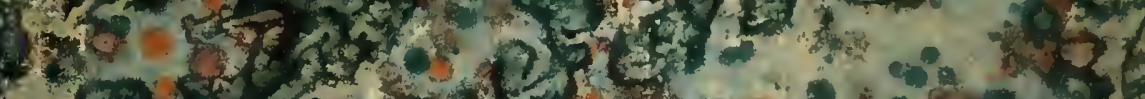

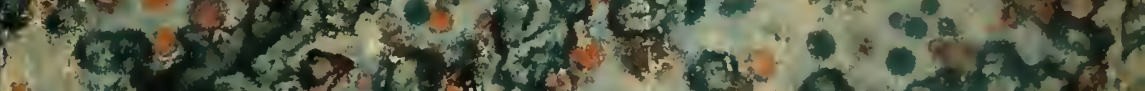

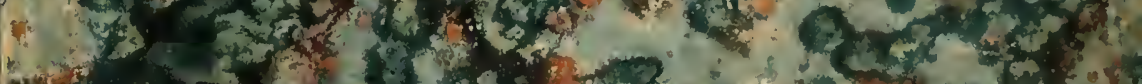

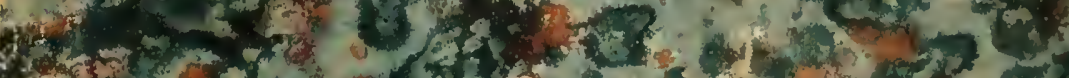

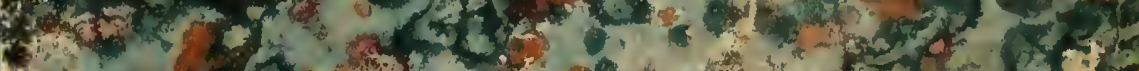

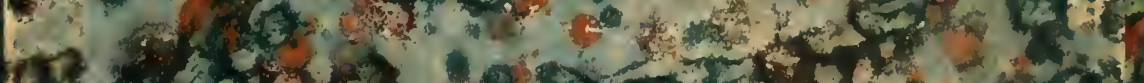

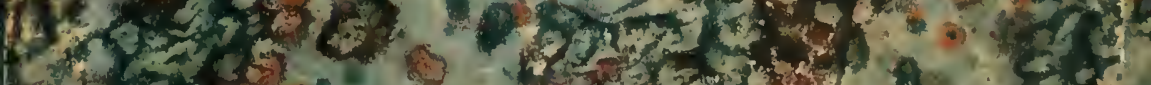

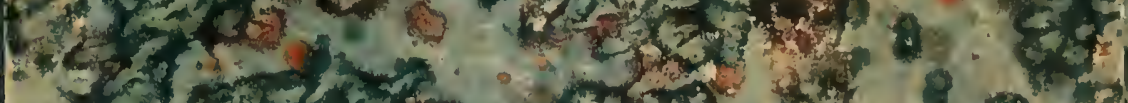

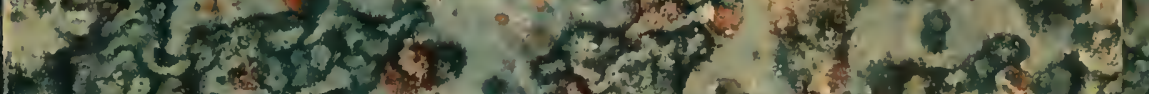

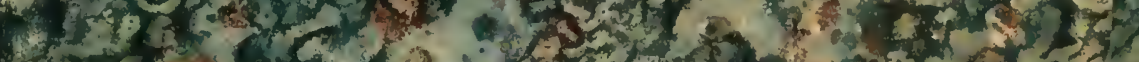

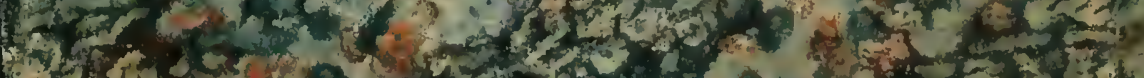

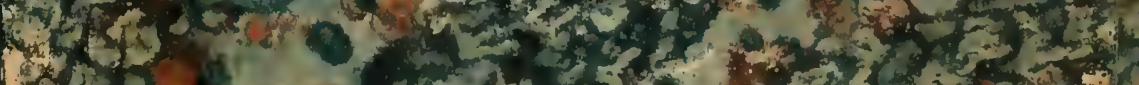

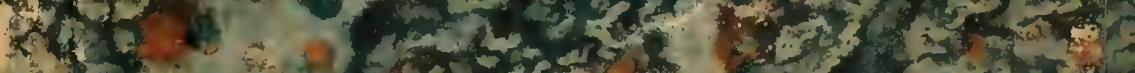

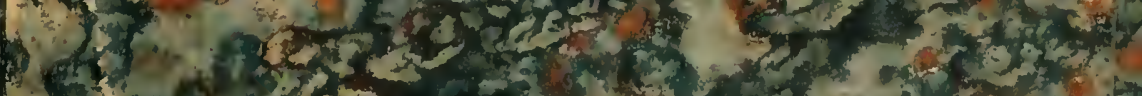
4. 


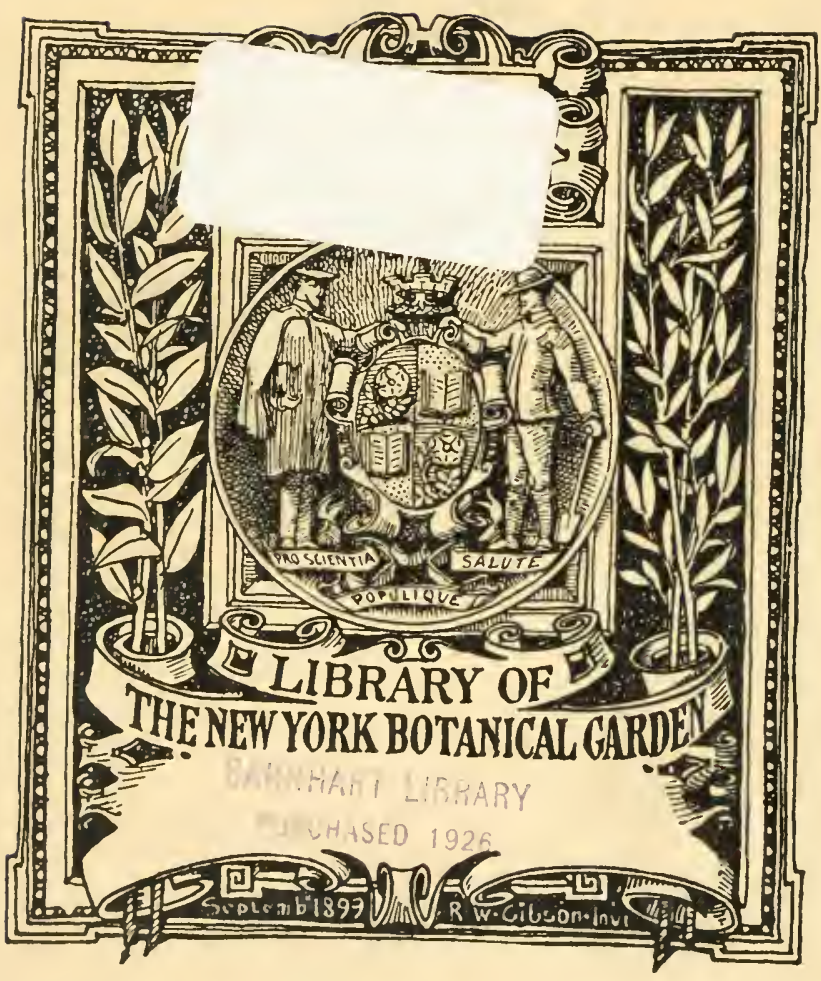






\section{ff) $\mathbb{1} \|$ i $(11$ d)}

Tes

\section{untüurlidgen Yilnuzenjuftents}

nad) allen feimen

Clallen, OCròumnen .und familien,

nebit

แaturgemäzer (S) rup

noer

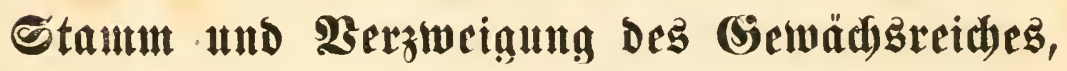

enthaltento

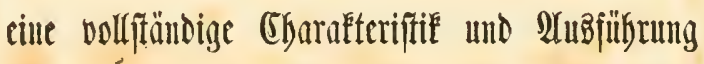

Det

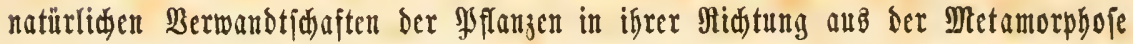
und geognoftifden Berbreitung, wie bie fortgebildete 3eit beren elnjofaung fordert,

lon

\section{Dr. fG. (G). findmin Reidentad),}

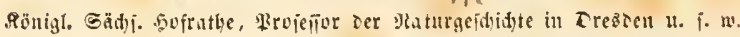

3 weite Angabe.

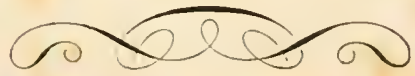

Rcipzig,

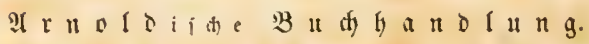
1850. 


$$
\begin{gathered}
+Q k 93 \\
R 283 \\
1850
\end{gathered}
$$

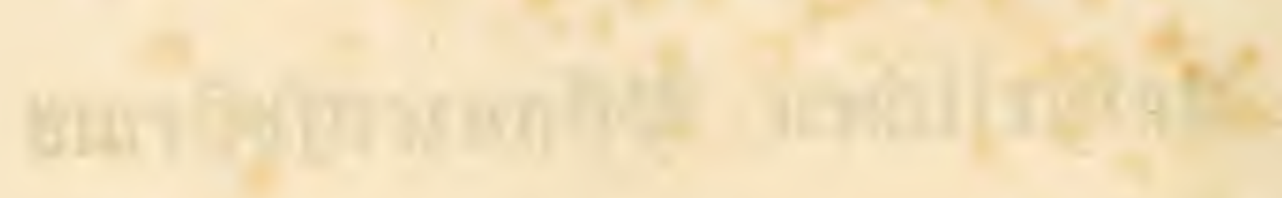




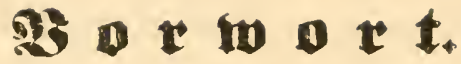

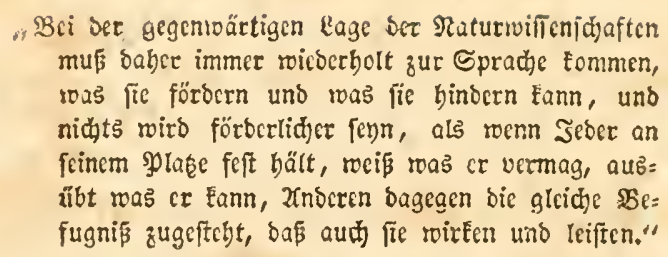

(अ.

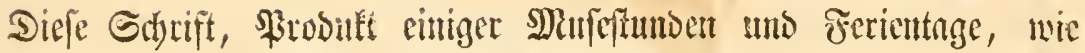
fie dem Berfoffer in feinem bewegten Gefobäftskereife nur felten zu Theil serón, Dürfte vielleicht befonderz den jüngeren sefern meines lieben

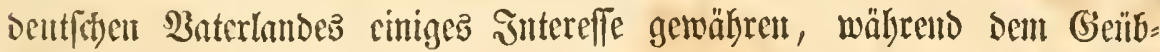

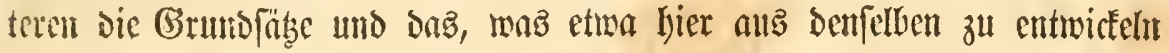
verfuct) worden, gewi eben fo gut und Manchem viclleidst nod beffer befaunt ift, als mir, wenn cr bie Duelle, alls ber ich) Altles forbüpte,

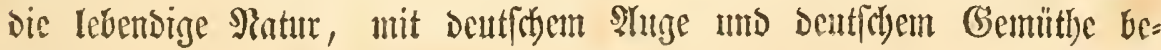
fithute.

Gollte aber überbanpt einer meiner geefrten Refer etwas $\mathfrak{B r a n d}$.

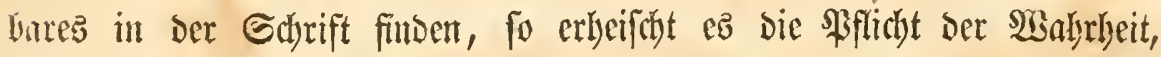

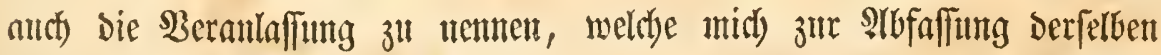
berufen. -

In einer thir erft menige Sage vor Sftern bei Durctifidt meiner Bibliothef im Fach Der Syftentatif micber zur Sall getommenen, frilter mie ith) mun fabl, nicht gemug von mir bendteten Srbrift, "Nixus" be= titelt, yon Serrn Lindley, Dr. Phil. und \$rofeffor Der Botanik an Der lluiverfitüt Rombon, fand ith cine mir gämblith entgangene, aber and 


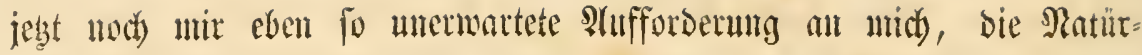

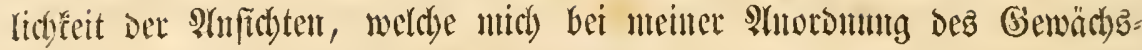

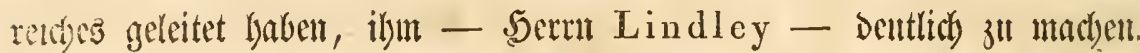

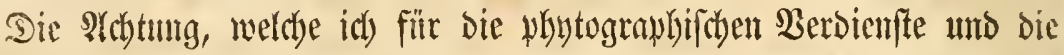

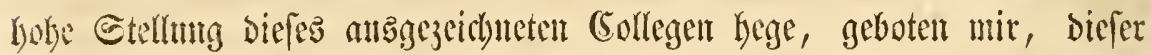

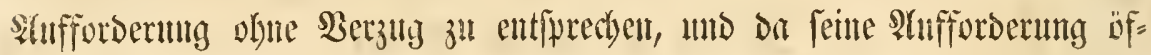

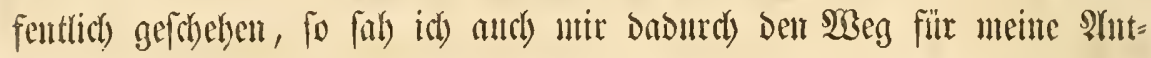

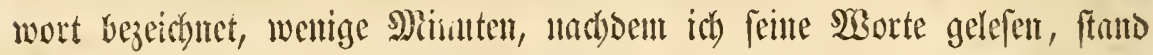

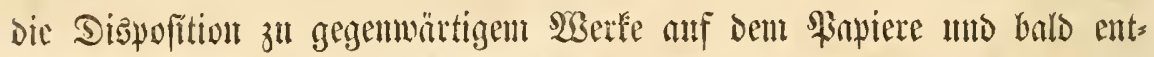
floffer die lïrigen Beilen der Feder.

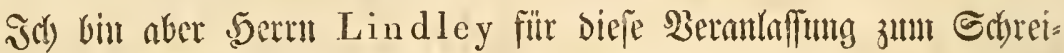

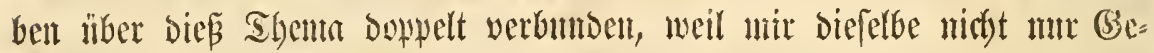
legenfseit giebt, ifm meime Bereitwilligfeit, ifwn gefulltig zu rewn, beweifut

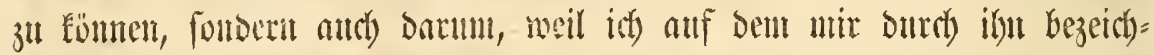

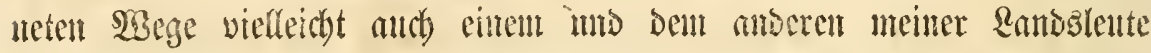

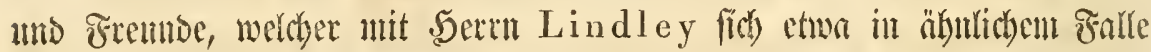

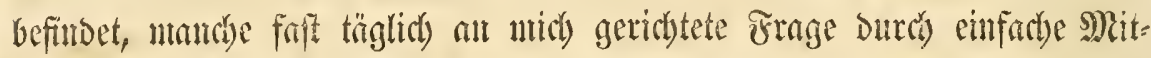

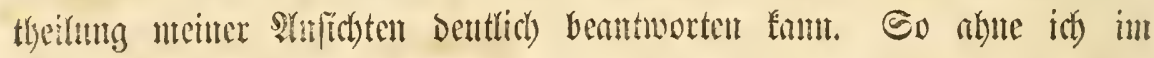

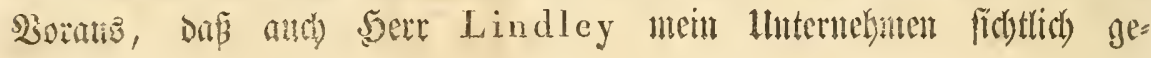
förocit.

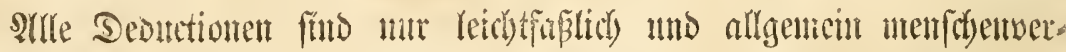

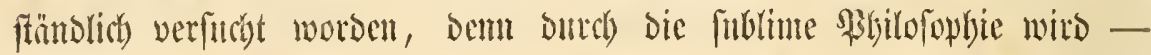
wie bereitz Göthe gezergt hat - Die Yebentsige Nantur weder enflärt

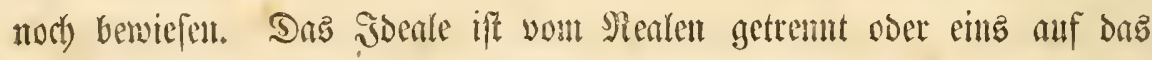
altoere bejogen, wie ç eben bie Sintur Der Sache verlangte.

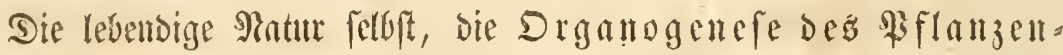

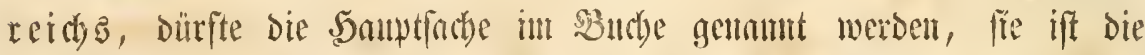

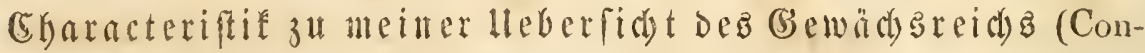
spectus regni vegetabilis. Lips, 1828). WGemt jetter Conspectus Der \$flanzengattungen Hach Den foron Damals befolgten, Dam in Der Flora germanica fït Deten 3mecte weiter autgedenteten, hier

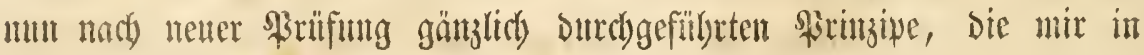




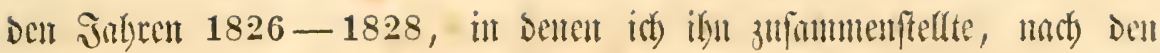

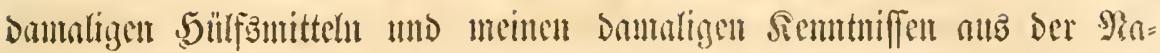
tur uns Riteratur, mir befinut geworbenen Formen in ifrem 3ufammen=,

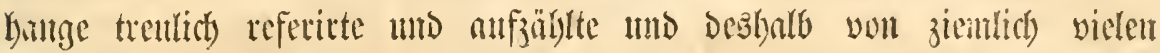

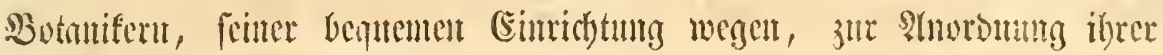
Serbarieit brandbur gefmben worden ift, fo boffe idh anch allen Befthern

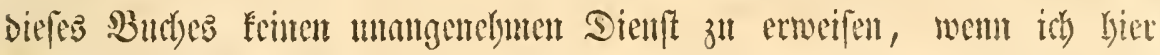

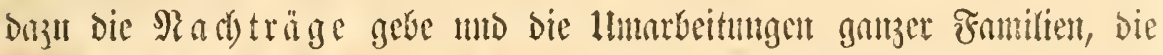

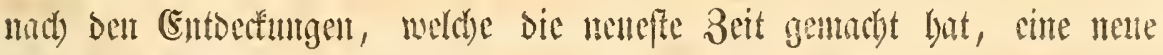

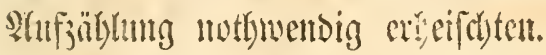

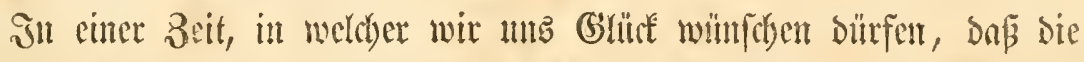

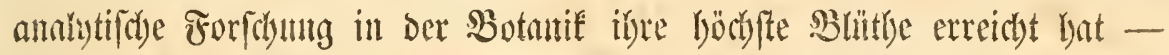
DeCandolle's prodromus, Endlicher's mo Meisner's genera plantarum füd Dafür Die redenden, irefflityen Bengen! - mag ce mot

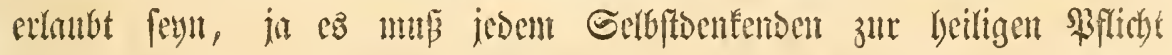

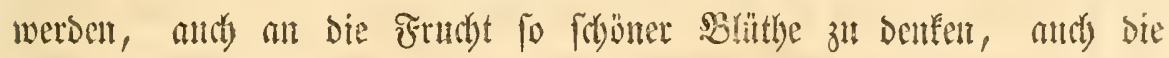

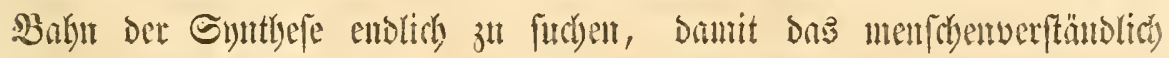

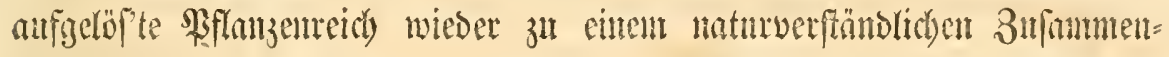

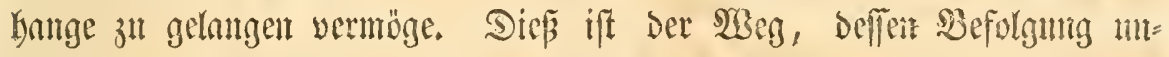

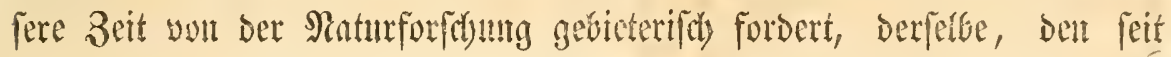

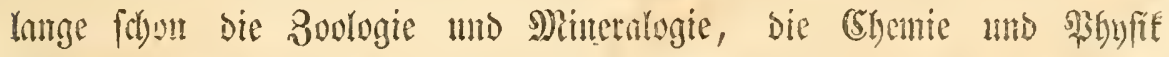

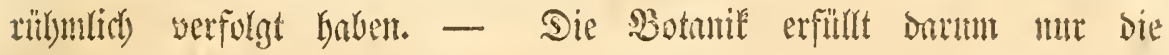
SYnfordentugen Des Beitalters, in weldyem wir leben, wenn fie im

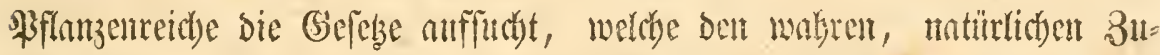

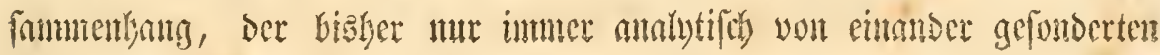
Jormen bedingest.

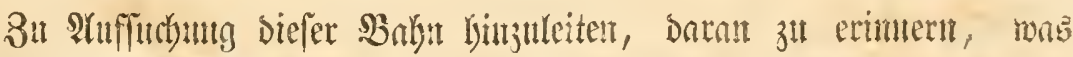

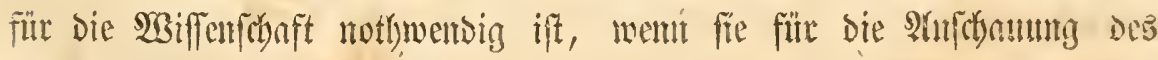

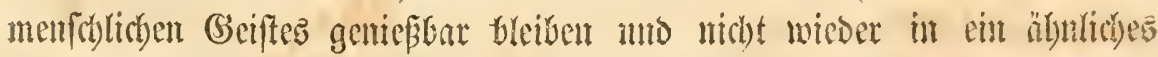

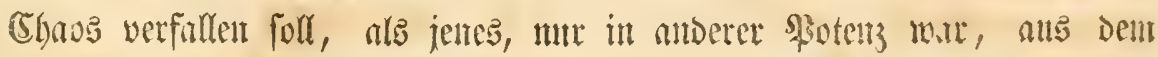

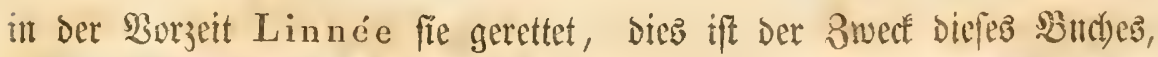

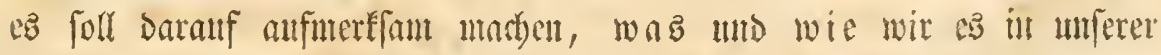
3eit an Der Sffanze beobachtent follen, unt nicht Des für Den Bufanment 
bung bes Gamzen möthigen Frabens verluftig zu werden. Darmm mird ieber die objective Bffanzennatur in Dem Buble wiederfinden, wenn ex fie felbft finmte, nder er wird fie darin erfemment lermen, wie er fie fin=

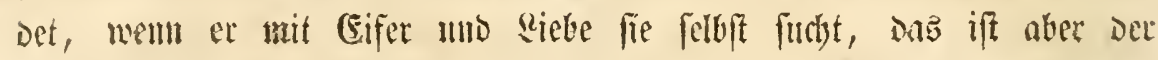

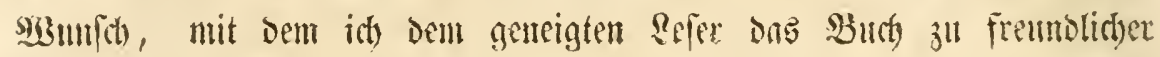

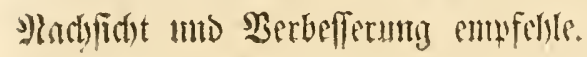

Der Berfaffer. 


\section{I th}

Stite.

5. Sotbereitenees.

Bride in bie Gefidide ber botanifden Sgftematit.

1.

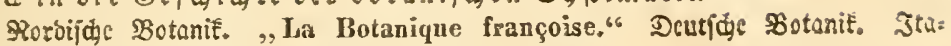

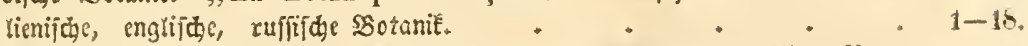

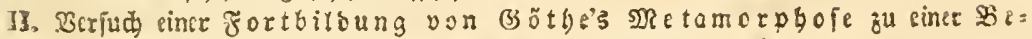
i daung bes gyflanzenceids in feiner Iotalität. . . . 19-96. * . . . . 19-25. Borläufige Einrörfe. . . . . . . . 25-26.

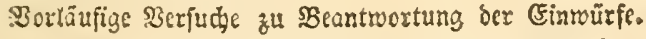

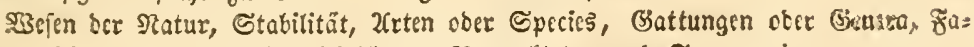

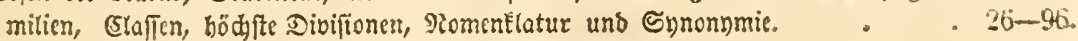

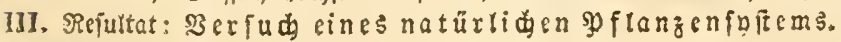

1. Cinleitenoes.
AA. Beróereitenoes.
BB. Nethodifdes.
CC. ॠe fultat.
A. Bon bre Fatur Birgabenes.
B. 3u ธतु)
C. Geroonacnzs.
- $9 \ddot{7}$
101.
$10 \%$.

11. Betraditendes.

A. Socales in ber Metamorphoje.

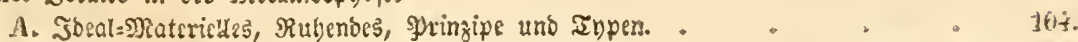

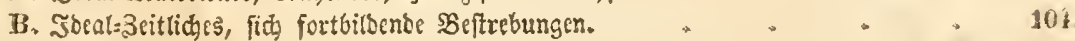

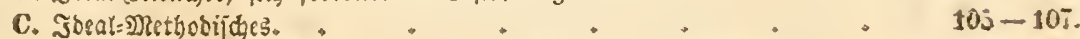

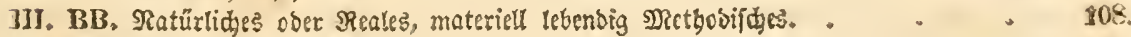

A. গูrãmillen.

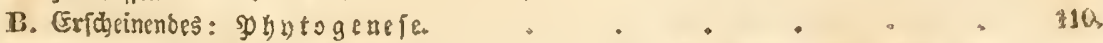

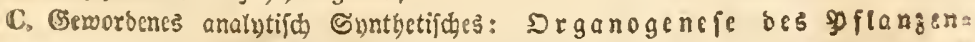
Ifich 5 ober Drganenentwidelung oer Sfflanzenmelt in ibrer Eongrueng als

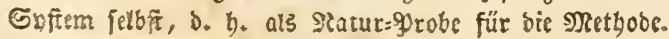

Gsufen und Elafen.

Dronuagen in ben erlaffen.

Reibs in ben D

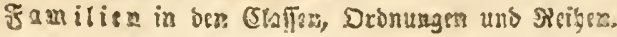

$112-115$

$116-126$.

$127-125$.

$129-319$. 


\section{(Erfte Rlalfe: \$ ilje: Fungi.}

Errife Srinung. Seimpitze: Elastouycetes. Esite.

1. Fam. urpilge: Praeformativi.

2. Fam. Branopitze: Uredinei :

3. Fam. T3axzenpilze: Tubercularii.

3reite Sromung. Fadenpilge: Hyphougcetes.

4. ซ̌um. Mrobcrpilz̆ : Byssacei.

5. శ̌um. Faferpilze: Mucedinei.

6. Fam. ๔đimmelpilze: Mucorini.

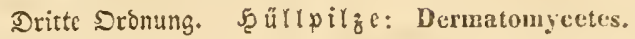

7. Fam. Sataudinge: Sphaeriacei.

万. Fram, Etrculinge: Lycoperdacei.

9. J్5um 5̧uttings: Hymenini.

3weite Rlaffe. Tiledert Lichenes.

Erffe Stonung. Seimfledten: Blastopsorae.

10. Fam. Ctaublecten: Pulverariac.

11. Zam. Gtatifrudtfleciten: Conincarpicae.

12. Fam. Malfledten: Arthonarite.

Bweite Dromung: Fabenfledten: Hyphopsorae.

Süđojenflectiten: Crateropsorae.

13. Fum. Ragelfledten: Coniocybeac.

14. Fam. Feldffledten: Calicieac.

๔. 132.

132. 17. Tom

Ropffectiten: Cepiralupsorae.

15. Frm. StaubEugelfl. Sphaerophoreae. 132. 18. Fam. Sinopfffedten: Cladoniaceae.

Dritte Sronung. Şülffledten: Dermatopsorae.

Fernffedten: Gasteropsorae.

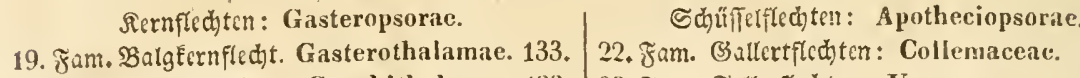

20. Fam. Finnenfledten: Graphithalamac. 133. 23. Fam. Tellerfledten: Usueaceae.

133.

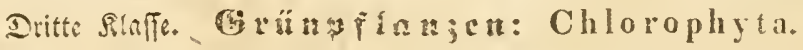

Erjef srtnung. 2r/gen: Alggae.

Snojpenalgen: Gongs Inplyyeae.

25. ซ্ৰam. Ballectalgen: Nostochinae.

26. Fam. Fabcnaigen: Confervacene.

27. Fum. Shlaudalgen: Ulvaceae.

135. 28. Bam. Batgalgen: Ascopleyeate.

135. 28. Fam. Betenffrudtalgen: Cerauiluceac, 106.

135. 29. Fam. Rernalgen: Florideac. 136.

136. 30. Fam. Tangalgen: Fucoideae. 136.

3weite Soronung. Moofe: Musci.

23sctetmoufe: Thallobrya.

Eaubmoofe: Phyllobrya.

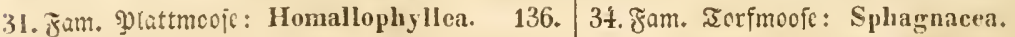

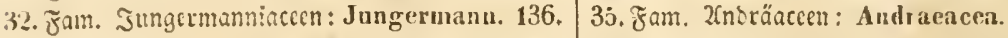

137.

13\%.

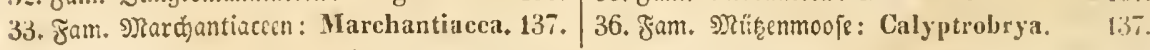

Dritte Sronung. Farrn: Filices.

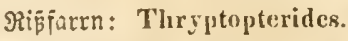

138. 40. Fam. Dsmunbaceen: Osmumterides.

37. శ్m. Salviniacecn: Salviniaceae.

38. Fani. Marfitiacen: Narsiliaceae.

39. ש̃an. Wretelfarrn: Pteroideae.

139.

138. 41. Fam. Eycabeacen: Cyeadleberile. 139.

138. 42. Zam. 3amiaccon: Zániweac.

Bierte Rlaffe. Eche iocupflanjen: Coleopligta.

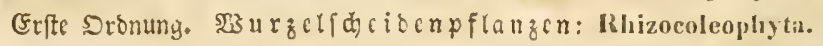

Tauchergesväçje: Limnobiac.

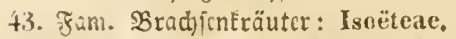

4. Zam. Mुafficriemen: 'Zostereae.

45. Jum. Arongemädjie: Aroideae. ๔d)lammutr

141. 46. Fam. Piftaccen: Pistiaceac.

$1+3$.

141. 47. Fam. शुafiertiejic: Alismaceac. $1+3$.

142. 48. Zam. Rixenftäuter: Hydrocharideac. $1+4$. 
Strite Dronung. Stammidjeioenpflangen: Caulo-Coleophyta.

Epclżngcwådje: Glumaccae.

49. F̊am, Broüfor: Gramineae.

50. Fam. Eypergräfer: Cypcroidene.

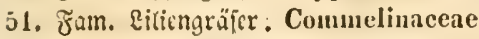

๔đimertelgomãch ie: Ensatae.

145. 52. Zram. Rohrfolben: Typhacene. 148.

147. 53. Zram. Shinertel: Irileae. 149.

145. 54. Fam. शarzifienichivertel: Narzissineae, 150.

Dr:tte Dromung. SBlattfde iocnpflangen: Phyllo-Coleophyta.

\section{Riliengewädje : Liliaceae.}

5j. Fam. Eimientilien: Juneacene.

56. Fam. Zauffintilin: Sarmentaceae.

57. Fum. Sirontition: Coronariae.
Fatmengewädic: Palmaceae.

151. 58. Fam. Drdition: Orchideae.

152. 59. Fam. Banancn: Scitamineae,

153. 60. Fam. Salmen: Palmae.
154.

156.

158.

\section{Sünfte Elaffe. Bweifelblumige: Synchlamydeae.}

Erfte Sionung. Rippenlofe: Enerviae.

Sajaben: Najateae,

๔duppler: Imbricatae.

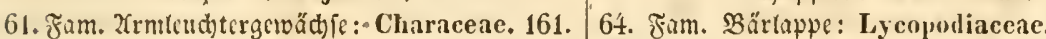

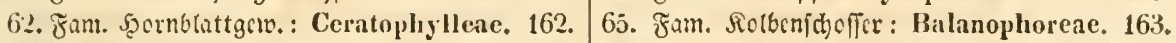

63. Fam. Noooftemoncen: Pollostemoneae. 16\%. 66. Fam. (5ntincen: Cytincae. 164.

3weite Sronung. Eteifolätterige: Rigidifoliae.

\section{ङd)tedttutütbige: Inconspicuae.}

67. Fam. @djad̆tchalme: Equisetaceae. 165. 70. Fam. 3apfenbäume: Coniferae. 168.

65. Fam. Giben: Taxineae. 166. 71. Fam. Froteaccen: Proteaceae. 169.

69. Fam. Santałacen: Santalaceae. 166. 72. Fam. Šcibcln: Thymeleacene. 169.

Dritte Sronung. 2coerblätterige: Venosae.

Unvorftommene: Incompletae.

73. Fam. গnticacsn: Myricaceae.

Blattricidfe: Foliosae.

170. 76. Fam. Dfterttzcien: Aristolochiaceae. 173.

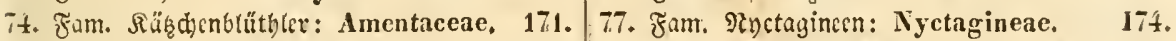

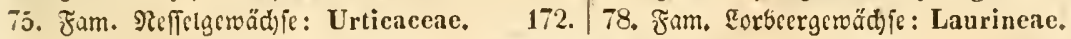

175.

Sechste Rlaffe. Gianjblumige: Synpetalae.

Crffe Dronung. Rö hecnblumige: Tubiflorae.

Seäufutblüthler: Aggregatie.

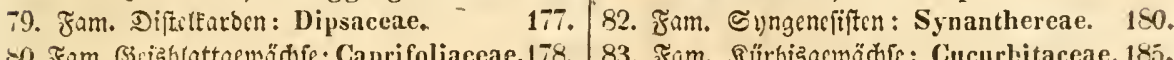

ßlocentüthter: Campanaceac.

81. Fam. Rubiacen: Rubiace 179.84 Fam. Btöftcr: Campanulaceac.

3weite Sroutra. Sdlunoblumige: Fauciflorac.

Röhrenblüthtcr: Tubiferae.

8.5. Fam. Rippcnblüthter: Labiatae. 187. 88. Fam. Şlobttariaccen: Globulariaceae. 195.

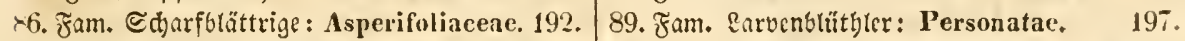

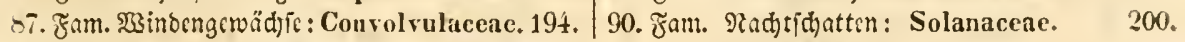

Dritte Srinung. $\subseteq \mathfrak{c}$ umblumige: Limbiflorae:

Bed)erstütgler: Crateriflorae.

91. Fam. Yjlumbagineen: Plumbagineae, 201. 94. Fam. 26зtepiabrn: Asclepiadeae. 207.

92. Fam. গुrimulaccen: Primulaceae. 203. 95. Fam. Drcbbtütlılcr: Contortae. 209.

93. Fam. Szeiben: Ericaceae. 205. 96. Zam. Eapotacen: Sapotaceac. 213.

\section{Siebente Rlaffe Seldblüthige: Calycanthae.}

Erfte Sronung. Berfdiebentlüt bige: Variflorae:

Rlcinbtïtbige: Parviflorae.

5ุüt/fenfrüdtige: Leguminosae.

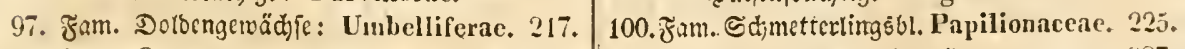

98. Fam. Sitcuzoorne: Rhanmeae. 222. 101. Fam. Eaffiaccen: Cassiaceae. $22 \%$

99. Fam. Zercbintbacen: 'Terebinthaceae. 223. 102. Fam. NRimojaceen: Mimosaceae. 227.

3roeite Dronung. 2(ehnlid)blütbige: Confines.

Ecoumbtitthige: Sediflorae. Rojenbtürbige: Rosillorae.

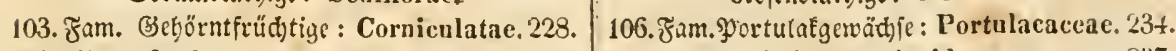

104. Fam. Roajaccen: Loasacene.

231. 107. Fam. 2rizoibcen: Aizoideae. $23 \%$.

10j. Fam. Çactusgeroăd fe: Cacteae.

232. 108. Zram. Rojaccen: Rosaceae.

$2+1$. 


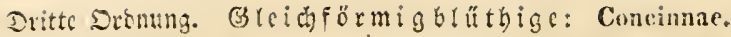

Saliotterzinbilithige: Onagriflorae. 103. Jam. Satorageen: Halorageas. 110. Fum. Siachtêtrgen: Ongrariae. 111. 75am. Mascitrictic: Lythrariae.
Nyrtenblüttbige: Myrtiflorac.

244. 112. Fam. \$ुolngatacen: Pol galacene. 249.

245. 113. Fam. Murtacecn: Hyrtacene. 252

247 114. Fam. 2(mugbaracen: Amygdalacene. 25-r.

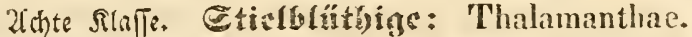

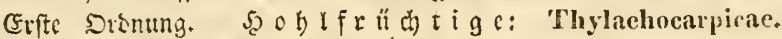

Siretzbtüthler: Cruciflorae.

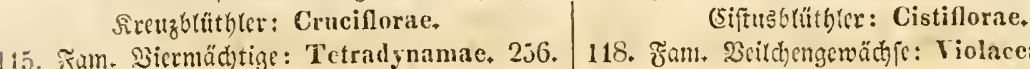

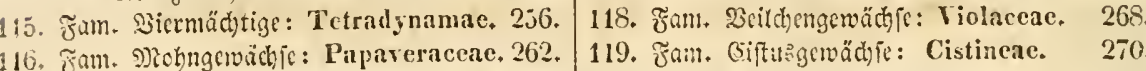

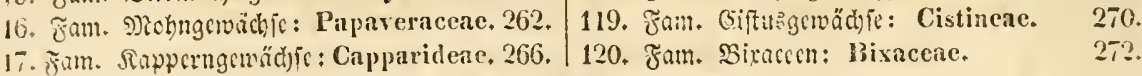

3woite Sronung. Spaltfrufthtige: Schizocarpicae.

jeanumétblïtbige: Ranuuculílome.

121. Žrn. RanunEetgew Ranunculaceac. 275.

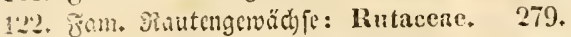

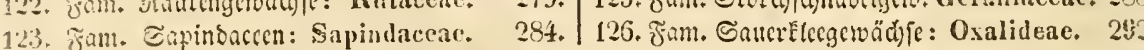
Dritte Dronmug. Säulenfrüch tige: Idlocarpicne.

Eintongtütgler: Tiliiflorae.

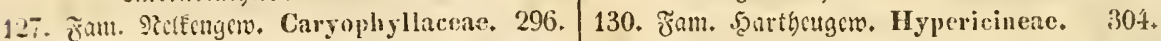

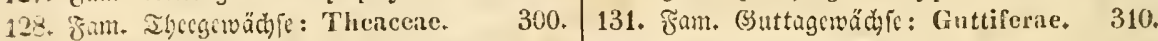

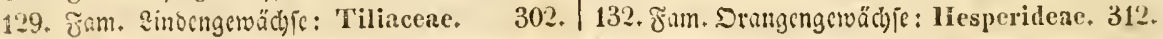

EvElärung uns Bitte.

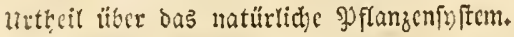

$\cdot * \cdot \cdot \cdot 321$.

๔chlujivert an ben Dpponentem. . . . . . . . . . . 322.

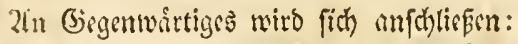

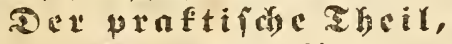

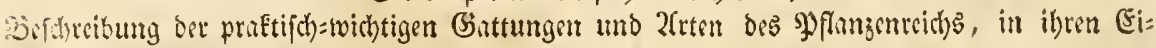

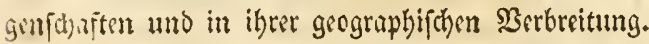

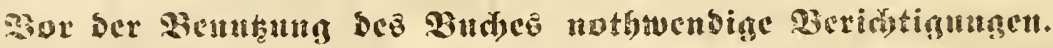

ฐcite 3. 3cile 6. v. 0. 1. be Eräftigte.

$22 .=11$. v. o. l. Jorale.

30. $=9$. v. o. l. Effentsart.

$31 .=17$, v. u. 1 . Verbis-ca.

$31 .=19$. v. u. 1. 2 2ftern.

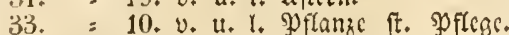

$37 .=9$. v. u. I. fici) wicocr zut 2c.

女3. = 15. u. 19. v. u. l, corolla gainopetala.

tt. = 10. v. o. $x$. atls, fand ich $2 c$.

60. = 2. v. u. I. Eitte ft. Scite.

$73 .=-13$. v. o. l. Tonrnefortiana.

$83 .=23$. v. o. l. organifirten.

$83 .=8, v$. u. $\mathfrak{l}$. Cirices in nur sine Spccicz $2 \mathrm{c}$.

84 . = 19. v. o. 1. Rchb.

$83 .=12$. v. o. . . zแl ocn.

$x^{5} .=11$. v. u. . IIngueninia.

107. = 7. v. o. 1. Beginnendes, 0. y. Synthesis zu vorigem.

$126 .=1.8 .2$ nm. 1. Sclizocarpium.

133. = 18, v. u. l. Marsiliaceae.

$139 .=17$. v. 0. $\mathfrak{l}_{\text {. Marattieae. }}$
Grite 140. 3rile 8. v. o. 1. Shloroplynten. $=14 t$. = 13. v. o. 1. phrnitogam. = $156 .=2$. v. o. $=$. bobarf t̂. barf. 189. = 10. v. u. \%. Chamaepitys. 191. $=7$. v. 0. 1. glatten ft. platten. $=201 .=18$. v. u. I. Verbasecac.

- 203. = 8. v. u. ftrcide $: 2$ bci Utrienl.

$=213$. = 2. v. u. I. convoluta f, contortá.

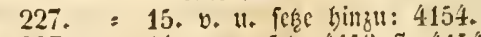

227. = 14. v. u. fę̧ 4153 ff. 4157 .

, 235. = 8. v. u. nad Lour.?) fesc Aylmeria Mart.

= 236. = 11. v. o. nadj Spergula fege: Alisne $L$. Drymaria $/ V$.

237. = 7. v. o. nadi Sesuvium L. feze caps. valvata: Ginginsia DeC. Colobanthus Burtl.

- 239. = 5. v. ut. nadi Pliytolacea $L$. fakc Gyrostemon Desf.

260. $=19$. v. u. ftreidbe Parrya $R \cdot B r$. $27 \%=20.0 .0$. ftreidge genuinae. 


\title{
Blide in sie (Gejolidnte
}

\author{
ber
}

\section{botanip̈ben Gypentatif.}

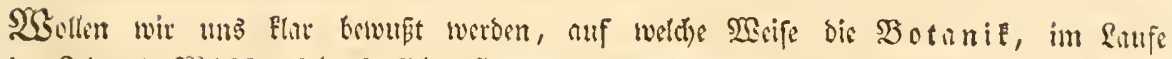

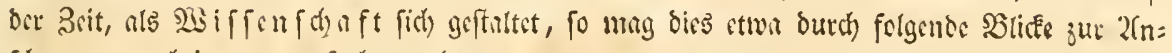

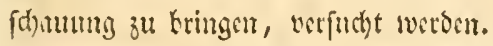

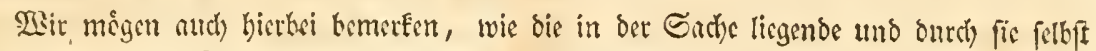

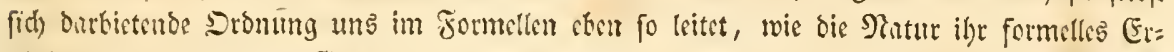

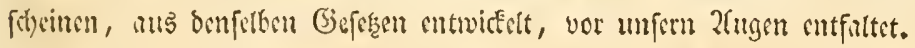

1. Thesis. Etroimm der Bafrbung, Reimung uno Snoppung. - Erffes Entwiffern

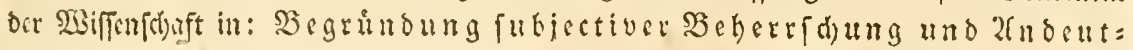
ung ciner mógliden bóberen, objectiven Befdaung oer Pyanjen= welt.

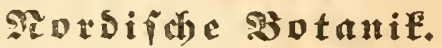

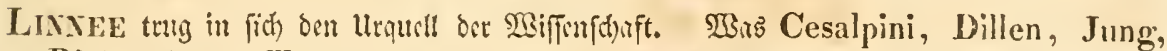

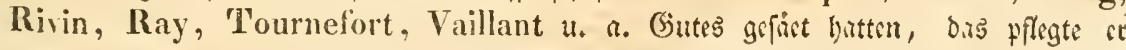

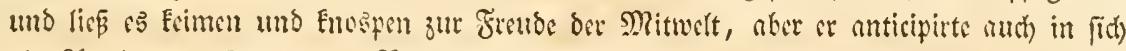

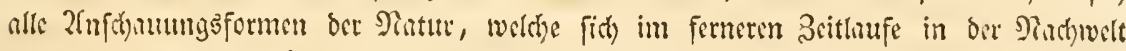

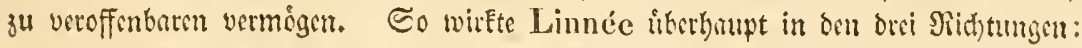

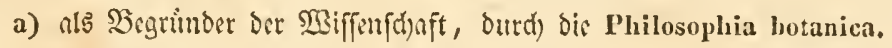

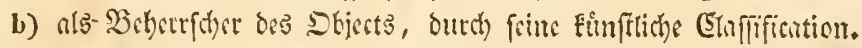

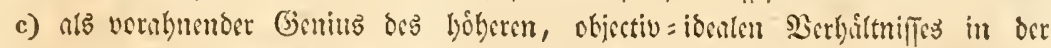

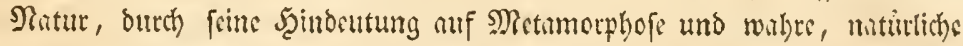
Bertwandtaduaften.

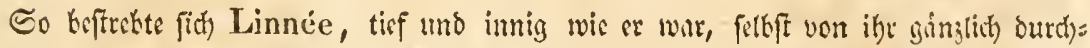

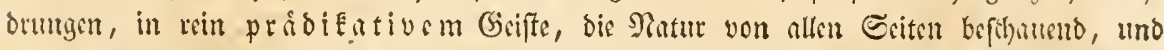

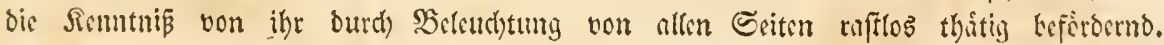

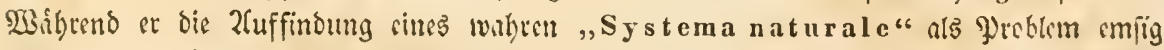

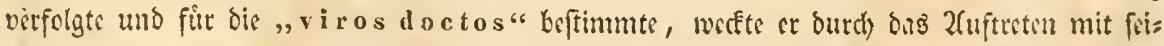

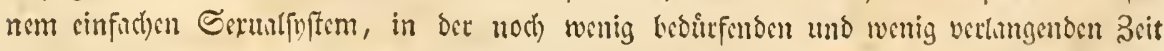

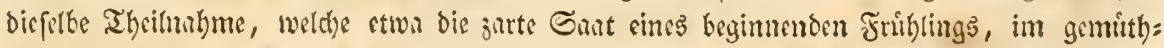

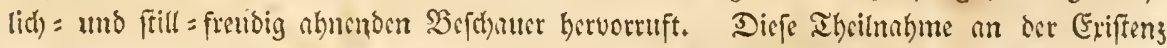

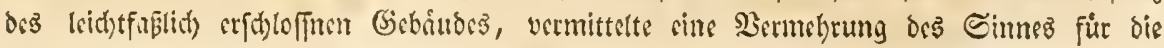




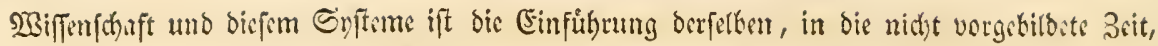

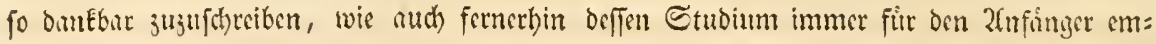

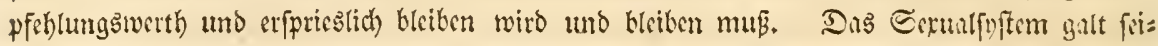

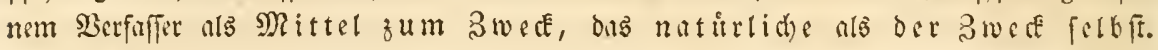

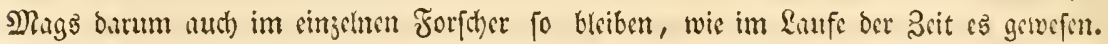

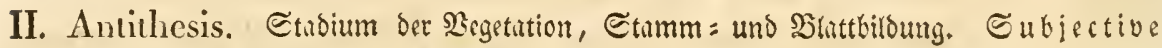
Bebertalung bes Dbiectes. -

\section{"La Botanique, françoise."}

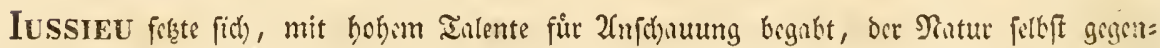

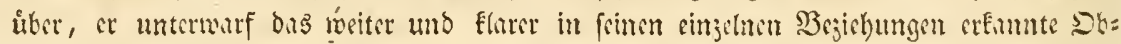

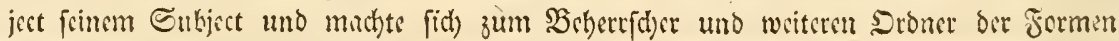
Der pflamblid)en Sobopfung.

IBas in feimen Sorgn̊mgirn, instefondere in Adanson und Linnée fic) vorge:

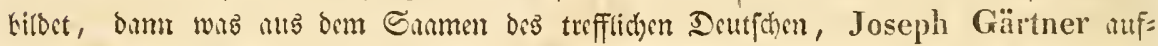

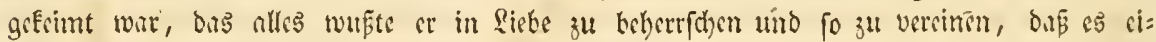

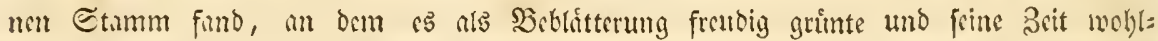

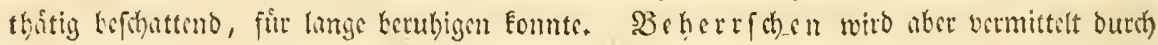

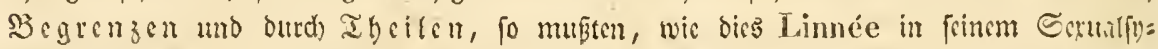
feme gethan batte, mit foincm naturlidgen Sifteme nber zu thun, nicht füt gut hislt, bie

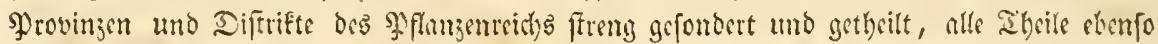

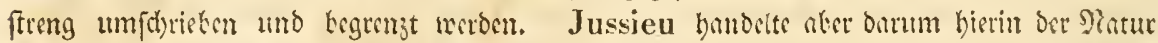

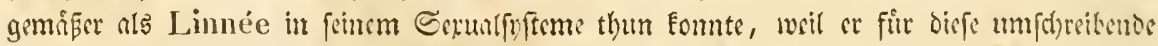

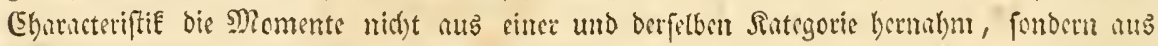

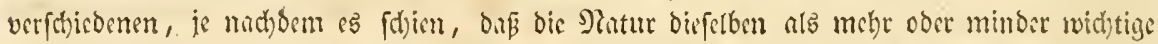

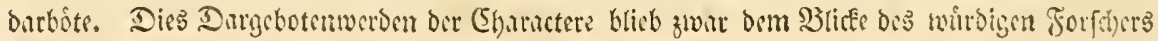

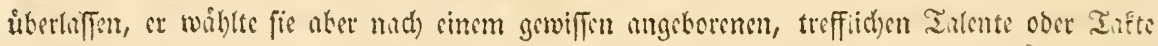

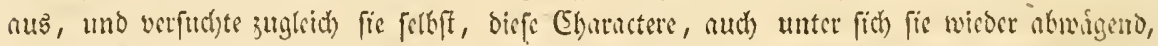

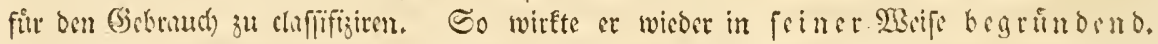

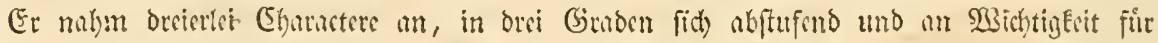

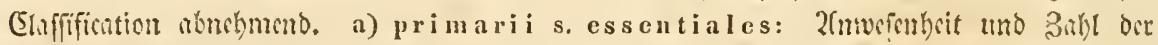

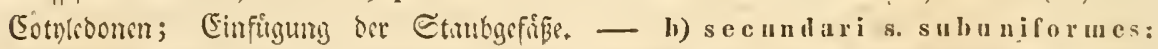

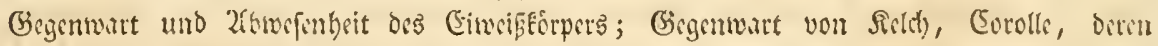

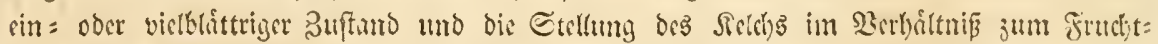

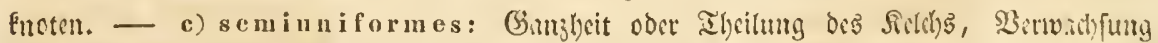

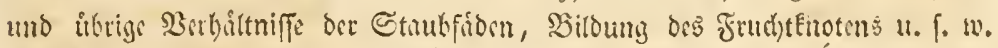

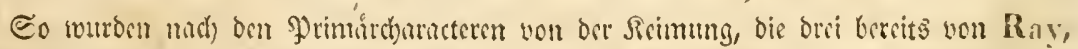
v. Royen unb andern gibrututern, von Linnée in ber Phflosophia botanica erhiuter=

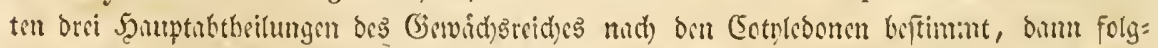

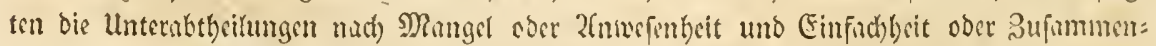

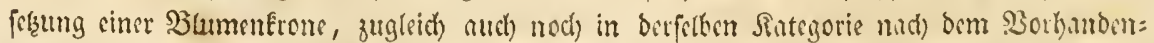
fenn einer Diflinie oder (Gifd)ledtstrennung. Dis britte Theilung beftimmte funfosth

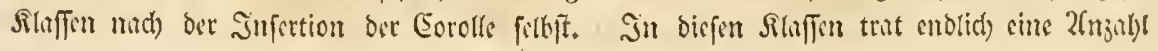




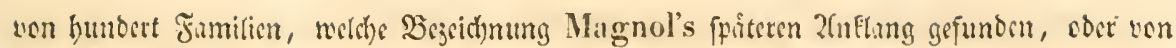

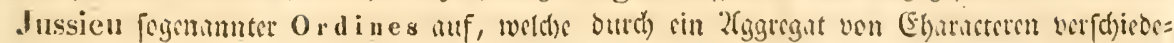

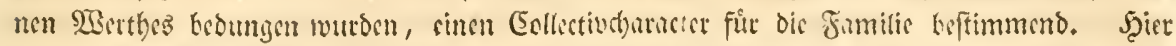
wart as untzitglich, wo Jussieu finen hohen Beruf glingend bewafhrte, das Serwinde

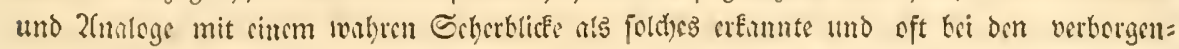

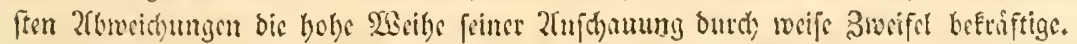

Jussieu fertef erwurb fid) nod) fermer bus großje Serbienft, in cigner Fortbildung

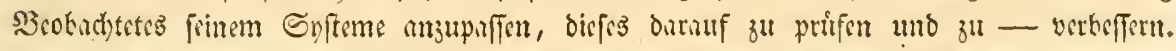

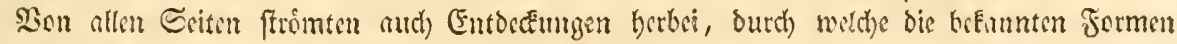

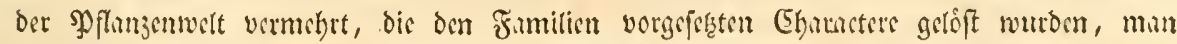

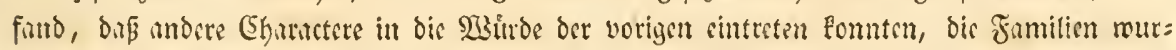
oen getbeilt turo mengebildoste in gleichen $\mathfrak{W e r t h}$ mit den alten geftellt, fo aber bie 2lygregnte

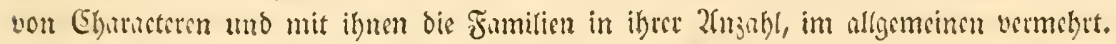

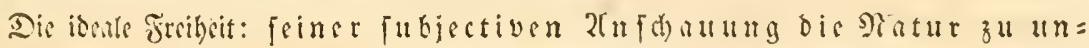
terwerfen, und fie, bic ferbft ibcale real ober pofitiv zu madyen, zsigte fid) als sin fid)

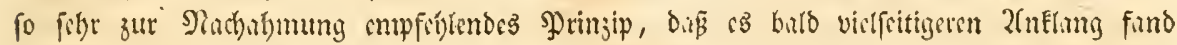

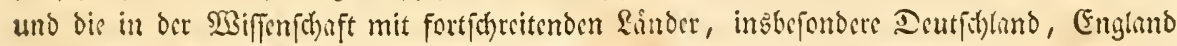

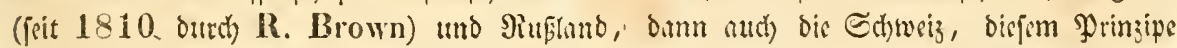

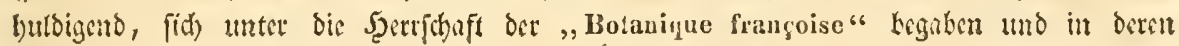
Sुrimsipe mit fortf(dritten.

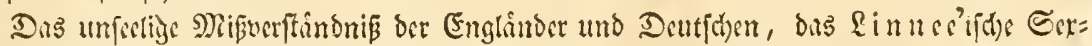
unlinftem milfe bie Sberband bebalten, tebte fid) zrwar nod) in sinigen (Geiftern keider Ran= ber bis in bie meneften Beiten bimen, nno nod) immer foreint Sem naturtid)en Syfte=

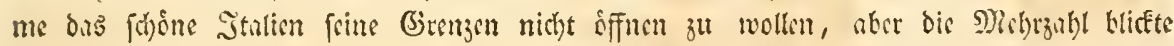

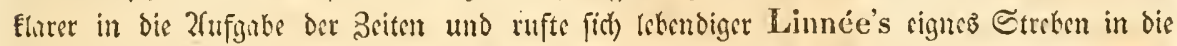

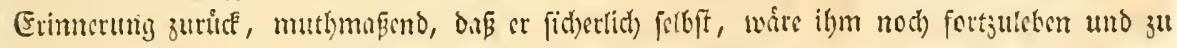

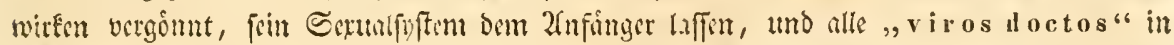

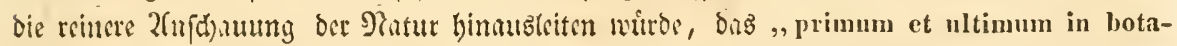
nicis desideratum" jut erfillen. Srin nimmer bebartendes Streben nath cigner Fortkild:

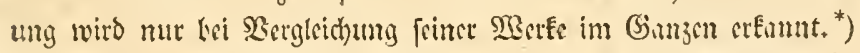

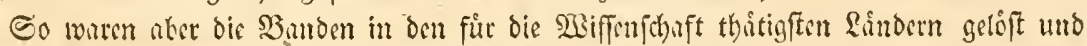

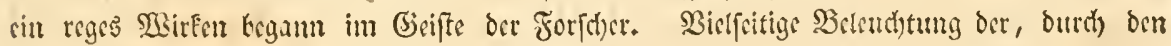

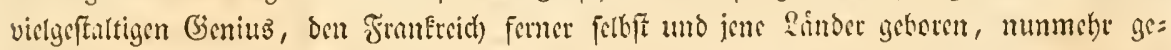

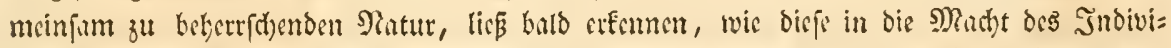

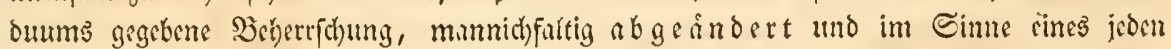

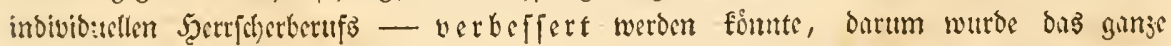

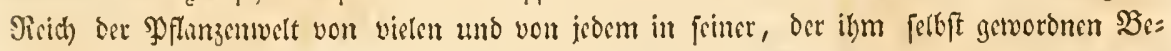

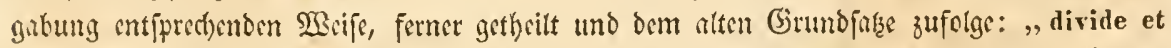

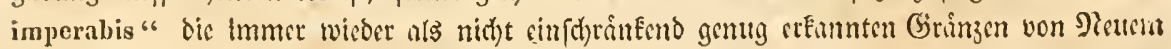

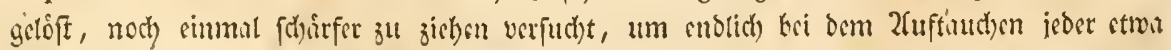

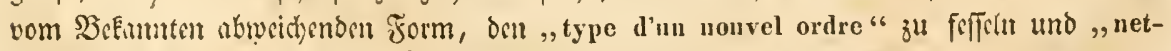

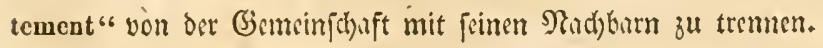

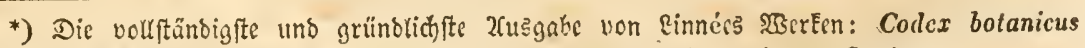
Linncanus cd. H. E. Richter, Lips. 1835. etc. gicbt am beften bavon Funde. 


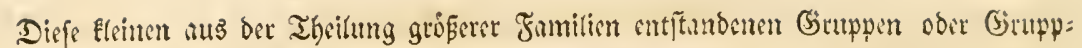

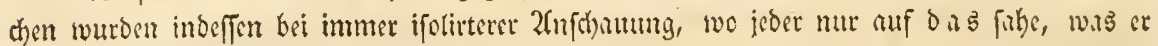

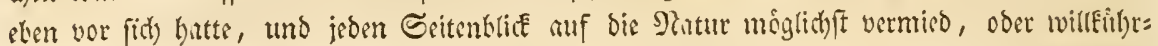
(id) umblifte - fur ifge Eonberung nom 3ufummenhange mit alten tefrumbeten Gemen=

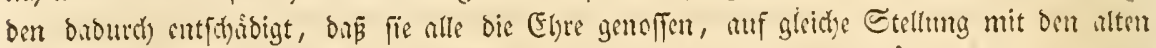
erhoben zu werdon. So baben fich bie bundert Frmilien ber Jufficulifiden Genera plan-

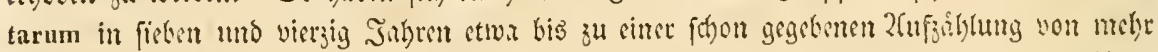

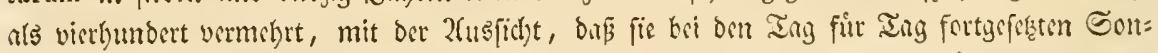

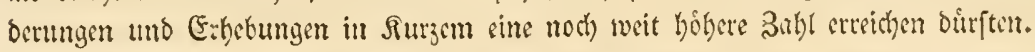

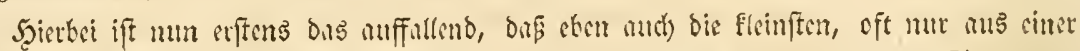

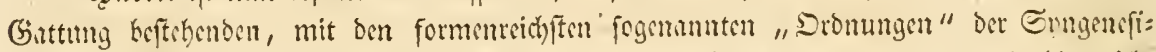

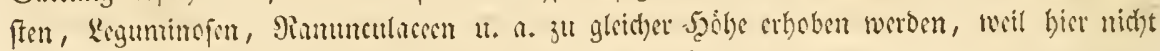

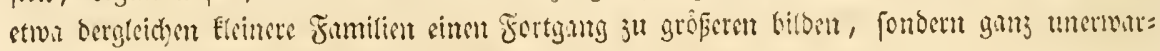
tet ifolirt unter ihnen auftecten.

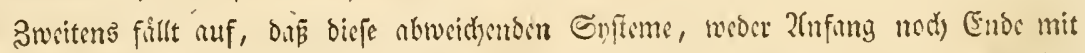

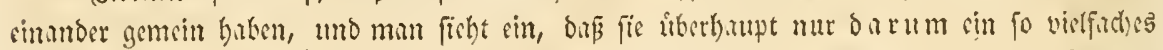

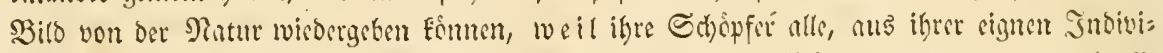

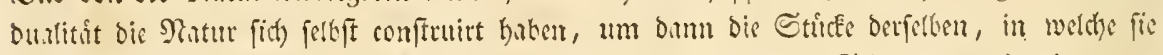

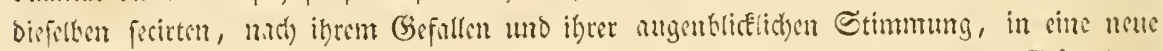

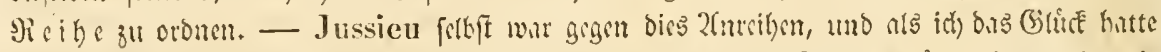

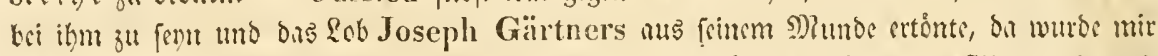

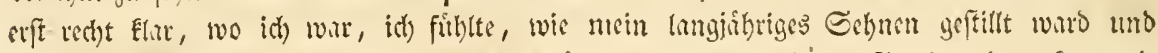

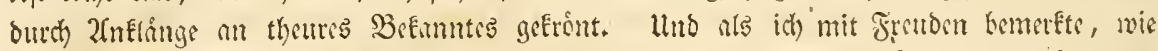

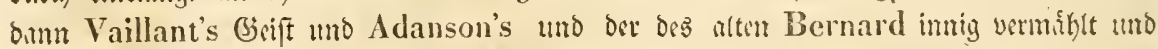

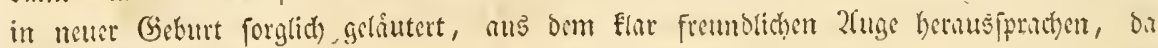

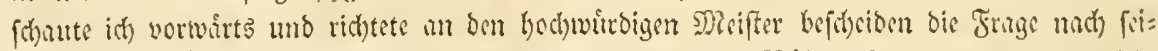

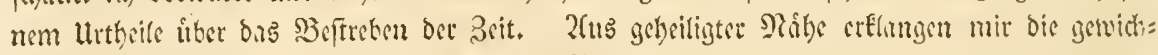

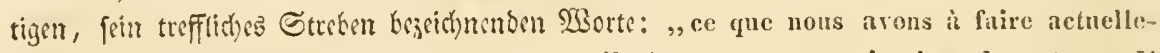
ment, c’est ì déterminer des groupes, car il n'y a pas une serie dans la uature. II faut tonjours $y$ penser que les productions de la nature, ne sont pas senlement en connexion en avant et en derric̀re, mais qu'il-y-a aussi lles rapports it eótè, et yque la carte de géographic est le seul moyen pour comprenlre ces rapports, entre les membres de la nature." "

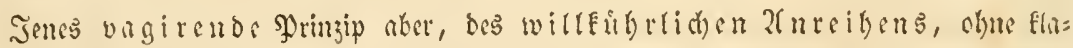

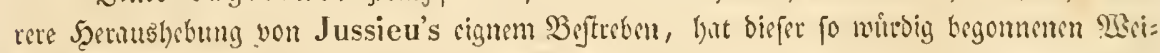
Pe, iffe 23 hrob wicoet entzogen - „series enim, quam dicunt linearem, est scientiale mors" fingt ber trefflidje Rocper.

Darum alfo betrete nut jeder, and) Trianon fermer mit beiligem E(t)uter. -

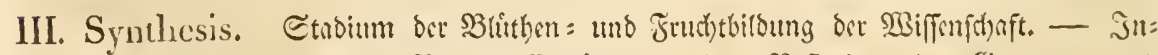

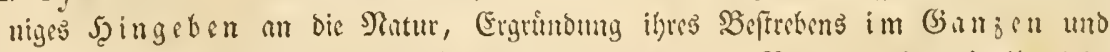

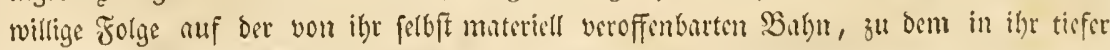

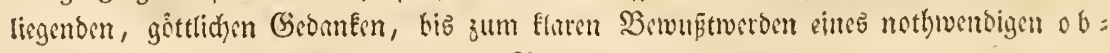
iectiven 3ufammentanges im ganzen, Matutrben. 


\section{(2) cutide 3 otatif.}

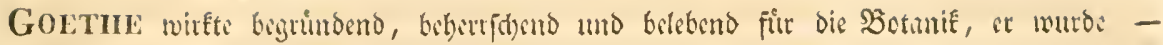
ous fortbiloende gatinzip furdas Doject.

Göthe (our kefiuntlid) unter Bor= uno Mitwiffung von Herder, Kant, Schelling, Schiiler, Wieland u. ?. bis beutfthe Eprad)e und bie beutiche, Elar gene=

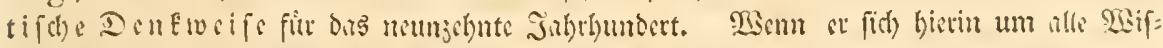

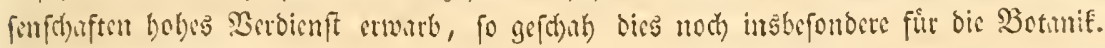

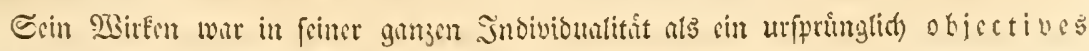

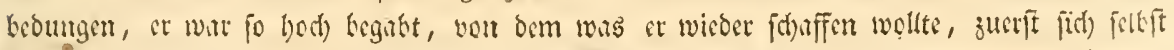

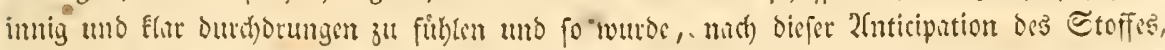

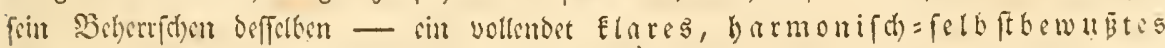

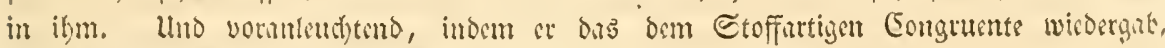

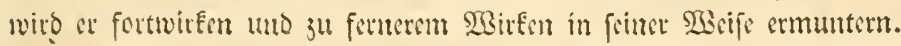

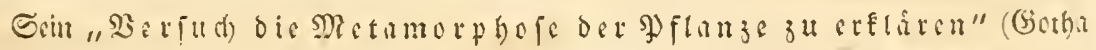

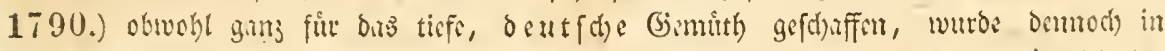

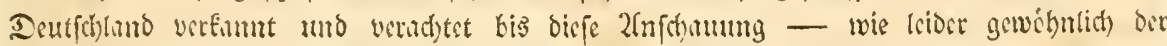

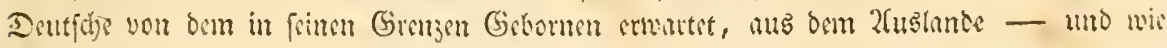

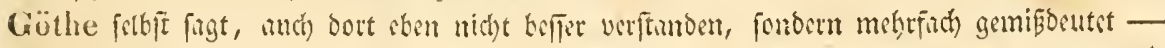

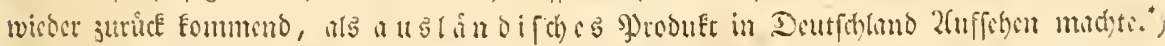

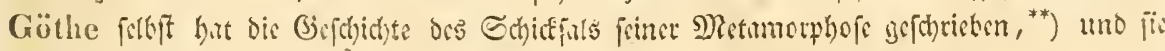

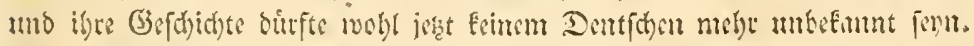

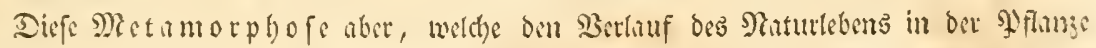

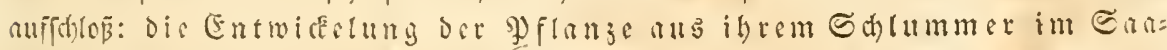

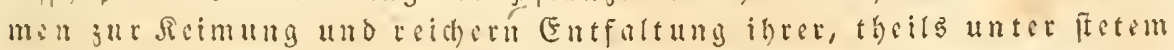

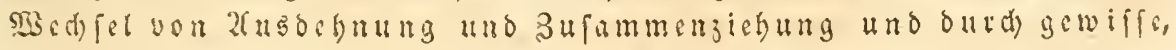

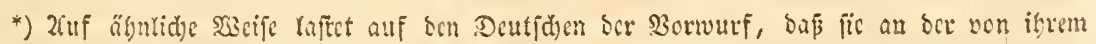

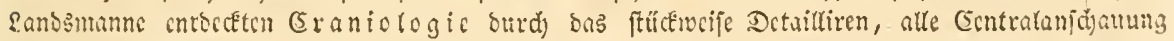

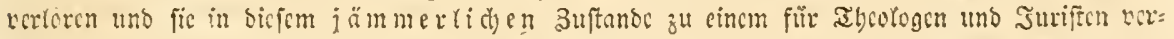

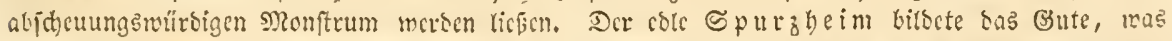

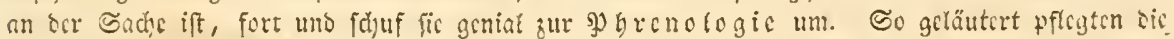

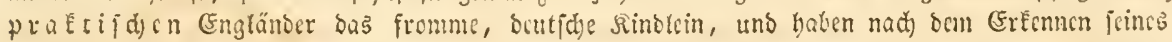

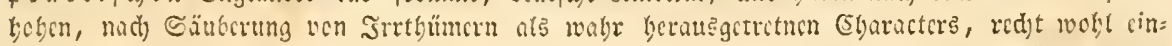

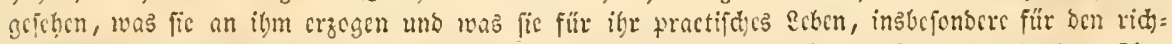

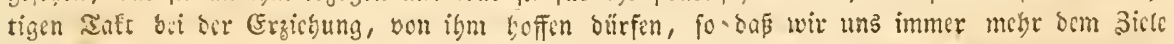

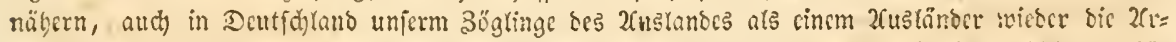

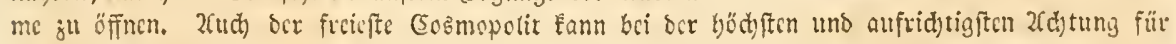

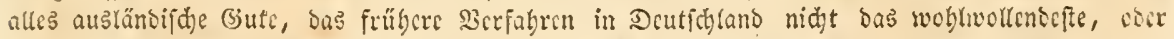

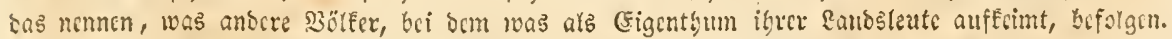

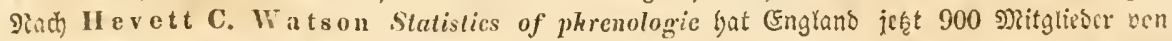

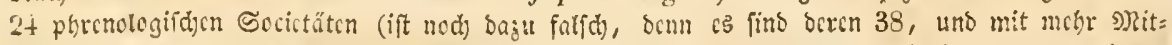

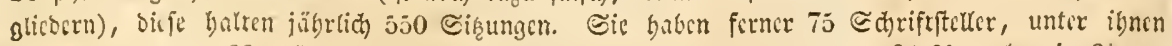

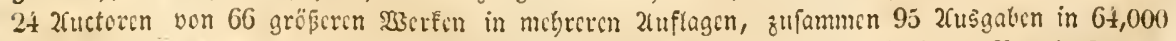

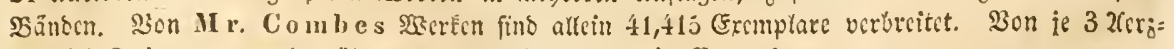
ten find 2, im gangen aber l̈bs 100,000 \$ुgrenologen in Englind.

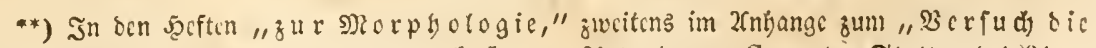
Detamorphofe ber gyfanzen zuertären. Zlügate von Soret. Ctuttgart 1831. 
ourd) Die Rebensfradien beoungenen und fie wieber bebiugenden 2lb=

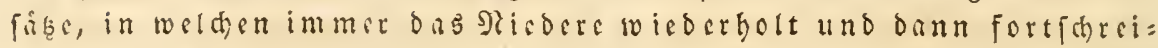
teno umgerandelt, 3 ub herer 2 ebeutungemporfteigt - Diefe nue in rei=

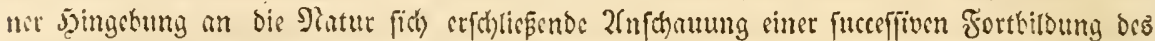

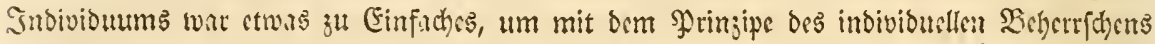
Dit Nirtur, fid) vermillylen zu hiffon.

Da ftellt fid bie Natur felbff vor umfere 2lugen, als geb a rende, dinn als lie:

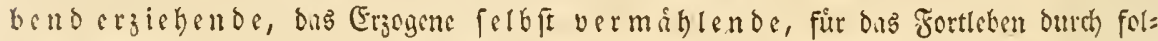
gende Ginerationen cs reifende Mutter. Sa geht alles in einunder unmerflich úker, wie ber Strom bes cigenen Lekens und wie wir im Menjofenleben nicht vermigen oburh

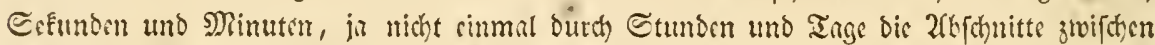

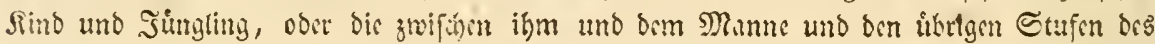

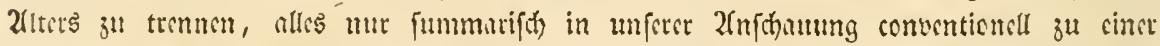

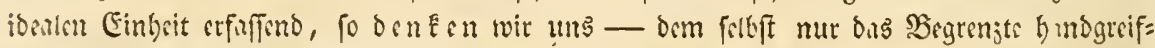

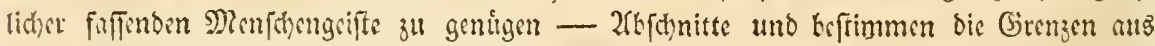

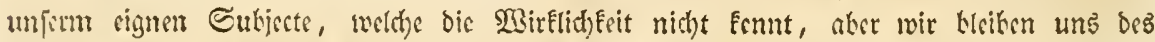

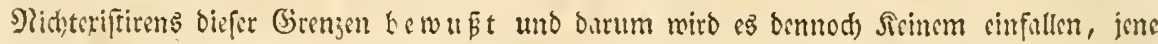

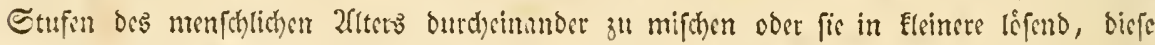

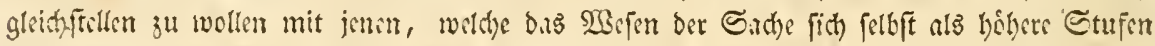
beoungen. Das cine Beifpiel bezcictinat ben $\mathfrak{B}_{\mathrm{ig}}$, wie wir allez, was jum Riben ge= bort, - D. h. altes was gottlich iff - bejhanen uno wie wir bei allem, obwebt

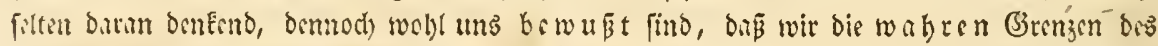

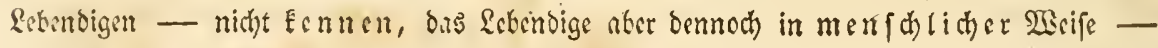
etfaffert.

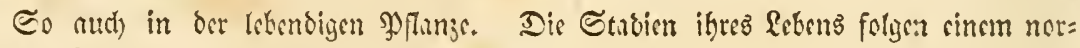

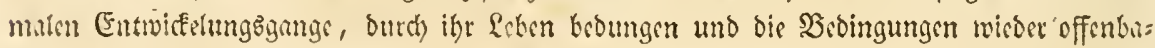

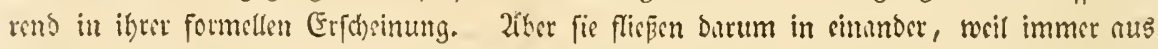

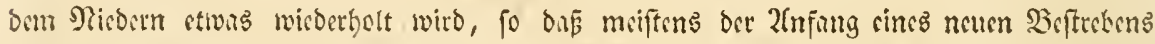
formell niebriger keginnt, als diss Formelle bes voranzgegangenen Befteckens gernoet, und

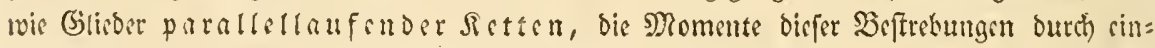

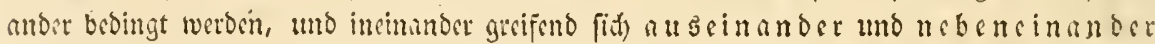
entfiltan, bildich finirbar bicfe Momente, stwa in ben zunclymenoen Grabon bes gengras phifd)en (bilobub. *)

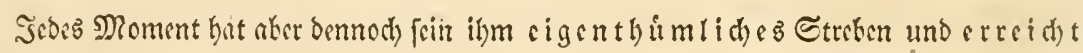

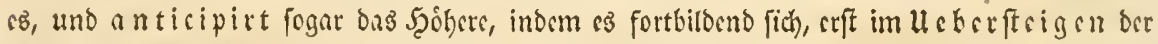

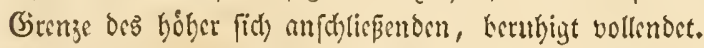

Co dem Naturlaufe treulich folgeno, crennen wir aber, wis bns Matriclle burch)

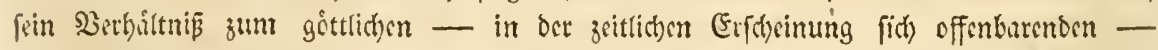

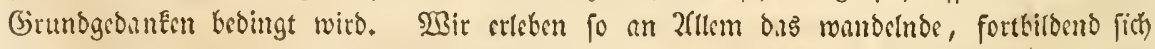

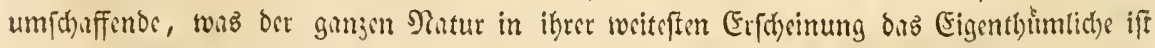

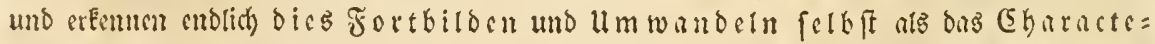

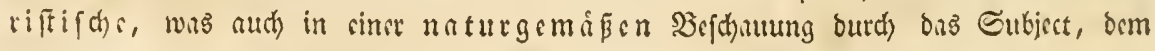

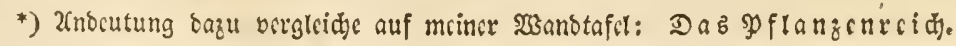




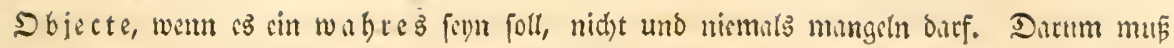
- bas burd) Brobard)tung ju crlangende, es mus die einfache, reine Erfintung, melde bie

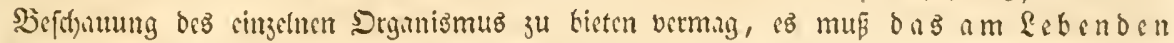
Erlebte in ber 2 nf

Co gehngen wir jul ber 2fufgube: aus ben Momenten, welthe bie 3 eob=

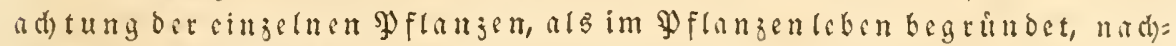
gewiefen bat, eine Befdaunng ber Sfflanjenwelt abjuleiten, in wel= d)er ourd Wiederbolung jener Momente, bie Spflazenwelt als Ein= beiterfdeint, einemigrer am bodf fenorganifiten Jndiviouen, in ih: rer fatfaltung vergleidbar.

Ch, G. Nees von Esenbeck barf, ohne irgent Einem zu nahe zu treten, atร

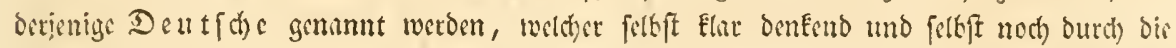

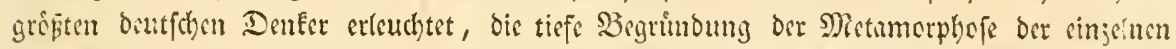

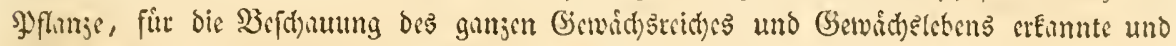
un ticfiten, am ausfübrich)fen analyfitent verfolgte.

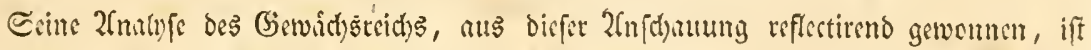

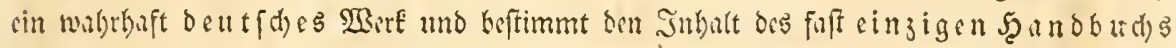
oer BotaniE, wetc)es aus ber beutfdien Eedule sutfprungen.

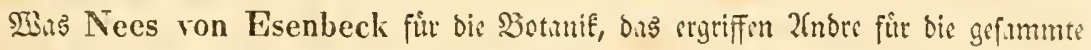

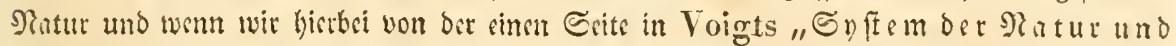

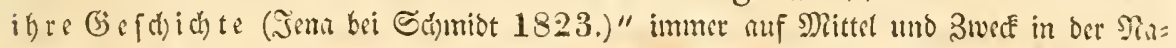
tut Eriftig und praftift) Gindeuten feben, fo tritt uns yon ber andern Geite aus Wilbrandts

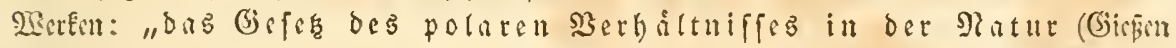
1819.)", "Darfellung ber gefammten Drganifation (Giesen u. Darmfridt

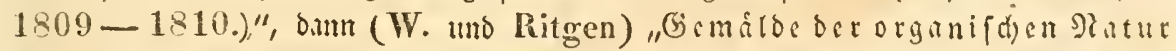

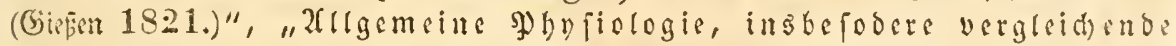

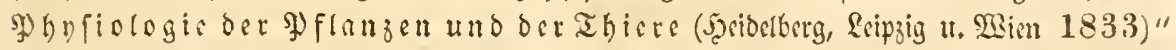

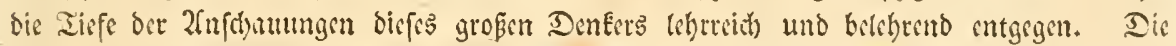

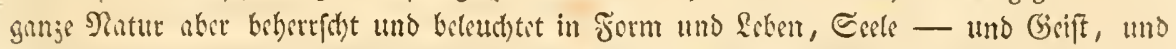

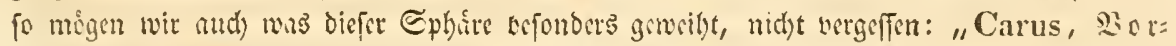

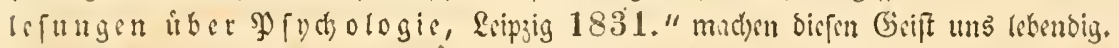

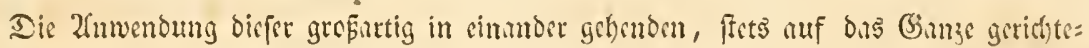

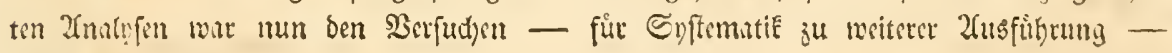
getroten.

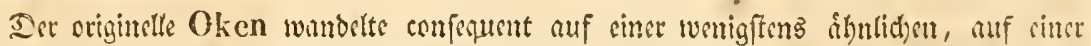

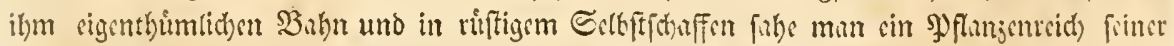

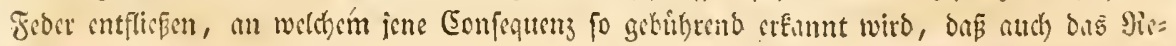

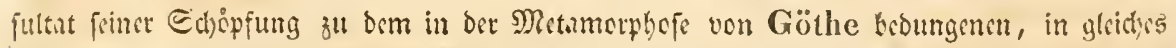

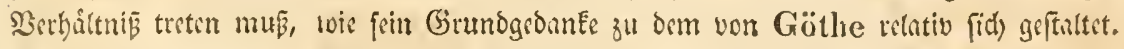

Ihm ferkft find wir aker barum vislfad) verbundon, weil st - als Eyftematifit cin

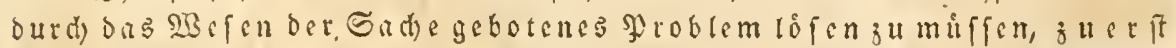

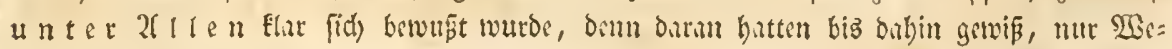
nige noch), ctwa groudst. 


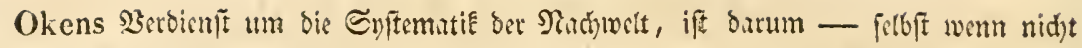

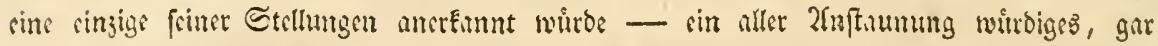
nidit wirtlich crmç̄ures.

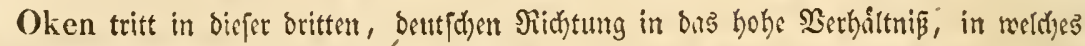

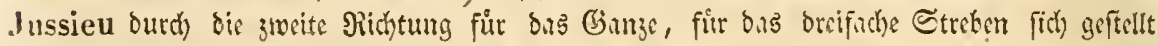

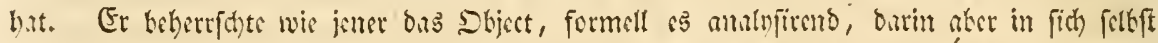

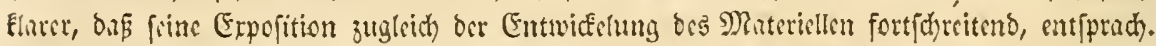

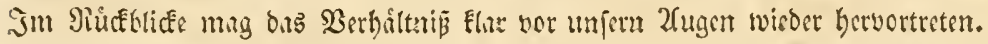

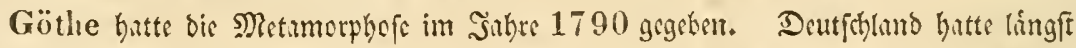

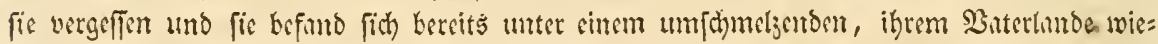
oer Sinterelie erwedfendon 2lete im 2lushande.

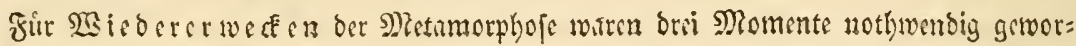
bill, wir erfimmen fie als:

a) thesis, Nees von Esenbeck wutroe der zweite Bater und Ergicher oer Mcti:=

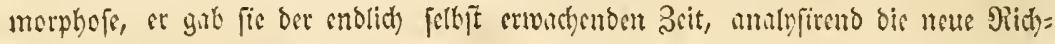
tung begrundend;

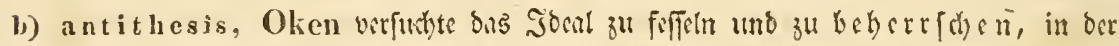

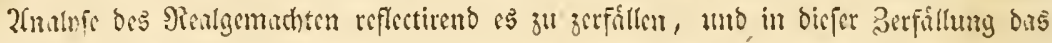

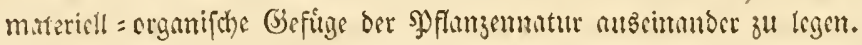

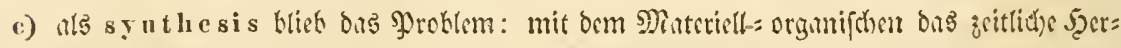

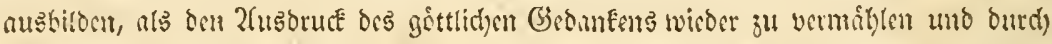

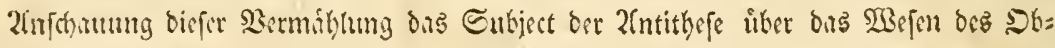
jects jut berathigen.

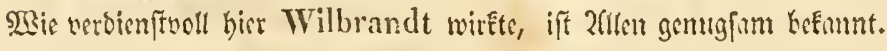

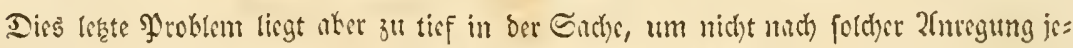

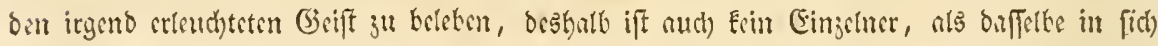

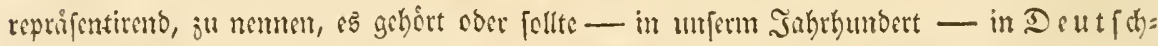
I a no uscrigftens, fur \&lle geboren.

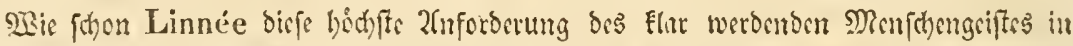

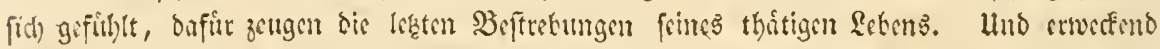
und anregend bis in bie ppiteften Beiten, werben feime, praelectiones", foine, prolepsis" -, , metamorplosis", frime, fragmenta methodi naturalis",

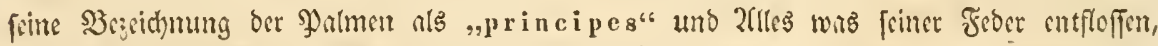
burd) Fitmftige Gisnerationen fortleten uno fortzeragen.

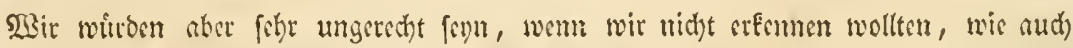

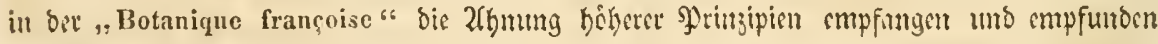
worben ifit.

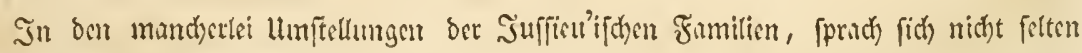

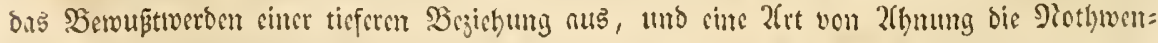

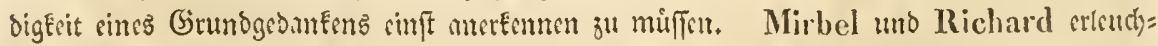

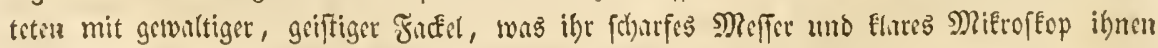

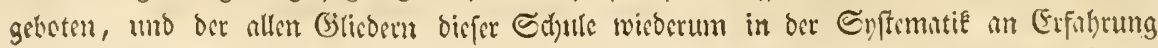

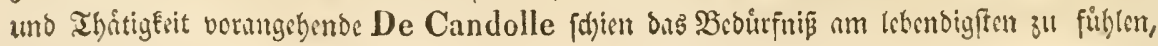




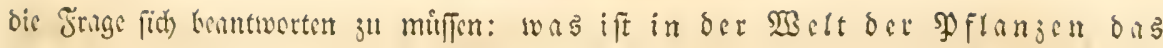
Slicocre tub was das 5ुoberezunenuen?

Er glankte dicie Pecrnsfruge, fribere 2lufithaungen von Desfontaines, Aubert du

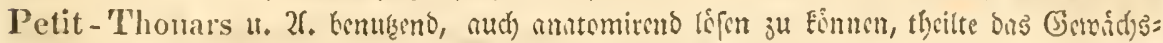
reich) in végrétax Vaseulaires ou cotylélonés, bicfe wirder in exogènes ou dicolyllédonés unt endogènes on monocotylélones, wifhrend bis jucite 2(ththeilung, bie végétaux cellnlaires on acotylédonés enthictt. In ber 2 Beife ber 26 fonderung alfo von Jussieu nicht;

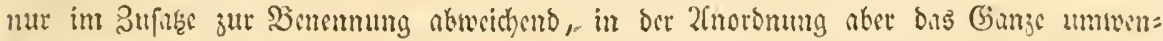
bano, von: Jussieu's Scerankildung zum Bollfommmen, fid) umEchreno, zum Linvollemm:

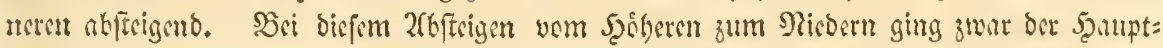

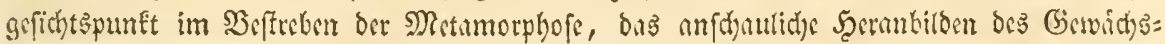

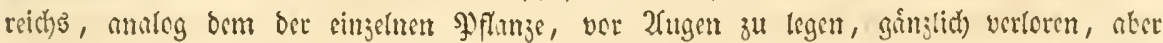

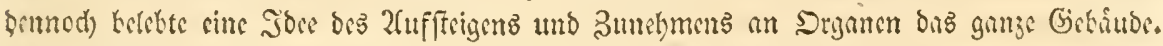

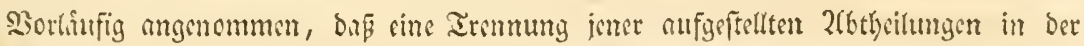

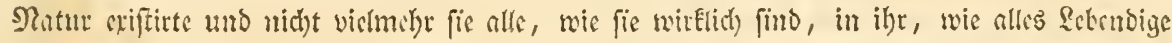

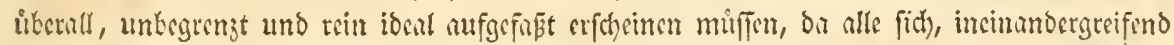

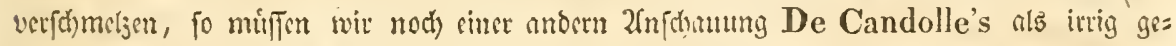

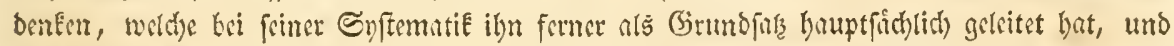

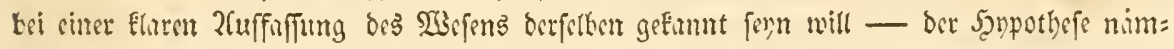
(ich), dus die abfolute 3 ermebrung gleid)namiger Drgane, bic bobere Srguni= fition kebinge.

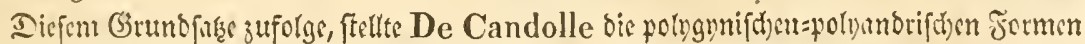

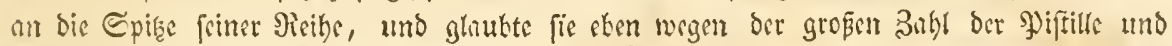
Gtuthgefíp zu biefer Etelle beredtigt.

23ir feben aus bicfer Stellung, bns ber ticf in Der Natur alles lebendigen liegende,

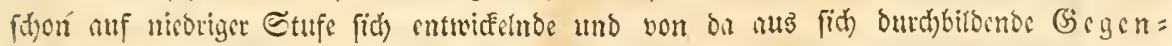

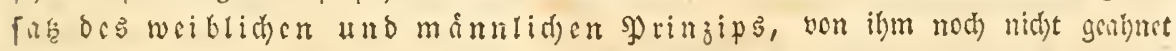
worbent ift.

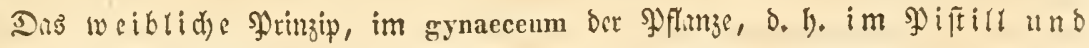

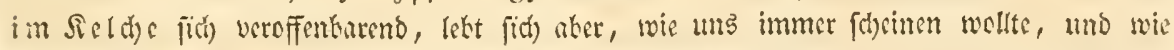

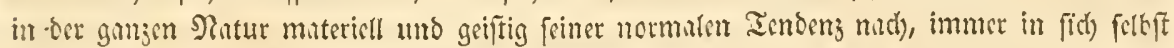

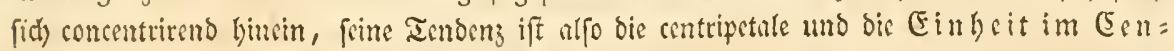

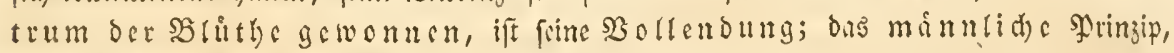

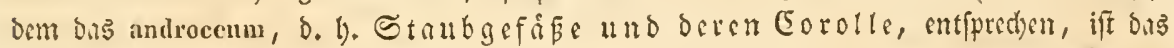

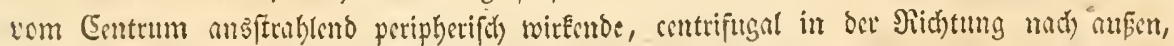

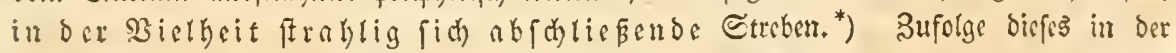

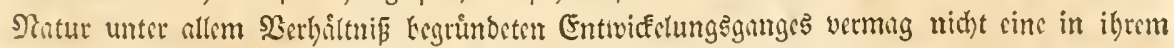

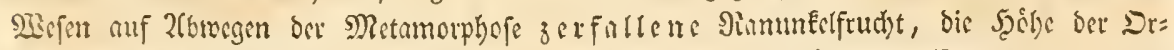
ganifation kedingen, fondern cine Frudt), weld e in oer bod) ften (5oncentration

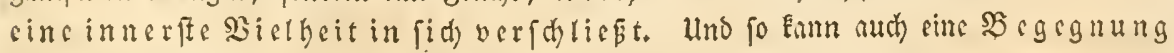

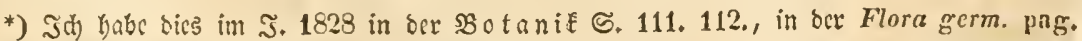

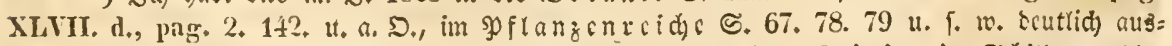

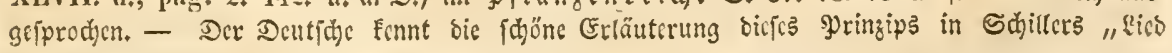
von bor Brocke." - 


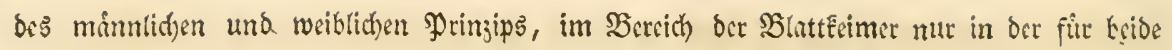

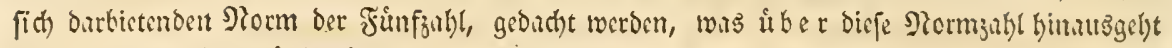

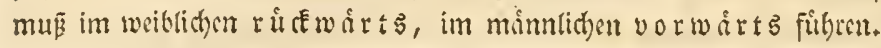

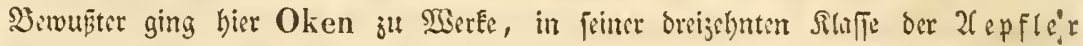

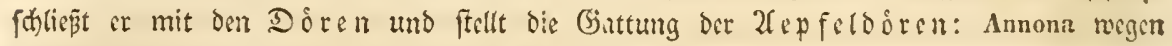

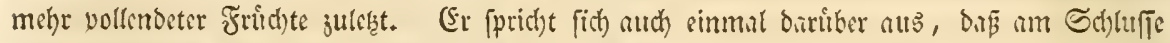

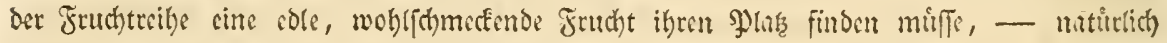
eine freie Frucht, denn ofhe morphologifhe Motivinung wire dats Prinjip bes 2 Bohtge=

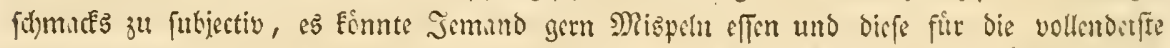

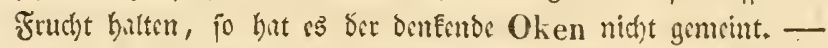

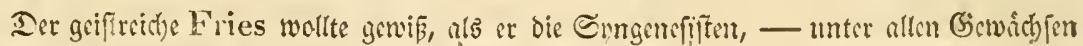

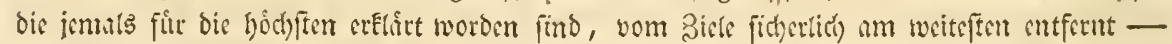
ju unverbienter Şobe erhob - feine Sefer nur priffen. -

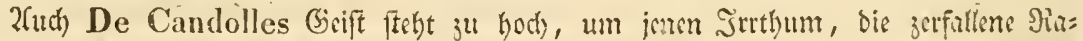

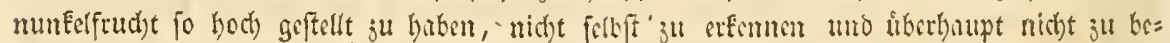

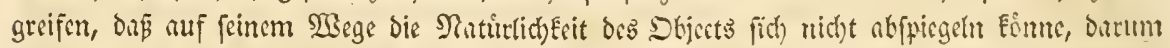

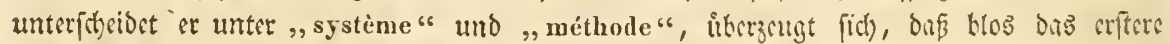

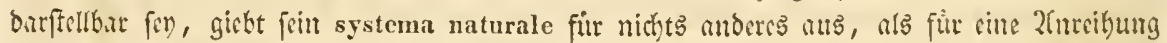

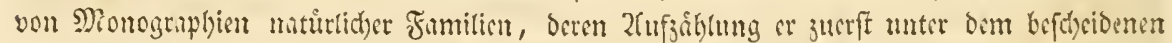
Titel barkot: Esquisse d'une série linciaire et par conséquent artificielle, pour la disposition des familles naturelles du règne régètal. "

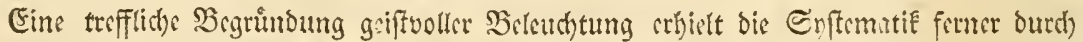

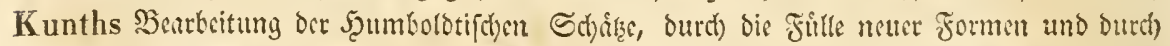

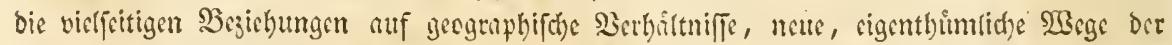

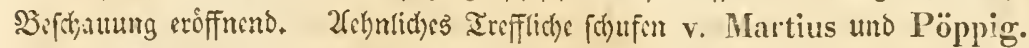

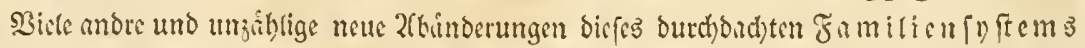

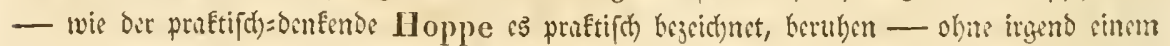
beftimmten Prinjipe zu folgen, ober 2fnfang und Ende rutiondl zu bedingen, obwobl bier

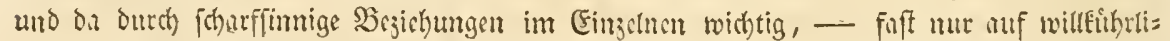

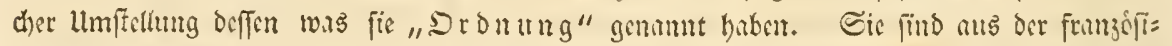

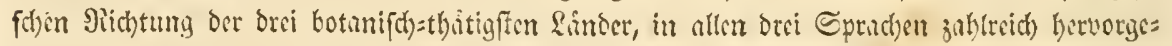

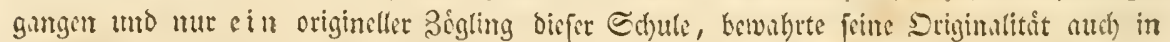

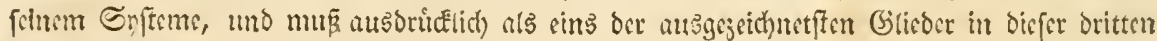
Sitthtung genumt metben.

Raspail war butch cigntes Brobadten zur (Erfabrung und 2(nit)auung mand)er Dif=

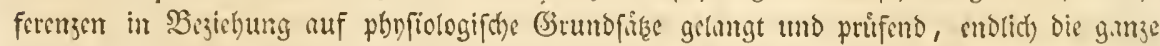

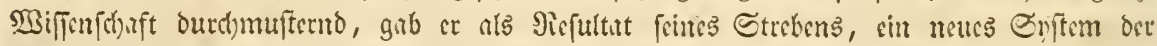

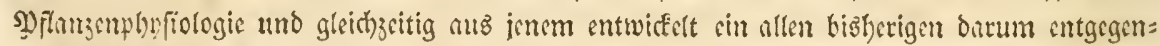

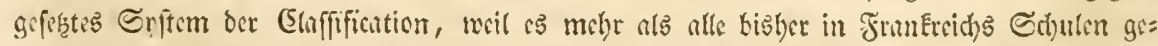

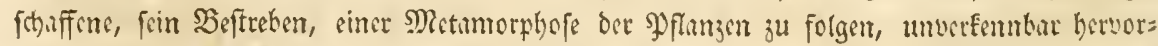

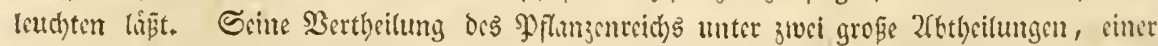

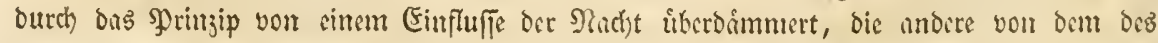
Tinges kefenchtet, ,Plautes nocturnes et plantes diurnes" ift irocm, wer begabt uno ge=

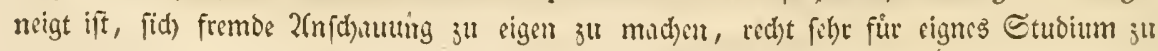




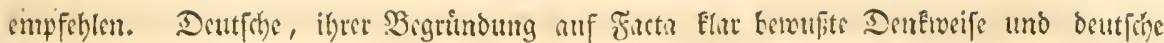

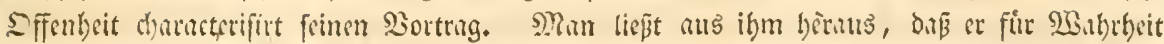

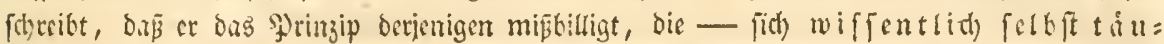
(d) en.

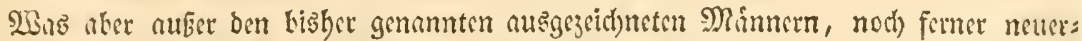
(ic) unter unfern bentfchen uno norbifchen Randzleuten: Agardh, Ahlquist, Alschinger, Ascherson, Bartling, Bernhardi, Betke, Biasoletto, Bischofl, Blıfr, Blume, v.Bönninghausen, Alex. Braun, v. Bridel, Bruch, Burneister, Bukhard, Chamisso, Corda, Cruse, Detharding, Dierbach, Dietrich, Döring, Dobcl, Ehrenberg, Fisengrein, Eklon, Endlicher, Engehmann, Eschweiler, Eysenhardt, Fenzl, Ficinus, Fingerhut, Fleischer, Fresenius, Fries, Fritzsche, Fürnrohr, Gärtner, Göppert, Grabovsky, Griesselich, Grisebach, Guthnik, Haberle, v. Hall, Harkart, Hayne, Hegetschweiler, Ilerbich, Heynhold, Gr. v. Hoffmannsegre, Holl, Hoppe, Hornemann, Hornschuch, Hornung, Hübener, Jäger, Kasthofer, Kaulfuss, Kirsclileger, Kittel, Klotzsch, Koch, Kölbing, Kosteletzky, Kunth, Kunze, Kützing, Lachmann, Lasch, Lehmann, Liṇk, Mann, Clamor Marquart, v. Martens, r. Martius, Mauke, Mautz, Mayrhofer, Meisner, Mertens, Meyen, C. A. Meyer, E. Meyer, W. Meyer, Mohl, F. Nees v. Esenbeck, Nolte, Opatowski, Opitz, Petermann, Pfeiffer, Pieper, Pöppig, Presl, Radius, Rabenhorst, Reinwardt, C. V. Reichel, Reum, Richter, Römer, Röper, Rohrer, Rostkovius, Roth, J.C. L. Rudolphi, Ruprecht, Ruthe, Jos. Fïrst von Salm Reifferscheid-Dyck, Sauter, Schabel, Schauer, v. Schlechtendal, Schimper, E. L. W. Schmidt, J. A. F. Schmidt, G. L.E. Schmidt, J. C. Schmidt, Schott, Schönheit, Schrader, C. Schubert, G. II. v. Schubert, Szubert, C. J. u. a. Schultz, Schübler, Schwägri-chen, v. Siebold, Spenner, Sprengel, Steudel, Succow, v. Suhr, 'Tausch, 'Treviranus, Unger, v. Vest, y. Vriese, Waitz, Wallroth, Weinmann, Welwitsch, Wenderoth, Wiegmann, Wimmer, Zencker, Zimmermann uno no () gubleriche '?fnocre, unter benen wir nber mit befonderer und imnigfter Domfbarkeit zu Kaspar v. Sternberg uno J. F. v. Jacquin uns bintoenden, diefer deut fben,

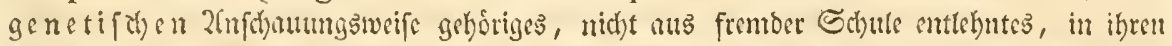

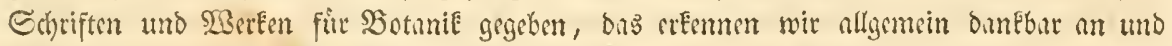

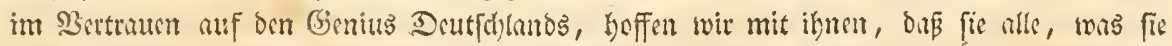

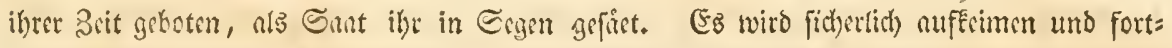
biloen unto wisoer fortgengen, in ber won Göthe fo flur bezeichneten Mitchtung.

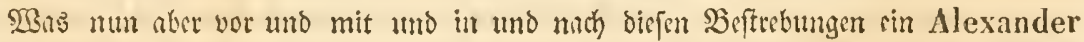

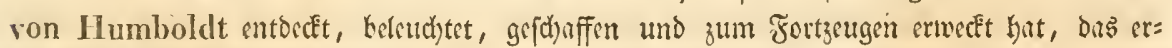

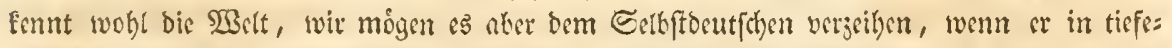
rem Gefuhle ber - Natur biefes Naments, im Fortbilden feiner cigenen 2lhnungen, jenç

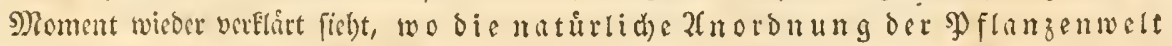

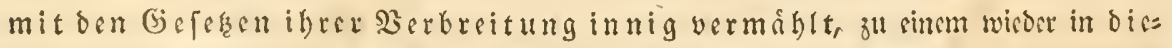

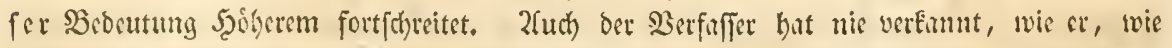

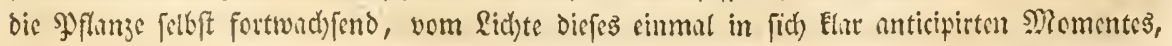
innig angegogen, eigentlid) nur ifm feine geringen Srifte gerwionet. $)$

*) Bergh. Botanil 1828. S. 364. 


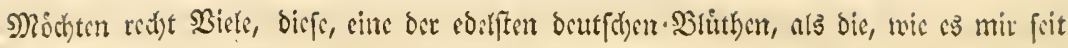

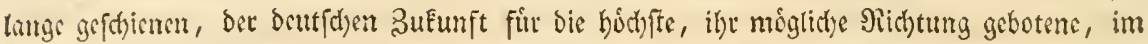

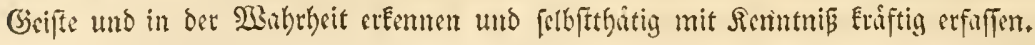

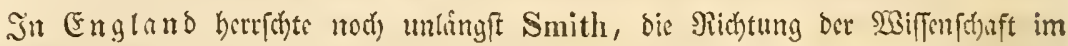

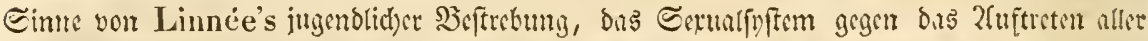

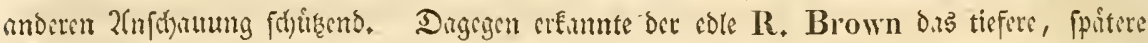

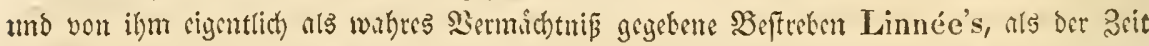
in ber wir leben, zum Fortbitden gethoten; er tithernafd)te Europa im Jahr 1810 burds

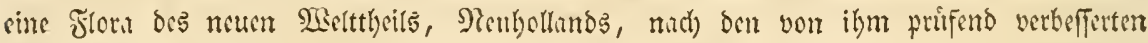

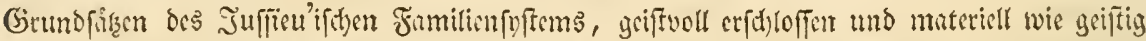

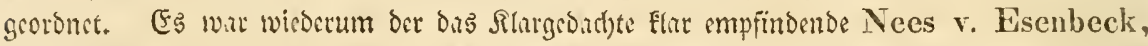

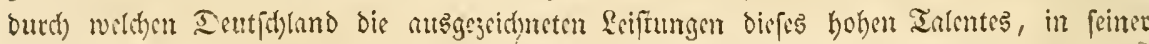

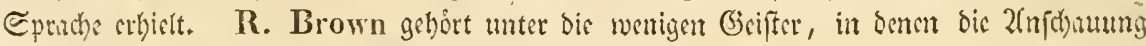
Ses Shjectiven fich in foltner Eongrtenj reflectirt.

Bentham, Don, Hooker, Walker-Arnot uno 2tnoers, thitig uno mit frumb=

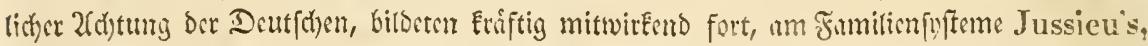

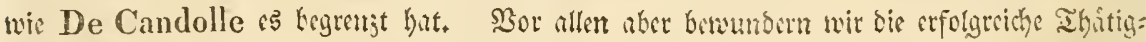

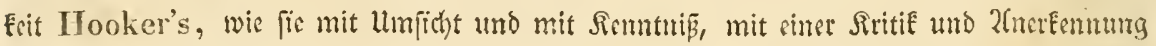

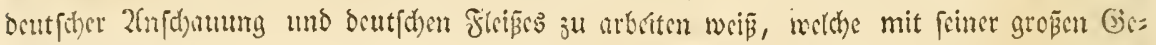

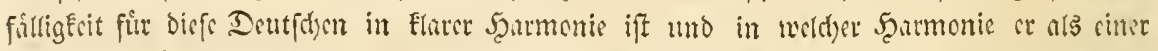

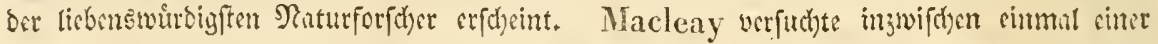

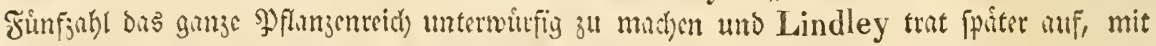

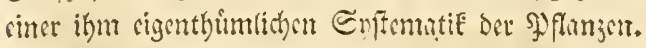

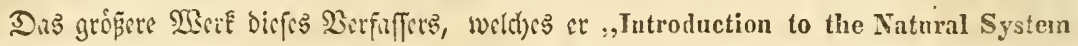
of Botany" genumt but, entruiffelt in foiner eigentlicher Einleitung bie 2lufgute ber ma=

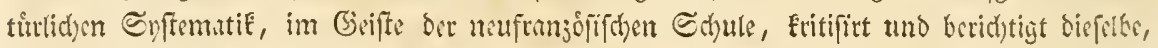

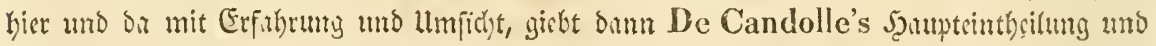

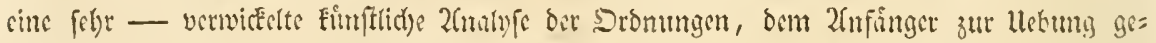

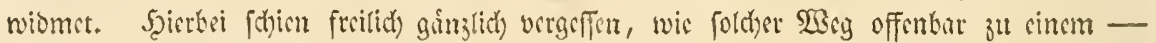

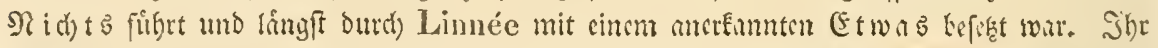

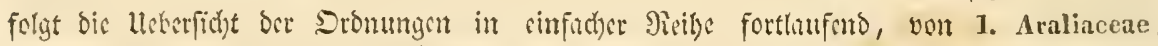
bis 252. Algae. Fin die cinjeften Fumitien findet fid) eine braudbute 3ufammenfteltung

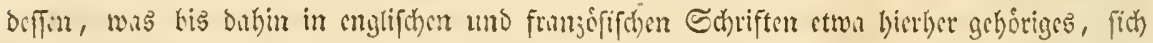

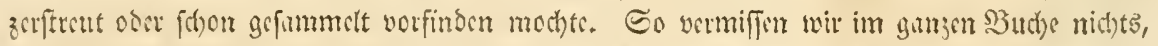

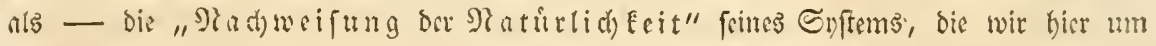

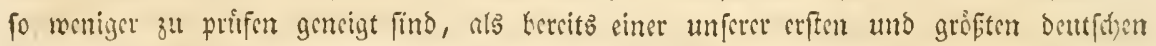

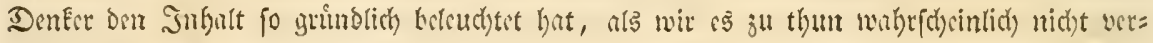
mocht haben wutoen. (23ergl. Wilbrandt in den Situnturblittern zur Flora od, allgem. bot. Zcitung. 1837. Scite 33. 49. 65. 81.) -

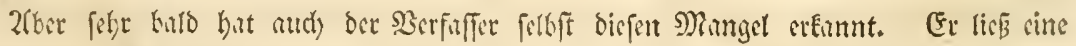

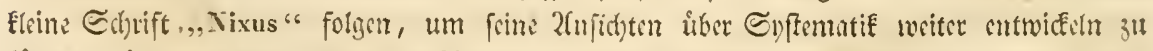

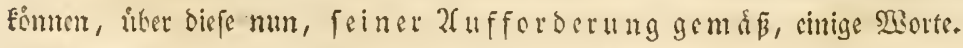

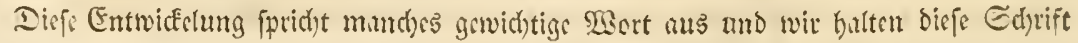

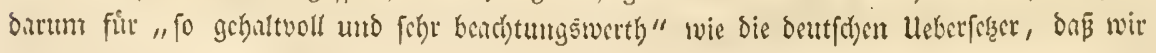




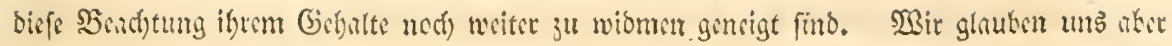

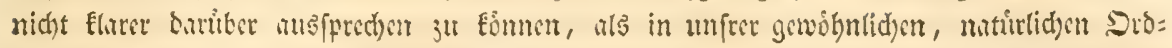
mung, moge alfo and bier, bisfe crhutht forn.

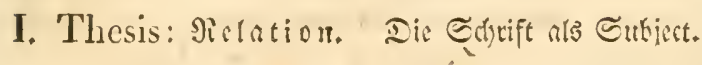

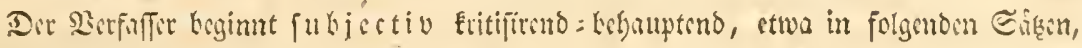
treulict) in folgenter Sromung.

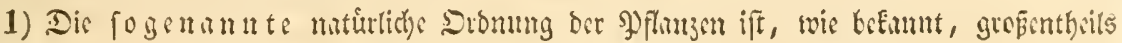

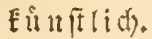

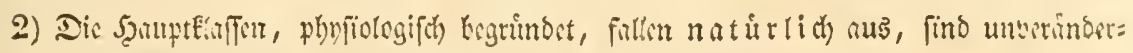
licte Grundhagn des Snjtrms.

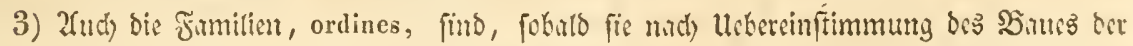

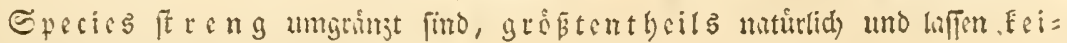
men wefentlid)en Tabel jut.

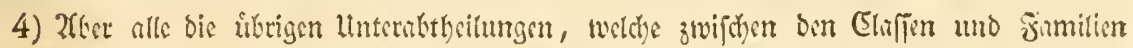

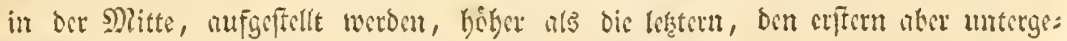

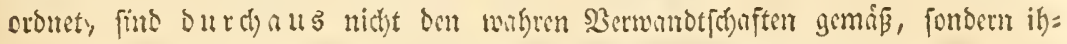

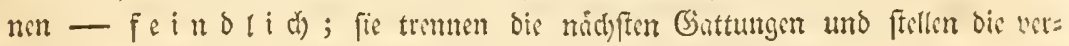

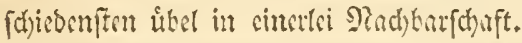

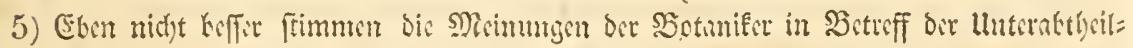
ungen ber Sinfen mit cinntor ubercin.

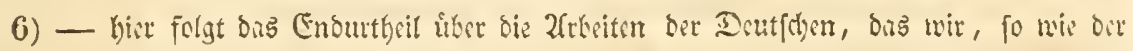

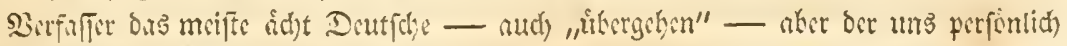

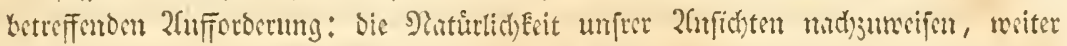
unten gern und willig ju entfpect)en, mach) Siriften bereit find.

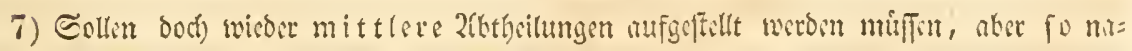
titulich, wis bic obirften und unterftert.

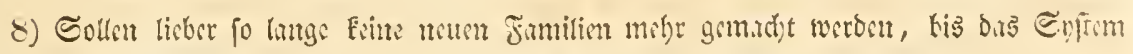

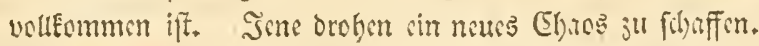

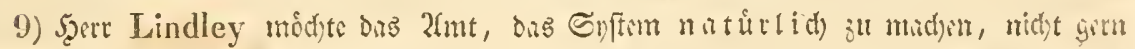

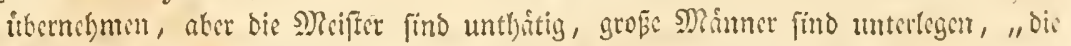

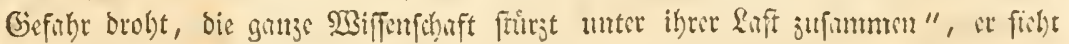

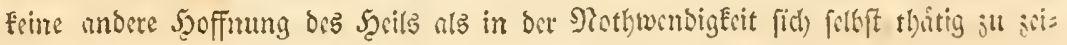
gen: in Serwerfung aller fenftidyen Theile bes Snfems uns in Erfegung derfelben burch eine neus, wirflich matirlide äer: theifutg der Framilien.

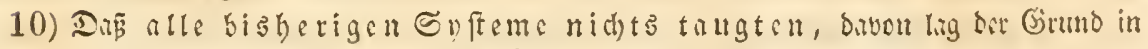

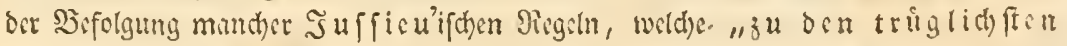
ge boren."

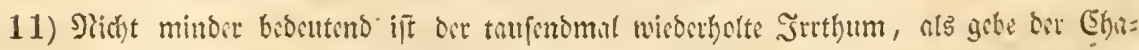

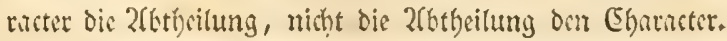

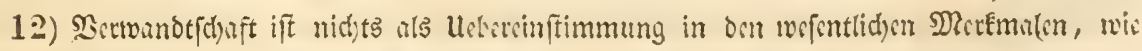
2fnalogie busjonige in ban jufilligen. 


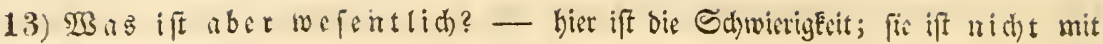

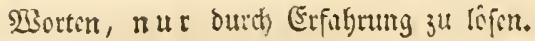

14) Şerr Lindley ftimmt nicht ber Sehre beree bei, welde meinen, bie Geffaltung ber Eharactere fei a priori zu beftimmen, und weldhe fagen, ber Grato ber $23 i d$ tigkert fen bem Gride der Entwidelung gleid).

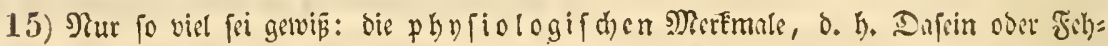

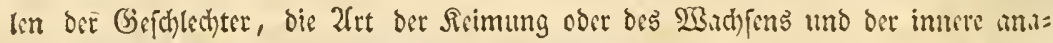
tomiftae Siul des Stammes durf Esinem andern nachgeftellt werben.

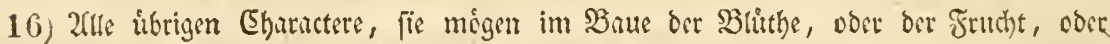

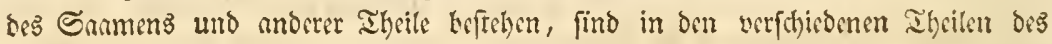

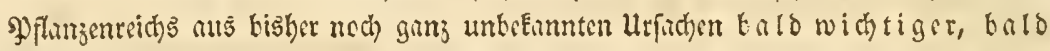
minder bedeutend.

17) Dis meiften 2futoren glatben, ss fri Esine 2tbtheilung von gewifjen und feften Grengen umpthioffen, nur die Spesies babe feft begrenjte Ebarnetere, - aber Erum mit Piect)t.

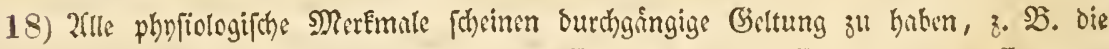

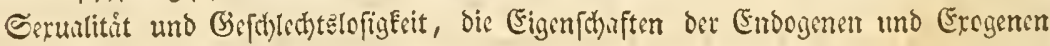

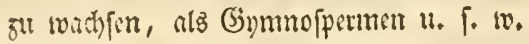

19) शiur bie vom Sauke bergenommenen Ebaractere find ungleid) ftundbaft, fie britcen แแน Ḃıftrebungen ณแรี: Nixus.

20) Soldye Éonnen Ésine Definitionen, nur Diagnofen erhalten.

21) Die fogeminnten (Ebaractere biefer Nixus beftefen in roeiter nicfts, als in 2frocut= ungen ber vorberrfdenden Formen, welde namlich in ben Iypen gefunden werden,

22) Sic find reidi an 2(usnabmen, weldye aber: ad characterem non ad affinitatem pertinent. $\boldsymbol{\Lambda} \mathrm{g}$.

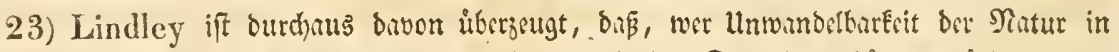

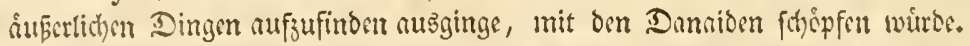

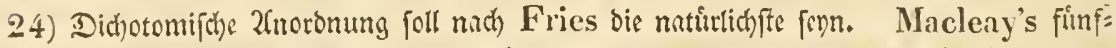
ziflige leuchtete weniger cin, jufállig wurde boch auch in feiner 2fuföblung ber Nixus, mandes finfzichlig.

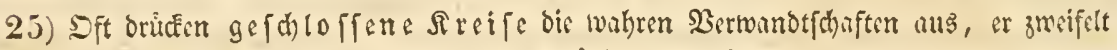

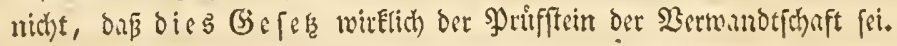

Die neue, "naturliche" Eintheilung folgt jefgt folbft.

A. Sexuales. a. vasculares. 1. Exogenae angiospernac, II. Lxog. gymnospermae (nimlich Cycadeae, Coniferac, Taxinae, Equisetaceac. Wits twir atwa baruker DenEen, folgt woiter unten.) III. Endogeneae. - b. evasculares, IV. Rhizantheae. B. Lisexuales, - Eż ghtht weiter:

1. Exogenac. subclas, completac. 1. pol ypetalae. 2. incompletac. 3. monopetal a e.

Dis fermere Finthcilung in Eohorten, Tixus und Familten entwicfilt nun dic Stell=

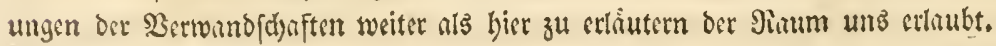




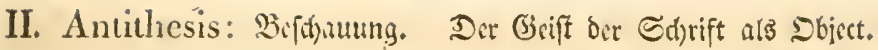

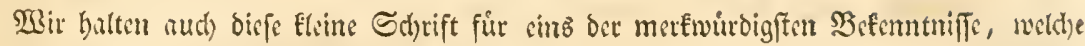
jimals in der Siteratur der BotuniE erîchienen find und wiometen beşullb bem Snhalte ihrer Einlitumg, fo viclin Siuum, als wit gethan.

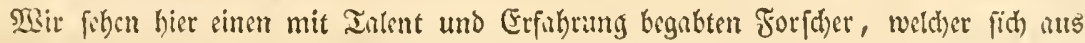

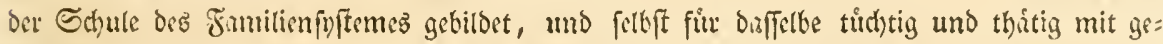

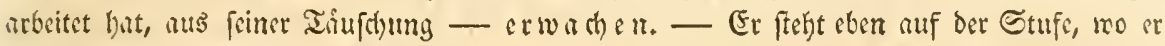
fieft, dar 2llks was, wir Göthe fagte, die Grumzofen pofitio machen, nicht pofi= tiv ift!

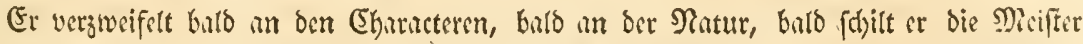

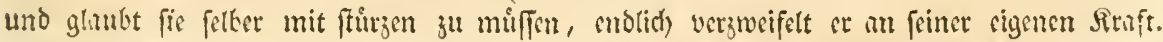

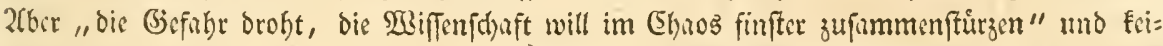

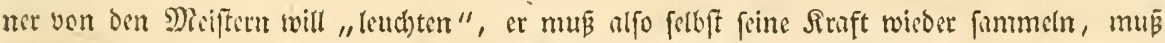

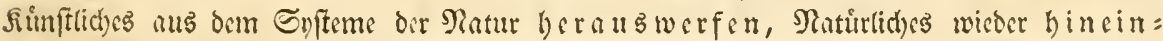
fef̧en, und wir fómen das lobenswerthe Seftreben - wenn audi) uninder oeffen Metive uno Sicjultite - nur chrem.

Cisft mun nimtich nach fold)en $\mathfrak{B o r g i n g e n ~ b a z ~ R e f u l t u t ~ a n , ~ f o ~ f i n d e t ~ m a n ~ - ~ c i n e n ~}$ nur fefr wenig abyecinderten Decandolle, man findet 3wifdenftufen, die erft feind:

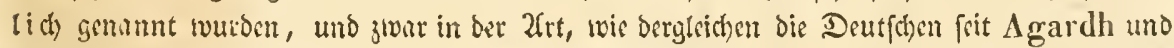
Fries und mir felbft uno dunn Bartling, lange fhon als Эiettungsmittel aus ber Unoromung der ", ordines" singefügtt bubur.

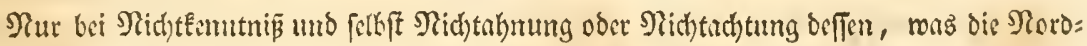
linter und Deutfhen getfin und ferner erftreben, Eunn man feine eigne Steltung in ber

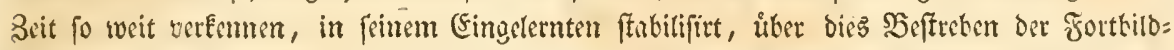

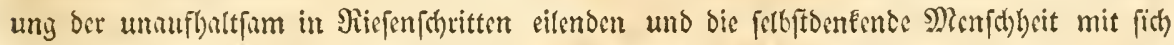
nehmenden Beit fo ju urthrilen, wie cine in Briffel georuckte, fo eben beute angelangte in Introuluction ì l'étude de la Botanique " Gierviber fid) susfpridyt: „, Je ne sais si la science a beaucoup gagné à ce travail de décomposition et de récomposition qui res-

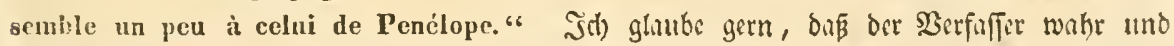

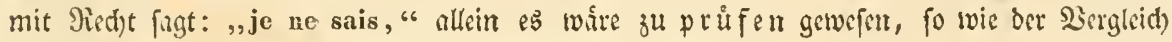

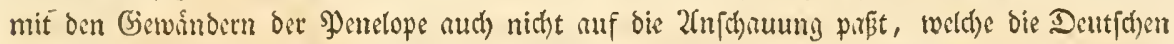

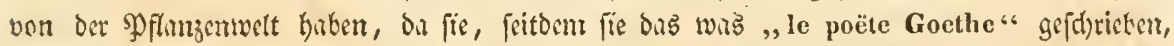

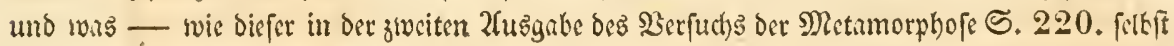
fangt - wieder etwas anderes war, als was De Candolle, wie auch oeffen Enftematif ges nủgend berweift, unter Metamorphoje gemeint Gat, beffer verftanden, fich eintilden, dus nuch) biefe Ş) lanzentwelt etwas Rebendigez pei.

2Ber aber feimen Stoff fo flar beberticht, wie ber $\mathfrak{B e r f a f f e r ~ j e n e r ~ , ~ I n t r o d u c t i o n ~ " ~ u n b ~}$ diffen in fo flarem Etyle zuruefégiebt, ber burf auch nid)t zweifeln, von ocn Deutfdicn nod)

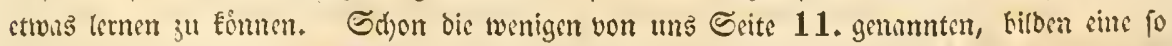
chrnulubige Nhacht, um ju verdienen, dar man unterfuche ob an ifrem Streben (5t twas

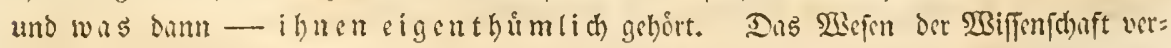

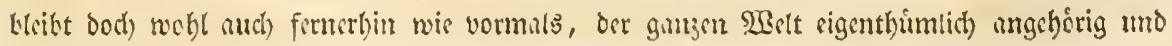
wo deffen (jilicder zerftreut find, fuche mim fie unpartbsiifd zufummen. 


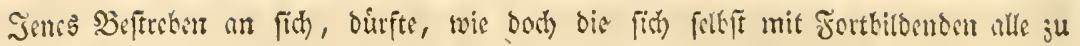

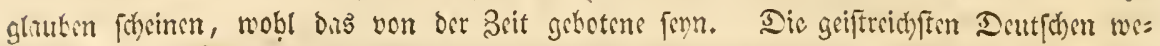

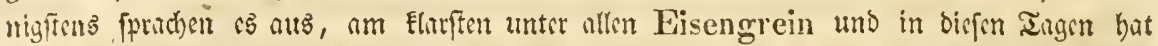

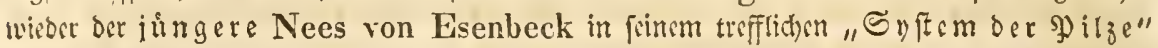
birs gethan und - wabrfbcintich - weif ar, was Agardh, Fries und Bartling uno Oken uno mine signen Eleimen Sethriften, bejonbers bic Flora germanica, feit zman=

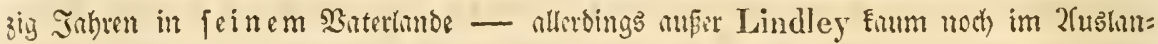

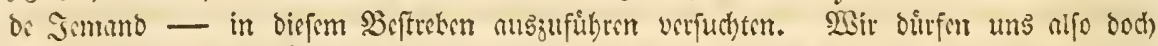

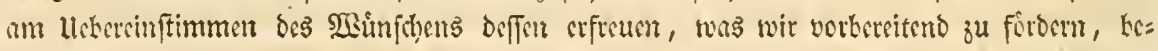
trits uns bemíhten.

Daß mut aber Lindley auf ben wir zurieffommen megen, ungeachtet feiner Spri=

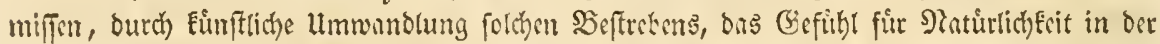

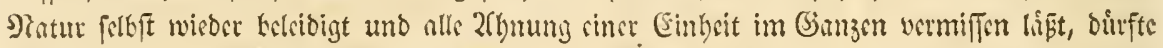
Eum fur jemmo twiberlegear erfdycimen.

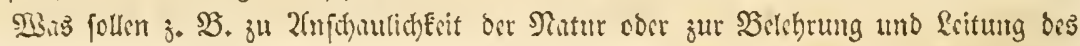
nuffuchenden Refers unter oen, ,racemosac" bis Bruniaceac thut? - Mis follen bie

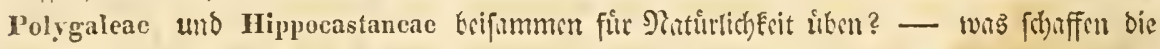

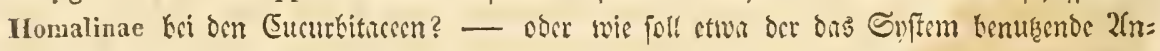
finger burd) bon ,, embryo circa albumen farinaceum arcenatus" bic Ginttungen Diantỉus

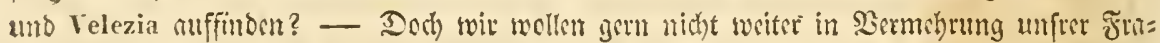

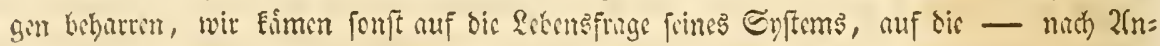
fing unt Ende.

\section{Sỵnthesis, SEcrinigung. SPridifatincs.}

„Car les sarans d'aujourd'hui ne sont jamais alversaires, “6

IR aspail.

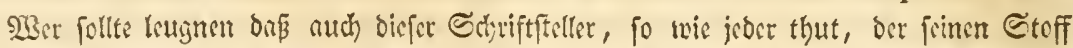

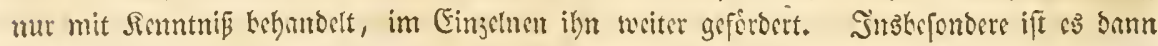

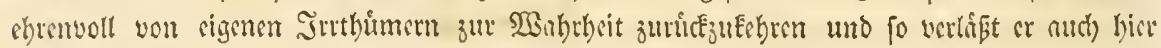

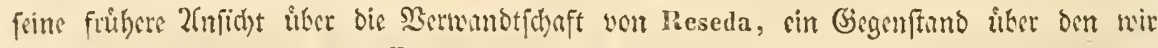
uns in ber signogenefe oer Cruciferen - wic wir glinken - weiter burchgreifend aus:

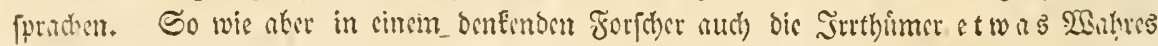

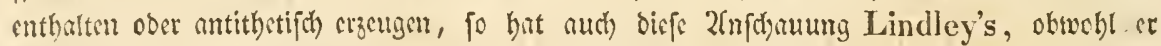

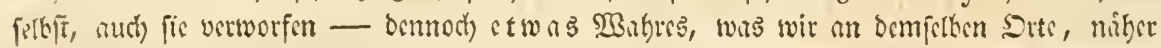

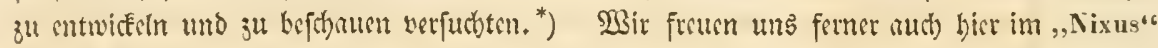

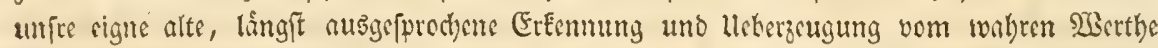
uno ber burd) ifh bedungenen Etslung ber Menifpermesit uno Euphorkincesn wisder auf=

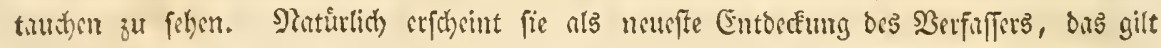

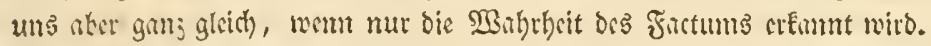

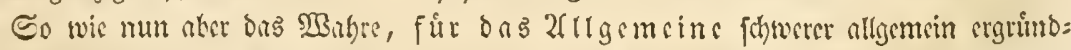

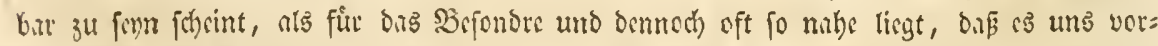

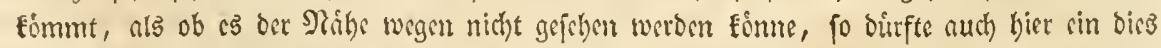
beftítigendes $\mathfrak{B}$ eifpicl uns in Lindley fict) Darkietent.

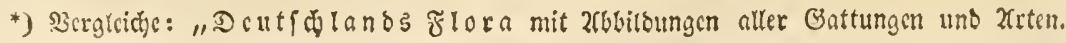
\&cipzig bei Socfmcifter 1837," 


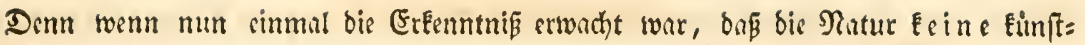
liden Fefleln trígt unb zu trugen verming, fo war es bod wobl nethwendig burnuch zu fra:

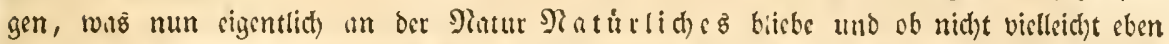
diefer 3 uffand feltif, welder keine Feffel vertrigt, bas Naturliche fei uno ob

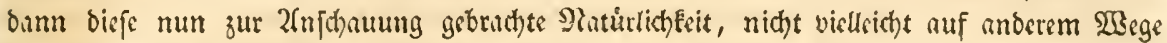
als auf bem in aller $\mathfrak{B e l t}$ bisher vergeblidh verfuchten - erén forme? -

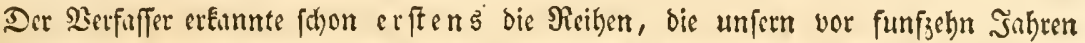
beftimmt und nod) baju mit 2(ngabe ifers \$primjips ausgefprodbenen, bumn mefrfach wie: ber auggefitiften "Formationen" ober "Bilbungzreiben" ber Tendenz nad) -

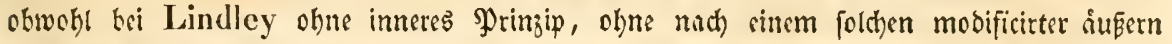
Erifheinung - entiprecten und bie er "Nixus" genunnt but; er abuete zweitens, ob:

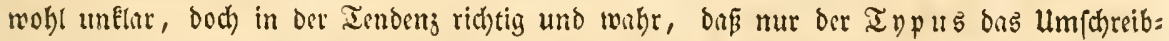

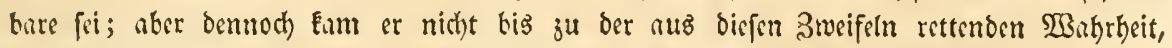
fondern - indem or unflur in fich felbft, bus ifm am naid)fen Sefreundete ubetwolleno herabiest und fo ben worken Segen friner ahat felber vertwitft - follt er immer tiefer in peine cigene $B$ erwidelung binein.

W3ir mitfen aufrithtig gefteben, wir wimfdhten, ber Berfaffer Gitte in biefer unElar: umentichloffenen, fohwankend=bezzeifelnben Stimmung feine Schrift nicht geforicken, fon=

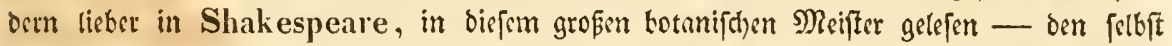

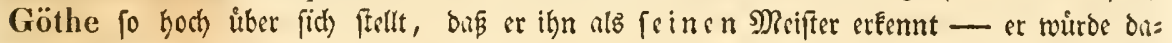

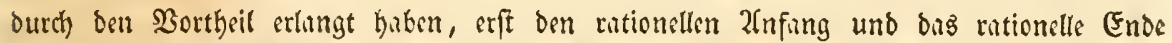

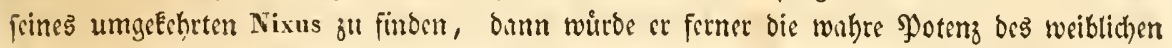

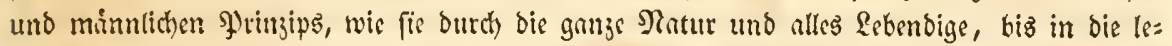

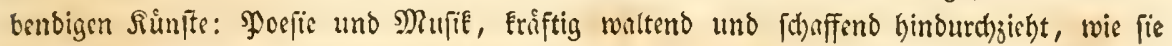

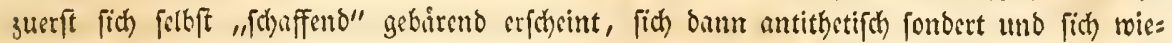
Der vereinigent, zettgend und fan affent und wiebergeboreno nuftritt, beutlich erforaut baben.

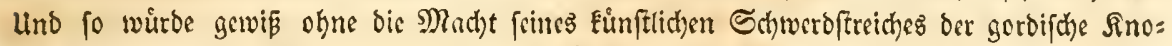
ten fich iffm in Siebe und natirlich gelof't baten, oenn er wire burch bie grofe lle:

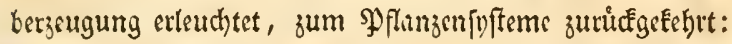

Dá ber CGarater felbit wie die Natur, bie er darafterifiren foll, cin lebendiger, ong heist, ein fo wie bie lebendige Natur, und mit igr fich fortbilbenoer, ihr alfo erf onn und badurh eben gleida $\mathfrak{a}$ tig getworbener, feyn múf

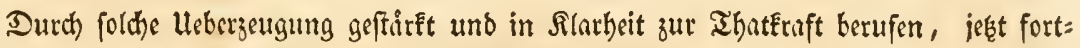

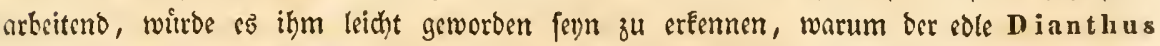
fhon als (Embrno fich nicht methr beuge, unter ben Eimftlich vom Berfaffer ifm befohlenen SbarnEter, benn was in oer Natur lebt, bas beugt fich audh nur in ber Babn, welthe

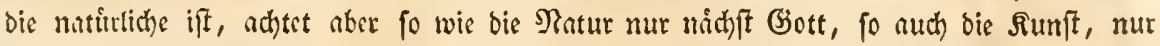
nidb) for Nitur.

23:as jeboch in ber Natur fich) nidht mit fortbilbet, bas erreicht wenigftens fein Biel

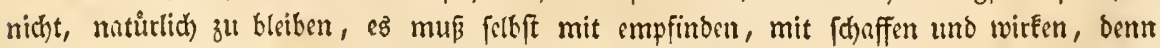

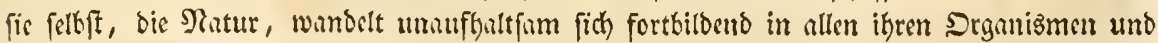

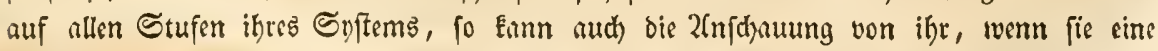


wabre fenn foll, immer und emig mur sine fid in fidj felbft fortbilbende, le= bendige jenn.

S(od) cine (peciellere von uns vertbcibigte, auf biefer allgemeinen Erfabrung berubende Grunbwabrbeit ber Eyftematie, oaß̄ die Species fich fortbilde, ift mun (Raspail „Annales d'observation" et nouveau systéme de physiologie II. p. 288.) in biejen Iagen "aud) nus Frantreid) gefommen und wir vertrauen jest fither ber Şoffnung, fie wiro nun aud) in Deutfoland anerkunnt werden.

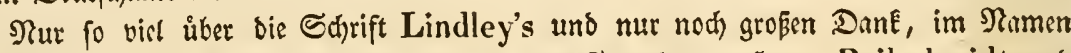
ber Deutfchen, unfern tordtern Lanbsteuten und Freunden, J̧eren Beilschmidt und

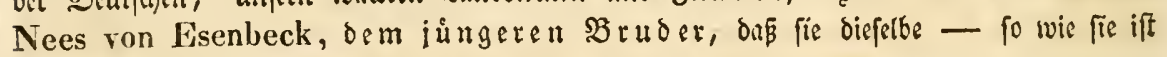
- auf beutfcten Boden verpflingten.

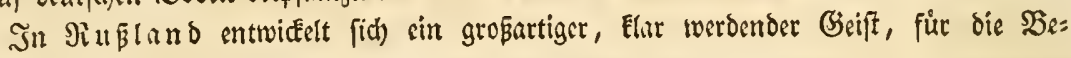
fchuuung ber letendigen Pllamzennntur.

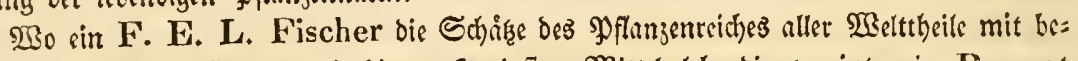

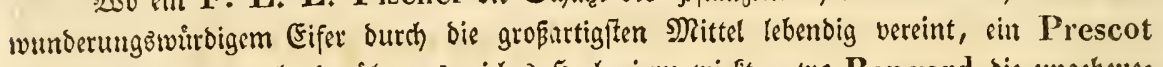
in gleidyer $\mathfrak{B}$ eife burch ein

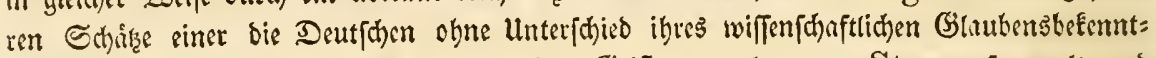

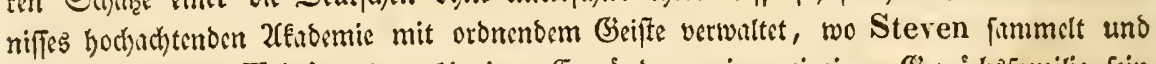
arbeitet, ber eble Trinius oer objectiven Ergrimbung einer eingigen (S) wewid)sfamilie fein gumjes $\mathfrak{E}$ eben geweiht, wo Ledebour's Thatigkeit in \&iebe gewultet, Dwygoubsky tehrte uno Schubert, Besser uno Eichwald allez neue priffend crfennen, da foun= ten aus dim Segen ber Bieifter eines Böber, Pallas, Stephan, eines Marschall von Bieberstein, einez Hoffmann, Goldbach und Mertens roht Minner erblühen unb mitwirEen, wie Andrzeiowsky, Bunge, Liboschitz, Maksimowitsch, C. A. Meyer, Schychowsky, Szovits, v. Trautvetter, "Turczanninow und 2fndere, de: ren fhoine Reiftungen zu Erwartutig now fdjönerer bered)tigen birrfen.

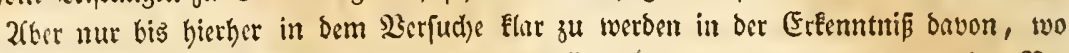
wir in urjerer Beit find. Und nur im offenen Erfeniren beffen, und im beutfden $\mathfrak{S}_{\mathfrak{e}}=$ ftreben nuch bem was von Gott und von ber Natur ůber uns gefegt ift - wurben wir Elar. - 


\section{Berfuch ciner Fortbildung}

\section{von \\ (0)"be's Metamorpboic

\author{
3t eincr $\mathfrak{B} e f(b) a n n g$
} \\ Des Sflaugneidos in feiucr Totalitüt.}

I. Thesis. Begrindung. - Etwas ưber Eignes voraus. - Beruf ૩ur Dbiectivitát? - Gefardrieben am Dltertage 1837.

$$
\begin{aligned}
& \text { "Riegt Dir Bseftern frar und offen, } \\
& \text { TBireft Du beute Eräftig frci, } \\
& \text { Ranmft aud auf cin Morgen boffen, } \\
& \text { Das nidjt minocr glïctetich jei." }
\end{aligned}
$$

(5).

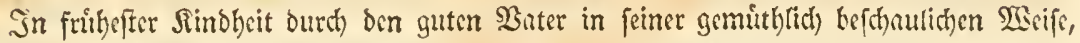
nuf bis rege Reken bes Drgnnifd)en in feimem Gistthen mo in oen Fluren ber dic Stndt

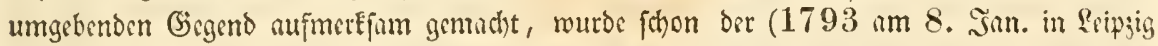

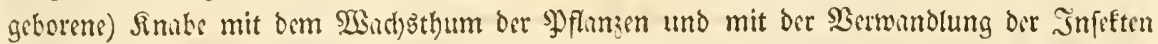

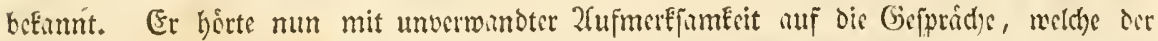
Biter mit Johann und Romanus Iledwig, und Capieux, und auth mit mand)em

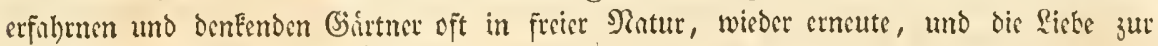
Beobachtung des Rebenbigan, war von hieran gegeben. Ein bunEel grabnetrs Etreken, at=

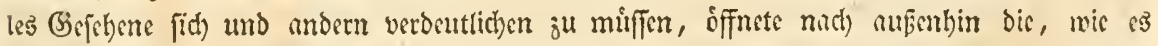

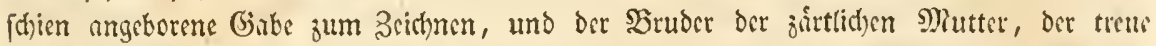

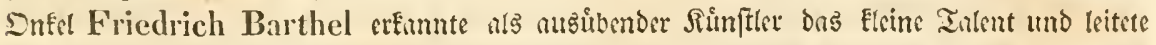

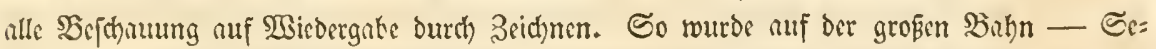

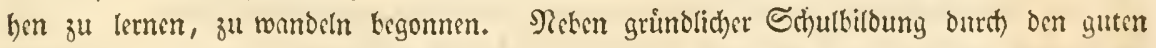

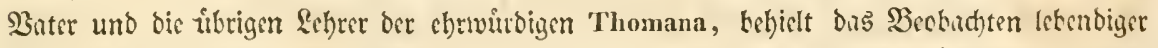

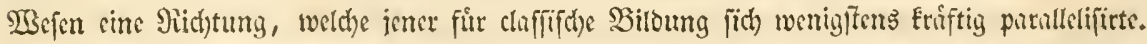

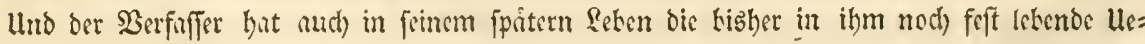

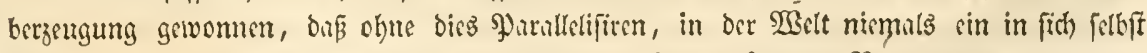

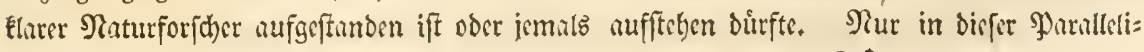


firung mag - und viellecidt fur bcibe Ridftungen - Slurfeit gefofft werben. Bech-

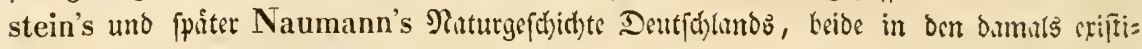

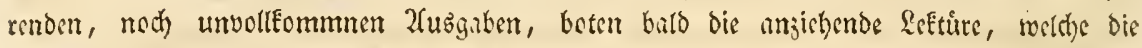

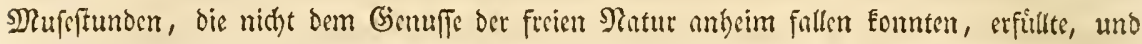

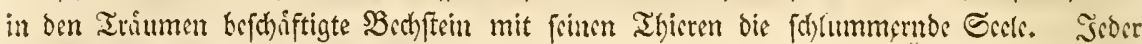

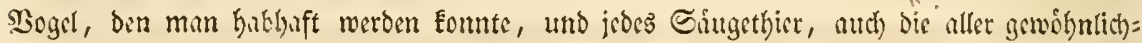
ffen nicht ausigeffloffen, und biefe maturlid) ofter als fpater feltne, wurben gepflegt uno

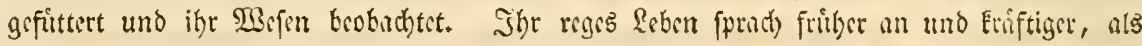

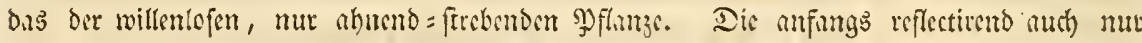

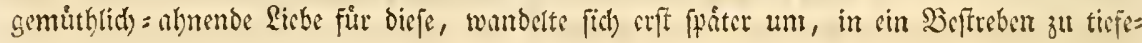
rem Sirftelen ifhes $\mathfrak{Z}$ sifens.

Dar Stubium Linnée's uno Schiller's erljoban ju gleither Scit bie in frillm

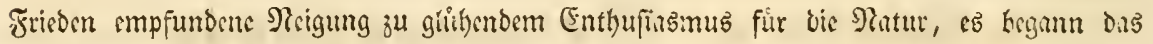

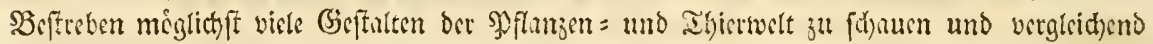

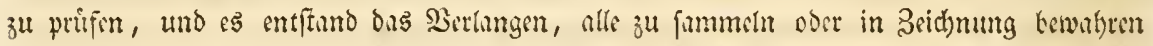
ju Fonnen. Sons früber fojon bejonnene Sammen, 'befonders von InfeEten, wurbe mit warmen Eifer fortgefsht und ein Eorrefpondenjzeris zuerft unter Entomologen eroffuret, in

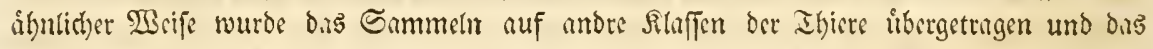

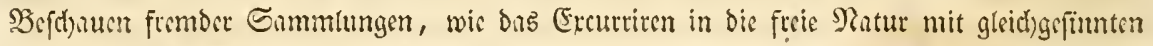

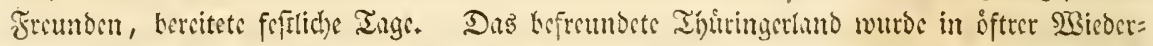

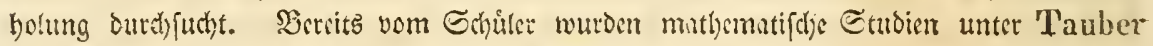

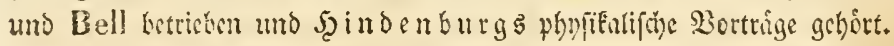

Die gumje Mithtung aber befimmte jum Stubium bet Medicint. So vorbertitit wurbe ber Şorfan bes witrbigen Schwägrichen und Ludwigg's betreten, wobei juetf

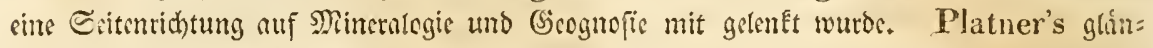

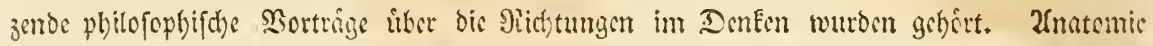

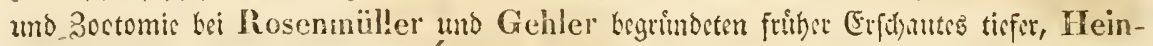

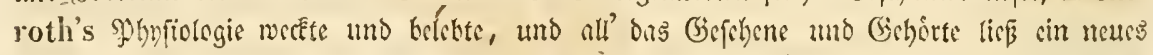

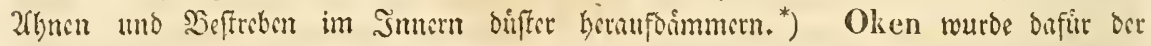

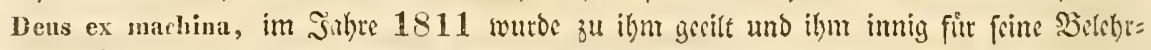

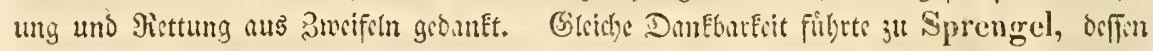

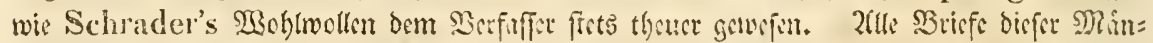

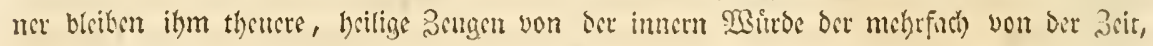
oor fie grhorten, in ifrem Bifteben Berfinnten.

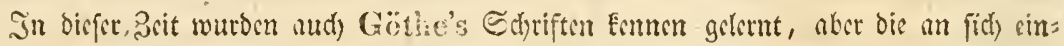

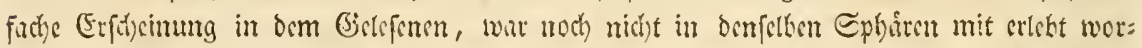

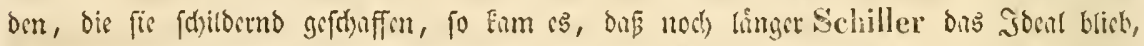

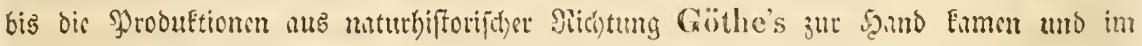

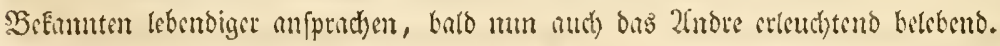

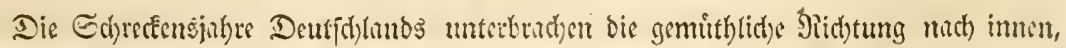

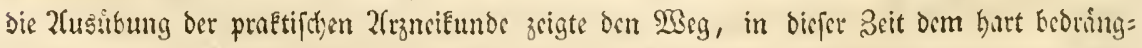

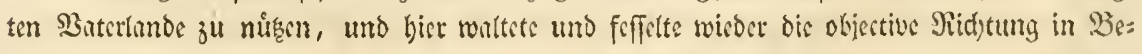

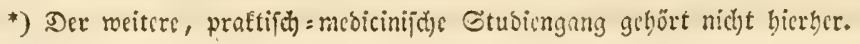




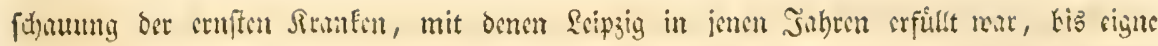

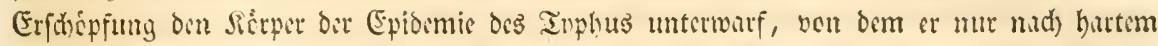

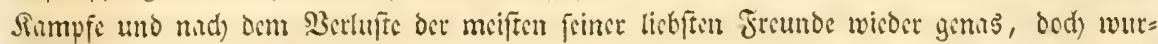
ben Choulant, Ehrenberg, Germar, Kaulfuss, Kunze uno Radius um to herjlict)er wicder begribit.

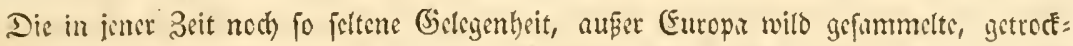

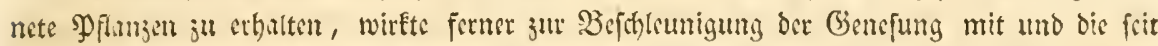

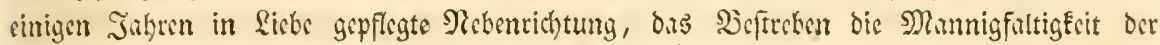

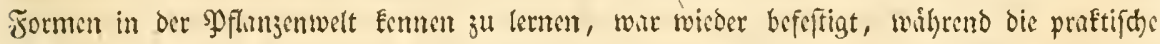

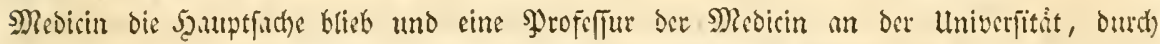
wohlmolienden Sorfhlig der whitdigen Eebrer, buld vou der Gnnde des Rönigs erlangt wurbe.

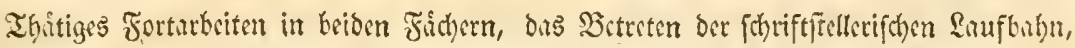

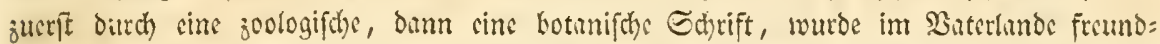

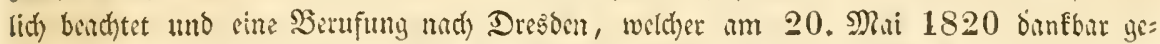

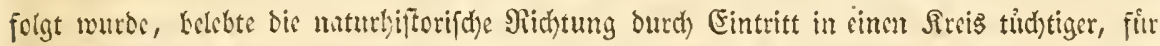

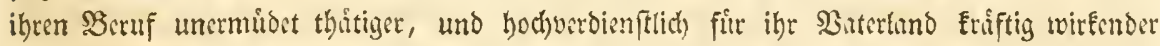

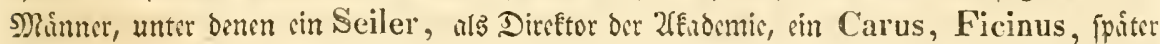

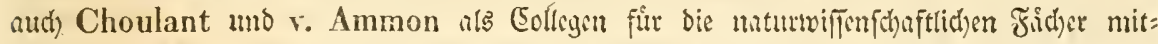

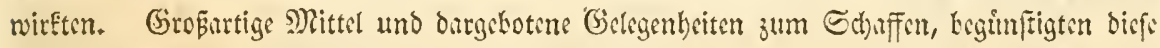

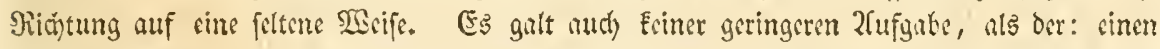

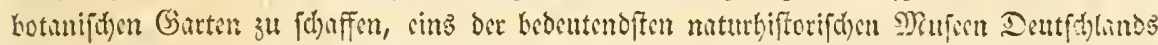

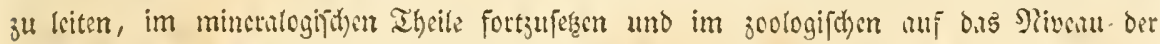

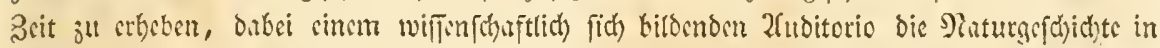
allen Sititutugen ju lebern. So war ber Beruf grgeten und alle Momente Diz Setens wurben iffm treulid geweiht.

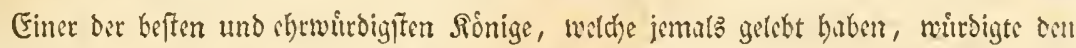

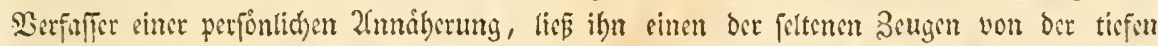

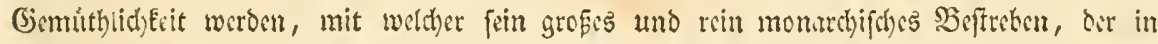

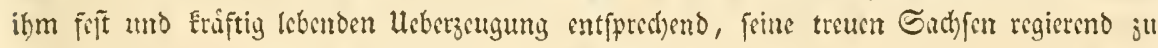

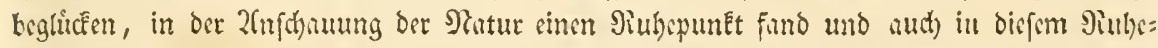
punfte bic bohe Eonfequenz ber tief wiffenfdaftlidben Grủndlichesit und bes in feiner wirbi=

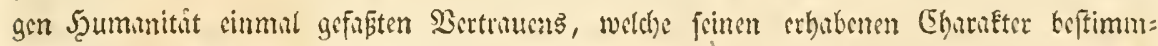
ten, bis zu frinem Syinfociben innig theitnehmens ind beftindig verfolgte. Co bci=

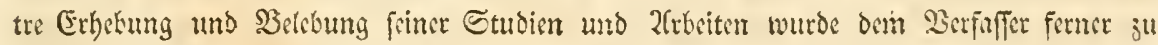

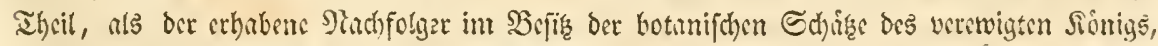

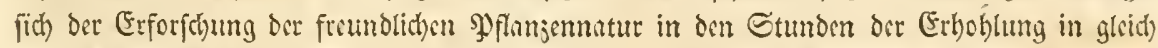

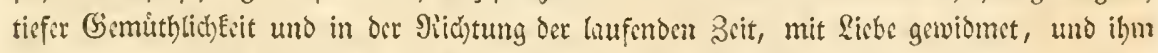

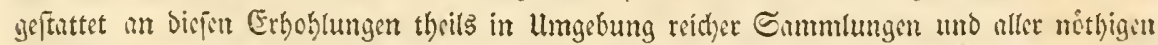

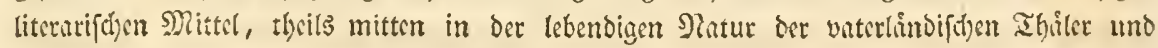
Berge, 2antheil nehmen zu burfern.

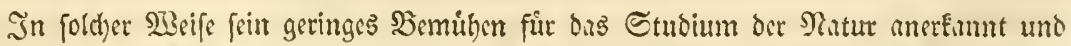

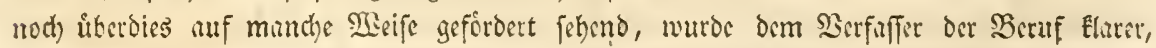

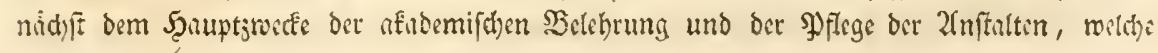




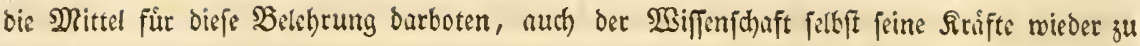
weihen.

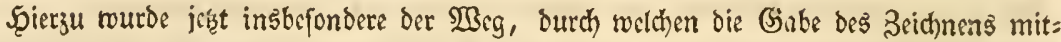
wirfen Eonnte, als ber paffende erkannt, fü die 2 Biffenfhuft alfo bas Beftreben, durch

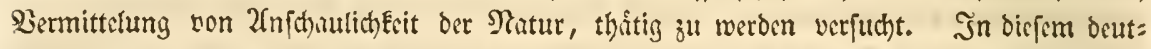

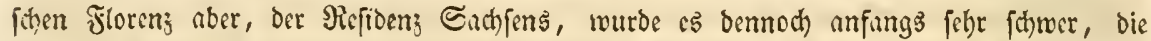

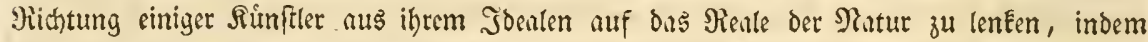
fogar Supferftecher ben Stich ber Beichnungen botunifecher (Segenftanto ablebnten, andre bie= ferm treu objectiben 2fuseinanderlegen ber Elcinen Realititen fo untreu wurben, Dos fie bie

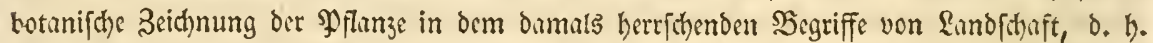

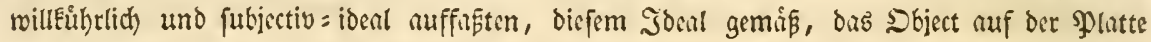
reflectireno.

Den Berfinfer Fonnten folche Şinderniffe nur noth fefter beftimmen. Theils ourch signes Miturbeiten, theils burch confequentes Fortfreten in 2fuffindung und Scerteiholung

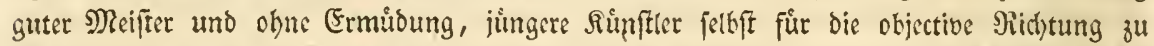
bitben, wurden nath und nath alle Schwierigkeiten itberwunden uno fo blickt er bernbigt auf bie Taufende von Darftellutgen ans der Thist = und Pflamzentvelt, melde sus peiner

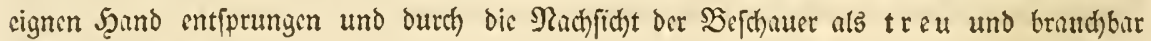
erfinnt moroen find.

Dicje Momente, donn bic faft tigglich gegebne Becunlaffung, alle bie cultivir= buren \$̧flumzenformen, weld)e bie GSirten Dresbens, - unter benen auß̌er bem eigent:

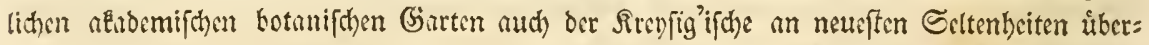
aus reich ift - und feiner Umgebungen, neff frer Fille entbalten, likenbig unterfuchen Ju fonnen, aud) bas baldige SBeEtmntwerden mit

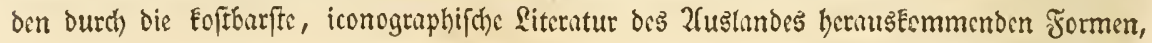
ein beitrer, an allen bicjen Beftrebungen innig uno lebhaft theitmehmender Familienteris,

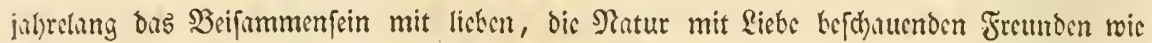
Bauer, Groh, 3euge der allererften Erpofition bes ๔rffems im $\$ .1820$, C. Schubert und J. C. Zencker, endlich uno itberhaupt bas Mitleben in sincm Rreife fir Sunft und

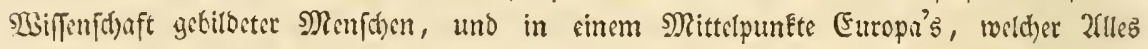

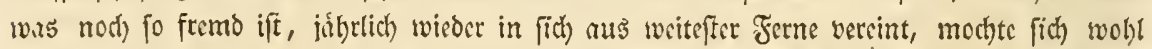

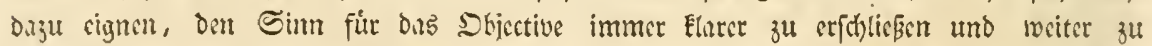

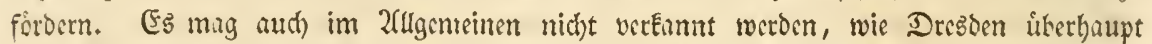

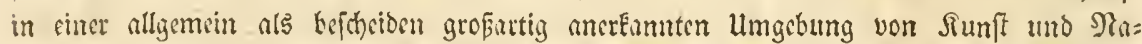

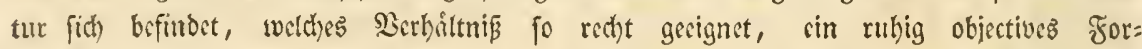
fdyen ju wecken und zu befordern, ben Neaturforfcher in friner geiftigen Ephite; bod)

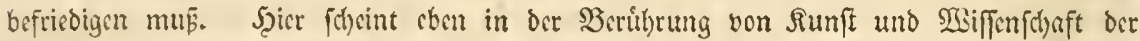

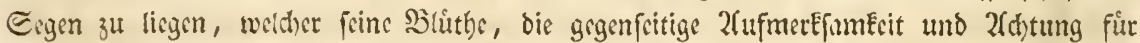

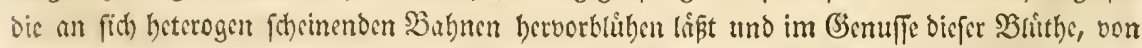

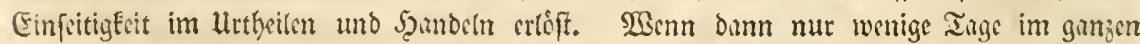

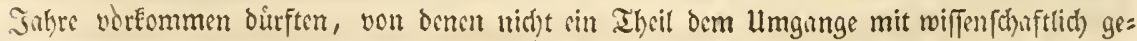

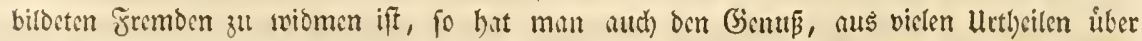

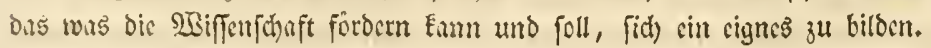

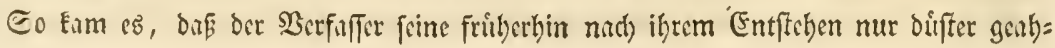




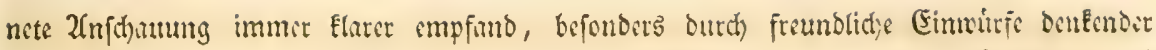

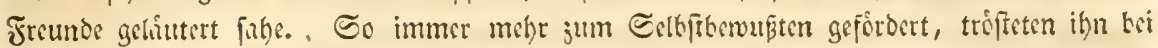

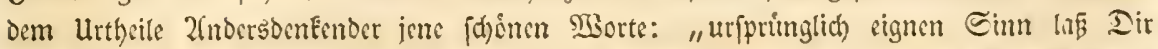
nictst truben, worn bie Menge glunbt, ift leid)t ju gluben," und raftlos verfolgte or

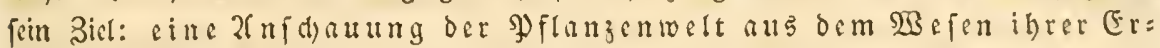

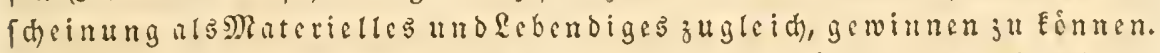

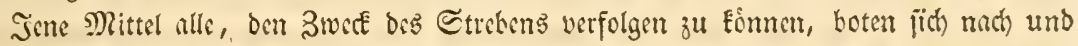

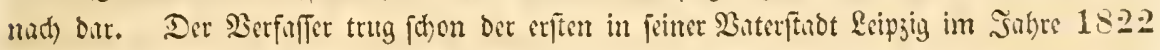

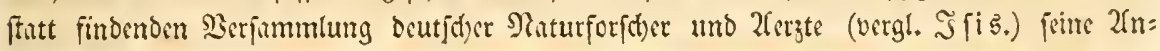

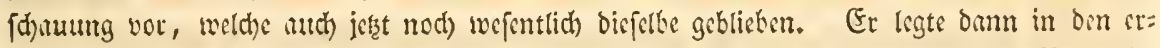

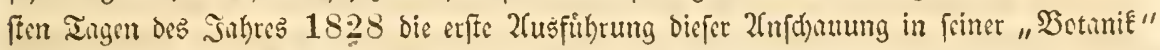

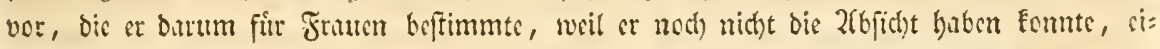
mem gelehten, Eritifitenden Sireife ben unvolleommnen Berfudy ju wiomen, bod) aber, wan

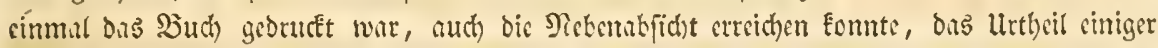

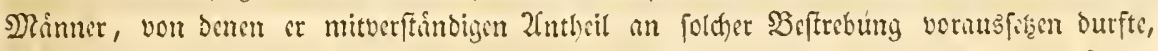

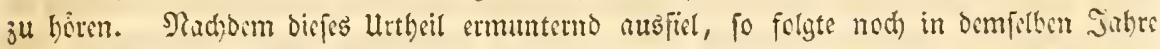
Der 2 emeis fur die Durcharbeitung feiner 2fufgnbe won allen Esiten, die "Uleberif(d)t

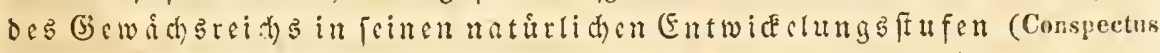

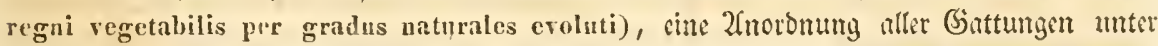

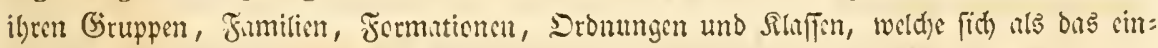

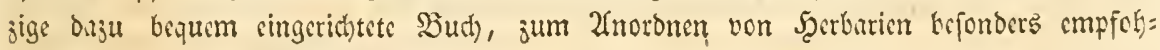
len bat.

Der Berfaffer freute fio der Scit, in ber fo vide Seleftoenfende ifn verftenden, in=

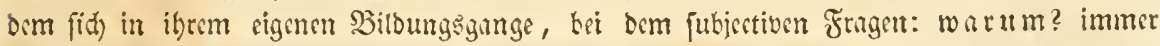

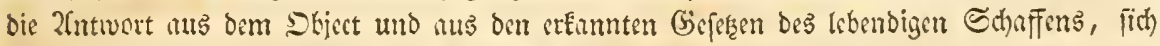

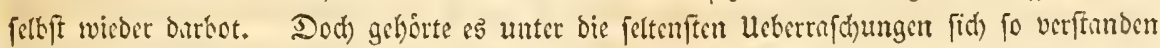

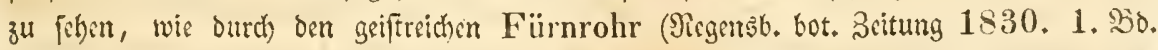

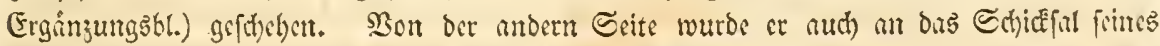
Borbildis, an bas von Göthe's Metumorphofe, auffallend eriumert, went 2lnore, immir

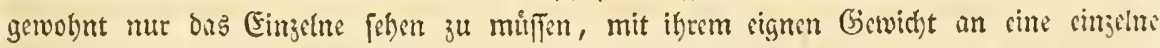

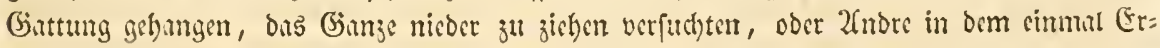
lernten beharreno, es unbequem funden, in bic meue, lethenbige 2fnfhatung fich erft bincin

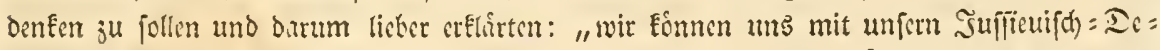

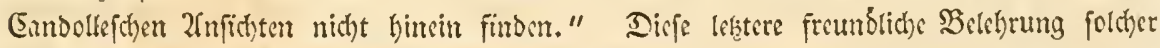

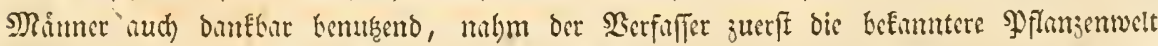
Deutfylunds unter ben Sieflexionsfpiegel ber Metumorphofe unb fand biburd bie Gemus=

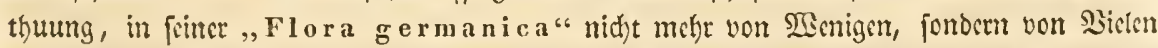
rerftanden zu weroun. Die (Sickraud)szettel zu biefem Epiegel - Die Einleitungen fir

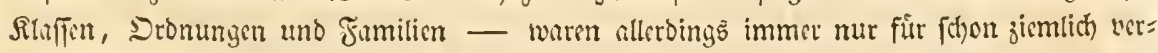

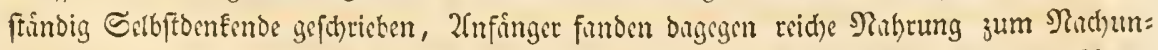

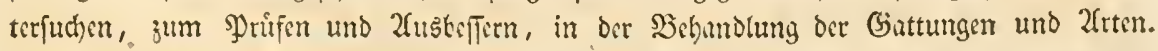

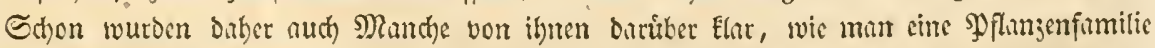

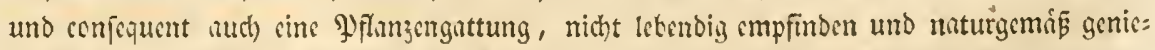

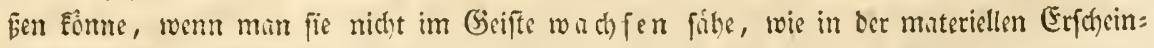

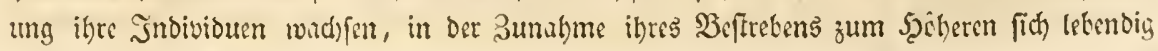




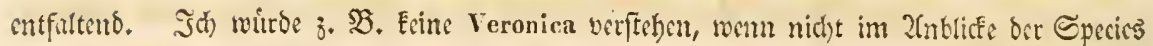

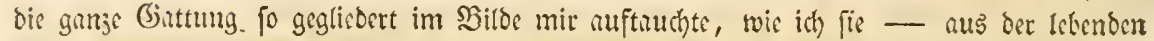
Slatur in ber Flora germanica wiedergegeten - vom sinfarbffen Seginnen oer ach felklit=

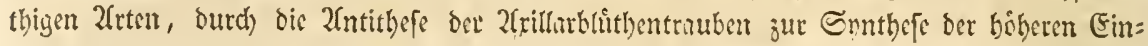

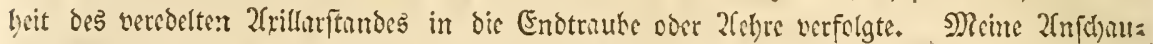

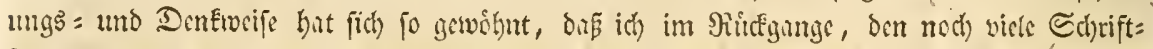

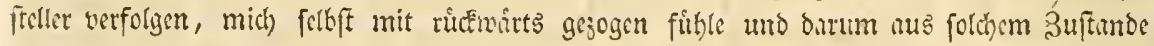
gern wieder binauseile in bie umgebung oes immer frei fich fortbitbendon \&ebens, ber freisat MRatur.

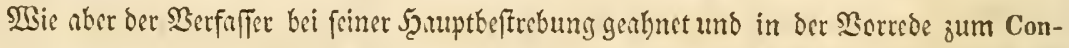

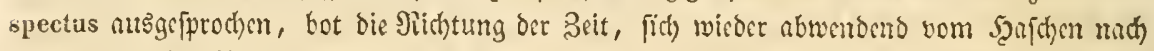

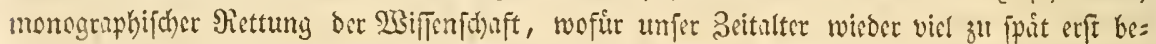

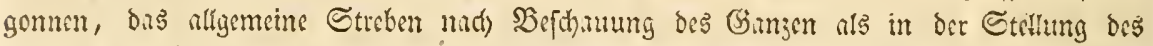

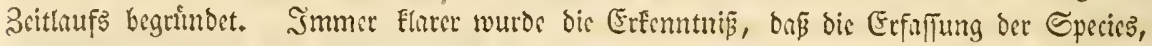

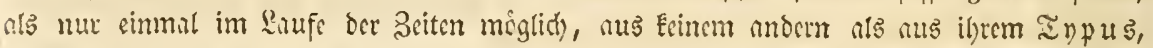

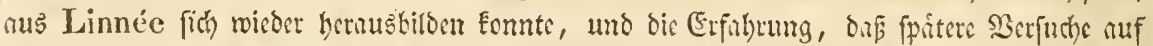

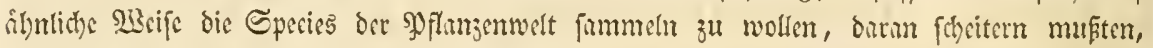
Dra die erften Theile vernlteten, bevor noth) Die mittlem erforienen, wurben ourch Röner und

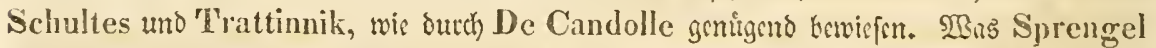
lofte, follte mur, wie ar, von Linnée's Erimncrung, fromm wie sin Ieutfolyer kegciftert,

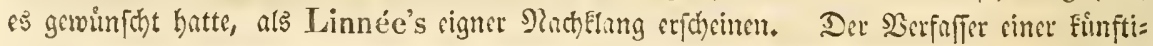

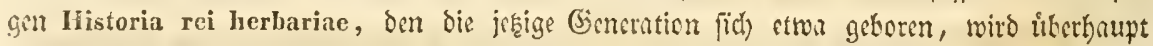
mod) ausfprectien milfen, was bic mittebonbe nidit ahnen will, was cigentlid) Sprengel Der SMifferthaft war.

Dis wabre SRichtung ber 3eit verfolyten nach und peit ifm andre bedeutende Grifter in

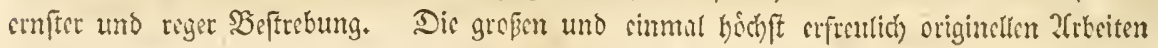

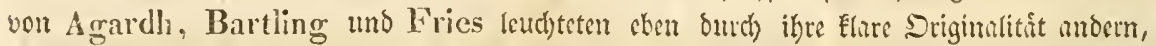

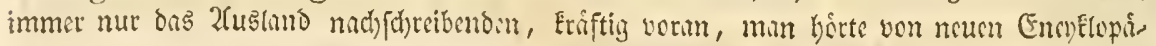

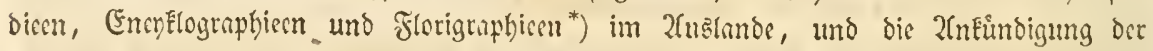

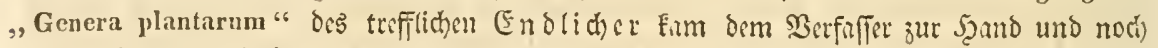

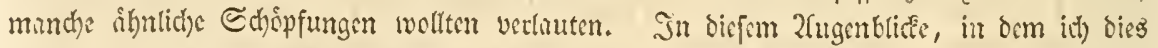
forteike, fommen anth Meissner's: ,plantarum vasenlarium genera " unb zcugen wicoer

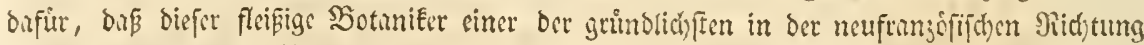
gertunnt weroen mur,**)

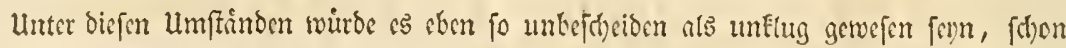

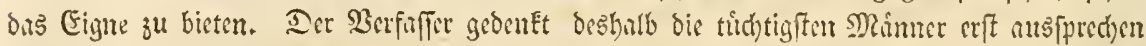
zu laffen, und bofft bumn wieder zlt Eommen, fobald er, was jene erffrebten, Flar wirb er=

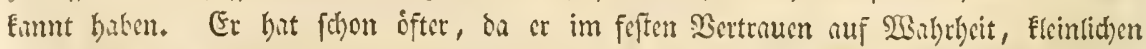

\section{-) Sit jam venia verbo!}

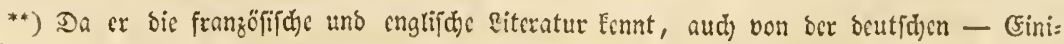
ges mit benutąt bat. Co war z. B. Dic (Sattung Aidelus f́cir 1828 - vergl. Conspectus regni

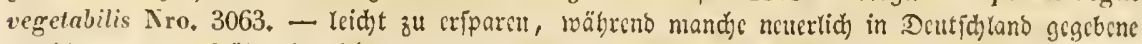

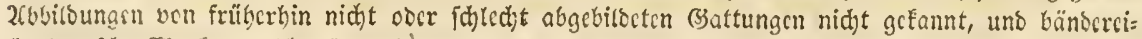

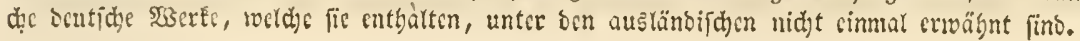




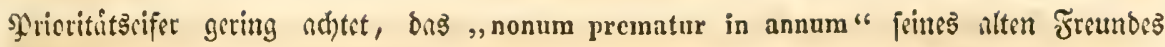
treulid) befolgt.

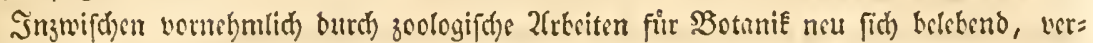

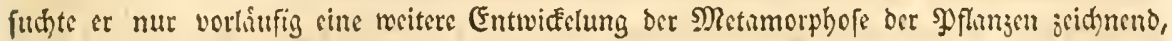

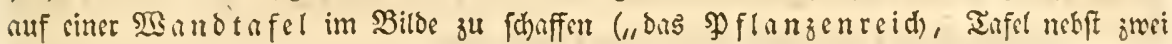

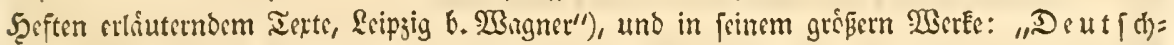

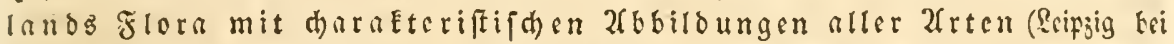

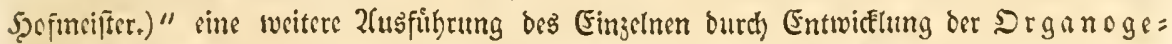

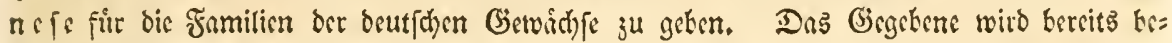
finnt pern.

Dic Fringe bie ber Serfanfer oft felteft an fid) that, of ar wobl aud) Göthe's 2fnf(t)au=

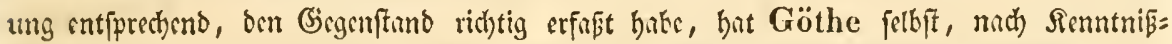

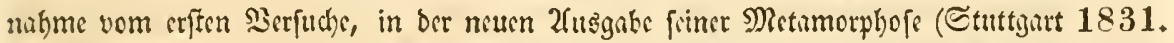

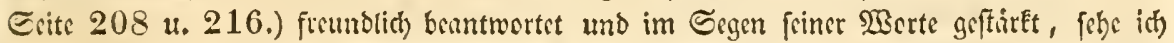

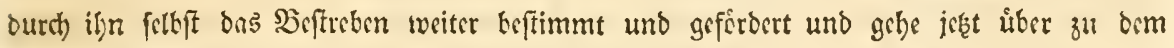
Sierfudje, befien Sicfultat annlyfirend wieber zu gebern.

II. Antithesis, Borlåufige Einmúrfe von aน

1) Göthe's Mctamorphofe iff cine $S$ bee.

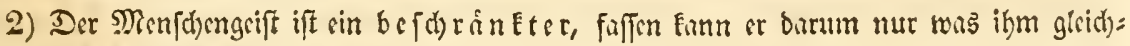

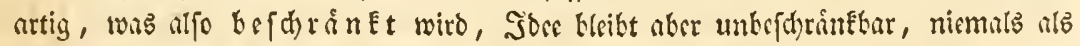
Sicules entfpredend erfactineno.

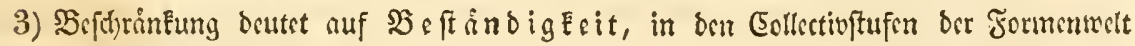
auf Stabilitit.

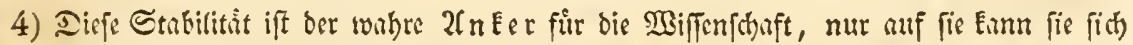

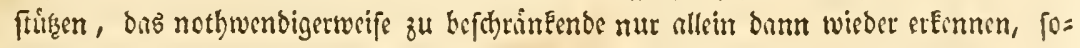
buld es ftab il ift.

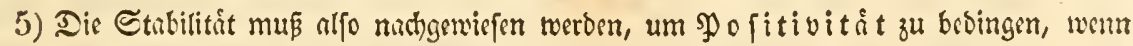

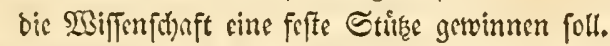

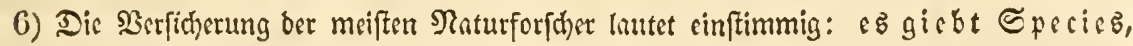
bie Speciez find ber Endzwe der Sdopfung und ber wahrena= tủrliche Snbegriff ocs Erfdafferen; bie Species wurben bes= batb uranfanglidi geforafen, fie pflanzen fid gleidartig fort und werben fich gleidatig fortpflanzen, fo lange bie 2 elt ficht.

7) Dirfe Species fint burth "getwiffe", in ben forthufonden Gencritionen "unab= ånocriche Meremale" zu erEennen.

8) Die Epecics werben butd) "gemeinfdaftlidhe Eharactere" zur B̧attung vereint.

9) Dis Ģnttungen fammen fid zu Familien: "man zirht nimlidy aus allen ben

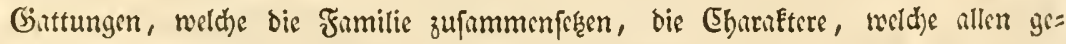

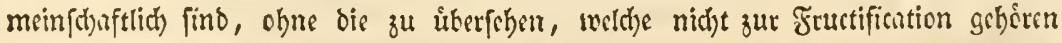

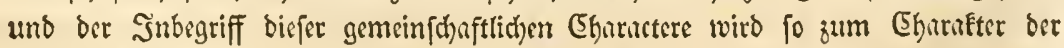

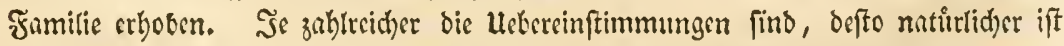
bie Familie und folglid' defto gewid)tiger wiro ibr EGarneter." Jussieu. 
10) Sramilien werben zu $\mathrm{I} l a f f e n$ burd) noch allgemeinere (5hanactere befeftigt.

11) Die 2 btheilungen oder gropen Divifionen, weldbe Slatjen in firf begreifen,

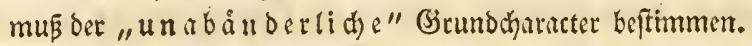

12) Nur jo ouf allen Stufen fiteng umidrieben, Eunn die Rlafifficition jelbft, sine Feftigkeit, eimen Eharacter gewinner.

1II. Synthesis. SBorláfige Berfuche zuB Bentwortung Der Eintourfe von ausen, nach benentfpedenden Siffern georonet.

"3u was $\Re e \pi$ icrm fino wir geboren, uno was bie inuce Stimme fpridyt,

Das täu[d)t auch bie bofinoc Seele nidgt."

ङdiller.

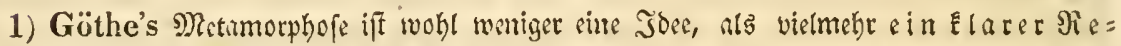
flex ciner Elaren 2 njobuung zu nenneu.

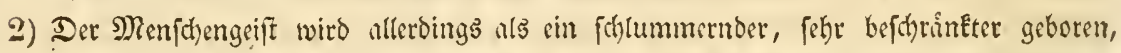
ermacht ift or aber — To twie alles \&ebendige - einer feten Fortbildung firbig,

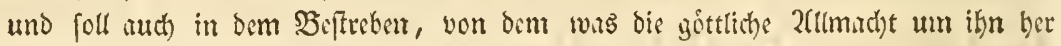
erfhaffen bit, eine immer flarere, bem 2 Befen bes Gefthuffenen immer mehr ent=

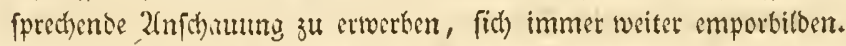

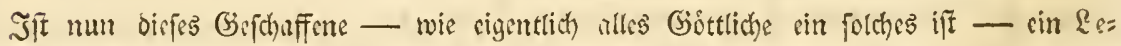

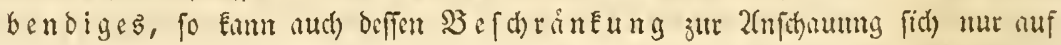
Nomente feiner zeitichen Erfdjeinung bejieken, Denn Fein zubites Rebensmoment firnı nututgemå im wambelnden Reben, in ber Stufe bes vorausgegangenen Mo: mentes beharren.

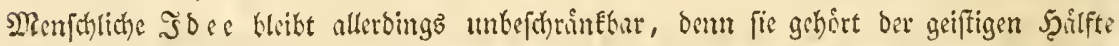

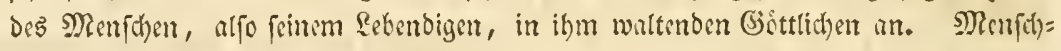
liches Soeal ift nker allerbings nicht rentijitkur. Dats Piente wito wentigitens nie bem

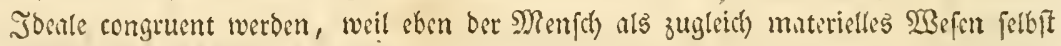

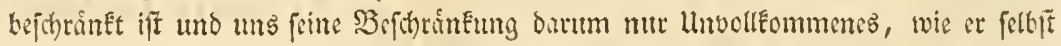

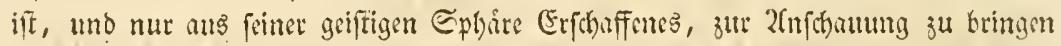
befinigt ift, da ber Menfech nie felbif der Gejipfer der Materie zu werben vermag.

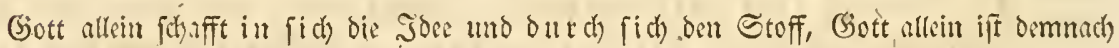

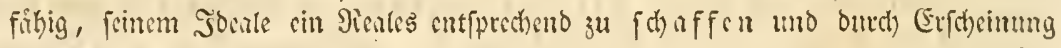

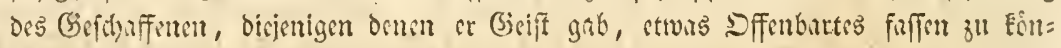

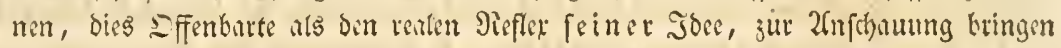
su laffer.

Die Shutur wat eine Soce, eint Giednte: Gottes, Gott fouf die Natur feinem Gioun= fen entprecheno im Stoff und im Seben, burnm ift: bie $\Re$ atur die eingige Erfdecinng, welche alseiner-aber nur oer gottlichen- Soce cat $\{$ predieno gedacht werben Eann.

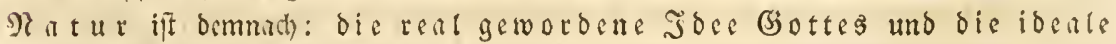
Rentitit für ben Menfert.

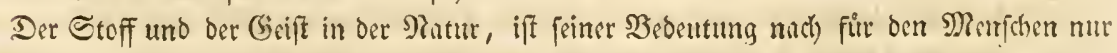
erfabbar nus der Erfoheinung, weldhe beide - Stoff und beift - ourch ibr gegenfeitiges Einmirfen anf einumber, bcoingend berbormfen. 


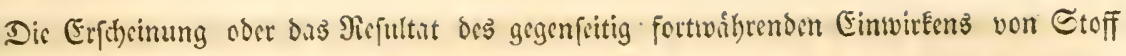

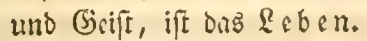

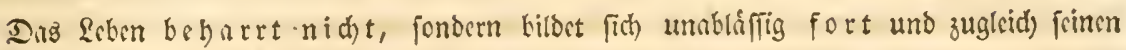
Ctoff $4 \mathrm{~m}$, rinem Bicle relatiucr $\mathfrak{B o l l e n d u n g ~ i m m e r ~ e n t g r g e n . ~}$

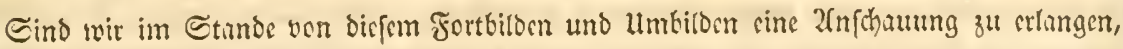

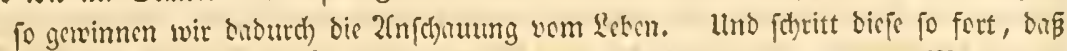

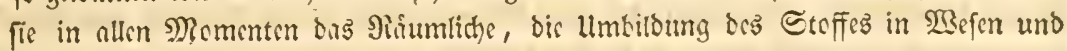
Form, mit bem 3citlichen, ber Entfintung oes Eekens paraliclifitt anjufuffen ver=

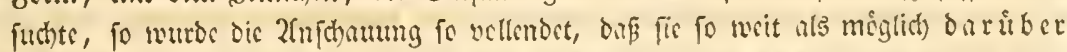
cufflicte, was itkerhaupt non Etoff und Geift ums von ber burch bribe kedunge=

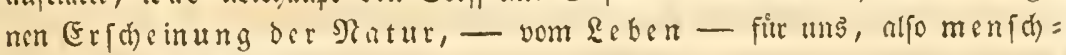
(ic) exfäbar jern biufte.

Bermag nber bicfe ?urchumung Esin begrenzter $\mathfrak{B}$ egriff, bem Etubilitit entperchen wurde, zu merden, fondern werkicibt fie auf ber Etufe ber unbegrenjten 2fnfhom= ung freken, fo wirb uns biefe boch zum entiperefenden Siefer ber felkft unke=

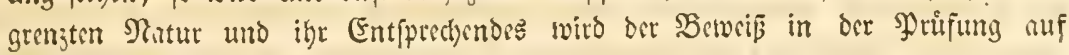
T3abrbeit.

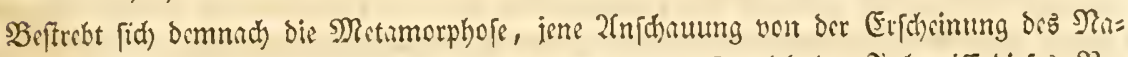

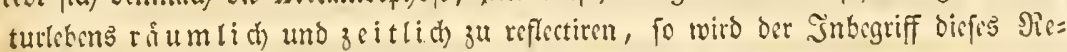

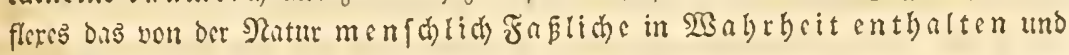
bieten.

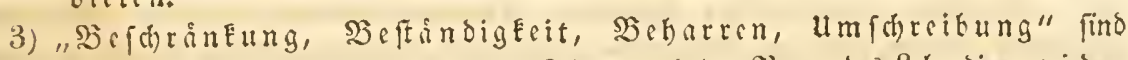
fimimtlic) Begriffe, welche bem bes Lekens und ber Slatur bes \&ekendigen wirder= freiten, nur bem Momente grbiren, wic im Sndivibuo fo aud im Collectiven und in ber Iotalitit bes Eolligirten.

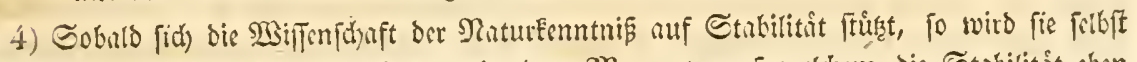

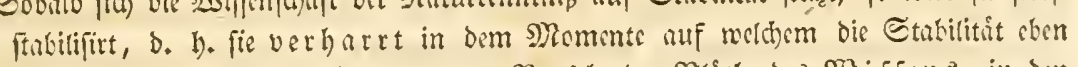

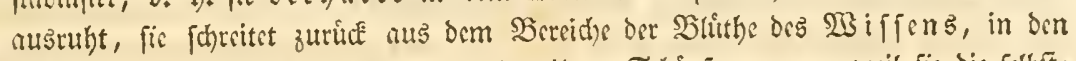

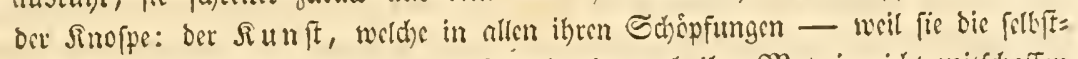

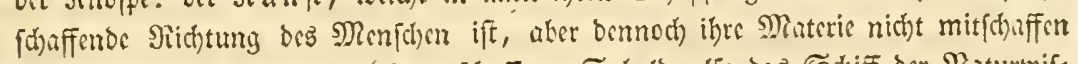

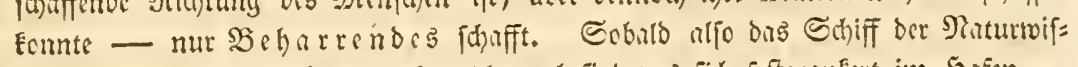

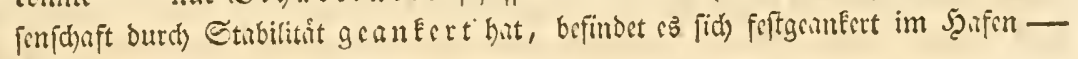
ber $\Re u n f t$.

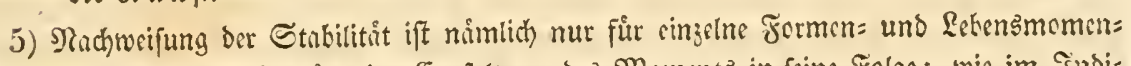
te moiglich, nicht fur cine Entfoltung bes \$oments in feine Folge; wie im Sindi= viduo fo auch im Eolligiten. Pुofitivitit oder Bernben auf foidser Etrbilitit be=

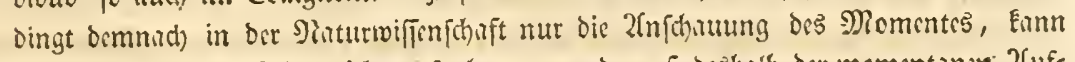

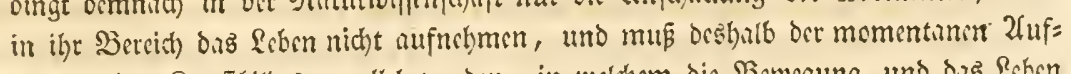
farfung bes Sunftbildes parallet merden, in welchem die Bewegung und dus Seken

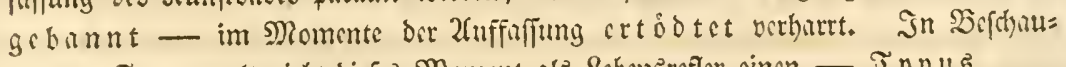
ung der Formentwelt gieft bief̧es Mement als \&eckensereflex cinen - Typu

6) Die Soce von Species in ocr Natur hat fich in ber Beit bis Menfhenlebens erfit jeit hundert Jahren entwidelt, fritorm nomblich Linnée zul lehten verfuchte cs gíbe "fo viele હpecies als Gott am 2Unfange der $\mathfrak{B}$ elt cribaffen. 


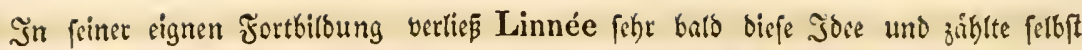

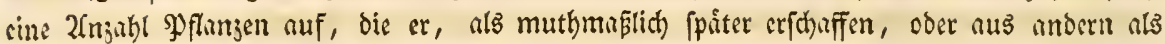
Mittelformen entftumbene, betrachtet. Wir find jef̧t von bem \$orbundenfern aller biefer

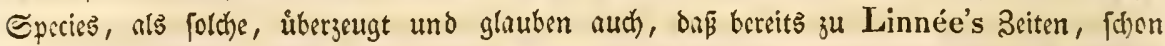
bie imsiften berfelben, feit lange beftamben baben mogen, aber - wie lange? bas wiro Shiemano in Bejirfung auf fie, wie auf andre beantworten fonnen. Linnée feibft fium

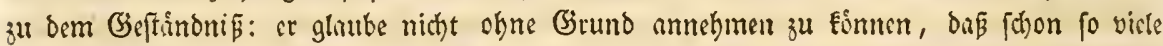

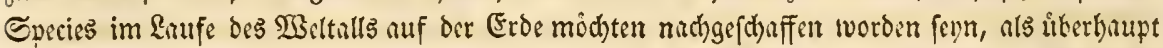
biefe hervorzubringen vermed)t babe; aud mige er nid)t barauf fdoworen, bas es vielleid)t

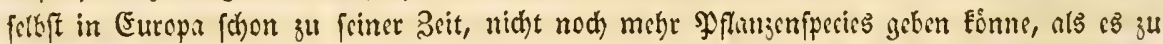
ber 3sit gegeben, wo Bauhin feinen Pinax gefforickett. Befonbers fagte er, bieten bie ar=

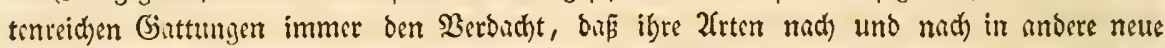
Formen fich umbilden. In Linnée ging noth fo weit zu figen: "Creator in ipso prim-

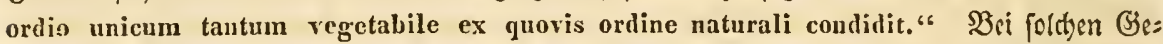

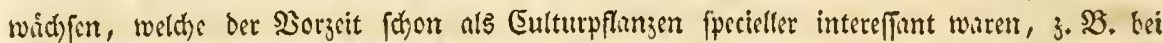

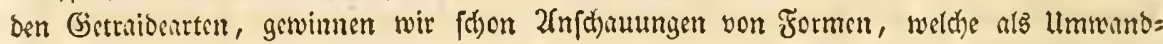
lungen ihres Inpus, ats febe wahted)einlich fich ausprágen, ja foft toitb fo ettwas, wis cs

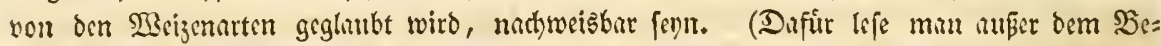
Ermntent, auch Raspail nouveau systéme de physiol. végét. et lle Botanique.) 2(ker wann mun hier ber Sultur allein fo großen Einfuß zufdereiben wollte, wie bei bem Berfdowinden Des T̃pus der Şumberaçen geftheben, fo mưrde bod) bie frcie Natur in ber Gattung Aster,'Aconitum, Amaranthus, Aquilegia, Carduus, Cirsium, Dianthus, Digitalis, Epilobium, Geum, Mentha, Polygonum, Potentilla, Primula, Rosa, Rubus, Saxifraga, Sempervivum, Verbascum und nod) unjấligen anderen im 2(uslande, immer mur bis:

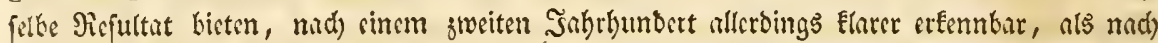

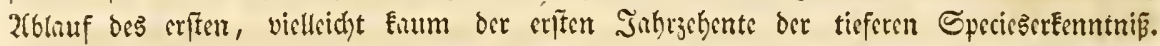

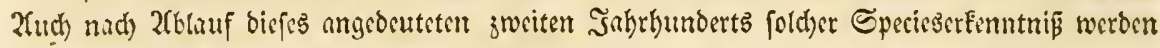
unfre నachfommen bie beutigen feitentangen Diagnofen fo vag und unbegrenjent finden,

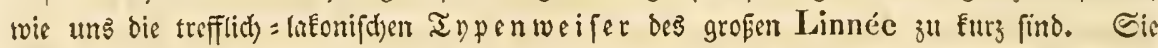

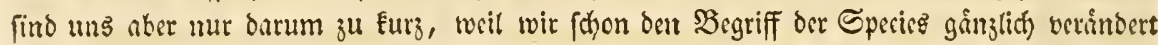
baben, weil wir vergeffen, baß fie in ifrem Typus einzig uno allein erEannt uno iu

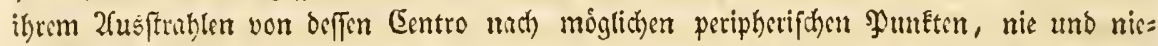
mats begrenjt werden Eunn. Se weiter ausgedefht wir bemnach ben Begriff der Specirs

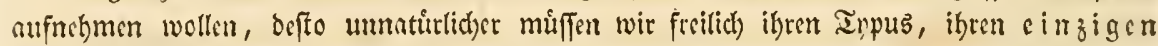

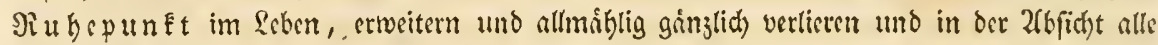

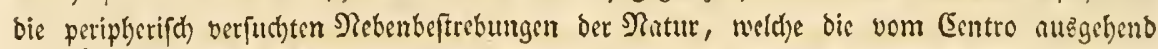

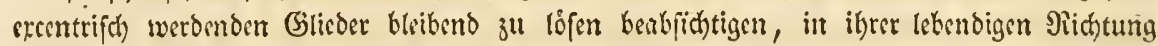

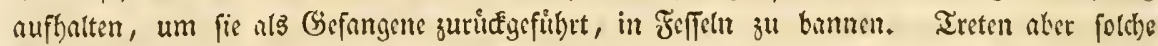

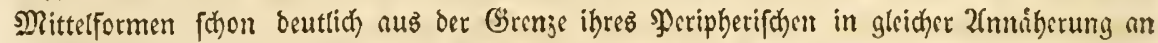
bie eimes andern, fo wiro es um fo unmeiglid)er fie in sine von beiben ju bannen, fie fohaffen (iid) fdon, wie ber netgetborene Şimmelsforper ihre eigne 2(tmofphire uno mit ihrer Selbft: frindigeit tritt fothon bas in ihrem Entfteben mit gegebene Beftreben zu neuer 2fubftrablung

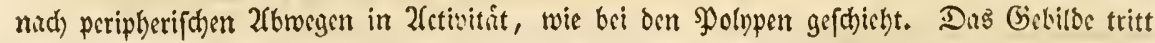

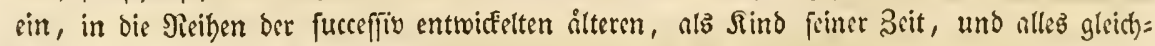

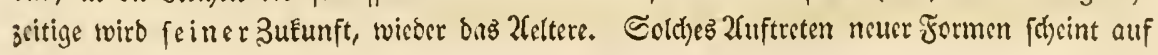




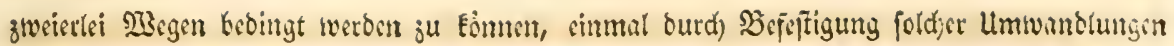

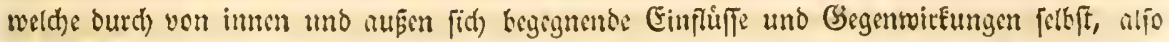
vegetabilif = geiftig wie vegetabifit) = materiell bebingt wurben, sin anbermal aker, vielleid,

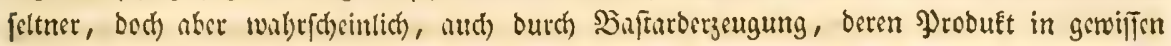

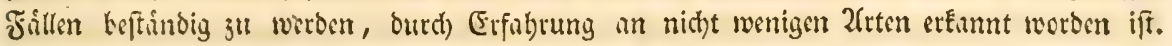

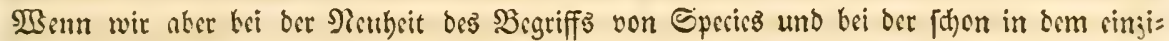

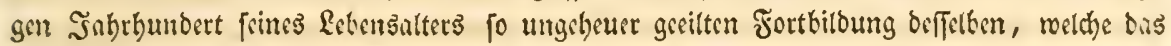
was im 2fufange Species bieß, febr bald (Semts, bunn aber aud) Familie, Sromung uno

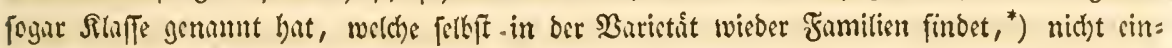
mal in Stunde fint, Géoffroy St. Hilaire's ber Stabilitit po grfabrorohenbe 2infid)t

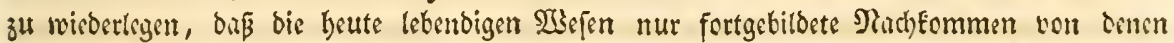

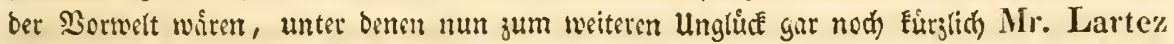

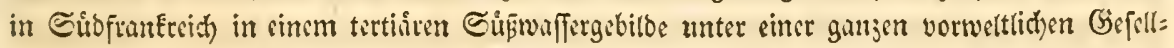
(d)aft von junnjig bisher nod) ginjlid) in abscuro verbliebenen 2feteurs aus ber Ifhierwelt

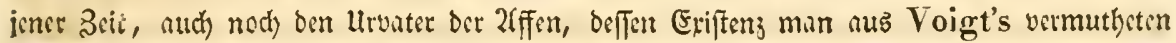

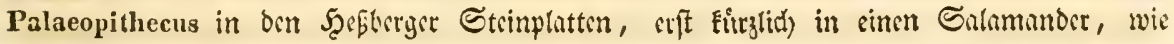
cinft Scheuchzers, homo diluvii testis" glucflich verwanbelt, wirflid) gefunden, 0

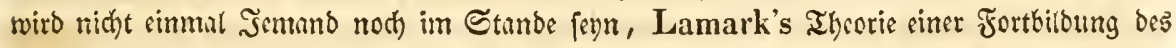
Srang Utung in ben Menjethen baburd) zu reiderlegent, bas er behauptete, beibe fenen ju glci=

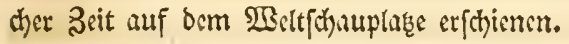

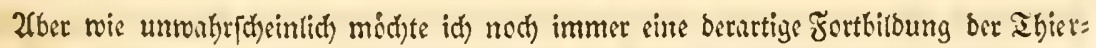
welt finden, wenn fie nid)t allein butch Elimatifhe Einfliffe und imnere Gegenmirfung anf

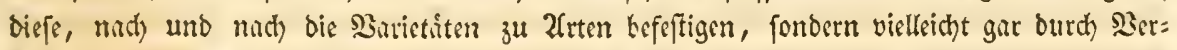
parang verjhicomer Epecirs, in ihrem Entfteben mit bebungen fenn follte. Das Thirr ift cin frei zwifd)en Şimmet und Eroe wan

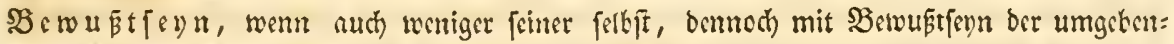

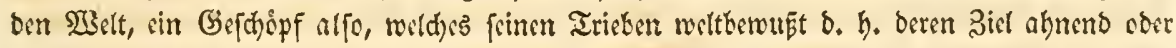
Eennend, nathjugehen vermig, es birrfte beshallb nur in feltenen Fâllen gefdehen und vor:

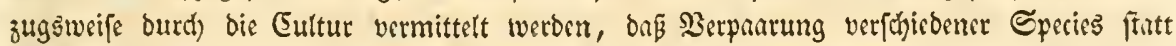

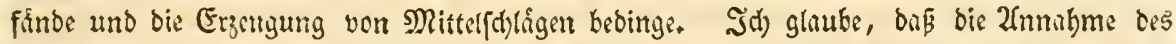
Bituustepnns im Thiere fdjon a priori uns binbert, an sine Fortbilbung ber Formen bir

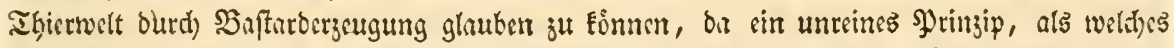

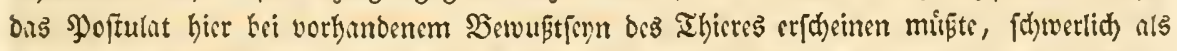

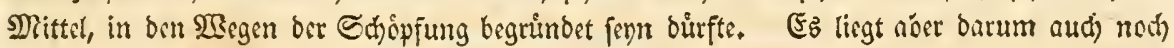

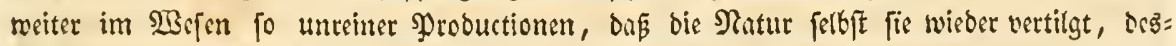
balb find foldje Giefjoipfe in ber Siegel nidjt weiter fortplantzungsfihig und fterken wisder

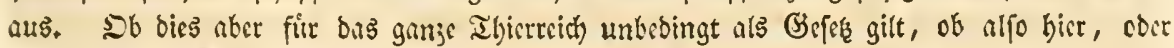
wo ponft dicfe (Srenzen fid) finden, ob aud) Cuvier's gróstentheils fich nid) cigentlid) bc:

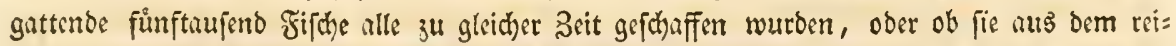

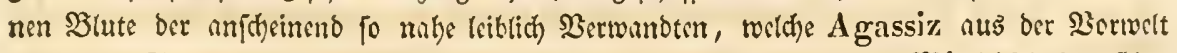

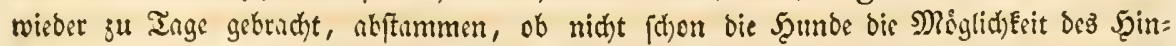

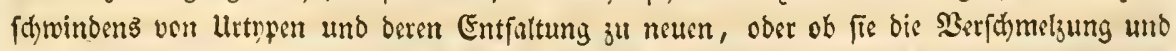

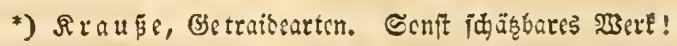




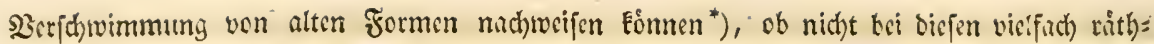

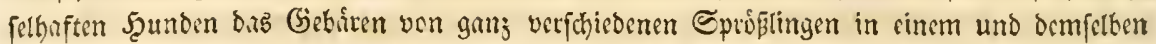

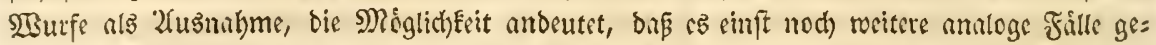
ben Eenne, ob auch bei ben nicberen Thieren, insbefondere den immer reipend vormitts aker bis jum Schtwinbelerzengen in ben Entomologen, fich in ifren 2frten vermehrenden und barum

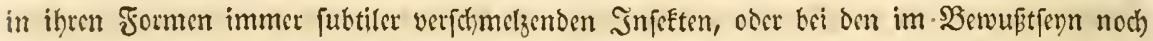

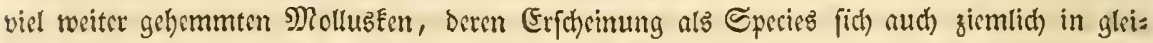

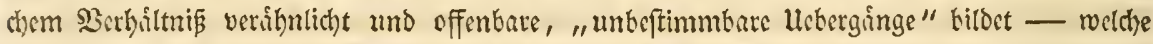

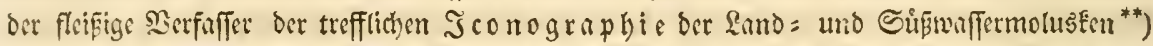

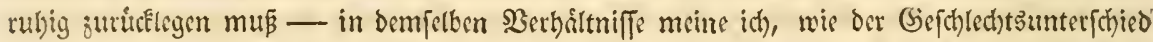

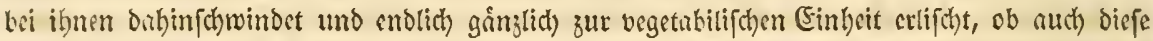
fich) zur Sgaurung noth alle mit gleidyer Strenge und immer zu fondern tviffen mógen, ob die fonderbaren (Egel und 9iegrnwuitmer bic fich pantweife befruchten um gegenfeitig wieber

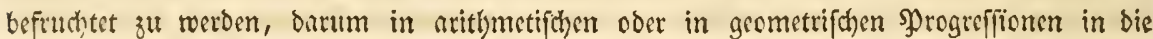
Epecics bes fdarfbidenden Fitzinger auscinunder gegangen, ob nitht bie ungloubliche Mamigfaltigfeit ber Formen und Ulberginge ber Mujdelgattungen Lnio umb Anodonta, welche man fü fich jelkif befuchtende 3mitter bielt, burd) Prevost's und Kirtland's und

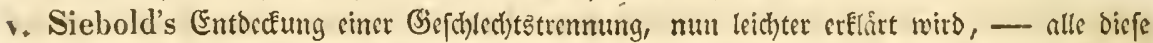

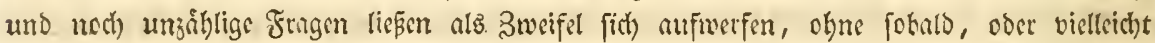
jemals, beftimmt aus Erfalyrung zur Flaren 2fntwort gelangen zu Eonnen. Ueber folche Dinge Eann mue bas Sukject im Menftyen fohell uno immer tereit riffonniten, cine

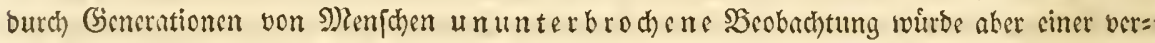
ftandigen 2fnniberung an cine verftindige 2lntwort vornusgehen múffen. 22er objectiv zu

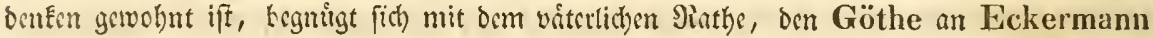

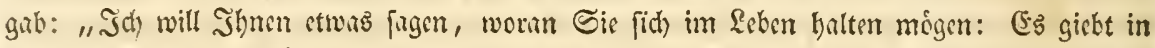

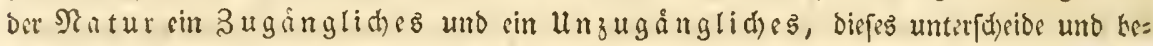
Dinfe man woht und babe Siefpeft." -

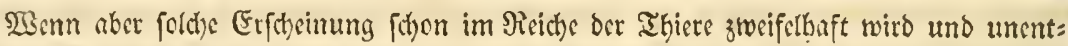

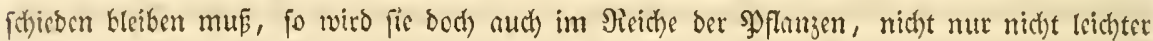

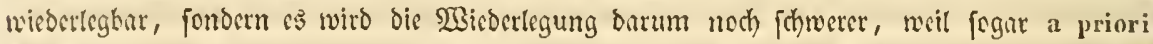

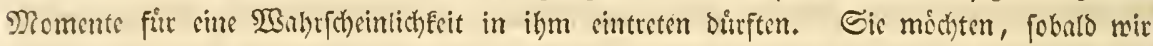
firt objective 2frrchanung Empfanglich Esit baben und nicht unfer Subject im Boruttheile fra:

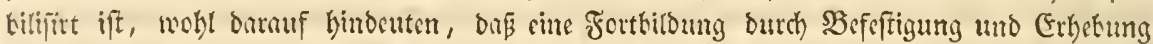

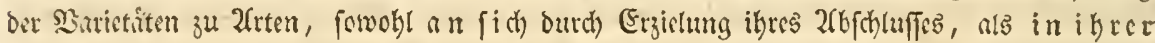
Erfa) cinung, für die 2lnfthaung bes Menfchen im conventionellen Bsiffe ber 3cit in

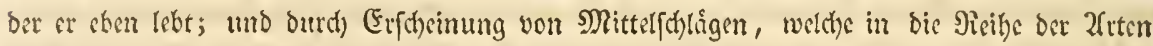

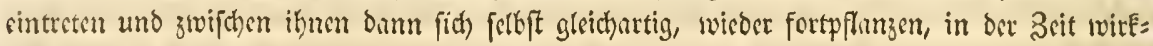
(ici) finttinden burfte.

Treu fich an bic Natur bingekende Beobadtung oer oben genannten Gisttungen von

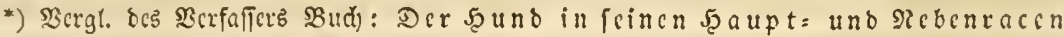

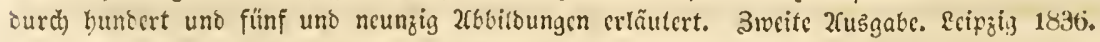

**) Rossmä s s c r Iconographic der Land - und Süsswasser-Mollnsken mit rorzïglicher Boricksichtigung der curopäischen noch niclit abgebildeten Arten. Dresden bei Arnold. 


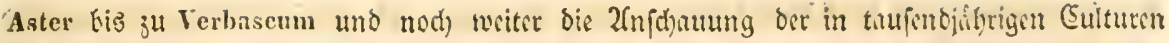
in sime linjahl son 2trten atscinmoer gegangener, ifren Trpte werloren bakenter Formen,

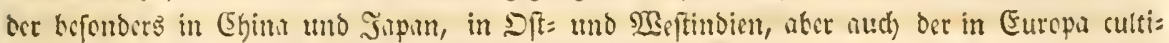

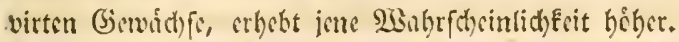

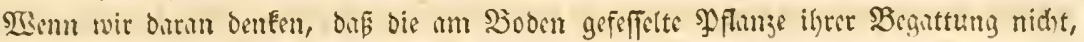
wie bis frei kemeglidge Ihier nothgehen Eann, bie Slatur aber immer fortfibth, fur Er:

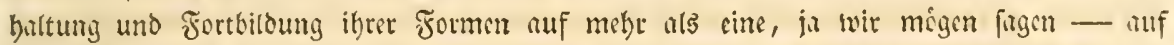

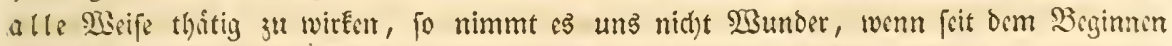

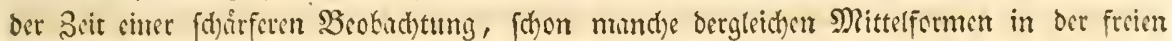

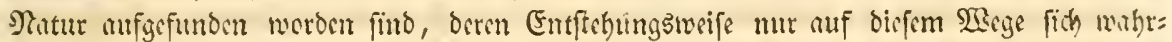

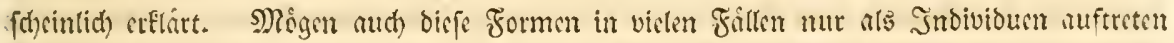

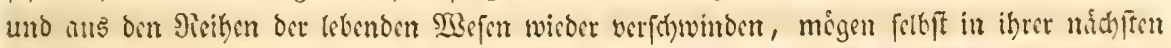
Gienerition, bufern fich bicfe twitflich vermiglicht, einjelne Jndivibuen zu ben Fermen dit

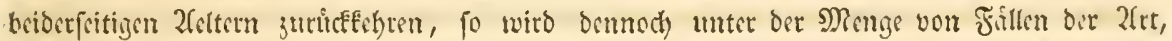

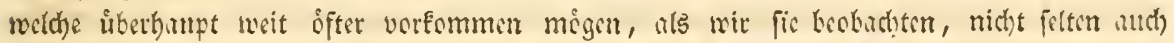
ber Fitl eintreten, dus ourch befondere, begunftigende ltmftinde, wohin vorzlighlit) der sl:=

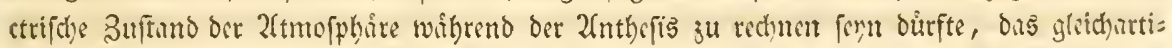
ge Fortbefteken bar Form an fich), allerbings gefidbert wird. Dicfe Eegunftigenden Umjtimbe

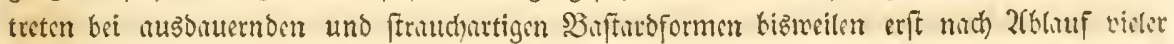

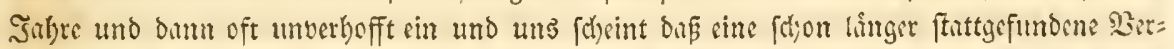

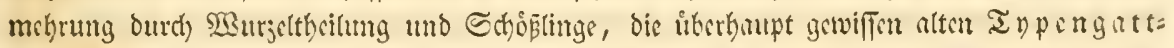

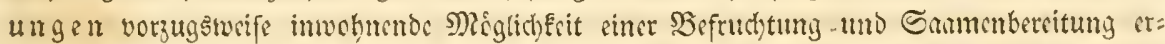

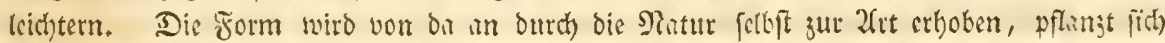

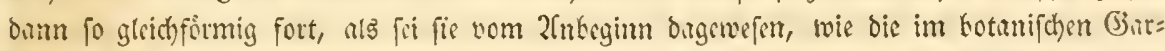

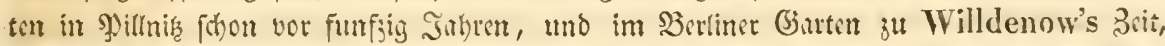

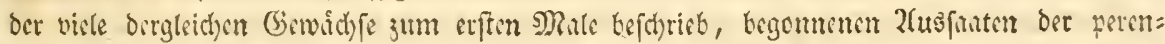

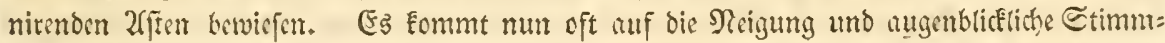
ung ber Betunifer an, ob fie bergleichen mun cimmal vorthandene und nickt wieder ants ber

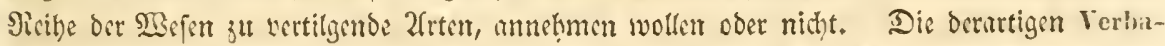

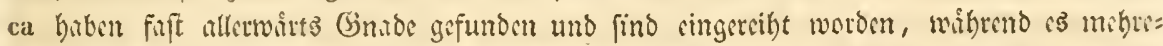

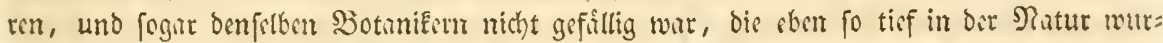

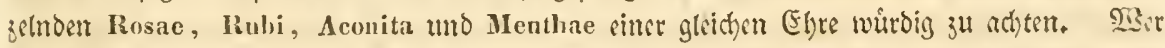

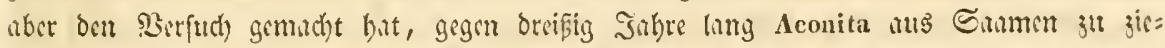

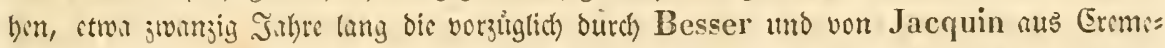

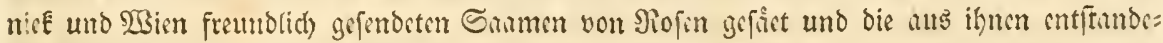
nen Pflunzen beofuchest hat und fis, fo wie Tenore's Menthae, aus dem Sarmen unter

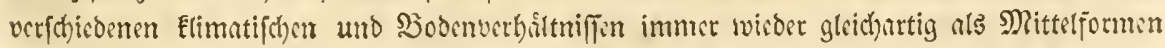

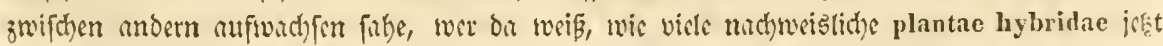

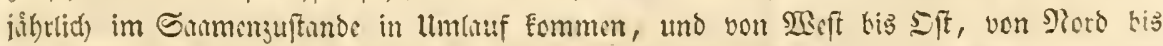

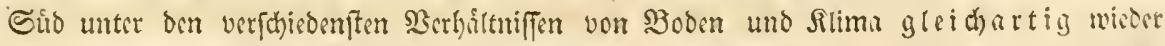

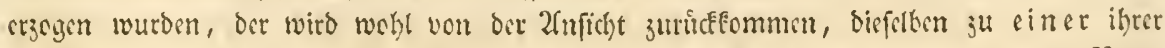

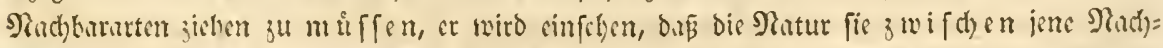

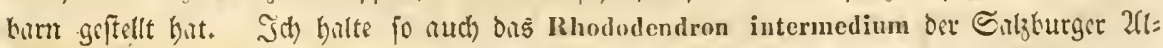

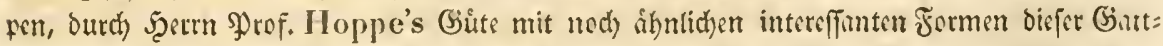

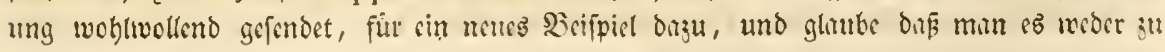


Rhod. hirsutum noch ju Rhod. ferrugineum zichen Eam, ob es ater fortbefteben und

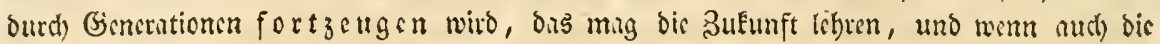

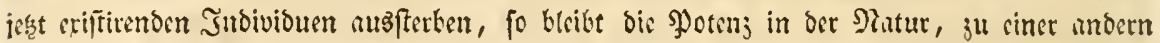
3rit oaffelbe פproduEt zu erzeugen, und beffen Fortbefteben vielleitht mad) Salyehunderten

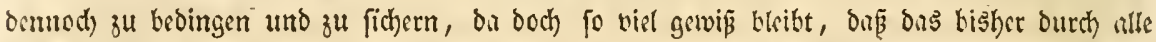

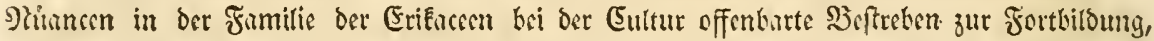

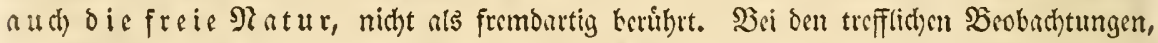

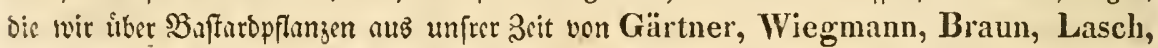

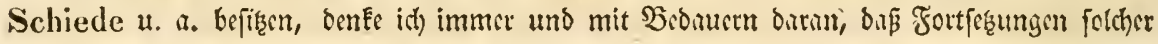

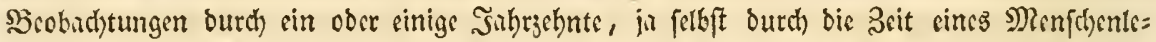
kens, fü bis Gsanje bes Naturlaufs, wie er feine Generitionen in Perioden von

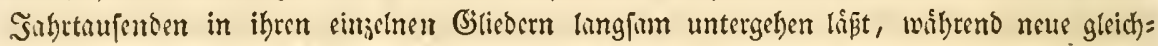

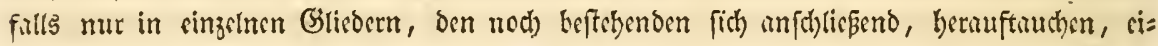

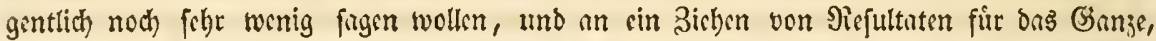

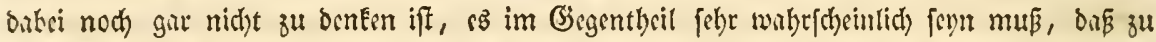

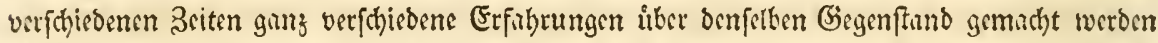

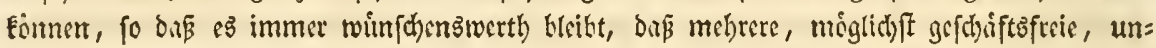

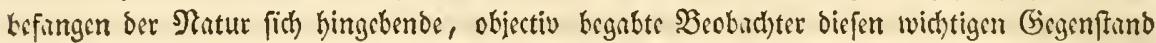

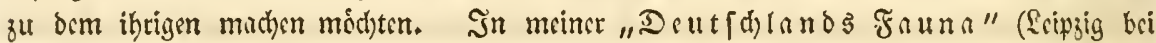

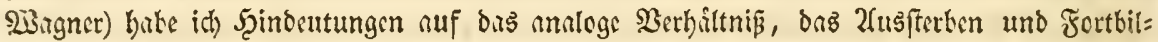

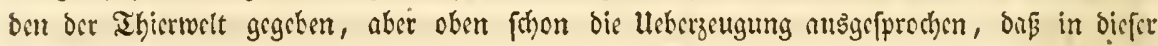

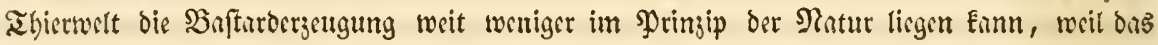

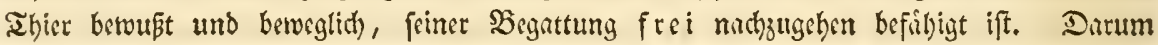

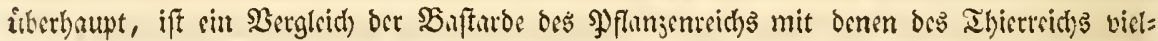

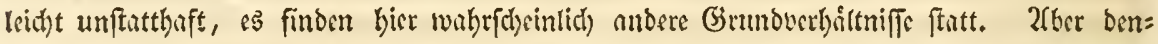
nod) veribuliden fich auch bicr, wic bon Eutomologen uno Drnithologen am Siften bes

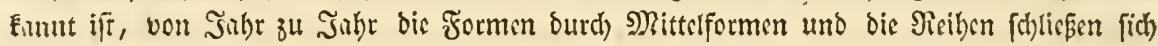
imniger burch biefe Mittclformen zufunmen, welthe bie alte 3sit wohl als $\mathfrak{B a t i c t i t e n}$ nn: zufpred)en vorzog. Dirfes Eintreten von Mittelformen mus immer vorfid)tig beact)tet wer=

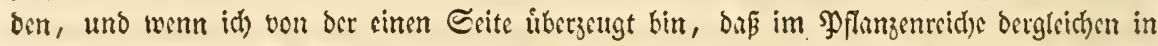

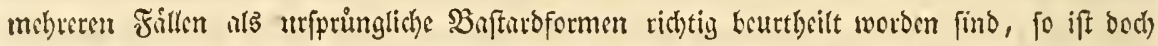

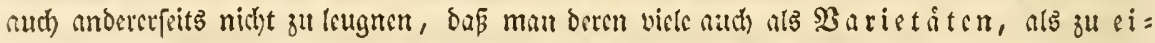

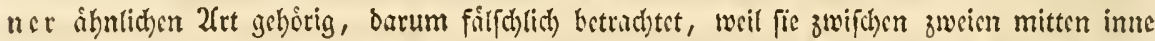
friben, ju bas man fie fogar unter bem Titel cincs "Ueberganges" zur Dereinigung z weicr in ber Natur gefonderter 2fren gebriud)t bat.

Die fubjective Joer von ben "Uebergingen," beren man fich ztur Sistrinigung

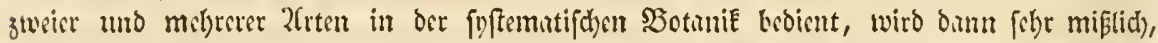

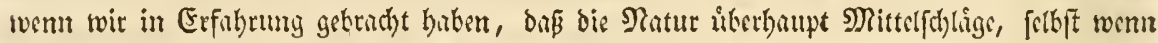

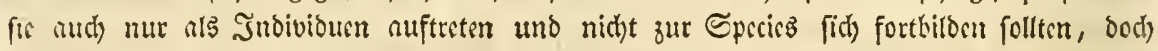

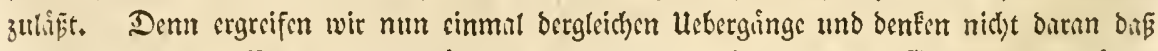

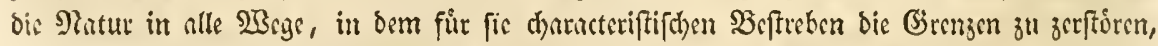

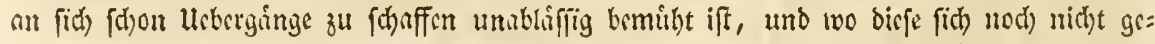

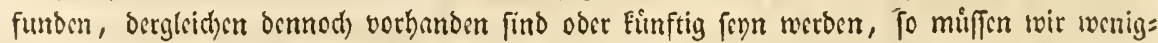

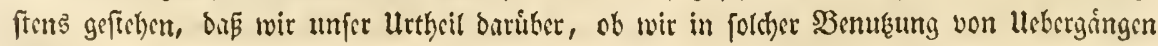

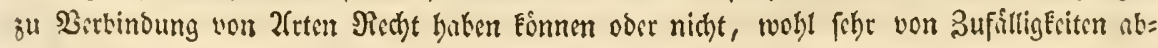




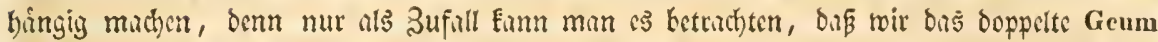
intermedium und bus Maulthier wie den Mautefel als Buffardersengniffe wirflid) Eenten gelernt baben und barum 2fnftund nehmen Geum urbanum und rivale, und 2 ferb und Efel

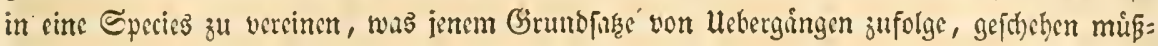

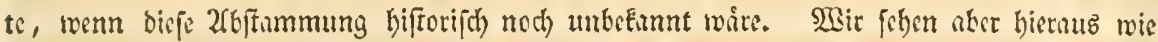

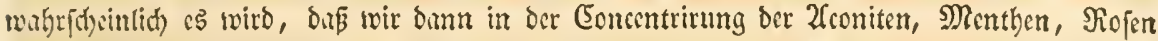
und Curifngen im Srrthume fenn Fonnen, on biefe und andere Ginttungen ibren 3wifhenfor: men wabrj(beintich mobl benfelben Ulepprung bereitet baben, wie bie Verbasca und Gea.

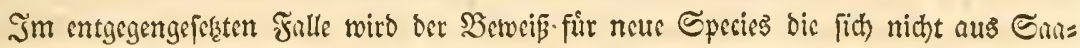
men gleichartig erjengten, was bus Sirterium für bie Erbebung zur 2frt cinigermasen bilden

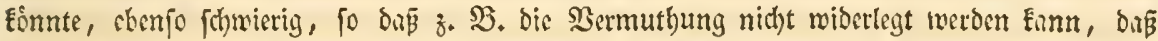
bie neuen Sempervira unferes wirbigen Koch, vielleidt burd) Sactburarten sinmal ver=

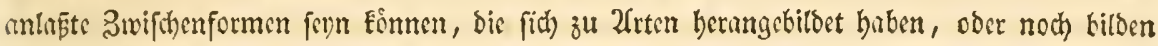
เwerdert.

Donß auch bier von bciben Sciten, mit sinem mathematifhen Serveife und ci= ner fubjectiven Bebauptung - po wie diberall in ber Natur - nichtsentfdic= Den werden Eann, Das bat Göthe fhon binlinglich crwiefen.

Scaben wir uns aber hier erlaubt dis "gewiffe" und bas "begrenzend =ab fon=

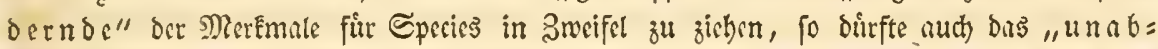

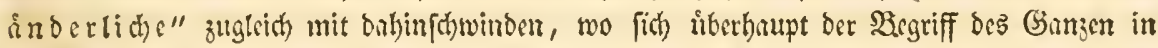

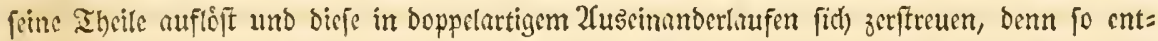
filtet fith ber Typus immer tiefer in feine Denterotnpen, bas 30 orbild fareitet in feine

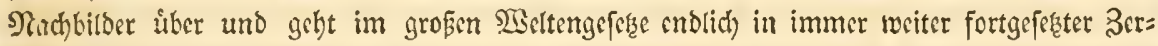
theilung fich anflofend und serfhwimmens - in ibnen unter.

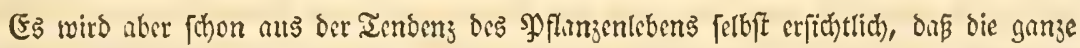

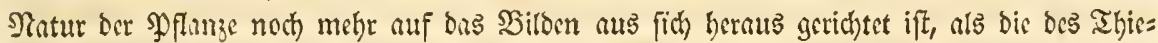

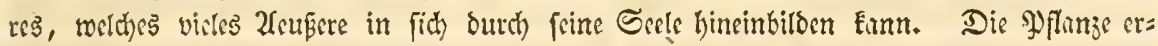

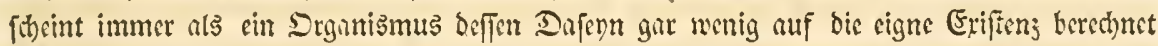

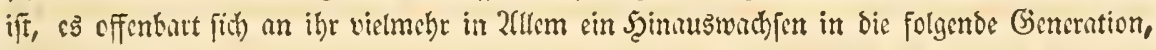

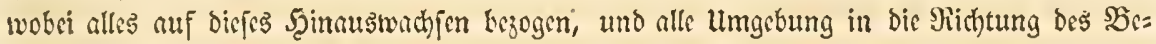
ftrebens, chen bicfes Rebenşiel zu erreichen, mit bincingejogen wirb. Darum alfo, da bie

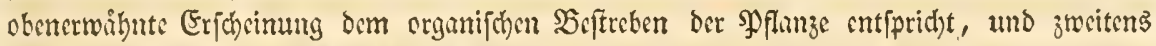

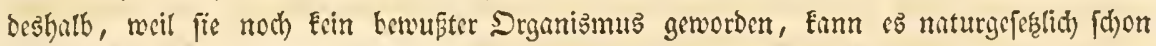

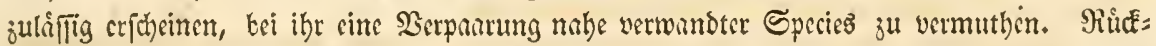

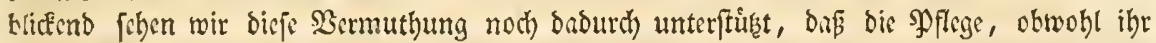

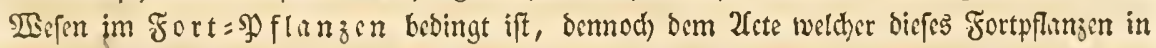
ber boheren Ephaire der Sarmenerseugung verwirflicht, nicht nadjzugehen, nidht wie daz Thier feinem empfundenen Tricke zur \$̧aranth, in Drtaberwegung zu folgen vermag, denn

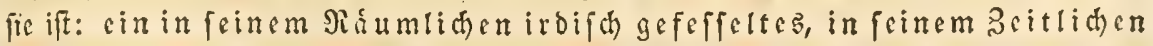

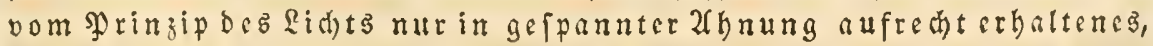
feiner Eriftenz nod nidht bewustes Ģefdipf.

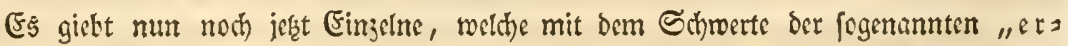

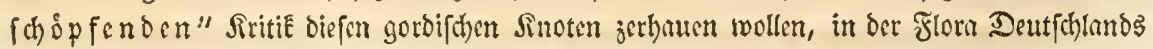

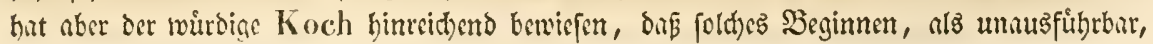


mobl immer ein blofes Soeal war, um fo mebr, wenn man in bie Seiten zuritcfigeht, wo die Eprcies auf J̈ndividuen beruhten, under hat trefflith ergrimbet, wie foldhe Individuen in

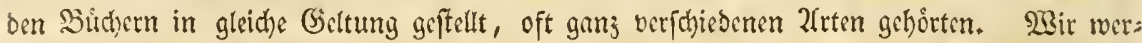

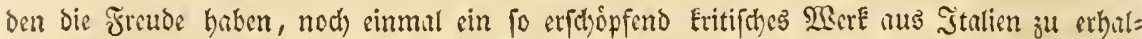

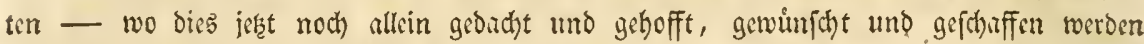
fann, - winn - wis wir herglid) wunf(hen - Der unermitdet thitige Bertolone

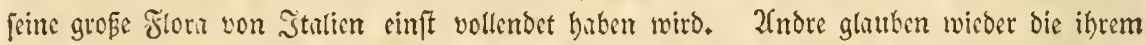

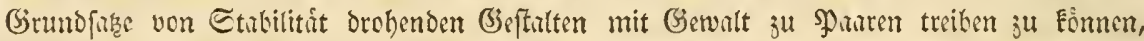

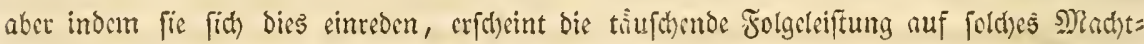
wort nut momentun, wie bas ganje \$ुorinzip der Stubilitit es aud) แut verhngt, benn bie Formen lofen ifre Fefiel und mathen fich frei um in immer nod) mekrern Formen fid) lofen

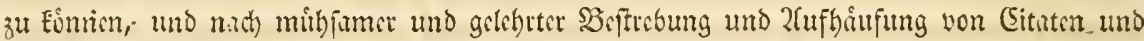
Cynomymen von Theophrast bis auf unjern wertfen Freuno Opitz und Serrn Ortmann,

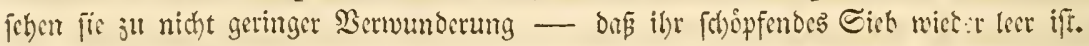

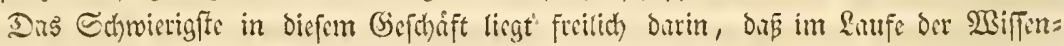
(h)ift voit Linnce bis auf Bernhardi nod) Eein Menfh gefingt bitt, was cigentlich 厄pe=

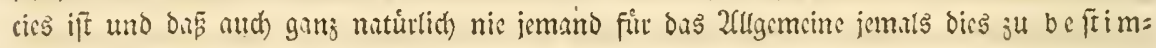
men vermitg, weil chen Species nichts beftimmbarez, fonbern ctwats lehendig, wie bis

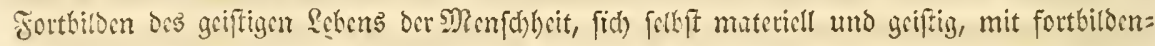

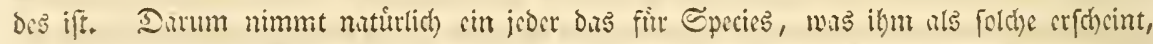

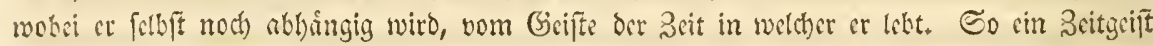

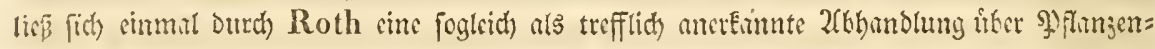

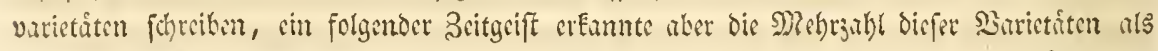

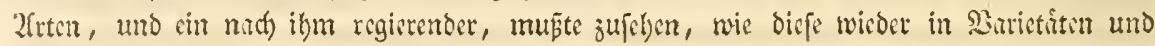
2litin fït) aufloften.

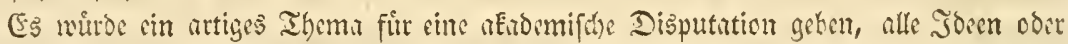

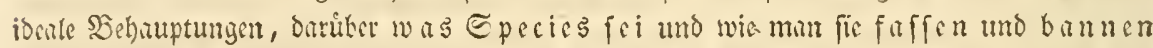

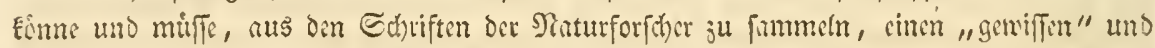

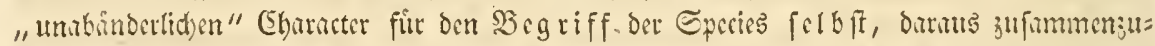

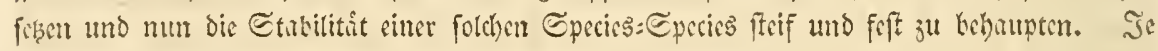

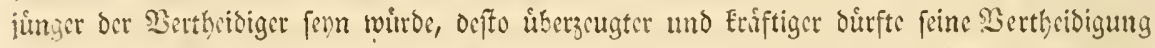

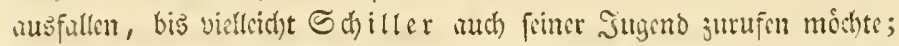

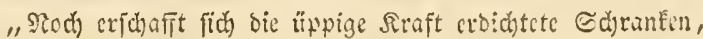

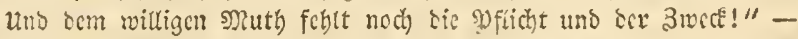

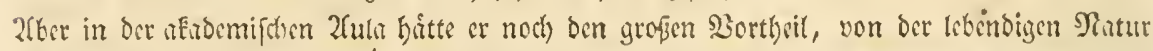

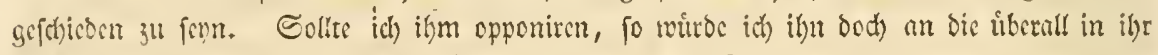
tonend: Ctimme erimern: jede Species hat ibren Iypus!

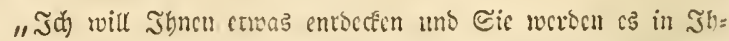

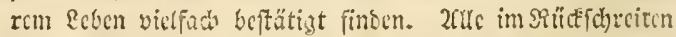
und in ber 2ufulbijung begriffenc Epod)en find fubjectiv, bagegen afer baben alle worjeyreitenoc (Eportacn sine ob= jective Richtung. Unjerc gange jeşige 3cit ift cine rülefichritento, benn fie ift cime jubjectioc."

(5).

7) Die "gewiffen unabauberliden MerEmale" liegen ůkerlyoupt nur in

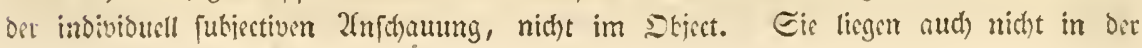


Dauer dez Beobactetns unb in ber Erfahrung, wie wire es fonft móglid, bis bie fo treff:

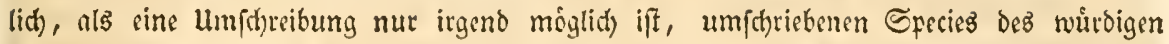
Koch, nod) nbermals burdh Spenner zufammengezogen werben foonnten? - batte un= fer trefflither, nummehr ourd) Oken's Segen perfönlid) fennen gelernter uno peitberm nur

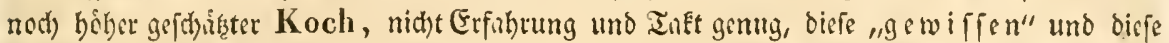

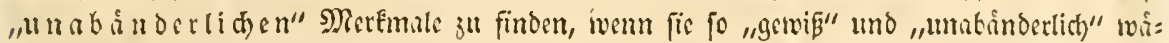

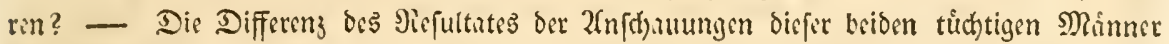

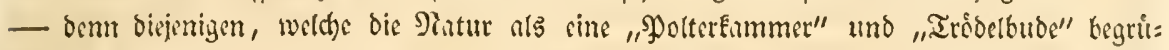

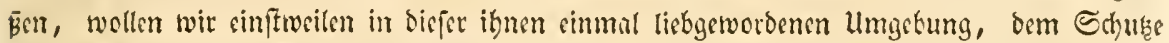

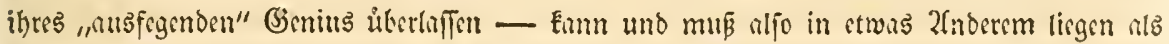

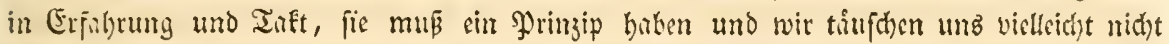

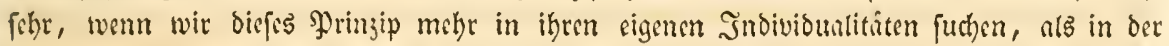

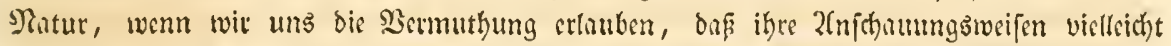

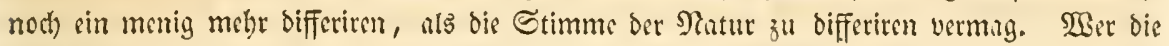
Differenzen in der 2fnfdonutng der Statur, von z we i tuddtigen Mannern gegeben lief't, dem

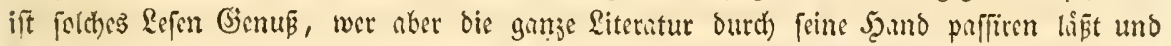

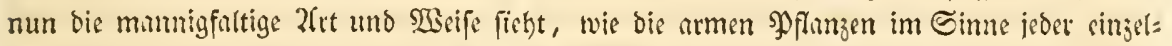
nen Eubjectivitít wisoser auf sine andere 2 seife erfinderifo) gemartert und gepsinigt werben,

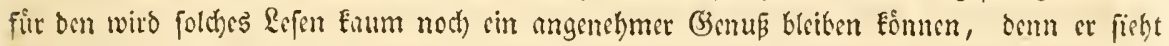

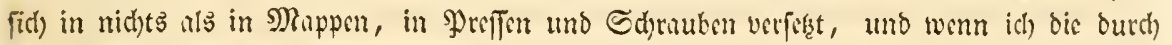

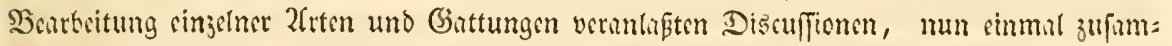

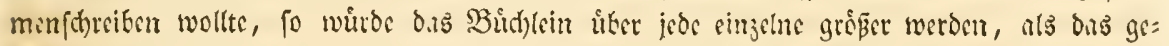

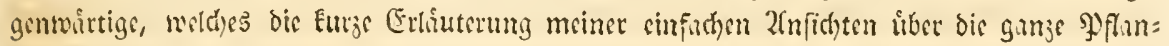

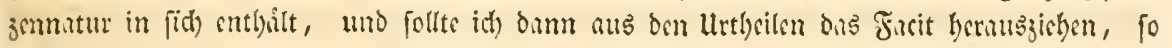

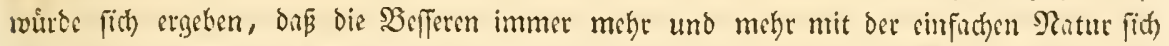
befreunben, und ber allen wirbig vorangebende Koch, aud Viola silvestris erkinnt bat, uno bie, wisder sinem ganz verfdjicbenen Inpus entiprungene V. Riviniana stenfo, wie bie in feiner Flora unterbrukte, Dann mich) Treviranus fremnolidger Erinnerung, Freunblith angenommene Cardanine lhirsuta, erfannt baben witrde, bitte id) cin cinzigess= mal bie Freube haben Eennen, dicfen werthen Eolfegen, wie ith mit fo munchem andern

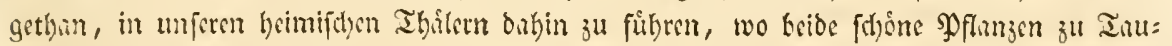

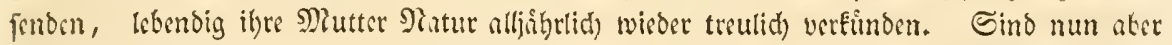

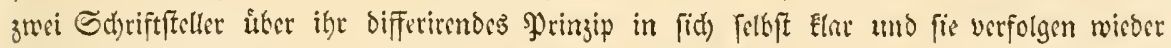

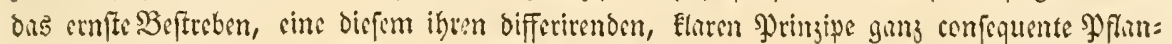

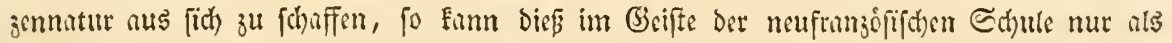

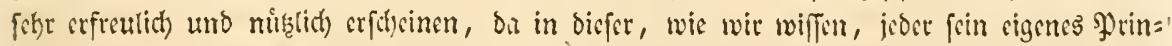

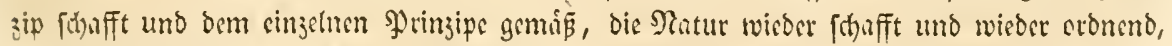

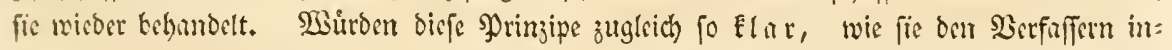
wohnen follen uno milfien, nach ausen wiebergegeben, fo follte umb mispte confequenter=

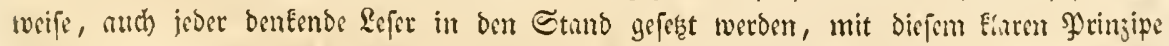

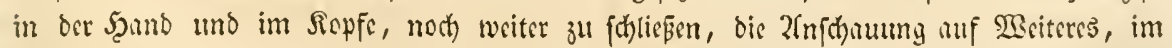

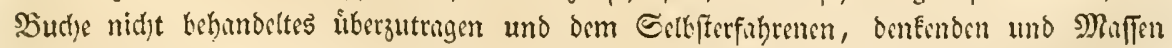

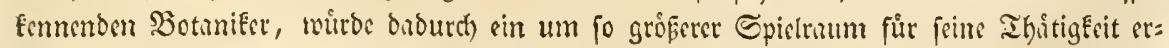

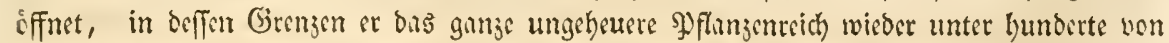

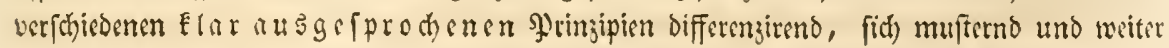




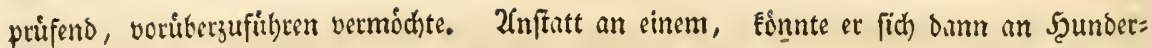
ten von nuturliden Specicz: Syftemen erferuen, wákgend es Adanson nur bis auf fed)zig Fumilienfy/teme geberatbt bate

Der 2fmfinger digegen wird, als foldher, boch immer etwas fowieriger llebung nod)

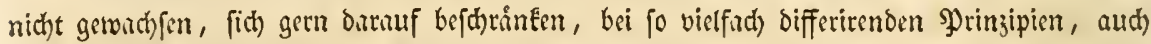

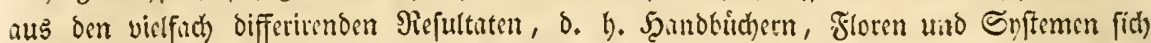
an einen Betribrëmunn zu batten und deffen treuer Speciesbotanifer zu werden, fo wie er virlleicht eines ganz 2fndern (Seweihrsmannes Familienbotanifer roiro, ba nicht 2alle ibre Epccies nad) Fumilien aufgefihgt buten.

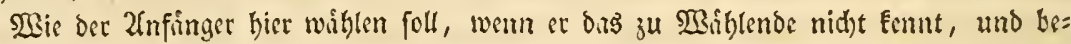

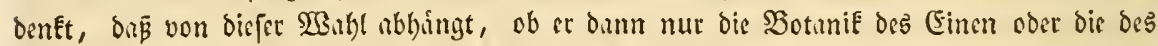

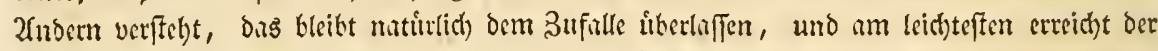
feinen Sweck, ber nicht wilhlt, weil er nicht wíhlen Eann, fondern bem folgt, wats ifm bus nichite ift, und dief dufiu, was ifm befhieden war, anfieht.

(Eg ift noch eine ruffallende, nber bod) vielfach) zu beftitigende Erfabrung, dor bie irrgften Eiferer gegen die 2(nerfenmer ber umpdutbigen 2(rten, weld)e diefe im Budt)e ber

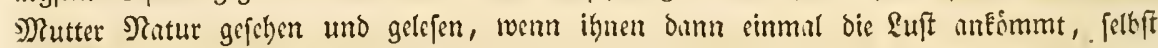
2frten zu muct)en, nichtz Befonderes zu $\mathfrak{B}$ ege bringen, weil fie fie bann eben nidjt alts ber Natur nebmen, fondorn nur uแb fich folkft. *)

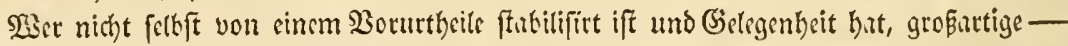

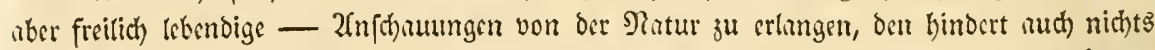
meist, eine reine und lebendige 2fnjid)t von ber ganzin Natur zu gewinnen. Der bertilfmte Ehrenberg fammelte feine ungeheueren Erfahtungen in brei 2 selttheilen uno weder dats

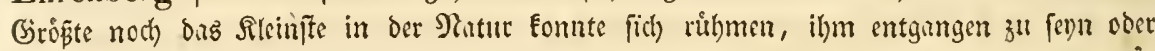
yon ihm bevorzugt zul werden, alles foste er mit gleid)er 2fufmerefomécit und Riebe in's

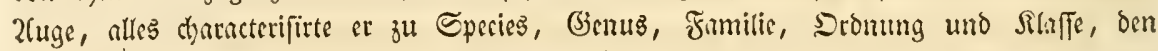

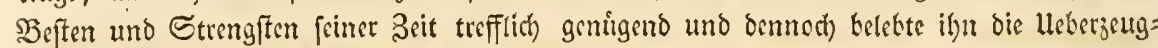
ung feines tieferen Seiftes: "Oenn wer um feftbegrenjte 2leten uno hber= haupt um unbegrengte Raturgefefe ftreitet, oer mub wohl in ber Natur felbit fíd nod) wenig umgefeben baben. Der einfiditabol= lere Naturforferer wird bie gottliche, von erinem Sterblid)en zu ers

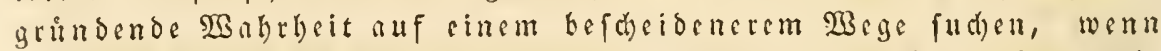

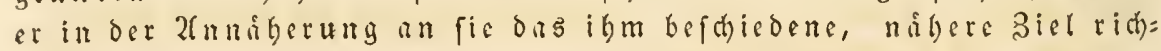
tig erEennt. (Qui enim de firmis speciebus atque uaturac quiluslibet firmis legibus ecrtant, in ipsa naturae contemplatione parum rersati sunt. Ingenuns naturac contemplator morlestins stulium in in impendit, ut veritati, quan praeter Deun, quem rocamus, mortalium nemo assecuturus est, peletentim suas appropinquat cognitiones in eoque animi oblectamenti nunquam attingendum, semper vero propiorem terminum lialset. ${ }^{* *}$ )

*) Einen neucn Berocis für bicfe (Erfabrung, gibt wicber bie in bicicn ragen angetangte,

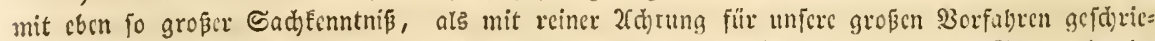
bene 2ibbanolung von v. Vriese (Wiegunan's Archiv 188\%, S. 112 - 118), 100 bemics

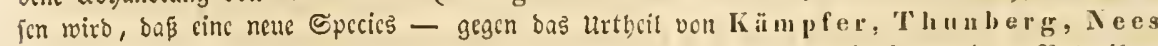

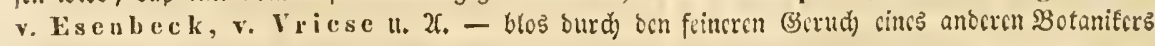
unteridicben roorben ift.

${ }^{* *}$ ) De canibus africunis. Symb. phy's. mannif. 


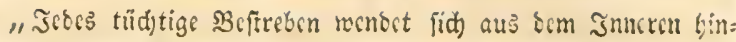

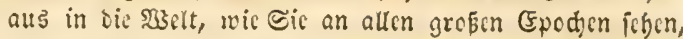
bis wirtict) im Strcten und 3orjdyrciten begrifien und alle objectiver Ratur find."

(5).

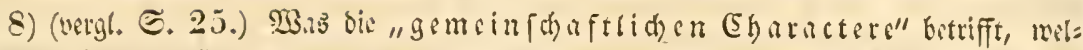

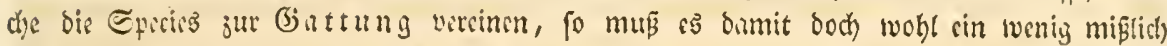

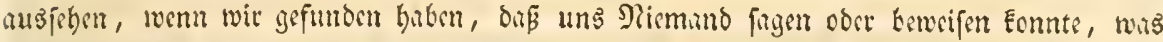

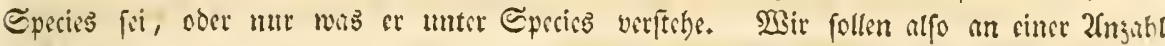
foidyer, dem 2futor, befien Epecicsbotmifer wir geworden find, belickigen Species, cinn

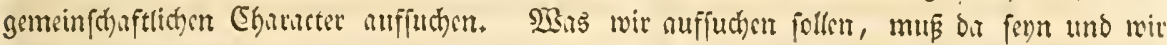

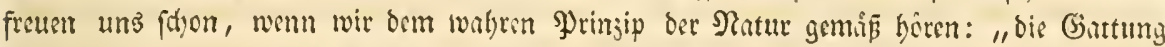
gicbt ben Egharreter," benn fie wirft fo naturgemis, objectiv auf uns sin. Der Ehal=

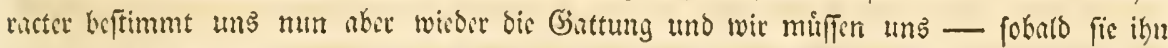

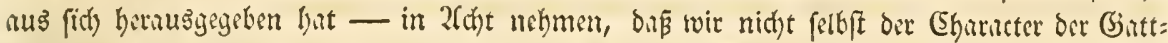

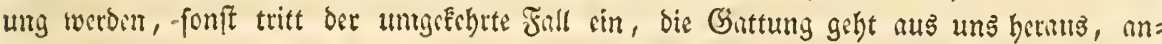
fratt it uns himein.

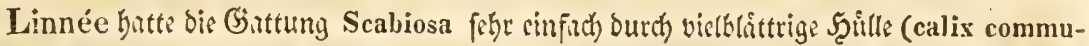
nis polsphyllus) und oberen boppeften befondern Rild) (proprius duplex superus), nimlith

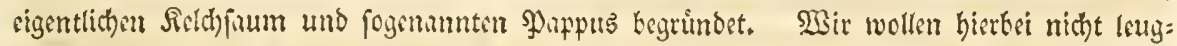

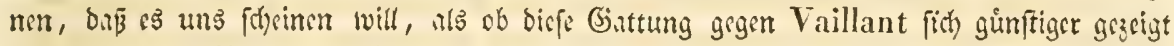

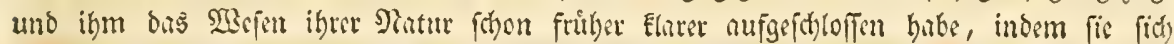

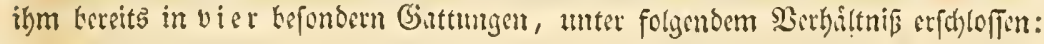

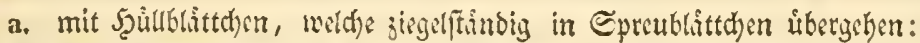

Succisa: mit viertbeiligem Gorollenfaum.

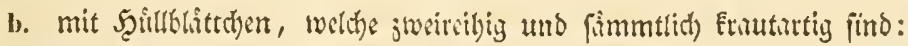

Asterocephalus: (mit fünftheiligem Eorollenpum, an ben tumb/tindigen wcit greß̈er.)

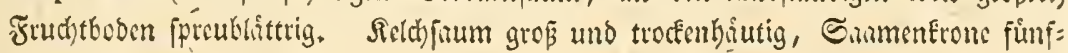
boritig.

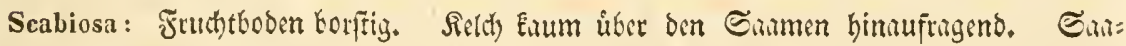
menfrome vielbortig.

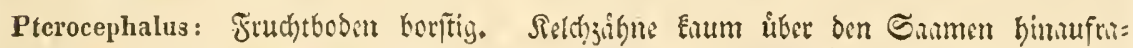
gend. Giumentrone fedurartig.

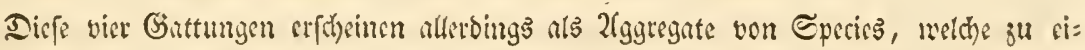

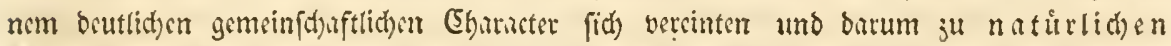

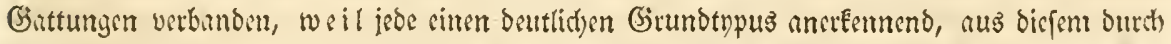

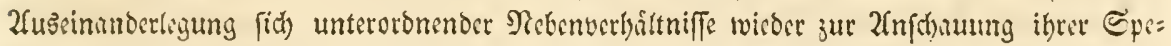

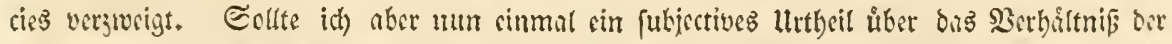
2rnjoumung von Vaillant uno Linnée in Bejichung auf bicfe (5intumgen, ausfpect)en, io

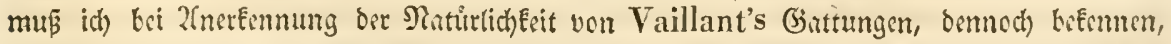

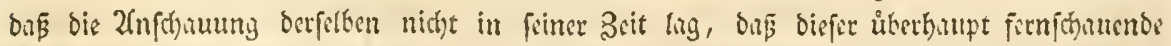
Geifit, in fich forcu sine Seit von La Gasca uno Schrader anticipirte, in bonen st

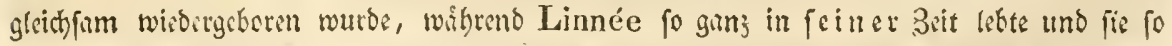

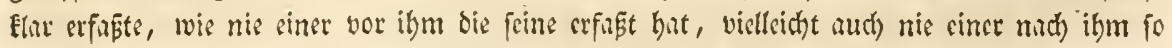

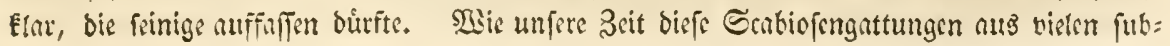




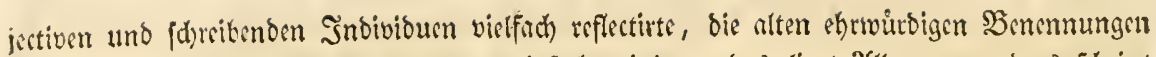

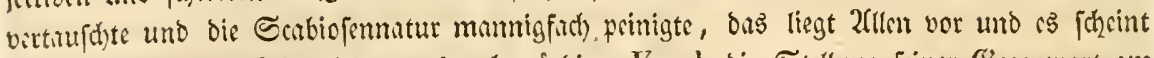

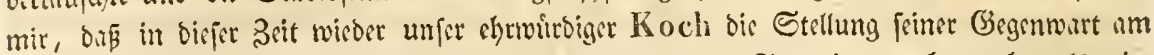

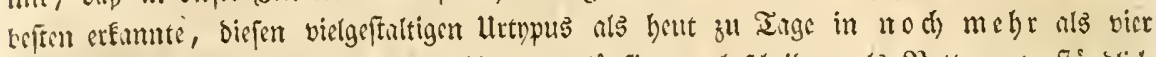

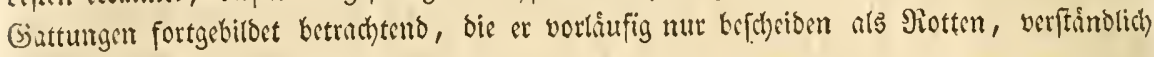
getrennt bat.

unjere waderen Bearbeiter von Dentfdhlunds Flora: Mertens uno Koch pprnchen

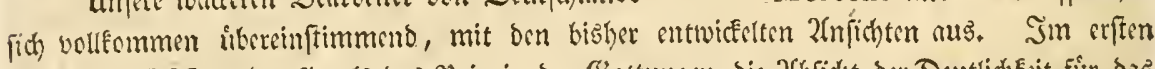

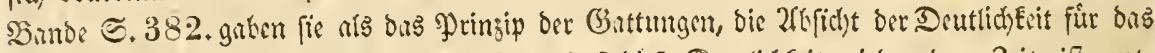

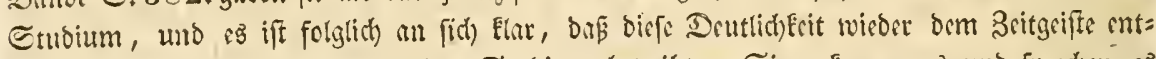

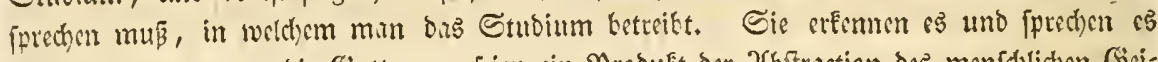

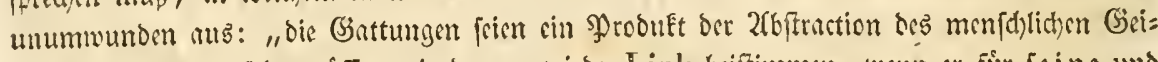

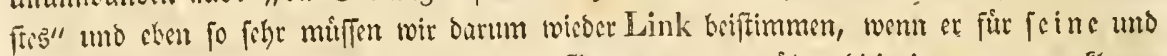

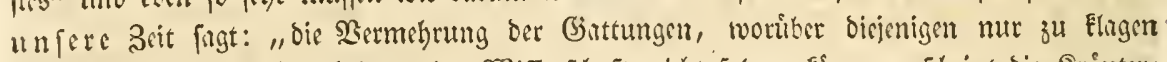

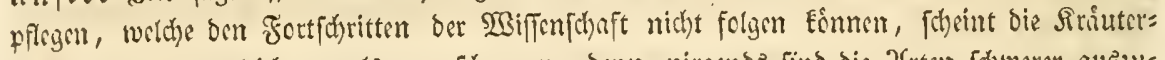

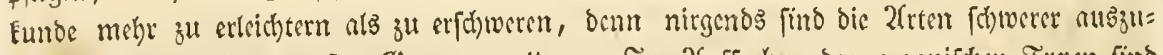

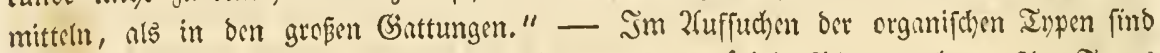

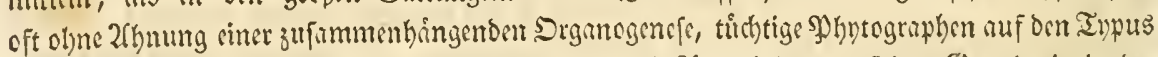

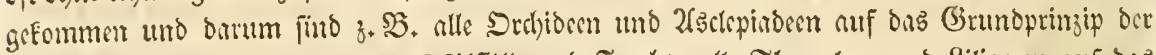

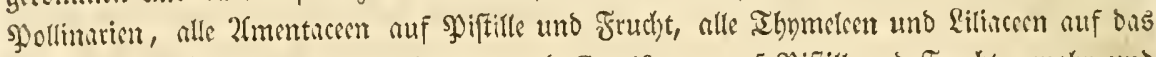

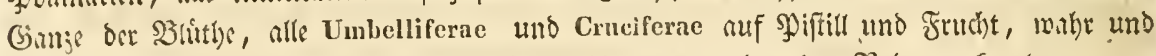

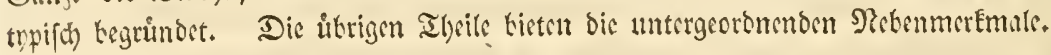

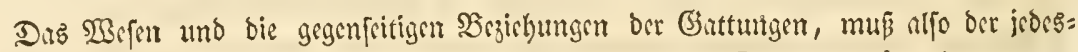

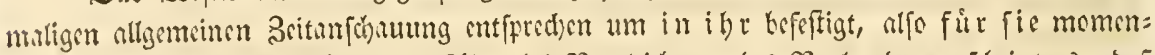

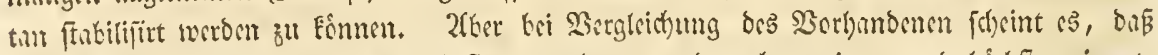

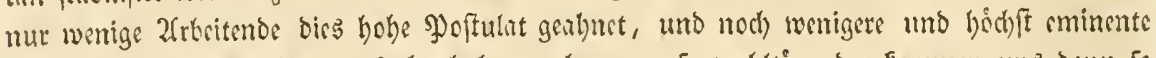

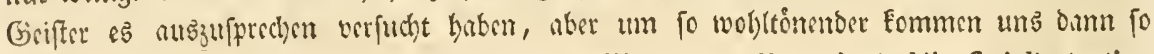
gervichtige $230 r t e$ entgegen: "quoique les familles naturelles aient déja fixé l'attention particulière d'un grand nombre de botanistes, leurs genres ne sont encore fondés ni sur des bases assez solides, ni sur des rapports assez naturels. C'e st cependant la découverte de ces rapports qui est le but principal quelondoit se proposer anjourd'hui dans l'étude de la botanique," Kunth Mćm. du Nlus. II. p. 62.

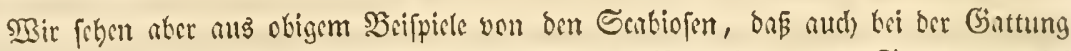

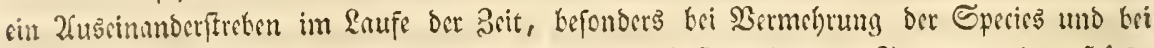

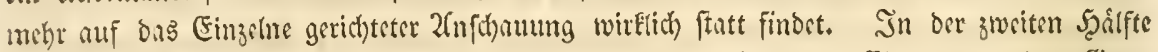

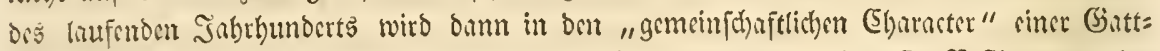

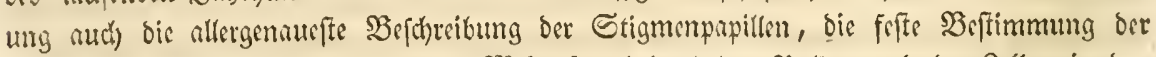

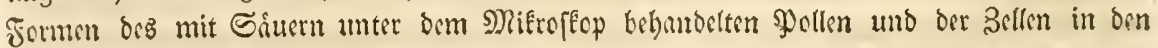

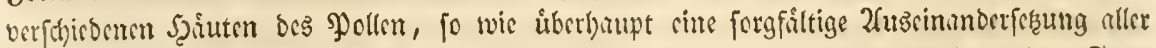

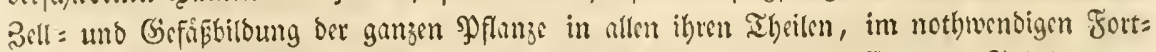

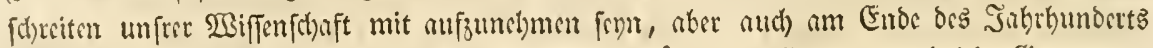

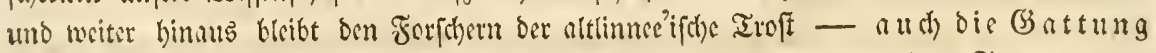
bat ibren naturlicben, mit fortfdreteno fid) entfaltenoen Iypus! - 


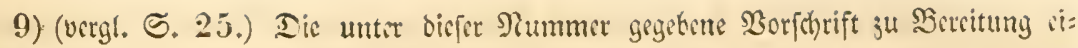

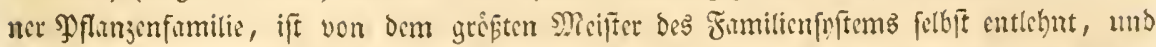
ficherfich auch bie kefte von allen, obwohe wir gen gefteben, buß bie meiften Berfertiger son

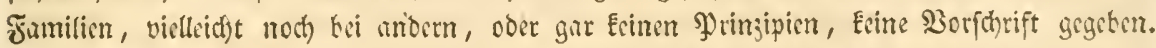
Dic Jngredinjen - bie Gattungen - merten dirin als gegeben vorutegefert, wir

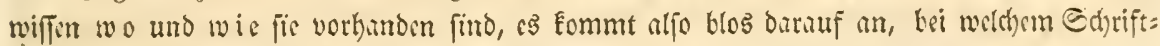
fteller - als unferm GirttungsbotaniEst - wir fie auffucten wollen. Jit ater dors

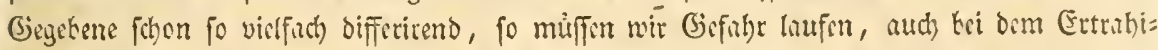

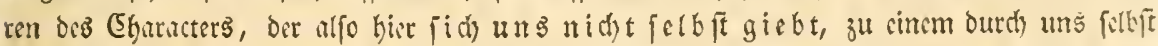

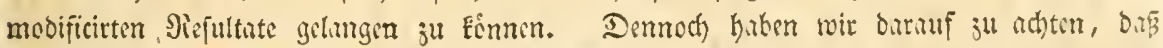

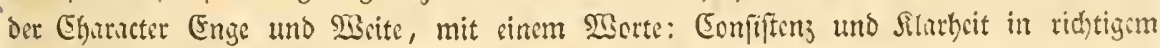
Ginde erlunge.

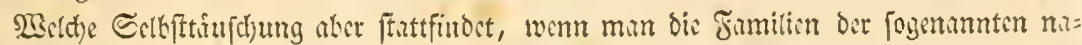

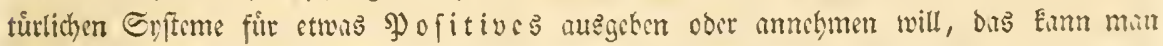

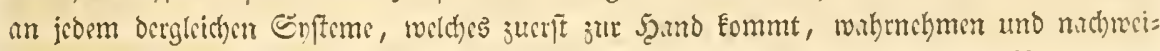

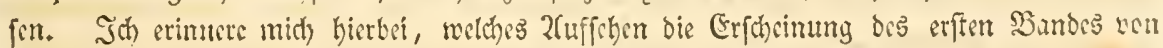

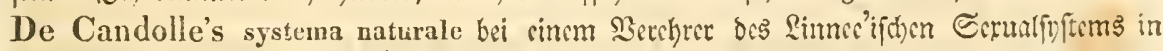
Ungarn gemadt, und wie es biefon vernnlast hatte, bie Unbeftimmtheit bes burin hert=

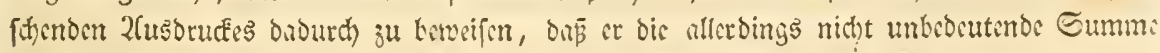

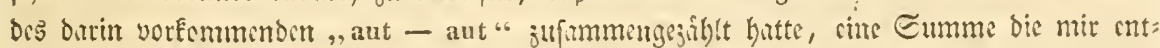

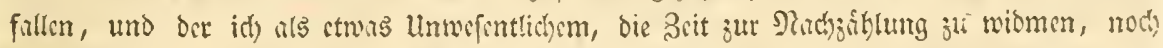

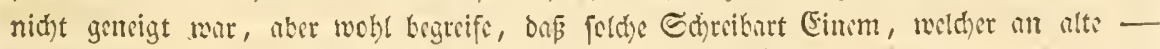

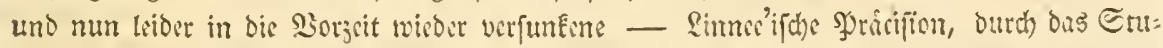

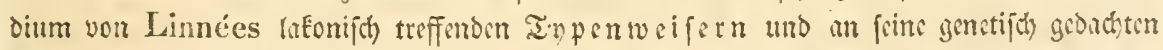
characteres naturales gewîhnt war, in cinen gewaltigen Edfrecken ju verferen, im Etmos

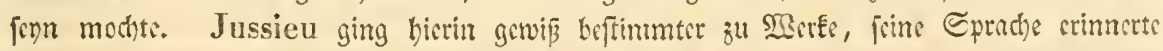

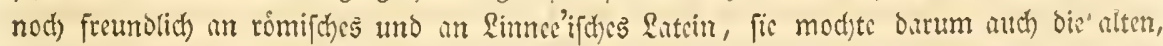

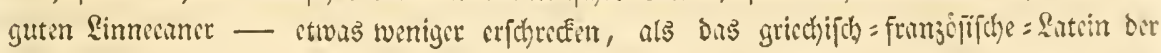
neufranjeifidyen Cdule.

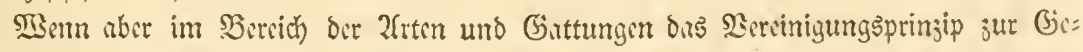

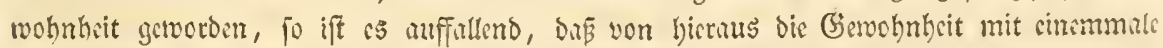

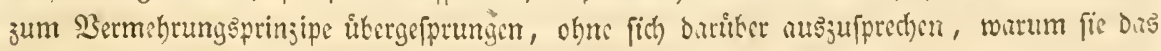

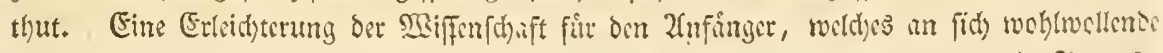

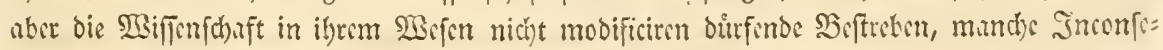

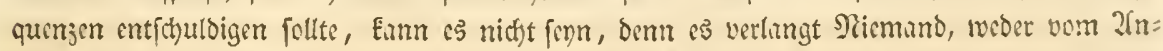

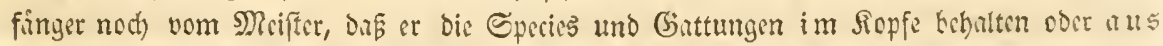

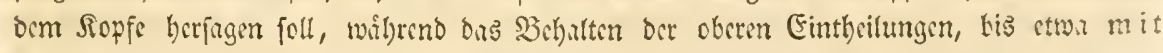

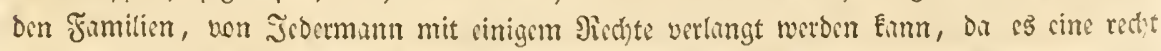

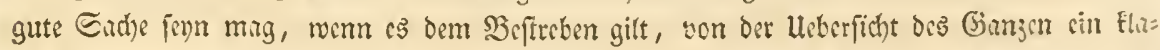

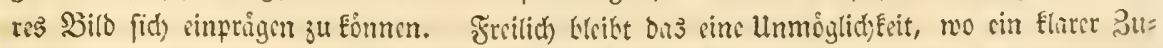

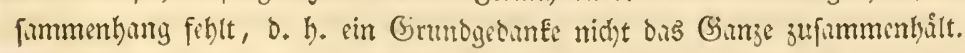

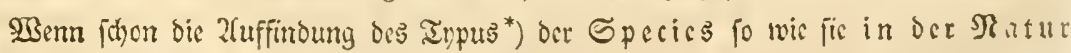

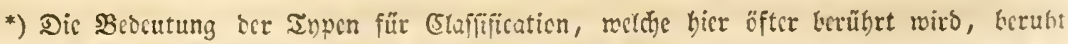

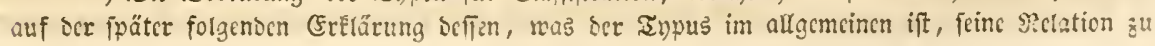


(iid) in oer 3ait in ber wir fie brobachten offenbart - wir wifīen bas biç nicht fưr bie Epcries in ben $\mathfrak{B}$ u fo ftellt bie Gattung, als Jintegriff noch mehrerer Momente, ihre 2fnforberung an unfere

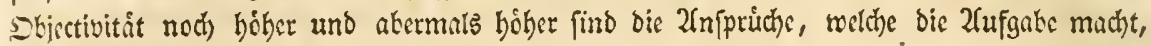
oen İpus der Familie in ber $\Re$ atur twirflich zu finden.

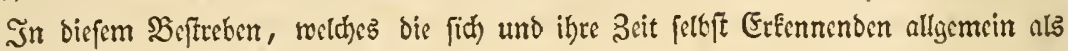

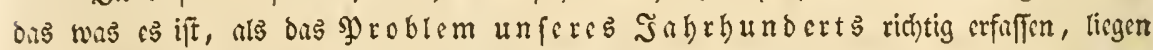
foron einige wenige, aber treffliche Seiftungen vor. Sh gefteke gern, bas mich aber, auser ben grofen 2rbeiten unjers licben Martius uno Mohl uno Nees von Esenbeck, Eum atwas aus Drren Bercich fo angefprochen bat, als Eisengrein's "Familic ber Schmetter=

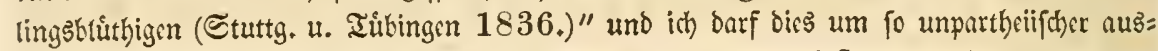
fpred)en, ats berfelbe meine Eleinen 2frbeiten nidht Eennt, wenigftens nur ben Conspectus citirt, wathrfectinlia) weil peine 21rbsit fhon fruhber vollendet wurbe, bevor meine Flora ger-

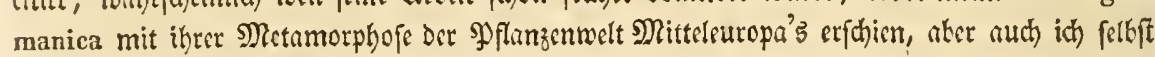
nur aus biefem feinen $23 e r e$, ifn Eenne. *)

oen Elajifificationgftufon if bercits in Dor Flora germanica p. XLVII., "rationes methodicae“

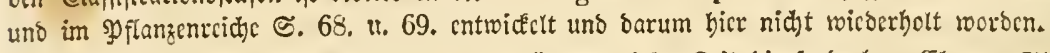

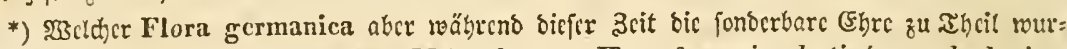

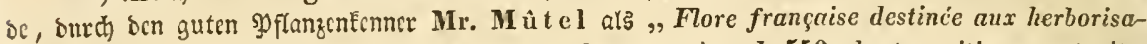
tions etc." uns mit cincm "Allas représentant les caractères de $\mathbf{5 5 0}$ plantes critiques extraites des belles centurics colorices de l'iconographic critique de Mr. Reichenbach" «. ฤ. mit jo victcn

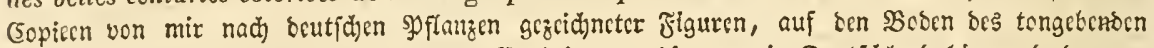

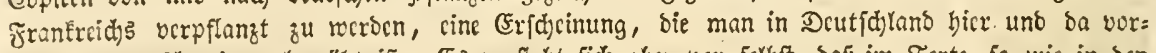

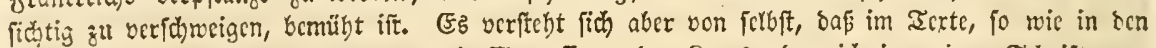

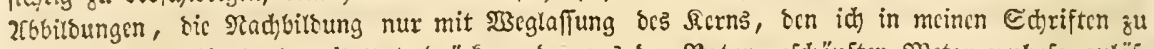

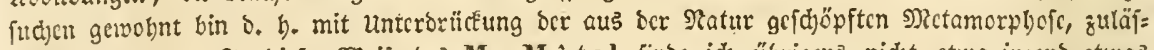

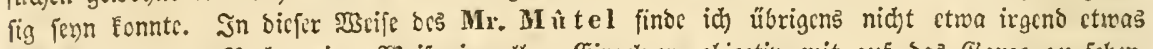

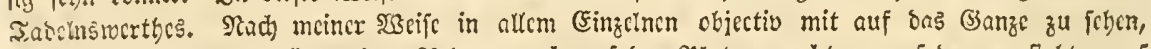

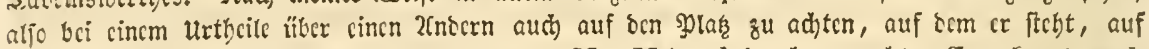

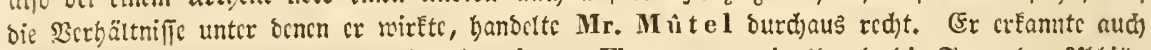

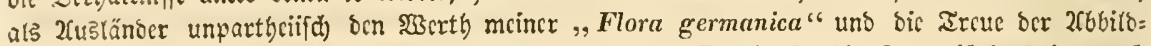

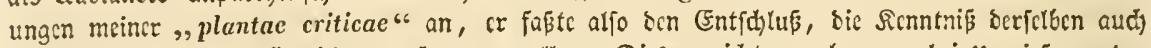

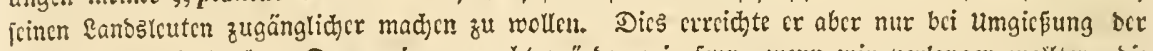

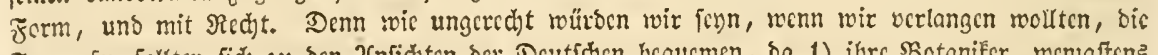

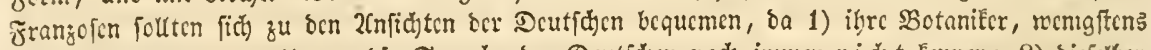

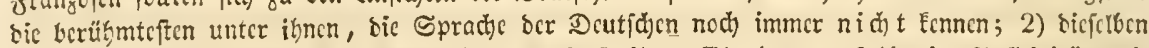

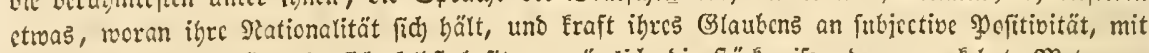

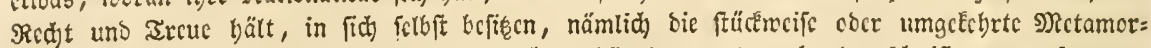

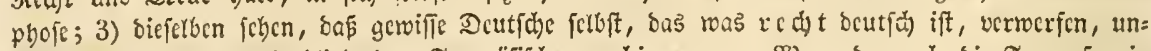

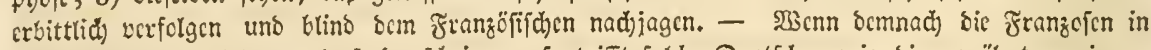

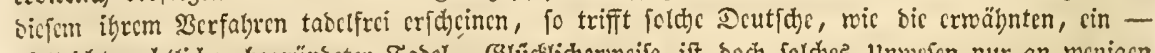

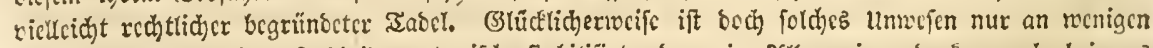

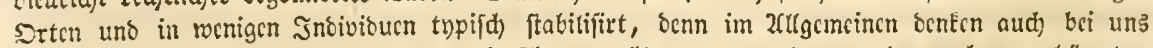

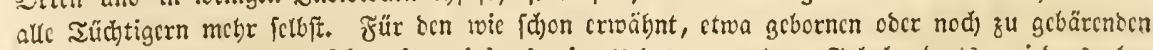
Eülffigen Serfaffer cincr, historia rei herbariae " bes neunzchnten Jalyrbunderts, wirb cs abcr

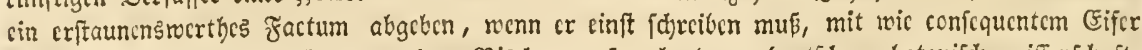

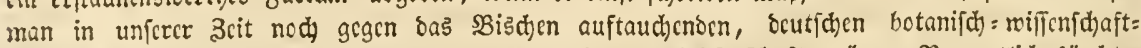

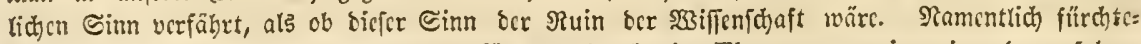

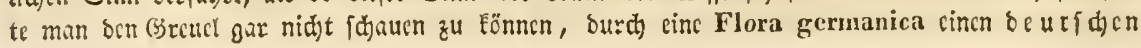


Eisengrein iff mir in feiner ausgejsidfneten Sdjrift als derjenige Botuniker erjdjies nen, von bem Vittorio Alfieri, jene hobe, cole Slatur, fdyon voribnend fagte: "o feltene,

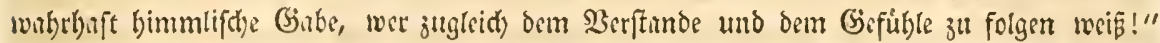

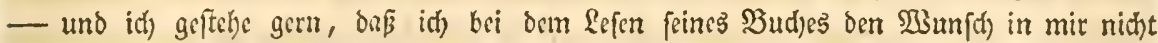

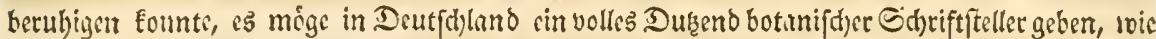

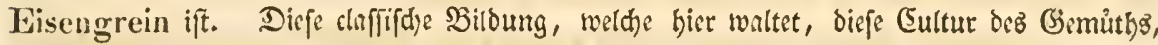

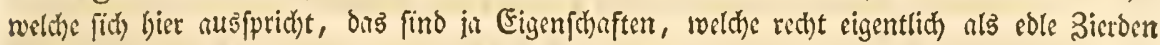
und Iypen bem Character bes Deutfhen geboren, warum ift denn alfo dis Dusend in

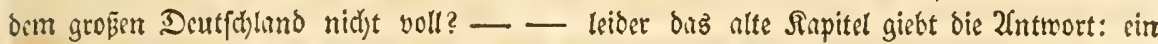

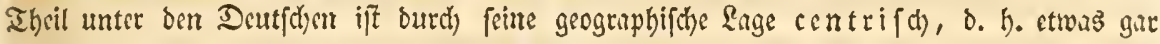

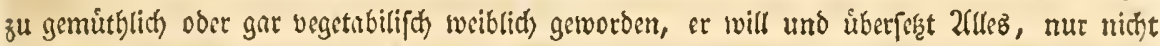
methr - fith felbft.

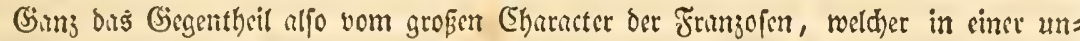

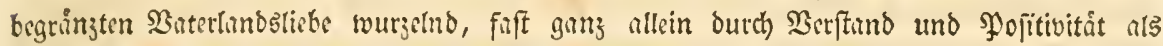
grobie SAtionalitit emporblitht und alles Fremonrtige nur bunn affimirt, wenn ec es minn: (id) ju beherrfaten vermag. So ftudirte und achtete Jussicu, wie er mir felbft fagte, un= fern Jos. Gärtner, um feine ciguen 2Cnfichten befriftigend zu unterfitusen und thist

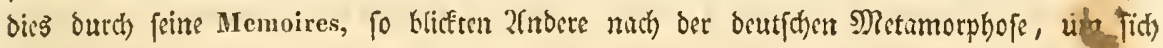
cine anbere zu machen, to tourde moine Flora germanica jur Flora française uno mei=

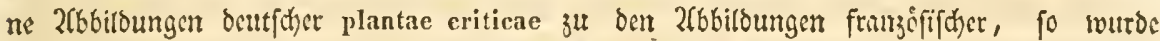

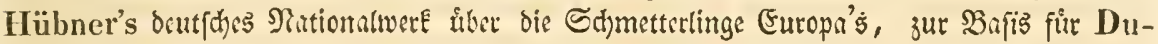

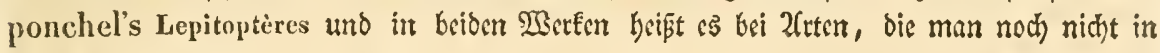

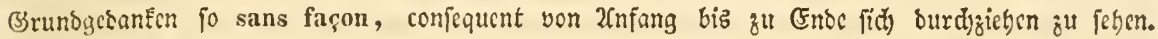

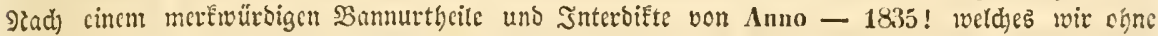

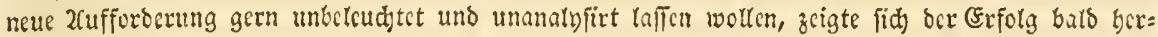

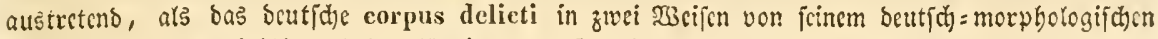

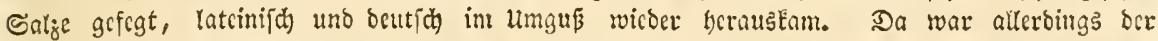

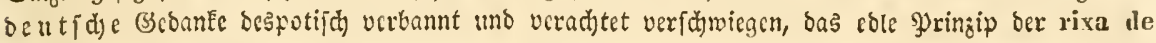
lana caprina wicber gewonnen, was ftatt Iaufender bon Seifpielen fifon bas cinzige von $21 n=$

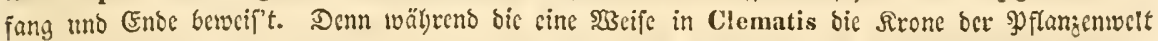

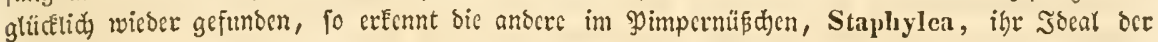

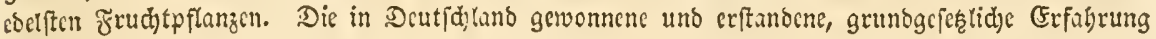
abcr, baß bie Berbreitung ber $\$$ flangen morpho:ogifd und geographifd in =

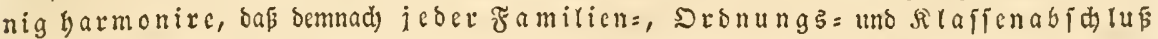
bas Scöbere morphologifd uno geographifd oexcint, ocm objectiv fehenten 2uge vor= fübre, baf వlcutfdlano alfo, ba.

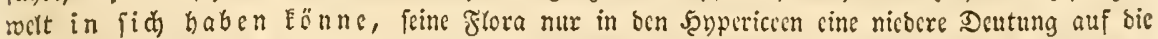

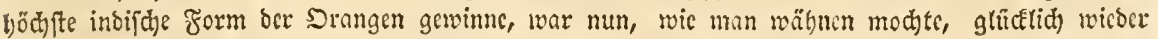

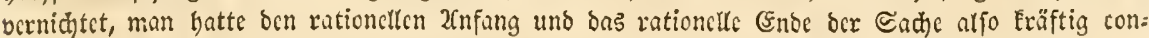

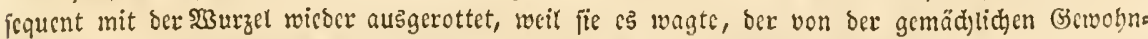

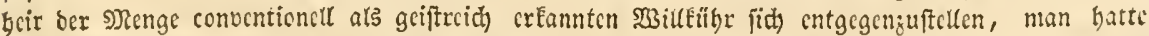

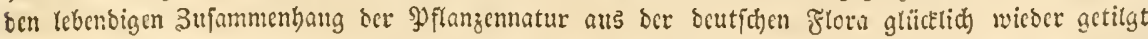

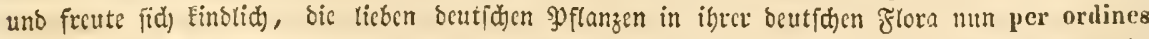

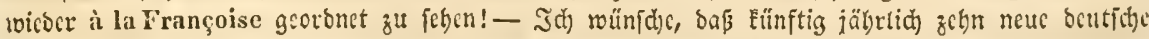

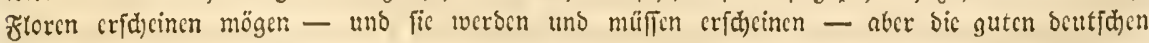

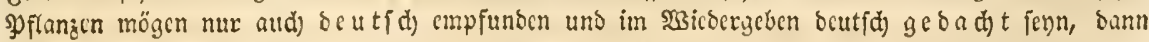

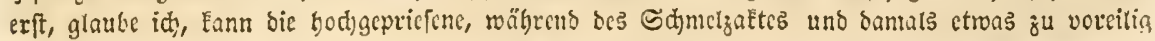

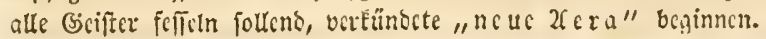


nerbulb ber franzofifaten Sirenzen gefurben: "man fand fie ba und bort und es mitb bas mit enden, baß man fie aud) in Frantreich nod) findet!" - 2ul biefe Erjocinung ift

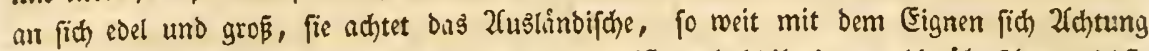

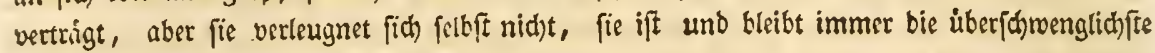
Baterlandstiebe, weldbe die $\mathfrak{B e l t}$ Eennt.

\section{"Zber baben benn bie \&eguminofen audh einen Typus?"}

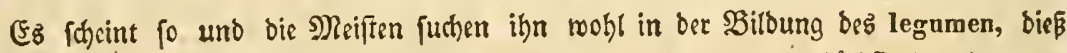

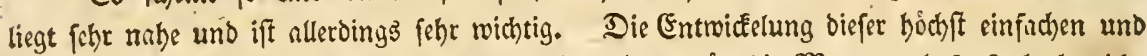
wenn man fie einmal rein beutfh=genetifh betractet, fur bie Metamorphofe fo hoch widh: tigen fruchtform, ift aber auch fdon fo gut und grundich nachgerwiefen worben, bus ich

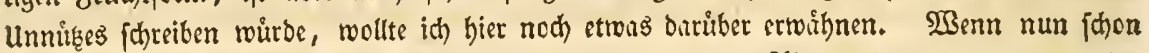

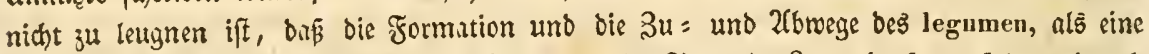

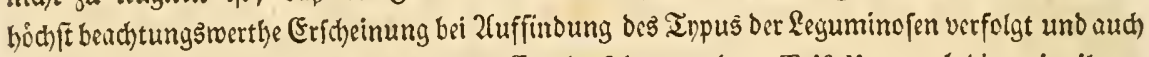
hier roieder, wic wir bei Betrithtung ber Gorolle fehen merben, Trifolium unbedingt in ibrem Lirptimipe, bie nicbrigfte Gattung fenn, uno diefe Ëtellung, bus Beginnen mit Trifolius,

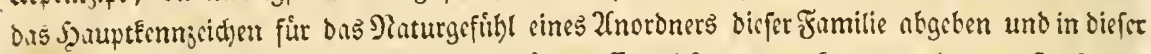
gisife die Fortbildung bes legumen zur höhern Entwicfelung, erfannt weroen mus, fo h,at mir boús immet gefdienen, nis ob burin, in biefer $\mathfrak{B}$ bilbung bes legumen, oer eigentiche

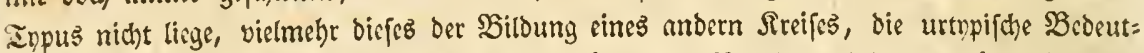

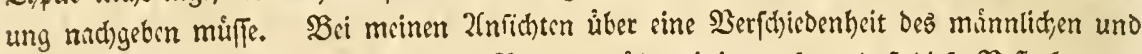

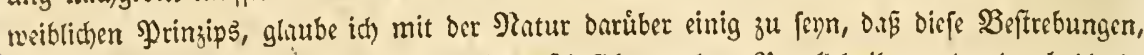

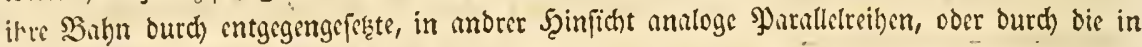
Den Sronungen neben eimunber gettetenen Formationen ber Slaffen, eine im rosikliden uno

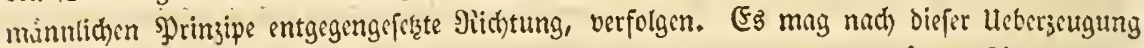

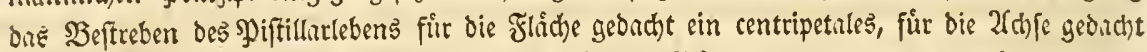
sin afrogenetijhes, bas minnliche bugegen fưr bie Flåche ein centrifugales, fur bie 2lchje ein amphigenctifches fenn. Den normalen und, wie ich glaube, vorwaltenden Berlauf ber

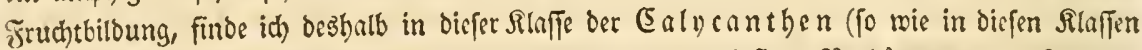

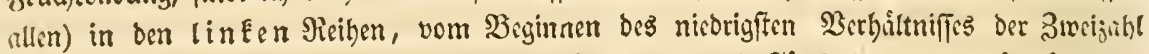

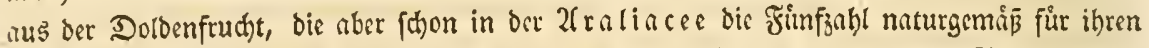

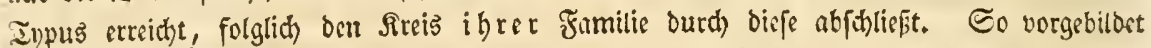

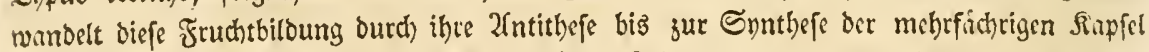
uno Beere, was cinftweilen hierber nicht gehort. Sch wollte mur barauf bindeuten, wie auf

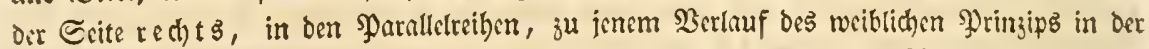

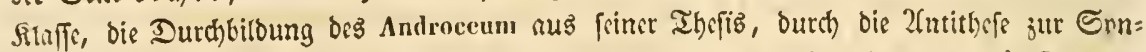
theie fith abichlieft, uno hierbei fpiclt bie corolla papilionacca eben cine grofe bochit bedeut= ungšbolle Siolle.

TSir móchten nun gern wiffen, was eigentlich biefe corolla papilionacea fei und mas

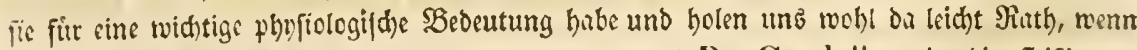
roir in bie Niemoires sur les légunineuses von De Candolie, in bie fleipig ge: arbeitete Carift von Bronn und in bas befte, liker bicfen Gegenftano erfdienene ESere, in bic

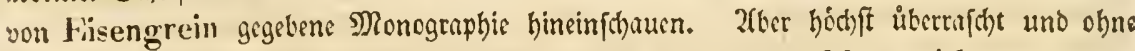

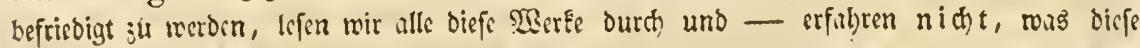




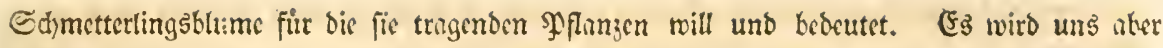
Dubci auffallend, twenn fo geifteche Mimner, um fid) bic Sadbe, bie ihnen fo nabe liegt, crftisen zu wollen, cine Menge fo geiftrciche Erelírungen und Doutungen aus fich entwi=

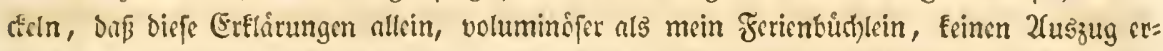
liwken. Der cinzige Oken folligt friftig felkffthaffeno, trie er gern ju thun pflegt, mit

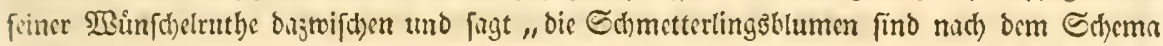

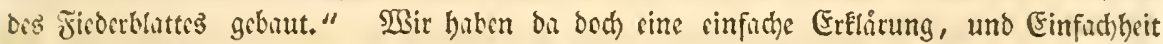
und Beftimmtheit ift immer eine gute Sache, wir firtoen aber in gegenwartigrm Falle fobr

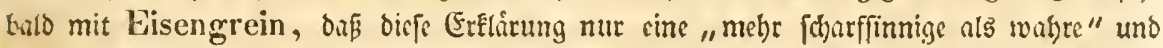

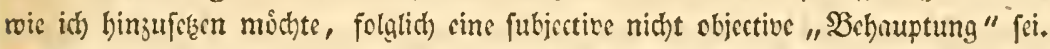

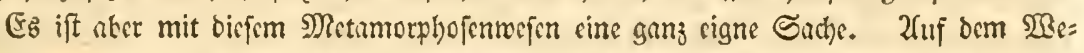

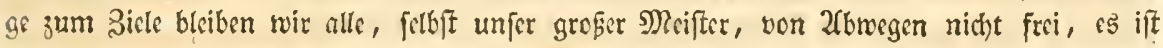

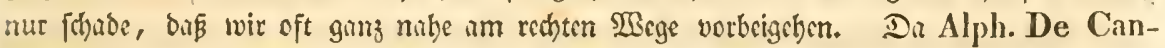
dolle in ber "Introduction" wicber abermals uno wicoer fehr richtig refcritt, daß Göthe's ,opuscule s'est trouvé femarquablement d'accord avec les observations et les théories de botanistes qui n'en avaient aucune eonnoissance, et en particulier de M. de Candolle, dans son mémoire sur les fleurs doubles (Miém. soc. d'Areneil) et dans sa théorie élémentaire (1813*) alfo M. de Candolle die Mictumorphofe obne Göthe entosctst

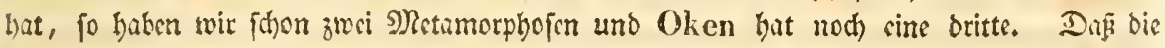
von $P$. de Candolle mirflid sin anderes Ding iff, als bir von Göthe, hat Göthe (2rugghabe 1831. S. 220. 221.) filbft gefagt und aufer bem bort gefigten - was mei=

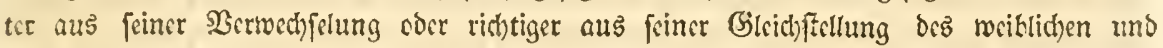

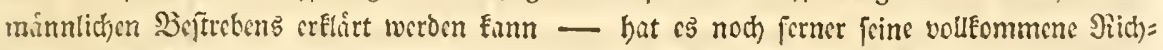
tigfect. Sdjon bie Entoccelung bes abortement, waldbes nad) Göthe's von unten nath oben oder yom 2fnfang zum Ende fich hinkerwegender Bafis, oa s's nicht burch vorgeitine Gicbirung zu Iage gelóft miro, als hebetatio, b. ち. Ertóbtung burch Stabilifation auftreten nußs, auch) die Corolla gamosepala, weldbe auf biefer $\mathfrak{B a h n}$ nur cine monopetala fenn und nicht eher ihre Gilieder vermåhlen Fann, bis fie vorber getrennt murben, berweift die entgegen=

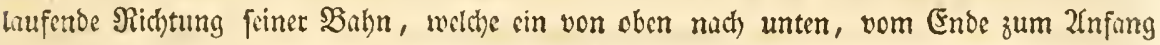
ruteftrebendes Syftem bedingen mufte. Der Calyx gamosepalus trat mun gegen die un=

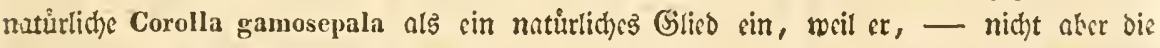

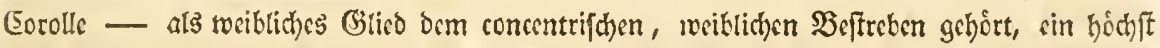
roidtiger (G)tund, ben Göthe von "Șcrman und. Dorother" an bis zu "Fauft" und

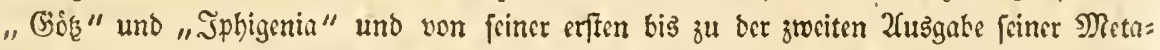
morphofe b. h. vom 2fnfang bis zum Ende feines grofen Schriftiftllertetens, - in wels d) cm allerbings zwifhen feinen botmifhen Stubien auch einige $\mathfrak{B e r f e ~ g e m a d ) t ~ b a t ~ u n d ~}$

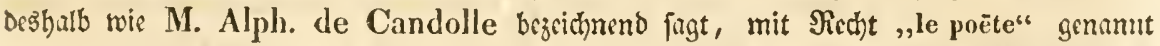
roerden Fann, bod) aber sigentlich in allen leinen profaifden und poetifthen Schopfungen

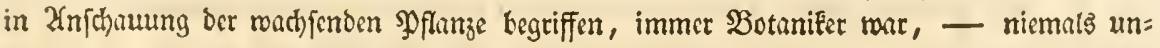
beachtet gelaffer.

•) Dr. J. Chr. Starke bielt übcr sie Metamorphofe im Jabre 1791 Sorlefungen auf

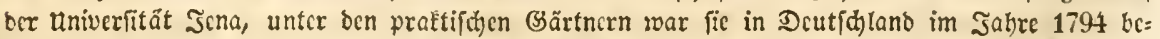
fannt, Voigt biclt bartibcr Bortcjungen 1803, Voigt uno Kieser ¡dricben barübcr 1808, Voigt

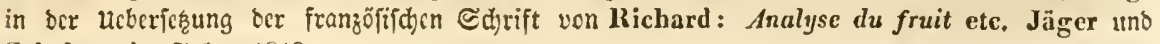
Schelver im Jahrc 1812. - 
Diefer unfer beutidjer Göthe alfo, hat in feiner Metamorphofe viclleidft aud stmas

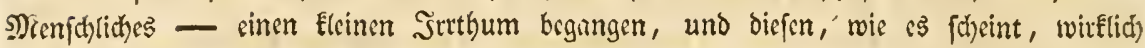

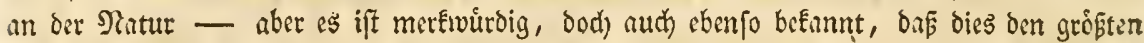
Geiftern gefdieht, Dar P. de Candolle's Metmmorphofe diefan Fehler mit der von Göthe

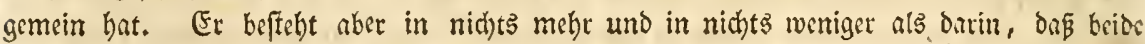

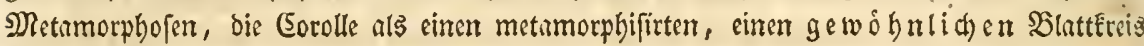
für alle F̧alle erEltiten.

Sd) babe in meiner 2fnoronung bes Spflangenreidfs frit febernjebn Jabren, alfo fo

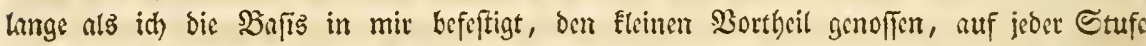
oeffelben, zu wiffen wo ich bin, yon meinem gralus naturalis aus fand, id immer bie cin=

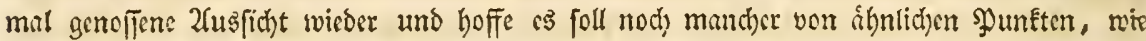
iifhrlich fo viele von unfern fdjonen Borghoben nus, hoiter fid umiehen.

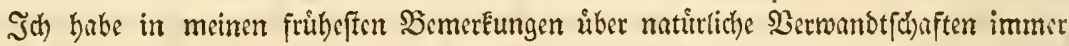
burauf hingedcutet, bas es recht angenchm fenn muffe, zu wiffen wos bic gute Natur in ciner folthen Sfflanjengefellifhaft, die wir Familie nennen, eigentlich vornehme, und id)

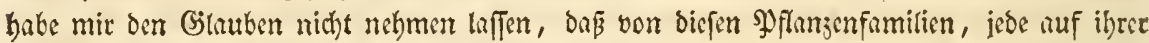

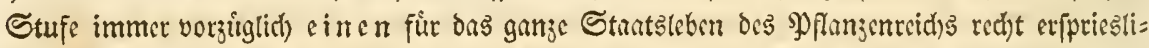

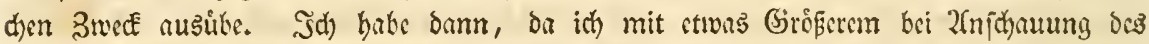

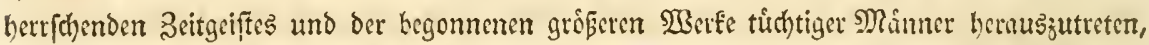
nicht gefonnen war, nur bie fleine Flora german ica und bie fleine bildidje Ueberjitht: das $\mathfrak{P}$ flanzenreid mit Erlúterung in 2 Sceften gegeben und in erfterer, in Der Flora, jeder Familie cinen ganj Eleinen, lafonifchen Typentveifer al’ graulus naturalis vorgejert, bem cigentlich nicber eime Seand als Fingergeig vorgefegt fenn follte, ba er eben baju beftimmt

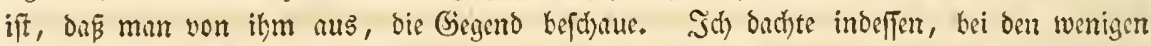

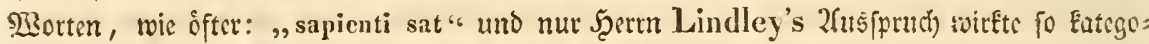

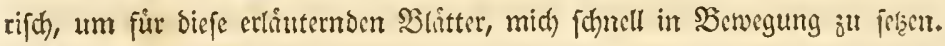

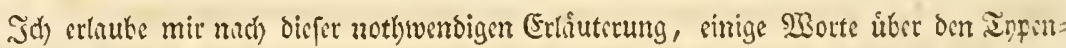
meifer fût die গ̧apilionaceen.

Er hcipt:

"Antherae absolvuntur."

$23 a 3$ heißst Das?

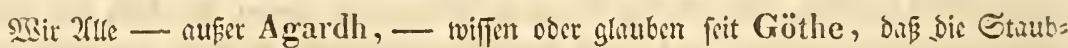

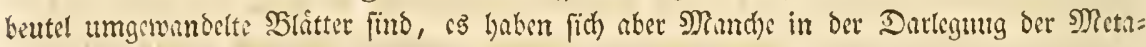

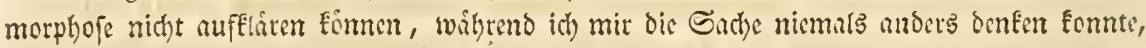

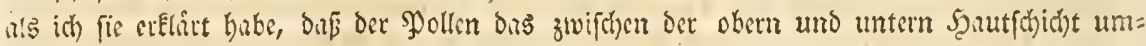

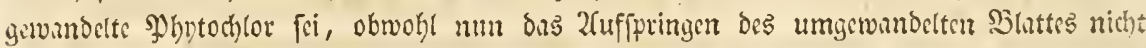

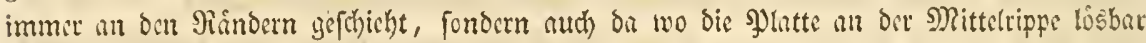

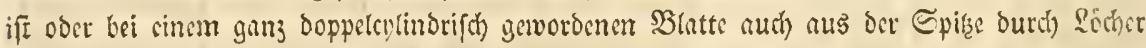

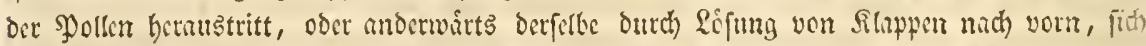

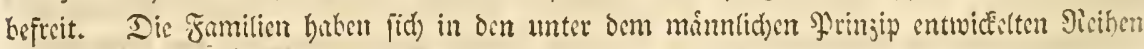

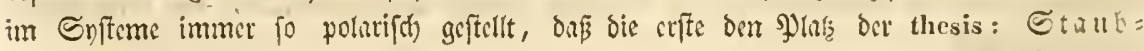
beutel, bie jweite antithesis: Strubfaden (Blattfiel), bie britte synthesis: Eorolli morphologif(t) stlautert.

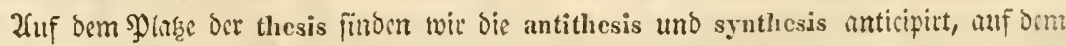
oer synthesis bann bie thesis und antithesis gamj maturlid wicoerbolt, tno wic bic anti-

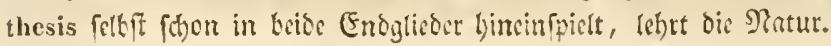


Wenn wir mun auf ben Stufen Der thesis, in Der Rlaffe, in weldher bie Entroidels

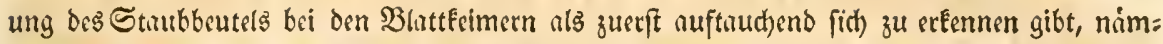
lict): Lycopodiacene, Strobilaceae, Aristolochiaceae, in ber folgenden filaffe ober in ber zwei=

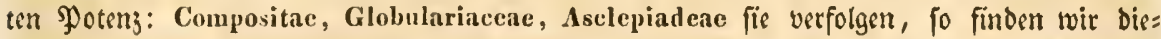
felte in ber wieder folgenden Siluffe, oder auf der britten \$ुoten, in den Papilionaceae, Portulacaceae und Polygalaceae nodimats (id) reicberbolend und weiter entroidelnd, uno na:

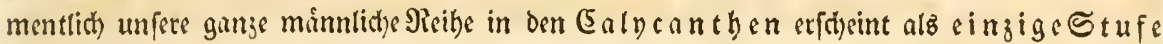

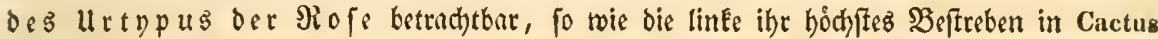

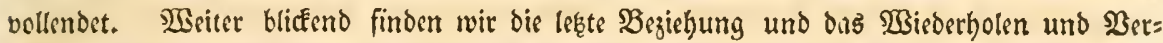
batren auf biefer 2(ntheren=Entmicfelung in ber vierten Poteng in Den Fumilien: Violaceae,

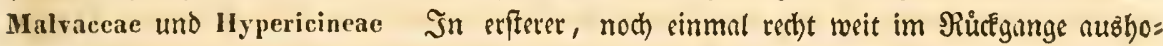
lent (mobei uns an bie Fifhformen von Whallfifh und Delphin unter Dent Saugetbieren, zu denfen wirber erlaubt ift).

2fber blicken wir mun auf bie biefem urtypus zugehórige Beziehung, ober bie fitr biefe Fumilien geltenden Deuterotypen, námlid) alß die 2fiticipation ber folgenben Iypen, der 2fn= tithefe, alfo des Staubfaden und ber Snntbefe, alfo ber Eorolle; fo erfeneinen beide unter folgenden einfinchen Mobificationen.

Die 2fntithefe burd) ben Staubfaben (Blattftel, baz wabre Blatt bildet fich) als ber

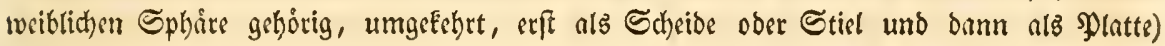
bilbet fich gehemmt, bie Sturbfiden entweber alle, ober bis auf einen freigebliebenen ver=

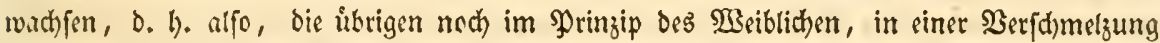

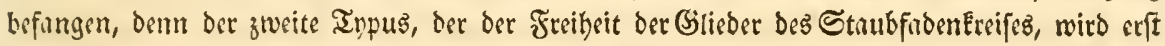
burth bie Eaffiace gefóroert und hier unturgemía, in zrveiter Familie, volleommen erreidht.

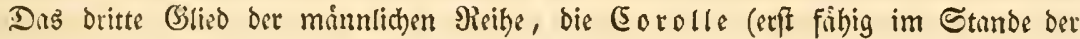
Sonnthefe, in der Mimofacee, fich ganz, o. h. reguliar zu vollenden) muß bemnach oben,

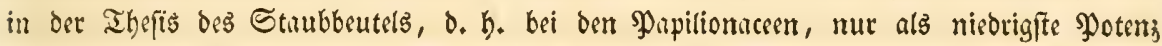
oeffen, was aus ibr werden farm, erfheinen, und fo nåkern wir uns jef̧t ber Fin = uno

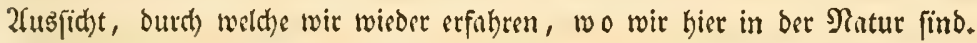

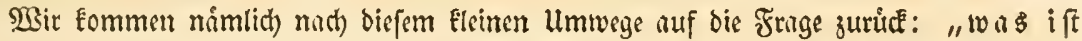
Sorolle?" Die brei exiftrenden Metumorphofen von Göthe und Nees r. Esenbeck, bie von De Candolle uno bie von Oken figen uns, fie fen ber mit Rielth und Strub=

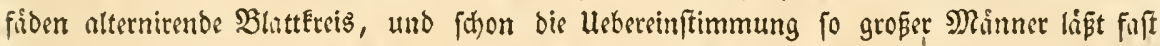
gar niemand wagen, an ber unmoglid) feit zu zweifeln, bas bie Sache noch anders gefunden

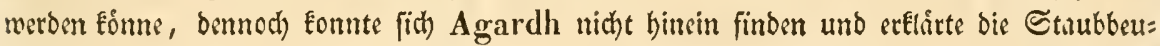
tel fừ verfirmmerte Sinofpen. Wenn fith nun wieber in biefe (Erklirung, die meiften nid)t hinein finden Eornten, fo mur ich geftehen, daß es mir mit berfelben eben fo geht und id bleibe burum bei ber 2fnfthounng, bie mir inımer alle jene, zu munchen Meitliufigkéten

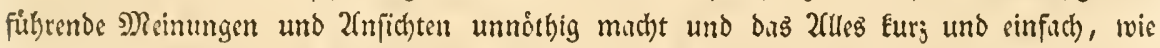

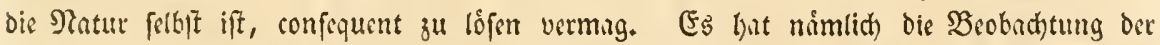

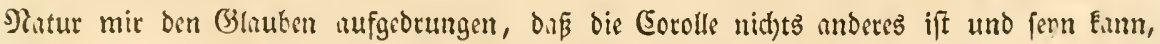
als: - - Der Stipularéreis fúr die Staubgefápblatter (Pflanjenrect)

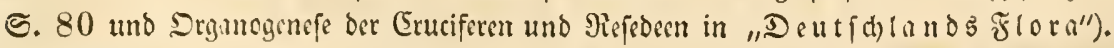

Seben wir uns in unferer 2fnoronung um, wo wir die Entfebung und Durchbild:

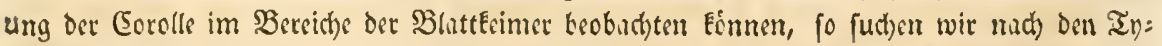
penveifern und finden in der Borbildung: Cytineae, Thymelcaceae, Laurineae, in dit 


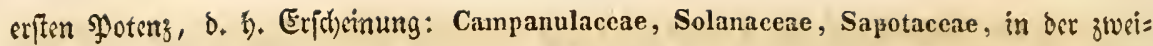

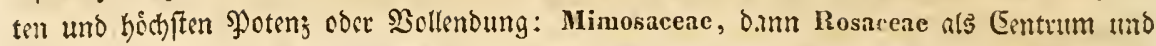
ginzliche Durdbilloung biefes orgnnifhen Prozeffes, und Amygdalaceac jeme wicherbolend;

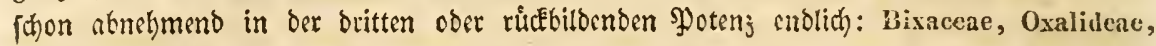
IIesperideae.

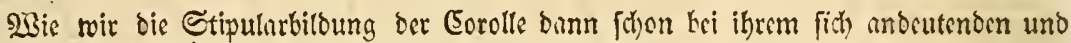

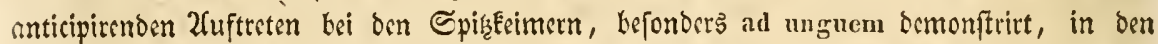

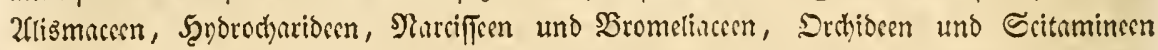

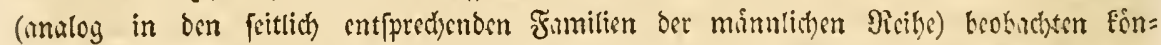

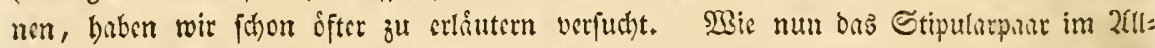
gemeinen fu einem Spetrlum amphigenetifh) jufammentritt, laffen bie Carjopisyllacea am beften in Silene (ogl, Fl. germ, p. 812) bcobachten. Hefersll too wir petala hipartita, bifida,

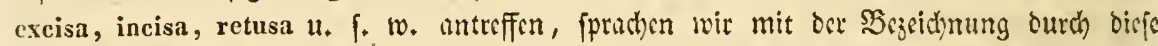

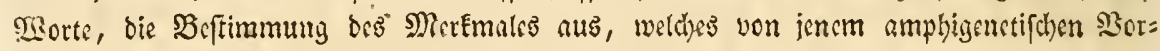
ginge jurúcteticb.

So boffe ith, find wir endich fo rocit, zu fethen wie bier in ben seguminofen

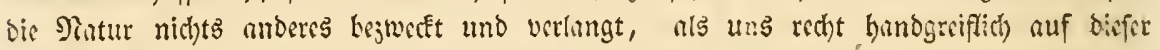
Etufe der erfen, o. h. nicorigften Entwicelung oer Corolla pleiopetala cir foldes offentartes Stipulargebilos, fo offentar wis ş aus oen hatbipiepformigen

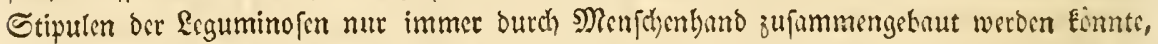
vor umer objectiv zu feben gewobntes 2lüge, zu legen.

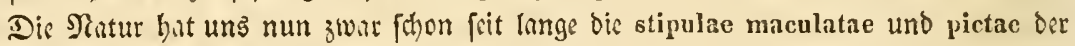

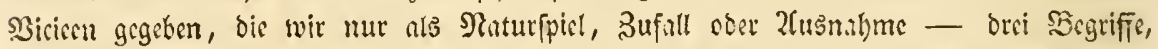
weldye ber beutfenen Metamorphofe ourchaus fremb find - erflirten, neer fie gab

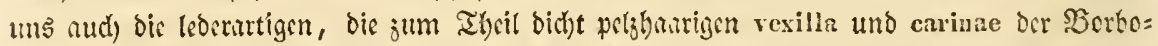
nim, bie oft grimen carinae uno vexilla ber Gompholobien, bei benen faff alle Stiputar=

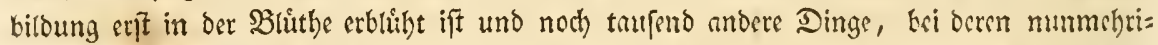

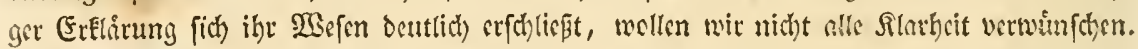

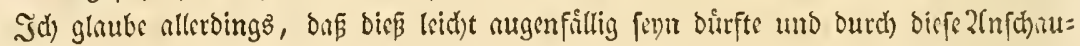
ung nicht nur bie Eorollenbildung ber Şapitionacen, fondern auth bis fo mand)er andern

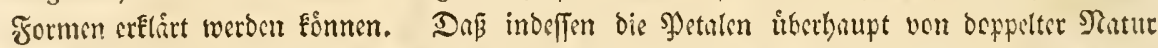

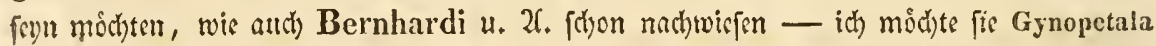

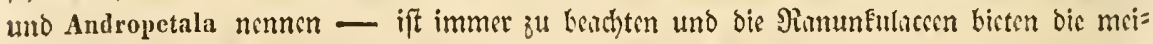

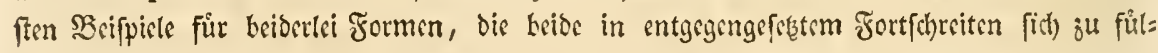
len vermógen.

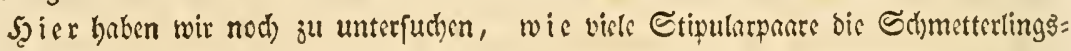
shume beftimmen.

Befanntlidy pflegt haut ju Irige nicmind mchr ju fagen, bie Corolla papilionacea keftinde aus vier Blumenklätern, wie wir vom alten Sefrer Linnce gelent buben, es

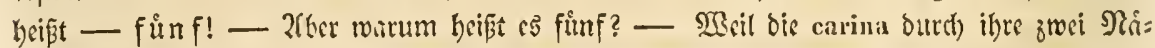

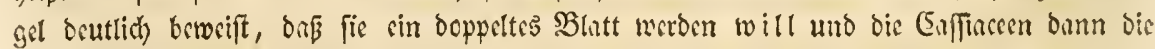

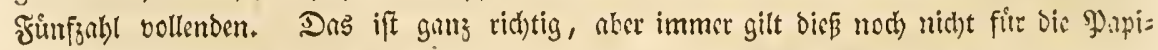
lionacent. Der BotaniEer foll zrour an Erembryonaten und, plantes cellulaires"6 glauben, bier aber auch einmal stwas ans ber Natur in fich anticipiren. 2(lle iene ?tu=

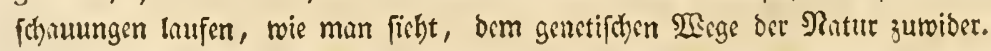




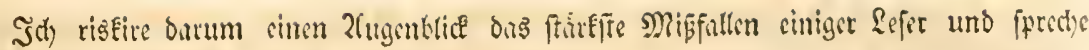
cinmul bie 3nlyl orei ans, aker - wie?

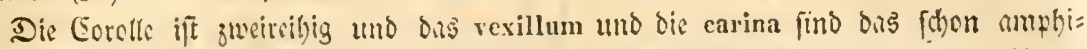

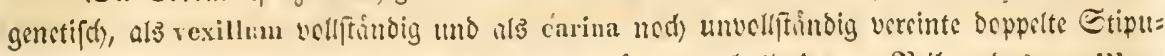

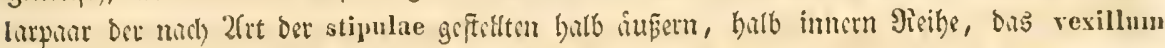

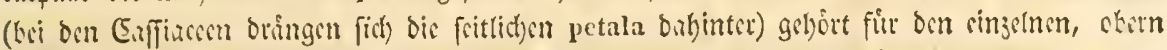

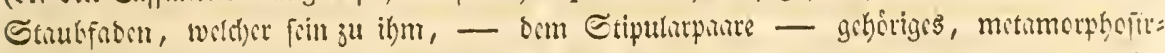

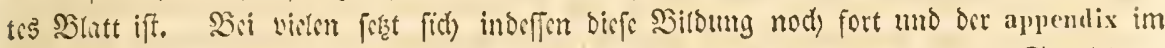

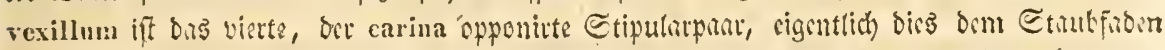

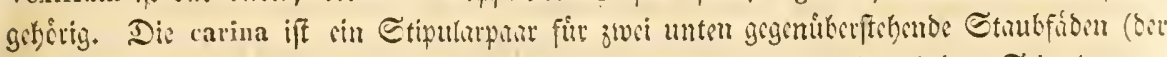

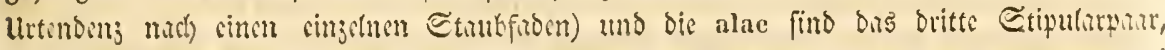

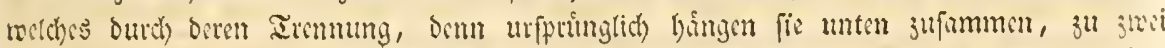

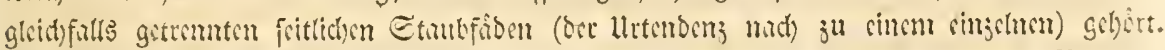

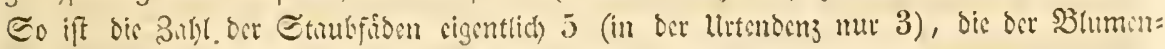

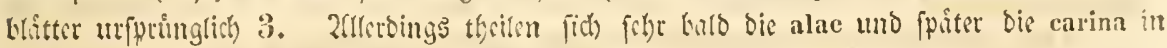

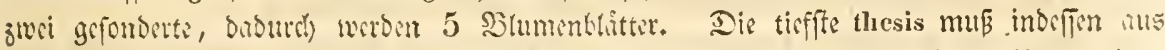
bet Corolla synpeta!n, bis alfo noch wicht in petala getremt ift, fict) berisusbitocn, bis =

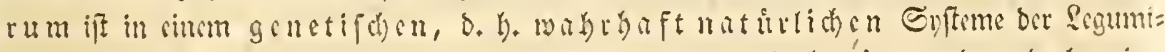
mofen, allemal wegen Silnme und Frudt: Trifolium bie beginnende, o. b. nifs=

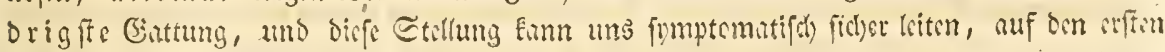

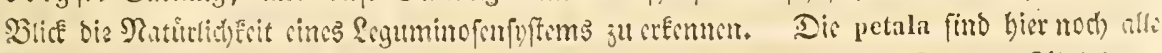

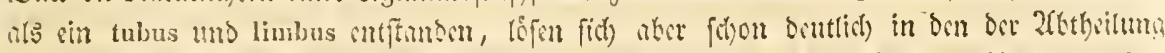
Lotophyllum (gek. 1824. Chronosemium geb. 1825.) gebirigen 2frten. Eehen wir jist

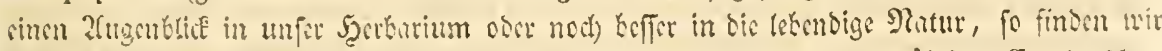

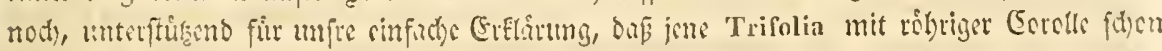

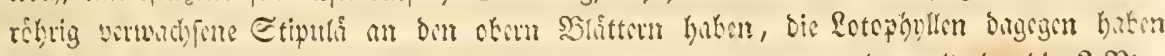

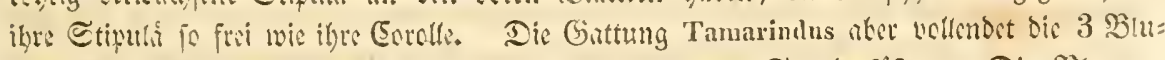

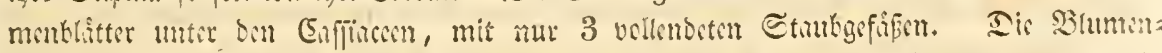

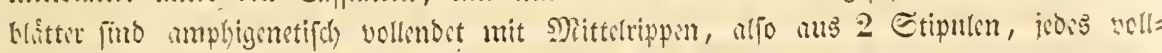

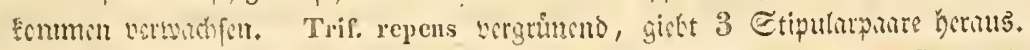

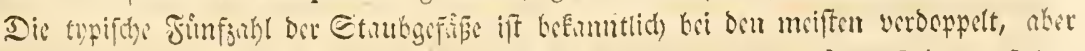

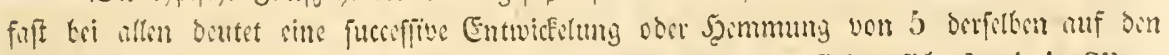

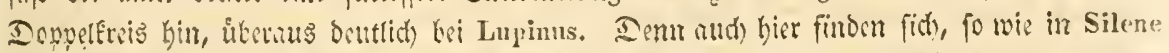
Diz corna, Lis cingelnon Gattungen 3. 33. Phaseolus Siubimente von Etipuhrpanran, dir

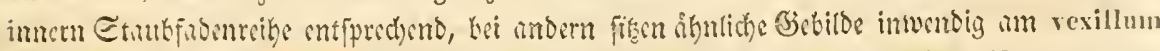

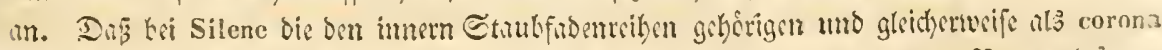

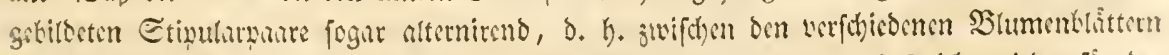

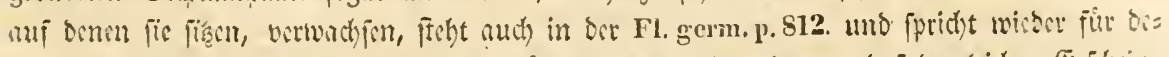

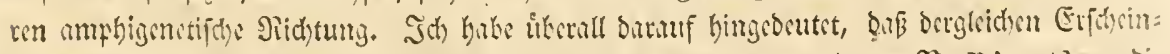

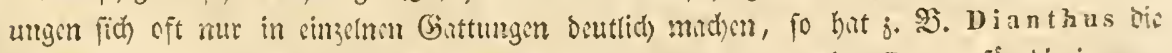

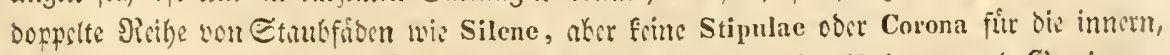

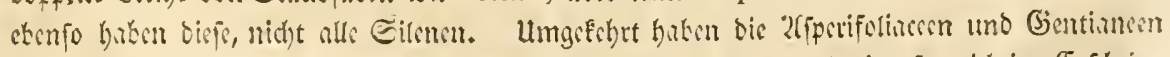

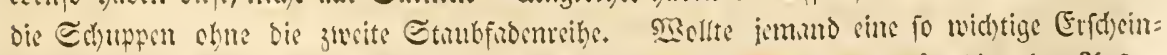

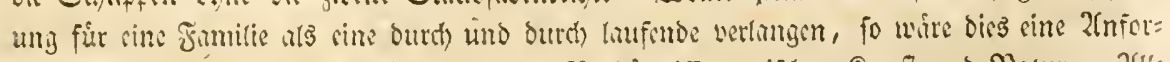

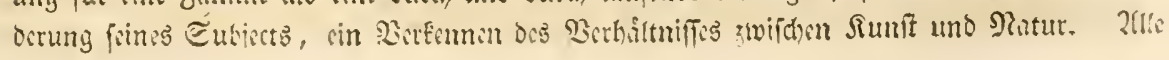




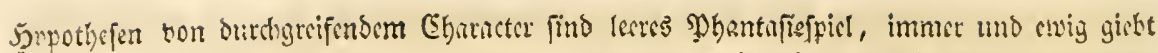

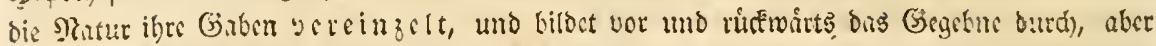

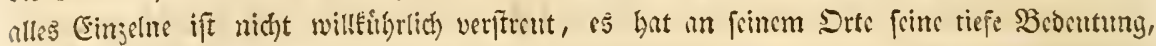

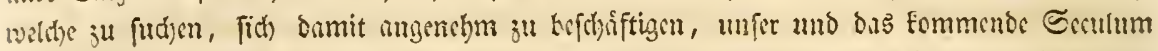

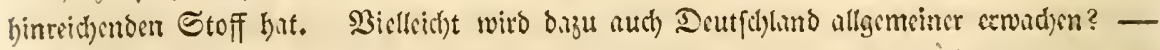

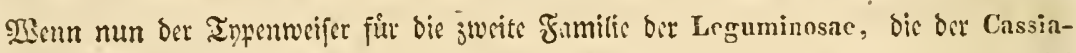

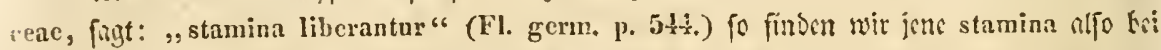

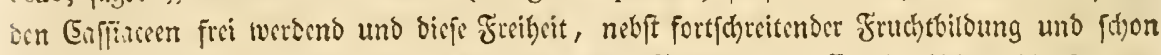

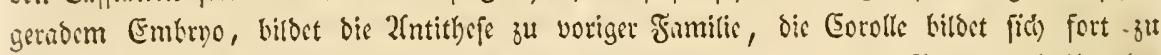

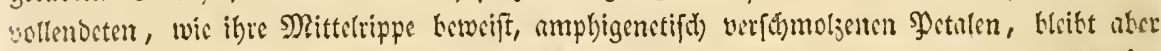

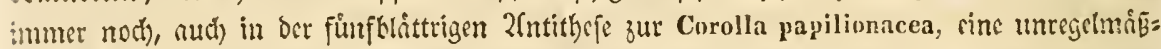

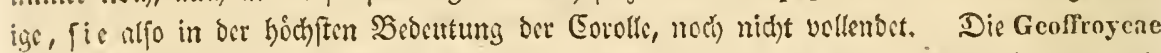

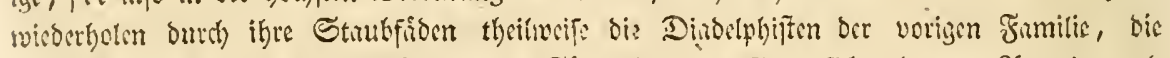

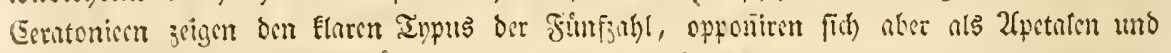

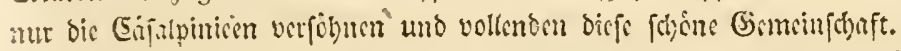

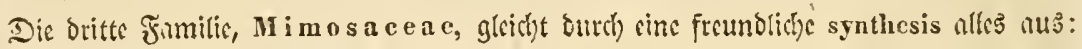
,androcei regularitas et libertas absolvitur staminilus hypogynis, corolliz valrata etc."

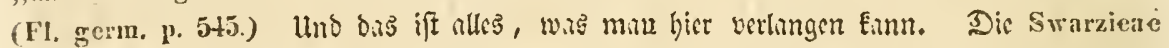

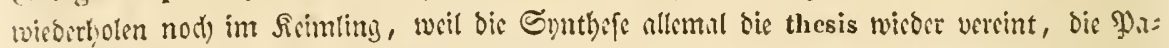

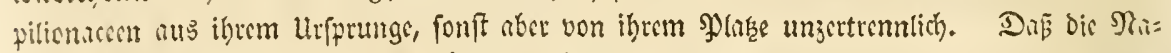

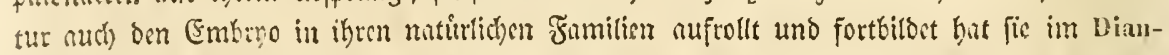

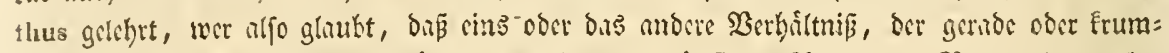

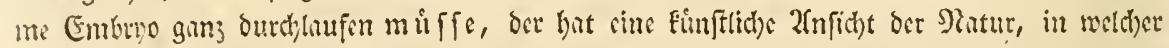

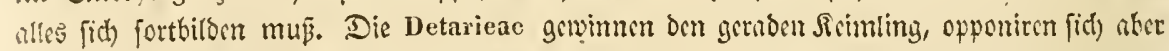

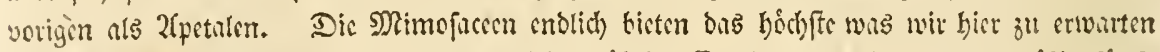

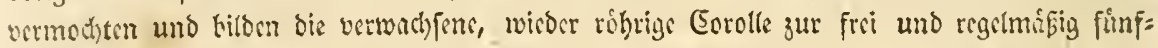

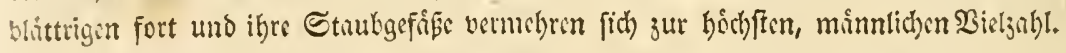

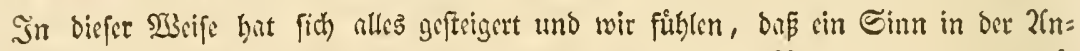
oronung licgt, wir megen fie foffen wo wir nur wollen, bas alte Naturgefes ber Spolaritít macht fid) geltend.

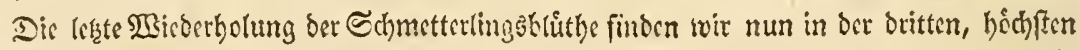

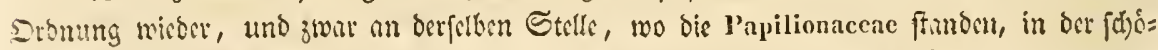

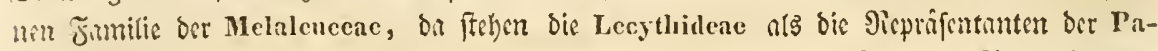

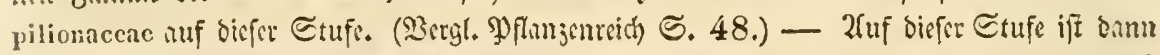

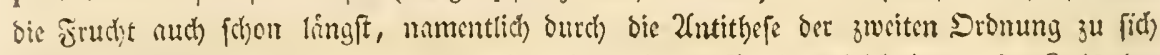

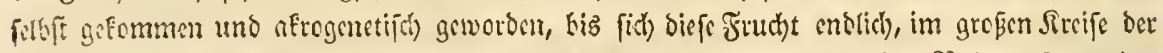
smigrenformation, in ben Lythreac unb Melastomeac, in bem ber Siofacenformation

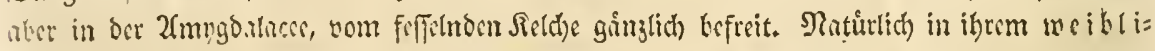

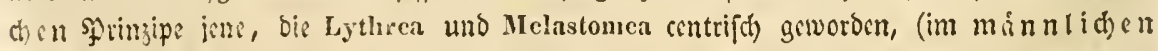

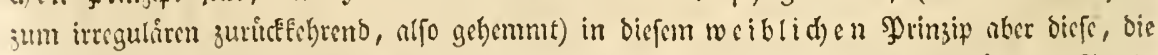

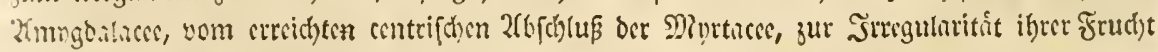

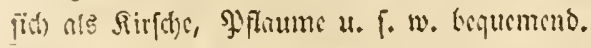

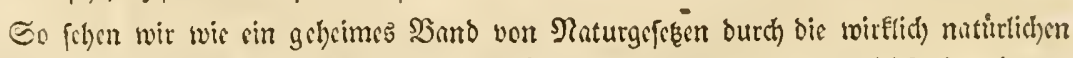

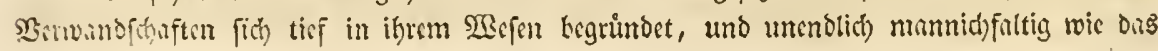


Nef ber Natur felter und fither sergweigt, fich bentlich bindurd) fohlingt. - Sch babe mir

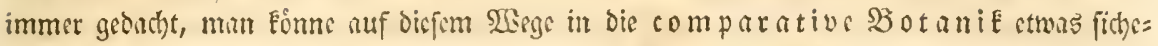
ret bincingelunget als gewoihnlich und dic Botumie fei es ber unferer Jeit, burd) dic compa=

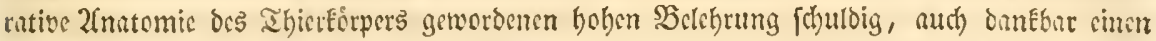

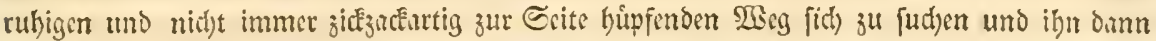
auf dor Bathn feiner Trpen, ebenfalls in Sithe ju verfolgen.

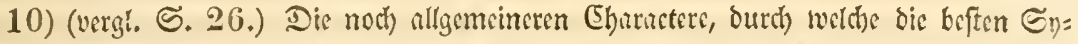
ftematifer dic Fumilien und Sitaffen vercinen, find vorzugsweife dic Infertion oer Etaukge=

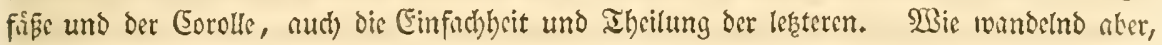
D. h. wie fich fortbildend dicfe Eharactere find, bả Fonnte bie cinz̧ige Giattung Saxifraga, die man dem Stunde dis Frnut)tEnoten nach, in zwei Siluffen hitte vertheilen milfen, fur den erften Fall und dic Familie der EriEaceen und \$ibodoraceen, mit ifren sinklittrigen Gorollen

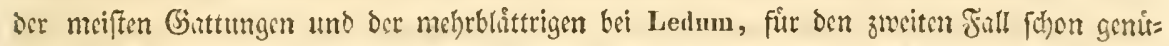

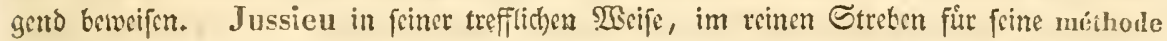

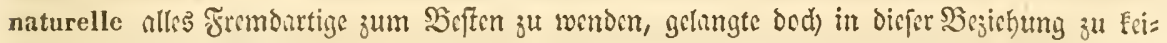

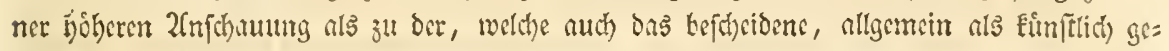

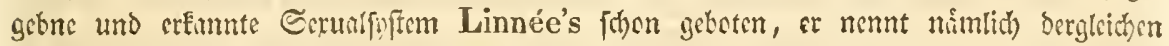
2abweid)ungen "2( fo zuhlreich vorfommen, wenn ir in ben Principes de la méthode naturelle fagt:, reiatirement aux exceptions nécessitécs dans eertaines classes, par suitc du choix forcé des caractères du second ordre, quelquefois variables, on pourra observer qu'elles sont plus rares dans certaines classes que dans d'autres, " 26ker fitbem uns Göthe auf

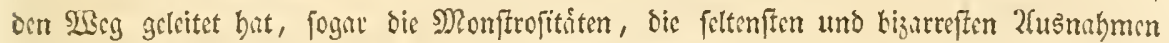

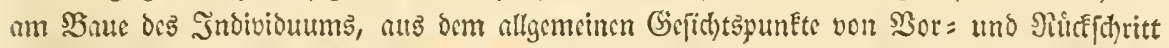

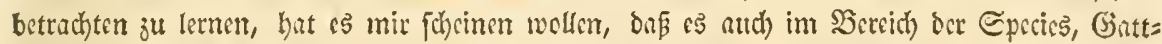

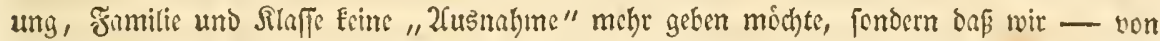

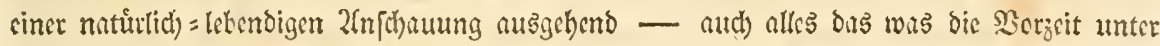

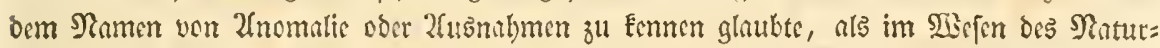
lebens und feiner formcilen (Erfbcinung bedungen kettudten burfen, dafern wir uns entichlis=

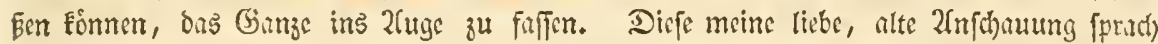

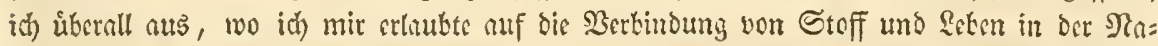

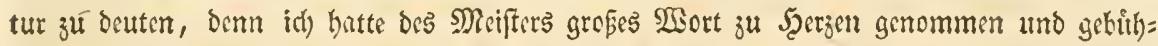
rend verefrt, wenner fagt:

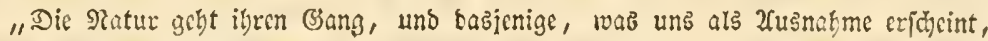
ift in ocr Ricget."

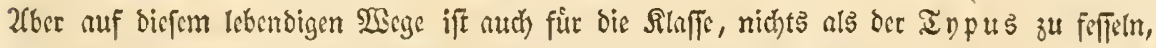

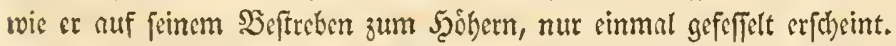

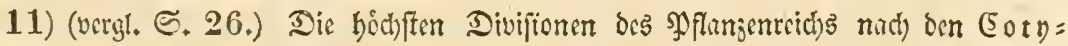

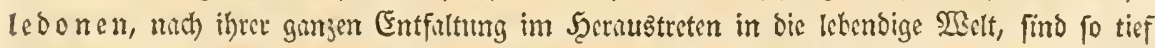

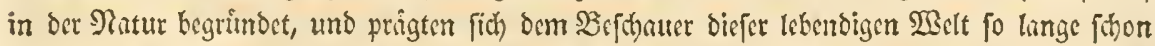

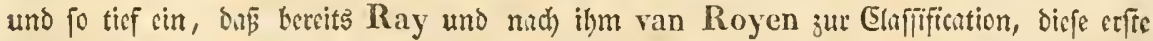

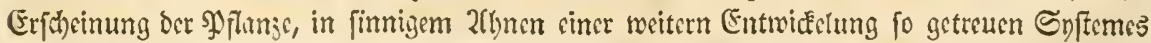

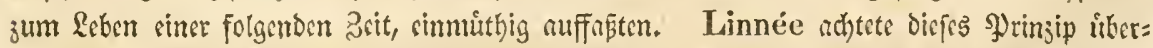
nus hoch und von Jussieu, pllegt bann bie cingetekte (Gerwohnheit der mitfprechenden Men= ge, beffen Erfindung ju leiten. 


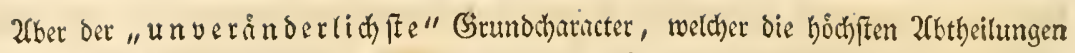

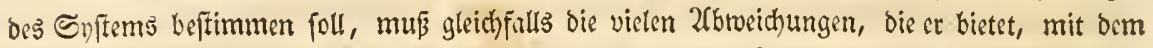

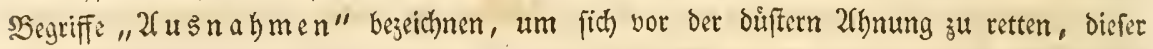

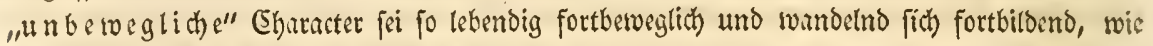
alle andern in ber Satur wirflich erjcheinen.

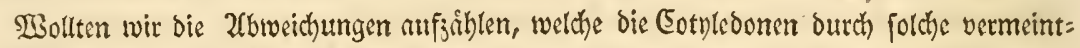

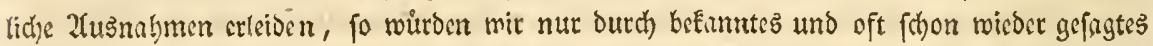
ermuiben. 2fuch hat Correa de Serra, uno oer feine ąeit weit und trefflich vornus=

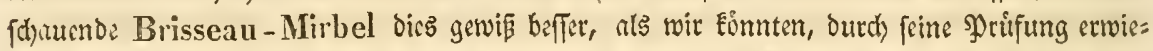
fen uno Aubert du Petit-Thonars, in deffen tiefen Blicken cinc lebendige Metamor= phoje der Spflinze, fidy berwust anticipirte, ber bocherfabrene Desfontaines und 2fndere

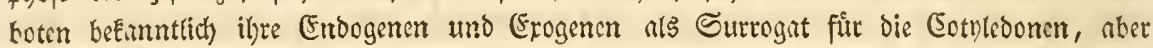
auch bus Surrogat ift fchon grumblich und vielfeitig geprift, und man bat auch bicfes — als ein lebendiges, fid) fortbildendes Eennen gelernt.

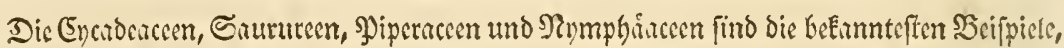

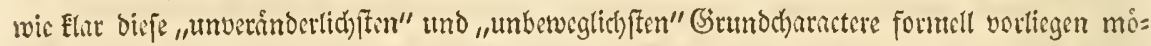

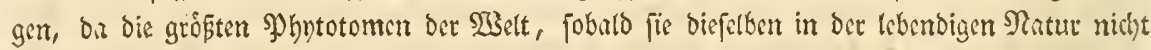

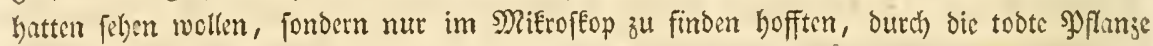

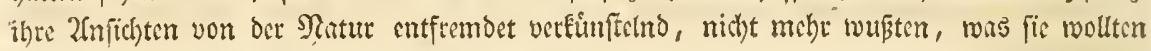
und polten, was fie fudteten und batten.

Wir wenden uns jefet nur noch mit wenigen 2 sorten jur Befthum oer verbeff= erten Szatuptabtheilungen De Candolle's uno Lindley's.

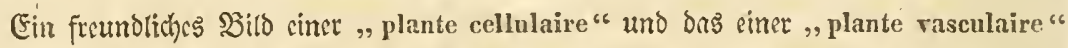

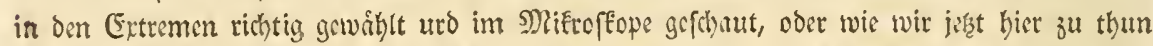

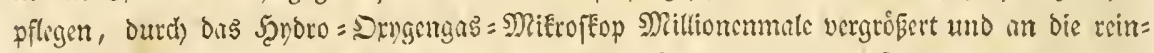

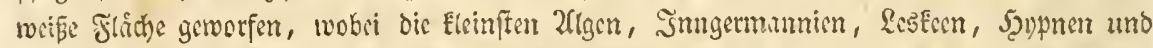

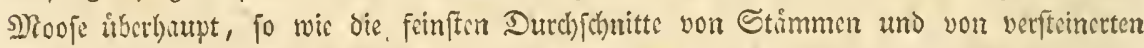

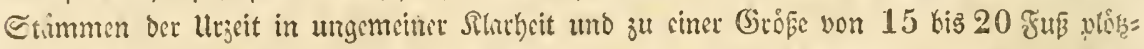

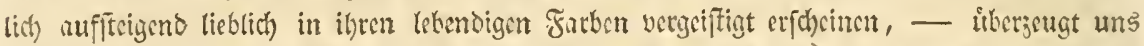

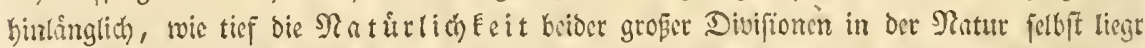

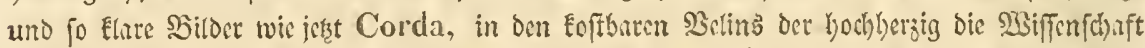

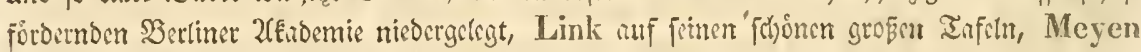

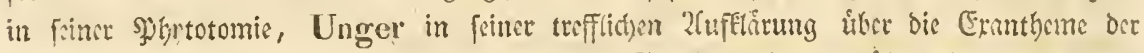

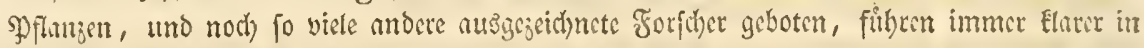

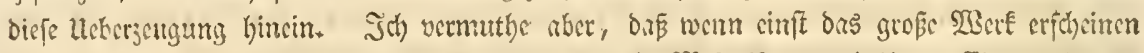

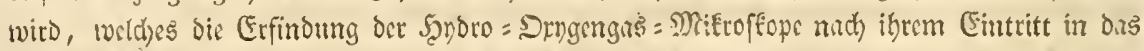
Seben, aไs unmittrlbure Entfaltung uno Fortbiloung ihres nothmenoigen Ricfultutes bedingt,

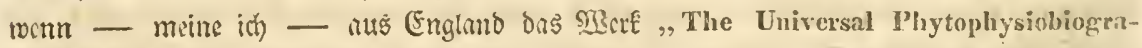

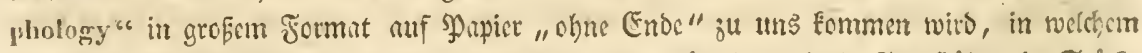

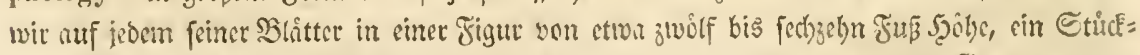
d)en Bellyetwebe uno Biffipbitbung, aus allon wenigftens bis jef̧t kefannten Battungen Des

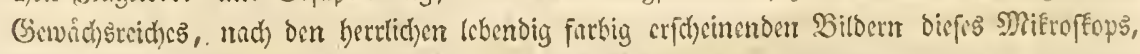

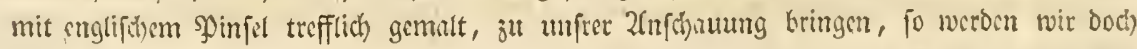

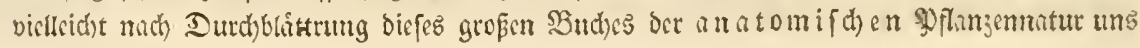




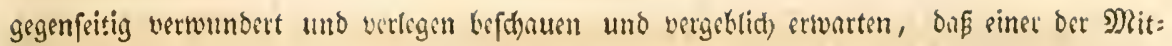
befoumer andeuten foll, wo bie "2rbichnitte" in oer Materie waren.

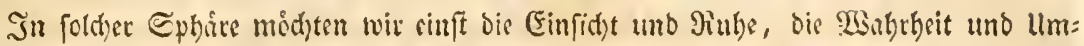
ficht unferes 'Treviranus burthmulten fehen! -

Die ,plantes cellulaires" find effens mit visten vereint, bei oemen von einer 3all= bildung aud) nicht bie geringite Epur fich andeuten fium, mit fold)en, wetd)e fid) ats unge= bildeter Maffenftoff Zenker's*), wibrend ibrer Eurzen Eskenszeit erbalten, in bus menjob:

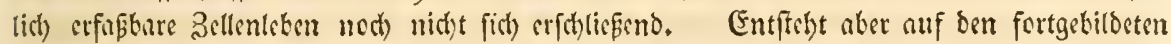

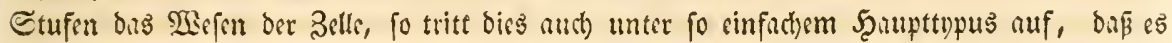

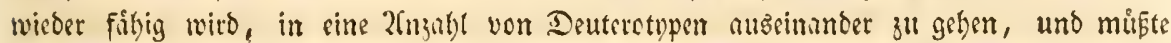

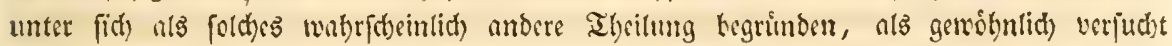

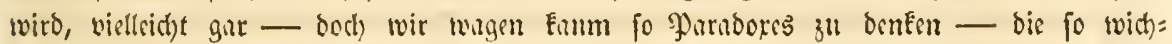
tige Frnge, ob die Bwifthenwand ber Bellen boppelt ober einfat) foi, ju Gunffen beioer Theile entfhrioen.

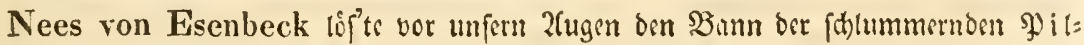

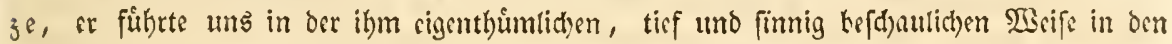

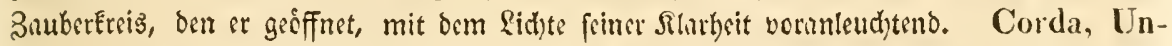
ger und Nees von Esenbeck bre jüngre Srnder, fitden fort und tereitrm von neuem

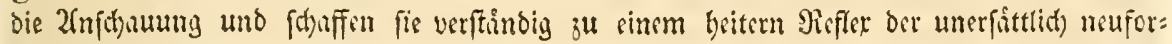
Dernden 3eit. -

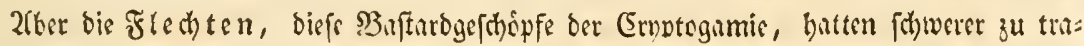
gen, bevor fis, in ifrem $\mathfrak{L}_{\mathrm{b}} \mathrm{en}$ beleuctet, burch Fties, W. Meyer und Wallroth orr

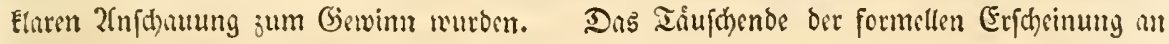

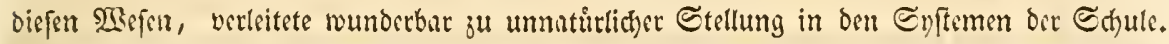
Bishen wir alles Sufirllige, fid) vor= und nadbbildende ab, fo bleibt ber Typus ibrer (Er=

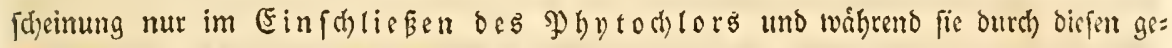

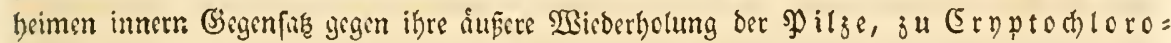
phyten werben, fo treter fie zwifhen bie şilze und zwifhen bie Ehlorophyten ats

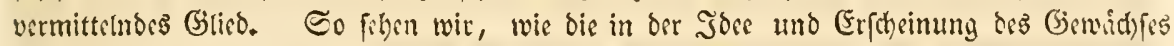

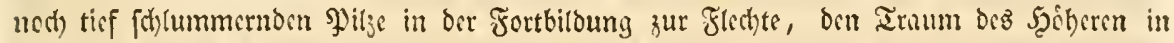
fich gewinnen. (5icid)wie ater im Reten bes Menforen ber Iraum im Serrich ber Socen= welt oft sine widhtige Folge für Natur und Reten entwicfelt, fo mag aud biefer untedeut=

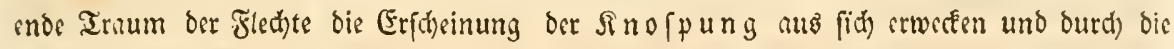
ganze 2 Belt ber Pflinzen bindurch, fich ausbreitend entfalten.

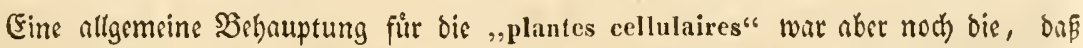

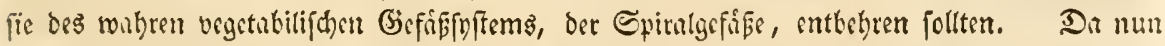

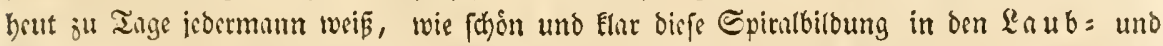
Rebermoofen brrauftaudt, ${ }^{* *}$ ) fo kedarf es weiter Eeiner weiteren Ertauterung bafir, d.r. jene $u$ mfdreibung oer ,plantes cellulaires" sine rein ibeale, in ber Platur burd)ins

*) Ratedismmus ๔. 7. 1524.

**) $\ b$ bie Gpiraten ber Trichiaceen, werdhe Corda entocefte, analog finb? 
nicht begründete ift. Unfere 2lnfdraung ging aber in biefer Erfabrung immer dabin,

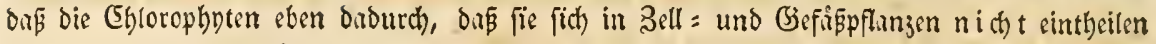

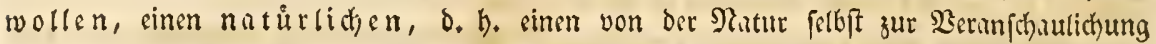
gebrichten İpus ror 2fugen legen. Diefer Iypus findet aber eben feine Barfis, D. h. feine momentme Stabilinung in einer ber grobirtigften, und in ibrer Fouttiloung in das Ungebeuere

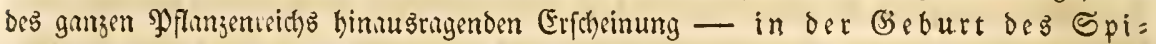
ralfy ftems! - Co uber alles menfoliche Begreifen erbabener Borgnng in ber Natur, erfcheint aher nicht wie ber $\mathfrak{B}$ lif, der felbft nut cin Eleines Symptom sines immer noch weit

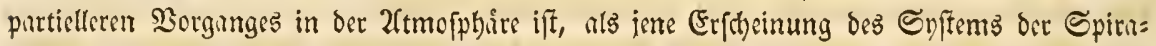

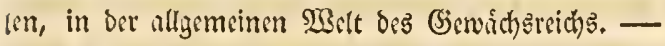

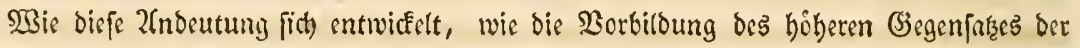
Farren bier auftritt, fdjon in den berrtichen Fornen ber Iange anticipirt wird, wie dunn

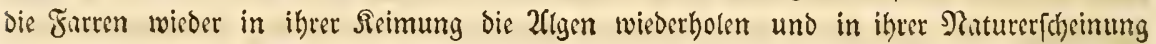
als Letendiges, iene wicber mit fich zu einem fojonen Naturganzen verbinden, wie zwirchen

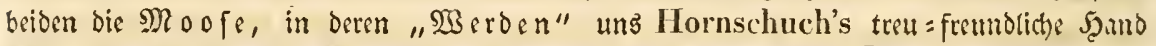
(N. Act. Leopoli. X. II. p. 513. t. XLVII-XLVIII., Dinn Nees von Esenbeck

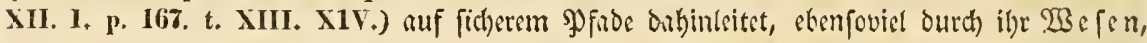

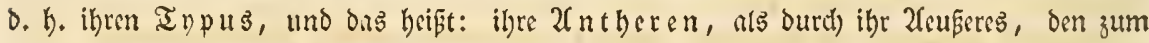

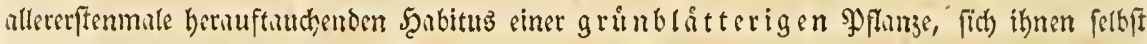

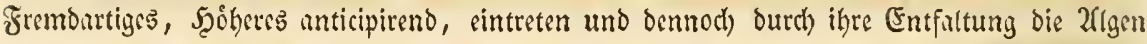
und Farren wieder freundich verfobnen, bas bute ich wohl auth angedeutet, allein man

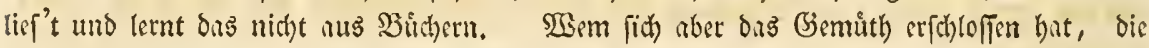

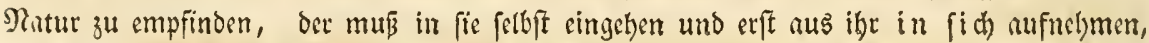

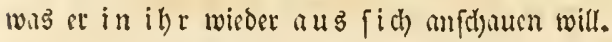

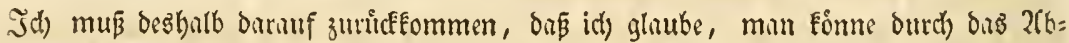

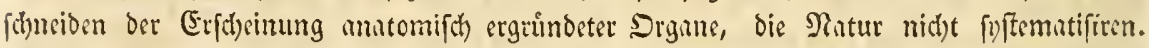
Man denfe, wie bie Formen, weldhe bie erften 9iubimente von Spiralen butbieten, unter

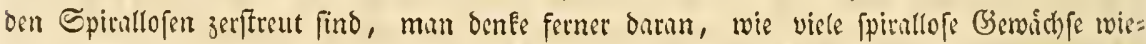

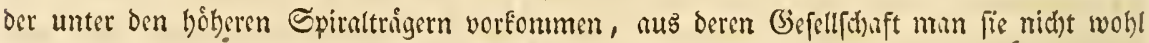

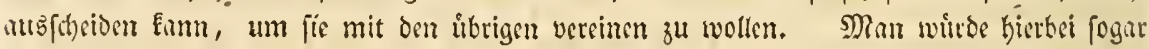
an Equisetum arrense fommen, weldtes in verfhicoenem Suftunde buld Spiralen zeigt, bald

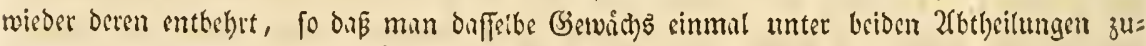
gleid) aufnehmen misterte.

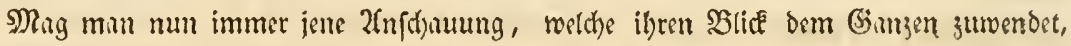

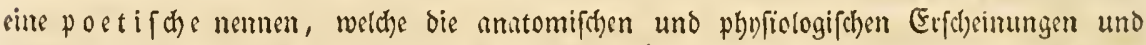

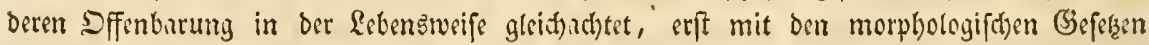
verbindet und bann bendhtet, waz aus diefem Sereine firt dic SyftematiE bervorgeht, fo

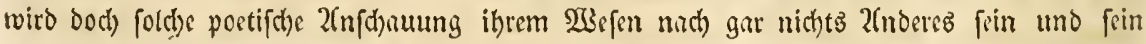

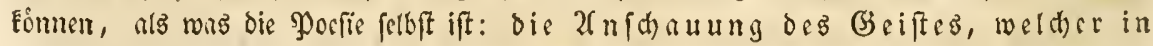
ber Erfdeinung oes Materiellen und 3eitlichen liegt, alfo die 2ffrimilation unferes eigenen Geiftes mit bem in der গatur.

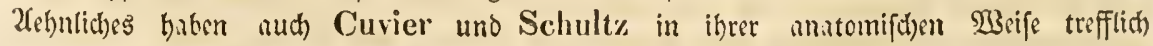
erftebt, die Comftruction deş gro 


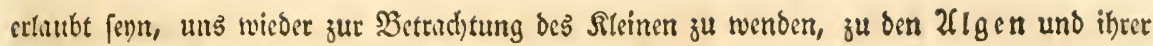
Stellung in ben Syptemen, daz beipt in ben Butchern.

Wiic wollen nidjt unterfuchen, wer sigentlich bie Soee aus fich entroifelt bat, bie

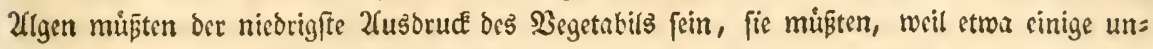

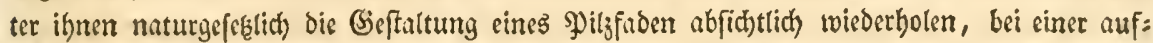
ffeigenoen 9icibe beginnen, bei einer abfteigenden aber befdhlięen. So burfte man in alter 3ait denten, obwobl weder Linnee nod) Jussieu to gedacht baben, es fhyeint aber auth) jest, als ob bie Melt fich nicht mehr bumit befrieoigen burfte.

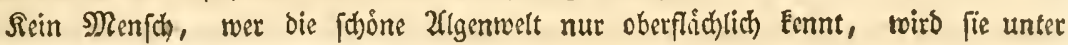
die Spilje und Flecteten erniedrigen, um ettwa mit De Candclle, Richard und Lindley ůbereinzuftimmen, bie mobl wenig daran gebadht haben, warum fie es thaten. Daßs bies nber falfh ift, berweifen fdon bie Syfteme, welde bie 2l(gentenner felbft madhten, wie Agardh, Fries uno J. C. E. Rudolphi, bie bodh woht tůchtigere 2f(genéenner find, als 2flle, weldhe bie 2flgen unter bie \$ुilge berabferzen, und woht wiffen musten, wieviel

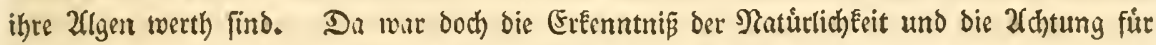

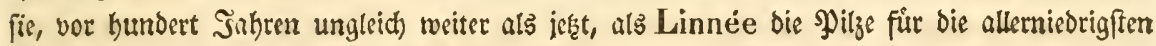
(Biewách fe erElinte, inderm er fagte:

Fungi: nomades, autumnales, barbari, denudati, putidi, furaces, voraces. Hi flora reducente Plantas lyematum, legunt relictas earum quisquilias sordesque.

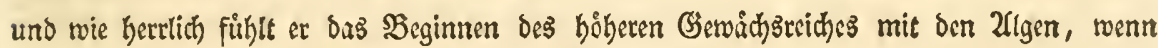
er meiter fagt :

Alga e: vernaculi, aquigeni, squalentas, redivivi, abstemii, nudiusculi. Ili inchnant cult uram primam vegetationis.

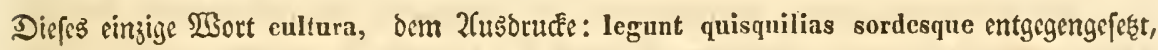

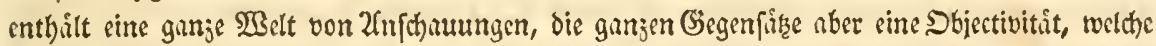
boch) begciftern mus, man fieft jis ben liebenstwurdoigen Forjher, wie er fich unter ben \$Dil=

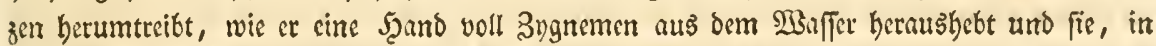

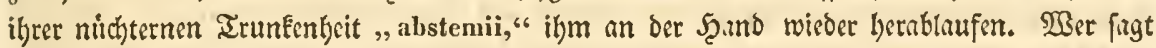

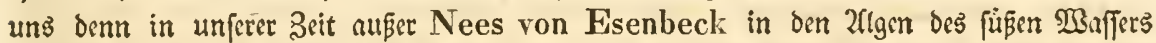
uno Martius in ber Reife in \$rafifien, vor allen ber eole Alexander von Humboldt,

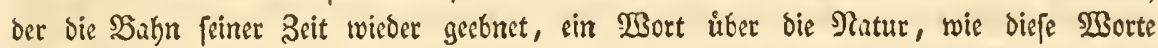
Linnée's?

Dưre, trockene Berftandesqualereien für bas jugendticbe (Semuth find manche Şand=

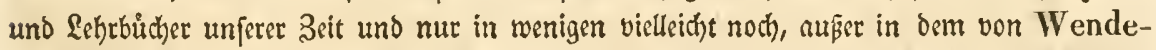

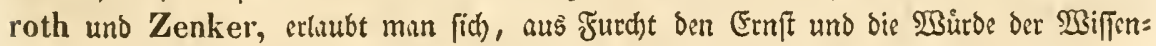
(d) aft zu vertieren, einen fdywathen Seitenblick für ben fehnenden 2(nfinger, auf feine lieke

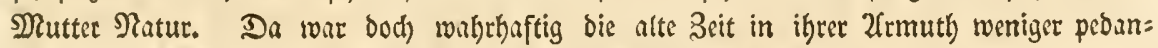

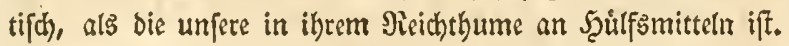

Berfen wir barum lieber einen, wenn aud) nur fluchtigen Btick auf bie Ģefammt:

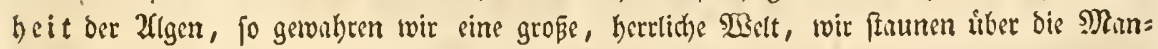

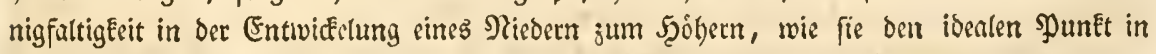

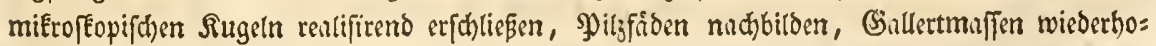
ten, burd) bie von Kützing mit unúbertrefficher Slarbeit exponirten Diatomeen bie freibe: 
twegliche Thierwelt beribren, in felkft in bieje hinuberforeiten, fich b.unn wurjelurtig ju jur=

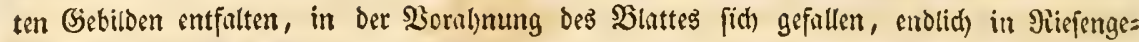
ftaltung auseinanderftrebend, bie Meere beberrichen. Denn bald lafien fich iiberall, wo

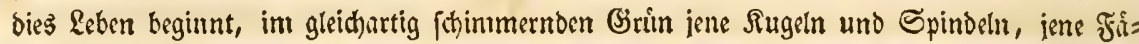

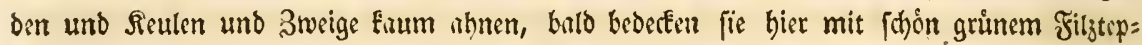

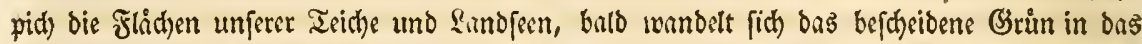

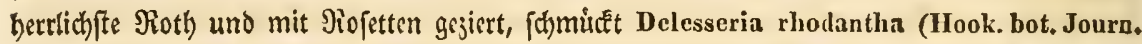
II. t. 126.) bie bie İnfel Mauritits umyebende See, bald futctuiren fie trieder bort in

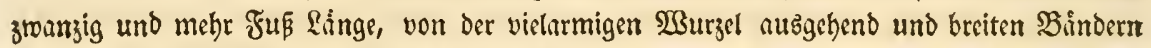

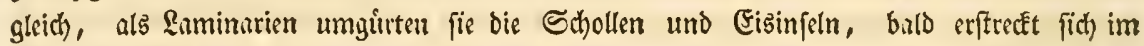
norblichen atlantifchen Scean Chorda filum zu einer 2lus̆bebnung von breisig bis vierzig

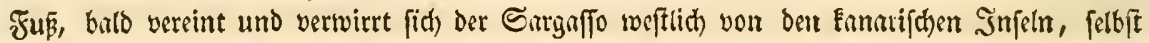
3u fdimimmenden Sinfeln von 160 Eeemeilen, Durdh weldhe bie burch cine 2lige beforgt ge= madten Sefdiffer bes ftürmenden Dcenns, mit ben Beilen fich Bahn bauen, bald endtich) (d)eint im ftillen Meere Macrococystis pyrifera, felbjt ưber bie Mlacht bes (Elementes bin= aużgreifen zu wollen, in taufend 2(rmen zur Linge von finfbundert, in funfzethntundert

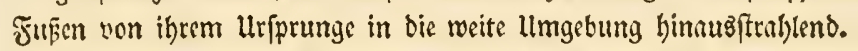

Ber follte aber nad) sinem folden lebendigen Şlicke auf bie lebendigen 2(lgen, nift

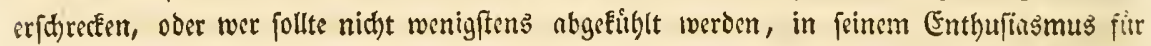

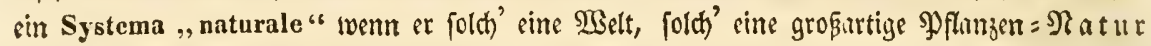
herabgefert fieht, noch tiefer als bie nod) tráumenoen Flechten, als bie nod) tiefer fchlum= mernoen Pilze? -

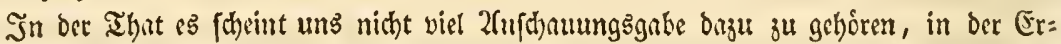

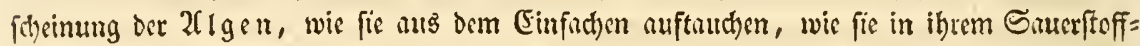

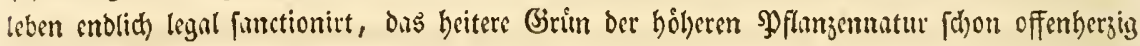

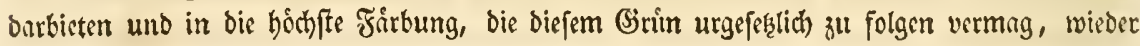
eż umbilden, in biefen lieblid) friedidyen 2figen, wie fie in ihrem ungeheuren (Elemente fich) in 3onen abtheilen und gejellig burdh bie fanften Uleberginge biefer Zonen fich fortbildend ju

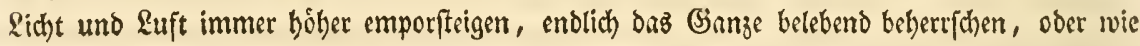

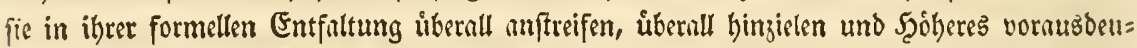

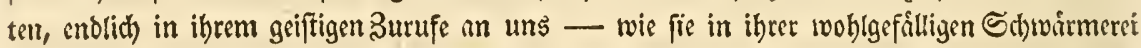
unz faft zwingen, jene auferordentliche Suvertire zu bören, welche ben Traum ber Flect)te

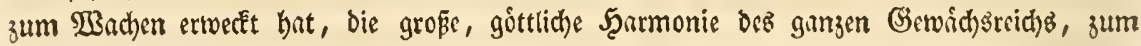
erftermale bem für folche Mufie empfónglichen Erofteife verfiunoend.

So erfcheinen uns aber bie 2flgen in ifrer innern Bebeutung wirflid) aud) immer und immer wieber als Scerolde ber böberen Pflanzennatur, bier und ba und bort und liberall nur

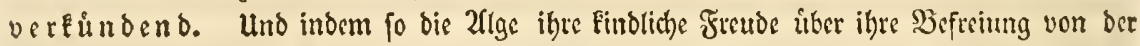

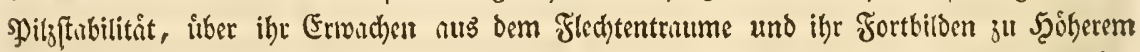

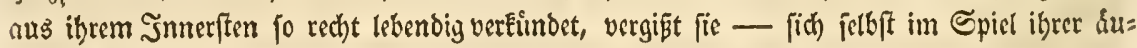
Berren Formen uno bewahrt uns nur inşgcheim ihren - Iypus..)

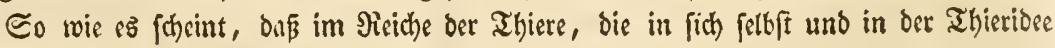

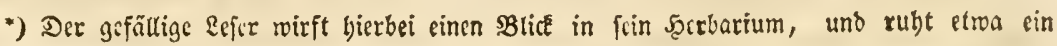

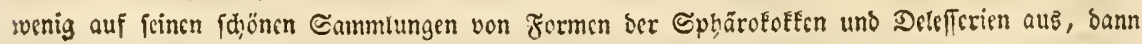
jagt ex wohl: 2tuce ift - 23 ahthcit! - 
noch tief follummernden, in Dem von ihnen beroobnten, ihnen witthlichan holbern Drgnut:

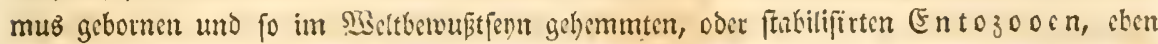

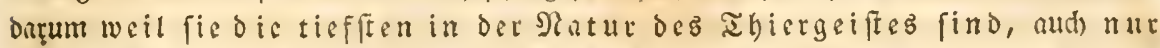

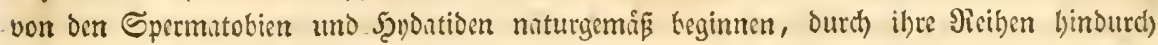
fic) Dann tweiter als Entozoen boher entfalten, in ben Eorillen bie nicberen Thiermefen baz Ihierleben triumen unt in oer freien Molluske, wie fie von oer 3witterwelt ber algenartigen

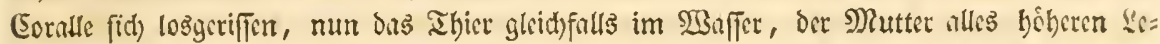

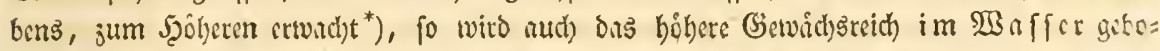

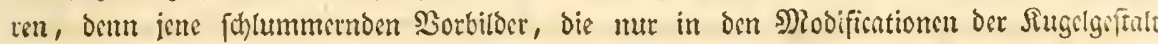
morphologird) materialifirten und materiall firiten und in ber Soee vom Mुflumjenteber pFlan=

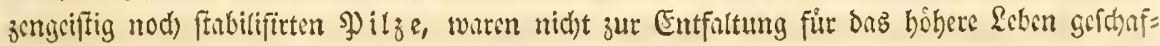

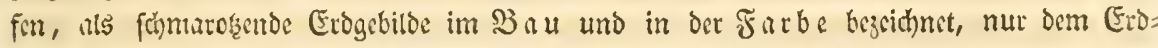
leben und Dem 2fufquellen des Saumen vergleichbar, beffen Eriftenj, als folcher, mis

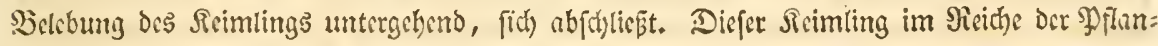
jen ift aber fich belebend - die 2rigs.

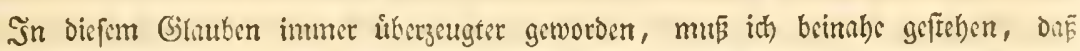
aud) in mir eine letendige Jose, faft ftatbil getworden iff, nimlich bie, dur alle Enfteme,

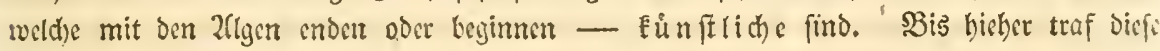

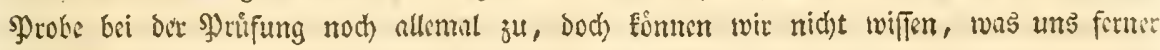
noch jugroucht ift.

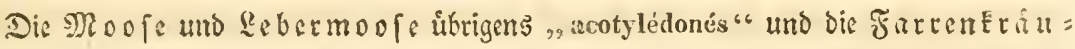

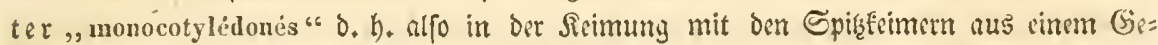

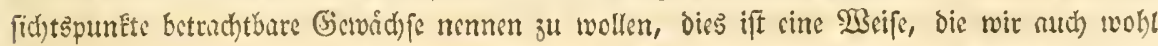

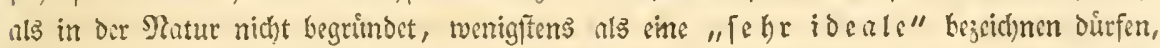

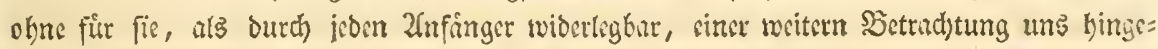
bin zu twollen.

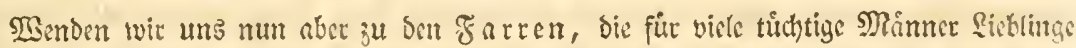
geworben und in benen wir allerbing bie licbenswirbigen 3inge ifger Sindheit aus ber $24=$

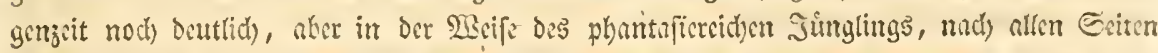
weiter anticipireno, beraustectend crblitêen. Şier banEen wir zuerfit utrerm gechrten Frrunde Presl, wilder cincr boben, objectioen Sigabung folgeno, biefe abermuls groburtige IEelt

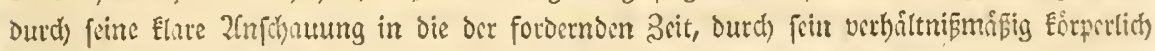

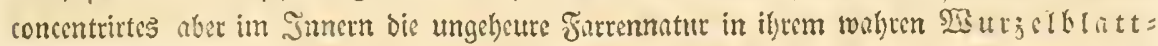

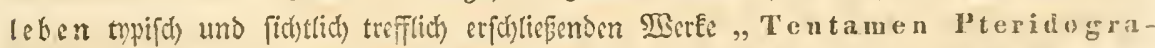

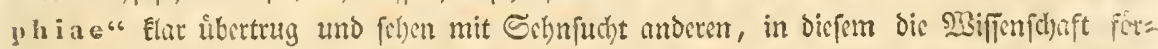

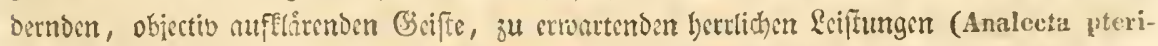
dographica) unferes theucen Sutgendreundes Kunze entgegen, wibend wir alles bus

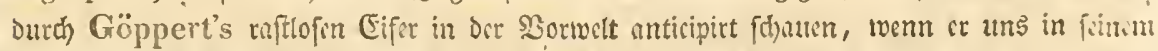
Systema Filienm fossilium (Nov. Act. Soc. Leop. Carol. XVII, vol. Supul.)

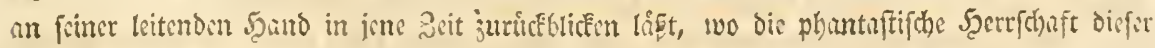

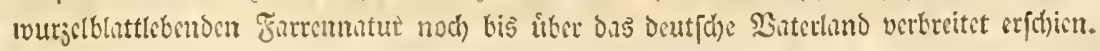

S3ie aber fo, tuchtige Merfe immer mehr bie Erfhermung des Formellen crliutern, fo

*) Sergl. min Süchlcin: 3oologic. Sittnu 1836. ङ. 166. 
bringen fie nuth bie 2fnfhauung von ben Hekergingen bes S̈nnern, benen endich nur bie

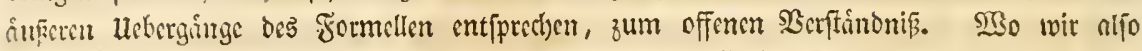
thir dic Gruppen bicfer - wenigftens einfertig phantuftifh idenl gefagt, - bie spat=

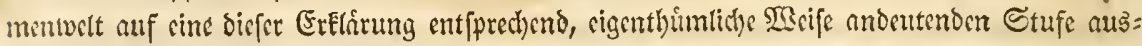

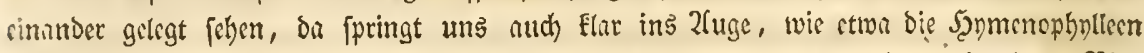
wirder in antatomifther wie in morphologifober Erfocinung als reales und unmittelbares \$in=

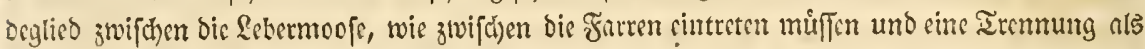
„,cellulaires" und, , rasculaires " nidht als sine umid)rsibbare begründen.

Silie aber noch mebrfach bie "plantes cellulaires" in ben Giberen Stufen ber , vasculaires" fid) wisderbolen, dafur liegen faft in allen Silafien die Dasifpicle vor.

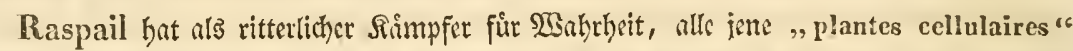

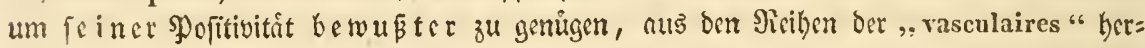

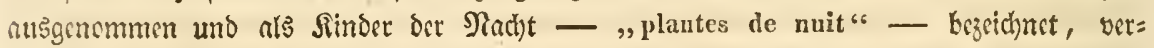
einigt.

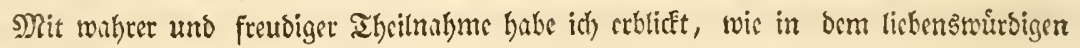
serfaffer ber, ,Genera plantarum secundum ordines naturales disposita“6

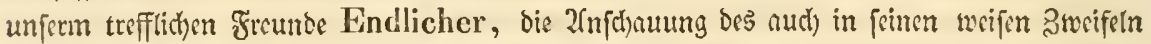

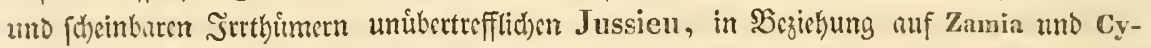

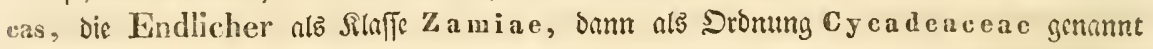

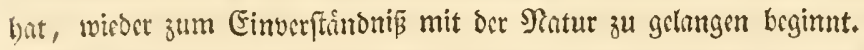

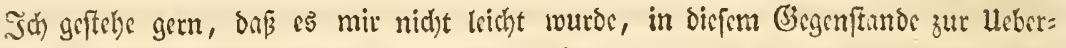

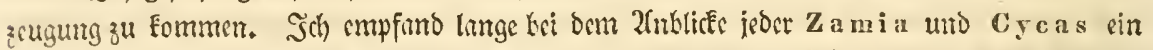

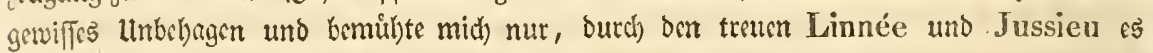

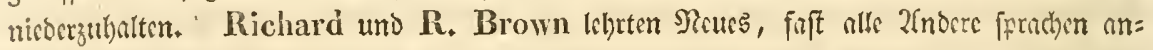
fitumeno mit, aber mir wurbe es im Jntuern babei nod unbebaglid)er und in nick)t geringer

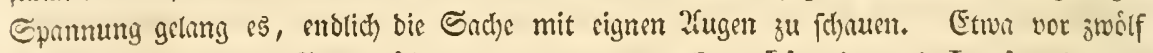

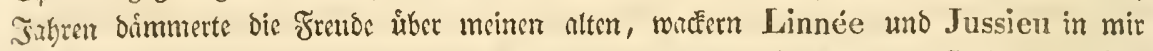

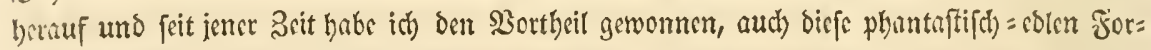
men mit wahrer Freube zu feben.

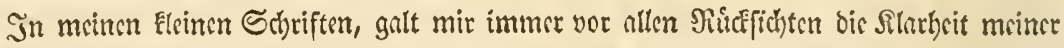

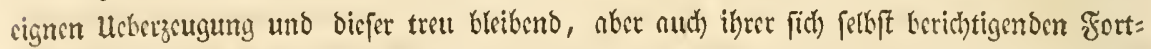

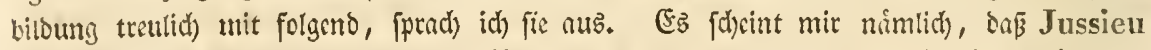

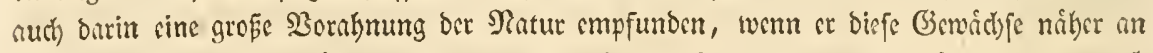

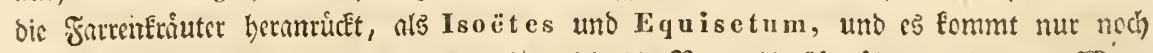
bitruf m, zu finden, wie uno wo eigentlich bic Natur bic 2(nniberung bictet. TSan=

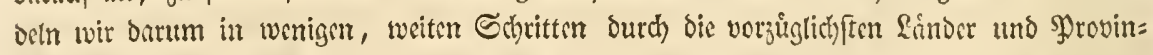
zen ocs Fartenreichs.

Der Untergang Der Moosanthere loft bie 2rntithefe ber Ehlorophnten und ber britte

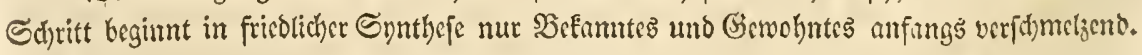

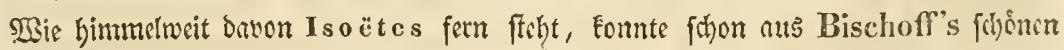

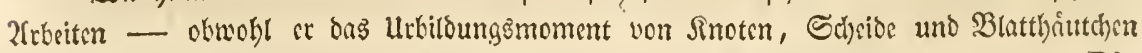

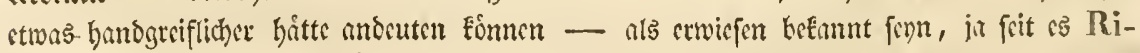

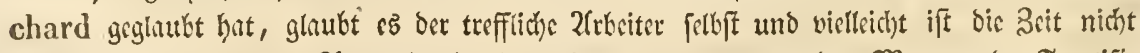
meft ferm, wo man im 2fustande cinfelyen wiro, das I s ö t e s bas Moment der Fructifi= 


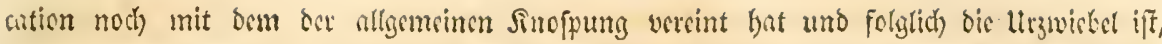

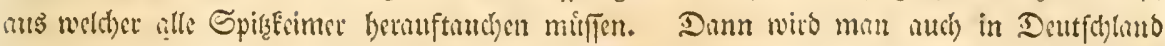

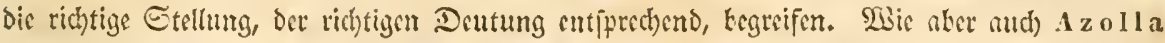

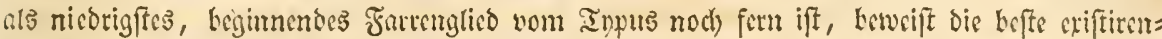
de Zanalyfe vom geiftridjen, water thatigen Martius.

Die Salviniacen und Marfiliacen beginnen mit ifr - mit Rzolla -

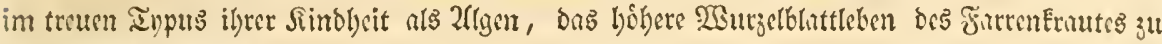

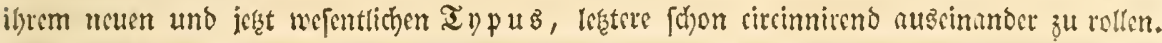

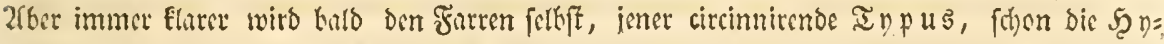

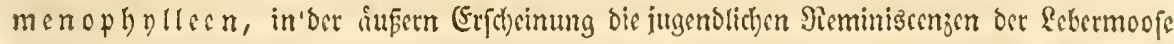

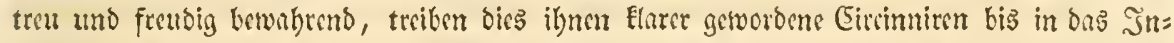

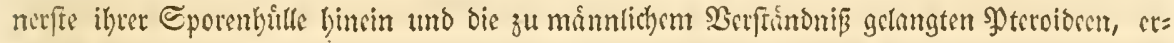

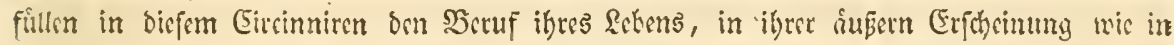

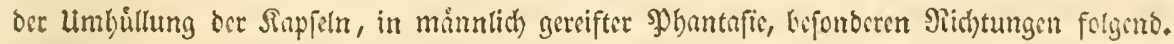

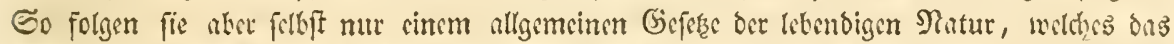

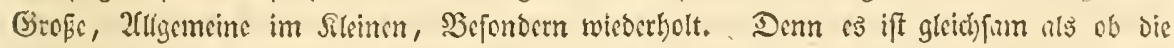

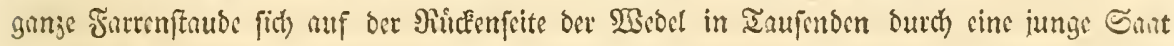

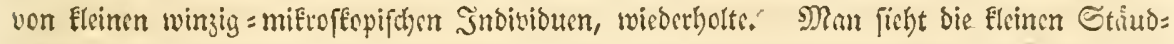
chen wiederbolt, wie in ifuen wieder von sinem ibealen Mittelpunle aus, die idenle UtEnofpe

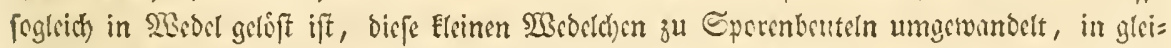

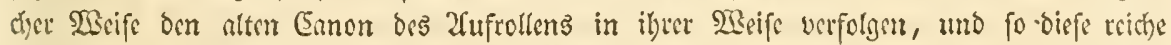

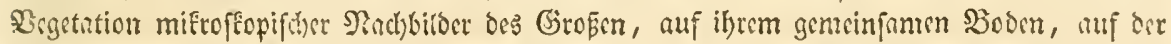

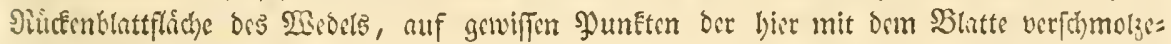

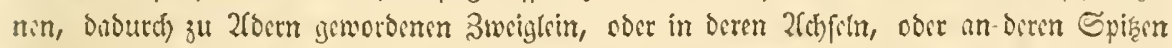

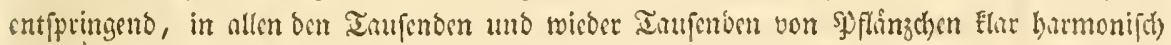

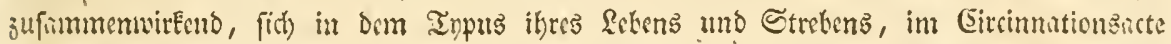

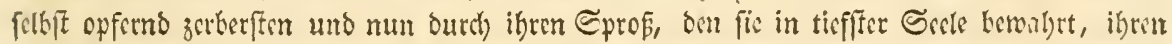

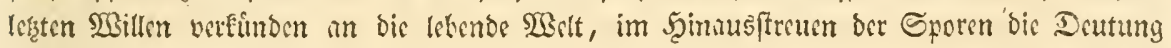

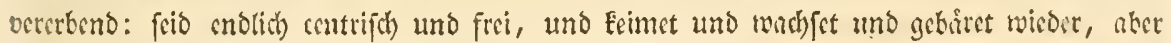
- nur circimirens, wie bie Mutter gethan.

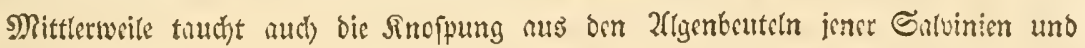

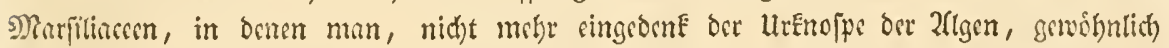
Gporen und - Sirner (?) zu finden beliekt bat ${ }^{*}$ - wirorr berauf und verliuft fids

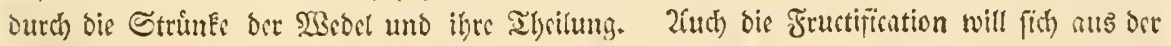

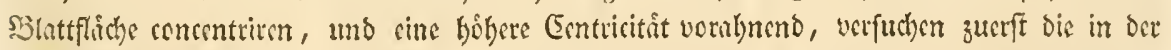

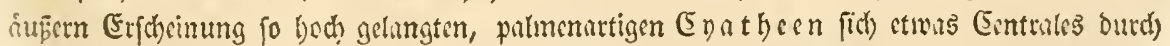

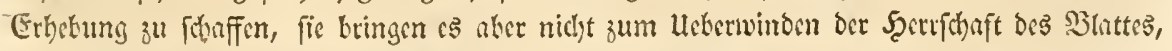

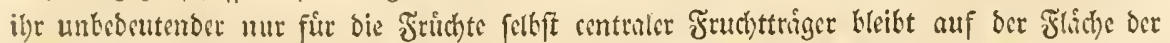

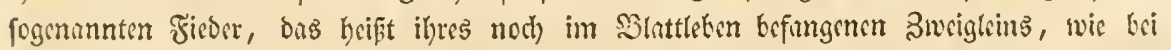

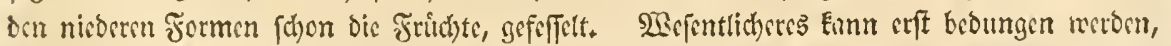

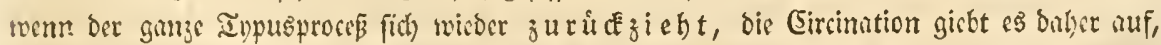

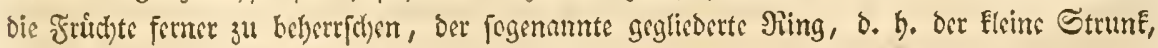

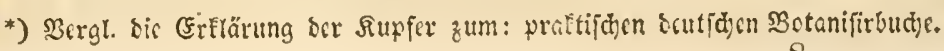




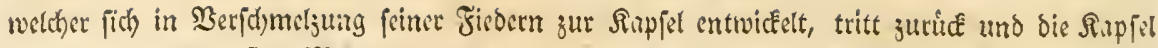

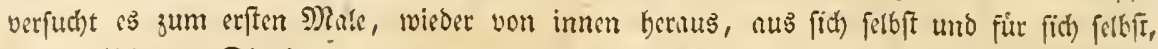

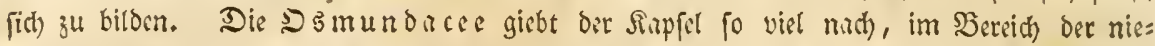

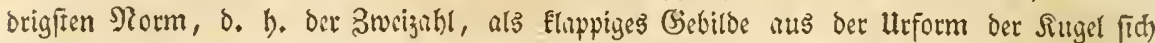

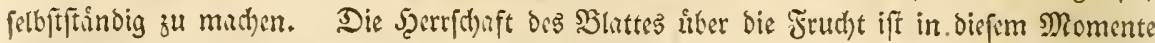

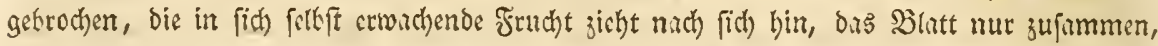
wie fhon nuf voriger Stufe bie edle Struthiopteris (boch sigentlith nur in geringerem (jinde)

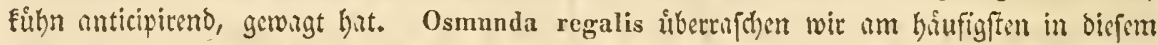

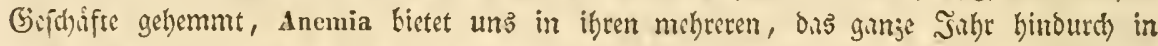

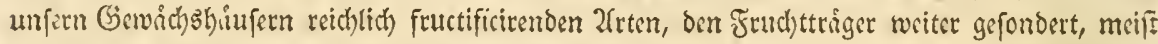

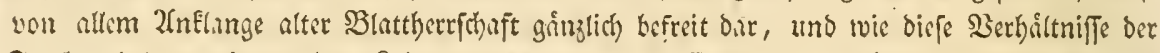

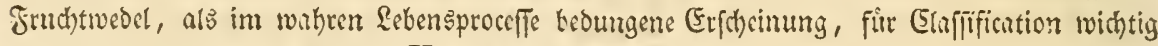

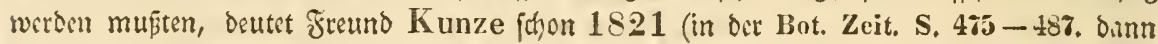
$492-503)$ weitse burch ppecisller begrủnoste 2(usfiulyrung an.

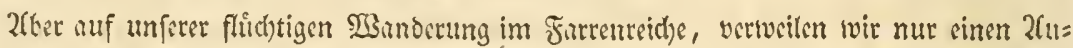

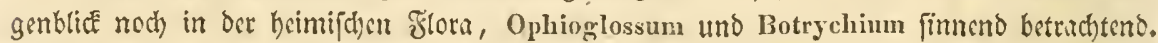

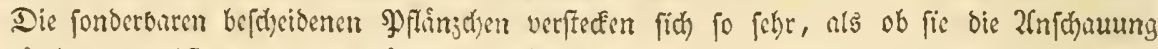

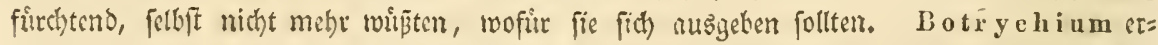
innert uns wobl noth an feine alte Mutter 0 smunda, aber bei bem Natteryinglein ming

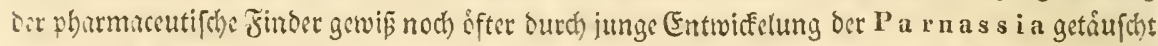

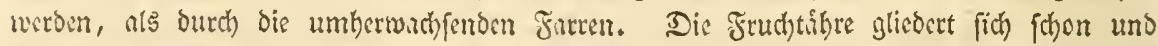

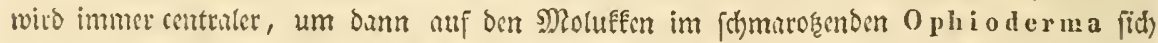

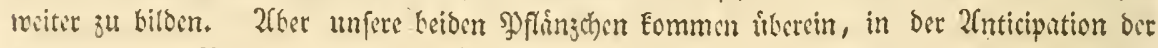
Simope in der \$̧afis des Ctrunfes.") Csit uns umfer guter Kaulfuss (Bot. Zeit. 1622.

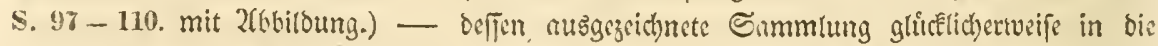

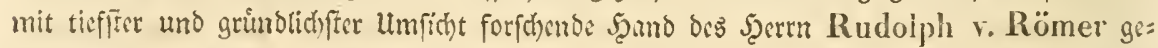
Eommen - durnuf nufureffam gemucht, baben wir bics wohl alle ofter gefeben, obne

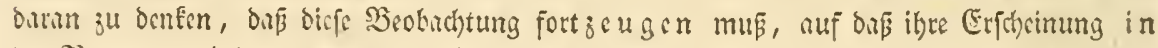

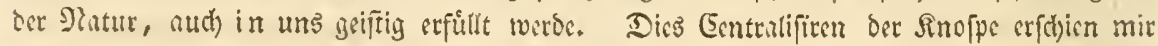

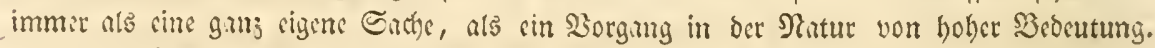

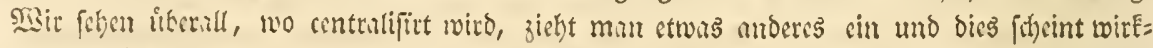
(it) sin Sisg ju finn, ben man - an feinem Drte - ganj bem Eaufe ber Natur ent=

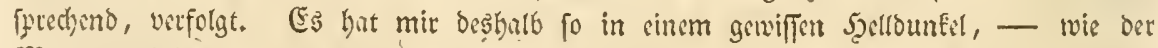

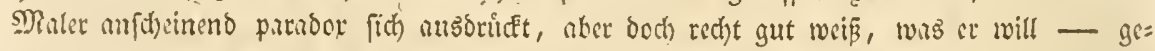

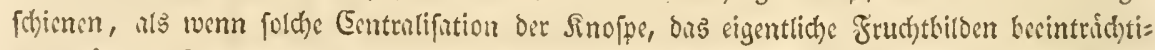

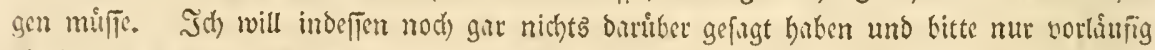

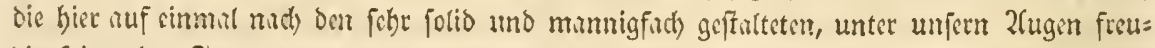

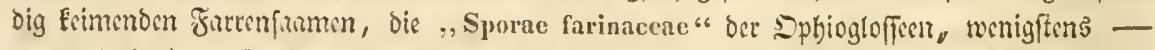

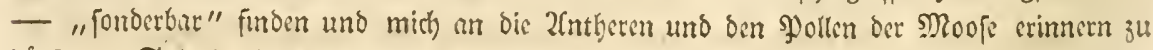
durfon. Cobato aber simem meiner ctwanigen Effer aus jonen, sporis farinaceis " cine boi:

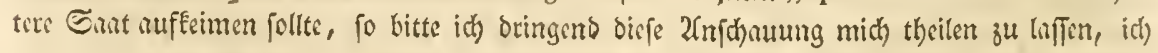

*) Sergl. Supferfammlung zum praEtifalen Botanifirbude, wo Tab XII, alle FarremErautformen ber beutiden flora nno mas ser gencigte \&efer etwa nod) bei ben farren benz langen mödite, analufirt fino. 


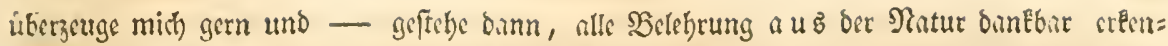
neno, meine Srtthumer, nls folthe, sin.

Es mig ober in allem Ermfte, und wir wiffen bies fogar beftimmt, vicle (jemich) in ber Natur geten, bei weid)en auth auf boberen Eufen, foknto ibnen auch beibe Fortpflin= zungşweifen zu I I beil geworben, bennoch immer sime burd) bie anbere gelyemmt wirb. Unfere

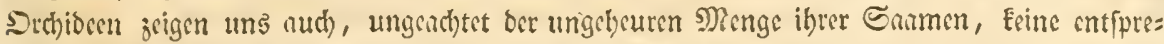

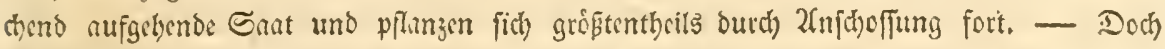
mir wollten anfangs auf Zamia Eommen umb Cycas.

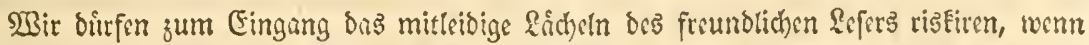

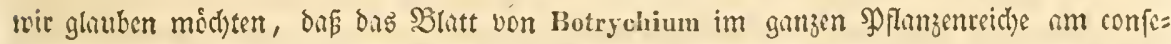
quenteften mit bem von Zamia vergleidhbar erjebeint. SBit wollen bunn burauf bindeuten, svie fich bei vollendeter (Eentralifinung Botrychium, in und nach) feiner 21tt, wenn es meglich)

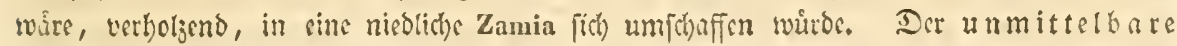
llebergang fu bicfer (5entralifation mig audi) Wabrjocinlidi) in Den Trimmern ber Sorwelt (iic) finden, bie wir shen an biefer Etelle nicht fparfam rotfinden. ${ }^{*}$ ) Findet man neer je= me 2fndentung noch zu fern, fo vergleidjen wir Helminthostachys und finden ba bie ganje

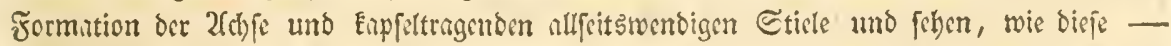

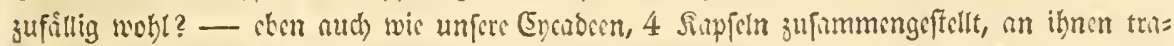

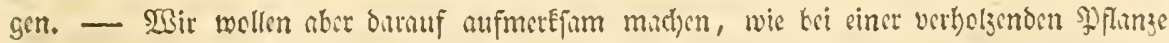
von biefem Inpus, bas Bange fpiralig georonet, im fortbilden in cine Eentralifitung oes

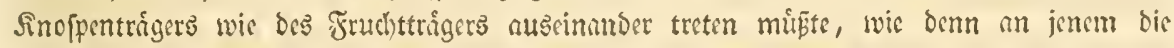

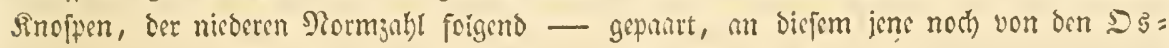

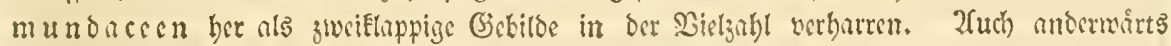

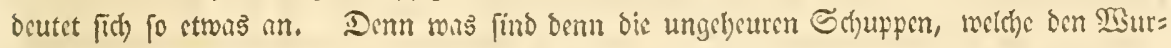
zelfteck grofer Farrenfrifuter, 3. $\mathfrak{B}$. Oer Marattia cicutaefolia Martius Crypt. Brasil. t. LXXI. fhon jirgelartig umginten anderes, als bie Usberklcibfel ber llmbitlung oer fincpp=

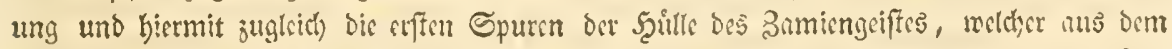

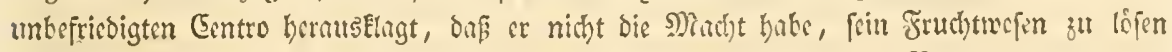
vom Btatte, um in fich ç concentritend, uns das fich verifhntid)ende Sitb ciner fogenann=

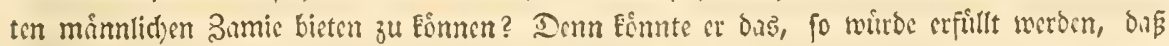

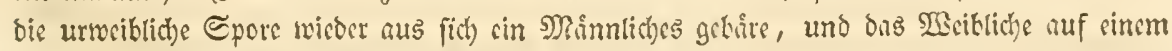
andern Stamme, ifm paraltel und neu fidy geftalte, im smigen Strchen zum Eentrum.

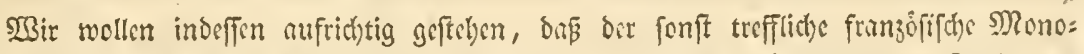
graph, feinen Şollen sin wenig teidht genommen, uno vielleicht hiberbaupt ber Swrifer er:

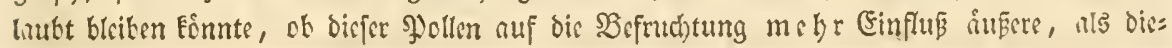
felben, bert aber sporae farinaceac genannten, rúcfgângigen Drganengetilde bei Botrs-

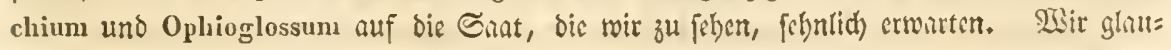

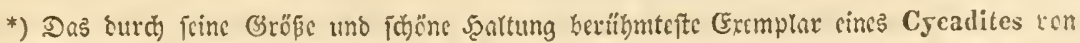

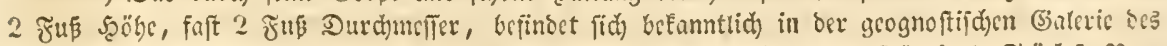

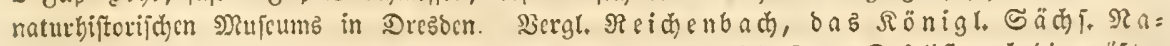

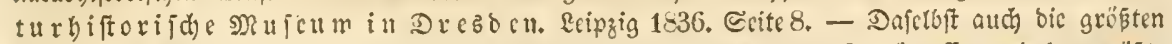

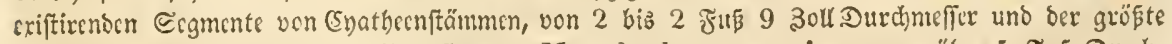

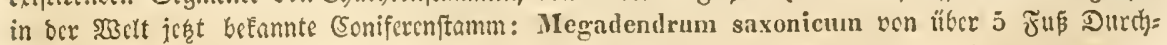

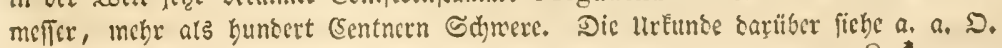




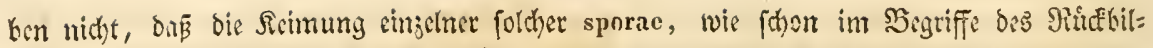

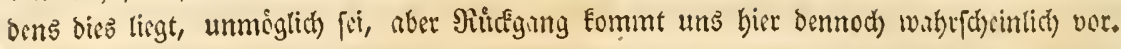

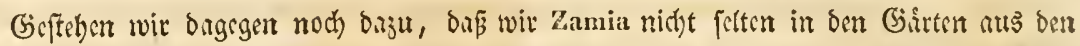
jogenannten Samen ergichen, won benen wir nidht methweifen founen, ob unb wie fie befrudtet worden, fo wurbe uns, wenigftens bis bierlyer betracbtet, Richard uns R. Brown noch nicht wirberlegen, wenn uns biefe, den Sinollen ber Equifeten - cini= getmap̄en menigftens - verglecthbaren Gebilde, fưt uns - vorthufig - als dic voll= endete $\mathfrak{A}$ nofpe der Faren erforimen.

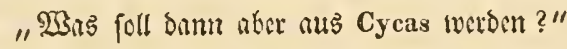

Cycas ligt allerbings dic alte Farrenmatur nod) weit Flarer und beutlicher auseinander, fo bas fie uns uber Deren Fortbildung fult zur Gewisheit bringt; uno wir begreifen nid)t, wic in $23 e r f e n$, in bonen einige, ordines" und Sinfen mehr fint, als in unferen fleinen,

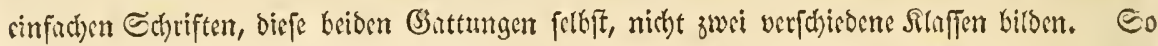

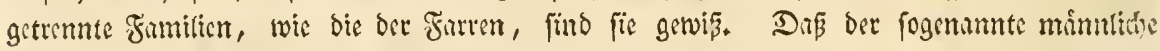

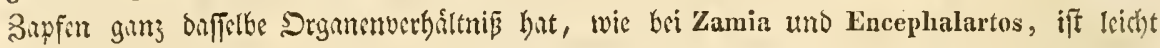
zu crÊtnncu. *)

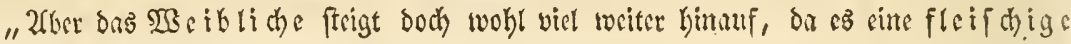
Beere jur Mist bringt!"

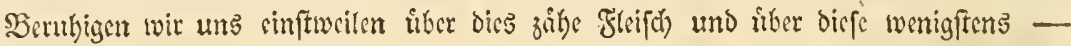

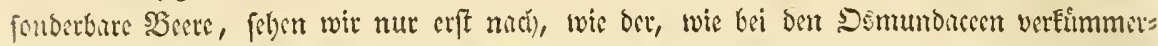

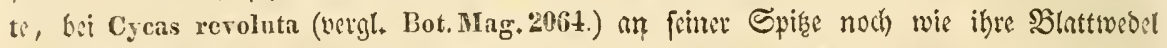

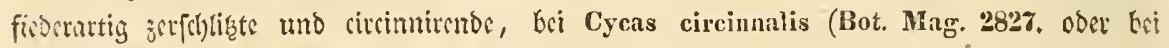

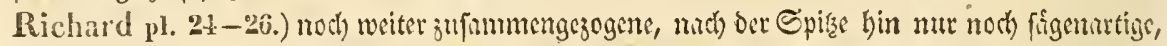

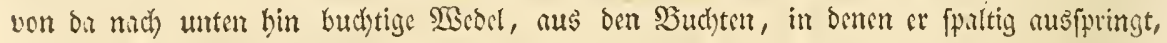

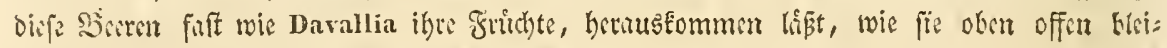
seno uno in ifrem Smern ganj freiliegent -

$$
\text { "mun bot) wobl ganj po, wic bci Taxus, Ephedra und Gnetum?" }
$$

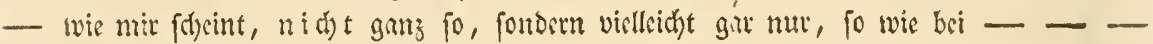

*) Jubcm id bicicn Ramen Enceplalartos ober (3) c birubrot (geborcn im J. 1834.)

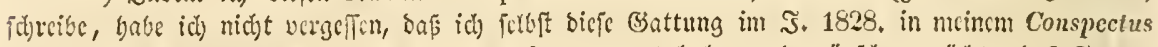

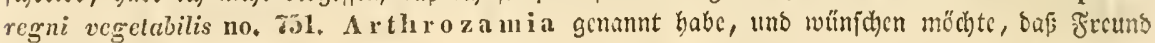

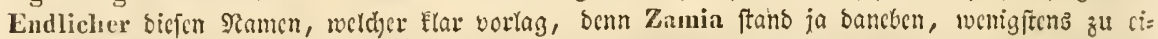
nem ridjtigen Eynounm gemadyt bätte. Sener stame Encephalartos ift abcr von cincm 2(utor gegcben, von bom id) ibn, wernigftens für beute, feleft annchme, und barum widit ben geringiten

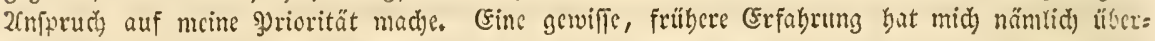

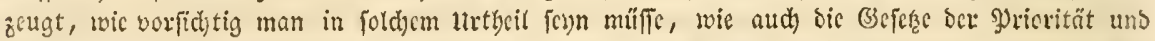

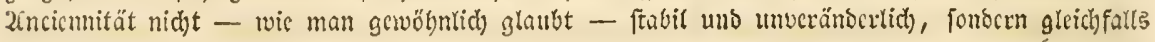

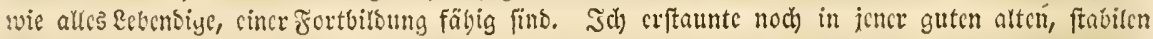

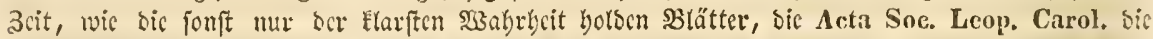
Fora ol. botan. Zeitung, bic Liunaca und nody fo mande andere Schriften, in cincr fubr widja

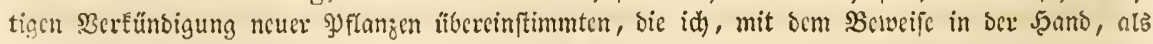

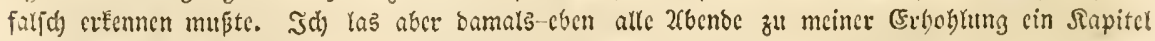

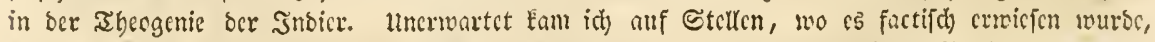

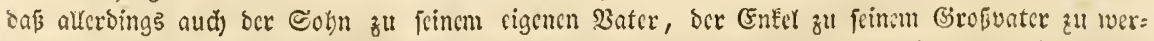

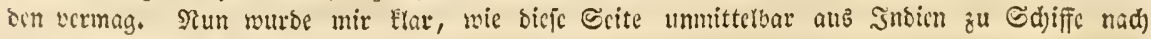
Soumburg geremmen, idj bad)te mie jekst: sapienti sat! und - fdywicg. - 


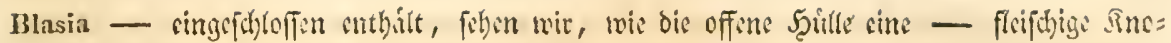

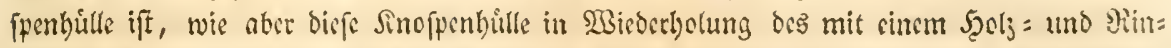

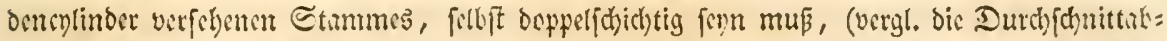

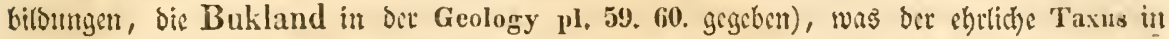
feiter 3 apfenbeere, in weldyer ber Metamorphofengnng fich bocts anders verbilt, und dic vou R. Brown viefverglidenen Gintungen Ephedra unb Gnetum nicht hernusbringen

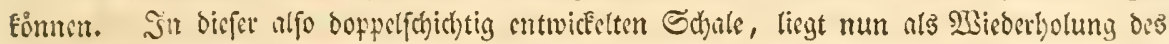

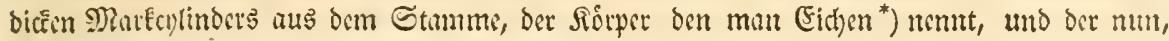

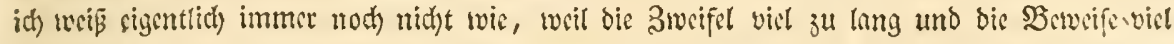
zu furz find, fo im offenen Fruchtenoten wom Spollen, Der felten ober vielleidyt faft nie ba

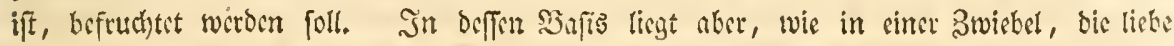

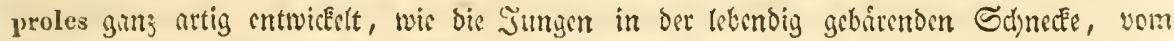

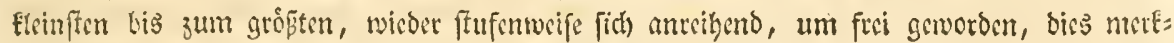

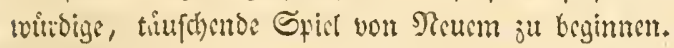

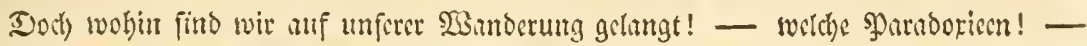

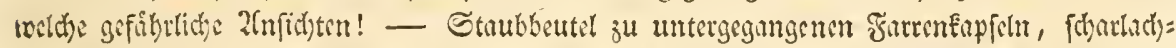

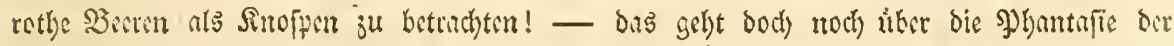
Fiarenwelt filkif!

Dic virkliche SBsfudtung ift nicht unmiglich und wirs nicht cben gelengutt, cs wiro

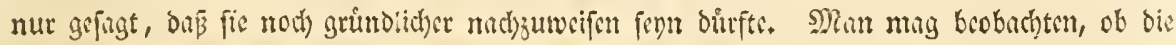

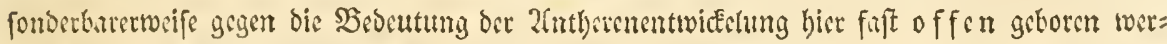

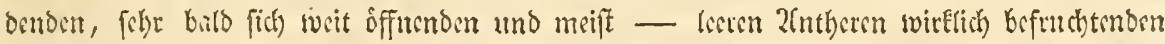

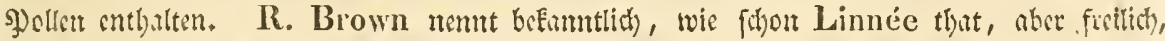
Damuls in feiner fpitiren, alfo fortbitbenden Ueberzetgung - was fur midy von bober

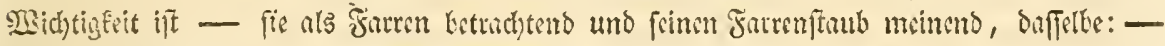

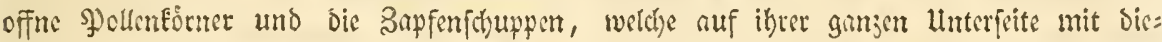

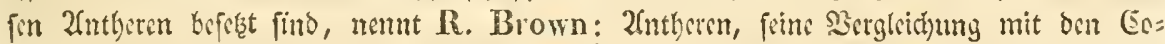
niferen verfolgens; Richard ment bas, twats uns bie 2fntheren oder untergegangens Swa

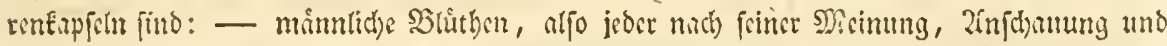

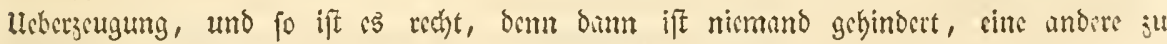
bitben.

Seitorm wir buth Nees von Esenbeck u. 2T. wiffm, was sine 2fntbare in

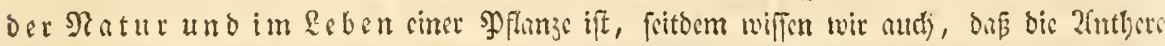

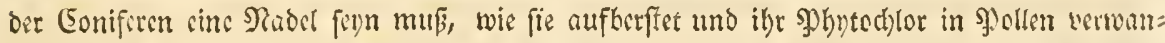

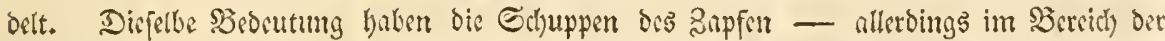

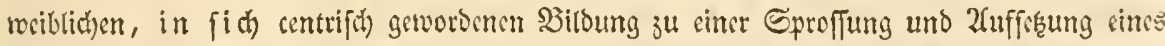

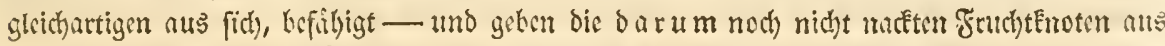

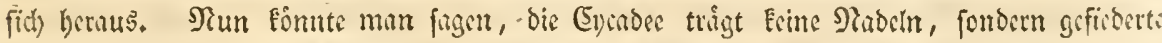

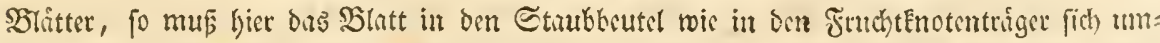
wandeln, und R. Brown's Ncinnmg - weldye a priori nidjt ittoufequent finn fonnti,

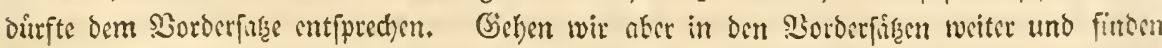

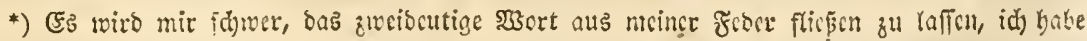

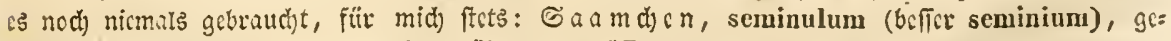

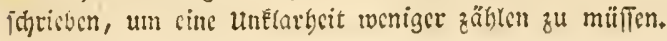




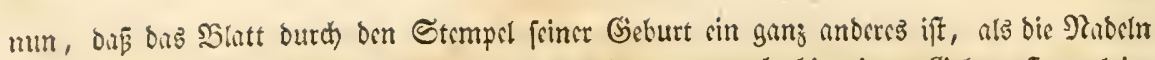
und SBlátter oer Soniferen offenkar find, fo wirb uns auch bier jener Beburtsftempel im

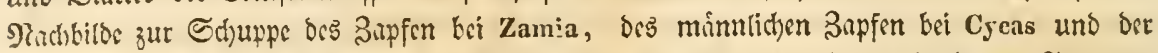

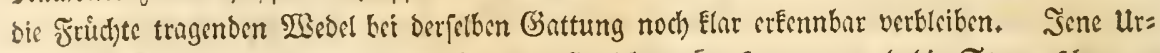

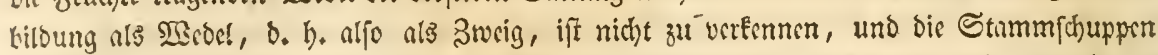
von Cyeas und Zamia, welthe nur Sajen von 20 eden verblicben, wirberbolen fid) als

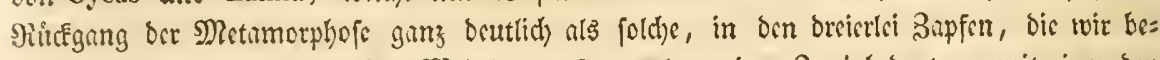

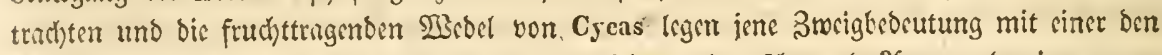
Eoniferen frembartigen Sinofpung Flar uno Deutlich vor bus fotumenoe 2(uge und crinnem an jene Sirrofpen, weldhe bei Kunze's Gruppe ber Gileitheniaceen vorfommen, weld)e Gilcidje: niacen bod) burd) ihre Mertensia fet)on bie Gubftanz und Unconfiguration des Encrocenwes bels corkilden dirften, oder an bic Der Woodwardia rarlieans, bus Polypodium proliferum, unb nod) niffer bic von Cystopteris, und crlauken sine 2 sergleichung mit ifnent.

"ת: morben?"

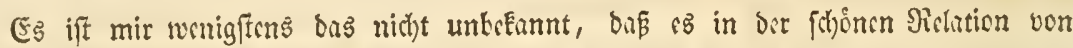
Hooker, bem wir, wie fo vieles Irefflithe, fold)es auch hierin verbanEen, unter andern beift: the female plants flowered at Seychelles, and Mr. G. Harrison, the Gorernement $\Lambda$ gent there, transmittel some of their roots to Mr. Telfair, in whose garden of. Bois Cheri, in the hisuritius, they have flowered, and being fecundated hy Professor Bojer, who touched them with the pollen of the male blosson, they bore

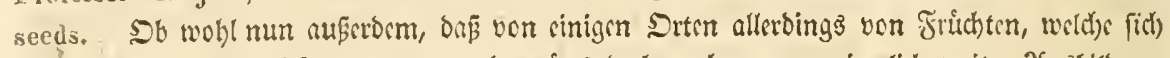

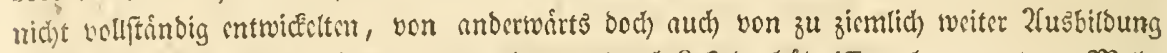

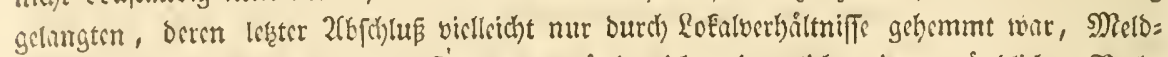

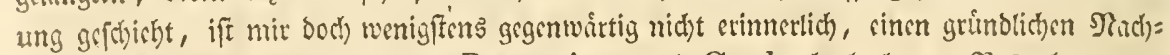

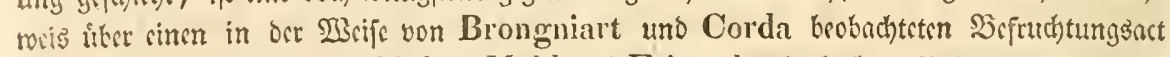

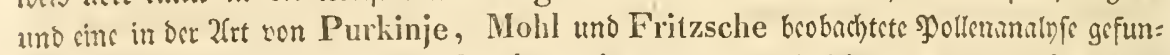

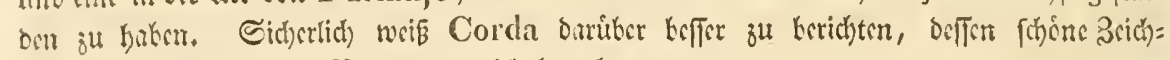
nungen bic 2fEabrmic in Docrtin, treulich berwabrt.

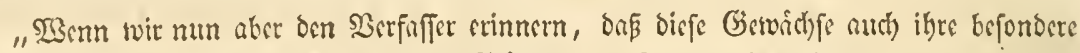

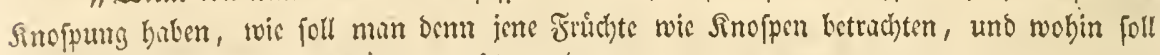
uns enolid) dics Sinofpentorén nod) fübren?"

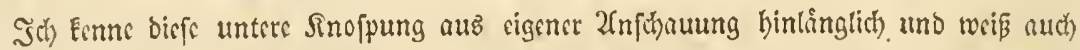
rectet wobl too cs beift: , the roots, howewer, hat, during that period, multiplied to twenty or thirty times the original quantity, and thus an opportumity was given for distributing them still more extensively, "ich fitte aber nactjuffefen unter welchem be=

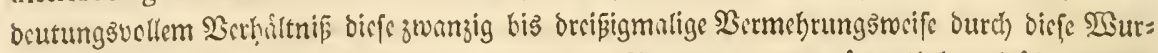

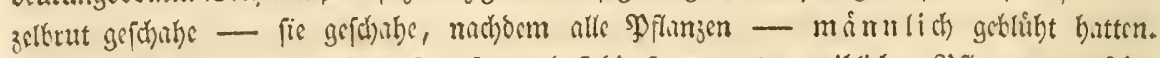

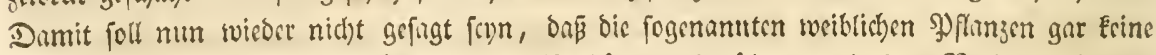

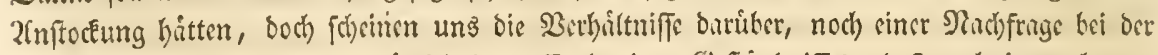

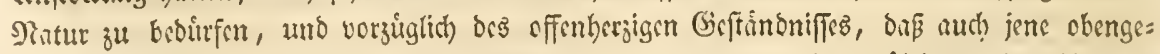
Jumnten Finren, wie Polypodium proliferum, Cystopteris and unzíblige andere bie bop=

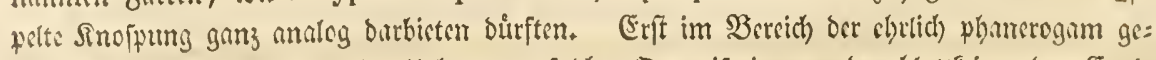

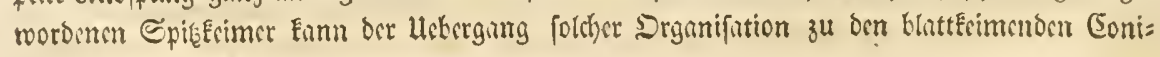




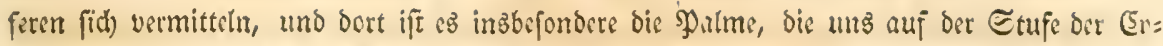
crobe naturgemás wieder begegnet, in meld)er jenes Sinofpenmefen zut ehtlid) phanerogam = ge=

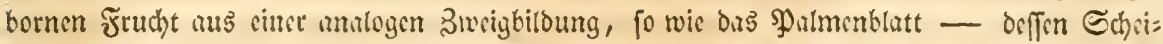
be bas lleblatt gewefon - Felbft cine pold)e ift und in Caryota bie Zamia nadjafmet, -

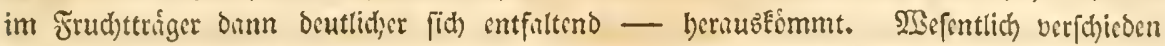
ift bie normale Snofpung ber Eoniferen, bod) aud) bei biefar tritt nad) gegebener Sirum=

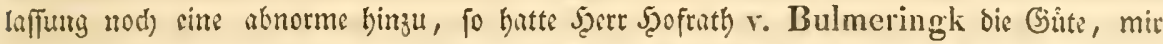

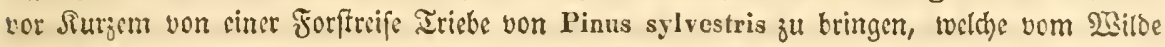

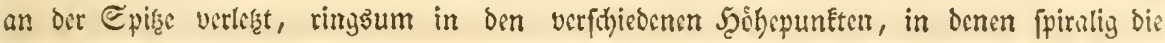

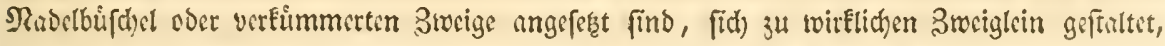

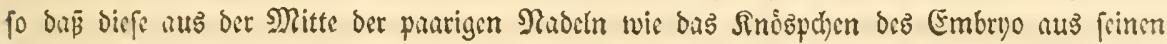
Eotyledonen heraustommen.

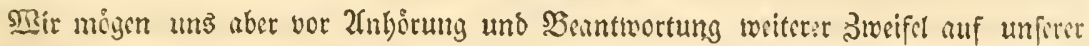

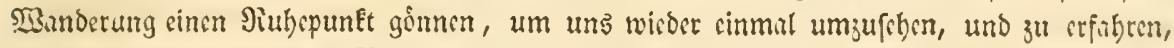
to o wir eigentict) in $\delta$ cr $\Re$ atur find.

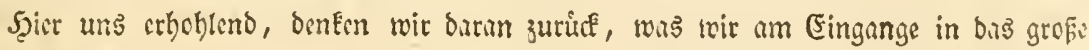

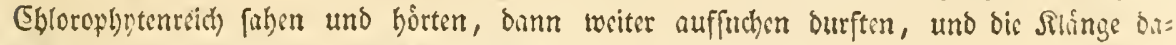
von, wars bie Suverture verEfindert, billen nod) in uns rvieder.

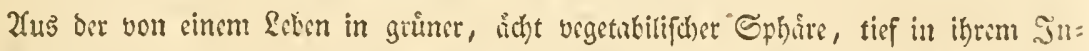

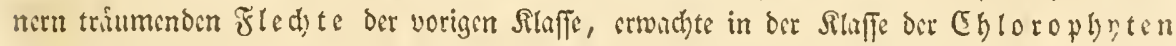

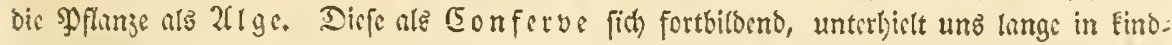

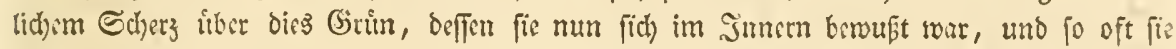

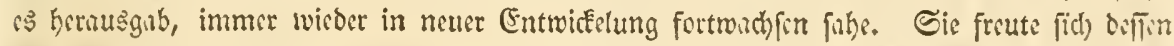
um fo mebr, als es ibr cinjiger $\mathfrak{B}_{\mathrm{r}}$ fif tour, bas (Einjige attd), was fie bingab, um in dir

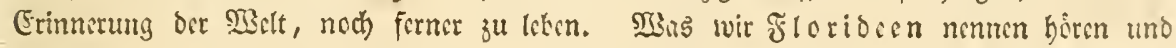

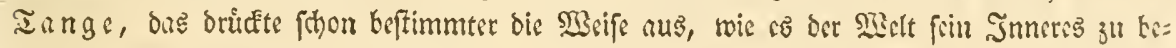
mabren und ju riberliefern gebad bte.

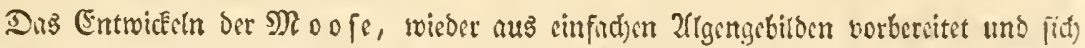

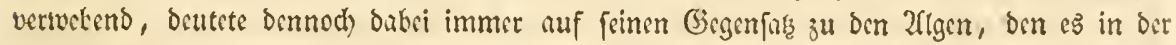

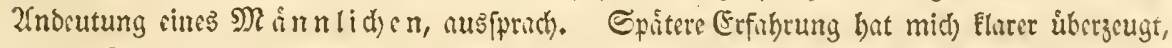

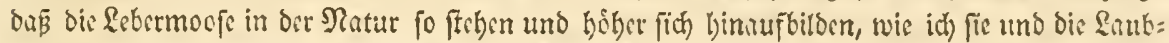

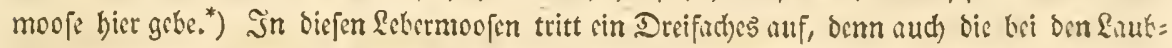
moofen biar und da frei werdenden Sinofpen treten bier fichon befimmt hinju, finpfeln und

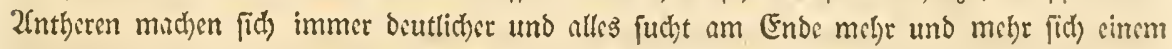

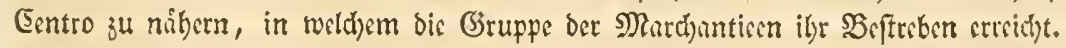

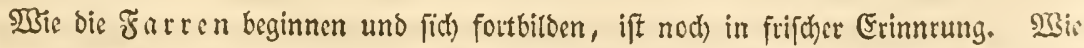
wher in ifnen bie in voriger Stonung vieder untergegangene Moozantbere fohlte, fubsu wir

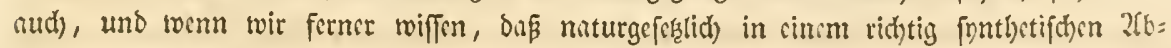

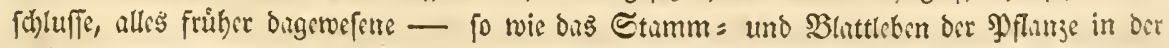

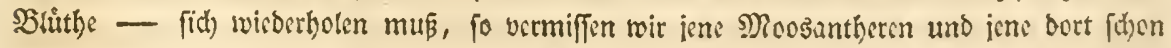

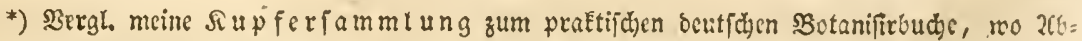

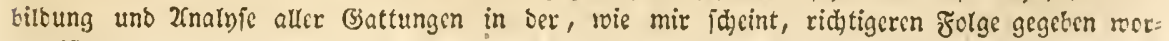
scon ift. 
fo boc) gebildete Sinofpe, fie miffen alfo ocn tieffen Naturgefesen zufolge, wicocr ct= idsinm.

S3cibes gewinnt aber bie Encabee mieder, die Metrmorphofe bildet, wie cs fdscint,

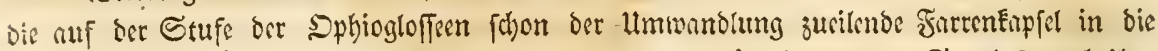

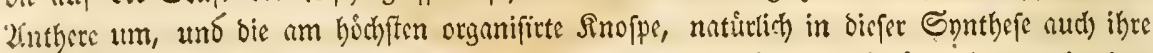

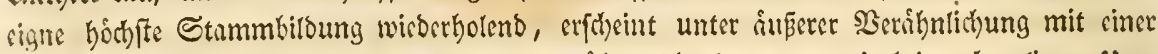

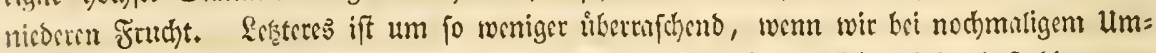

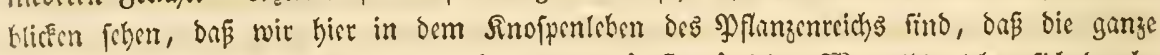

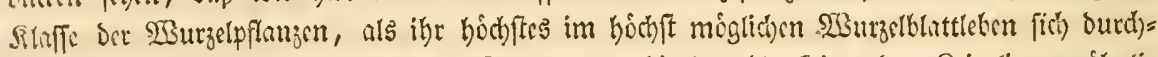

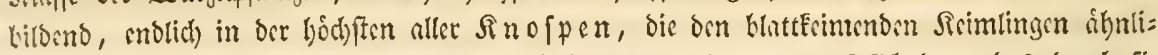

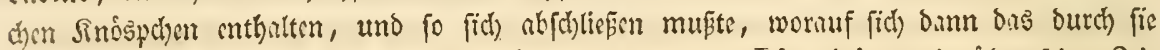

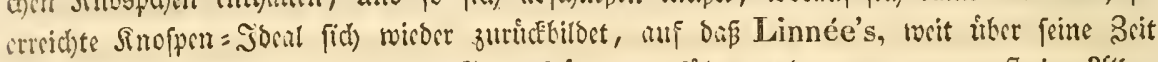

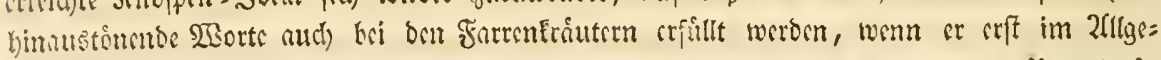
mciman figt: , alias partes habent crassiores et convolutas, novac plantac rulimenta in se continertes, - hac vel ad basin, vel ad latus, vel in sinu, - labent reras gemmas" - bimn beftimmter auf unfern Fall pafferb: "gemma est pars plantac - quae occultat sųuamis, foliorum rudimentis, embryonen futurne herbae" uno "gemmae perinde ac

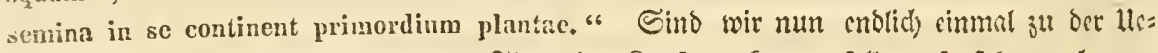

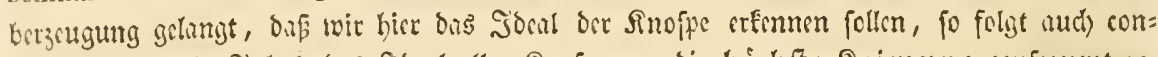

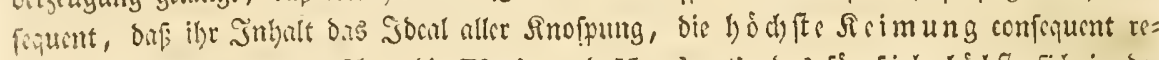

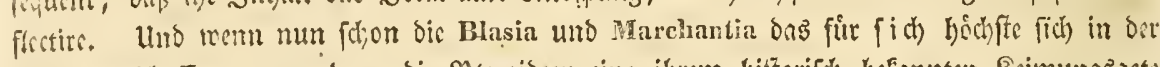

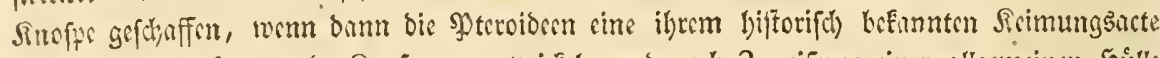

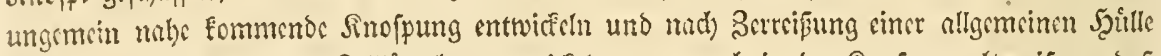

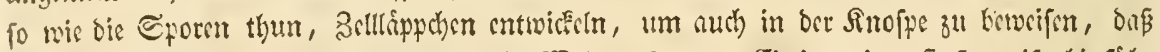

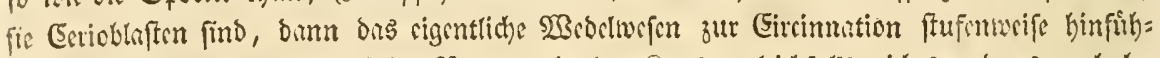

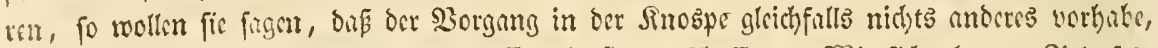

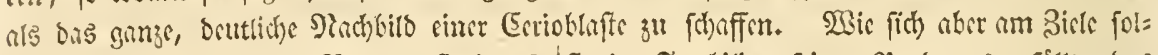

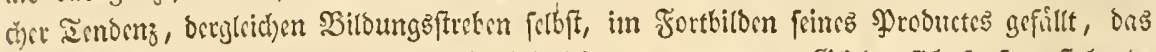

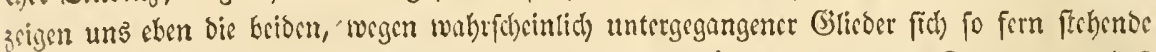

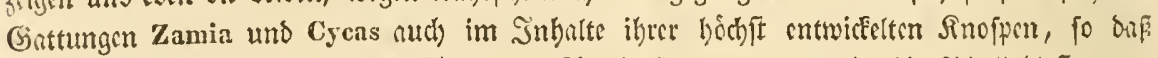

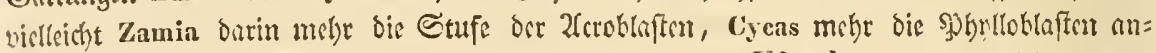

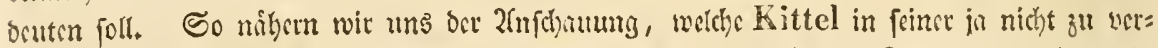
geffinden 2665andlung (Bot. Zeit. 1830. no. 39. S. (i23.) gegeben. Deffen ungerichtet labe

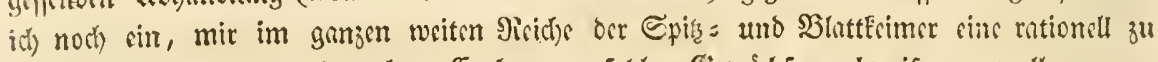

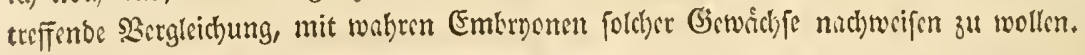

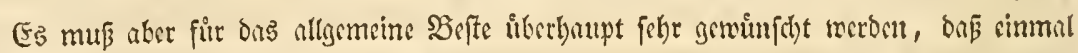

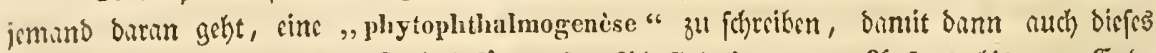

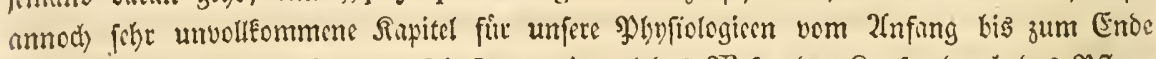

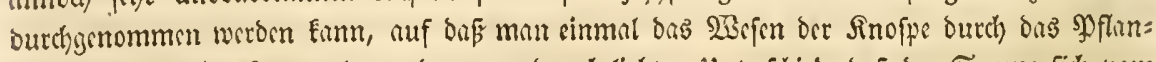

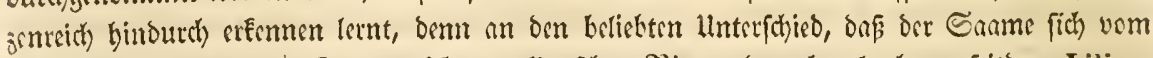
Stumme lostrenne, bie Jinofpe nicht, wollte fhon פirmano mebr glauken, fritbem Lilium tigrinum fo biufig in bic Giarten gefommen.

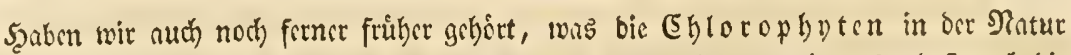

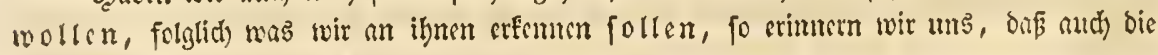




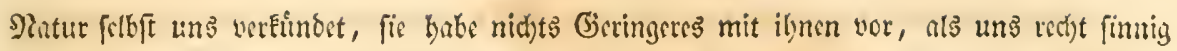

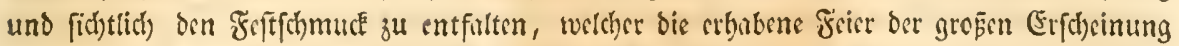

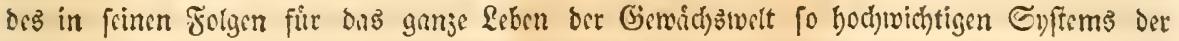
Epiralen whitoig begeben zu luffen, walbrbaft beiligen moge.

SBic num bicfes gewaltige Syftem orn ungebsuren, die ganze funftige Natur burch)=

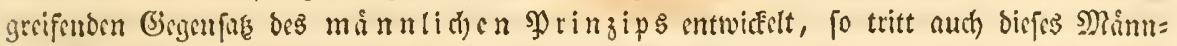

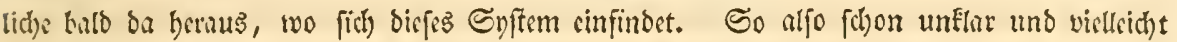

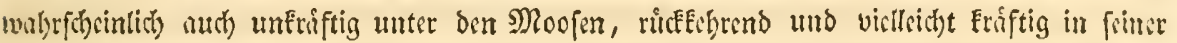

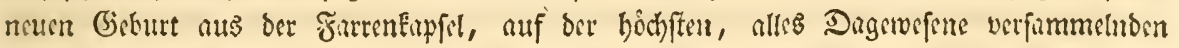
Stufe ber Everoben.

"Shu follen wir alfo boch wieder baran glauben, boß bier 2 efruet)tung ftitt findet,

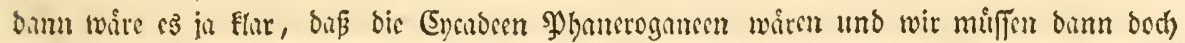
lisber Richard, R. Brown, Brongniart uno Lindley folgen, welche fie ju ben Co= niferen gefteilt baben."

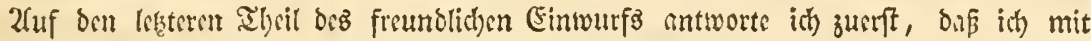
von mir fo hoch) verebrten Mormern weit licker libercinftimmen, ats von ifnen abweichen

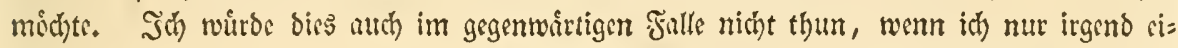

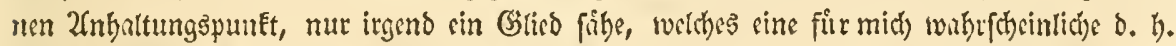

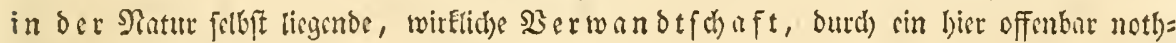

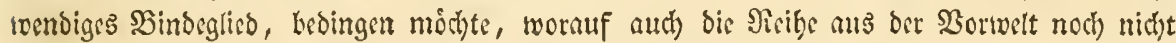

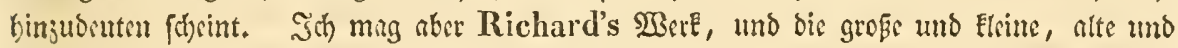

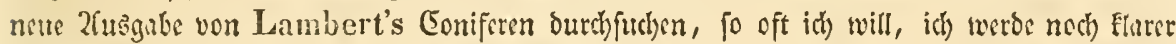

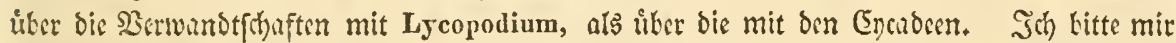

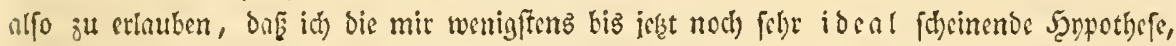

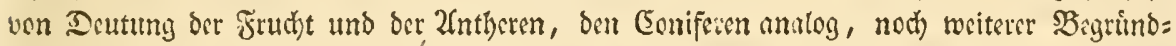
ung empfeblen barf, und vor ber Şand Seern Lindleýs \$ercinigung — felbft wenn fie Dus einzige origindle Moment feines "maturtidyen" Syftems wire - noch niobt foigen mus.

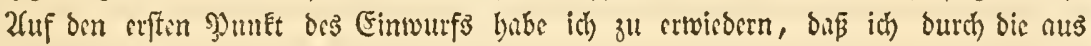

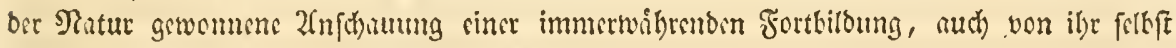

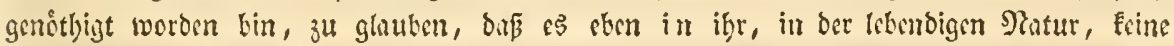
Eryptogamen geben Eunn, well fie fich nicht zu Shbanerogamen binanbilbeu burfen,

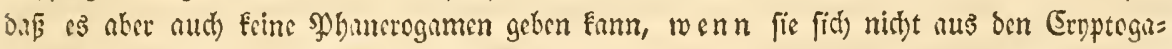
men beraufbiloen. (Sisergl. hinten bie Tubelle.)

Vaillant, Henschel unt Brongniart verbanfen wir bie unfterbiche, aนร lef̧te=

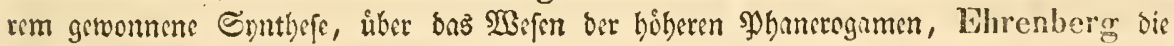

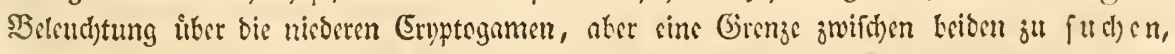
fiel fo geweiheten Priftern ber Shopfung nitht cin.

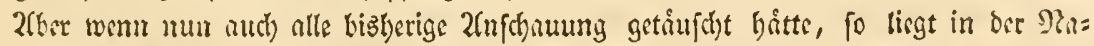

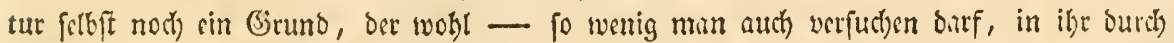
mathematifhe (sirunde etwis lebendiges mathemutifa) bemeifen zu wollen - mit sollem

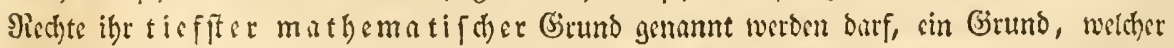

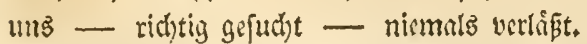

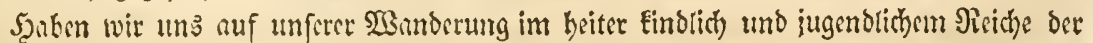

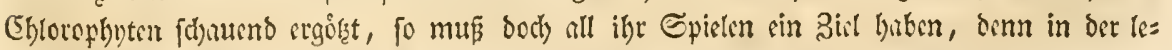

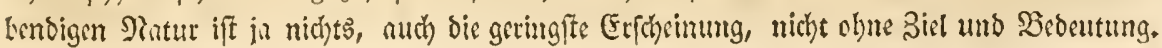




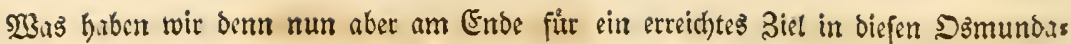
ceen und in biefer fich palmenartig bunEenden Enathee gefunben? S̃n ber That, wir múffen gefteben, now) Eeines, in ihnen allen ift noch bie leere SPbantapie, bie fich im Sireife berum= breht und immer und immerfort in Ewigkeit aus der UrEnoppe wie bie Reuthteugeln aus der

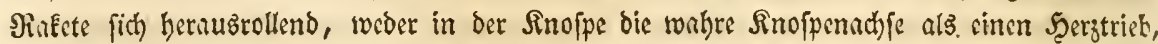
nod) in ben Jweigen, obwohl blatt = und frudtttrngend zugleich, nicht einmal ein feitliches Eentrum als eine Elare Sinoppenbedeutung geninnt, fo alfo nur bafdend nach dem (entro,

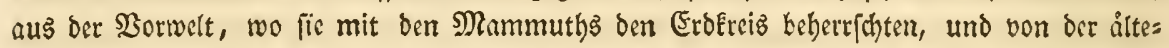

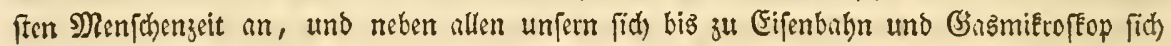

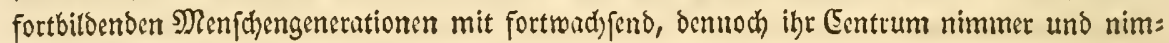
mer erreidjten.

Soldjes Spiel hig woh! nidyt im gottlichen (bidonelen ber Schipfung, in bem alles

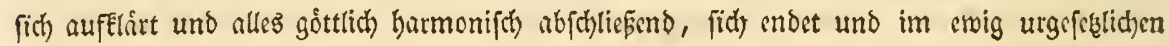
Enclus, an feinem Ende bann, ben 2Cnfang wieber begrüpt.

2lber fo breken wir uns aud felbft fo lange mit in Spirulen und phantuftiphen Srci= fen herum, bis wir in ber (5yeabee und in ber 3 amie endid): bas Elar gewordene Eentrum ber Ehlorophyten erfenmen. -

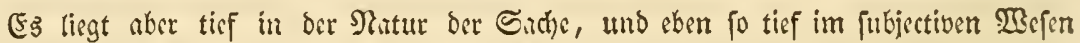

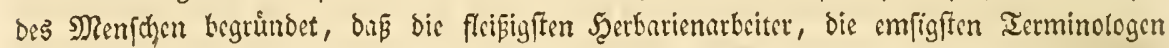

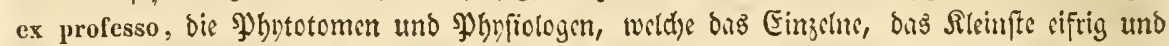
immer wicder und anbalteno und lange befhauen, in bicfes Finzelne und Sicinfte fich felber mit innig bineinleben. So betreibt aber bann aud ein grö̈er Thail ber Sylfemetifer und

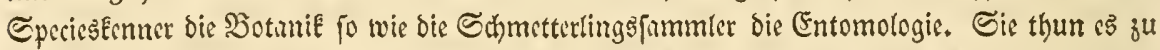
ifrem Sergnigen und fic thun Daran ganj recht. Nur ift babei zu bemerten, bas aus fo

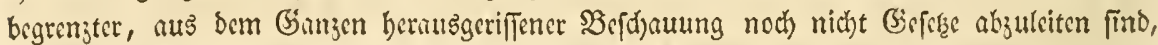

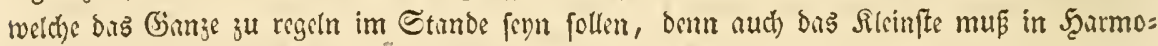

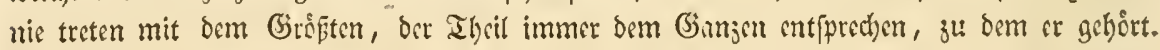

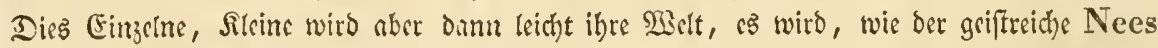
v. Esenbeck bics Streben to tief und grumblich erfarfend, cinmal hefonifh treffend be: zeid)net, bie fleine Somm in ihrem Bimmer, fie ziehen nun alles in biefe, felbft noch Soch:

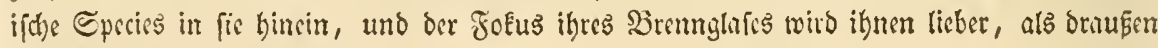
bie grope Conme am 5̧immel.

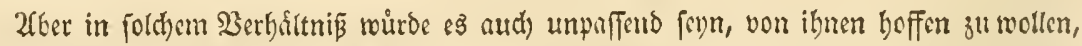

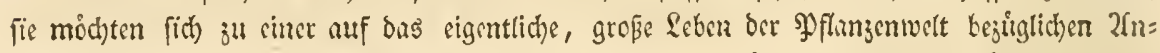

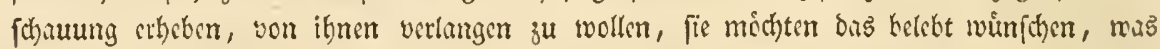
ned̆) feinet (Ertootung und 3erpaltung weit leid)ter und bequemer fich bandbaten liept. Dis

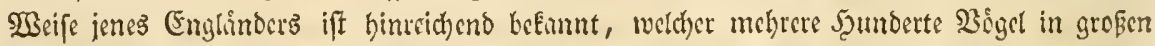

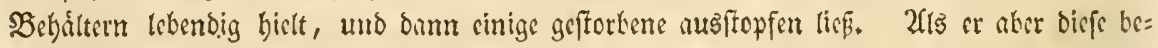
feben, gefieten fie ihm beffer als bie lebendigen und gefieten ifm fo febr, dor cr num alle todtfolitigen uno nusftopfen liç.

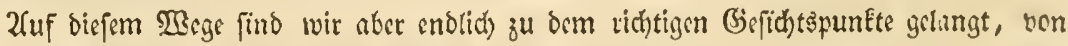

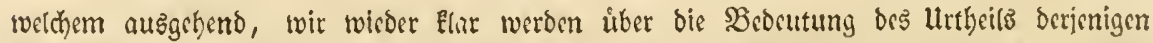

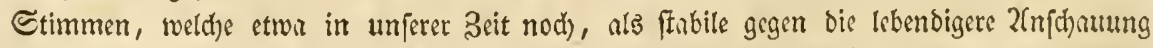

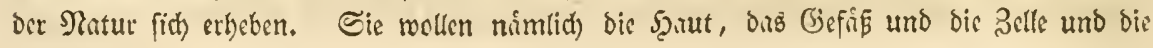




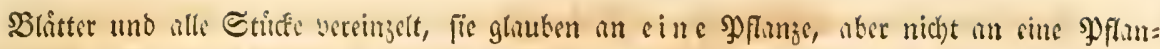

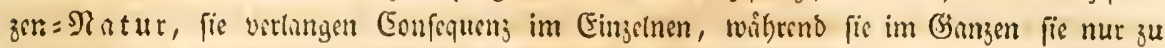
nhmen verubjheuen, fie fordern befpotifh Familicn zur "Dronung" unb leugnen Keharrlich sine stonung in ber SPflanzenwelt fetteft!

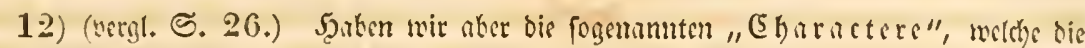

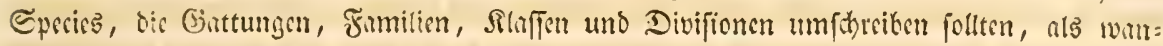

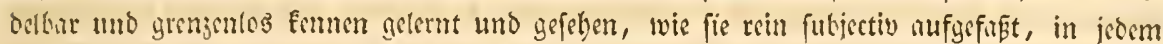

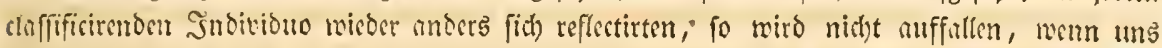

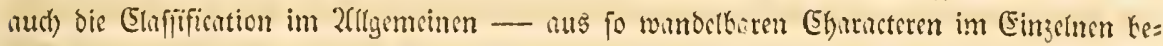

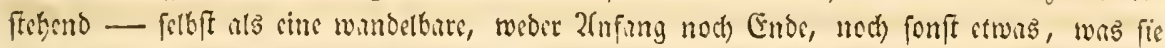

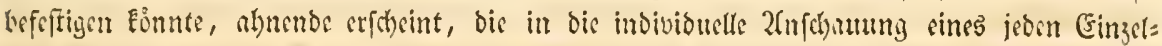

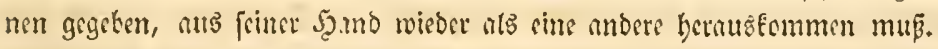

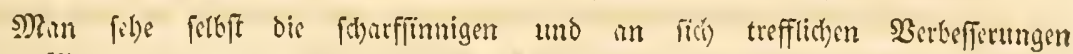
Bernhardi's an ben Rumunculacen, wie De Candolle diefe im Systema naturale

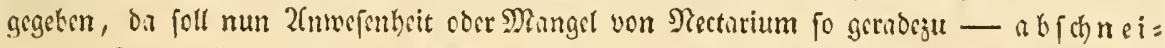

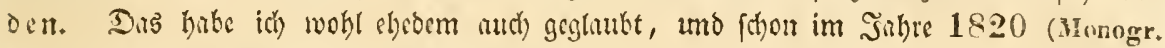

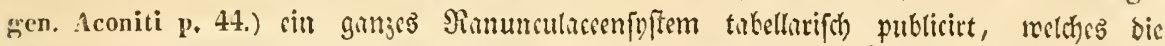

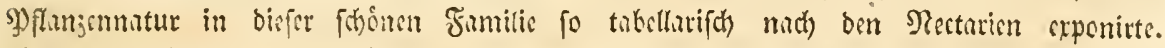

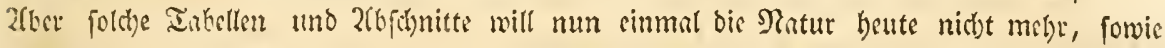
fie nie unb niemats etwas fo gerndeju abfontribet, fondern immer altes nur mad) und nach cutrwickeft und lingfam fortbilbet, umb ba freut man fich zul lefen, wenn ber tullentwolle Meisner mit frimem bentifhen 2fuge fisft: quum vero Cimicifuga, Actaeae proximum genus, petalis gatideat nectariferis, Caltha autem nectario careat, characterem lunc solum an distinguendas tribus illas haud sufficere, - in aprico est." Das , in aprico" foll wenigftens wathefbeinlid) heipen: im Somentlaren, und bunn ftimme ich ganz gewíz damit literrin.

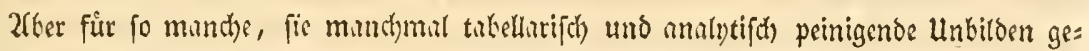

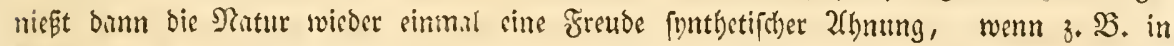

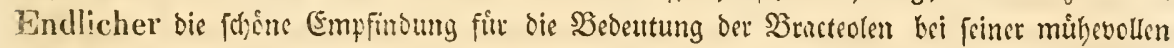

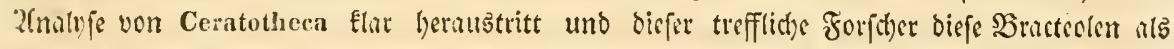

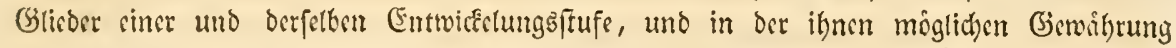

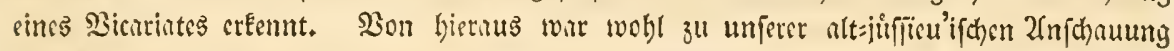

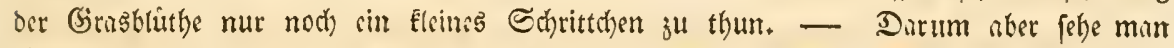
Eunftig in bie Srganogence des Naturtebens und frige bie ticbe Natur, was fie mit ibren Frmilien und Binttungen felb ft will.

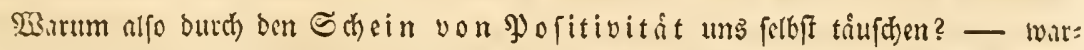

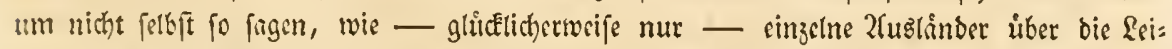
ftungen bor Deutfchen fich nusbricken: ,Ce sont des idées, ce ne sont pas des faits" ober in offenlyergigem Deutfd: wir beuten in allen unfern umfdreibungeu nur auf ben Typus bin, alle unfere Species und sattungen, unfere "ordines" und flaffen, fammt ihren gropen Divifionen, find nid)t zu umfdreiben, fie baben in ber Ratur igren Typub, in ber gegebenen 2lusbebnung und Begrengung find fie aber, fowie ibre, charactères immobiles" nur eine uns allein geborige, fubjective soee. 


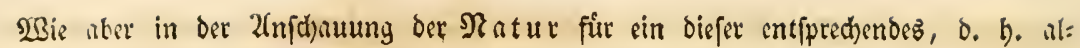

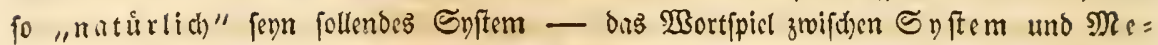
thode beritefichtigen wir nicht* ${ }^{*}$, benn De Candolle mannte baffelbe , système", wwis Jussieu ,methode" genannt hat, fo wie er baffetbe, wab biefer ", monocotylédonés " uno "dicotylédonés" nount, als ,endogénes uno exogenes" lieber uno, wie or ju glauben faeint, ftrenger umfhreibeno**) begeichnet, - wie für sin foldhes Snffem alfo,

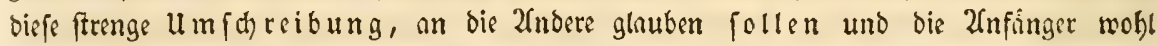

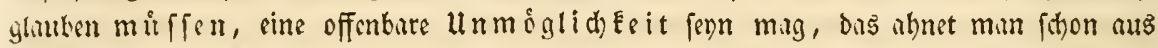

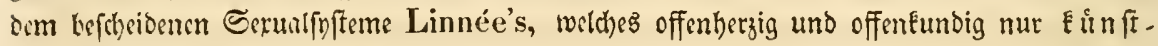

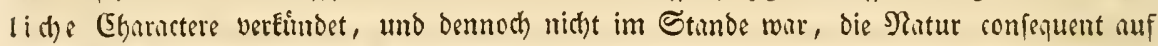

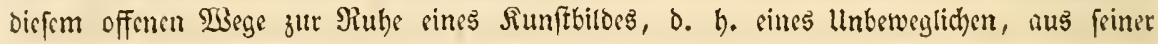
umrahmung nid)t mehr hernustretenocn, verweifen ju fo̊nnen.

Die geiftige 2luffaffung und materielle Berurbeitung bes Stoffes uno die buraus er= folgten Siefultnte, waren burch) Jussieu's, glucflichertweife in feinem wurbigen Solnne Adrien de Jussieu erbaltene und fortgebildete Begrbung, gunz in ihrer 3eit beoungen uno wurben trefflich begrindet, barum mig sine biroon etwa abweichende neue Beit, bei

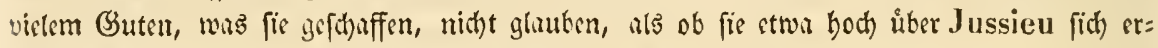
bohen, ober woht gar "au niveau" ber jegigen 3eitforderungen fïh befände.

Raspail zeigt uns gleichfalls, wic bis Joce bes natiurlichen Syjtemz fajon wahrbaft

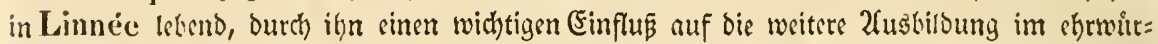
bigen Jussieu gewonnen und figt unter andern: Linnée, toujours nodeste, paree qu'il étoit toujours l'ami inséparalıle du vrai, Linnce ne se donne pas comme l'auteur, mais comme un des collaborateurs.

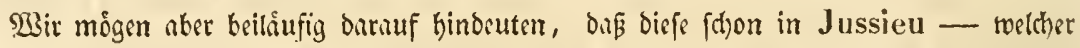
von Linnée fagte: "il a plus fait pour l'histoire naturelle que ses prélécesseurs" lebende, in Raspail jef̧t wieber Elar geworbene und, watz noth mebr fingen will, endich

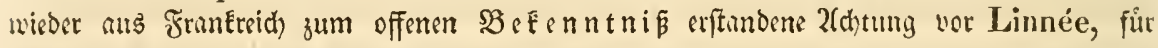
uns sine bedeutende Erfheinung ift, und wir vermuthen, bas wir nus biefer 2fntityefe,

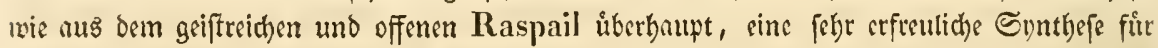
die'neue "Botanique françoise" zu erworten, berechtigt fenn durfen, benn in jebem in=

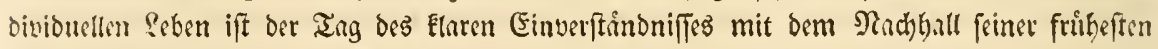
sindbeit, nur sin Iing Des Gilucfes und ber Freude.

Mng hier auch anbangstweife zu ber Befchauung ber Pofitivitat unferer 3eit noch

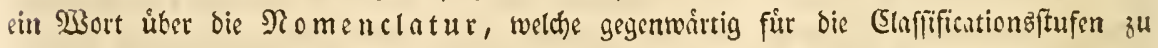
frauben Sitte gewoorden, Eurz zu erwihnen erlaubt fenn. Die Meiften ffimmen damit

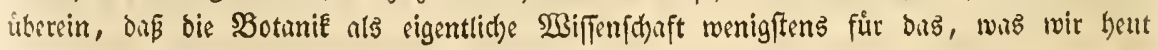

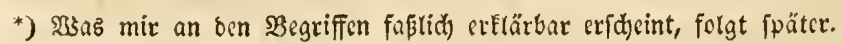

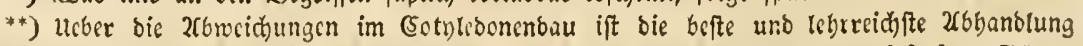
von Bernhardi: Ueber die merkwürligsten Verschiedenheiten des entwickelten Pflanzenembryo und ihren Werth fïr Systematik. Linnaca. 1832. S. 516-613. 


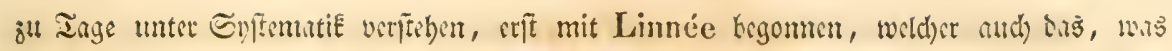

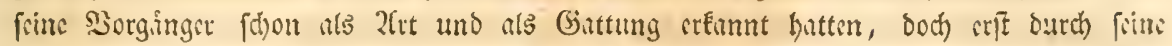

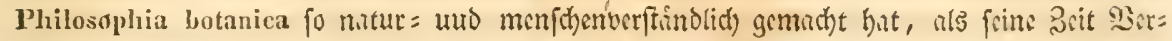

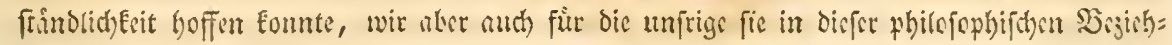

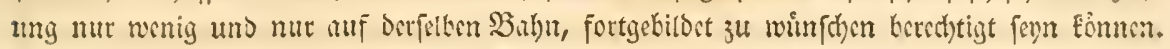

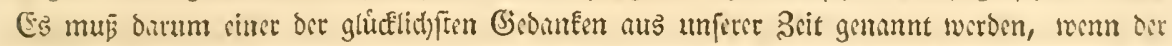

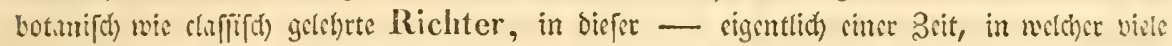

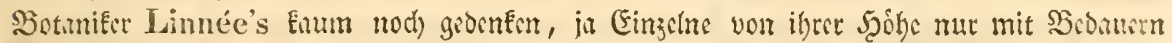

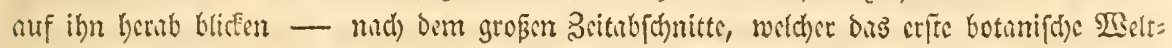

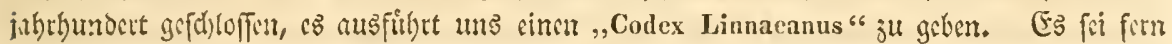

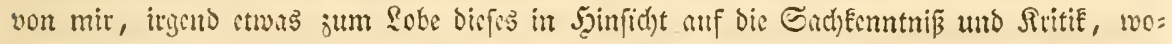

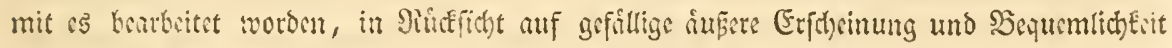

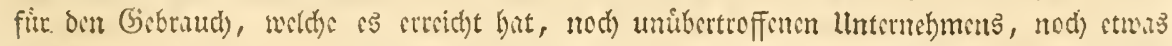

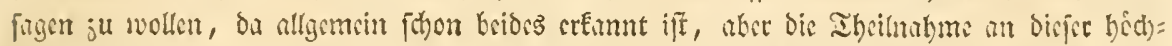

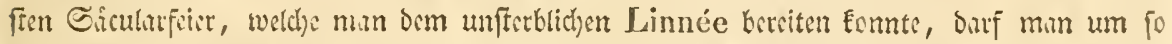

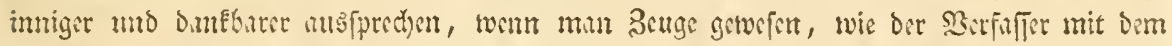

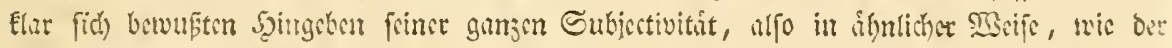

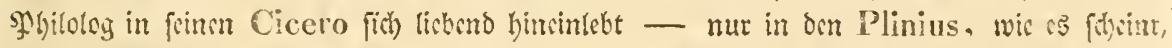

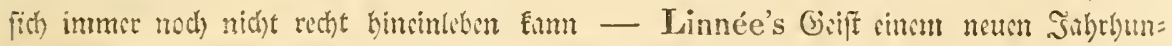

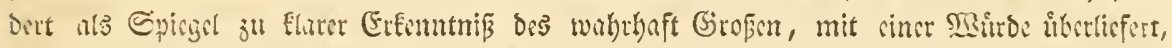

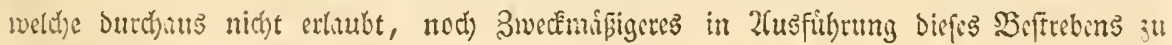
Dinken ober zu mimidion.

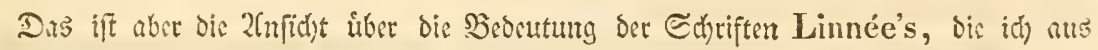
vollem S2arjen mit allen ltntefangenen mit cmppinde und theile.

Ith furt meinen Ibeil butte Durtm Richter's: Codex Linaeanus und Fndlicher's:

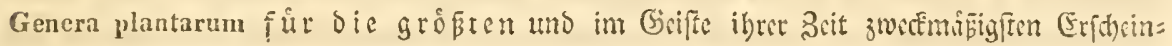

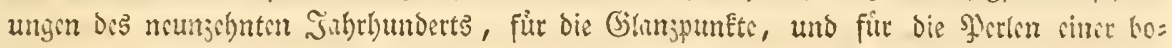

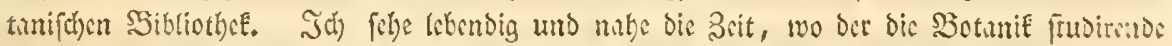

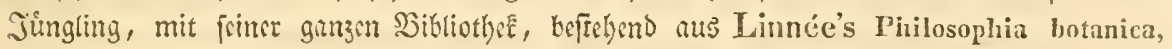
Richter's Codex Linnaeanus uno Endlicher's Genera plantarum und irgend cincr Flora,

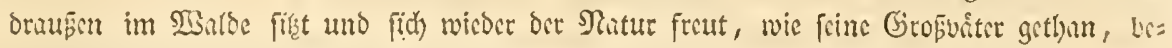

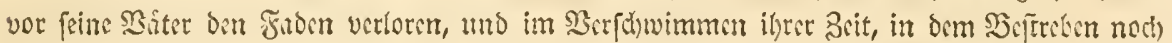

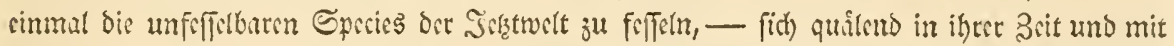
ihr - verfd)wanmen, weil bie Natur burd bie naturtidy Syfteme attsinumbergegangen.

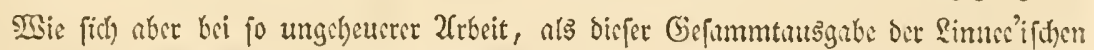

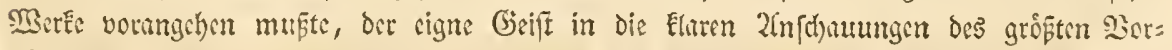

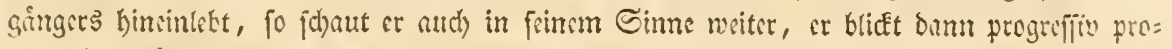

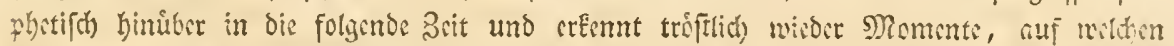

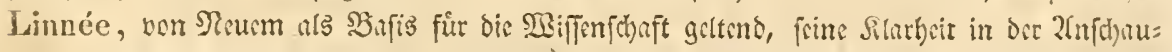

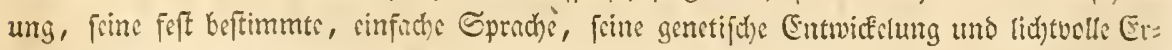

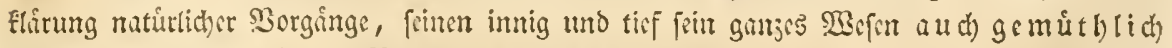
belebendeit Fricreifar für bie Natur, wicder vererbt hat.

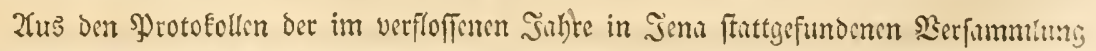

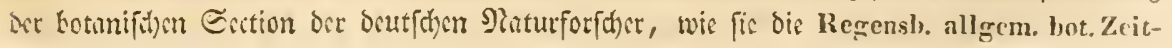




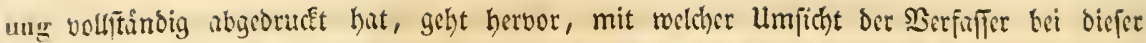

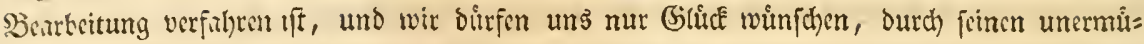

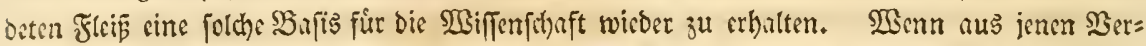

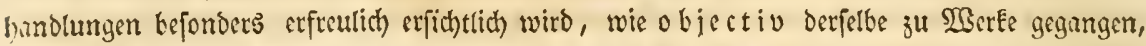

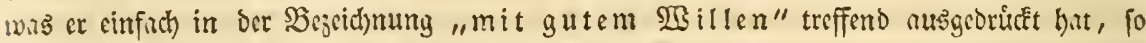

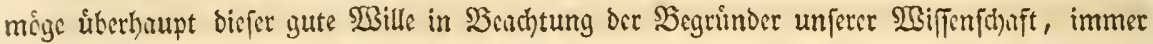
in guter Erinnerung verbleiben.

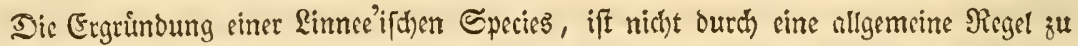

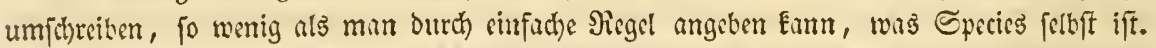

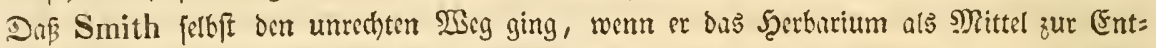

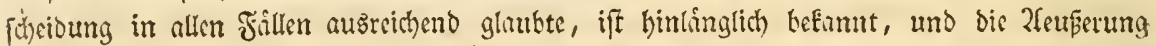
vou Koch, er moge nicht in allen Fiflen nach feinem Scerburio beurtbeilt fenn, unterforei= hen mit inm, wir getwis alle.

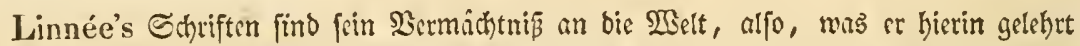

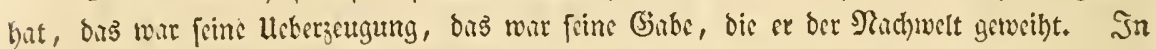

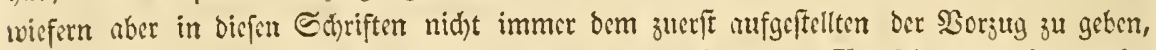

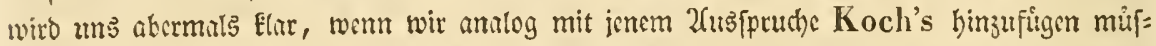
fen, wir mogen aud) nicht mehr nad) unjern erffer, fonbern mach unjeren fpiteren, uno aนs cigner Fortbildung entfproflenen Uebergeugungen und aus beren Wirdorgabe in umeren

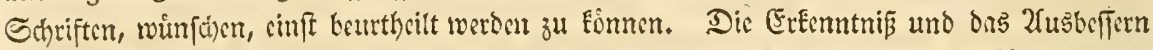
umperer erkannten Fibler if jit in unferer Fortbiloung beoungen, und biefe 2lusbefferung felbjt Dü Sefte, was Lubcrhaupt an uns fenn Eimn.

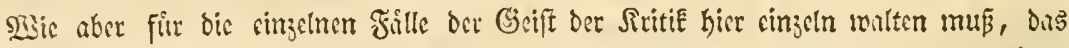
mus ocm literlaffer bleiben, weldher in biefen Geift fith hincin geldkt bat, burum móchte

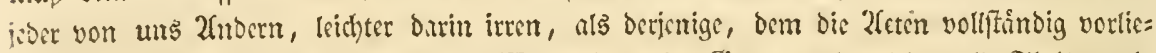
gen und ber butchbrungen ift you ber $\mathfrak{W}$ ahtheit, "im Ğnzen aber leben alle Theile, lebt

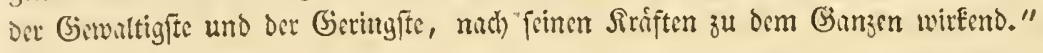

Finzelne Srrthimer uber fich felbft, folitest nic dus Seben eines Snbividumms, fo lunge cs sin menfolithes ift, von fich auts, birs verfteht fich von felbft, und bas biefe itobi= vibucli zu keurtheilen find, bas verffeht fith aud) von jelbff.

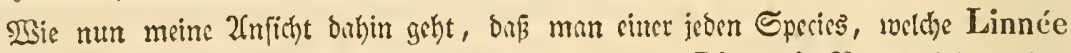
gefannt und unter bom bcibefaltenen Irivialnamen bat, aud Linnée's Namen beifece, bas bate $(t)$ in ber Flora germanica zu zeigen verfucht, und aud) nod) oudurch ben 2utor fitr

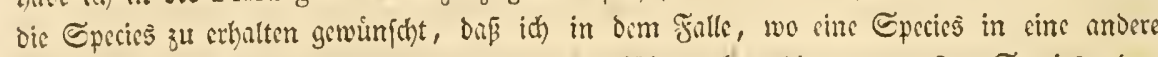
(Gattung verfect morden war, biefer mun generifo nothwendig umgetauften Epecies, ben

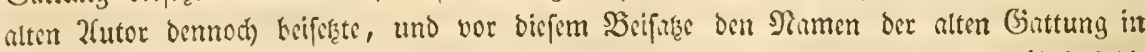

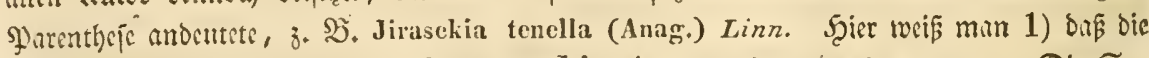
Epccis von Linnée ftrmmt, 2) duf fic Linnće Anagallis ténella nannte. Die $S_{y}=$

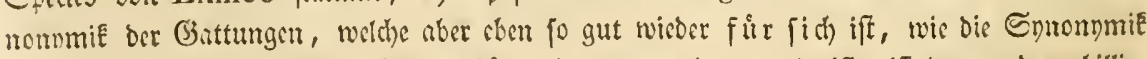
Dir Frmilic einer befonocren Stufe gehort, benn wars cinem rect)t iff, iff bem andern billig, lefte mich, bus dic Giattung Jirasekia nicht von Linnée, fondern von Schmidt ift. Die Entomologen effreuen fid) feit langen Sanhren des tooblthitigen (Erfolges dicfes practifech be= twifhten Shrinjipes, um beffen Finfithrung, wenn id) nid)t irre, Freund Germar cin gro=

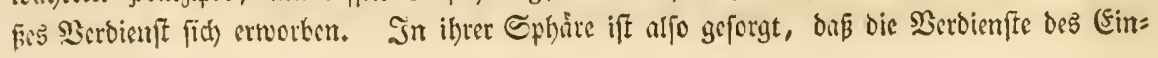


zelnen um dic Epecies der Species, bie um, bie Ginttungen den Gattungen verbleiken. Dazs ift, benke id), fo flar wie bas Eentrum im Sireife.

Wo freilich fpecielle Namen von neuen 2futoren fobon recipirt waren, babe id biefs beibebulten uno nut cir unfreundlicher Wille fonnte cinzelne, vielleid)t begangene Irrthủmet

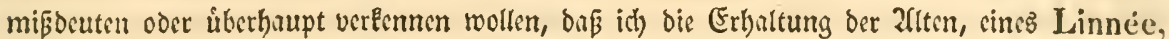
Scopoli u. a. der Sciferzung meines neueren, eigenen Namen vorzichen wollte.

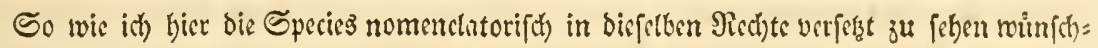

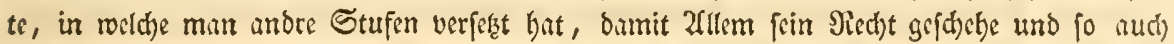

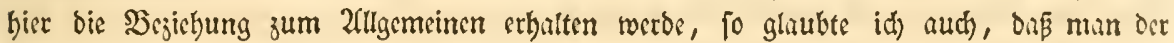
(Battung folde Red)te cinriumen mulfe.

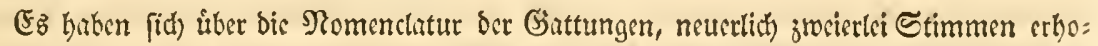

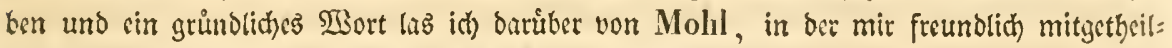

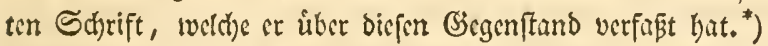

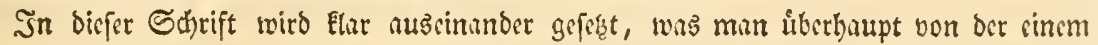

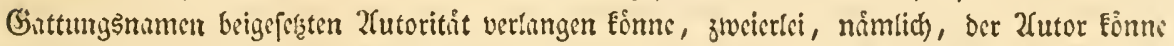

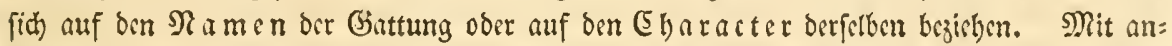

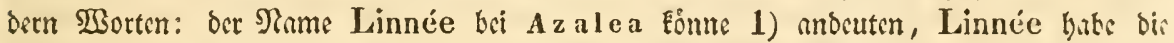
Azalea procumbens in feincm Sifteme Azalea genannt, 2) aher aud, Linnée bute ben Ebarneter ber Ghittung Azalea nad) biefer Azalea procumbens beftimmt.

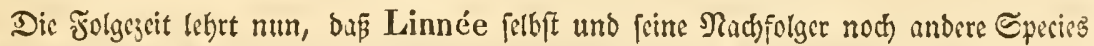
in bic Giattung Azalea gered)net baben, wie z. B. A. pontica, viscosa, nudillora น. F. w.,

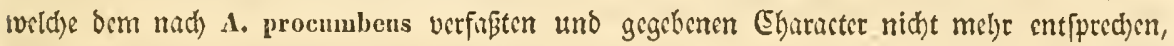

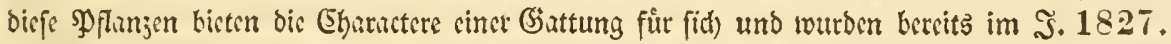
nโకె Anthodendron (in Mössl. Handb, 2te Ausg. S, 214.) gcjondett.

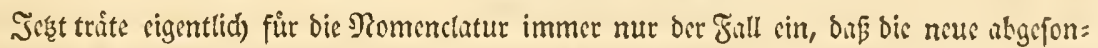

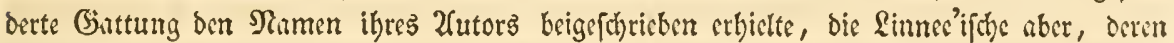

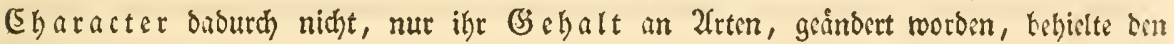
Bsifát Limnée.

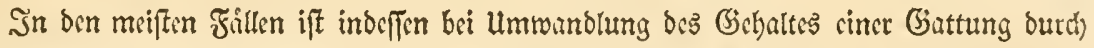

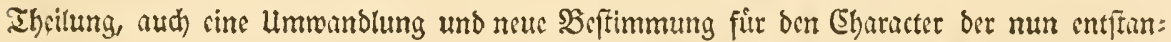

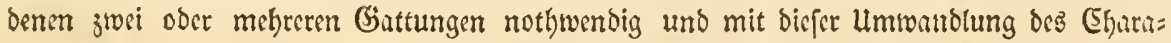

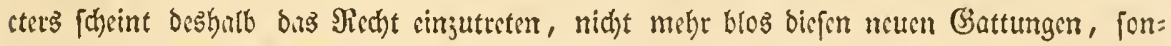

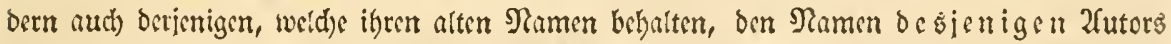

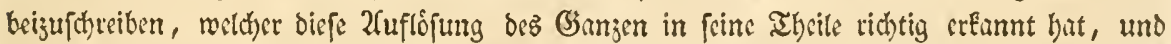

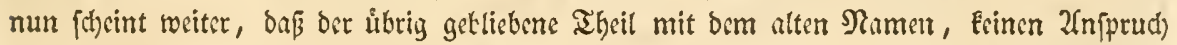

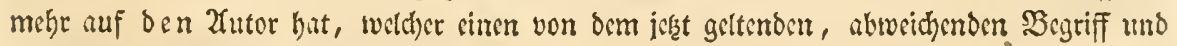
Gichalt o.mit verbunden batte.

So bit fich, um ein Baifpiel ju grben, Richard das unkeffreitbare Sirbianf cr=

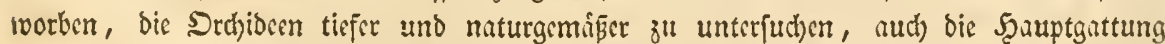
Orchis in mefrere Ginttungen zertfeilt. Nad)bem er num aber Anacamptis, Nigritella, Gymnadenia yon Orchis gcjontort, und bicfe mit frincm Namen untedingt bejeichnet wet=

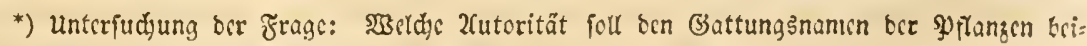
gegcben roeroen? - Tübingen 1836. 


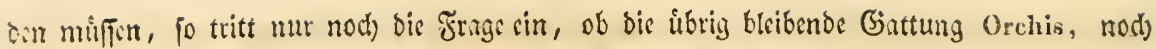

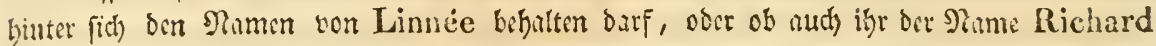

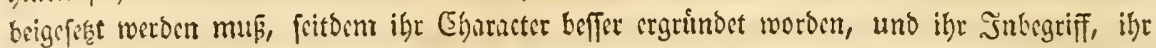

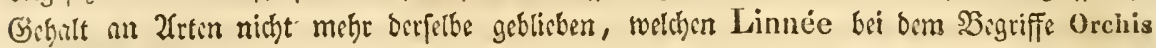
umfaret hat.

Die Folgcleifung ber lefteren 2 Beife, D. \%. alfo bas nunmeht, wic s3 phisn, Noth=

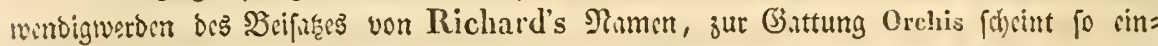

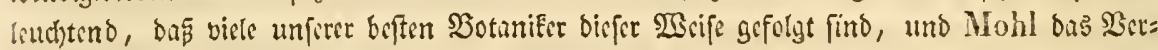
bienfit bat, bie Sache weiter beleuchtet zu baben.

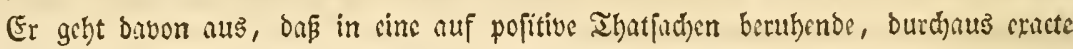

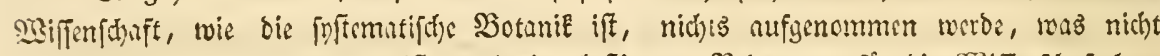

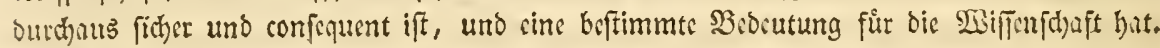

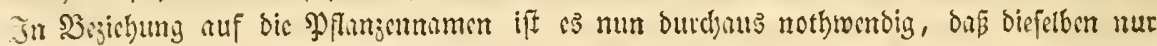

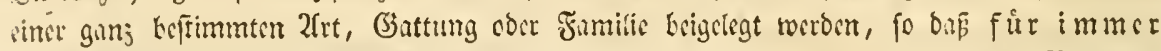

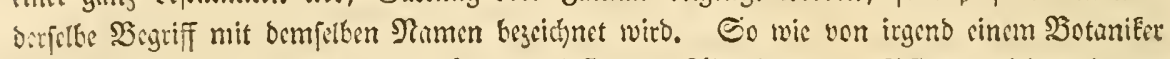

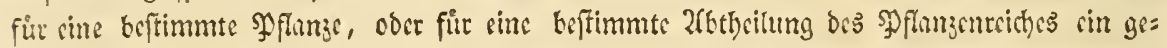

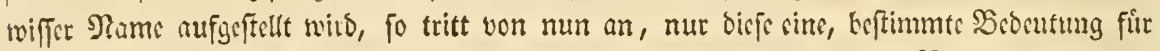

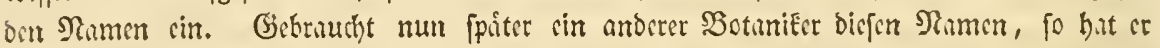

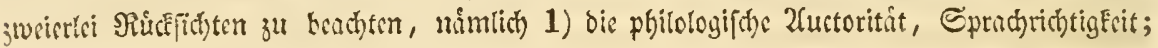
2) bie botanifole 2fuctoritit, Sactbedcutung.

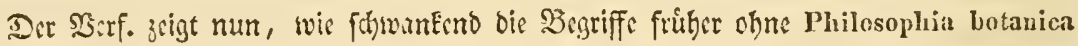

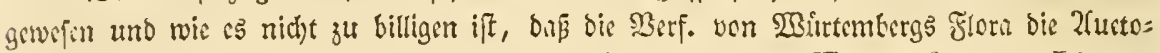

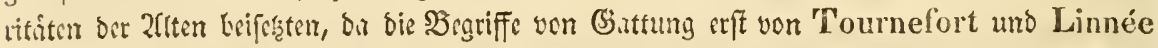

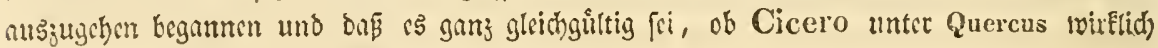
sime (Eidge verftandon bake, ober ob birs nid)t fri.

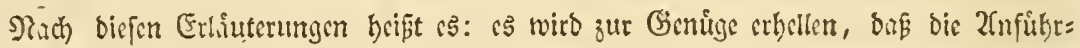

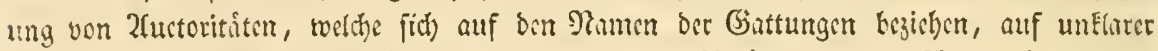

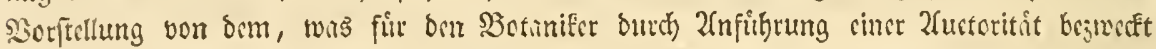

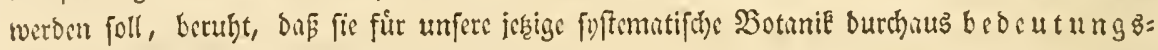

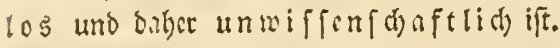

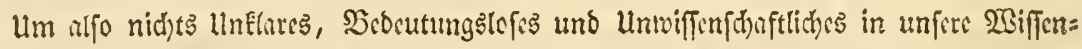

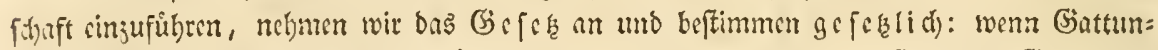

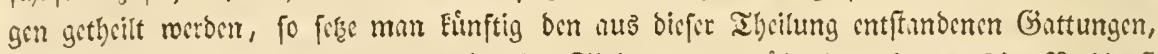

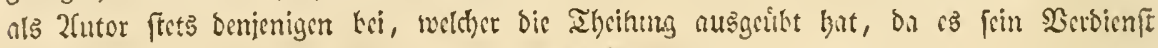

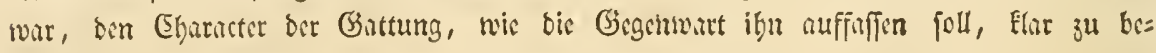
fitimmin.

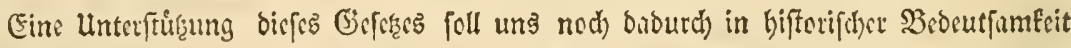
grgaben werbon, daß̈ Linnées, Haller's, R. Brown's uno De Candolle's 2uctori=

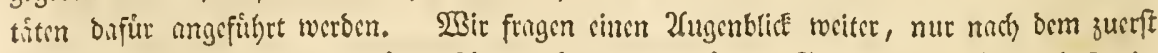

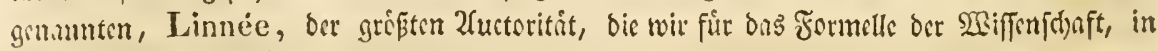
ihm, als beren Sdjipfer, erfennen.

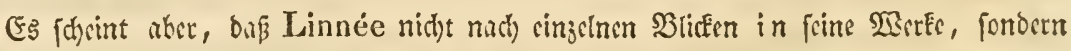

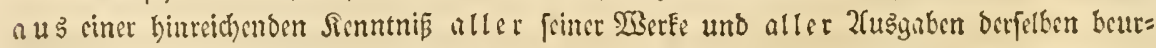

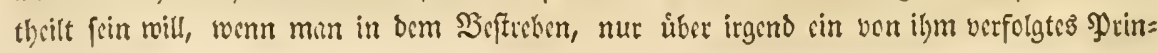




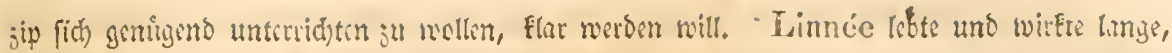

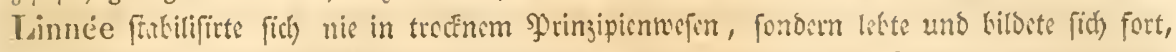

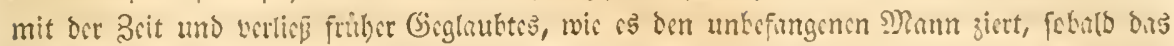

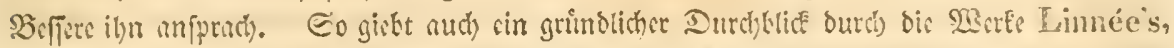

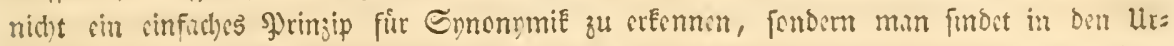

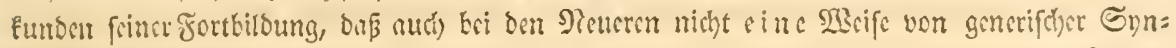

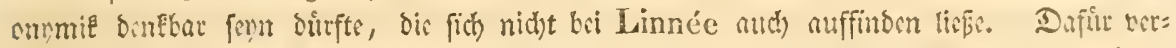

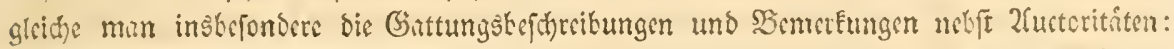
Genera I. II. I, VI. -- Systema I. und II, - Philosophia botanica p. 120-201. befon= Ders §. 210. etc, -- Mant, I. und II.

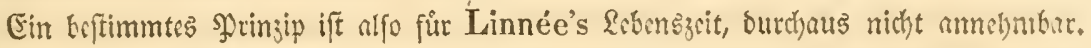

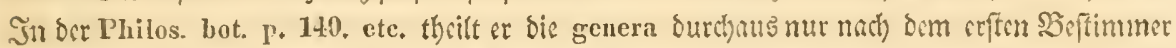

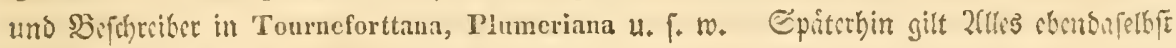
allin bem Sencnner. Linnée furcikt aud):

Cyperus Mich. Tourn. now) Dam fo, als ar fdon in ben Coroll, gen. Die Cyperella Mich. unt Holoschocnus Mich, abgetrennt bat, und als Schoenus fortheftehen liffit,

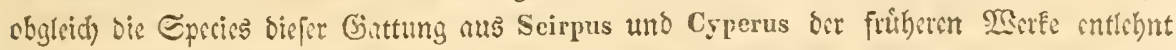
find. Derfilbe Fill findst fratt mit Passerina: Struthiola; Cerinthe: Onosma; Polcmonium: Ellisia; Convolvulus: Evolvulus; Campanula: Canarina; Cephalanthas: Nauclea; Lonicera: Chiococca, Ioranthus, Diervilla, Triosteum; Terbascum: Celsia; Celastrus: Ceanothus; Illecebrun : Achyranthus; Ornithogalin: Albuca, Hypoxis; melde wenige Sseifpicle indeffen Gier nur bie Ctelle von Gunberten zu vertteten, genugen.

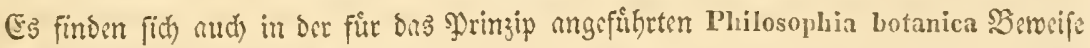

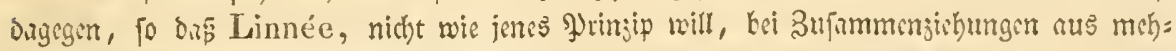

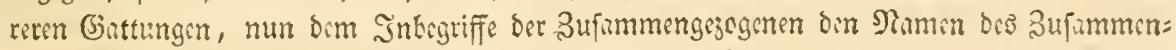

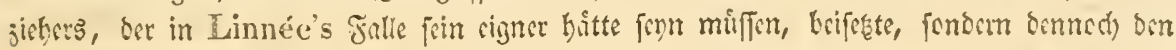

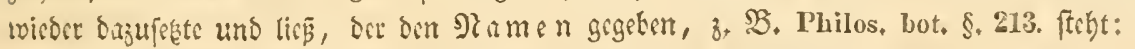

$$
\begin{aligned}
& \text { Citrus } T .=\text { Aurantium } T \text {. Limon } T \text {. } \\
& \text { Pyrus } T \text {. Malus } T \text {. Cydonia } T \text {. }
\end{aligned}
$$

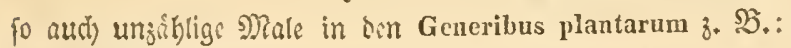

Cistus $T$. Cistus T. IIclianthemum $T$.

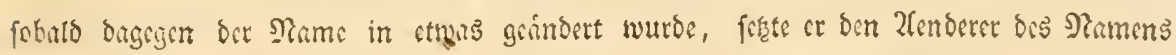
Esi, j. $\mathfrak{S}$.

Ballota (L.) Ballote T.

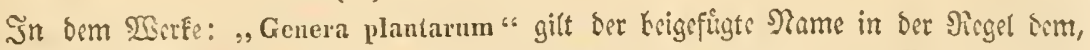

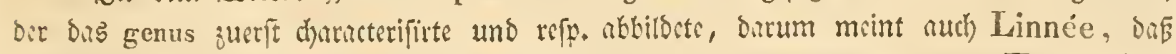
'Tournefort's guter 3ciduncr oft mebr Berdimft um bie Gittungen babe, ats 'Tournefort

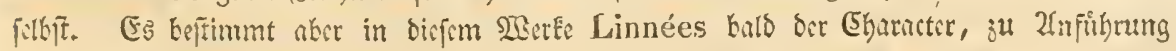

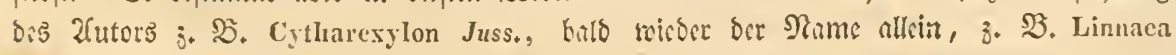
Gronoi.

Sn Syst. I. finbt Corispermum Juss. mit syn, Stellaria Dill, dagegen in Gen. 1. I1.

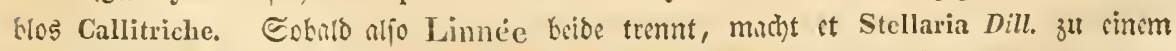

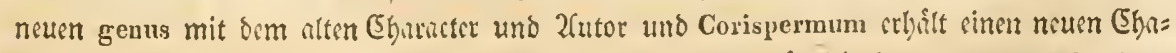
ructer. Ebemfo iff der Eharacter von Alsine Gen. I. II, wortlid) ber ton Stcllaria Gen. V. VI. Dugegen bee ven Alsine Gen. Y. VI. ift nelt. 
Dis yon Mohl S. 23. seftrittene, ex parte" findet fith bei Linnẻe un unjihli=

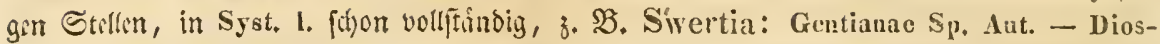
ma: an Anisi stellat, sp. - Rhinanthus: Pellicularis spec. Tourn. (Gen. I. - VI.) Pedicularis: Pedicularis spec. Tourn. (Gen. I. - V'1.) - Turnera: Onagrae sp. Fcvill. (Geu. I. II.) - Crinum: Lilioasphodeli sp. Dill. (Gen. I. I1.) - Andiemetla: Erieae sp. Tourn. (Gen. VI.) - Amaranthus: Aniaranthi sp. 'Toum. (Gen, VI.), -

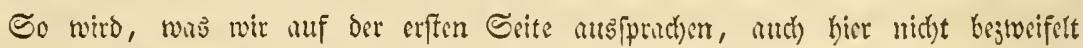

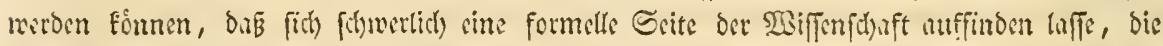

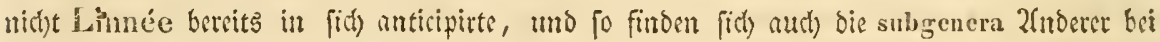

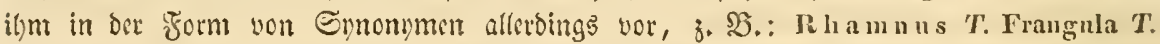
Alaternus T. Paliurus T. Cervispina T. el. XXII. - Primula: Pr, veris T, Auricula ursi $T$. etc, - Gction in Syst. I. findon fic fich auch biswoilen abgetrennt, als $A$ nomalae alins classis, (anderwitts boch bie Species fo georbnct) z. 3 .

Staphylaca: Zanthoxylon Catesb. als anom. el. V. 2.

Stapliyla ea: Staplyylodeutren $T$. und ats anom. ber filaffe $V$.

Enplurb i a: Titlymalus T.und Euph. Eluteria Pet. Gr. als anom, cl. 入. 3.

In Din Generihus plantarum und Cor. generum werden fie dimn oft von cincm genus zum andern beriber verfect, 3. 2 . Satureja uno Calamintha erferes von Thymus weg, ats novum genus, lefectes von Glecoma ful Melissa. Beit Th ym as $T$, hat Linnée in Syst. I. erft dic syn. I. Satureia 'T. 2. Serpyllum 'T. ย. Thymbra T. - Ninch=

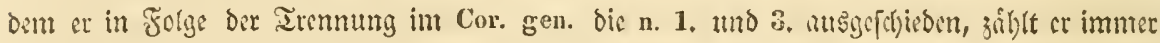
noch ju 'Thymns 'T. Serpylhum 'T. Acinos Riv. Mastichina Boerh. Sercteren bat er jwor cigentlid) ingwiforen (in Spee. I. Syst, X.) zu Satureia gezogen und erft in Spee. II. sq.

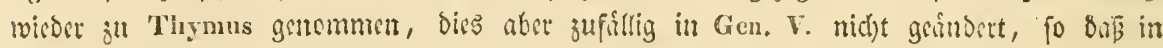

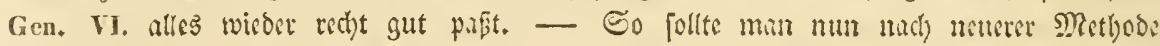
Fityreiben:

Thymus Linn. sp. I. non Tournef. nec Limn. Syst. I, ne: Linn, Cor. gen. nec Lim, spec. II. ser!.

Das waine richtig, aber body - ctwas peintid und fir bie Sache an Ende ofne gre: B̊n Errolg.

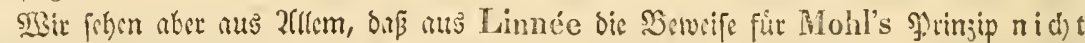
entwicfolt werdon Finnen.

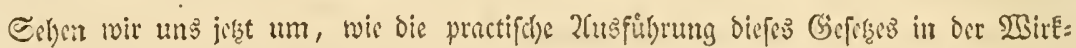

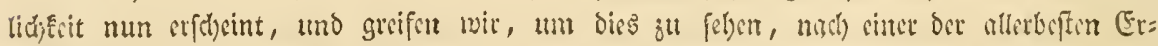
posinungen umferer Satt: Nees von Lisenbeck Gencra piantarum Florae Germanicae, fo finden wir afferbings confequenterweife nicht melge Anagalis Linnce fondern wegen Iron = ung von Jirasekia, nur Anagallis schmill, nid)t mebr Lysimachia Linnée fondrn Lysimachia Mönch, wegen Natumbnrgia Mönch.

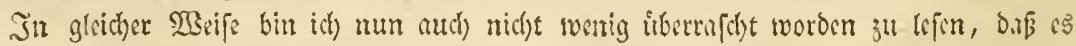

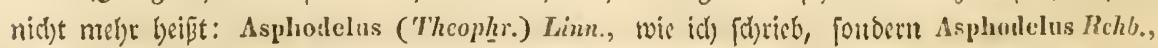

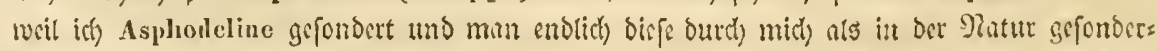

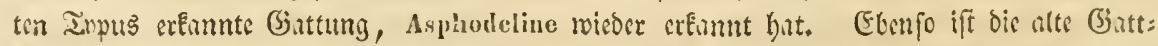

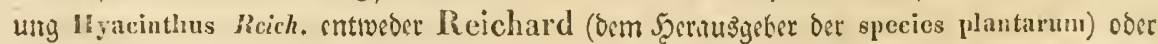

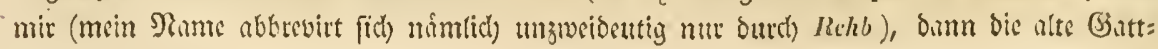


ung Scilla (megen Agraphis) Link, Dic alte Ginttung Osuithogalum megen Mrogalum cken= fulls Link, bic alte Ginttung P'olygonum aker (Iwegen E'ayopyrun) Giürner, bie Ginttung Calla (wegen Richarlia, Iunth uno Convallaria (wegen I'olygonatum etc.) Desfonlaines 3ugeforicten worder.

Co meit fobeint es alfo, bar alles recht confequent ift uno musficht, wir mollen aber

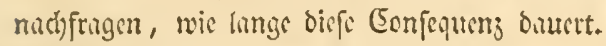

2llfo bicr nur cin paur Bsifpicle.

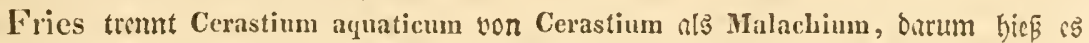
im Pfrimip nid)t mobr Cerastium Linnce, fonocrn Cerastium Fries. Jd) forleft finde mid) miner lnterfuchung, daß aud Cer. manticum dazu gehort, und bie Ginttung Malichinm mun in ifrem boppsiten Şabitus, mit Stellaria (wie St, nemorum umb IIolostea cour grauinea) fich purrallefifitt, noume is Malachimm manticum (Cerast.) Linn. Sef̧t fonute man fagen Cerastiun Rehb. Şerr Dr. Fenzl tritt aker auf, nlteritt fich beftig liker bic=

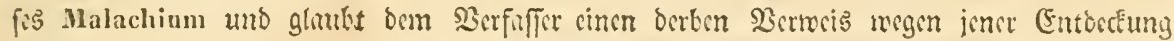

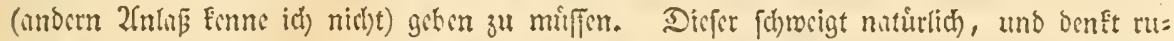

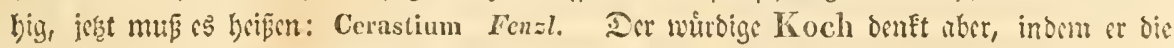

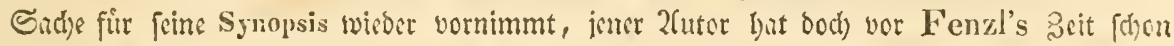

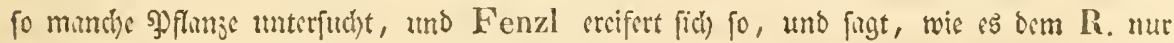

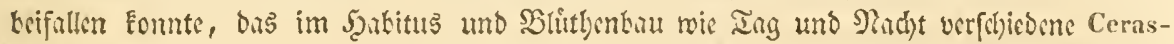
tium manticum uns Malachim arquaticum in sime Gintung zufummenjuffllen, begrcife

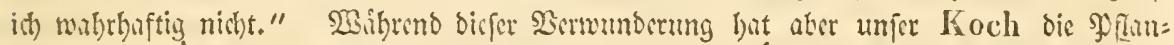

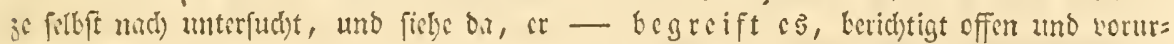

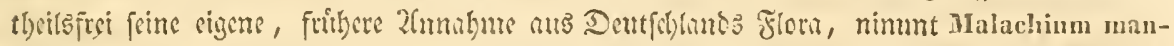

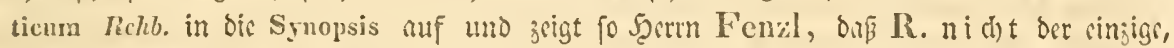

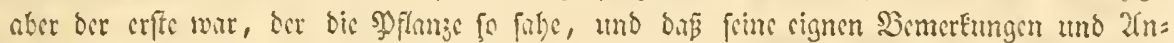

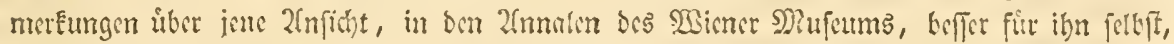

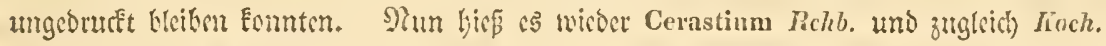

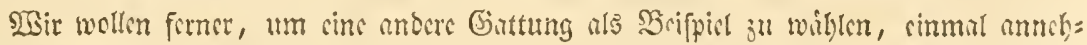

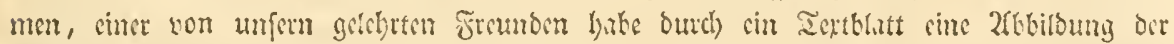

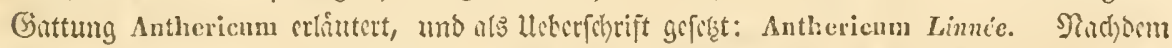

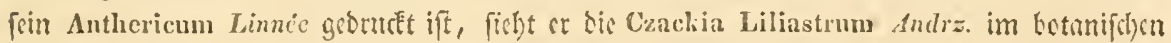

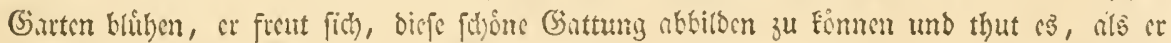

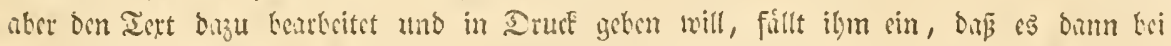
Antherinum anftatt Linnie riffiger Andrzeiowsky bsipen muffe, on Andweiowsky die

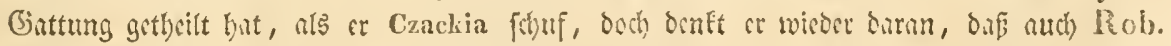

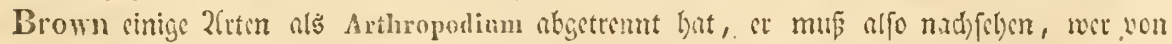

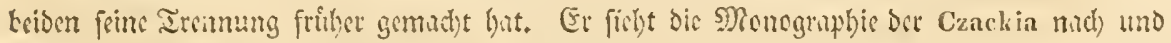

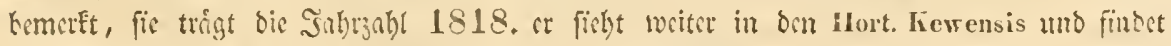

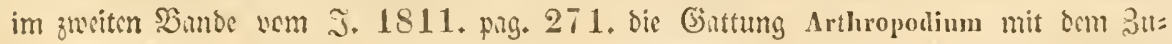

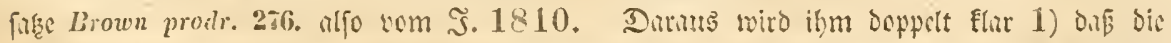

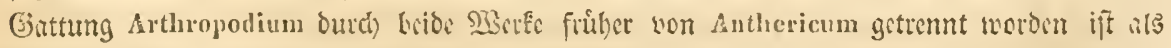

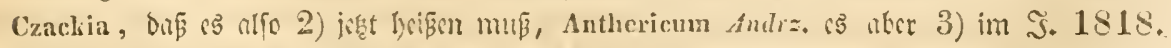

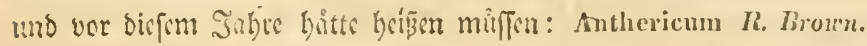

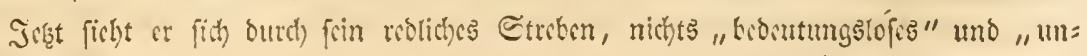




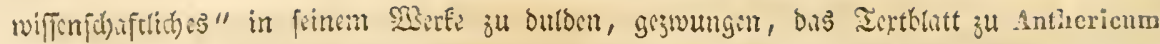

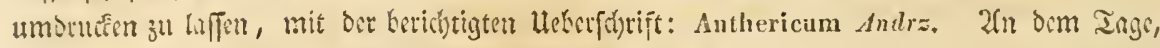

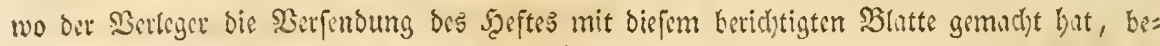

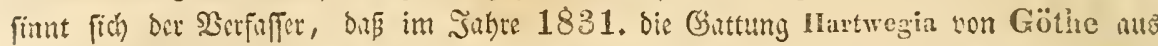
Anthericum Sternbergianum Schultes gebildit, in Den Act. Leop. Carol, publicirt worden

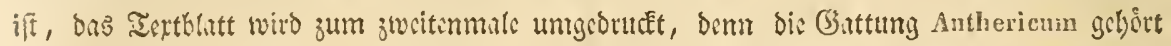

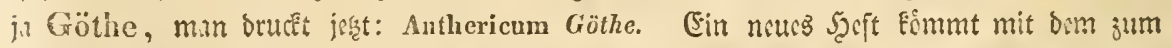

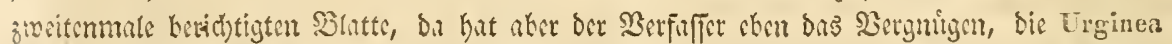

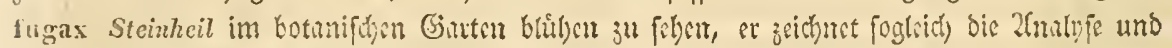

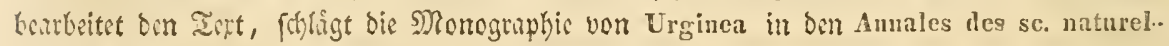

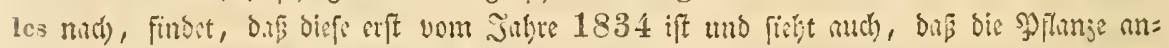
fungs Anthcricum fugax Moris gchsifien. Dos ift ihm etwus unungenthm, beun cr

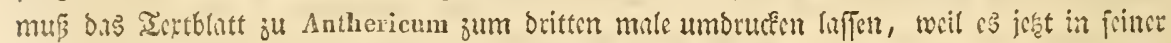

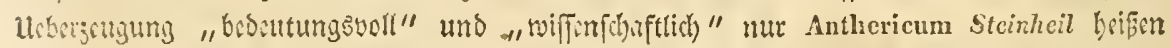

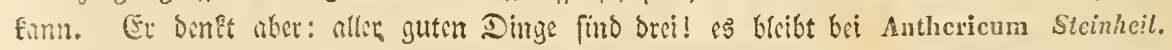

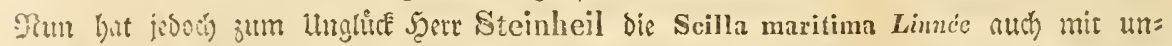

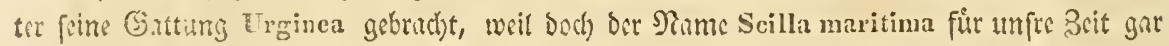

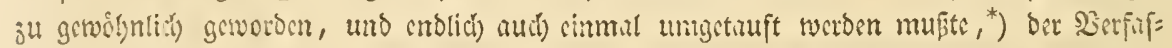
fir billigt birs und ficht fich folglici) geztwungan, in feimm sajt wiffenfouftidyen Etreten,

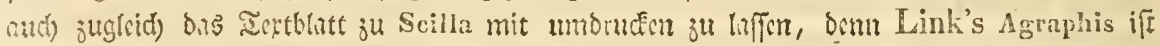

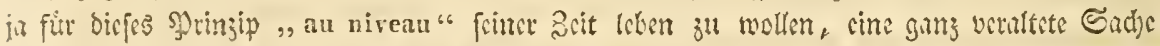
gerwordin, dit fie fothon im Sabre 1829, folglid) noth vor ber cigentlict) neucn acit, in

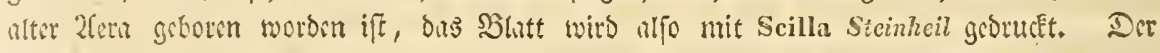

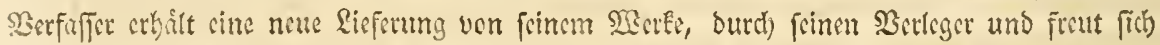
zut twiffen, das er nun feimem Seftrchen zum drittenmale genitgt bat, indem cr chen on feincm 2(rbeitstifd) beflyiftigt ift, bie neuffen Binbe vom Botanical Registcr butchjufeben.

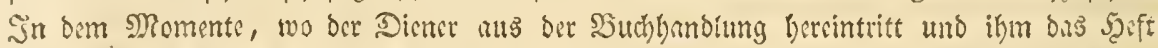

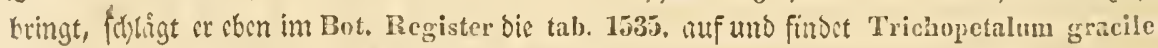

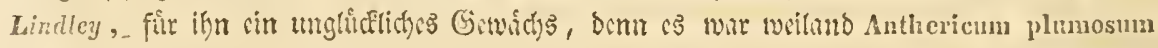

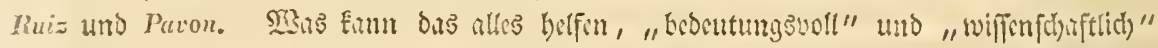

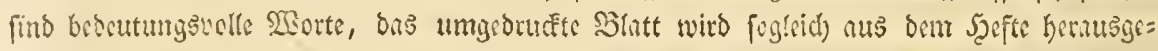

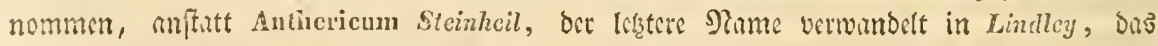

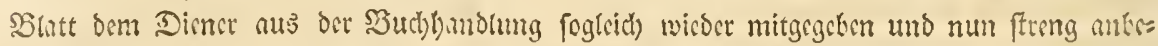

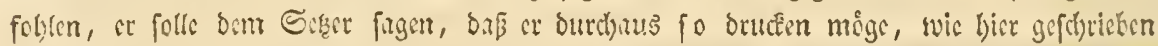

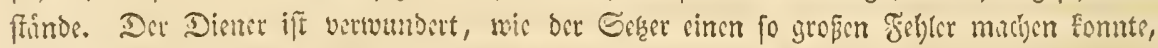

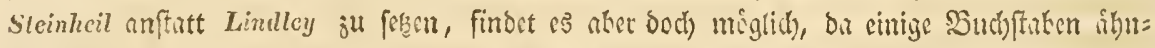

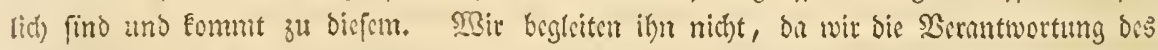

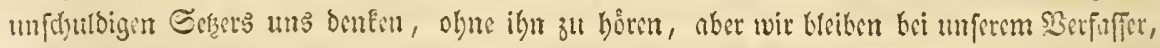

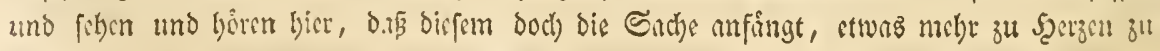

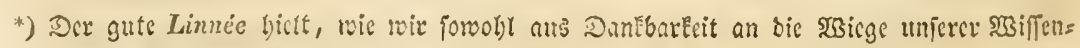

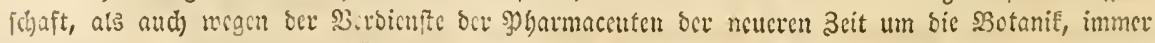

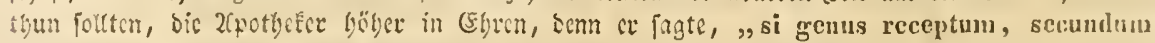
jus maturac et artis, in plura dirimi debet, tum nomen antea communc manebit rulgatissimae et of ficinali plantae. 
geben, er denft, id) batke nun viermal nact)gegeben und umbruteen lafen, und es bleibt nun= mebr bei Anthericum Lindley, mag mun nod) fommen, wer ba will. Dabei fillt ihm inbeffen ein, sinmal nadjuffehen, was biffe ihn fo bamonifd quilende Ginttung itberbaupe

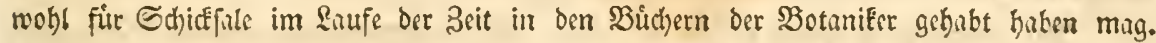
Da fieht er denn erftens, daß̧ Linnée fdon felbft im IIort. Cliff. bis Giattung 13nlline als von Anthericum gefondert betrachtet, uno ihm fo dis erfe Snconfequent bes befolgten Sprin:

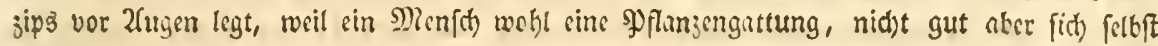
zu theilen vermig. Die Gittung Bulline wurde aber wieber verlaffen, Jussieu und Lamark nanten fie wieber Anthericum und bie andern Antherica, mit Tournefort

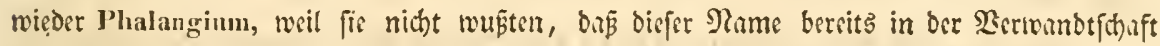
der Epimen, für Ihiere fanctionirt worden war. Seşt batte man alfo als Anthericum bas jut beurtbeilen, was Linnée Bubine genannt batte, und l'halangium mit. Willdenow,

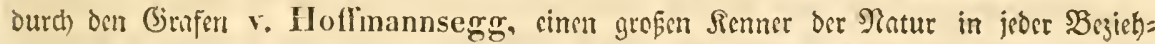

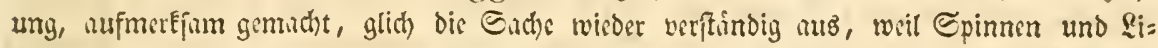
lien bods jwei virfechicome Dinge find und forn miffen, und fo batten wir Anthericum Willl. - Hudson tremte Authericum calyculatum als Tofielda, $\mathfrak{s}$ bies mun Anthericum Ihudson, aber autb) Möhring trennte $\Lambda$ nthericum ossifragum als Nartlecium, ę

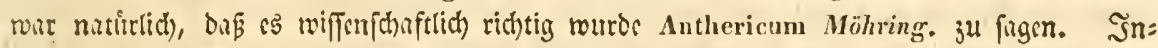
Deffen Firm wicder Lamark und madte bus Anthericum Liliastrum ju Ornitlogalum filiforme. - Da wir uns um ben Namen gar nicht befimmern, und nicht darnn ju benéen braud)en, daß Lamark bie Biattung Phalangium tennt, fondern unz ons fo einerlei fenn "mus, als ob "Cicero untr bellun Sirieg oder Frieben verfteht," fo fehen wit auch bier,

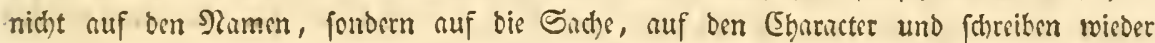
Anthericum Lam. গun Eommt auch wicder Persoon und macht Anthericum reflexum ş Conanthera Echeandia. 23 as fur Lamark red)t wat, if fü Persoon, und zrour

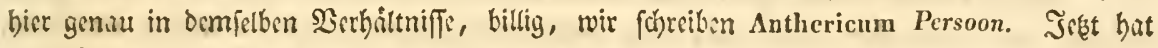
aber Ortega bie Gattung Echeandia gemacht, wir find unermidoet zu foctsiben: Antheriricun Ortega. De Candolle erfennt inzwifhen in cimer anderen Gpeciez, dem Phalangrium ramesum Burmann, fsine Binttung Diasia uno De Candolle wiro fúr Antheri-

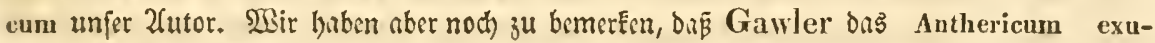
viatum aus der Giattung entfernte, indem or $\mathfrak{C B}^{\mathrm{CB}}$ ju feiner Albuca exuriata mad)te, folglid) Den Inbegriff von Anthericum wirderum indert, tuno wir, um nid)t "unElar" ju

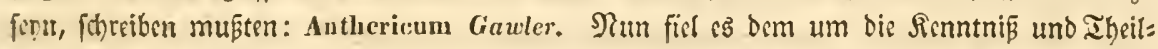
ung ber Sribsen, Narziffern uno \&iliaceen hodynerbienten Manne sin, fich felbft umjutaufen, er fuke wher fort Botunifer ju jenn, nahb unjer Anthericum serotinnm unter feine Gagen auf, nunnte fich felbftKer und wir wurden genotbigt, wollten wir unfere "wiffenjdaftliche" Ehre behaupten, Anthericum Ker. jull forciben. Willdenow batte cinftreilen. bieferbe Sflange Ornithogalum striatum genunnt uno Salisbury diefelbe jur Gottung Llo y dia gemad)t. SLit haben hier mit einem Eleinen Beitaufwande nod ju ergrinden, weldem bon diefen breisn, ober für wie viele Iage sinem jeven bie Egre zu geken ift, hinter Anthericum fieben in Dirren.

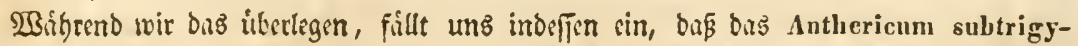
num Jacquin's von Willdenow Leimanthium pallidum genunnt, von Walter, Desrousseaux und Aiton unter Melantlium, von Michaux unter Helonias gebrad)t worden

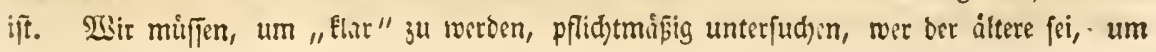


zu triffen, wem oder wem wieder und zum wievielten Male einem von ibnen bie Ehre ges bưfrt, ber 2futor von Anthericum "bedeutunggvoll" und in unferm Prinzip für bic Minuten un ferer 2fijoruung bleiben zu Eonnen.

Die Unterfuchung weiter gefübrt zu feben, will ith gern meinen geebrten Refern erlaf= fen, benn fie find fchon zu ber Ueberzeugung gelangt, dus diefes fdjon etwas zu lange Bei=

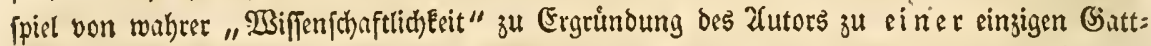

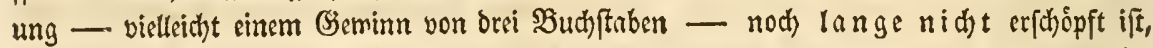
und bas mil gar vieles nicht cinmal eingefallen fenn mag, was in bic Unterfud)ung gehort biatte. -

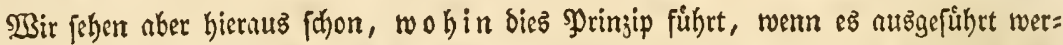

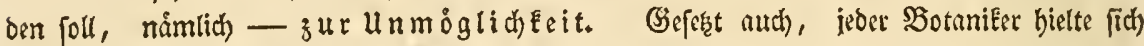

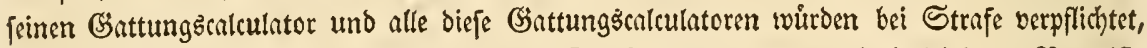

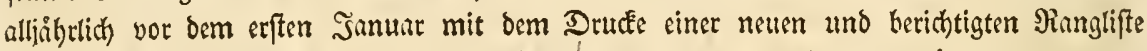
fư bie im verfloffenen Silfre zertheilten (Sattungen fertig ju wirben, fie Eonnten allefummt

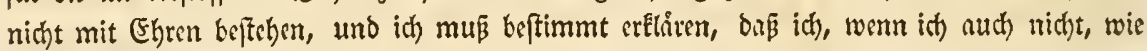

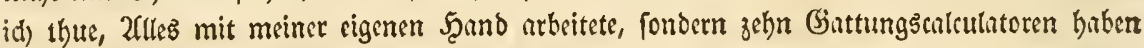
finnte, im Fall ich, zu biefem "wiffenfobaftlichen" und "bcbeutungšollen" \$rinjip gezwum= gen wourde, nicht adt Tage lang BotuniEer feyn móchte, weil mir ein Menrchenleben zu

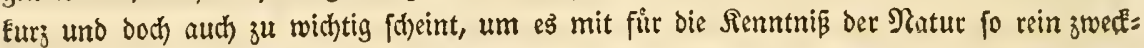
lofen uno fo ginglid) unnüken Dingen, verlieren zu mogen.

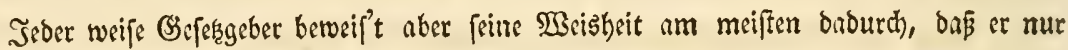

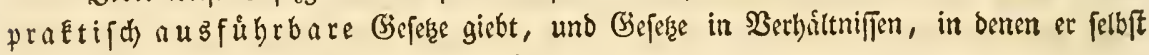
lebt und reldhe ifm eigne Erfahrung gegeben.

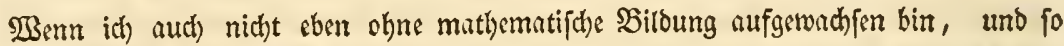

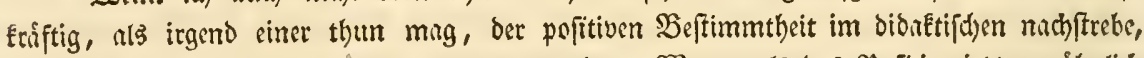

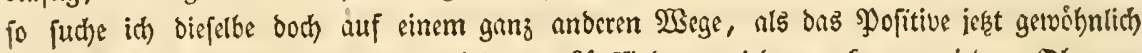
gefucht und dann leider audh, oft bei dem 2fuffinden, wieber verkinnt wird. Sb man aber jenes genjtid (Sirenzenlofe, und die, wie bie Sterne am Şimmel in ifrem unermes= lid)en Blau, berumflimmernden Slamen ber unglitelicten 2(utoren, welche eine netue SPflan= zengattung aus eimer alten ridjtig erfemnt baben, nod) bis auf eine Spur von "Bebeutung"

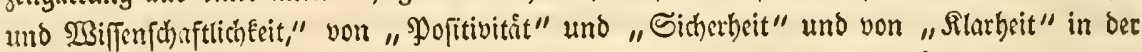
ulten Battung verfolgen, oder nur mit irgend einer "Sonfequenz" auf linger als auf ein=

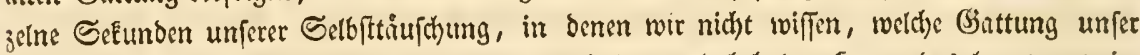

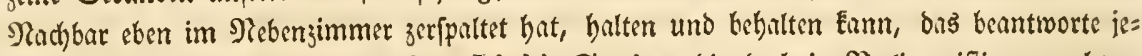
ber fith ferbft. SRicht einmill über Link's Species, bie boch in Serlin eriftiren modhten, fonnte fein trefflicher Eollege, Kunth unz belehren, um wieviel weniger ift bas mit ben (bisttungen moglich, bie einer, oft ofhe fie zu fehen, fo eben zertheilt.

Die wabre, Elare Pofitivitat ift von unferer vornchm thuenden Sobeinpo=

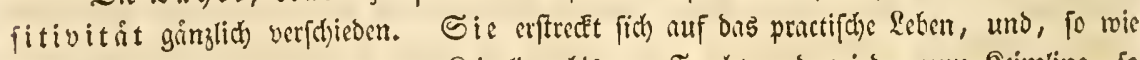
Die Metamorphoje uns leitet, vom Seimling bits zut Frucht und wieder zum Sermling, fo

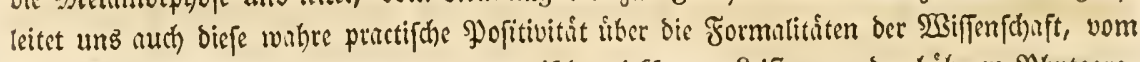

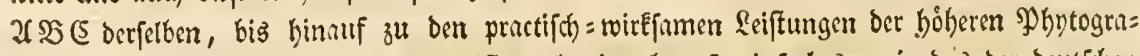
phie. 2fber ihe Filum ariadneum ift auth sin chen fo sinfadjes, wic bas ber beutifhen 


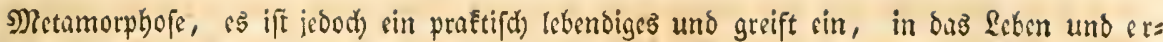

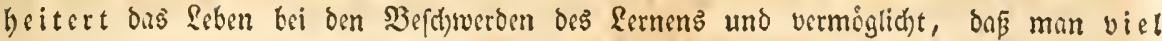
lerne und viel wieber fhaffe, benn es ift felbft bie wahre Sronung bes eetens, aber freilich verdirbt es feime 3eit nicht, mit dem Ergribsln in oen Jrrwegen bes \$en= ichenverftandes, fondern es wendet fich offen und vertrauensooll felbft an bie Natur.

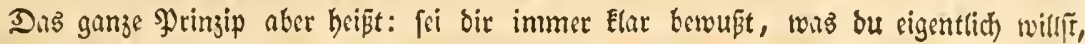
fude den 2lufang und erforfde das Ende! -

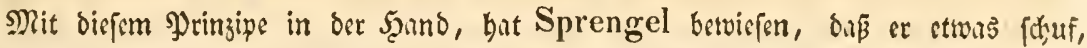

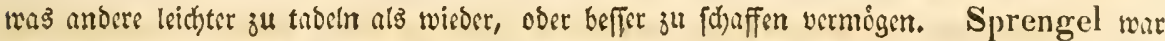

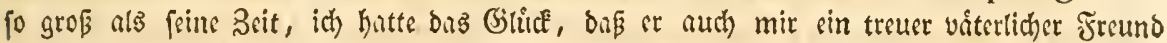

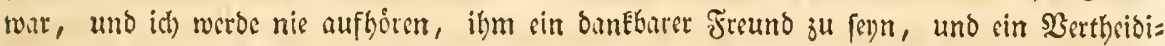

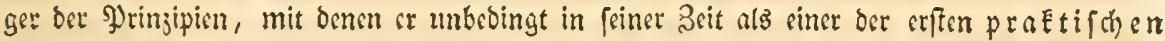

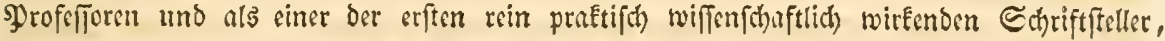
von feimen Sduilern und unbefungenen \&efern erfannt twurbe.

Shne cinen 2ufang ift aber bie Sinonymik nicht zu benken, in jenem Sुrinjipe feklt aber aller 2rnfang, es breft fich immer und ewig um's Ende;

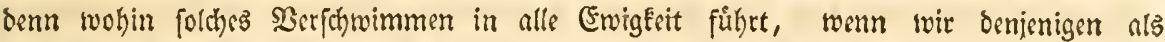

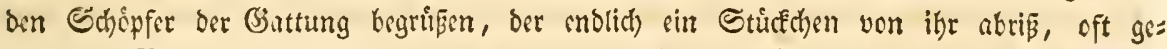

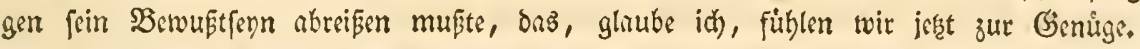

2(ber aud) Esin Negiment indert barum feinen Namen, wenn es cinen Rann

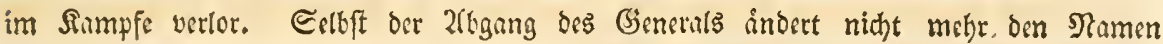

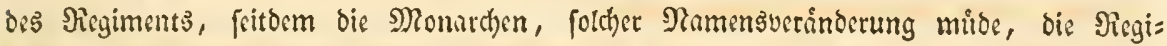

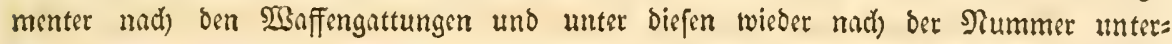

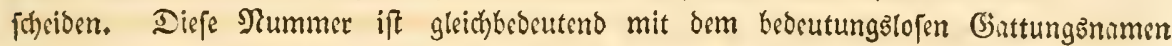
in ber BotaniE, von bem fehr richtig Mohl pricht, Denn es Fommt nur barnuf an, ben 2

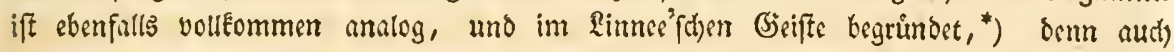

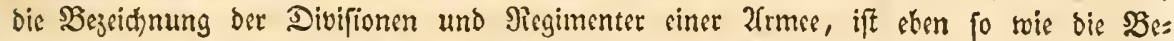
3cich)mung bes SPflunjenteichs in Frinen Fumilien und Giattungen nichts anderes, ats

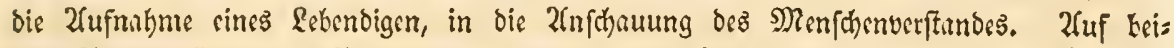
sen Seiten ift daffelbe Fortbilden uno diefelbe Berinderung im Raufe ber Seit. un= pere Genernle, deren Shamen den Ginttungen verbleiben mulfen, find die Zutoren, die зuerif fie gefohnffen, nid)t bie, welche einen ober ben andern Mann bem Negimente entfůbrten; uno ber Rame jener verbienffvolleren Gienerale, verbleibe der Ginttung, jo lange die lebende $\mathfrak{W e l t}$ nod eine Speciez derfelken lebendig Eennt und erbaitt, bie ih:

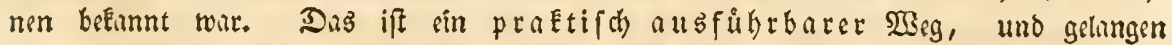
mir auf biefem 2 sege nod) überbies bajll, unfere gropen Sorfahren in Erinnerung zu

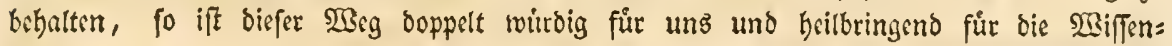

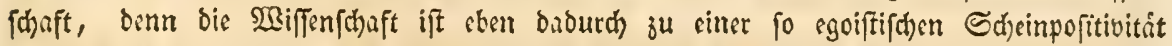
berabgefunfen, weil fie nur bas Neue will und nlle Plietít gegen bie 2atten verteug=

*) Principes, nobiles, proceres, milites, servi etc. 


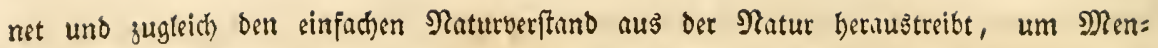

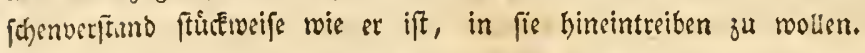

Beginnen wir alfo dis Begeidnung ber Garttungen mit Tomrnefort und Linnée, ift abor nod) beut zu I.lge siner unter uns, mit fo grumblidyen biftorifd philologi=

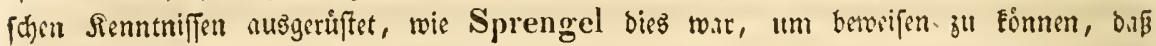

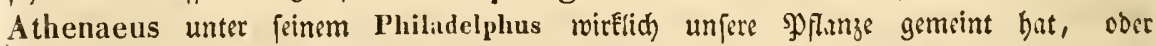

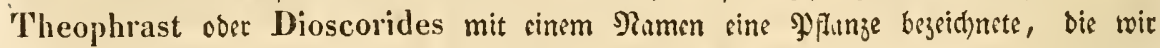
mit Tournefort uno Linnée nod) beute fo nemen, fo migen wir es gern fehen,

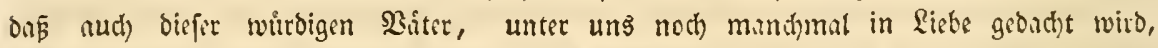

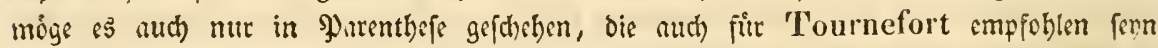

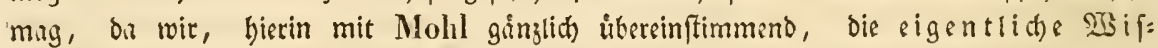
fenfd)aft, wie wir mitfen, bell uno Elar nur mit Linnée zu begrifisen vermogen. Dirs conferontive Şringip aber Ennn, wie idh benfe, von allen Seiten billigem 2fnpprutbe genitgen. Es genight aber auch unferem SBeftreben in Linnèe's Giefte zu

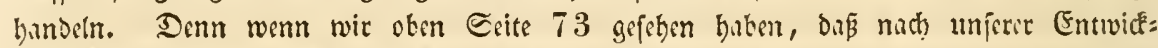
clung der Sarbe, Linnée's Grumbgeduke ber war: wer ben Namen zuerft (in

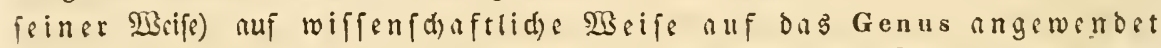
Gat: iff autor generis! fir ihn war es nlfo meift Tournefort und Plumier, für unz, in unferer Denftweife: Linnee, und dicjenigen feiner Nadhfolger, welche duns

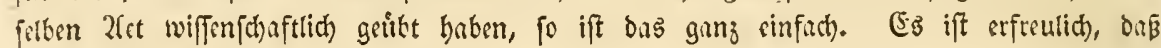
Fudlicher in frinem bertichen Wierfe: „Genera plantarum" Das "bedeutungstofe" uno "unmiffenfhuftlithe" Prinzip befolgt, weldfes bas unfrige ift; mit Sprengel und mit ifm und mit noch butndert andern tůdhtigen Mannern, wollen wir uns trófen, uno

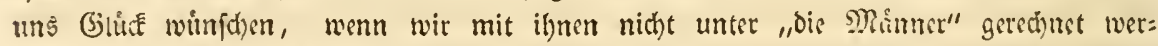

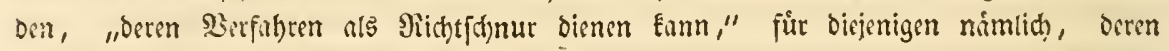
Prinjip nicht 0.15 unfrige ift.

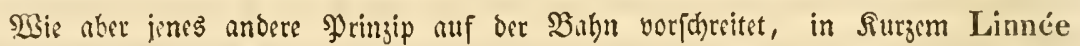
ginglid) aus der Nomenchatur zu verlofichen, das ift an fich flar, bem twenn bereits Gittungen, wie Orchis, Rosa, Triticum, Anemone, Kanumeulus u. a. nidht mehr

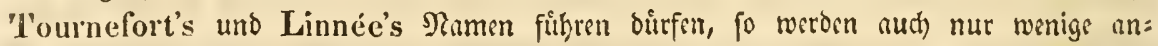

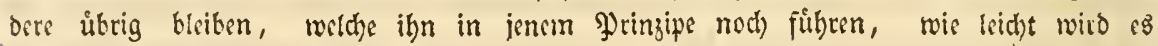

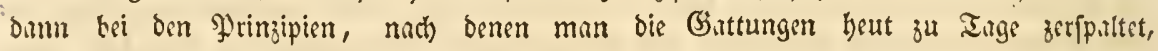
nuti) ieme menigen nod) theilen zu Eomen und nad) foldsen Prinzipien, wie etwa bei Bhexia, Melastoma, Oenothera u. n. ge(d)chen, thcilen ju milfen. Mag aud) nod) man= d)es Geppaltene ourd) Reduction wieber vereint werben, fo find und breiben wir bod) mit jencm Prinzipe fohon conjequent nuf ber Bashn, alle Ginttungen mit neurn 2futorm зи bezeidgnen, unt unfer Linnée ifit ganz unrettbar auf bem ficheren 2 Eege, sine biftorifde SRythe zu wetoen, wie man bereits oen 5̧omer uns genommen, uno

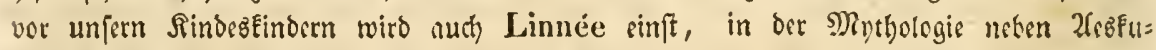
lap patiobiren.

Fragen wir hier noch sinmal barmach, worin ntm, neben biefen hohen Girwinne atts unferem atten Prinzipe, neben biefer Erbaltung einer Erinnerung mons wilbre, felbftbs:

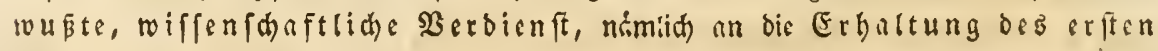




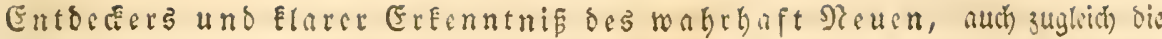

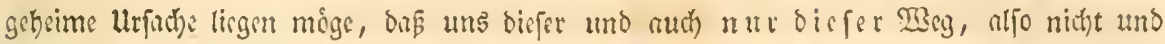

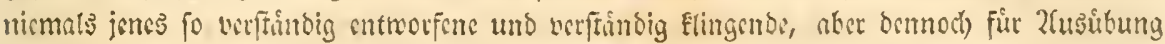

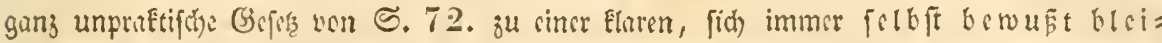

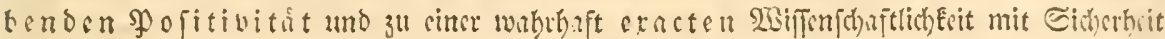

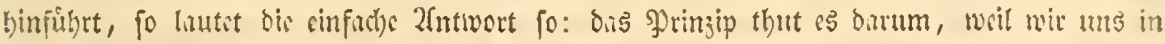

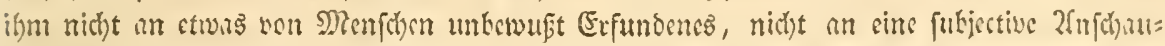

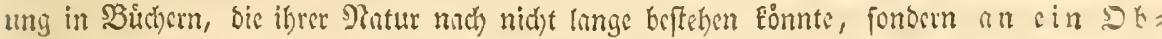

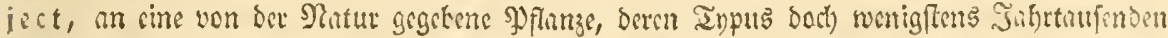

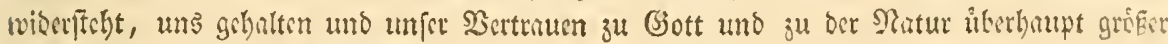
jimn barf, als ju ben Menchiben.

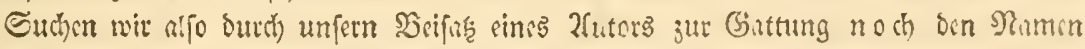

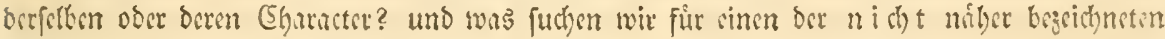
Ebarnetere, Deren wir orcicrtci baben?

"Num, boct) wohl nur ben naturlichen, ba beut zu Iage alles "matiolich" jenn

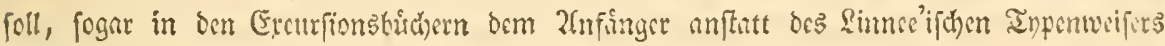

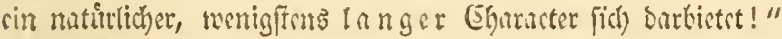

(3)ut, nebman wir aljo an, ş miffe ber natůrliche Cbaracter gemeint forn. Sirfer

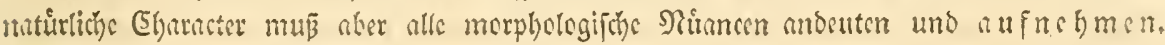

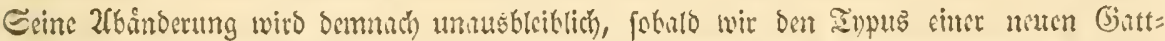

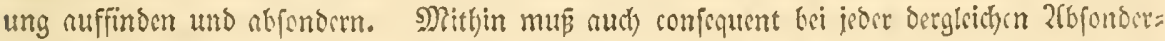

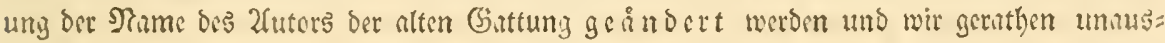
Hriblich in ben Strubel bes mgeduteten Wirtwarts. Suthen wir alfo lisher nicht methe

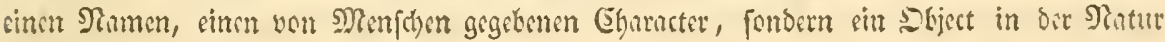
- cine Pf flanze.

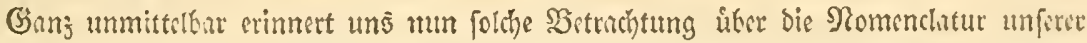

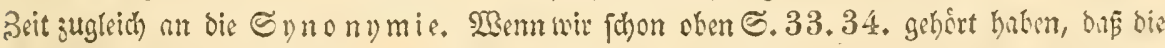

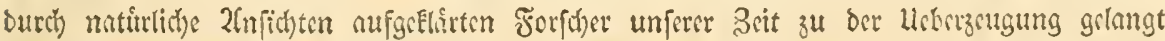

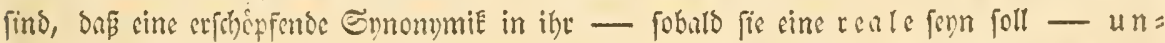

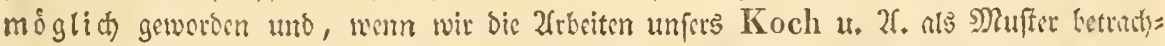

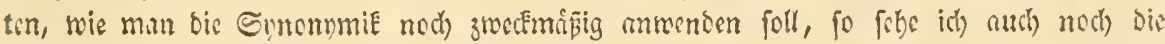

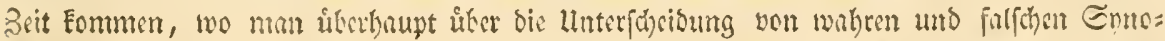

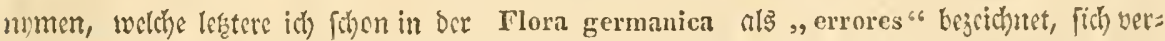
frindigen wird. Richter gieft oen erroribns bie bezeichnenten 9lumen Dys-onjma uno Cac-onyma, und id) bin mit Koch hiketgengt, dor fdyon beut ju Tage sine Ennonnmis weldite alles vereinigen will, nur nod) cine nominelle femn forne, aller realen Sacoutuny

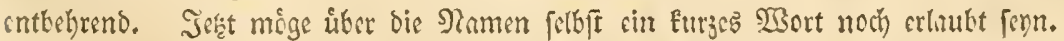

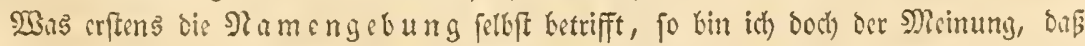

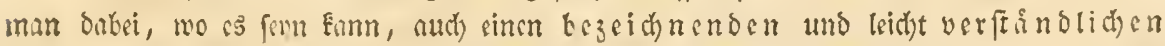
Ditmen wible. Es lisgt sins ichon naturliche, obwohl oft unflare 2thmung im Sfrincip

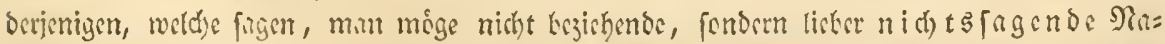

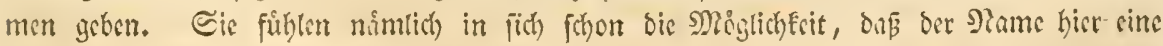

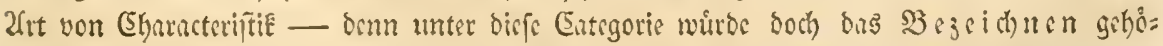

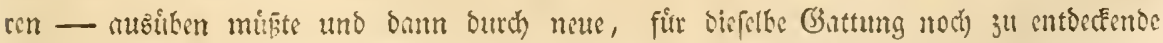

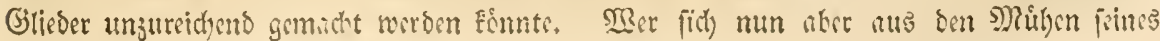




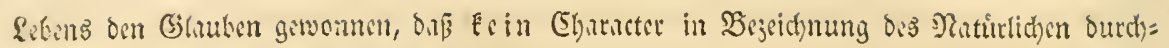

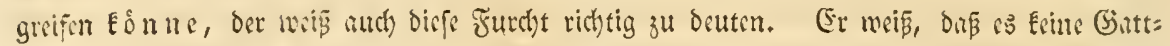

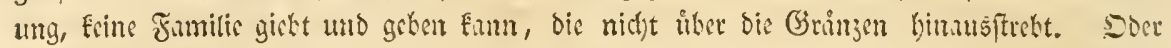
foll man ben Ramen Leguminosae batum aufacten, weil einige Trifolia einen utricnlus

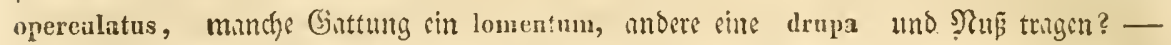

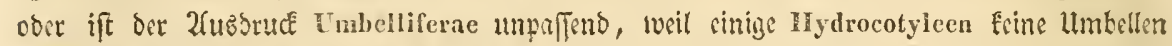
b.ıben? ober boiren wir anf bie Asperifoliaceae bei ifrem Stamen zu nennen, weil cinige Cerinthe-2futen Engl uno glatt fint? oder burfen wir nidht mehr Papilionacene fagen, meil Amorpla Feime Corolla papilionacea hat? nicht mehr Sungeneriften, weil bie Stauk= beutel boi Elentleranthera Poit. und Corymbium $L$. frei find? nidft mchr Saxifraga, tweil

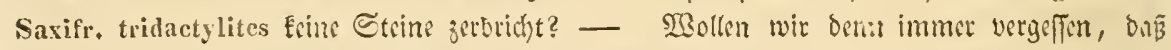

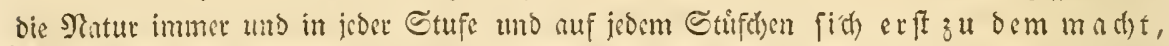

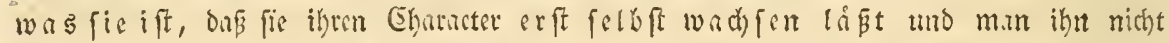

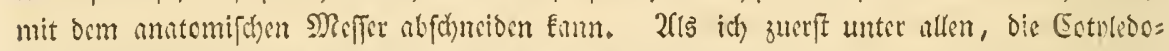
nen von Braya alpina im Solfre $182 \frac{4}{2}$ in Sturm's Flora, Jeeft $34 .$, abbildete, fo jeigte

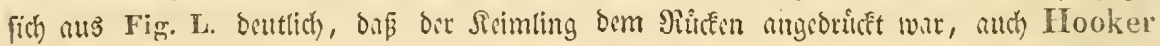
and Gay foffen fir fo, und ich) ftillte fie d.urum in Mössl. Handb. p. 1103. zwifchen Malcolmia

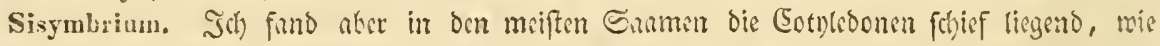

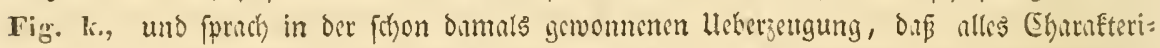

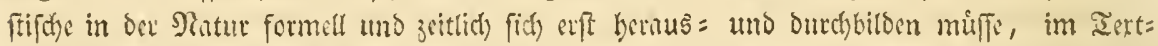

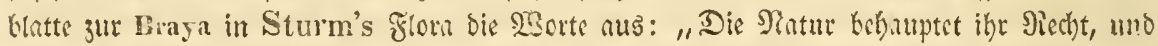

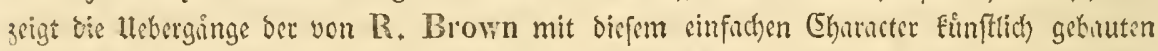

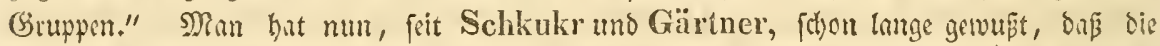

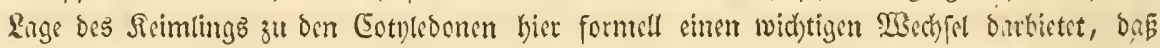
aber ocrferbe bei fonftiger l!chereinftimmung oer 'Tetradynamae uns nidjt zu ciner Sertheil=

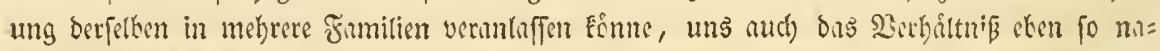

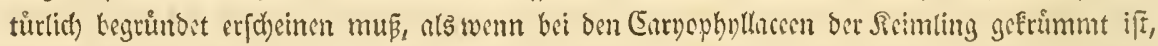

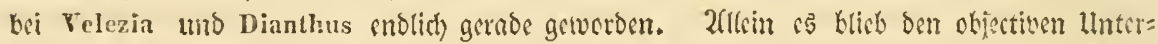

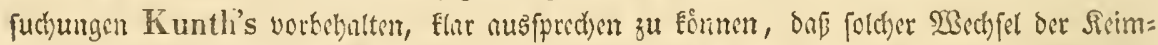

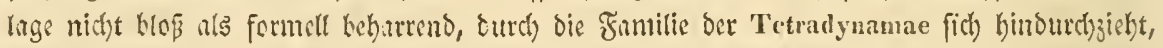

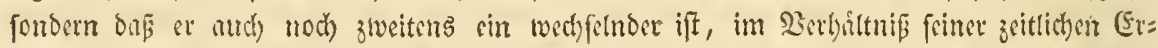
fochanm, und fo alfo burd) Kunth (Wiegm. Archiv 1837. II. S. 292. fo chen crft

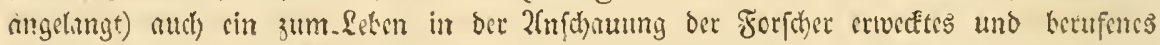
Faftum geworben. Soei fo fujoner und num manthe Differnz im Erkbten Flar machenden

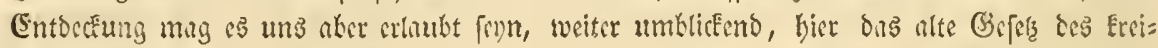

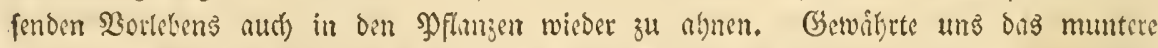
Dreten oer Embryonen in Den Ficrn von Lymmaeus stignalis, wie fie, mit ihrem Ectynte

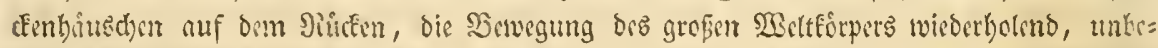

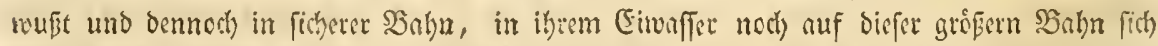
zım das cigane Eentrum brebcno, kerwigten, (Carus, Nov, Act. Leop. Carol. XIII. II. P.

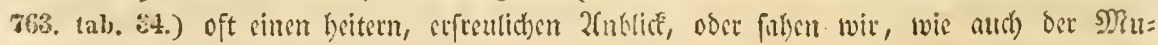

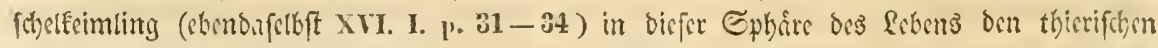

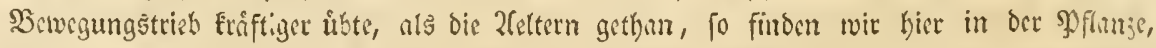

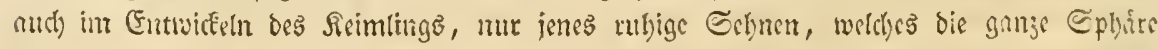

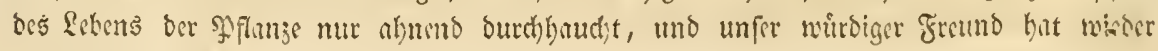




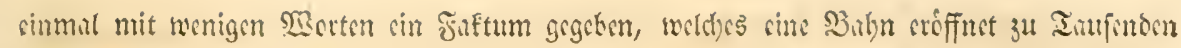

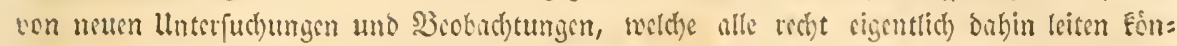

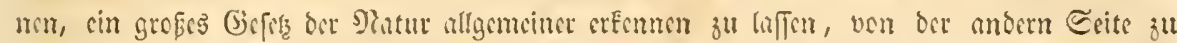

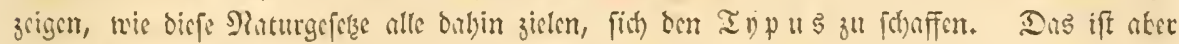

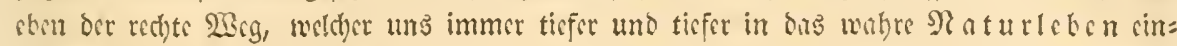
fillyte.

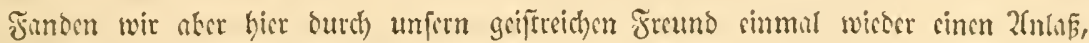

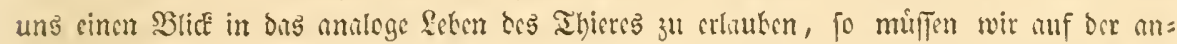
ocm Esite cin ginjlidyes Serfennen alles wahren Naturlebens burin finden, wenn 2fndere

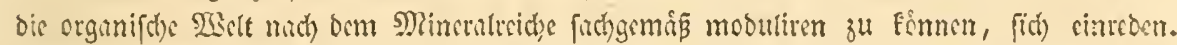

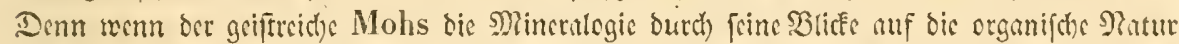
treffich gefordert uno, wie jeder Unkefungene ficht, offentur und and anregeno fortzengeno verwirts geknoht hat, fo mag man barin mur nicht SBerchtigung finden, dis Eache vice versa ju nchmen. Die lekendige Natur ftcllt man nid)t mif ben Sioff, mis cinen

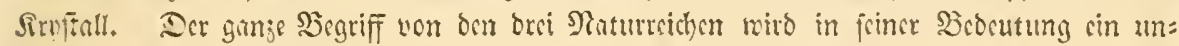

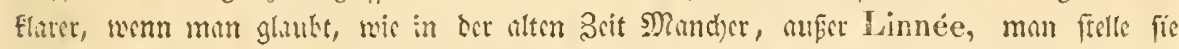

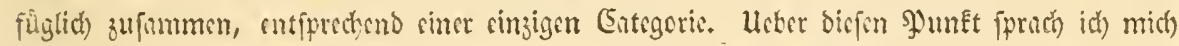

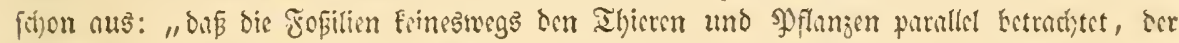

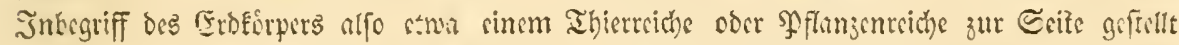

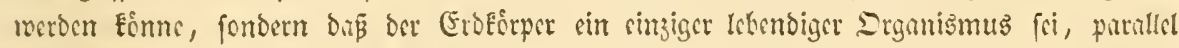

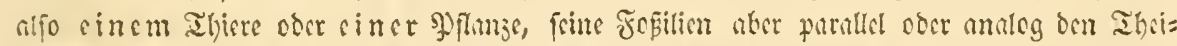

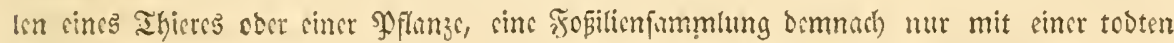
Sinohenfunmlung, Scoljfummlung, oder mit cincr Sammlung aud) im unorganifitten Sórper, in since Pflanje ober in cincm Ibiere erjengter Mineratien: Eoncrementen oder Firrfferlen,

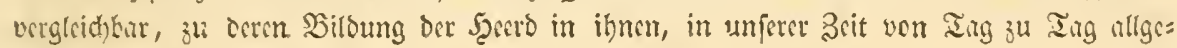
meiner inerfiunt wurde."

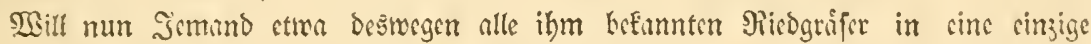
Epecies: Carex nultiplicata, alle Srokundecn in cine Orobanche polymorpha, alle Mlen= then in cine Nentha verticillispica, (wril eine 2trt alletoings in oor Natur aud) bie Enn=

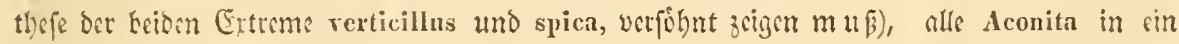
Aconitum varigaleatum, (meil aud) hicr, wic in allem was lckt, bic Enntlye? ihre Ertreme

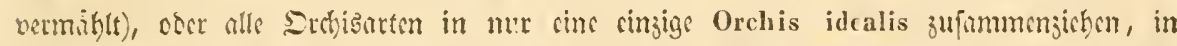

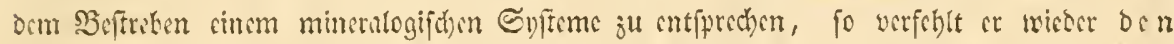

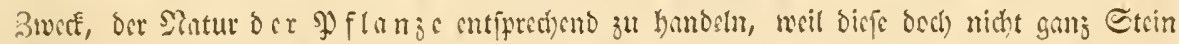
it, fondern auf oer Etufe ber Gottung ocm gemaltigen Drange ibicr Natur Folge zu leiften

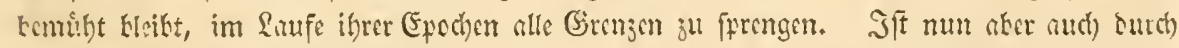

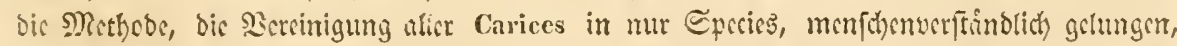

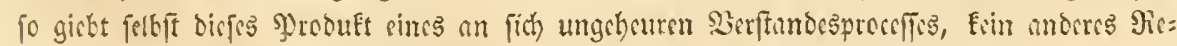

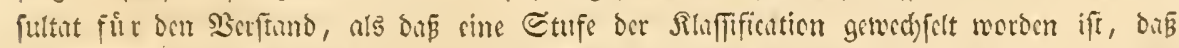
mit nun das Epccics nemmen, was fonft Germs genumnt worden ift. 2Kker nir gewinnen aud) nichts, senn unterfdyciben miffern wir noch immer bie Formen, bie wir 2frt nunnten,

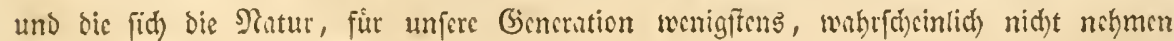

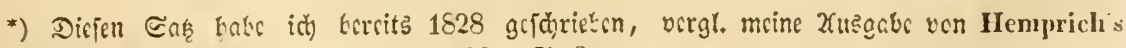

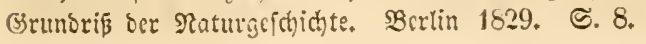




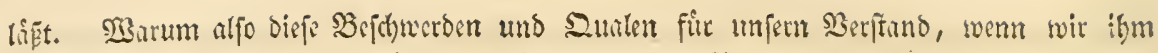
rinfinder uno leichter fo ju genugen vermigen, wie bie Niatur uns letendig bie 2frten ibres Carices kietst. 2tis llebung mognt wir indoffen folches Seftreken niemals vetmerfen, Das

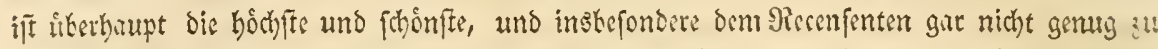

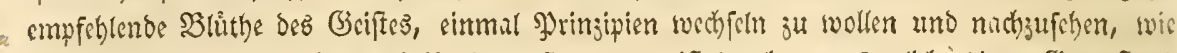

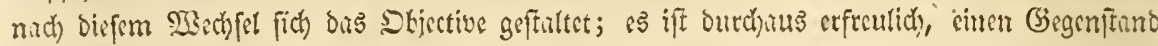

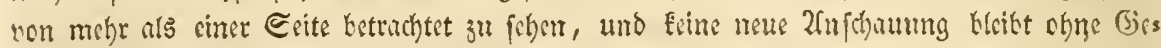

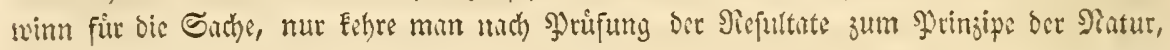
willig nathgebeno, felkit mit jurick.

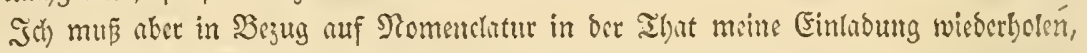
nir eime Familie ober Gistumg, wethe fich) kereits in ifrem Trpus entfaltet bat, nemen zu

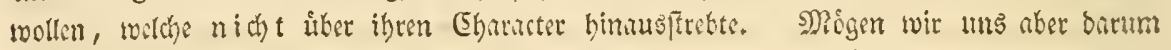

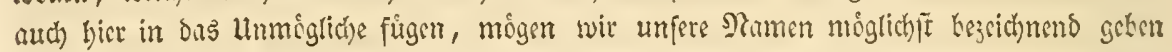

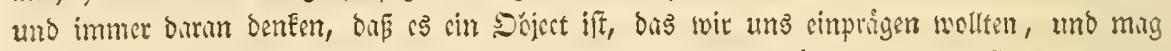

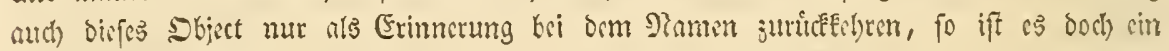

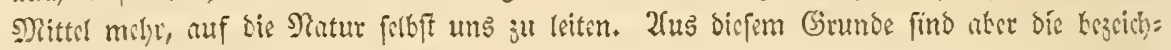
nenden Namen bie beşten, die Nirmen, aus Mentichennumen gebildet, find okjectiv weniger werth, ater fie crinnern uns an Serbicnite um bie Cache und find immer nod weit keffer, als bie finnlefen, die manche Sctyriftffeller lisken. Dic Giattung Adenogramma Recich.

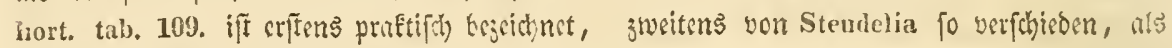

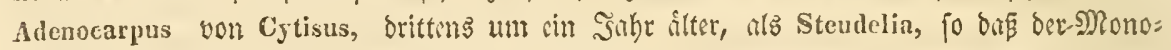

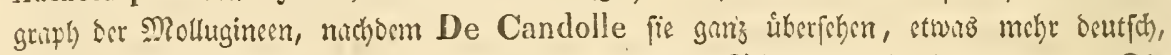

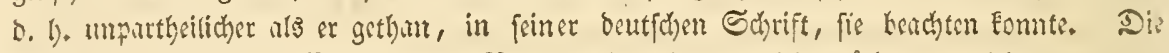

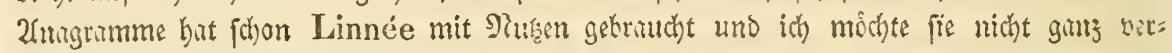

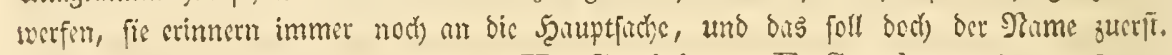
Migen wir alfo die 2(nigrumme yon H. Cassini und E. Spach arfnefmon, fo gi: frefon wil, b.ris wir burch Rehis und Anogra, ourch Torysca und Roscyna, fotirls

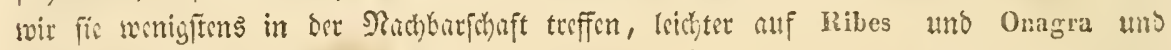
Ascyron fommen, als wenn es gilt, bic 2fufgate zu lôfen, was Calylophis beifen folle unt

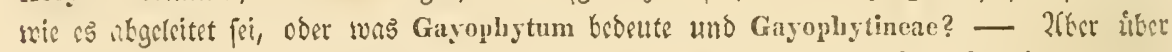
bis, wic Mlanche fagen, "viclen" und "fiche vielen" (Gattungen von Spach witroe id) mir

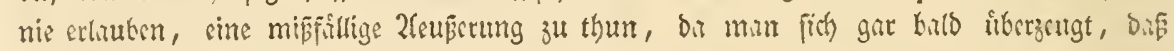

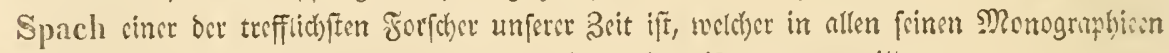

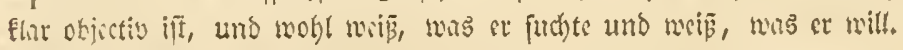

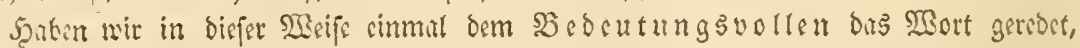

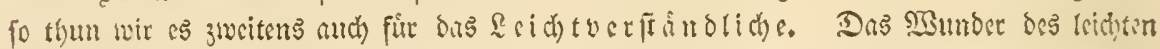

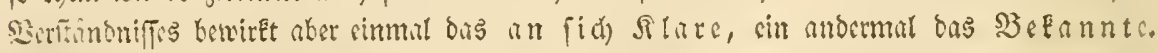

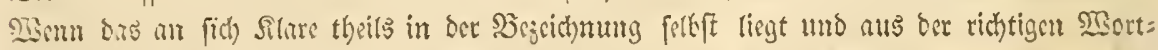

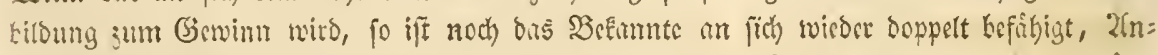

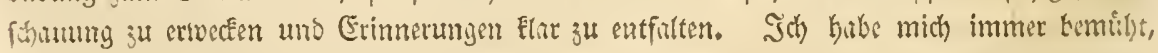

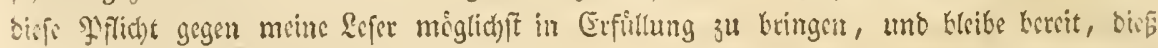

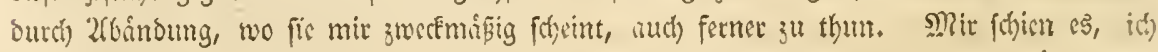

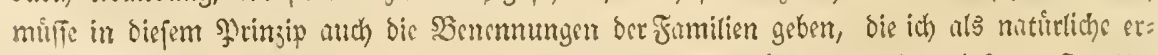

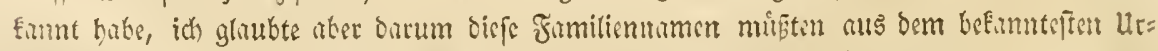

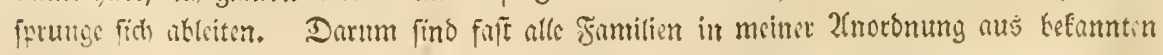




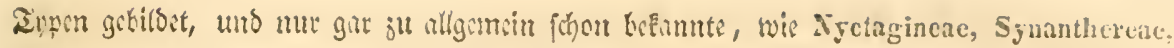

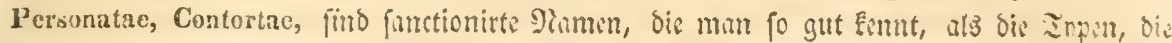

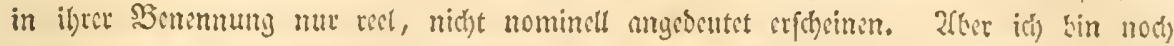

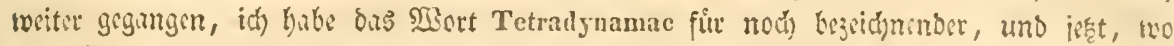

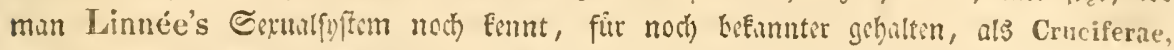

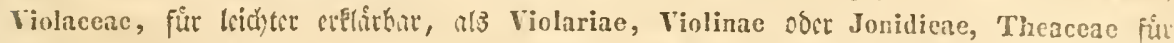

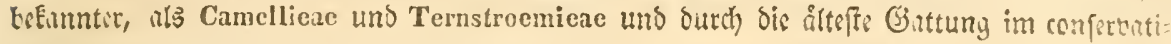
เen গु)

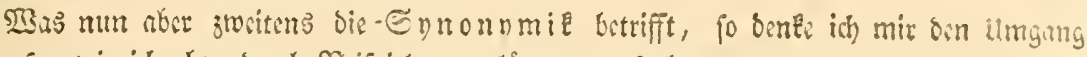

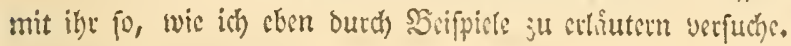

A. Sỵn-onyma: 8 usnnungen, o. b. im Ssithufe lege naturac ti tilis.

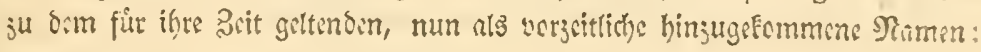

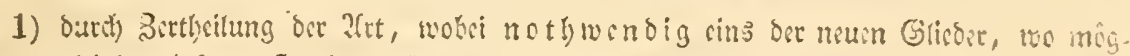

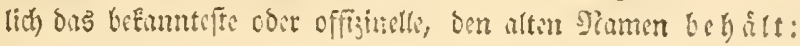

Cerinthe major I inn. theilt fich in C. niajor $L_{*}$ asjera Roth. strigosa Rch3.

Sedum Telephium Linn, theilt fith it $\mathbf{S}$, maximum Sut. tmb Telephim 2 . und $\$$, I is baria Lock.

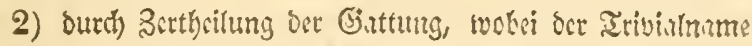

a) Det alte bleiten muß:

Leonurus Marrubiatrum $L$, wiro Chaiturus Marrubiastrum Ekrk.

Antirrhinum Cymbalaria L, wiro Linaria Cymbalaria Mill.

Anthericum ossifragum $L$, wird Tarthecinm ossifragum Măh,

Saxifraga paradoxa Sternb. tyitd Zahllirucknera paratoxa Pchb.

Nerglciche funte in ber Flora germanica bic Gattungen: Vignea, Colonoprasum, Ine-

rnm u. a. m.

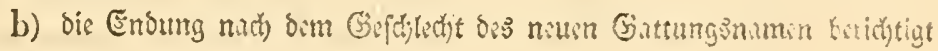

Asphodelis luteus $\boldsymbol{L}$, wito Asphodeline Iutea Rchb.

Conyza sordida $L$, wito Fhagnaion serdidum $R c h b$.

Ornithogalum lutcum $L$. wird Gagea luter fícr.

Lotus hirsutus $L$. wird Bonjeania hirsuta fichb.

Lotus microphyllus Hlook. wiro Bonjcania microphylia $/ 2 c h$ b,

Sisymbrium tanacetifolium $L$. wird Hugneninia tamaetifo!ia Rchb.

Valeriana rubra $L$. wiro Centranthus ruber $D_{e} C$.

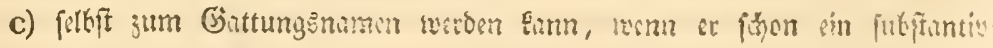
appellativar iff:

Angelica Archangelica L. wiro Archangelica officinali.; Boffri.

Antirshinum Linaria $L$, wito wicder Linaria rulgaris Bauk,

Ophrys Corallorrliza $L$, wiro Corallorrhiza inuata $R . B b$,

Pinus Abies L. wird Abies excelsa DeC.

Puus Larix $L$. twito Larix europaea $D_{C} C$.

Rhannus Frangula $L$. witd Frangula vulgaris $R c h b$.

Vaccinium Oryeccos $L$. mito Oxyencens palustris Pers. 
d) ganz megfallen muE, fobald ar

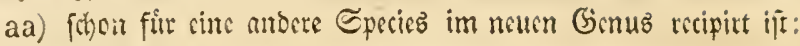

Cucribalus Behen $L$, wito Silene infiata $S m$.

Nyosotis squarrosa Kit. wirb Echinospermum leflexum Lchm.

Cineraria alpina $\beta$. $L$. wiro Senecio Iyratifolius $R c h b$.

bl) fobato or borm Giattungsnamen gleid)hutet:

fuentodon Tarasacum $L$, mird Turaxacum officinale Mnch.

Serner obige Saifpiste von c.

cc) fobald er brm Gonttunganamen ganj gleichbedoutend if:

Arctostaphylos ura ursi $S_{p}$ r. wirb $\boldsymbol{\Lambda}$. officinalis Wimm, ct Grab.

dd) fobsto or bem Ginttungsnamen theitweis gleichbeduteno und ifhn= (ich) lonteno ift:

Ophrys spiralis Linn, wito $\left\{\begin{array}{l}\text { Spiranthes antumnalis Rich. } \\ \text { Spiranthes aestivalis Rich. }\end{array}\right.$

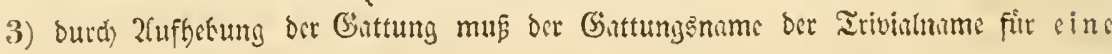

- Epcics merden:

Periclymenum germanicum Bauh, wirs Lonicera Periclymenum $L$.

Caprifolium italicum Dod. miro Lonicera Caprifoliuu $L$.

Xylosteum Dodonaei mito Lonicera Xylosteum $L$.

Sytuplioricarpos fol. alat. Dill. wiro Lonicera Symphoricarpos $L$.

Dicrvilla acadiensis $T$, wirb Lonicera Diervilla $L$.

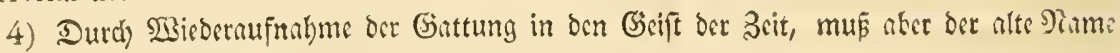
wicber als Gisttungsname borgeftellt werden:

a) firt fich allein:

Lonicera Caprifolium $L$. wiro misocr Caprifolium hortense Lam.

Lonicera Xylosteum L. wirb mirber Xylosteum dumetorum Mönch.

Lonicera Symphoricarpos $L$, wiro wicder Symphoricarpos rulgaris Mchx.

b) mit Berwandlung oes bisherigen Ginttungsnamens zum Zrivialnamen

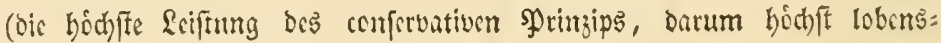
wertin!):

Lenicera Diervilla $L$, wiro Diervilla Lonicera Mill.

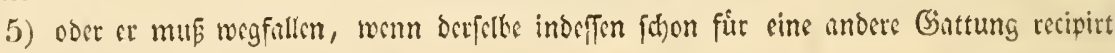
worden if:

Coronilla Securidaca $L_{*}$ mito Bonaveria Securidaca Scop., tocil foton cine Securidaca $L$. exiffirt.

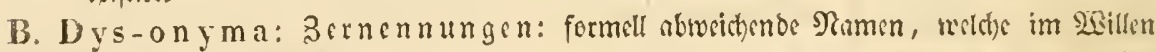

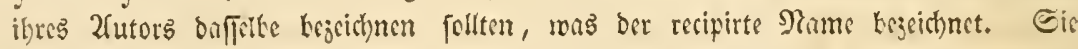
entitanden:

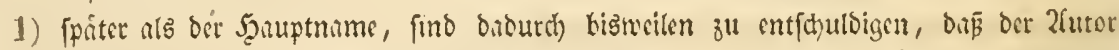

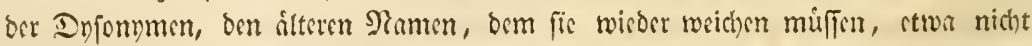
Eemten mochte, in mandyen Fiflen wirflich nidyt Eenuen fonnts. Eine Girenze buzwifchen anzugeben, ift unmegrich, besbalb gitt bie 2fncimnetit.

Melaleuca canescens Otto.

Melaleuca tomentosa Colla $\}$ M. incana $R$. Br.

Melaleuca myrtifolia $t^{\circ}$ ent. iff M. squarrosa Sm. 
Melalenca obliqua Hort. ift M, styphelioides $\mathbf{S m}$.

Mlclalenca decussata $W$. Lin.

Melaleuca ellorantha Bonpl. $\}$ ift M. diosmifolia Andi. vergl, Schauer's Monogrinphis.

Melalenca foliosa Dum. Cours,

Acacia prominens $\boldsymbol{B}$. Mag. 3502 (1837) war fhon A. decora $R$ chb. ic. 1993. (1828).

Acacia taxifolia Lodd, (1828) mar fdjon A. Oxycedrus Sieb. Fl, anp. (1824) et Rchb. DcC. HIook.

Crepis macrorhiza Lowe Mser. B. Mag 2988 (1830) wart fajon Schmidtia quercifolia hichb. (1828) Florula Lusitano-MIader, cum (liarnosi ")

Crepis maderensis Lowe Mscr. (1820) war fdyon Schmidtia anethifolia Rehb. (18:88) Fl. Lusit. mad.*)

Erysimum lancelatum $R, B r$. (1812) war fiton Erysimum Cheiranthus Pers. (1807).

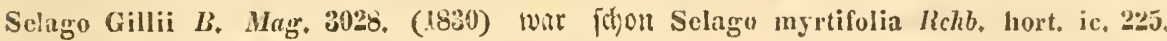
(182!)

Stachys altissima Desf. I. Par, (1828) mar fhon Stachys sibirica $L k$. (1822.) Rchb. pl, crit. 508.

Stachys inscripta Rchb. Lort. t. 40. Tout fuhon Stachys hirta $L$. ef. Fl. germ. 22163.

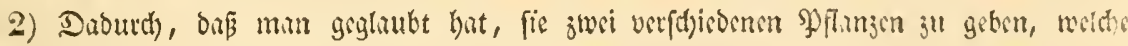
aber sineclei finto:

Lotus uliginosus Schk, if Lotus major Scop.

Sisymbrium hybridum Smith.

Sisymbrium islandicum Gunu,

Sisymbrium palustre Leyss.

Cino Nasturtium palustre DeC: $^{\prime}$

Sisymbrium terrestre $R$. Br.

Orobus venctus Mill. Ser. DeC.

Orobus variegatus $T e n, S_{c r}$. $D_{c} C$.

Orobus multiflorus Sieb. Ser. DeC.

Orobus serotinus Prsl.

Orohus rigidus Lang.

Orobus pyrenaicus Scop.

Orobus vernus b. Latifolius Roch,

Hypericum maculatum Walt.

Hypericum naculatum Walt.
Hypericum nicranthum Chois, fino II. punctintum Lum.

fino 0 . venetus Clus.

$\left.\begin{array}{l}\text { Asprella Schreb, } \\ \text { Homaloconchrus Micg. }\end{array}\right\}$ fino Leersia Solander.

Ellebocarpus Kaulf.

Teleozoma R. Br.

Cryptogenis Rich. $\quad$ find Ceratopteris Brongn.

Furcaria Desv.

Cryptogramma Grev.)

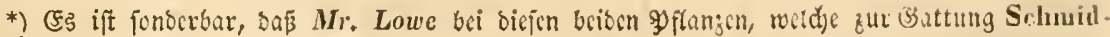
tia Much, ganz ficher gehören, fragt: "where are these described?" Da cr mcine übrigon

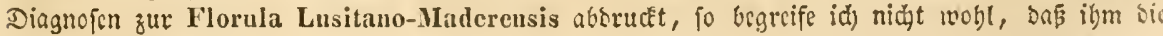

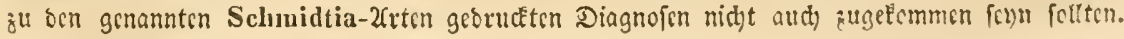




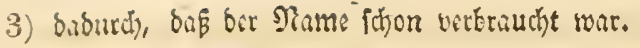

Harrisonia Hook, (fhon Adans.) murto Bastera Rchb, consp.

Hippion Spr. (fónon Schmidt) mutó Slerogtia Rchb, consp.

tiriope Ilerb. (fition Loureiro) rutoc Liriopsis Rchb. consp.

Uronia Filliot (jibon Nuttal) murbe Seutera Rchb. consp.

Mirenia rent. (fhon R, Br.) wurbc Genlisia Rclib. consp.

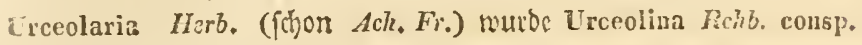

Turpikia Ilumb, und Bonpl. toutoe Fulcaldea Poir, und Voigtis Spr.)

Turpinia Pers. wurde Poiretia I'ent.

Turpinia Rafin, wurde Sclımaltzia Desv.

Rochelia R. S. wurbe Echinospermum Sw, Lehm, - Rochelia Rchb. Glib,

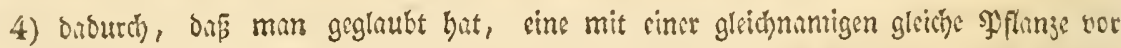
fich zu haben:

Ledicularis adscendens Gaud, iff P. Barrelieri Rchb, nicht P. adscendens Schlcich.

Pcdicularis cenisia Gaud, if $\mathbb{P}$. gyroflexa Itill. nicht $\mathbb{P}$. cenisia Vill.

Pedicularis rostrata Jacq, ift P. Jacquini Koch, nicht P, rostrata Linn.

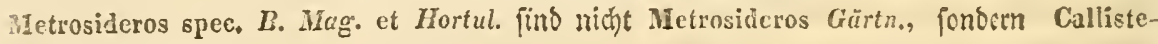
mon $R$. $B r$.

Tetrosideros spec. Sm. etc. fino nicht Metrosideros, fondirn Angophora Cav.

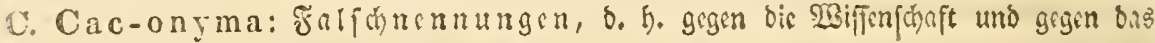
conferbative Prinzip verfosende Plamen. 2tipo:

1) fubjective 9ieuerungen:

Adenostyles riridis $H_{*}$ Cass, onftatt $\Lambda d$. alpina (Cacal.) L.

Adenostylcs albida $H$. Cass, nnftitt $\Lambda$ d. albifrons (Cacal.) $L$.

Allenostyles candilissima II. Ciss, anftatt Ad, leacophylla (Cacal.) $W^{\text {. }}$.

Phagnalon triceplalum II. Cass, anftatt Pla, sordidum (Conyz, sordida) $L$.

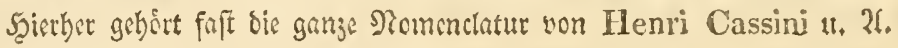

2) ilferflúfitige:

Neottidium $R, B r$, war und Elribt allcin Ncottia (Neffpflanze) $L$.

$\left.\begin{array}{l}\text { Securilla Pers. } \\ \text { Sccurigera } D e C \text {. }\end{array}\right\}$ war anto ktrift Bonareria Scop.

3) Namen keĔantet Thicre:

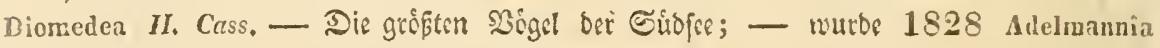
Rclib, consp.

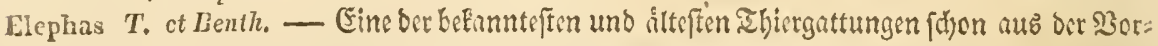
milt; - wart auch fot on lingft lilinanthus L. Ilall. All.

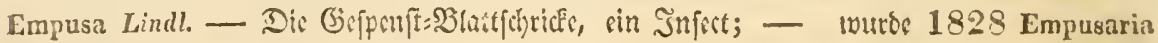
Rchb, consp.

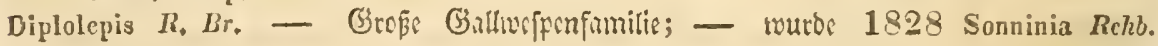
consp.

Gymnocephalus Schwägr. - Eine Fifch = und eine Negeignttung; - Mar Orthopyxis P. B. wutbe Aulocamnion Schwëgr.

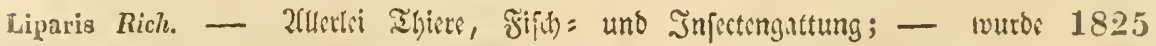
Sturmia Rckb, pl, crit, IV, p, 69.

Segcstria Fries, - Eine Spinnengattung; - wurbe 1828 Sphacromphale Rchb. consp. 
Trachys Pers, - Eine Rifiergottung; - routbe 1828 Trachyozus Rchb. consp.

Tritonia Ker. - Nattirlich Secthicre, bctunnte MollużEn; - murde 1827: Waitzia Rchb. Tascheulb. f. Gartenfr. ङ. 98. น. 102.

Phalangium Juss, - 2ffterpinnen; - murbe micber Anthericum Linn.

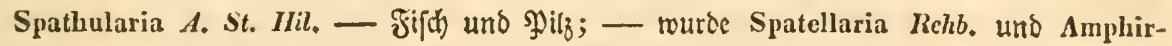
rhox Spr.

Șerglo aud Holl in ber Flora botan. Zeitung. 1823. 5.495.

4) auf falfdie Dbjecte úbergetrigene Namen:

Larbrea Seringe ift gar nid)t Larbrea A. St. Hilaire.

Arthrozamia, als syn. bei Endl, ift gar nicht Arthrozamia Rchb.

Hyphopsorae, ałs syn. bci Eudl, fino gar nicht (Coniothalami Licheno) Hypliopsorae $R \operatorname{ch} b$.

5) falfob abgefchricbene:

Astrolobium DeC. muß̄te Geį́en: Arthrolobium Desv,

Borkhausia Lk, muß̈te bciß̨n: Barkhausia Mnch.

Diclytra DeC. muß beißen: Diëlytra Borkh.

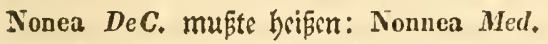

Sternebergia Herb, mußte heipen: Sternbergia $W$. $K$.

Potentilla Weinmanni Lodd. muß̧te bciß̨n: P. Wiemanniana Günth. Schumm.

Ebenfo 2futornamen:

Heyne ffatt Hayne if faft ftereotyper Fehler beutider Edoriftifeller.

Bissch. und Bisschoff ftatt Bischoff.

Paliss, und Palissot ftatt Palisot.

Schmied fratt Schmidt.

Höll fatt Holl.

6) ungrammatifalifthe unb barbarifas:

Anigozanthus Labill. wurde Anoegosanthus Rchb, Consp.

Loroglossum Rich, wurde Himantoglossum Spr.

Cliniphila Nutt. wurde Chimophila Radius.

Diarina Rafin, wurbe Diarrhena $P, B$.

Diplogon Poir, murde Diplopogon R. Br.

Gypsophylla Quorund, wurbe mieder Gypsephila $L$,

Hypoelyptum Vahl. murde Hypoëlytrum Rich,

Abumon Adans, wurbe Agapanthus L'Herit.

Ciju-puti Adans, wurbe Melaleuca $\mathbf{L}$.

Huttum Adans, wurde Barringtonia Forst.

Roram Adans, wourde Echinaria Desf.

Einft wird die Zeit fommen, wo man, den Synonymen kereits untertiegend, die Sla=

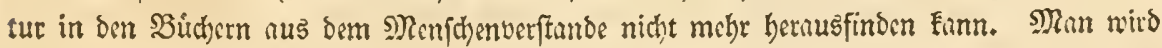
endich) Das heroifthe Mittel crgreifen, die Dysonymen und Sinfonymen nidyt mehr mit in

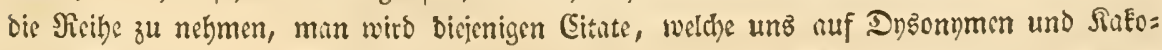

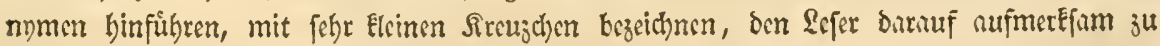

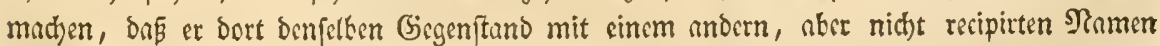

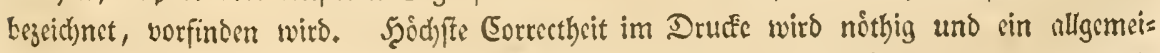

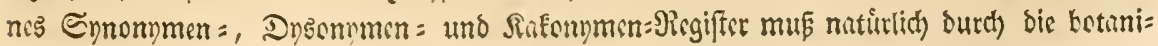




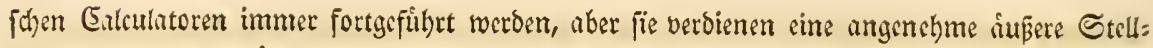
ung, demn es gilt für fie, ihe Reben blos Namen und den Srrungen bes Nen[chenverftan:

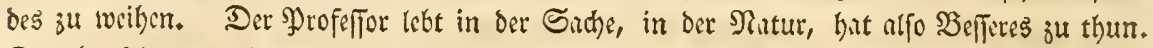
Da aber jedes von beiben, Sactie und Name, nicht obne bars anbere gedeift, fo mus aud Der Gilculator ein botanifot gebildeter Mann fenn, ber dem \$profeffor fdinelle Nadymeilung ju geben verming, fo wie wieder der Profeffor den Ealculitor berathet und leitet. Son

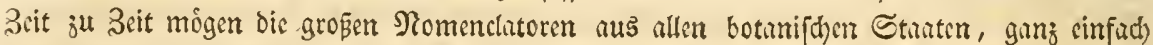
und ettwa fo, alphabetifch georonct, exforinen:

Rame (recipirter), 2Uuctoritit und (5 itat a) zum Samen; b) ju befter Dingnofe oder

Sefdoreibung; c) zu beften 2(bbildungen. Batertand. Da uer.

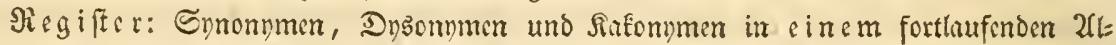

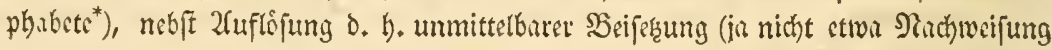
burch Biffern u. Dergl.) des Scattptnamen.

Zus allen biefen oon ben cinzelnen Ealculatoren sefertigten Nationalmomenclatoren girbt bum cinmal cin Scauptcalculator mit ber 2fusdurur unferes Steudel, uno vorjitglith

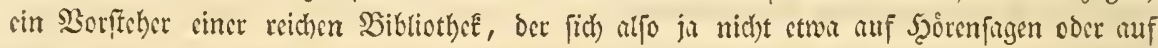

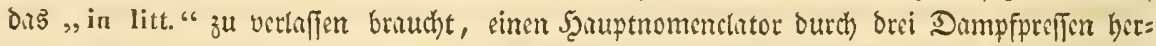

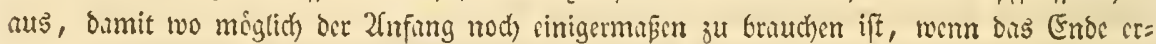

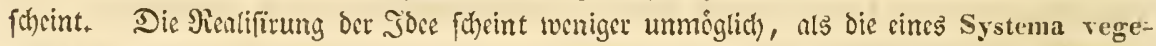
tabilium oder einer 2 uşgabe oce Species plantarum, und obwoht dic 2fusfihtung auch

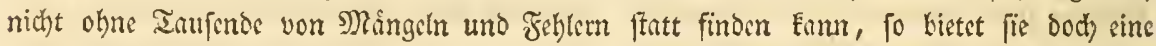

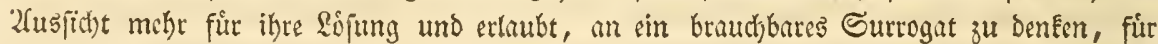
Das, twas wir nicht baben fơnen, oder wenigftens fo lange erwarten muiffen, dak bars En:

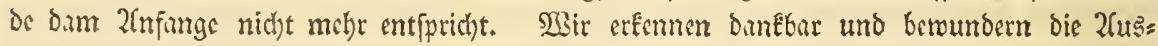

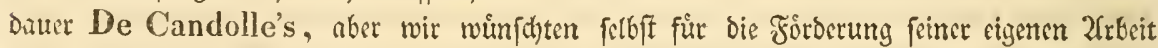

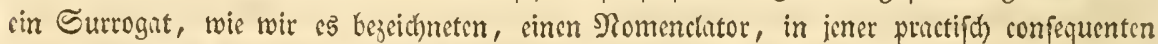

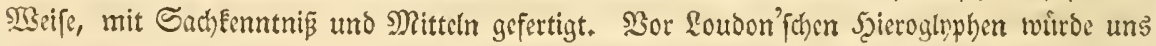

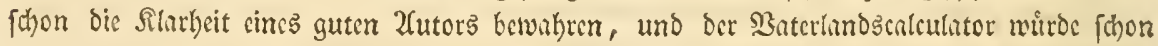

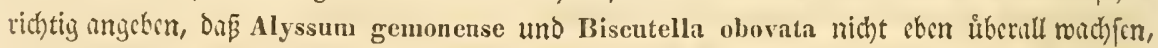

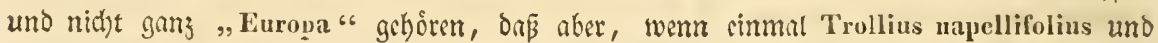
Astragalus Cicer „Europa" gefoitt, dann Trollius europaens uno Capselli bursa pastoris

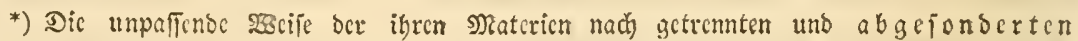

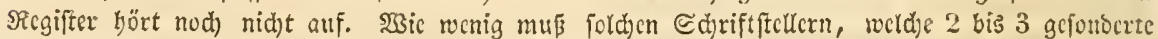

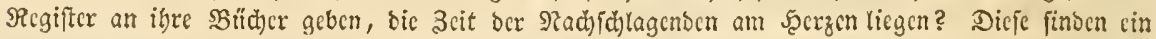

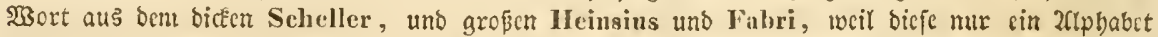

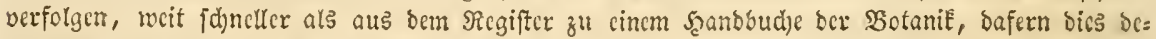

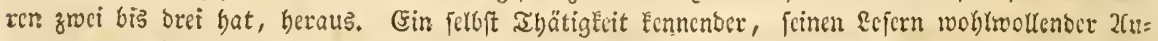

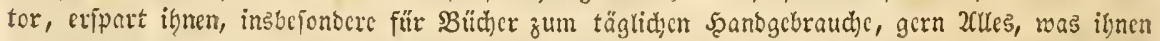

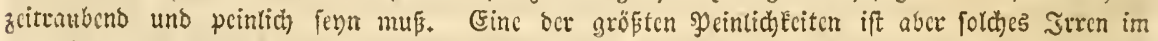

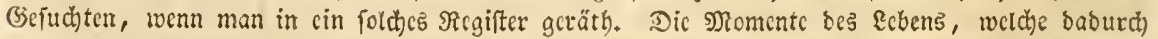

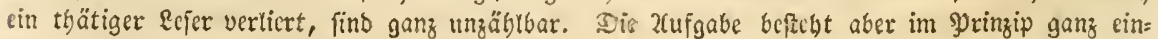
fad) nur barit, man foll fid Elar objectio in bic Stcllung bes Ecjers und folglich auth bes aufs

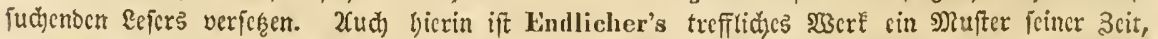

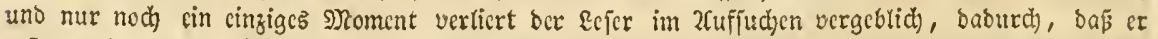

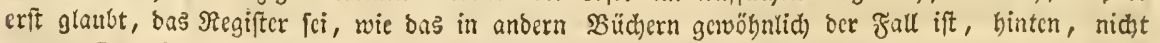

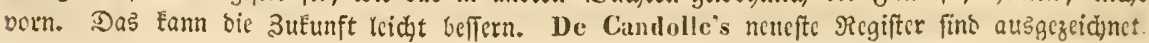


auch noch ausec "Britmnien" zu finden fern burfte, fo wie Biscutella lacvigata auch nod) ausicr ,Italy" wict) ft.

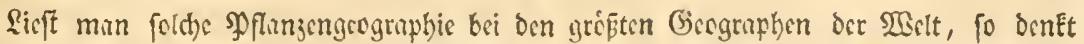
man doppelt banfbar an Alexander von Humboldt, uno erEennt aud) Doppelt erfreut,

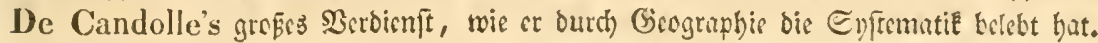

Saaben wir uns nun aber fo red)t nach beftem $23 i l l e n$ und (Scroiffen treu abgemuiht,

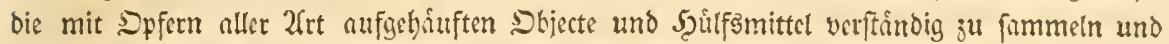
ju kenuten, baben wir Maffen von lebendig in ihre Scit bincinktithenden, fo wie von "lege artis" gepreften \$flanjen beifammen, fiken und ffeben und berwegen uns jwifchen

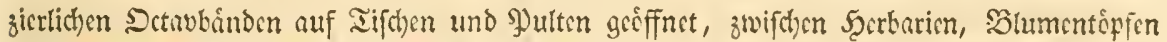

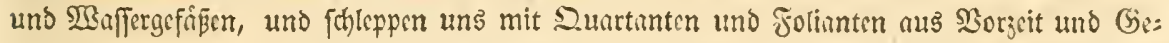
genwart emfig und sifrig berum, fdeuen bie Mithe nicht, sinzeine. Fragen an unfere Freun=

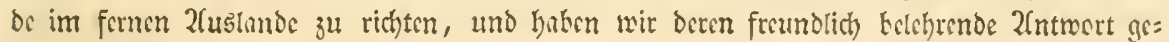
buldig crwartet, fino wir snolich aud) mit unferm Eulculaior gant verftindigt, baten Enn=

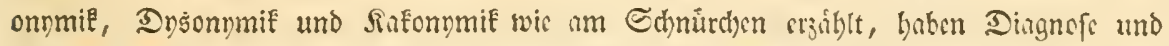

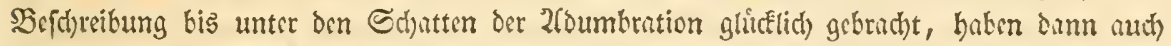

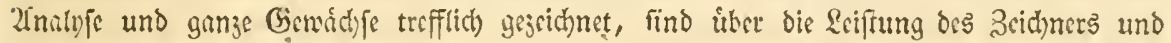
Siupferfechers uno Coloriften entzuckt, bezablen vielleicht als ebrlich gemitthliche Deutfhe, die

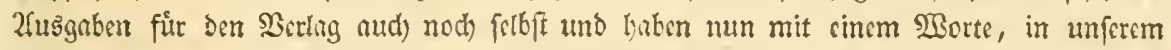

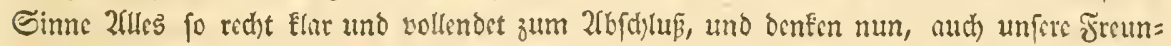
de wicose mit sinem Etuffedyen Sintur zu erfreucn - fo fommt bennod) viclleid)t nod) cinmal bie liebe Slatur, und fendet uns in ben $\mathfrak{e r}$ ften 2 pril, Des Botanical Registers numlid), Deffen Secft vom erften 2fpril vor 8 Ingen in Sondon erfesien, und bas wir to eben auffelugen, und zcigt uns $D_{a}$, wie fie cinmal alfe unfere Mube mit sinem cinjigen Schlage micder vernichtet, wie fie fereinbar trefflich begrindete, - jemand wurde, und

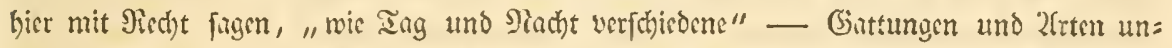

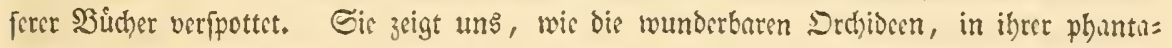

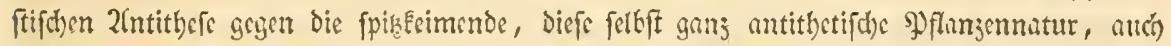

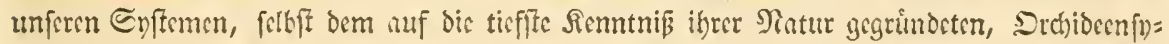

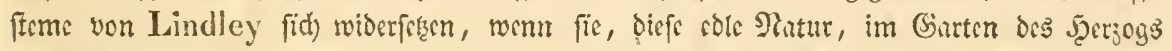

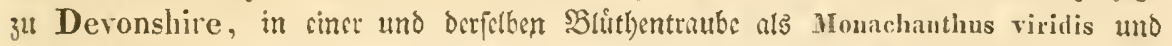

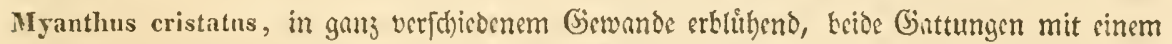
Male frmmt ifrem Gisfolge vou 2frten zur Mutter Catasetum, lisbend zurúckfithrt, in= oem fie noch siner britten (Gottumy, Mormodes, freundich winft, ifr ju folgen.

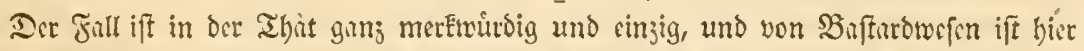

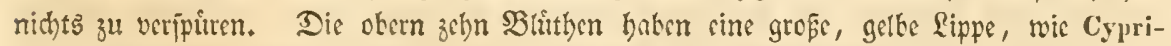

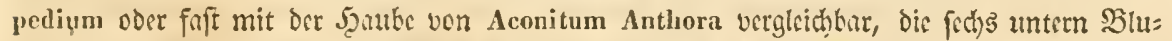

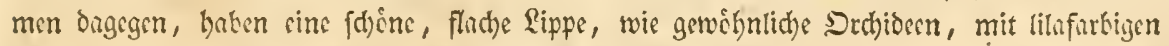

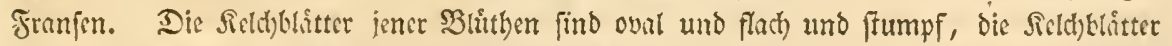

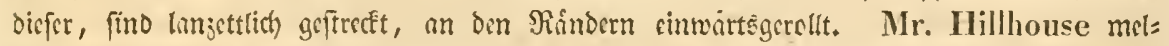

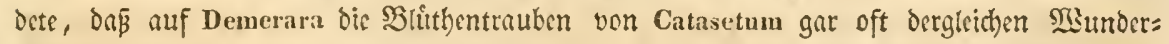
slithen, vercinigt gekiren. Fuch blihte, wie ber berubmteffe Fenner ber Srdjiben, M. Lindley, berichtet, im Scerffte 1836 im Garten der Horticultural Society cine Pflunje von Crnoches und trieb von siner Esite cinen Etenget mit oen befunnten uno wobl= 
riechenden Slutthen von Cynoches Loddigesii, votr ber andern Seite einen, mit ben geruch: lofen Blutthen von Cynoches cucullata.

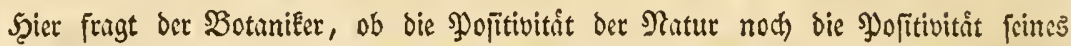
Subjectes ift, beruhigt fith aber wieder und erEennt bejcheiden, mit dem grofen Selbftoenter Agardh: Die Natur hat ibre eigene logiz! -

STir miffen uns bemnath, in mehr als einem Falle, in unferer fonderbar fortid)rei= tenden 3eit, baran getwofnen letnen, oft das, was wir für "bedcutungstos und unwiffen=

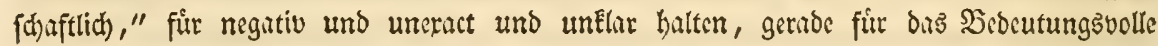

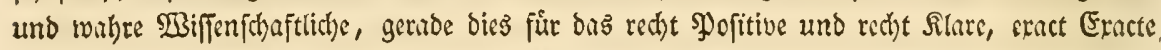

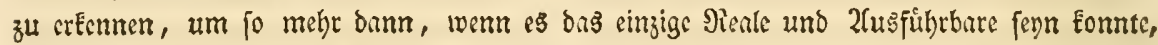

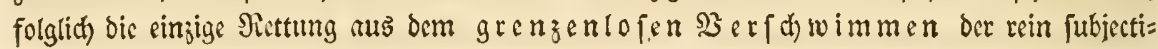

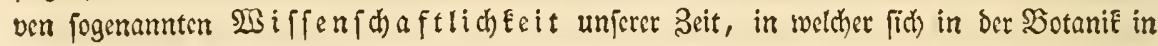

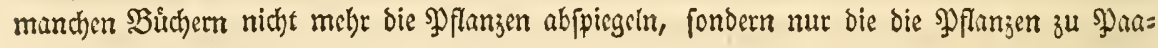
ren treibenden Menjchen.

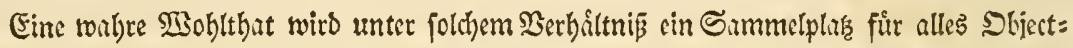
ive, ras in ben ber Natur treuen Naturbefthutern noth auftaucht, und foldhe Sammel=

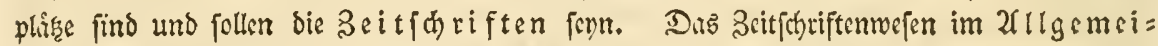

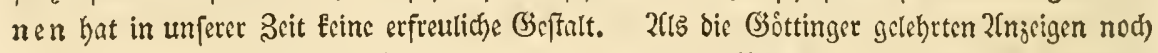

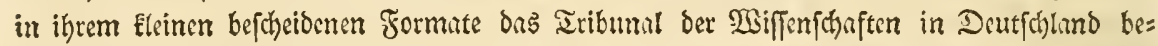
ftimmten, lingen auth bie Slaturwifferfthaften in einer noch fo befcheibenen 3uritefgezogentsit,

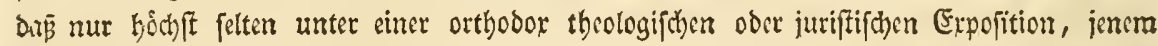

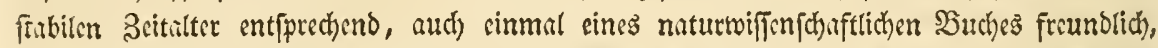

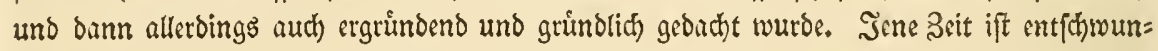

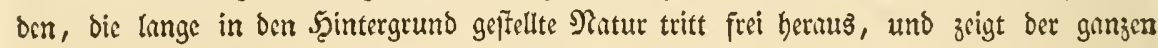

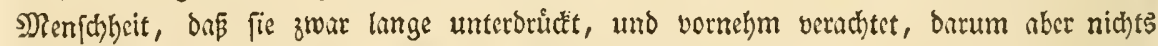

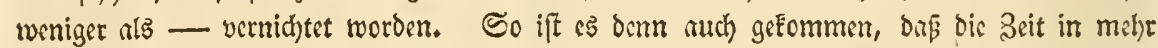

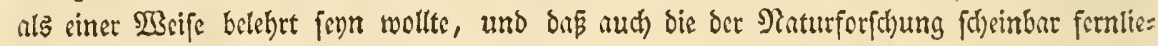

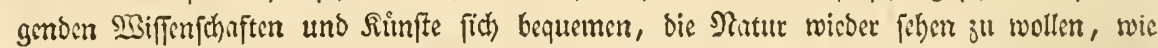

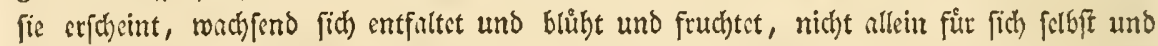

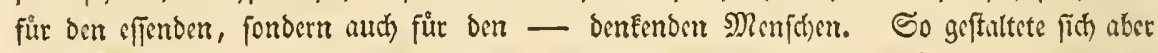

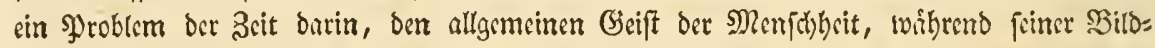

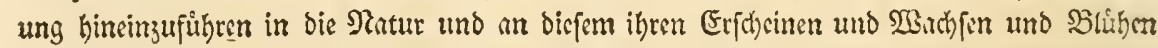

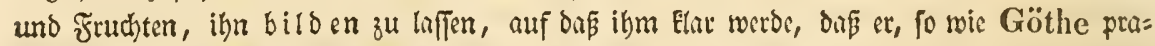

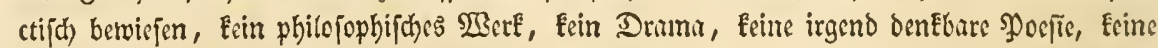
Biograpbie, Eeine 2fnalyje cincs claffifdycn 2futors, Esinc Chria Aphthoniana, ja Eein Edul= erercitium Elar begreifen und ourd)/fhauen, am allerwenig/ten freilich von allen geiftigen

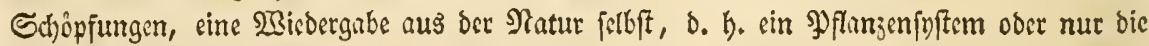
Erpofition ciner Slaffe, Sromung, Familie, Gattung und 2(rt naturgemǻ in fich auf=

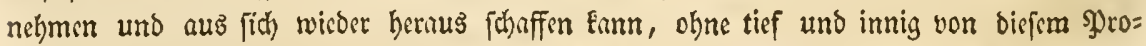

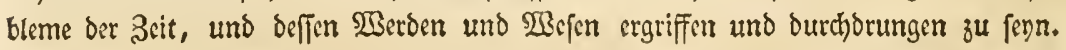

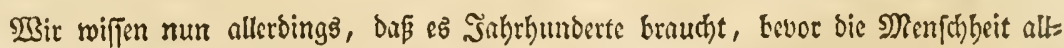
gemcine $23 n h r h e i t e n$ begreift und Linnée lefrte, daß man bie natürlithen Framilien nicht mit ber Echecre vom Faden ber

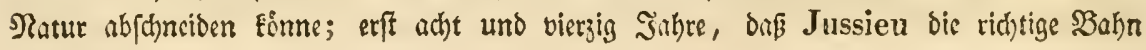


zeigte und gare erff fiebenunbvierzig Sahre, Daß Göthe biefe Babu mit feinem Elaren \&ichte zum erften und dann wieber zum leşten Male in feiner Metumorphofe, auberdem aber fein

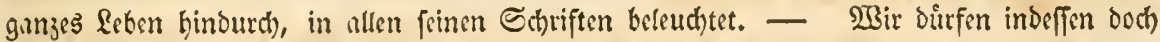

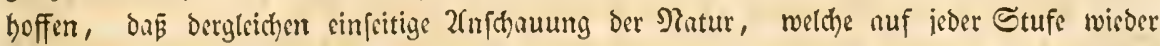
neue Drgane finden will und fid) terechtigt ghutet, biefe wieber mit neuen Namen belegen

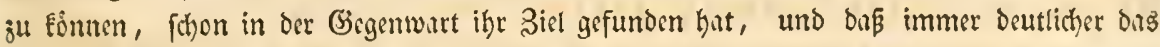

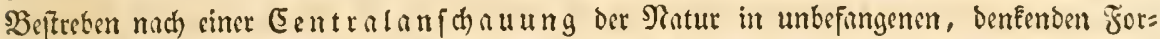
id)ern fich fund giebt. Unter Şunderten von Beifpielen, aus biefer Elur naturtid genetifchen 2Beife entiprungen, móge nur das eine erwithnt feyn, wetches Röper gegeten, deffen Mic= ouctionsftreben einft von ber allgemeinften 2enerEennung gekront werben burfte. In jeinen Sebriften waltet jugleid) Der lebendige Umblick auf bie ganje Natur und allis (Finjelne tritt 10 mit bem Gianjen in harmonifhen Einflung, indem wir burtin bie Balfn erfennen, wethe

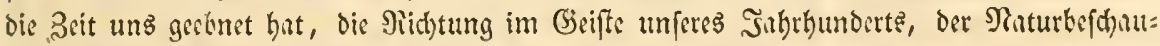
ung objectiv forfdsend, uns jelber ju weityen.

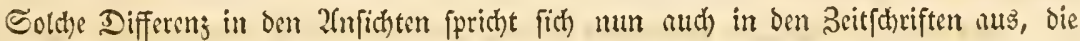

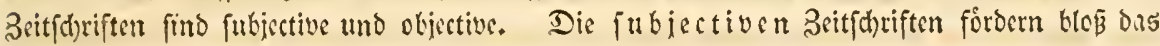

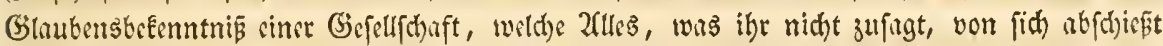
oder entfernt. So ift es getommen, Daßj bentfhe Siternturblatter und 2fnnalen, welche

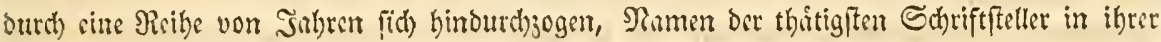

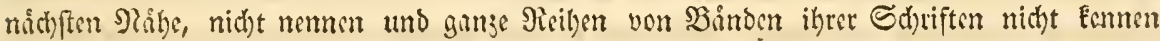

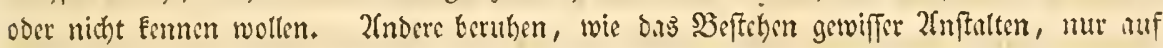

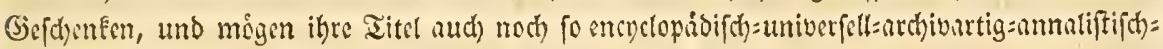

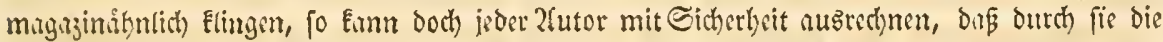

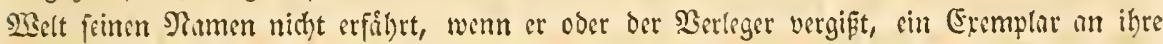

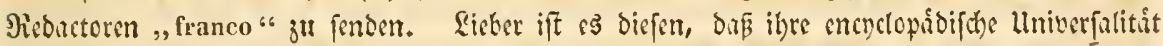

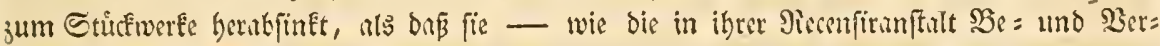
urthsilten thum miffen - sin Bud) fich anfdhaffen follten. So burfte man ben beflugin,

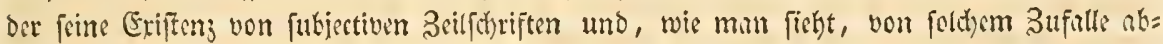

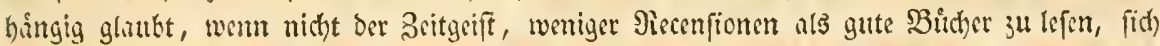

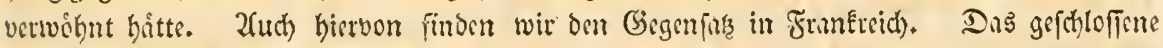
Bulletin von Ferussac, in weldyem bie beutichen Sdriftin neue unt munchmal gon; curiofe 2futoren und Iitel erbielten, und bie trefflicten Annales des sciences naturelles von Brongniart, Guillemin uno Decaisne nefmen parthcilos das grumjolifdye auf, von

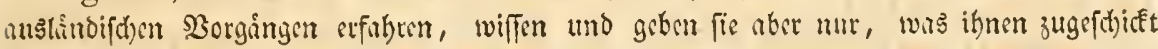
wird, und balten fich oft mur an cimen Botinter in Deutfdhland, unbekimmert, ob nod)

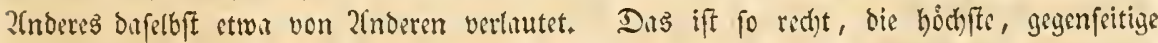

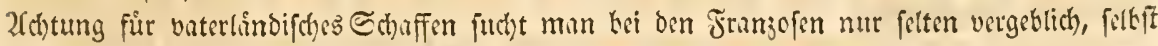
Sppofitionen rourzeln in Datertandsliebe. Der Franjos und Sd)weikęer Eennt und lieft fein Baterhand, wie "'eine Ration bus ibrige liebt.

Die objectiven Aeitfdriften find bumaner, fie bulten fid mikr an bie Suche. Die am meifen unpartbeiifhen, rein objectiv= Gummen Bsitfchriften für Botanie, ober 
Tie cinfoliefend, find in Seutfoluno bie Akten der liaiserlich Le opoldinischCarolinischen $\Lambda$ kademie der Naturforscher - sin mibthaft fariferlides Birmict,tní, und bic Flora boer allgemeine botanische Zeitung der Königlieh bayersehen botanischen Gesellsehaft in Regensburg. Wir glauben anci) Wiegmann's Arehiv für Naturgeschichte auf biefer trefflithen,

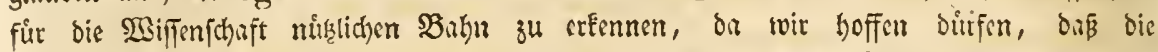

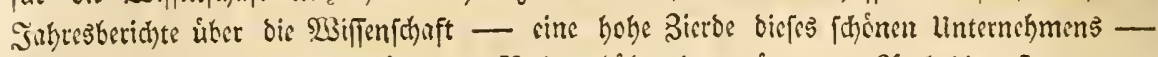

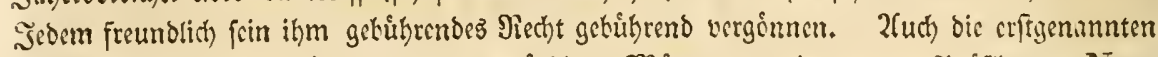

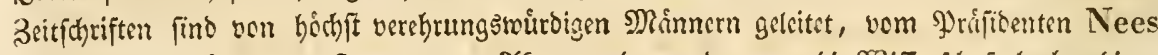

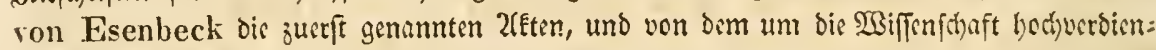
ten গु)rof. Hoppe und dem geiftrcichen Dr. Fürnohr die jwsite, die Flora.

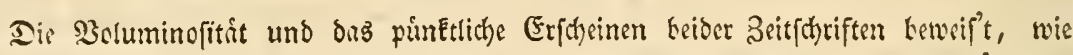

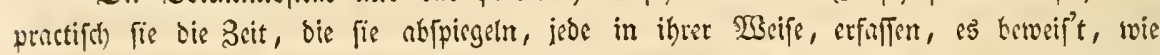

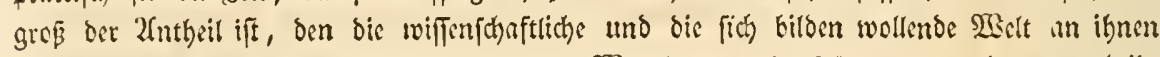

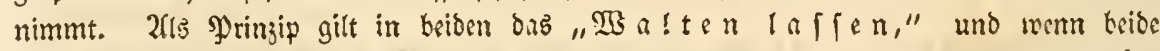

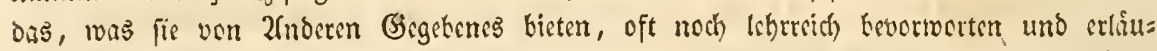
terno cinfithen, in bie Peflerionen der Beit, fo ming in ber sinen and dots (Einutten gern gefehen, und es ming freundich erfannt werden, wie fie cine Sct)ule murbe, burch

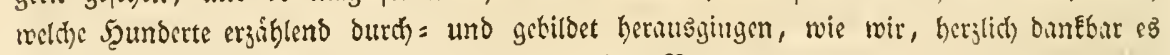
cmpfindend, in wenigen 2 Borten auf bem zmeiten Blatte unferer Flora gemanica, diés

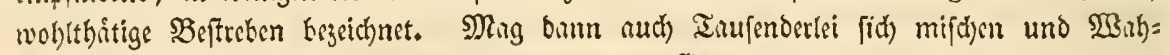
res und Falides begegnen, immer theikt boch ber Gerwim an Shjectivem ein grober und wather.

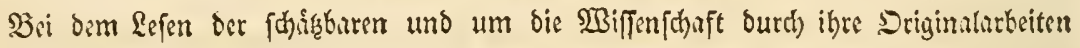

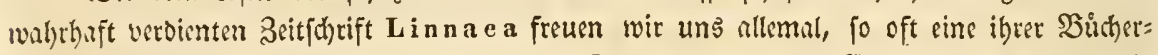

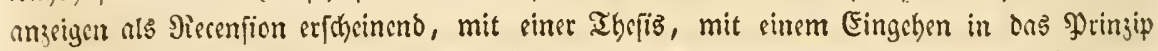

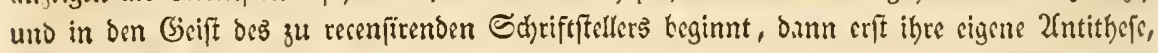

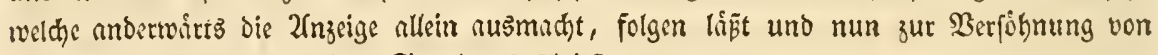
Shiect und Subjest mit einer Syntheje bejhliest.

$23 e n n$ aber Dierbach's Repertorium uno Pressl's Repertorium nicht etwa burch andermeitige, oringende Bepchaftigungen bicfer alleroings immer ribmlid thitigen Berfafer,

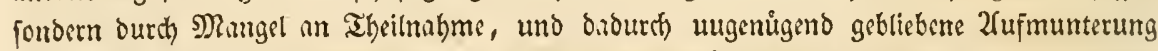

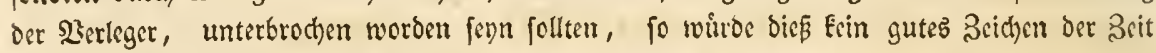
fern, benn beide Unternebmungen waren, wie in ifren Pringipien, fo audh in ifren Ien=

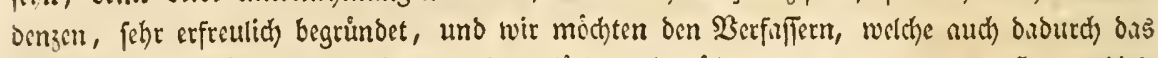

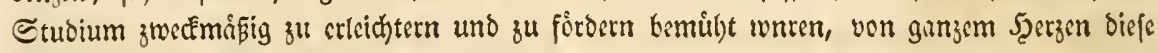
2fufmunterung twinfthen.

(Es ift num aber in ber That, wenn wir nod) sinmal sinen leşten Blide auf ots

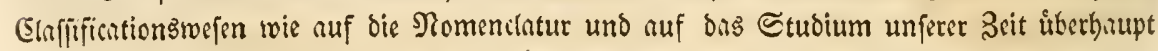

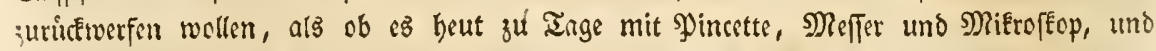

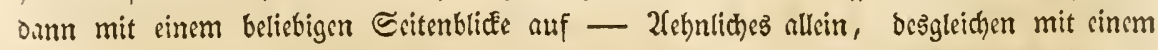

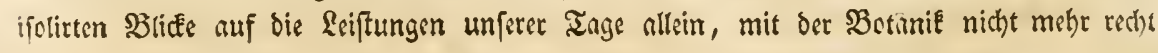




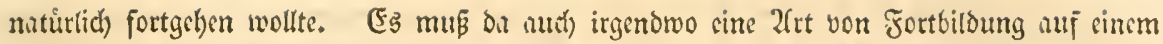

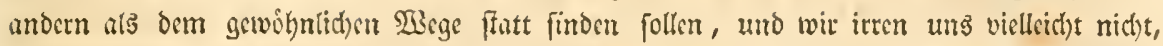

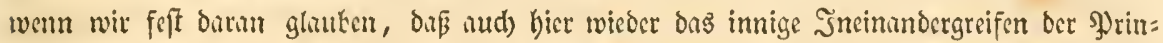
zipien Linnée's, Jussieu's uno Göthe's, den befferen $23 \mathrm{cg}$ unt in Frieden geeknct.

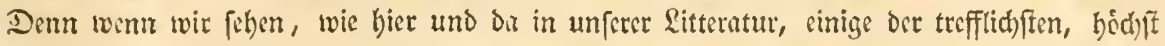

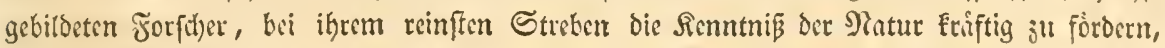
thititig und ruffig fothufferd, dennod) zu febr im Formellen gefeffelt, das practifd =lehendige

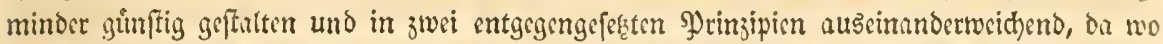
dis Natur bie Einbciten will, fich im 3ertbeilen gefillen, oa wo fie alle Feffern ju jerfpren=

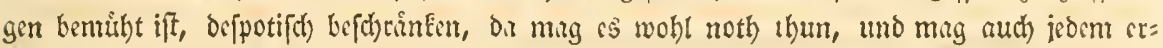

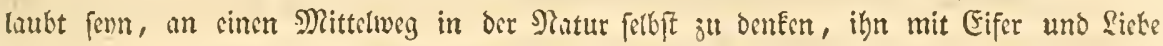
ju futchen, und Eraftig und thitig mitjutvirEen, um iłn fid)er ju finden.

Das iff abereben ber hobe Gegen ber beutiden Metamorphofe,

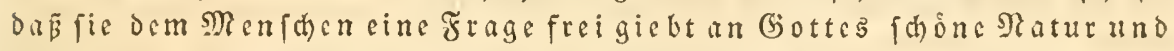

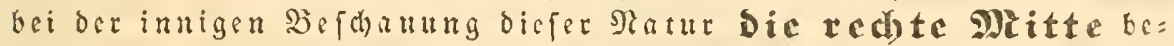
wahrt. - Darum dic Morte in Egren:

„Unfre Nutter beist Natur,

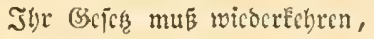

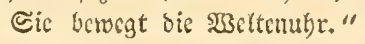

\section{Sachball ber Statitheie.}

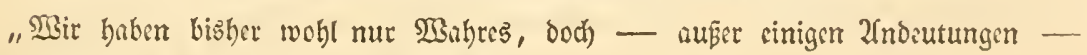
nid)ţ Nicues, nur uns lingft bek̂nntes gelefen.

Mir fonnen uns bietoutd) nod) nid)t bewogen finden, umfere Şoffmung ouf bus In: pentwefen zu fersell, uno geftebert wirderbolt, wie wir Eraft unjeres 2fmtez uno Berufz, dic wabre, pofitive Sififenf(b)aftlid)Esit aufred)t ju erbalter und barum fo alte, nad) bundert

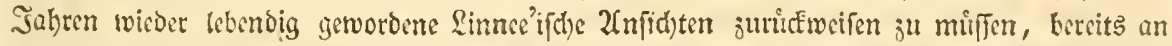

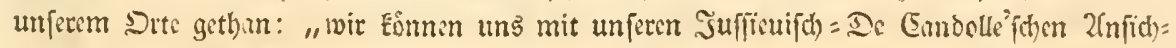
ten nicht binein finden."

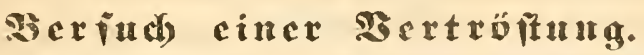

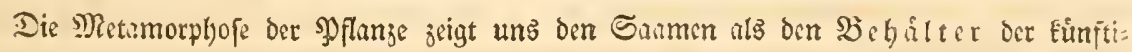
gen Şflanze.

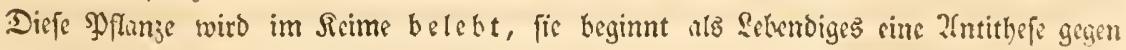
jene Eriften; zu verfolgen, fie twith it.

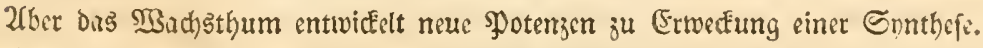

Dire Snnthefe erblúbt, fruber Dageroejenes twiederbolend, Folgandes in fid uer= [d) melicns.

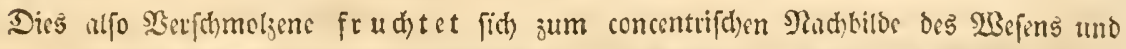

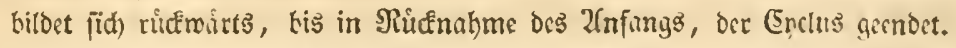

Sene Mlomente fins ater, wie fur bas (Sanze gemeinfdiftlich, fo audh jedes in fict) fel=

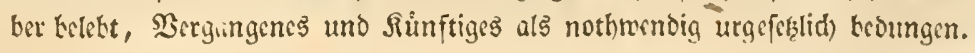




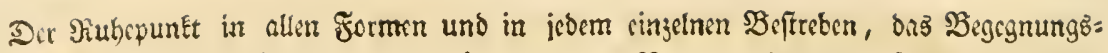
.moment von Sicoerem uno Şiherem, von నergangenbeit uno 3ufunft - als Begrenjung demnad) im Seginnen des Micberen: bas erfte Moment des Beginnents

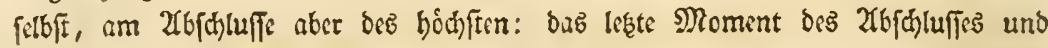

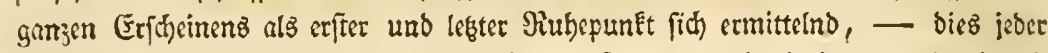

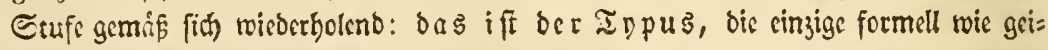
fitig erfaperere Erfocinung in ber Natur.

\section{3wciter Rabhall ber Intitbefe.}

"Dab ming fhon beffer Elingen, wir birten fhon ben Begriff $\mathfrak{E}$ egrenzung, und hoffen biraub nod) ouf Stabilitit.

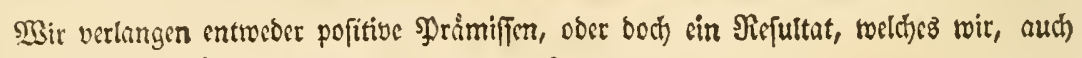
in unferer Beife geprift, als ein pofitives crfennen."

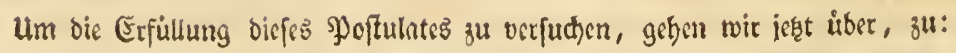

IIT. Synthesis. SEerfdmelzung ofr gegenfeitigen $\mathfrak{X} u$ d a u ung zur Me: tb 0 Dof. 


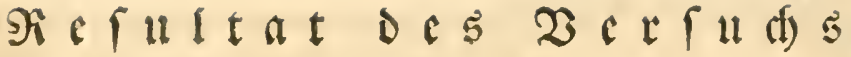

3น

Fortbilsung Scr Metarmorphoic

alह

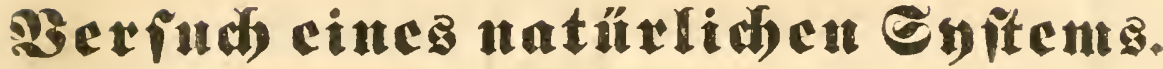

\section{I. Ėinleitende}

\section{AA) Thesis:}

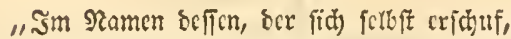

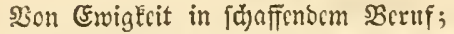
Wn feinem Namen, ser ben Glauben forlfft, Sertrauen, Riebe, IJätigfeit und $\mathfrak{i n a f t}$, In icnes Ramen, ber, fo oft genannt,

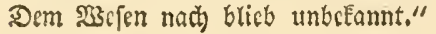

(5.)

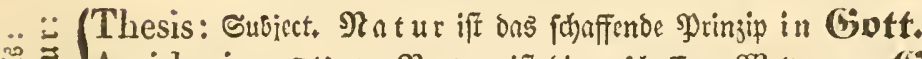

Antithesis: Dbject. $\Re$ atur iff bie erforffene Melt yon Gott.

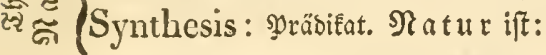

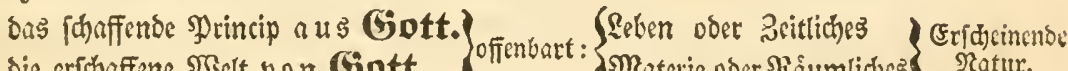

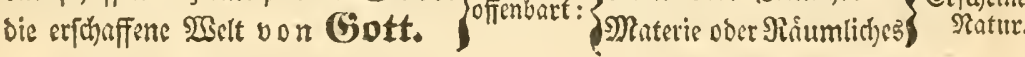

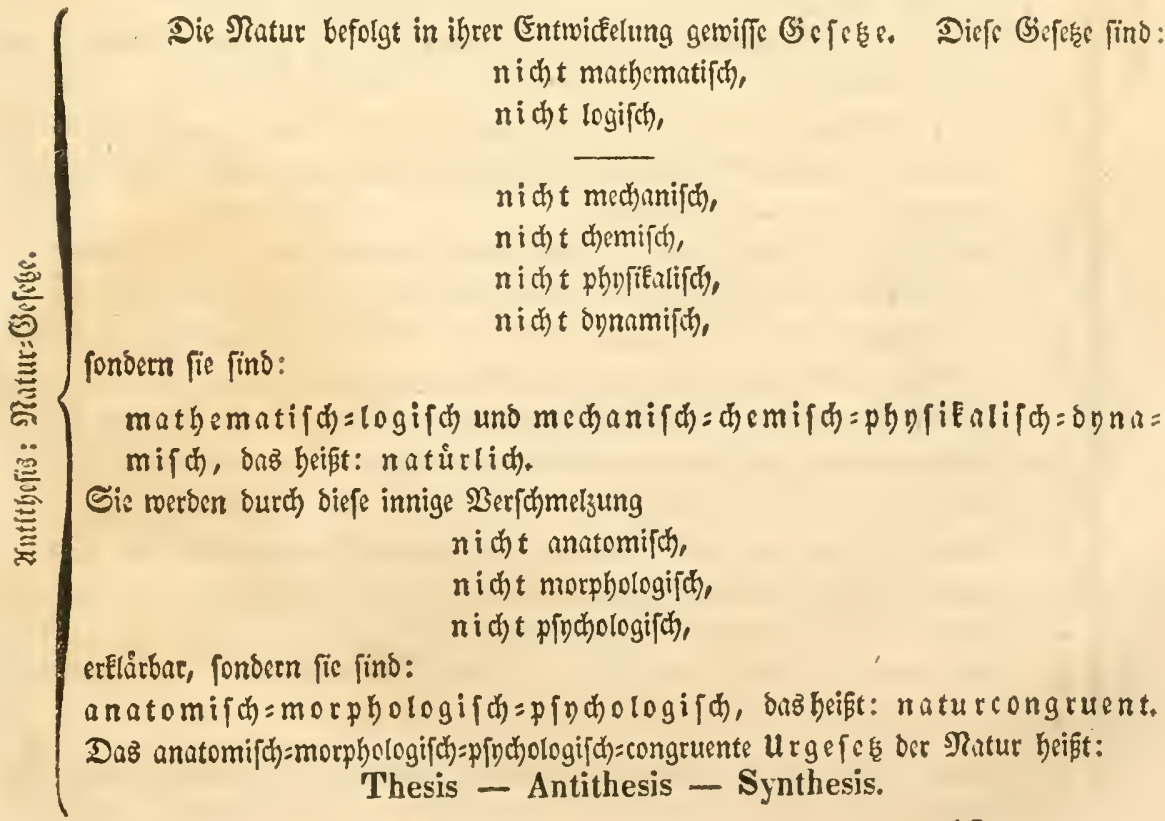


Dis $\mathfrak{R}$ atur hat ihre Bafie oder:

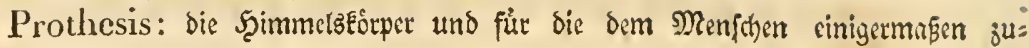
gingliche Natur - Den Fro Érper in feinem Gefiuge, feiner Geftilltung und

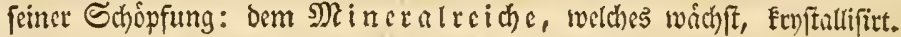

2uf biefer rubenden und fich rud = und umbidenden, fortwatefenden Bafis lebt bie organifde ßelt:

Syftem iff ber nothwenbige 3ufammenbang eines Gsanzen burch deffen Mefentliches, unter natiutichen und naturcongruenten (Sepesen bedungen.

Das $2 \mathfrak{B}$ efentlide ber SPflumbe ift materiell und lebendig zugleith, denn

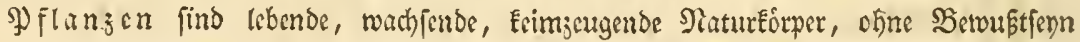

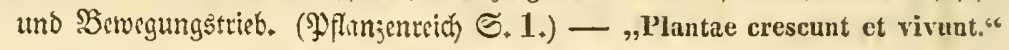
Metbode iff bie formelle 2turseinanderlegung bes Suftems.

Snifem und feine $\mathfrak{N e t h o d e}_{\text {ift }}$ entweder:

Eunftict): auf materielle (b. b. ráumlicte) Momente, ober

naturlid): auf materielle und lebendige (b. h. zeitlide) Momente ju= gleich) begrưndet.*)

(3) emifdte Syfteme find logifd undenktar. 2flle bafü ausgegebenen, fo wie alle "matiulich" genamnten, ofne Folgeleiftung ber zeitlichen Entridfelungs" momente, find - flinfticlid.

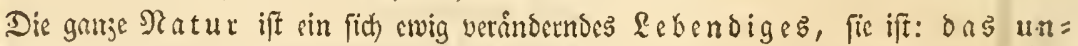
veranderliche Prinzip ber veranterlichen Erfdecinung.

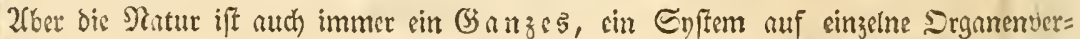

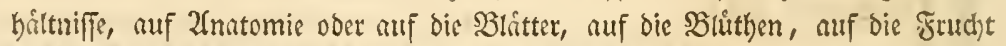

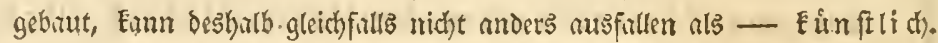

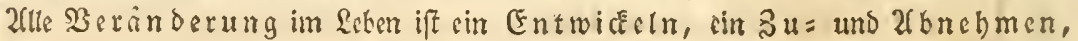

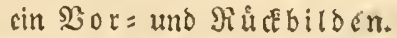

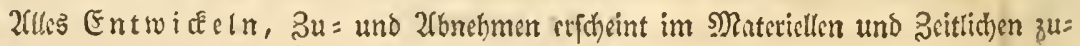
gleich) kedungen, 'es verfolgt gewiffe Inpen und Etadien.

Die Iypen find die erftreteten SiuhepunEte der Studin.

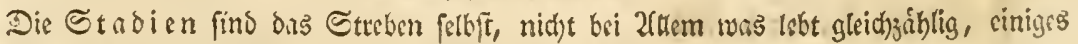

*) Diefen cinzigen roabren uno baltbaren unteriduico gab ich aud in meiner um=

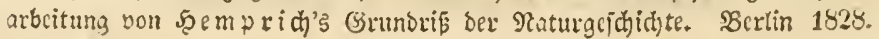


Lebendige zsigt mur wenige, anderes mebrexe Ctrbion oder Entwifelungs: frufen, in Finer (Erfd)cinung im Retern.

Meniger ober mehr Stidien in ber Entwickelung und in beren Erfosinung be= bingen die relativ minder doer mehr gefteigerte Bollendung.

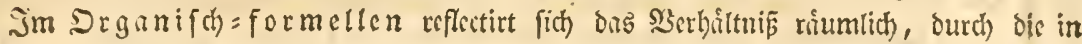
gleichen Sielutionen zugleich mit oen \&ebenserfdeinungen vermebrten un= gleichnumigen Stgane. 2flles cinzelne muß cinmal vorwalten. 2flles an fincm, it)m getrithrenden SPlaf̧e.

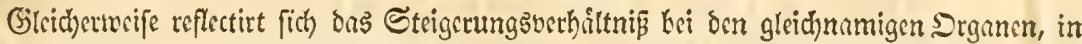
Der ihnen sigenthumlictyen Entwickelung, welche wirber befondern, in ibrem

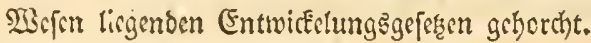

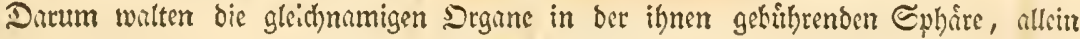
vor, olyne bubei ciner Seitenbegiebung auf bie ungleichnamigen verluftig ju weroen.

So bedingt fid) der Begriff cincs Niebern und Ş ó heren.

Das "natur $\mathrm{rli}$ (d)

1) in Elarem und nothwenbigen Jufunmenhange fehcat, mit bem Univerfum ber

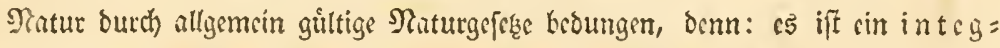

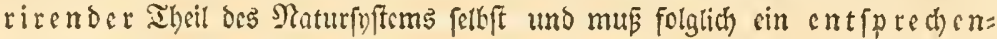
ber Theil fenn. - Ės mur

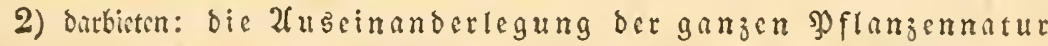
in ber Gefammtheit ihres Materiellen und Lebendigen gleich.

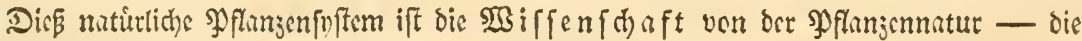
bobere Sotanif falbft.

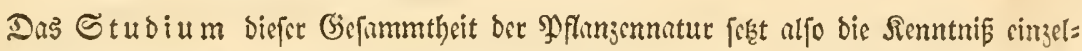

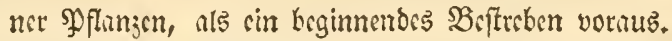

Die Sinofpe ber 2 Biffenfohaft war, ift und bleibt - bie $\Omega u n f t$.

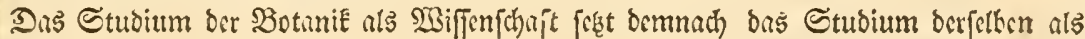
Sunft voraus.

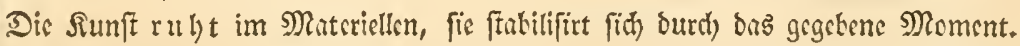

Dus Fimftiche SPflungenfyftem beroabrt und bietet gegchene ftubilifite No= mente.

Der gelungenfte $\mathfrak{B e r f u c h}$ bicfer Stubitifation aus nllen 3siten und furt alle 3citen, ift - bas Rinnee'ifde Serualipftem.

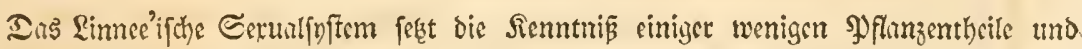

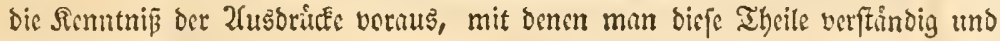
verffindich bezeidinet.

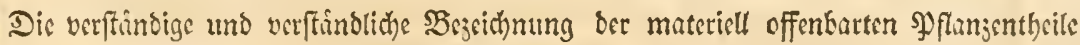
ift bie Rinnee'ijate Ierminologie.

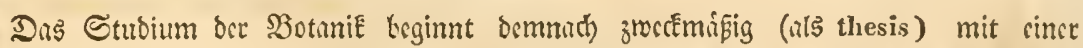

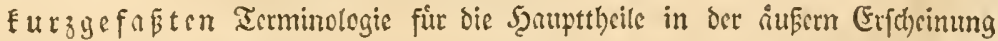

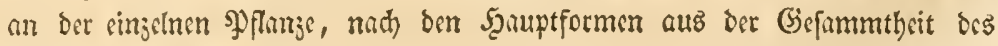
গicidys. 


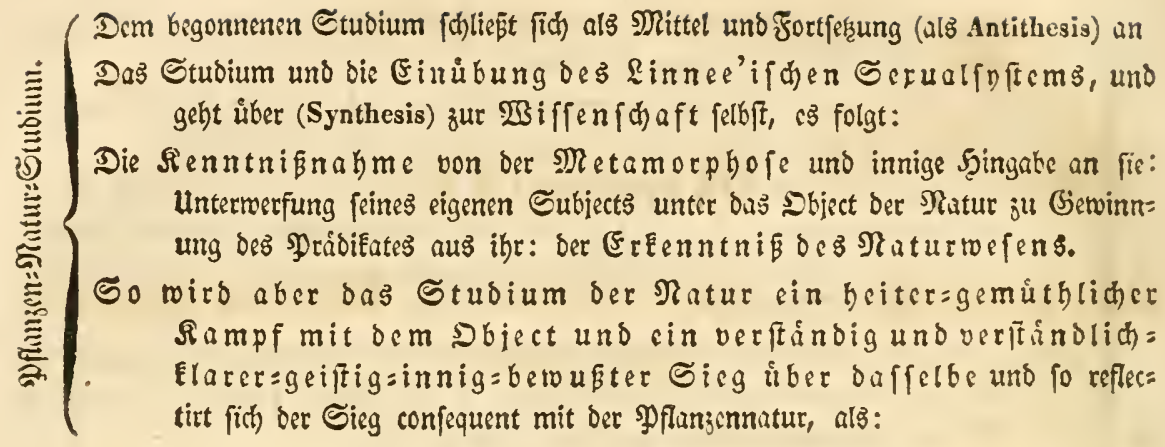


BB) Antithesis: Methosifidyes.

\section{3) tait.}

\section{Res herbaria - Phytognosia - Historia naturalis plantarum.}

2115:

Thesis: Fornt.

Antithesis: MZethode.

Synthesis: Reben.

A) Thesis: futzgefapte, vortecritente, Sinnceiffhe Terminologie furt:

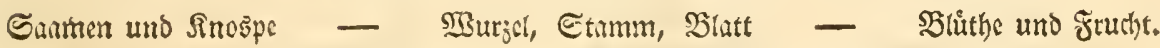

B) Antithesis: Rimme's Serumlintrom:

Sonftruction - Zinoronung und Einutbung - Girit.

C) Synthesis: Sुำtorganognofir.

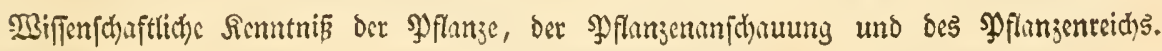

a) Thesis: গু flanj

Individuclte গ̧hytorganognofie oder:

Sghytorganognofe.

Srganomorphie.

\$phntotomic oder

Rnatomic bat Sfllanzen.
Drganographic.

höbere Terminologie und beren

2fnwenoung: S3hytographic.
Srganobiotif.

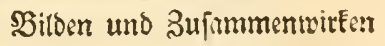
ber Drgume:

Phntophyjiologic und produtt: Şbytod)emic.

b) Antithesis: Methods.

Siteratur. SBibliographie, Gichidtc.

c) Synthesis: פf flanjenrciof.

Generdle \$ghytorganognofie oder

gु $\mathfrak{y}$ to

Drgamonomic.

Entmidelungsgeferfichtsit

im Formclien.

इrganomorphoje

Elaffification.

Evitem und Methods.
Siganoonnamit.

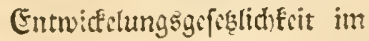
estrnoiaen. 


\section{CC) Synthesis: Reiultat.}

A) Thesis: Bscgcbcnes.

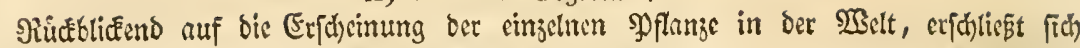

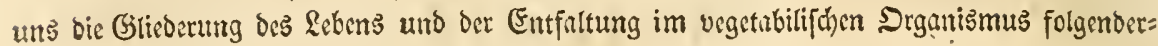

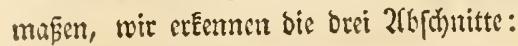

Seimleben, Segetation, Fructification.

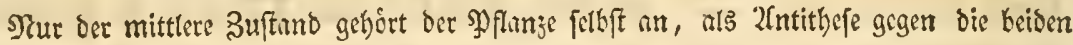
Endjuftande, benn bicfe gehoren als im univerjum ber Natur $\mathfrak{r}$ hende und beruhenbe

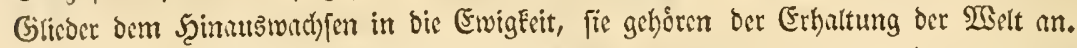

Seber biefer 3uftande begreift aker Stabien in fith, welthe burh orgunifhe Bebilloe reprifentirt merdea. Das Ganje ordnet fith beshalb in ber lebendigen Slatul folgender= māen:

\section{Seimleben \\ oder}

Şorbilbung, Prifor:

mation, als
Segetation

Stockbildung.
Fructification ober

Shluthen = und Fruchtsiloung.

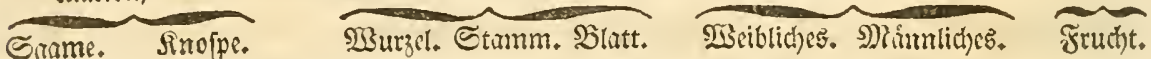

Co bictirt ung aber bic গlatur ben

Cautout fur bas eeben und formelle Erfbcinen oer spflanze:

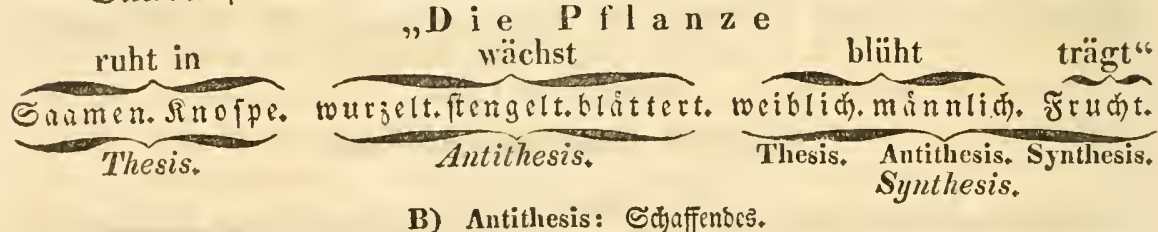

Den von ber Natur unz ferkft bictirten, thetifdyen, objectioen Ganon fiffen roir auf,

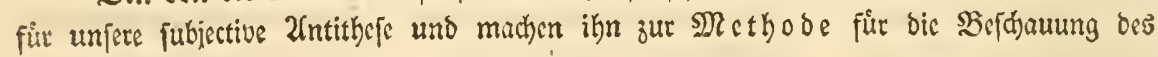
Gianjen.

C) Synthesis: Brroonnenes.

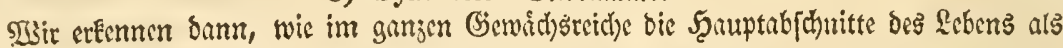

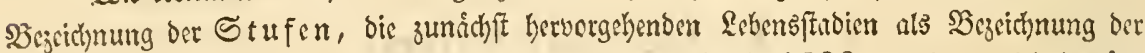
Elaffen fich reflectiren. So entwiefelt fich - wie ich jeit 1822 zu zeigen mich bemitht

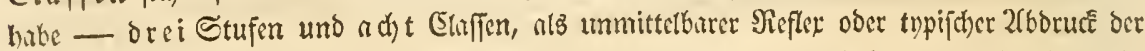

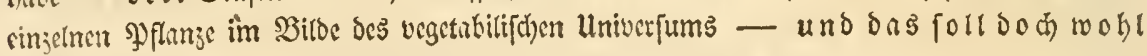
sin $\sigma_{1}$ ftem jeyn? -

I.

Faferpfinnzen.

Inophyta.

Stelechophyta.

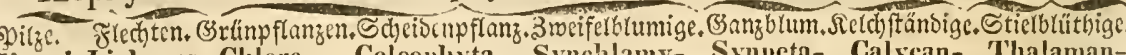
Fungi, Lichenes, Chloro- Coleopliyta, Synchlamy- Synpeta- Calycan- Thalaman-

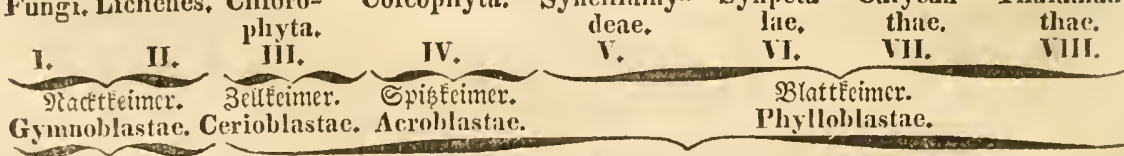

Gymmoblastae. Cerioblastac. Acroblastac.

Silkp fิimรen.

Hemi-Protophyta.
III.

Blutben = Antho-Carpo-phyta. 


\section{Betrachtendes.}

\section{AA. \\ Socules in ser setanotpore.}

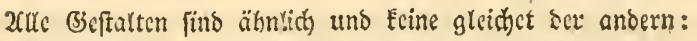

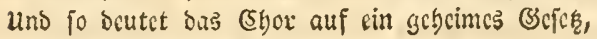

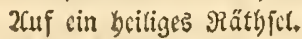

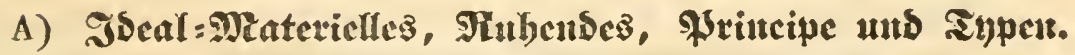

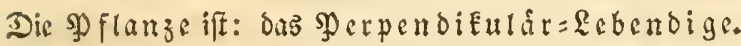

Die Şalbp flanje ift bie organifhe Jugel in ibren Modificutioner.

Principium anandrum.

Der Pुilz ift die in oer Sugelform und Deren Segmenten ftabilifirte P̧flanje, mit innerer 2 (nthithere ats Regel.

Principium protogynum.

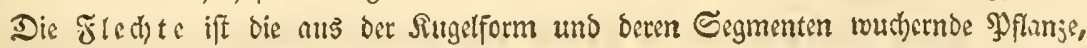
mit nand) ausen geÉefyeter 2(ntithefe.

Principium heterogynum.

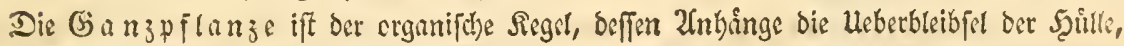
von frinem 2lustritte aus Der UrËugel.

Principiun androgynum.

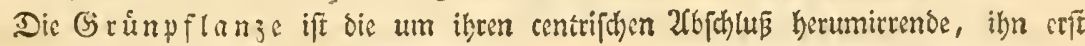
am Ende erreichende Binjpflange.

Principium Amphigynum.

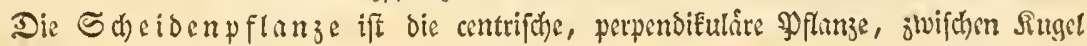
und Reger: Enlinder, die acrogenetifhe Pुflanze. Principium gynandrun.

Die Slattecimende if die nad) unter und oben, nach innen und außen geroad)= fene - amphigenetif(b) \$ुflamge. Principium hermaphroditum.

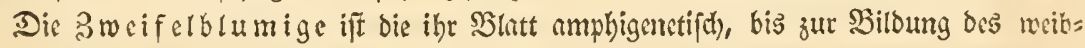

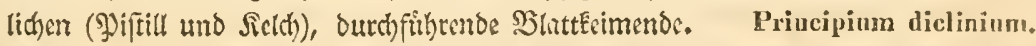

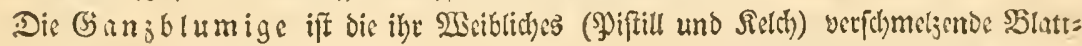
Émenoe.

Principium centrogynum.

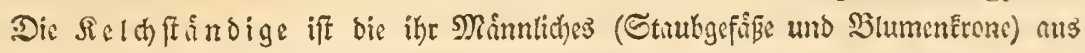
bem vollendet Siseiblichen (Piffill, Seldy) emporhebende 2 lattecimende.

Principiun masculinum.

Die Stielbluthige bat bie Frudbt vom Silthe befreit und wieder von ber (Fin= beit Ourch 3rocizaht und Spaltung wieder zur Einbeit ber fitgelform concentrirt.

Principiun centro-dicho-schizo-inliogynurn.

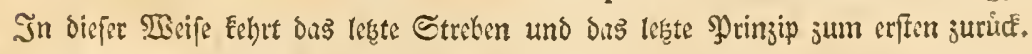

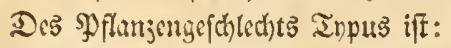

1) Thesis: protogynus; 2) Antithesis: masculinus; 3) Synthesis: her in a proditus. 


\section{B) Iseal = 3eitliches, fich fortbirsenbe Seftrebutgen.}

Die şflange ift: baz im Srbifden wurzelnbsgefeffelte, vom fidte in abnender Spannung erhaltene Rebendige.

Die Şalbpflange ift die in ihrem 2fhnen bem Gemicht bes Sroifhen unterlegene Pflunje.

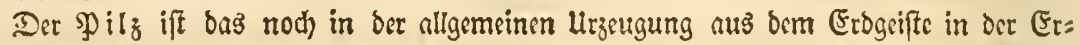

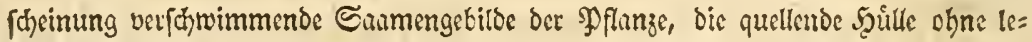
benoigen Sisim.

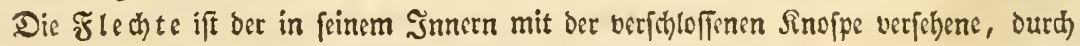

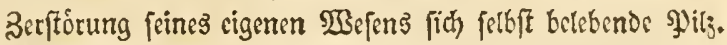

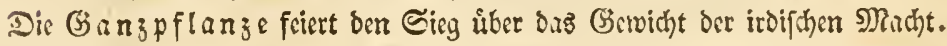

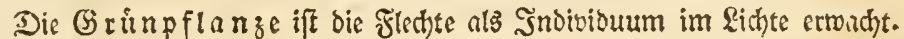

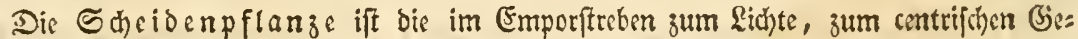

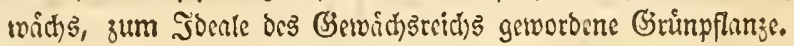

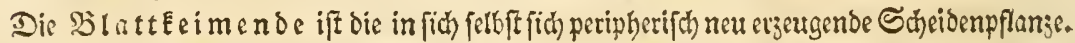
Die 3 weifelblumige ift bie fich felbft nue abnende, an fich felbft verzweifelnoe IBeiblichEcit.

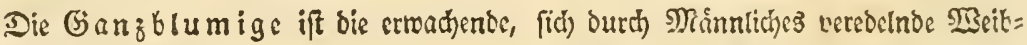
lid)Eerit.

Dii Neldbluthige ift bie mit bem vollendet Minntiden gluctict vermáhlte SBsiblidyét.

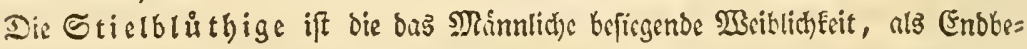

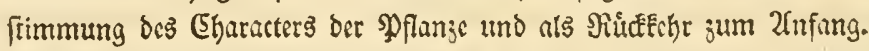

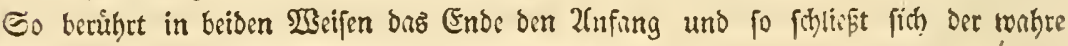

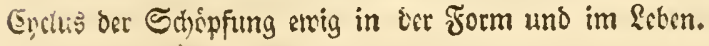




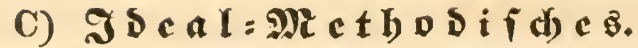

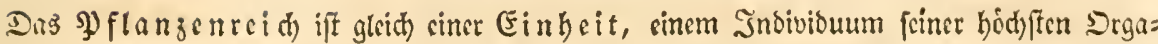
nifationsftufen, cinem immergtunen, inmer blitgenoen, immer fruchtenden Baume ber toirmeren Soni.

Der Saum erfocint

a) materiell, als ráumlides (jebilde im Momente relativer Mollenoung ftabilifirt, alfo formell:

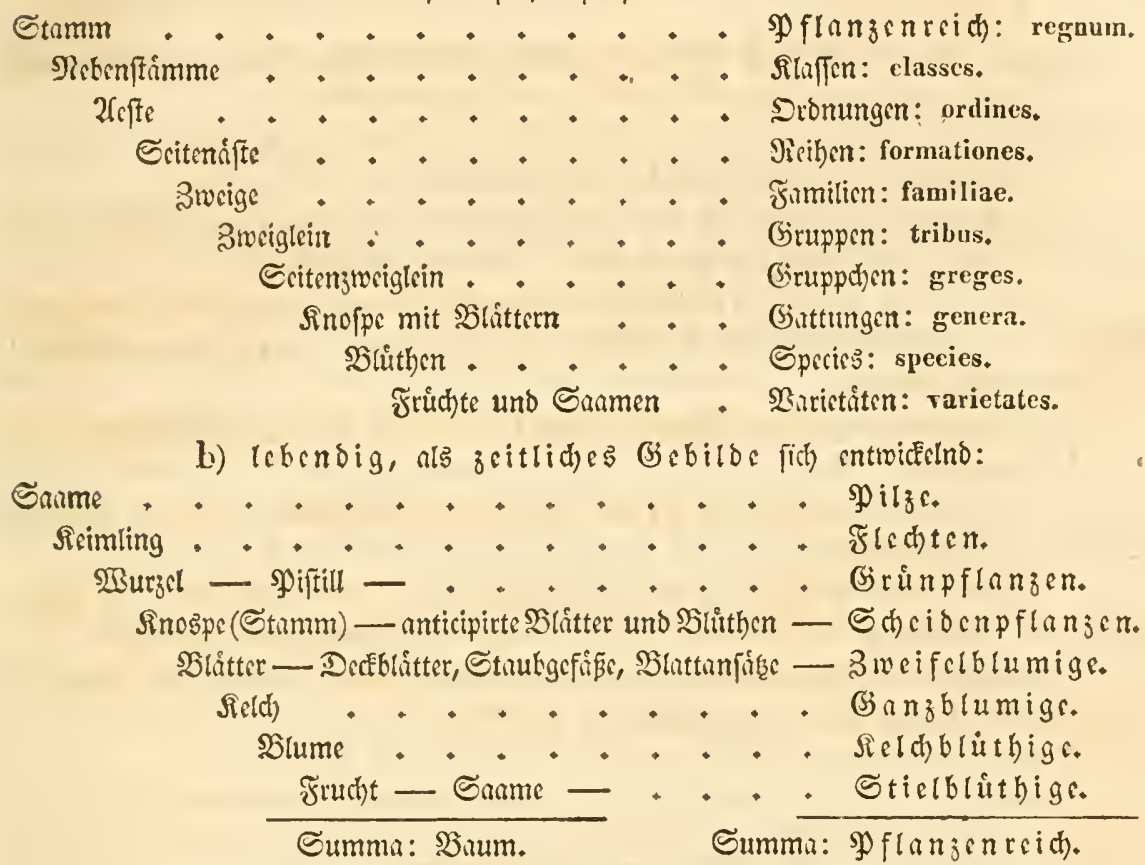

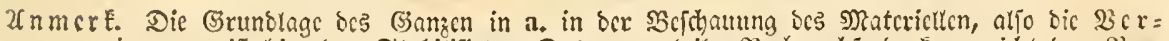

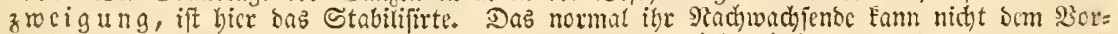

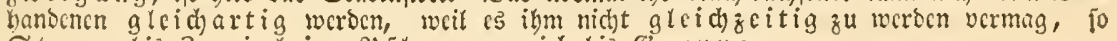

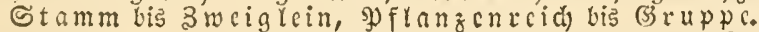

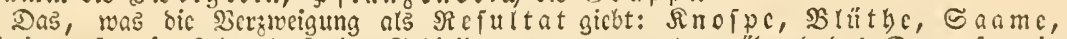

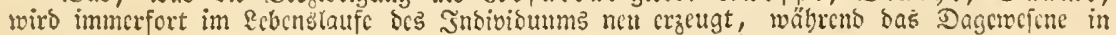

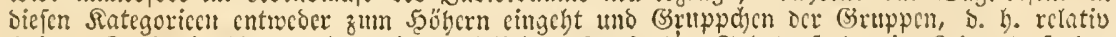

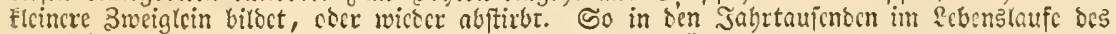
(Sicröd)scrichs dic Sattung, Spccifs und 2 arictät.

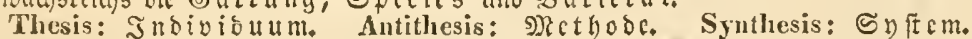

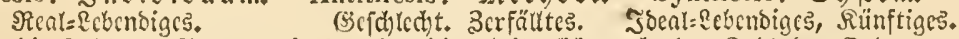

2) Gchon bic 3abl ber (3ruppen in ser Famitie theilt fich nach ber 3abl Der Drommgen ber (Slajle, ba fie bicfe Sronungen ganz naturgemä̈s wicberbolen, folglich in fich felbft cinc Thesis, Antithesis uno Syuthesis in ocr Entwicelung ocr Familie nachrocijen, uno bicfon ur= gefere dor Statur folgen minfen.

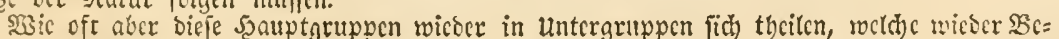

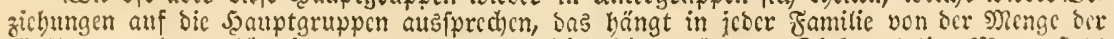

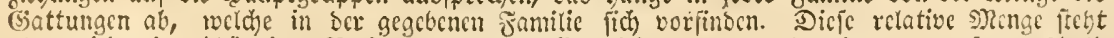

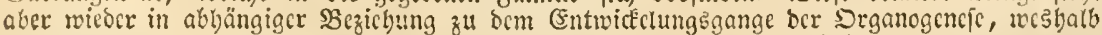
bie meiften (3attungen ba find, wo bic widitigften Spomente fich surchbilocn.

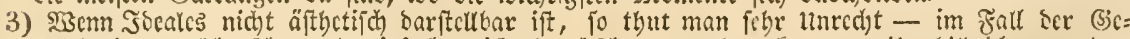

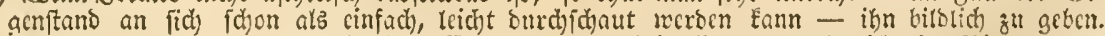

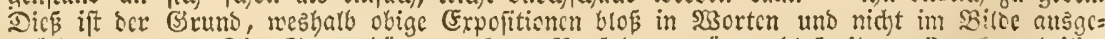

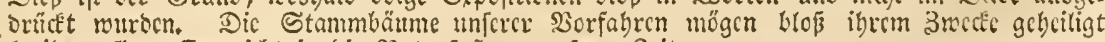
bleiben, fie paffen nidyt in bif SRaturfyfteme unferer 3eit. 
c) Urithmetifd = Soealez outd) bie organifden Saflen.

\section{1) Sn Form und \&eben.}

Ein5. - Einfind ift alles Beginnen.

$\mathfrak{B}$ eiblid.

Thesis: Der ionale PJunEt verÉorpert fich als Sugel.

Beifpicl: Reimpunft, Caame, spily.

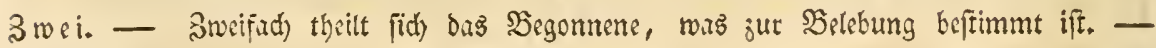

Minnlich.

Antithesis: Der Pुunft wird jur Rinie, bie Nugel jur Epindel, Die Ringendimenfion crmedft juci ஒoole.

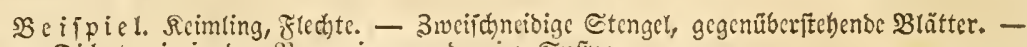
Didjotomie in ber șerkroeigung ober im Enfrem.

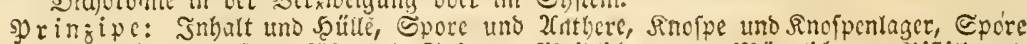

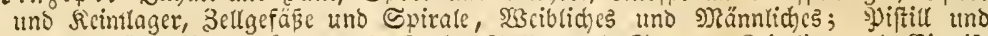

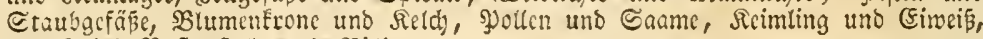
NarE und SBaft, Scolz und Ritribs.

Drei. - Drcifuch gefituttet fich das Reben.

Sind!ich.

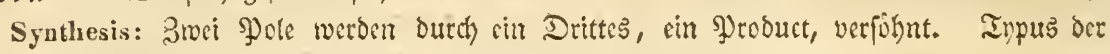

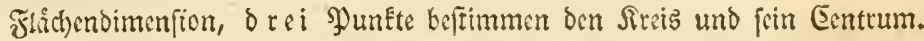

Scifpicl: Inofpe, 2rnthcre uno Epore in Scr (3rtinpflan;s. - Rormzabl ber Echei= Denpflanzen in Etengelgeftaltung, SBlattffellung, SBlüthe uno Frud)t. - Trichoto:

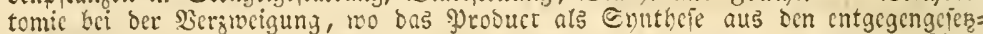

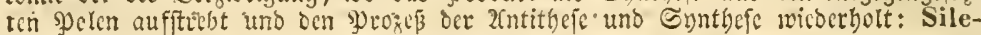
ne nemoralis. Gbenio als Reflex im Evftene.

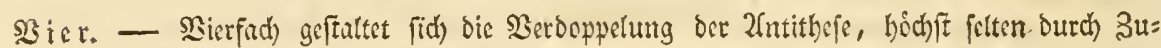
rechnumg einer Einbrit zur Jerizabl. - Doppeltsantitbetifd. - Wird

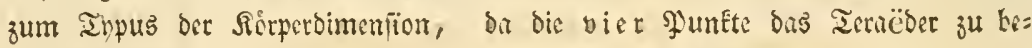
ftimmen vermógen, vetharrt ater meift im Suirl oer Jlíd)e.

Scifpiel: Sporen ber Agaricinae, (nad Ascherson's Bcobactung) Jungcrman=

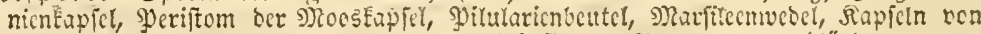
Gleichenia, Platyzoma, log. Untberen bei Cycas, Yjotamogetencnblütbe 2c.

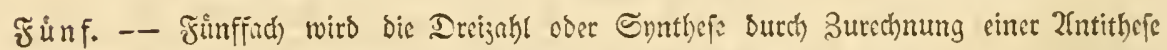

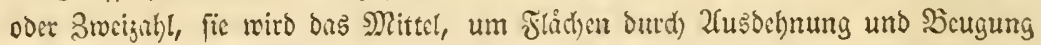

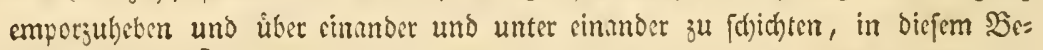
ftreben wird buta) fie bie Epiralberwegung beoungen, ourd) orren momentanes

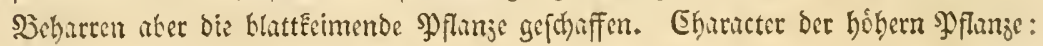
Doppelweiblid $=\operatorname{minn}$ (id).

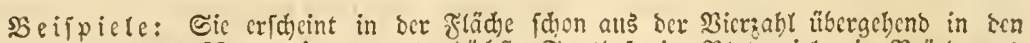
Siapfeln von Mertensia, - alb bödite Énnthefe in Blattquirl, in Stüthe uns Frud)t der SBlattecimer.

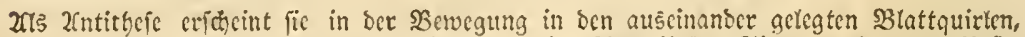

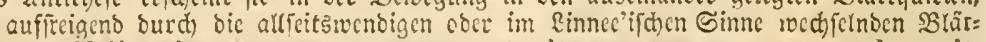
ter: Folia alterna, qunm unm post alterum tanquam per gradus exit, - sparsa, quum sine ordine copiosa. -

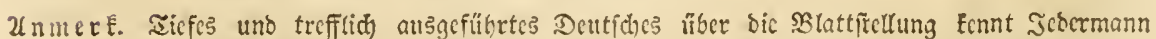

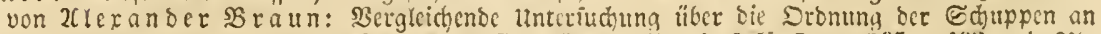
den Zamnenkepfen u. F. to. Nov, Acta Soc. Leop. Carol, IV. 1. p. 195-402. mit $2(b=$ bilb. - So wie in ber ticf grünbliden, äd)t beutjoben 2frbsit von Martius tIber, , lie Erivcanleac ${ }^{6}$ N. Act. Soc, Leop. Carol. XVII. 1. p, 1-72, wo ber Berfaffer, obne cs zu merten uno zu benterfen, S.70. gan; genau unjerer nadh cigener Unterfuchung im Jabre 1028 conspect. gegebcnen Etclung ber Lriocauleae gefolgt ift. 
c) 2ritbmetifh: Socales ourd bie orgunifoen 3ablen.

2) in ber Erpofition des Enftems oder der Methode.

aa) Matiurtiche Sirie:

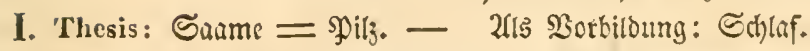

Antithesis: Inofpe $=$ Flechte. - 2tל belebtares. 2llp:

Samin : Sinofpe $=$ Sুil $:$ Flechte.

11. Thesis: $\mathfrak{B u r z c l}=$ Grumplanze. -21 2 Leben=Beginnenocs.

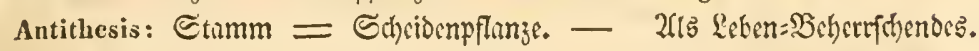

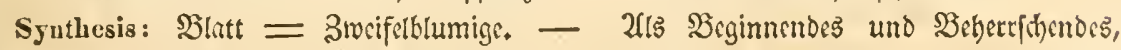
Ecben=నcreinigcudes. 2lipo:

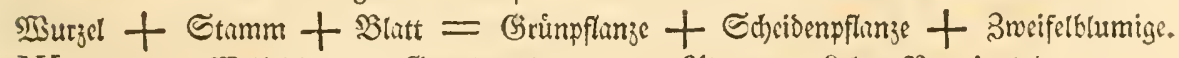

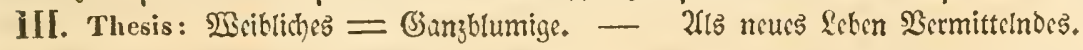

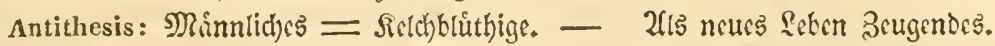

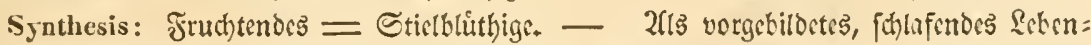

umfaffendes. Das Ende nimmt den 2(nfang wicoer anf. 2lipo:

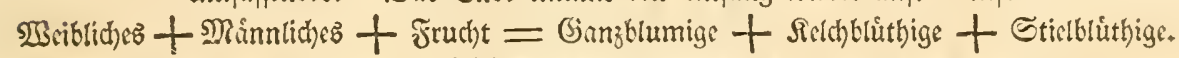
bb) Siunftide Singe:

I. Thesis: Şill.

Antithesis: $\mathfrak{F}(\mathrm{c} d) \mathrm{te}$.

Synthesis: Grimplanje.

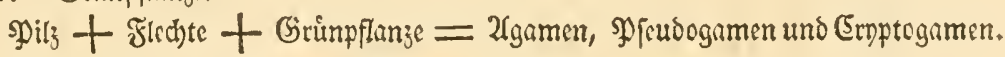

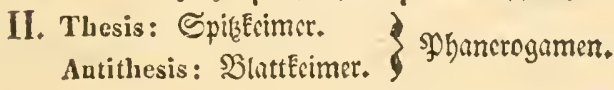

Thesis: 3tweifclblumige.

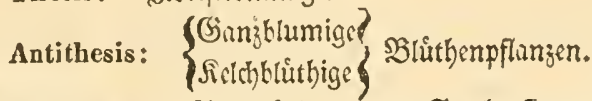

Synthesis: Sticlbluthige $=$ Frudthflanjen.

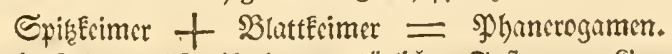

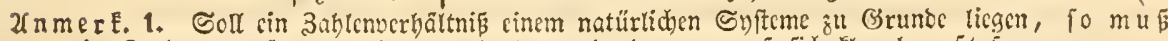

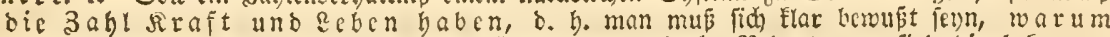
fie barin ftect. Sbfteme mit 3abyenverbältniffen obne foldhe SBcocutung, find bie befannten

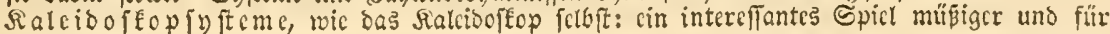
müBige \&eutc. -

2. Sidjt cinmal im Socalen ift cine Irennung von Eryptogam uno sybancrogam in oce lcben= bigen satur benfbar ober juläpig für - unfere 3cit. @ie mar von Linné in je in ev

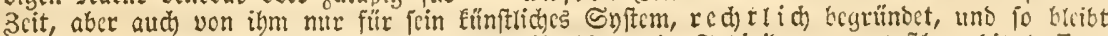
fie aud für bie erften Iage tes botanifden Stubiums im Jnoivituo unantaftbar, bis ballette

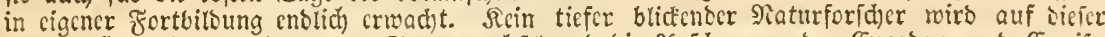

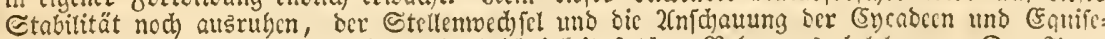

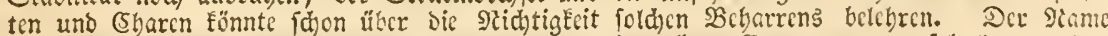

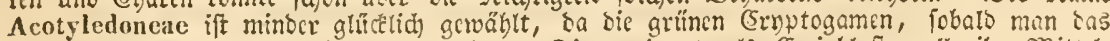

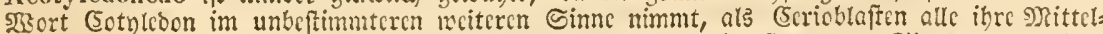
Gebilbe, ihren 3cllecim cocr Cotyledonislium berauggcbin, in fircngeren Sinne abir, jotalo

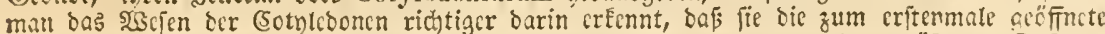

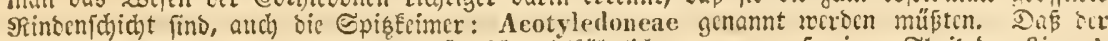

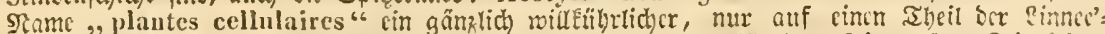

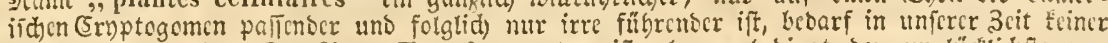
SBiocrlegung mebr. Der Stame Exembryonatae if aber umbeoingt oer unglüctlichfite von

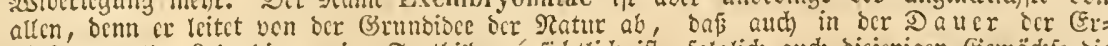
fidcinung alles \&ebenoigen, sine Fortbiloung fidtlid) ift, folglich aud) bicionigen (Semädhfe ti:

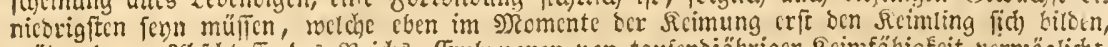

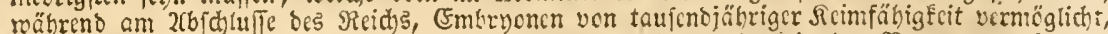

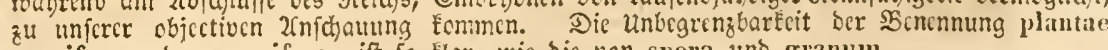
sporiferae obce graniferae ift fo Elar, wis bie von spora und granum. 


\section{III. $\mathfrak{A} \mathfrak{a} \mathfrak{i} \mathfrak{u} \mathfrak{i} \mathfrak{l} \mathfrak{i} \mathfrak{d} \mathfrak{e} \mathfrak{E}$}

\section{BB.}

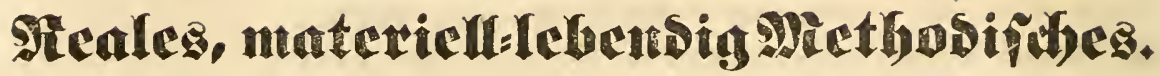

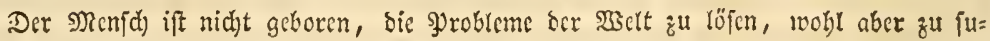
den, wo bas Sy roblem angegt und fid fobant in ber (s)enge ocs segreifliden zu halten. - Die Seandlungen bes uniberiums zu me $\}=$

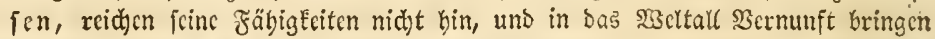

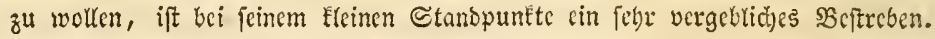

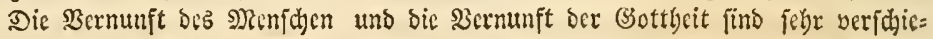
bone Dinge.

\section{A. $\mathfrak{g} \mathfrak{r} \mathfrak{a} \mathfrak{m} \mathfrak{i}\{\lceil\mathfrak{c} \mathfrak{n}$.}

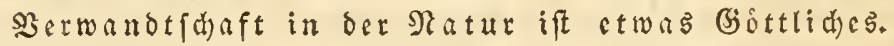

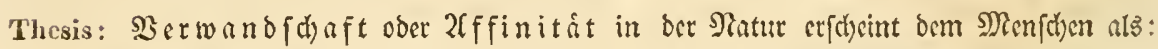

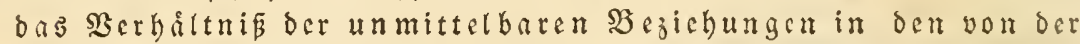
Thesis burch bie Antithesis jur Synthesis fortfdreitenden $\Re$ eiben: , les rapports en avant et en derrière." Jussieu.

Seitenuerwandtfdaft ober (Sonfinitat (2̂nalogic) srfdyeint dem Menjhen ats

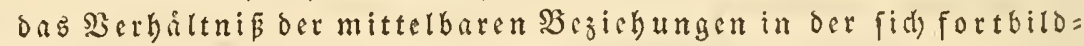

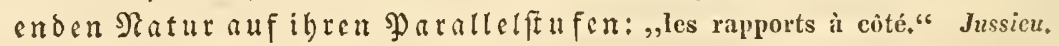

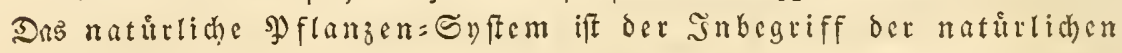
Bermandthaften nad beren rofumlicher und jeitlicher Entwi= afelung.

Antithesis: bas natůrliche Syftem ift nicht $\delta_{a j u} \delta_{a}$, um bic matcriellen 2(njangs: grimbe oor Botanie aus ifm zu erternem, fondern um bie griondliche (Er= lernung berfelben burd) bas maturtidye Syftem in bie lebendige Natur itberzutrigin und zu begriftigen.

Limnée's Gerualfyftem iff die beste, eriffirende 2futithefe gegen bas natiutitye Syftem,

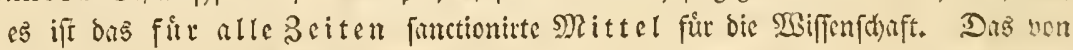
Limnée geabnete, lebendige, natůrlidye Syftem iff ber J

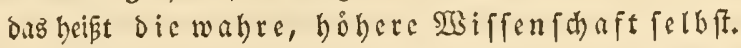

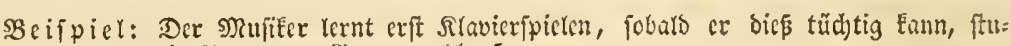
birt er mit Suken - Seneralbaß.

Synthesis: Die jufinftige Scit mag barmf binarbiten nod sine Drganogencfe ju

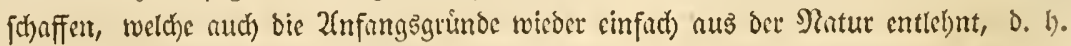
nid)t nus ber Mitte ober von binten beginnt und bann, von zu vielen Menfohen=

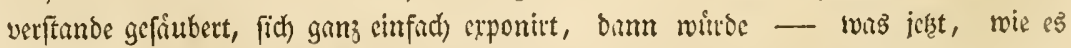

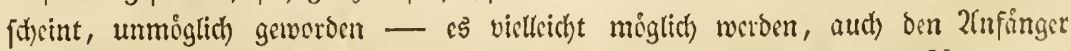

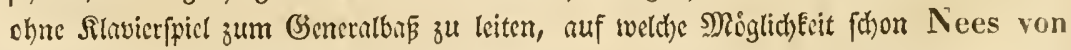
Esenbek's Şandbuch binleiten fơmte. 
Uhnung. Der geredte und geiftecide Biograph Jussicu's, in ocr Wugsburger allgentinen

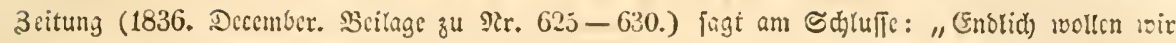

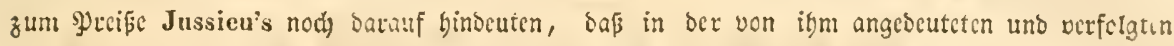

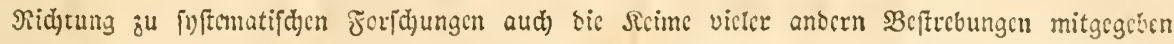

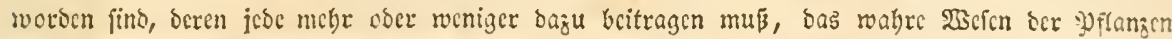

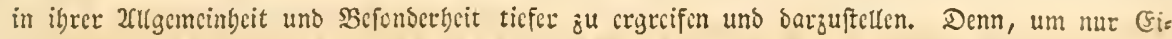
nes ou bemerten, to wie in Jussicu's Beifte bic Joce bes 2enalogen, oç Befreunecten

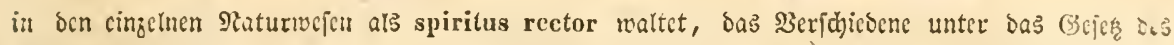

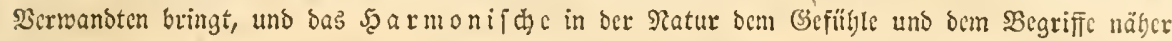

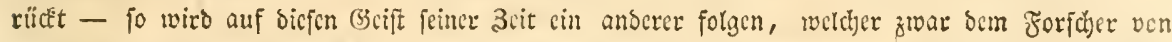

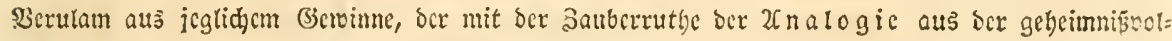

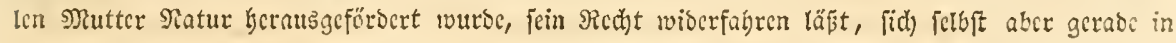
Scr intgegengefeften Rid)tung beivegt, und nidjt bas 2(naloge, Fondern bas Disparate in tem

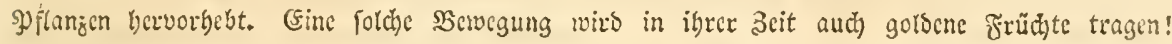

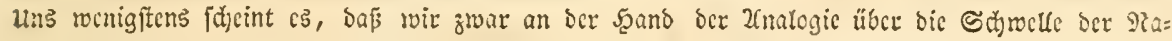

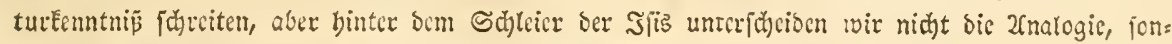

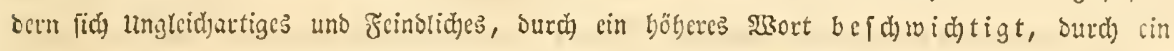
mäd)tiges Sanb nadburlid) berubigt." - W3ir täufchen uns wablicheintid nicht, menn mir

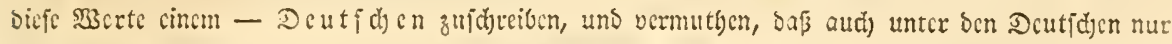

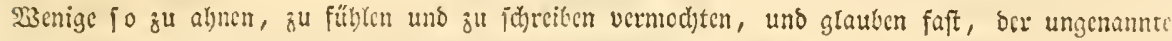

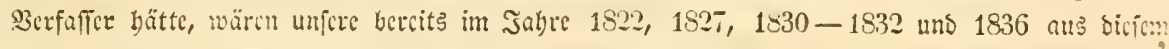

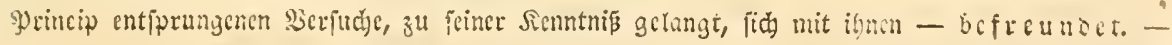

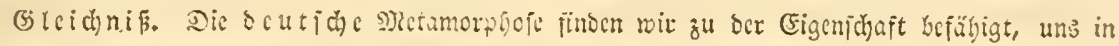

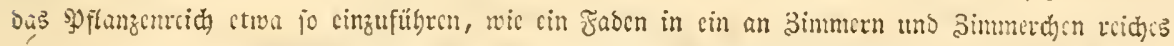

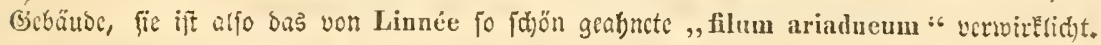

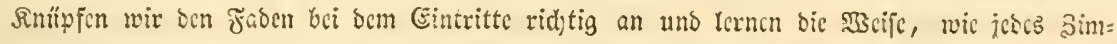

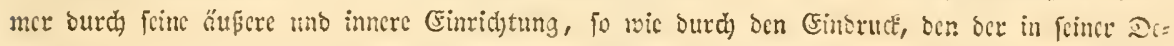

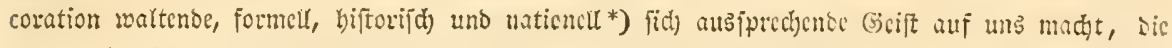

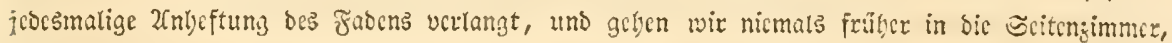

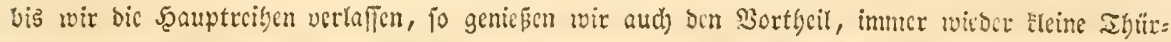

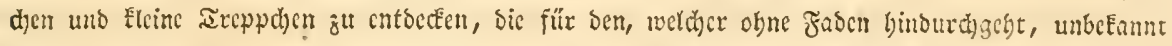

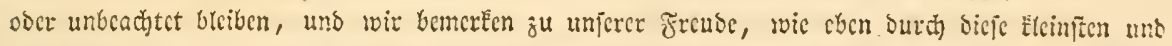

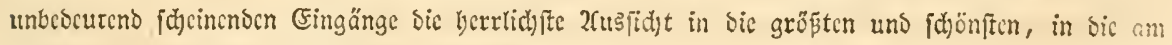

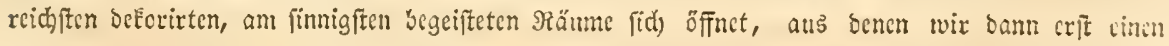

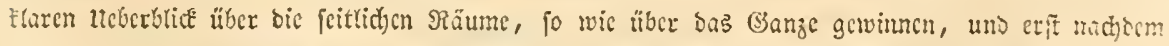

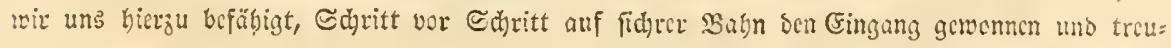

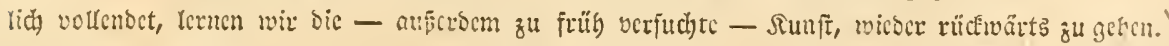

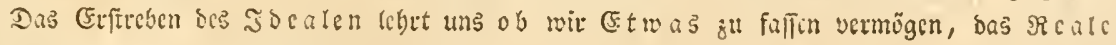
wiro bam bas zu foffende fetbft fey)t.

*) Diutct aljo auf Gectritung, Eeben und geograpbifdge Berbreitung bci bcr Wyflangcufamilic. 


\section{B. Er \\ अ by to ne te.}

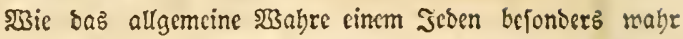
gerworbon, muß man von ifm ferbft erfabren. (5.

Die Tnpen der Pflunje im Romente oer Reimung erfakt.

\section{Protothesis: \\ I. Nactifeimer: \\ Gymnoblastae.}

⿰מilze und FIlechten, oder

Fraferplanjen:

Inophyta.
Rebendigez oder 3eitlidhes. Inpen.

\section{Inpen.}

Thesis: Maffenftoff, Urz̧cle. (JicimÉrndhen.) Thesis: UrEeimmafle fondert fich.

Antithesis: verlingert.

Antithesis: wird von Den Elementen zum Reben gejogen, momentan ftabilifitt, dounn politifitrt.

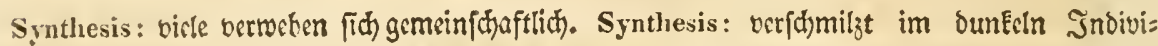

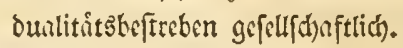

Thesis:

II. Bellfeiner:

Cerioblastae.

Grimpflangen:

C h 1 o r o p h y $\mathrm{t}$ a.

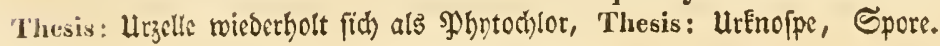

bei ber Reimung berftende Sdyale (mit

(d)on orgunificher Fullung).

Antithesis: Beflgcbilde aus Phytodjlor.

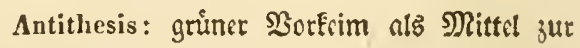
Indiviburlitit.

Synthesis: aus dem 3ellgebilde bie Inofpe. Synthesis: Indivibum.

Antithesis:

III. Epitzfeiuter:

Acroblastae.

\section{Scheibenplanzen:}

Coleophyta.

Thesis: Raimling und Eimsí, boppelfhali= 'Thesis: polare Feimung.

ger Saame.

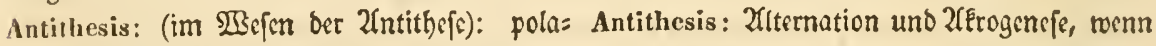

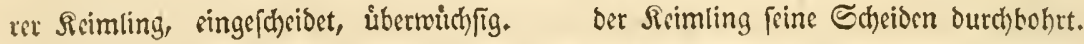

Synchesis: Snoten unb Sinoten ůbereinanber. Synthesis: 2fErogenefis uno 2fmphigenefï in Silutbe und Frucht vermihlt.

\begin{tabular}{|c|c|c|}
\hline sis: & IV. PIatffeincr & Phyllo \\
\hline $\begin{array}{l}\text { Srocifclb?umige, } \\
\text { Synchlanydie, }\end{array}$ & $\begin{array}{l}\text { Sangblumige, Ricldbbrithige, } \\
\text { Synpetalae, Calycanthae, }\end{array}$ & $\begin{array}{l}\text { Eticlblüthige. } \\
\text { Thalamanthac. }\end{array}$ \\
\hline
\end{tabular}

Thesis : Orcifhaliger Saame. Eimeis fhwinbet. Thesis: 2fmphigencfe, Doppelwirchfigfert.

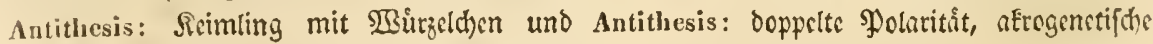
Sinoüphen, gegenuberftebende (Eotrleoonen. und amphigentifde.

Synthesis: Jinoten uno 2(ntitbcfe ber Blitter Synthesis: Spiralitit, dann in Blutthe und

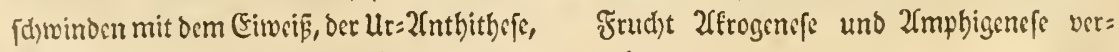
Difitu, zur 2fiternation, D. S. Epirnlftillung. mihlt. 


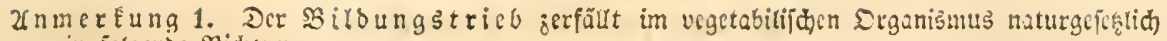
in folgenoc Riditungen:

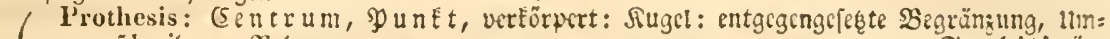
id)reibung, Bcharren,

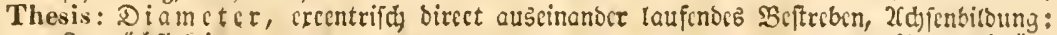
årroüchjighteit,

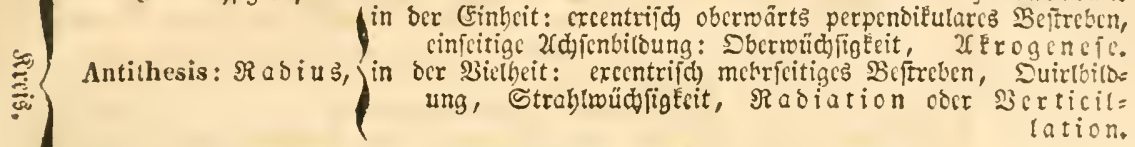

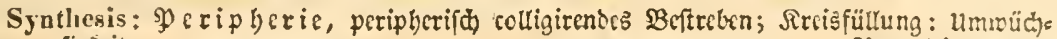
figteit,

Metathesis: Spirale, Lebergang aus bem Eentrum zux Peripherie, 厄chneckenbildung: Spierwüdjigkeit,

Spiralität.

2. Bei allen $\mathfrak{D e r g l c i d u n g e n ~ b e r ~ a l f g e m e i n e n ~ D r g a n o g e n e f e ~ u n d ~ b e f o n d e r n ~ D r g a n o m o r p h o f e ~ c a r f ~}$

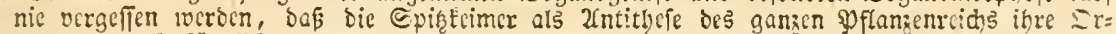

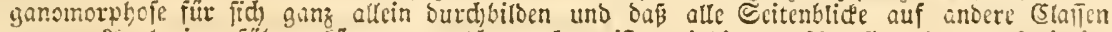

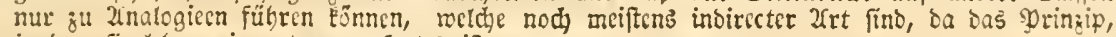

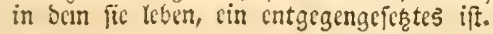

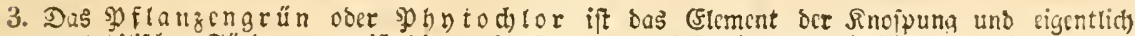

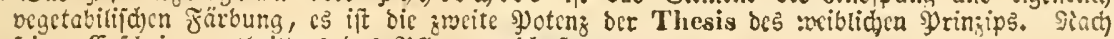

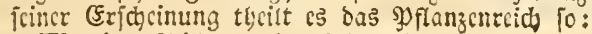

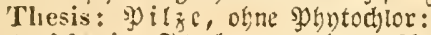

Antithesis: Ffedten, mit verfhlofienem Sghntod)lor: Cryptochlorephyta.

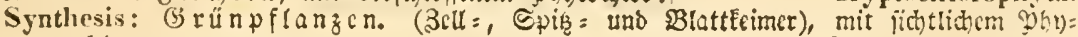
tod)(or: Phanerochloropliyta.

unter ifurn bie micberholung:

Thesis: Die groB̈e शlate ber Grünpflanzen.

Antithesis: Entinecr, Balanophoreen, Srobandace u. f. ro.

Synthesis: Equisetnm arrense, beffen fruchtfamm: Achlorophyt, fteriler Etumm:

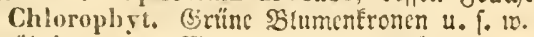

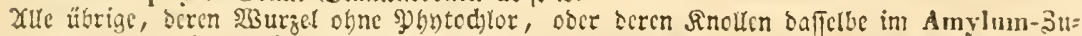

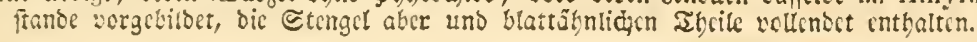


C) Germotiencs 2inalntif $=$ Snnthetifdes.

\section{5 t Dis}

\section{PJlanjenteithos}

oder

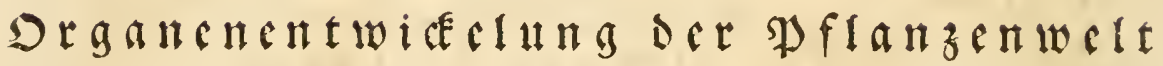
in ihrer Congruens als Eyftem feldeft,

Das hcipt als

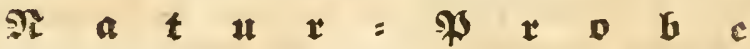 fúr bie \\ Methos.}

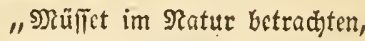
Jmmer (Fins wic 20lles adjten."

(s.

"Im Banzen aber $\mathfrak{l} \in$ ben alle Ihrile, Rebt ber Bemaltigfte und der Berringfte,

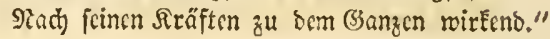

v. Şatirft.

Stufen und Rlaffen.

Etufen: gradus, werben butch bie brei $\mathfrak{S}_{\mathfrak{s}}$

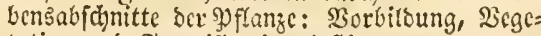

Organonomia. totion und Fractification beftimmt.

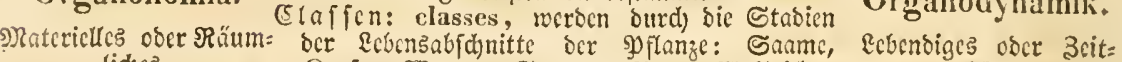

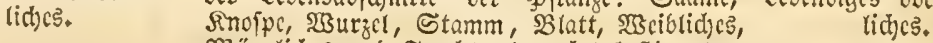
Männlidjes und Frudbt als a d) $t$ beffinmt.

\section{Iทp $\mathfrak{n}$. \\ (E) $\mathfrak{x}$ e tuf \\ Torferpilanzen: INOPHYTA.}

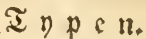

Norbildung von

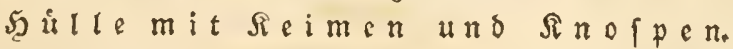

Sie fino:

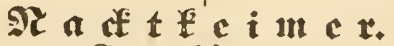

Gymnoblastae.

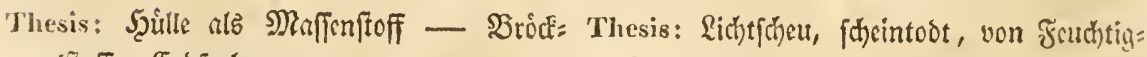

sliftoff. Erofarben.

Antithesis: Urgelle, utenoppe.

Sỵthesis: Reimlager, Sinofpenlinger.
Ecit aufquellend.

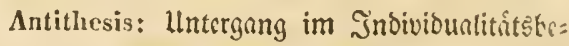
frrcten, fterben bei oer Gseburt.

Synthesis: Borffilubung, Inoppung. 
Srgantonomic,

Stufen und אilafien.

Thesis: im Bereich Det Sugelgeftalten fta: Thesis: wie oben. (Inophyta).

bilijitt.

Antithesis: Urgellen (ohne Urfnoepen).

Antithesis: wie oben.

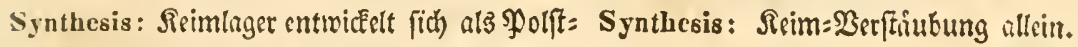

sr, Frnoengefled)t, Marfmaffe mit åußerer

Şüllte (volva).

\section{Slaffe II. $\mathfrak{F}$ (e}

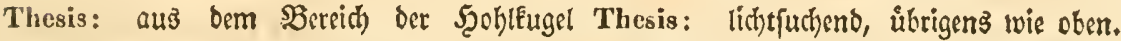
rucijernd.

Antithesis: Urjelle † UtEnofpe, biefe als Antithesis: ourch UrEnofpung, sorediu, fich sphntochlor, Farirbeftoff eingefbloffrn. zerftótend.

Synthesis: Sicimlnger + Inofpenlnger. Synthesis: Sisitwerftäubung + Sinopung.

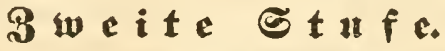 \\ Otodfufrnzen: STELECHOPHYTA. \\ )atericlles ober アäumlidị. \\ I $y p$ e n. \\ Durdbildung von \\ Şurzel, Stamm und $\mathfrak{B l a t t , ~}$ mit 2Untithefe als Siluthe. \\ Rebendiges ooct acitlidues. I Y $\mathfrak{P}$ ?}

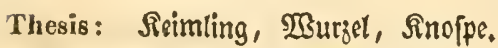

Sellifoff, ⿰מgntochlor, Shiftill.

Intithesis: Spirale, Spolten, 2(nthere.

synthesis: Spore, Saame, Frudt.
Thesis: Jicimen, Saugen, Inoppen.

Antithesis: 2(thmen, Befruchten, 3eugen. Synthesis: Єduffen, Giebihren, Bewabren.

Sis find

凡 ก

in ben

brei mogglichen Pfotenzen:

Silaffe III. Ģ r un flanzen: Chlorophyta.

Thesis: Jinofpe und Seimling mit ein= Thesis: zerprengt bie Şưlle, feimt cin= facher, endlich Doppelter Şưlle. fritig.

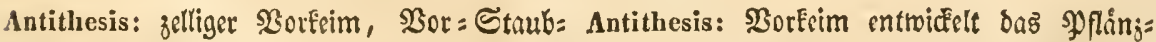
beutel. Splint: $\mathfrak{B l t t t}=\Im_{t a m m}$. ()en aนร fich: Aelléêimer, Cerioblastae. Sorbildung Des Mlännlichen.

Synthesis: Sinofpe, Epore, Sporenbeutcl. Synthesis: fprofiend und gebirend. 
Srganonomíe.

Srganobnnamif.

Stufen und Elafien.

Gradus et Classes.

Slafte IV. Sdeidenpflanzen: Coleophyta.

Thesis: Inofpe: Inofphen, Boden und Thesis: zerfprengt bie Sdhaalen, Ecimt po= Doppelte (endlich breifact)e) Şülle. lat.

Saume: Sisimling uno Eiroci巨̄, Şaut und

Sobale.

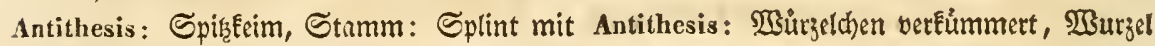
Mark und Bajt tibercinander (3. B. Grố= fer) ooer in eine Eingeit verfotmolgen wiro exceritrifh, Fontbiloung nach oben (verbolzende).

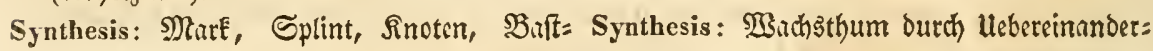
(5y)(inder, B̉latt und Sielch. Frudtetenoten, Griffel, Marbe. Dlattjachioe, Silattbåutchen, Blattplatte. Staubbeutel, Strubfaden, Gorolle.

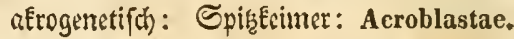
Frucht: Saame, Gaumentríger, $2 \mathfrak{b a n d . ~}$

\section{Sitre V. হueifelblumige: Synchlamydeae.}

Thesis: Sinope: Sinofpchen, Boden und Thesis: Sproft mech oben und wurgelt im orcifiche (cnolich vierfache) Şuille.

Saame: Reimling, (Fiweiß abnehmend), Sernbaut, Fleifhbaut, Echate.

Soden nach unten, in $\mathfrak{B a f f e r}$ und $\mathfrak{Q} u f t$ excentrifh, Sieim zerfprengt bie Schaalen, feimt nach unten und oben.

Antithesis: Gotyledonen: bie zum erftenmale Antithesis: vergweigt fith als $\mathfrak{B u r g e l}$ und

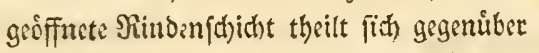
over quirlartig. SpFathlourzel, Stumm Dreiphidtig: Mark, Splint, Sinde ge=

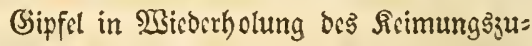
ftanocs gegenuber oder quirlattig, Dann aiternirend und piralig.

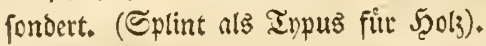

Synthesis: Eplint und Rinde als Scohlcnlin= Synthesis: Pfaḅtroutgel wád) nath unten Der umgeten dirs Mart.

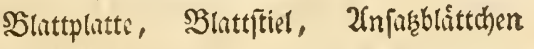
fort, der Stamm nath oben, biefer zugleich (Stipnlac).

Strubbeutel, Gtiubfiden, Gordle. von ber Mittelfobicht nach innen und au= Ben: Amphigenae.

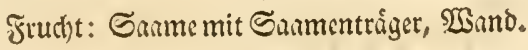
Blutthe, Sefrud)tung, Frucht. 
Srgnonomie.

Etufen und Elaffer.

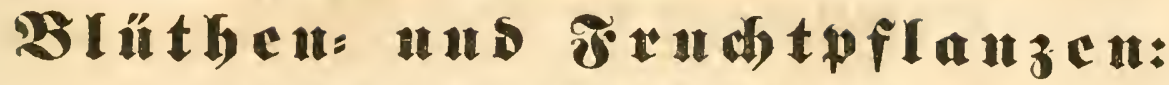 ANTHO-CARPO-PHYTA.
}

\author{
BlattEeimer mit Durbbilbung \\ von
}

Matericlles ober Räumtiđes.

I $\mathfrak{A} \in \mathrm{n}$.

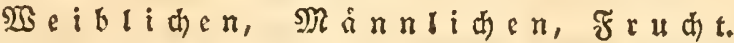

Silaffe VI. Ģanzblumige: Synpetalac.

Rebenoiges noct 3ెcitlid)es. I $p \in \mathbb{n}$.

Thesis: গुiffill, Relch.

Thesis: Mreibliches fich concentrirent.

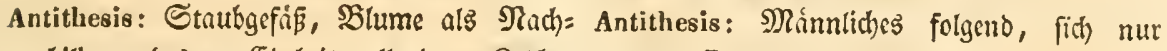
bilbung oes zur Eingeit vollendeten Fieltbs, zum Excentriren beftrebend.

noh einblâtterig gebemmt.

Synthesis: Şơbere Frutchtbildoung beginnt. Synthesis: Meibliches bas Minntiche ůber= Meift Idiocarpium. wirgeno, Frud)tbildung erfiter Şoteng.

\section{Silaffe VII. Sieldblủthige: Calycanthae.}

Thesis: झुiftill, Felch.

Thesis: Weiblidyez untergeoronet.

Antithesis: Staubgefífic und Slume nuf Antithesis: Niinnliches fid) vollendend, bas bem Sielche auffitzend, B̈lume mebrbláttrig. IBseibliche beherrichend.

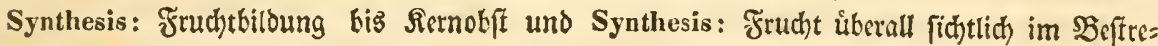
Strinobft.

ben fict) vom Rislde zu lófen, zweite Pुotenz.

Silafte VIII. Stielblưthige: Thalamanthae.

Thesis: গुifitill, Sielch.

Thesis: Reibliches vom Månnlichen ging= lich gefondert.

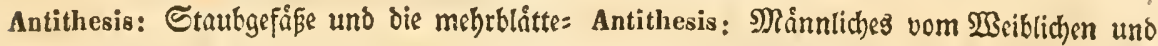
rige Blume vom Siclde gefondert, auf von ber Frucht gainzlich befreit.

bem Stithenfticle auffifiend.

Synthesis: Fruchtbildung bis jur Drange. Synthesis: Frudbt ber bodffen Pूotens, im: mer ginzlich frei. 
Srganonomie.

Srganobnnantif.

Dronungen.

Ordines.

\section{Sronutyen in sen Clapon.}

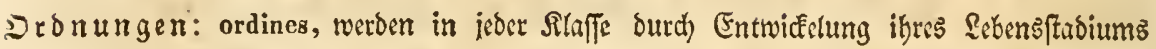
nach bem Brrundgefere der Thesis, Antithesis und Synthesis, als orei beftimmt.

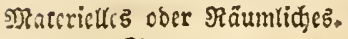

זทp $\mathfrak{x}$.

Ecbensiges ober 3cittiches.

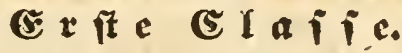 \\ अ i z c: Fun $\mathrm{g}$ i.}

Erfte Dromung.

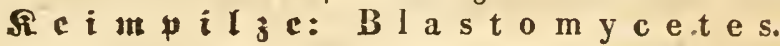

Thesis: UrÉcimmaffe.

Thesis:' Niedere Elementarzetgung (2Baffer und $\mathfrak{Q} u \overline{f t}$ ) renlifirt unformlidhe organifit: bare Mafer.

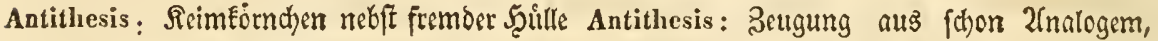
(aus ஒुflinzenzellgencbe). 2ebendigem (einerझुflanze) tenlifitt ben Seim= punEt.

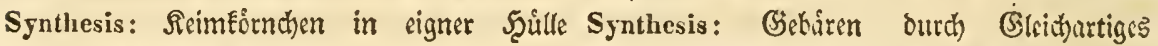
(stroma) auf Srganifhem.

(tubercularia) auf (S)leichartigem (ciner PJflange), Rebendigem oder Todtem.

3roeite Sromung.

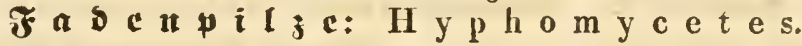

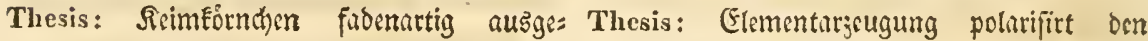
behit.

Seimpuntet jum Fridin.

Antithesis: Faben fondert Durd Theilung fich Antithesis: Das clementur Pुolarijitte fondert felbft in Reimérnchen.

fich, bejouders auf Drgmifchem.

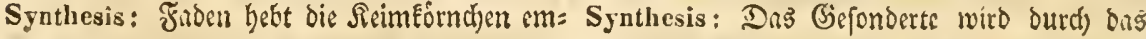
por. 2(eußrerlich)= P̧olarifirte terminalifitrt.

\section{Dritte Sronung.

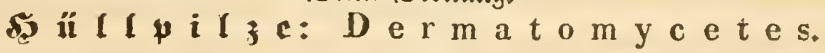

Thesis: Reimfornd enen einfad), bei folgenden Thesis: Hemoment in ber Ulmbillung ber boppclt umbưllt. Şưlle meift Eugelgeftaltig, fortgebildeten Urform.

fchleim = oder Enorpelartig.

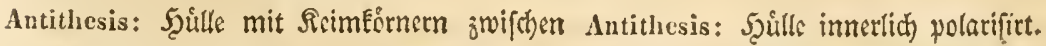

Scaurgeflecht geftreut im Sัnnern.

Synthesis: Şưlle Eugelgeftaltig oreifact), Synthesis: Şille fid in ber ltrform ooppelt (hymenium, pileus, volva), Stamm in= wicderholend, bas \$ुolarifitte úferwindeno. merlich getworden. 
If $\mathrm{fgabc}$ Persoon, dic Bcbrtiber Nees von Esenbeck, Ehrenberg, Fries, Kunze, In-

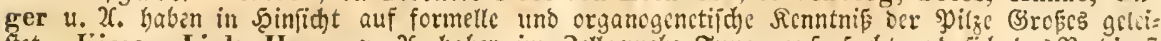

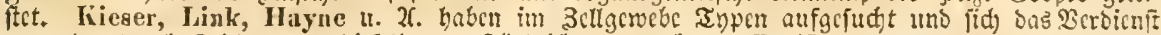

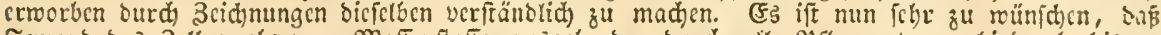

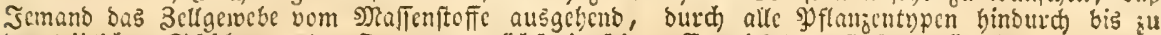

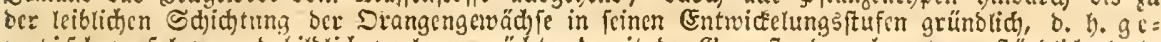

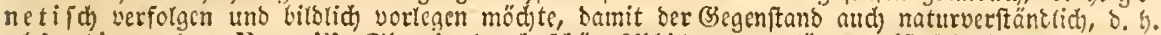
objecriv weroc. Raspail's IGcorie, ourd, fdöne 2(bbilbungen crläutert, ift febr bcađtcnstocrth.

\section{3 weite EIafic. Flediten: Lichenes.}

\section{Erjte Sronung.

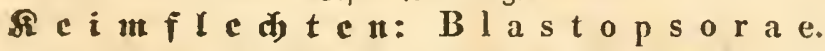

Thesis: אeimEnopper unbegrenzt. (Pulver- Thesis: Şihere Elementarzeugung (\&id)t) aria.) realifirt dic Reimfnofpe.

Antithesis: Seimfnoppen und frembe (vege: Antithesis: Seugung aus foher 21nalogem, tubilifabe) Şütlle. (Arthonia.) Rebendigen. (P) flutţe.)

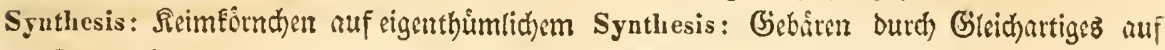
Ritojpentoden. (thallus crustaceus: Co- Ģleidfartigem. (PFflanje.) niocarpon.)

3.toite Sronung.

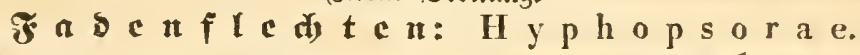

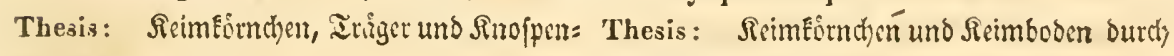
booin (thallus).

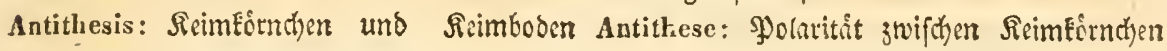
Dem Snoppenboden genitfert. und Sinofpenboden unterdư⿱乛龰t.

Synthesis: Reimfornchen und Reimboden Synthesis: Doppelpolaritit:

(cephalodium) JeimEnoppen auf Irńger Reim + Reimboden : Tríger = Triger und Sinopenboder. : Surpentoden + SinofpenÉctuchen.

Dritte Sronung.

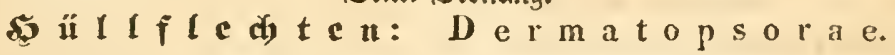

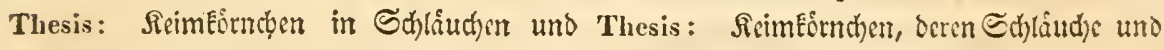

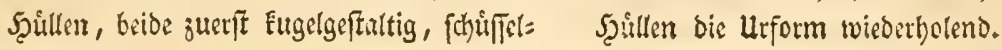
artig, rimnenartig, tellecurtig.

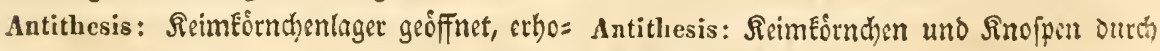
bert, Sinoppenlinger zulef̧̧ ffraud)artig offig. Triger polarifirt.

Synthesis: Reimfoinchenliger gefdloffen oder Synthesis: Doppelpolaritit:

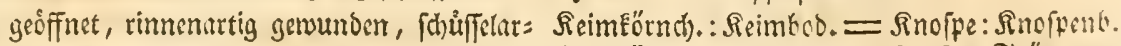

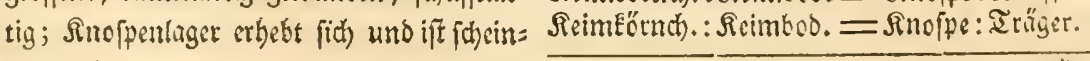

blatturtig.

Snofpe: Snofpenboden = Fnofpe: Irïger.

2fufgabc. Micheli, W. Meyer und Wallroth haben dic Fortpflaniung ocr frecten be=

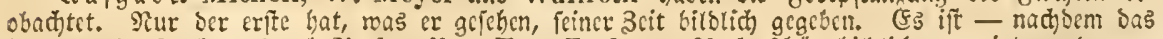

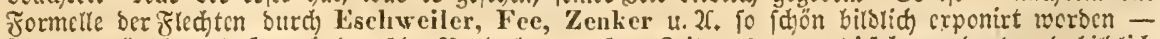

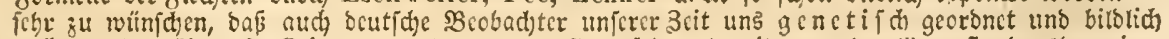
erlä̈tert bas über dic Reimung vorlegen, mas fie gefeben, bamit man ocn (segenftand allgemeiner Elar zu erfafien vermag. 
Srganonomic.

Sronungen.

\section{Dritte Elatie. \\ Ginunp}

Srganooynamif.

Ordines.

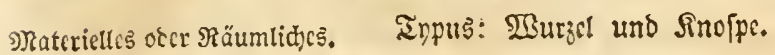
I $) p \in$ n.
Eebendiges obce 3eitlides.

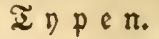

Erftre Dronung.

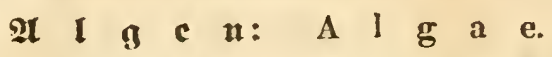

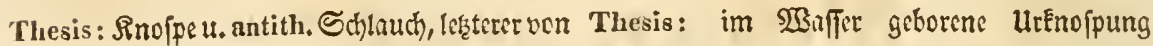

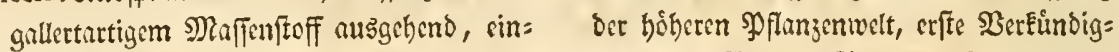
fact), gegliedert, zufammengefert:zellig, rip: pig, forft Erautartig (nur toot: lederartig). Farbe graggrun, in bie mogglidien Modifi= cationen biefer Z̧arbe vertaufend。

Synthesis: Enolid) Beutel mit Snofper und Glitederfíben im Innern. ung ifter 2 Surzel, Stamm =, Stwig = und 3itattform. Spiralenvorbeutung in ber Sirospenlagerung ber angnemen, mit ge=

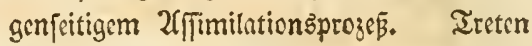
auf als ifolitte Bellgefópe und burchlaufen oic Sellbiloung ofme Spiralen zu ethalten.

\section{3̧weite \$ronung.

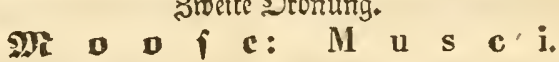

Antitlesis: Snofpe und Eporinkinfel gefon= Antithesis: erffe fich aufblatternde Sinofpe Dert, als Begenfar fur beide ein 2fnthe= renuorbild. Synth. Etamm mit Utrblittern.

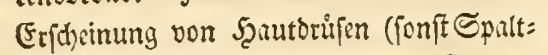
offnungen: stomata genunnt). Drppets mit centrifher Sprofjung, Sordcutung ei=

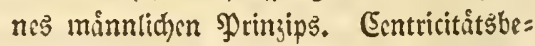
frreben in erfter Syotenz. Spiralener= ideinung. artige Slüthen. ,

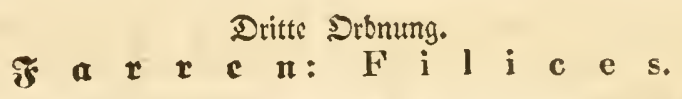

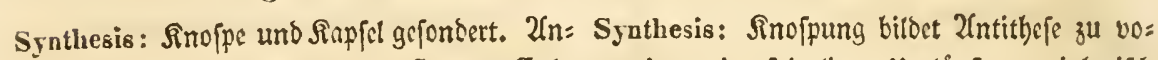
therenvorbild fehlt, tritt erfit am Ende (Cycadeae) wieber sin. Stamm aus peripherifoth ppiraliger Sinoppung vermaurt. Sinoppen wandeln fid in einfeitige blatt= ftielfor SBtattgerwebe (20ebel), beren 3weiglein in Der SBlatffubftanz Rippchen bilben und un= terfeits in inren 2 deffeln oder an ibren Spigen bie Fruchthoiufdyen tragen, bei höheren Formen zieht fid bon ben Frưd)=

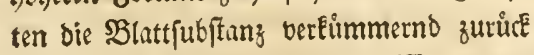
und bann erfochinen fterile 2 scost als riger, in fpiraligen Umliafen peripherifo irtend und (id) ercentrifoh aus der UtEnoip: ung beraus und donn fith feltbft aud) feit= (id) auseinanberrolleno (circinnatio), erff am Ende der Sobnung bis Gentrum (in Zamia) erreidyeno. Frůd)te bei ben tnpi= fden Formen gleidy falls aus Eleinen circin= nirenben Slattchen gebildet und ourd) 2(n=

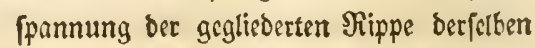
im trodenem Suftande zerreisend und bie Eporen nujftreuend, Deren Siemung fiid) ber Sinofpung veribntidyt. Slattter. 


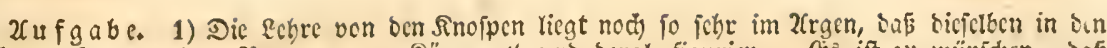

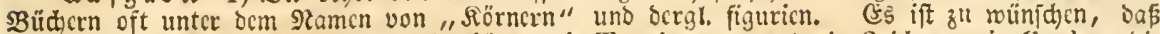

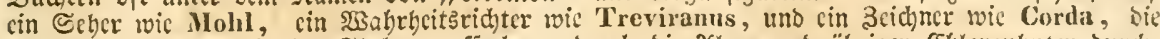

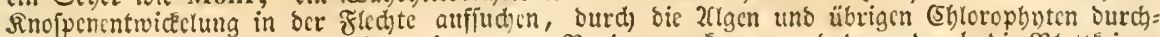
fübren, in ocn Episfeimern wicocr im neucn Srginnen crfemen und bann ourdy bic SBlatteimer verfolgen, fo genetifd mil oer relativen sicimung zufammengeftellt, gut bilolid) bargeftellt ver: legen möge.

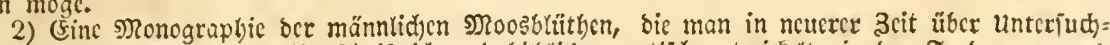
ung ber Sapicln vergeficn, ift, ideriftlid) und bilslid, genetijd) entwidelt, in ben forberungen an unjere 3cit mit beoungen. SRannigfaltiges ift ba nod) zu crwarten.

\section{\$icte EIaife. Erheisenpifanzen: Coleophyta.}

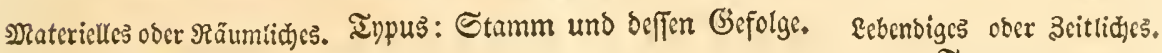
Iy

I $y p \in$.

Erfte Sronung.

\section{:3urjel = Gdjcisenpfanjen: Rhizo-Coleophyta.}

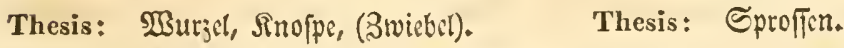

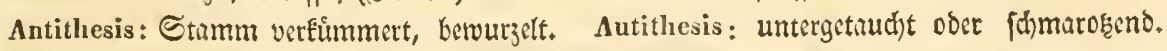

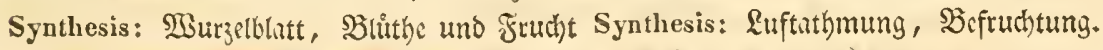

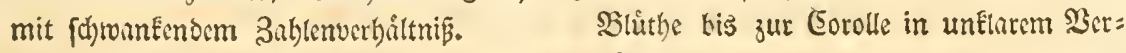
bâttniß̄.

\section{3tweite Sronung.}

\section{Ctamm= Cofiscispflanjen: Caulo-Coleophyta.}

Thesis: Frourzel, Inofpe, (నtwiebel).

Antithesis: Enotiger Etamm mit 3twifdency= linbern (internodia).

Synthesis: Stengclbhtt.

Blůthe mit oreigihligem 3ahlenverbåltniß,

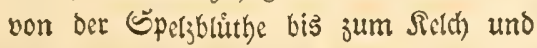
zur Corolke: Narcissus, Pancratium.
Thesis: Eprofen, 2fnrifen (pl. cespitosac).

Antithesis: 2flternation, \&uftpflanzen.

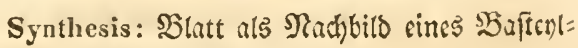
inders des Etummes.

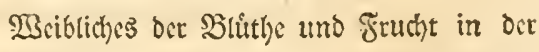
zmeiten Pुoten;, Mannlictes in oer żoci= ten Pुoten: Die Eorolle im Eluren $\mathfrak{B}_{\mathrm{er}}=$ hốtnis vollendet.

Dritte Dronung.

\section{B!att = Grbcisenџfitaujen : Phyllo-Coleophyta.}

Thesis: Trurgel, Sinofpe, (3wiebel).

Antithesis: Stamm aus verfd)molgenen $\Re_{n}=$ ten verholzend.

Synthesis: Błlattform vom (Finfachften bis Syuthesis: SBlntt bis jut hoodffen \$oten?. jum burch Serreīung Gieficoerten uno banofortmig Strabligen.

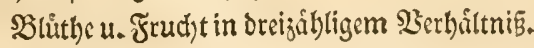

Thesis: Sproffer, 21nrafen.

Antithesis: 2fttemation, Serticillation, Epi= ralitît. Ruftpflanjen.

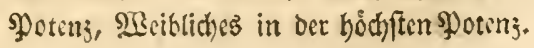


Srgmonomic.

sorbungen.

Srgannognamif.

Ordiues.

Unmerłung. Ncine im Sakre 1822 und 1827 vorgerd̆lagene Benennung: Coleophyta

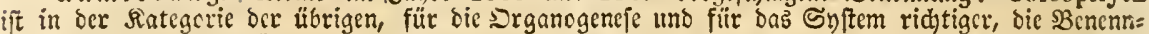
ang Acroblastae gebört in die \$ghntogencie, wo fie auf Iabelle 8. gebraucht und erläutert wurbe.

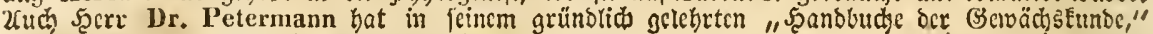
ecipzig 1836, bicran mich freundlid erinnert. -

Sieje (slaffe, als bie grobe Xntitheje des ganzen Pflanzenrcids, bat ibre Drganogeneje

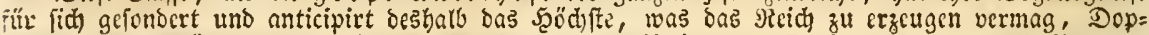

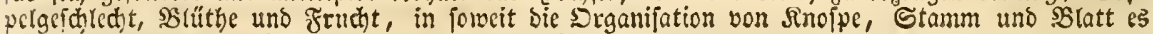

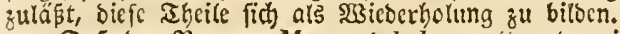

Das orr Name, Monocolyledoneae " naturviorig fern mus, on bas, roas man bier (Soty)= teson nennen toollte, etruas in ocr Drganogencie meicntlich veridiebencs ift, von bem, mas man bei ben SBlattéeimern Eotyledon genannt hat, bebarf Eciner Ereflärung, wenn man Der Srganoge=

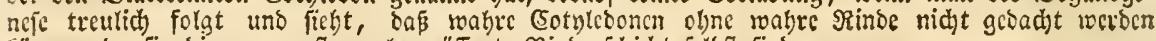

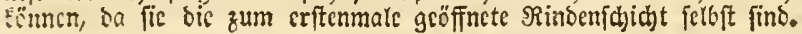

\section{F it $\mathfrak{n} \mathfrak{f}$ e CIafie. \\ Bweirelolnmige: Synchlamydeae.}

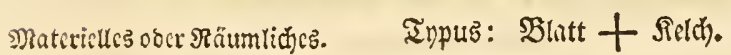
I $p \mathfrak{p} \mathfrak{e}$.
Rebendigrz ober 3citlidbes. I $y \in \mathbb{n}$.

Erfte Sronung.

\section{$\boldsymbol{H}$ i p $⿻$ ul}

Thesis: Sinope und Reimling cinfad be= Thesis: Sinoppung und Rcimung fort al= ginnend, bunn blattEeimend. Şiftill be: genartig, bannvollendet, wie in biefer Elnffe. ginnt und bildet fid) Dutch, erboilt Griffel . Wustzelleben vorwaltend. uno Narbent.

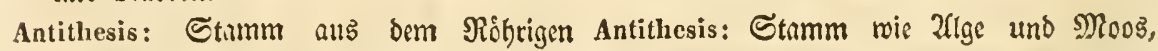
(Grobzzelligen) zum Rocker = und Didt)tzelli= gen fid fortbildent, meift ohne Spiralen, endet mit folchen in Mannliches: nls 3̆tweílappige Unthere beginnend. Sonn vollitandig.

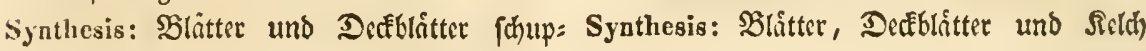
penurtig, meift rippenlos. SBlittgen diellinif̨̆h. Jiuschen, Sirpial. untergetnutcht oder fumarosecno, $23 u r z c l=$ pflanzen. Moosantbere wieberbolt, bann vollftínoiger.

erffer Spotenz. Sefuchtung in ben niebern G̈licbern zweifellyaft, bilbet fich bann burdi) niebere Stufen von $1=2$ = bis unbeftimmt zóblig.

3meite Sronung.

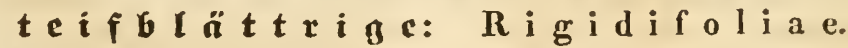

Thesis: Sinopperls Inoten beginnend, bann Thesis: Sinopung und Seimung auf bie beutlid) amphigenetif क. Şiftill beginnt noch eimmal ofne Marbe, bunn bis zur 2fFrogenen beutend, bann beutlid) amphi= Eentricitit nurbig. genetifक. 
Antithesis: Stumm verholgt in coneentrifaren Antithesis: Stummlebenvorwalteno. $\mathbb{1}$ udbs Schidten. Minnliches roicderbolt fidf ncu und bưllenloz und bildot fich bis zu freien perignnifhen Staubgefípen auf $c 0=$ rollinifd) $\mathrm{cm}$ Reld)e. fdeinbar afrogenctifh, Minnlides roic Moosantheren beginnend, fich vollendend. 23eibliches Sieldygebilde geftaltet fich bis zum Sdbinmånnlid)en.

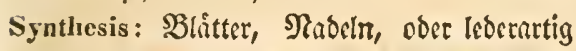
faum gendert, (mit wenigen Ulbergingen

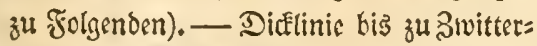
blutbar. Fruddtbildung: Sammelfrud)t

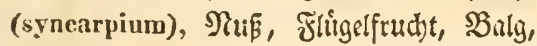
Strinfrudt.

Synthesis: Blitter, Dedfblatter uno Sidl):

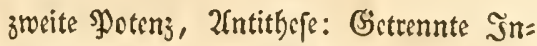

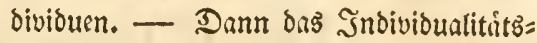
befteben burd) bie 3ivitterbluthe erreicht. Frud)tformen nicorig, gefellig vertwath fend beginneno, enolich indiviouell.

Dritte Sronung.

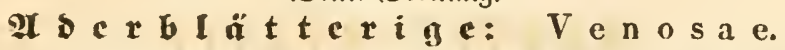

Thesis: Sinofpe und Reimling amphigene Thesis: Scochffe BlattEnofpenbiloung ift crs tifd) vollendet, (Piperaceae, Aristolochiaceae meniger). Piffill mit excentriforer, Dann centrifher Rarbe.

Antithesis: Stamm verbolgt concentrif

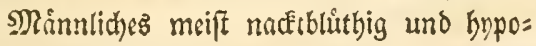
gnnilch.

reidjt, felbft im Ricimling.

Siftill vom Spaltnarbigen zum Ganjunr= bigen ủbergehend.

Antithesis: $23 u(b) \mathfrak{s}$ geht in bas beftimmt Umphigenetif be (auß Piper u. Aristolochia) úber.

Mánnliches 2 = und 4:, am Ende 5= zirflig. Beginnt in ber Antithese (Aristolochia) epigynif(b), meift hnpogynifd).

Synthesis: SBlatt durbläuft alle Formen, Synthesis: Blattbildung uno folglid) Reld)= wird biufig nervig ober ftark geadert, bis zum fojilonerbigen.

Diâllinie bis ju 3mitterblittben. Frud)t beginnt als $\widetilde{S} a m m c l$ frucht (Syncarp-

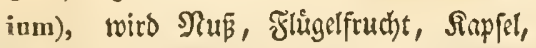
Stcinfrudt. bilbung in oritter Potenz: Synthese nljo

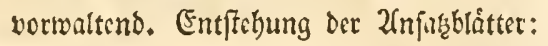
stipulae.

Scillen, Silthe bis corollinifd), weiblitise Bedcutung mit $\mathfrak{B}$ c|ftrcben zu Mánnlicbem. Frudit von entgegengeferter, o. 5. månn= (id)er, verticillirender झidftung von ciner allgemeinen 2 d) [e aus burd) Spiralitit fith concentrireno uno in oer 2Antitbefe des Minnlidyen (Aristolochia) cine Mittel= fiule getoinnent.

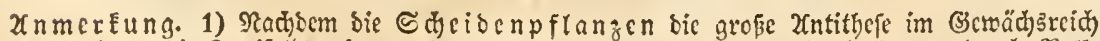

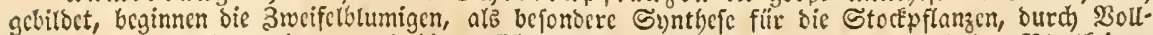
cnoung ocr Blattformationen, und bieten fid, zugleid für bie allgemeine Snntbefe ber Blattecimer

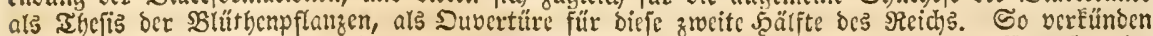

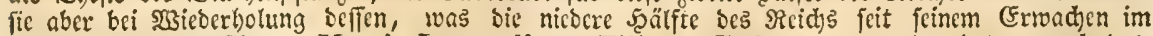
(Sirunen (Algae: Chara, Musci: Lycopodium: Filices : Podostemoneac,) geboten, aud bas,

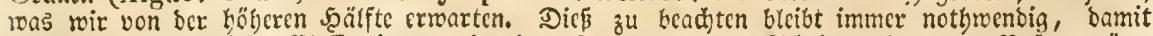
nicht Jemand in biefer (5lafie eine unmittelbare fortfęuna ber Édjeisenpflangen auffudjen möge, bic er, Elaren গaturgejęen zu Folge, vergetlid fudocn nü̈ste. 
Srganonomie. sronungen.

Srganooynamif. Ordines.

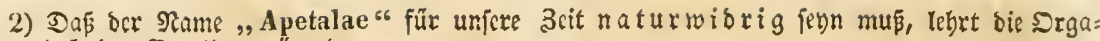
nomorphofe ber Sorolle gentigend.

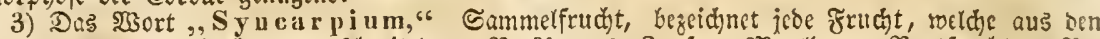

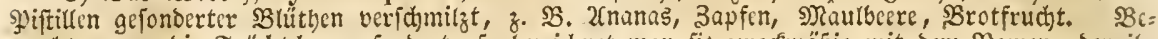

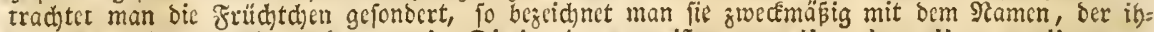
nen, ihrer Statur nad), zuÉommt, im Diminutivo: meift samarella, drupella, nucella, capsella, baccella.

\section{Eed B te Ela fif. \\ Gontofrmige: Synpetalae.}

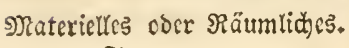

I $\mathfrak{p} \in \mathfrak{n}$. \&ebendiges ober 3acitliches. Iypen.

Erfte Soromung.

Möbrenblumige: Tubiftorae.

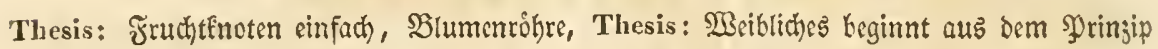

Staubgefifise exfter Pootenz (Snngefiften). Des Mânnlichen: zerfâllt.

Antithesis: Narben getrennt. Reth mit ber Antithesis: Naknnliches beginnt aus dem Jruht verichmoliser. Srinjip des SLeiblichen: verichmolisen. (B̈lume aus cinem unten róbrigen Sande: Compositae.

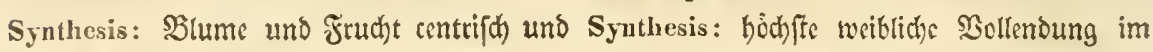
regelmâäig.

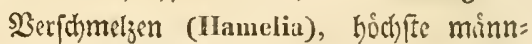
liche im 2usftrnhlen (Nichausia).

3weite Sronung.

\section{Edulunsbluntige: Fauciflorae.}

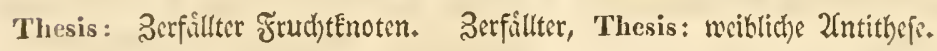
frcier Sicth.

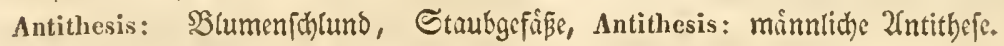
zrocite Potenj.

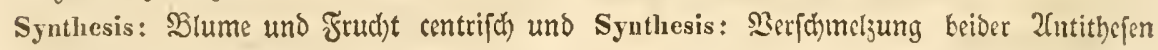
regelmifífig. zur Snntbefe in Der jweiten গjotem.

Dritte Sromung.

\section{Eaumblunige: Limbiflorae.}

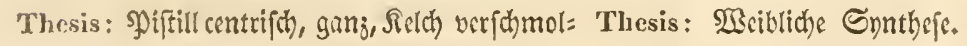
zen, centrifh, frei.

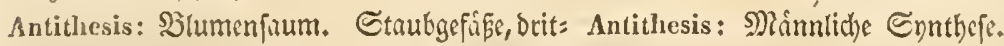

ti Sुotenj.

Synthesis: SBlume und Frucht centrifh und Synthesis: Doppelinntbefe, oritte Pुotenz fur regelmisig, frei, nur in ber 2fntithefe cin: gerwathin.

Blitthe und Frudyt, bie SBlume in Der minnliden Pisibe (Asclepiad. Passifl. Contort. Sapot.) aus boppeltem EtipularÉrcif́ getilloct. 
2fnmerEung. Dic Sonennung, MIonopetalae, "woldhe in Linnée's uno Jussien's 3cit for richtig war, ift für bic unfrige natur wiorig, folglich unjüäjig gewotocn, fcitocn mir

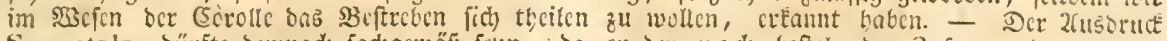

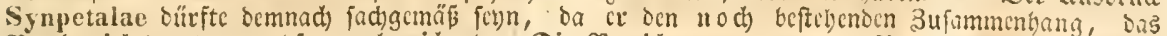

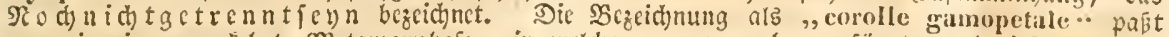

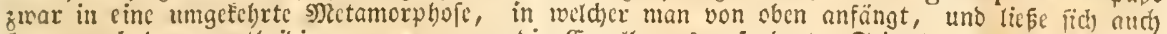

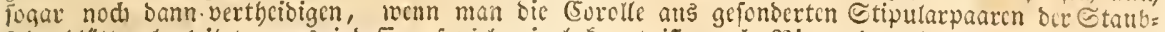
fabenblättcr berleitete, was indeffen, foviel mir befannt ift, nod) Niemano getban bat, inde/fen iff

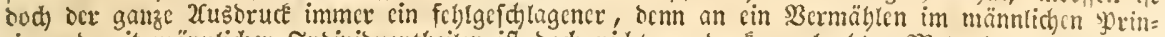

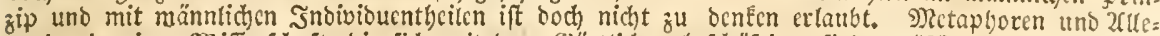

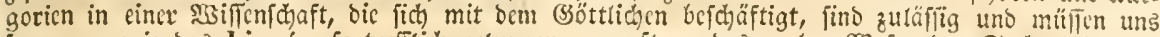
poaar - wic bas linnée fo trefflid) gclungen - oft an bas wahre 23sefen ber Eache crinnern, aber fie milfen ouch - treffen, fouft pallen fie nidht in bic scutide Śdule uno Netamorphofe.

\section{Birbente Elatifie Reldoblinthige: Calycanthae.}

Matcriclles obcr Räumlid ç.

T $\eta p \in$ n.
Rebensiges ober 3eitfiches.

I $y \in \mathbb{n}$.

\section{Erffe Sronung.}

\section{3erjodicscublüthige: Variflorae.}

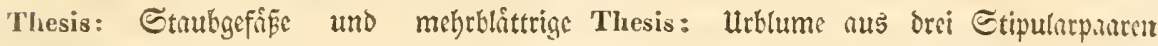
SBlumenErone (wenigen mangelnd), ver= machien und unregeimipigig beginnend. für bie Strukgefípe, unregetmin̄ig, dann regelmiafig merdend. (Leruminosae).

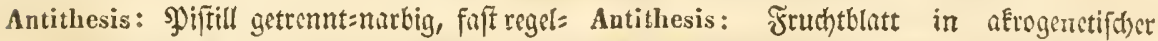

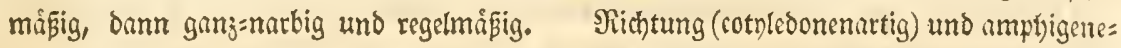
tifher Silchtung (foliolun von Papil.) zufammengelegt, bildet bie beginnenden Frudbtformen (Umbelliferae, Papilion.)

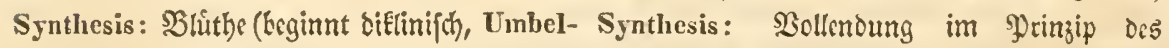
lif. Rhamn. Thereb.), meift zuitterlich.

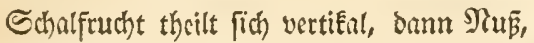
Minntichen, Frucht im Snnern vollendet, fünjählig, sentrif(h): Araliaceae.

Şưlic: am meiften regelmásigig vollendet Dic Steinfrucht.

3rwite Sronung.

\section{T(chnlidbbüthige: Confines.}

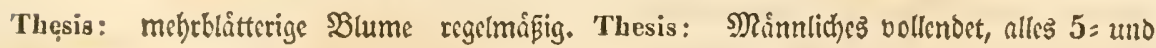
vielzåblig.

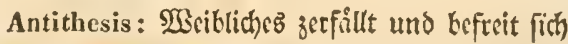

Antithesis: Diftille und Fruchtficher gefon= aber vom Sield), verfomilgt auch tvicoer bert, bann wieder ganj. Durch Şülfe bes Sieths.

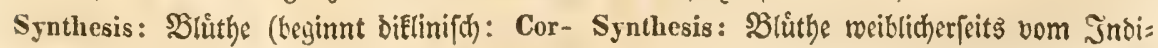


Srganonomie.

Srgmobynamit

soronungen.

Ordines.

niculatae, wiederbolt in Poterium) meift stritterlich, ift fo roie bie frudht, centrifod.

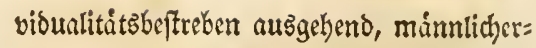

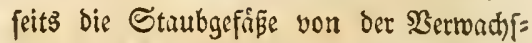
ung(Portulacaceae), montadelphifh), dann

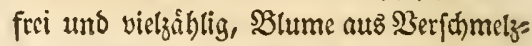
ung (3. BB. Cotyledon) in ihrer 3erfîllung hober vollenoet, Frucht nus ihrer Berfill: ung (Corniculatae) jur bohkeren Eingeit erhoben (Cacteac).

Ditte Dronung.

\section{Gireichiourntige: Concinnae.}

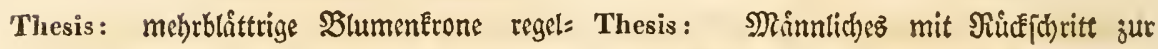
mápig (mangelt anfangs). 3iweiz̧nl(Circaea).

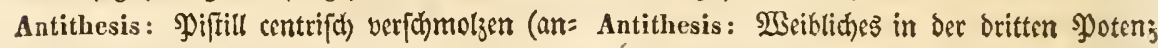
fungs zerfâlltt). centrifich.

Synthesis: Blưthe (beginnt biflinifh) ift Synthesis: Blưtbe weiblid)erfeits vom İn= jtwitterlich, centrifo

Frudbt centrifó), biz jut fíchrigen freien divibualitititzeffreben außgeheno, mánn= Rapict und boch vollendeten freien Stein= lici)erfeits von ber Berwad) fung (Polygalaceae) frei rerdend, zwitterlich, centrifor. frucht. Frudt in fich) vollendet, audh von ber Şarridaft dez תelds) fich befreiend.

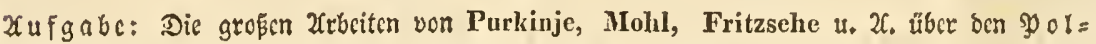

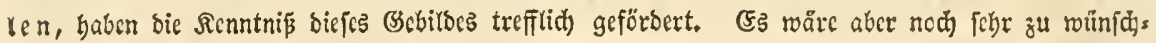

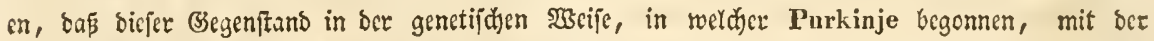
tiefen Berfolgung, wic Mohl und Fritzsche ing erfaßjt baben, vom erften Momente feincs

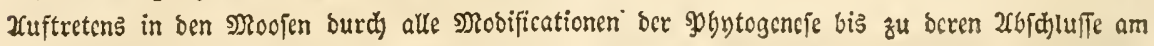
Ende Der slattfeimer burd)gehend, bildstid vorgelegt rerbe. 
Drganonomie.

Dromunger.

Srganooymamif. Ordines.

\section{I d $t \mathfrak{c}$ EI $\mathfrak{a}$ i门c. \\ Ctichulinthige: Thalamanthae.}

SRatcricutes obcr $\Re$ ăumlicycs.

Ty $p \in$ n.
Rebenoiges oocr 3citlidges. I $y$ P $\mathrm{n}$.

Erite Sronung.

Soblfrüd)tige: Thylachocarpicae.

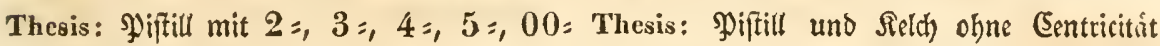

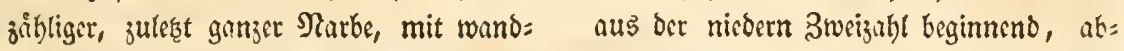
ftindigem Sanmentrióger. faltend, biz zur centrifd)en Fünfzaht yor= fohreitend, bann bleibend.

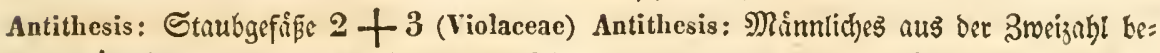

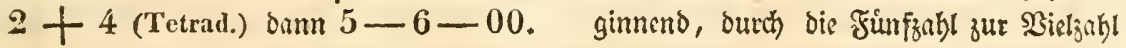
SBlume 4 = bis 5 blittrig. gelingend.

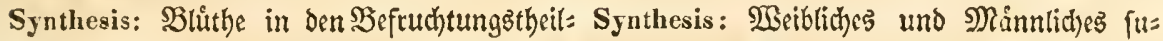
en unregelmị̄ïig, dann regelmísig vollen= Det, endlid) bie Błtume wieder rudfigingig, d)en die frumfabl, obne cinander gegenfet= tig in ihr begegnen zu Eonnen.

(Flacourtianeae).

Frucht unregetmåsig, dann regetmás̄ig und centrif

\section{Bmeite Sronung.}

\section{Epaltirühtige: Schizocarpicae.}

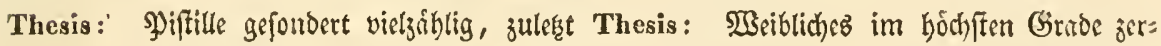

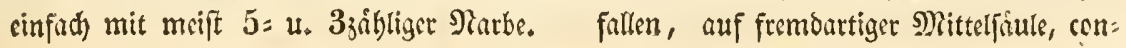

Sicld) viclzaiblig zerfallen - (nuie bas Şif= till), minnlictes (Corolle) nathafmeno. centrirt fich quitlartig und ppiralig, endlich quirlartig vorliufig zur Einbeit bollendet

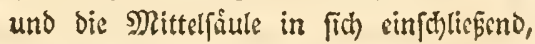
bie Giriffel veriftme!zend. (Ruta).

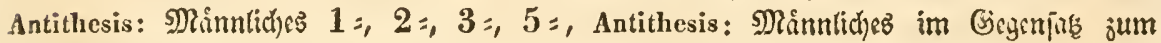
00 zillig. (Euphorbiac, Rutac, ete.) wath)= $\mathfrak{r} \mathfrak{S}$ Blumenkrone cinrethig (Helleborus, $\mathrm{Ni}_{-}$ gella.) ober fehlend. zerfî́llten Meiblid)en, vertwad) fon beginnend (Malvaeeac), dann fich roicder jerfíflend, die normale fimfzahl gerwinnend.

Synthesis: Bhiothe (bei wenigen untegelmaipig) Synthesis: Mainnliches und MBeibliches ge= rege(miñig, Reth) getbcilt, Frucht regelmá= sig, vicltheilig, zulef̧t ganj. genfeitig in ber Funfzabl cinander begeg: nend, (Rutae. Geraniac.), dann dus

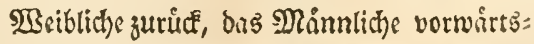
fd)reitend. (Sapind, Oxalideae: Bombac. Rhizob).

Dritte Dromung.

\section{Gäulenfrüdigtige: Idiocarpicae.}

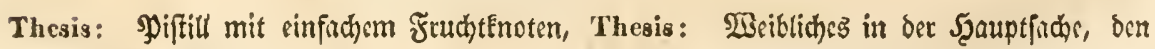


Srgattonomic.

Srganobyuamif.

Sronungen.

Ordiues.

mit getrenntin Grtiffeln, endidi mit cin:

FruchtEnoten fidon vollsnbet beginneno, fircham Griffe?. mur nod) in oen (Griffeln uno Narben getrennt, in fincr juetten Stufe verfomel= zend, in ber britten vollendet.

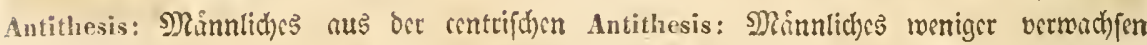
3-5:3ahl zur Siclzahl frei werdend, am Ende rieder polnadolphifd). Eeginneno (Caryoph. Hyper.) unofrei= twerdeno bis zur Shlume aus boppelter Etipularrcibe (Silene, Tilia), aber am Ende die Staukgefápe mieder verwad)fen. (Hesperidae.)

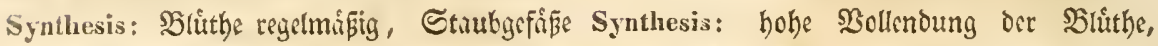
viclzithlig, frct, zulegt wieder verwachien; Frud)t regelmifig, beide im Tnputs Der Fünjaitht vollendet.

Dieje \$ollendung im Minnlict)en wrid)t Dann oer hochften Sollenoung oer Jrucht,

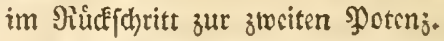

Linmcre. Die Epaltfrudis: shizocarpium, als alfgcmeinc 2entitfefe aller Fruchtbildo

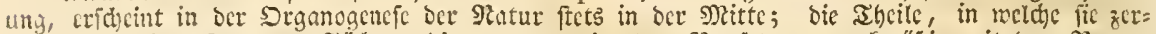

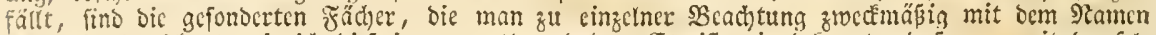

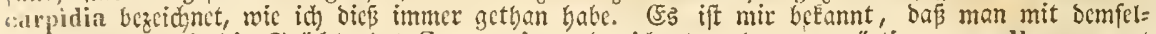
ber: Stancn auch bic Fitid te des Syncarpium bezcichnet uns gegemvärtige carpella genannt hat. Jone múficn indeffen, ba fic boch wabre Frudfformen für fich find, genauer beftimmt und

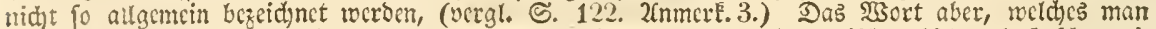

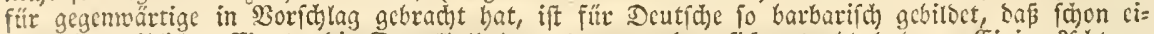

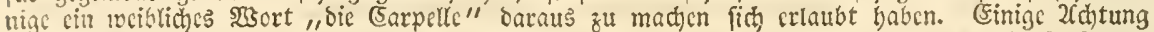

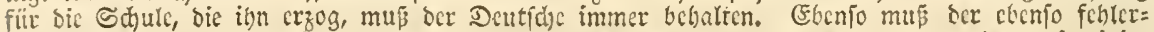

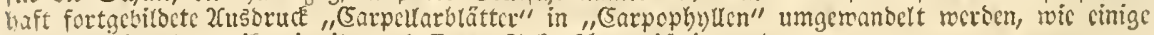
fprudentroige Sotanifer in ihrem befferen Ialt fohon richtig gethan. 
Srganonomic.

\section{Meifen in Sen Orsmangen.}

Reihen oder Formationen: Formationes, werben beoungen burd) bis Reomutten

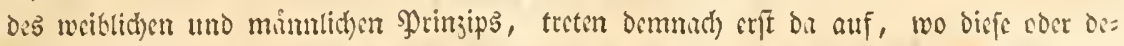

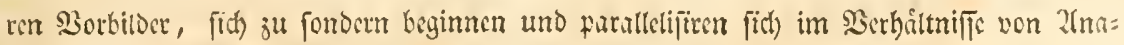
logien im entgegengeferten Serlaufe.

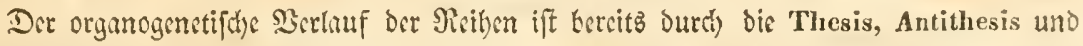

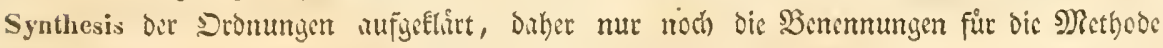

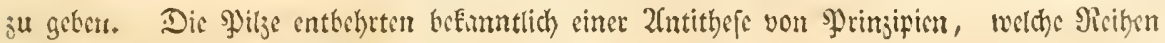
bedingen, wit beginnen demmad) mit

\section{Nia f f e II.}

F l c dy t a : L i c he $n$ e s.

Sron. II. Trasenferisten: IIyphopsorae.

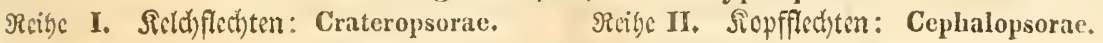

Ston. III. Sulfiffedoten: Dermatopsorae.

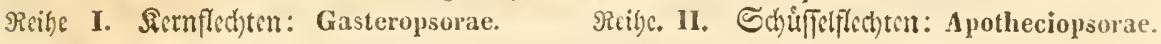

Staffe III.

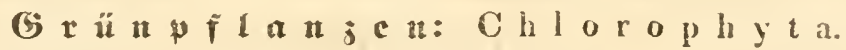

Dron. I. Mgget: Algae.

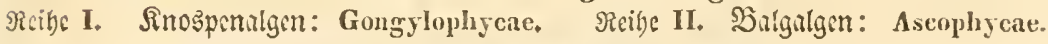

Dron. II. Meopie: Musci.

Reihe I. Medolmoofe: Thallobrya, Reige II. Bhluttmoofe: Phyllobrya.

Sro. III. ஐrarn: Filices.

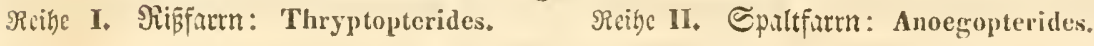

Silfie IV:

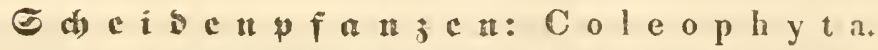

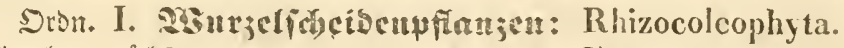

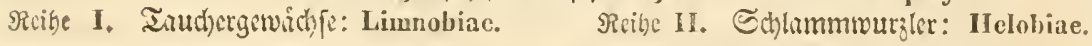

Sron. II. Etcugeliobeiberpfonjen: Caulocoleophyta.

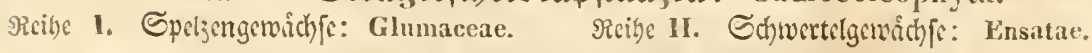

Sron. Hr. Sintêdjeibenpitanjen: Phyllocoleophyta.

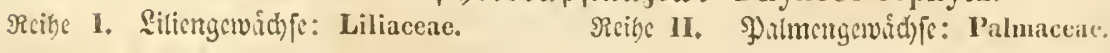

S la fic V.

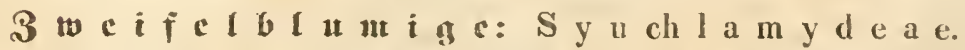
Sro. I. Păippenloje: Enerviac. 
Srganonontic.

Reiben.

Sron. II. Etcifblättrige: Rigidifoliae.

Srgandmumit. Formationes.

Rcige 1. Shlethtbluthige: Inconspicuae. Rribe II. Doppelbeutige: Ambiguae.

\section{Srbn. III, 2Abcrblättrige: Venosae.}

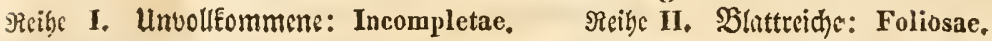

$$
\text { Slaffe VI. }
$$

G a

Sron. I. Röfrenblunige: Tubiflorae.

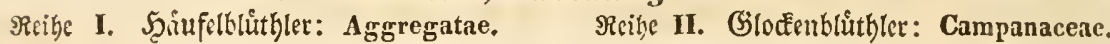

Sron. II. Goblunbblumige: Fauciflorae.

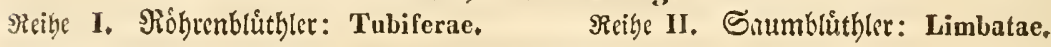

\$ron. III. Caumblumige: Limbiflorae.

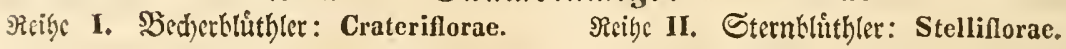

$$
\text { Il a f f e VII. }
$$

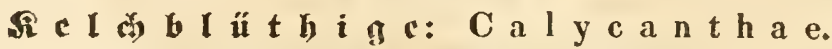
Sron. I. Serfodiesenblifthige: Variflorae.

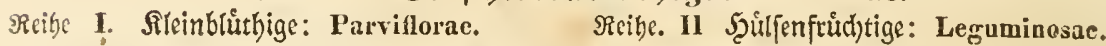

Sron. II. Acthnlichbrüthige: Confines.

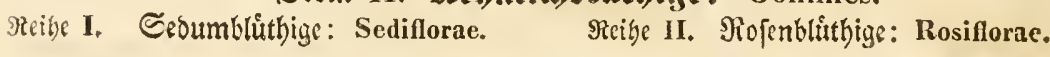

Srơn. III. Gleidfförmige: Concinnae.

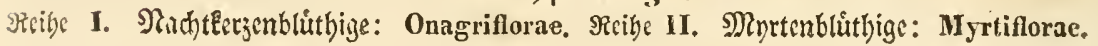

\section{§la f f e VIII.}

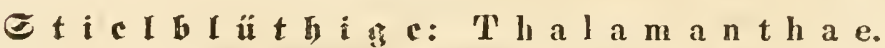

Sron. I. Sçoblìrürotige: Thylachocarpicae.

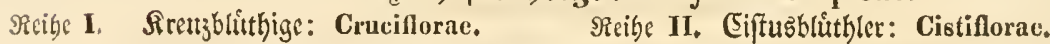

Srdn. II. Epaltirühtige: Schizocarpicae.

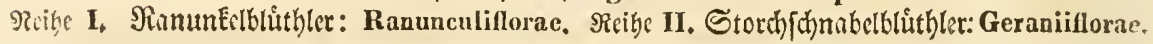

Sron. III. Gäulenfrǘt)tige: Idiocarpicae.

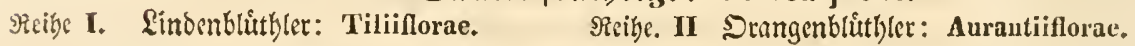

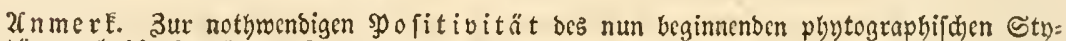

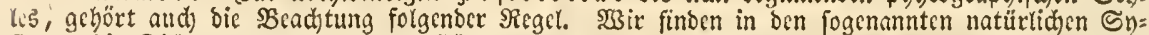
ftemen bic Differenzen ber Sormalzuftänbe, welde fid) bei 2(nalyje ber (Sattungen ergeben, burd)

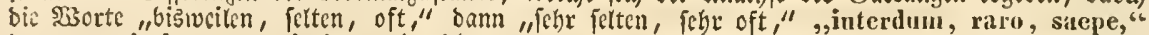

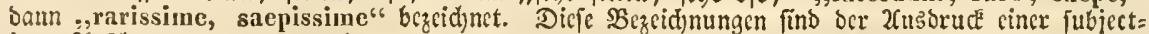

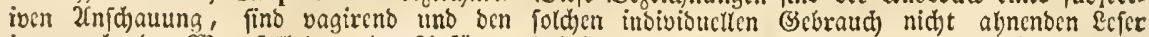

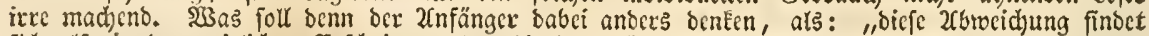
[id] alfo in ber zeitlichen (Fricheinung ber bierfergebörigen $\mathfrak{P}$ flangen $m a n d$ d 5 s] al, bie eine offer, bie andere feltener!" To if es aber bod nidjt gemeint, man will bier nidet von zcit=

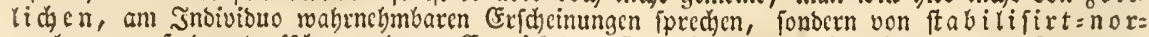
malen, auf ber typifd gegebenen Cntwidelungsftufe d)aracteriftifd fixirten, was follen alio bier jene unverfändlich:vagirenoen 2fusbrücte, warum nidht bier objectio fagen, wic man bic

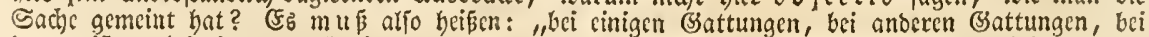
ben meiften, bei ciner (weldye in Parentheje genaunt wirb), bei viclen, bei mebrern, bei febr vic= len, bei ben meifter, meiftens, meift," arfo: ,quibụsdam, aliis-aliis, paucis, in genere quodam (indicato), in multis, permultis, pluribus, plurimis." - So will es ber Genius ber Spradje, fo fdicts idj immer und fo hat man immer geroust, was idb wollte. 


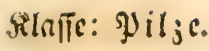

\section{Er ite EI $\mathfrak{a}$ íc. \\ Srounng: Raimpilize. \\ P i I c: F ung i.}

Erite Dronung.

Rcimpilje: Blastomycetes.

1. Familic. Utrpif̧̧e: Präformativi.

2. Familie. Batundpilze: Uredinei.

3. Familic. 23arjenpilge: Tubercularii,

3weite Sronung.

Fabcupilje: Hyphomycetes.

4. Familic. Moderpifye: Byssacei.

5. Familic. Farerpilge: Mucedinei.

6. Familie. Eshimmelpilge: Mucorini.

Dritte Dronung.

Siillpilje: Dermatomycetes

7. Familic. Sdylauchlinge: Sphaeriacei.

8. Famitic. Strulinge: Lycoperdacei.

9. Famitic. Şutlinge: Hymenini.

1. Famili. Urpil;e: Praeformativi.

lltferimmafli.

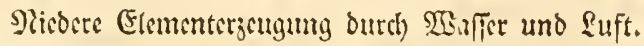

Srganifïbar. - Gallert = ftrub = Enotpelartig.

Bruppen: Conspectus p. 3, no, 1 - 6 .

2. Fanitic. Siranopilje: Uredinei.

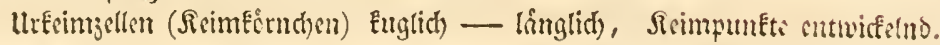

Erjengung innerbalb vegetulitiphen 3ellgewebez lckender Pflumjen.

Im Drgunifitungş=:Deftreben untergebend.

(3ruppen: Conspect. p. 3. no. 7-85 b. Botanisirbuch t. II,

3. Familie. 25:arjenpilze: 'Tubercularii.

Reimfermdion im Reimpoliter.

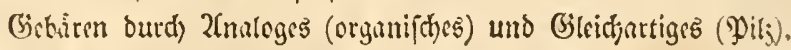

Stgunifitt. 2Barzen=flocken=artig.

Bruppen: Conspect. p. 4. no. 36-52. Botanisirbuch t. II.

4. Jamitic. 2hoderpil;e: Byssacei.

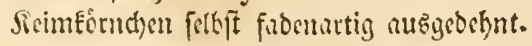

Elementurersengung pohrijït den Scimpunft jum Fnoent.

Sm Drganifitungsbeftreben untergehend.

(stupprn: Conspect. p. 5. no. 53 - 62. Botanisirbuch t. 11. 


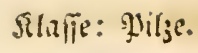

3. Familie. Fapirpil;e: Mucedinei.

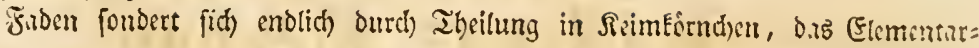
Polifirte trennt fich wieder, befonders bus auf Drgunifacm fupents.

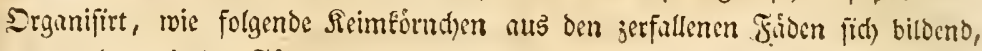
ober mit ben Fidoen erjeugt.

Bruppen: Conspeet, p. 5. (i3 - 123. Botanisirbuch t. II.

6. Familic. Echiuntulfie: Mucorini.

Finen bebt Die Seinforndhen in frinem Jmern empor.

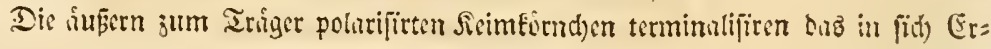
jeugte.

Reimforndien in Gillert ober Bhlupen.

(Sruppen: Conspect. p. 7. no. 198 - 145, Botanisirbueh t. II.

7. Ëmitie. Ed)laud)linge: Sphaeriacei.

Srimzellen in cinfucher fdileim= oder Enorpelartiger, ounn kei ben volfomm= nern in boppelter lumbüllung,

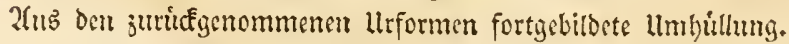

Sirimjellen bei sinigen nod) ungefondert, bie llegeftaltung (J̈am. 1.) wieder= holend, bei undern beutlid) in Şiglen (Tuber.) und Sebliuthen.

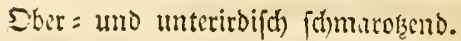

(Srufpen: Conspect. p. 8. no.146-103. p. 10. no. 2209-299. Botanisirb, t. II.

8. Familie. Etreufiatge: Lycoperdacei.

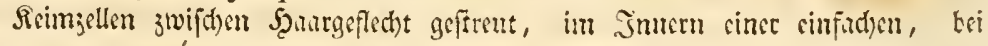
vollemmóner Formen in ciucr Deppelten Soullle.

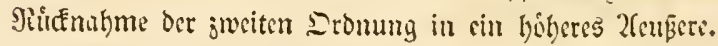

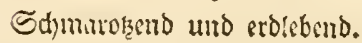

(Sruppen: Conspect. p. 8, no: $164-228$. Botanisirbuch t. II.

9. Familie. \$sutliuge: Hymenini.

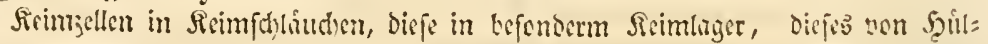

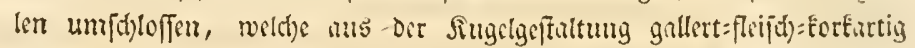
jinto.

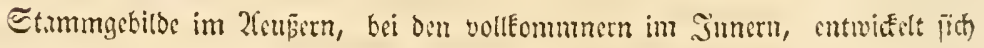
inety.

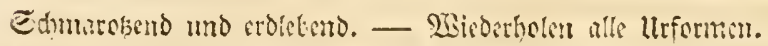

(Bruppen: Conspect. p. 12. no. $2 \$ 0-$ ü19). Botanisirisuch t. 11. 
Silajle: Flled)tcu.

\title{
3 weite Elaife. Fledtent: Lichenes.
}

\author{
Erfte Dronung.
}

Ancimfectign: Blastopsorac.

10. Familic. Etmbflechten: J'ulverariac.

11. Famitic. Etinthirudtfferd)ten: Coniocarpieac.

12. Famitic. Matflit)tin: Arthonariae.

3 weite 2 ronu

Fascuffeditem: Hyphopsorae.

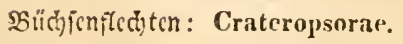
Ropfif(c)ten: Cophlailopsorac.

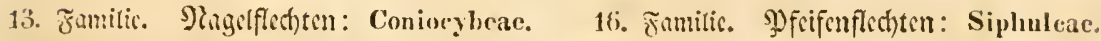

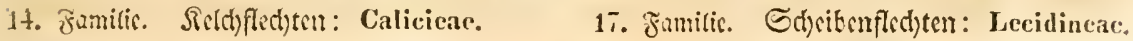

15. Famitic. Stubbugelfedten: Sphacro- 18. Fumilie. Snopffledten: Cladoniaceac, phoreae.

Dritte Sronung.

Siülifichten: Dermatopsorae.

Scrnflcolten: Gasteropsorac.

હdüfiffifchten: $\Lambda_{\text {potheciopsorac. }}$

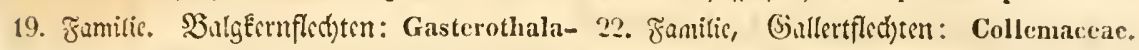
mi. 23. Zamilic. Ifllerflchten: Usneaccae.

20. Famitic. Minmenfrchten: Graphithalami. 24. Familic. Edjuffeffedten: Parmeliaceae,

21. Fumilic. Sntursiflecteter: Gyrothalami.

10. Fumitic. Etanbifterbten: Pulverariae.

Reimtnofpenhafen (soredia) unbegrenjt, butlentos.

Dutch bibre Elementarjeugung, ourch Qicht realifitt.

2luf Etrin, todtem Şolje, Simtor.

(3ruppen: Conspect. p. 20. no. 350 (35t - 35!)?). Botanisirbueh t. 111.

11. Tumitic. Etaubirabtfedyten: Coniocarpicae.

תcimforndyen (sporidia) auf Eeginnonom Sirmhger, jwifd)en cincm dimn: Ermftigen finospentuger (thallus). Dewhiutó)en jerpringt.

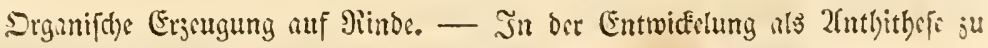
ben Nindburgliedern.

(3)ruppen: Conspect. p. 19. no. Зึّ1. Botanisirbuch t. III.

12. Famitic. Pinfficedeten: Arthonariae.

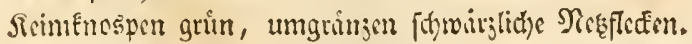

Srigunifthe Erijeugung.

2uf letendiger Siinde ber Eiden u. T. m.

Gruppen: Conspect. p. 19. no. 353. 
Silaile: Flect)tert.

13. Familic. Magelflediten: Coniocybene.

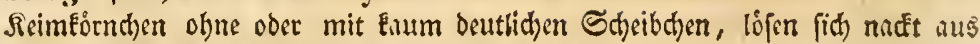
Euglicher, Eork̂ntiger Şuille.

Şîlle beftimmt geftielt, in fruftigem Snosppentager.

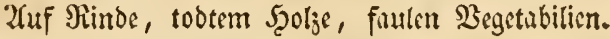

Bruppen: Conspect. p. 19, no, 361.

14. Gamitie. Sicldjflediten: Calicieae.

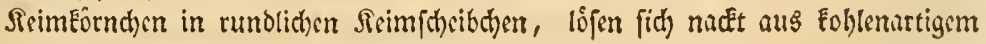

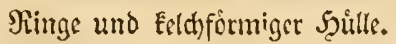

Şillle in Snospenliger eingefentt ober nus cinem ftielartigen Iriger (podetium) oben becherformig gebildet.

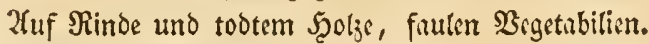

(3ruppen: Conspect. p. 19, no. 330.

15. Fumilic. Etaubfugelfed)ten: Sphaerophoreae.

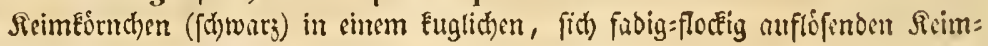
liger.

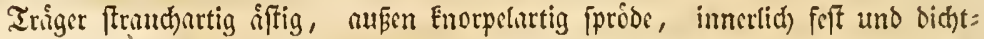

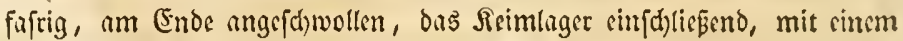
\&och auffpringend und zerreißeno.

Snospenlager nidht entwicfelt, Sinospung bem Iriger gleidhurtig, forullenibulid). (sruppen: Conspect. p. 20. no. 362.. Botanisirbuch t. III.

16. Famiic. Wieifenfited)ten: Siphuleae.

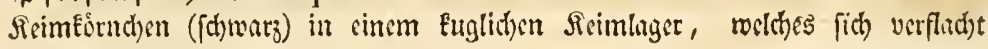
und j(t)windert.

Trigger, effig, bidstfaferig, an ben Enden angefdrwollen, bas Seimliger cin=

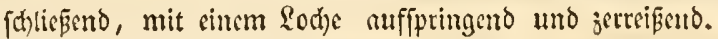

Sinoppenlager - fehe ich) wernigftens - nid)t entwickilt, sinofpung osm Tríger glecthartig, fornllenáhnlid.

Bruppen: Conspect. p. 22. no. 406.

17. Familic. Edyeibenflechten: Isecidincae.

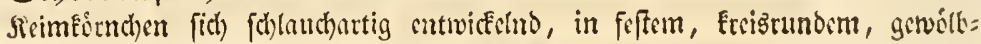
tem Sieinlager, welebes unterisits verfoloffen ift.

Iriger ungewoibnlich und unbedenteno, ocm Eruftigen, bei sinigen attvits

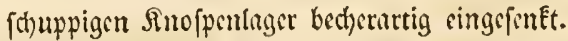

Bruppen: Conspect. p. 22. no. 411. Botanisirlonch t. III.

18. zamitic. Snupiftedsten: Cladoniaceac.

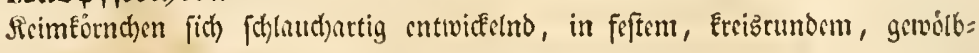
tem Sicimliger, weld)es unterfits offen iff.

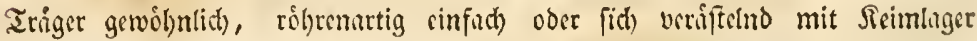
am Enbe, oder bederformig offen und fproffend mit Seimlinger auf bcm Mhúubungsinnde.

Sinoppenluger fó)uppig=blíttrig.

ङruppen: Conspect. p. 22. no. 412 - 415. Botanisirbuch t. III. 
19. Famitic. Balỵfernfierlyten: Gasterothalami.

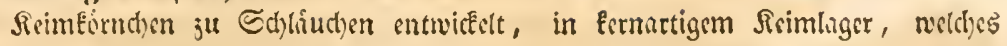
fdomindet.

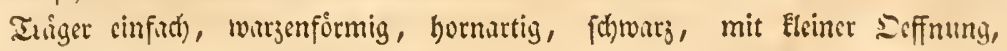
Den Rern cinjeltiefend.

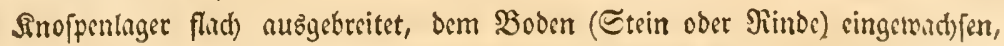

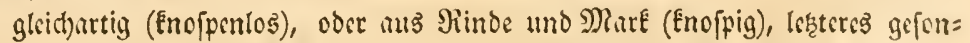

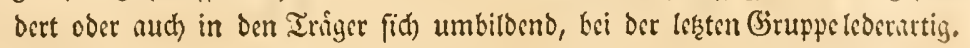

(3ruppen: Conspect. p. 20. no. 363 - 381. Botanisirbuch t. III.

20. Fanilite. Minuenfled)ten: Graphithalami.

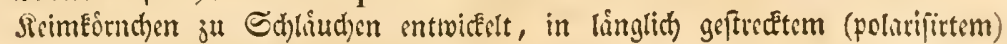
Reimliger.

Iriger rimenurtig bas Reimlager umgekeno, jidt) iffnend, werhirtend.

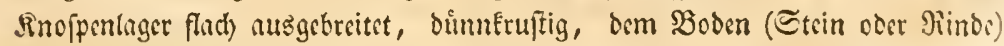
cingerwattsan, Enofpig.

Brtuppen: Conspect. p. 21. no. 382 - 392. Botanisirbuch t. 11 .

21. Familis. Snauclfiedsten: Gyrothalami.

Sicimfónd) mundenen Sisimlagern.

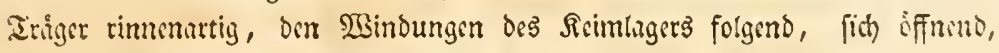
verhiutend.

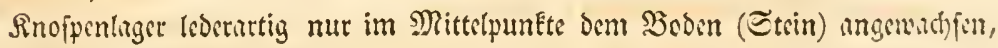
Enopipi.

Bruppcn: Conspect. p. 21. no, 393 - 894. Botanisirbuch t. 111.

22. Familic. Gallertflediten: Collemacene.

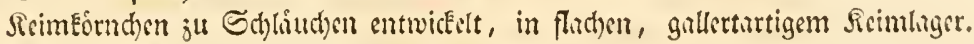

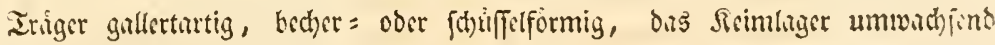
und mit ihm verfochmeljend.

Sirrofpenluger gallertartig, (trocken jum Theil băutig) maşig oder luppig, mit bem Iriger veridjuneljent.

(3ruppen: Conspect. p. 21, no. $395-398$.

23. Familie. Ieflerfled)ten: Usneaceae.

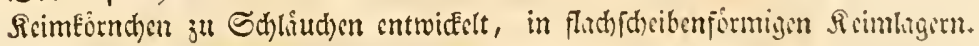

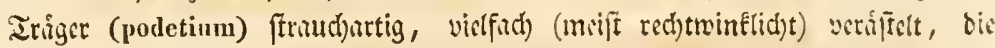
Seimlager tellerirtig offen umgebent.

Snoppentager (thallus) Elcinfduppig, aus $\delta \mathrm{cm}$ Iringer entwidesit.

(3ruppin: Conspect. p. 5. no. 8\%, uno p. 22. no. 405 - 410. excl, 406. Botanisirbuch $t$. III.

24. Zamilis. Edunifîlifted)ten: Parmeliaceae.

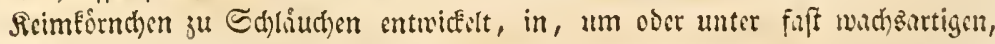
anfungs wazzenifgnlidjen, bum ufrghasformigen, fidy verfluchenoen Sdjeiken.

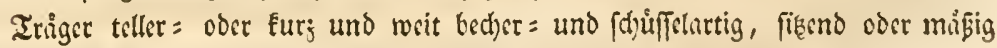
geffistt, aus Erugformigem Juftande fich verfludent, libergehend in 0.35 : 


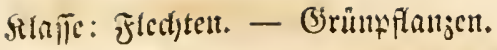

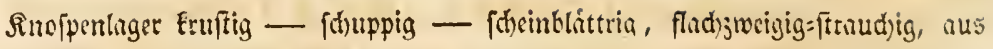
Shinbe und Mare (mit Sinoipen) in beiben Eubftinzen.

Bruppen: Conspect. p. 22. no. ¿̈99 - 40t. Botanirsirbuch t. III.

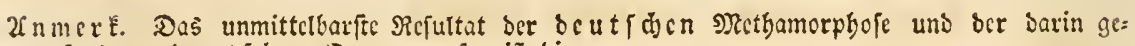

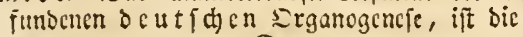

\section{S $\mathfrak{r} a \mathfrak{a}$ u}

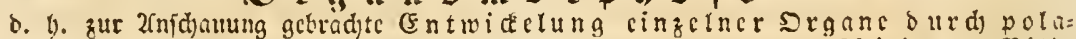
rifirte gotengirung auf homologen Etclen ocr verfdicocnen Birs:

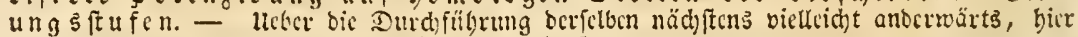

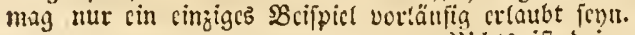

\section{,1) ididts if oriunen, nichts ift oraupion:}

Denn was imen, bas ifit aupen.

\section{Thesis. Ant i thesis.}

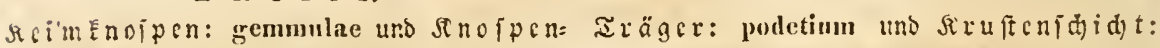
lager: stratum medullare als uriplints und urblattsiloung.

Erite Spotenz, hod dife.

10. Familic: allein vorbanocn, nur mit atnorm 10. fohlt. cutrvicfecten gemijd)t. 11 . beginnt.

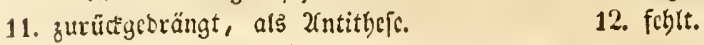

12. wicocrvorwaltens, überwiegend.

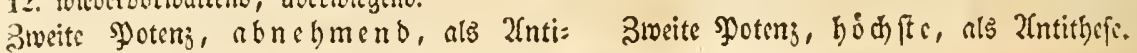
theje fur bas Giante.

13. 16. untergcorsnet.

14. 17. vorwaltend.

15. 18. untergebeno.

Dritte Spotenj, alles vereinigeno.

19. 22. vorthanben, fid ausbilberto.

20. '23. unterorücét.

21. 2t. bödflt sollendet uno vormaltenb. stratum crustaceum, als urkaut = unb $u r=$ rindenbitbung.

Erjte Sotents, beginnenoc.

13. 16. Fohr ausgcbildot.

14. 17. untcroritidt als 2sntithcis.

15. 18. bödjit vollendet und vermalteno.

Dritte Potenj, vermittelnd abach= medoc.

19. 22. unterbrïlet.

20. als Rinne $\mathfrak{u} r=$ \% . 23. Frrauchartig ver= walteno. ntr. ๖े.

21. 2t. abrictumeno und untcrgctycno.

S y n the s i s.

T ll $\quad$ e 8 : i 8.

Seimétönden; sporac.

Ertte Potenj, beginnendo.

10. fohlcmo.

11. vorbanben, übermicyent.

12. unterbriedet, uncntroidtelt.

Swcite Potenz, hod) fte.

13. 16. vorwaltems.

14. 17. unterortëcet.

15. 18. vorivalteno.

Dritte Potenj, abnchment.

19. 22. unterdrület.

20. 23. vorbanden, uีbervicgen๖.

21. 24. (ourch Soredien-sBirbung) unterbrü čt. secimlager: sporophorum, lamina proligera Ach.

10. follt.

Erfe গুotenj, beginnend.

11. begiunt.

12. follyt.

3rwcite Şotenz, bo dh fte.

13. beginnt. 16. Euglids.

14. 17. Eximfernartiges ธdjeibdyen.

1j. untergchend. 18. Euglid).

Dritte গुotenj, beftimmende.

19. fuglid). 2'2. fchlent.

19. vortvaltens. 23. abnchmens. (ur $\begin{aligned} & \tau \\ & \text {. }\end{aligned}$ 


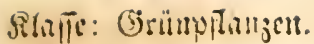

Sromung: จllyen.

\section{2 rittc Elaife. \\ Corunpranzen: Chlorophyta.}

Ertite Dronung.

Qufgen: Algane.

finofpenatgen: Gongrlophycae.

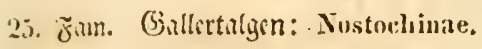

"2i. Fim. Fabmalign: Confervacene.

2-. F̆am. Ed)huthalgen: Ulraceae.
Balgatgen: Ascophycar.

28. Fam. GilcnEfrtid)tuligen: Cramiacere.

29. Fam. Rernalgen: Florideac.

30. Fam. Iangalgen: Fucvideae.

\section{3tocite Dronung.}

Mènje: Musci.

Wedclmoofe: Thallobrya.

31. Fan. भु) attmoofe: IIomallophyllea.

32. Fam. Jungermumuiaccen: Jungermanniticea.

33. Fam. SDhurchuntiuceen: Marehantiacea.
Raubmoofe: Phyllobrya.

34. Fam. Torfmoofe: Sphagnacea.

35. Fam. 2fnorcuccn: Andraeacea.

36. Fam. Mhlų̧nnoofe: Calyptrolirya.

Dritte Dronung.

Trort : Filices.

Risfarrn: Thryptopterides.

37. Fum. Gatviniucen: Salviniaceac.

3ะ. Fam. Shatfiliacen Marsiliaceae.

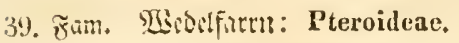

Spattfarrn: Anocgopterilles. 4c. Fum. DEmundrcen: Msmundaceac. 41. Fam. Encrocitcen: Cycateaterat.

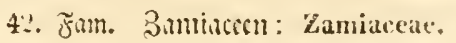

25. Fumilie. Gaffertalgen: Nostochinae.

Jisimfnopen farblos, roth, blut, grim, obne betutitis oder in Fimlager

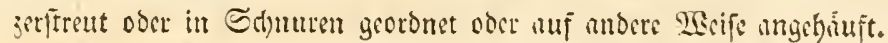

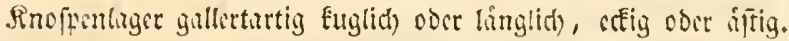

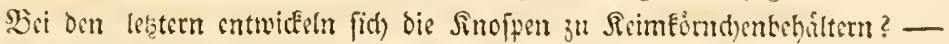
(sruppen: Conspect. $416-439.414-43 \%$. Botanisirhuch. t. II.

26. Fumili. Fastualgen: Confervaceae.

Sieimenofpen ber niedrigften Formen farblos, fadenattig, jerfillend, dir biteri= gen grin, in frobigem Snoffenlager.

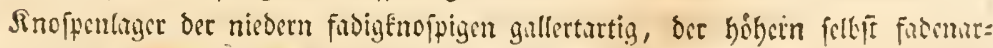
tig, einfact) oocr kffig, unterhrodgen oder giglicdert.

(5rupper: 1. Oseillatorinae (Leptomiteae, Hyrocrocinae, Osillarite).

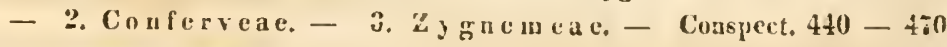

botanisirbuch t. $\mathrm{V}$.

27. Fumitie. Edylandighen: Ulraceae.

Jicimfnofpen fitrtig, meit grim. 
Riafien: Orrünflanjen.

Srónung: IIgen.

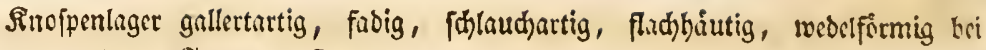
Den lesten Formen geftielt.

(Srippen: 1. Vaucherieae (Protonemeae, Etcospermeac; Bryopsidene). - 2. Ulveae. 3, Caulerpeae. Conspect, $470-480$. Botanisirb. t. $v$.

28. Fumilic. Gelenfirudutalģeu: Ceramiaceáe.

Reimfornd)en forblos, follauthartig entwickelt (nur in ber 2fntitbefe ober bem zweiten (Siliebe cinfach) Euglich) bis zum Decéelitufipringen, innerbalk

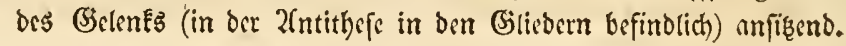

Reimlager fodenartig gegliedert, Gelenke cingerchnưrt ober bautig. (Gruppen: 1. Lemaneac. - 2. Lomentarieae. - 3. Ceramieae. Conspeet, 461. 484. Botanisirbuch t. VI.

29. Famitie. Siernalgen: Florideae.

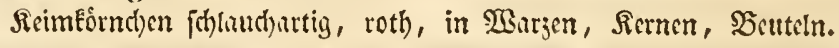

Seimlager bei ben begimnenoen fabenntig, gegliebert, quirlajtig, bei folgenoen webelurtig frei veriffelt, trokfen Enorpelurtig.

(Gruppen: 1) Cladostepheae. - 2) Polyideae. - 3) Rhodomeleae. - Conspect. 481 - 502 (excl, 484.) Botanisirbuch t. VI.

30. Familis. Faug̣algen: Fucoideae.

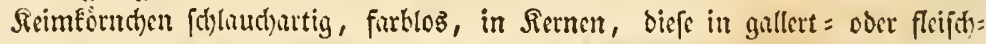
artigen, meift warjigen (troden fehr barten) Şodfern, zwif̣dyen geglicoerten Fiom.

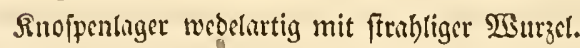

(Sruppen: 1) Laminarinae. - 2) Fucinac. - 3) Sargasseac. Conspect, 503 - 514. Botauisirhuch t. VI.

31. Familic. \$rattmoofi: Homallophylleae.

Resmenopen grin, in jelligen Šeuteln, bet cimigen bether = ober fronenartig iid) $\delta$ ĩ़uent.

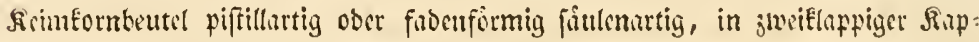

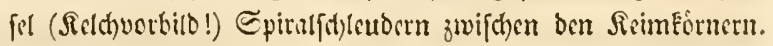

Sinofpenlager platt, weoclattig, zellig, Mitteltippe undiutids.

(situpen: 1) 12 iceieae. - 2) Targionieae. - 3) Anthoceroteae. Conspect, 515. 518 -526. Botanisirbuch t. X.

32. Familic. Jungermantacen: Iungermanniaceac.

Seimfnofpen grun, bei ber nicbrigften (Battung nod in cincm Beutel in den IScoel eingefenft (Blasia), bei ben ubrigen zu Ropfd)en angebiuft, tsi cimi= gen bie Ropfot)en gefticlt.

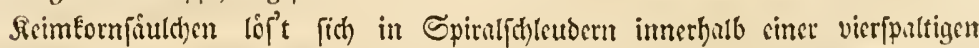

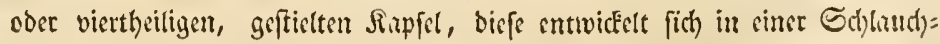

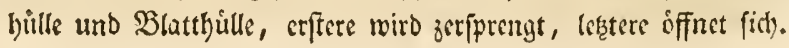




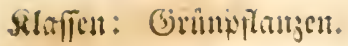

Sroniutg: MRooje.

Sinoppenlinger wedelartig, mit Mitteltippe, blattirtig zellig, platt uno gabcl=

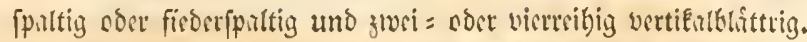

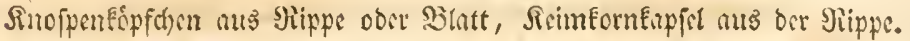

(Gruppen: 1) Blasicat.- 2) Geocalyceac.-3) Jungermannicac. Conspect. 530 - 532. Botanisirbuch t. XI.

33. Famiti. Pintobntiaceu: Marchantiaceac.

Seimfnoppen grin, in fiet) iffnenoen $\mathfrak{B e d} e r n$.

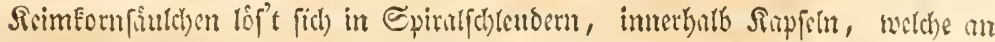
ibrem Simbe vertifal, ober quar ringenm oder vicrpaltig aufpuringeno,

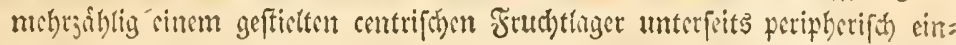
gifintet fitto.

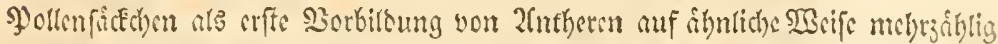

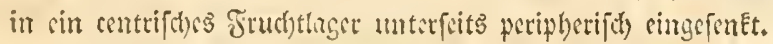

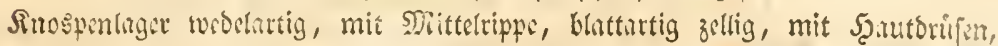
platt uno gatelpsittig.

(Bruppict: 1) In nularina e.-2) R cbouilliariac.-3) Marchanticace Couspect. 528 - 529. Botanisirbuch t. XI.

3t. Fumilic, Zurrutoofe: Sphagnaceac.

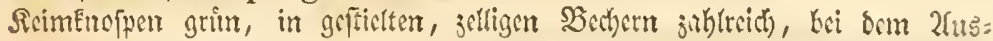

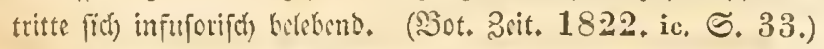

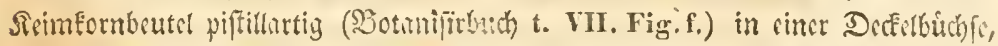
welche ffiterthin mit oun Seimefunern angefillt ift, (vergl. Fig. g.). Dis

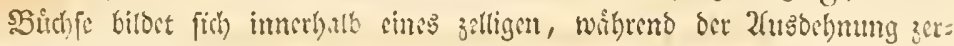

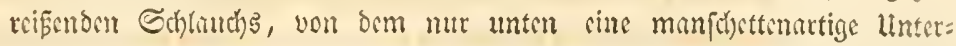

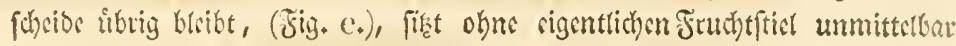
mi cincm 3̂vigleitr.

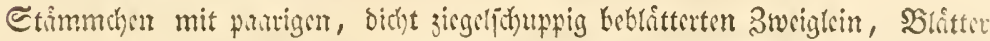

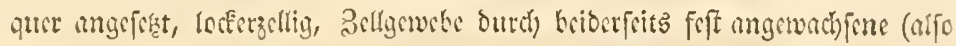

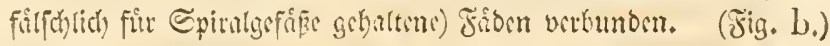

Gruppcn: Sp hag a am. Conspect. 533. Botanisirbuch t. VII.

35. Familic. Sttseräncent: Andraeaceae.

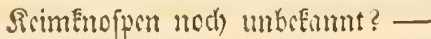

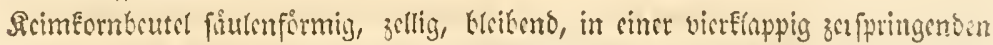

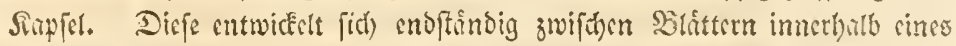

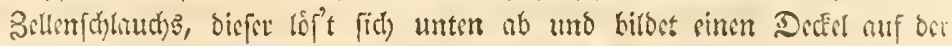
Saptch.

Ctimmethen mit zelligen Slittern bifjt jirgelartig befest.

Gruppen: $\Lambda$ n I raceace. Conspect. 534, Botanisirbuch $t . \mathbf{x}$.

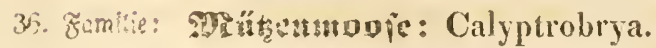

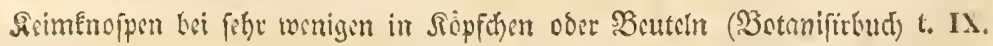

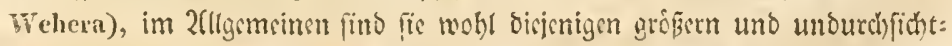

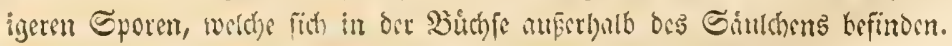


Silnfie: Brüupflanzen.

Srointng: Mooje. - Farrn.

Seiméornbeutel fiuldenformig, imnerbnlb bes boblen Frudtffieles emporfeigen in eine mit Deckel ringsum auffpringende, meift an Der Mủnoung mit

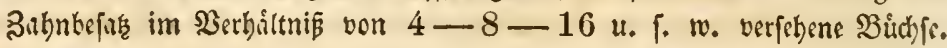

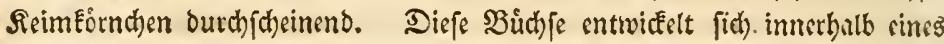

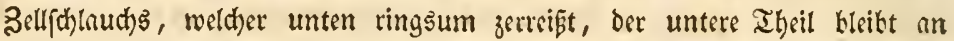
der Bafis des bohlen Frudtftieles (seta) als Sdjeidchen (raginula) vergl. Botanifirbuch) t. IX. Polytrichum), den oberen bebt bie Bưcbfe als 2Rithe (calyptra) mit fich empor.

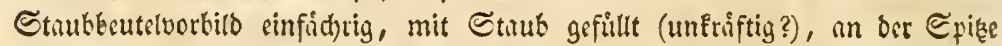
nuffpringend, zwifchen gegliederten Fidoen (Ur:Sorollenandeutung).

Stimmathen mit zelligen, meift eintippigen 3 lattern bicht befergt, bei febr we= nigen vertiknl, bei nllen ůbrigen queer.

(3ruppen: 1) Eutophyllocarpica, - 2) Acrocarpica. - 3) Maschalocarpica. - Conspect. 535-681, Botanisirbuch t. VII-X.

\section{Zumilic. Ealvinincecn: Salviniaceae.}

Seimknofpen zuthlreid) in zelligen Şúllen, bieje geftielt, zohlreid) am Mittelfúul= d)en in Beuteln, welde gefficlt am Stammoten unterfeits anbingen. Ent: nidfelung ift befinnt. (t. XII. Fig. 9.)

Reimécrnbeutel geftielt, jublreich an Mittelfîuldben in befondern Beuteln, meldje geftielt am Stimmothen unterfsits anbingen. Seimung unbekennt? Şollen?

Stämmden fowwimmeno, Blitter gefondert, zellig, flich).

(3ruppen: 1) Azollea e, - 2) Salviniacea e, - Conspect, 516-517. Botanisirbuch t. XII.

38. Familic, Dearjulacect: Marsilaceae.

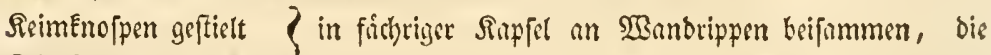

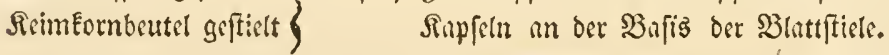

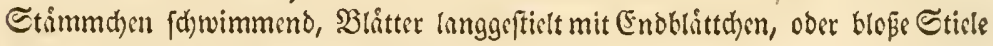

(Pilularia). fich fpitutig nuštollend: circinnireno.

Gruppen: Conspcet. (ii2-(i73. Botanisirbuch t. XII.

39. Familic. X3toblfarru: Pteroideae.

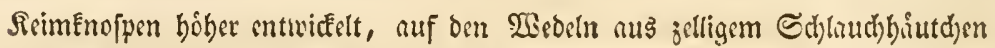
ball wedclurtig fid) wicber entfultend.

Reimfornbeutel gefticlt, juhtrcich) buffldfformig beifummen (sorns), Etiel als

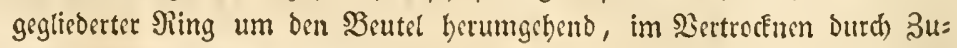

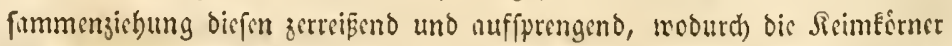

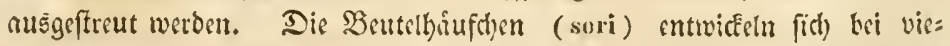

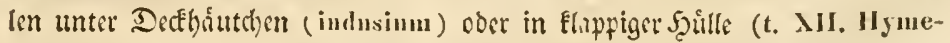
nophyllum), oder in Becherbille (wie Nooskichfe, bei Trichomanes).

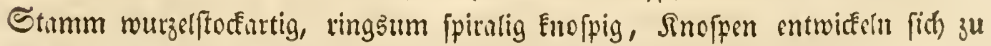

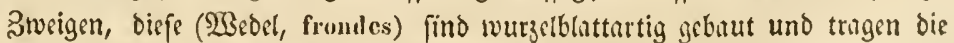
Beutelbiufden alf ber Miteffeite ibrer vertifal an ber Piippe und beren 
Berżweigung verf̧chmolgnen $\mathfrak{B l a t t m a f f e , ~ a n ~ b i n ~ S p i k e n ~ o b e r ~ i n ~ b e n ~} 2(d)=$ frin der Miippd)en (3weiglein).

(Sruppen: 1) Hymenophylleac. - 2) Polypodieae. a) Gymnogrammene, b) Adianthecte, c) Peranemaceac. - 3) Amphibolocarpae. a) Cyathoideae, b) Gleichenieae, c) Parkericae, Conspect, 67t734. Botanisirbuch t. XII.

40. Famitie. Traubeurarn: Osmundaceae.

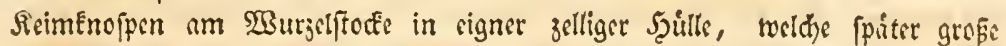
Schuppen bilbet, oder im 230 edel fetbft (Botrych. Ophiogloss.), bunn fajt centrijd\%.

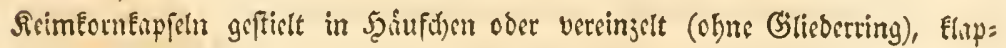
pig soer mit beftimmten ?idjern aufipringend.

Ctrmm wursclffockintig, in ber Entwidestung bem ber vorigen s.tnlith, aber

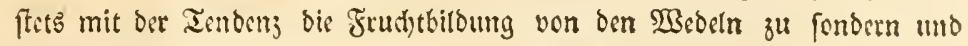

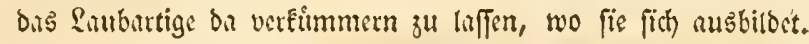

(3ruppen: 1) Osmudeae.-2) Mlarattiaceac.-3) Ophioglos seae. Conspectus $847-749$. et $735-746$. Botanisirbueh t. XII.

41. zamilit, \$alntcujarru: Cycadeaceae.

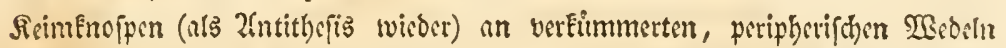

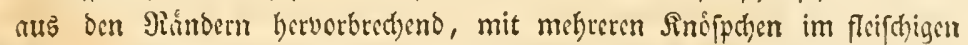

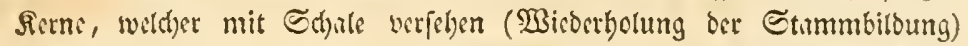

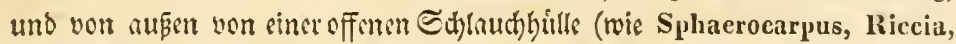
Blasia) umgdben ift.

Seimfornkipferth auf getrenten Indivibusen, zweiklappig, ganj wie bei ben Itrukenfurm, zu $3-4$ jufummengefseltt, (wic bei Helminthostachys), auf ber Untirfeite won Ed)uppen sines centrulen 3apfens.

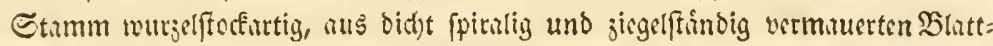

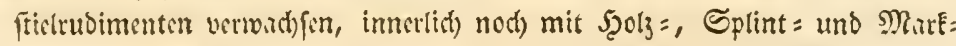
cylinder. Wreder wieder jum $\mathfrak{B}$ tutt getrorden, aber nod vollfommen cir= cimirend.

(Sruppen: 1) Palacocycadeac? - 2) Cycadeae. Conspectus 752.

4․ ร̌anitic. Зฉpicnjarı! : Zamiacene.

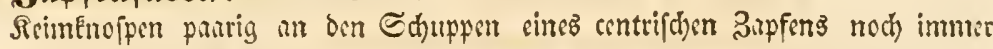
oft grim und fproffend wis bsi andern Farten (verg!. aud) Zamia pumila 13. Nitg. 2006.) Sinoppe bri ber झicife fleifdig, faft roic bie kei Equisetum, im Imuen mit sirópdisen.

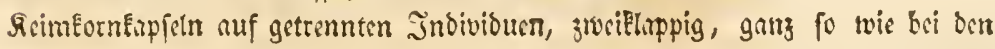

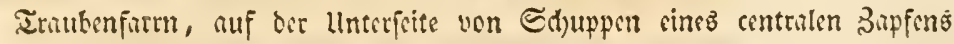

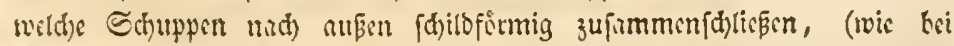

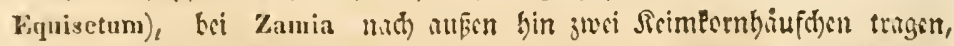

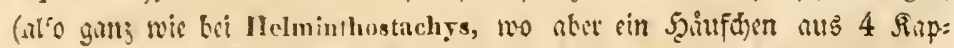

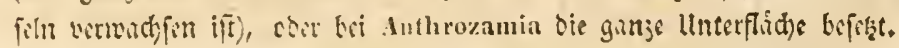


Rinfie: Edjeibentiflanjen.

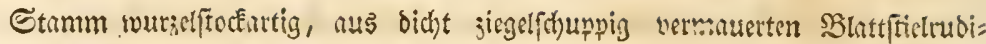
menten verwichjen.

Gruppcn: 1) Palaeoplyta? - 2) Arthrozamieac. - 3) Zamieae. Conspect. $250-251$.

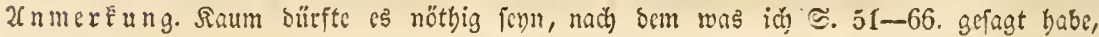
noch cimmal barauf aufmerEfam zu mad)en, wic Elar natürlid) sie Ratur fid bartïber ausfpricht,

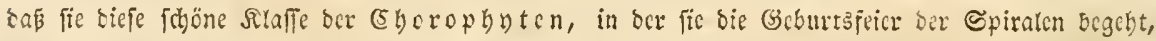

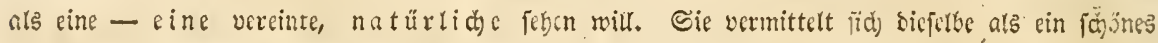
(Banzes burd) forgende Nomente:

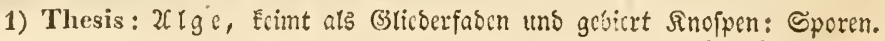

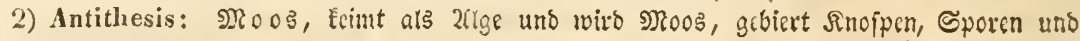
2intheren!

3) Synthesis: Farrneraut, Ecimt 1) ols 2uge, 2) ats Ecbermoos, 3) ats Farrentraut,

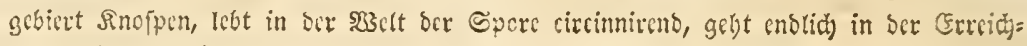
ung ser (Sentralität unter.

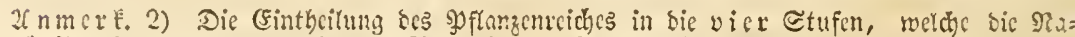

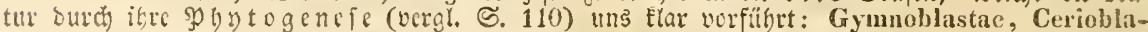

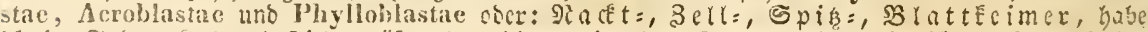

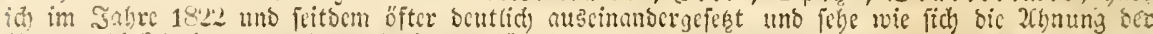

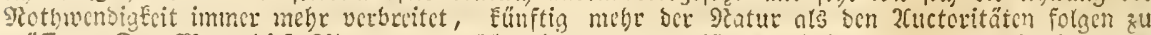

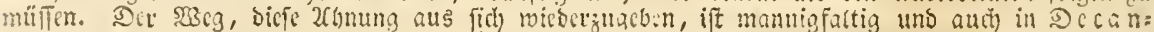

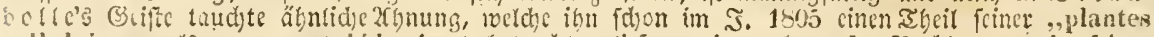

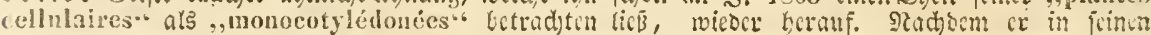

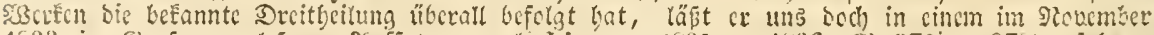
1833 in Senf vorgctefmen 2fufiake (wergl. Limiaca 1835 - 1836,

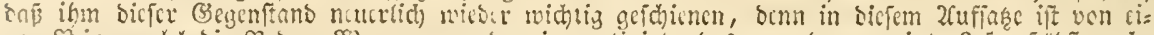

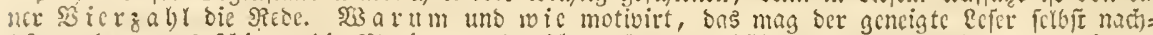

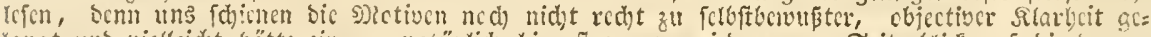

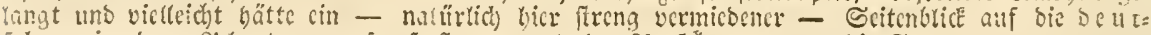

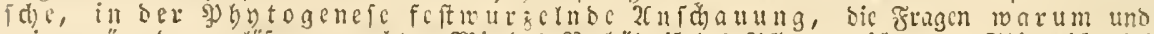

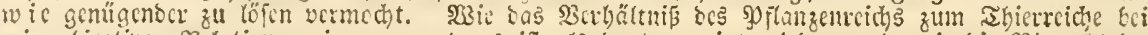
zin objectiven Refationen cin gan anberes ift als bort gemeint roiro, uns wie bie Nitrzabl im

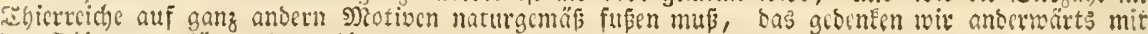
ien Sojecten, erlänternל zat bicten. 
Mafle: Styciocmplanjen.

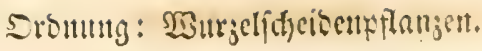

\title{
Sicte Elatic.

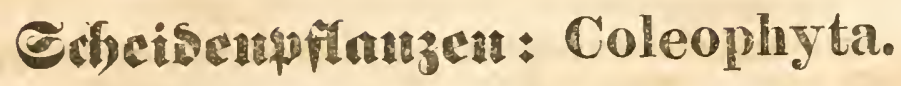

\author{
Erfte Sronumg.
}

Szurzeliogeisenpilanjen: Rhizocoleophyta.

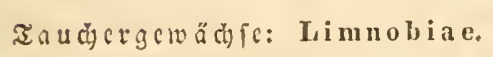

43. Fum. ŠratifnEtifucr: Isoëteae.

44. Fัan. 23affrriemm: Zostereae.

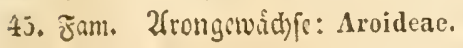

Sd rammurzer: Helobiac.

46. Fam. Nુiffincen: Pistiaccac.

47. Fam. $\mathfrak{B}$ nfferlicfors: Alismaccae.

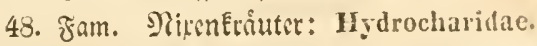

Sweite Sronung.

Etnutupaciocuptinuzen: Caulo-Coleophyta.

ङpclzengetuäde: Glumaceac.

49. ชัum. Girifir: Grimineae.

50. Fam. Eupergrifjer: Cyperoideae.

51. Fam. Riliengraifre: Commelinaceac.
(5) wertergeruach fe: Ensatae.

52. Fam. Siohrfolbon: Typhaceae.

53. Fam. Sd)mertil: Irideac.

54. Fam. Narjiffenffrnertal: Marzissineac.

Dritte Sronumg.

Blattidgeisenpranjen: Phyllo-Coleophyta,

\section{Riticngeroädfe: Liliaceae.}

55. วัam, Cimfuntilien: Juncaceae.

56. Fan. Saruteulition: Sarmentaceae.

57. Fam. Sironsnlilien: Coronariae.

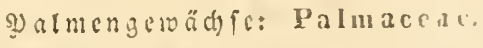

58. zam. Srchibern: Orchideise.

59. Fam. Ccitanimen: Scituminteat.

6ก. ₹m.m. Shalmen: Palmae.

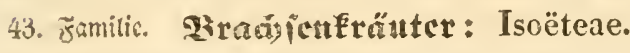

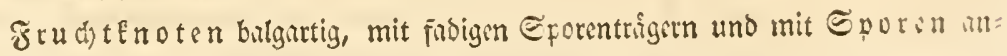
geifitt.

Etaubbeutel balgartig, mit fadigen গুollentrigetn und mit গুollen ungefütut.

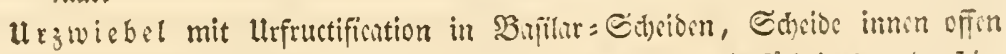

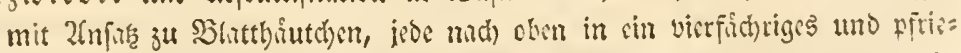

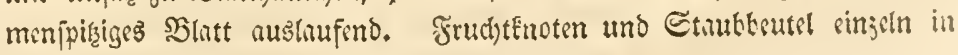

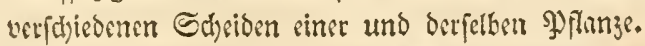

Bruppen: I s o e te s. Couspect. No. 753. Flora germ, p. 5.

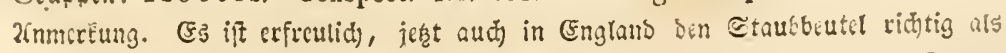

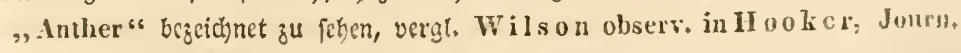
of Bot. IV. p. 312.

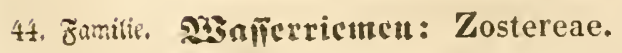

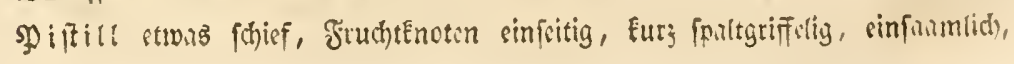




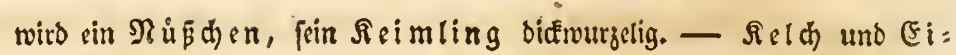
weís fehlt.

Staubbeutel balgartig, mit fabigen Pुollentrigern unb mit Şollen ange= füllt. - SBlume fichlt.

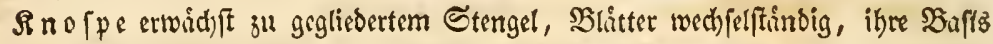
iffnet fich innerfeits als Schcide, worin sin Siolken, wolct)er Fruchtenoten

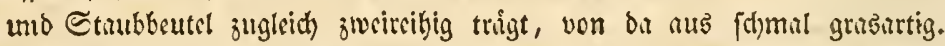
Phucagrostis (Cymolocen Kou.) bitbet mit Siucfnalhme von lsoetes cine

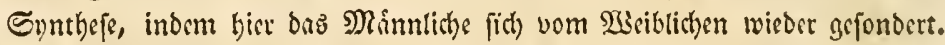
Der vietfadse Stmubbrutel (alfo weiblich verfdymolyen) bebt fich auf einem Staubfaben empor, bus Wribliche theilt fid) in ber 3wcijall, zwci bicfe FrudhtEnoten (wie ber cine bei Isoetes) arfolyenen in ber Bafis oer Sdyei= ben, aber jeber trigt, wie bei Zostera, ben gefpaltenen (Griffel. Der Pollen ift frinfibig, wic bei Zostera, uno ber Saume, wie bei ihr, eimjeln.

Brupps: Z 0 stera etc. Conspectus No. 75 - 756. Fiora germ. 2.

4j. Fumilie. Etrongetwäd fie: Aroideae.

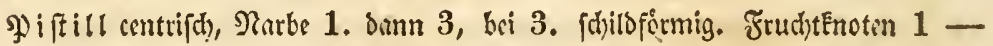

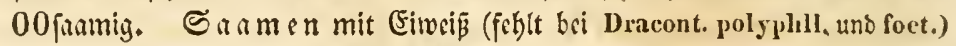
Reimling griffelformig, athfenftindig, mit Spalte für bic Sicimpilze, thi

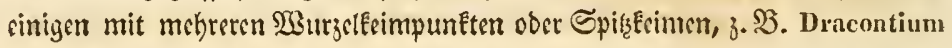

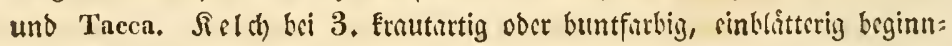

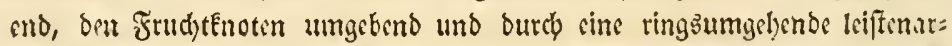

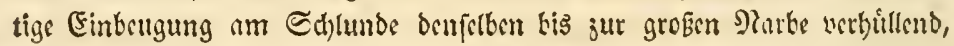
Saum fect)spaltig, bei Attaccia uno Tacea ber ganje ficld fetbstheiliy gefondert.

Staubbeutel entitshen zucrft (3. B. Caladiam) peripherifd) (nus umge:

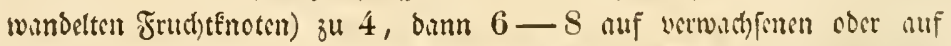
freien Stubbfioen vom \$iftill entfernt ober bei folgenden um buffethe ber: umgeffellt unb bei ben Taceen nuf bem Siclite am Jnnenrande bes

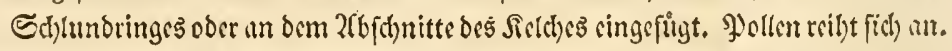

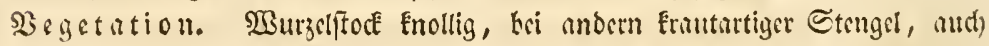
verbolzenoer, bewurzelter, Eletternder, felymarofzender ober aufrethter Stimm, (3. S. Pothos). B latter unten fithcibig, wir bei Zostera teginneno (Arum temifol.), bunn lanzettlid), bis fpießs= und fobilbformig, ficberfpult=

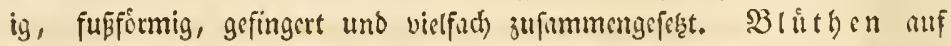

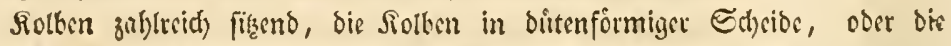

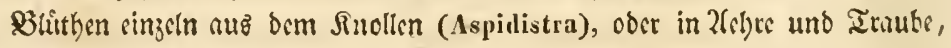
enblid) Dolbe, bei ben ubbrigen Iaccuceen.

(Sruppen: 1) $\Lambda$ raceac, a) Ambrosinicac, b) Dracunculinae, c) Caladicae.

2) Callacea e. a) Richardieac, h) Calleae, c) Oronticae.

3) Taccaceac. a) Tupistreac, b) Peliosantlicae, c) Taccene. Conspectus 766-785. 756. 780-781. 784. 785. 787. Flora germ. 1 . 9 - 10. excl. Acoro. 
Rtaffe: Edjeibcupflanjen.

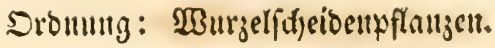

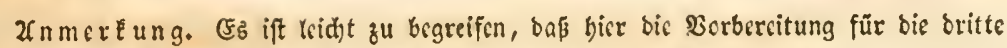

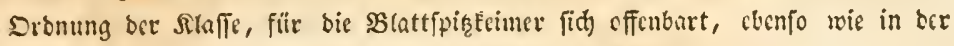
furciten Familie bic Sorbcreitung für bic zucite Dronung, bie ber ๔tamm: pflanzen unb in?befonbere ter Bsiäfr.

46. Familie. \$iptincen: Pistiaceae.

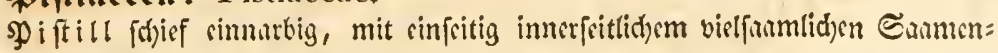
triger. Ea ame mad) Der 2fußentwand jugrkehrt, horizontallicgent, cen=

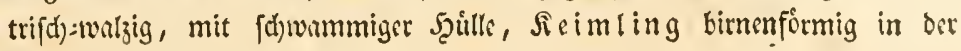
Epilįe des Eimciéres.

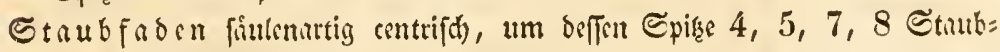

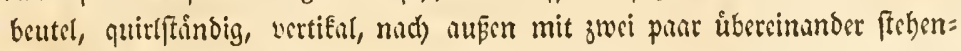
oen Sichern auffpringend.

Begetation. Etrnget gegliedert, eifig, fdywimmeno, mit Gestenfwurgel= birfochn, Siojetten aus umgekebst sirunben, nervigen Blattern, Blathen=

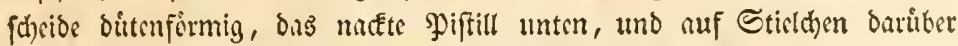

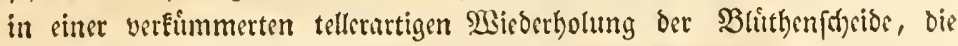
Staubfidenfialte. Die ganje \$flunje ift nach Blume's tieferer unter= furthung burd)aus mit (von Lindley hiber(ebenen) Spiraten burd)sogen.

(5ruppen: P istia-2futen ugl, bci B I ume: Rumphia vol. I. - Conspect. 765.

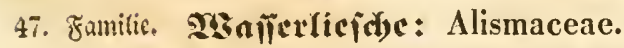

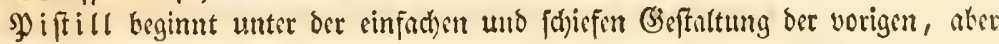
mit innerre 2(nlinge zur Theilung, mit $2-4$ aufrect)ten Sinmen in bin=

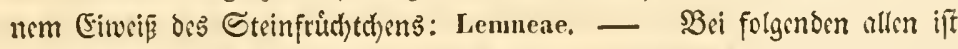

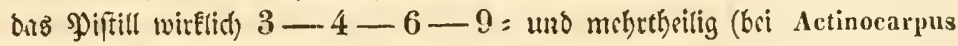

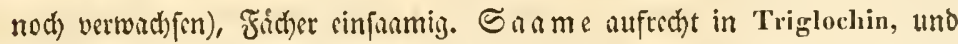

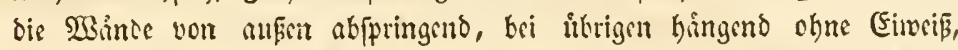

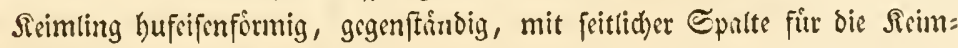

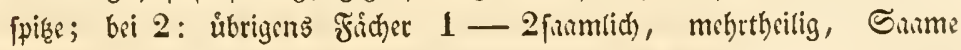
obne Eiwsiä, Seimling hufeifenformig, glcichftandig; bei 3: vielfanm=

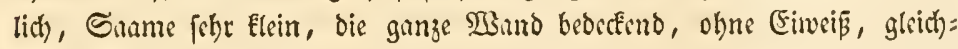
ftíndig. - Edyside, $3-4=$ und $6=$ bis mehrthsiligem Iir (d).

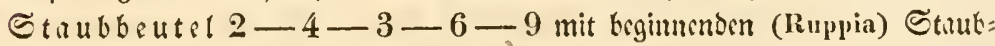
fáden, nllein ober um ben FrudtEnoten, siuffidrig bei Zaunichellia, bsi

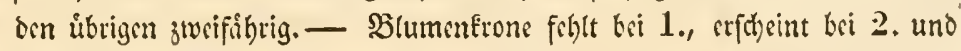
3. brciblittrig, finbig.

Begetition. Stumm und 3 hatt bildet fid). Lemna bat nod alles ver=

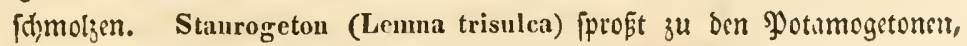
birr frobenartiger, wectjel = bis gegenftinndig beblitterter, flutbenoer Etenger,

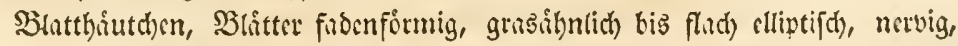

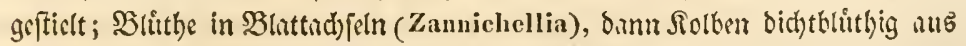

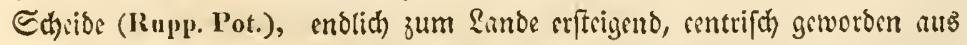

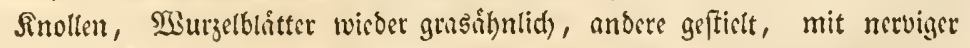
Slutte, Sdurft einblüthig, trinbig; quirltrnubig, quirtrifpig - bolbig. Frudyt meift eine Ifoiltêpfel: schizocarpium. 
Siañe: Edjeibettpflangett.

Sromut g: Marzelfacheibepflanzen.

\section{(Sruppen: 1) Potamogetoneae (Lemneac, Zamichelieae, Potamere. - 2) Alismeae, a) Philydrum, b) Triglochinae, c) Alismae. \\ 3) Butomeae.}

Conspect. 764 uns $591-801$, nebit 1306 uno 1288. Fl. germ. p. 6 - 912.

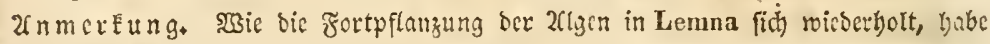
ich faton früber beriditet. Sebermann Eann fich fetbit bavon tiberzeugen.

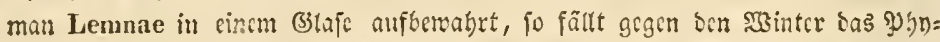

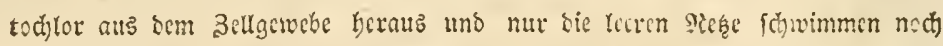

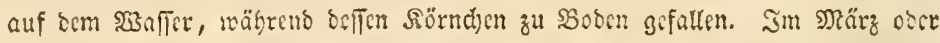

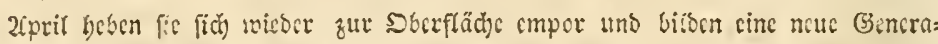

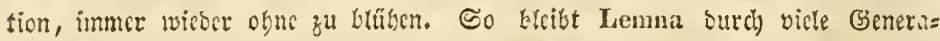
tisnen cryptogam und wirb nur burch befonderc Begünftigung phancrogram,

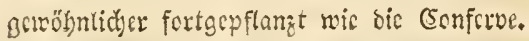

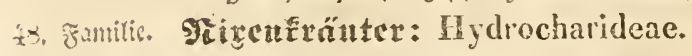

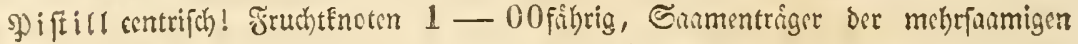

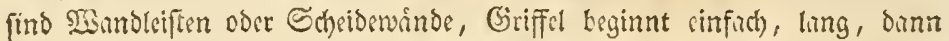

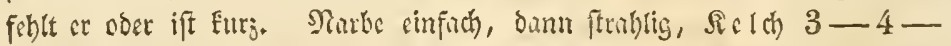

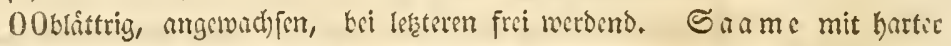

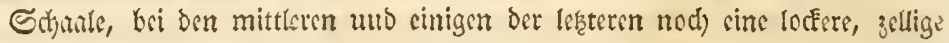

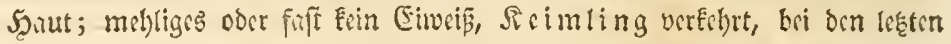
Giattungen mit zwcielinpigem Edjetionfack (nicht (5otyleobnen).

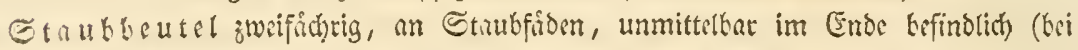

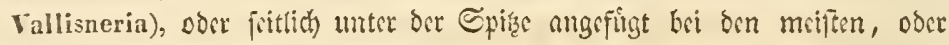

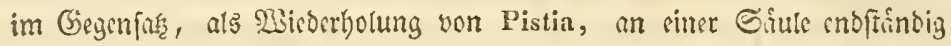
Enmuelurtig: Nepenties. Schcincorolle 3-00klattrig, bei ben lefgtern gethe ber Relly jicgetartig in bie Sirone itter.

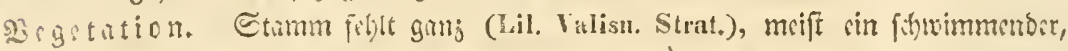

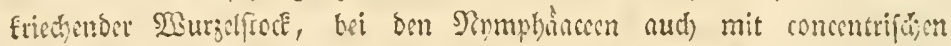

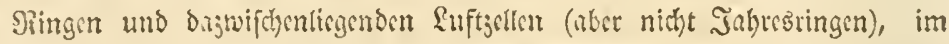

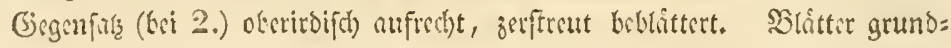

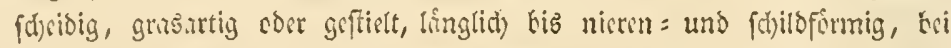

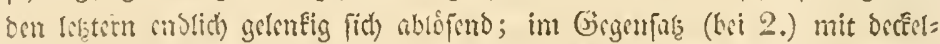

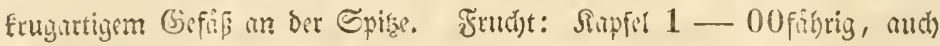

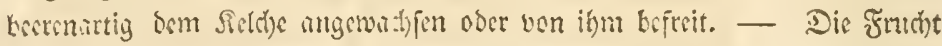

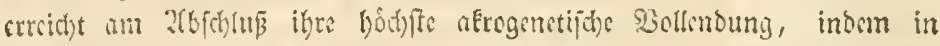

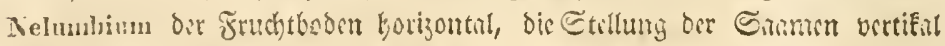

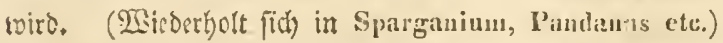

(Sruppen: 1) Stratioteae, (Lilaea, Flodea, Vallisn, Stratiot.)

2) Nepentheae.

3) Xyuphacaceac. Hydrocharene, Nyuphinene, Nelumbere.

Conspect, 802-818. - Flora germanica p. 13.

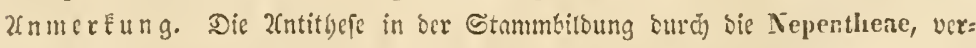
mittelt fich für jeccmann Yeid)t baburch, baßs bas Männlidje sic Pistia, altes

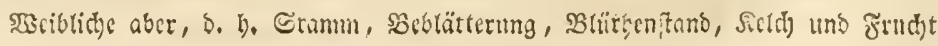


von aupen nidgts anderes ift, ats bie Fortbiloung uno böbere Entrvickectung von Yotamogeton, wäbrend Frudt unt Saame bie Gruppe mit bon IIyilro-

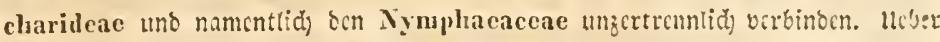

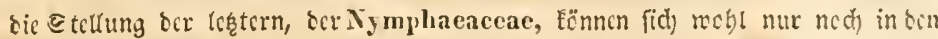

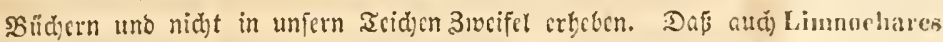

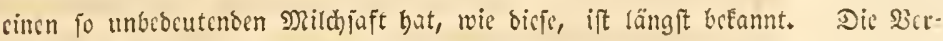
glcichung for sslïtle mit Magnolia geft nid)t cben tief in sas Dbject unto waß Dccandolle samit gcroollt hat, daßj feine Monocotyledonen feime folia convolutira bätten: ,Foliaçne convolutiva etc. caracteres in endogení nondum olsservatos "babe id nicmats verftonecn, weil in meinem exemplare feincr Théorie élémentaire p. 3̈2 fteft: Fenilles, ,eonsolutives - par cxemple le Bananier, le Balisier ete. idh atth an alten Ecitaminecn nis

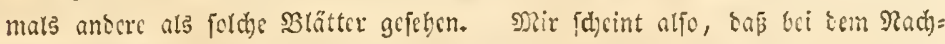

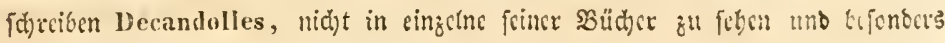
bic siatur folbft zu befragen ift.

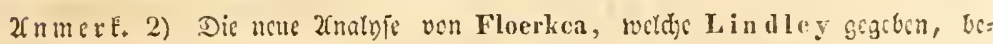

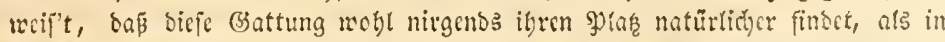
ber Bermandtichaft ocr Geraniaceen! Li ud l e y räpt fie burch vicle Familicu

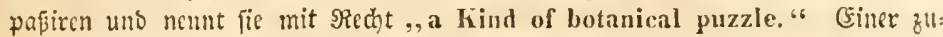

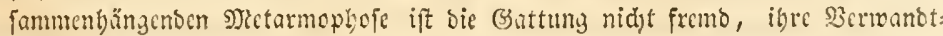

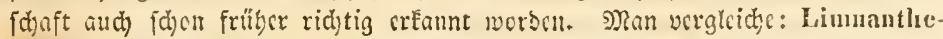
ae $R$. Br. bcil Tropacolene jutäăjt.

\section{Familis. Gráịx: Gramineae.}

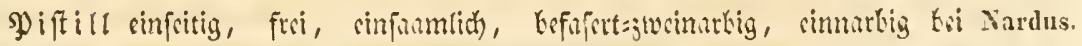

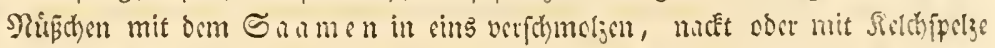

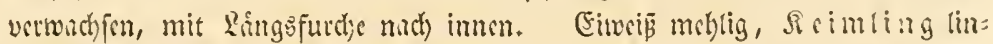

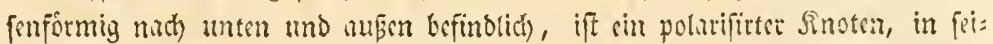

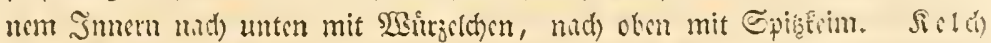
26littrig, ס.

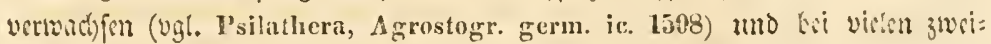

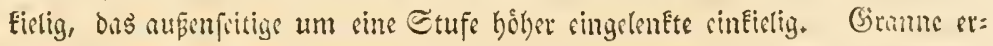

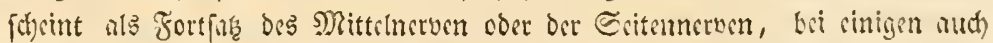
vom Relchbatte getrennt ober bei andern ohne burlelte, 3. 23. Andrepogan.

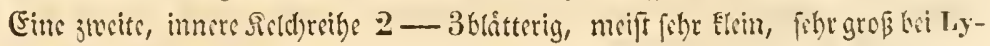

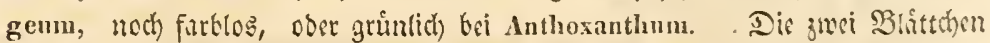

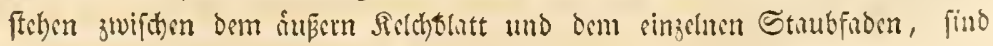

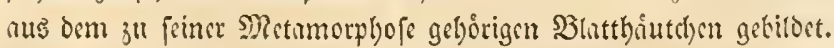

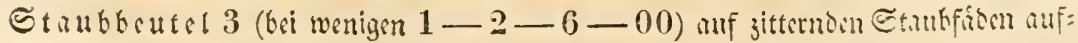

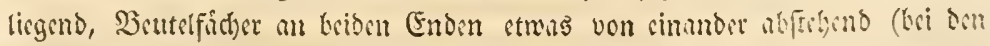
meiffert, lings ober oken auffpringend. Der vorbere Etunbfidon tri sinigen

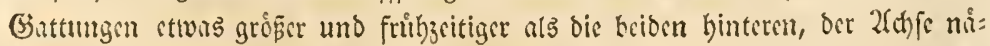

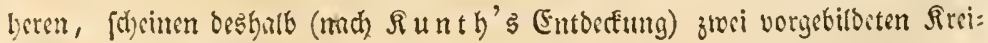
fin anjugchiren. 
Silaffe: Cd)riocupflanjen.

Dronutg: Stammidjeibentflanjen.

Begetatiou. Stumum ein ro̊briger Şalm, Enotig, die Blatter gehen vom obern

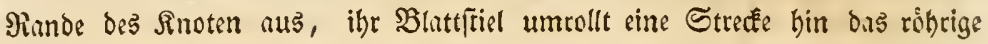

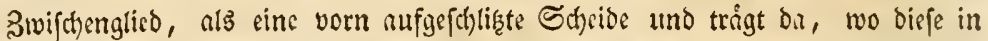

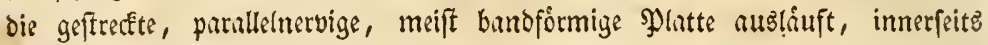

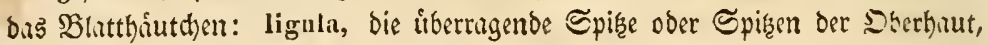
welche bie Scheide innerlich atselecibet. Der leģte Sinoten lof't fid) in bie Bluthenache auf, biefe ift fortgeferst ober gegliedert, ihtenurtig, traubig, finger= ihtig, rifpig (zufammengejogen ats Straup), Blitthen in Scheide: gluma,

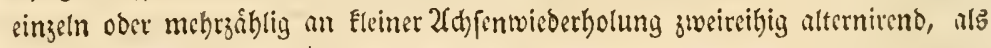
(3inz̧ithrohen: Epicula. *)

(3ruppen; 1) Agrostide ae (Agrosteae cum Chlorid., Avenaceae, Arundinaceac).

2) A udropogoneae (Oryzeace et Phalarideae, Paniceae, Saceluarinae).

3) Festucace ac ('Triticeae, Festuceac, Bambuseae).

Conspect. 819-1062. - Fl. germ. p. 15-54.

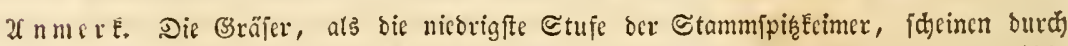
bie Ratur ben 2fuftrag erbalten zu baben, uns ben Sorgang ber erfiten, einfachften Stammbilos ung Flar ver Xugen zu Yrgen und fo wie fie 1) aub ben beioen Etementen: Fnoten und Röbre,

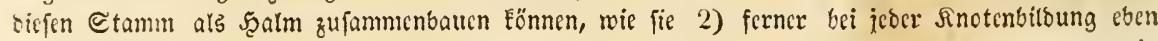

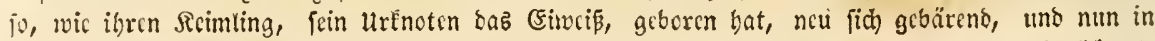

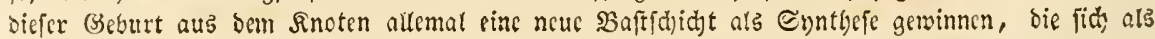

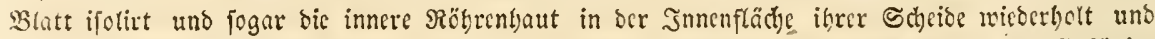
alb Blattbïuthen becnoet, fo find aud bife (Bräfer 3) befugt und berufen, Eraft ibrer Erichein= ung an biffer Stelle, nod) eine andere nothwendige Folge ifrer Sorganifation vor unfere 2lugen

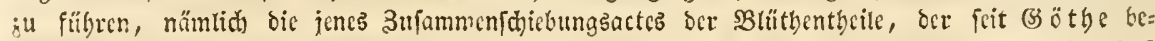

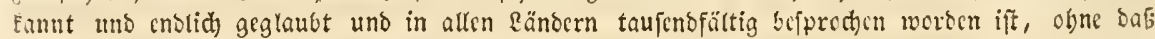
man baburd) geneigt worden, biefen sprozç ba feben z̆l wollen, wo bie Ratur ihn als norma!

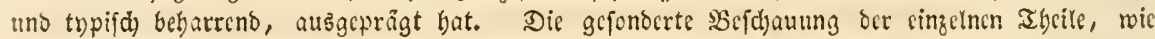
fie in neueren 3eiten geroöbnlid gemorden, hat alferbings immer ben slice auf bas saanze im Shaturleben gefloben und of ideefle Metamorphofen anticipirt, wo bic Shatur fie noch nicht as: boten. Limuée und Jassieu baben bagegen aules fehr ridftig erkannt und nur in ber Deutuny

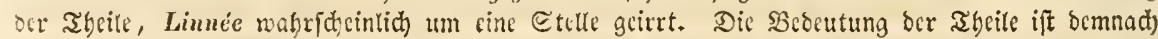

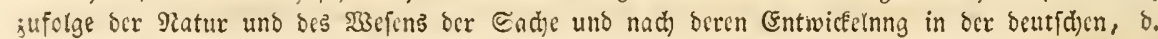
6. burd) bus ganje Taturleben verroärts gehenden Srganogenefe forgente:

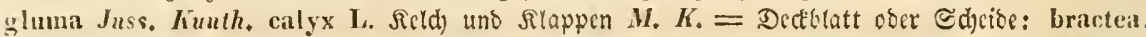
calyx Juss. corolla L, gluma Rich, palea ext. et jut. Kuth, SBtume und Spergen M. L. $=$ Ricldi: calyx exterior.

nectaria Schreb. squam. hypogynae $R, B r$. lodiculac $P, B$, Deféfpelzen $M . K$. $=$ inneret Sict(d): calyx interior.

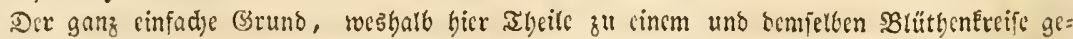

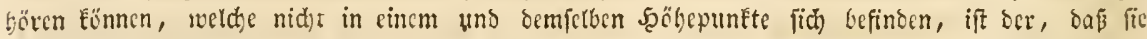

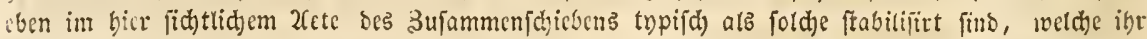

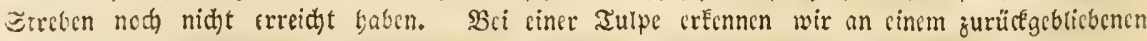

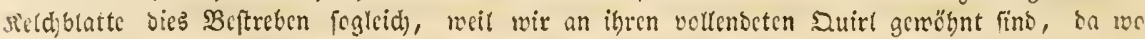

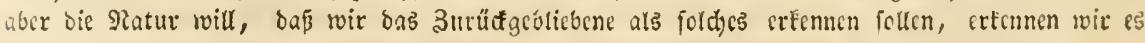
nidt.

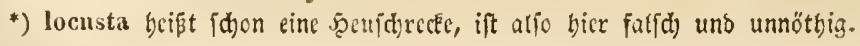


50. Zamilie. Cupcrg̣näír: Cyperoideae.

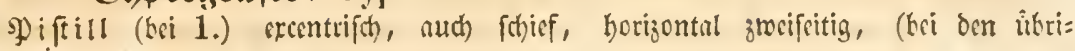
gen) meift vertifill, Fruchtenoten centrif do brcifeitig oocr rundid), (Briffel in 2 bis 3 befiferte Fudemnarben gefpalten. - Selch (bei 1.) zmeifperzig, grêpere Epelze unten, dic fleinere obere umfaffend,

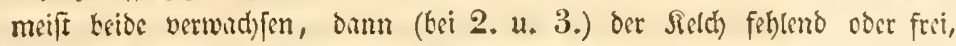
Sield)blitter 2-4-6-00, borfenformig ober linerlifh, ringsum ober an Den Pisudern baferig, wie bie Binttrinder der fluchbláttrigen Ginttungen.

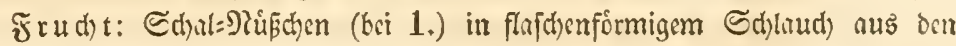

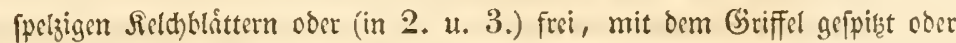

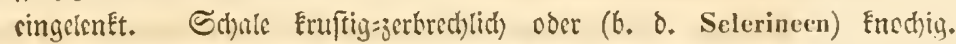
Eiwois fültt oen ganjen Innenraum, fieimling in oefien Baffis uno (alls 2fntitbefe beffen ber (Grifer, fo wie bie gamge Cyperoidee) in sin yo=

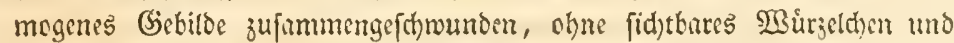
ohne Expižkim.

Staubbcutel meijt 3 , bei menigen 2frten ober Ginttungen $1-7-10$ - 12. Find jweifiddrig, oben bie Ficher parallel und yertienl lings des Staubfiden fo angewadtjen und biefen Theil bes Etrubfiden nuth vorn

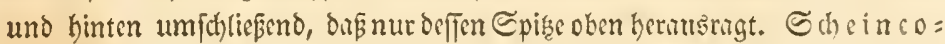

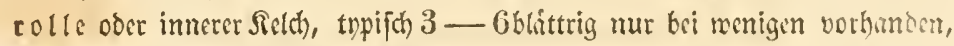
von ben mittcleuropaiifchen Gisttungen hat fie nur Malacochaete $N$. v. E. (vergl. Deff. fdjone 2rbbildoung: Gen. Fl. germ. fase. IX.), two indofiert mur 5 Corollenbliftter gezeritgnet find. Daß bier nicht un meibliche, o. $\mathfrak{h}$.

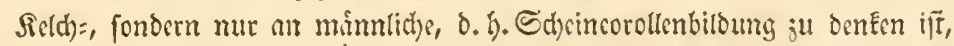
bemeif't die homegene Bitbung der Staubfadenfpize, mas N. v. E. ourd) Beifas der Fig. 4. 5. Deutlich berwiefen biat. (Diefe 2frt von Eorollenfor=

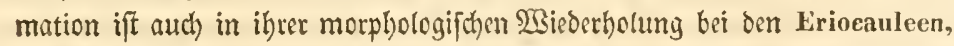

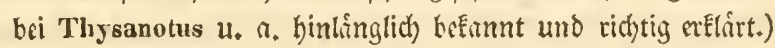

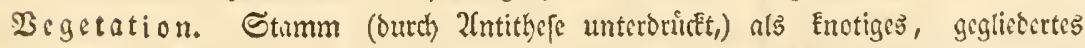
Sibizom soer geringetter Sinolle ober rafig anftocfend, centrifon gerrorben, unterirbifich, bei wenigen nod) fidttid): Carieeae, Selerineae. Sblitter aus ringsum gefdlofiener Scheide beginnend, bei cinigen nod grasthtt= sibnlich und nervig wir bei ben Paniceae, auch mit 2 latthintd)en verfeben:

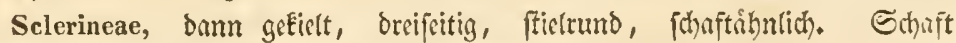

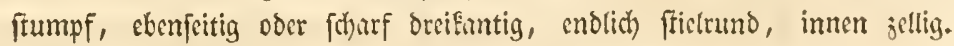

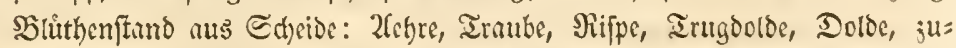
fammengefecte Dolde, 2fehrd)en aus Sdyeiden ober Decfblittern zicgrhartiy

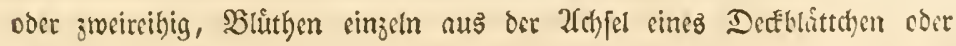
S(d)uppe: Gluma.

(3ruppen: 1) Caricinae (Cariceae, Clrysitriccae, Sclerineae).

2) Cyperinea (Cypereae, Schneneae, Dulichieae).

3) Scirpina e (Fimbristylcae, Scirpeae, Fuirencae).

Conspeet. $1065-1129$. - Fl. germ. p. $51-80$. 
Slatfe: Sdjeibenpflamzen.

Sroum : Stammidjcideupflanjen.

51. Familie. Riliengräier: Commelinaceae.

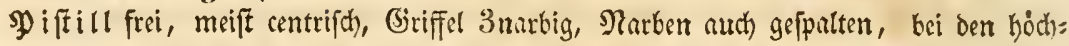
ften (Commel.) endlich vollendet einfidt), Frud)tenoten bildet fith ju 3 : (bei Lirioc.

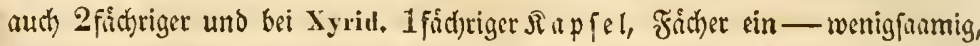
bai Xyrideae viclfanmig, Sa amen bảngent, bei Commelineae mit langem, linealifhen Nabel, bei benfelben auch mit Decfel auffpringend. Reimling

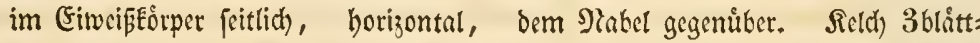
rig, bei Restiaceae aud 2 und $3 \mathfrak{u} .3$ alternirend, fpelfig, bei Eriocauleae 2 nach vorn und 1 mach hinten.

Staubbeutel $3-6$, bri Rest. $1-6$ find 1fichrig, bei ben übrigen 2= fichrig, bei Commel. mit breitem Steg. Etnutfifocn bei Rest. nuf bcm

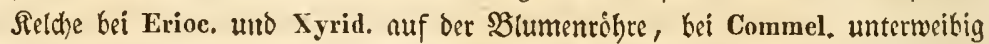
uno bartig. Scheincorolle fehlt ober fpelsig bei Restiaceae, bei ubrigen 3 blittrig, aud) robrig vertwadben, himfillig.

Begetation. Stumm bei Rest. uno Commel. granartig, Enotig, mit Fufer= uno Ed)eibenblittern, auch $\mathfrak{B l u ̛ t h e n f h e i b e . ~ B l u t h e n ~ j w i t t e r l i c h , ~ i n ~ 2 ( e h r e n , ~ I r a u = ~}$ ben, SRifpen. Bei (ber 2(nttithefe) Xyrid. wic bei den Cyperoideen Eein ober niojt Enotiger Ctumm; SBläter grunoftheibig, fothmal, meift bart und reitend;

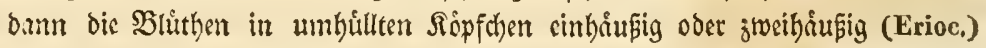
oder bei ůbrigen zwitterlich in zapfenartigen 2fehren (Xyrideae).

(3ruppen: 1) Restiaceae.

2) Xyridea e (Eriocauleae.)

3) Commelineae.

Conspert. $1130-1175$.

52. Familie. Mefrfolbcu: Typhaceae.

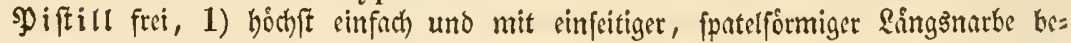
gimnent, 2) in ber 2fntithefe nus biefer cimpeitigen \&ingšnarbe (Sparg.) mit Neigung zur 2 - 3sipaltung, bann 2) in getoonnener (onsentration von 2 ju 3, auch $2-3$ B̧andplacenten abjegend (Freyc.), bei Pandanus wieder afrogenetifh centrif(t) werdent (wie oben Nelumbo) erfeheint bie Fruetht 1) bei

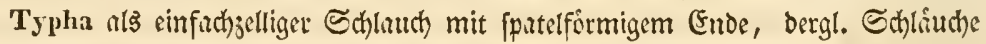
Eommen nudb) viele leer oder fteril vor. 2) bei Sparg. Freyc, uno I'and, ift bie afrogenetifhe Eroffrung gewonnen: Dectelftapfel. — Sanmen: Seim:

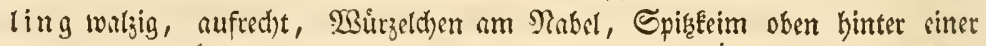

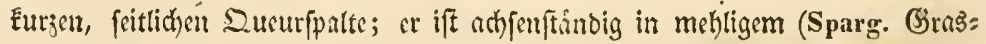

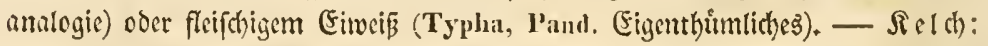
1) zurtgegliederte Fióden, Dem Fruchtid)laudye unilog gebildet umftellen die $\mathfrak{B a f i s}$

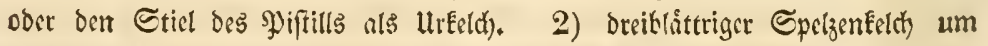

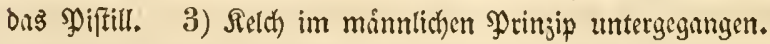

Staubbeutel: 1, bei Typha ans auf gemeinfidaftlicten Stielen zu $2-3$

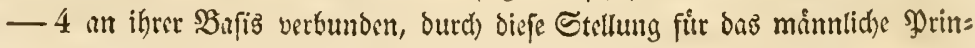
jip umgebildete \$iftilker tritt an jedem auf jeder Seite gegeniber cinc zmei=

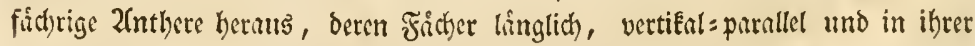
ganjen \&ange auffpringen. Das fterite \$yiftill und fein Eolfiger brüfiger 


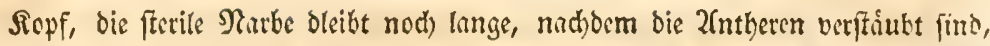

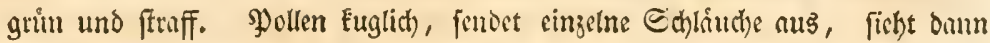
pilzförmig aus. Urcorolle: $3-4-6$ zart lincalifdye, contimutrlidse

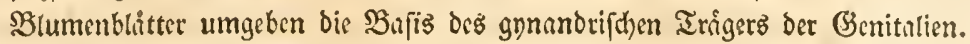

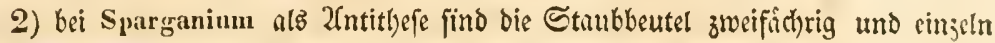

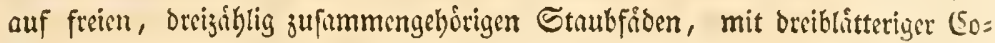
rolle unterfftúgt. 3) bci Pandanus find die Stanbfíden vicder zu cinem

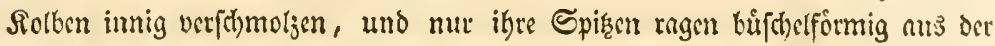
Serfotmelzung heraus, ebenfo bei Freycinetia, wo bie aus ber allgemsinen

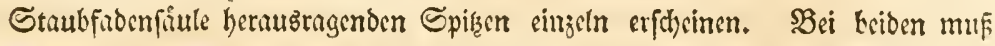

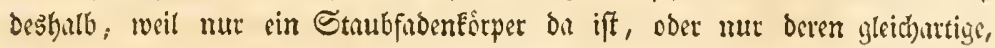
wie bei Freyc. beifummenftehen, die corollinifde Bebcutung in ber, kei Pandanus cinblittrig, bei Freycinetia nber mieber vollenteten uno farbigen,

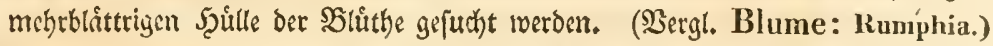

Begetation. Stamm. 1) Intoten bei Typha, Sdhaft blatttrig, Blâtter Föribig' fohwerdofortmig, oben ein weiblidser, unmittelbar barůber ein minntither Solben, jeber aus cinjelnet, ulternirender Sdyeide. 2) Sparganium im (5iggenforge, mit auß einem Sinoten verthingertem, Etraturtigen Stengel, wenige rinnige Blittter,

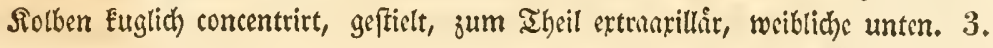
Pandanus uno Freycinetia baben holzige, baumartige, jogar oben áftige Ettim:

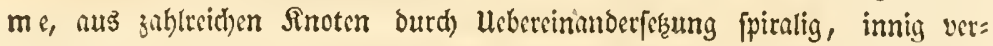
fojmolzen, baber bie Blâtter, wie bei vorigen, rinnig und gefielt, bier aber bei Freyc. jum Theil und bei Pandanus (Erinnerung an bie Bromelinceen) ginglich) nimperartig=fígentandig, fohr beftimmt fpiralig geffellt find.

(3ruppen: 1) Typheae.

2) Sparganieae.

3) Pandaneae.*)

Conspect. 790. 791. 1270. 1281. 1273. Fl. germ. gen. X. et XI.

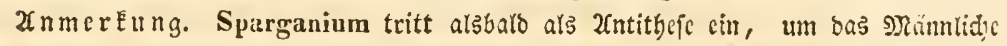

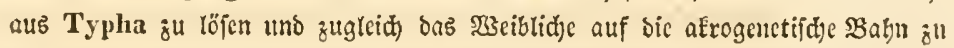
Leiten, bie in Pandanus burch aufredten ভaamen verbarrt, wäbreno Freyci-

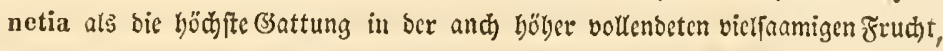

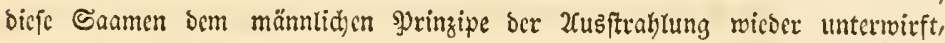

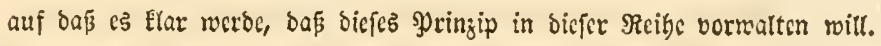

53. Familie. Edytwertel: Irideae.

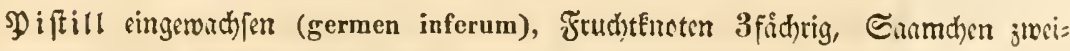

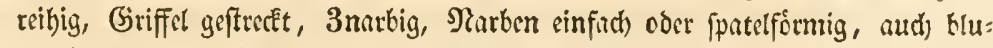
menblattuntig, 8. B. Iris, oder 6 theilig und gefrumft bei Ferraria, Relch unten róbrig, um ben Frudjffnoten herumgetwad) jen, Saum 6thitig, dic 3

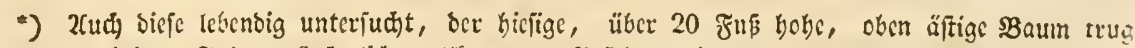

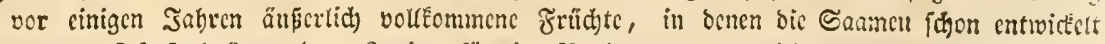

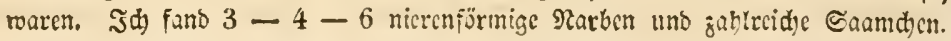


innern 2(bjanitte ober alle corollinifa), bei 1. und 3. regelmápig, bei 2. an= tithetifo unregetmásig. Sapfel orciflappig, oreifáchrig, אlappen in oer

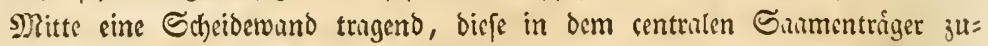
fammenftopent. Saamen an beffen Leiften zweikeibig, Jeimling líng= lich, gefrummt, inmen auffreigend, in groferm fleifhjigen ober bornartigem (Fi= mci巨. -

Staubbcutel 3, anfrecht, zuerft auf Stnubfabenfáte gemeinforaftlich zufammen= geftellt oder verwadhen (MBicberfolung von Typha), bann frei und bie orei Staubfáden mur mit ber $\mathfrak{B l u m e n r o b}$ re verwachfen bei Iris $u, n, 2$ ) in ber Silumenrobte unregetmápig, wic bie $\mathfrak{B}$ lame jelbft, bci Gladiolus ete. als 2fnalogon ber Esrifer, man denfe an Diasia, Watsonia plantaginea, triticea

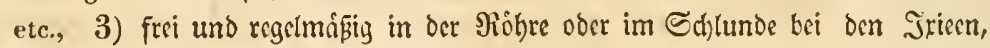
mit regelmip̈iger, meift ganz corollinifher $ఠ$ deincorolle.

Segetation. Stamm borizontal Érichend, geringelt:Enollig, aus fo viclen Fino=

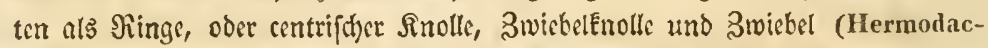
tylus Fl. germ. p. 83.). - Schuft oder beblatterter frutattiger Stengel,

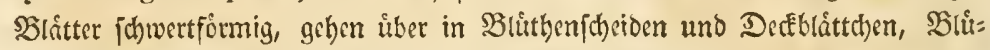
then cinjeln, ahrenartig, trutbig, gebifchelt trabbig, alternireno, oichotom, rifipig, fopfig, Doldig. Strmm holjig bci wenigen, z. $\mathfrak{B}$. Genlisia, Witsenia.

(3ruppen: 1) Ferrarieae. -

2) Gradioleac. -

3) Ixicae.-

Conspectus 1186-1209. Flora germ. p. 80-86.

\section{Familie. Marjifienidjwertel: Narcissineae.}

Siffill singemidfen, bei wenigen in 2. uno 3. (Tillanls. Pitc. Wachend.), frei; Giriffel geftreckt, গlarben breilappig (bei einigen Bromel. groreht), thi ben bich)=

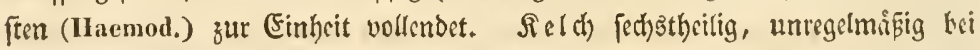

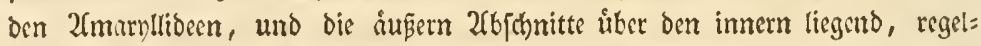

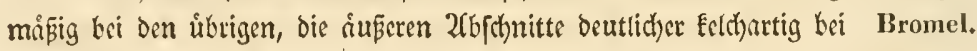
Burm., alle faft gleidh, theils verwadj fen uno meift aupen wollig bei ben Hypoxid.

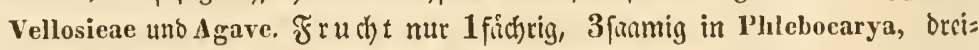
fáchrig und vielfanmig, aber nidjt auffpringend bei Hypoxilcae, ưkrigens meift

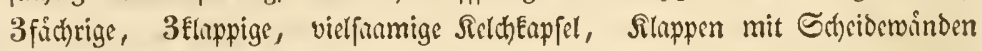
in ber Mitte, fie bat von ben Reldhabfthnitten berablatfende Flught bei ben Burmannieen, bei menigen beerenartig, alle verfămelzen becrenartig z. B3. zu einer Sammelfrucht (synearpium) bei Ananassa, und ift endich wicber vom Sicthe ganj frci bei Tillandsia, Piteairnia uno Wachentorfia. Die Fácher find 1-2-00jarmig, die Sa amen fluch, z. B. bci Cyrtanthus fuft pa= pierartig, ober fotildformig bei ben mebrfaamigen Hacmodoreae, oder flich und rundlich oder narkig bei andern, fehr Elein bei Bromel. uno geffreift bei den Burmann, Gdhale bủnn, bei ben Hypoxid. fobwatz= Enuftig mit feitlidfen

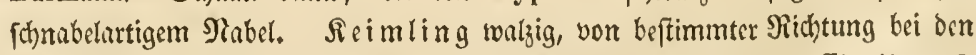

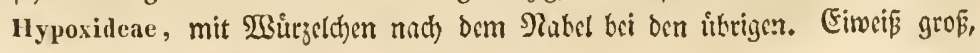


Silatie: Sdjciocmplanzen.

Dromung: Etammidjciocupflanjen.

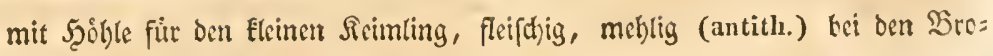
meliaceen uno Şämodoreen.

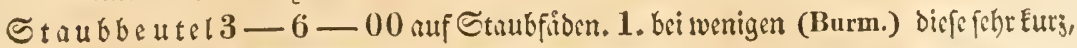
nur 3 Scutel beutlich, mit brcitem Steg (analog Commel.) queer auffpringend, im

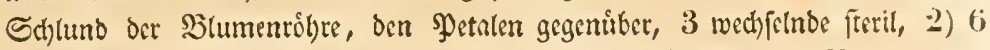
vollemmene in ber Mióbre oder dem Salund ber Slutthe bet ben Narciffen uno úbrigen, febr tief im Re(d) bei Hypoxid. Bromel. und Vellos., uno juhlecid)

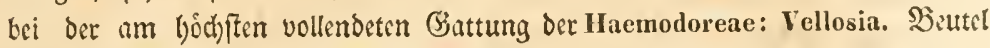
auflicgend oder meift aufrecht, zweifichrig und läng auffpringend. $\mathfrak{B}(\mathfrak{u}=$

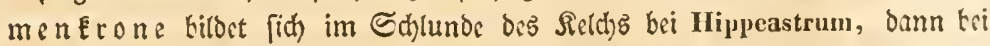

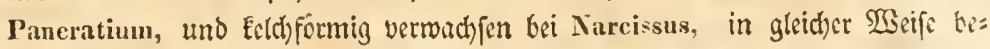

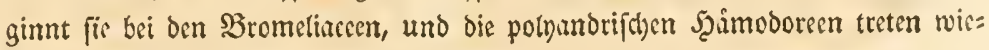
ber ganz fdycincorollinift) auf.

Segetation. Stumm: 1) 3iviebel bei den 24marnllideen treibt reitende Blitter

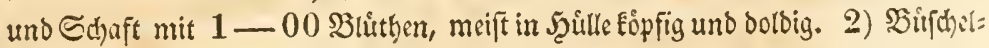
artige $2 \mathfrak{S u r z e l}$ mit reitenden $\mathfrak{S}$ lattern bei Burmanniae, ober mit alljeitig ge= wendeten, nervig fultigen Blittern und Sthaft bei Hypoxid,, ober mit ๔tamm, melcher aus dicht in einunder gejthobenen finoten verholzt, bei Doryanthes unter ben Narcisseae, bei ben Bromelieae, Eaum bei Cureuligo (Hypoxid.) aber bei

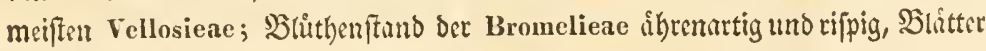

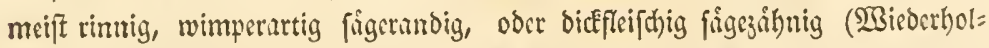
ung von Stratiotes) bei Agave; bei Vellosieae rifpig (Wachenl.), trugbolbig bei ben meiften und entlich) tvicber einbluthig polyandrifch und ganjnartig auf

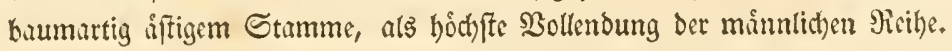

Sruppen: 1) A maryllideae, (Cyrtantheae, Amarylleae, Nareisseae.)

2) Bromelieae, (Ananasseae, Tillandsiene, Agaveac).

3) I a emodoreae, (Burmannieae, Hypoxideae, Vellosieac). -

Conspect. 1210-1284. - Fl. germ. 86-90.

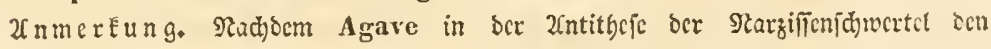

Stamm wieber gerwonnen, muß jie ifn in oer Syntheje ber Bromeliaceen wirder

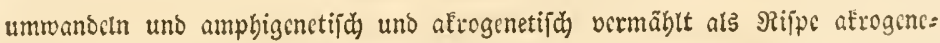

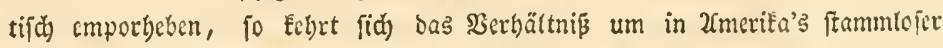
Agave, bor gröfiten und am weiteften verbrciteten von allen ber Battung.

5̌. Familic. Eimienlilicn: Juncaceae.

Pुiftill beginnt und chbet mit simfud)em Fruthtenoten, bei allen mittlern Giattungm ift Derfelbe 3theilig jerfillen. Narbe bei 1. a. Itreitfolbenformig, bei 1.b. '‘. und wieder bei ben lef̧en $3 \mathrm{c}$. oreitheilig. Sield bei 1 a. zweifpelzig, bei 1 b. c. zrosireifig 3 ppetzig, alfo alternirend 6 fpelzig, kci 2 . Etrutartig, kei 3. corollinifh, bei kciben alternireno 6theilig. $\mathfrak{f r u d}$ t bei 1 a. cin sinfanmiges

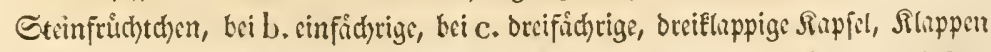
in ber Mitte bie Sdbibetwand tragend, SBinde am Snnentande 1-wenigfau= mig; bei 2, uno 3. fint bie 3 Sippiclfächer meift hûtfenartig=getrennt, 200faumig, fpringen an ber Snmennath auf, an beren Riubern fie bic Eau= men trugen, bei menigen fo roicoer wie bei Juneus eine Edjeiderwand in oet 
Shañe: Edjcibentlanjen.

Sromung: Blattiheibemplangen.

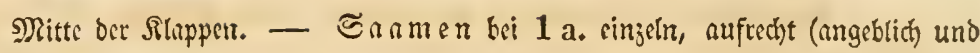

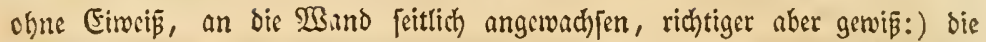

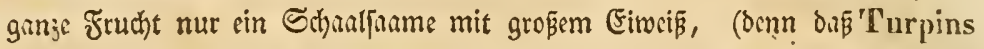
"tissu corpusculaire de l'embryon "Eein Fleif einç Embrno, fondern ei= nes Firweī̄es ift, burfte Eaum zweifelhaft fonn, leider finde id an meinem Eremplare Eeine Frucht), bei 1 b. und c. weniģihlig, nufrecht, mit fleifdigem

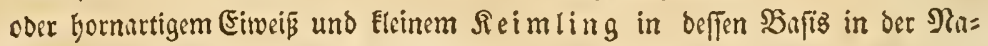
belgegend; bei 2 , in jedem Fach 2 Saamen aufredt, ohne. (Eiweí, Reim= ling mit Ringşpalte fü ben Spifketm; bei 3a.b. baben bie Sanmen ein fleijhiges Eimeiß und ber Eleine watlige fieimling liegt in Demferben (wie Commel.) dem Saamen gegenitber, bis endich bei 3 c., als ben am bochliten

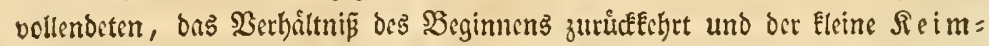
ling in orm einjellen Sanmen jeocn Fadbs in ocr Bafis uno Nabelgegend in bornartigem (Fiwcis bewahnt wirs.

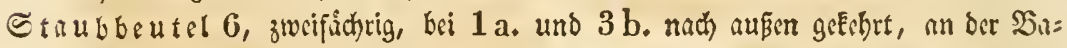

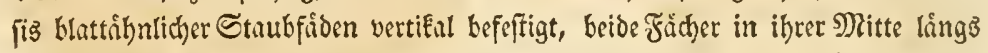
aufpringend; bei allen folgenden eintwirts gerichtet, rundlich, herzformig, láng=

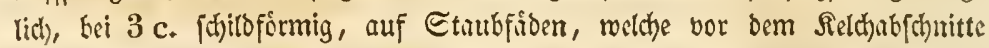
cingefingt find, aufrecht; und wo beren 3. vertirmmern, find dieß bie vor ben

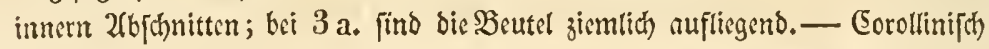

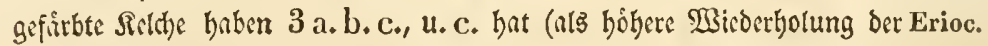
und Xyrideae), in ber minnliden SBlithe cine breityeilige Sdycincorolle.

Begetition. Stam:n. Rbizom gegliedert, horijontal Eriedend, bciandern (Sattungen centrifoh, jwiebelartig, mit Schaft, endlich mit beblettertem Stengel, welḑer Enautartig wirb, bei den am meiften vollendeten: 3 c. fraudbartig verholyend. Slitter foheibig, vom zofterenartigem 3uftande 1 a. zum pfriemenformigen, fåchrig articulitt=zelligen, grasurtigen, bann pabibig=rinnig, flach rippiggefaltet (Veratrum), endich bart und reiteno bei ben frnutbartigen Xeroteen.

(Sruppen: 1) Junceae, (a. Posidonia, b. Luzula, c. Juncus).

2) Scheuchzeria.

3) Melantheae, (a. Colchicene, b. Veratreae, c. Xerotene).

Conspectus 1285-1315. (excl. 1306.) Flora grerm. p. 90-99. (exel, No. CXXXVII.)

56. 8nmilie. Brufenlilicu: Sarmentaceae.

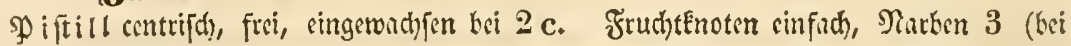

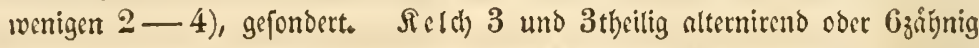
6tbeilig, bei wenigen 4theilig (Paris quadrif. Roxb. Maianthem.), frautartig ober ber innere Sireis oder daz ganze Giebilde corollinifh; frei oder bei 2 c. um

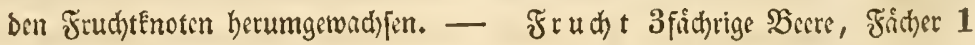

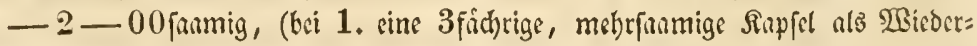
holung von Junens, bei 2 b. cine einfächrige, 2kluppige, vielfanmige Sapfet), boi 2 c. sine 1 - 3fádrige gefliggelte Rapfel, mit geflingelten Saumen. -

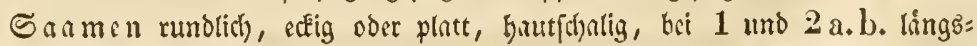

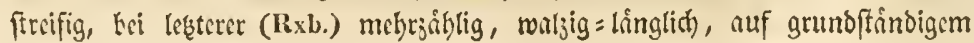


id)tammign Sanmentriger alle aufrecht. Eimeiß fleifhig, Feimling Elein,

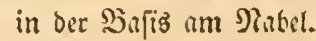

Staubbeutel 6, (bei wenigen 4-8), mublich) ober geftrectt, zmeifichrig, Fact)er an oder über ber Bafts der Staubfíden bei Paris und lzoxburghia, bei leşterer gefondert, innerfeits an corolimifden Strubfíoen, bei beiden ragen bic Enden

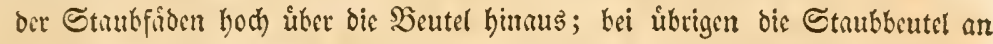

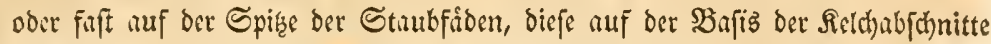
und vor iffen in ber Mitte, ober in ber Selchróhre bei andern, bei 3 b. Jinglict) verroid)[en. Scheincorolfe 3blatterig in Trillium uns Ruscus, vict: bis fintuloltterig in Paris.

Segetation. Strmm. 2fus Eriechendem vielfproßendem ober entrifhem, bei cini= gen ungehcuerem, meift mehligen Sinollen Eraut= oder ftrauchartiger, alfredtet oocr Eletternoer Stengel. Sllitter meift flich und nervig, firend ober gefitielt, twenigen fa)eibig oder rimnig (Acorns, Ophiop.), theils leberartig, bei cinigen

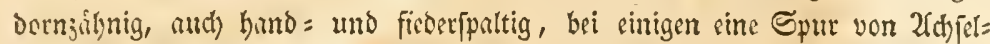
blittern (Rusc), quirlftindig bei 2 a. uno wenigen von 3 a., fonft figend oder meift gefticlt, gegentiber bei $2 \mathrm{~b}$. ober bei den übrigen wech felnd; Shlutbe bei 1 .

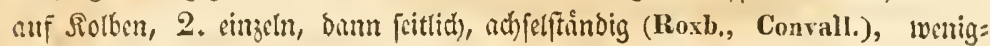
b!tiţig auf 2(ch)felfticl (Boxb.), achfeltrubig (Diosc. Smil.), enotrubig (Smil.), unter oder auf bem SBlatte (Strcptop. Rusc.).

(Bruppen: 1) Acorinac.

2) Dioscorinae, (a. Parideac, b. Roxburghia, c. Dioscorcae.

3) Smi la cinae, (a. Convallariae, b. Rusceac, Ü. Smilaceae.

Conspectus 786-789. 1316-1811. Fl. germ. p. 99-101.

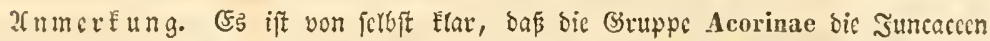

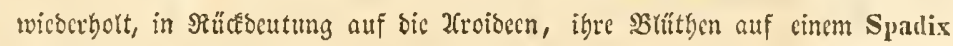

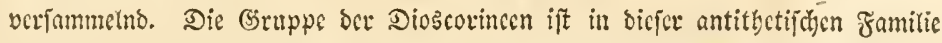

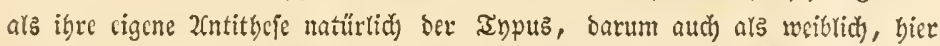

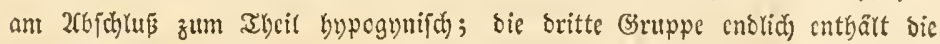

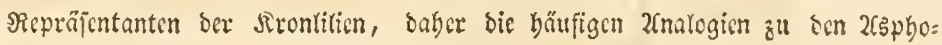
Seleen. -

\section{Fumilic. ßronlilicu: Coronariae.}

Sูiftill centrifo, nut ein wenig fobief bei den beginnenten formen von $1 \mathrm{a}-\mathrm{c}$.

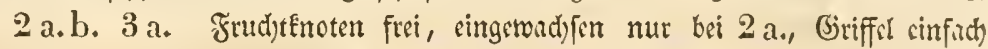
(Foblt bei Tulipa), গarbe aus 3 ver(chmolzen ober cinfact). Selth) 3 und $3=$ theilig wech/felnd, bei wenigen (Gattungen (unter 2 a.b. uno 3 a.) Etruturtig,

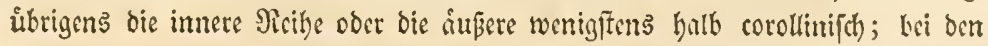

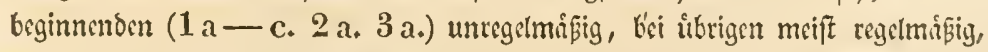

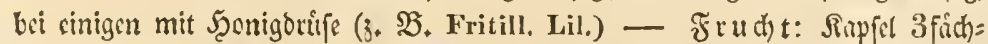
rig, 3Eluppig, (bei Miersia mur an ber Spike auffipringend), Edteidewånde mittelftindig. Sarame aus ber Mittelfiule, zweircihig, meift 00 zíhlig, beffen

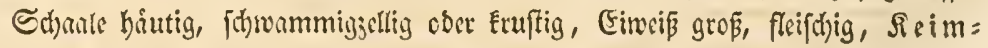

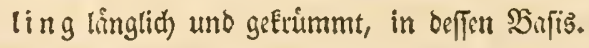


Echeibenplanjen.

Srouung: Blattidjeibentlanjen. Sirontiticn.

Staubbeutel 6, (bei Gilliesia feblen 3), eintwirts, nufred)t oder aufiegend nuf

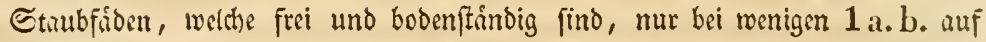

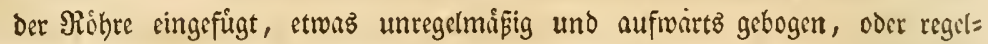
måfig bei ben meiften, ganz fojief uno verwachjen und nur'3 mit Beutel bic

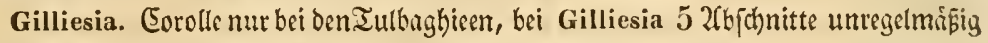
lappig beginnend, bei Miersia 6 gefpaltene, bei Tullaghhia entlid) bicfe 6 ge=

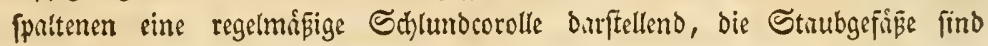

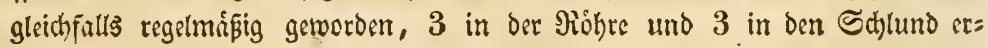
hoben. *)

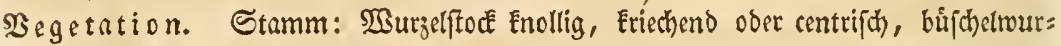

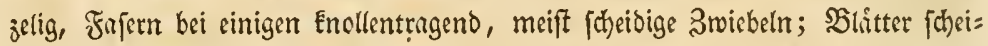
big, meift fohmal und rinnig (bei wenigen fo breit wie bei Massonia), auch) reitend (Nartheciu'u, Phormium), faftig frautartig, leberartig ober bickfeijchig, wurzelftindig ober ftengelftindig und fikeno, wenige geftelt 1 a. verbebt, aud

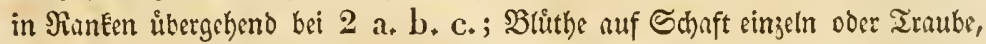

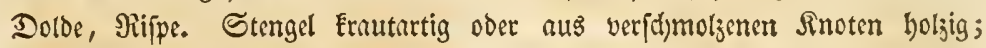
Sluthenftiel bei $3 \mathrm{c}$. meift mit cinem Rnotengelent.

(sruppen: 1) Hemerocallideae, a) Pontedereae, b) Poliantheae, c) Hemerocalleae.

2) Tulipaceac, a) Alstroemericae, b) Methoniceae, c) Tulipeac.

3) Asphodcleac, a) Tulbaglieae: Gilliesia, Miersia, Tulbaglia, b) Scilleae, c) Dracaeneae.

Conspectus 1343-1413. - Fl, germ. 101-118.

58. Familie. Ordhibect: Orchideae.

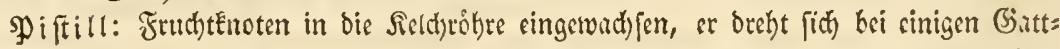
ungen von 1. zur Blittyenzeit in einem ganzen Sireife herum, Giriffel als Siobre

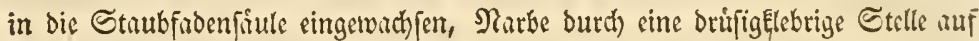

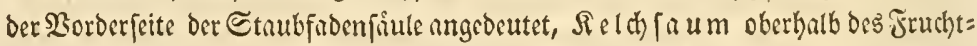
Enotens 3theilig, meift farbig, unpariger 2 bbfanitt oben, itbrige feitlit), nods 2 Blattet)en als zmeite Siethe zwifhen jenen nad) oben, neben ber Staubfa: benfiule. Frucht: cingetwadyfene, meift mit bem Sield) getronte, (Jeldh) abfall= cllo bei Vanilla), cinfichrige Sapfel, 3 - 6rippig, cinficthrig, nur bei A postasia oreifachtig, 3 Elappig, אlappen in ber Mitte lings mit Samenteifte, zwifd)en Sippen abjpringerto, welche geftellartig beifammenbleiben. Saamen úberaus zablreich), fein fpreuformig, meift mit madh beiden Enden fich) fort ferender, fd)hffer, zelliger Schanle, Reimling linglid), in oer Bajis cines fleifthigen Eimeipez.

Staubbeutel 1. endftindig auf biffem Strubfaben, welcher meift fur ift, auf

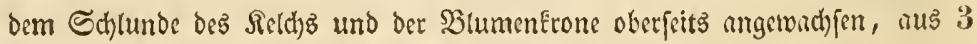
verwach]en, ber Beutel vorn ummittelbar fortge[est, $2-4-8$ fid)rig, bei ci=

*) Miersia und Tulbaghia nad) unterfuduung im Eeben. 
nigen ber Beutel cingelenft, aud) mit Defel; hei Cypripedium ber Endbeutel blumenthtturtig wueberno, und von zwei feitlichen Fortfikgen triggt sin jeoce sinen queriber zweifichrigen Stutbbentel, bei Apostasiene firt 3 Stinbbeutel

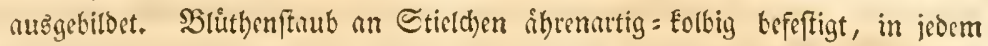
Seutet $2-4$, Etaub pulverartig oder machäartig, Eticl bei vielen auf ciner

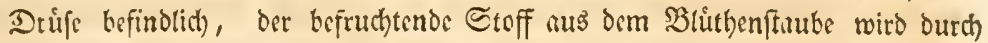

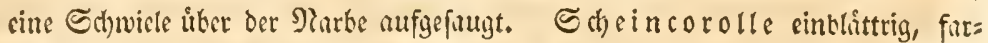
big, bei sinigen gefpornt, cine Sippe als Gegengebilde oer Etnubfidenfoutule,

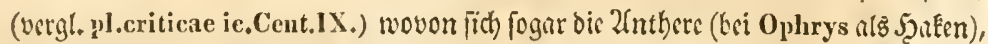

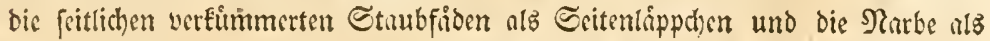
SRectarbrufe oder anders gefirtbter Fled twicber abfpirgelt.

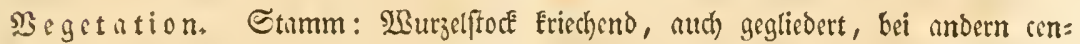
trif(d), fleifd)iger Sinolle, bei andern cin Enollenåbnlid)er Sinoten, yon Sllattern (d)eidig umbitlt, Etenget Erautartig oder verbolgent, Dam auth fohmurokend, mit bicken Ruftwurzeln. SElatter jetyeibig, bei vielen reiteno, gerno= oder Etumm:

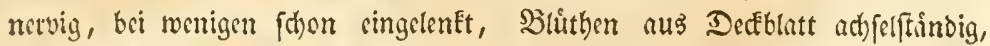

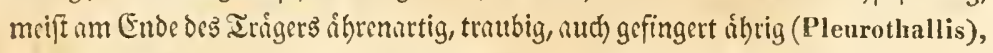
auf Solben (Megaclinium), bei ben fnotentrigenoen auch acj)elftintoig, aud) rifpig. (Sruppen: 1) Orchide ae genuinae, a) Ophrydeae, b) Epipaeteae, c) Malaxilleae, - Epillendreae, Vandeae, Vanilleac.

2) Cypripedieac.

3) Apostasieac.

Conspect. 1414-1584. - Fl. germ. 118-135.

\{nmerfung. Die foböne Familic ift nun burd) Wallich's und Blume's berrlide Ent= occkung ber Apostasia uno Neovidia enolid) natür tid) geworbon, b. b. fic bat bas 3icl ibrcs

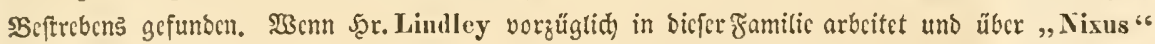

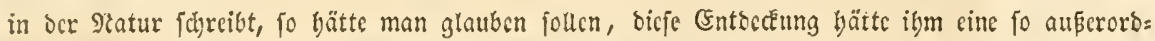

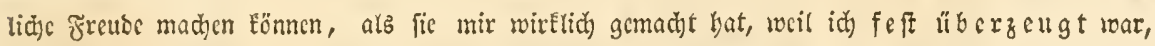

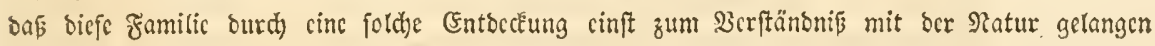

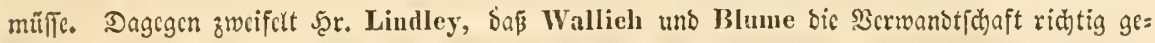

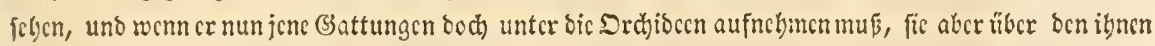

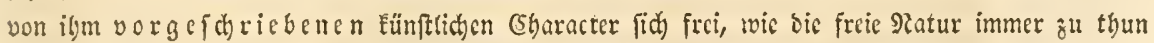

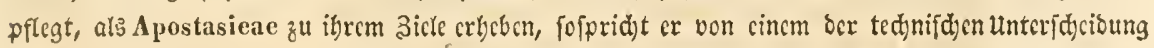

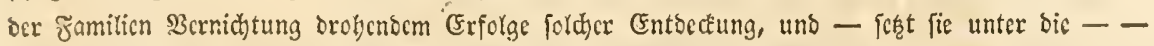
Xnomalicen! - - Dic ur= Mctamorphofe bcr Familic berubt übrigens bicr auf ber Srga= nogenefe ocr 2(nttgere, bas crfte Moment bor (Fintheilung fann alfo nur burd) bic Scauptfufe bor

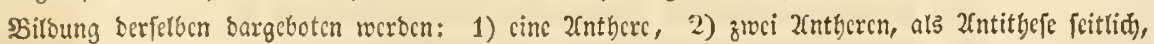

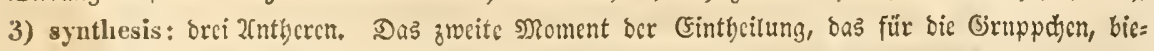

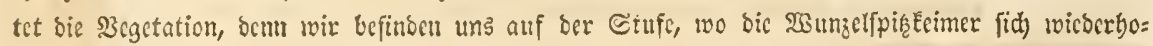

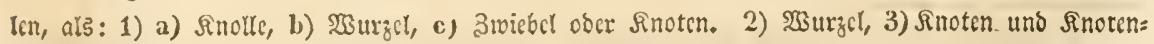

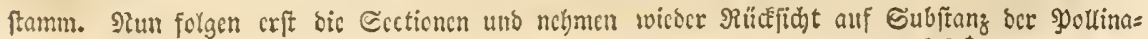


rien, auf sippe mit ober obne Sporn u. \%. w. - \as barf robl Eaum angebcutct zu rocr

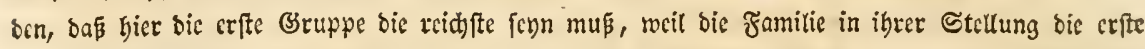

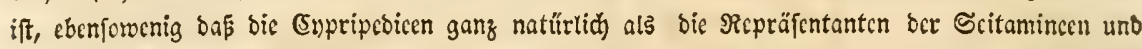

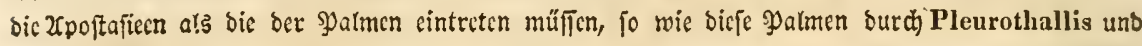

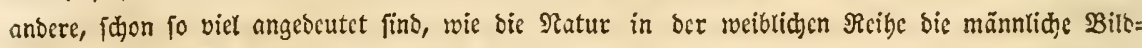
ung oon Tulbaghia in Gilliesia vor 2fugen getegt bat.*)

\section{Familie. Bananen: Scitamineae,}

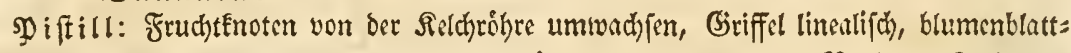
artig, bann fadenartig mit tridfterfómiger ober getheilter $\mathfrak{R}$ arbe. Reldfiaum

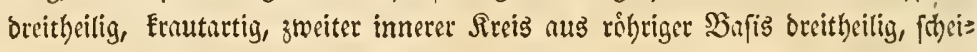
Denartig, farbig, crfterer geht unter in oen Mupaceen, lef̧zerer bleibt ourdb bic

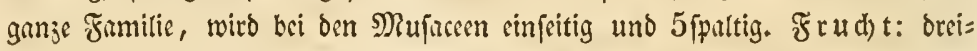

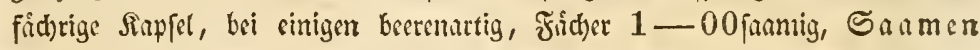
yon ber Mitteladje ausgetheno, aufred)t, meift ziemlich groß. 1) Euglidh,

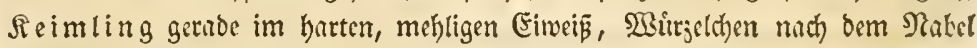
gerichtet. 2) meift ccfig, bei cinigen mit Sanntel, Eimeis feblt, Reimling in

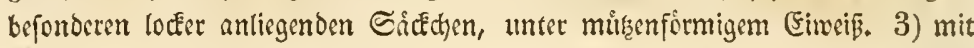

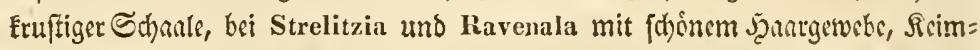
ling mitten im mebligen Eiwein.

Staubbeutel: 1) cinfichriger, $\delta$. b. butber Strubbeutel an ber einen Scite

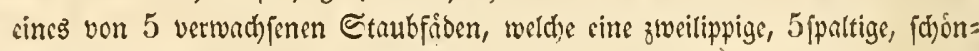

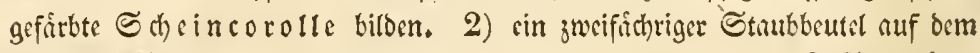
mittleren Etaubfaben, nuf jeorm ber jeitlidjen ein balber, dieje 3 Stnubfíben bilden die Sberlippe der Sdbinblumentrone, deren Unterlippe jwei Eleine, ocn

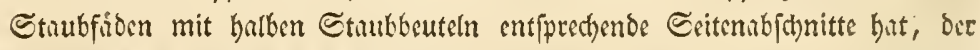
mittlere Ctaubfiden bialt zwifhen den Find)en peines Stmbbeutels den zartfa=

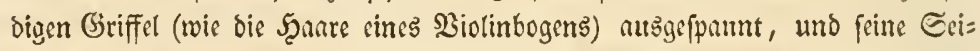
temanbingfel baben noch nicht bei allen den bulken Stmbbeutel erlangt. 3) Die Sdyeincorolle bat fich ju 5 Stmubfiden nusgebildet bei Strelitzia und ber

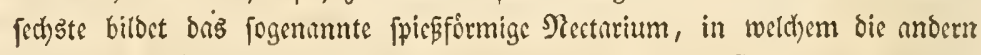
liegen; Der (5orollenfeld) vollendet, 3theilig. (Jeldy meift auf cin sinzelnes

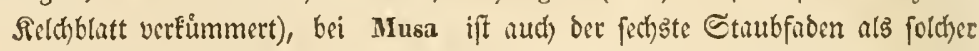
vollendet, oer S3eutel bieibt aber fteril, Der Corollenteld twird cinfeitig 5fpultig, und Der Reldy eine cinfeitige, jenem gegeniberftebende Schuppe. 2fuf ifntithe IBeife etEliart fich Heliconia uno Ravenala.

Begetation: 23uzzelftock Enollig, Enotig geringelt, foraftreibend oder centrifd an=

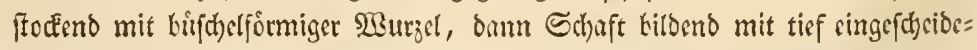
ten, fọpibig langgeftielten ficberuervigen 23 lättert, endich in Ravenala

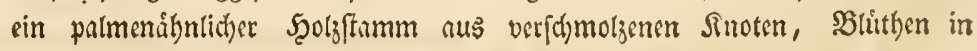

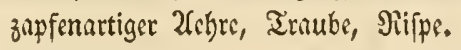

*) Die ferten blülyenbe Renanthera coccinea blühte hicr im vorigen Jahre 1836 vom 2 uu=

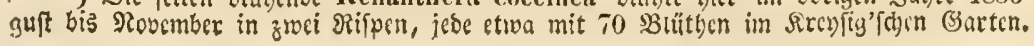


(3ruppen: 1) Сапиеве.

2) Amomeac.

3) Musaeac.

Conspect. 1588 - 1612.

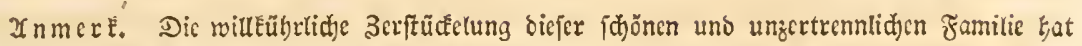

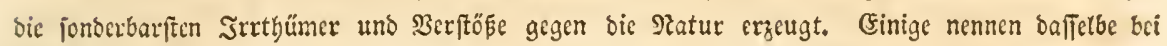
Strelitzia હorolle, was fie bei andern (sattungen Seldh nennen. Sugleidh nchmen andere ben

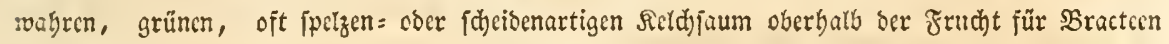
uno glauben, biefe [eyen unter ber Frucht entftanoen unb mit in fie bincingewactifen, was burch

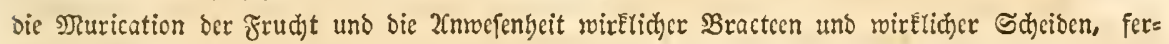

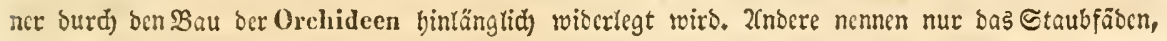

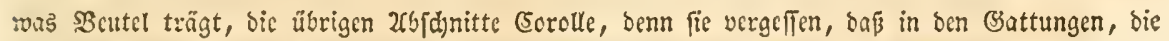
fie ats Eünfllide "ordines" davon gefonbert haben, Dieferben Statbfäben ifre SBeutel erbalten.

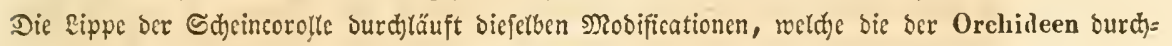

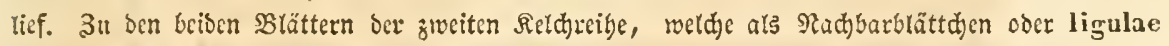

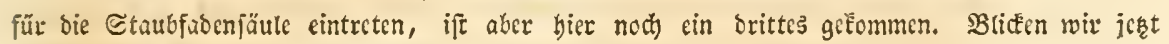

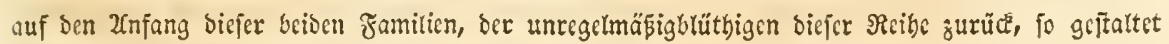
iid) bic Netamorphofe folgendermapen:

\section{O r c h i d e c.}

S c i t a m i n e e:

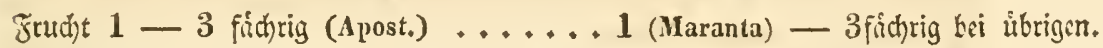
Sieldfaum 2 reihiy: 3 u. 2 theilig ......2 2reihig 3 u. 3, Dann (Helic.) ${ }^{*}$ ) 1 4,2 น. 3,1 u. $3,1-5,0=$ u. 6 theilig.

Strubbiutel $1-2-3 \ldots \ldots \ldots . . .1-1 \int^{1} \int^{1} \int_{2} 1$ u. 5 fterile (Mnsa ${ }^{\circ}$ ) 3 น. 2 fretile - 5 u. 1 fitriler.

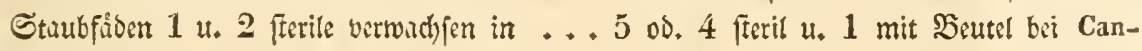
Orehilleae genuinae, endifindiger fif: ril, feitlicher mit $\mathfrak{B}$ eutel bei Cyprip., alle 3 mit Soutel: Apostasia. na, 3 fteril u. 3 mit $\mathfrak{B e u t c l}_{\text {bei }} \mathrm{Amo-}$ meac, 1 fteril $u .5$ mit Bentel ksi Strelitzia, 5 fferil $u .1$ mit $\mathfrak{B c u t e l}$ bei Musa 9 , alle 6 mit Santel bei Musa $\overbrace{\odot}$ -

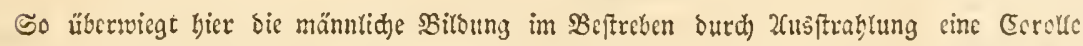

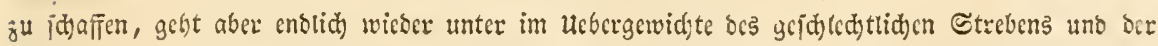

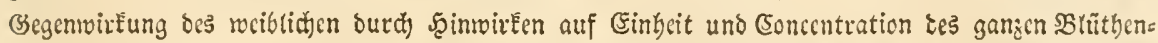

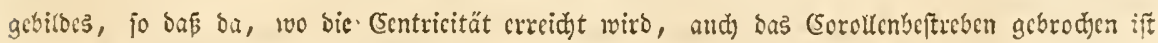

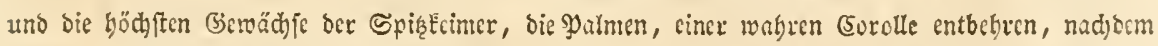

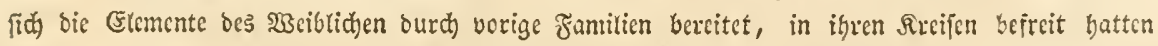

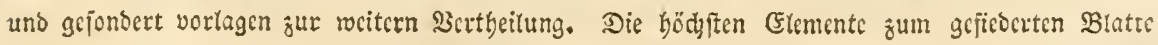

*) Şei 6 fdjon anwejenben Gtaubfaben Eann nämlid) bie für cinen fteriten @taubfacen ge= baltene Sdjuppe IIook, exot. 190. Fig. 3. 4. nidhts anocrs fenn, als ber vierte, obere

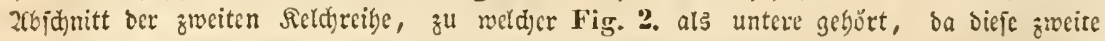

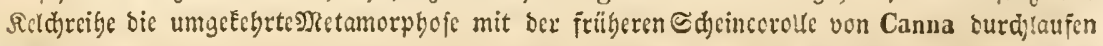
muß̧. 
find glcidfalls gegcuen, ber Riefenftamm oer Ravenala ift geroonnen, und fo ift nur noch ein cinziger ๔djritt zum sicke, zu ber:

56. Familic. \$almen: Palmae.

SPiftill frei, Frudtenteten 3 finchrig oder 3theilig, Firher einfanmig, bei Cyclantheae nod) 1 fifchrig uno vielfiamig; (Griffel feblt ober cinfind), Narben 3 oder sime. Reld): 3 und 3 blittrig wechfelno. Frucht: Stcinfrudt mit ver= Eehrt jiegelfhuppiger, mit fajeriger oder fleifhiger Schrle. Sarmen: hart= (d)arlig, mit großem Eimeis, Eleiner Reimling, welther vom Nabel fern bei ben meiften in Der Mitte des Riuckens vom Eiweī̄e eingelagert iff.

Etaubbeutel 6. zweiffichrig, Ficher parallet ober bivergireno (j. $\mathfrak{5}$. Gnestum). Fidoen verwach fen und aufgeridhtet oder frei und ausgebreitet, den 6 Relchab=

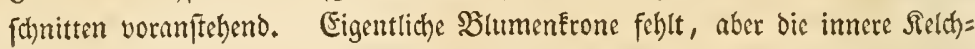
reihe meift corollinifh, bei Cucifera thebaica bie Bhume Der ł̇nriben beut= (id) nachuhmeno, robhrig, mit orcitheiligem Saum uno 6 Stuhgefâ̄en im ๔ahlunde.

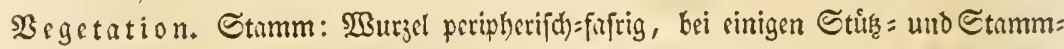
toulzeln, Stamm aus bicht verichmolzenen Snoten verholzend, einfach sin= Enopig oder bei cinigen (Cucifera, Hyphacne, wie Pandanus:) gabelaftig mebr= Enofpig, Şolz hornurtig, nach) innen weid)er, in ber Mitte MarE. Endtrieb jîhrlich central jich ermeterno; Blåtter mit halbumfaffenden Blattfitelen, hei vielen bebornt, jeocs auß jeinem verjhymolzenen Sinoten fpirutig um die Snofpe

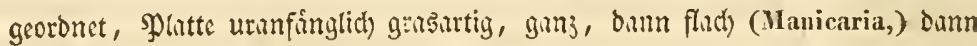

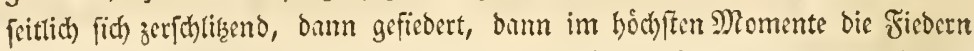
enditandig zufummenge[choben: gefíchert. Sluthentriger immer aus Scheide, als centrifther Solben beginnent, bann adj felftinbiger, fid) atternirent lofender,

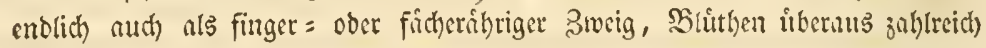
eingefenft (Elaeis, Astrocar. Acroc.) ooer in ber Z(d) fel von Defeblittern

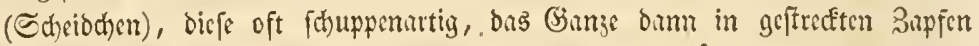

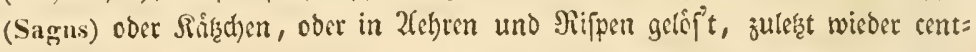
rifich.

(3ruppen: 1) Cyelantheac.

2) Phoeniceae.

3) Palm a e genuinae: a) Sagoinae. b) Cocoinac et Arecariae.

c) Coryplrariae, aa) Sabalinae. blo) Borasseae. cc) Coryphineae.

Conspect. 1613 - 1663. - Flor, germ, p. 136.

2nmere. Bloiefprad bes Satanophilus mit ber Ratur über bas Thema: "roas foll id

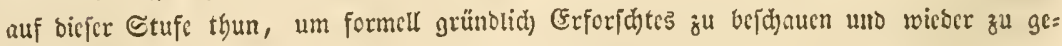
ben?" -

Bot anophitus. Wुo find wir jest in bcr Pflangennatur?

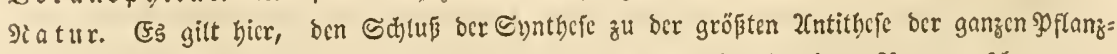

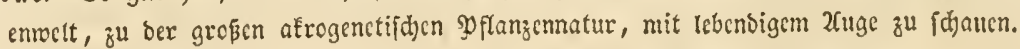

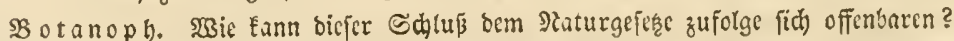

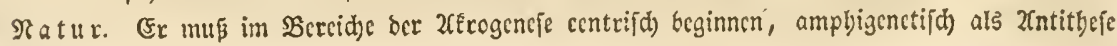




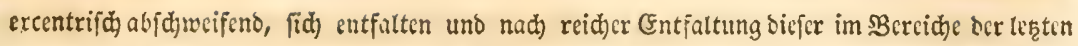

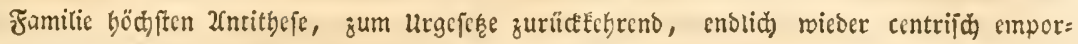
ftreben, bic Iegzte Resigung zur 2entithefe verjöbnens.

Sota noph. W3er mirt, und mo in ber פyflanzennatur, fo hobe Zufgabe löfen?

Ratur. Sudje bie hobe Röiung fo bohen \$problems auf beiner \$3anderung burch bie Diflang: enwelt, nur in ber säbe bes 2fequators, uno ou trittft bann grwifdyn ocn Tropen in bie

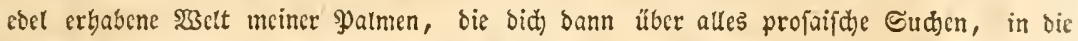

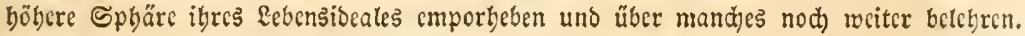

Botanoph. Worüber follen bie gyalmen mid nod weiter belehren?

Ratur. Eie follen Eunb madjen, baßß ber Etreit ber Drdjibecn uno Ecitaminecn über weib:

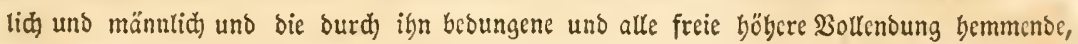
Berwodj)fung oer Frudjt, in Riebc getöp't ift.

Botanoph. WBer hat ifn gelöpt uno modurch?

Ratur. Es wurbe bercits butd bie sBanane entidfieden, bas Männtide foll fortbeftehen

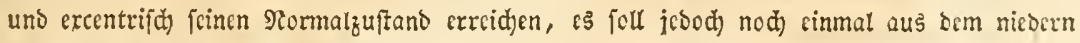

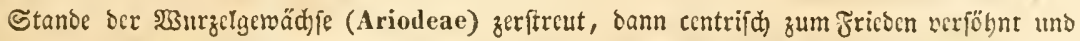
in einbrübriger $23 e r f$ chenelzung - wie cinft Sysirhynchium uno Marica - roicber begin.

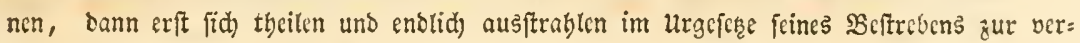
boppetten Dreizabl, boch altes bies nur unter zrwei şesingungen, 1) fo oft als mögridy

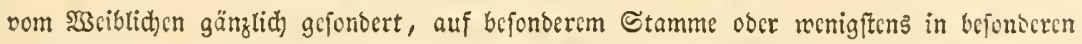

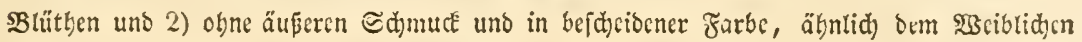
fetbft, ogne Erinnerung an bas ftolge Befteeben ber Srdjibee und Banane, eine csorolle

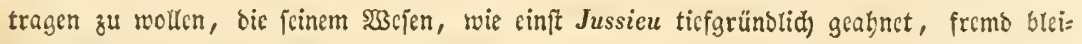
ben mus.

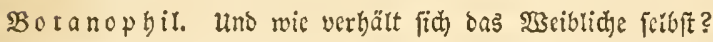

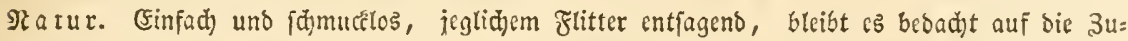

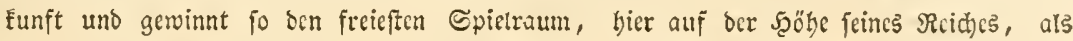

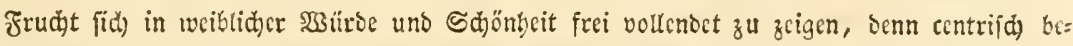
ginneno uno nur einmal nod) in ocr Dreizab! (Phoenix uno Rhapis) im prinzip des

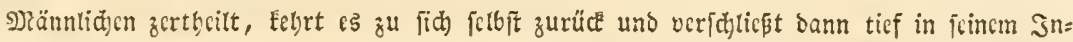

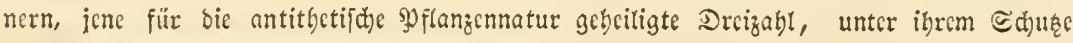
ben ๔prós Eünftiger '(Senerationen borabyend.

Botanophil. Wुo beginnt abcr all' bics SBcftreben?

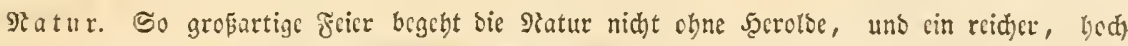

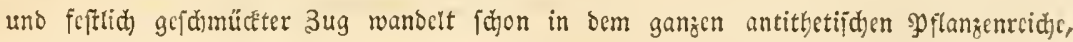

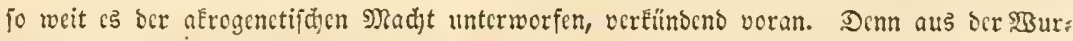
zetmelt bicten bercits Caladium, Dracontium, Candarum :. a. Den Rotben uno sas

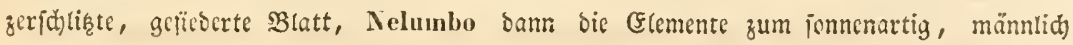

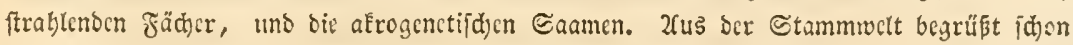
Zea, nit Scleria uno Fuirena, fid in lciđłtem Sorfpicte gefalleno, währeno Pandanus, Freycinetia, und mit Genlisia, Witsenia auf ifrem Etamme fid folger emporbebun und Agave ben Rotben burch bie 2Cebre ber Littaea zur preramioalcn Rijpe cntfaltet.

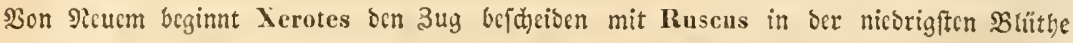
und Frud,t, und mit bem tild)tigen Stamme Dracaena uns Yucca, bis Pleurothallis

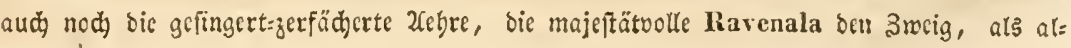




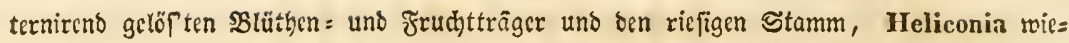
ber uno bic rohlivoluenoe Musa, ben eentrifd gewordenen Siolben, noch cinmal geivonnen und burch fiedernerviges \$Blatt bie Elemente für bas Fiederblatt ber şalme vercinten.

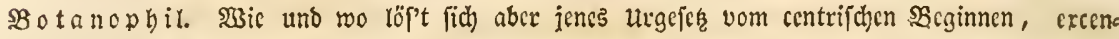

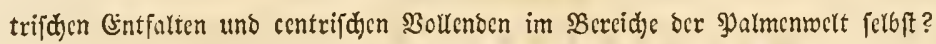

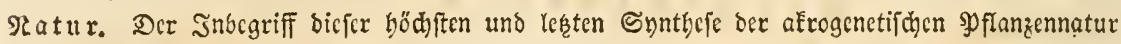

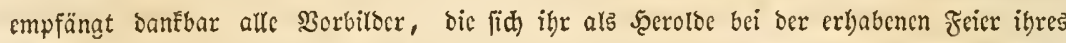
Eebensfeftes geboten uns veräbntiăt fich ithnen, fo vicl fic nur Eamn. Schon jenes 233 ur $\bar{z}=$

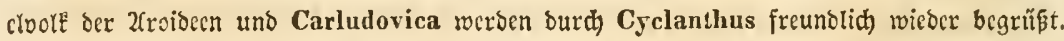
2tue 2rogeorbncten jener gras = und lilienartigen StammbölEcr werben burch bie reichfte Entfaltung ber \$phoeniccen, ber Gagoinen, Socoinen und (Soryphaecn gebührend em=

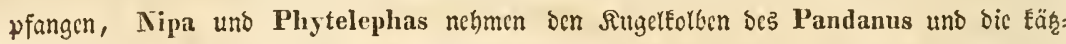

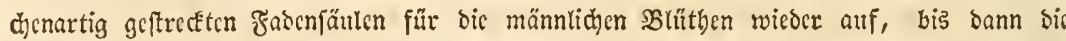

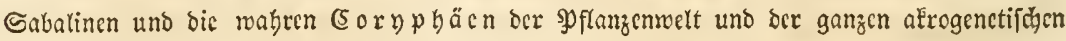

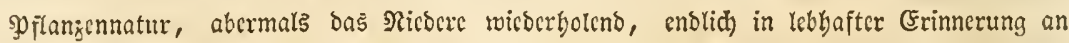

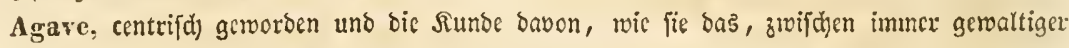

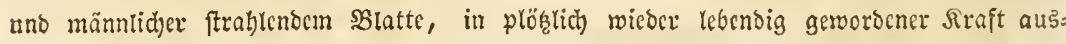
ftrahlende amphigenctifdye Befes, mit Dem ocr 2ferogencfis freundrich vermählt, in Der un=

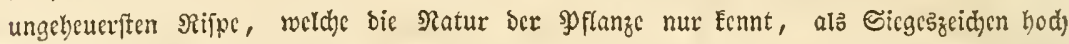

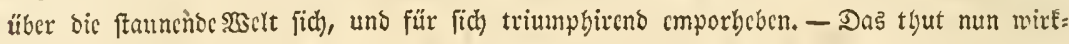
lid) - Corypha!*) -

*) Die colen Sdjirmpalmen auf ben Mroluffen, Malabar und Ecnlon, (Corypha umbraculi-

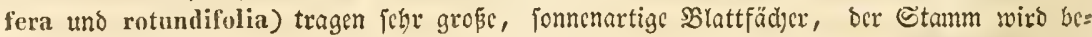

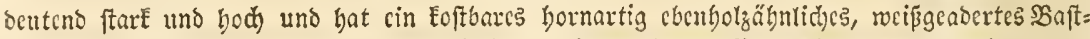

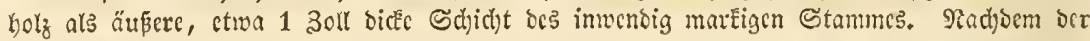

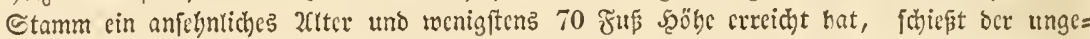

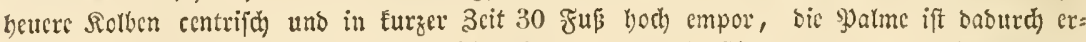

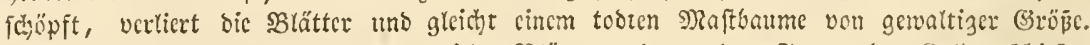

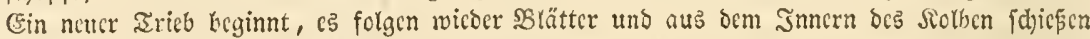

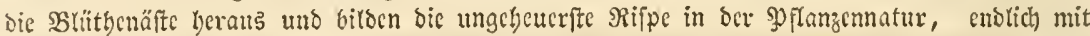

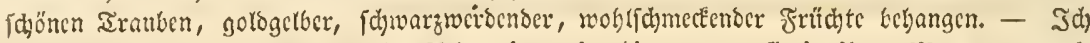

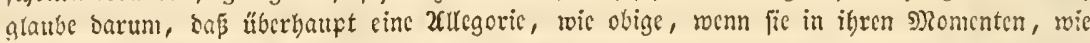
in ibren Sbjectin, ridjtig motivirt ift, ber lebendigen SRaturanfdyaumng nicht frembartig cr=

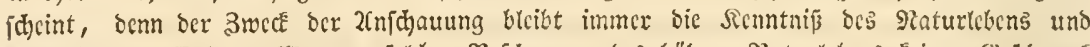

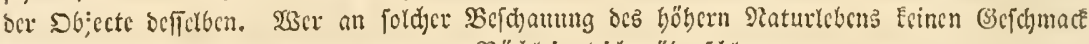
findet, fann bicfe Ecite oter bas gange Büdilcin tridyt übcriflagen. 


\section{Fit it $\begin{gathered}\text { it } \\ \text { E I }\end{gathered}$ \\ Bweifelblumige: Synchlamydeae.}

Erite Srbmung.

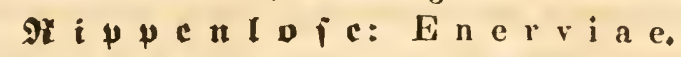

Rajaben: Naiadeac.

o1. Zam. 2(tmleuchtergewidef fe: Charaecae.

62. ₹am. Şornblattgewaid) [e: Ceratophylleac.

63. Fam. গyodoftemoneen: Podostomoneae.
Sthuppler: Imbricatac.

64. శam. Birtlappe: Lycopodiaceac.

65. Fam. Solbenfdyoffr: Balanophurcae.

66. Fam. Eytinecn: Cytineae.

3weite Sromung.

Etcifblät terige: Rigidifolia e.

5 dechtblüthige: Inconspicuae.

67. Fam. Shad)trithalme: Equisetaceae.

63. Fam. Fiben: Taxineae.

69. Fam. Santalacen: Santalaceae.
Doppelocutige: Ambiguae.

70. Fam. 3apfenbiume: Strobilaceae.

71. Fam. Protenceen: Proteaceae.

72. J゙am. Ssiosln: Thymeleaceae.

\section{Dritte Sromung.}

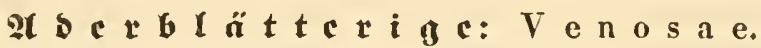

unvolreommene: Incompletae.

73. Fam. Muriciceen: Myricaceac.

74. Fum. Rafichthenblitthler: Amentaceae.

75. zam. Neffelgewid)fe: Urticaceae.
Bbattreide: Foliosac.

76. Zam. Dfterluzeien: Aristolochiaceae.

77. Fam. Nyctingineen: Nytagineac.

78. Fam. Rorbergewaidbfe: Laurineae.

61. Zamilis. 2(rutleurbteragetwär)je: Characeae.

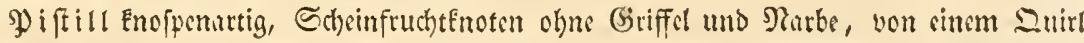

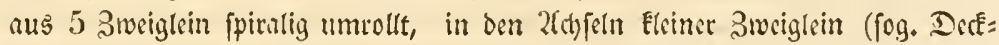

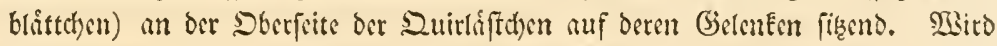

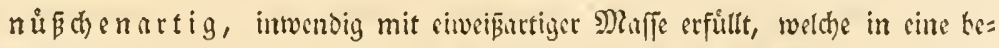

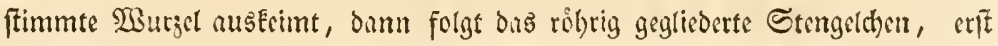

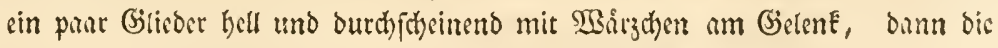
Gilieber mit Bhtttgrin.

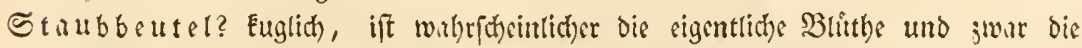

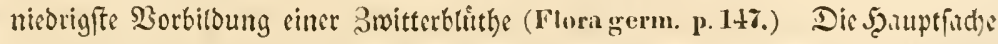

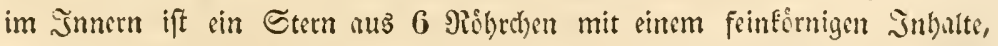

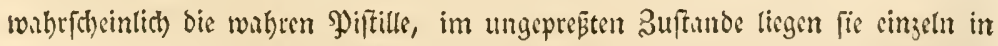

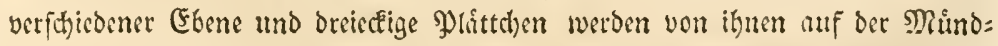

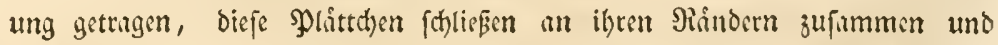
bilden eine gefdloffere Siuger, in Simern find biefe Wlittd)en mit vom Mittel= 


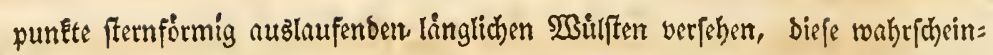

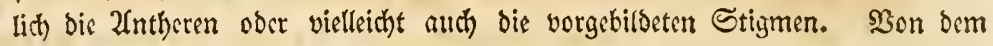

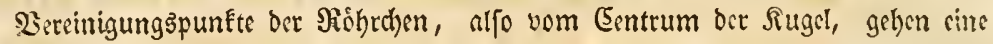

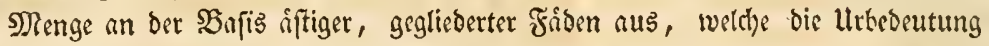
von Stanbfíben, oder von Blütbenbillle haben moger.

Şegetation. Strmm grgliebert, auts cinfuchen Rishren getenfig zufammengefrest ober bie (s)lieber aus um eime Mittelróbe langs peripherifót herumliegenden Siohren gebildet, quirlaffig, bie 2fefte tringen die fogenunnte 2futhere zwifhen Eleinen Decklatturtigen 3meiglein. Der frnutartige Stumm ohne Spirnten verzmeigt fich quirlattig oder bitfotom, in Den hoblen Riumen ber Niebren beroegt fich

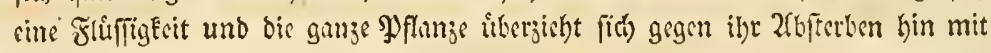

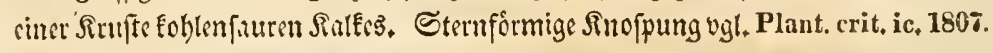

(Seruppon: 1) Nitella.

2) Cli ara.

Conspectus N. 1663--1665. - Fl. germ. p. 147-150.

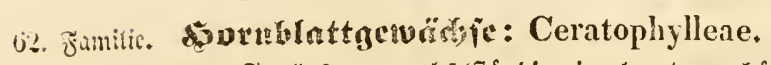

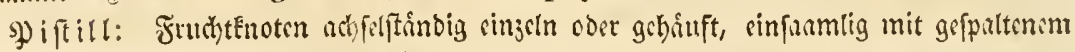

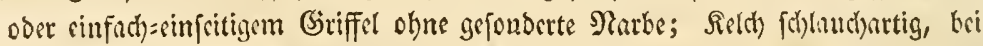
Cerntophyllum vielthrilig. Saame sinzsin, bei 1) uno 2) alfrecht, feitlid)

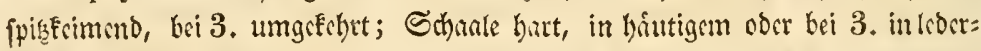

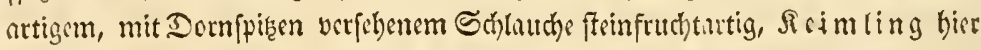
victtbcilig, Federchen ropettemartig viclthcilig.

Staubbcutel: in 1) und 2) aus cinem centrifen Staubfaden (minnlid) umge=

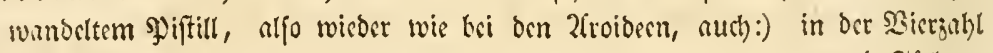

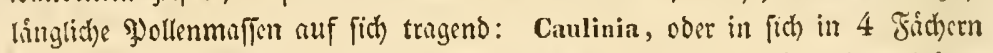
enthaltend uno 4Flappig amfpringent: Naias, obre dic Staubbenterklitter fdjon anfings getrennt, ropettenartig verwad) fen, zwoi = bis orcippirig uno jeocs inmerlid) zweiffichrig: Ceratophyllum. Bai 1) uno 2) ciute fichliudyartige jer=

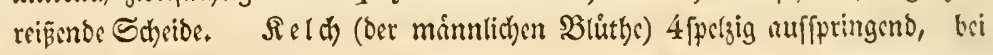
$3)$ ein 4 theiliger Reldh.

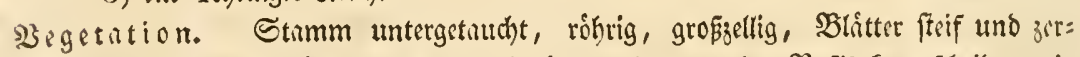

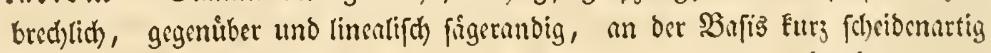
erweitert, bei ben Eeratoplyplern quirlftindig, gabetthoilig und fígezálgnig. Bluttbe bei 1) sinbåufig, bei 2) zweibiulig und bei 3) miedre cinbaituig.

Gruppon: 1) Caulinia.

2) Naias.

3) Ceratoph jlleae.

Conspectus N. 1666-1668. et 1669? - Fl, germ. p. 150-152.

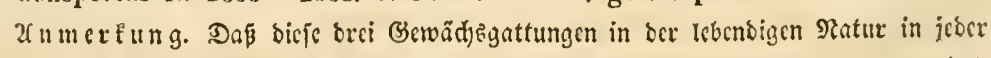
Bezichung inniger zufanmenbalten, ats in ben $B$ tïdern ibnen zufammenzulyal= ten crlaubt wird, bas ift wohl feine Fragc.

63. Zumilic. \$oboftemonecn: Podostemoneae.

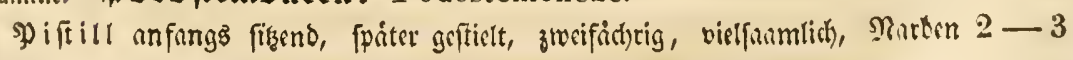




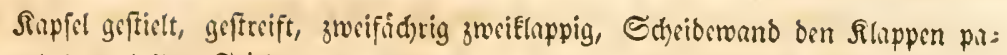
rallet an beiben Flád)en fummentringend. Sarme feht Elein, Reimling nod) unbefinnt. Blatthe feldtlos in Ed)eibe, welche jerreipt, bei Lacis sin fdup: perturtig visttheiliger Siclds.

Staubbcutel 2-5-00, zweiffidrig, lings mefpringeno, auf vertwathfenen

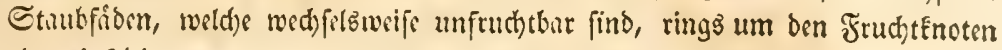
oder sinfeitig.

Begetation. Stamm Erauturtig, fiftig, untergstiutht, Blitter bei 1) verfd)mol= zen berablaufend, bei 2) wechisiftindig, bidyt ziegeturtig, audh am Etengel ber:

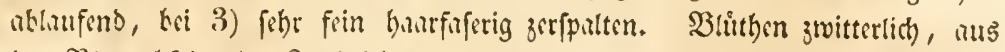
bin Bhattud)fern oder 3meigipianen.

(5ruppen: 1) B Landorieae.

2) Marathreat.

3) Lacideae.

Conspect. 1680 - 1655.

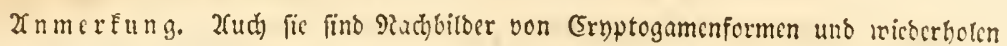

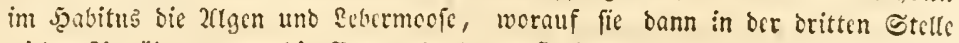
micter 2fnnäbsrung an dic Eeratophylucen crifteben.

64. Familie. SBärIappgewädyje: Lycopodiaceae.

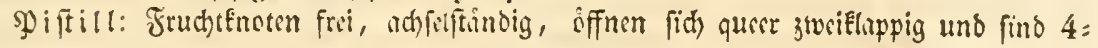

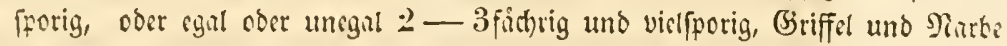
foblen, bie Eporen fino fuglids mit grubigzzstliger Edhale, buken 3 in sinen

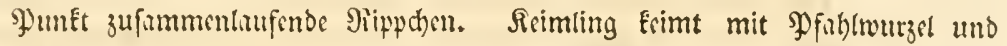
zwei gegenuber|tebenten 2 blittchen, nidht als Bellferimer.

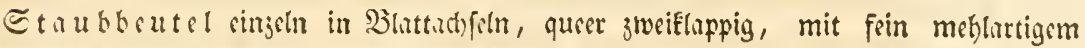

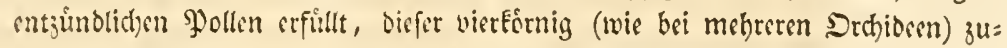

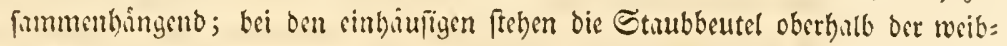
lidfen 3 luttherr.

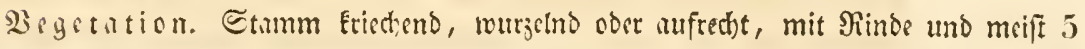

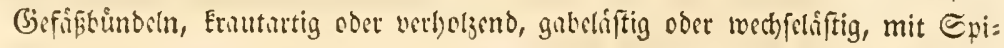

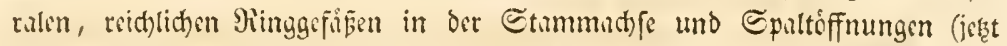

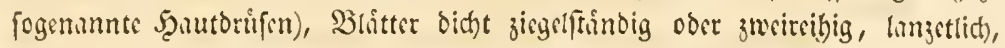

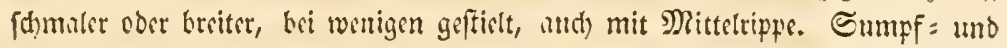
Şaidepflinzem.
(5ruppen: 1) Selaginelleae.
2) Planantheac.
3) Bernhardieac.

Conspect. 1656-1678. - Fl. germ. 152-153.

63. Zamilie. Sivlbenidyoffer: Balanophoreae.

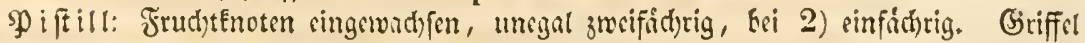

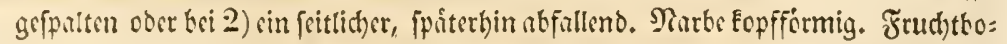

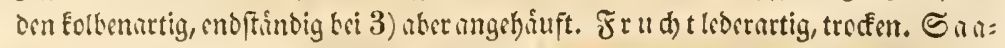

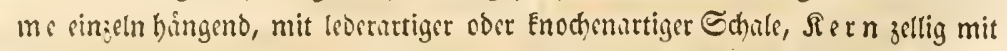

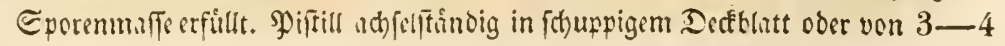


Sthuppen (wie in voriger Framilic bei Bernhardia mit 2.) Eeldyartig um= gebert.

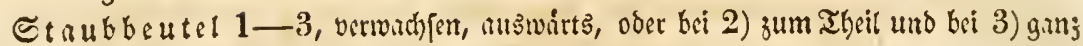
frei, 1 = oder unegal 2fick)rig, lings auffpringent. SPollen (wie bei vori=

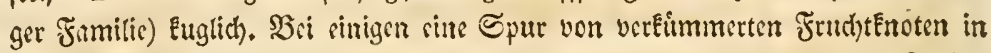

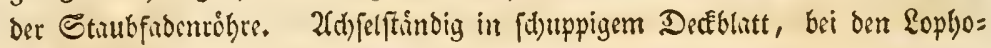
phuten aus beren Epalte entppringent.

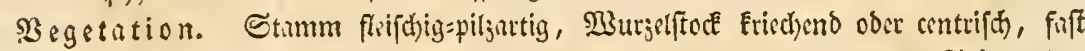

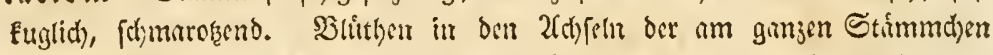

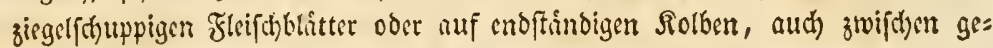
fticlten Schilbjouppen, cin = ober zwribintifg.

(Gruppen: 1) II elosie a e: Langsd. Hel. Scybalium Schott. Endl. Scynopsole FindI.

2) Cynomorieac.

3) Lophophyteac: Lophophytum Schott. End1. Ombrophytmm $\mathbf{P} \ddot{p} \mathbf{p}$.

\section{Conspect. 1679-1682.}

66. 'zamilie. Cytincen: Cytineac.

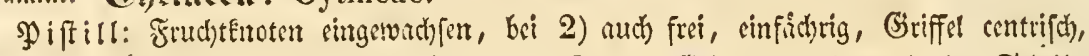

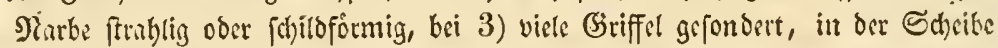

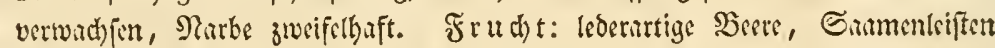
00 unt 00 fanmig. Sarme pefr zahlecich, in Bra, Sdymle ledrartig oder Eci 3) Eltod)entart, gefeldert, Sicrn zellig, Eei 3) aud) fabig.

- Strubbeutel aus centrifiter Sanlle, auswirts, 2-00fichrig, Fricher geribe oder ungleity bogig, langz anffpringend, bei 3) bie Strubsutel 00 gefondert

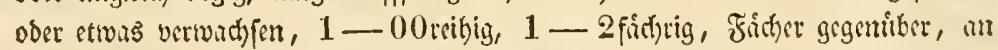

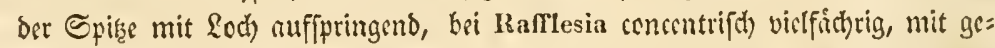

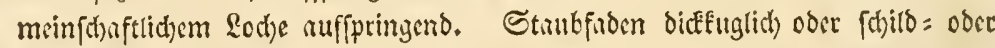
fátenformig, Sollen fur bie Siefruchtung wieder fo viel ober fo wenig werth) als bei Lyeopodium.

Begetation. Stamm fleiphig pilzurtig, die Slutbentbeile innerbalb groß̈er und fleifrtiger Sduppen, ganj auf cin 3 humenphantom rebuit, nur in ber 2 ?n= titbefe (c) uppenblittrig und viefblutbig (bei Cytinus Sareophytum) boldentraus big ober rippig zertheilt, fajmarofeno. SBlithen cin = uno zweifiujig.

(sinppen: 1) H y duoreac.

2) Sarcoply te a e: Cytinus, Sareophytum.

3) Rafflesiea e, Frostia, Zippelia $\mathbf{R}$ c h b. *), Raflesia.

Conspeet. 1683. 1686. 168\%. 16i85. Fl. grerm. p. 153 et 843.

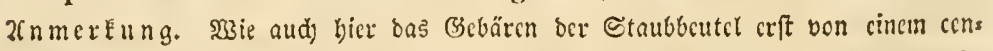
trifd 23 eiblid)cn ausgent (Aroideae, Pistiacene, Typliatcae, Orchilene),

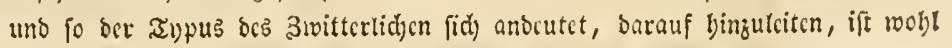

*) Zipuelia Brugmansia (Brugm. Zippelii B I ume.) liegt eben, in bicfen Tagen aut Juva angelangt, in allen Entroickelungsftufan vor mir. Der Prame Brugmannsia mar sergeben. 


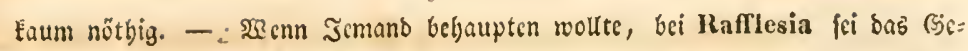

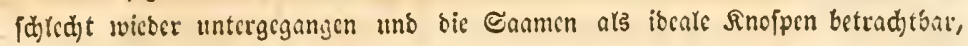

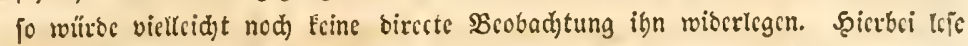

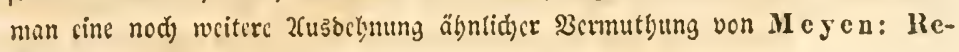
gensb. bot, Zeit. 1829. p. 49-63.

\section{Familic: Erd)ad)telhafute: Equisetaceae.}

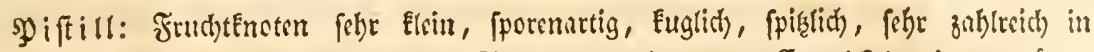

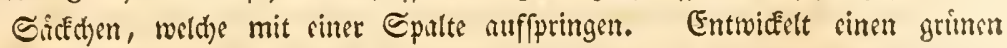
Sisimling, wolltier fid zellig quirfformig nusbreitet.

Staubbatel 4, unvolleommen fpatelformig, an ben Enden zrocier Fitoen, in

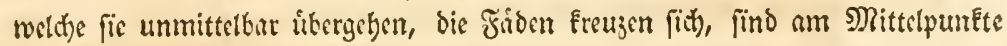

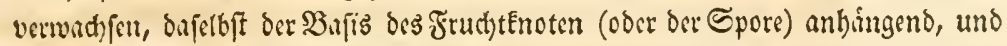

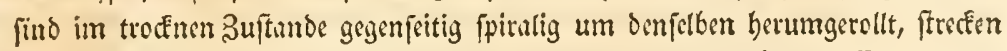

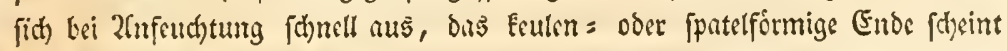
Şollen andocuten zu wollen.

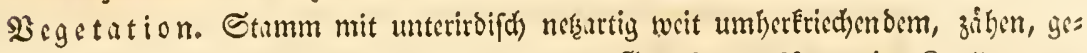

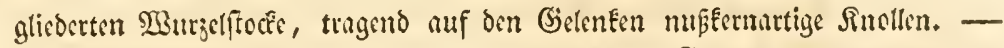
Etengel gegliebert, bohl, mit Efeltet aus Riefelerde (Struve: de -silicia in plantis. Berol. 1835.), birttich, geftreift, an jebem Bicterte sime Edjeibe aub

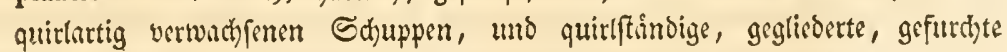

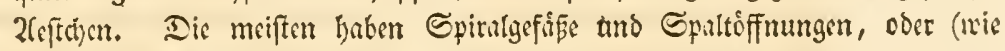
Eq. Telmateia und ber fruchttringende Stengel von E. arvense), entbehren bic

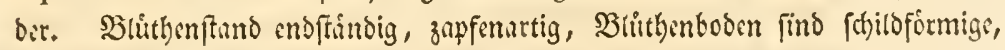

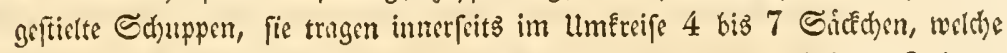
nach) dem MittelpunEte hin auffpringen und mit jenen vorgebilbeten Switter=

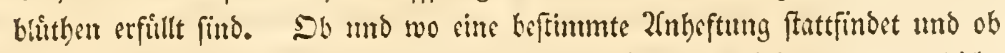

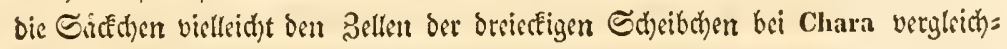

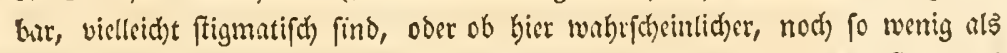
bei einigen Formen der erfen Dronung, an Befruchtung zu benken ift, burf weiterer Unterfuctiung empfoblen weroen.

(5ruppen: 1) Equisetum.

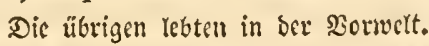

Conspeet. 1689. - Fl. germ. p.153--155.

68. Familic. Eiben: Taxineae.

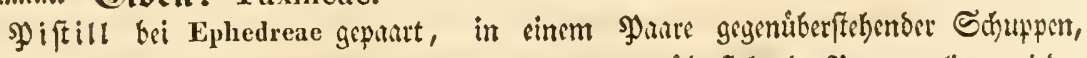

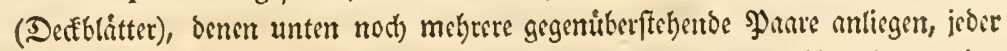

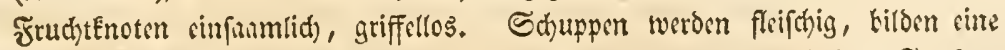

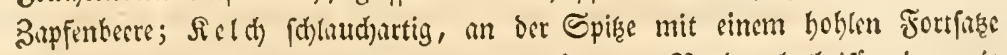

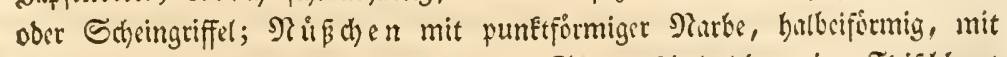

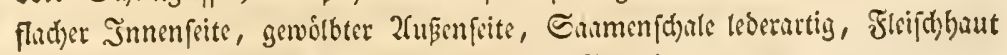

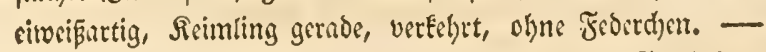

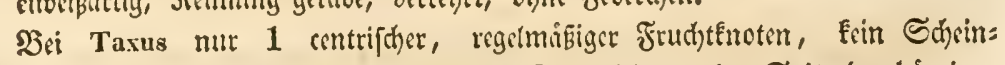

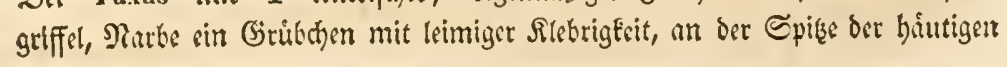


3meifelolmmige.

Droแtแก: Etcifblättrige. Eibcn.

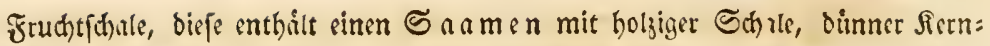

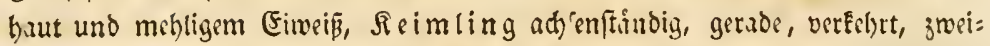

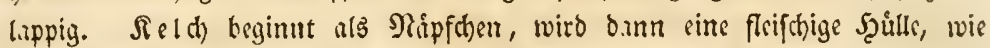
Beere, fur ben Frudttnoten, uno bleibt oben offen.

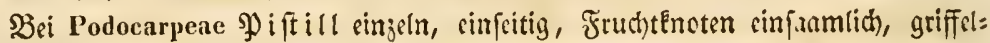
los, Sarame bri Poulocarpeae umgefibrt, bei ittrigen aufrect)t. Seld

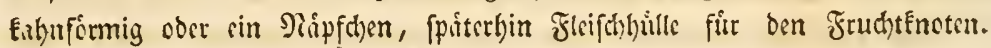
Scimling zwcilappig.

Staubbeutel: bei 1) mebrere an ber Spige siner Strubfiobnfiute, aufredt, zmci=

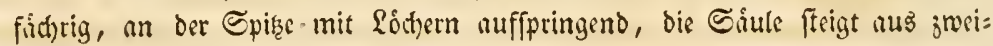
Elippig vertiEal halbgeoffuetem Sislche.

Bri 2. ift bie Stuubfitonfiaule am Enbe in sin horizontules, frumpfifernform: igrs Schilo ausgebreitst, biss iffnet fich unterfates in 5 (auch $4-8$ ) Fiddern, weld)e ben Eugeligen ภु)ollen entbulten, (dentt m.n fich bie Stuubfíben gefon= Orrt, fo gehen fie alfo fo ummittelbar in oen Fुollentriger utber, wie bri

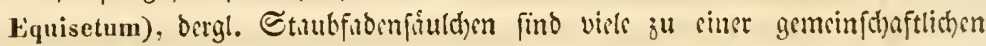

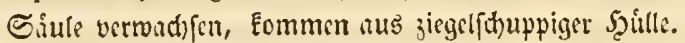

Bei 3. Find bie Staubbentel 2fitchrig (Podocarpus, Dacrydium). cocr 1 fíct)= rig (Salisburia, Jhyllocladus) maift mit foupponutigem '2Tnfar.

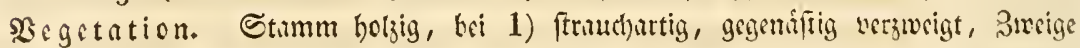

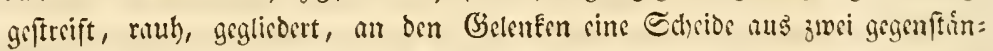

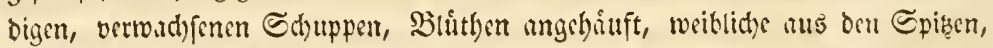
minnlict)e aus ben Gislenfen.

SBi 2) und 3) Etamm vollendet holzig, frriudt) = ober boumartig, zmeizeilig

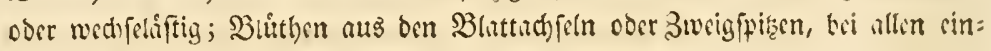
oder zrosibiufig.

(Sruppen: 1) Éphedreae: Eplicilra.

2) Taxcae: Taxus allin.

3) Podocarpeac: Podocarpus, Dacrydium, Phyllocladus, Salishuria.

Conspertus 16!)1. 1693-1695. 1719-1728. F). germ. 1. 155-156.

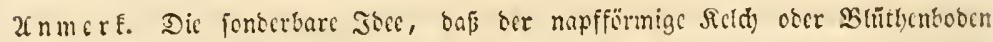

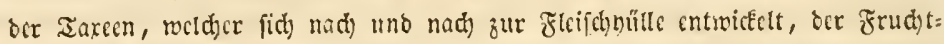

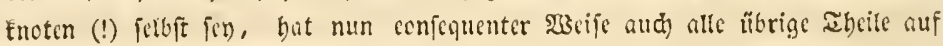

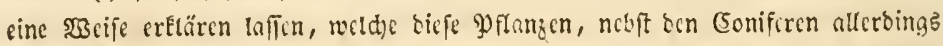

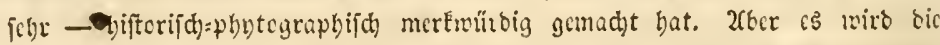
Joee von Esymnojpermen, weldye vicfleidit nur von ben Encabecn ausging, für bieje gegenuärtige Etufe wahridgeintid ned) ferner fo parabor blciben, wic fic

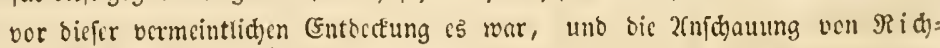

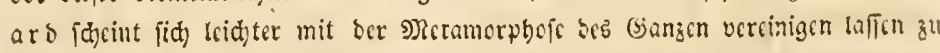
fömen.

69. Fumiti. Eantalacen: Santalaceae.

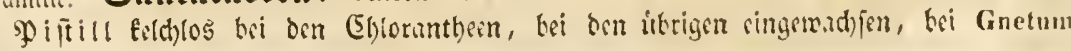




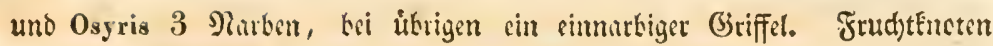
cinfanmig, wiro jut Eteinfurbt nit cinem Saamen mit fleifhigem (Fi=

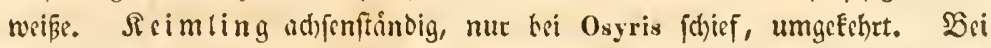

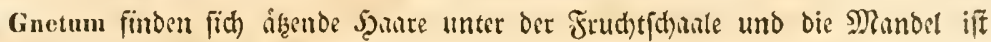

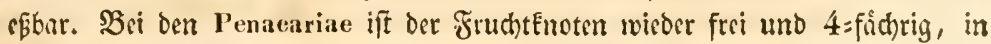
jroem Fadje 2:faumig, Saamen zu 2 unten an der Sheibewano, mit breiter Nath und Reimwarze. Sield)róhr'e umwichfit den Fruchtfmoten, hei 1) ohne Saum, bei 2) und 3) ift ber Saum $3-4-5$ =theilig.

Staubbeutel zum Theil einfíd)rig und auf ciner Schuppe cingewadfen hei Chlorantheae, tibrigens 2=fichrig, lings auffpringend, auf Staubfioen, welche bit 2) in den (SelenEen siure gegliederten 2(ebre, bei 2) und 3) im Selchidlunde unter Drufenf(huppe, vor den Saumabjhnitten, gleidzåblig fteben, kei Nyssa in ber minnlithen Blittle boppelzithlig.

Begetation. Etumm Fraut = oder fraud) = uno baumartig, 3rosige und Slatter (bci Chlorantheae, Santalum und l'enaeariae) gegeniber ober (bei likrigen)

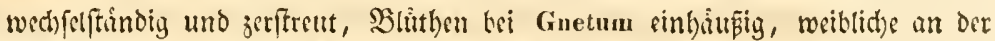

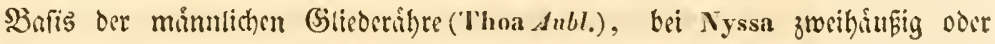

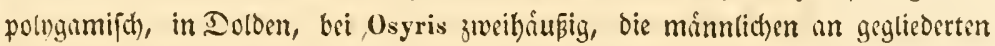
Suveiglein (faft wie bei Guetum) in ben Gelenten gegeniber und sine um

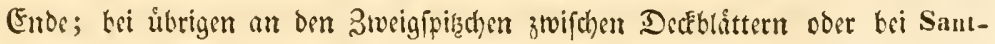
alum in freien orcitbeiligen \$iifpen. SBlatter lederartig einrippig oder fieders rippig.

(Sitppen: 1) Chlorantheae. $1954-1958$.

2) Xysseac. 1712 .

3) S a t ta le il e, a) Exocurpeae. 1701. 1707. - b) Osyrideac, $)$ Ophireae $1790 \cdot 1790^{\prime} \cdot(3)$ 'Thesieae et $\left.\gamma\right)$ Santal. genuinae. 1802 - 181L. - c) Peuneariae. 3041 et Sarcocolla Rchb.

Flor. germ. $15 \overline{1}-158$.

Unmer E. Gnetum fhrint erft bie Chlorantheae zu vollenden, bie Ephedreae wic= berhotens, roçhalb audh woht bie \$crwandtidaft ber Bruppen erft ourd jene (Battung Elar

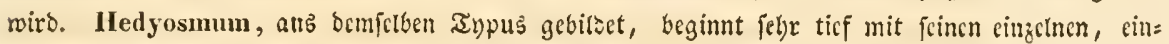

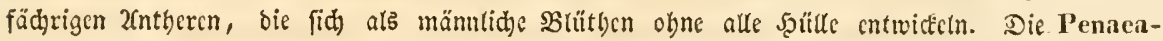

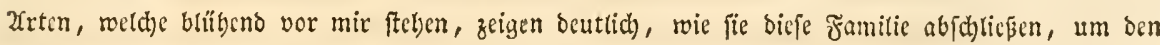

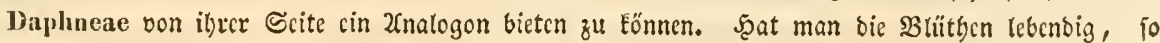

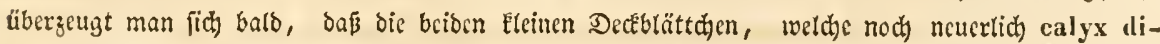

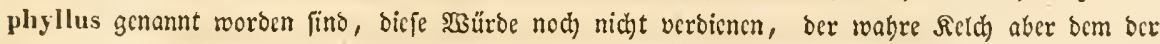
Daphneae analog ift. (Eine allfallende Erifdeinung finb bie groficn Drilifentörper bci Penaea mueronata, weld)c bie Form ber Staubbcutel babcn, bie wabren bciocn flcinen Etaubbcutcffäd)ce vber nur innerfeits unten auf fich tragen, bei ben fä)ön rotbblübonoen 2(rten P. squamosa uno

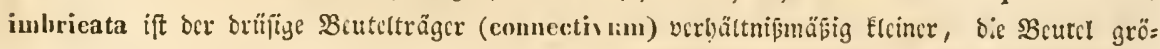

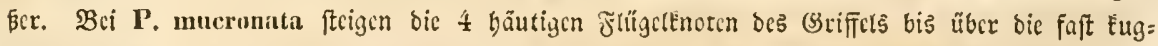

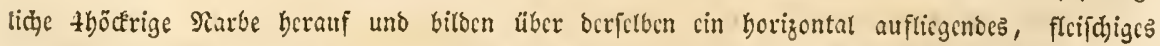

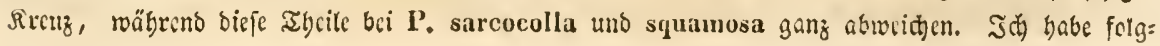


תln|ึe: 3weifslblumige.

Dronung: Etcifblütterige. Eantalacen.

Ende vor mir: Penae myrtilloides $T h b$. fruticulosa $L_{\text {. }}$, nucronata $L$., Sarcocolla offici-

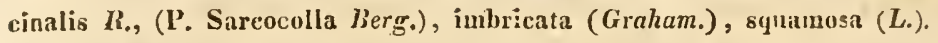

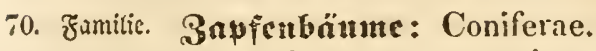

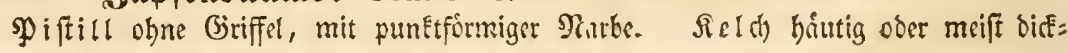

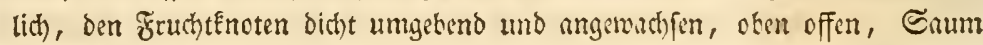
fehlt ober (Alictinae) zweifpalttig. Shlitthen fiesen auf Schuppen aufrecht

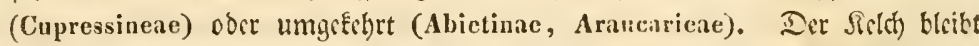

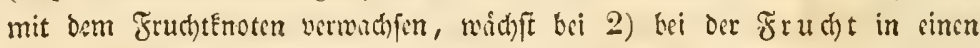

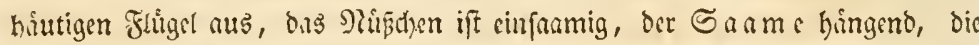

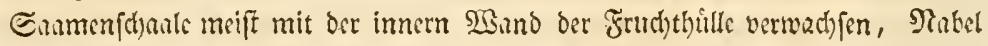

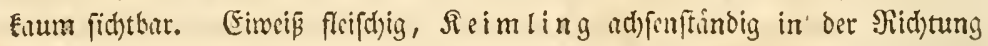
bes Snumens; mit 2-3-12=quirlftindigen Sotnlebonen, bei einigen aud) Das Federdisn fichthar.

Staubbeutel aubmirts gekflyt, bei 1) cinfichrig, Elappig aufpringent, in ber

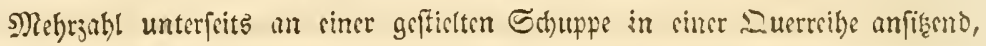

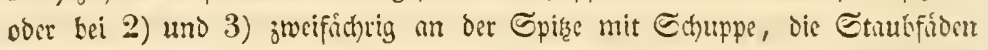

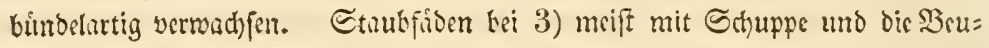
tel endidid bei Agathis uno Cumninghamia obereits. Woollen brcihâutig.

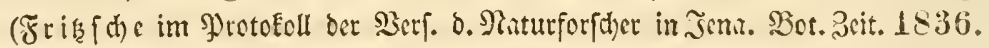
ङ. $703-706$.

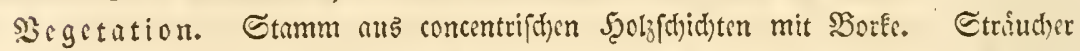

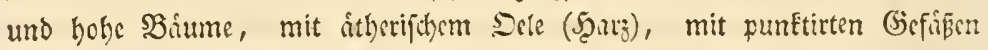

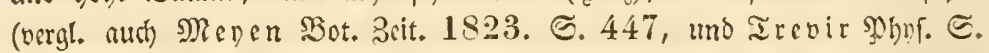

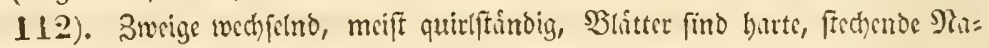

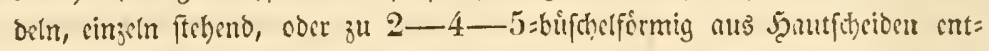

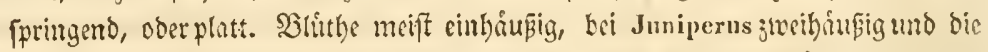
weiblichen zu 3 cnoffinndig, bci librigen bie weiblichen fo wie die minnlichen, birfe auch Eci Juniperus, in Jupfon, D. h. 2fehren mit Ed)uppen (metumorpho=

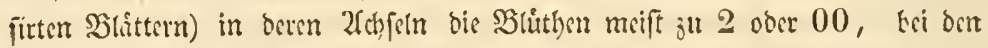
2fraucsticen 1 ober 3 anfitert. Die Ethuppen meroen holjig uno bildon Brpfen, mur kei Juniperus merben bie 3 ङ Sieldybere.

Bruppen: 1) Cupressinae.

2) Alietinac.

3) Aracarieac: $1730,1728,1729$.

Conspect. 1719 - 1730. - Flor. germ. 158 - 162.

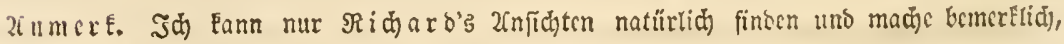

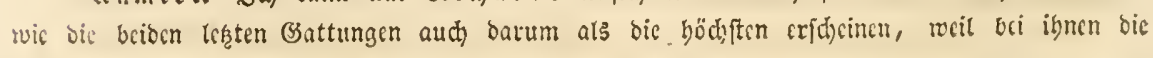
ভtuubbeutel wicoce zur Dberfeitc getangt find.

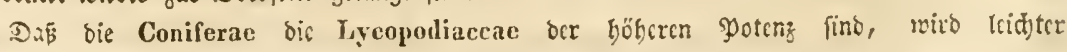
Elar, wonn man, roic man, un das cınpfinsen zu Eünnsñ, thun muß, alle Battungen vor fich 


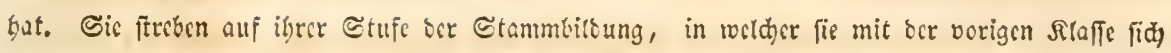

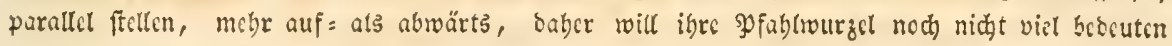

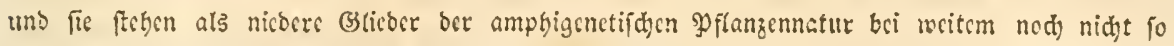

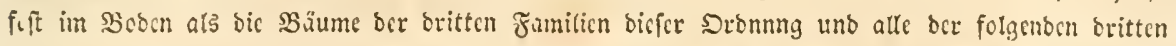

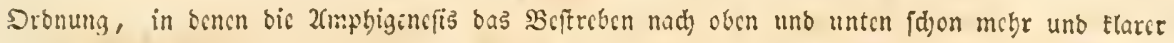
in fich auggleidgt.

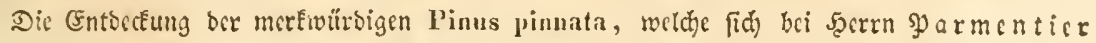

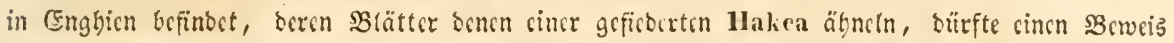

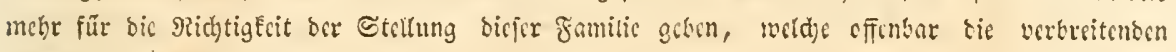
Gstiber für bic sproteacesn entyätt.

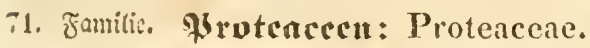

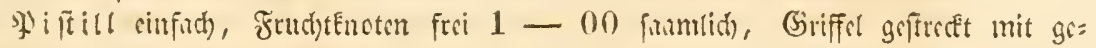

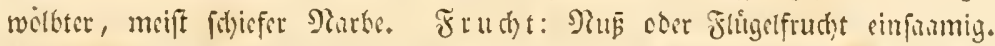

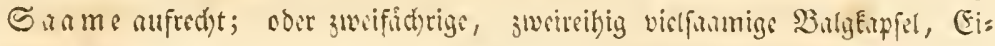

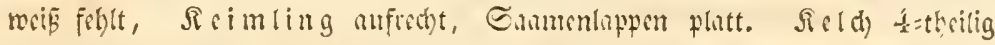

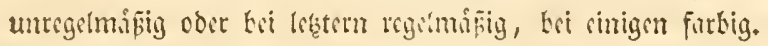

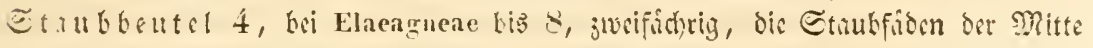

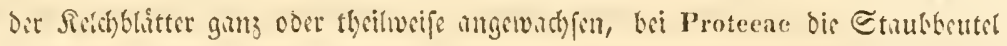

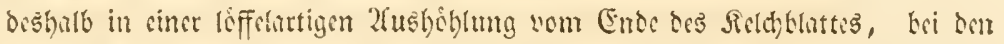

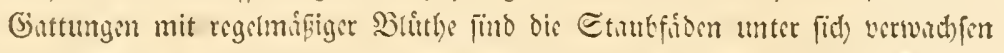
(symphyonema of. Rchb. hort. t. 107.) oocr g.mj frei (Persoonia), kei ber

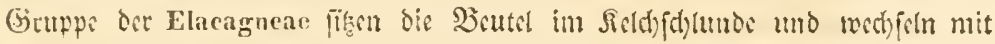

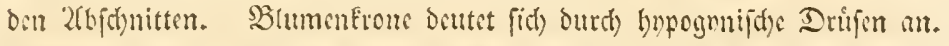

Sigetation. Etumm holzis, Etriuct)er odar Bitume, Etumm ciniger unten Ecocutend werbicft, 2fefte quirlurtig oder zerftreut, Slitter lederurtig, bei legets

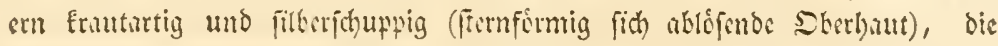

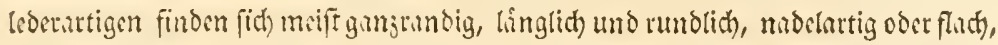

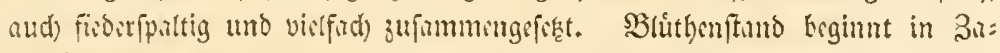

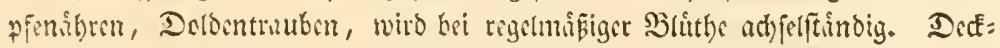

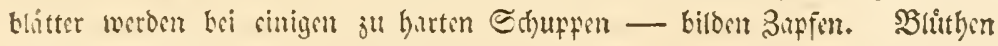

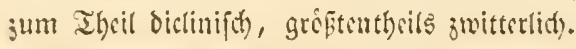

(3) rupcn: 1) Proteeac: - irregulares, a) nuciferac 1332 1746, b) folliculares: 1955 - 1950.

2) Persoonicae: regulares 1748 - 195.

3) Ela eagnea e: alternantherae, 1815 - 1818. - Flora germ. 162 .

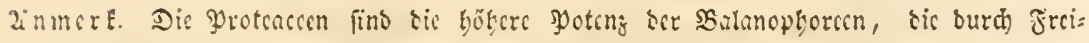

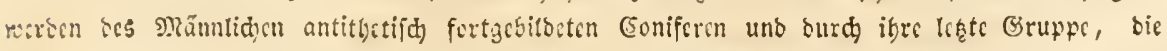

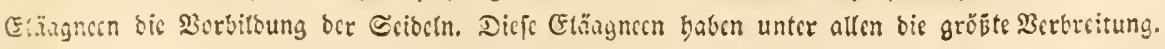

\section{Famitic. Ecibeln: Thrmeleaceac.}

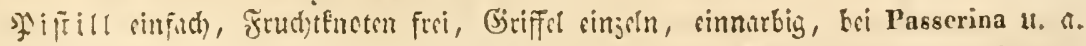
fiitinftindig. Stcinfrud t troffin oder faftig. Saame cinjeln, bingend,

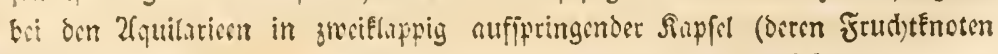


3roifelblumige.

Sromutg: Etrifglätrige. ভribstn.

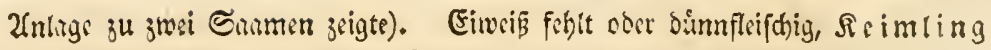

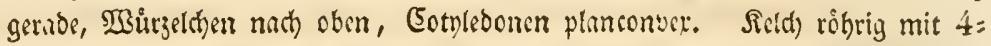

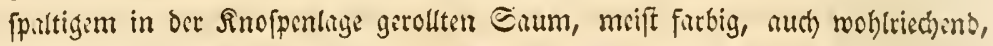
auf ber Frud)t bimwelfend.

Staubbeutel beftimmtzáblig, mift 4 ober 8 (in wenigen 2, auch 10), zmai=

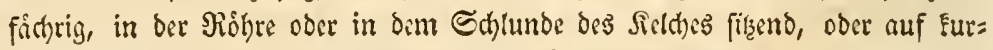
zen Strubfiden. SBlumentrone durd) Drufin im Boben der 2 litthe ober in

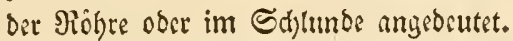

Begetation. Etamm bei eimigen Etruturtig, bei oen meiften bolzig, frraud $=$ uns

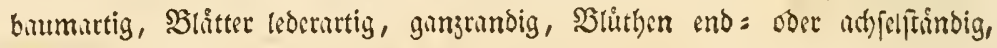
Diclinifo, boch meift jwittertid).

(3ruppen: 1) Darwinieae: $1781-1782$.

2) Daph nea e: 1772-1783, nefft Forestiera Poir, Dirca, Lagetta, Cansiera.

3) Aquilarina e: $178 \frac{3}{-}-1785$ b.

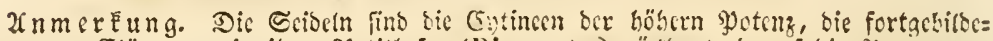

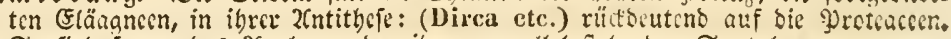
$\mathscr{E}_{e}$ find ferner bas 2enalogon ber itmen parallet feclemben Eantalacen.

73. Familie. Masuricacent: Myricaceae.

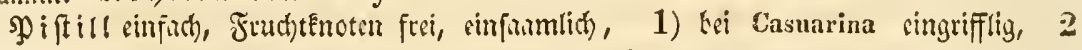

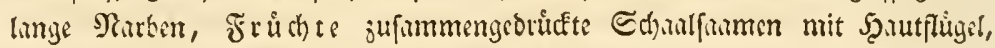

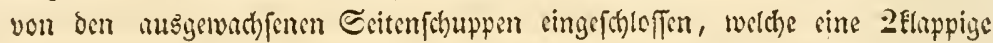

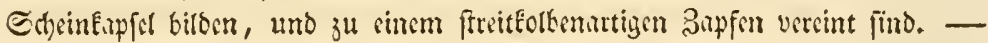

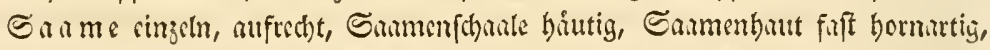

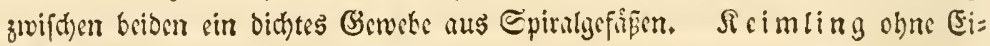
roeiß̧ (verkehrt und mit (Eiweiß bei 3).

2) bei Hyriceae 2 pfriemenförmige Marhen, Steinfrichte einjeln, mit flif d)

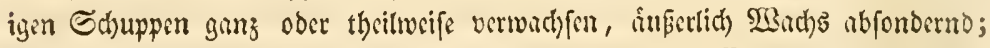
Sanme obne Eiveis, Whitzelden fehr Furz, nach oben, Eotnledonen plan=con= vex. Sryuppige Drefblitter anftutt sines Silches. -

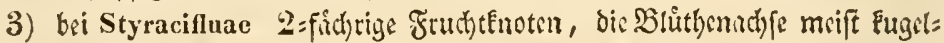

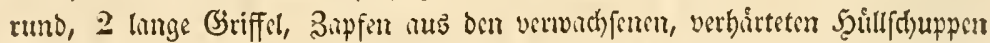
Der Btuthen, in beren Bertiefung die verfelgtt Eegelformigen Sapfeln eings=

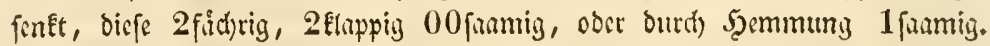

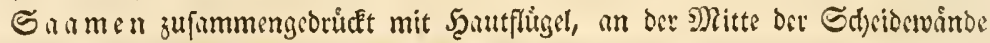

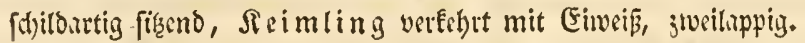

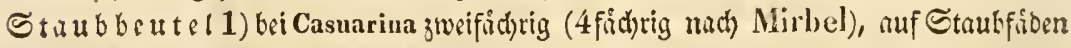

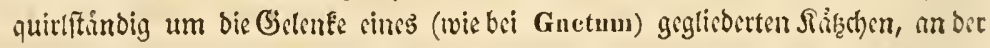

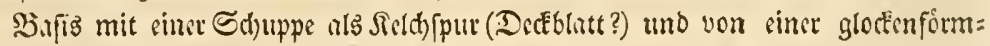
igen, zuhnfpultigen Sabibe des Gistents umgeben, bie Beutel heben kei iber Entwiffelung eine 4theilige (Ealyptra cmpor (Feld)? (Eorolle nach Richard).-

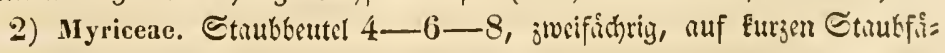

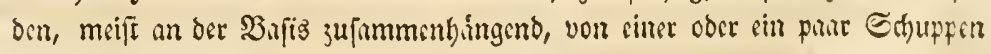

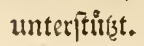


3) St:̈raciluae. Etnutheutel umgrEfhrt berzformig, 2 Elappig, cinzein nuf

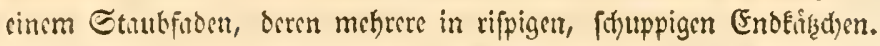

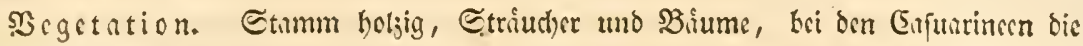

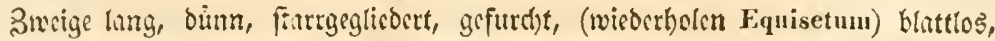
bei 2) uno 3) Blitter wethfirto, meift lcoerartig, fingentandig, bei Liqnidambar bandformig, kei 2) mit bargigeat \$ूun€torifen, bei 3) triofelt der Balfim

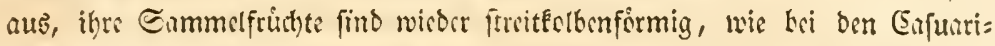

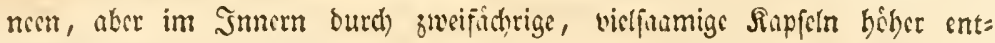
ridfelt.

(Gruppen: 1) Casnarinca c: 1690.

2) $1 \mathrm{yriccac:} 16992.16 \mathrm{~s}-1700 \mathrm{~b}$.

3) Styracifluac: $2 \overline{7}$ J. 1831.

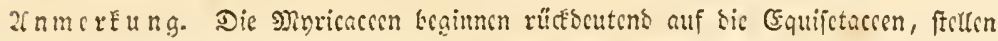

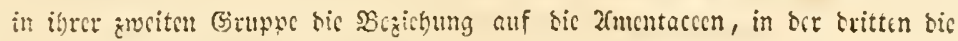

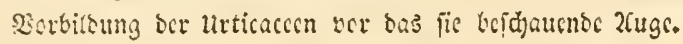

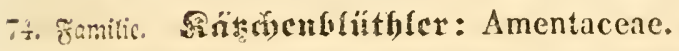

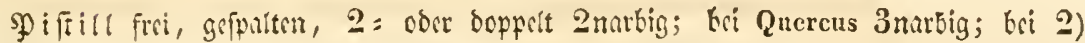
meî̃ mit 2 Şutrflingeln bci 1 und 3 ungcflugelt.

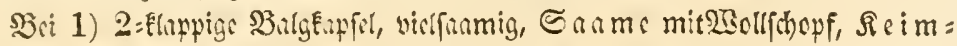

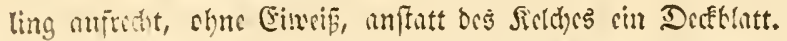

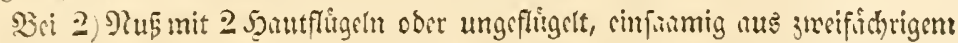
Fruthtfnoten mit cinfaumigen Fichern entfanden, Canme bingend, obne Ei=

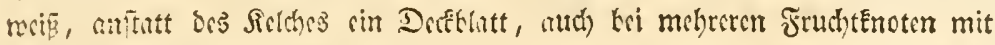

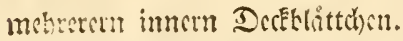

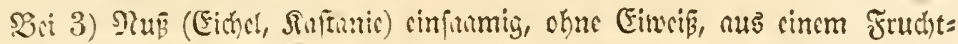

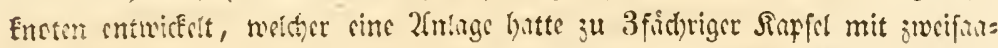

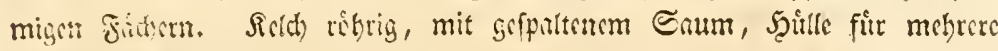
Shtutben 4kentrig.

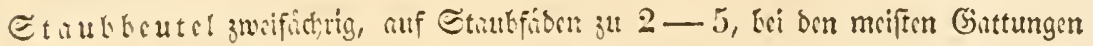

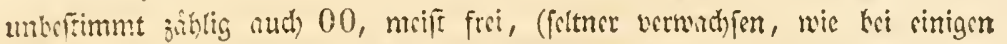

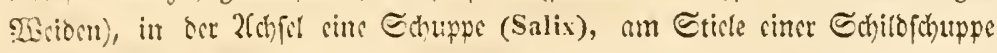

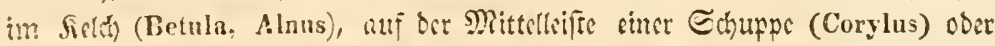
wuf cimm gifpultenen fietd) (Quercus (ctc.).

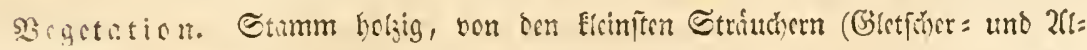

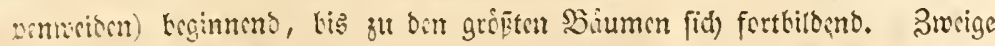

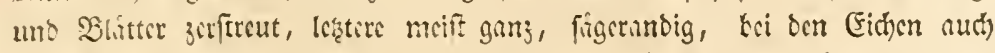

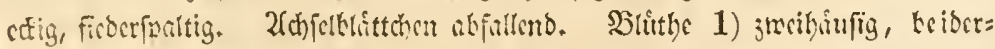

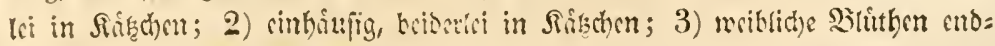

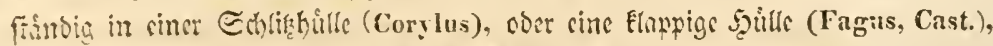

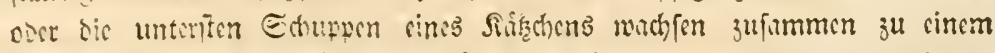

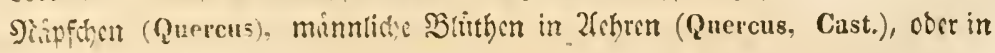
Sicpouncn (Fagus). 
3worfelblumige.

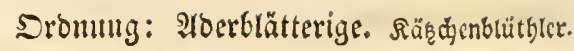

(3ruppen: 1) Saliceae: $1787-1789$.

2) Betuleae: $1790-1798$.

3) Fagineae: 1795-1798, et Lithocarpeo Blume.

Xnmerkung. Dic Cariccen begimen ben neuen Inptrs in Berfdumcizung des

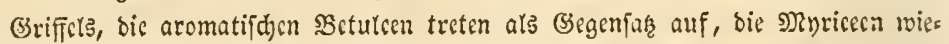

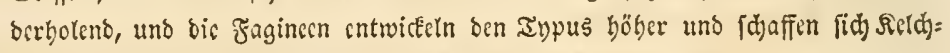

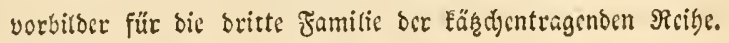

75. Familie. Stefielgewädje: Urticaceae.

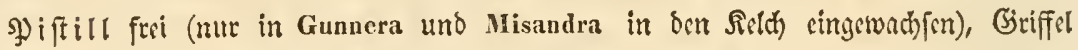
Eurz ober Eeiner, Narben 2 und orr Fruet)tEnoten zweifanmlidy, oder sinfact), aud) ftern = ober freitfolbenformig und der Fruchtfnoten cinfanmitio. Selch $4-5$ thsilig (bei einigen 3 thsilig) oder fehlend. Frucht in Echurlfamen oder Etrimfructet)en. Sanme aufecht oder bingend odre feitlich. Eimeís bei oben genamenten Sinttungen vorbanden, hibrigens fehlent, A e i m l i n g gerube oder

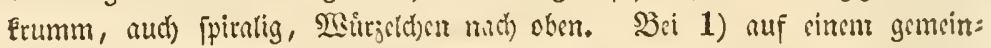
f(t) aftlichen Frudftboden bicht zufunmengebringt, bicfer Euglidy oder linglich

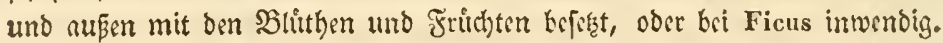

Staubbcutel 2 -4fichrig, meift 4 (boi wemigen $3-5-6$ ), lings auf= fpringend, auf freien Ctnubfiben, welche bei einigen cingetrummt find und

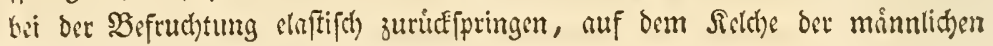
SBlithe eingefingt, vor beffen $2(\mathfrak{b}$ [a)uitten fretert.

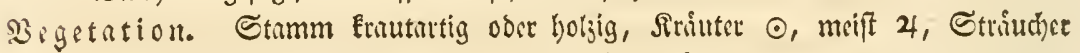

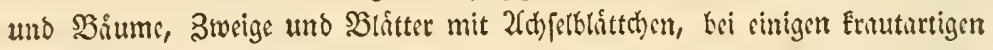
gegeniber, bei ben meiften zerftreut, f(b)urf aut) mit \$rennbraren, meift ge=

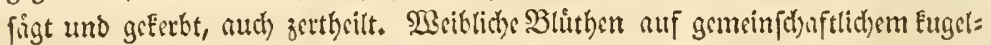
runden oder linglichen Fruchtroden bei ben 2frtosarpese, bei orm (5ruppchen c. inwenbig im anpen umgrbogenen ober (wie bei Ficus faft gefblofferen

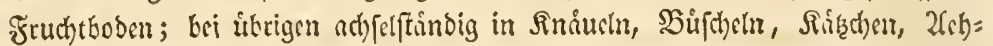

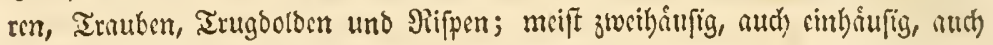
mit S'vitterbluthen gemif̧ct.

(Sruppen: 1) Artocarpene, a. Plataneac: 1894. - b. Moreac: 18131815. et Maclura $\mathrm{Nutt}$. - c. Dorstenieae 1811, 181\%; 1816, 1817. -

2) Urt i ce a e, a. Lnpulinac: 1810. - b. Gunnereac: 1818 et

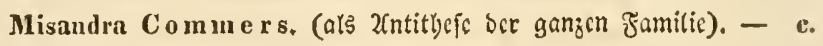
Cecropieac: 1899-1809. 1819-1821. -

3) Ulme a e, a. 1827 - 1829. - b. Celtileae 1830 - 1831 ? c. Lacistemene: 1826 et Synzyganthera Rz. Pav. (Didymandra W.).

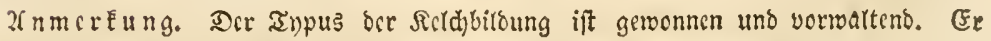

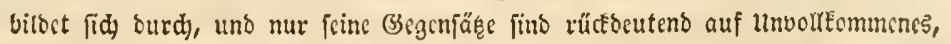
wie es war. Die 26rtocarpeen wicberholen bie Myricaesen, bic Utrticen aber bie 3(mentaccen, uno bic Bollenbung ober bas Centrum fällt in bie britte Bruppe. 
76. Famitic. Oiterfujeicn: Aristolochiaceae.

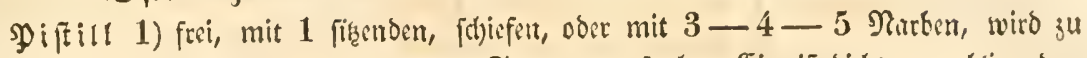

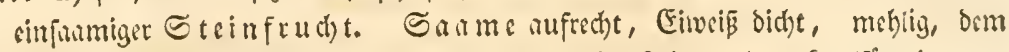

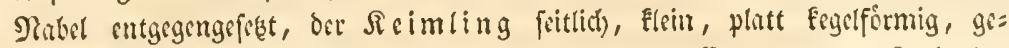

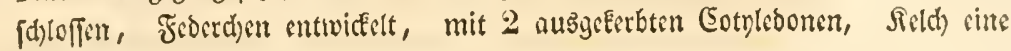
feitlidye Sdyuppe.

2) Frud)tfnoten singewnd)

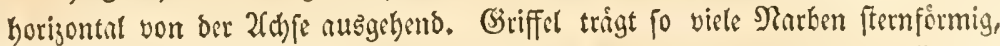

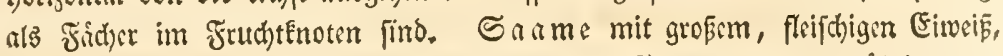

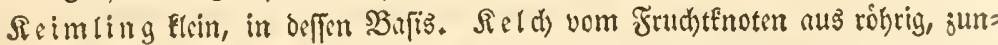
genformig ober bútenartiz, audl) mit Destel, ober breilappig, intoenoig farbig.

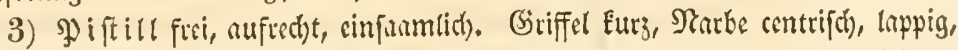

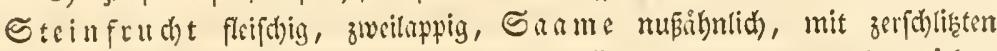

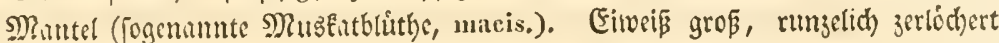

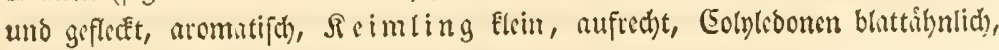

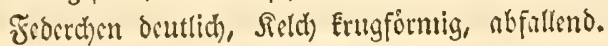

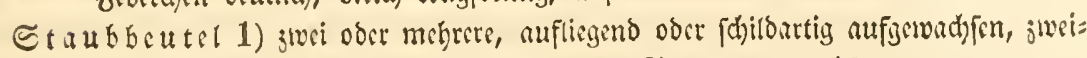

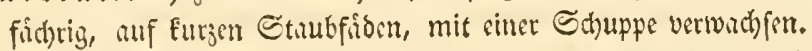

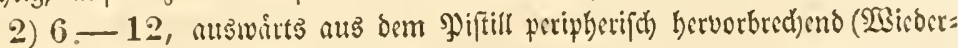

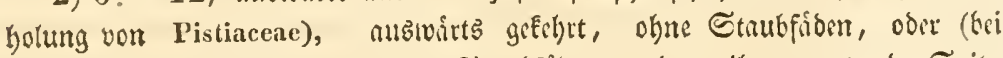

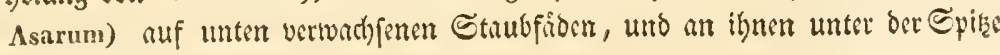
ziwifiritiog Gervorberdgend.

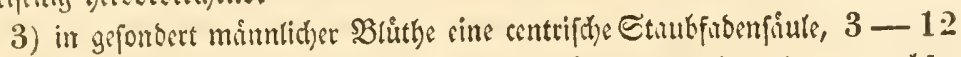

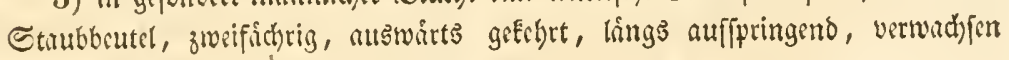
ober gefondert. Ferthputum briellappig.

Begetation. Etamn 1) Etrut= ober frrutchartig, Enotig geglicoert, F̧olfbillo=

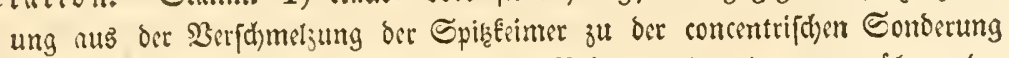

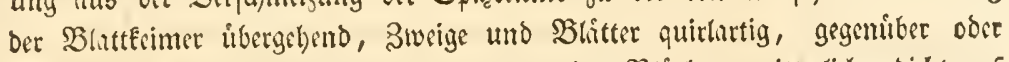

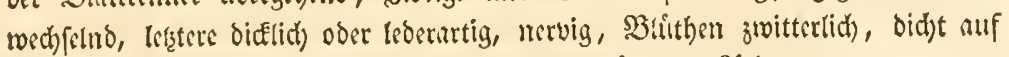

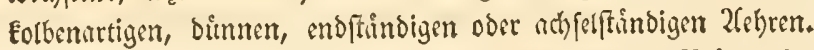

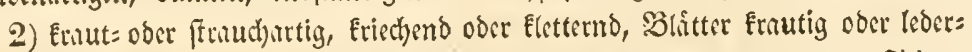
artig, aberig, sinfuch, auf ber 2(ntitt)efe aud getheilt, mit blattartigen Etipu=

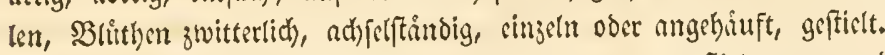

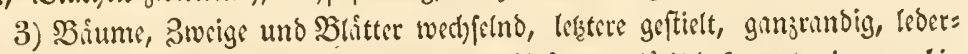

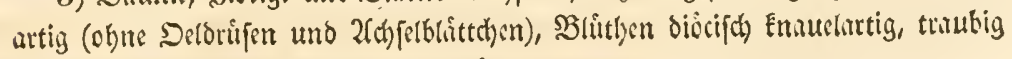
ober rifpig, mit Eappenartigen Derfblíttern.

Bruppen: 1) Pipereae: $1854-1855$.

2) Aristolochiae: a. Braganticae, b. Pistolochiene. Şicricr Aristolochia: Glossula, Pistolochia, Endodeca, Siphidia, Einomeia, Dictyanthes R a fin. - c. Asarineac, - 1856-I861.

3) Myristiceac.

In reicber den Typus. Die \$ipereen geben ben aon an, bie 2rriftotodieen umbül=

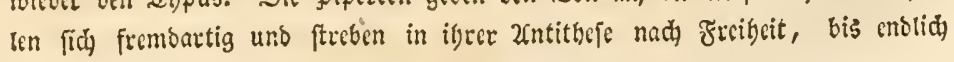




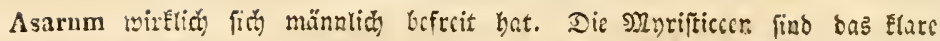
Bersitb dir fautrinecn. (Bargl. Dehansia Blume.)

\section{7.. Famitic. Nuctagincen: Nyctagineen.}

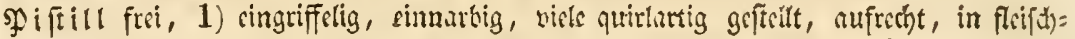

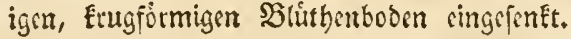

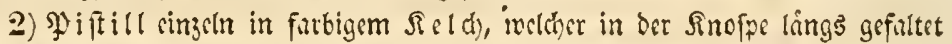

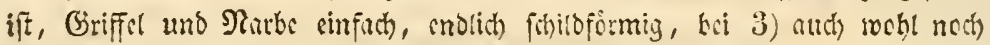

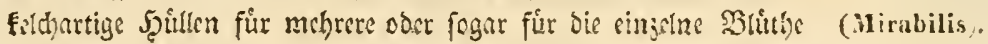
Frukt t kri 1) und 3) cine bagebuttartige Sammelfrtd)t, bei 2) cinfath, bic

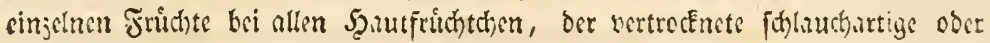

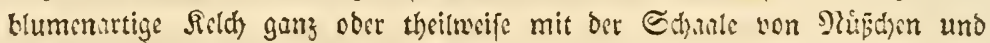
Sar men innig berfomolion. Dod) kemeif't bie miglthe Sondertung yon ber

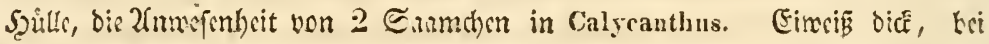
Dionimiere, Atherospermeae, Jyctagincae, fiht fohon in den Galranthim.

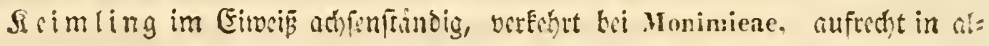

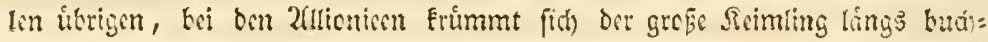

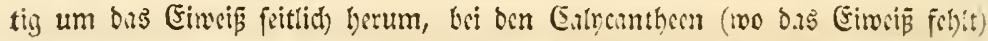
githt bise budtige Sirummung in bis 3upammentolung liber.

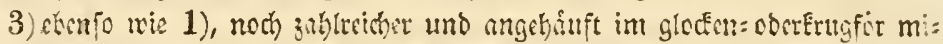

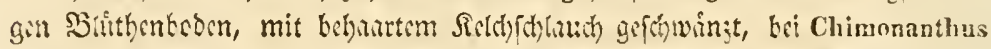

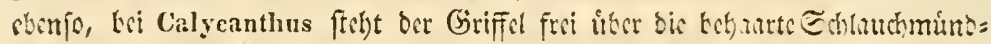

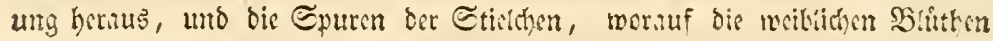

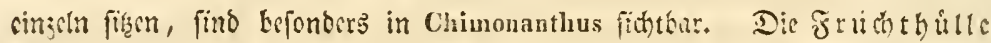

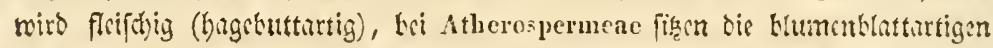

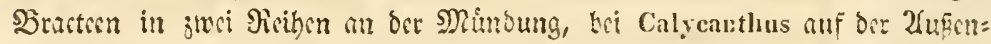
flidje des Erugformigen Fruchtbodens firarrig unto abfallent. (petala A uct.)

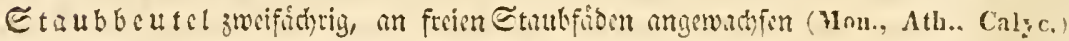

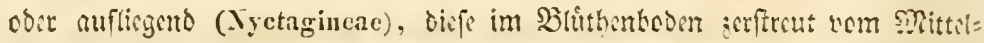
punEt: bis zum Sintic (Monimicae), im SoEen allcin (Atherospermeae), mit

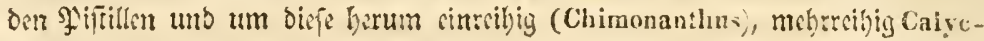
anthus).

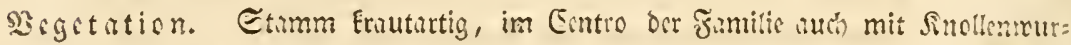

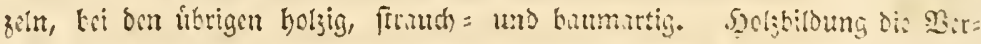

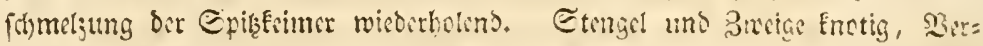

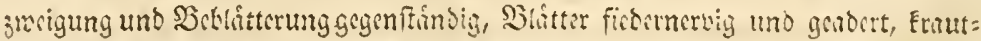

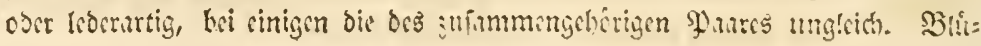

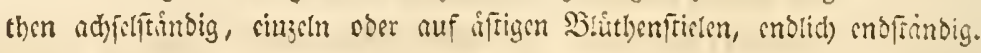
Gruppen: 1) M on im i ca e: 18:2-153.

2) Allionieae: a. Eriogoneac: 4292 . b. Eocrhanieac: $1830-$ 1843, c. Pisonieac: $18+4-1833$.

3) Atherospermune: a. Penueat: 1438. 1838, b. Chimonantheae 1833 , c) Calycantheae: 1832.

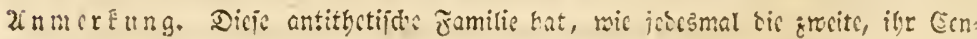

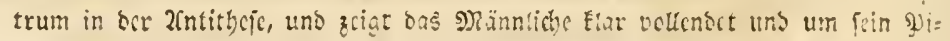




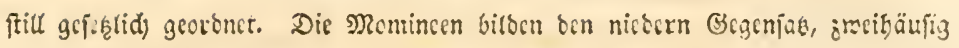

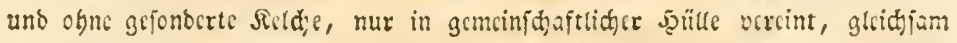

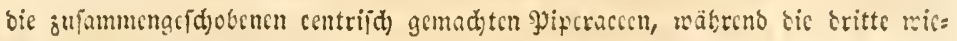
ocr aromatijace Gruppe bic Raurincen fijon andoutet.

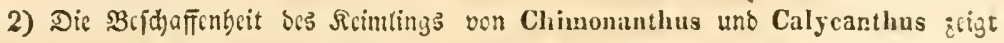
wie bicfer auf ber bäbe feincr antityetifdon Familie bas Fimeis übcrmunten

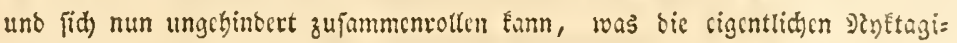

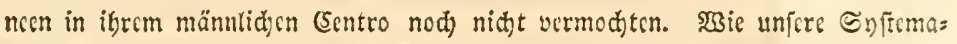

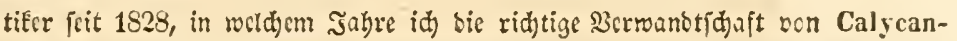
thus im Conspectus unb ben Character in maincr Bcarbsitung von Hössler's Şanssud) gegebon, nodi mit Calycanthus umgciprungen fins, uno wegen scs

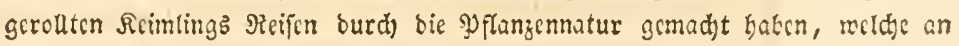
bie ber Flörkea aus ben Scybrodjaribecn erinnert, bus liegt überall als cinici=

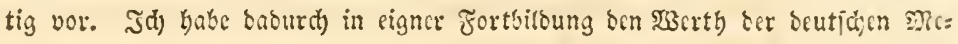

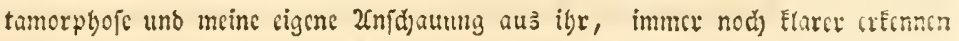
geternt.

\section{Familis. Eurbecrgetuädje: Laurineac.}

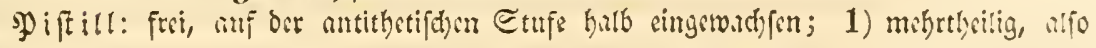

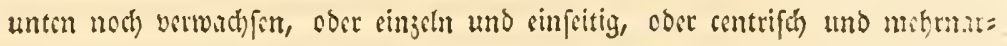

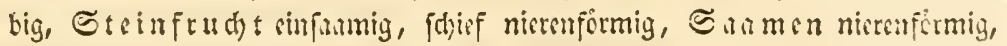

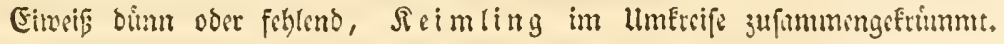

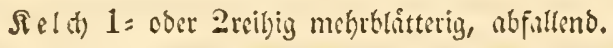

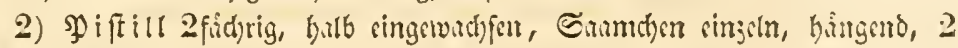

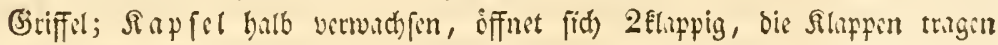

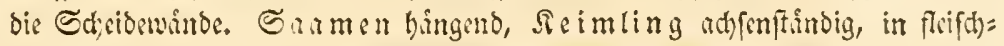

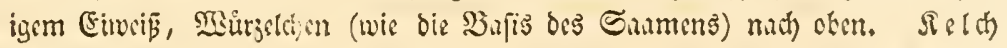
virtbcilig.

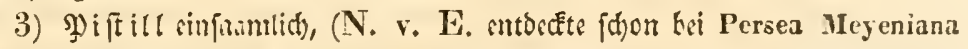

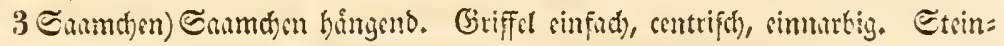

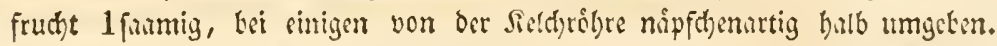

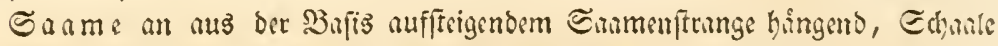

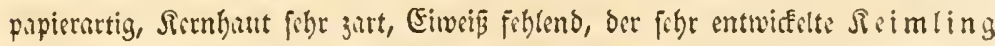

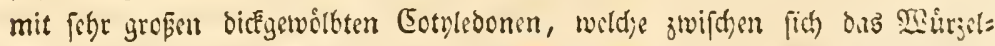

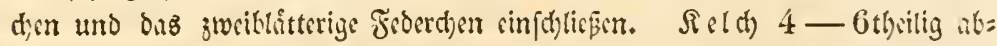
fallend ober beffen Sarfis bleibend, in ber Sinofpe die inuten ?tefonitte teu ben cusern bedouft.

Staubbeutel jweifidity, nuf Stuuffiden, weldye bei einigen Menispermecr ters wathfen find, bei allen übrigen frei.

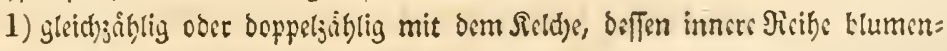
blittirtig iff.

2) 4 Etaukgefine fruchtbar mit Beuteln, weld)e fich 2 fichrig mit Shafen offneu, bie Sluppen fallen ab, 4 Etanbfídon siner iuseren Sisige unfrudtbar

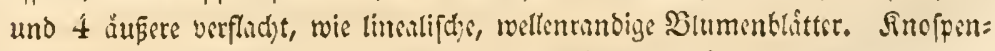

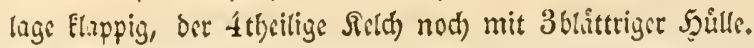


3) Staubgefópe Eeldyftindig, meift boppelt oder orcifuch zoiblig in 2-3 Sici= ben, Seutel angerwact) jen, einwirts, zweifád)rig, mit Silappen aufpringend,

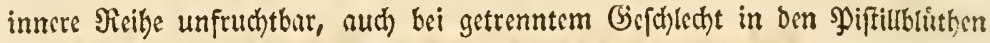
verfitmmert vorbanden.

Sogetation. Stamm frrauch = und baumartig, 1) ranEend und Eletternd, kci 3) in ber 2 Bicderbolung nur Cassytha, tvindend (nuth 2 (rt oer Cuscuta), para:

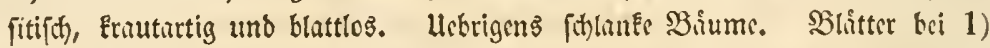
uno 2) meift Erautartig, bei 3) meift lederartig, nervig oder gendert. SBlithen adjel = und endftindig, in Sipfdhen, SBifdelu, Irauben, Mifpen.

(Sruppen: 1) Menispermea e: $1865-1898$.

2) H a mamelideae: 1899-1902.

3) La ure a e: 1904-1916. Suppl. cf. in N.v. E. syst, laur, et Blume Rumphia.

Znuct Eung. Don beridtigten Sharakter von Ilamamelis batte id bercits in

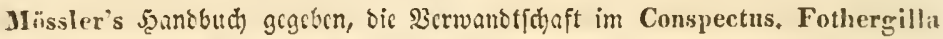

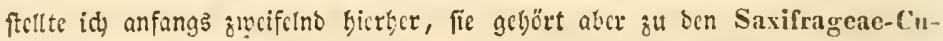
noniaceac, mit bencn bic Hamameliteac nar babituclfe 2fchntich Ecit hoben.

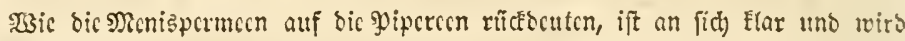

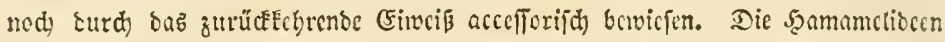

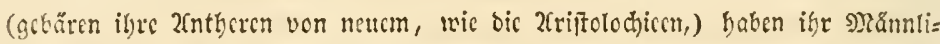

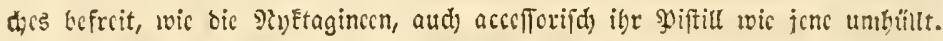
Dic Eauren fino bas rcid) auszcltattete Gentrum, bit colcre Fortbilsung, in welder Laurus nobilis fid meiter entfaltet, fic vellenten wabrbaft nokilitireno

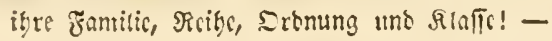


(S)an; blumige. Famitien.

\section{Ecch \\ Ganjblumige: Synpetalae.}

Sinpetalae. Fimiliae.

Erite Sromung.

9öbreublumigze: T'ubiflorae.

Ђุãufclblüthler: A gegregat ac.

Gaumblütbler: Campanaceac.

79. Fram. Diffelf́troen: Dipsaceae.

82. Fam. Sntgencfiften: Synanthereae.

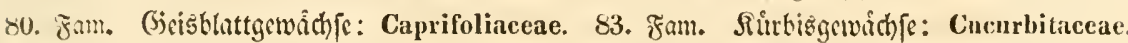

81. Fram. Эubiaceen: liubiaceate.

84. Fam. Gilôteler: Campanulaceae.

Edhlunbblumige: Fanciflorae.

Söbrenblüthier: Tubiferae.

35. Fam. Sippenblithler: Lalliatae.

86. Fam. Scharfblittrige: Asperifoliaceae.

5i. Fram. W3indengewidd [?: Convolvulaeeae.

Saumblüthler: Limbatae.

88. Fam. Gilobulariacen: Globulariaceae.

89. Fanı. Rarbenbluthler: Personatae.

90. $\widetilde{F a m}$. গadhtf(d)atten: Solanaccac.

\section{Eaumblumige: Limbiflorae.}

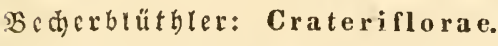

11. Fam. Phumbugincen: Plumbagineac.

9). ซึam. भु) rimutaceen: Primulaceac.

93. Fam. Jૃciðen: Éricaceae.
Stcrnblütblex: Stellifloiac.

94. Fam, 26stleptinden: Asclepiadeac.

95. Fam. Drchbluthler: Contortae.

96. Fam. Gapotacen: Sapotacene.

-9. Familic. Diftelfarben: Dipsaceae.

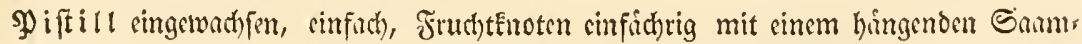

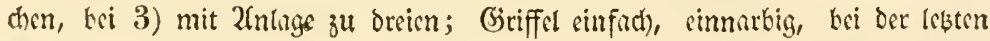
(biattung fehlend, aud) in 3 Rarben getof't. Frutht ein Stharlfume mit dop: peltem \$oppus gefreint, bei den legeten (Sattungen (Valerianella, Sambucus) (a)windet ber immete Pुappus und die Frucht entwickelt 3 Fadjer, aber erft in der leften Gattung cine breifanmige Etcinfrud)t. Sanme verfehnt mit bin=

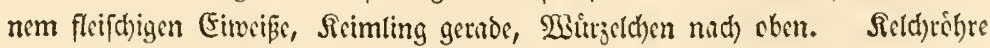

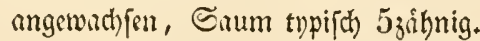

Staubbeutel 2fóchrig, atf freien Stnubfioen in ber Blumenfrone eingefugt,

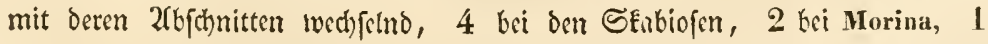
$-3-4-5$ bci den Balerianeen. Slume róbrig, Mumbung $4-j=$ ppaltig, bei ber lef̧en Gattung burch) bic nod) róhrenblumigen (Vilurnum-Solenotiuus) glokeknformig, endich) radformig 5theilig. (Der Eamm hat brmm bie britte Dronung anticipirend, bie hóhre litertwunden).

Begetation. Stamm frutut, zuleşt fruudburtig, Enotigsgelenfig, bei $\mathfrak{B e r b o l j = ~}$ uni noth mit bem dicken Marécylinder; Blitter gegeniber, cinfact) ober ge= firdert und zufammengeferct, $\mathfrak{B e r z w e i g u m g ~ b i c h o t o m ~ ( V a l e r i a n e l l a ) ~ o b e r ~ a t s ~}$ 
Sppofition oreitleitig bei ben meiffen. Blitthenfrand fopfformig mit Setille, rird quirlurtig bei Mirina, aus bem Eopf = und quirtformigen trugdoldig und rifpig bei den $\mathfrak{B}$ allerianeer.

\author{
Bruppen: 1) S cabiosea e: 1981 - 1936. \\ 2) HI orineac: Horina $L$. \\ 3) Valerianeac: a) Valerianeae genuinac uniloculares: Centr- \\ anthus, Valcriana, Triplostegia Wall. Betckea Dec. - b) Va- \\ lerianelleac bi-triloculares: Astrephia Dufr. Fedia Mnck. 'Plect- \\ ritis Lindl. Valerianella 'T. etc. - c) 'Sambacente istig- \\ maticae drupaceae 1-3-Iocnlares 1 - 3-spermae: Viburnum. \\ Samlucus.
}

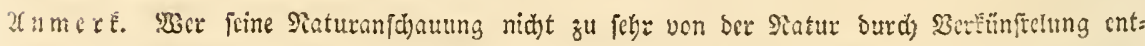

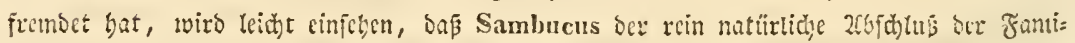

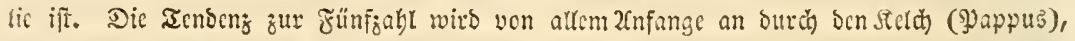

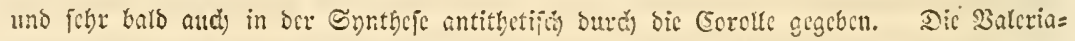

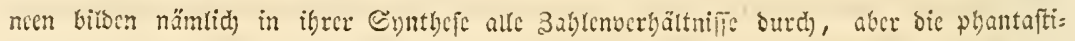

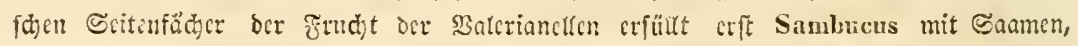
Eenn ber Frühtenoten, welcher Gier ber Inpus ber Familie ijt, vertangt feinen centrijdjen

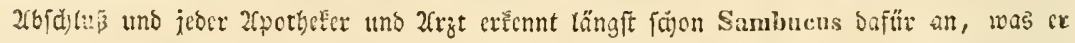

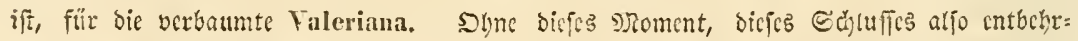

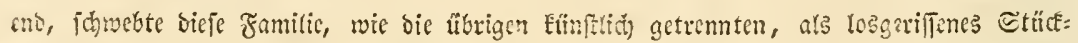

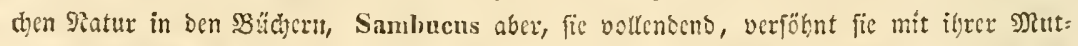
ter Satur. Morina beutet übrigens auf Caprifoliaceae, bic Vulcrianeas aufRubiacear.

¿v. Familic. Geisblattgetwể

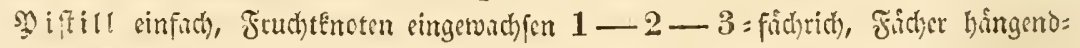
1 -2:fanmlich, Griffel fisltuno, SRarbe cinfuch, bei Rhizoph. gefpulten. Eteinfucht 1-2-3=fichrig, gefront, meift faftig, kei wenigen (lihizoph. Diervilla, Linnaca) trofin. (Fin spigynifdes 9lingpolfir bi Lorantheae. Samme bangend zu 1-2, Schate bart, Eimein fleifhig, bei

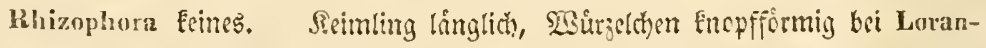

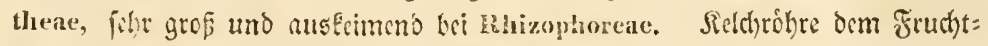
fnotrn gumj angewathfor, Earum furj und ganj oder linger und $4-5=$ theilig.

Strubgefá̧e 5 (4-6-8) Beutel odr Strubfibon bri Lorantheae auf

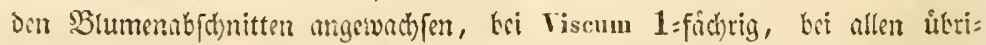

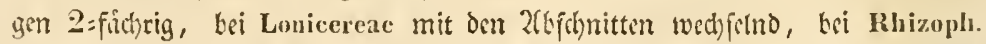

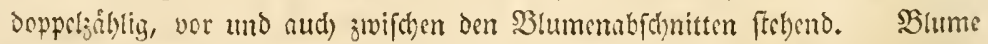
róbrig bai Lorantheae, 2(bfd)nitte Elappig, bei sinigen ticf jertbeilt, chenjo ker Rhizophoreas; róbrig obcr glofig, bie 2lkfdgnitte umeinunder gelegt, bei Lonicercae.

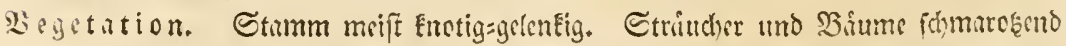
wie Lorantieae, friect)end wie Lintaea, fletterto oder am Erefirunde (der Tropenlinde:) im Ed)hamme murgetntes Didfid)t bildend: Rhizophoreae.

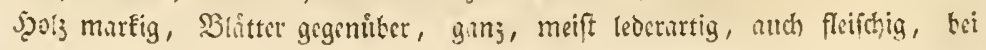




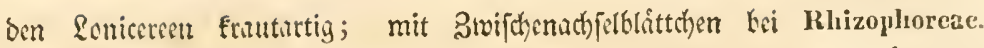

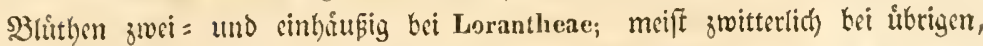

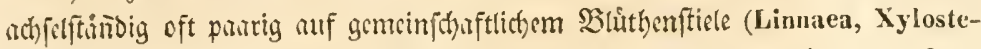
uin), biiphelartig uno trutbig (Lorantheae, Symphoricarpos) quirl = uno fopf= artig (Lonicera.)

(3ruppen: 1) Lorantheae: 1959-1968 et suppl.

2) Lonicereae: $1957-1985$ et suppl.

3) Rhizophoreae: 1967-1969. (Cassipouren videtur Chrysobalanea).

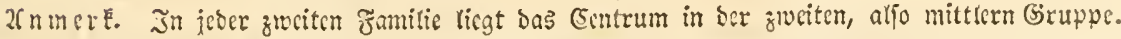

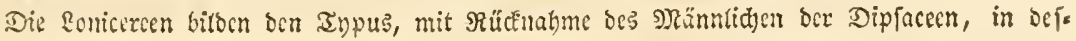

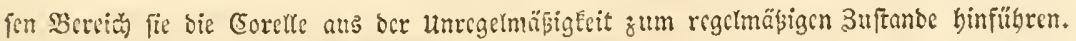

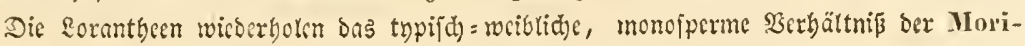
neae, als zrocitcr Bruppe bcr erften Familie uno bic Rhizophoreae tretcn in' bie Elurfte

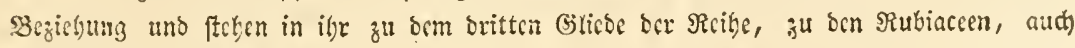

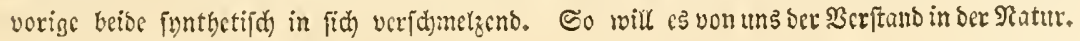

81. Familic. DEtbitrest: Rubiaceae.

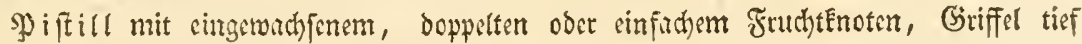
getheilt ober cinfach, Narben 2 fopfformige (Stellatae), ober 2-5 plitt=

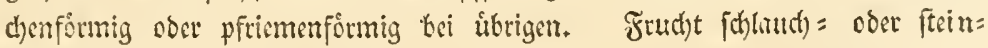

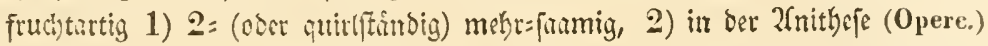

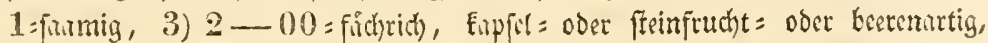

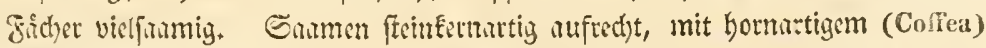

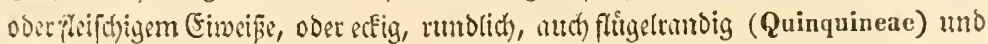

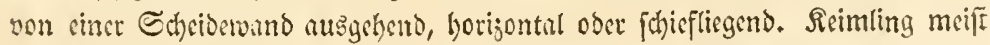

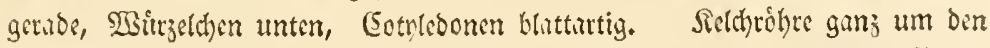

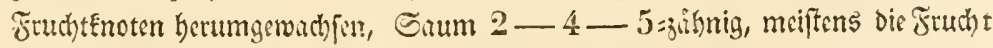
Erôneno, (jhon bet don Stellatae: Sherardia) oder fihtento.

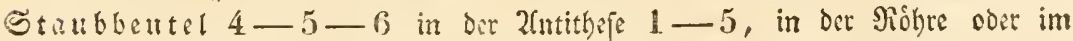

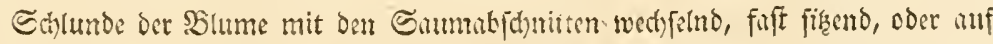

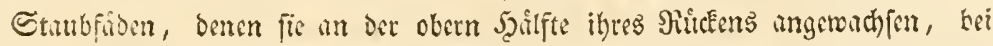

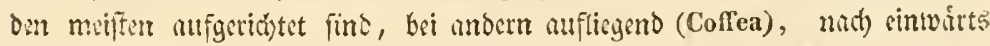

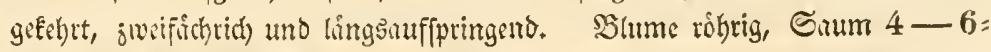
fipaltiy in ber sinofpe kerumyelegt.

Bigetation. Sráuter, Etríuder, Bisume, Stonget ober junger Stamm Enotig= getentig, SBtâtter ungetbeit, bei 1) quir(ftânsig, bei 2) uno 3) gegeniftándig,

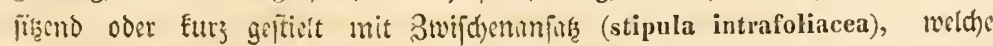

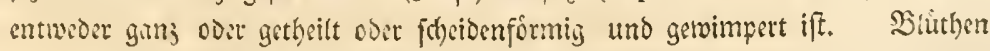
meift zwittertid), hei wenigen biclinifd) (Vaillantia, Galium Cruciata) in don

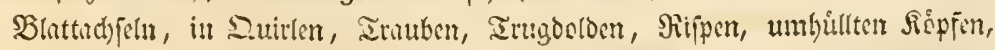

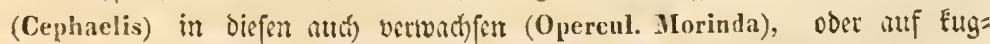
tichun Frudotboden nugehiuftet (Cephrilanthus, Sarcocephalus, Nauelea).

Bruppcn: 1) Stellatae: sphacrostigmaticae, 1986-1991. Fl, germ. P. $201-210$. 
2) An thospermeae: schizostyles (Stigmata filiformi-subulata, aliis lirtella.)

a) Opercularinae: I-spermae. - Operenlaria Gïrt. Pomax Soliand.

b) Anthospermeac: capsula 2 sperma. - Anthospermum L. Ambraria Cruse. Galopina Thnb. Phyllis $L$.

c) Coprosmeae: bacca nuculis osseis monospermis. - Coprosma Forst.

3) Coffeariae: schizostigmaticae (stigm. lamellatis aut subulatis) 2-polyspermac.

a) Spermacoceae : platystigmaticae 2-4-pyrenaceac. $\left.{ }^{\propto}\right)$ Putorieae: Putoria $P$. Plocama Ait, Rchb. hort. t.11. etc. $\beta$ ) Euspermacoceac. 1998 etc. - \%) Cephalantheae: Cephilantious etc. -

b) Coffeinae: 2-5-20-pyrenaceae drupaceae. $-{ }^{\infty}$ ) Cephä̈lideac: capitatae involucratae. Cephaëlis etc. - $\beta$ ) Psychotrieae: Fl. distincti. Psycliotria, Coffea ete. - $\gamma$ ) Guettardeae: drupa $2-6$ aut bacca pluriloculari loculis monospermis. Griettarda, Cordiera. -

c) Cinclineae: 2-00 loculares polyspermae. «) Hedyotideae: 2-loculares capsulares apterospermae. Hedyotis, Rondeletia, bicrber auch Houstonia $\boldsymbol{L}$. etc. - $\beta$ ) Quinquineae: 2-loculares pterospermae. Cinchona, Exostemuna. Nauclea etc. $\%$ ) Gardenieae: 2-6-00-locul. drupaceae v. baceatae. Isertia, Hamelia, Gardenia etc.

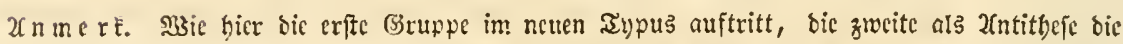

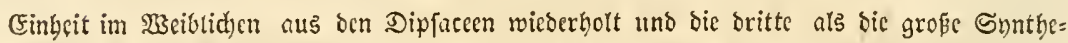
fe uns bas inrex Etethnng als britte Familic, entfprcthende Eentrum, alles wieder auf fich

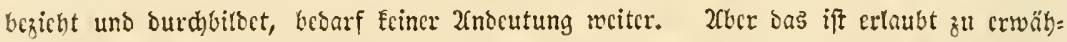

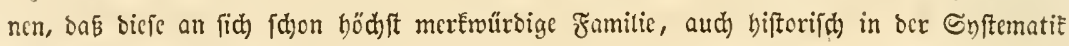

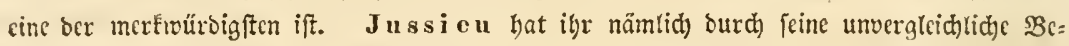

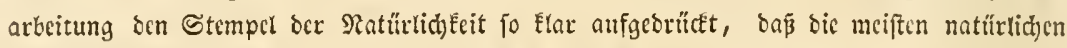

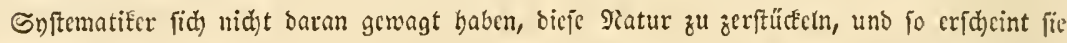
in Decandolles prollomus systenatis naturalis als cine rcine natürlide fá

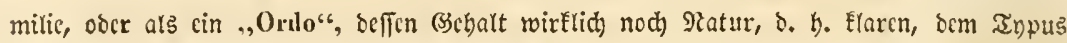

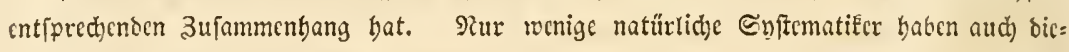
ic Siatur fünftich zu zerreifen verfud)t, abcr Decandolle bat hödff verticnftooll

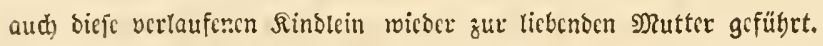

\section{Q2. Fantitie. Enugenep̈iten: Synanthereae.}

1) iftill: Frudhtenoten cingewad) fen, sinfach), sinfinumlich), (Siriffl cinfach), am (Ende

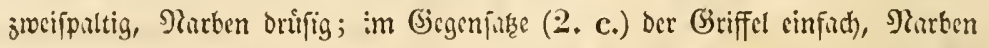

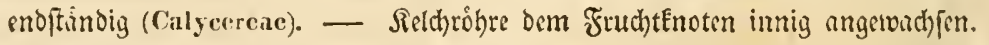
Saum tildet eine Sanmenfrone (pappus), wenig ober viclftruthlig, ein= ober

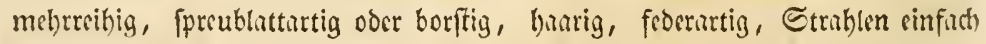
oder gefpalten (Carlini:). Im Girgenfuse (2.) dic Reld)e zufammengewachfon

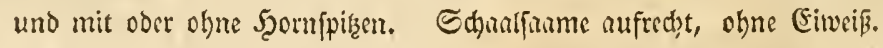

Staubbeutel 5, linglich), zmeifichrich, einwirts gefefhrt und lings aufipringeno,

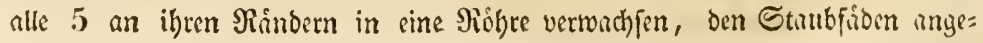


warth fen, rocldye unter ibnen frei, aber weiter unten mit oer Blumenribe

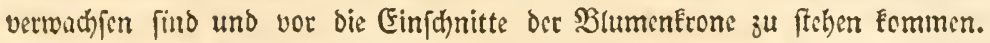
Im Gigenfake (2.) find die Beutel frei und dis Füben vermadfien. Sis

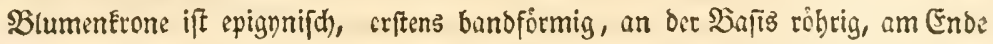

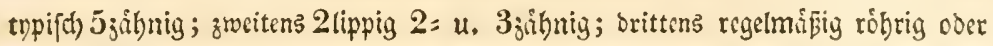

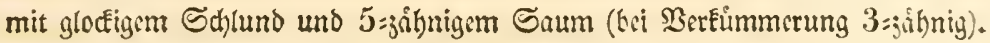

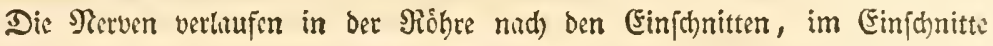
theilt fich jeder gabelartig und verliuft in ben গand von 2 kenadjbarten Jîh= nen. (2fmphigenetifhe Stipularbildung ber (Sorolle).

Negetation. Stamm fraut= oder ftruchurtig, Slitter gegeniber, bei wenigen quirlartig, meiff wedbictno uno jerfifteut son allerlei Subftanzen und Formen. Sluthenftund ein Ed)ibenfopf (compositum), burd) bie verfürjte Slutben= ach fe oder biejer gemeinjd)aftid)e Bluthenboden (receptaculum) ift Eci rrenigen Eolbenformig (Rudbeckia), bei ben meiften getwolbt ober flad), mit Girukdhen

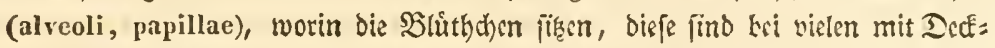

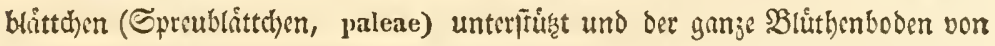
auper mit cinet Şưlle (anthodium), welche bei cinigen nod) eine befondere Blatt= bưlle (involucrum) bat, umgeben. Dieşlittchen ber erjeteren (squamac) find cin=

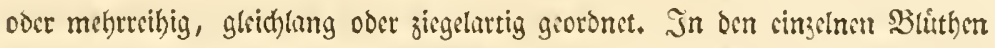
cines Scheibenfopfes diejer Compositae finbet fich das (jejhled)t veridhieden, bei einigen ein = und zmeihiusig vertheilt.

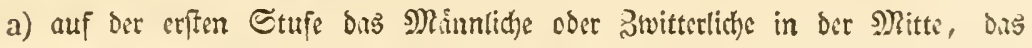

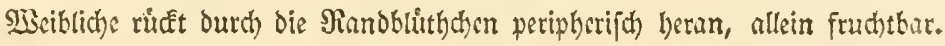

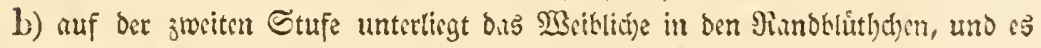
finden jich in ifmen unfrud)tbate ober gar Eeine (jriffel und Narken uno nur bie Slůthdyen ber Sdeibe bringen ifre Saamen jur Sieife.

c) auf ber britten Stufe ift alles ausgegliden uno bie verpobnten Gejhledter

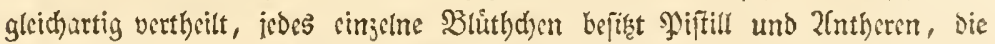

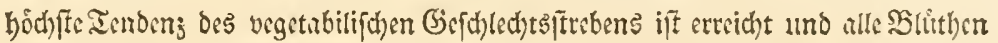
bringen frudjtbare Sirmen.

Der Begenfats (2.) trennt bie Gejd)letter wieder, fo d.rẽ in a) und b)

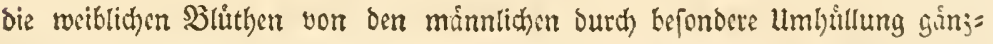

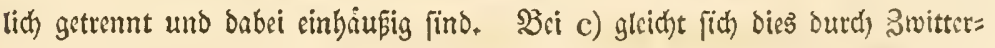
blüthen wieder aus.

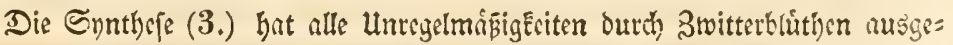
glichen, behart aber dibrigens noch im Inpts ber Snnuntheren, mije nuт

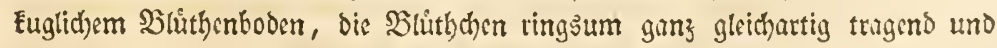

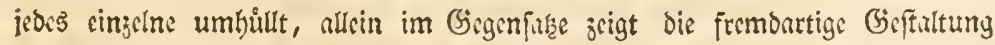

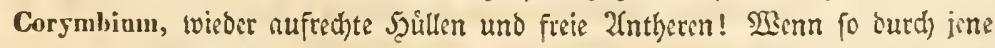
Die Bebeutung bes etwat gejtwungenen Berbiltniffes bes Cobibenfopfes it einer vollendeten Sugclperipherie endlid in Sricben getô't und bic Blithen sercinjelt roorden, fo werden auch hier bic Stutbeutel fret und in ber 2 seife ifhes For=

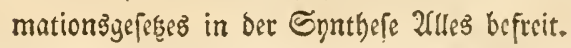


(3ruppen: 1) Composit a e: synanthereae genuinac.

a) Amphigymanthae, (Syng. necess, et superfua Linn.)

a. liguliflorae: Melampodieae.

B. labiatiflorae: Dipterocome Fisch, et Mey. Perdiciun, Leria etc.

$\%$ tnbuliflorat: Gnaphalieae.

b) Amplicenianthae. (Syng. frustcanea Linn.)

a. liguliflorae: Heliantlicae.

ß. labiatiflorae: Bacazia, Zoegrea, Stockesia etc.

$\%$ tubuliforae: Centaurinae.

c) Homoianthae (Syng, aequalis Linn.)

a. liguliflorae: Cichoriaceae.

6. labiatiflorae: Mutisieac (et Nassaurieae).

$\gamma$, tubuliflorac: Cynarcae.

2) Syncarpicae: elcutherantherae monadelpliae.

a) Xanthiene: androcephalae, floribus masculis in receptaculo globoso segregatis.

b) Ambrosieae: androstachyae, floribus masculis pluribus in involucris spicatis.

c) Calycercae: hemaphroditae semine inverso.

3) Segregatae: synanthereae et eleutheranthereae homoianthae floribus singulis involucellatis.

a) Eleplantopeae: compresse-involucratae squamis alternis conduplicatis, corollis palnatis.

b) Corymbieae: verticillato-diplyyllo-involucratae corollis 5fidis, antlerae demum liberae.

c) Echinopeae: exinvolucratae spliaeroceplaalae coroll. 5-partitis regularibus.

Conf. Conspectus No. $2121-2744$.

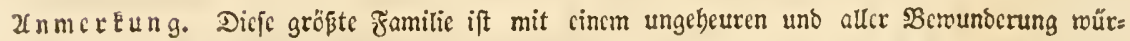

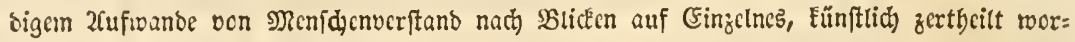

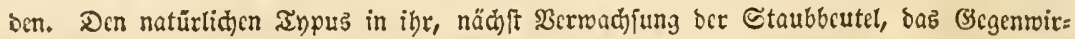

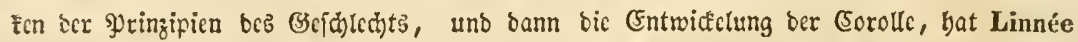

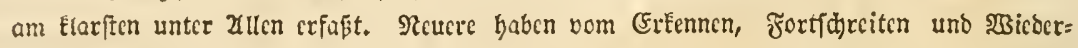

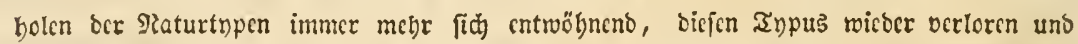

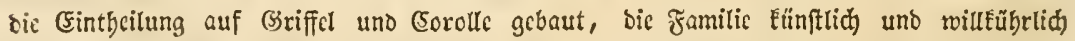

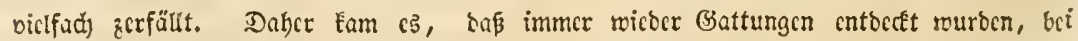
benen man ric Fischer uno Meyer bei Dipterocome fagen muß̈te: ,genus in nulla ex tribubns a cl. Cassin i conditis apte collocandum" uno folde Tribus müßten zur

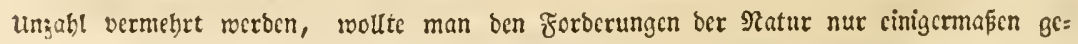
nügeno verfalyren, einen aypenmeifer uno genetifdje Entwicéclung roürbe man ocnnod) 


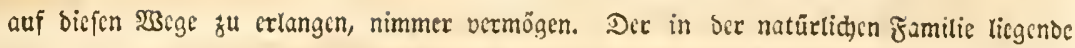

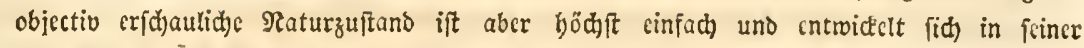

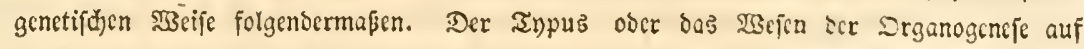
bicicr Stufe beftebt nämlid:

1) in Entrodelung ore 2fntycren:, , a therae oriuntur" Flora germ. p. 211. Diefe 2fnthercuentwicketung cridscint auf bicfer Stufe fyngenctifh, b. b. in Berwadjung,

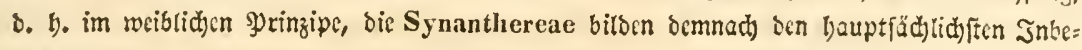
griff ber Familic auf allen orei Şauptifufuen.

2) folgt bas lebendig fortfdyreitende \$alten bes allgemeinen (Scid)led)tstypus, wie oben

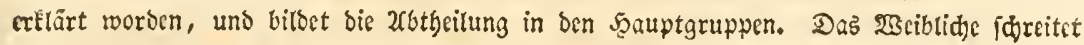

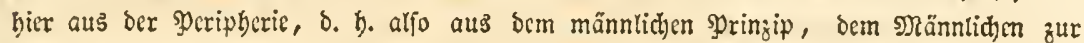
Bermäblung entgegen, bis die Homoianthae alle Differcng ausgleidjen uno bie Echino-

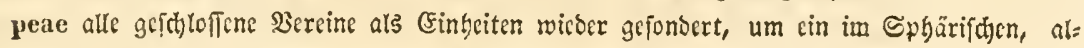

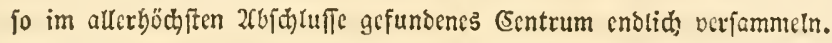

3) madt fid die 2enticipation ber (5orolle aus ber sritten Familie, gelteno und geigt uns cine parallele Entrickelung mit ber Eorolle ber (5ampanulaceen, nur mit bem unter=

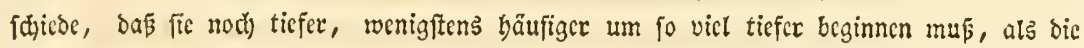

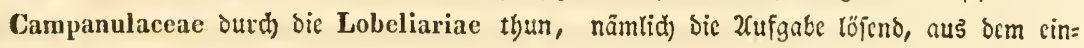

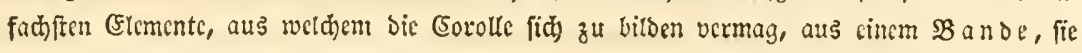
zu fdaffen. Diefe Bungenblïthdon Eommen barum bier fo bäufig vor, bod̈ allamal nur auf ber nicorigften Stclke. Shr 3ufammenrollen bringt anfangs mur bic unregctmäß̈ig

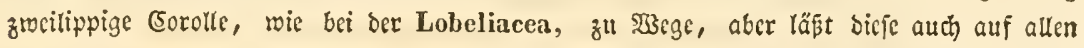

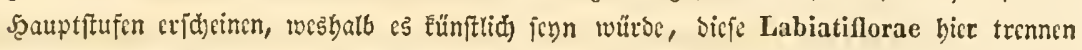

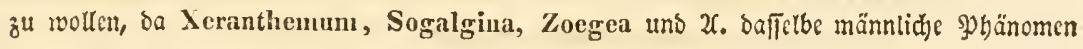

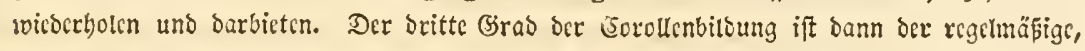

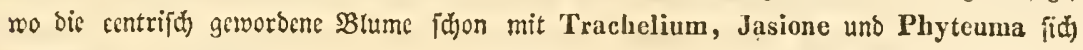
zu verglididen vermag.

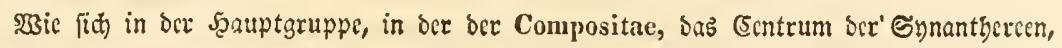

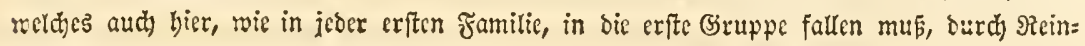

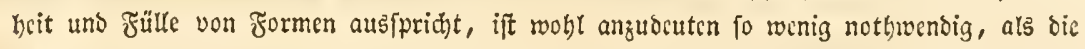
SBģ

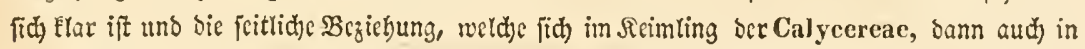

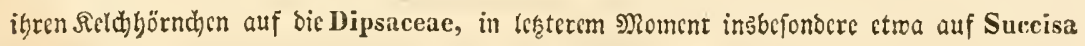
corniculata u. a. ausfpridjt, aud bürfte bie ber Segregatae auf bie Campanulaceae tridht

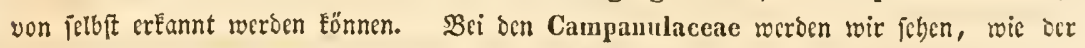

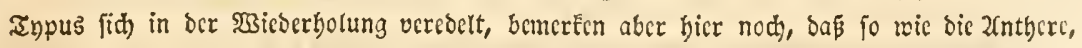

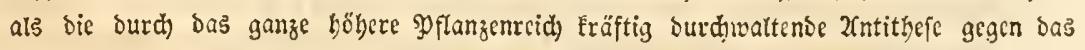

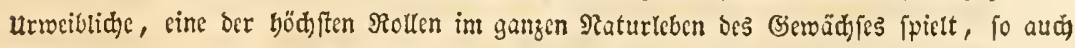

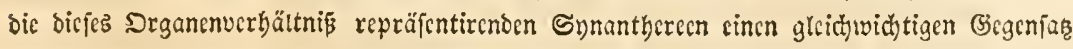
gegen bie ganze böbere Pflanzennatur bilden, und unter allen ben Familien, weld) Derie!l= ben Typus: ,, antherae oriuntur." ałs ben ihrigen erfennen, die ftänffete, uno gerabe biet auf bicjer Stufe barum bie ftänffite Familie bes 2rntbcrentyptts find, weil bier bas

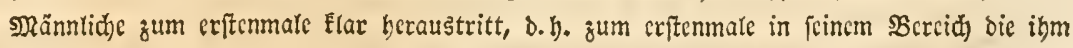

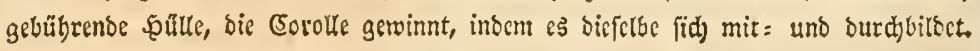


Gianjblumige.

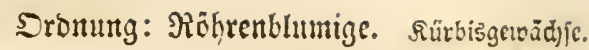

\section{Familic. Sürbig̊gcwäbị: Cucurbitaceae.}

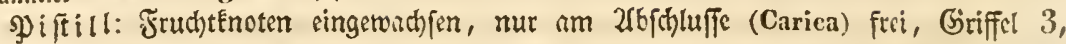
find gefondert oder zur (Finbeit verfomolzen, meift fpaltnarbig. Reld)= róhre dem Jruchtennoten angewachfen, bei Carica frei, Schlund die Slu= menfrone tragend, Saum 5:\{paltig. Frutht fleifhig rinbig=becrenartig:

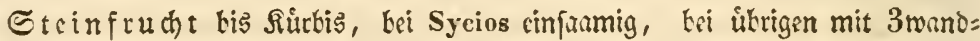
ftindigen $1-2-00$ faramigen Samentrígern, weldhe hatbe Echeide= rainde bilden, Saamenftrang am Sanmenende verbift, Saame aufrecht, 2Bürselchen am Rabel, Reimling ohne Eimeif, Eotyldoonen fart blatturtig. Staubbeutel 2=fichrig, austwirtz bei Nhandirob. uno Cueurbiteae, einwirts bei Cariea, bei Nhandirobene uno Carica zu 5-10 auf freien $\Xi_{t a u b=}$

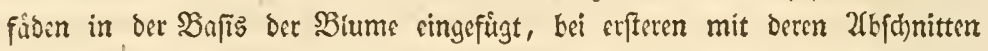
wechfelnd, bei Cariea doppeljabliy, fo das Beutel auf Staubfiben an ber

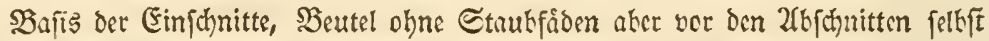

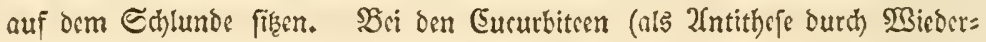

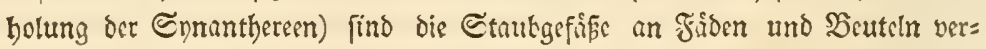
road) $\{\mathfrak{c}$, bei einigen jur Dreizahl verfimmett Cueumis, Cucurbita), Bacutcls

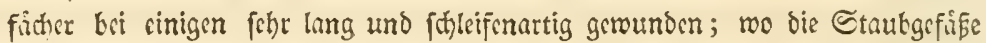
gefondert find, find auth dicfe Fiad)er noch methe gefondert. BlumenErone

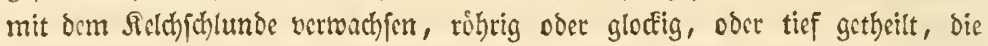

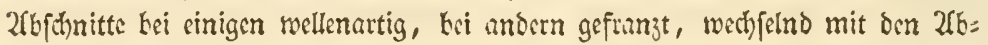
[dinitten bes Riciths.

Begetation. Stamm. 2Burzel meift cinjährig, bei sinigen (Bryonia) Snollen

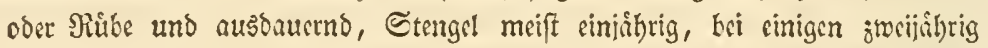

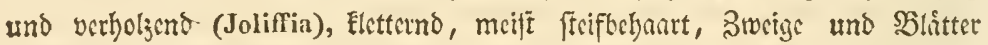
zerffreut, bicfe geftelt, bri cinigen cinfach) (Zanonia), meift bandformig jer=

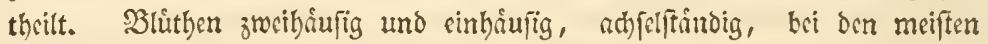

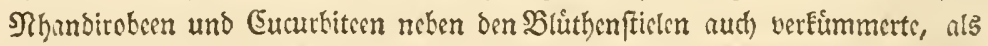

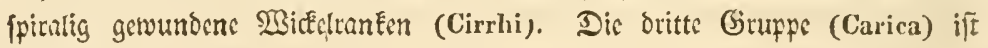
kaumartig.

Gruppen: 1) Nhandirobeae: epiggnae eleutherantherene introrsae, 2871 -2872, 2850. (Kolbea est Passiflorea).

2) Cucurbiteae: epigynae synanthereac r. eleuth. extrorsae. 2746-2765.

3) P арау асеас: hypogynae diplostemoneac introrsac, 2883.

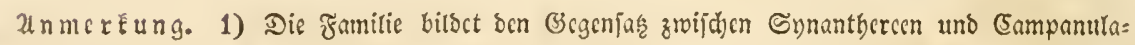

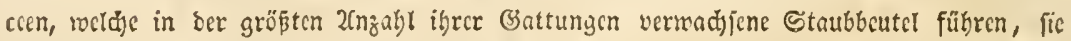

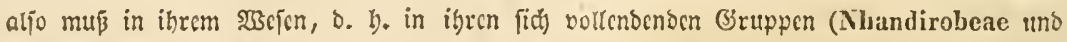

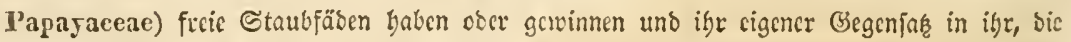

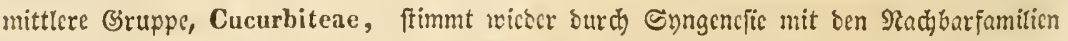

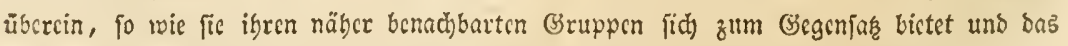
(Sentrum ber Familie burch bie meiften Formen beftimmt.

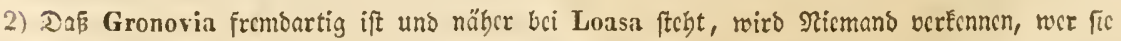

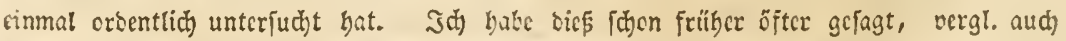
Hort, bot. 11, p. 9. Berghl. Loasueeac. 
Gianjblunige.

Sronuแg: Jiöโrenblumige. Sürbisgcroäd)

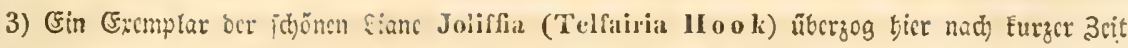

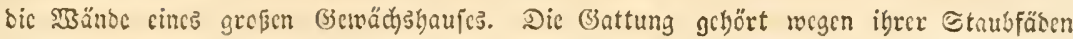
mit gerabcn, gcjonberten $\mathfrak{B} e$ tutcln, allerbings wic id fie geftellt babc, nähcr zu Feuillaea als zu Trichosanthes, weldfer Icegteren fie nur burd) bie gefrungte Scrolle babituell äbn=

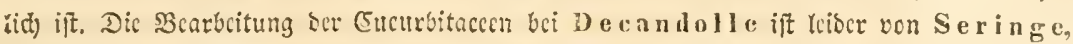
baher crfährt man bort gar nicht cimmal, ob Juliffia 2fnthcren hat und crfähret in ber gunjen Fantitie nichts, liber bic bice gerabe fo rvidjtige Richtung ber 2(ntheren üterhaupt,

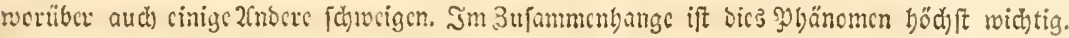

4. Fumitic. Givadfrer: Campanulaceac.

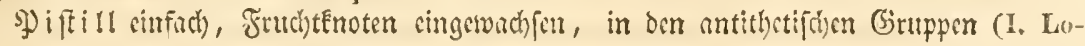
belicae, b) 子. S. Byrsanthes, 2) Styliliariae, b) Scaevoleac, 3) Campa nuleae, b) Canarincae) freimerbens. (jifffel aus $2-3$ verfd)molyen, mit $1-2-3-5-6-8$ Siarben, bei den Sobclien, હane= volern und Bjoobenicen mit bedjecurtigem, am Simbe gefurertin Siblcier los: bar umgeten, bai úsrigen biefe Fufern unter ben Jarben über ben Gitiffer

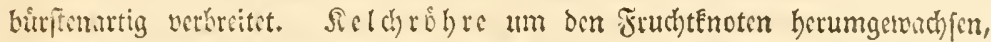

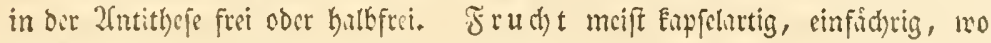

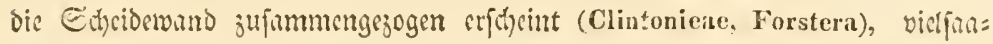

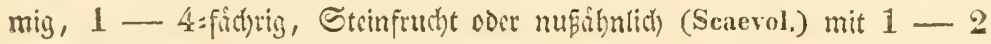
aufrediten Sanmen, tei den itbrigen $2-3=$ finthig siclfamig, mit Defel aufpringeno (1lypsela, Sphenoclea), aufreiß̄no (j. S3. Prismatocarp), mit Richern umfipringend (Campanula), Docr beerenattig und nid)t auffpringend,

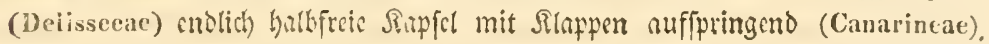
Cunmentriger 2. wambftindig odr auf oer breiten Eabcidewand verfdwimm:

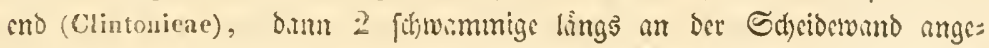

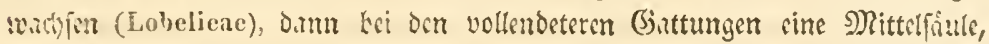
bei Jasione ballb, bri librigan gans, mit fownmmigen Sanmentrigan,

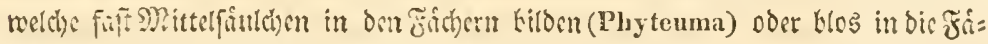

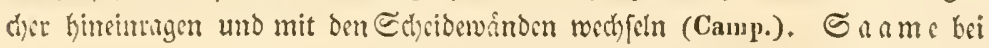

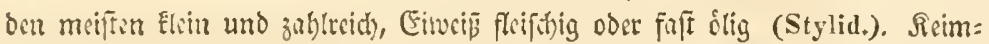
ling in ber S3iftis anfrect)t, gerobe, lingliche, bei Scaevol. unb Good. rund:

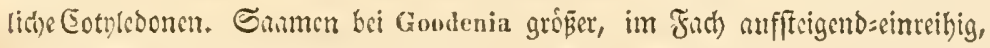

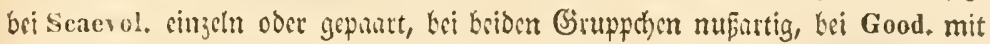
vertiEnlem f̈ligelitume.

Staubbeutel bei Stylidium 2:pantig, parallet und forizontal hiber ber Sarbe liegeno, bei úbrigen 5, vertikal, nufrecht, jweifáchrig, bei eimigen $5-6$ - 8, bei Lobeliariac und Stylidiariae meift an ben Rindern verwatbjen, bei Campanulariae frei, mur bei einigen (z. 23. Jasione, Phyteuma) wábreno Der Entwidelung jufammenbingend. Ctubfitben bodenftindig, verwad) jen, bei Stylidium ginglid mit dem (Grifet verid)molzen, bei ber britten (Stuppe frei. Slume rofhrig, mit Eluppig 5=\{poltigen Saum, unregelmis̈ig, zwei=

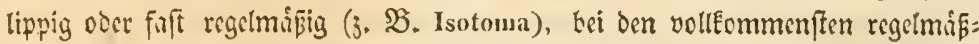
ig erfit idhmal 5:theilig, bunn glofkenformig, endlich fternformig (Michauxia). In ber 2fntithefe ift bit Satum wellenrandig und übergeiegt. 
(5ianjblumigs.

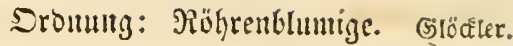

Degetation. Stumm: Warzel bei vielen Enollig, bei ben meiften aftig, Etengel Ernuturtig, bei wenigen holjig (3. 2. Goodenieae), Blatter zerfterut und wed)= feind, bei Canarina gegentiter, bom lineulifhen alle lingliche, herzfôrmige und rumblict)e limriffe burchlimfend, bei wenigen fleifthig (Scaevoleae) oder boht (Lobelia Dortmanna), Fibl ober verfhicosnartig befleioct. Blithen zmitter= lid), bei cinigen achfel: bei andern enoffinoig, Eopfartig, umbuilt, faft Rolben (Sphonoclea), 2lebre, Traube, Silpe.

(3) upwen: 1) Loheliariae: synanthereae microspermae.

a) Clintonicar: 1-loculares operculatae vel 1 - 3 valres. Ilypsela Presl. Lysipoua Kuth. Clintonia Dougol, Gramnuatothea Presl.

b) Lohelieae: 2-loenlares hivalves, Metzleria Presl. Myopsia Presl. Dobrowskya Presl. Monopsis Salisb. Rapuntuu T. 'Tylomium Presl. Sulenopsis Presl. Lobelia $L$. (cum Siphoeampylo Cham.). Euchysia Presl. Byrsanthes Presl. Isetoma $\mathrm{P}, \mathrm{Br}$.

c) Delisseeae: baceatae (hacca siceav, succuleuta). Trimeris Presl. Pratia Gaulich, Macrochilus Presl. Delissea Gaudich. Kittelia Rchb.*) Rollandia Gaudich. Clemontia Gaudich. Centropogon Presl.

2) Stylidiariae: gynaulrae et synanthereae macrospermae.

a) Stylilieae: gynanirae. Conspect. 1918 - 1921.

b) Seaevoleae: synanthereae hypogynae, locul. 1 - 2 spermis. Conspect. 278: - 278t.

c) Goolenieae: synanth. aut eleuth, seminibus seriatis, Couspect. $2854-2881$.

3) Campin uleae: demun eleutherantherae, nicrospermate.

a) Pongatiene: ex apice operculato disseminantes. Sphenoclea Giärtn.

b) Cimpinuleae: e lateribus laceris disseminantes. Phyteuma L. I'etromarula Dec. Prismatocarpus l'Herit. Campannlis L. Specularia tec. Tracheliun L. Adenophoria Fïsk. Symphyimdra Dec. Musschia Dumort, (Chrysangia Lk.) Mereieri Dec. Michnuxia l'Herit.

c) Cimarineae: ex apice lacero vel plurimis libero, valvato dehiscentes.

a) Jasione $L$.

B) Lightfootia l'Jlerit. Cephalostigma Dec. Campanumoea Dec. Collonopsis Dec. Canariua L. Platyeodon Dee. Wahlenbergia Schroul.

y) Lkëlla $L$.

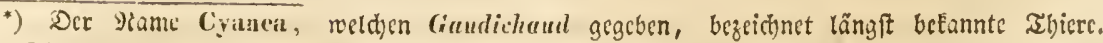

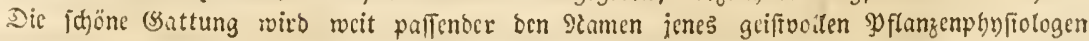
rragen, weld)er in gegcnwärtiger Familic, auf bie unterfdecibung our Bruppe ber Jajioncen aufmerffaum machte. 
(5antsblumige.

Dronutg: Sï̈hrenthumige. Btöeter.

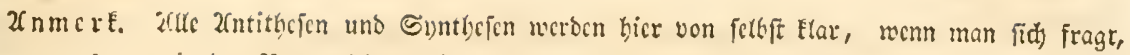
was man it ber Satur felyen wirb, uno nidjt bloz, was man ctwa fehen wilt. DaF

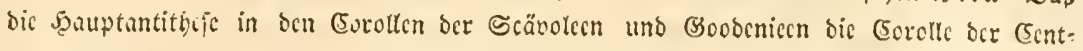

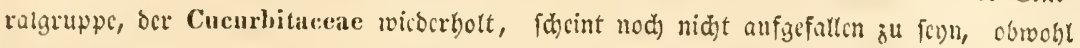

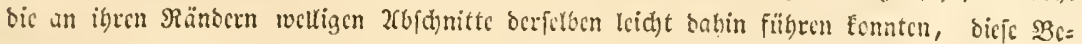

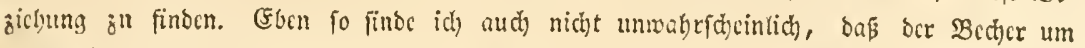
bas Stigma dic mit Soncentration bes ๔rigma mitconcentrixtc umgebung ocr Etigmaten

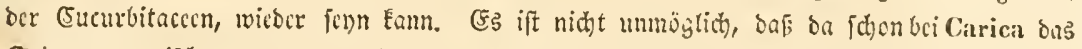

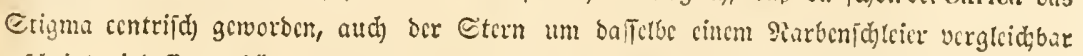

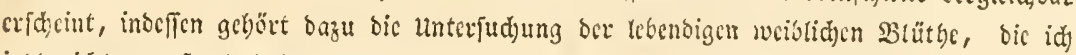

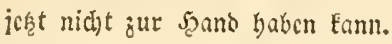

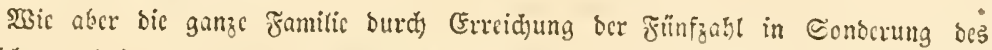

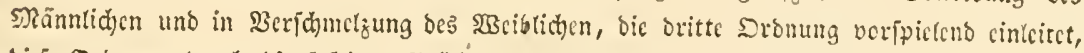
bicfe Dromung ourdy bie faftigen Früd)te ber Deliffen uno bic ftcrnförmigen Brmentronem

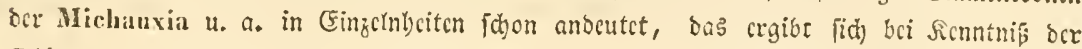

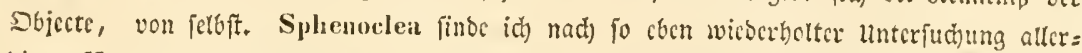

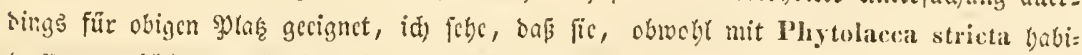

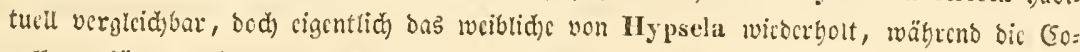
rolle vcrtünm.rt if und bie Eurzen Gtaubfäben fici.

85. Familic. Rippenblítrler: Labiatae.

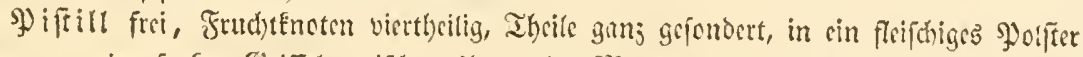
cingefenft, Griffel zwifhen ihnen im Dittelpmete singefrect, geftecft, verti= Eule: borijontal gefpaltene 9larke. Basi den vollendeteren oar Frnthetenoten

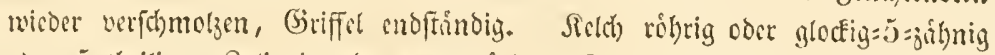

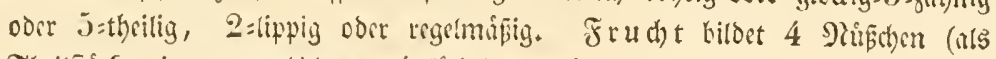
Theilftucke einer vorgebildeten 4=firthrigen, 4:framigen Sinpfel, bei Prasiun Etimfruthtchen, bei ben Berbenem toird bie Frutht jum Echluuth, jur fich:

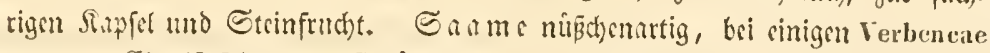

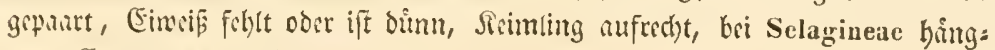
culd, Cotyledonen fluch.

Staubbeutel 4, bei wenigen 2, fie fint bei cinigen sinfitetrig, bei ben miften

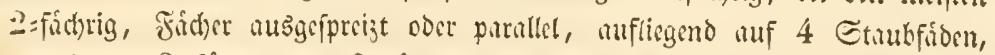
von denen 2 furger und 2 linger find, kei Salvia die Sacutelfict)er parrallel buth) sinen langen Eteg (Commectivum) getremt, welther im Ëhutmierge:

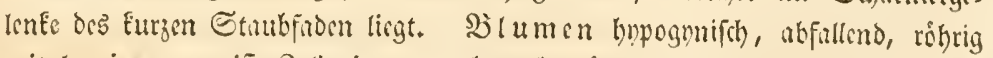
mit lappigem, meift 2=lippigen, aud) wachenformigem Saum, von dem bir Sberlippe cingefirbt und die Unterlippe 3lappig ift, bei benmeiften mit deutlichsm Echlund. Dic Miobre trifgt dic Stnubfíden. Bei ben Berbenen wiro dic Blume in einigen Battungen tridter= und prifentirtellerformig, ber Saum re:

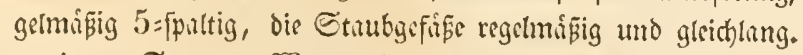

Segetation. Stamm: 2 surgel cin = und zweijibrig, msift perennireno, Strngel uno jüngere 3weige vierkntig, Berzwcigung und Bcklifterung gegenuber, Shlitter mit Delgribet)en, meift geftielt uno Eerb= ober fígermoig, in lánglid)en Umriffen oder in runden, bei fibr wenigen bandformig oder firocripaltig (Leo- 
nurus, Nepeta pinnata, Phlomis pinnata) geudert, bei vielen rungelig. \$Bci Verbeneae auch einige quirlartig und lebcrartig glinjend, bei menigin gis

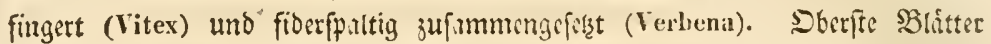

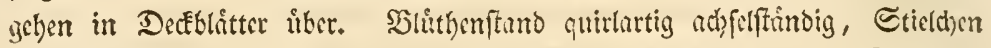
an ber $\mathfrak{B a}$ [is oder an ihrer Ifscilung mit Deceblittchen $1-2-3-5=$

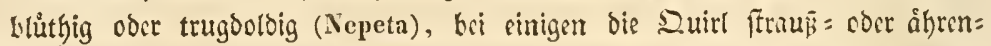
artig gedringt, fheinenoftindig, bei bon Berbeneen anjer bicfem Blithenftande (Vitex) auch Sipfchen, zum Ifycil mit Duirlfitlle (Lantana), und endfindige wirfliche 2 (ef)ren mit alternirenoen unb jerftreuten Blittfen (Verhenil), fogur in flifichige 2loffe cingefenft (Staehytarpheta), oder in enoftindigen Doldentrauken (Verbena Aubletia), Inngooldeu (Volkamera) und Traben (Duranta) uno Miifpen (Aloysia, Tectona). Blutthen jwitterlich), bei menigen polagumifith,

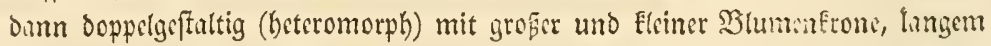

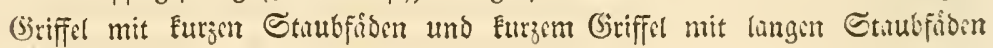
inocrno.

(jruppen: 1) Leiosehizocarpicate: nneilae laevigatac.

a) Tepetariac: ealyee subregnlari 5 - 10 dentato.

a) Mentheae. - Lyeopus L. Mentha L. Preslea Op. Isanthus Mchx. Colcbrookia Rxb. E'erilla $L$. Tetradenia Benth. Elsholzia (Elsh. Cyclostegia. Aphranochilus) $\boldsymbol{W}$. Dysophylla Bhune. logostemon Dsf.

B) Origancae. - Sitturcia L. (Sit. Tragoriganum, Pyenothymus), Origanum L. Majomana $T$. Amaracus Bnth. Monardella Bnth, Pyenanthemum Mchx. (Tullia, Brachystemum). Bystropogon l'Herit. (Byst. Minthostachys). Cunila, $L_{0}$ - Ziziphora $L$. Blephilia Rafin. MIonarda $L$.

r) Tinpeteac.

a:c) Staclydeae: Galeopsis L. Anisomeles R. Mr. Betonica L. Stachys L. (Alopecura, Eriostachys, Catostachys, Stachiotypus, Olisia, Chamaesideritis, Ambleia, Zictenia). Spincele Brtl. Chasmonia Prsl. Lagochilus Bulh. Liminm L. (Lumiopsis, Lamiotypus, Orvala, Galeobdolon). Leonurus $I$.

33) Marmbicae: Anisodontium 'Rchb. Lagopsis Bnth. Harvibium L. Sideritis L. (Eusideritis, Lupedoclea). Lavandulia $L_{\text {. }}$ Craniotome IRckb. Ballota $L_{\text {. }}$ (Ball. Beringeria, Acanthoprasium). Panzeria Inch. Lasiocorys Linth. Ioylea Wall. Otostegia wienth.

ry) Phlonitene. Lencas R. Br. (Ilemistoma, Loxostoma, Ortholencas, Astrodon, Plariostomil). Leonotis Bntl\%. Phlomis (Phlomitis R. Phlomis). Notochacte Bnth. Eremostachys Bnth. Eriophyton Bnth. Moluccella L. Achyrospermum Bnth, Colquhonnia Wall.

b) Salviariat: calyec irregulari bilabiato. 
«) Melisseae. - Burgsdorfia Much. (et Mesiodea Mnch.) Calamintha 'T. Much. (Acinos, Calam., Calomelissa). Mulissa L. (Meliphyllun, Macromelissa, IJeteronelissa). Thynus h. (Serpyllum. Mastichina, Psendothyubra). Micromeria Bnth. (Piperella, Hesperothymus, l'seudomelissa). Hedema l'ers. (Hed. et Mosla). Gardoquia Rz. Pav. Glechon Spr. Kieithia Bnth. (lieithia, Gymnocylix). Eriothymus Bnth. (et Rhabdocaulon). Thymbra $L$. Diceraudra Bnth. Jogngyne Buth.

3) Ocymoileae, - Iyptis Jcq. (Hypenia, Siagonarthen, Umbellaria, Buddleioides, Mintlidium, Pectinaria, Mísosphacria. - PoIydesnia, Cephalohyptis, Santhiophaea, Trichosphaeria, Oocephalus, Eriosphaeria, Cyanocephalus, Cyrta, Plagiotis, Apodotes, Spiearia, Gymneia). Marsypiantlus Mart. Peltodon Pohl. Mosthosma Rchb. Orthosiphon Bnth. 'Acrocephalus Bnth. Vlesona Blume. Geniosporum Wall. Ocimmu L. - Pycuostachys Hook. Aeollanthus Mart. Anisochilus Wall. Coleus Lour. (Solenostemon, Aromaria, Calceolus). Plectranthus l'llerit. (Anethystoides, Pyrauidium, Isodon, Melissoides, Meterueylix, Colcoides, Germanea), Hoslundia $L$.

y) Salvieae. - Audibertia Bnth. Salvia T. L. (Plethiosphace, sethiops, Horminum T. Eusphace, Drymosplace, Hymenosphace. - Ilemisphace, Gymosphace, - Heterosphace, Notiospha-

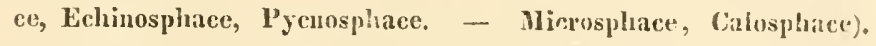
- Meriandra Buth. Rosmetinus 'T', L.

c) Prasieae: calyce bihahiato rel subregulari post anthesin ancto vel inflato.

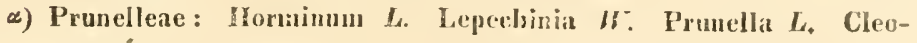
nia L:

3) Melitteae: Melittis $L$, Physosterria Beuth. Macbriblea Benth. Symandra Nutt. Eriope Benth.

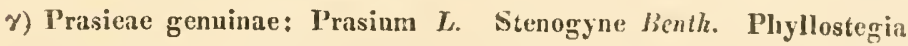
Benth. Gomphostegia Benth.

2) Trachyschizocarpicae: nuculae subcohacrutes reticulato-foreolatae aut granulatae.

a) Teucrieae: - Ajuga L. (Chamaepytis, Bugula, Pseulanisomeles). Amethystea $L$. Trichostemma $L$. (Streptepodium, Orthopiulium). Teucrium T.L. (Scordium, Chamaedrys, Polium, Scororlonia. Staclyyubotrys, Pycnobotrys, 'Teucris, Teucropsis, Leucosceptrum Sim.). Plileboanthe Tsch. Cymaria Benth. Lophanthus Benth. (Vlechia Rafin.) Hyssopus $L$.

b) Scutellarieae, - Scutellaria L. (Galericularia, Masclalustachys. Stachymacris, Meterantlesia, Lupulinaria). IIymenocrater Fiseh. et C. A. Mey. Ierilonia K. II. B.

c) Prostanthercae. - Xemigenia R. Br. Westringia Sm. Micro- 
Gimjthlumige.

\section{Drganogeneje.}

Soronung: Sd)lunbblumige. Rippenblüthlcr.

eorys R.Bi. - Hemiandra R.Br. Prostanthera Lab. Cryphia R. Br. Cliilodia R.Br.

3) Angiocarpicae: stylus germini concreto apicalis, drupa $r$. capsula.

a) Verbencae: drupaceae orthospermac. - Collinsonia $L$. Verbena L. etc. cf. Conspect. $2894-2940$.

b) PI a tun ieac: baccatae. - Monochilus Fisch, et Mey. IInlmshioldia Retz.

c) A c a u th i r i a e: capsulares capsula retinaculis etastice dehiscens (calycem, corollam, stamina habitunque tribuum antecedentium repetcutes).

๙. Acantheac: semina retinaculis uncinatis suffulta, - Iygrophiteae: Hemialelphis N.v. E. IIygrophila N.v. E. Ruellieac: Echinacanthus $N, v, E$. Dipteracinthus $N . v, E$.

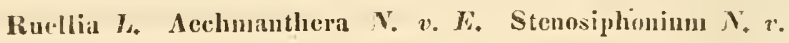
F. Strohilintlrus $B l$, Goldfussia N. . F. Asystasia $B$. Petalidium N. v. F. Dyschoriste N. $v$. E. Leptacautius N. $v, F_{*}-$ Phlebophyllum N. $v, F$. Adenacanthus $N . v$. F. - Barlerieae: Asterantla N. v. E. Barleria L. Loplustachys Pohl. Aetheilema R.Lr. Lepidagathis $U$. Menracantlus N,v, F. - Acantheae genuinae, Bleplaris Juss. Acauthus L. Dilivaria Jass, - Andrographideae, Erianthera N.v. F. Haplanthus $\boldsymbol{N}, v, F$. Anthographis $\boldsymbol{H}^{a}$ ll. - Diclipterae: diandrae dissep. solub. Rumgia $N, v . k$. Dicliptera Juss, - didynamae: Blechmm Juss, - dissep, adnato: Amphiscopia' N. v. F, Peristrophe $N, v$, $V_{\text {, Hypoës- }}$ tes Sol. Raphidospona N.v. F. - Justicicae Eranthemcae:

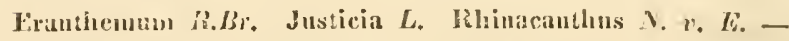
Just. Linelliariae: Cryptoplnrigmiun V. v, F, Phlogacanthus $N . v . F$. Loxinthus $N, v$. F. Endopogon N. v. F. Crossandra Sulisb. - Justic, Gendurusscne: Mostellularia Zahb. *) Henichoriste $N, v$. F. Griptophyllum $N . v . N$. beloperoue N.v. li. Adhitoda Herm. Geudarussa Rumph. Leptostachya N. v, L. Gymustachium N,v, $\%$

6. Llytracieac: retinacula in pipillate lomam coutracta, scmina parva scrobiculata ferentia nec fulcientia. -

«u. diandrac. Elytraria I uhl. Nelsomia $R B r$.

$\beta \beta$. didynamae. Ademosma R.Br. Eberusaiera N. v. F, Elytracauthus N.v. K.

$\%$ Thunbergieae: seniua retinaculis in cupulam corncam di-

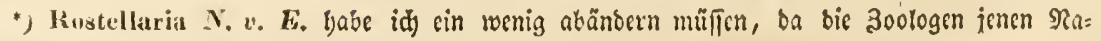

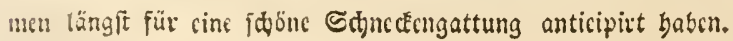


latatis seminique adnatis suffulta. Thunbergia $L$. Yeyenia N. v. E. IIexacentris $N, v . E$.

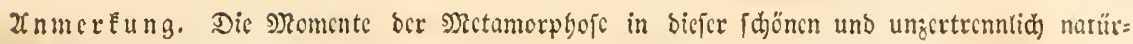
lidgen Familie find folgenbe:

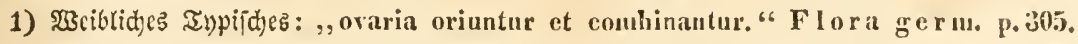

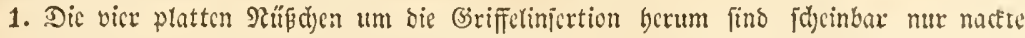
Caamon: Leioschizocarpicae Nepetariae - Salviariae un bilbon fid fort ju Stcinfrühtdyctr: Prasieae.

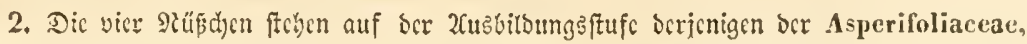
in bor zwcitcn Gruppe: Trachyschizocarpicae. Mandjertci formen fpicten vor, bei melyeren bic ber Anchusa ete, bei l'eritomia fogar bie von ben (5ynoglofien.

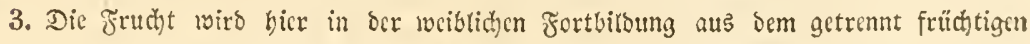
3uftanbe zu cincr (s)anz fund)t, aus bcm sehizocarpium cin idiocarpium, bartm in un=

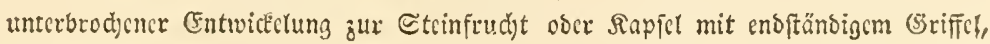
swie bei ben verwandten, Girr anticipirten 2efperifoliaecen und Convoluntacecn, atio 3) Angiocirpicac.

Das unmittetbare Binorglico ift Collinsonia, in bor jungen $\mathfrak{B}$ tüthe cine Leiuschizocarpica, zur Frudjtzcit sine Verbenea mit eilziger cinjaamiger Escinfrucht, mit Epur vom (Enogriffc!!

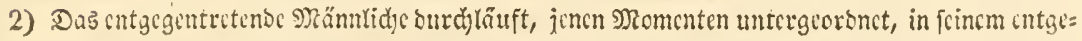

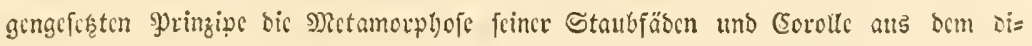

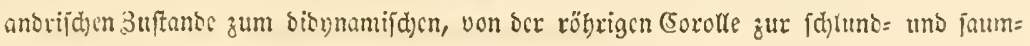

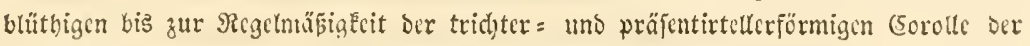
Eonvolutacen in ocren birr erftanoenem Borbilbe Thunbergia.

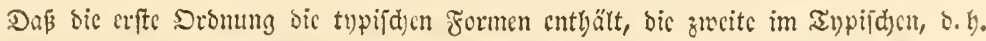

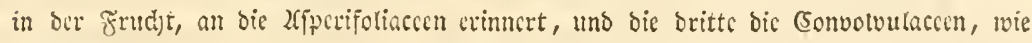

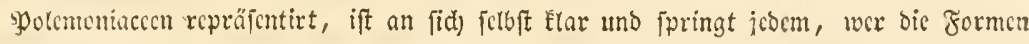
Ecmnt, in bic 2(ugen, ex barf nur nachichen, wic bic Siattungen Jf exacentris uno Thun-

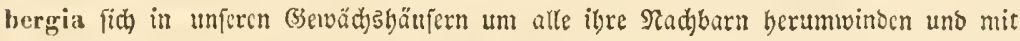

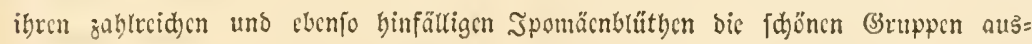

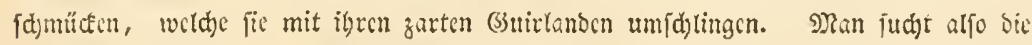

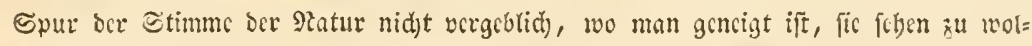

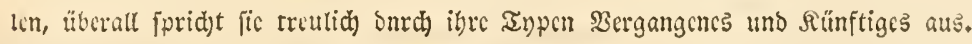

3) Wseiblid)eb 20nticipirtes: Stigma und Reld).

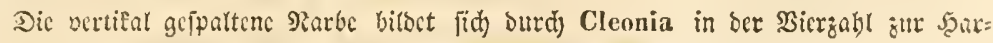

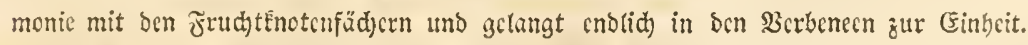

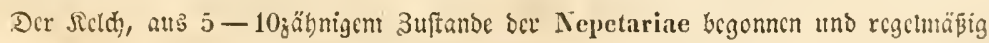

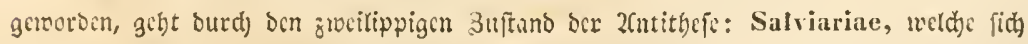

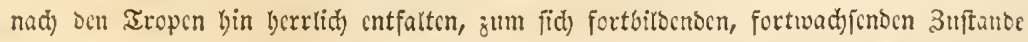

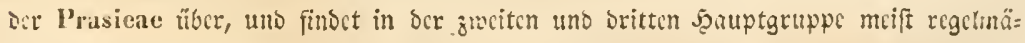
pitge, Dollcnoste formen. 
Gianblumige.

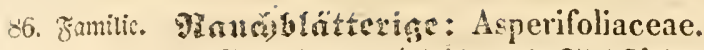

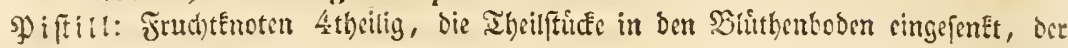

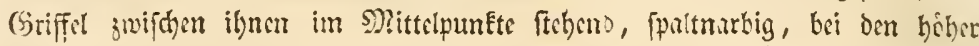

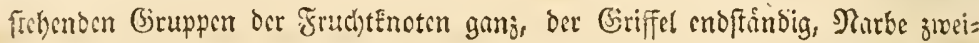
Eugclig oder gabchrtig vierffaltig (Cordieac), oder rinfach. Seldh 5theilig, mrift

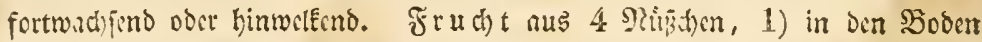

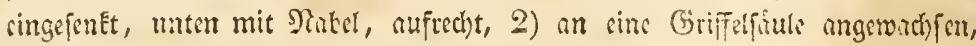
bingend (Cynogloss.), 3) Eapfctartig, fpater 4fid rig aufiptingeno (lleliotrop.), Sammen hangeno, 4) Stcinfutht mit Etcinfernen, Jern cinfamig (Tornefor-

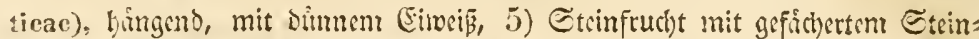

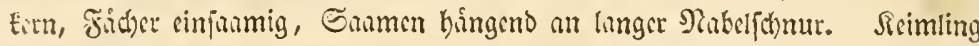

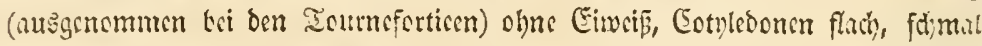
an griffeiformigen Sicimling (Etydrophyllene), lingsgefaltet (Corliaceac), 4 Entyledonen nur bei Benthamia.

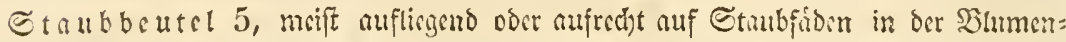
robe mit den Saumakfentten wethelno, beginnen (Echium) bionnamifi)

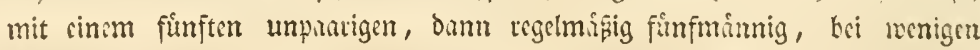
(Corilia) 6-7mannig, in bir Jinoppe eingebogrn bei Fiylrophylleae. S3tumenftone róhrig, mit Schluno, Saum bei ben erften jweilippig (Eehium), bei oent úbrigen proffentitteller = ooer trichterfornig mit regetmáf: 5ypltigem Caum, in ber Sinoppe umgrlegt, bei weniigen rno = uno fremfor: mig (Borrago). Im Eshlunde pitsen bei sinigon unter ocr Mitte bor Gaum=

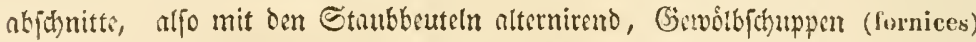
welche bei einigen bicht zujummenid)tiesen, bei wenigan Fulten (Heliotrop.), bei den wenigften Pinfel (Pulmonaria).

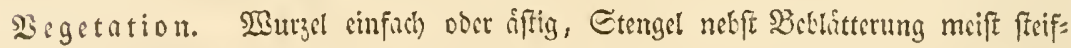

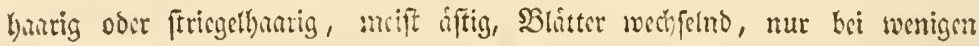
(IIydrophyllum) gegeniter, cinfinche linglide formen, nur kei tvenigen

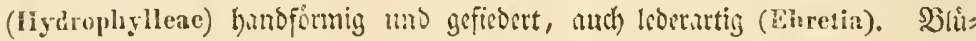

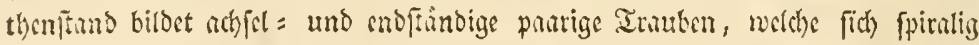

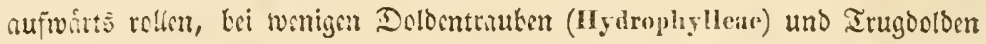
(Tournefortieae, Cordia). SEtithen zwitterlid), auch beteromerph wic bei vo= riger Familie, (vergl. Pulnonaria Fl. germ. et pl. erit.)

(Sruppen: 1) Schizucarpicae, stylus inter earpidia.

a) Heliene: nuculac basigenac, faux perria; 29-4e-2060 et Echiopsis Rehb. (E. fruticos etc. cor, regulari, stigunate sinplici differt.)

b) Borragineae: umenlac basigenae, faux fornicibus instructa. Irosotis $L$. Anchusa $L$. Stomatechium Lehm. Solenanthus Lecd.

Exarthena R.Br. Borrago L. Symphytum $L$.

c) Cynoglosseac: nuculae styligcuae faux pervia: Plagiohotrys Fisck, et Hey. Arucbia Forsk, 'Trichodesma R.I3r. Rinleria 

Pall, faux fornicibus instructa: Rochelia Rehb. Echinospermum Sw. Cryptantha Lehm. Botryospermum liunge. Asperngo L. Omplablodes 'T. Mattia Schult. Cynoglossum $L$.

2) Idiocarpicae: stylus terminalis, fructus juniores integri demum dehiseunt aut Irupacei indehiscentes. Hlores cymosi.

a) Heliotropiceae: muculae membrana denum partibili inclusae, stigma simplex.

Tiaridium Lehm. Hcliotropium L. Preslaca Mart.

b) Tournefortieae: nuculae albuminosac in drupa, stigma bifidum. -

Messerschnidia $L$. Tournefortia L. Rhabdia Mart. Beurreria $J c \%$. Whretia $L$.

c) Cordieae: nuculae 1 - pluri-loculares in drupa, stigma quadrifilo-dichotomum.

Cordia L. Patagonula $L$. Varronia $L$. Cardiopsis II $a$ milion.

3) Capsulares: capsula 1-2-locularis placentifera oligo-poly-sperna, semina peudula albuminosa, embryo styliformis demissus.

a) Ellisiene: caps. bilocul, in dehiscens 4-sperma, Ellisia $L$.

b) Hydrophylleae: caps, 1-locularis bivalvis, placentis basigenis 4-sperma.

Pliacelia Juss, IIydrophyllum $L$.

c) Nemophileae: capsula 1-locularis bivalvis 4-00sperna, seminibus invicem supra sibi impositis. -

Nemophila Barton, *) Eutoca R.Br.

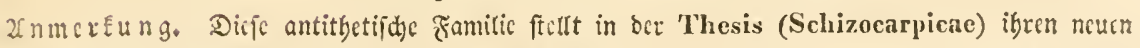

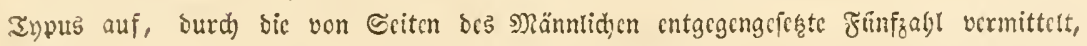

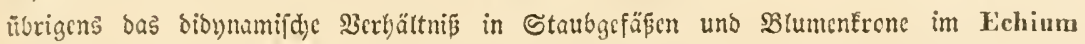

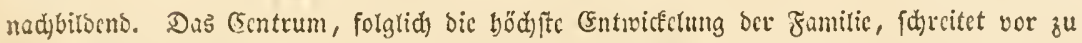
ben tropifdyen Formen ber şauptgruppe (Idiocarpicae), nad) bem fdon in ber verigen bic (5) $)$ togloffen bics Sentrum für bic Frudibitoung angedcutet unb erftrebt batten. Die $26=$ thifme vom Inpus repräfontirt fich) burdi) bic Orittc (jiruppe (capsulares), bolde im un=

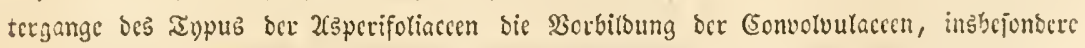
ber Dुolemoniaccen crfâfen, benen ibre (sattungen fo nabe treten, bas mur ibr antitbetifa) umgec Ecbrter Scinling fic von ifnen trennt. 2chnlides madjen fdon bic Cordieac flar.

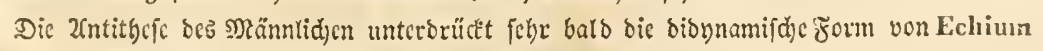

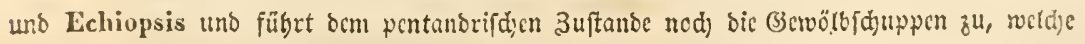

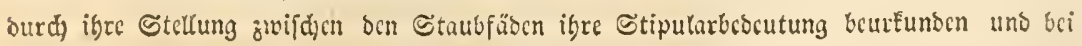

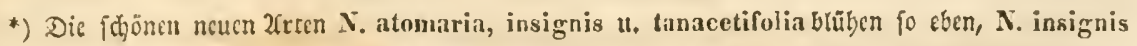

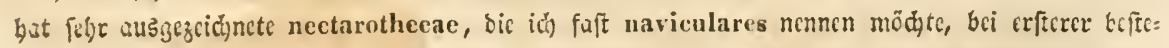

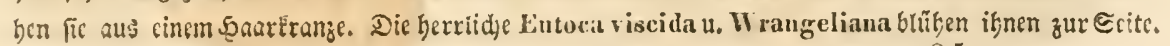


Banjllumige.

Dromung: Edyłunbblumige. Raửblătterig̨.

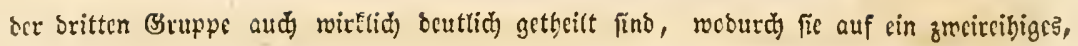
.rft in ber britten Sromung möglichft vollendetes Berbättnis ber Sorolle binbeuten.

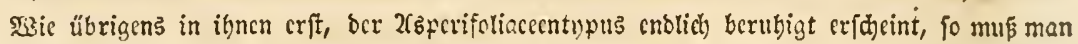

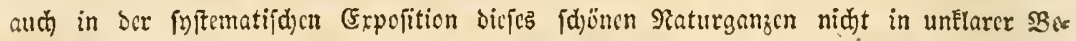
ichautung bas Einzerne zerftücectt begchren, man mup ber Entfaltung bes anpus rwillig fol= gen, fo woit in ifm bie Siatur ferbft fich verzmeigt. Das war cinft Jussieu's trefflidye Wsife.

87. Familie. จ3̧iubengetoridofe: Convolvulaceae.

Piftill sinfuct) und frei. Fruct)tenoten $1-4-3=$ fiochrig, mit Drưfenpolfter umgeben, (sirffel cinfact), mur in ber 2(ntithefe (Hydrol.) tief getheilt, Narke jweilappig oder dreifpaltig, bei andern centrifch fopf = oder fdjilbformig. Felth

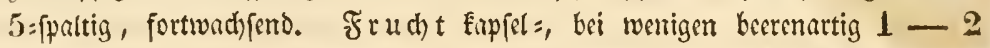
- 4 - 3:fâdrig, Gdyeidrwand am Siande ber Slappen in den centraten Samentriger ưbergsheno, Finther $1-2-00$ =paumig, San= men meif Eantig, cufrect)t, Eiwweip fparfam, fchleimig, Scinlling gerade,

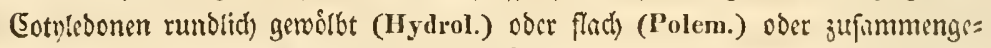
rollt (Conrolvuleae), Sammenfhatle lof't jid) in Ed)lcim mit vislen Spiralen bei 1)

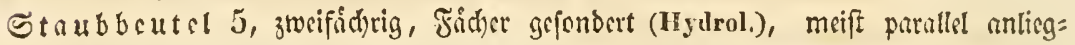
eno, långs auffpringend aufliegend, bei ber Şauptgruppe aufred)t. Staut=

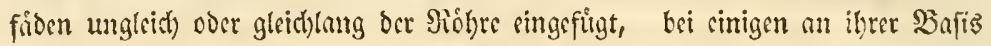

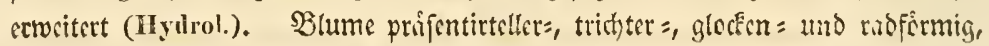

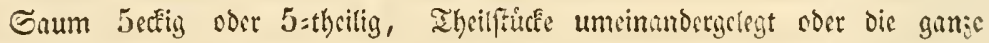
Slume langs gefaltet, vor und nad) dem Slithen zufammengcorcht (Convolv.).

Segetation. Ctamm: Warzel bei cinigen Enollig, bei andern cinfad oder iffig, Stengel aufrecht oorr windend, meift Exautartig, aud) milcheno

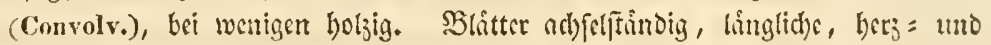
fpiefformige umriffe oder getheilt und geficoert. SBlithen zwitterlict).

(5rupprn: 1) Polemoniariac: tristigmaticae, cotyledonilns planis.

a) P'bloginae, suboblique tubiflorae oppositifoliae: Phlox $L$.

b) Gilieae, rectitubiforne opp.rel alternifoliae oligospermae: Welwitschia Rchb. (Hügelia Benth.) Linanthus Bculh. Leptosiphon Benlh. Fentylia Benth. Gilia Cav. (Dactylophyllum, Ipomopsis, Eugilia). Aegochloa Renth, Courtoisia $h c h b$. (loculis biserialipolysp.) Collomia Nult. Toitzia Juss. Cantua Juss.

c) Polemoniene, campaniflorie polyspermae, loculicidae: 1'olemonimm $L_{*}$ - septicidae: Cobaen C'av.

2) II ydrolecae: schizo-2-8-styles, 2-8-1-loculares polyspermae, cotylelonibus convex is: Wigandia $K, Z$. B. Sigonpi Aubl. Nama L. IYydrolea $L$. Codon $L$.

i) Convolvuleae: holostyles 2-1 stigmaticae, cotyled. corrugatis, Conspect. 2992-3016. (excl, 3000 et 3001 ).

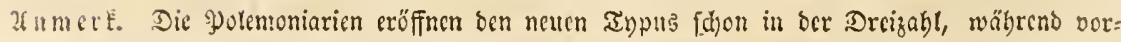
ige auß ber nicbern 3meizahl bervorgingen. Sie beginnen mit Rücknahme gerrilfer Ben bereen aus ber Familie ber Rabiaten; bic bci sinigen, z. S. Phlox setacen fehr fochief gebaute $\mathfrak{S l u m e}$ und bie unglcidhe sänge ber Ctanbföben, io wic bie form bor Canmen 
bentet wefentriad) barauf bin. 2(ud) find bic formen von Plulox faft bic cinzigen, mit, wic bei ben Eabiaten, gegemübcrftebenoen $\mathfrak{B t a ̈ t t e r n . ~ D i c ~ G i l i e a c ~ u n b ~ l ' o l e m o n i a ~ r r i c b e r b o l e n ~ b a n n ~}$

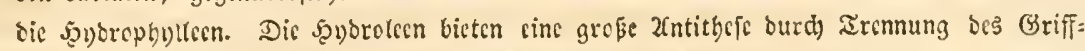

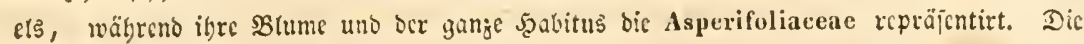

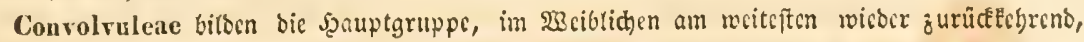

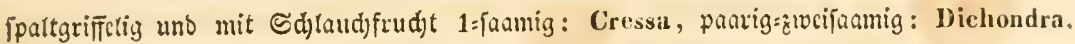
mit 4 Stürdyen: Falkiil, bis in ber reidyferr Battung Convolvulus alle formen fict ver= cinen urb ausglcichen. Courtoisia vergl. im Hortus bot. 182!) ic. 208.

88. Fanilic. Gilubufarinecen: Globulariaceac.

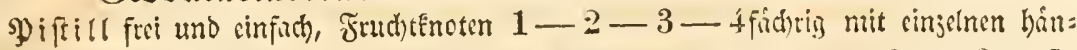

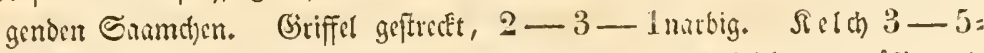
theilig, frift fpeljig oder framenfederartig (Brumonia), enolich regilmâpig mit

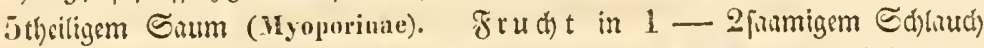
(Glob. Ilebenstr. Selar.), oder Etrinfrudt mit $2-3-4$ firthrigem

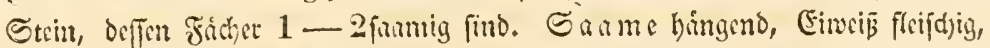

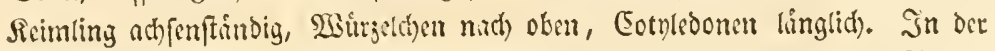

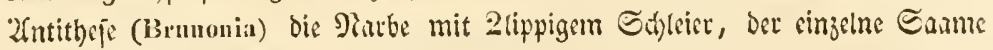
nufrecit und obue Eimeís.

Staubbeutel cin= bis zweifichtig, 2 (Anathelepis) bis 4 anf dionnamifdsen Staubfidoen, weldye am Miunbe der Lippe anbingen (Hebenstr.), tief in ber Sishre eingefugt find (Gloh. Selag. Stenochil. kicmoph. J'holid. Bont.), cno=

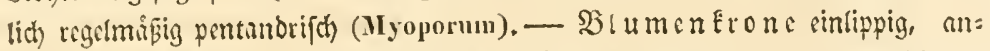
ftutt ber llnteriippe mit bypogynif̧er Drufe (Hebenstreit.), oder jweilippig,

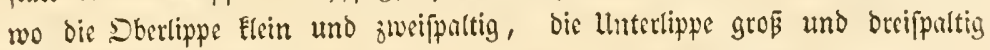
(Glob.), ober bie Dberlippe kreit uno 4 jihnig, bie Unterlippe Eleiner uno cin=

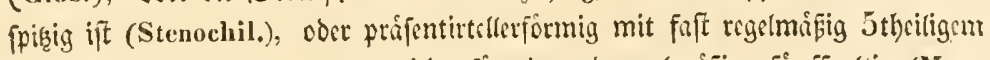

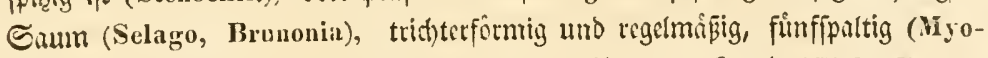

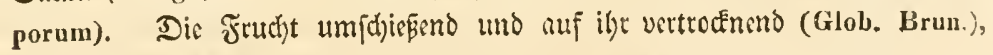
oder abfallend (Myoporum).

Begetation. Stumm: Misurget einfirth oder aftig, menige cinjibrig (Hebenstr.), meift perennireno obcr holgig, Sibaft (Brmouia), Etengel Etautartig, bod)

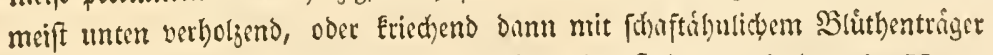
Glob. nudicaul.), bei Librigen frtuchurtig und foft baumurtig (Bontia, Myop.

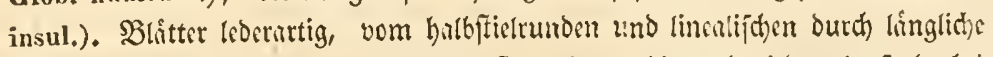

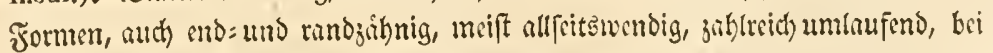
menige ngegenuber (Selago myrtifolia Rchb, hort.ic. 223. Myopor. oppositi foliuni

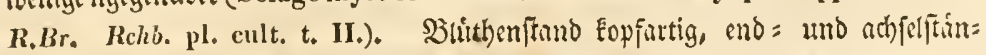
Dig, mit Şuille (Glob. Brum.), affrenatig (Hebenstr.), bofdentraubig uno trugboldig aus 2(ebren (Selago), aud) einzelm oder gepantt in ben Slattreb) fein (Myop.). B lutben jivitterlich.

(3ruppen: 1) Globulariene: didynamae, antherae 1-loculares, utriculus 1-2 spermus.

a. stamina in basi tubi, limbus bilabiatus: Globularia. $L_{*}$

b. stamina in margine labii suporioris, lab, inferins ad glan$25^{*}$ 
dulam hypogynam reductum, Diaudra: Agathelepis Chois. didynamae: Dischimia Chois, Polycenia Chois. Microdon Chois. Hebenstreitia Chois,

c. stamina in tubo, liubus subregularitur 5-partibus: Selago $L$.

2. Brunonie a e: pentandrae, antl. 2-loculares syngenitac, stigma bilabiato-indusiatum, utriculus 1-spermus, semen ereetum exalbuminosum: Brunonia Sm.

3. I yoporiuac: didynamae et pentandrae, antherae biloculares, drupa $1-3-4$ sperma.

a. didynamae loculis monospermis: Eremophila R.Br. Pholidia $R, B B r$. Stenochilus $R, B r$.

b. didynamae pyrena biloculari: Bontia $L$.

c. pentandrae: Myoporum Banks.

2(nmertung. W3as geht bicr in ber Patur vor? - ", antherae oriuntur!" - Eie ent= ftchen aber nidyt in ber Thefï zufanmengeffellt, wie in Der erften Dronung, fondern anti=

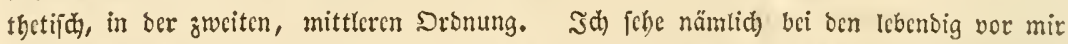

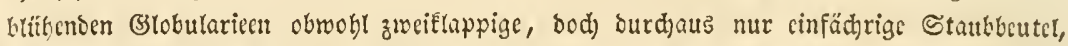
wie ich in der F1, germ, fie 'bejd)rieben uno in sicfer Erfahrung fchon im Compectus sic (Sruppe zu ben Calcginect geftelt habe, weldhe \$erwandtict)aft, aud DeC. im prodrom, IV. p. 664., natürlid) obne ocs urfprungs $\tilde{o}^{\mathrm{u}}$ crwähnen, ats bie rabre crEannt hat. Bartlings Irrennung ber Globularineae burdh 2fächrige 2(ntheren berubte bcmna hỉ auf sinem,

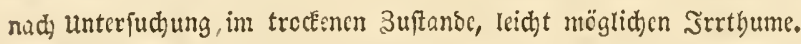

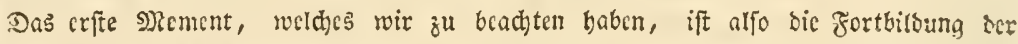
Etaubbcutcl, weldje ganz cinfach obcn aus ber Spişe ocr Ctaubfäber beraus trichter =

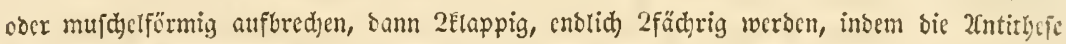

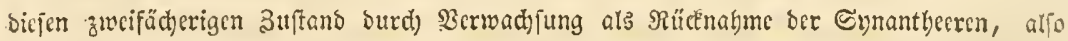

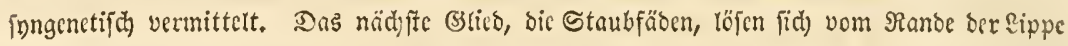
bei Hebenstreitia (Relıb, hort. t. 133.), bei ben übrigen trägt bicfetben bie Röbrc. Die (5orolle beginnt bei Globularia im 3ulftande ber Eynantbereen und gebt in ben ber vere

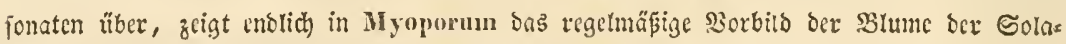

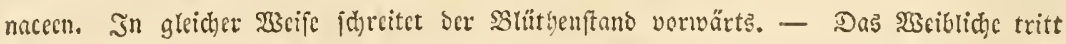
entgegen aus oen Synantberecn, aber im Cbaracter ber zmeiten Stonung: vom feldye bis

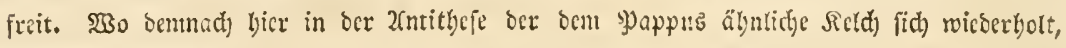
rie bei Brumonia, ou fteht er unter bem Frudtefnoten. Die Eprcublättcr ber Eynge=

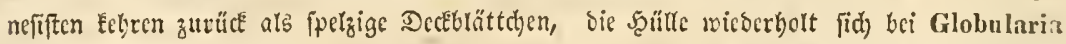

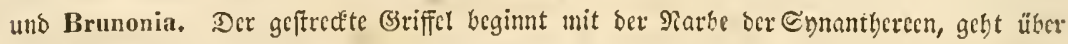

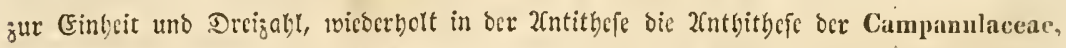

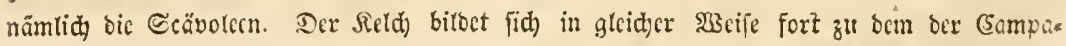
mulaceen, aber frei, wie Seacvola, wetche in ibrer 2entitycje oie gegentwärtigen Myoporinae

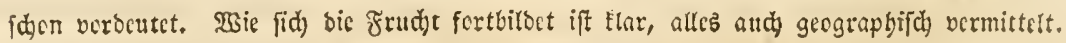


Gianjbluturige.

Sromung: Sdylunbblumige. Inrvenbtüthter.

\section{Zुamilic. Sarbeublüthler : Personatae.}

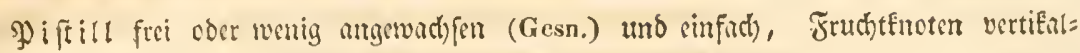
2fichrig, bei wenigen 1 fíchrig, Grifel geftecft, Narbe borizontal geipalten,

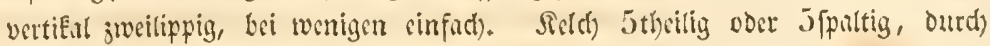
Serfimuterung 2blitterig (Orob.). Fruch t finpfel = bei einigen berrenartig, 1fict)ig 2flappig, Sanmentriger wambftindig (Orob.), ober an ben eingebo: genen Shappenrindern (Rhinauth.); oder meift vertiEal=2fotchrig, Gaumentriger in Der Mitte an ourd)gefender Echeidewano (Scrofular.), oder bei wirder ge=

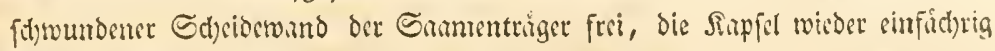
(Linterniariae). Sa amen in ben Fichern auftedt, wenigzinlig (Melamp.),

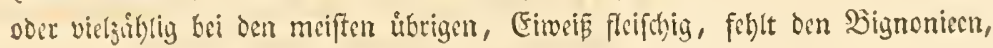

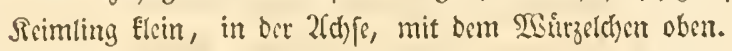

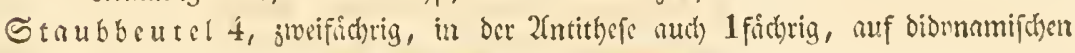

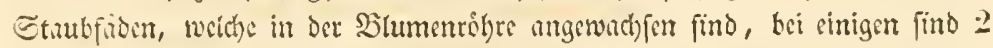
Soutel verkimmert, bei andern tritt cin funfter Stnubfaden unfrudbtbar (1'entstemon) ober fruchtbur (Verbasenm) binju. Disanilra Gat 7. B lu me rohrib, anch mit Edyluno, bor Eaun zweilippig, lowenmanturtig ober trichterformig, glockig uno prafientirtellerformig, boch feibft im regelmáfigen 3uftinde in out Sinoppenlage unregelmiöig [Bndleia], *) bei einigen bleibt ein Shing son ber ver=

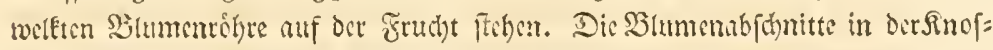
pe harumgelegt, Sherlippe cingebogen, luterlippe auf juner arfliegeno (pl. crit,

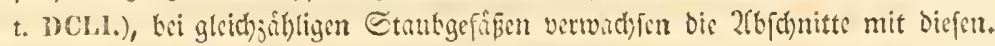

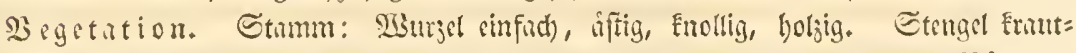
artig, eist = und jweijaffrig, perennireno, ftrud)artig nebft sinigen Bisumen. Imeige und SBlitter jum Theil gegeniber, bei untoern unten gegeniber und oken jer|treut, bei andern iberill zerffreut. Slitter vom ltngetbeilten burd) bus

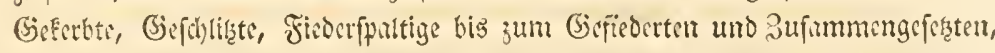

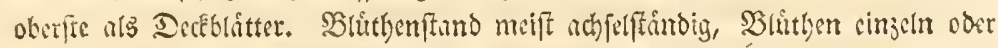
in Xeben, Irauben, Miipen, zwitterlid).

(Sruppen: 1) Rhinantheae: antherae infra mucronatae, capsulae margines inflexi seminiferi.

a) Mclampyreac: capsula bilocularis oligocperma: Melampyrum T. $L$.

b) Tozmieac: capsula 1-locularis bivalvis 1-sperma: Tozzia $L$.

c) Pedicularinae: capsula bilocularis polysperma : $3085-$ $30 y 1$. et Siphonostegia Bnth.

2) Scrofularinae: antherae 1-2-loculares, capsulae, ulti-

*) Ino jo Diutet bic Statur wie überall immer babin, wir follen erft nadjfeben, wo fic her= fommt, bevor wiv uns baran balten Ë̈men, wo fie bimvill. Dic MRetamorphofe ber Ratur bee

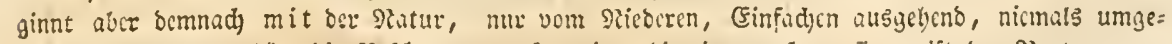

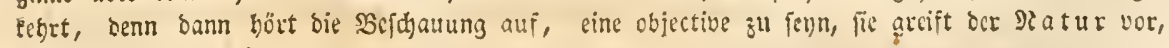
und riro jubjectio. 
inis baccatae dissepimentum utrinque placentiferum, semina albuminosa.

a) Antirrhinariac antherae biloculares.

a) Veroniceae: eorolla irregularis, rotata vel.campanulata et infundibularis: Cochlidiosperma Kchb. Veronica $L$. Paederota $L$. Wulfenia Jacq. Calorlıahulos Benth. Gymnandra Pall. (Lagotis Giörtn.) - Picrorhiza Royle. Geochorda Chom, et Schlecht. - Sibthorpia L. - Disandra L.

B) Antirrhineae : corolla labio saccato, personata aut ringens: Triphysaria Fisch. et Mey. Phylacanthus N. et M. Hemimeris $L$. (Diascia Lk.) Angelonia K. H. H. Nemesia Ient. - Anarrhinum Desf. Linaria T. Mill. Antirrhinum L. Galresia Juss. (Agassizia Clavv) Maurandia Jcq. (Usteria Cav.) - Lophospermum Don. Rhodochiton Zucc.

y) Digitaleae: corolla infundibularis ant campanulata limlo planilobata.

us) caps. septicila: Collinsia Nutt. Pentstemon L'Herit.Chelone L. Elmigera Rchb. Ourisia Comm. (Dicliroma Cav.) - Digitalis $L$. Isoplexis Lindl.

$\beta, \beta)$ loculicidae: Centranthera $R, B r$. (Razunovia Spr.?) Glossostylis Chum. et Sehlecht. Seymeria Pursh. Gerardia L. Macranthera Nutt. Virgularia Rz.Pav. - Physocalyx Pohl. Escobedia Rz,Pav.

b) Bignoniariae: antherae uni-biloculares, semina exalbuninosa.

a) Sesameae: capsula lignosa seminibus apteris numerosis, indefinitis: Sesamum $L$.

ß) Martynieae: capsula lignosa oligosperma: Pedalium $L_{\text {, J }} \mathrm{J}_{0}$ sephinia Ient. Pretrea Gay. Rogeria Gay. Sessea $h z$. lav.? Martynia Houst.

y) Bignonieae: vapsula lignosa plurimis quasi siliquosa, semina alata. Conspect. 325t-3274.

(:) Caprarieae: antlıerae 1-2-loculares, cor, subregularis, semina albuminosa. P'lurinis folia crenata vel serrata.

«) Verbascarine: antherae uniloculares:

жu) Lentibulariae: personatie sem. exall. peltatis: Utrieularia $L_{0}$ - aut albuminosis basifixis: Jovellana Cav. Baea Commers, Calceolaria $L$.

$\beta \beta)$ Scrofulareae : labiatac exalbuminosae: Pinguicula $L$.Brandonia Rchb. Alsuminosac: Ceramanthe Rchb. Scrofularia $L$. 
yr) Verbascene: subrotatae: Isanthera N,v, E. Alonsna Car. Celsia $L$. Verbascum $L$.

3) Gratiolene: antherae 1-2-loculares corolla tuluata limbo plurimis explanato: Limosella L. Gratiola Lo Beyrichia Cham. Schlecht, Achctaria Cham. Schlecht. Bonnava Lnk. Peplidium Delit. Nlicrocarpaea R.Br. Dopatrium IIamilt. Lindernia L. Torenia L. Artonema Don. (Diceros Pers, non Lour.). Vandellia L. Tittnannia Rchb.") Matourea Aubl. Sphaerotheca Cham. Schlecht. Herpestes Gürtn. (IInnuiera Mich. Bramia Lam.) - Uredalia $R B r$. Mimulus $L_{\text {, }}$ - Morgania R.Rr. - Limno-

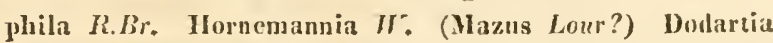
L. - Stemodia L. (Mollestia et Diamoste Cham. Schlecht. Lindenbergia Lk. (Brachycoris Schrat. Rovea Dscsn.) Pterostigma Lnth. - Erinus L. Xyeterinia Don. Manulea $L$. (Nemia Berro.) Palmstruclia Rlz. fil. Suterit Roth. Buchurra L. (Caupuleia \%. P. Th. et Piripea dubl.). - Russelia Jcq. Capraria L. Freylinia colla. Budlleja L. - Browallia $L_{\text {. }}$ Franciscea Pohl. Hlmiphragma Wall. 'Teedia Rul. Halleria $L$.

y) Brunsfelsicae: antherae biloculares, coroll: infundilutaris, placenta centralis, emiryo eurvulus. Heterathia. r. E. Anthocercis Lab. Brunsielsia $\mathrm{h}$.

3) Oro!baclueac: parasitae é tuberosae placentis parictalibus.

a) genuinae: antheris loasi mueronatis: 3051 - 3mio.

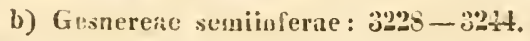

e) Cy rtandreate, hypogynae septo valvari utroq̨ue utrinque revoluto placentari : Ramondia Rich. Rchmannia Libosch.**) Henkelia spr. (Rottlera $V$. Didymocarpus Juck. Sureptocarpus $L i n d l$.) Loxonia Jack, Lisionotus Don. Aeschynathus Jach. (Trichosporm Don. nec Fries.) Leueocarpus Don. Cyrtandra Forst. - Llazcenta in pulpam soluta: Csescentia $L$.

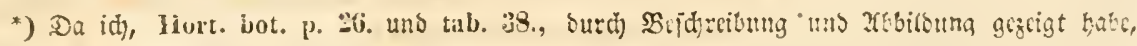

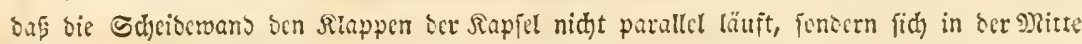

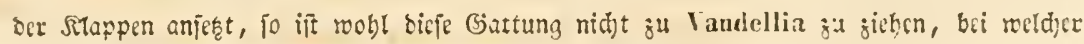

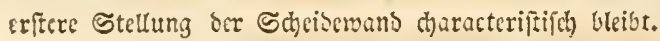

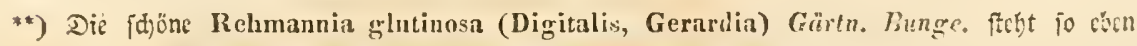

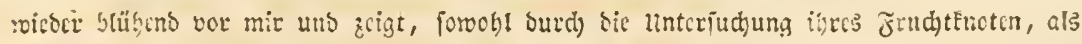

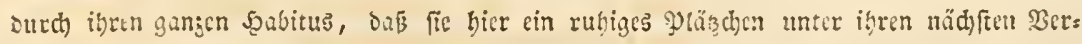

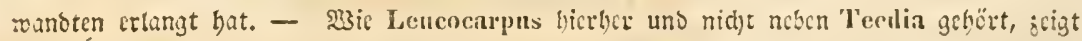

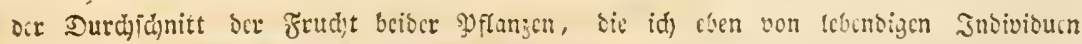
sntnchme. "Bei erfterce finsen wir bie ungeryo!nte (Erfd)cinung ciner Jleiffjmafie, aus der

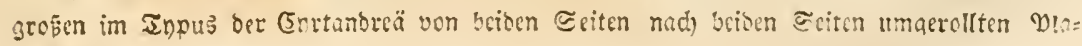


Banjblunige.

Sromung: Edylunoblumige. Earbenblütgltr.

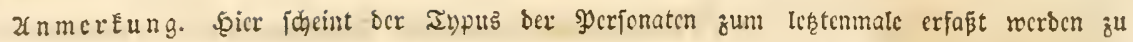
Eöunen uno in ben ber Colanaccen binäbcrzufpicten. Das Beginnen jeber Şauptgruppc

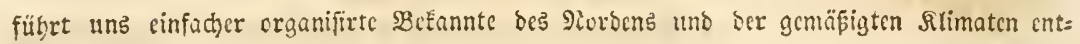
gegen, ocren Formen fich forrittweife fortbilben zu weiterce Entfaltung, bie fie unter tro=

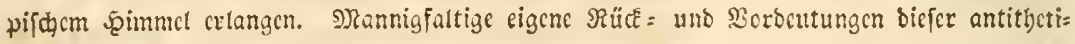

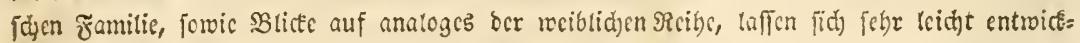
eln, id will abcr bom geneigten \&ejer in bicfer Betrachtung nibit vorgrcifon. 2fucs wies ierbolt fich bcutlidy, fogar bie Betbascen in Ramondia, wie bie Gratiolecn in ben üteri=

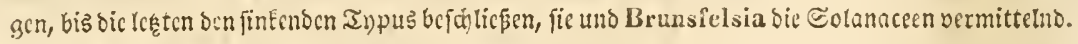

90. Familie. 9zactstidyaten: Solanaceae.

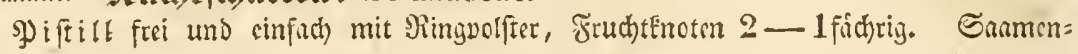
triger auf beiden Seiten ber Echeidewano, oder frei unb central. Griffel ge= freeft, Narbe zweitépfily oder burd) cine Furche getbeilt oder einfach). Seld) $J=$ oocr mehrtbcilig, fortwachjomb.

Staubbeutel 4-5, zwcifichrig, aufredt ober angewarthen. Facher an ocr Spise docr meift lings aufipringend, auf Ermbfioen, weldhe entweder biss= namifd) (Salpiglossis) oder bibnnumijh mit fimftem Etaubfaden (Atropis),

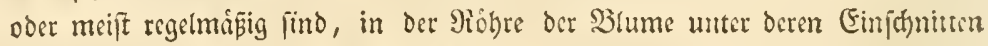

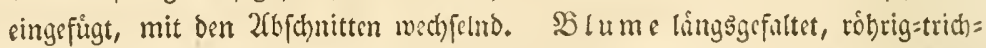
terformig glockig, thoformig, Saum unregelmisig bri ben beginnenden Gatt= ungen, 4-5lappig, bei ben folgenden regelmẩig 5 ppultig oder Jtbeilig, bei wenigen umgelegt, bei den meiften linggéngefultet. Bas cinigen finbet fich) an

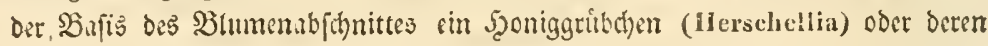
żuei (Duleamara).

S3egetation. Stumm: 23 urzel bci wenigen Enollig (IIerschellia tuberosa), fnollentrageno (Solan, tuberos), meift einfuch) uno affig; sinjibrige, zmsi=

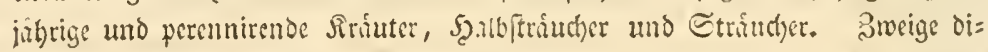

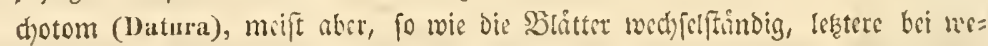

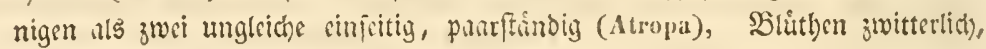

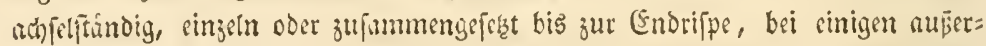

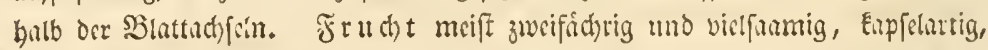
mit Deffel aufipringend, meift mit filazpen, oder gefd)loffen bieibende trofkene (Capsicum) oder meift faftige Beere, nur bei Nolaua finden fich Etcinfrichte mit einjaumigen Firchern.

Gruppen: 1) Dolaneae, pyrenaceac. Nolana $L$.

2) Luridac, capsulares et baceatae placenta amphigena (antithctica).

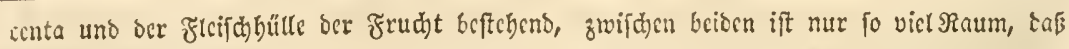

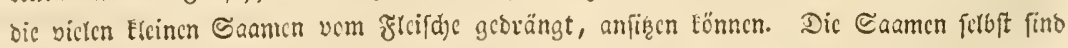

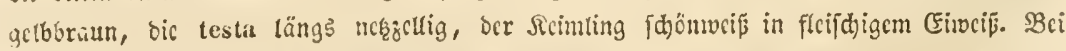

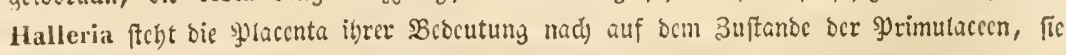
twiro yon iffen ๔aamen feripherifd) umyeben. Crescentia zeigt in ocr Frudt bic Fort= biltong won Leucocarpus, in bon ciwribtofon Saamen bic Sollendung ocr Bignoniaceate unb gremat an bie Colinaccin. 
Sromuttg: Snumblumige. Raütidutten.

a) II yoscyameac: operculatae (placenta amphigena). Hyoscyamus $L$. Scopolina Schult.

b) Nicotianeae: valratac placenta utrinque adnata simplici. -Schizanthus Rz. Pav. Salpiglossis $R z$. Pav. Nierembergia Rz. Pav. Petunia Juss. Lchmanuia Spr. Nicotiana $\boldsymbol{L}$. (Nrctagella, Tabacum et Tabicina 1 .). Datureac: valratae placenta utinque duplicata. Stramonium Gürtn. Datura L. CeratocauIos Bruh. Brugmansia Pers.

c) Solaneac, baccatae placenta (amphigena antitletica).

a) Atropene: campanulatae placenta livisa. - $\Lambda$ tropa $L$. Anisodus Lk. Nicandra Gürtu. Solandra Sw. Desfontainia Rz. Pav. (Linkia P.).

B) Physalideac: infundibuliflorae placenta unita, Saracha Rz. Pav. Physalis L. Iferscliellia Bowd. Nectouxia $K_{\text {. }}$ II. B. Dierbachia Spr. (Dunalia $K$. H. B.) Lycium $L$.

,) Solana: rotiflora, omnes placentarum variationes repetentia, ut supra iam Nicotiana. - Solanum L. Nycterium Ient. Lycopersicum $T$.

3) M andragoreac, baceatac placenta libera (synthetica).

a) Mandragora $T$.

b) Jaborosa Iuss. ${ }^{*}$ )

c) Cestrum L. Vestia $W$.

26nmerE. Die Nolaneae bicten bie RücEseutung nuf bic Myoporinae, dic Luridae bilsen

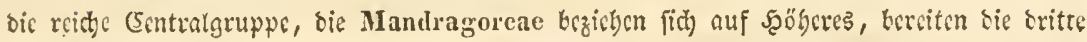
Sronung idjon vor.

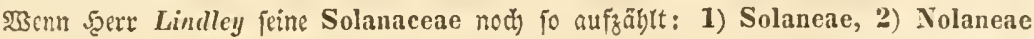
Rchb. 3) Verbaceae uns bamn in bor 2(nmertung fagt: ,Rchb. zicht bie erfter uns sie

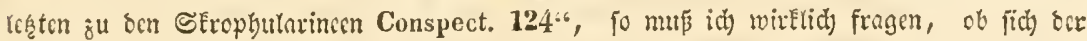

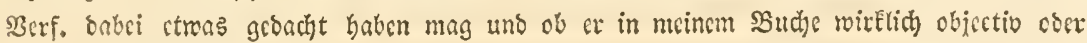
nur jubjectio gctejen bat?

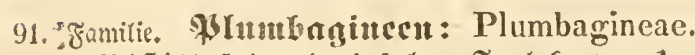

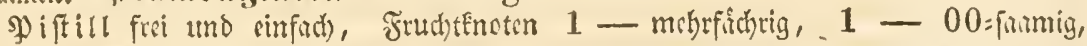
Griffel geftrectet, Narbe pfriemenfpişig (Plantagin, uno Stilbe) oder $5=$ :paltig (Plumbagineae) odcr Fopfformig (Epacridene). Feld aub fuft fpeljigen zicgelfitandigen Schuppen (P'aut. Epacrid), in ber 2(ntithefe voltendeter, réhrig

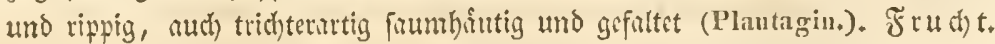
Schluuth cinfuamig (Littorella, Arm. et Stat.), ringsumnuffpringeno, San= mentriger fcheideroandartig iroerfeits 1 - 4 = farmig (Plant.) odir freuj=

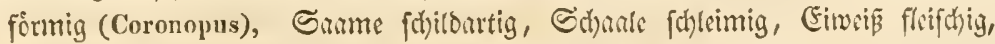

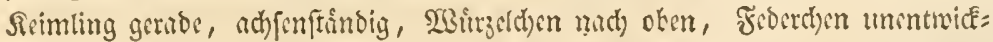

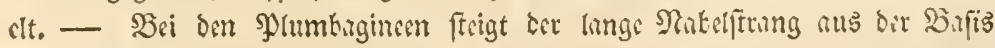

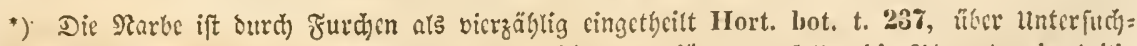

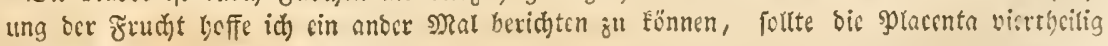
firn? 
Der cinfaumigen Rappel empor, (ber Saume alfo eigentlic) aufrect) aber ber Saame bingt von oben berib, Eiweis mehlig, Feimling lainglich und flach, W3urzetchen mach oben. - Bei Stilbe unb ben Styphelieae cin = ober we: nigfaumige Eteinfrudbthen, bci Epacreae mebrfichrige, fachppaltige Sarpjel

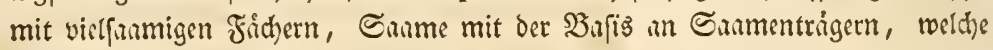

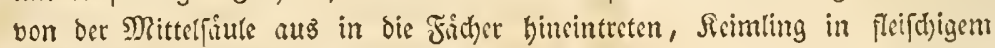
(Eiveipe ad) fenftindig, aufred)t.

Staubbeutel $4-5$, zweificbrig, aufliegend kei leşteren aufred)t, mit ben Slumentbfonitten med) felftindig auf langen Strubfáben tief in ber Blumen: robre cingefügt (Plantag. und Stilbe) oder auf Eurgserm nichft borm Echlunde (Styphel.) oder im Schlunde fift ftaubfindenlos firsend (Epacrid.) - in Der 2fntithefe (Plumbageae) vorimftehend, entweder bodenftindig (Plumbago) oder ben Rigeln ber foft ganz gefonderten Bltumenblatter anthingend (Armeria). Blumenfrone robrig, trockenbiutig, bei cinigen (Plant. u. Epacrid.) nuf ber Frucht vertrocknenb, bei anderen (Plumbag.) abfalleno, mit ppif̧ig 4-5: ppaltigem in ber Snofpe umgrlegten Saum (Plantagineae, Stilbe), sbenfo aber aนళ dem Ğrintichen in bas Farbige ưbergehenb (Styphelieae, Epaereae), auch tief zerthcilt (Sprengelia), nebft 5 bodemftindigen Schuppen bei (Epacris); zarter prifentirtellerforemig uno ftumpflappig (Plumbago, Statice monopetala Dracophyllum etc.) in Der Sincipe gedeht ober tief zerthcilt 5 blittrig (Armeria).

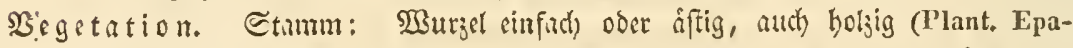

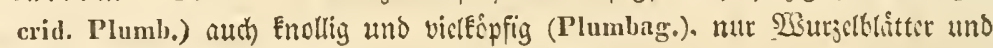

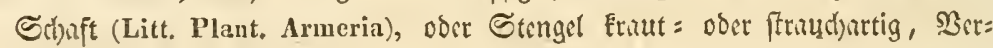
ziveigung uno Beblitterung gegenuóor (Psyll.) gubel= (Stat.) oder wed)filiftig.

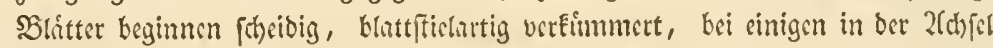
wollig (Plant., Stilbe) fdomal, runolich oder breikuntig uno linealifh) (Litt.

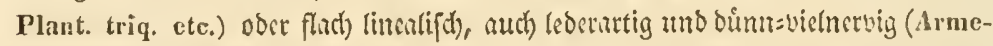
rin, Epaerid,, Dracophyllum), ober mit beutlicher \$hlatte Erauturtig nerbig (Plant. major. etc.) ober gendert (Plumb.), auth) ledirartig und gendert (Stat.)

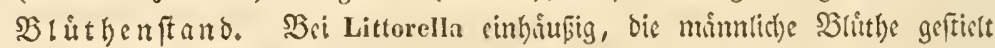

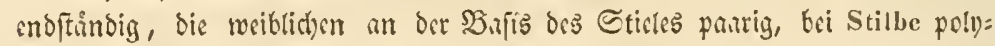
gumifa) in Fopfurtigen (Endifbren, (bei Psyllinm und Armeria) in umbillten

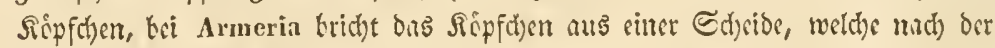

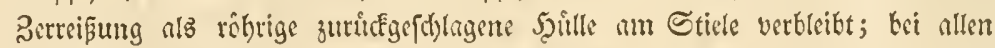

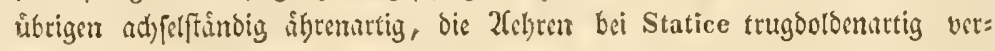
bunbern.

(3ruppen: 1) P l a n ta g in e a e : scleranthae, stamina corollae scariosae alterna.

a) Littorelleae, monoicae monospermae. Littorella $L$.

b) Psyllicae, op positiflorae et oppositifoliae dispermae. Psyl. Iinu $T$.

c) Plantageae, sparsifforae et sparsifoliae 4-8-spermac. Coroupus T. Plantago $L$.

2) PI umbageae, stamina corollae tenuis limbo obtuso, in pracfloresecntia conroluto, anteposita. 
a) Armericae, pentastyles scapiforae, spathaceae, capitatae, Armeria $W$.

b) Staticeae, pentastyles spiciflorae. Statice $L_{*}$ Aerialitis R. $\mathrm{Br}$.

c) Dentellarinae, holostyles calyce herbaceo glanduloso, Plunbago $T$ :

3) Epacrideae, stamina corollae demum coloratac laciniis (utplurinum) acutis alterua, semina basifixa erecta aut porrecta.

a) Stilbeae, scleranthae, polygamae, monospermae: Canpylostachys Knth. Stilbe Berg.

b) Styphelieae, drupaccae aut capsulares oligospermae. Conspect. $3322 y-38+2$.

c) Epacreac, enpsulares polyspermae. Consp. $3343-3352$.

Xnmerf. Dic Patur fagt bier ganz cinfach, baß bic Plantagineae cinen netten İpus be= ginnen, den fie burd ifren urfprung, D. b. Surd ibre Gaamen, ats ben ser Primula-

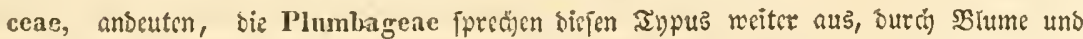
Staubgcfäpe und deren Etellung, bie Epacrilene wicderbolen crft durdy Stilbe bon un= tergeorbucten ãypus ber Plantagineae uns erinnern burch inge Styphelieae an bie Plumbagene und Primulacene zugleid, währeno bie Epacreae als offenbarę \$orbild

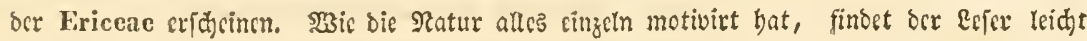
in feinem Scerbario und noch beffer im botanijajen Barten.

92. Zamilic. Strinulacen: Primulaceac.

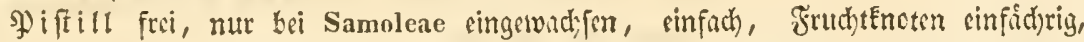

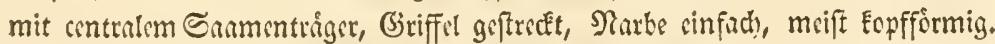

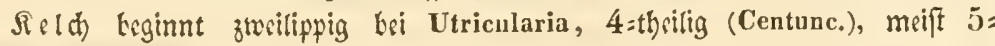
fpultig, glockig ober rubformig, 6=tberitig (Naumb.), 7 =theilig (Trientalis.) $\widetilde{F}$ u mit Deckel (Sold.), mit Bainnen (Cycl. Prim.) ober Elappig (îrrige Primuleae und Lysimachieae) ober singetwadjom, an ber froin Epire mit Bifhnen auf= fpringend (Samol.) ober freic Eteinfrutht (Myrs. Jacq.). Saamen 5:zínlig

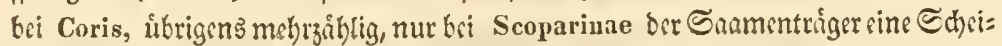

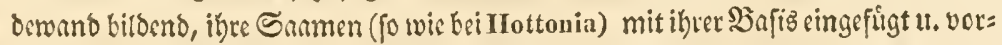

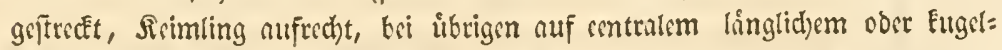

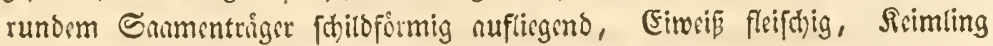
quer, linglich), ziemlid) getrode, ober gefrủmmt (Myrs. Jacq.)

Staubbeutel 5, (2. bei Utricul., 4, bei Centunc., 6. bei Naumb., 7. Gai

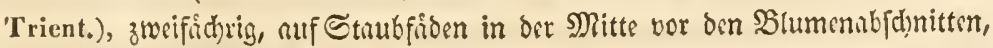
aufrecht, ftumpf ober gefidnabeit (Sold. Cort.), bodenftindig und frei (Glaux),

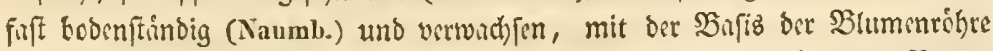

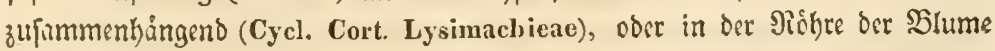
singefingt (Primuleae). $\mathfrak{B}$ lume fohlt (Glaux), unregelmínig (Coris), bei als Inn' librigen regetmáfig, entrosoer prófentittellerférmig (Androsaceae) ober trifhterfírmig (Sold. Cort.), gloctig (Samol. Theophr.), rabformig (Scoparia, 
Gantzblumige.

Sronutg: Sauntulunige. Pgrimulacecn.

Cycl. Anagallit. Myrs. Jacq.), Saum $5-(4=6=7=$ theilig, Shlund nadet oder mit Drufin (Aretia, Androsace) ober mit Şanern (Scoparia) ober mit Shuppen zwifden den Staubfíben. (Sold. Samol. Jacq.)

Se getation. Stumm: Wurzel Enollig (Cycl.), meift cinfad) ober vieffipfig uno affig, Safuft (Primuleac) oder Stengel, Ermuturtig oder ftrauthartig, Serzwcigung und Beblatterung gegenitber und quithrtig (Anatgallid.) oder ziegelftindig jerftreut (Coris, Aretia, Ardis. Jacq.), SBlitter ftaut = ober leder= artig, genoert, ungetheilt, bei cinigen eingefthnitten (Cort. Primula sinens.) oder mur bas geficoerte (Sertippe (IIottonia). SBlit thenftand: 2lehre (Coris), Sibuft cinblithig (Cycl.), endooldig mit Scille (Androsaceae, Cort.) achjel= ftinbig einzeln ober trinubig (Anagallid,), adfel = ober enotraubig, trugboldig

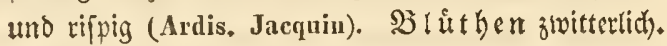

(3ruppen: 1) Primuleac: tubiflorae, tubo staminifero.

a) Corideae: irregulares labiatae, spicatac, Coris $L$,

b) Anlrosaceae: regulares lypocrateriformes 3359 - 3361 .

c) Cortusene: regulares limbo camp, et subrotato, oxyantherac, Cyclamen $L$. Dodecatheon $L$. Cortusa $L$. Soldane!la $L$.

2) Lysimachieae: rotiflorae, basistemoneae.

a) Scoparinae: corolla 4 partita, caps, bivalris placenta septante. Scoparia $L_{\text {r }}$ Sphaerotheca Cham.

b) Glanceae: apetalae valvatae. Glaux $L$.

c) Anagallitcac: rotiflorae operculatae et valvatae. Centunculus $L$. Jirasekia Schm, Anagallis $L$. Naunburgia Mnch, 3อ2ง. $3325-3028 b$.

3) J a cquinie a e: drupaceae.

a) Samoleac: drupa infera apice capsularis dentibus aut non dehiscens, Samolus $L$. Schefficldia Forst. Bacobotrys Forst.

b) Ardisieae: drupa libera monosperma, Bladhia Thb. Ardisia Sw. Cybianthus Mart. Embelia Burm. Purkinjia Prsl.

c) Myrsineae: drupa pleiosperma. Myrsine $L$, Jacquinia $L$. Theophrasta $L$. Leouia $R z$, Pav.

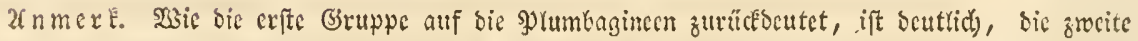
ift bic Ecntralgruppe, igre 2entithcje ppaltet bic 3 tumentrome wicber bij zum 5:blättrigen 3uftande, bie Jacquiniaceen wicberbolen alles und verföhnen alle Differenjen, inbem fie zu=

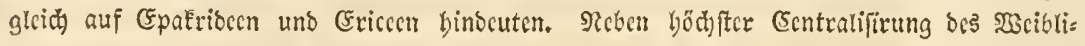

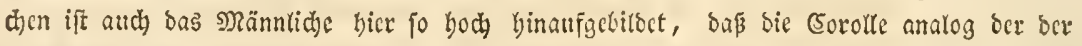

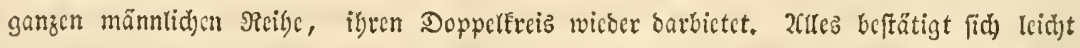

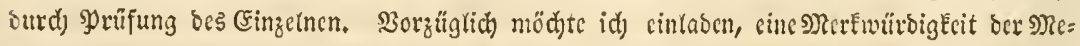

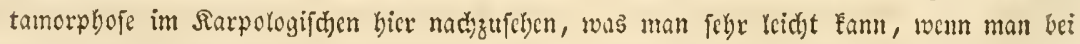

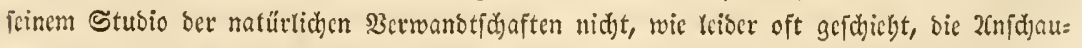

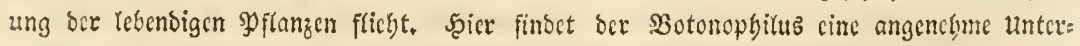

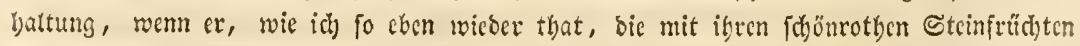
reichbetadene Ardisia lentiginosa (crenulata IIort.) bor fid hat. Jd) felly bier in bics

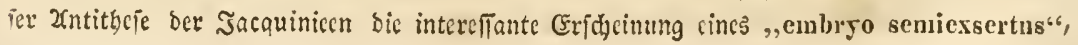




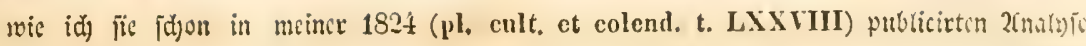

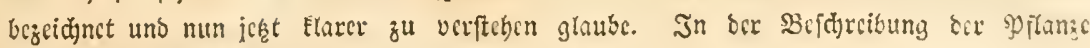

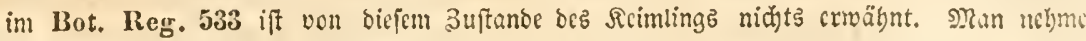

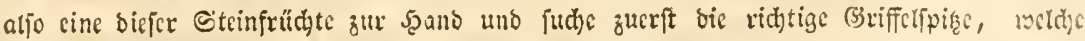

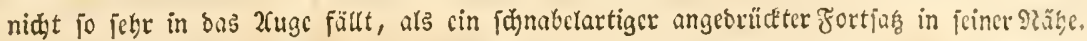

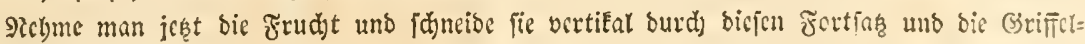

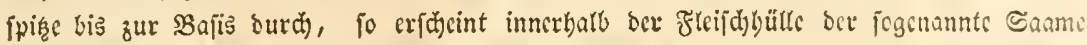

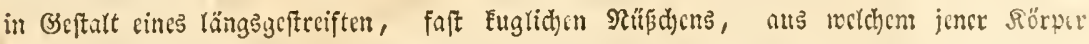

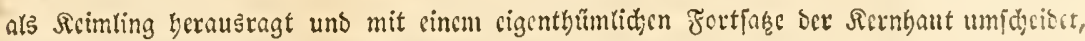

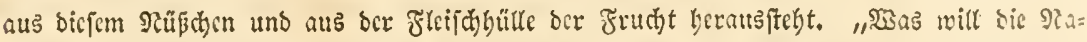

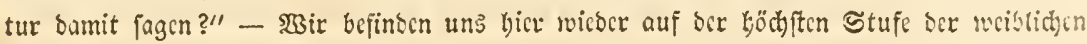

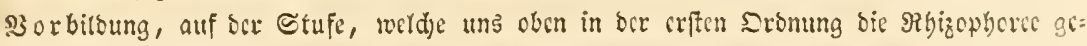

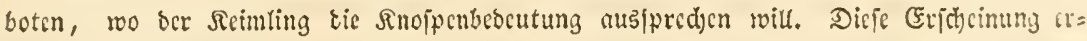
läutert aber zuglcid) cine anocre Fortbilsung in ber Eorpologijdhen Sphäre. Sie bcutit

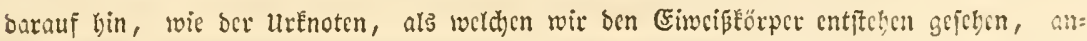

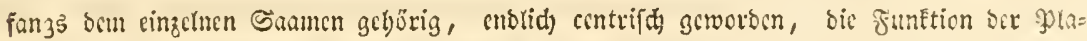

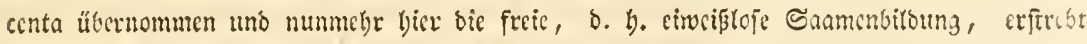

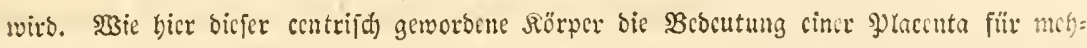
rere Saamen, wirktid) baben mag, zeigt Ardisia humilis (Anguillaria zeylanica)

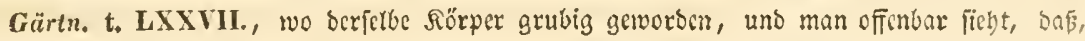

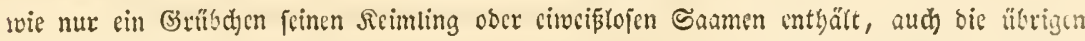

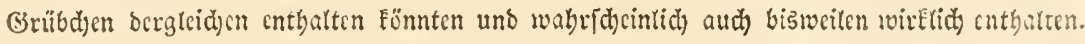

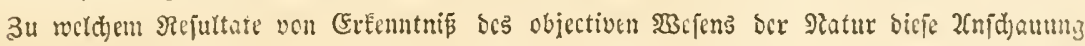

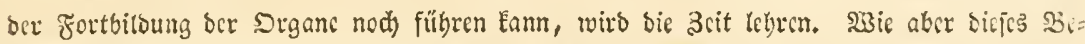

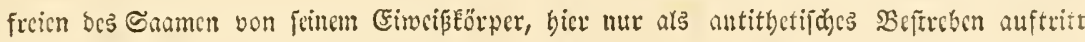

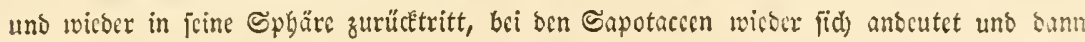
erfit in forgenden Rraffen bcimifa) wirb, bas legrt bie Betradjtung bes 3ujammengangcs im

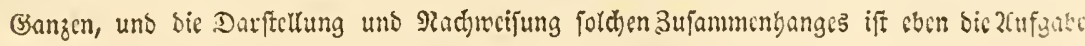

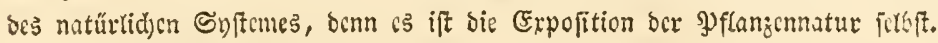

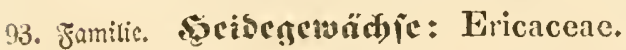

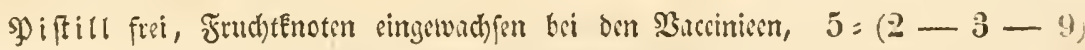

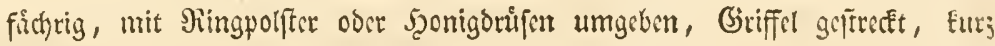

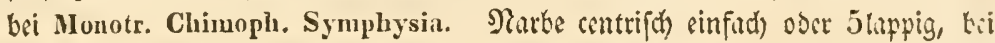

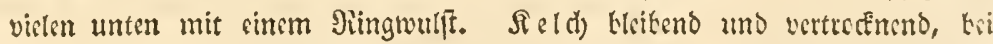

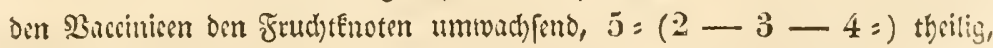

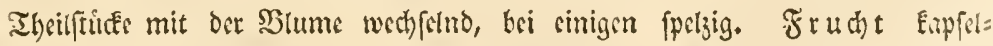
artig, fei andern beccuntig, $5=(2-3-4-8-9 \Rightarrow)$ fichrig

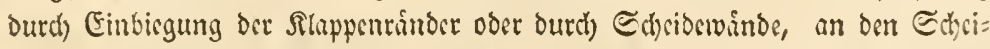
Derwinden oder in ber Mitte ber Filappen auffpringend, Sanmentriger centrijō,

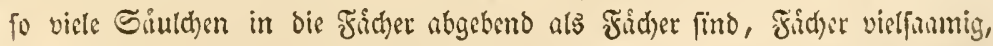
(nur Fri Arbuteae und Gaylussaceae wenig= odcr 1 famig), Ganme Elcin, frilftuburtig, Fei cinigen mit locferet zelliger Szant umgeben (Monotroy. Pyroleae)

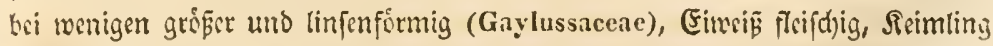
aufretit, geribe, Eotnledonen bulbrumb. 
(5) הlijblumige.

Sronung: Enumblumize. Saribegerwädy fe.

Staukfeutel 10, bei wenigen $4-8-5$, in vielen Bantungen bie Ficher gs= fpornt, an ber Spibe oder lings auffpringend, 2, auch 4 fichrig (Sympliysia), fïeno (Symplyysia), ober auf Staubfioben tief in ber SBlumenróbre singefiggt, coer bodenftindig, mit ben Saumabjhnitten der Blume gleich) = oder boppel= sifflig und wed) felno, mcift frei, mur bi Synactinia Rchb. (Erica monadelpla) in eine Mióbre verwachfen, an beren Miunoung bie Seutel sinen borizontalen Etern bilden. SBlume auf ber Sieldbbrifis oder bem Slluthenboden, robrig,

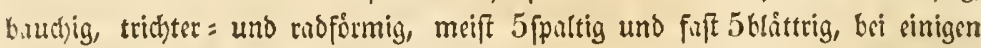
4-Spaltig, in ber Sinoppe berumgelegt, am meiffen unregelmipitg und 2 lippig bei Rhodora, librigens wenig unregelmå̄̄ig, meift regetmá̄ig.

Degetation. Stamm: Wurzel (bei Monotropeae fhmarofend und der Strngel

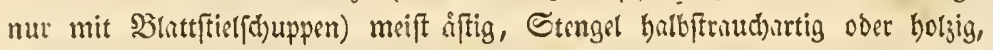
frtutu $=$ und faft baumartig, Serzweigung und Beblitterung bei einigen ge= gintiber uno quirlartig, meift zerftreut, Blitter nabel = ober lederartig unge=

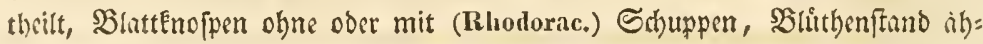

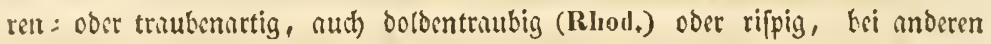

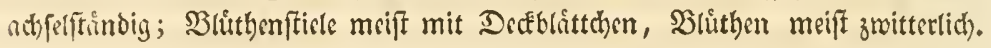
(3ruppen: 1) Ericariae: capsula loculicila (in sola Calluna septicida), aut bacca supcra, cernua (in Monotr. et Andromela caps, crecta). a) Lriceae: antherae crectae, corolla synpetala.

๙) Calluneae: septicidae. Calluna Salisb.

f) Andromedeae: loculicidae. Sympieza Lichtst. Blairia $L$. - Synactinia Rchb. Erica $L$. Bruckenthalia Rchb. Menziesia Sm. Phyllodoce Sulisb. Antromeda $L$, Lyonia Nitt, Gaulthicra Kalm. Encyantlius Lour.

r) Arbutene: baccatac. Arctostaphylos Adans, Arbutus $L$. Pernettia Gaudich.

b) Monotropeac: antlicrae incumbentes subpeltatac. Monotropa L. Schweinitzia Ell. P'terospora Nutt.

c) Pyroleae: antherae crectae aut incumbentes apice perforatac aut rostratac, corolla sub 5-petala.

Diapensia L. Pyxidanthera Nclux. (Lepuropetalon Ell.)

Chinophila Prsh. em, Rad. Pyrola $L$. Clethra $L$.

2) Vaccinieae: bacea infera.

a) Myrtillcae: faucillorae, bacea loculis polyspermis.

Oxycocens Pers. Vacciniun $L$. Ceratostemma Juss. Thibaudia $R \approx$. Pav.

b) Symphysicac: pleiopetalac calyptratac. Symplysia Presl.

c) Gaylussacieac: synpetalac unultiloculares, semuibus singulis majusculis lenticularibus. Gaylussacia $K, I l, B$.

3) Rhodoreac: capsula supera all valvularum inflexos margines dehisecns (in a, et b.) erecta.

a) Chamacledeae: regulares apicicilac. Epigaea $L$. $\Lambda$ zalea $L$, Kaluia $L$. Ammyrsinc Prsh. 
Sronnng: Sanmblumige. Sqaitrgatädje.

b) Rhododendreae: irregulares apicicislac, Rlodora $\boldsymbol{L}$.

\section{Antlodenilron Rchb. Rhododendron $L$. Rhodoliamus Rchb.}

c) Ledeae: regulares pleiopetalae basicidac, Ledum $L$.

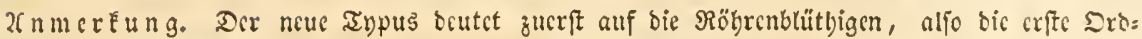

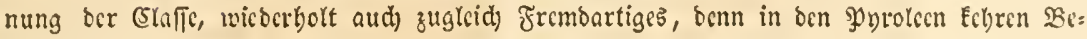
ziclungen auf Esonvolvulaccen (Polemoniaceae: Diapensia) u. auf bic Primulacecn zurnice,

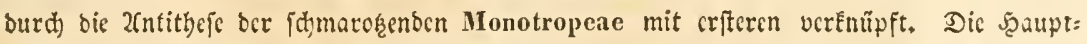

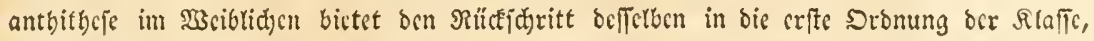
Sic Saccinieen tvicberbolen nämlich) dic Roniccteen: z. S. Linnaea und Symploricarpos. In fid felbft cutwoideln fie fid) antitbetifd, fd)liefen aber mit eigentyümlid) linjenförmigen Caanicn, an bie 2 rbutecn crimmermb. Die Ryoborcen jeigen dic Ericacen in ifrer bödten

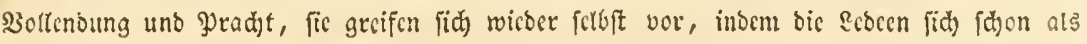
פolnpetalcn voluenoen, darin aber eben von ber \$olkendung bes cigenen Iypus wicocr zurück: gehen.

94. Zamilic. 213̈repinscen: Asclepiadeae.

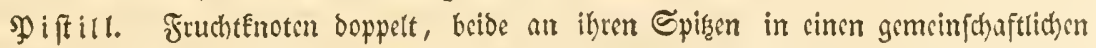
Decel vercint, welcher mit bem StrubfadenErange verwadb) fen ift und math au=

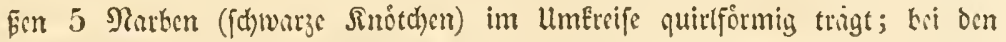

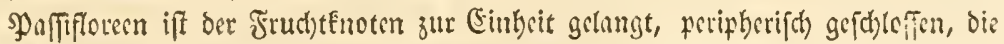
(B)iffel brcizallig und frei. Sitd) 5theilig, meift rabformig. - Frud)

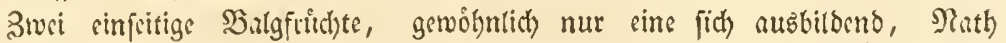

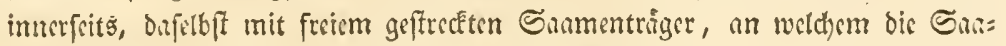

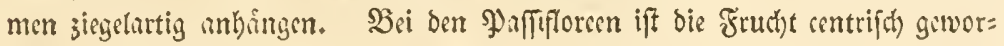
ocn, orciflappig auffpringend oder beerenurtig gejctloffen, Saumentriger mano= flinbig: Sa amen meift an langem Samenftrung, weldyer fid in Scioenfión nufló?t bei 1) u. 2), obcr ủter den Sanmen als wulfigige Nappe (Modecea) fich fortge=

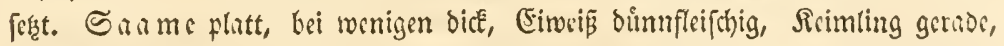

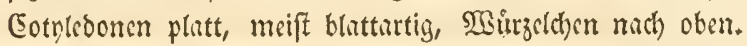

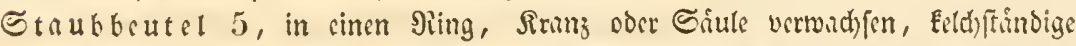
Stubfiben offnen fich lings math ausen in 5pantigen Fid nochmals gethoilt, nach oben mit sinem Bipfel verfsen, welche Sipfel fid)

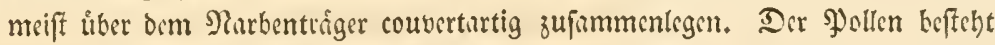
aus ciner watbzantigen Maffe (pollinarimm), welche fich in jedem Fach), alfo partig ober boppclpanig in jrocm Etaubbentel, bejindst, mit sinem fonnatel=

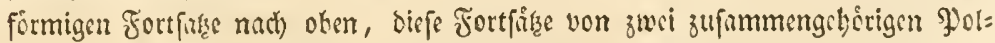
limarien find gegen simmoer gerid)tet und versinigen fich zur Scfrud)tung mit

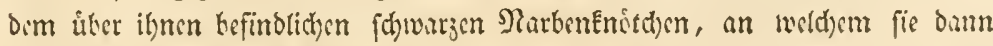

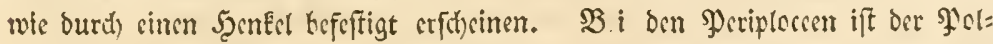

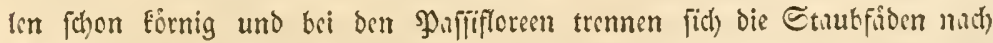
sber, indem fie bloz unten verwachfen blsiben, und tringen bie auflicgende 3neifid)rig vollendete 2tuthere frei auf fich. STlumenfrone 5theilig, in Der Sinoppe Elappig, auch trid)terformig (Cerop. Cryptostegia), bei den Şaffiflo= ren umgetegt, meift ftern= und rabformig. Die Stnubfíben bilden num jwifchen 


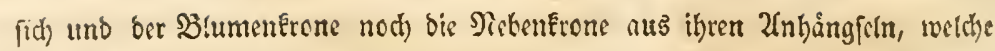
mit ihnen und mit ber (gynopetalen) Ştumentrene alterniren.

Legetation. Wutzel bei cinigen fnollig (Ceropegia, Aselepias tuberosa) bei an= bern bidf biffbelfoferig (Vincetoxicum), ober bei ben meiften sinfad) und affig.

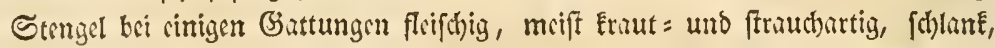

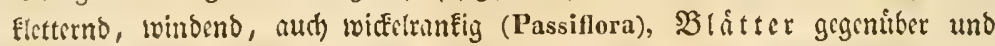
gunjrandig, mcift geftielt (Asclepias) uno abfalleno, nur bei menigen quirl= artig uno irechfelno, bei ben Paffifloren immer wect)fino, bei vislen unter ib=

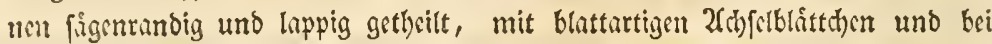
cinigin mit Drifen. SBtithen adbfelftindig, bei wenigen crtrantllar, in ge=

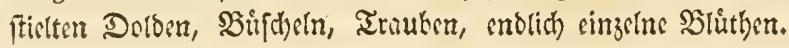

(5rtupen: 1) Aselepicac: pollinaria ceracea.

a) Stapelieae: antherae apice exappendiculatae, pollinaria erecta $3108-3415$.

b) Cynancheae: antherae membranaceo-appendiculatae.

«) Pergularinae: pollinaria erecta. 3416-3422.

B) Gonolobeae: pollinaria transversa $3+123-3424$.

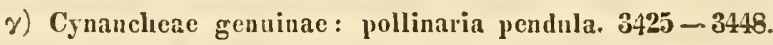

c) Astephancac: tubus stamineus exappendiculatus. $3 \pm 49-$ $345 t$.

2) Periploceae: pollinaria granulosa.

a) Itemidlesmene: monatelphae. IIemidesmus $R, B r$.

b) Periploceac genuinac: synantlereae, parapetala subulata. Periploca L. Gymnanthera $R$. Br.

c) Cryptostegieae: pentandrae synanthereae, parapetala furcata. Cryptostegia R. Br.

3) Passifloreac: antherae 5. biloculares perfectac, liberae, stigmata 3 perfecta.

a) Malesherbieac: capsula elongata, apice trivalvis, Malesherbia Rz, Pav.

b) Paropsieae: Capsula inflata sessilis, 3460-3462.

c) Grainadilleac: fructus stipitatus trivalvis aut baccans. 3468 $-3172$.

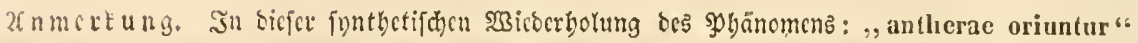

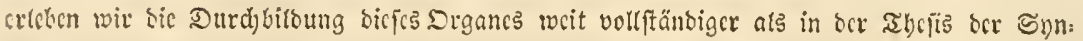

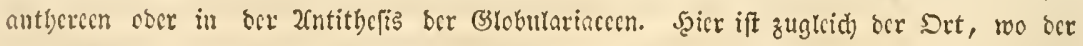

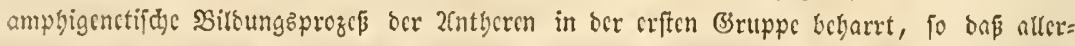
sings żmci zufammengcthörige झुoltinarien in zwei benachbarten 2entheren befinblid)

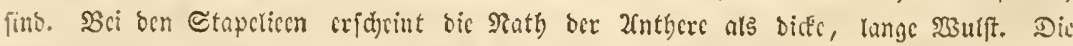

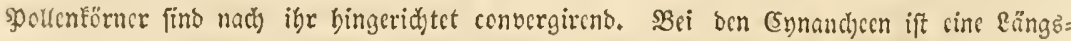

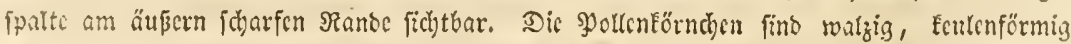

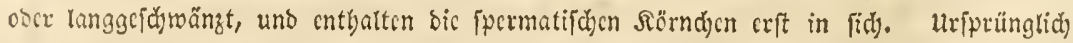

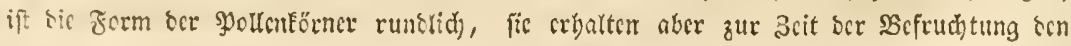




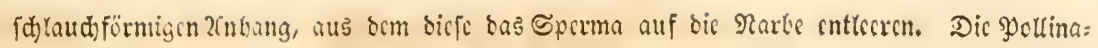
ricn find aljo folbft von cincr feinen şaut boutelartig umgeben.

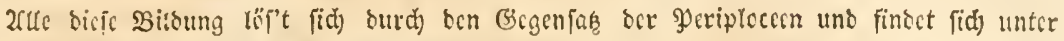

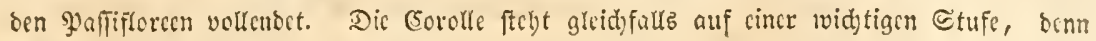

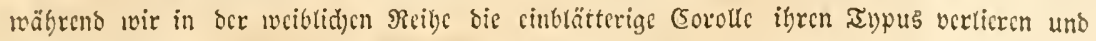
(idi) zute fünfblätterigen fortbilben fahen, fo geht hier bis bisher bagenefone corolle in

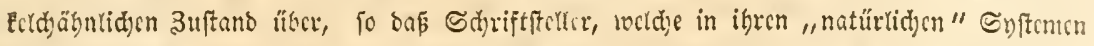

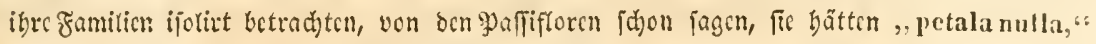
bafür aber ifmen cimen ocppclecifigen Seld ertbeiten, obne zu fragen marum coce nober?

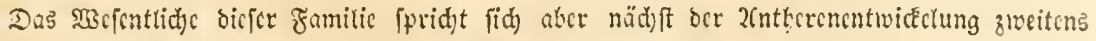

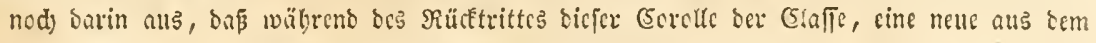

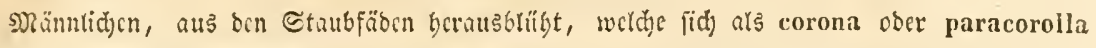

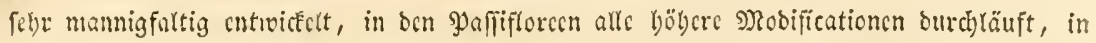

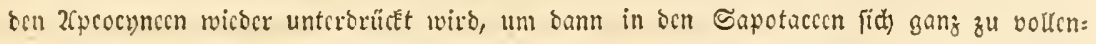

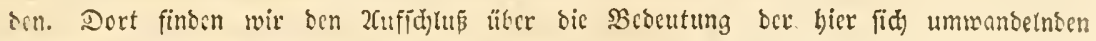

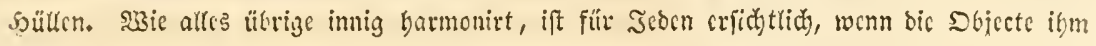
vorlicgen. Insbcjontcie wirb cs leidjt Elar, wic bic Maffiflorecn von ber Ratur berufon

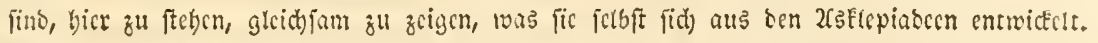
Gie beutct babci natürlid) sinigermaßen auf bie (Eucurbitacen zurücé und giebt nun ber

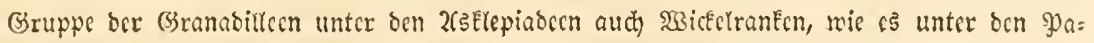

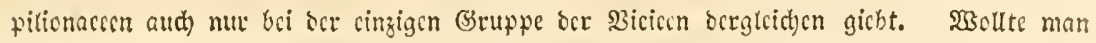
aber beswegen fdon bie Cucurbitacen und gaffiflorecn zufammenftelen, fo witro man

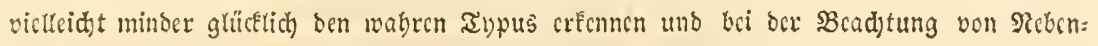
bingen ben şlice auf ben 3ufummengang bos şanz̧en verticren.

\section{Familic. Prebblüthler: Contortae.}

Pुiftill boppelt. 1) meift in eins uerfhmoigen, Frutdetenoten bann 1 = ofter 2 fächrig,

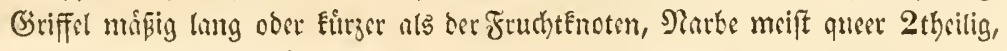
(allo ksi gencigter Bluthe vertiEal gefpalten und borizontal mugherecitet); -

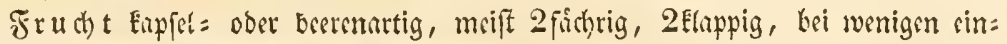
fíchrig uno nicht nufpringeno (Menyanthes). Saamentráger bei ben

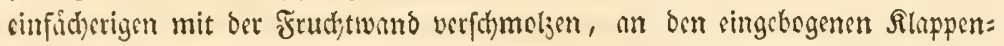

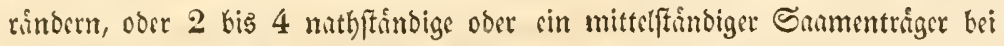

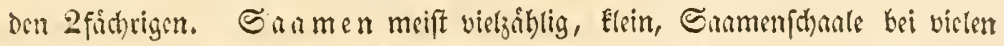

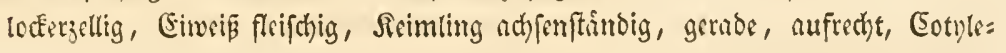
bonen nicht blatturtig.

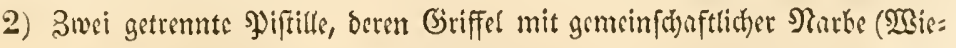

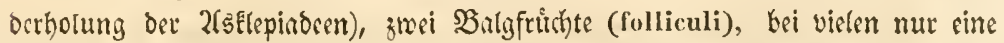

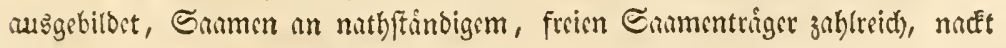
ober mit Şararchopf, Eimeis fleifchig, Seimling gleid)ftionoig, Eotnlroonen blattartig.

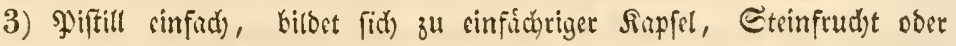
Bere, Sanme platt, Eimeis fleifhig ober bornartig. Scimling wie bei vorigen. Á (d) 5thrilig, bei wenigen $4-8-10$ theilig, Heibent.

๔taubbeutel 5, (oder $2-4-8$ ), fo vicle als 3 Gumenabldnitte, mit benen 


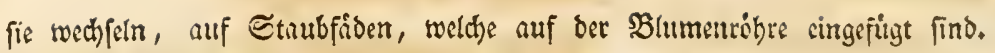
Bెeutel zweificherig, fpringen lings auf, bei wenigen anfangs alle zufammen= bingeno (Gentiana), Błume in ber Sinofpe rechtšum georeht, umgelegt, Elap: pig, trichter - prifentitteller - rabformig, bei einigen im ๔ẹlunde mit jer=

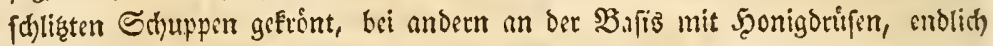
bei Parnassia 56littrig, bie 5 doppelten Sconigorifen von ben Blumenblat= tern gelof't nno mit geftielten Drifen gewimpert. Duripenpolfter jubifhen Refth und $\mathfrak{B}$ tume bei siner 2(btheilung von Chironia: Tracheanthera uno bei Echites.

Begetation. Stamm meif 4kantig, Enutntig, Blátter gegenuber odar quirl= artig, paralletmervig und ganjrandig, nur bei Swertia und Parnassia rect)= felno, Gei Heuyanthes auf Der SBlattfitielpise (metumorphofirtem 3tociglein),

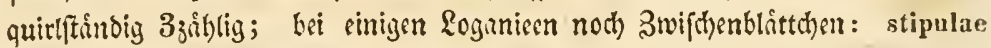
intrafoliacene, (Deutung allf Rubiacene). SB(ůthen meift ad) sclftínbig, kei Menyantheae Traube oder Dolde, bei Parnassia sinjelne $\mathfrak{B}$ litthen enditandig. Sci 2) und 3) aud Stráucher und Bäume, einige mit Milchjaft, Blithen

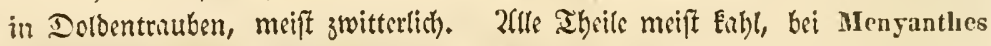
bic (Sorolle innerlid) faferig=zottig, bei Mitrasacmec uno Logania Stengel und Slitter wenigftens nech) Dem SBluthenftunde zu, bebiurt.

(Gruppen: 1) Gentianeac: valvati-et contorti-florae holocarpicae, cotyledones in semine nondum foliaceae.

a) Menyant leac: induplicatac.

a) Menyantheae genuinae: Menyanthes $L$.

B) Limnanthemeae: Villarsia Vent. Limnanthemum $G m$.

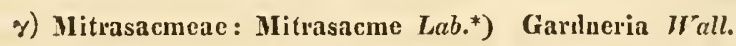

b) Gentianeac genuinae s. Chironieac: contortae monocarpicae.

a) Sebacariae: placenta centrali demum libera. Schüblera $\boldsymbol{H}$. Sebaca $R, B$ ). Exadenus Grieseb.

B) Erythraeariae: placentae suturales demum liberae discretae $6-2 \%$.

A) apparatus glandulosus in funilo corollae nullus.

1) Stigmata bilamellata $v$. bicornia stylo imposita.

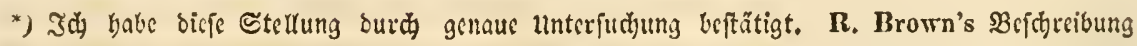
Der (Sattung ift unzursidicno, aud) Griselnacls batte nidht fo volfftändige Excmplare vor

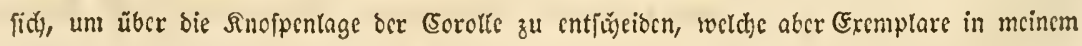
IIerbario ganz beutlich als iuluplientiva zrigen. Die Saamen finben fid böd) fetten, in mebr als 20 unterfuchten Fapfetn fanb id nur nod) ctros 3 vor. Cis find Elcin, glän=

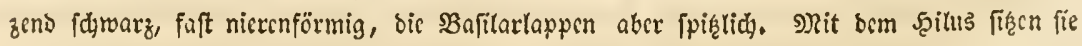
faft fojilbförnig allf. 2fn Berronnotidaft mit Veronica, wic Griscbach glaubt, mödhte

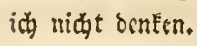


«) cor. (infundibuliformis) demum supra capsulam torta. Erythraea Renealm, $P$. Canscora Lam. Orthostemon $\mathrm{Br}$.

B) corolla demum circa capsulam marcescens, rarissime decidua, Sabbatia Adans. Clilora L. Dejanira Chan. Schlccht. Scluultesia Mart. Lisianthus P.Br. Irlbachia Mart. Helia Mart. Coutoubea Aubl, Prepusa Mart.

2) Stigma indivisum (vel subbifilum) ovario impositum. Centaurella Michx.

3) Stigma integrum! stylus ovario distincte inpositus. Cicendia Adans. Voyra Aubl. Lxacum $L$. Slevogtia $\left.R \cdot h b .^{*}\right)$ Chironia $L$.

B) Glandulae epipetalae corolla rotata, antherae inmutatac. Agathodes Dou. Frasera Walt. IIalenia Borkh.

C) Glandulae hypogynae, corolla clavata $r$ infundibuliformis antherac inmutatac. Crawfordia Wall. Tachia Aubl.

જ) Swertieac: valvarum ipsi margines semiuiferi.

«x) Glandulac epipetalic uullae. Pleurogyne Esckch. (Lomatogoniunı $v, B r$.). Gentiana $L$.

$\beta \beta)$ Glandulae epipetalae. Auagallidium Griseb. Swertia L. Oplıelia Don.

gr) Glandulae liberae ciliato-glanduliferae. Parnassia $L$. e) Loganieae: convolutae substipulatac. Logania R. Br. Geniostoma Forst, Ustería Lam. Pagamea Aubl, Gärtnera Lam. 2) Apocyncae: contortae schizocarpicac phyllocotyledoneae.

a) Echiteae: comespermae ecoronatae (Asclepiadearum repetitio). Consp. 3512 - 3526, et Kiamettia Kostlz.**)

b) Vinceae: gymnospermae ecoronatae (Centrum). Conspectus $3533-3540$.

c) Nerieae: comespermae coronatae (Sapotacearum prolusio). Conspect. $3527-3532$.

3) Carisseac: contortae holocarpicae phyllocotyledoneae.

a) P o ta li c a e: calyce et corolla inaequinumeris, seminibus scutellatis numerosis. Potalia Aubl. Anthocleista Afz. Fagraea Thnb.

b) Rauw o If i a e: opposite dispermae, Conspect, 3551- 3559.

c) Stryclu neac: calyce et corolla aequinumeris, fructo baccato (paucis sicco) 1-00-spermo. Consp. 3541. 3542. 3560-35i9.

*) Die Battung Slevogtia batte ber Monograph der Eentianeen verfaunt, crkannte fie aber in minem Serbarium [oglcid) wieder an: Slevogtia viscosa Rchb. waar chederm Exacum Ait.

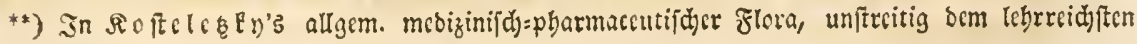
unter allen prattifden 5̧anbbüdjern. 


\section{(3)anjblumige.}

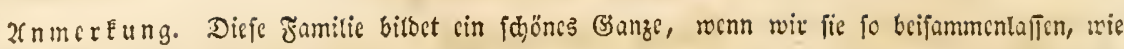

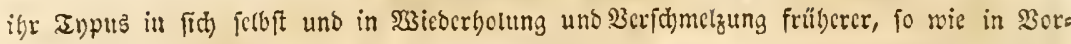
scutung fünftiger Bslieber, fich) verzweigt. Während bie ganze erfte Gruppe ben neten

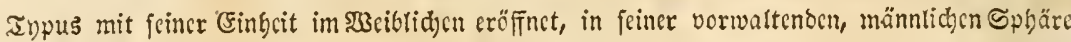

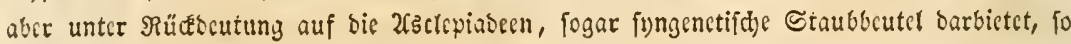

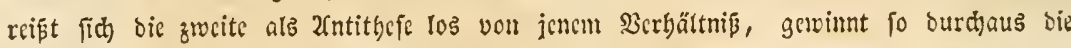

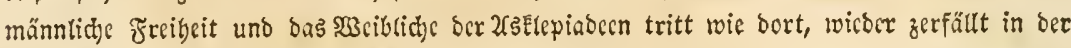
3roeizabl beraus, bie Bruppe ferbft aber entbält bie reinften II)pen ber neuen Beftaltung,

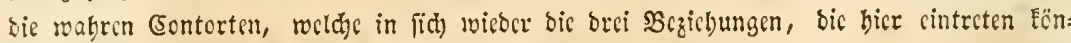
nen, für sen viffenden Forider lcidyt offenbaren. Dic britte (Sruppe tritt in flare $B c=$ ziegung zur britten Familic, wäbreno fie alles bagewefene Iypijale wisbergolt unb ver: sint. -

Die Şlätter faft in ber ganzen Familie cricheinen mebr als slattffielgcbildo uno bei Me-

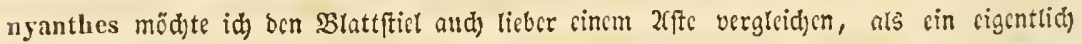
oreigäbliges Blatt annebmen, da bic Blättdjen quirlartig borizontal ftebcn (roic bei Mar-

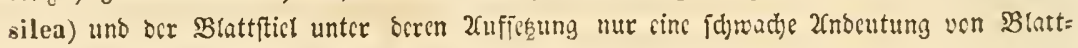

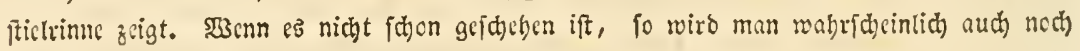

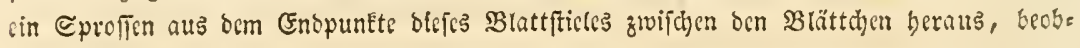
adtcn Eöntrn.

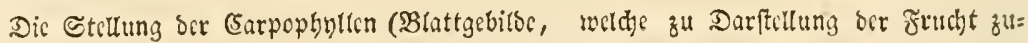
iummentretcn) ift typifd widjtig, aber fie wirb als eine einfeitige $\mathfrak{B}$ ezichung auf sas Formelte inmer unzureidend blciben, wenn es getten joll, aus ith sin Pflanzeninftem zu crbaucn. Ein foldes, auf bicfe \$afï erbaut, würbe erftens ungemcin verwickelt auš

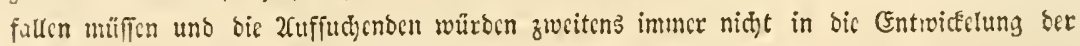

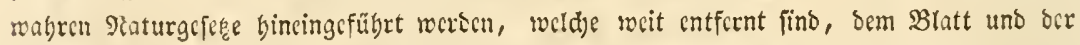

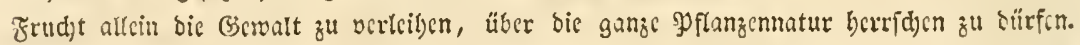

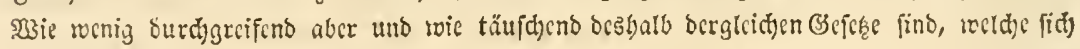

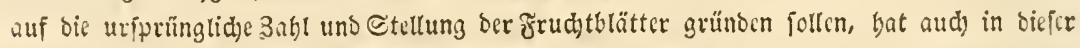
Familie ber treffich bcobad)tende Grisebach, ganz unferer cigenen Erfabrung entfprechend uno gleidylauteno in feiner Dissert. inauguralis: observationes qunedam de Gentianearum familiae characteribus pag. 27, beuttich gemad)t. - Derferbe wiberiegt Lindley's

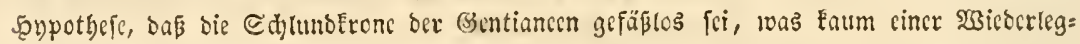
ung beburfte. (Eben fo soenig möge man aber aud) verEennen, baß́ bies nette Eorollenge: bitbe bei ben 2fpocnncen cbenfo befichaffen, in ber neucn Bcocutung since corolla pleiopetala immer Elarer als Etipulargebiloc für bic Etaubgefäße beraustritt uno bercn Künf=

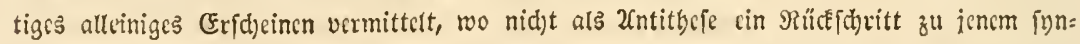
petalen 3uffanbe ober zu Bitbung von \$petalen, weldje unmittelbar binter ocn ๔taubfäben

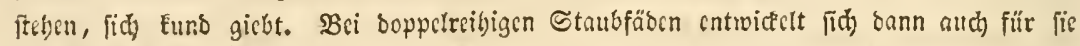

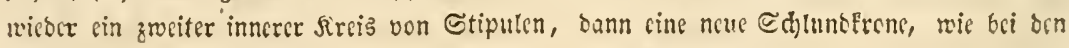
Eitenen, bier im analogen Farl fidon in ber (Sruppe ocr Capoticn. 
96. Fumilic. Envutacern: Sapotaceac.

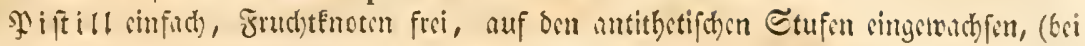

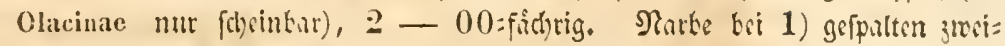

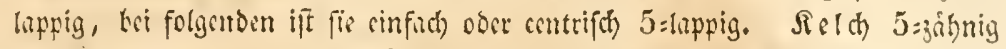

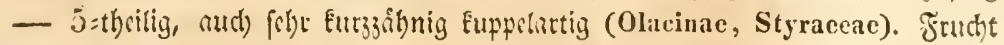

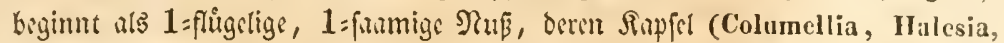
Brexia), Berte (Jasminum), bei den allermeiten eine Steinfrucht, 1-00=

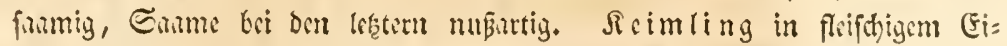

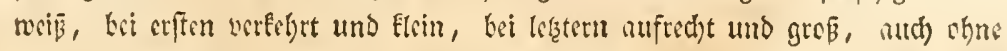

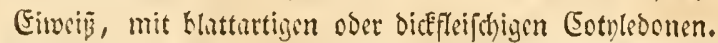

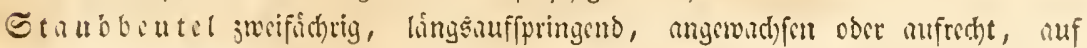

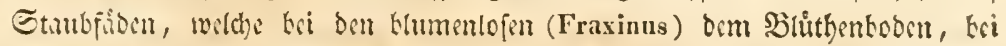

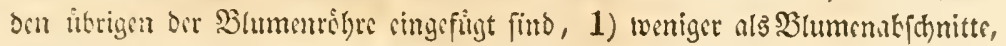
nimlich) mur 2 bsi Jasmineac, 2) glcidjoiblig, namlid) $4-5-6$ un

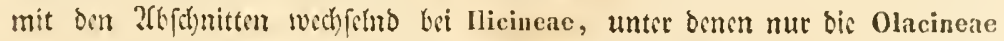

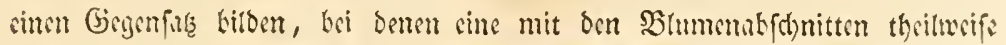

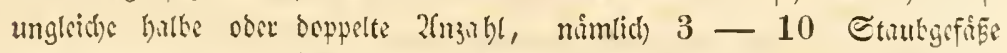

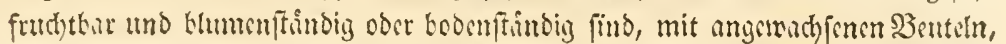

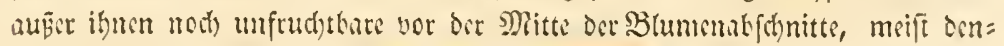

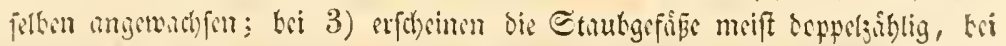

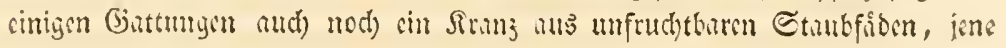

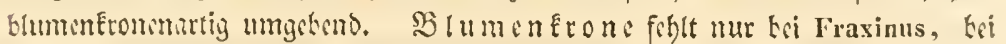
folgenosn trichterfirmig ober prifentirtellerförmig mit 4-5-6-8palti= gam Summ, odir (wie fchon kci Ornus) febr tief, wie mabrklitterig, jerthsilt,

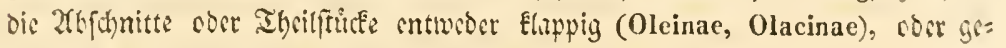
rollt (Jasmineae), auf = obsr umgeligt (bei allon übrigen).

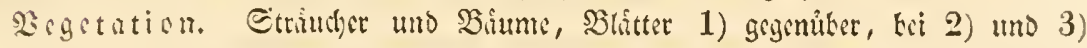

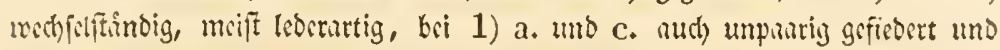
fiederfpaltig, (Fraxinus, Jasminum, Leea), Esi ůbrigen ganj, ganjrandig ofer

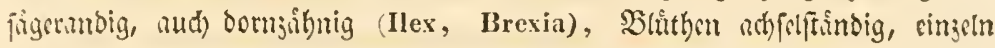
(Olacinac), ober maif in Bufdyeln, Irnuben, Dolden, Jitipen, Eci cinigen

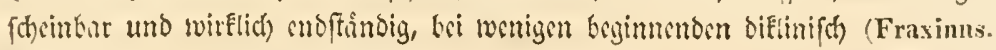
Binspyrns), meilt jtwitterlid).

(Gruppen: 1) Jasmineac: hemistemoneae i. e. diaudrae, oppositifoliae.

a) Olein a e: cor. ralrata, germen liberum (biloculare, seminia pendula, albumen carnosum, embryo brevior cotyle. donilous subfoliaceis). Fraxinus $L_{\text {, }}$ Ornus $P$. Chionanthns L. Linocicra Sin. Notelaea Fent. Plillyrea T. $L_{\text {s }}$ Olea T. 2. Xoronlia Sladm. et A. P. Th. Mayepea Aubl. (Cerantluts Schreb.)

b) Columelle a e: cor, rotata, germen innatum, capsulabiralvis marginibus inflexis polysperma. - Columellia $R \approx$. Pav,

c) I o gorina : cor. contorta, germen liberum placenta centrali. Syrimga $L$. (Tilac $T$.). Lienstrum $I$. Forscthia 
Iahl. Mogorium Juss. Menodora Hb, Bpl, Jasminum T. $L$, Nyctanthes $L$. (Parilium Gärtn. Scabrita $\dot{V}_{a h l}$ )

2) Hicinae: isostemoneae i. e. 4-5-6-andrae, alternifoliac. (Opponuntur Olacinae staminum numero incongruente.)

a) Aquifoliaceae: cor. imbricata, pulvinar nullum, germen liberum 2-6-loculare, (Seminia definita, pendula, allumen magnum carnosuru, embryo parvus bilobus radicnla supera). - Cf. Conspect. 3620 - 3628.

b) Ol acin a e: cor, valvata anisostemonea. (Germen liberum tristigmaticum, calyci cupulato demum aucto immersum, 1-loculare, seminia subterna e plac. centrali pendula, drupa 1-sperma, scmen pendulum, alb, magnum carnosum, embryo parrus non evolutus). Conf. Conspect. 3610-3617 et 3618?isostemonea glomeriflora: Barreria Scop. (Poraqueiba $\mathbf{A u b l}$.)

c) Brexieae: cor. imbricata, pulvinar hypogynum annulatum dentatum staminiferum. (Germ. lib. 5-loculare, loculis e placenta centrali biseriali-polyspermis. Radicula supera, albumen nullum!) - Brexia Noronh. (Venana Lam.) lioussea $\mathrm{Sm}$ ? -

3) Sa pote a e: (iso-et) diplostemoneae, stamina corollae alternantia et altera serie anteposita, plurima genera tandem exalbuminosa, embryo magnus evolutus.

a) A e g i cereac: isostem, 5-andro-monadelphae, polline acervulato. (Cal. contortus, fruct. follicularis 1-spcrmus, semen in funiculo longissimo calyptrato - ex Konig Annals of Bot. - crectus, cotyledones radicula maxima duodecies breviores, albumen nullun! Aegiceras $L$.

b) Styraceac: diplostemoneae, germen innatum (pluriloculare, seminia pendula et alia erecta).

Conspect. $3590-3594-3618$ et 3619.

c) Sapoteac genuina e: iso-iliplo-et triplo-stemoneae, germen liberum pluriloculare, scmina definita erecta, plurimis pyrenacea exalbuminosa. (Opponuntur Diospyreae stauinibus corollae lacinits antepositis aequinumeris, subdiclines alluminosac seminibus pendulis, ligro turissimo).

a) Chrysoplyylleac: 4-5-andrae, stamina corollae laciniis anteposita. Samara $L$. Buruelia Sw. Ilunteria Roxb. Sideroxylon $L$. Sersalisia R.Br, Chrysophyllum L. Manolilla Juss. Lucuma Juss,

3) Diospreac (Ebenaceac): 5-8-10-12-16-andrae subpolygaunae, staminibus uniserialibus. Pentandrae (sem. crecta Giürtno): Leen $L_{*}$ Lasianthera P. B. - octandrae: Diospyros $L$. Cargillia $R$. Br. decandrae: Royena $L$. 
Turaria Mol, - 12-16-andrac: Paralea Aubl. Visnea L. (Mocancra Juss.) Embryopteris Gürtn. (Cavanilla Lam.)

જ) Miunsopeac: 6-8-10-00-andrac, staminibus bi-triscrialibus - 6-8-audrac: Achras L. Imbricaria Commers. Mimusops L. Bincetaria Forsli. - decandrac: Inocarpus Forst, - dodccandrac: Bassia Kön. - polyadelphate et polyandrac: Symplocos L. Onphalocarpus P. $B$.

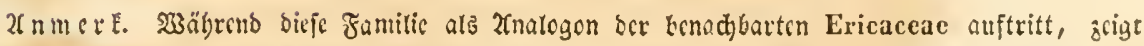

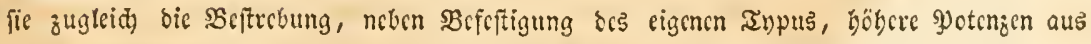

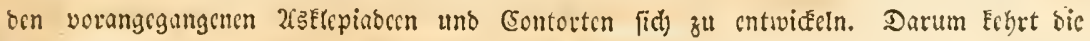
corolla valvata, sic corolla contorta zurück, barum wits bict cin polfen iccrvulatum in ber Aegiceras mögtich, währeno ocren fonocrbare bornförmige Frudt, wethe ifr

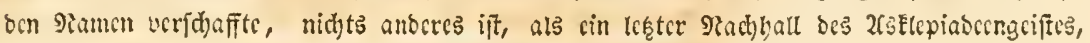

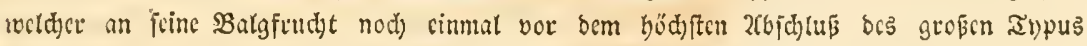

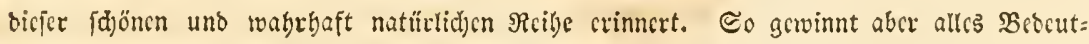

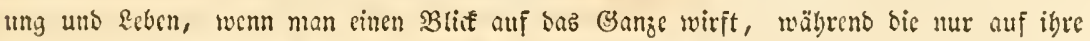

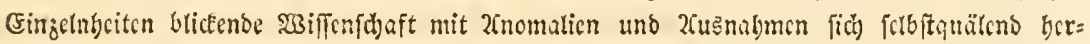

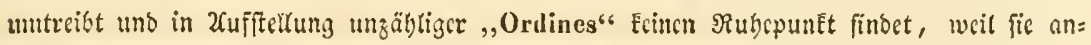

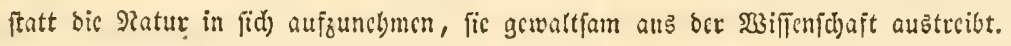

Die srittc Brufpe zcigt bcuttid), wic fic übcrbaut allç vicberbolend verfögnt und

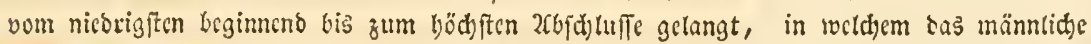

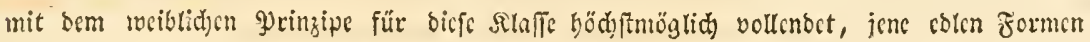

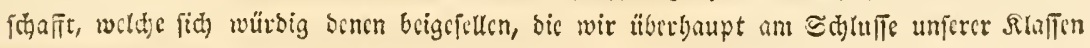
glt tre Tin gervoknt find.

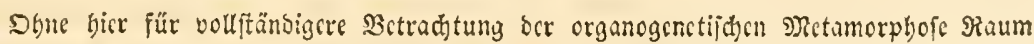

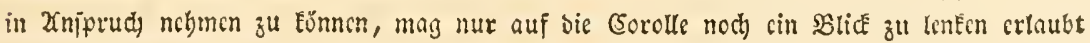

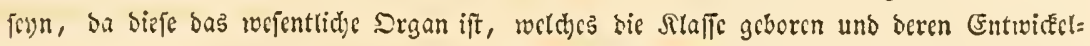

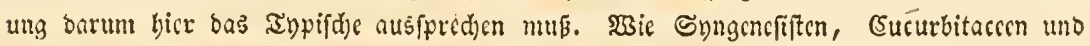

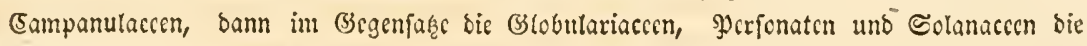

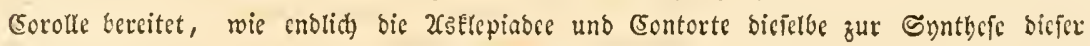
Sapotaceen gciührt, bics liegt vor, indellen wollen wir ncbenbei bejortbers beadten, ixie

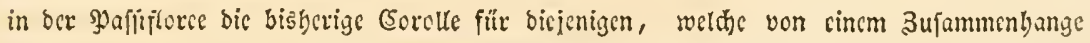
ber Ratur nidjts wifien wollen, (vergl. S. 15), aud) bie Csorolle ber Syaffiflorcen ver: licren, wie rwir fdjon wiा̄en. - Die rigentbümlicten Borbitber sines zwciten innern

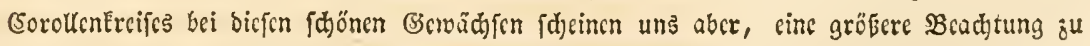
verbienen ats ibnen gewëbnlid ąu I

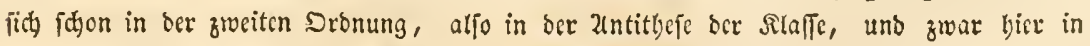

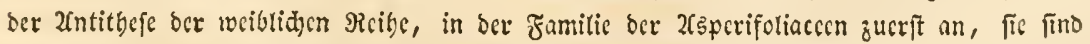

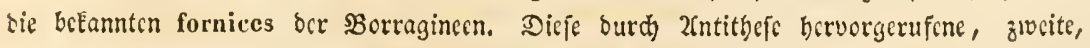

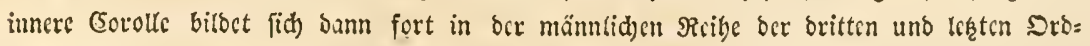

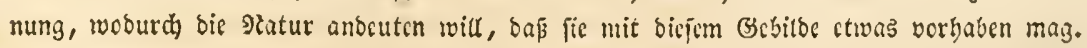
(Fs fdjeint nämlid) - id will nidjts behaupten - als ob bic fynpctale (5oralle bcr jeţ=

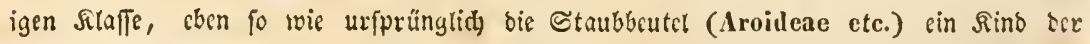

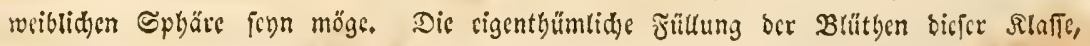


Banjelumige.

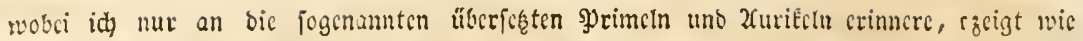

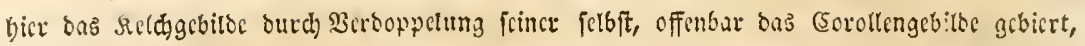

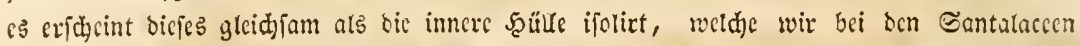

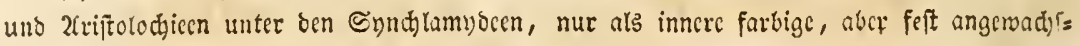

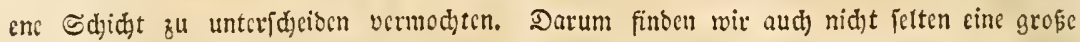

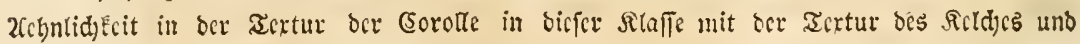

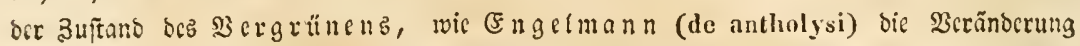

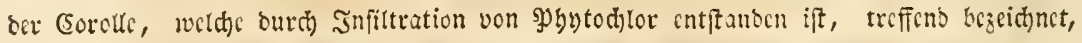

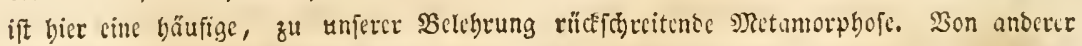

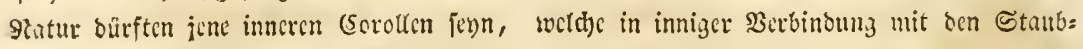

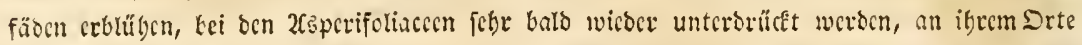

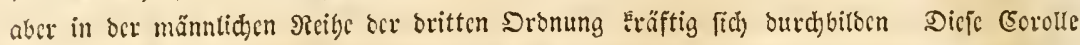
ift vom urfprunge aut, cine jencr entgegengefegefe gu nennen, fie ift dus nidht männlich ge=

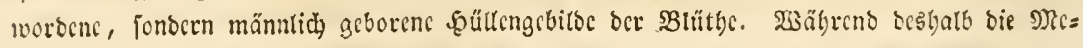
tamerphofe jencr (Sorolle nadi oem Centro binftrebte und aisz fich bic Staubfäben Bebar,

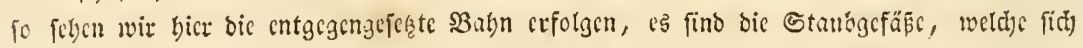
biffe innercn Sorollen gebärcn uno bicje in Irenunang foglcich mebrblätterig beginnende

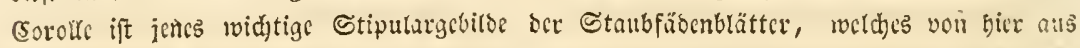
ourd bie gumze Segetation uns begleitct, ce ift bic andropetale 50 rolfe, weldhe ime

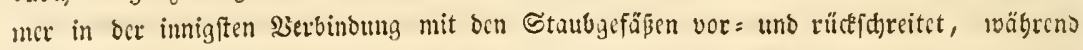
jenc gy nopetalen (5 orolfen nur bier uno ba wieber bommond (Ranunculaceae) für bic albropetalen eintecten Eönnen. So geht alfersings ebenfo, wic bic 92atur bic Raupen= baut abfrtecifin läpt, um ibr Inncres cruvecten zu föıncn, bie alte Corcils unter, um cine

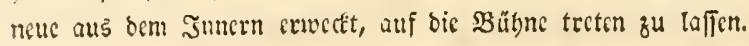


Rald)blitifige.

Caljcantlac. Familion.

\section{Eicbcute EIafic. seldbblítgige: Calycanthae.}

Erifte Sirnung.

\section{2eridisicsenblüthige: Variflorae.}

Srcinblüthige: Parriflorac.

97. Fam. Doldengeduid) [e: Unbelliferac.

95. Fam. Fintujoont Rhamneae.

99. F̊m. Trebintfacen: Tcrebintlnaceae. 102. Fam. Mimofacen: Mimositceac.

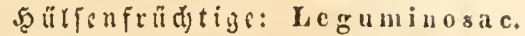
100. Fam. Echmetterlingsblumige: I'apilionaceac.

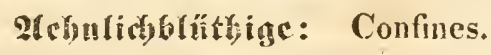

Ecoumblüthige: Selliforac.

103. Fam. Geborntfrichtigr: Coruiculatae.

104. Fam, Loaficren: Lonsacene.

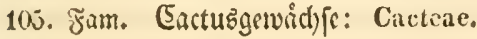

101. Fam. Enfliucen: Cabsiaceac.

Rofcnolumige: Rosiflorac. 106. Fam. Fortulaknorn: Portulicaccac.

107. Zam. 2lizoidien: Aizoitleae.

108. Fam. Siofuccen: Rosaccae.

Dritte Sronung.

\section{Gleidởumigublutbige: Concinnae.}

Staćlectzenotüthige: Onagriflorae. 109. Fam. Şalloragent Ilalorageae.

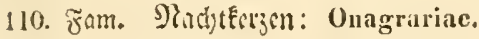

111. Fan. 23sibridye: Ly thrariae.
Mntcublüthige: Mŕrtiflorac

112. Fam. গ̧olıgaluceen: Polygalaceae.

113. Fam. Myrticern: Myrtacene.

11. Fam. 2lmigoalncent: Amygdalacear.

97. Fumilic. Dolsentotuädyfe: Umbelliferac.

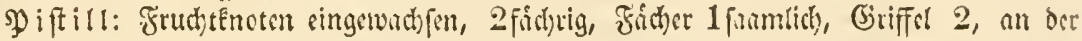

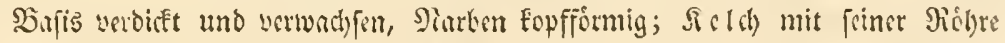

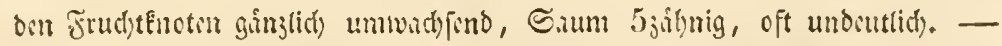

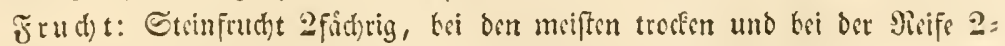

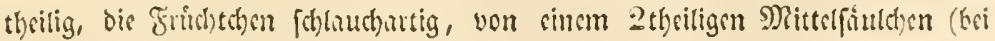

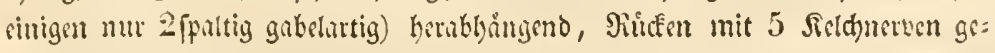
rippt, (Miippen, costae), Esi andern treten noch) Bwifd)entippen (costae se-

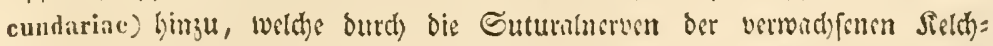

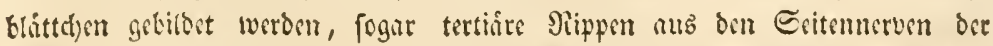

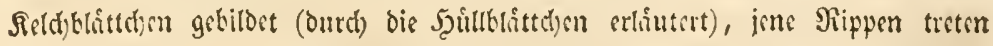
mebr ober weniger berwor, bei sinigen flitgelartig, Eummurtig, igelftud)clig, oudh

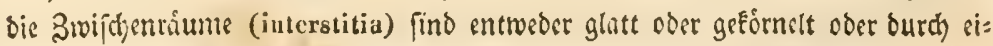




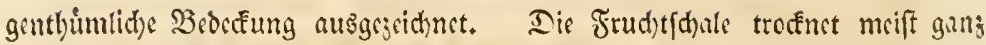
aus, bsi wenigen (3. B. Suyrnium) foftig, sine becrenartige Etrinfruct ; bis

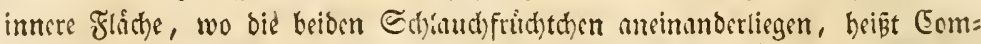

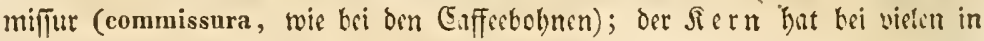

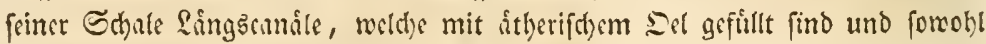
auf dem Silcken als nod) efferer auf ber (5ommiffur (j. B. Herarleum) beut=

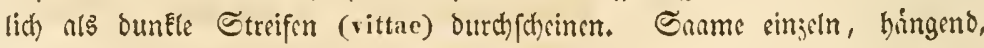

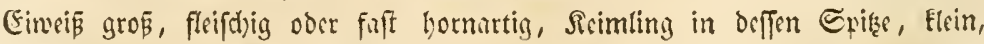
Wirizetden nach oben, Eotuledonen linglid).

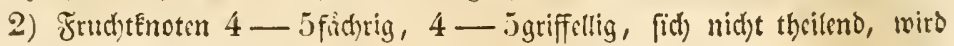
meiff fiftige Strinfrudt mit $4-5$ Gtrinfernen.

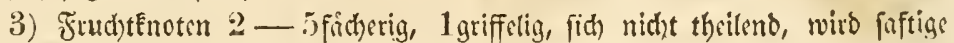
Strinfrud)t mit Stcinfermen, ober enolid) freie, 2fâtbrige Becte (Vitis) mit 2 oufrechten Ganmen in jebem Fndh.

Eta bbeutel 5, foft berzformig aufred)t coer auflicgend, zitterno, lóngz muf= fpringeno, nuf frein Etrubfíoen, welde unterbub des Drufinfolfterż einge: filgt find, vor bem 2tufblithen einmérts getogen. Blumentlátter 5, mit ben

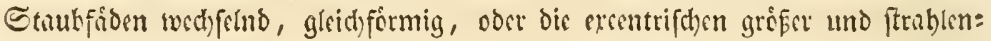
artig verliongert, meift ausgeferbt, mit liber bie Rerbe bincingetegener růf =

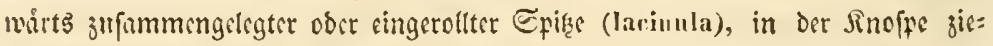
gelartig, fo wie bie Etaubfidon singsbegen, bei andern ganjtandig und weni= get eingeboger. Sasi Cissus 4 , bei Adoxa $8-10$, bei lledera 5

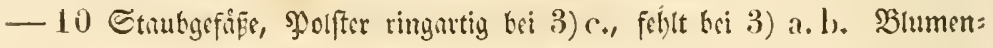
blitter bei 3) fort fluppig, bei Sciadoplyyllum und Vitis oben zufammen= bảngend, mingenartig itd) ablifend.

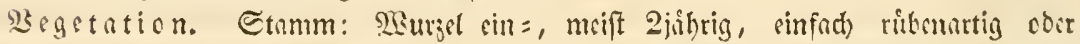

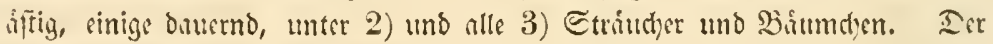
Eraturtige Etangel róbtig, Enotig, mit Echcidrwinoen, leer ober matfig; Blitter zerftreut mit fheibigen Blattfielen, meift zufummengefsht, hei sinigen

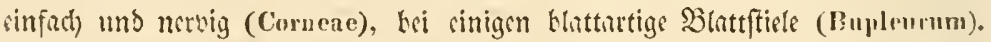
Bliutbem|trand sine endftandige, zufammentgeferte Dolbe, bei sinigen ad) fel = oder

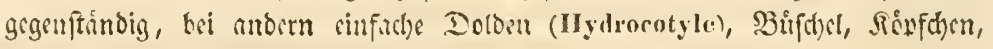

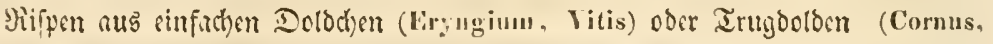

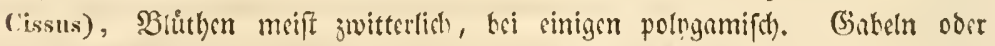

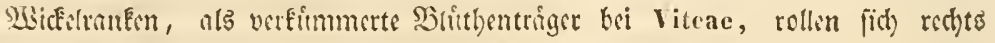

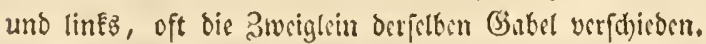

(3)tuppen: 1) Umbelliferae genuin a : digynae seluzocarpicae(Lagoecia semidigyna exordium sistit umbelliferarum).

a) Coriandreae: indehiscentes, mungyna et digynae albuune incurvato-gilibo, antice excavato-inflexo. Lagocria L. - Coriandrum L. Alrenu $D_{C} C$. Astomaea $R\left(h_{b}{ }^{*}\right)$ Hifora $H o g m$.

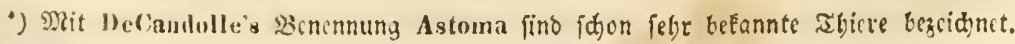


b) Solenospermae: dehiscentes bipartibiles albunine involuto, versus commissuram longitudinaliter sulcalo.")

a) Scandicine a e: paucicostatae elongatac.

Scaudix L. (Scand, et Wylia Hoffm.) Muthriscus $P$. Chaterophyllum L. (Cacusciatlium et Clacroph.) BiasoIcttia Koch. Caldasia LaG. Sphallerocarpus Bess. Mo. lopospermum hoih. Velatea DeC. Myrrhis Scop. Osiuorrliza Rufin. Grammosciallium $D_{e} C$.

B) S m yruie a e: pancicostatae turgidie.

Olivieria Tenl. Auisosciadium DeC. Echinophora T.L. Evoacantlit Lab. Arctopus $L$. Cachrys $T, L$, Prangos Lindl. Colladonia DeC. Lecokia DeC. Matgydaris hock. Ifermas $L$. Conium $L$, Vicatia $D e C$. Arracacha Bancr. Plenrospermum Iloffn. Grafia Rchb. (Hadnilia $K$, non 1h. - G. Golika [Athan.] Hacq.). Hymenolieni De C. I'lyssospermum Cusș. Smyrnium L. Perideridia Rchb***) Scilligeria DeC.

r) Caucalinae: multicostatae costis armatis ant alatis.

Caucalis L. Turgenia Hoffm. 'Torilis Aduns. Spr. Flacoselinum Koch.

c) l'l is y permate: dichiscutes bipartibiles, albumis coumisura plani,

x) unbellato-nubellulatae paucicostatae.

au, I nuincac, contractae et teretiusculae (apterae et iumarginatile).

Ammincite gonninae s. contractae: Trinia Iloffu. Helusciadiun hock. (Mauchartia Neck。? et Cyclospermum LaG. Trachysciadiam $D_{e} C$.) Discopleura $D_{c} C$. Leptecaulis Null. Piychotis K. (Ptychot, et Trachypleurum $L k$, Meteroptycha $D e C$.) Falcaria Riv. Haladnihia lichb. Sison LaG. Schultzia Spr. Ammi $L$. Aegopodium L. Carmo L. Bunium L, (Chryscuu, Caroides DeC: et Conopodium K.) Cuninum C. Bunh,L. Trepocarpus Nutt, Cryptotaenia DeC. Pimpinella $L$. (Anisum Al. Tragium $S p$ r. Tragoselium T.) Siun $L$. (Sisirum Riv. et Berula h.) Rumia $H_{0}$ ffm. Cicula $L$. Lizia $K$. Pentacrypta Lehn, Apium T.L. P'ctruselinum

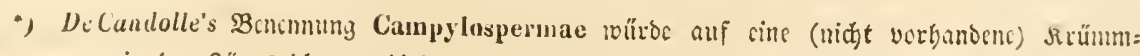

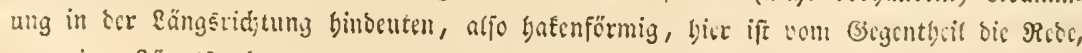
von cincr \&ängs̆furdje.

*) Der Siume Eulophus DeC. (Nutt.) gegörte lange vor 1825 Ectunnten Jufefton, auf waldje

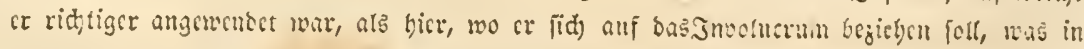

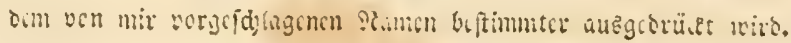


IIoffm. Wydleria DeC. - Trachyplenrum Rchb. Bupleurum L. Heteromorplia Cham. Schlecht. $-\Lambda$ mmineae Seselincac s. teretiusculae: Crithum T.L. Conioselinum Fisch. Pachypleurum Led. (Gaya-Gaul.) Meum T:L. Wallrothia spr. Silans Bess. Ligusticum T. L. Athamanta $L$, Trochiseanthes $K$. Thapsium Nutt. Cnidium Cuss, Coenolophinm $K$. Libanotis Criz. (Eriotis $D_{e} C$. ct Lib.) Soranthus Letl. Seseli $L$. Dererra DeC. Kinndmannia Scop. Foeniculnm Altens. Acthusit L. Cynosciadium DeC, Dasyloma DeC. Sclerosciadium $K$, Anesorrhiza Chan. Schlecht. Phellandrium 'T.L. Oenanthe L. Ottoa K.I.B. Lichtensteinia Cham. schlecht.

$\beta \beta)$ P'eucedanea es, alatae: genninae s. lipterae:

Iferacleum L. (Weudtia Hoffm. Sphondylium IIoffm. Trichogouinm DeC. Carnelia DeC. Heracleum HIoffm. 'Tetrataenium $D_{c} C_{\text {. }}$ ) Zosimia Hoffm. Polytaenia DeC. Johrenia DeC. Astydamia DeC. Pastinaca T.L. Opopanax K. Archemora DeC. 'Tiedemannia DeC. Capnophlyllum Gärt. Cortia DeC. Anethum T.L. Palim-

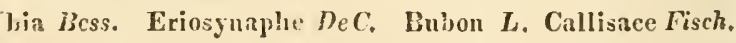
Impcratoria L. Cervaria Riv. Oreoselinm Clus. T,M. R. Pterosclinum Rckb, Thysselinum Docl. Riv. Spr. Pencedanum L, Ferula T.L. (Fernla et Ferulago $\boldsymbol{K}_{\text {.) }}$ ) Dorema Dou. - Angeliceac s. tetrapterae: Levisticun J. Bauh. et $K$. Selinum $L$. Ostericum Hoffim. Angelica I. Arehangelica Hoffm.

yr) Tordylineac: annulatae s. toroso-marginatac.

Tordyliun 'T, (Tord, et Contylocarpus Hoffm.) Irasselquistia $\boldsymbol{L}$.

B) umbellato-umbellulatae multicostatae.

$\alpha \approx)$ Silerineac: leuticulares s. deplanae.

Siler Scop. Lirubera Hoffim. (Ulospermum Lk.) Agaș̣llis Spr. Galbamu Dou.

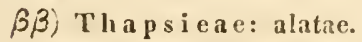

Laserpitium T: L. Cymopleris Rafin. Thapsia T. Z. Lophosciadium $D_{C} C$. Melanoselinum $I / 0 f m$.

yy) Daucineae: armatae.

Baueus I. (Carota, Auisaetis DeC, Platyspermum IInffin.) Orlaya $I f o f m$. Artedia $L$.

\%) imperfecte rel irregnlaritr umbellatac.

as) II y d o o o t y le a e: complanatac.

Hydrocotyle $T . L$. Crantzia Nult. Dimetopia $D_{\mathcal{C} C}$. 
Erigenia Nutt. Micropleura LaG. Trachymene (T. incisa Rdg.typus!) Rdg. Hïgelia Rchb. (Dilliscus $D_{C} C_{\text {. }}$ ) Catepha Leschen. Astrotrichia DeC. Xanthosiia Rudg. (Leucolacna R.Br. Cruciclla $L$ cseh.) Bowlesia Rz.Pav. Fragosa Rz.Pav. Azorclla Lam. Plectophytum K.H.B.

$\beta \beta)$ II u line ac: parallele biscutatac i. e. deplanatac.

Bolax Commers, Mulinum $P$. Drusa $D e C$. Inanaca Cav. Diposis DcC. Spananthe Jcq. Yozoa LuCi. Asteriscium Cham. Schlecht.

ry) Saniculeac: turgidae suboratac.

Sanieula T.L. (San, et Sanicoria DcC. itlem, cf, Flora germ.) Petagnia Guss, Macquetia Nock. (Donilia Spr.) Hohenackeria Fisch. et C. A. M. Astrantia T. L. Actinotus Lab, Alepidea La Roch. Horsfieldia Bl, (Schuhertia $B l$.) Eryngium J.L.

2) Araliacea : 4-5-gynae holocarpicac.

Adoxa L. (Mochatellina T.) - Panax L. Cussonia Thub. Maralia A.P. Th. Gilibertia Rz. Pav. Gastonia Commers. Toricellia DeC. Paratropia Blume, Aralia L, Sciadophylhum P. Browne.

3) Cisseae : monogynae sublibere holocarpicae.

a) Hederace ac: pyrenac 5-10-1, scuine 1. pendulo.

IIcdera L. Arthrophyllum $\mathrm{Ll}$.

b) Corneae: pyrena disperma, semina pendula.

Cornus T.L. Aucuba Thb. (Eubasis Salisb.) Votowitn Aubl. (Glossoma Schrcb. Guilleminia Neck.) Mastixia Bl. Polyosma Bl.

c) Vitea e: semina pyrenacea in bacca demum libera subgeminata erecta.

Cissus L. Ampelopsis Michr. Vitis $L$.

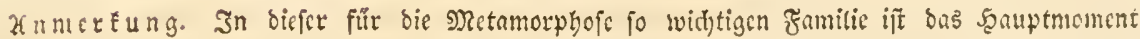

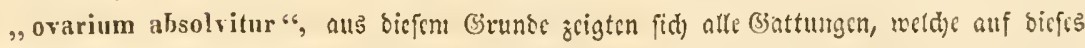
Mournt gebaut wursen, ats natürtich, bicjenigen, werchs man von antersantigen Theilen

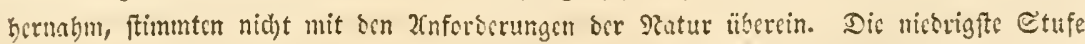

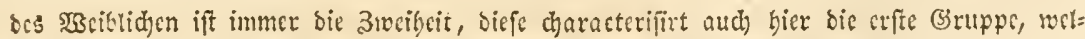

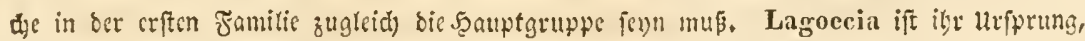

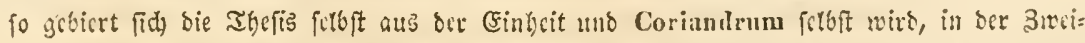

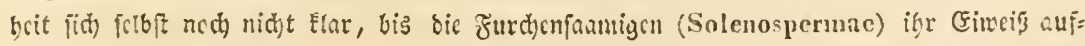

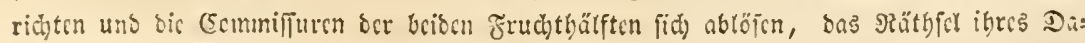
icuns crefennend. S3ie von bicraus bas 2 ent

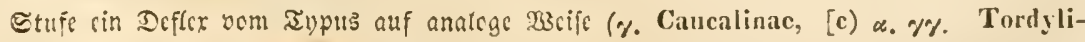
neac], $\beta . \gamma \%$. Daucineac, $\gamma \cdot \gamma \gamma$. Saniculiuac) fid) offenbart, cbenfo wie bic erften unb

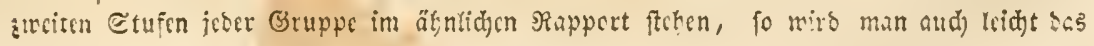


Ref(t)elüthige.

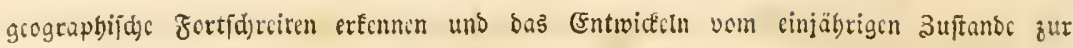
ङ taude und zum verbotgenion Strauth.

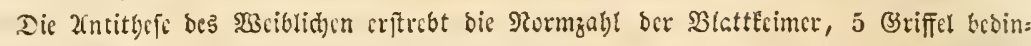

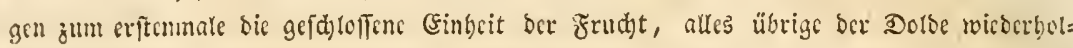

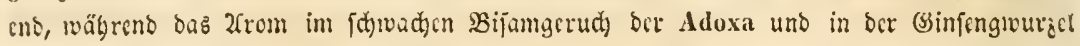
ertiji, und den Gaamen nidyt mebr inneroobnt.

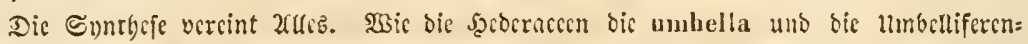

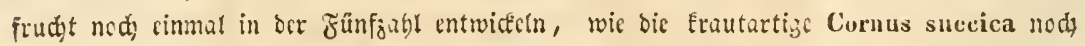

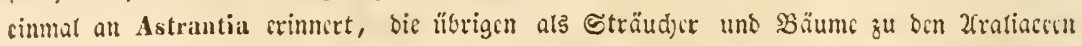

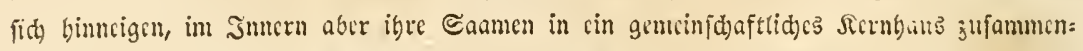
gegegen, fo entfaltet fid) Cissus, Ampelopsis und litis in tegter Erinncrung an bie

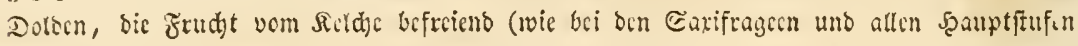

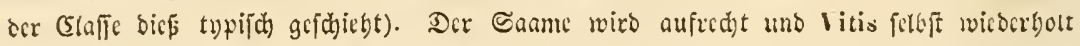

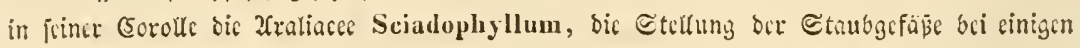

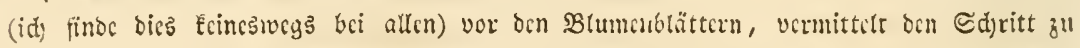

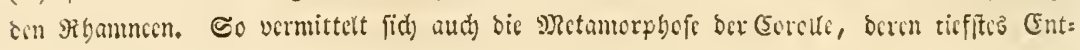
fitcher Lagoecia jeigt, bis zum Diflex in ocn forgentocn Iy)pus.

\section{Famitic. Phaumeen: Rhamneac.}

If iftill: Fruditenoten eingewadfien, butb ober gans frei, von fleifthigem Sholfter

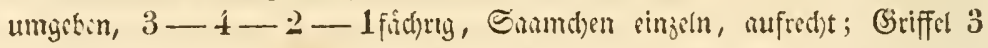

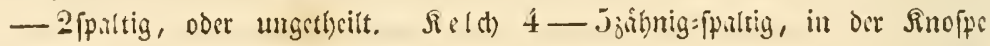

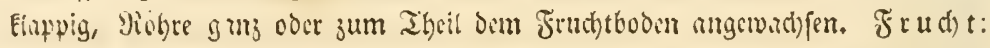

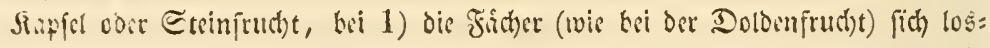

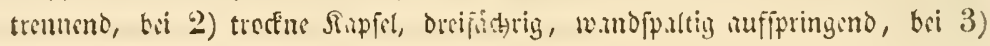

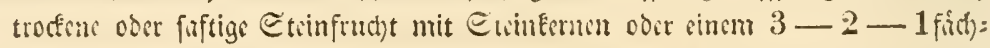

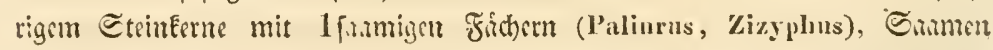

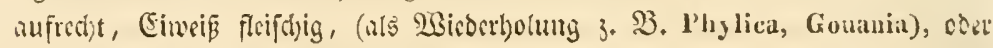
dimm, bit den vollenditeren frblino.

Etaubbaute1 5, bei rocnigen 4, aufredbt ober nufliegend, anf Stmbfidoen, weld

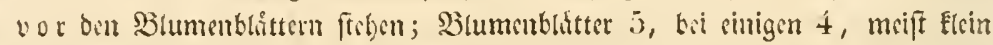

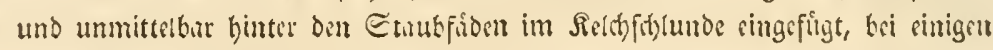
E.lppenformig.

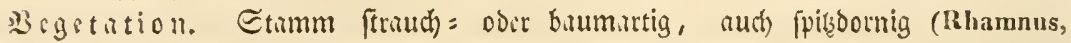

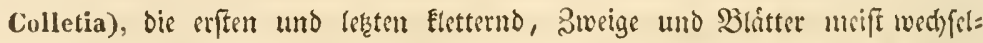
ftiurbig, ungetbeilt, bei vielen fígernnbig, bei sinigen gegeniber, mcift mit

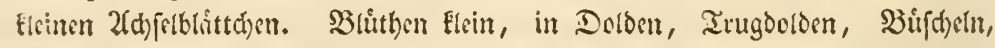
Rutueln, bei sinigen Iruben, meift zwitterlich), bei sinigen zmeibinufig (z. $\mathfrak{B}$. R:hiunus).

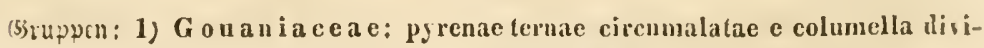

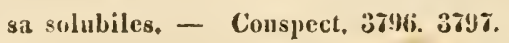

2) Ceanothea e: capsula trilocularis septicida. - Conspectus $3854 ?-3809$. 
3) Frangulaceac: drupa sicca aut succuleata, senina suberalhuminosia. - Conspect. $3310-3822$ - Icerija Rxb. Parilia Dennst.

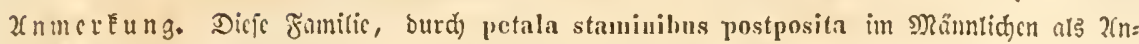

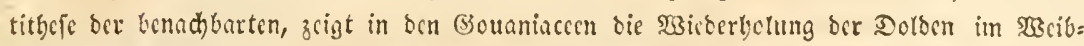

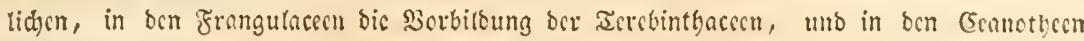
ibren sigenen Typus. Dap unter ben Frangulacen, recldje bic Gynthefis birben, Palinrus bic Bounniacsen, bie mit breifäd)crigem Stein verfebenen Battungen bie Eeanothen wicberholen, bie tibrigen, im Inpus dor Eynthcie verbarteno, auf bic ahbrebinthinaceen binbcuten, ift von felbft klar.

99. Familic. Terebiutbacen: Terebinthaceac.

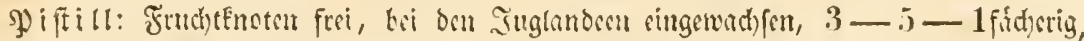

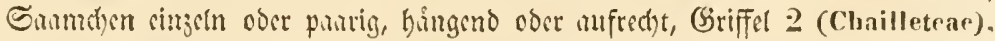
5 (Conuareae und Spondieac), oder einfad) mit $2-3-5$ Sharbin, hei

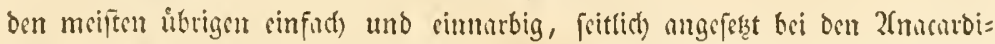

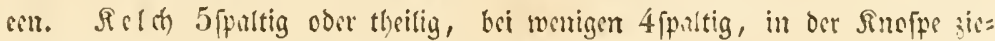

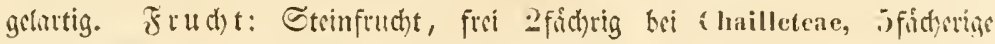
Sappel, imen mafpringend bsi ben Eonnuren, Etcimpucht mit 5-32-1 Strinfern, ba ben Eponbircen cin Strinfern mit 5-2 Fichern, kpi den Sughuorn sine untere Etrinfrud)t mit 2Elappigan Etriukerne. Sanme hingend, bei anderm anfrecht, mit fleifdrigem Eimcis (Ehailletern, jum

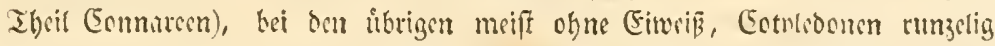

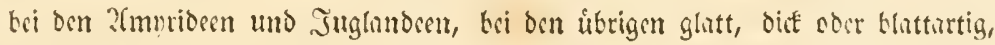

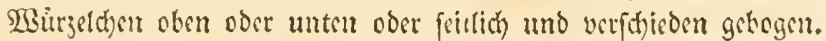

Staubbeutel 5, bei den 2fmyriocen 8. bei andern 10 (Comnareae, quaediun Imyrideae, Spondiaceae, Anararlieac), for bon Juglunbecn unbeptinmt zifhlig $3-36$ und hupognnif(t), bi Sorimlein A. P. Th. Fommen 16-28 vor,

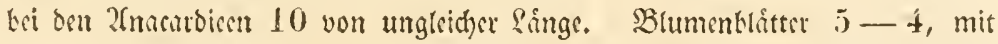

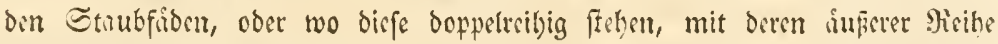

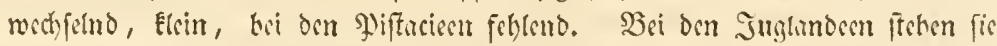
unmittelbar vor oen Seldybfdynitten in oer weiblichen Bltithe uno offentar

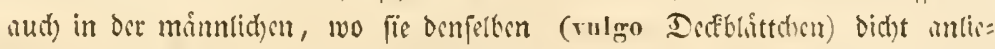

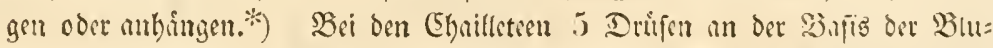

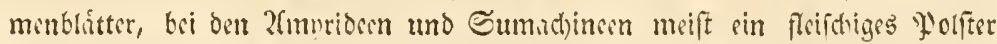
um den Frud)tfnoten und ju Befeftigung ber Etuubfiódn uno Silumentlitter, bei ben Juglandeen und Connarem fiklt es.

Begetation. Stráuher uno Bitume, Berzmeigung uno Belutbung ierftrem,

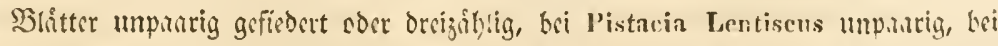
Chailletia, Duraun, Anacardieac ungetbcilt, Eraututig oocr nteift leocrittig

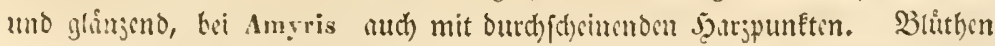
Elein nur bei wenigen zwitterlich), meift biflinifd), adjel ober enditinoig, in

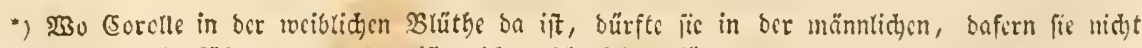

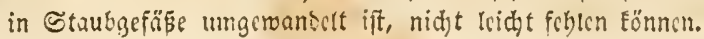


Seldolithige.

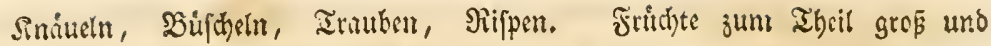
jurtig.

(3ruppen: 1) Chailleteae: 2-listyles drupa biloculari sublibera, seminibus solitariis pendnlis carnoso-albuminosis, Stam.5. pet. 5 . excisa. (Flos umbelliferarmm repetitus, cyma e petiolo. Conspect. $3823-3825$.

2) Connareac: 5-styles (stigm, capitita), caps. 5-locularis intus dehisecns, scmina geminata ereeta. Stan. 10, pet. J. integerrima. Pulvinar 0. Albunen carnosum ant 0 . embryo inversus! (Repetit, Aral, et Ceanoth.) Conspectus 3826 3829 .

3) Terebinthine ae: 1 styles (stigma lacero-divisum, $2-3-$ 5-lobum) drupa libera, in antithesi innata, 1-locularis 1-sperma aut $3-5$ pyrena aut pyrena 5-2 locularis.

a) Amyrideac: germen liberim $3-5-2$ Joculare (tot stiguata), drupa $8 \mathbf{- 5 - 1 - p y r e n a , ~ q u i b u s l i m ~ c o r t i c e ~ v a l - ~}$ rato, albumen 0. cotyled. contortuplicatae, aliis (ut is Schwägrichenia) plano-convexae, radicula supcra. Conspect. 3835-3849.

b) I u g l a d d a e: drupa 1-sperma infera, cotyled. corrugatie maximae, ratlicula supera, plumula pinnata. Conspectus $3831-3834$ ?

c) S u m a china e: drupa 1-sperma (in antithesi pyrena $5-2$ locul.) libera, cotyledones plano-conrexae. (Sem. e funiculo adsceulente crectum ant pcululum, albumen 0.)

a) Pistacieac: 1-spermac isostemoneae, cotyl. foliacéce, radicula in commissuram inflexa aut obliqua. Conspect. $3850-3858$. $3856-3860$.

B) Spond ic a e: 1-pyrenae, pyrena J-2-loenlaris, sem. 80Iitaria penlula, diplostemoneac.

Conspect. $385 t-3855$.

y) An acarli i a e: 1-spermae, cotyled, super radiculaun replicatac. Integrifoliae!

Conspect, $3861-3868$.

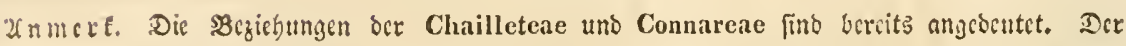

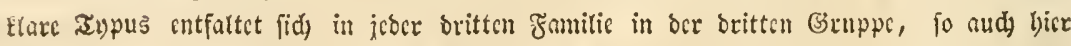
bie veiden Formen ocr Balfambäume in ben Amyrileae bon fï̈d)rigen 3uftumb ber vor=

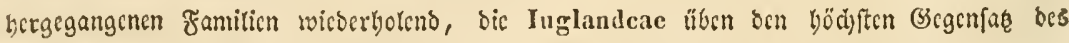

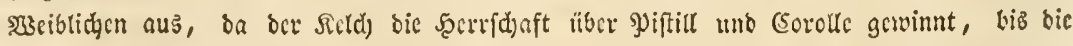
Sumachinae alles löfen umb fürocrn. Dic nod) ofne Corolle beginnenden Pistacieae

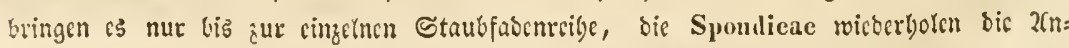

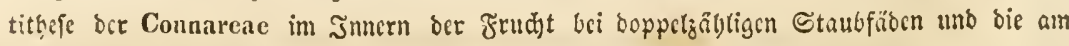

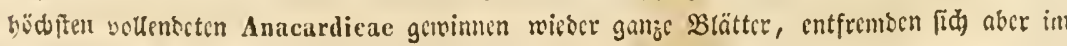




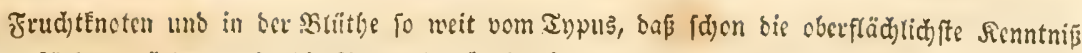

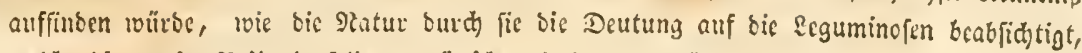

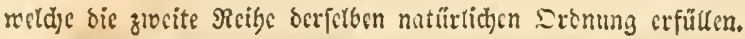

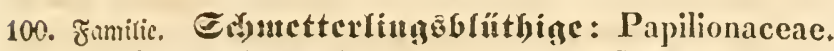

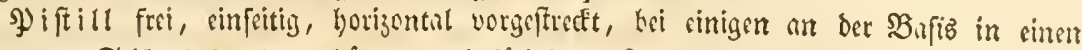
Etift (stipes) verbinnt, cinfít)rig, 2 Piftille bet Diphaca, Wisteria, Sinmdien an ber obern Nath, biefe bei menigen (Astragaleae) eingezogen und 2 Rangsfict)er bildeno, Briffel auffteigend, Sarbe meift innenfeitig; Sield) un=

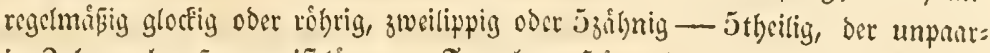

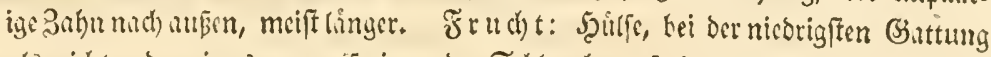

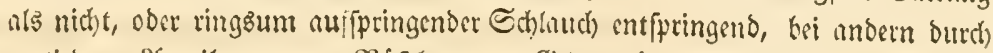

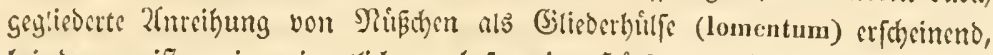
b.i ben meifen cine eigentlid)e mehrfanmige Ştilfe nus 2 Sllappen, deren

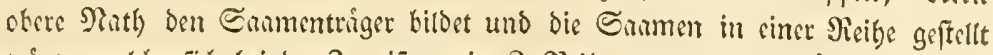

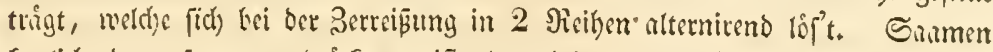
Euglide) oder jufammengrbrickt, meift glutt, bei wenigen fornig (Vicia lathyroi-

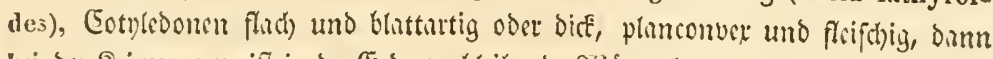

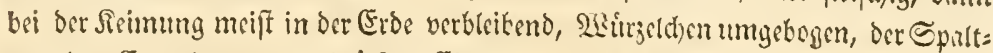

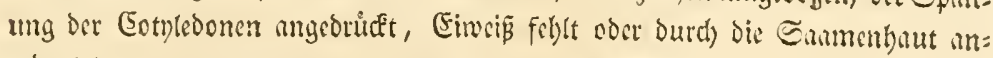
grocutet.

Staubseutel 5 und 5 , zmeifokit)rig aufrect)t und lings aufpringento, auf in

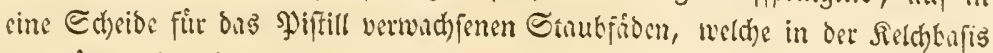

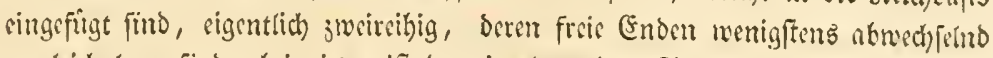

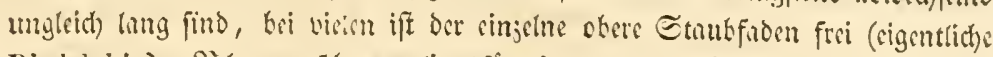

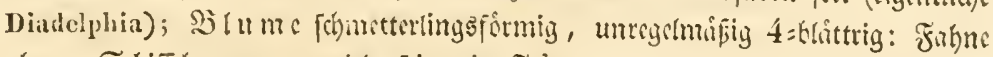

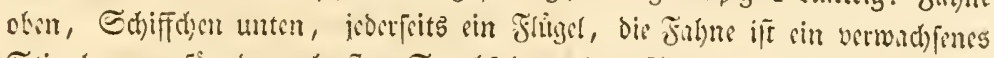
Stiputhrpant fur oen oberften Etutufinden, ons Sdifflyen ift sin verwachfo

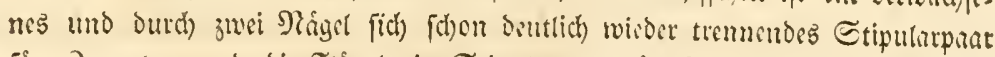

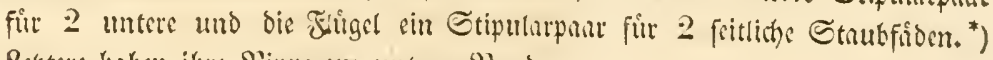
Selzerere baben ifre Sippe am untern Siande.

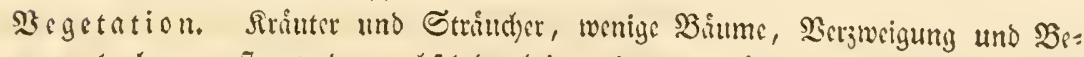
laubung jerftreut oder wech felno, bei wenigen gegenuber (Scottea, Platylohinm

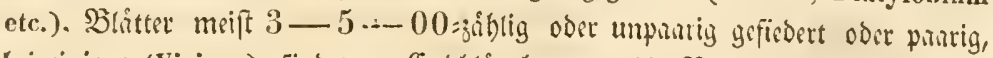
bei sinigen (Vicicae) find vom Endklittdyen nur bie Nerven vorhanden, eime Wisfeltrunfe bitbens, hei wenigen cinfadt) 2 litter, durn bei wenigen getheilte (Pothlob. staurophyll.), bei cinigen bic Ficoern boppelt und breifach (Oxytropis vertic.), bei Polytropia Prsl. aus oen 2(b)feln ber unterften Blattchen sine

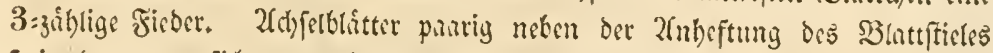
frei oder unter fich verwad)fen, oder ifm angewachfin. Slathen jwitterlid)

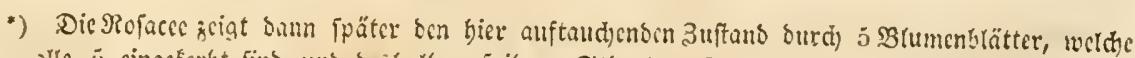

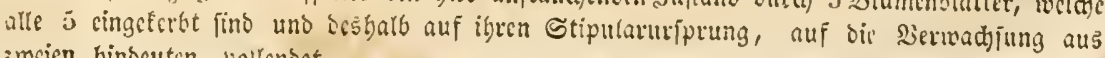
jrocien binseutin, wollenbet. 


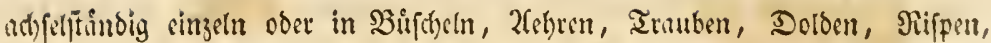
feltener endftündig, SBlittyenfticle meift mit 2 Decétlittchen.

(Bruppen: 1) Loteac: diadclplrae, utriculatae et leyruminosae.

a) Trifolieae: synpetalae et 4 petalae, ntriculus aut legumeu uniloculare, cotyledones foliaeene.

a) genuinae: cor, tubata, folia primordialia alterna similia. - Trifolii subgenera melius exposuit cl. Presl.: cor. synpetala: Calycomorphum Pr. Galearia Pr. Mistyllus $P r$. Paramesus Pr. Trifolium $L$. - rexillum liberum, alae cum earina connatac: Hicrantheum Pr. Amuria $P r$. Lupinaster $P_{r}$. -

§) Trigonelleae: petala libera: Lotophyllum Rchb. (Amarenus Pr.) - Reliqua conf. Conspect. 38(i9 - 3!18. et Bonjeania Rchb, pl. erit. ic, 1331. Fl, germ. p. 507.

y) Galegeae: pet. libera, folia primorlialia alterna ant opposita, dissimilia, altero piunato. Conspect. 3887 - 3916. Peritropia Prsl. etc.

b) Astragaleae: cotyledones foliaceae, legunen suturae impressione subbiloculare. Conspect. 3879 - 3885.

c) Fa ba cea e: cotyledones crassae.

๙) Vicieae: folia paripinnata cirrata. Conspcct. 3918 3924 .

B) Phascoleae: folia trifoliata et impari-pimnata, primorlialia opposita, legunen septulatum. Conspect, 3925 3958.

y) Glycineae: fol, primord, opposita similia, reliqua varia. Conspect. 3960 - 3986.

2) Cienisteae: mouadelphae et decandrae leguminosae.

i) Geniste a genuinae: moutelphae, cotyledones foliaceat. Conspect. 4029 - 4058 .

NB. Requienia interponenla numeris 4043 et 4044 .

h) Inthyllidea e: monatclphae, cotyledones carnosae epigeae. Authyllis $L$, l'ogonitis Rchb. Lupinus $L$.

v) Sophoreac: deeandrae, cotyledones foliacenc, Conspect. 4059 - 4091.

3) II edysareac: diadelphae nucamentaceac et lomentaceae.

ii) Coronilleae: fasciculillorae, lomentum teres ant compressum, cot, poroso. foliaceae (ut in b.) Consp. 3978-698\%.

b) Ou brycheae: spici-v, racemillorae, nucanentum indehiscens 1-spermum aut lomentun, Conspect. $3988-4010$.

c) Dalbergicae: racemiflorae, legunen 1 - 2-spermun indehiscens, cot. carnosate Conspect. 4011 - 4022.

2f nmete. "Anamorphosis e corolla coalita ad lihere papilionacean, ex ntriculo at legume'l, e nuce ad lomentum, e herlis al fintices arboresque." $\mathrm{Nl}$ germ.

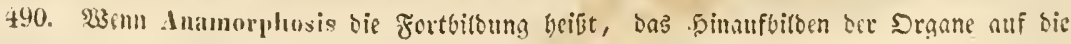




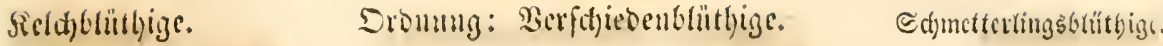

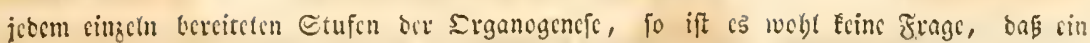

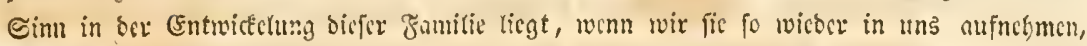

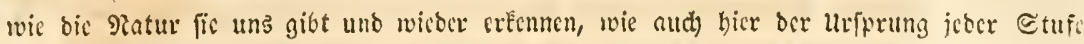
im vicrocn beginnt unb nad) bem 2(equator fortichreitens fith weiter cntfaltet. Eimiges über bas Einzcine bir 9)etamorplofi ift fajon in ocr Eintcitung reforitt worecn. Fic

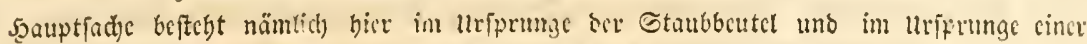

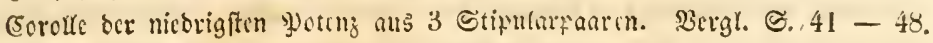

\section{Fumilie. Enimiacen: Cassiaceae.}

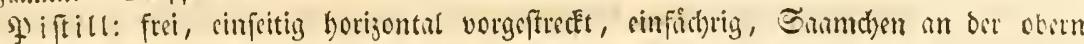
3)ath, (Sififfl auffteigend, Narbe centrift)! bsi Caesalpinia digyna 2

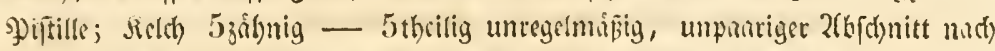

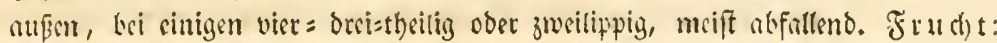
Syitfe ooer geficherte Gilieorrbulfe, Eurmen un ber obern Sluth, innere Ein:

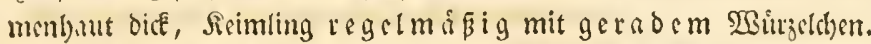

Staubbeutel 10, 2=fictyrig, lings ober an orr Spike nuffpringend, auf 1)

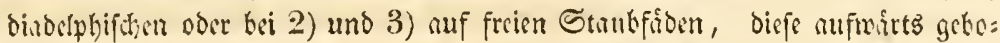

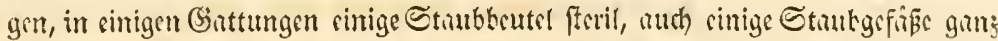
febleno, bei Ceratonia mur 5, bei Tamariudus mut 3. SBlumenfronc 1) fdrmetterlingsformig, 2) febleno, 3) fdintterlingsformig mit $2=b$ lattri= gem $\odot$ (h) iffden, 23 tumenblitter mit Mitterippe.

Segetation. 2Benige Sirsuter, meift Striuder uno Biume, Blifter (nid t) orriginglig) sinfid), gamz (Parivoa) und zwcilnppig (Banliuia), gezweiet (Hy-

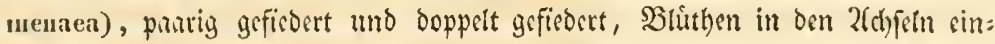

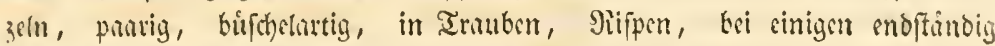
(II) menaea), meift zrvitterlich in ber 2(ntitheje (2.) biflinifth.

(Brmppon: 1) Geoffroyeae: diadelphac et monadelphae (sarcolobene). Conspect, 4096 - 4102.

2) Ceratonieae: 5 - 10 - andrae apetalae. Cuncpect. 4103 - 4108, (4109 pertinet ad 5399).

3) Ca es is lp in i e a e: 3-10-andrae 5-petalae. Consp. $4110-4154$.

\section{2. ₹̧amitic. Màinojacen: Mimosaceae.}

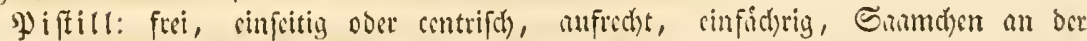

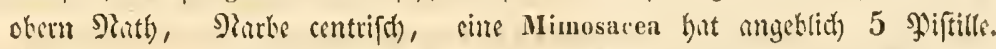

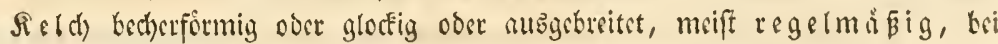
Swarziene uno Detariene in ber Sinofpe Euglich, oum $2-3-4-5$ Elappig fid) offueno, bei hibrigen Elappig 5zốhnig - 5theilig, unpanariger

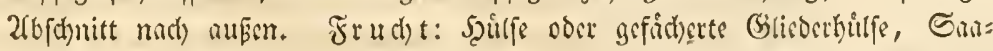
men an bet obern Nath, innerc Gaumenhumt bick, Reimling bci 1) mit

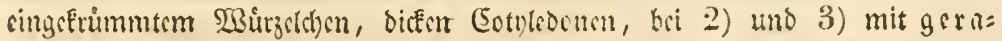

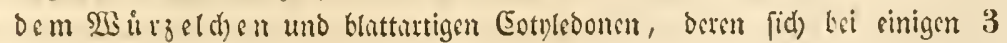
bis 4 findocit.

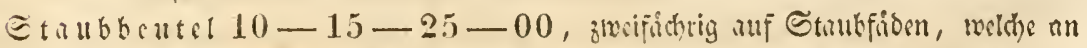

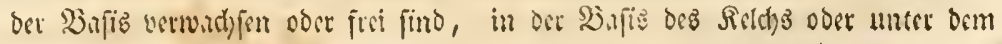


Frud)tenoten (Swarzieae) eingefügt, aufiterigend (Swarzia) ober meift nuf= rectht. SBlumenfrone fehlt obet ein einfeitiges \$Bltumenbtatt (Swarzia), oder Elappig regelmaisig 4-5blittrig, audi unten rögrig verwithjen, bei Mimoseae.

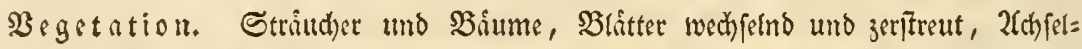
blalttchen oft Dornattig, 23litter $1-2-3$ firdh, aud) verbunden und gefingert gefiedert, bei einigen auffallend empfindlich, bei vielen Acaciae aus

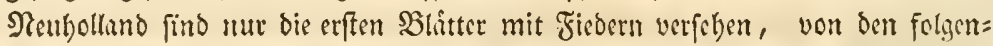

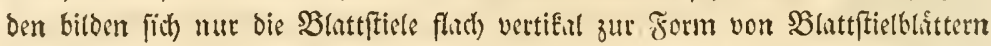

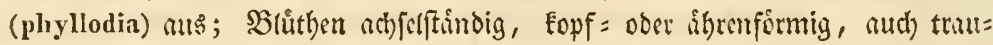
big, aud) butch) zufummengeftellte Riopfden trubbig uno rifpig, moift zmitter=

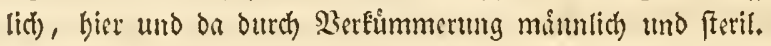

(3ruppen: 1) Swarzieac: stamina hypogyna adscendentia, petala 0-1-3 embryonis radicula incurva, Conspect. $4157-4160$.

2) Detarieae: stam, irregnlaria, petala 0. Iegim, carnosum, embryonis radicula recta. Conspccl. $4155-4156$.

3) Mimoseac: stam. et cor. regularis! entbryonis radicula recta. Conspect. $4161-\frac{4172 .}{2}$

24 nแ

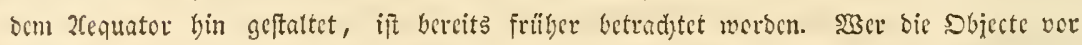

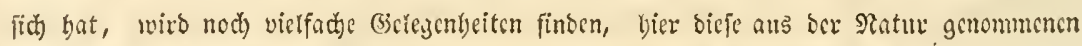

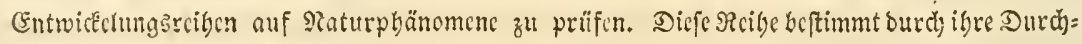

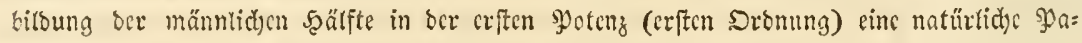

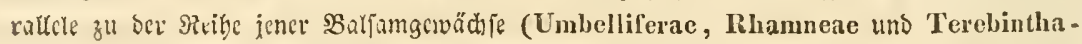
ceae), welche bas weiblid) in ber erften s)otenz burd)bilbete, zcigt von bor andern ङsite

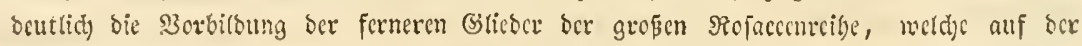

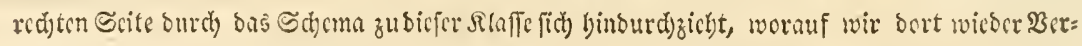
anla îung finben werben, binberten zu Ëm

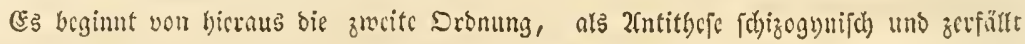

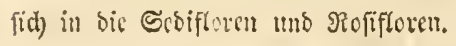

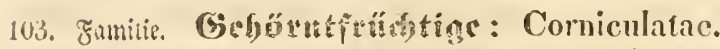

Piftill: Frudtenoten singewathper, bei boberen Gittungen und im Siaginfarse (2)

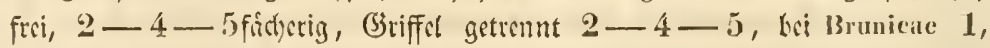

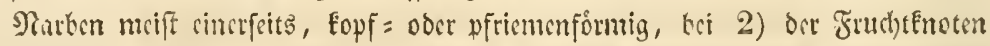
mcift bis jur Sisfis in feine $2-4-5-10-12$ Finther getheilt oder

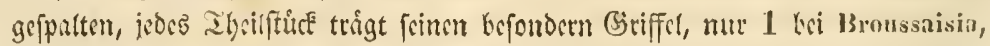
Brunia, fehlt bei Galacineac. $\operatorname{Iel}($ ) $5=$, bi einigen $4-6-8-12=$

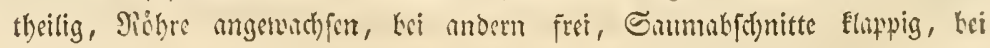
Chrysosplenimm. Zahllurucknera u. Ceplualotes intwendig furbig. Fruct t 1 -

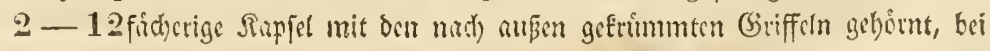
2) bie Fiober getremt, balgartig, an ber innern Shath faumentrngend und 
Duferbft anfipringend (boi Diamorpha an bor ausern Nath), bei wenigen fuftig

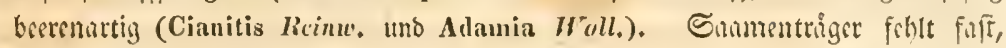
wo bie Gamen aus dom Bodan anffteigen (Chrysosplenium, Mitella) coir

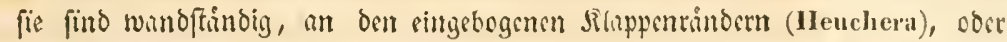

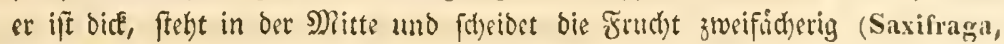
Brnnia), oocr ift mittelftandig und bat mebrere Fincher um fid) (Francoit,

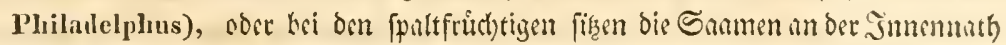
oer Fidice in zwei Sisitgen (bei Tillaea nut 2, bei Cephalotus nur 1 Gatame). Sarmen zirmlid flein, meift horizontal, bei Chrysosplenim, Nitella unto

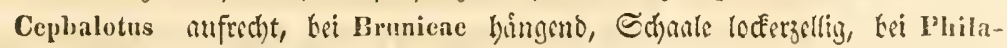

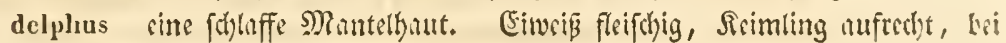
Philialelphus verfergrt.

Stanbbeutel $5-10-12-15-00$, zwriffiderig, lings nuffiringeno,

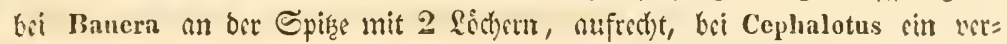
tiEal fotheibenformiger, f(t)wummiger Strg oose conneetivum, mit getrenuten

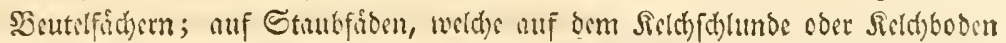

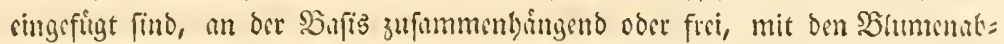

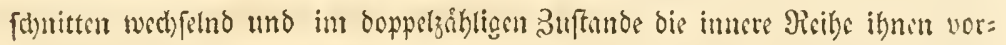
unftheno, fituzer uno fritbzeitiger entwicfelt; bei Philadelphus uno Baucra

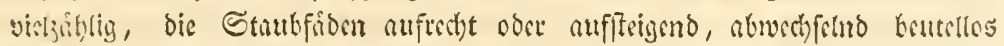

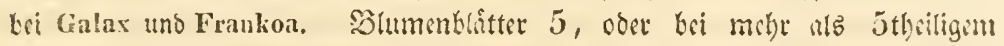

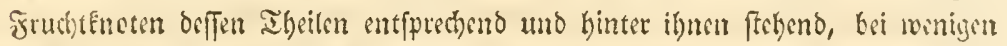
die SBtumenblatter róhrig uertwathen (Cotyledon, Bryophyllum) oder an Der Bjafis zufummentingend (Rochea, Granmanthes). Bari Chrysosplenium, Astilbe, Zahlbrucknera, Cephalotus, Callieoma umb Belangera Esine Gorolle.

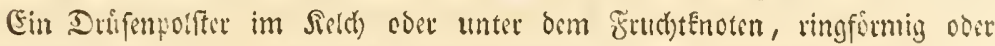
jeriç)lif̧t, oocr atb cinzelne Drufen (Crassularieae).

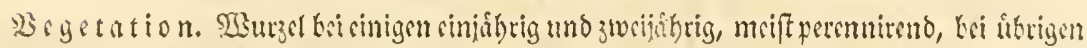

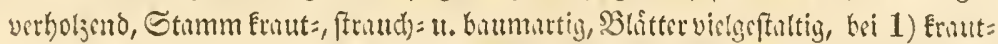

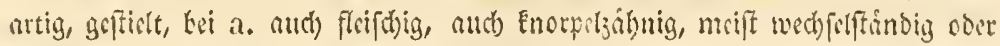

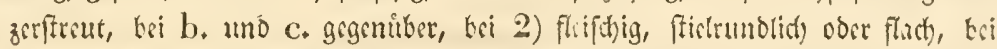

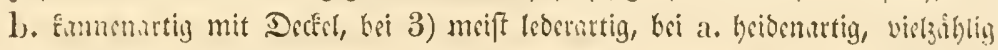

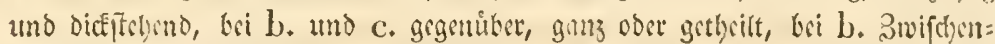
acbjelblîttc)sn. SBlitben in bethitterten Trugbolocn (Clurysosplenium, Hy-

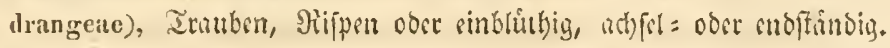

(3ruppen: 1) Saxifragariac: schizostyles (oppontuntur: a. $\beta$. stignatibus sessililus) herhaceae et fruticosac diplostemoncae (opponuntur Donatia, Heuchera et Drummondia isostemonea) exstịnlatae.

a) Saxifrageae, herbaceac.

๙) II c u eherca e placenta basilari subnulla, lateralibus aut centrica. 
«a) Chrysosplenieae: apetalae calyce colorato. Chryso s- $^{-}$ plenium $L$. Astilbe Ilamilt.

$\beta \beta)$ Mitelleae: $3-5$ petalae. Lepuropetalon Null. Drummoudia $D e C$. Tellima R.Br. Mitella T. L. Lsptarthena $R, B r$. Heuchera $L$. Tiarella $L$.

y) Vahlieae: 5 petalae placenta centrali soluta.

Vahlia Thb. (Bistella Decf.) Rchb. hort. t. 91.

B) Galacine ae: stigmata sessilia, placenta centralis.

Galax L. Frankoa Cav.

r) Saxifrageae genuinae: placenta septante, ult. multisiliqu.

Donatia Forst, Zahlbrucknera $R c h b$. Saxifraga $L$, Micrantlies Haw. Robertsonia Haw. Bergenia Mnch, Eriogyuia Hook.

b) Philadelpheae: fruticosae icosandrae, oppositifoliae exstipulatae. Philadelphus $L$.

c) II ydrangecae: fruticosae diplostemoneac oppositifoliae exstipulatae 2-5-1-styles, 2-5-loculares.

Ilydraugea L. Sarcostyles Presl. Cianitis Reinw. Alamia Wall. Deutzia Thub. Broussaisia Gaudich.

2) Crassularia e: libere schizogynae, carpidia calyci alterna, antherac erectae, embryo rectus.

a) Sedeae: carpilia libera pleiosperma intus dehiscentia. Conspect. $4173-4190$.

b) Pentloreac: carpidia basi concreta interne dehiscentia: Pcuthorum L, - externe: Diamorpha Nult.

c) Ce pla lote a e: carpidia libera monosperma.

3) Cun on ia riac: schizostyles lignosac.

a) Brunieac: 3-2-1-styles 5-antrae parvillorae capituliflorae, (drupa sicea, seminia pendula) fol. inbricata exstipulata, habitus ericoileus.) Consp. 4221 - 429?9.

h) W e inmanie ae: 10-aulrae parviflorac (fiores in capitnlis, spicis, paniculis, folia opposita, stipulac intrafoliacene).

Codia Forst. Belangera Camb. Callicoma Aizti. Dieterica DeC. Weimmannia L. Cumonia $L$, Arnollia $B l$. Ceratopetalum $S m$.

c) Baucrea : icosandrac grandiflorae (flores in pedunculis unifloris, fol. opposita composita exstipulata.

Baucra Andr.

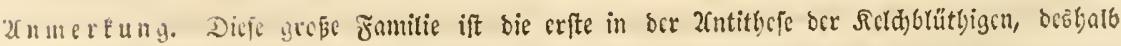

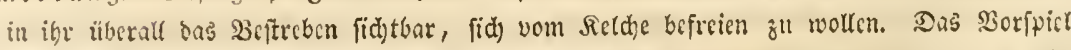
ber seudjereen witb burd bic nicbrigfte Battung Chrysosplenium auf cine fehr cinfad) gescije begonten, gleidjan aus Iydrocotyle und Ailoxa ber verigen Drsmung, Brüthc 


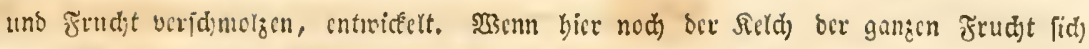
ats Boben barbittet, fo baben fdyon Leptarrhena, Tiarella, Robertsonia u. a. bas \$ुi

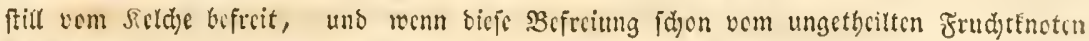
crffrebt wird, fo gelingt fie nod) melyr ba, wo bor Frudjtfnoten fid ferbft in feine Fächer

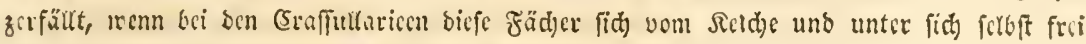

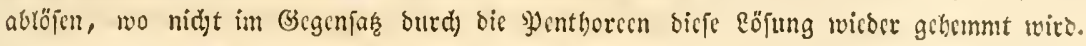

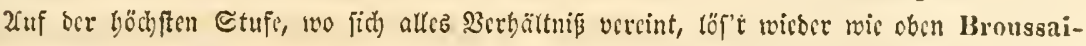

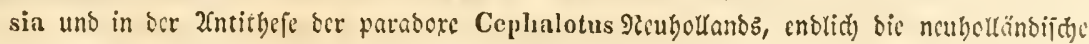

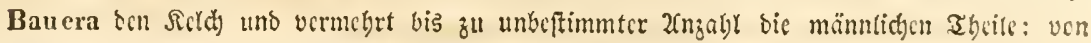
7-10 B tumenblätter, uns bis 60 Staubgcfäße entbartens.

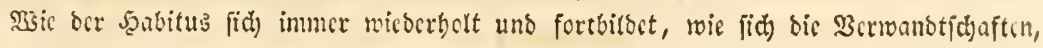
ber Sorbercitung analog, and) geographifol) vermittern, bas alfes fpringt leicht in sie 2 (u=

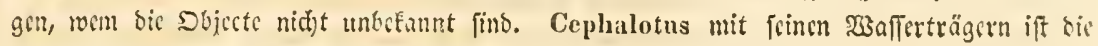

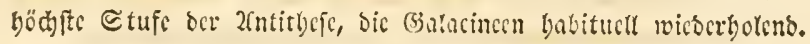

\section{Famitie. Ronforectl: Loasaceae.}

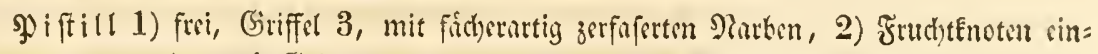

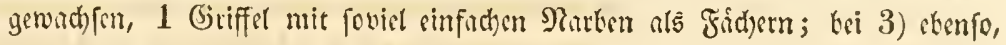
aber Frudtencten frei. Fis (d) röbrig, trid)terartig, glockig, 5theilig, firtig und abfallent, in ber Snoppe auflisgend, bei 2) 5zaiblig, fleifhig, in oer Inofpe offen, bei 3) 5blitterig, bleibend, zirgeldecfig. - Frudt Enpfelur= tig, 1) cinfidyrig, oben 3klappig, Sanmentróger 3, mittenwandftindig, virlfamig, Enamen von biutigen Mantel balbumbillt, Shante mit dinner

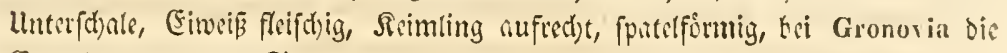

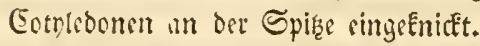

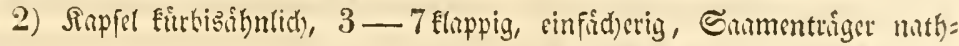
finoig, 1-4-00fanmig, Gammen obne Mantel uno obne Eimpiä; Seimting getroe, aufrect)t, Eotyledonen Flein uno flich).

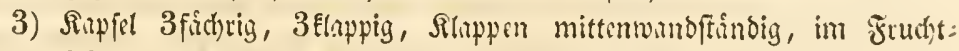
Enoten 00 : bei ber Aleife aker wenigfanmig, Sanmen bingeno, flutb), flitgel=

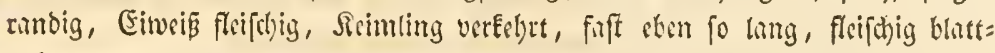
artig.

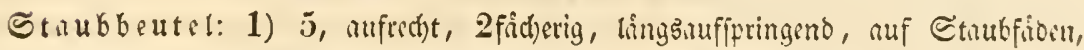

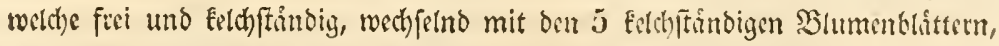
meldye in bie Sinope gederbt find.

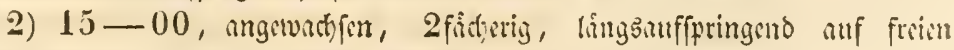

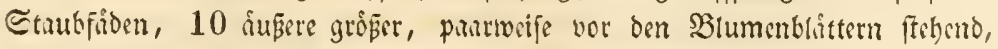

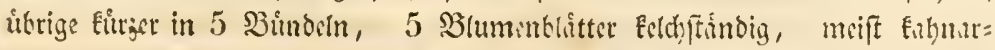

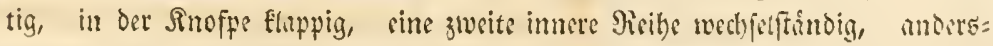
gettuitit, rimnenutig, mit bouftenartigen Fortfinen (Loasa, Blunenbachia).

3) $10-12$, jwifict)erig, lingshuffpringeno, ati langen berausfthbon=

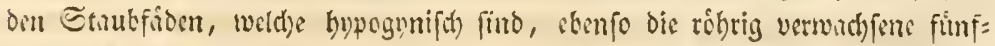
fpultige Silumentrone.

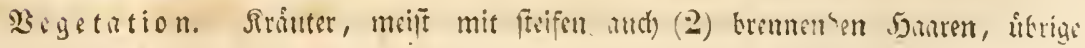




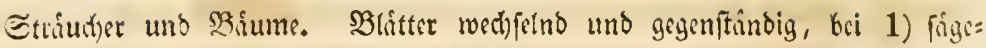
runbig, bei 2) tuppig zertheitt, biunflrifhig uno wie bie ganze gुoflunje jujtig

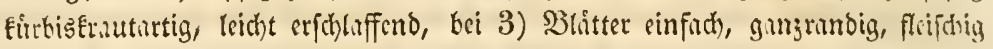

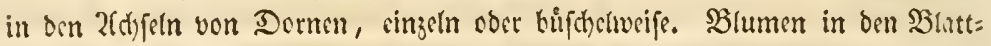

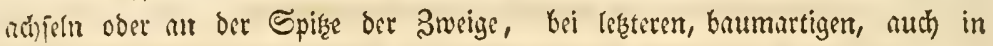
Siifpen.

(Swipen: 1) Turuereae: 5-andrae contortae (herbae schizostyles axilli - et petiolifforne).

Piriqueta $A n b l$. Turnera Plum.

2) Loascac:5-00 andrac epigynae (herbae urticantes).

a) Gronorieae: 5 andrae (drupaceae 1-spermae cotyledonilus apice infractis).

Gronoria $L$.

b) Blumenbachieae: ralratae parapetaliferae.

BInucuhichial Schrad. (et Helicterojiles $D e C_{\text {. }}$ ) Loasa Adlans.

c) Ji entyelie a e: planipetalae iubricatae (stamina piorina 10 -25e, paripetala mulla).

hlaprothia K. II, B. Sclerothrix Prsl. Mentzelia Phim. Bartonia Sims.

8) Fo uquicrea e: $10-12$ andrae hypogynae (frutices et arbures terminalispicatae et panieulatae tuibllorac).

Fouquiera K.H.B. Bronnia K.H.B.

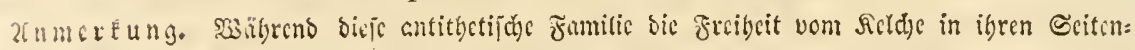

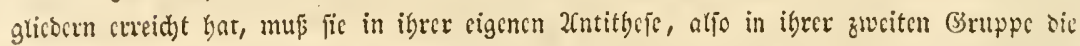

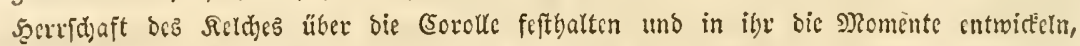

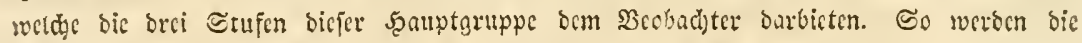
Blumenbadjicn bas (Entrum, uns nad) beiocn Rid)tungen von ba aแt motivitt fid) ber

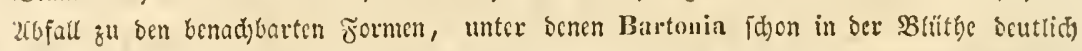

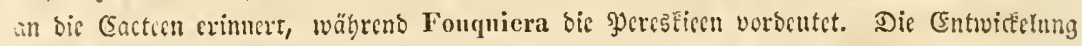
won Blumenbachia habe idy llort, bot, t. 121, gegeben, bajerfft auch p. 9. Sie mere miltosige sane ber (5otyletonen von Gronovia befdridfen.

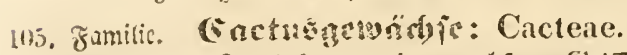

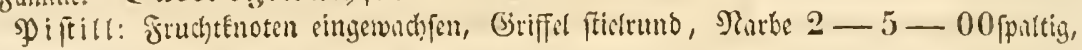
bei 1) aufrecht, bei 2) 2 Sharben borizontnl, falt velfchmoligen, bsi 3) $5-$

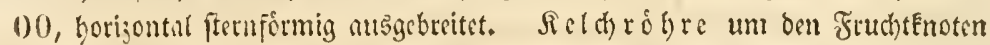

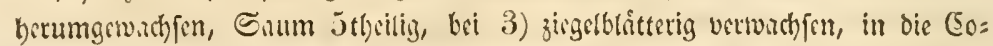

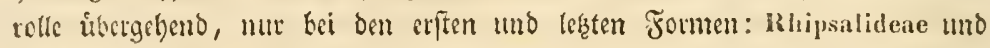

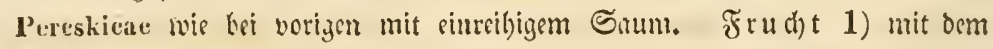

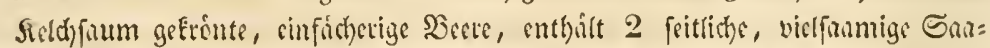

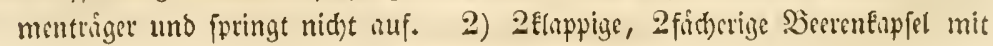
Sield unb (Siriffel getront, bie Slappen beugen fich an ber Slath ein und bitbin

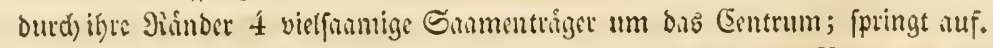
3) Beere flifhig cimfoctyerig, mit viclen Sarmen in fuftigem 3 tei zerftrut.

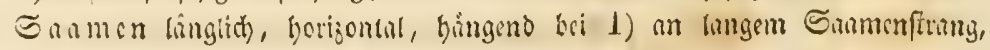




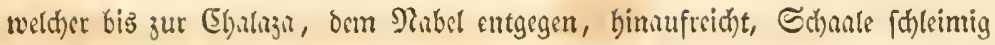

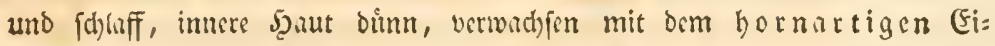

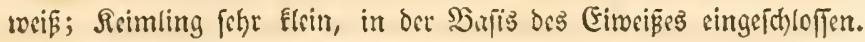

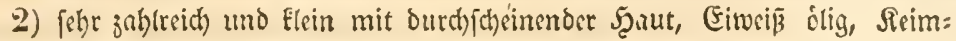

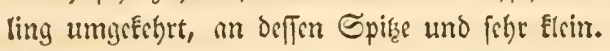

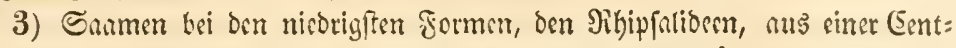

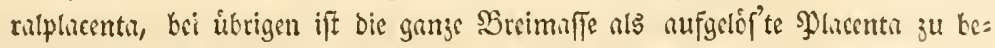

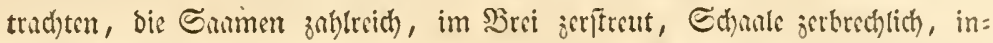

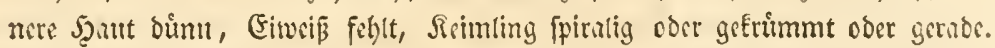

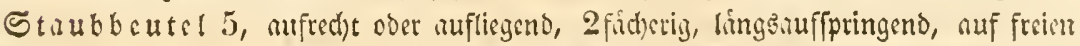

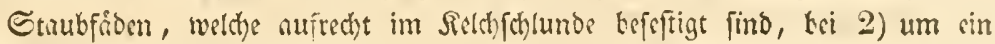

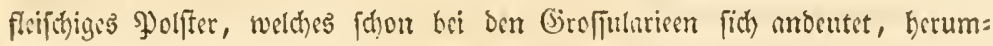

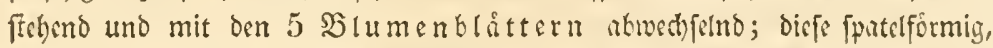

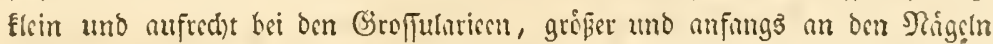

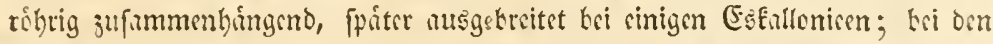
Escteen geht ber zirgelblitterige Field) unmitterbar in sine mebrribige, jirgels artig virlstitterige SBlumenftone hiber, wäbrend bie exfen uno leşten Formen,

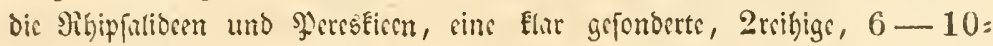
blitterige Corolle entriefétn.

Begetation. Striudser hotsig und veriffelt, ober kei ben Enctem fleiphig und

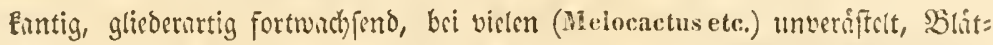

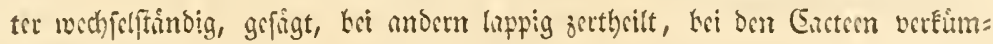

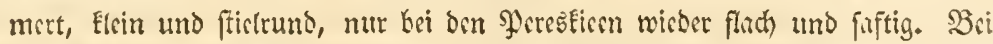

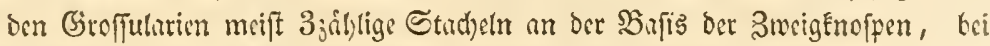

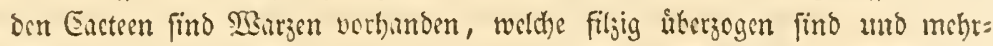

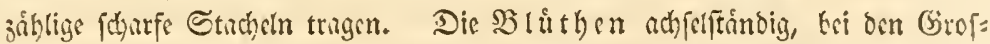

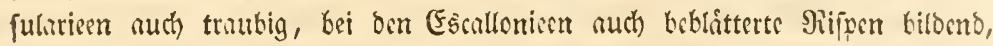

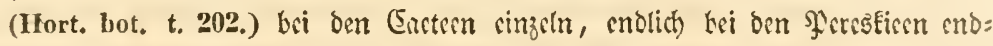
ftinnoig.

(5ruppen: 1) Grossularieac: schizostyles baccatae.

Grossularia T. Robsonia Berland. Rebis Spach. Ribes T. Botry caspum Rich. (Coreosma Spach.) Calobotrya Spach. Chrysobotrya Spach.

2) Escallonieac: schizostigmaticae dehisentes.

Escallonia Mutis. Quintinia DeC. Forgesia Commers. (Defforgia Lam.) Anopterus Labill. Itea I. (Diconangia Mitch. Cedrela Lour.)

3) Cacteae genuinae: actiniostigmaticac indehiscentes.

a) Rhipsalibeac: calycis limbo uniseriali, petalis 6 , placenta columnari.

Rhipsalis Gärtn.

b) Opunticac: calyx imbricatus in corollam imbricatam transiens.

Síanamillaria Hav. Echinocactus Salm-Dyck. Melocactur 
Relḑblüthige.

C.Bauh. Cactus L. (Epiphyllum Herm. Cereus Juss. Cereaster DeC.) Opuntia 'T'. ('Tuna Dill.)

c) Pereskieae: calycis limbo uniscriali, petalis biserialibus, placenta in pulpam soluta.

Pereskia Plum.

2rnmertung. Das Fortbitben bicfer Formen, wie es in morphologifder und gcographifder Sarallete fich vermittelt, ift an fich Elar und bie Sbiccte allbefannt, daber bic 2fnocutung

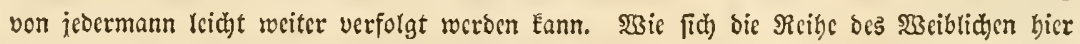

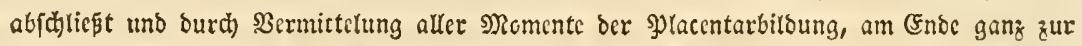

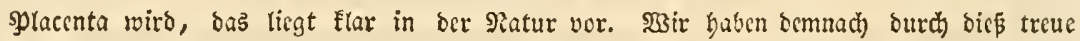

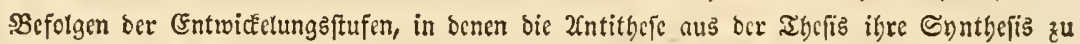
reiderem Inbalte vermittelt, cine natürliche Familie, uno burdi fie wieber ben fontheti= \{đen Sdlü cince natürliden Reibe.

106. Familic. \$ortulafacen: Portulacaceae.

DSiftill: meift mit 2(ndeutung zur Irennung in 3 , bei cinigen in $2-5$ Frucht= Enoten, batkfrei, bei ben begimmenden Selerantheen und in ber 2(ntitbefe ber Begonieen unterftánbig, (Siriffel meift $3-2-1$, bei wenigen 5. Narbe meift Eopfformig, in ber 2(ntitheje auch zerfafert ober zettheilt und zartwarzig. Ielch 5 theilig begimend, bei folgenden abuchmend, $3-2$ theilig, in ber Intithefe zmeideutig, zum Theil corollinifa), bei 3) wieder beftimmt und von

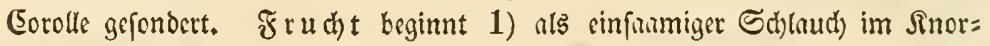

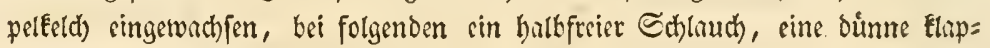
penlofe oder Elappige Siapfet mit centralem Sarmentringer und ofteter 21nbeut= ung zu 3 - 5ficheriger Theilung, ein freiwerdendes 3 Enntiges einfaumiges Nüs=

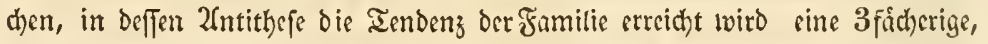
vielfinmige, untere Sarpjel mit centralen Sanmentriger, weld)er in iroes Fach mit einer blättchenartigen Seiffe (anderwirts boppelt) nusliuft; auch) faftige Steinfrud)t mit Etein, welcher bie Fricherthcilung andeutet (Coccoloba). Bei 3) wicber sine zarte Siapfet mit beftimmt = ober unbeftimmtz̧ihligen San= men, weldhe nidft, ober ringsum, ober Elappig nuffpringt und wieder sinen centralen Sanmentriger gewinnt. Sarme Eugelig oder nieren = uno linfen=

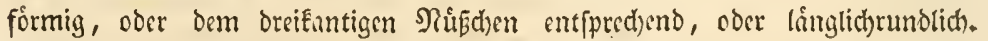
Echanle hart, Eimeís meift mehlartig, bei wenigen fleifhig ober hornartig (Polygonum, 'Tiniaria, Persicaria, Avicularia mo Begonia), rungclig bei Coccoloba. Seimling ringartig gefrummt, bei ben allermeiften um bas

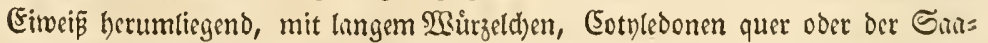
menfliche parallel, bei wenigen achfenftandig (Begrouia und Coecolobeac), dunn bei einigen berfelben blattartig flach aud) gebogen, burd) daz ganze Eiweí bin= Durchzieheno (Fagopyrum).

Staubbeutel zwoifacherig, linglich, bei wenigen pfeilformig (Coelanthum Fzl.), langżuffpringend, aufrecht, aufliegend, auch zitterno, bei wenigen angewach)= fen (Begonia), nuf Gtaubfäben, welche meift frei jïn, den Siclchabjhnitten voranfteken, ober eine zweite Sicihe mit ifnen wechfelno, dicfe bei cinigen ohne Beutel (Herniaria), oder panrweife vor den Feldabfhnitten, oder unbeftimmt 


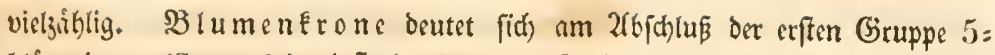

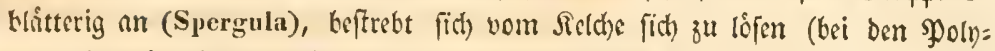
goneen) und tritt endlich) vollendet hervor (Portuluceen), 5blastterig, mit ben

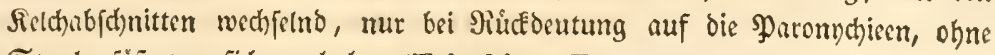
Staubgefä́ré vor fich zul baben (Telephieae FI, germ. p. 574.).

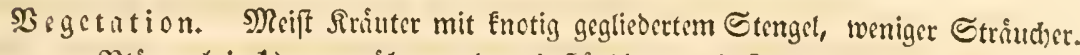
Shtitter bei 1) gegenitber und quirlftindig, bei 2) trodene hiutige Iuter (ocreac), Dem $B$ tattfficle innen angewant) fen, bei wenigen noch) aüerdem $\mathfrak{B l a t t =}$

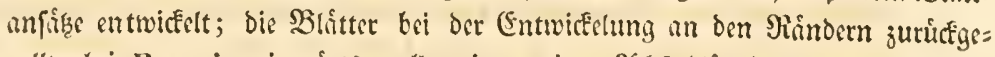

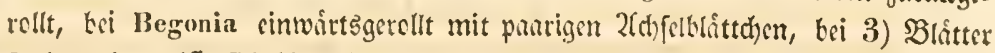

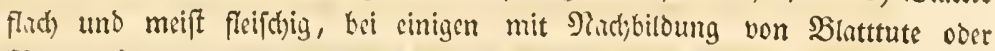

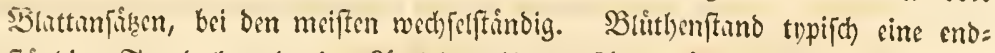

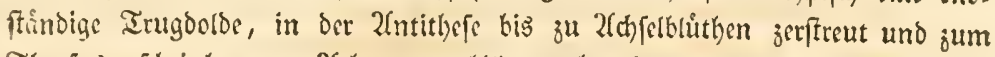

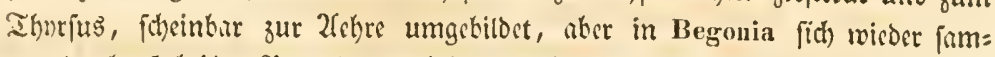

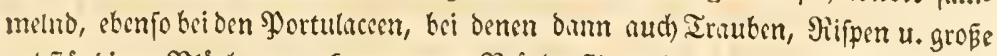

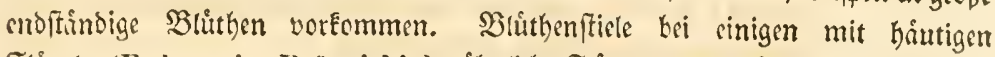
Fthigetn (Podopteris, Brïnniclia), ihntliche Flingel limfen liber bie Frucht bei Begonia).

Grupprn: 1) Paronychicae: oppositifoliae et subverticillatae, calycis partitiones scarioso-marginatae.

a) Sclerantlueae: utriculus 1-spermus calyci indurato innatus (apetalae vaginatac).

Scleranthıs $L$. Mniarum Forst. (Ditnca Bnks, et Gü̈rtn.) Guilleminia K.II.B. Pollichia Solend. (Nekeria Gin. Meerburgia Mnch.) $^{-}$

b) Illecebrae: utriculus vel capsula emersa evalvis aut trivalvis (apetalae scarioso-stipulatae).

a) Il eruiariae: monosperuae.

Herniaria T.L. Gymnocarpum Forsk. Anychia Mchx. (Queria Gärt.) Illecebrum L. Paronychia Juss. (Chaetonychia, Eunychia et Acintlionychia $D_{e} C_{\text {. }}$ ) Cardionema $\mathrm{DeC}$. (Bivonaea $\mathrm{Fl}$. Mer. nec. al.).

B) Polycarpeae: polyspermae evalves,

Polycarpaea Lam. (Hagaea Vent. Mollia $W^{\text {. }}$. Lahaya R.S. Hyala l'Herit, Anthyllidis sp. Adans, Polia Lour,?) Stipulicida Mchx. Ortegia Loeffl. (Ortega $L$. Juncaria Clus.) Polycarpon Loeffl. (Trichlis Hall.) Cordia Fl. Hex, et DeC.

y) Loeff 1 ingiea e: 1 -00-spermae capsula s-valvi. Queria Loefl. Minuartia Loeffl. Loefflingia $\boldsymbol{L}$.

c) M ollugine a e: capsula emersa membranacea 1 -3-locularis apice loculicide dehiscens.

a) genuinae: apetalae capsulis 3-5-Ioculari. 
Mollugo L. Coelanthum Fnzl. MallogonumFnzl. Pharnacenı L. Hypertelis E.Mey. Balardia Cambess. Orygia Forsk. Glinus L. Axonotechium Fnzl.

B) Steudelieac: apetalae, stamina 5 ealycis partitionibus alterna, capsula monosperma indehiscens,

Adenogramna Rchb. (anno 182\%. hort. bot. tab. 109. Conspect. No, 4411, et Spreng, syst. et gen.) Steudelia Presl.

r) S perguleae: 5-petalae, capsula 1-locularis dehiscens. Spergula $L$.

2) Polygoneae: ocreatae perianthio ambiguo, pistillo monospermo diviso (opponitur Begonia polysperma).

a) Rnmiceae: monospermae perianthio definite biseriali, utroq̣ne lierbaceq.

Gxyria Hill. Emex Neck. Runex L. (Acetosa T. Lapathum T.). Ihleum L. Polygonella Mchx. Tragopyrum M.lB. Atraphaxis $L$.

b) Begonieae: polyspermae capsula infera triloeulari, embryone basilari erecto (diclines polyandrae stipulatae). Begonia $L$.

c) Polygonea genuinae: calyce ambiguo corollino,

«) I'ersicarieac: embryo lateralis, cotyledones accumbentes (alhumen corneum).

Koenigia L. Centinolia J. Bcuh. (Avicularia Meisn. nou Gesn.) Persicaria T. Tiniaria Meisn. - (albumen fariuosum:) Aconogonum Meisn. Bistorta $T$.

B) Amblyoneac: embryo lateralis, cotyledones incumbentes (albuncu farinosum:)

Amblygonum Meisn.

y) Coccolobeac: embryo axilis complanatus (albumen farinosum:)

P'terostegia Fisch. (: A. Mey.*) Fitgopyrum T. Podopteris $I l b . B p l$. Brimnichia Gärt. Triptaris $L$. et Blochmanuia Weig. Coccoloba $L$.

3) Portulaceae: corolla definita! - (repetito apetalae: Cypsclea, Trianthema, Sesuvium in antillesi).

a) Telepliea e (I'aronychieas repetentes): calyx 5-partitus,

") Die niedliche çattung l'terostegia Fisch. C.A. Mey. febt fo cben blitheno und fruchttrags

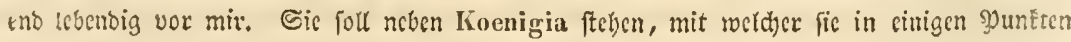

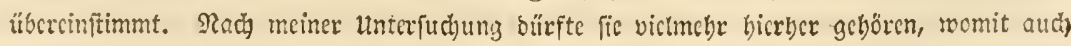
inre Sebaarung, ser slangel ber eigentlichen Iuten, wie fie bei Coccoloba und Triplitris

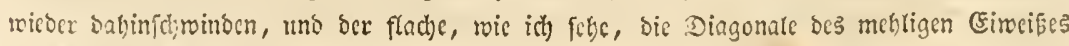
ausfüllenoe Sitimling übcreinftimmt. 
stamina 5 calycis partitionibus anteposita, petala 5 alssque staminibus.

Corrigiola $L$. Telephinm T. L.

b) Scsuvicae (Polygoncas repetentes): calys 5-7 partitus subcorollinus, capsula circumscissa:

Cypselea Turp. Trianthema $L$. Sesuvium $L$.

c) Portulaceae genuinae: calyx bi-partitus, ultimae 5 - 7 partitus, stamina petalis anteposita.

a) Monticac: capsula 3-sperma, valvata, stamina lefinita $3-5$.

Montia Mich. $L$. Leptrina Rafin, Claytonia $L_{.}{ }^{*}$ )

B) Portulacellae; capsula circumsissa, semina et stamina intefinita.

Portulaca $L$.

y) Talineae: capsula valvata 00 -sperma ealyx bipartitus.

Calandrinia Knth, Talinum Ad. Anacampseros Sims. Grahamia Gill. Hook. Portulacaria Jacq. - calyx 5- z partitus : Lewisia Pursh.

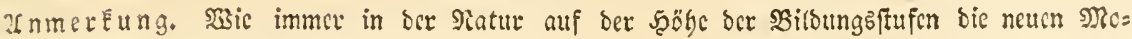

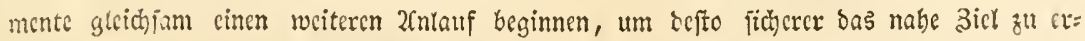

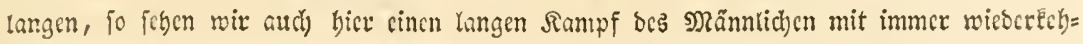

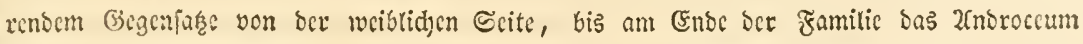

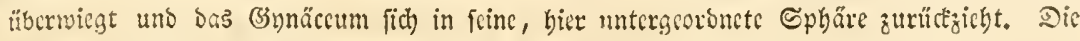
Exüftigfte Sppofition übt Begonia, bic Slüthe ton Atraplaxis wieberboleno, afer im

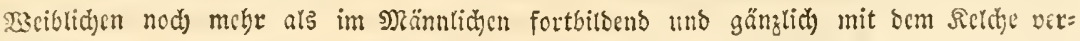

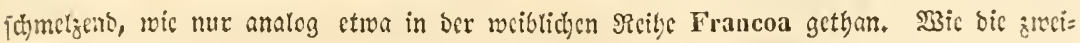

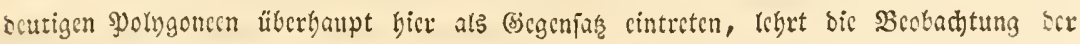

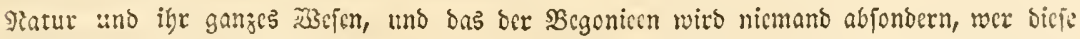

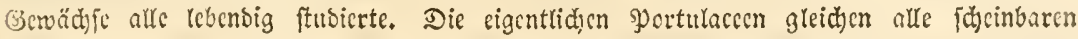

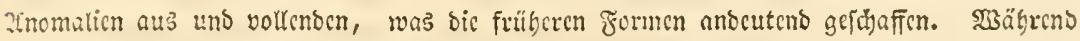

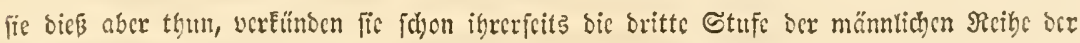

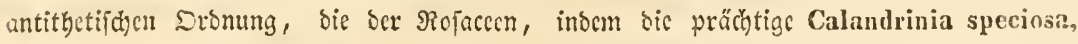

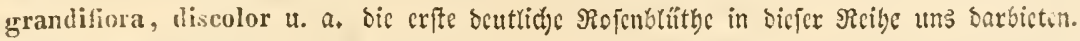

107. Familic. 2íijoidecu: Aizoideae.

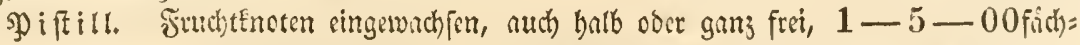
crig, Ficher 1-00funmig. Griffel Eurj 2-3-5-00thcilig, Nar=

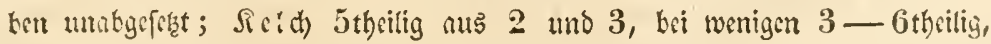

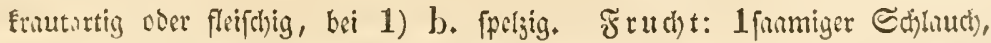

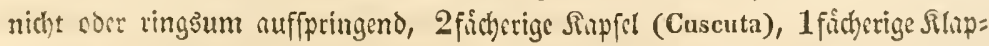

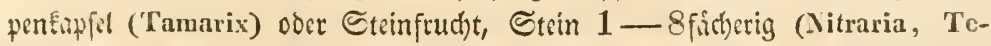
tragonia), typifd vollmbet cine afgeftuşte 00ficherige Sapfel, mit centrifolen

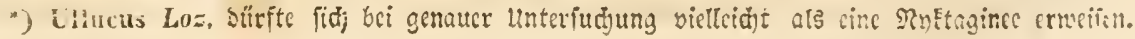


Sanmentriger, fternformiger (sirips, an oen Innennithen auffpringend, auth

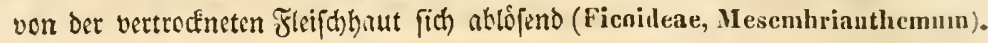
Sanmen nieren = oder linfenformig, hei menigen linglichrund und birnen=

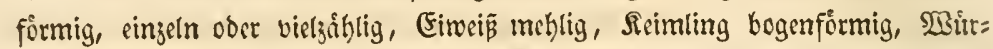
zeldyen nach unten ober nad) oben, ober horizontalliegend, aud fothecfenartig eingerollt, bei 3) gernoe und aufrecht, nur bei Nitraria veréebrt, bei metgreren (Salsolene, Tamariscinene) fehlt bors (Eimcif.

Staubbutel 2ficherig, (bei Poranthera 4 ficherig), lingsaufipringend, $5-00$,

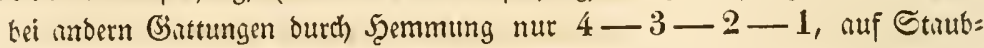
fíden, welche verwathfen ober frei find, auf dem Sielche cingefugt ober mit ben Shlumenblittern feitlich) verwacbjen (Achyrantheae) ober hopogynifit), bei Tamarix

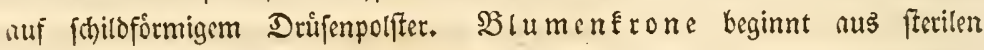

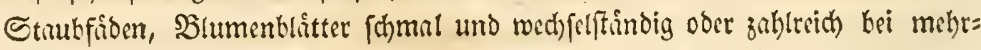
reibigen Stuubfidon (Ilesembrianthemum), bci ber lef̧ten (Gruppe endid) sine

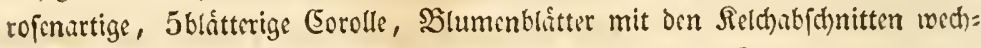
felnd, langtid) oder fidicf brifformig (Reaumuria), in ber Snofpe umgelegt.

Sigetation. Strmm Erautartig, auch) gegliedert, getentig (Salicornia), Blitter

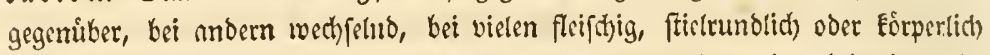
vielgeftultig (Mesembrianthemmm), obsr fluch uno meldenartig, bei vielen in Der Iugend ober fitr immer mit wafferhellen 23 sirjthen, wie Thautropfthen befestrt (A tripl. Chenop. Tetragonia, Mesembr. cristall. glac. etc.), 2(ch)elblitt:

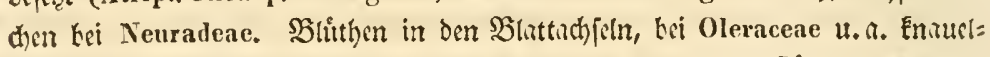
artig oder áhrenattig, trubig (Phytolacceae), rifpig und endfrinoig (Mesembrianthemum, Tamariscineae.)

(S)ruppen:

1) Oleraceae: apetalae nonospermae curvembryae.

a) Che nopodea e: calyce herbaceo demun aucto quibusdam baccato aut membranaceo-appendiculato utriculum tencrum monospermum includente, semine albuminoso (in Salsola, Anabasi etc. exalbuninosu), embryone circulari aut spirali, Conspect. 4319-4353.

a) S a licorneae: articulatae spicatac, flores conformes, semen verticale albuminosum embryone circnlari.

Salicornia $L$. Halocnemum $M$. B.

ß) Atripliceae: continnae glomeratae diclines difformes, semen verticale albuminosum embryone circulari.

Atriplex L. (Atripl. et Obione Gärt.) Halimus C. Bauh, Wallr. Diotis Schrcb. Ceratocarpus L. Axyris L. Spinacia T, L.

y) Che nopodea génuinae: continua hermaphrotitae volygama suliglomeratae, Fores conformes 5-partiti.

ac) Kochieae: embryo circularis in semine albuminoso horizontali :

Tcloxis (Ch. aristatum) Moq. Tomt. Cyclolepis Moq. Tant. Clienopodium L. Blitauthus Rchb. 1823. (Acroglochin Schrl. 
Lecanocarpus N.v. F.) Kochia Rth. Suacda Pall. (non Forsk. Sclerochlaena R.Br, Kochia II, Willemetia C. A. V, nom Neck. et Brongr.).

$\beta \beta$ ) Bliteae: embryo circularis in semine verticali:

Panderia Fisch. et C. A. M. Monolepis Schrad, Blitun L. Orthosporum C. A. M. Beta $L$. etc.

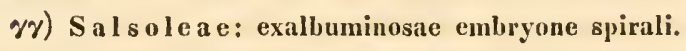

$\alpha \alpha \alpha)$ Anabaseac: articulatie squamulis hypogynis (potalorum rudimentis) auctac, semina verticalia.

Brachylepis $C, A, M$. Anabasis $L$.

$\beta \beta \beta)$ S a l s o l ea e g e n i in a e: continuae ecarinatae testa membranacea, eal. $3-5$ sepalus bibracteatus, semen verticale:

IIalogeton C.A.M. Halimocnemis C.A.M. semen horizontale: Salsola $L$.

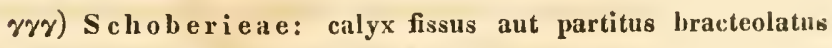
carinatus, seminis testa crustacea,

Seloberia C. A. M. et Led. Sehanginia C. A.M. et Lett.

b) Amarant lieae: calyx scariosus 2-3-bracteolatus (Petiveriae 1-bras:teolatus).

a) I marantheae genuin a e: hypogynae 5 (1-3)-andrae.

Polycnemum $L$, Camphorosma $L$. Corispermum $L$, Amaranthns $L$.

B) Achyrantheae: diplostemoneac sulumonaliphac, antherne 5 (1-3), filimenta alterna sterilia aut petaloidea fimbriata. IReliquae ex $435 t-4383$.

g) Petiverieae: spicatae apetalae $6-7-8$-andrae, seuen rectum erectum cotyledonibus involutis.

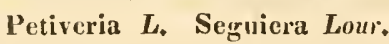

c) Phytolacceac: calyx subcoloratus, stamina perigyna $4-5$ aut bypogyna 4-00.

a) Cuscuteae: petala squamulosa (cf, pl. crit. ic, 690 - 691.) staminibus alterna utriculus capsularis bilocularis, semina gemina emhryone spirali.

Cuscuta $L$.

3) Baselleae: petala nulla, utriculus 1-spermus, embryo spiralis. Boussingaultia K. H. B. Basella $L$.

r) Rivinicae: petala nulla, stamina subliypogyna, drupa vel hatcca 1-0(1)-locularis, loculis 1-spermis.

Cryptocarpus $K, H . B$. Microten Sw. Ancistrocarpus $K . H . M$. (Potamophila Schrk.) Bosca $L$. IRivina $L$. Phytolacca $L$.

2) Aruideac genuinae: succulentac 5-00 anlae capsnlares currembryae.

a) Gis e kiea e a petalae 5-andrae, capsula 5-partita, loculis 1-spermis: Gisekia L. - 2-3-partita, loculis 00-spermis : l'oranthcra Rudge. 
b) Ficoide ae: corolla 0 - aut 00 -petala, capsula angulata, truncata aut pyrena 5-locularis 5 valvis 00 sperma.

a) Tetrag ou ie a e: corolla 0 , pyrena in calyce inclusa monosperma: Anredera Juss, (Clarisia Abat.) $-3-8$ locularis singulisperma: Tetragonia $L$.

B) Ficoidea genuinae: corolla 0, capsula 5-locularis00-sperma. Aizoon $L$. (Ficoidea Dill.)

y) Mesembrianthemea e: corolia radiati-00-petala, caps. 00-locularis Joculis stellatim ad suturam intus liberam dehiscentibus, endocarpio membranaceo demum secedente.

Mesembrianthemun Dill.

c) Neuradeae: corolla 5-petala, capsula stellati-10-locularis singu- lisperma sem. exalhuminosa, folia stipulata. (Rosacearum prolusio!) Neurada B. Juss. Grielum $L$.

3) Tamariscineae: corolla alternipetala murcescens, stam, 5- 09 , semina rectembrya albumine rel nullo rel (in antithesi b.) parco farinoso.

a) Nitrarieae: stigmata 3 , drupa e germine 3 -6-loculari singulispermo matura 1-sperma, semen pendulum exalbuninosum.

Nitraria $L$.

b) Reaumuriea e: stigmata $\mathbf{2}-4$ aut $5-6$, capsula $\mathbf{2}-4$ ant 5 -locularis, 2-4 aut 5-valvis, semina geminata erecta subalbuminosa villosa.

Hololachna Ehrnb. Reaumuria $L$,

c) Tamarisceae: stigmata 3, capsula 1-locularis 3 -ralvis placentis parietalibus 00-sperma, semina comosa.

Myricaria Desv. Tanarix T.L.

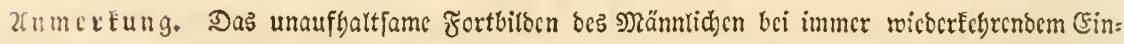

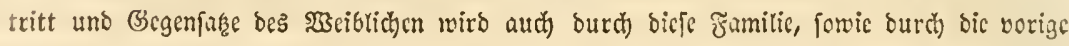
tlax. Șäbreno ich aus jener Familie in einer uno berfetben Battung von Calantriniis Inonanủra bis zu grandiflora, speciosa, tliscolor, ocn 2cbftaub von 1 bi弓 $j u 40$ un 50

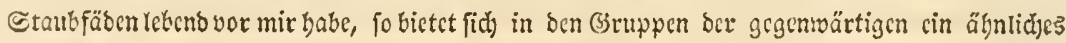

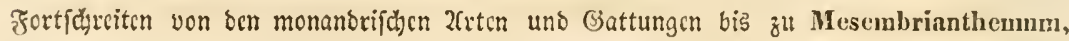

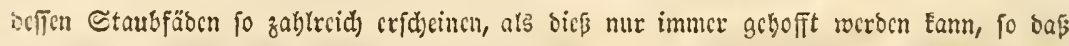

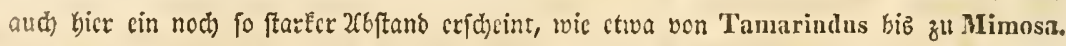

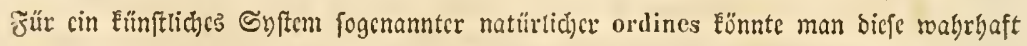

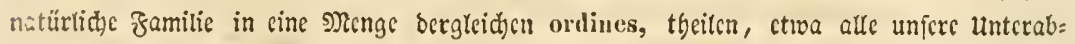

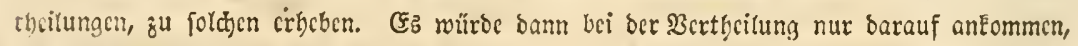
of ber Eintbrilende auf irgend sin igm beticbiges Drganenuerbältrii mehr obcr teeniger

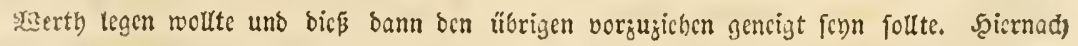

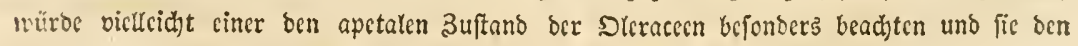
nur analogen, niđht aber verwanbten urticaccen annäbcrn, sin anberer Eönnte ien 3uftano

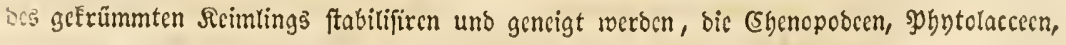
zimarantbecn unb andere Bruppen ber yorigen Famitie mit oen Earyophyllaceen verbinoen 
Seldhblinthige.

Sronung: Relyntidg thäthige.

Kizoiben.

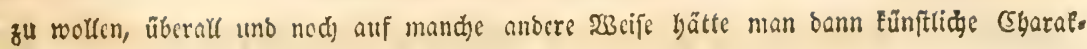
tere zun limfdyreiten ber 9katur (id) entrwickelt.

פRir folint bie Entfaltung cines gropen und fdjönen Tupus in bicfer Familic zut lie,

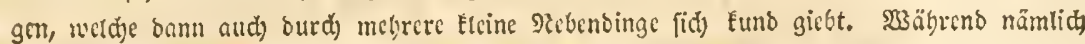

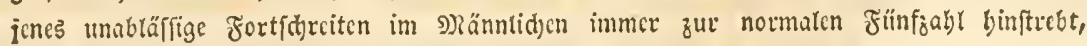
fic enolich ats Mesembrianthemum in alfen Sireifon übcridgreitet, aber bald burch Fir. tritt des Rofentupuls für bie Eorolle wicocr befhränft wirb (Neuradeae), fo bcrubigt fich aud ber Jircis ber Staubgefäßc enotich in ben verbaumten 2(izoibecn (Tamarix)

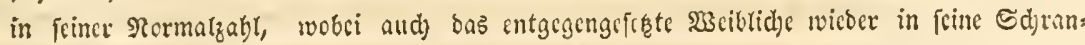
fen zurücétritt.

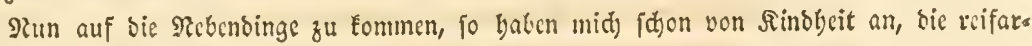

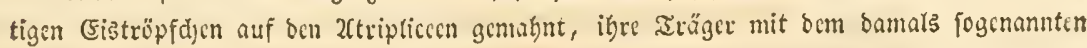

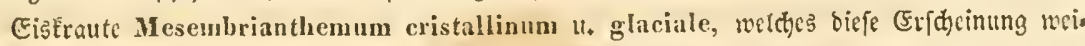

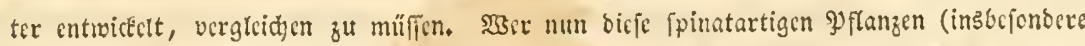
Salicornia, Altriplices, Spinacia, Amaranthus oleraceus, Tetragonia, Mesembrianthemum cristall, etc.) als Epcife genofien hat, ber wirb fid nody burdy mebr als cinen

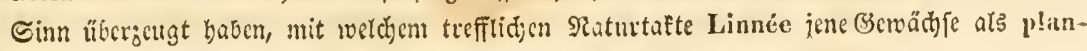

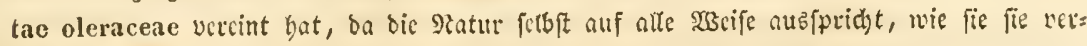
cint fegcn will. So wie aber bie ভympetalen ilber igre Sphäre binattsftrebten, fich poly.

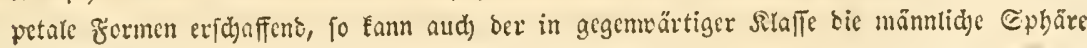
bemmenoe Feld nidjt verbinbern, bas bicfe von ifm fid befteit, ja wir möd)ten eben bas

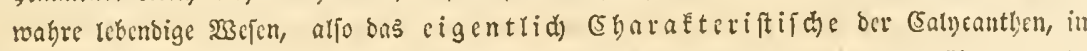

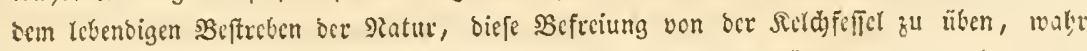
uno ridjtig erfennen. Unabjebbare Siünftelei vürbe confequent für bas b̧anze bedungen, wollte man cine ober bic andere Brtuppe ober Baattung wegen bypogunifajen 3uftandes

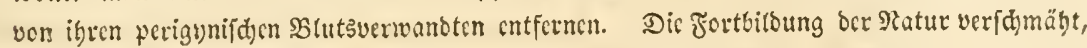

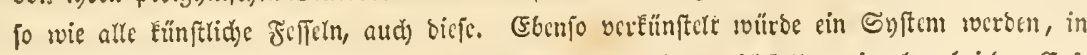
bem man wieber verfennen wollte, daß̧ ber હaame Gier gleidjfalls, wie oben bei ben (sai=

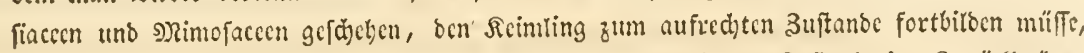

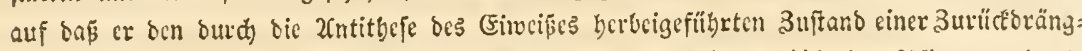

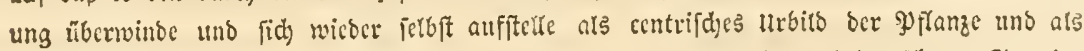

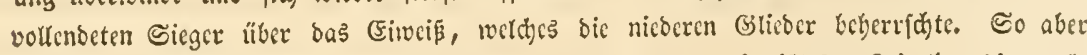
vorbcreitet, cmpfängt bie corolla rosacea mit gersbem, eiuwiß̄ofen Seimting bic greßße Rofacenfamilie, als bic bödjfte Solfendung ocr antitbetifdjen Srbnung ber Rlaffe.

\section{Zamilie. Plojacecn: Rosaceae.}

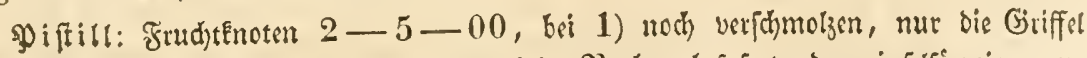
ziveitheilig ober einfertig (Cliffortia), Slarben befafert ober pinfelformig, nur

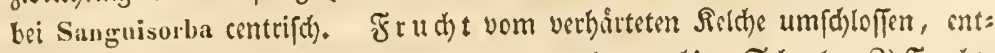
widelt fid) zu 2-1 Strinferne mit glatter ober grubiger Sthanle. 2) Frucht=

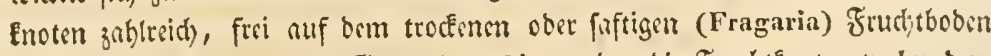

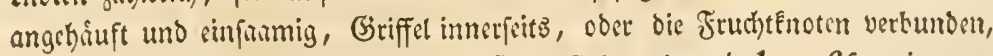
als Shapfefficher quirhartig zufammengeftellt (Spiraea) uno $1-6$ finmig, nn ber Sinnerumith auffpringend. 3) Fruthtenoten 2-00 in der Feid)róbre

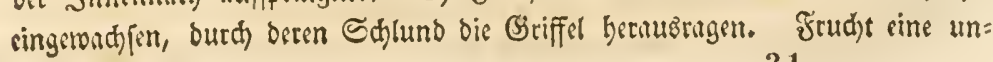


tate Stcinfrud)t mit $2-1$ Saamen in ber Nuß (Agrimonieae), Şagcbutte mit vielen 1 fannigen Steinfernen in Brei (Rosa), ober bie Sanmen ju 2

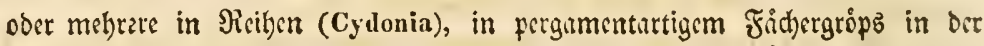
fleifhigen 2 fpfelfrudt.

Ea amen bängend, Seimling gernde, Bürgelden nad) oben, Sotylebonen

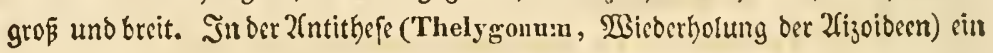
einzelner, grubiger, vertiEaler SteinEern mit Ercisformigem fisimling, um sin vertikares fleifhiges Eimeís auf beiben Seiten bernbgetogen, bie reifen Samen

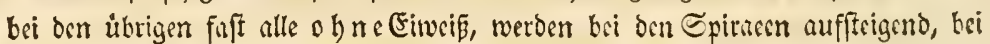
Den (Elifforticen und Sুomaceen aufred)t. - $\mathrm{Rel}$ (d) $5=(4-3-2)=$ theilig, bei ben Sanguiforben und Plotentilleen mit Dectblettthen, bicpe bei ben

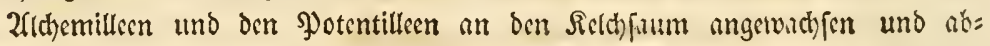
wechfelnoe 3mifhenzipfel barftelleno.

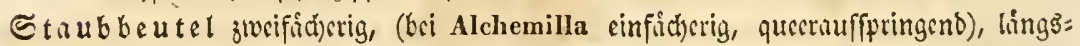
auffpringend, meift rumblich) und Eurz, aufred)t oder etwis amflirgent, bei

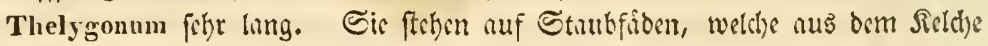
entipringen, meift frci oder menig verwachfen und in ber Snofpe sinwaitt ge= bogen finb, unift zu 00, bei menigen beftimmt $5-10-15-20$, bei Alchemilla uno Sanguisorba 4, bei Acaena 4-2, bci Aphanes 2-1. Blume bei 1) fchleno, bei 2) uno 3) rofenartig, 5 Slumenblitter mit Fur=

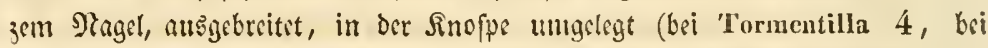
Dryas $8-9$, bi Cotoneaster uno Chamaemeles mufrecht).

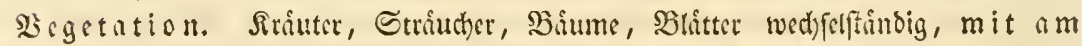
Slattfiele uno Zweige. angewad) fenen T(b) felblattern, meif gefingert unb geficdert, aud) unterbrod)en geficoert, ficocriputtig, bandformig, bei ben bie Gruppen befohießsenden Gantungen aber ungetbeitt, figerundig. -

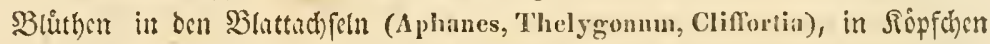
(Poterim, Acaena), 2fefren, Irmuken (Agrimonieae), SRifprn (Spiriea),

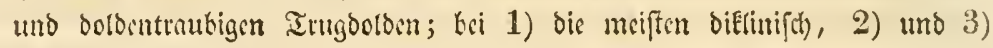
meift jivittertici).

(B) ruppen:

1) Sanguisorbeae: apetalae 1-2-styles lyypoclerocarpac. (Portulacacearum Sclerantlicarum repetitio).

a) Sanguisorbea genuin a : scmina prudula rectembrya.

a) Alchemilleac: antherac-1-loculares, stigma capitatum, bracteolae calyeis limbo adnatie. Fol, palmata, digitata.

Aphanes $I$, Alchemilla $L$.

B) Poterieac: antherae biloculares, stigmata penicillatit, bracteolae calycis tubo subjectac. Folia pinnata. - Conspect. 44224426.

y) Fothergilleae: antherae biloculares, stigmata subulata, folia integra. Drupa 1-sperma:

Cercocarpus K.II.B. - disperma : Fothergilla $L$. fil. 
b) C y ocrambeac: pyrenaceae embryone circulari verticaliter utrinque deflexo, (Aizoideas revocantes). Folia sueculenta.

Thelygonum L. (Cynocrambe T.).

c) Cliffortieae: drupaceae semine erecto rectembryo. Folia uni-trifoliolata.

Cliffortia $L$.

2) Potentilleac: corollatae schizo-eleutheroearpae.

a) Potentilleae gen uinae: caryopsides siccae 1-spermae in receptaculo aggregatae. Conspect. $4433-4+46$.

b) Rubeae: caryopsides succuleutae (acini) in receptaculo aggregatae. Cylactis Rafin, Rubus $L$.

c) Spiraceae: capsula loculis follicularibus verticillatis $1-6$ spermis. Conspect. $4449-4458$.

3) Roseac: corollatae schizostyles et schizogyna hypocarpae.

a) Agrimonieae: spicatae-fasciculatac, schizostyles sclerocarpae.

Agrimonia T. $L$. Aremonia Neck. (Amonia Nestl. Spallanzania Pollin.)

b) Roscae genuiuac: centriflorae in calycis tubo carnescente po- Iypyrenae.

Rosa T.L. Huiltenia Dumort. 1825. (Lowea Lindl. 1829)

c) Pomaceac: schizogrnae (opponitur Crataeg. monogyna) pomiferae i. e. prrenas osseas aut eapsulam membranaceam in calycis tubo carnescente includentes, seminibus erectis.

Conspect. $4460-4421$.

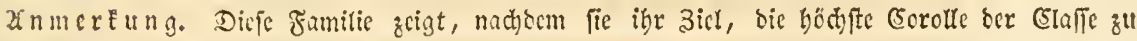

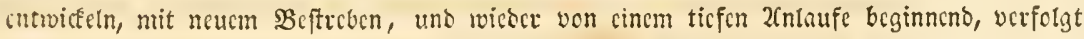

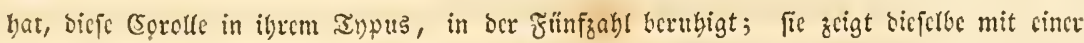

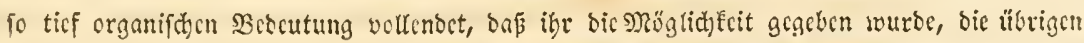

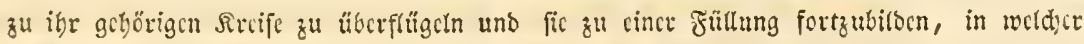
wir fic ats bas ฐocal cincr $\mathfrak{B l u m e}$ crÉEnnen und licben.

S3it bic Bruppden ber crften (sruppe atts ben vorkergegangenen Familien fich ber:

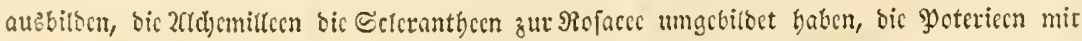

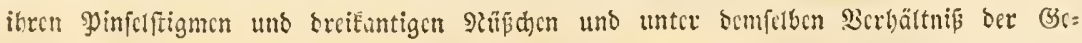

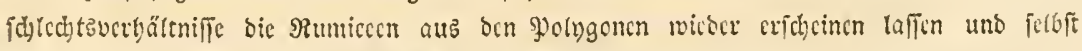

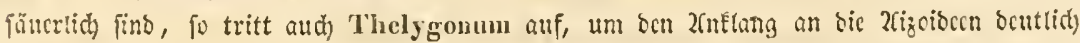

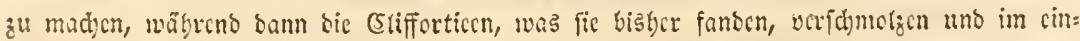

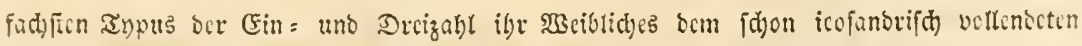

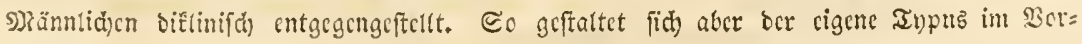

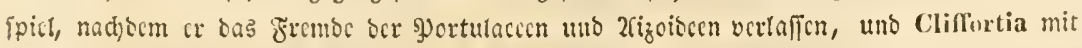

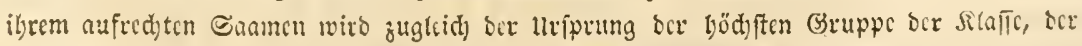

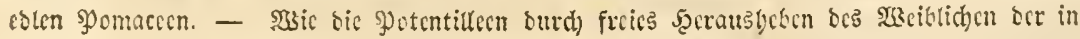

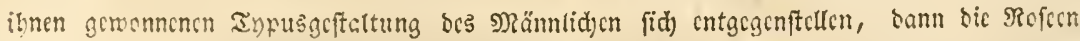

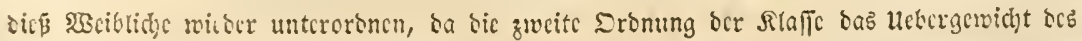

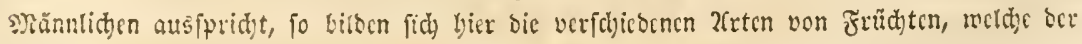


Reldblütthige.

entgrgengefergte 3uftand geftattet; bie vollendetffe, b. h. freicfte Frudtet ift bic Brombcre

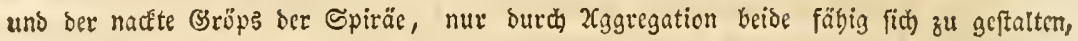

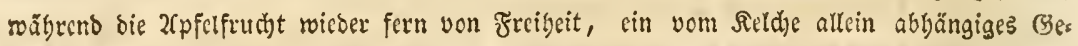

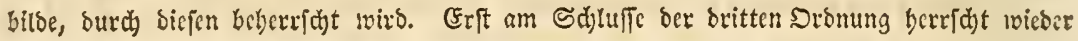

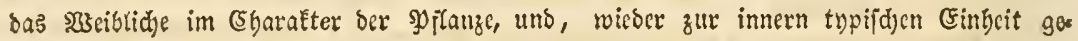

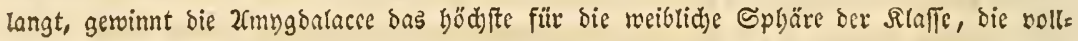

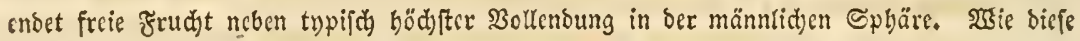
britte uns tef̧te sronung beginnt und fid) fortbitbet, eröffnen uns bie beiben folgenden Raciben ber Dnagrifloren uno Myrtifloren fogtcict.

\section{9. zamilic. Saloragen: Halorageae.}

Siftill: Fruct)tenoten eingewadfen, einfach) ober aus mebreren verfd)moljen, mit

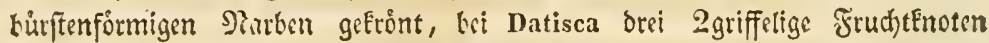

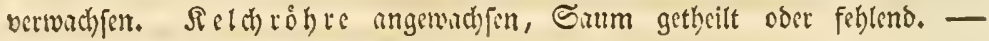

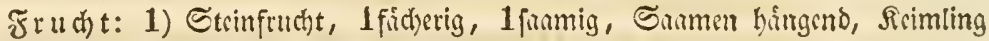

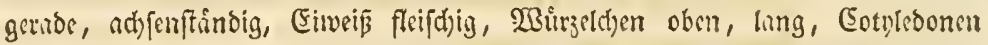
Elcin, 2) melbr=, 3-2-4fidherig, cinzelfarmig, Sanme ebenfo, 3) 1: fitherige Sippel mit 3 wambftindigen Sanmentrigem, Saumen zultreich,

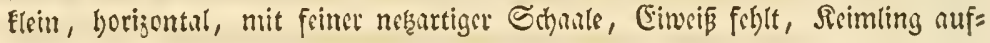
vectit.

Staи6ьсиtel: $1-4-8-15$, find 2 fofcherig, lingsaufpringent, aufrect oder aufliegento auf meift Eurzen Ctaubfíben, weldje nuz ocm Boden oez Sicldes entiptingen. Silume feblt, oder bei 2) 4 blitterig Ereuzftinoig, mit

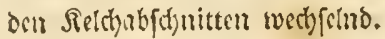

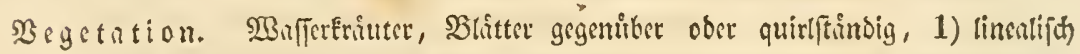
und gambrandig, 2) chenfo oder gefigt und frin ficberfpaltig (nts Silnttgerippe), bie Ruftbritter and) lanzetlid) und tief gefiggt oder bei 3) geficdert und bis

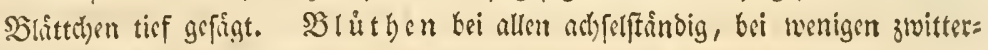
lid), bei ben meiften cin= oder zweib åt

(3rippen:

1) Hippurideac: apetalae monandrạc, styltis unilateralis, semea 1, pendulum, calycis limbus integer minimus.

Hippuris $L$.

2) Myri opltylle a e: $0-4$. pctalae, $3-4-8$ andrae, schizostyles $3-2$ -4 Ioculares singulispermac.

Serpicula L, Proserpinaca L* (Trixis Mitch, Gärt.) Myriophyllım V'aill. Goniocarpus Kön. Meionectes $\boldsymbol{R}, \boldsymbol{B r}$. Haloragis Forst. Cercotlia Murr.

3) Datis ceac: apctalae polyspcrmae exalbuminosac.

Tetrameles R.Br, Datisca $Z$. 


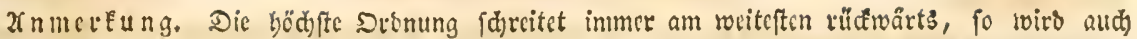
bict aus ser nicorigften bas nicbrigfte (slied, bie weiblidge Eingcit, wicocrbolt, nit ägns

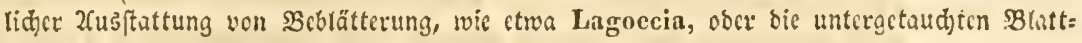
gerippe von Sium u. a. Datisca findet in ber gamzen \$j flanzennatur Eeine nähcren Ber: wandten als hicr, ift aber mit mandisen nodj byer zu vergleidyen, als mit ber von einem

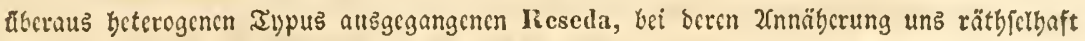
bleiben würde, roorin eigentlid Berwandtjdaft gejucht weroen follte. Banz dicjelbe Forts bildungsweife bes 2 seiblidjen, von ber cinfaamigen હteinfudjt mit eiveiphaltigem Sanmen zur vieffamigen Rapfet mit cibcißlofem Saamen, bieten aud bie folgenden Famitien bar und fie ift bic gewöbnlidje Entfaltung ans oer einfuchften Frudbtform, wie in biejer Flaffe,

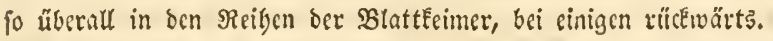

110. Fimilie. Stad dytferjet: Onagraceac.

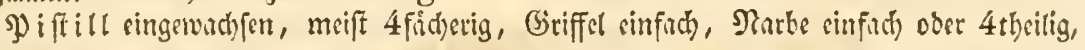

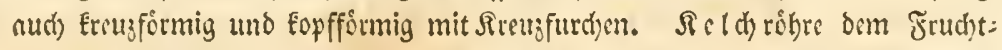
Enoten angewady [en, bei einigen (Ebilobieac, Fuchsieac) nod) itber ben Frudt) fnoten himas verlingert, Saum 4theilig, bei Circaca nur 2theilig, bri ben bơchften Formen (Combreteac, Alangieae) auch 5 theilig uno Eluppig (Granateae),

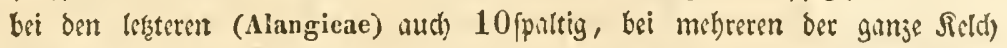

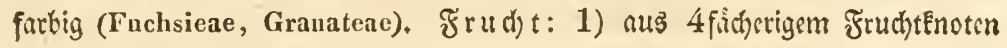
sine 1 faumige tußartige Strinjutht, Saume bangeno, groß̈, obne Eitwcis̄,

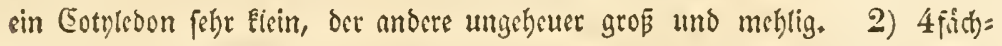

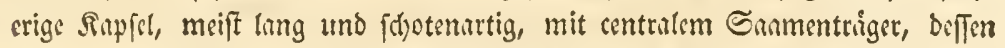

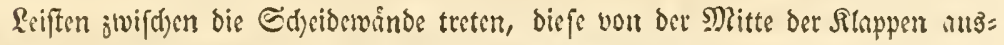
gehend; bei andern beerentrtig und nicht auffpringent (Fuchsieae). Sa $\widetilde{S}_{a}$

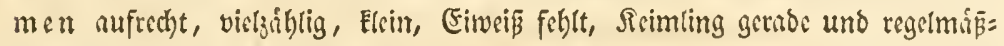

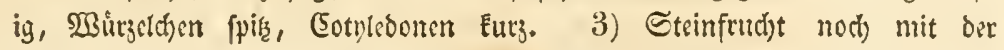
Neigung an oer Spike nufzufpringen, gefligeit (Combretum), ober rippig uno

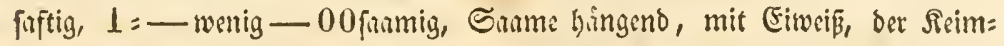
ling gernoc, (5ety)lebonen bif und gefaltet (Combreteae gen,), ober zufim= mengeroflt (Terminalieae, Granatcae), ober berzformig und fluch (Alangiene).

Staubbeutel: $2-4-8-10$, nur bri siner Gittung 00 (Punica), an=

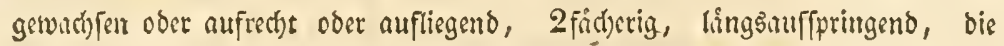

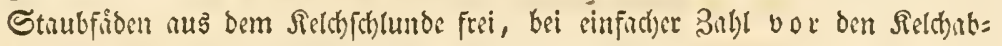
fdhitten eingefigt, bei boppetter fteht die zweite Reibe vor ben Btumentittern, bei mehreren Giatturgen mit sinem Drifenpolfer wed)felno, weldes bei sini= gen (Circeae) ringfórmig ift. $\mathfrak{B}$ lume fehlt krimenigen (Isuardia, Termina-

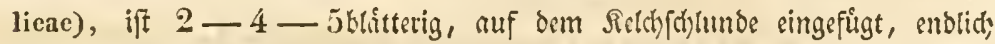
onppeljiblig (Alangium), meift ausgebreitet, in der firtope umyelegt ober um: rollt, bei Fuclısia coccinea etc. fo bleibeno.

Digetation. Siruter, Stråucher, Bioume, Bergwcigung und bic ungetbeilten fiedernervigen Slåtter gegenuiber ober wectyjefftindig, bei viclen gejăhnt Trapeae, Oenothereac, Lopezicae), bei úbrigen meift ganjranoig, bci 1) bie un= tergetutudeten mur als Blattgerippe. Blitben jwitterlid), bei wenigen biflinifd) (Montinia, polngamifhe Terminalieac), aus ben Blattuchfeln sinjeln obir 
Selthblunthige.

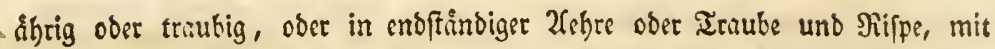
ober obne Deckblattthen. Sei Trapa die Reimung monofotylebonifh, brr grope Eotyledon bleibt anftatt cines Eimeipéforpers im Saamen, bei ůtrigen

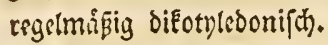

\section{Gruppen:}

1) Trapea e: semen 1, in drupa infera pendulnm, cotyledon altera minima germinans, altera maxima albuminosa et restans.

Trapa $L$.

2) 0 enothereae: erecti-00-spermae capsulares et baccatae.

a) J ns sic u e a : germen calycis límbo coronatum, capsula, Isnardia L. (Dantia $A, P, T h$ ) Ludwigia $L$. Prieurea DeC. Jussiena $L$.

b) Epilobieae: calycis tubo ultra capsulam plus vel minus prodncto, deciduo. Capsula $\propto . \beta$. sessilis, $\gamma$-stipitata.

«) Camissonicae: processus tubi calycini brerissimus, limbus refiexus, stamina alterna longiora, stigma indivisum.

Gayophytum A,Juss, Camissonia Lk. (Oenothera: Sphaerostigma DeC. Agassizia et Holostigma Spach.) - Calylophis Spach.

f) Onagreae: processus tubi elongatus, limbus reflexus, stigma crociatun.

ux) Stamina aequalia, capsnla 00-sperma. Anogra (antea Baumannia) Spach. Oenothera L. Megapterium Spach. Onagra T. Pachylophis Spach. Larauxia Spach. Hartmannia Spach, Kneiffia Spach, Xylopleurnm Spach.

$\beta \beta)$ Stamina inaequalia, capsula subdrupacea oligosperma,

Gauridium Spach. Gaura L. Schizocarya Spach. Stenosiplion Spach.

yr) Stamina inacqualia, capsula siliquosa 00-sperma, semina appendiculata aut comosa.

Cratericarpum Spach. Boisduvalia Spach. Godetia Spach. Phaeostoma Spach, Eucharidium Fisch, C. A, Mey. Clarkia Prsh. Chamaenerium $T$. Fpilubium $L$, Crossostigma Spach.

ข) Zauschnerica e: processus tubi elongatus, capsula stipitata 1locularis 4 valvis 00 -sperua.

Zauschneria Prsl.

a) Fuch hieac: calycis tubo producto, a bacea deviduo.

Brebissonia spach. Lyciopsis Spach. Kïirschlegeria Spach. Fuchisia $L$ Schufia Spuch. Skinnera Forst.

3) Circaeeac: drupa sicca vel baccans.

a) Lopezieac: petala $2-4$, drupa erecti-2-00-sperma, corolla 0 aut irregularis, stamen $\mathbf{1 - 2}$.

Riesenbachia Prsl. Cirenea $L$. Lopezia Crv. 
b) Combreteae : petala $0-2-4-5$, drupa, semina pendula al buminosa, in $\gamma$ exalbuminosa.

a) Combretea genuina e: corollatae 4-5 petalae, 8-10-andrae, embryo rectus, cotyledones crassae plicatae.

Combretum Lüffl. Cacoucia Aubl. Quisqualis Rmph, Lumnitzera Willd.

B) Terminalieac: subapetalac 10-andrae, cotyledones convolutac. Conspect, 45u2 - 4546.

y) Granateae: 5-6-petalae 00-andrae, cotyledones convolutae (semina 00 in balausta: pounum corticatum coronatum, infra diaphragma subtriloculare, supra 5-9-loculare dissepimentis membranaceis, placentis parietalibus).

Punica 'T.L.

c) Alangica e: petala ealycis laciniis dupla (10), drupa 3-1 sperwa, semina pendula al buminosa, cotylelones cordatae.

Alangium Lam. (Angolamia Scop.)

Uf nmertulng. Dic Familie tritt burd) bic (Fridycinung ber Corolle als 2intithefe für bie vorige auf und biefer Diganentreis bildet in ifr fich burdy, bỉ zur నormzagl ber $\mathfrak{B l a t t =}$ Ecimer, biefe logar enolich verbopperno.

23ic bie Trapeen die exfte Familie in bic cFrinnerung zurinceführen, bic Denothereen ibern reinen Iypus Elar entfalten und mannigfaltig geftalten, bic (Sireacen endrid Fremo:

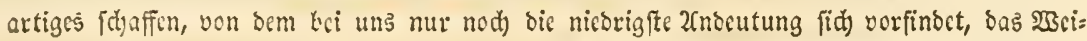

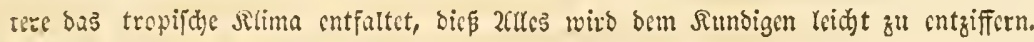

11. Jamitie. 23et\$ride: Lythrarieae.

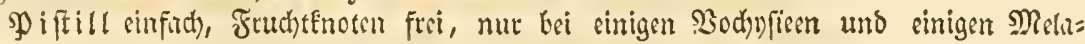
ftomen ganz ober theitweife cingewach fen, Birifer einfuch mit Fopfformiget

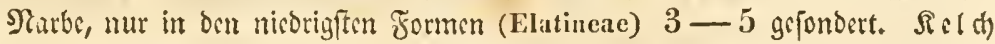
bei ben Elntincen ticf 3 - 5thcilig, bei librigen rôbrig uno rippig, am Saume

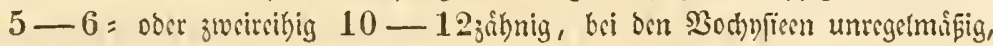
oberfer 2(bjd)nitt gefpornt. Frudi)t: 1) Sinpfet mit centratem Sarmentrigar,

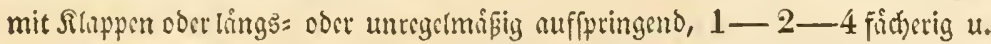

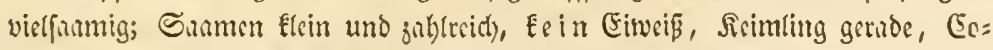
ty)(roonen fluch und biatturtig, 2) Sinpid mit contrulem Sanmentriger, 3=

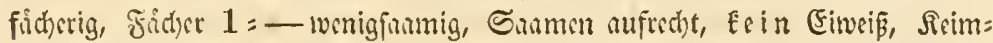

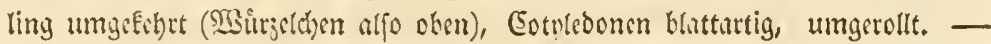

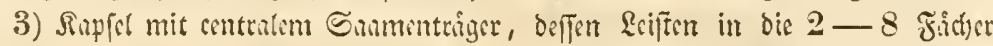
cintreten und vielfaumig find, mit Jiluppen aufipringend, welthe in bir Mitte

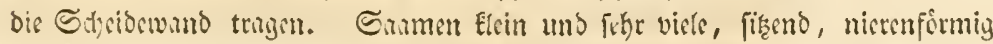

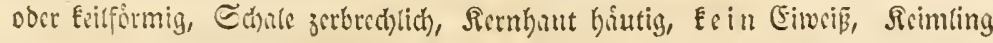
bogenformig oder geribe, gefputten.

๔taubbeutel: 1) 2ficherig, $3-6-8$ oder $5-10-12-15$, dir

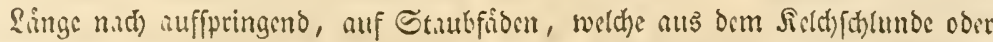

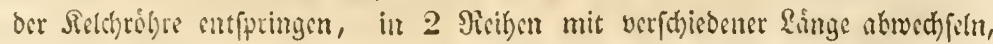

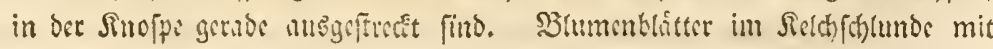




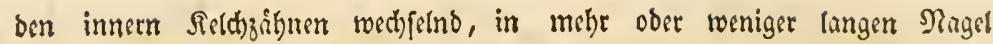
verfdimåtert. - 2) Stubbfiben $1-5$, tief im Sielche entfpringend, since mit 4 fåd)erigem Seutel, hibrige unfruct)tbar; Błtumenblitter $1-2-3-$

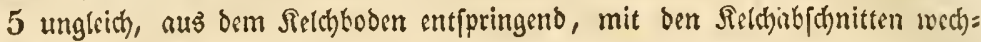
feinb, in einen Slagel verfómailert, bis mittlere am grésten, breit und aus= geferbt. 3) Stuubbuttel linggeffrecêt 2fácherig, an ber Spife mit 1-2 Sodpern ober Spalten auffpringend, ber Eteg (connectivum) nad) unten ver: lingert, bafelbft verdiaft und parig geppornt, auf bem Strubfaden singeicnet, abred) felno unvoltsommen ausgebildet und unfruchtbar. Staubfiden boppel=

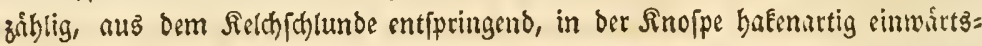
gebogen, sBlumenblitter $4-5-6-8$ in ber Sinofpe umeinandergelegt, Damn mcif auggebrcitst, bei einigen ein buinnes Fleifdppolfer.

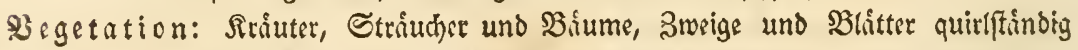
ober meift gegenuber, bei einigen zugleidh wechfetffíndige (Lythrum Hyssopifolia), Blatter meift glatt, glinzend und ganzrnonoig, auch leberartig und fiedernervig, bei ben Melaftomen nervig und meift fein rwimperartig figennoig und run: zelig. SB túthen zroitterlid, in ben Siattachfeln, auch âbren = und trauken= artig beblittert, bei ben boberen Formen auch einzeln entoftandig und in End: trauben uno Siifpen.

Gruppen:

1) Lythreae: orthostemoneae, schizo - aut holostyles, calyx regularis, semina obtusa.

a) Elatineae: sehizostyles, -

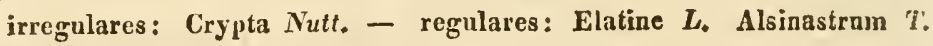
Bergia L. Tetradiclis Stev. Merimea Cambess.

b) S a licarieae: holostyles, semina obtusa, calycis limbus plurimis alternatim dentatus.

Conspccl. 4550-4552. 4555-45i6.

c) La gerstroemie a e: holostyles, semina alata (pterospermae), calyx ralvatus. Conspect. $4577-4578$.

2) Vochysieac: orthostemoneac, holostyles, calyx calcaratus irregulariter partitus. (Corolla irregulariter $\mathbf{1 - 5}$ petala, stamen $\mathbf{1 - 5}$, unius anthera 4-loculari, reliquorum nulla. Capsula Iibera aut sublibera 3 locularis 3-valvis loculicida, semina singula ant pauea pendula embryo inversus, cotyledones convolutac, albumen uullum).

Consp. $4506-4516$.

3) M cla stomeac: campylostemoneac, (stamina duplicata, antherae calcaratae, alternae steriles. Consp. 4580-4618. Ewyckia Blume etc.

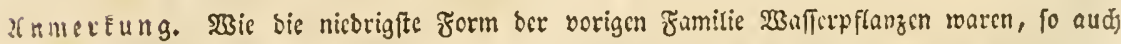

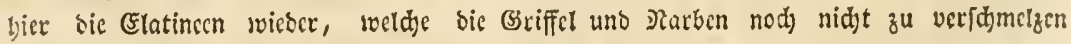
vermodten. Deren 26nerfennung als Blicber biefer Familic, wie id fie im Jabre

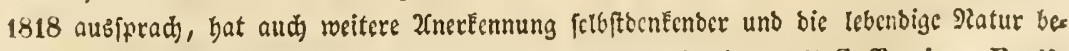
fádauenber Forfder gefunben. Die Fortbilbung ber Ealicarieen mit Sufrenia, Peplis unb Lythrum beginnend, erfhliept immer flarer ibren Typus, bis in ber Jentit)efe bie 
Erinnerung an bie (5ombretecn, atb bie Bollenoung ber Şauptantitycje bicfer Rcihe, wicber

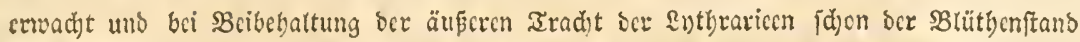

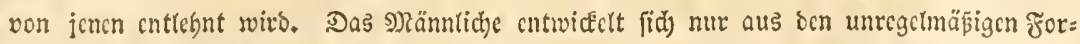

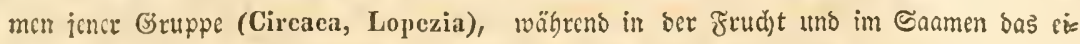

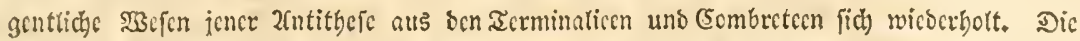

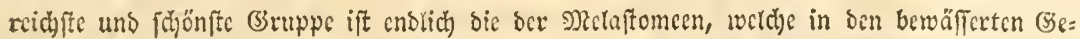

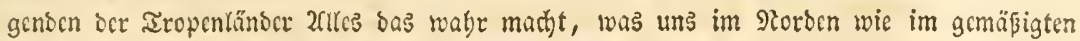
Ranbe, unfer Lythrum bcjdjciben verfünbet.

112. Familic. \$3olygnlnce tt: Polygalaceae.

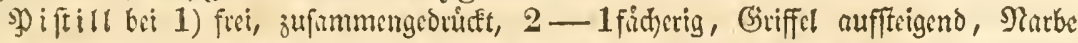

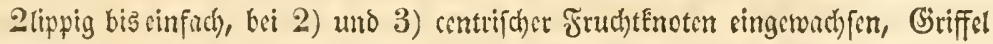

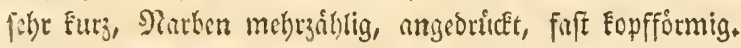

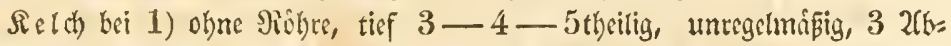
fdynitte sufferlid, bavon siner oben, 2 nach unten gerichtet, bei einigen fpect=

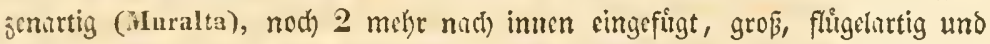
farbig, oder alle 5 Selthabfonnitte innen farbig (Krameria), bei 2) und 3)

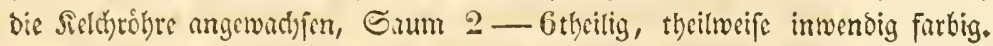

Frudt: 1) Sapfel ober Eteinfrud)t, 2Elappig ober Elappentos und leber=

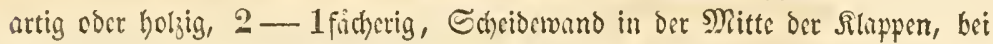

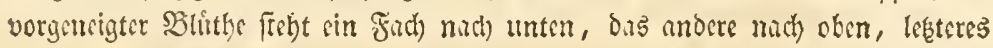
ksi vielen verfilmmert. Eanmen cinzeln, unter bor Spiąe bernbhingend,

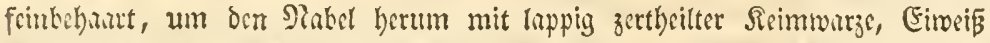

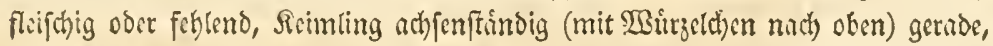
bsi Tírameria der Eaume gejphnabelt unit erbabener Linie an ber Jnnenfeite,

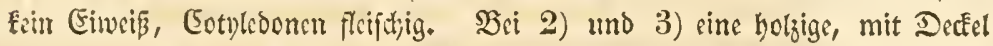
aufipringende, oder beercnartige, nid)t auffpringende Siapfel, 2-6findherig, vielfaumig, Saumen aus der Mittelfâtule, kei einigen (Couroupita) in Brei,

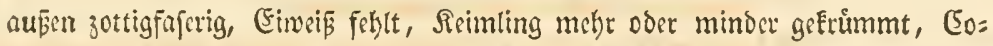
tyledonen fleifotig, bei einigen verwad)fen (Lecythis, Bertholletia), bei andern bhettrrtig und gerdort (Couroupita).

Staubbeutel: 1 a) ju 8-10, gleidfiam batbe, 1 fócherig, Eculenformig, an ber

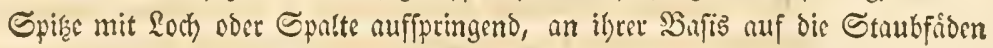

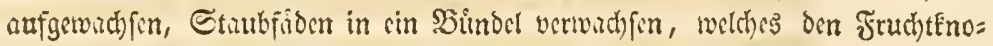
ten von unten fdyeidenartig umgiebt, in Der Mitte gefpalten (biaderwhijd) uno mit den freien Enden ber Etaukfiden aufwirtşgebogen ift, bei andern

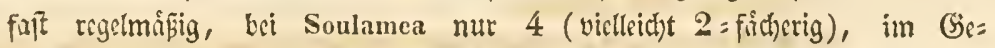
gmfar bei b) Tramericac find 3-4-1 bupognnifhe Staubgefípe, sin:

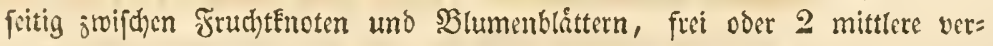

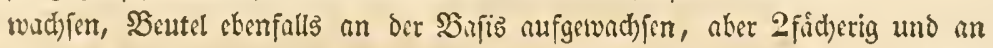
Der Epitie mit 2 Ridhern auffpringeno; bei c) Tremandreac 8-10 Beu=

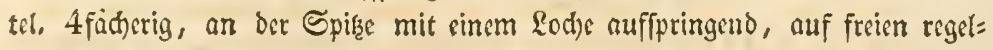

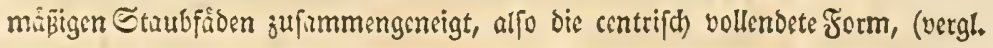
hort. bot. t. 38.).

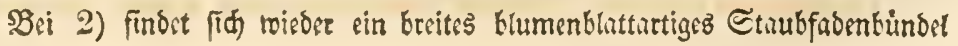


vom centrifh) geroorbenen Poiftll nus mad) unten, von da auffeigeno (es iff bie Fortbiloung Des Mittelftick's vom petalum barbatum von Polygala) Eabn= fờmig an feiner Spike zeróftelt (gennu twic bie carina bei Polygala speciosa, myrtifolia etc. etc.) uno 00 z̧âlylige, 2 fficherige, aufrechte Beutel tra=

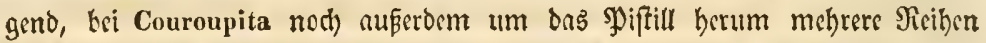

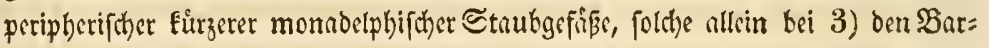
ringtonieen, (zu denen Couronpita bas Bindeglico ift).

Blume bri 1) beutliche Biederbolung ber Sdymetterlingsblume, Sdjif:= d)en am beftimmteften ausgrbilbet, mit cinfachem Ragel, bas Staubfnden= bindel umpd)liesento, an feinem Ende aufferigend und jeriffelt (barba, crista, fterile Staubfóben), bei andern ift biefer 2fnhang klumenblattartig (Muralta, Comesperma); Fahme zweiblitterig uno Elein, meift jebes Blittchen mit zwei ungleithen Bipfeln, Flügel zweideutig, zugleich als innere Sicldbribe betrachtet,

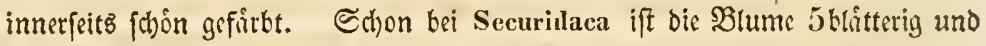
noch verwatbfen, bei Kraneria (erfte 2(ntithefe bes 2Bsiblid)en) wieder nur 3= bis 2blitterig uno anfrecht, in Capit. Beeches voy. t. V.), bei Tremandreas foron 4-5blitterig tno rigetmápig gemorden. Bei 2) und 3) die Sblume meift etwas ungleich), 6= blâtterig, auch wobl dic \$lumenblitter verwadffin (Couratari), bei ben lef̧ten

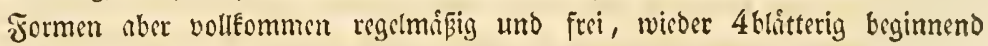
(Barringtonia), ' dann 6-8blitterig.

Bigetation. Sirutuer, Striucher uno Binme, Blitter wed)felftindig, bei meni= gen gegenuber ober quirlartig, meift Enbl uno ganzannoig, leosrnttig glinzeno, bei cinigen mur nervig, bei andern fieosrmervig uno neşroberig (Polygala sect. Senega, Lecy thideae, Barring tonia) uno weidjbchaart, bci sinigen burdjidsineno puufftirt (Polygala scet. Timutua, Polygala glandulosa etc.), bei boberen Formen (Tetratheca glaudnlosa, Lecythideac u. Barringtonia), unch) fígcrun=

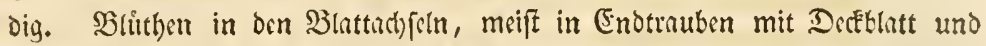
einem Part gegeniberftehender Doceblåttchen, an ber Bajts bes Bluthenfticles bei den Ginttungen mit freiem Fruchtenoten, an ber Bafis ber Seldhroihre bei sinigen mit singewadt) [enem Fruchtfnoten (Couroupita), in oer Mitte der $\mathfrak{B l}$ lu= thenfticle (Gustavia), ober endid) febleno (Barringtonia)?.

(Siuppen:

1) Polygaleac: capsula aut drupa verticaliter compressa2-1-loculario, loculis 1-2 spcrmis.

a) Polygaleac genuinac: antherae 1-loculares poro aut fissure simplici dehiscentes.

Conspect. 3029) - 3040 .

b) Kramericae: antherae 2-loculares, poro duplici dehiscentes.

Conspect, 3042.

c) Tremandreae: antherae' 4 -loculares, poro simplici dehiscentos, flore regulari.

Conspect, 5422 - 54\%3. 
2) Locythideae: capsula regularis 00-locularis, loculis 00-spermis, corolla irreguharis et stamina unilaterali-monadclpha (in Couropita siunt peripherica).

Conspect. $4649-4653$.

3) Barringtonicac: capsula indehiscens aut bacea 00-locularis 00sperma, calyx, corolla et staunina regulari-peripherica.

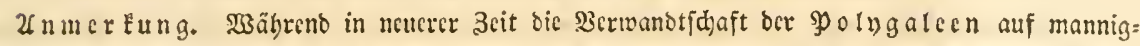
faltige 33 sife gebcutet wutbe und bie 3uftammenftellung mit ben Droferaceen, Biolaccen, Fumaricen und Ecguminofen mir nidjt ticf in ifrem \$3efen begrünoct eridjienen, fo labo

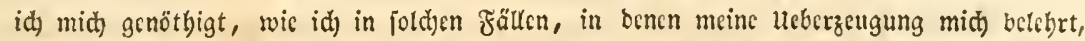
Daßs id über cinen (segenftand nidjt ganz flar bin, gern zll thun pflege, ber von mir am

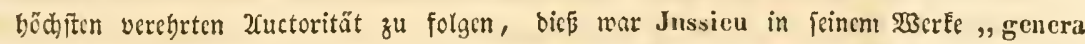

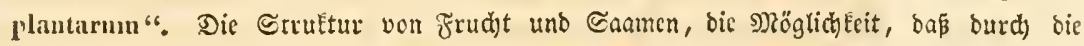

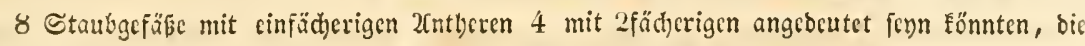
möglidje 2 ergleidjung ber Corolle mit oer von Stenochilus uns noh mandjes babituclle,

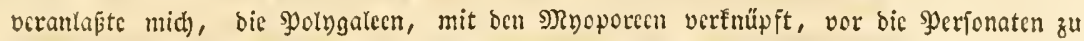
feşen, mit benen wieber sinige 2(nalogiecn burd) bic Metampyrecn fid barboten. Dffen

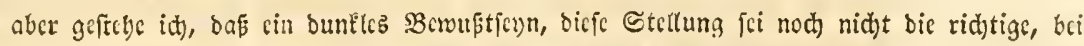

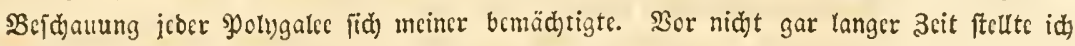

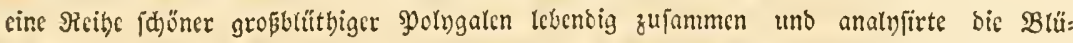
then von Polygala myrtifolia, macrophylla, oppositifolia, bracteata, speciosa, virgata, Muralta Ifeisteri und mixta, und verg(id) bamit getroctencte 20rten und Battun= gen, weldhe lebendig nidjt vorftanden. Die Fortbitoung ber Formen ourch Nuralta, Monina, Comesperma, Securidaca, bamn bic 2fntitbcje ber Krameria wurben balb Elax,

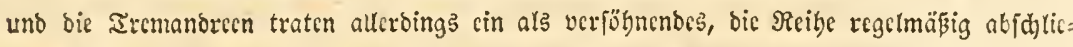

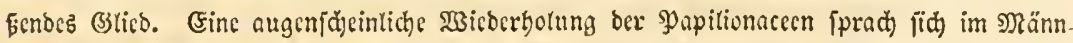
lidjen ber Sporngalecu zu beutlid aus, um verfannt werben zull fönnen, ber İpus: , an-

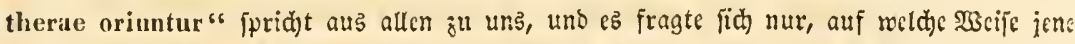

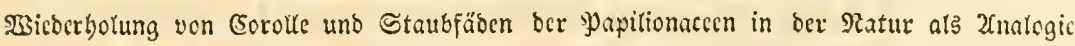
ober Confinität motivirt fci. (Fin Durd)muftern ocr männliden \&eguminojenrcibe fübrte

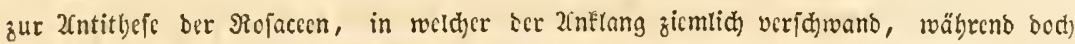

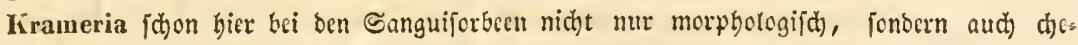

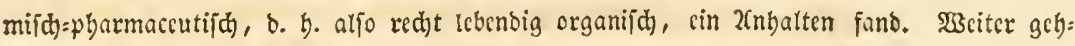

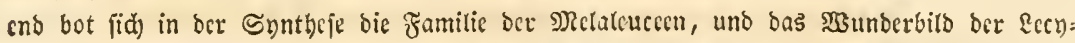

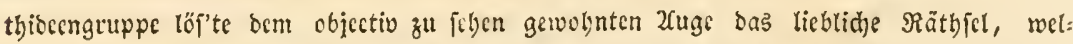
des bie Polugaleen burd) bie fchön geformte crista ober barba inres unteren $B$ lumenblat: tes barbicten. Dic \&centhibeen jagen unz, bas in oer ganzen \$pflanzennatur fein bomoge:

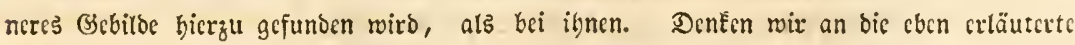

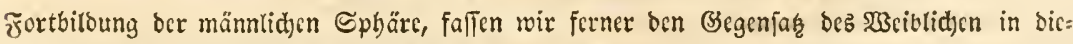

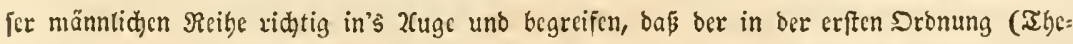

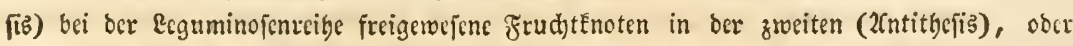

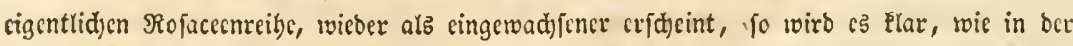
oritten Dronung (Gynthefï) obige Berbättniffe fid) alle vercinen, wic hier ber, ebenfo twic bei ben Eeguminofen, vertifal zujammengebrnicete Fruditenoten ber Sgolygaleen bann burd)

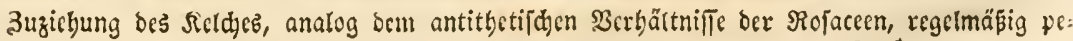


Seldyblüthige.

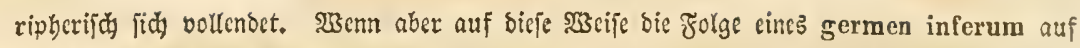

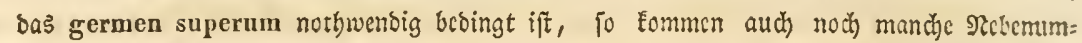

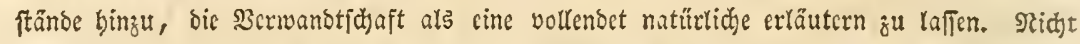

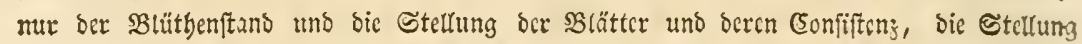

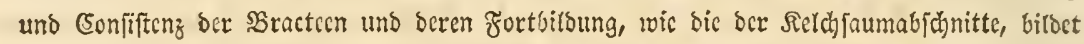

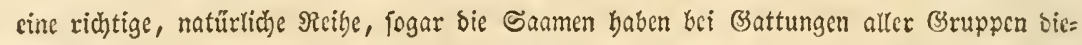

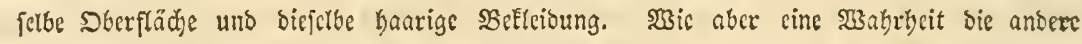
gebiert, fo lebrt aud bicje aufgefundene Berwandtichaft, bas sie Melatencecn, obrobl bie

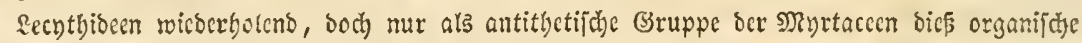
Berbältniß wicber beraujhcten fönnen, ba fic bon ber Familie ber Myrtacecn nur unna= türtich getrennt baren, bic morphologifdjen Notive jolvobl, als bic dyemifd)=pharmaceuti= fiden, fie abcr zu bicjen wieber zurüdefillgren.*)

.i3. Familie. Minrtacen: Myrtaceae.

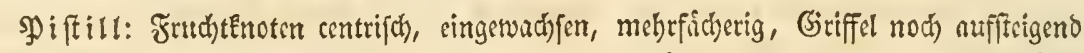

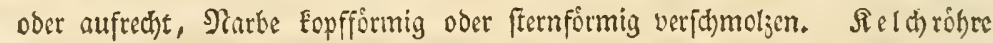

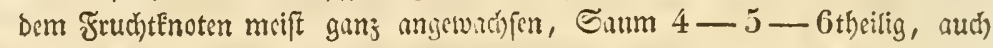
abfallend, bci wenigen (Eucalyptus, Calyptranthes) obme fich ju theilenrings: um abfpringeno und milęcroformig emporkebend.

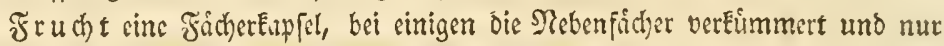

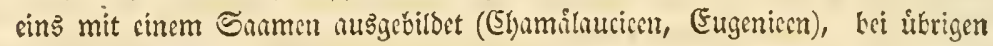

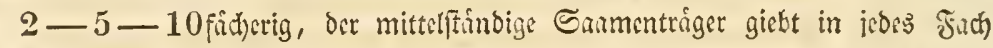

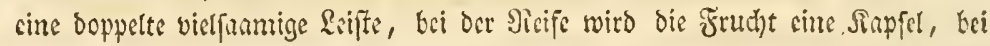
ben Meliteuceen holzig und bei sinigen viele unter fich und mit ber 2ffrtinde,

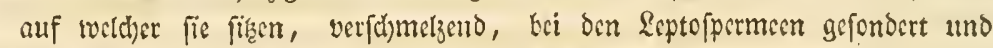
meift Eurj gefticlt, bei Minrtecn fleifhig, becremartig.

Saamen febr Elein und foilftubifnnlid) bei ben Mclalcuceen und Rep= tofpermeen, ober gröser, Euglid), länglich, nierenformig ober eckig, mit bogen=

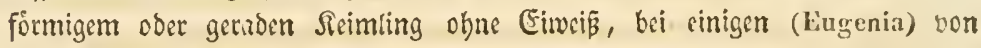
\{chongrimer Farbe.

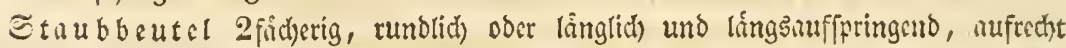
ober aufliegeno nuf Staubfiben, welche polnabclphifh (in Sitmoel vertachfen) ober etwas monade(phific) oder frei find, bei cinigen sinreifig beftimmtzinglig

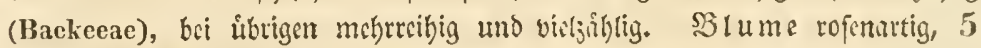

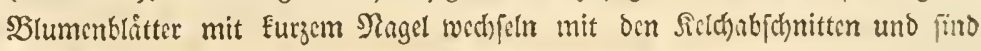

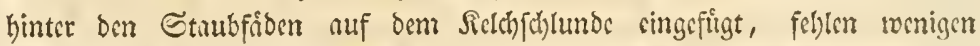
(Eucalyptus), oder fino flein und finum fichtbar (Calothamnus), ju ciner Mhize verwachfon, welche abfitlt (Eudesmia).

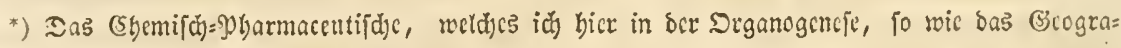

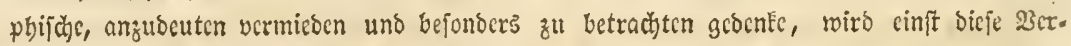

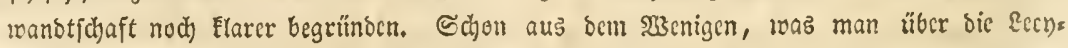

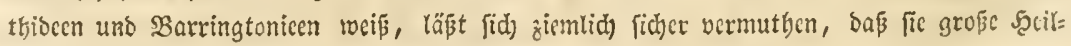
mittel barbicten bürften, bencn ber \$ुolngalen annalog, ganz veridjicoen von benen att ben Mintecn. - 


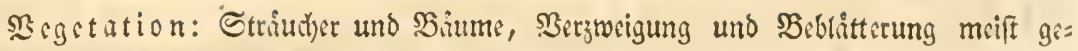
geniber, bei sinigen wect)fetmo (Esptopermen und einige Euculnpten im 20

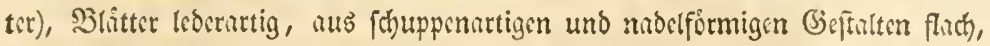
mift lanjetlid) und ganjrandig, bsi wenigen breit, mit zablecid)en Fiederner=

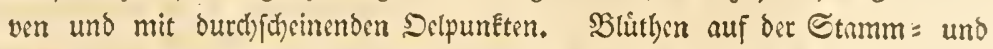

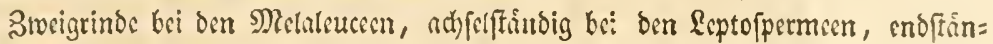

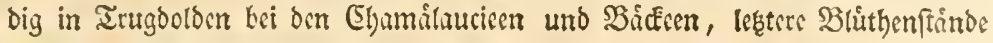
wisderbolt bei ben Miyrteen.

(5ruppen:

1) Melaleuceae: stamina polyadelpha (plurimis colorata et petalu parvula superantia) ultimis libera.

Calothamnus La B. (Calothamnus et Pentaphalanx). Billotia Colla. Lamarkea Gaudich. Beaufortia R.Br, Melaleuca $L$. líunzea $R c h b$, Callistemon R.Brr.

2) Chamacla ucieae: stamina uniserialia definita, libera aut subpolyadelpha, drupa e germine pluriloculari 1-sperma semine erecto.

Pileauthus LalB. Genetyllis $D \in C$, Chamaelaucium $D s f$. Verticordia DeC. Calythrix LaB.

3) Myrte a e: stamina uni-pluri-serialia sublibera, capsula apice deliseens aut bacca (certe junior) plurilocularis.

a) Baekeeae: stamina uniserialia 5-10-25, capsula $\Perp-4-5-10$ cularis.

Bartlingia Brngn.' Baekea L. Astartea DeC. Eudesmia RBr. Tristania $\mathrm{RBr}$. Lophostemon Schott.

b) Leptospermeae: stamina libera indefinita uniserialia capsula 2$4-5$ locularis.

Leptospermum Forst. Agonomyrtns Schauer. Fabricia Gürln.

c) M y r te a e genuinae: stamina indefinita pluriserialia.

z) Angop horeac: capsula 2-3-locularis.

Metrosideros Gärtn. Angophora Cav.

B) Eugeniea e: bacca drupacea matura 1-2-locularis loculis 1 2-spermis.

CaIsptranthes $S w$. Myrcia $D_{e} C$. Syzygium Gürt. Caryoplyyllıs T.L. Acmena DcC. Crossostylis Forst. Eugenia Mich. Jambosa Rmph。 - et (?) Catinga Aubl:

r) Psidieae: bacca plurilocularis, loculis 00-spermis.

Myrtus L. Jossinia Contmers, Psidium L. Camponanesia Rz. Nelitris Gürt. Souneratia L.*)

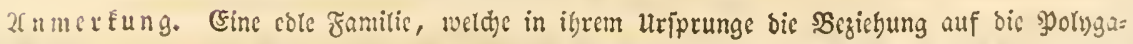

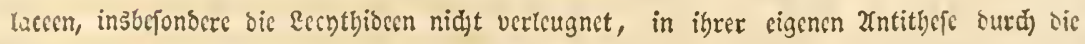

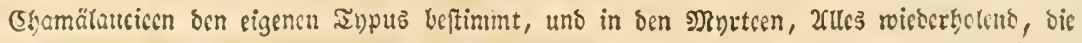
böbcre Syotenz ber Rofaces vertünoet.

-) D6 Viviania Colla (frübcr Melanopsidium Colla) uno Myrrhinium Sckott. bicther ge:

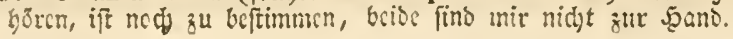


114. Familie. 2(umggonfnceru: Amygdalaceae.

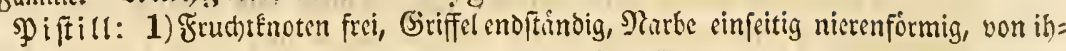

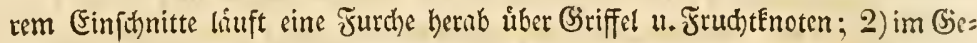
genfafec (Homalinae) ber Frudtetenoten etwas eingewathfen, oben aber Eigelfict: mig und frei, Griffel $3-5$, pfriemenfpifig; 3) bei ben Ehrnfobaluneen ift ber JrudotEnoten etwas zufammengeorictet, ber Griffel nuf Der einen Sirnte tief

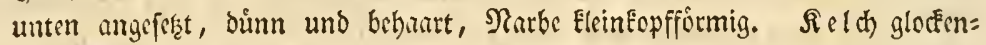
formig, Saum 5theilig, bei cinigen Scomalineen andi) mebrtbeilig, abfalleno.

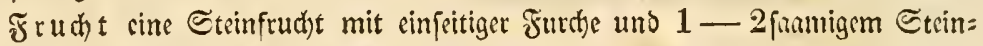
fern, hingeno, brahalb ber Snamenftring von ber Bafits entfpringend, um

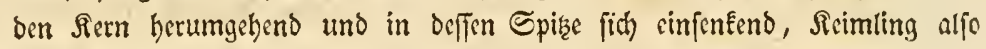

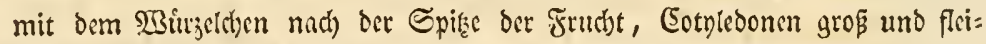

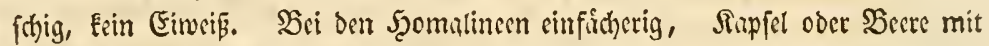
3 -5 wandftinndigen vielfaumigen Saamentrigern, Saamen horizontal,

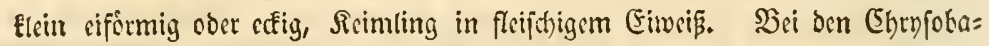
lanen cine pflaumenifnnlid)e Etcinfrud)t, Etcinfern rinnig gefurd)t, 1 fumig, Reimling groß́, mit fleifchigen Eotyledonen, in fleifhigem Eiweí (Ilirtclla). ober obne (Fimeiz (ưbrige Ehrnjokalineen).

Staubbeutel: zweifiof)erig, lings aufipringeno, auf Staubficoen aufrecht, tweldie auf bem Sicldfallumbe ringsum ftehen, bei 1) zahtreich und regelmifigig ver: theilt, monabelphiff, bei 2) ju $1-3-6$ vor jrocm Silumenbintte, frei, bei 3) ringsum $3-5-10-15-20-40$ uno frei, ober bei Thelyra uns Acioa cinfeitig, meift raubbanrig. SBlume 5blitterig, Şlu=

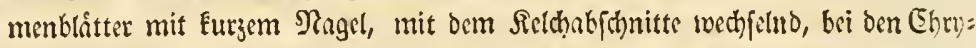
fobalaneen feht Elein uno binfállig, auch feblend (Stylobasinm), bei ben Şo=

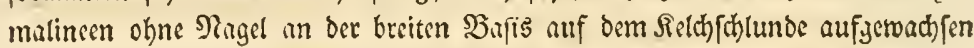
und mit rundithen Drufen akwidhfetnd, aud) boppelziatlig.

Begetation: Strifucher, meift Satume, Blitter wedffelfínbig, fiebernervig, viel=

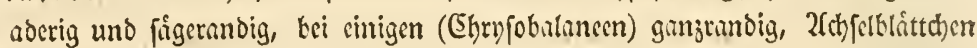
parig an ben jungen Trteben, Ppiterbin abfallend; Bluthen ans ben Slatt=

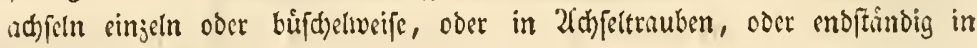
Irugdoiden (Şryjobalaneen).

(Bruppen:

1) Ceraseae: acro-holostyles (stỵlo apicali simplici).

Conspect. 4705-4i09.

2) Il omalinae: schizostyles (stylis $3-5$ ).

Conspect. $4679-4704$.

3) Chry 80 ba laneae: pleurostyles (stylo laterali subbasilari).

Conspect. $4517-4525$.

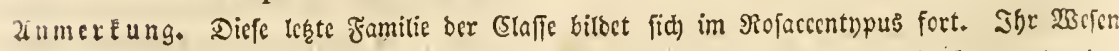

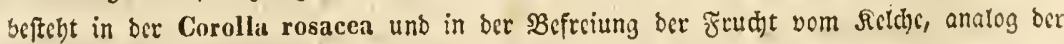

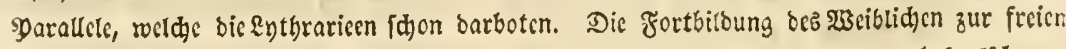

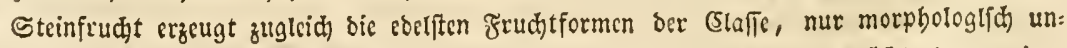

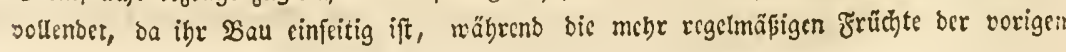




\section{Seldbblüthige.}

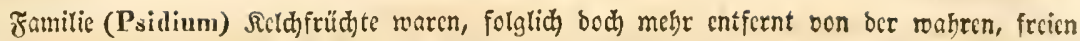

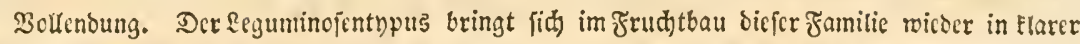
2xnocutung zur crneuten Erinncrung uno läßst fie als feine bödhfte Fortbitoung im Etrcber

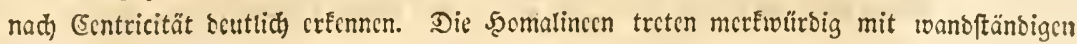

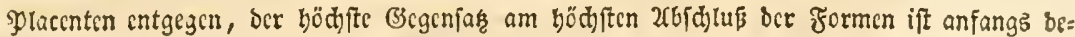

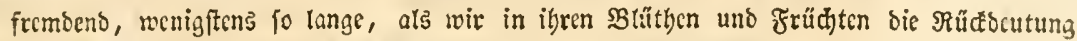

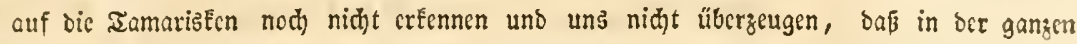
spflangennaiur Ecine (Sruppe verwanstere Formen cnthält, ałs bic ber Shryfobalaneen, bercn Grangeria u. a. mit bon Scomalinecn innig veriłdmetzen. Die Slumenblätter bei biefer Gruppe für innere sicldreibe zu nchmen, idjeint nid)t in ihrem

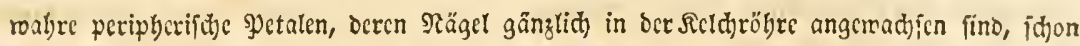
Napimoga berocij't bic vom Ricld ganz verifhicocne Icrtur und Betcibung, und fobato

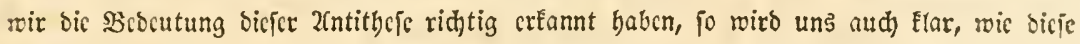
syctala bicr ebcnfo wie bci Tamarix, Reaumuria u. Hypericum nebft ocm ficldje fachen

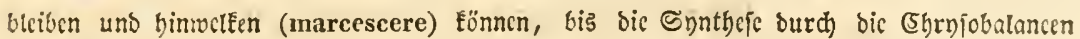

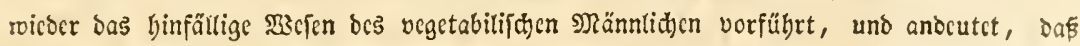

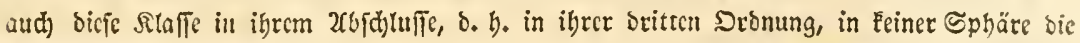

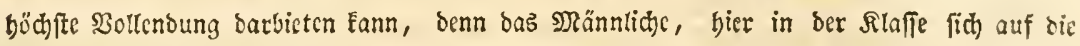

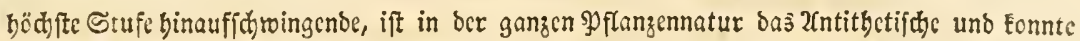

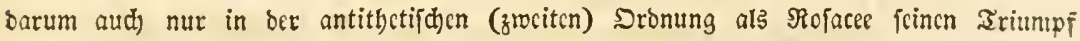

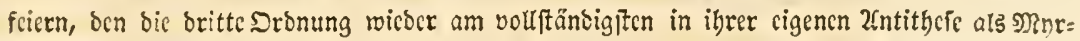

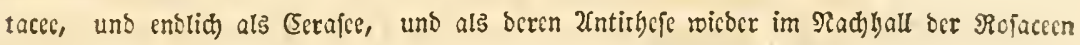

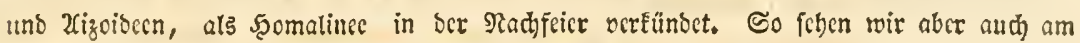

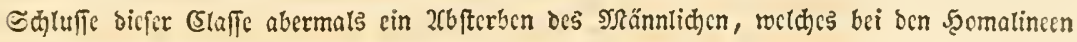

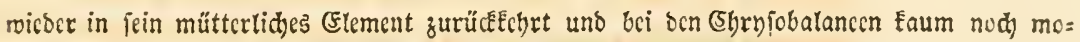

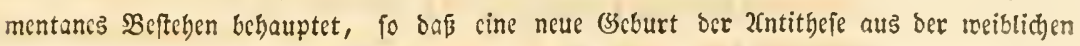
Ephäre burch bie leşte Rlafie fỉ wieber vermittelt. 
Etielblüthige.

Jamiticn.

Thalamanthae.

Familiac.

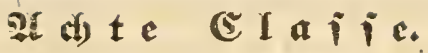 Eticlblíthige: Thalamanthae.}

Erifte Sronung.

Şoblfrüdige: Thylachocarpicae.

Fieugutithler: Cruciflorac.

115. Fam. Sicrmidutige: Tetradynamac.

116. Zam. Mobngenádbje: Papaveraccae,

117. Jan. Rapperngetwåd 5 : Capparideae.
(Siftusblüthler: Cistiflorae.

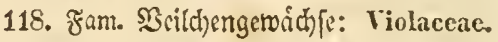
119. Fam. Gifrusgetwåd)fe: Cistineae. 120. Fam. Biraceen: Bixaceae.

\section{3ิtocite Dronung.}

\section{Epaltirühtige: Schizocarpicae.}

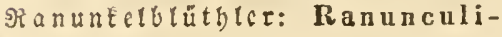
fl or a e.
ङ tor $\{$ (a)naclblüthler: Geraniiflorae.

121. Fam. RanunEelgetoidafe: Ranuncula - 124. Fam. Malvengewichje: Malraceac. ceae.

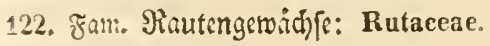

123. zum. Sapindrceen: Sapindaceae.

12.. Fam. Etordyfjnabelgctuidjfe: Geraniacere.

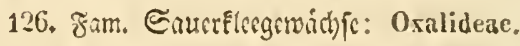

Dritte Sronung.

Cäulenfrüthtige: Idiocarpicae.

cinbenblutbler: Tiliflorae. Drangenblütblex: Aurantiflorae,

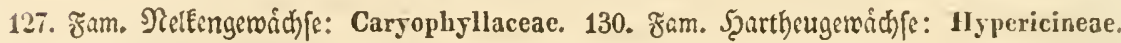
128. Fam. Thesgewádhfe: Theaceac. 131. Zam. Guttigemichfic: Guttiferae.

129. శăm, Lindengewáchf̣: Tiliaceac. 132. Fam. Srangengemichfe: Hesperideac.

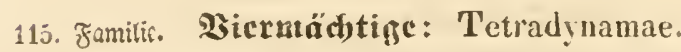

Piftill: fri, sinfach, Griffel meift Eurj, Narbe meift zweilippig, bei wenigen fopf:

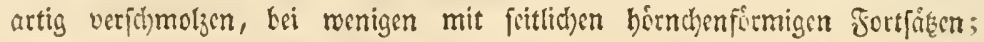
Saamentriger 2, gegenuber, Sängstippen bilbeno, oben in bic Etigmen ver= laufend. Rcld 4 blatterig, abfallend, ie 2 Reldblatter gegenuber, dic 2 ctmats mefje auperen bie 2 mehr inneren beckend, nur bei Ricolia uno Savignya alle Elluppig uno den Saamentrigern des Shiftills entfprectend, ge:

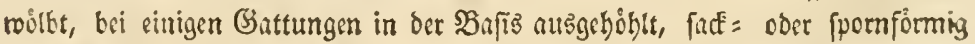

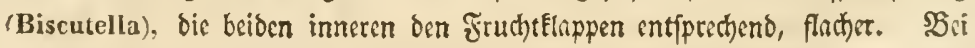


cinigen bleibt nutb 2(bfall ber Sicld)blatter sin Jitanj fteben (Teesdalia), bei

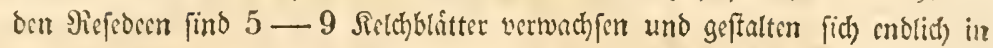
Ochradenus zu fhutfelfirmigem, 5zühnigen fielche. Frutht: 1) nidjt

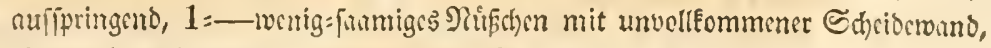
ober mefrere bergl. angereift, hei ber Sieife queer abpringend; 2) Echothen oder mehr geffect: Edhote, befteht nus brei Theilen: Scheiberband und 2 Silmpen, bic Edhcibewand ift sin Durd) bic rippenartig auffteigenden Sammen= triger gebildeter Slubmen, beffen Siundleiften tuten am Uriprunge und oben im Siriffel vercint fint und jwifdsen fith sin zarteż Şatutthen ausgefpannt balten,

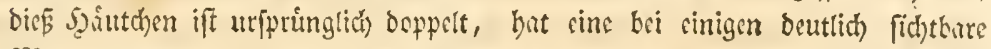
Mittelrippe (Sisymbrium Sophia, Ilïgueninia pl. erit. ic. 1330), butber es bei

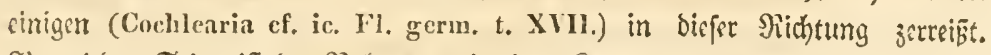

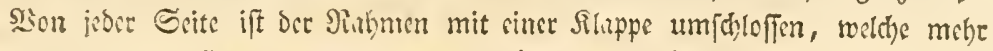

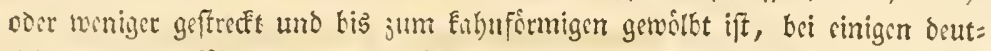

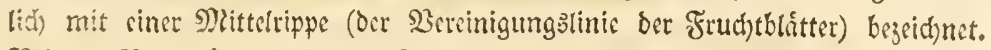

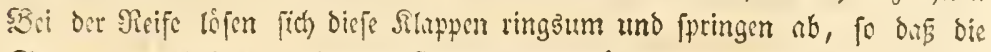
Carmen frei liegen und auggeftreut weroen Eonnen; bicfe find meife bang=

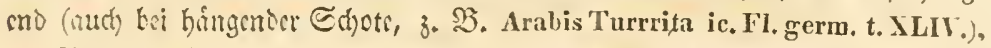
an Canmemftringen atls keibon Sanmentrigern zu beisen Seiten eines jeden

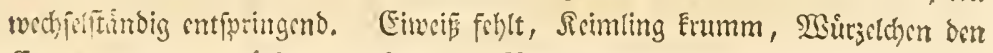

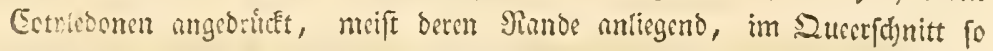

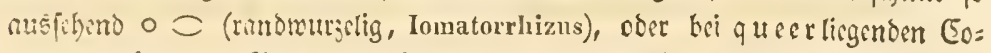
trlebonen liber bie Mitte bes Siuckens von sinem beribergebogen: $O$ () (rưféen=

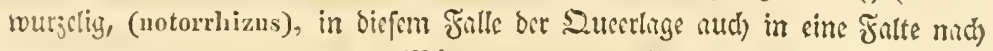
vort jufammengelegt und Das 2 Sitrzettyen in bie Falte bes sinen bincingrbo=

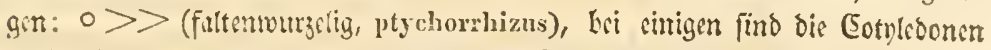
math fümal unb ling, bunn bei jenen sagen such woht fpitulig eingerolit (Fl. germ. ic. 4159. 4161.), oder bogig (IIort. bot. tab. 55.), bei viclen

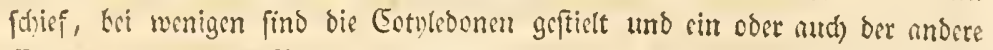
Cotnlidon an sincm Slumbe singephlugen, (margine inflexae: Dentaria ic. Fl. germ. 4314. 4315. 4319.), ober rund herum singercllt (Calepina in Fl. germ. 4163. Fig. F.). Sei Tetrapoma, (Tetracellium) bat burs Sdyotchen sin $4=$

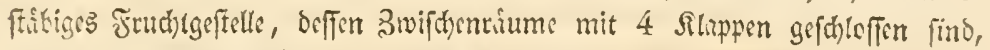

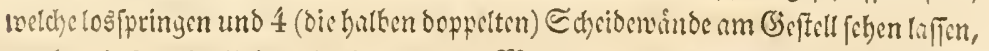

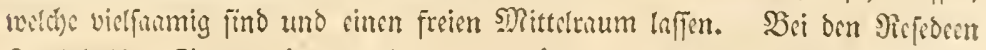

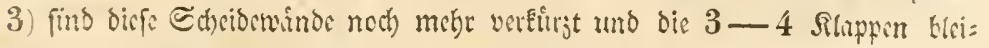

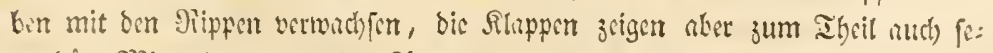

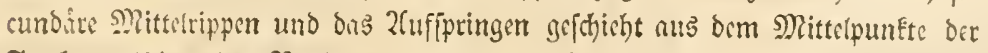

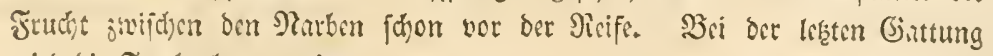
mito die Frutbt berrenitig.

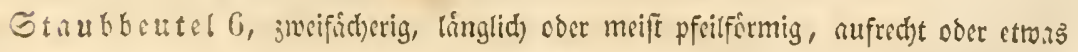

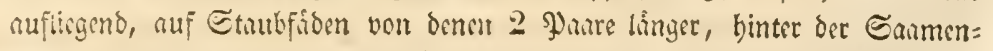

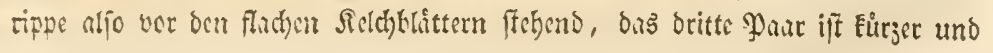


fteht jeber eingelne Staubfaben vor dem hocerrig getwólbten Feldjblatts, alfo hinter ber frudtelappe; bei reenigen find bie beiben Strubfíden bes lüngeren Şaares verwadbjen (Anchonium, Sterigma), bei anderen verkủmmert das

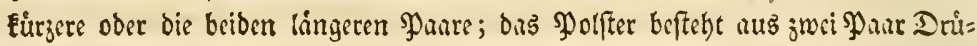
fen, jeber ber Eurrzeren Stuubfïben fteht zmifchen biejem \$laur singefught, bei

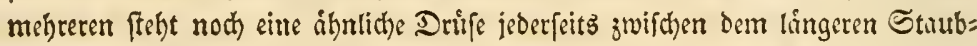
fabenpare, biefe 4-6 Drưfen find fleif̧hig und forongrum, bei anderen (Biattungen fommen nur 2 vor (Lunaria), auth 10 (Selenia). SBlumen= blatter 4, parig binter Den langen Staubfidenpanren ftebeno, in sinen

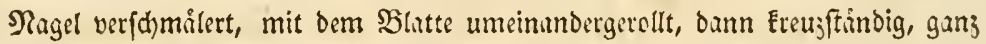
oder ausgeferbt ober fiederfpaltig (Sehizopetalum); alfo je jwiften einem han= gen uno einem Eurzen Staubfaben eins nad) außen gewendet, bei menigen paralld nach ihrer Saite (Berteroa Fl. germ. ic. 4284) oder bis Syliar dir cinen

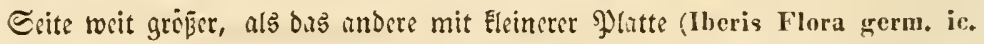
4191-4201.) S3ei 3) Den Siefedecn find die Drúper des Şolfters ju sinem

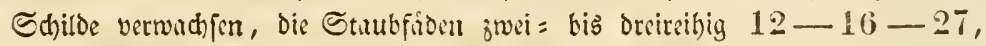

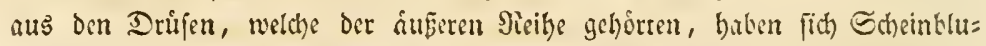
menblatter $3-5-6$ entwiffelt, tocld)e nus bickem, noch orüienurtigen, einem verwachjenen Stipulatpaare gleid)enden Pangel sine hanbformig jertbcilte jufammengefaltete Platte auscinunderlegen, lef̧tere das zertheilte Filument.

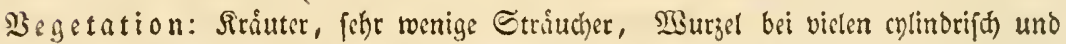
fpinbelformig, meift sin = und jweijifhrig, wenige peremnitend. Sllitter all=

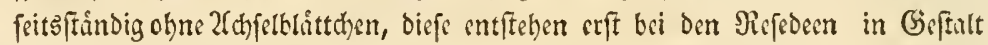
yon sin parar Drưfen, Shlitter nur bei Subularia obne Şlatte und piriemen= formig, meift Eoblartig, gez̧ihnt, fiederfpaltig, leierfortmig, bei wenigen ftrauch= artigen lederartig uno ganzrunbig. Sluthenftand boldantrubbig, widh)it jur

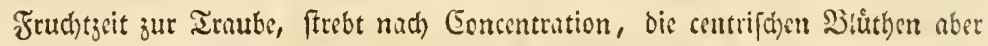
meift fotwindend. 3mitterblutthen.

(Sruppen:

1) Syuclistae: non aut transrersin articulato-dehiscentes.

a) nucamentaceac: 1 - 4 spermae seplo nullo aut incompleto.

a) Euclidieae (et Anastaticene): of lomatorrhizeae.

Septo subnullo: Euclisiam R.Br. Ochthorlium DeC. Pugionium Gärtn. - Septo ineompleto: Anastatica L. Morettia DeC.

B) Isatideac (et Buniadeae): o() notorrhizene.

Cotyledones non inflexae: 'Tetrapterygimm Fisch.C. A. Mcy. Isatis L. Thysanocarpus Ilonk. Tauscheria Fisch. Sobelewskia M. B. Myagrum T.L. - cot. clongatac inllexae: Bunias $L$.

r) Calepineae: $0>>$ ptyehorrhizeae; cot, margine involntae:

Calepina Adans, - cot, non involutae: Zilla Forsk. Muricaria Desv.

b) articulatae: lomentum transversum dehiscens. 
«) Cakileae: oᄃ lomatorrhizeae.

Cakile T.L. Chorispora DeC. Corilylocarpus Desf.

B) Groldbachieae: o () notorhizeac.

Goldbachia $D e C$. Anchonium $D e C$. Sterigmostemon M.B. (Sterigma $D_{e} C_{\text {. }}$ )

y) Raphanistreae: $0>>$ ptychorrhizeae.

Rapistrum Boerh. Didesmus $D s v$. Linarthrocarpus Labill, Raphanistrum $T$. c) continuae siliqua coriacea aut spongiosa.

li a phaneac: $0>>$ ptychorrhizeae.

Crambe T.L. Raphanus $L$.

2) Amphisehistae: utroque latere valvuli dehiscentes: siliculosae ct silitquusae.

a) siliculosae angustiseptae, i. e siliqua abbreviata, septo valvis transverso.

a) Thlaspideac: oO lomatorrhizeae.

Naviculares s. Iberideae: Teesdạlia R.Br. Thlaspi Dill. L. llueris $L$. - Biscutatae s. Biscutelleae: Biscutella $L$. Menonvillea DeC. Cremolobus DeC, Negacarpaea $D_{e} C$.

B) Lepidieac: o() notorrhizeac.

Sencbiera Poir. Noccaca Rchb. (IItutchinsia RBr. [non Ag.] FI. gern, ic. 4222-4223.). Lepidium $L$. Eunomia DeC. Jonopsidium DeC. Rchb. pl. crit. t. 874. Capsella l'ent. Aethionema $R . B r$. Hexaptera Hook. Hymenophysa C.A.Mey. - Cotyled, biplicatae: Brachycarpaea DeC.

r) Psychineae: o> ptychorrhizeac.

Psychine Desf. Schouwia DeC.

b) siliculosae latiseptae i. e siliqua abbreviata septo valvis parallelo.

a) Alyseac: o Clomatorhizeac.

aa. Clypeoleae seu silicula septo demum evanido non dehiscente:

Clypeola L. Peltaria L, Ricotia L. Redowskia Chum. Schlecht. bb. Drabeae silicula dehiscente compressa:

Erophila DeC. et Draba L. Petrocallis RBr.

1) Alysseae genuinae, silicula acuato-marginata diseo valrulaLarum plus minus convexo:

Meniocus Desv. Alyssum $L$, Odontarrhena C.A.M. Psilonema C.A.Mey. Ptilotrichum C. A. Mey. Aurinia C.A.Mey. Koniga $\boldsymbol{R}, B r{ }^{*}$ )

2) Siliculae inflato-rentricosae:

Aubrictia Adans. Schiverebia Andrz, Vesicaria Lam. Berteroa $D_{c} C$.

3) Siliculae complanatae:

Farsetia 'Turr. Selenia Nutt. Lanaria $L$.

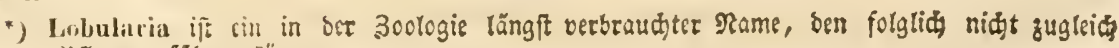

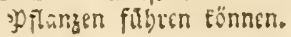


ce) Armoracieae, silicula torosa, dura vel subossea, semina subglobosa immarginata:

Rhizobotrya Tsch. Kiernera Med. Cochlearia L. Armoracia G. M, S, Fl. Wett.

B) Camelincae: $\circ$ () notorrhizeae.

Neslia Desv. Eutrema $R B r:$ Aphragmus Andrz. Orobium Rchb. (Oreas Cham. Schlecht, nou al.), - silie. bilocularis compressa:

Subularia $L_{*}$ Stenopetalum $R_{*} B r$. Matthewsia $I l o o k, A m$, Eudema Ilb. Bpl. Platyspermum Ilook, - silicula inflata: Camelina Crtz. Hymenophysa $C, A, M$.

g) Vellcae: $0>>$ ptychorrhizeae.

Silic, loculi 1-2 spermis: Vella $L$. Bolcum Desv. Succowia Med. - silic. loculi 4-00. sperwi: Carrichtera DeC. Savignya $\mathrm{DeC}$.

c) siliquosae i. e siliqua oblongata ant lineari donatae.

a) Arabideac: o . lomatorhizeae.

aa) Mathioleae:

Luperia $D e C$. Pinaria $D e C$. Acinotum $D e C$. Matthiola $R, B r$. b) Chcirantheae:

Notoceras $\mathrm{R}, \mathrm{Br}$. (Diceratium et Tetraceratiun $D_{c} C$.) Sterenia Adams. Cheiranthus $L$, Streptanthus Nutt. Oudneia $R, B r$. Macropodium R.Br. Parrya R,Br.

cc) Arabileac gentinac.

a) rerae: Abasicarpon Andrz. Arabis $L$. (et Turrita et Turritina). L'arrya $R, B r$, Turritis $L$.

B) Cardamineae: Anlrzeiowskya Rchb. Arabisa $l c h b$. Cardaminc T.L. Pteroneuron DeC, Dentaria T.L.

भ) Nasturtieae: Nisturtiun C. Buuh, R.Br. Leiolobia Rchb. ap. Mössl. Brachyolobos All. - 'Tetrapoma (Tetracellinu) Turezann.

3) Sisymbreac: o() notorrhizeae.

a) Hesperinae:

Malcolmia R.JB, Deilosma Andro. Iiesperis L. Leptaleum $D_{e} C$. cot. elong. flexuosis: ILeliophila $L$. Chrmira $T / h b$ ?

hb) Erysimeac: Braya Stmb, Hpp. T'aphospermurn C. $A$. Mcy. Suclowskia C.A.Mey. Dontostemon C.A.Mey. (Andreoskia DeC. Erysimum L. Syrenia Andrz. Stanleya Nutt.

cc) Sisymbreac genuinae:

IIalimolobos 'I'sch. Sisymbrium $L_{*}$ (Velarum Plir. Kibern Adlens. Arabidopsis DeC. Sisymbrium gen, et Norta Adans.) IIugueninia Rchb. pl. crit. ic. 1330.

y) Brassiceae: $0>>$ ptychorrhizeac.

aii) Diplotactcae: Diplotaxis DeC.

b) Sinapicac: Eruca T. Sinapis L. Erucastrun Rchb. 
cc) Brassiceae genuinae: Moricandia DeC Orychmophragmos Bunge. Brassica $L$.

3) Acroschistac 5 . coilocarpicae: ex apice inter stigmata disseninantes aut baccatae.

a) Resedeac: ylacentae et valvulae $3-4$ prismatico-connatae, stamina 11-27, parapetala $3-5-6$.

Resedella Rchb. (R. dipetala, glauca), Luteola $T$. Reseda $T . L$.

b) Asterocarpea : placentac $4-5-6$ valrulas utrinque rumpentes, demum senina singula raginantes, in gynophoro stellatac.

Asterocarpus Neck, (Sesamoilles T.).

c) 0 chradenea e: bacca indehiscens (calyx patellaris 5-dentatus, corolla nulla).

Ocluradenus Dclile.

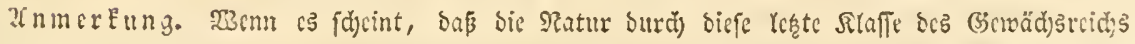

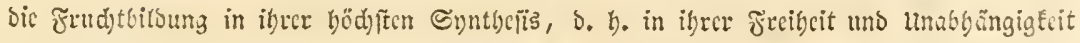

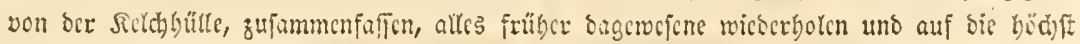

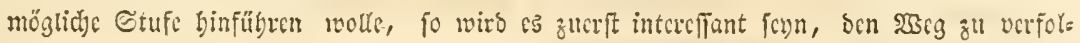

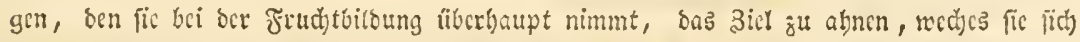

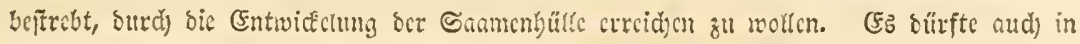

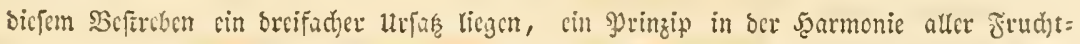

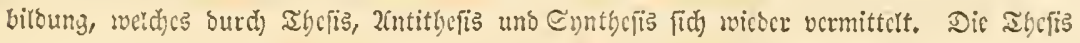

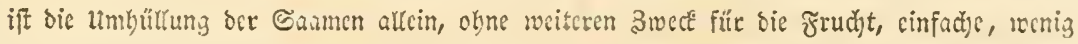

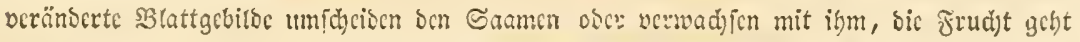
unter, bei ser Scimung ber Caamen verwitterns, fic opfert fid ifgm. (Fin zuveiter böbse

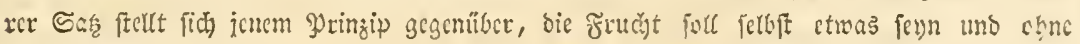

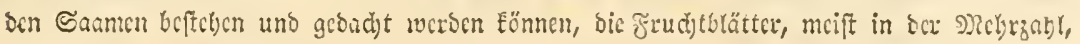

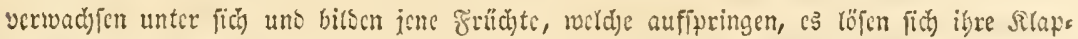

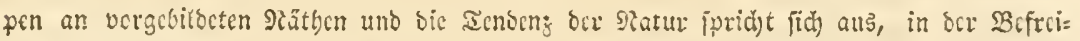

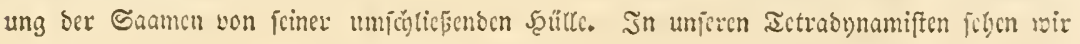

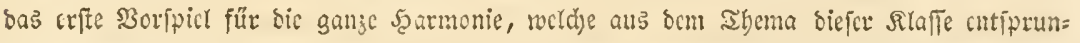

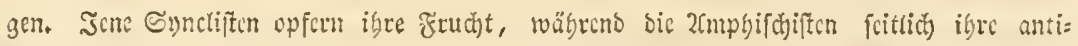

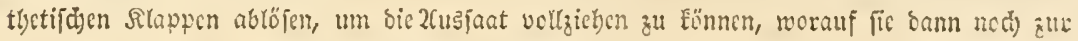

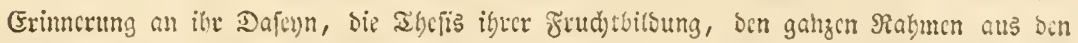

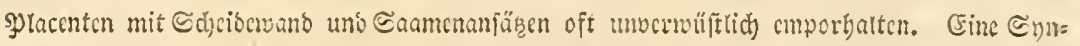

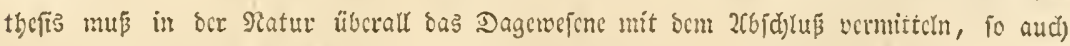

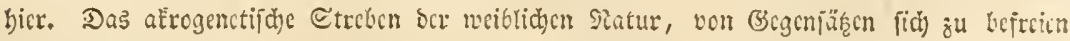

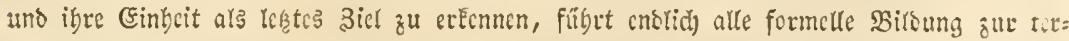
minalcn (Eonecntration, jo wic wir bie getrennten @tigmate enslid) centrifi) verrinigt $\mathrm{cr}=$

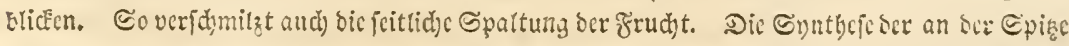

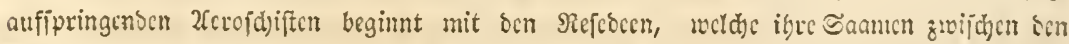

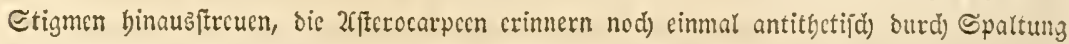
an bic foitlid) aufipringenocn 2(mpsijdiffen, abcr fternförmig zur Finbcit gelangt, bis enslich Ocliracicuns wicber alles 230 ciblicbe jur bödjten Sesteutung ber frudjt in ser

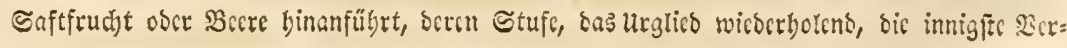




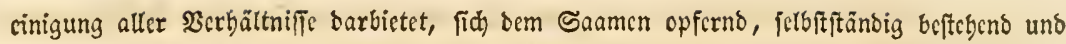

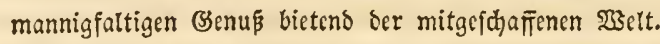

Behen soir num in bie (5lieberung ber Brruppen, fo müfen biefe fich fo an einander

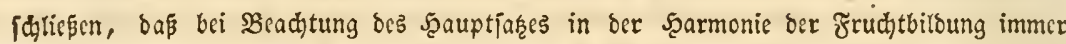

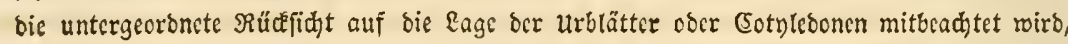

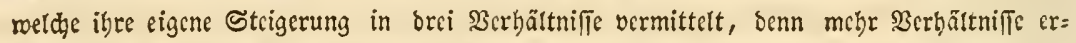

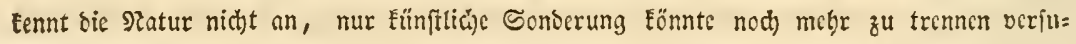

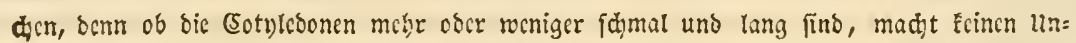

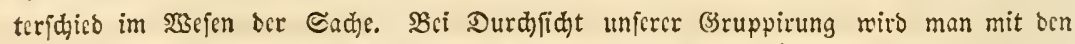

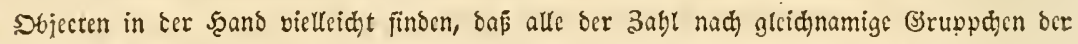
verfdicbenen Bruppen fid roieber entipred)en und bas Bange bie roabrhaft natürlid ver=

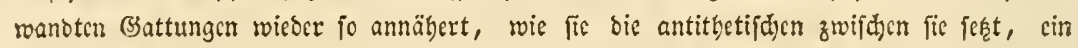

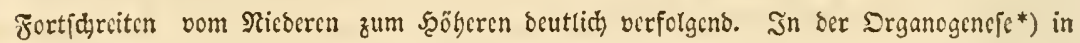

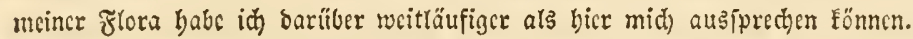

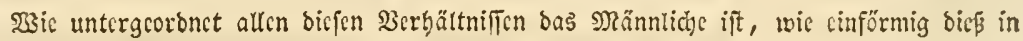

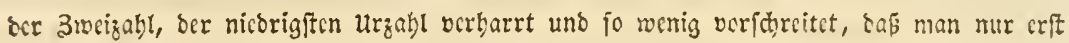

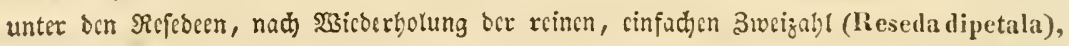
bas Fortforciten zur Fünfzahl burd sparapctalen, uno bas fortbilocn ber Staubge=

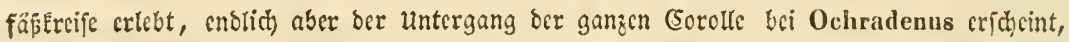

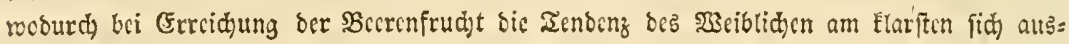
fpridgt, bicép zulus licgt yor. -

\section{Familie. Miobugetwäbje: Papareraceae.}

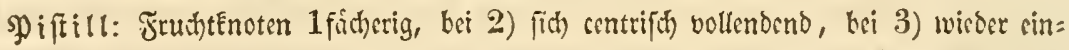
peitig, Saumdhen horizontal, enblich bei Berbereae aufredt; Griffel Eurz obcr

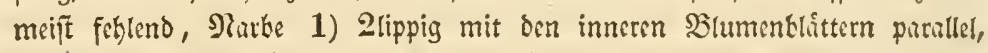

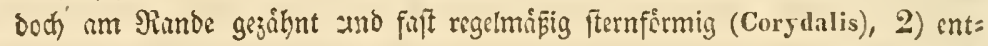

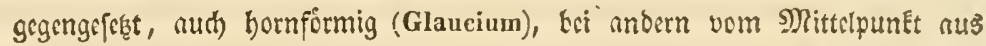
frci, faft aufredt (Hypecoum uno Platystemoneae) oder fternformin auf cin Edjild aufgerwahfen (Papaver); bei 3) iff ber Eurze (Siriffel ober bie balb= runoe ober rundiche Narbe auf der cinen Seite aufgelest, anf wether ber San= mentriger fich befindit, bei einigen (Berbereac) ziemlith centrif b). - Seld):

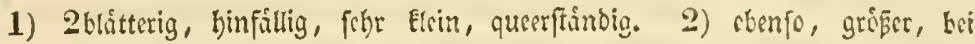

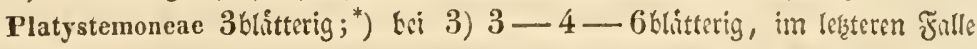
2reibig, abfalleno, audi) mit fohuppigen Dectusttchen. - Frudt: 1) als

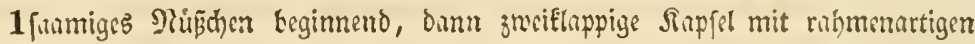
mehrfanigen Saamentrígern, bei Dactylicapuos cime Besre, Sanmen bori=

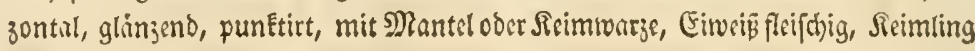

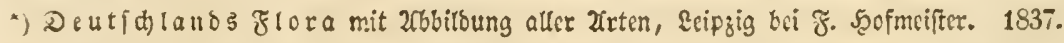

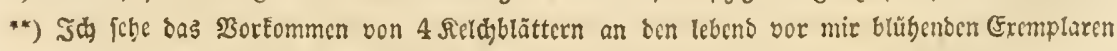
vor Platystemon lineare Benth. nur aโรื 2rušnabme an, und cine Entfernung sicfer (5attung bon bcm innig vcrivanoten Ptatystigma californicum, wic fid bicfe bei Meis-

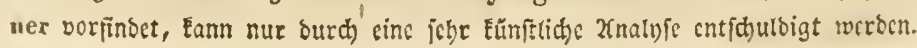




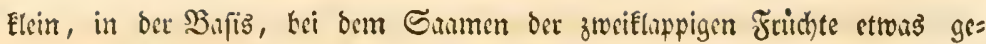
Erưmmt; 2) beginnt als fdotenfórmige Sirpfel geglicbert (IIypecoum), mit 2 Silappen, weld) uom rathmemartigen Sanmentriger lóngs abfpringen (Chelido-

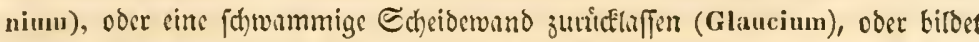
fich) fort alb mehrfinppige Sapfel, von beren Sammentrigergeftelle bie Slappen abfpringen (Roemeria, Argemone); ober dic গুlacenten als bulbe Scheidewinde

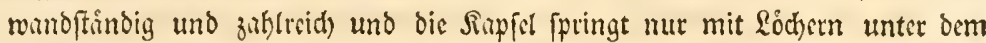

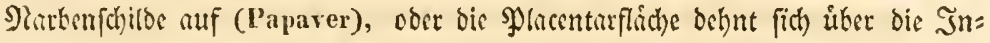
menfeite ber Silappen aut? (Platystigma). Sarmen zahlreich), meift mit Seim= wrrze und auf ber Dherfliche getornelt, bei cinigen glatt und glanjend

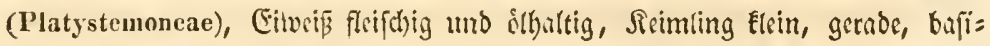
lar. 3) brginnt wieder fobotchenformig (Bocconia, Jipimedimu etc.), Dann blufenformig (Leontice, Caulophyllum); bunn lederartige ober faftige Serre

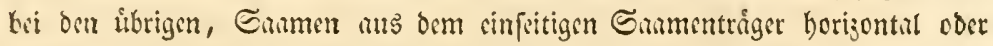

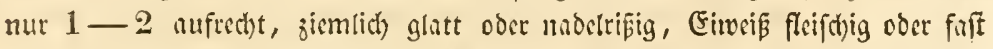

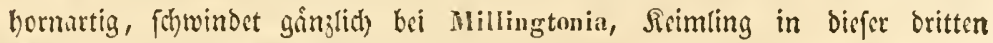
Gruppe am boichften vollendet, faft fo ling alg ber Samen, Sotyledonen blattartig.

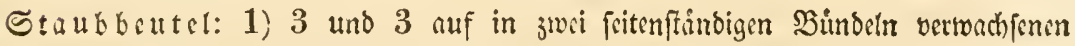
Etrubfíden aufrect)t, immer ber mittlere Beutel 2 fircherig, bie beiden jeitlictsen 1ficherig. 2) aufredt und 2fitcherig, lingsiuffpringend, 4 auf froin Etaubfáden (Hypecoina) oder jublteich auf freisn Staubfioen in mebreren. Siribm, biefe Etrubfioen bei ben \$ुlntyffemoneen mit febr breitem unterfas, bei 3) a. ckenfo, mur fdmaler, fhon bei Jeffersonia bie Seutel Elappig auf= fpringeno, bsi b) und c) ben Blumentlittern gleid)3afflig und ifnen vorunfeth=

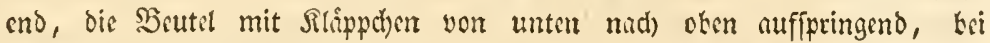

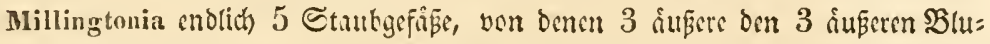
menblittern anhingen, 2 innere mit Eugelrunben queerauffpringenden Find an prófentirtellerfórnigem Steg. - Slume 1) 4brítterig, 2 grép̈ere mit

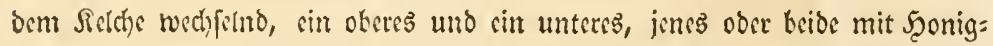
fporen, beibe feitliche fleimer und fluth), meift alle etwas zufummentingent. 2) 4 glcid)e $\mathfrak{B l u m}$ (unblitter, Ereuzftindoig, bei Hypecoum bie 2 inneren orci= lappig, bei menigen 5 (Argemone), in ber Sinope jerEnittert uno eingegwingt.

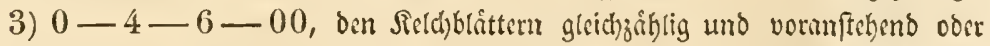

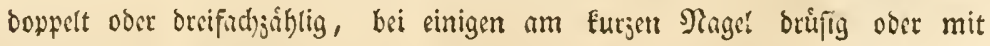
Schuppechen verielen.

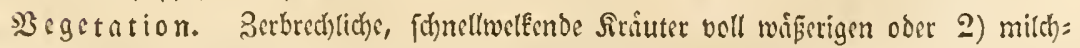

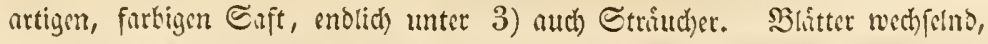
fein zufammengefest oder firderfpultig, bei wenigen sinfact) unto quirlftinnoig (Platystcmoneac), bei 3) auch hanbformig uno gefingert, Euchtig ficoerfpaltig,

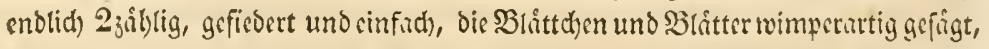
bei sinigen auth lederintig. Blutthen in 2fefren und Irauken in ben contri= fd)en Gruppen auch encftíndig und botbenurtig (Chelidonium) und trugberbig (Diphylleia). 


\section{Sruppen:}

1) F a mari ea e: stigma bilabiato-substellatum, Irupa, capsula siliquiformis ant bacca, stamina diadelpha definita, corolla calcarata.

a) Fn marie a gen uinae: unicalcaratae indehiscentes monospermae.

Fumaria L. Platycapnos DeC. Bernh. Discocapnos Cham. Schlecht.

b) Corydaleac: unicalcaratae biralri- dehiscentes pleiospermae.

Corgdalis I'ent. Bulbocapnos Bernh. Phacocapnos Bernh. Capnoides

Gärln. Cysticapnos Boerh. Sarcocapnos DeC.

c) Diëlytreac: bicalcaratae (bivalves et baccatae pleiospermae.)

Adlumia Rafin. Eucapnos Bernh. Diëlstra Borkh.*) Dactylicapnos Wallich.

2. Papavereae: stimmata opposita aut stellata, capsula lomentacea, aut 2-3-5 valvis, aut foraminibus dehiscens, stamina $4-00$, corolla regularis $4-8$ petala.

a) H specoina e: schizostyles, lomentaceae tetrandrac.

Hypecoum L. Chiazospermum Bernh.

b) Chelidonieac: oppositi-stigmaticae, raivatae, polyandrae.

Chelidonium C.Banh.L. Glancium T. Roemcria Hudik. Eschscholtzia Cham.") Hunnemannia Sureet. Dentromecon Benth.

c) Paparereae genuinae: stigmata 3-00 stellato-excentrica.

a) Argemoneac: stigmata horizontalia libera, capsula valvis teliscens.

Stylophorum Nutt. Meconepsis Iignier. Argemone T.L.

B) Porocarpae: stigmata scutello radiatim allnata, capsula poris deliscens.

Paparer T.L.

\%) Platystemoneae: stigmata libera arrecta, capsula ralrulis ipsis placentarilus dehiscens.

Platystigma Benth. Platystemon Benth.

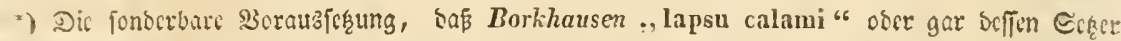

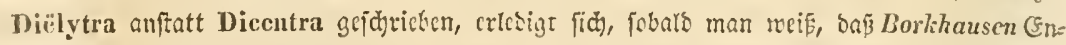

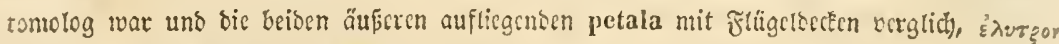
foll ja gar nid)t Sporn bisiscn.

"*) Die Şenerfung von 5eren Lindley Bot, Reg. 1948., unter woldger Siummer ce tine neuc

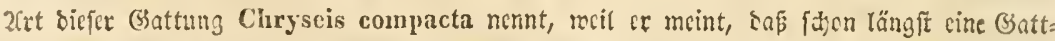

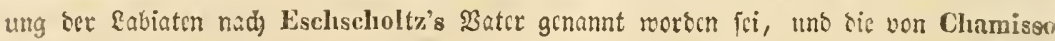

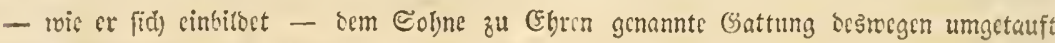

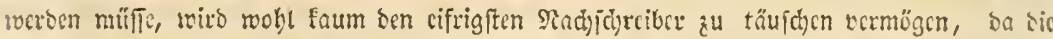

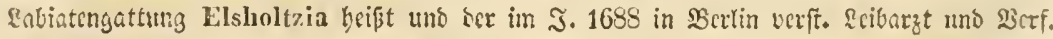
soc Flora marchica Joh. Sigism. Elsholtz nicht wohl ber \$atcr bes am 1. Not. 1:93 in Dorpat geborenen, Iribcr vicl zu früh verfterbenen Joh. Friedr. Fschscholtz feyn founte. Det

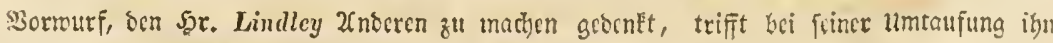
ferbit, Senn Cliryseis ift finon cine altc Enmantbercengattung yon Ilenri Cassini. 
8) B erberide ac: schizo-holostigmaticae, flos regularis, fructus siliculosus aut irregularis placenta unilaterali, in antithesi (b. Berbereac) regularis, baccatus, seminibus erectis.

a) Bocconie a : stauniua inlefinita, antherae longitudinaliter dehiscentes, ultimi generis (ut sequentium b. c.) valvatae.

a) Corolla nulla, capsula siliculosa bivalvis $1-6$ sperma:

Bocconia $L$, Macleaya $R, B r$.

B) Corolla 6-12 petala, capsula siliculosa bivalvis 00-sperma:

Sanguinaria Dill, $\boldsymbol{L}$.

ช) Corolla 6-8-9 petala, capsula baccans placenta 00-sperma uniIaterali non, aut ultima operculato semi-dehiscens,

Podophyllum $L$, Jeffersonia Bart.

b) Berbereae: stamina definita petalis anteposita, germen 1-loculare.

æ) Epimedieae, siliculosae polyspermae.

Epimedium L. Vancouveria Morr. Accranthus Morr.

B) Cauloply yleac, vesicariae seminibus $3-4$ erectis,

Leontice Mchx. Caulophyllum Mchx.

y) Berbereae genuinae, baceatae hexandrae.

Diphylleia Mchx. Nandina Thnb. Mahonia Nutt. Berberis $L$.

c) Milling tonicae: 5-andrac5-petalae, 2-antlerae. Millingtonia Roxb.

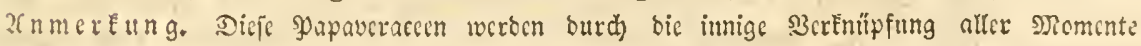

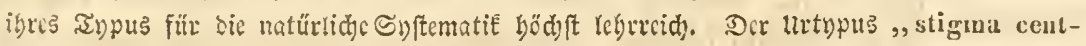
rice absolvitur" (Fl, gremm, p. 696.) fällt in biffer antityctifdan Familic in ibr (con= trum unb bas Fortfdreiten zu Errcichung biefes Bictes liegt überall vor, jebe Grappe rie:

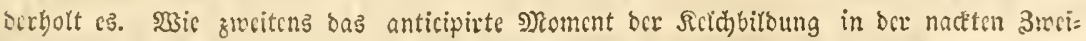

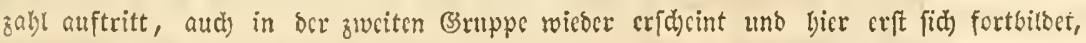

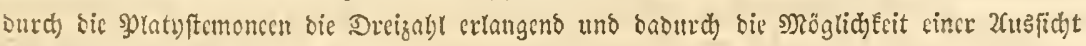

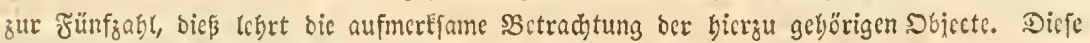

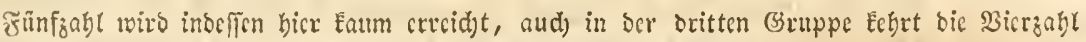

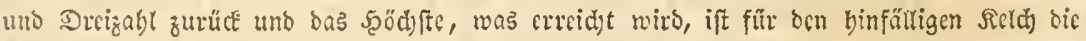

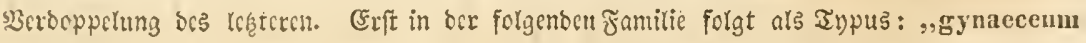

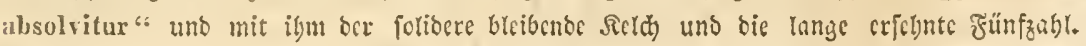

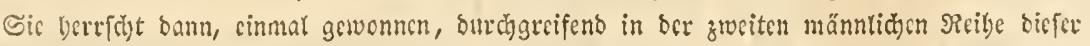

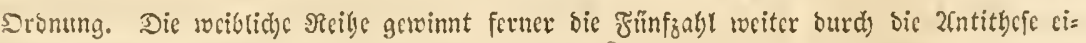

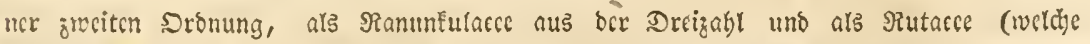
feloft antityctifch auf bic erfte Sorbnung zurücébcutct) aus ber Bicrzabl.

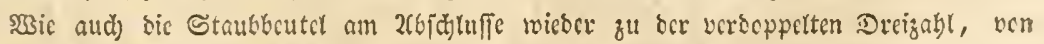

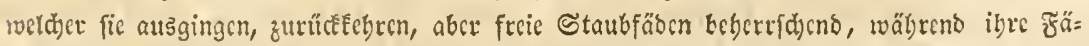

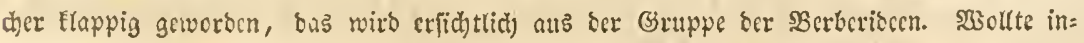

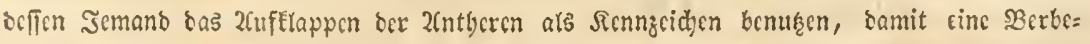

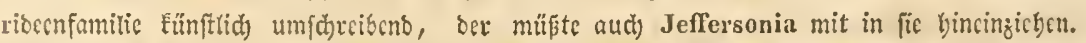

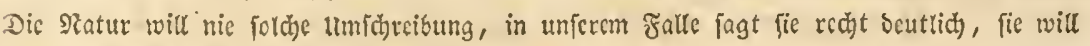

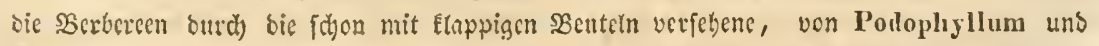

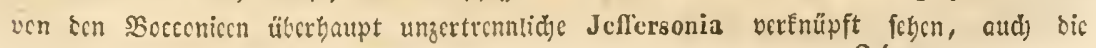


Etielblintthige.

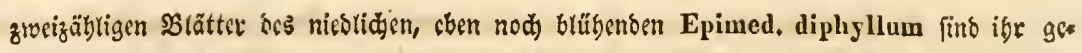
geben.

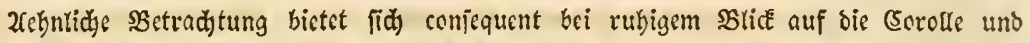

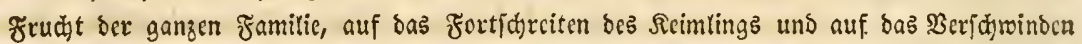

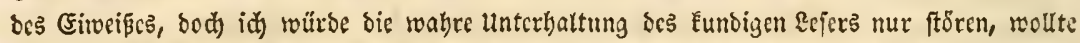

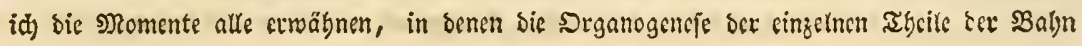
ber Natur folgt.

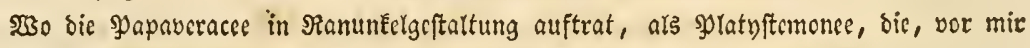

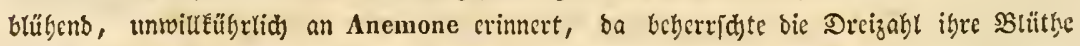

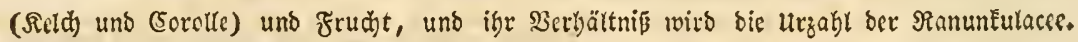

11\%. Familie. Snquerngetwädjic: Capparideae.

Piftill: Frudtenoten bei 1) und 2) auf einem Stift emporgeftreckt, cinfifherig, 2

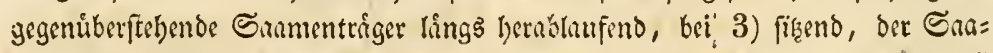

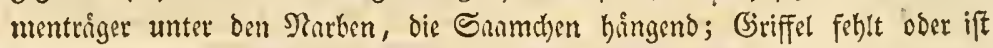
furz (Stigmarota), Slarbe fiseno, cinfach ober feernformig, 2-4-5$6-8$ frrablig getbeilt. Ifeld) $3-4-5-7$ fpaltig ooer theilig, ats fallend ober bleibend, Eappenfiermig und Elappig bei Denhamia, bii útrigen

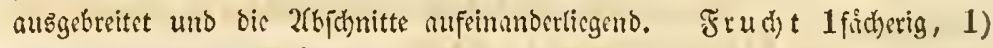

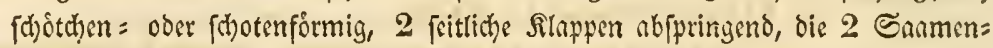

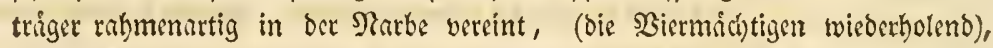
Die Saumen $1-2$ = reibig, nicrenformig, glatt ober geformelt, (Fi二 toris fehlt, Seimling gefrummt, Sotnledonen batbundtid) uno queer,

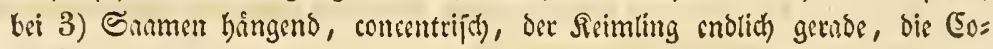
tylesonen flict)er.

Staubbeutel: 2fickerig, rundlich oder linglich, nufrecht ober aufliegend, anges warbjan bei Melycitus, lings and (Kïggelaria), beftimmtziflig 6 (vertimmert zแ 1 bei Dactylaena), - 8

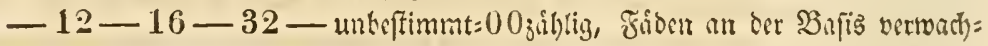
fen (Gyuandropsis), bei kitrigen frei, cimbetme Druifen um bie Staubfiben lsi Cleomeae, geferbtes Singpolfter bri Roumea. Błıme 4blatterig unregel=

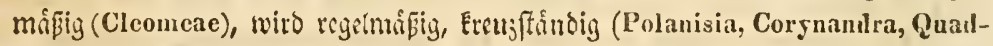

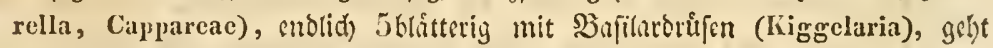
trieder unter und felylt ginnjlict) bei Flacourticac.

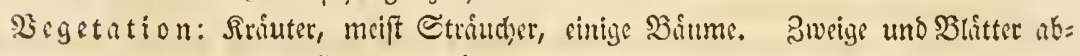
wechfelno oder zerftreut, oreizáblid) und gefingert (Clcomeac) odir eimfud) (cinige (Eleomeen unt faft alte ůbrige), bann meift gamjmonbig bei 3), aud) Eerb= und fógerandig, 2febfelblíttchen fehlend oder anftatt oeren cingelne (Flacourtia) oder doppette Dornen (Clcome spinosa ete. Cappareac). Blithen jwitter

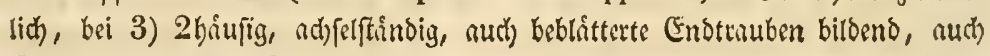
Dolbentrauben und Siifpen.

(s)ruppen:

1) Cle om ea e: capsula siliculosa aut siliquosa, stamina 1-6-00.

a) Peritomeac: calyx 4 dentatus demum basi circumscissus. Peritowa $D_{c} C$. 


\section{Stielblůthige.}

b) Cle omeae genuinae: calyx 4-8epalus, stamina definita adscendentia, corolla plurimis irregularis.

Dactylaena Schrad, Cleomella DcC. Physostemon Mart. Cleome L. Gynandropsis DcC. (Pollogyne HIfgg.) Polanisia Rafin. Quadrella $D_{c} C$.

c) Corynandreae: calyx 4-sepalus, stamina indefinita erecta cum corolla eruciata regularia.

Corynandra Schrad. Rchb. hort. t. 147.

2) Cappareae: bacea indehiscens, flores hermaphroditi.

a) Morisonieae: calyx 2 fillus, 2 sepalus aut $4-8$ partitus, stamina 00. Morisonia Plum, Busbeekia Findl. Maerua Forsk. Thylaehium Lour. Rhinanthera Blume.

b) Cadabeac: calyx 2-fidus $4-8$ sepalus, stamina 4-8.

Tovaria Rz,Pav, Stephania $W_{*}-\Lambda$ tamisquea Miers. Cadaba Forsk. Schepperia Neck, - Hermupoa Loeffl.

c) Capparene genuinac: sepala 4-libera, stamina 00 indefinita (ultra 12).

Boscia Lam. Nichuhria DeC. Crataeva L. Ritcliea $R B r$. Capparis $L$.

3) Flacourtiancae: dioicae.

a) Melicyteac: calyx $3-5$ dentatus, petala 5 eglandulosa, placentae 1-2-spermae.

Deulamia Ucisn, (Lencocarpus Rich.) Melicytus Forst,

b) Flaconrticae: calyx $4-5-7$ partitus, petala nulla, stamina indefinita (subcompacta).

Stigmarota Lour, Roumea Poit. (Koelera $W$. Bessera Spr.); Flacourtia Commers, L'Herit.

c) Íigg claricae: calys 5-partitus, petala 5 sepalis alterna ungue glandulifero aut squamuligero, stamina definita 5-10-20, placentac 00-spermae, - styli 2-5: Kiggelaria $L$. - stigma peltatum: Hy lnocarpus Gürtn. - Pangium Blune.

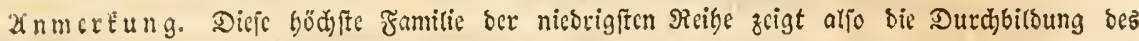

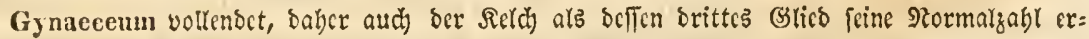

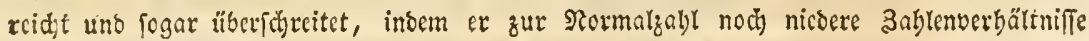
sviscrbolsnd bingufügt.

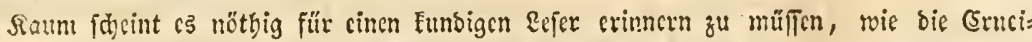

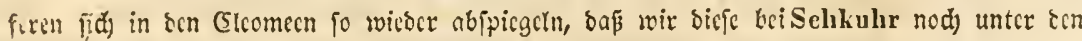
Tetrabunamiften antreffen. Die rabre \$3icocrlyolung crefennt aber der unbefangene vorgüglich im

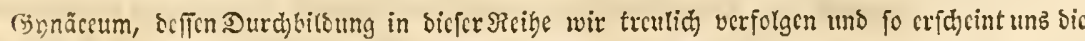

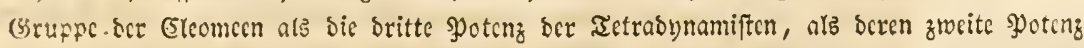

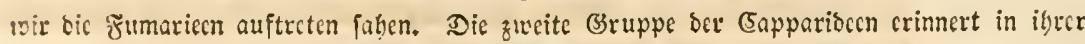

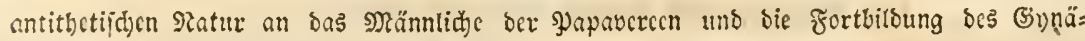

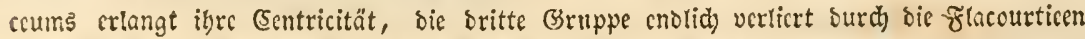

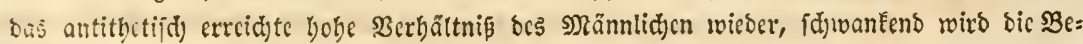

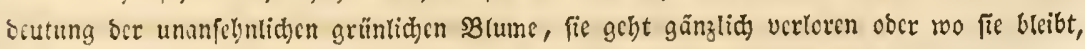

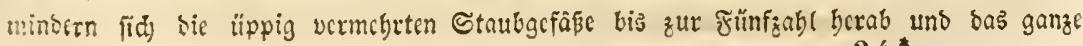




\section{Etielblinthige.}

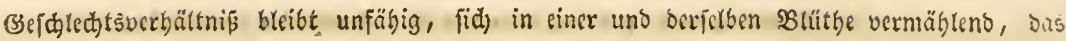
böbere 3icl ber \$fflanze zu idaffen. W3ie aber bie frudth dabei gervinnt uns cine concent= rifde Etellung größerer Saamen mit volfendetem Reimling gewonnen, bas lebrt idjon Flacourtia.

\section{Familie. Beildsengetwädje: Violaceac.}

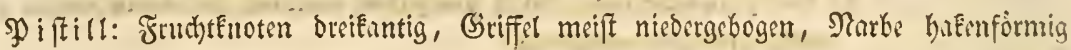
bei andern ausgebofhlt, bci Piparea 3ppaltig, bi Pittosporeae aud zmeieopfig. Seld) 5blitterig, jweircigig ober cinteifig, in oer Sinofpe aufliegeno, bei 1)

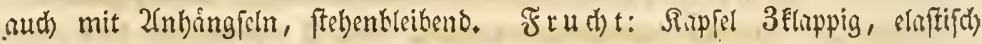
aufpringeno, Slappen in oer Mitte långs faumentrageno, Sanmen meift jub̆l=

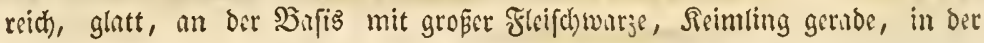

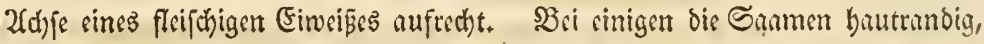
bet 2) c. Saurageseae: die Sinamen an Den Jilappentinoern, bei 3) Pittospo-

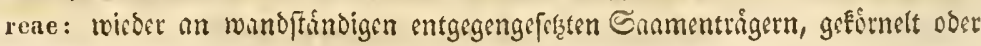

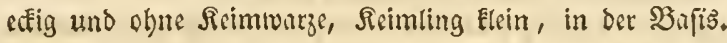

Staubbeutel 5, bei 2) c. aud) $10-15$; platt, meif pfeilformig, angewarch fon, einmirtsgekelytt und anfangs stroas zufummentbingent, bei ubrigen nabe anein= anderliageno aber frei, atswhitts bei Corynostylis, longsauffpringeno, nut kei Luxemburgia at ber Epife mit 2 Rỏhern aufpringeno. Stauffidoen meift Furj und flich, kei einigen monadelphifh, bei ben meiften frei, bei 1) meift bie beioen oberen mit pornartigem Safilntfortfas, welcher fid) in ben Eporn

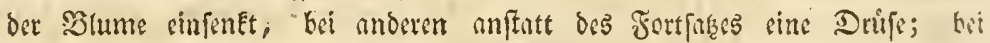
Sauvagesia mechfeln Stmbfión ohne SBeutel mit oen keutsttringenoen mo Ginter biejen ftelht eine 5flitterige Niebenfrone, bei Lavradia biefelke flafd)en=

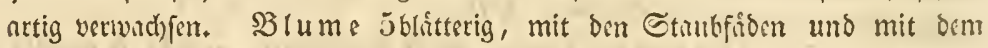
Sielthe wechfeino, untegelmáfig, 2 nach oben, 2 nuch ben Seiten, cincs mich

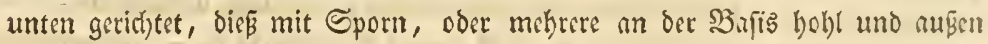
bơcéerartig, bei 2) uni 3) regetmápig uno flad), nut bei Billardiera nod) an oer Sajis geboblt.

Begetation. Wenige ein= und zweijâtrige, neift ausbuuernoe Artuter uno Stráucher, Blaittel wurzel = oder ftengel = uno zweigftindig, zerftreut, bei me=

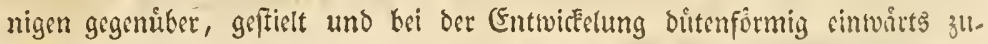

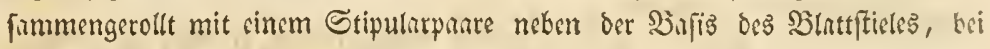
2) und 3) bie Silitter leocentig, bei lef̧eren obne Stipula. Şlithen

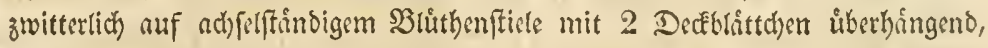
einzeln ober mebrete aufect)t, traubig uno audj boldentraubig, endidi) (Bursera)

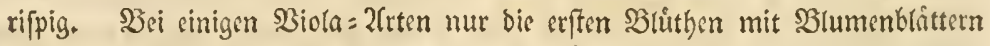
und unfructstbar, bie fpateren blumenlos und fruchtbar, bie Stumenblatter in Der Sinope faft bet allen ubercintundergerollt.

(3ruppen:

1) Violea e: corolla irregularis 5-petala plurimis calcarata, stamina 5. antherae subcohacrentes, capsula valvis tribus medio placcutiferis elastice dehiscens, stipulae. 
a) Violeae genuinae: antherae introrsae, sepala auriculata, capsula 00-spermia,

Viola L. Grammeionium Rchb. (Jacea Commers, Mlelaniun DeC. non $R B r_{\text {.) }}$ Dischidium Ging, Schweiggeria Spr. (Glossarthen Marl.). Erpetion DeC. Sweet.

b) Corynostyleac: antherae extrorsae, sepala esiuriculata, capsulia 00-sperma, semiua plana.

Corynostylis Mart. (Calyptrion Ging.).

c) Jonidieac: antherae introrsae deplanae, sepala exauriculata, capsula plurimis 00-sperma.

a) Hybanthe ae: nonadelphae.

IIybanthus Jacq.

B) P o mbalie a e: pentandrac, petalo infuno maximo Iabififormi.

Solea spr. Pigen DeC. Jonidium Vent. Pombalia Vand, Anchistes St, Hil. Noisettia K,II.B.

r) A mp hir hogeac: pentandrac subregulares conniventes (transitus in Alsodeias), folia quibustam opposita.

Amphirrhoge $\left.R c h b *_{*}^{*}\right)$

2) Alsodineae: corolla regularis ecalcarata, stamina 5, autheris apire appendiculatis (in antithesi 5-10-15 exappendiculatae, filamentil pirtiu alterna sterilia), capsula valvis tribus medio (in Saurageseis in margine) placentiferis, stipulac.

a) Alsodineae genuinae: pentandrae, valvulae medio placentiferae.

2) Cerantlue e e: moualelphae capsulares.

Alsodeia A.P.Th. Ceranthera P.B. Prosthesia Blume. Lentalobit Lour. Pliysiphora Soland.

B) Co nohorieae: libere pentandrae capsulares.

Conohoria Aubl. Rinorea Aubl, Tachilota Aubl.

y) Pental obeae: pentandrae, subliberae, baccatae.

Restaloba Lour.

b) Il ymenant hereae: 5-andro-monadelphae, stamina parapetalis anteposita.

Hymenanthera Banks,

c) Sauvageseae: 5-10-15-andrae, filamenta altcrna sterilia ant paracorolla intra stamina corollamque.

a) $\mathbf{P}$ i pareanae: parastemones monadelphi hirsuti. Piparea Aubl.

$\beta$ ) L uxemburgicac: antherae 5-15 subscssiles, milateraliter monadelphae, filimenta sterilia minuta.

Luxemburgia St. Hil,

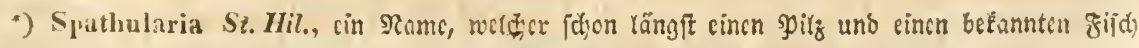

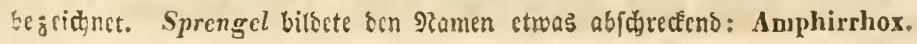


भ) Lavradieac: paracorolla, praeterea filamenta sterilia nulla aut alterua.

Lavradia Ielloz. Sauragesia Jacq.

3) Pittosporea e: corolla regularis ecalcarata ant petala basi concarogibba, stamina 5 libera, antherae exappendiculatae, eapsulac aut baccee placentae 2-5-00-spermae. Stipulae nullae.

a) Billardiereac: petala basi concaro-sulsconvoluta, fructus indehiscens coriaceus aut baccans bilocularis, septo utrinque placentifero. Flores ut in Violis bracteolati penduli.

Billardiera S $\mathrm{S}$. Sollya Lindl. Pronaya Hüg. Mariantluus Hüg.

b) Bursarieae: capsula compressa bilocularis dehiscens, Flores paniculati.

Bursaria Cav.

c) Pittosporeae genu inae: capsula 1-locularis ralrulis medio-placentiferis. Flores erecti corymbosi.

Senacia Commers. Pittosporum Banks.

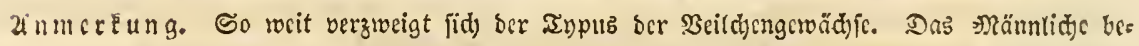
ginut in ber aus bem

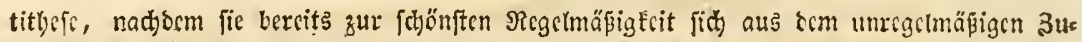

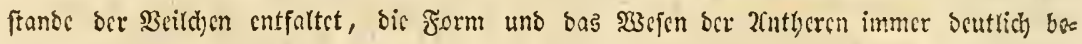

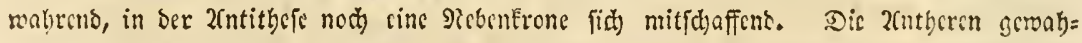

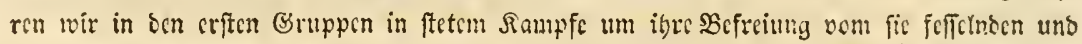
siber fic hinausragenocn Eonncctivum, won bom crft sic böhcren Formen befrcit find.

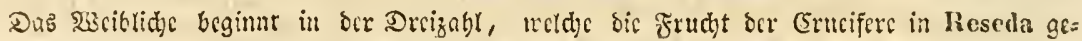

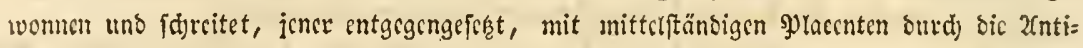

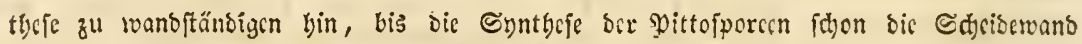

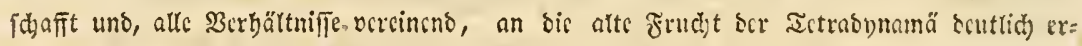

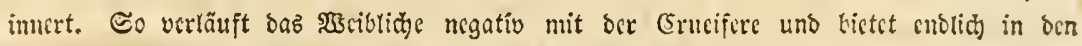

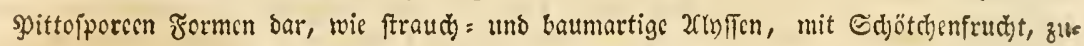

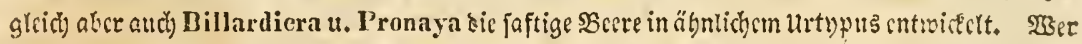

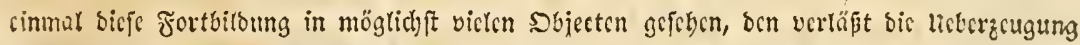

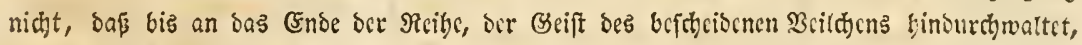

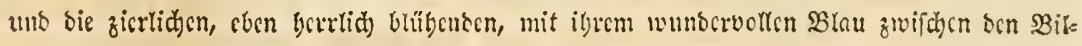

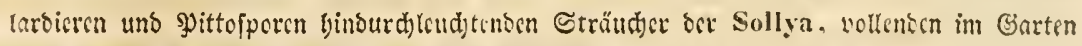

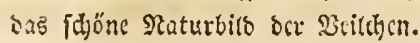

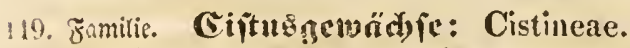

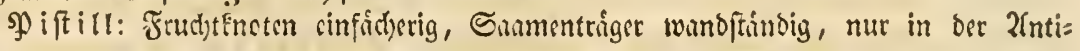

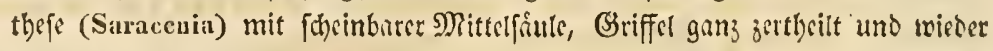

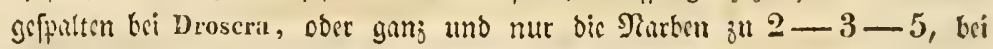
Saracenia fchilbfortmig, bei ben leģteren Guattungen Fopffirmig, endidi sin=

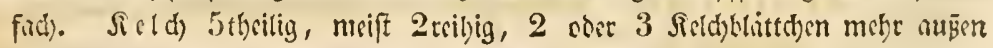
unb aufliegent, ober cinblítterig mit vortretenden Mittelrippen unb mehr cose

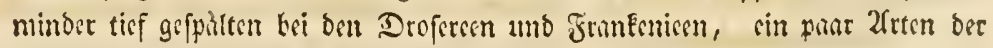

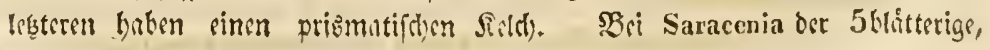


farbige Reld) nod) mit Eleiner 3thlitteriger Şillte. BZei IIelianthenum und Cistus bie immeren Relthelitter in ber Sinoppe gedecht. Frud) t: 1ficherige

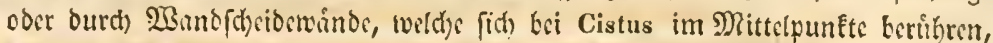
5-10fricherige, flappige Supfel, bei Dionaea Elappenlos beerenurtiy, bci Saracenia eine fdsinbure Mittelfiule, von welther Sarmentriger in bie Fifs (b)e einzeln cintreten und in ibnen fich) zu Doppelleiffen [pulten, aber bod) diefe Sarmentriger gefteflutig jerfpringend an ben Sdyeidewandon in ber Mitte ber Silapen, fich anlegend; bei Fraukenieae die Sinmen an den Piandern ber Slappen befeftigt. Sa amea zaflrcid), Elein, bei Hndsonia mur $1-3$; glatt oder geformclt. Eiwsib fleifobig meblig, Jeinting Elcin, Eci Drosereae,

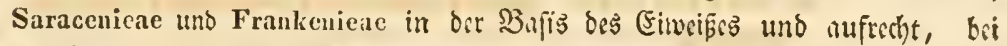

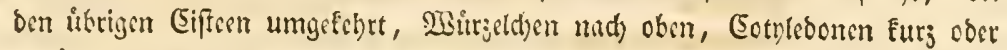
veriingert uno bafig (Hcliauthemum) ober in oer Fortbilbung fpirnlig (Cistus) singerollt.

Staubbeutel 2fritherig angerwathen oder aufrecht, limgs ober mit Sichern an oor Spiffe (Roridulene) auffprimgend, von ber Gunfahl auşgebend, mit den SBlumenblittern wed)felno, zur Dreizuht herubfteigend, aud bic Dreignfl ver= doppelnd (Frankenia), dic Simfzafyl vermefrend: $10-15-20-00$.

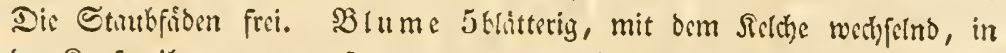
ber Sirofpe ifm entgrgengefeft umgelegt, nur in Lechea 3blitterig; bei ben

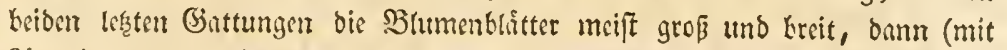

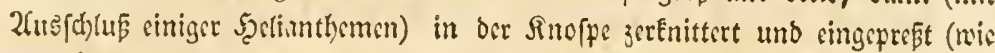

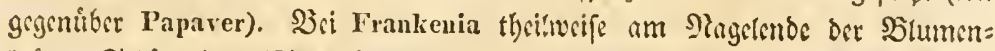

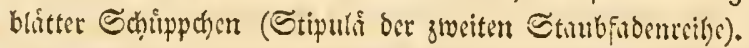

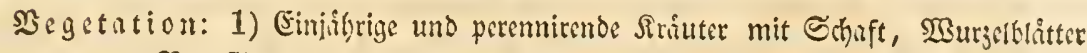

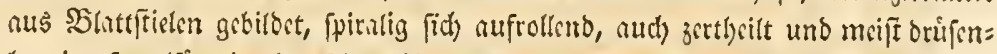

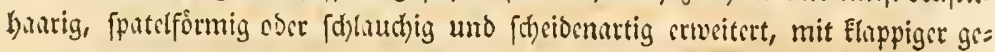
gliẹbert aufgeferęter, getwintperter Şlatte (Dionaea), ober Eappenformig crwei= tert (Sarneenia), bsi anocrin. (Droserae exoticae, Aldrovauda, Roridula)

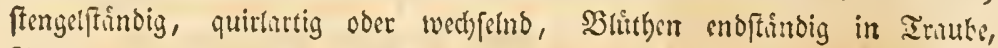
Irugbo:de, endlid) sinjeln (Saracenia), dic Cticle fid) ebenfalls mifrolls

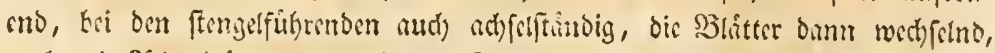

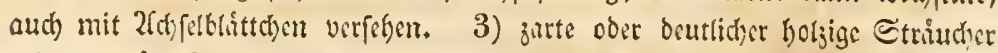

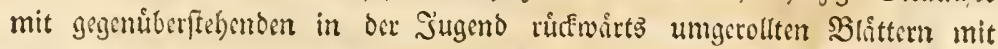

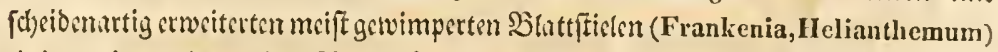

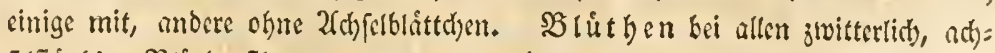
felftindig, Sluthenjticle ein = ober mebrblittbig.

Gruppen:

1) Drosercae: schizostyles et schizostigmatica 5-20-andrac, folia circinnantia.

a) Rorelleac: schizostyles antheris 5-10 longitulinaliter dehiscentibus.

Aldroranda Monti. Drosera L. Drosophyilum Lli. 


\section{Stielblüthige.}

b) Roriduleac: holostyles, antheris 5 , poris apicejdehiscentibus. Byblis Salisb. Roridula $L$.

c) Muscipuleac: lolostyles $10-20$ andrae, fructu 1-loculari 5 -valıi subbaccante.

Dionaea Ellis.

2) Sarracenieac: lolostyles stegostigmaticae.

(Ordinis antithesis summa, anicissima tamen Drosereis).

Saracenia $\boldsymbol{L}$.

3) Cisteac: holostyles schizo- et cephalostigmaticae 3-6-00-andrac, petiolis vaginantibns, foliis in plurimis margine revolutis.

a) Frankenieae: stigmata 3-capitcllata, stamina 5-6-7, petala 5 longeunguiculata ad basin laminis coronulata. (Calyx 5 partifus, 5-fidus, 5-dentatus, capsula 1-locnlaris 3- [2-4] valvis, valvae margine seminiferae Sauvageseas rerocantes!).

Frankenia L. Beatsonia Roxb.

b) Leche a e: stignata et petala 3 , semina panca, stamina $3-12$,

Lechea $L$.

c) Cistea genuinae: stigma simplex, stamina $15-00$.

Hudsonia $L$. Helianthemun $T$. Cistus $T$. $L$.

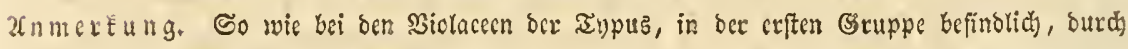
bie zlucite und sritte fid weiter entfaltet, fo fehen wir bicr in ocr 2(ntithefe ben Iy)pus

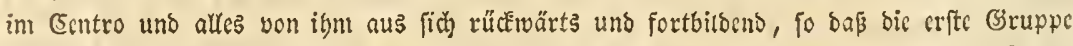

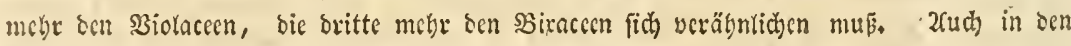

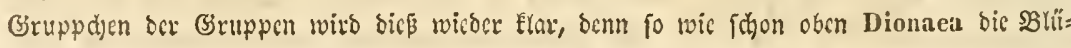

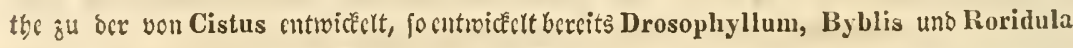
antithetif

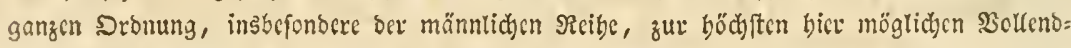
ung crbebt, Dionaca beutet aber zuerft auf bie frucht ber Biraccen. In ber britten s (Sruppe begimen dic von ben folgenden unzertrenmlidyen Franeeniecn, nuf bie Sauvagefen ber

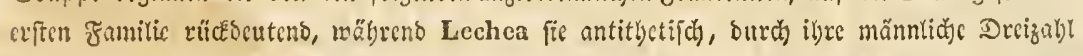

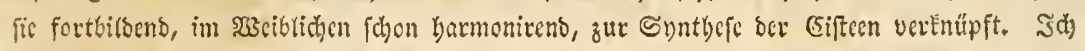

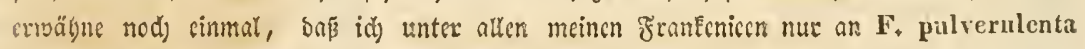

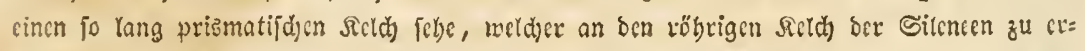
innern vermöd)te, bei ben übrigen ift er tiefer zerthcilt. Saci Lecliea febe id fo reenig

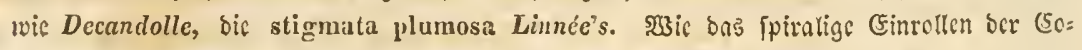

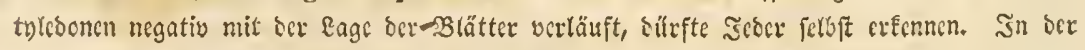
exften (Sruppe rollen fide nämlid) bie Blätter fpiralig auf, ocr Acimling beginnt gerabs,

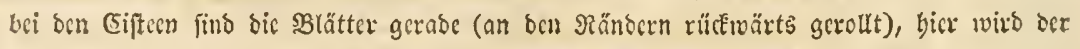

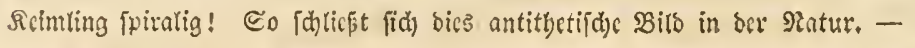

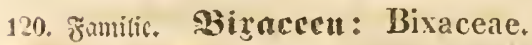

Piftill: Frudtetnoten sinfáct)erig, (Griffel sinfad), Natbe $2-3-4-5$ ppultig oder harbeugclig verfdimolzen. Feld) $4-7$ blitterig, SBtittd)en anfeinan: berticgent, boi 1) 4 -56litterig uno innen farbiy, bei wenigen flappig

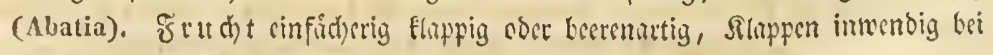




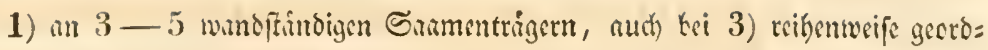
net, kei 2) all oer ganjen Ĩnnenfliche werbecitet, Einmentriger alfo bier nef̧= artig veriffelt. Enamen meift in Brei oder fecteft mit Elctrig Eecrenartig

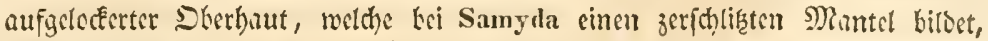

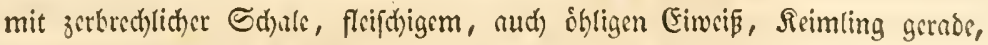
bei 1) ift dor Reimling umgefshrt und ber Same bat cine ticfe Reimgruke, bei 2) und 3) ift ber Scimling aufrect), bei allen bie Cotylebonen flach, auf

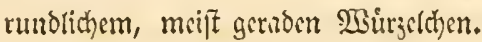

Staubbeutel: 1) berjformig, $8-30$, innerfits lings nufpringend, auf in

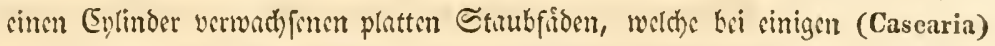
afwechfetnd folbupenartiy und benteltos find, bei c. Patrisieae find bic Staub= beutel langgefteckt und pringen an ifren Scitent nuf, dic Etubfiden fhon rund(ich) uno frei, wie bei alten folgenden, und, wie bei ihnen, auch viclzath= lig, bei hisrigen find fie Fitrger, meift rundtid) und fpringen etwas feitlich) auf. Blume feblt bei Samydeae umb cinigen Bixeae, bei ubrigen iff fie 5 klitte= rig, ciftusurtitig uno bei einigen 2 =, auch $5-7$ blítterig.

Begetation: Strîucher und Biume, Sllitter wechpelftindig und cinfad, bei 3)

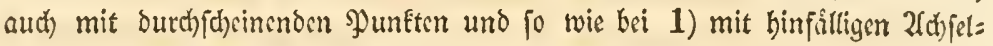

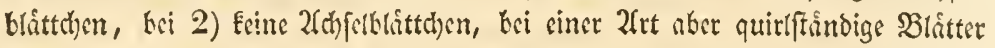
(Erythrospermum verticillatum Lam.). 3 wittetblithen ach felffándig, meift geftielt, cingeln oder dic Stiele veraftelt und mebrbluthig bis zur Dolden= trinte und Sifpe.

Bruppen:

1) Samydeac: stamina $8-30$ in urceolo monadelpha in c. libera 00. (auth. cordatae, in c. elongatae, cor. 0. eaps. valvulis $3-5$ coriaceis dehiscens, in ultimo genere bacca, semina 00 e medio valvularum baccaeformia, embryone inverso; in a. et b. stipulae.

a) Samydeac genuinae: filamenta singula antherifera, pulvinar nullum.

Guidonia Plum. Sanyda Loeffl.

b) Casearieac: squamulae antheris in urceolo alternac.

Casearia Jacq. (Anavinga Rheed. Chaetocrater Rz. Pav. Jroucana

Aubl. Pitumba Aubl. Melistaurum Forst. Athenaca Schreb. Lindleya Knth. malvac.).

c) Patrisieae: filamenta libera urceolo circumdata aut nullo, capsula aut bacca, stipulae nullac.

Patrisia K.II.B. Ryania Tahl. (Ryanaea DeC.).

2) Erythrospermeae: stamina lihera brevia, ut petala definita 5-7, stigmata 3-4. Fructus indehiscens subhaceatus, in placentis romosodiffusis 00 spermus, embryo erectus, stipulae nuilae.

Erythrospermum Lam.

3) Bixineae: stamina libera 00 indefinita, corolla $0-5$-petala, ultimis generibus $3-4-6$ petala. Embryo erectus. Stipulae nullae. 
a) Prockieae: stigma simplex, petala nalla, in medio genere rarius quina.

Prockia P. Brun, Laetia L. Abatia Rz. Pat.

b) Ludie a e : stigmata 2-3-4, petala 0 aut 5 .

Ludia Lam. Trichospermum Blume, Lindackeria Prsl.

c) Bixcae: stigma simplex, petala 5, aliis 3-4-6. - Capsula au: bacca.

๔) 8-6-sepalae, $3-6$ petalae:

Ascra Scholl? Banara Aubl. Christiania Prsl.

B) 4-sepalae, 4-petalae: Dasyanthera Prsl. Echinocarpus $13 l$. Xyladenius Hamili.

y) 5-sepala, 5-petala: Bixa $L$.

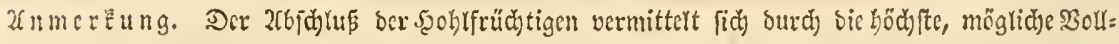

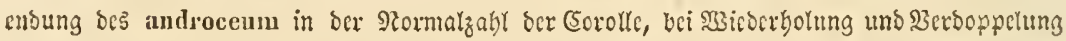
ber Urzablen, mit binen bicfe Sronung begonnen, faft überall aber bic Etaubfäsen in Der

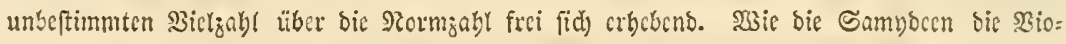

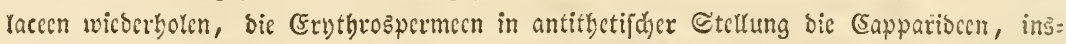
bcjonocre bie Flafourtiecn, ałs analoge paralkelifiten, mno jene Samydecn żu ben wahren

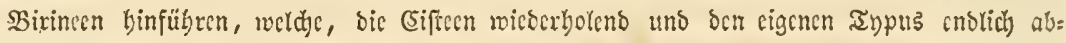
fdylicßent, bic Soronung matürtid volfenoen, liegt flar vor.

In ber erfen Didnung waltete aljo begimeno bas einzerne frudetblatt in ten

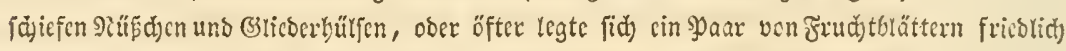

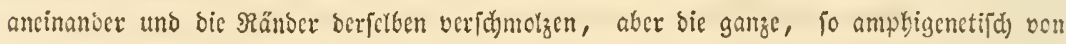

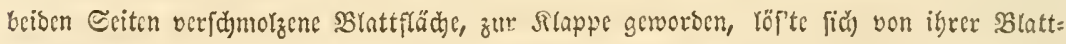

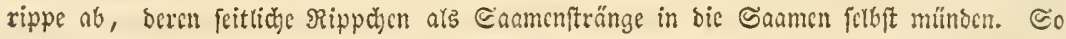

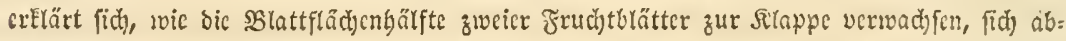
löfen Eonnte, fo crElärt fid aud), wie fie auf ifrer fecunsärcn Nippe, bem Bercinigungs= rande, bci ganzranbigen Blättcrn, wie bei Isatis, glatt uno ganzransig feyn, bci gczăfyn= teri ober fdjrotjägeförmigen 3uftanbe ber unterften $\mathfrak{B l a ̈ t t e r , ~ i m ~ F a l l ~ a u c h ~ b i e f e r ~ s u r d ) ~ b i e ~}$ operften ganzranbigen längft fajon geföwwunben, bennod) in biefer Silappemippe ber Frudjt

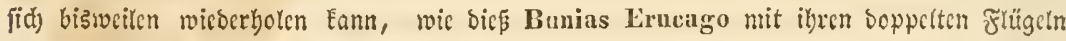

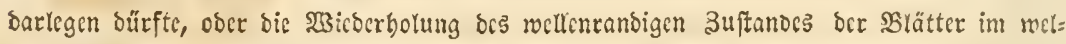

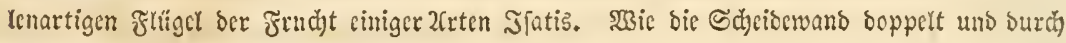
Mittclrippchen, fdon in Der uroeutung zur Ircnmung befäbigt, besbalb in Tetrapoma

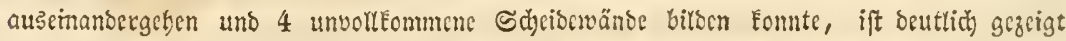

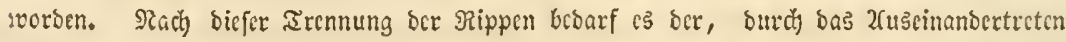
ber in ber erfen Enffebung fich gegenfeitig bicht anlicgenoen Blattrippen entfandener Scheibewand nidjt mekr und bei Reseda erfheinen bie Ripwen allein, auch bic Fumarieen,

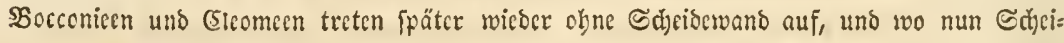
Dewände wieder entįtehen, wie bei Papaver, find biefe bie zu Eciften geworbenen 3 tacenten.

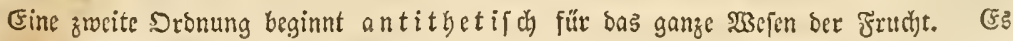
ift nidjt mehr jencs fricolidje Bufaumentegen von Fruchtblättcen, um mit ibren Rippen bie Saamen gemeinjuaftlid) für bas (sanze zu tragen, fondern jebes frudytblatt roill fith jetbft eine Frudyt bilben, cin aus ber friebliden. Finbeit feindlid) zerfaltener 3uftand bringt 
nur Echigocurpien bervor. Bci 2lggregation biefer Frud)tblätter in mehreren Recihen ober

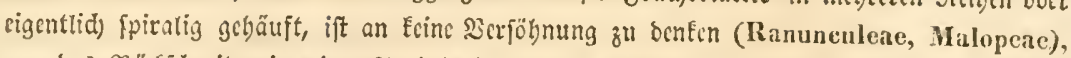

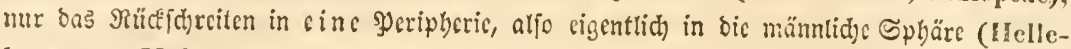
boreac et Malraceae cyclocarpae), vermittelt Berjöhnung fur (Finfeir, uns aus ihr

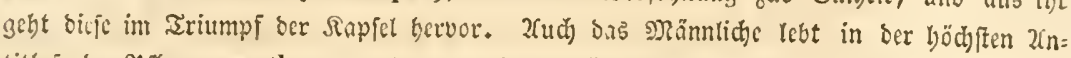
titbeje ber Y̧flanze: antherae extrorsac, beren \&öjung bic Siatur fich vermittelt, benn alle

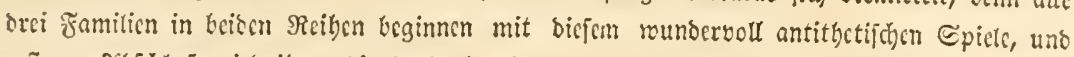

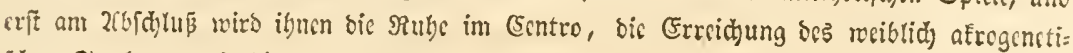
fdien ๔trebenz, wic bic ser antherae introrsac.

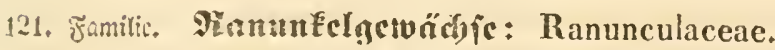

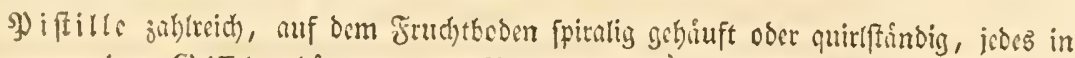

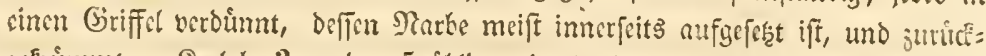

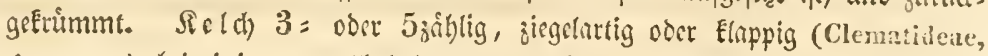

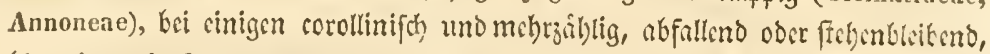

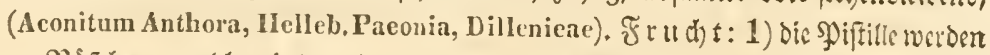

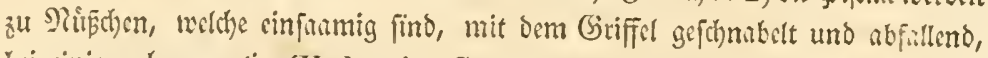
bei cinigen kecrenartig (Hydrastis, Guatteria ete.). 2) bie \$ुiftille werben jut

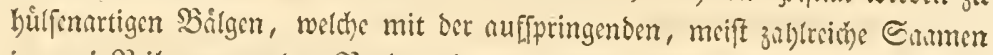

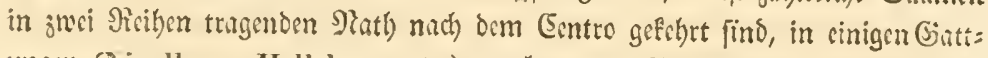
ungen (Nigelleae, Helkeborns etc.), auth) an ber $\mathfrak{B a f i s}$ verwad) jent, in ciner (Erobatos) cine Doppelfapfil bildond, in cimer (Actaca) auch kecremntig. 3)

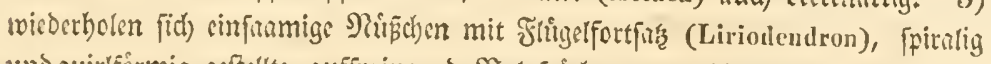
unt quillformig geffellte, auffpringende Balgfrud)te, unter-dicfen wieber becrenat:

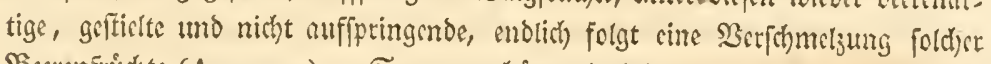
Secrenfrid)te (Annoneae). Sanme bingend bei Anemoneae, kei librigen meift aufrecht, glitt ober mit rauber (Isopyreae) querfaltiger (Nigelleae, Aconitum) Dberbaut, bei vielen mit ciner fielartigen \&eiffe bom Nakel aus, bei sinigen mit groß̈er Srimmarze (Calthal, Dillenieae), Eiwsis fleifchig, faft horn=

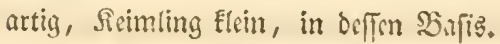

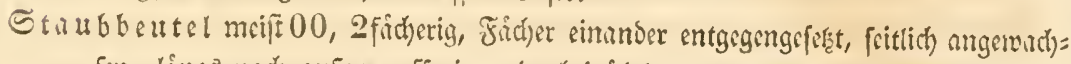
fen, lings nad) ausen auffpringent, bei dibrigen nad) ber Eeite und nad) vor=

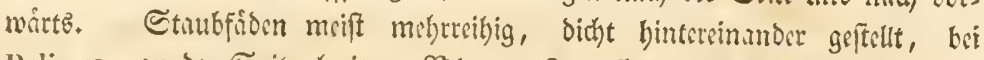

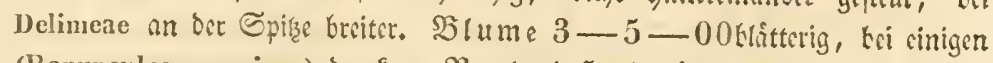

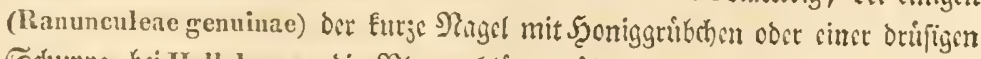
Schuppe, beillelleboreae bie SGlumenblitter róbrig, meift jweilippig ober trich= terfôrmig und in Der Sarfis Sconig abfonderno, bei ükrigen entweder Feine Slumenthitter (Actacariac) oder biefciben jicgelintig obcr flappig (Annoncae).

3egetation. Einjifhrige uno perennirende Srótter, Xantlorrhiza und alle 2) und 3) find boljig, diefe vom flcinftraucjigen bis jum baumurtigen Buftunde.

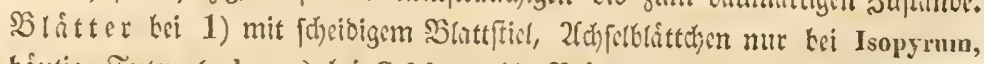
biutige Iuten (ochreae) bei Caltha; bie Slitter folbft burchlutfen nlle For= 


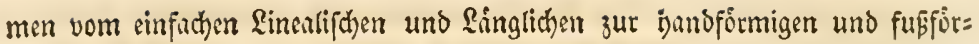
migen Ifyeilung, 3 = und 5 zathligen 3ufammenperzung, find wurzelftíndig kei

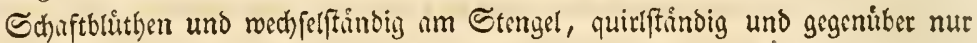
bai Anemonene. Basi 2) und 3) find bie Sblattftiele meift Eurz und ohne Echeiden, 2(d) felblittthen bei cinigen um bie SBlntténofpe getollt (Magnolieae genuinae, Illicieae), ober fehlen bei ben ůbrigen, die Blatter felbft meift leder= artig, ganj und ganjrandig, ober ecfig ausgrbud)tet (Liriodendron), geferbt (Dilleniene), aud) aromatifh mit burchfitheinenben Delorifen (Hllicieae). -

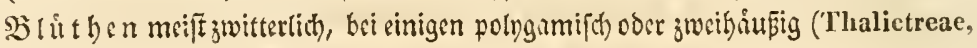
Delimeae).

(3ruppen:

1) Ranunculeae: herbaceae exstipulatie. (Xanthorrhiza fruticosa est, Isopyrum stipulas liabet, ochreas Caltha).

a) Ranuncilea genuinae: carpidia 1-sperma, semen erectum, calyx defiuitus, petala definita, ungue foveolato aut squamuligero nectarifera.

a) Ceratocephaleae: stamina pauca subdefinita:

Ceratocephalus Mnch.

B) Ficarieae: stamina indefinita, calyx ternarius:

Ficaria Dill. Casalen St.Hill.

भ) Ranunculariae: stamina indefinita, calyx quinarius:

Batrachium DeC. Ranunculus L. Krapfia DeC.

b) Ancmonea e: carpidia 1-sperma, semen inversum pendulum, neetaria nulla.

a) Adonideac: stamina, petala et sepala indefinita. (Carpidia in spicam compacta, folia linearia aut multifido-composita.

Myosurus $L$. Adonis $L$.

3) Thalictreae: petala nulla, caryopsides stipitatae longitulinaliter nerrosae. (Flores multis polygami, folia quinato composita). Sepala 4, exigua:

Thalictrum L. - sep. 5-9. petaloidea: Syndesmon Hffegg.

y) Anemonea genuinae: sepala corollina imbricata aut uniserialia valvata.

aa) Pulsatille ae: sepala corollina iubricata. (Folia uultifila, piunata, palmata, ultimis caulina involucrantia verticillata oppositave.

Callianthemum C. A. Mey. Auemone L. Hepatica Dill. P'ulsatilla $T$ :

$\beta \beta)$ Knowltonieac: sepala biserialia heteromorplıa herbacea. (Folia palmata, composita).

Hamallyas Commers. Knowltonia Salisb.

ry) Clematideae: sepala uniserialia valvata. (Folia opposita.) 
Viticella Dill. Clematis L. (Flammula Rupp.) Viorna Pers. (Cheiropsis $D_{\mathcal{C} C} C$ ) Atragene $L$. Taravelia $D_{\mathcal{C}} C$.

c) Helleboreac: carpidia pleio (2-00)-sperma, (Hydrastidi et 'Irautretteriae 1-sperma), calyx definitus, petaliz plurimis tubulosa nectasifera (Actacariis nulla, l'aconiis explanata absoluta).

«) Is opyreac: petala scpalis alterna concara aut infurdibulari-curnuta. (Folia ternati-compositi).

Leptopyrum Rchb. Enemion Rafin. Isopyrum L. Aquilegia $L$.

B) Delphinieac: irregulares.

Delphinium $L$. Aconitum $L$.

9) He 11 c boreac genuinae: regulares, scpala trpice 5, petala tubuloso-bilabiata sepalis plura (Actacariis nulla, P'aeniis explicata).

ax) Nigelleae: carpidia comnata (ultimo gencri capsula incarcerata), petala geniculata.

Garidella L. Nigellastrum Mnch. Nigella T.L. Erobatos $D_{e} C$.

$\beta \beta)$ Trollie ac: carpidia aggregata 1 -00-sperma, petala glandulosa ant tubuloso-bilabiata.

Trollius L. Gaissenia Rafin. $\Lambda$ phanostema St.Mil.

yv) Hell eboreae: genuinae: cyclocarpicae.

«s) Actacariae: apctalae racemosae (primum genus et secundum 1-pauci-flora).

Hydrastis L. Trautvetteria Fisch. et C.A.Mey. Actinospora Turcz. Botrophis (Nacrotys) Rafin. Actaea L. Petalorum loco glandulac 5. furcatac: Xanthorrhiza l'Herit.

$\beta \beta \beta)$ Helleborastra: petala tubuloso-bilabiata.

Cimicifuga $L$. Coptis Salisb. Eranthis Salisb. Helleborus $L$.

ryy) Paeon ieac: corolla in calyce corollino nulla aut calycem persistentem superans alssoluta.

Psychrophila DeC. Calthá $L$. Paeonia $L$.

2) Dillenieae: lignosae, flore quinario (calyx utplurimum persistens).

a) Delimeae: filamenta extrorsum dilatata, antherae loculi discreti subrotundi.

«) ge uu inae: stamina 00, carpidia 1-00 sicca.

Detima $L$. Tetracera $L$. Davilla Vund. Curatella $L$.

B) Recchicae: stamina definita $\mathbf{1 0 .}$

Recchia Moc. Sessé.

y) Doliocarpeac: stamina 00, carpidia baccantia.

Doliocarpus Rol. Empedoclea St. IIil. Trachytella DeC.

b) Hemistemoneae: stamina unilateralia.

Hemistenma Juss. Pleurandra LaL.

c) Hibberticae: stamina peripherica (filamenta extrorsum non dilatata), antherarum loculi oblongati. 
«) genuinae: stamina 00 , carpidia sicca.

Hibbertia Andr. Dasynema Schott. Othlis Schott. Wormia Rottb. - polyadelpha: Candollea LaB.

B) Adrastaeariae: stamina Jefinita $7-10-15$.

Pachynema R.Bi. Adrastaca DeC. Acrotrema Jack.?

g) Dillenieae genuinae: carpidia carnosa aut coalescentid.

Dillenia $L$. Colbertia Salisb. Capellia Blume.

3) Magnolicac: lignosae flore ternario (calyx utplurimum deciduus).

a) Magnolic ae genuinae: carpiủia spiraliter aggregata, flos inbricatus, folia impunctata stipulata.

«) Liriodendreac: carpidia samariformia indehiscentia.

Liriodendron $\boldsymbol{L}$.

5) II anoliariae: carpidia dehiscentia ant secelentia aptera. Magnolia $L$. Michelia $L$. Manglietia Blume.

y) Talaumeac: carpidia coalita.

Talauma Juss. Aromadendron Blume.

b) Illicieae: carpidia verticillati-stellata, flos inbricatus, folia puetata stipulata.

a) Illicieae genuinae: flores hermaphroditi, carpidia follicularilignosa.

\section{Illicinm $L$.}

(0) Tasmannicac: flores liclines.

Tasmannia R.Br.

y) Wintereac: fiores hermaphroditi, carpidia baccantia, Drinis Forst. Temus Mol.

c) An noneae: curpilia aggregata aut verticillata, ultimis coalita, fus valvatus.

a) Cardiopetaleac: petala lusi coalita (ultimi generis calyptrata). Cardiopetalum Schlecht. Ilexalobus A.DeC. Miliusa Lesch. Hyalostenuna Wall. Orophea Blunte.

6) Guatterieae: petala subclansa aut libera, carpilin 1-sperma. Anaxagorea st. Ilil. Artabotrys R. Br. Dugnetia St. Hit. Polyalthia $\mathrm{Bl}$. Guatteria $R \approx P a v$.

y) A n no n a riac: petala libera, carpidia pleiosperma aut coalita. a) Uvaricae: stanina inclefiniti, carpidia libera,

Sylopia I. Brum. Coclocline A. DeC. Habzclia A. DeC. Unona I. Lvaria L. I'orcelia Rz. Pav. Asimina Ad. Trigynaca Schlecht.

F̧) Boc a geac: stamina 6 , petalis antepiosita.

Bucagca st. IIil.

ry) Annoneac grenuinae: stamina 00, carpilia coalita.

Lolsocarpus Whigt. Arn. II cnsclielia P'sl. Monodora Dun. Rollinia St.frit. Anona $L$. 
3nmerEung. So writ legt uns bic Ratur bon Rammfertupus Elar vor bas foridyente

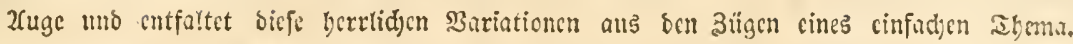

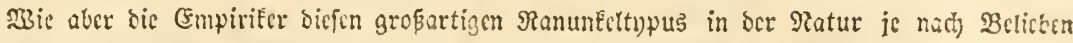
für fich zerfpalten, uncirgetenk anberce İypen, beren Integrität fic erfannten, wic ctro

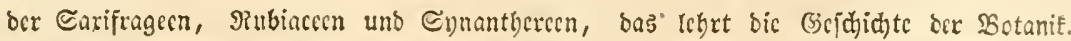

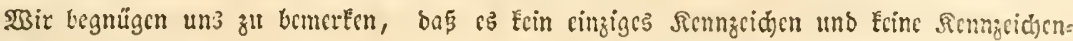

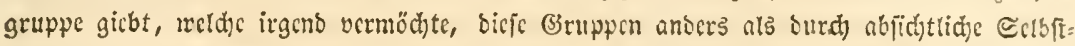
täufđung unb ous (Eonvenicnz cincr gcfälligen Mittälifdung, von bcn anbern a!s Fantiticn

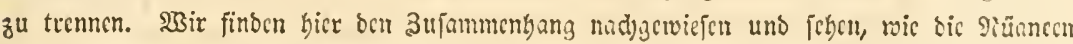

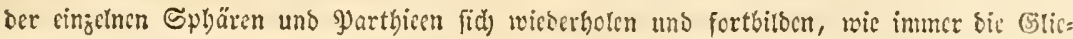

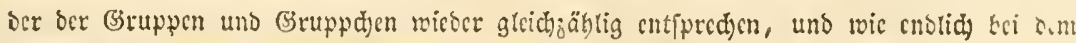
böd) ften Fortbitten bes 23siblichen bas Männtidge abuinumt.

Darum mar ber lurfás ber Familic: , Ovaria oriuntur plura separata "Fl. germ. p. 717. unb wir Saben gcichch, wic bie Ratur fid beftrcbt bat, bicfe ovaria plura jum

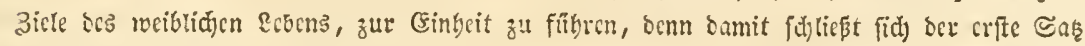

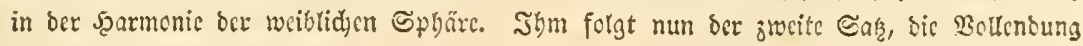
von Griffel und Starbe.

\section{Familic, Suntengetuäbje: Rutaceae.}

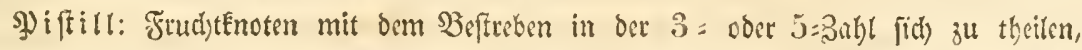
bei menigen in 2 (Mercurialis) ober in vicle Frocther (Hippomane), bei cinigen

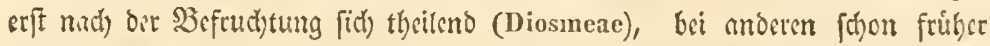
gefondert (Ochneae). (Strifel bei 1) meift tief 3 = ober 5thcilig, bie 9larken aud) woht 2theilig oocr bie gumze fternformig, bei 2) uno 3) oct (Siriffl (an=

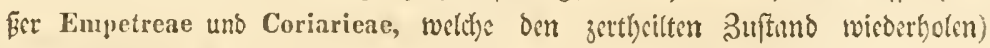
meift cinfud), bann mur an ber Şnfis getheilt, aus jedem Fruchtenotenfad) mit sinem Sdyen Eel entfpringeno (Ruteae unb Diosmeac) ober zwifcten ocn gefon:

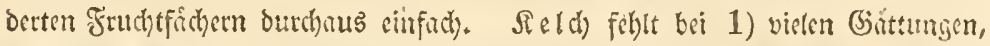
bei Euphorbia fünd die naEten Sluthen geftelt von cince Ecldhartigen Şille, mit 4

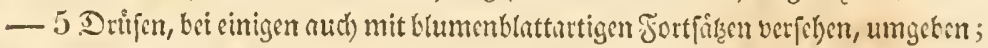

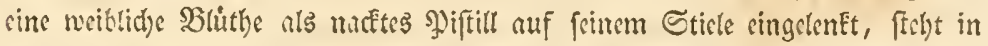

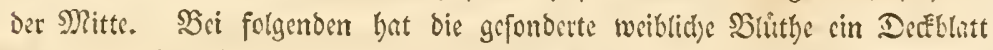

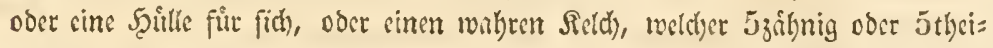

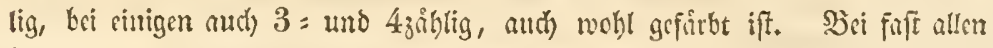

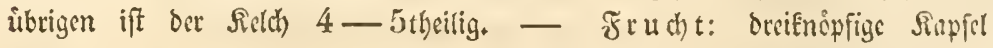

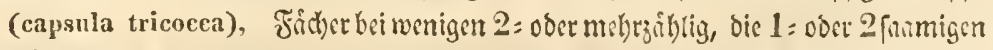
Fåcher fint burd) cine Mittelfiule verbunden ober theitweife frei, iffucn fich

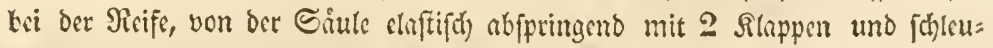
bern ben Gamen aub, bic Sarmen find bangend und haten cine flcifdige

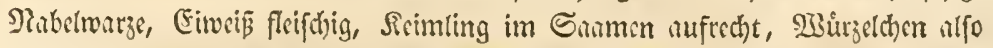
im Scingen nady oben, Eotyldoonen fluct). Baci andern ift bic frucht nicht auffpringend, hotzige Sapfal obcr fleifdige Socere (IIura, Hippomane), wie bei den (Empetreen, fid) wieberholt. Sei 2) pringen bie Fander an bie

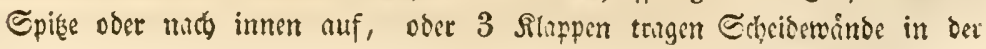


Mitte; mebrete Diosmean fpringen ebenjo shaftifd nuf als bie vorigen, fis

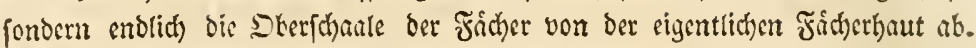
Canmen wie sinige verige, Eimeiß beginnt fdon bei einigen zu feblen unb fehlt bann bei Simarubeac ganglith.

Staubbeutel 2facherig, aufrecht, bei einigen nod) angetwadben (Pachysandra),

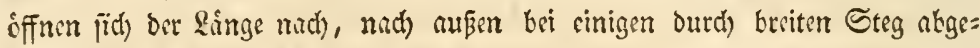
fondert (Didsmandra, Monotaxis!); kei 1) meift unbeftimmtzåhtig, in dem

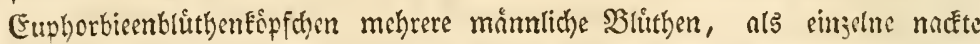
Strubfitoen, jeder auf peinem Stirlden, weldes an ber Bajits fein Decks

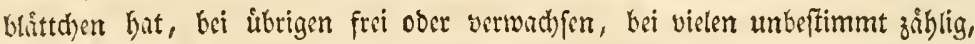
meijt, wic unch bei 2) uno 3) zu $3-5-10$, kei wenigen $2-4-8$,

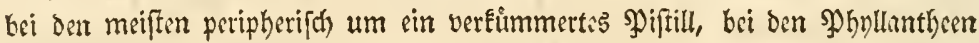
im Mittelpunfte ber Blithe bie verwadbfonen Etaubfíden. SLlumen frone kei wenigen vorhanden (Crotoneae uns Cluytieae), 3-5blitterig, Blumen=

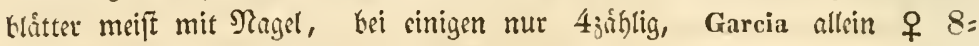

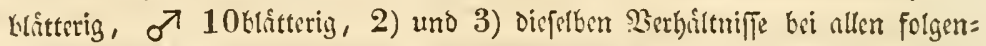
oin, bei einigen fehtent, aud) bei Ochna 10 britterig, bei sinigen bic \$letala mit oen গligeln jufammentaingend (Stackhousieac, Cusparieae), kei Dictamnus unregelmápig, mit nufferigenden Staubfíton, hei cinigan cime Pieige un= fruchtburer Staubfidoen blumenblattartig (Barosma, Agathosma).

Sigetation: Sirâter, Strâucher uno Baume, Slitter meift jerftreut, bei weni: gen gegenuber ober quithartig, meift sinfich), von fdimalen und linglichen For= men, kei eimigen geigenformig (Eupl, cyathophora, Jatropha), bandférmig getheilt (Ricinus) oder gefiedert (Phyllanthus, Dictamnus, Boronia, Zan-

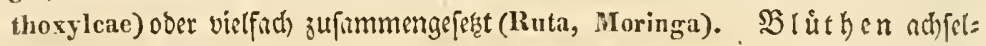
ftandig beginnend (Callitricle), zu Sippfchen in Şülld)en bicht vercint, dicfe in wiederholt gabeliffigen Dolben (Eupliorbia, Anthostemma, Monotaxis), in

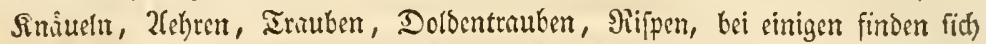

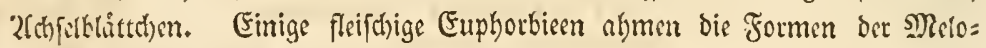

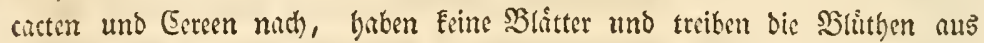
23arzen. Bei einigen find die gefiederten 23 litter gegliedert (Quassia), $\mathfrak{B}_{\mathfrak{e i}}$ ben Diosmeen find bie Blitter meiff oriffig, bei einigen ourchfichtig punEtirt.

(Gruppsn:

1) Eupliorbieac: apetalae diclines. (Crotoneac et Cluytieae corollatac opponuntur).

a) Euplorbieac genuinae: apetalae loculis 1-spermis, floribus omnino incompletis bracteolatis.

a) Callitrichineae: flores axillares solitarii : Callitriche $L$ :

B) Tith y maleae: flores in anthodio calyciformi glandulifero aggregati, femineus centralis. 
Euphorhia L. Pedilauthus Ncck. Hendecaudra Fschsch. Anthostemuil -1. Juss. Monotaxis Brngn, Findl.

\%) Ricineac: flores glonerati, spicati, racemosi et paniculati.

ак) H ippomineac: spicatac, -

demun drupa 1-sperma: Stilago $L_{0}$ Antidesma $L_{0}-$ drupa 2-4-sperma: Styloceris A. Juss. -

capsula tricneca ant drupa vel lacea 3-sperma: Maprounca Aubl. (Aegopricon L. fil.) Commia Lour. Lxcoecaria L. (Gymuant us Su, prod. Scbastiania spr.) Hinmalanthns (Onalanthus) A. Juss. Triadica Lour. Stillingia Garden. Sapium Jacq. Cnemidlostachys Mart. (Microstachys A.Juss.) Omphalea L. Hipponane $L_{*}$ (Hancanilla I'lum.) Hura $L$.

$\beta \beta)$ A calypheae: glocaeratae et glomerato-spicatac.

Mercurialis $L$, Acalypha $L$. Caturus $L$. Anahaena A. Juss, Dalcchampia Plum. Tragia Ptum. Hecaterium $K z$. Mappa $A$. Juss, Macananga A.P.Th. (Panopia Noronh.) Claoxylon A.Juss. Concercibum Rich, Alcliornen Sw. (IIermesia Bupl.)

ขr) Ricinea genuinae: fasciculatae, racemose, corymbosac, paniculatae.

capsula 4-cocca: Pluknetia Plum. - 2-E cocca: Rottlera Rxb. Gelonium Roxb, - 3-cocea: Adelia L. (Bernardia Housl.) Acidoton Siw. Adriana Gaudich, Ilisingera Hell. Amperea A. Juss. Ricinns L, Manilıot Aslans, (Janipha K, H. B. Cuidoscolus Pahl, (Jussieua Houst. Bivonaca Rafin.) Siphonia Rich. Niabea $A u b l$.

b) Crotoneac: corollatae loculis 1-spermis (plurimae aromaticae),

e) Micrantlı cae: tripetalae.

Micrantheum Desf.

B) Argytlonmeac: 4-petalae.

Argythammia P. Brw. -

y) Crozoplorea e: 5-petalae.

drupa 1-sperma: Griseliuia Forst, - capsula aut bacca dicocca: Mozinna Ort. (Loureira Cav.) Aleurites Forst. - Jrupa putamine dicoces: Anda Piso. (Joannesia Felloz.) - enpsula tricocca: Ditaxis Iahl, Crozophora Neck. Croton L. Crotonopsis Mchr. Caperonia A, St.IIil. Codiaenm Rmph. Ricinocarpus Dsf. (Echinosphaera Sicb. Räperia Spr.) Jatropha $L$. Elacococca Conmors. - flos \% 8-pet. o 10-pet, Garcia Rohr.

c) Buxeac: apetalae et corollatae loculis 2-spermis.

a) Phyllantheae: apetalae mesostemoneae (synnema s. filaucuta connata in centro).

Conspect. 5069-5082. et Geblera Fisch. et C. A. Mey. elc.

B) Cluyticae: corollatae mesostemoneae: 
Antrache $L$. Cluytia dit. Briedelia $W$. et peristemonent: Satvia $\boldsymbol{W}$. Richeria I ahl.

y) Buxeae genuinae: apetalae peristemoneae (stamina pistillo hebetato peripherica).

lechacea: Pachysandra Uch.r. - fructicosae: Buxus L. Securinegil Juss, Amanoa Aubl. Flïggea $W$. Ilyaenanche Lamb. ('I xicodendron $T / h b$.) Sareococea Lindl. Tricera Schres. Thecacuris A. Juss, Drypetes Fall.

2) Rutaria e: herinaphroditae corollatae. (Exordiuntur e dielinilsus, apetalis in antithesi adversintur).

a) Empetrea e: schizostyles.

a) Empetrea genuinae: diclines tripetalae baccatae.

- Empetrun L. Cyminosma Gürt. (Jambolifera L.) Cetatiolia kich. Corema Don.

3) Stackhousieac: 5-6-synpetalac, drupa aut capsula costata. fl. monoici : drupa 1-locularis demun 1-sperma: Psendanthus sicb. - f. $\not$, capsula loculis dispermis: Stackhousia Sm. Tripterocoecus Findl.

भ) Cneoreac: flores $\breve{q}$ tripetali, capsula tricocca :

Cheorum $L_{\text {, }}$

b) Zanthoxyleae: diclines schizogynae loculis 2-4-orulatis. (Sapindacearum praecones!) -

Consp. 5125-5140. et Ailanthus Desf.

c) $\mathbf{R}$ u tea e: holostyles 4-5-petalae, (plurimis) capsula 4-5-coccis.

a) Ru tea genuinae: petala unguiculata, capsulae loculi pieiosperni ad suturam dehiscentes.

Peganum L. (Harmalum T.) Haplophyllum (Aplophyllum) $A$. Juss. Ruta L. Buenuinghausenia Rchb.

ß) Diosmeae: schizocarpicae loculorum andocarpio bivalvi clastice Ilehiscente.

cos) genuin a e: quinariae petalis liberis:

Dietannus $L_{*}-$ Calodendron Thub. Barraldeia $A_{*} P$.Th. (Barraultia $s p r$.) Polembryun $A$. Juss, - Adenandra $U$. Coleonena Bartl. Diosma Berg. Euchaetis Barll. Acmadenia labrtl. Barosma $W$. Agathosma $W$. Macrostylis Bartl. Milocarpus Iahl. Almeidea St. Hil. (Aruba N.v. E.) - Diplulatria R. Br. Chorilaena Endl. Plicbalimu Veut, Crowea Sim. L'riostemun Sm. - monadelpha: Philotheca Rudg.

$\beta \beta)$ Cusparieae: synpetalae, petalorum ungues connati aut cohaerentes.

Monniera Aubl. (Aubletia Rich.) Ticorea Aubl. Krytrochi- 
ton $N$, et $M$. Diglottis $N$, et $M$. Galipea Aubl. Spirantlera $\mathrm{N}$, et $\mathrm{M}$.

જy) Boron ieac: quaternariae, petalin libera:

Erolia Forst. Melicope Forst. Zieria $S \mathrm{~m}$. Boronia $\mathrm{Sm}_{*}-$ pretala counata aut cohacentia: Correa Sm. Antonmarchia Collor.

y) Moringea e: holocarpicac 1-loculari-trivalves. (Boenuinghauspniae los repetitus, fructus inlicans 'Zygophylleas!)

Moringa Lam.

3) S ina r ub a a e: indehiscentes exalbuminnsue. (Polyg. et $\not{q}$, apet. et coroll.)

a) Coriarieae: schizostyles germinilus conmatis (Kuphorbieas illen rebcrintes).

Curiaria Nissol, - ? Pedicellia Lour.

b) Guas ie a e: holostyles, stylo e geminibus oriente, superne simplici (ibutae typum sequente).

(onspect. 5181 - 5180.

c) Ochucac: holostyles, stylo inter germina sejuncta pulvinari impoxita centralis, in antithesi Elvasiae stylus terminalis. (Fauriliae scopus: integritas styli stiguratis(jue!)

*) Castelea e: semina pendula albuminosa:

Castela Turp.

«) Elrasieae: stylus germini pluri-loculari terminalis. kisasia $\mathrm{DeC}$.

y) Ochneae genuinae: albunien nullum:

Walkeri Schreb. Diporilimm Burt. Ochna Schreb. Gomphiat Schreb. ? Philomeda Norouh.

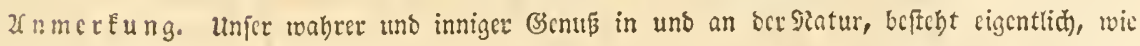

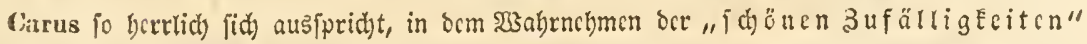

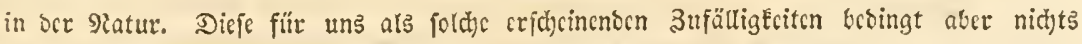

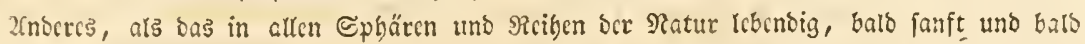

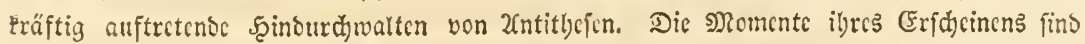

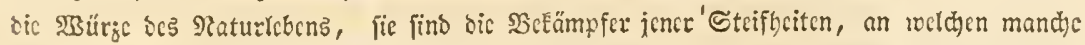

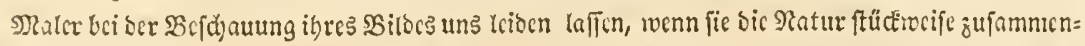
દ̌le

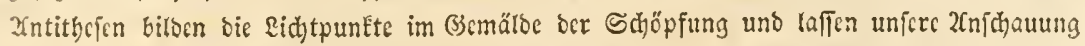

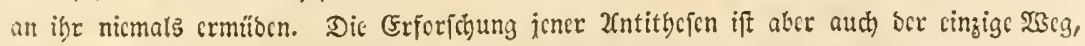

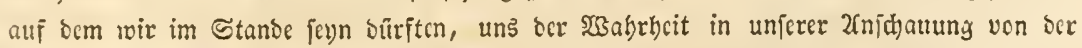

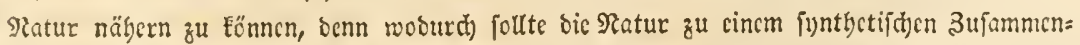

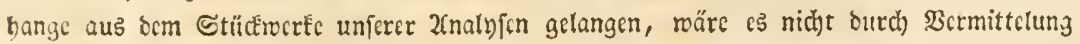

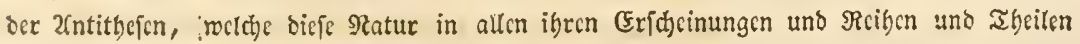
rege belcben? - Jch möchte atch) wohl ben deus ex machina febcn, metdicr für bicic=

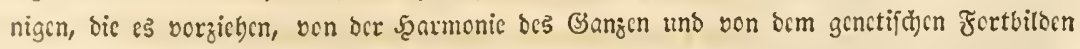

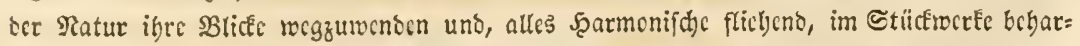

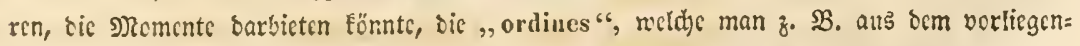

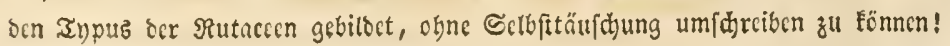




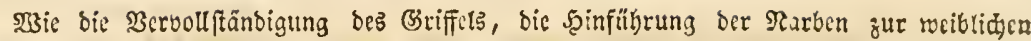

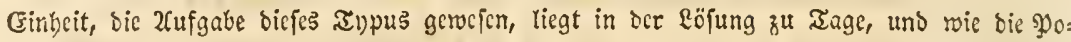
tenzen fich innig verketten, bas Tehrt bie Bruppirung. Dic gange Familie ift cine 2uati= thcje zrwifden ifren Rachbarn, beibe endlich freunolid verbinoend.

Nit fonberbarer Einfeitigécit hat man bie Euphorbiacen coroltentofe genannt,

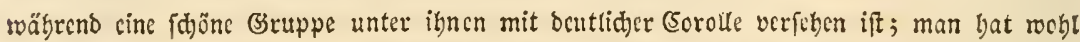

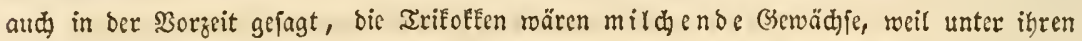
vielen (3attungen eine ober cin \$aar wirklid) mildende vorkommen. 33 ürbe man fic nidjt

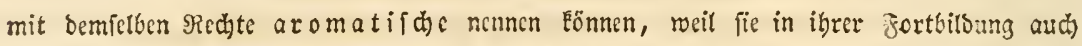

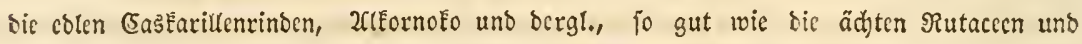

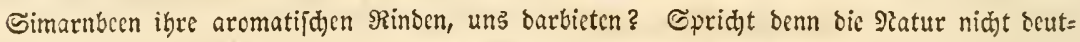

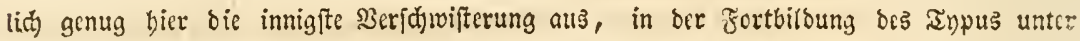

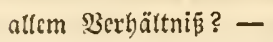

123. Familic. Enpinsacen: Sapindaceae.

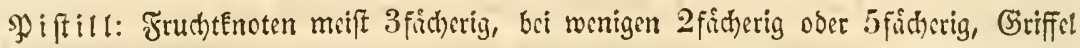
endftindig, bei vielen cinfeitig und aufffeigend, Narben 3 oder $2-5$, aud) einfach. Seld) 5 blitterig, in ber Sinofpe libereinanderliegend, bei sinigen 2 nupere ober 2 innere 3 líttchen Eleiner, bet andern 2 cbere nod) nid)t ge= trennt, aljo 4blitterig ober 4tbeilig, ober 1 blatterig und nur $4-5$ zábnig ober fpaltig (Ihippocastaneae). $\mathfrak{F} r u d) t$ : 3 ford)erige, bautige oder boljige Sapfet oder fleifhige Eteinfrutht, Eutmen am Mittelwinkel ber F̧äher, einzeln ober wenigjátlig, meift aufred)t, bei wenigen mit Mantel (avillus), bei ben meiften mit groß̄em Seimfleck, diefer bei cinigen wulftig. Seimling beginnt

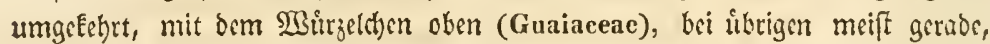

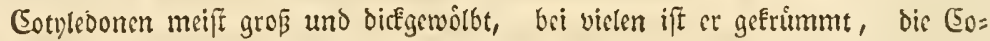
tylebonen mieder abwaits gebogen, bei ben 2fereen und Dobonicen fpiralig:

Staubbeutel 2focherig, innerfeits lingsinffpringend, meift 8 , bei wenigen 5 7 - 10, bei cincr Binttung 20 (Prostea). Etaubfioen frei, bei weni=

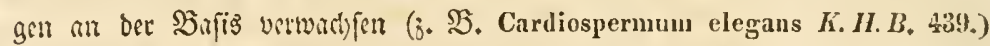
bei ben meiffen anfiteigeno und ungleich, bei andern anfuecht und gleich. (Fin

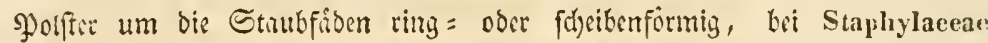

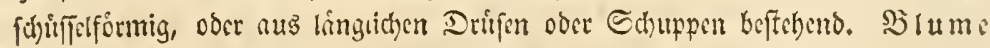
4 -5blitterig, unregelmípig ('Zypophyllum, Ilippocastaneae, Cardiospermun, Koelreuteria etc.), bei anderen regelmábig, fiblent bei cinigen (Dodonaea, Llagunoa, Stadmannia), bie Blumenbtâtter bei mebreren (Cardiospermum, Cupania, Koclrenteria), unten an ber Băfis nat) innen mit einer b) iutigen Sdhuppe.

Begetation. Sriuter, Strioucher, Biume, Serjweiguny, uno Seblitterung bei einigen gegeniber (Tribuleae, Guajaceae, Staphyleac, Acereac, Ilippoeastaneac), bei ben ubrigen wechfelno, bei einigen 2(thfilblattchen (Tribuleae, Guajaceae. Staphyleac, Mcliantheae), bri andern Winfetraneen (Cardiospermeae), bci

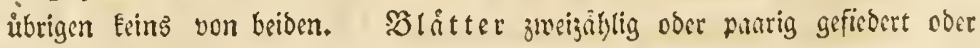
gefingert (Ilippoeastaneae), bei twenigen oreizáblig ober umparrig geficdart 
(Kinelreuteria, Staphylaeeae) und einfidf) (Dolonaca etc.). SBlithen jwittertid), bci 2) b. c. uno bei 3) polngamifd, kei wenigen sinjeln sodjelftin= big ('Tribuleae, Zygophyllene), bei den meitīn in Irauben und Tiiffen, aut) endiftinoig.

Bruppen:

1) Zyophylleae: capsula typice 5-locularis, folia opposita stipulata.

a) Tribuleae: indehisecutes cxalbuminosae.

Tribulus T.L. Elirenbergia Mart. Kallstroemia Scop.?

b) genuinac s. Guajaceae: dehiscentes albuminosac hilo subnullo. Conspect. 5194-5200.

c) Stapluy a e a e: dehiscentes exalbuminosae hilo magnotruncato (iam familiae typico).

Staphylea $L$. Turpinia $T_{\text {ent }}$.

2) Panlliniea e: capsula membranacea costata ant alata (samara) 2-1locularis, in antitlesi etiam 3-locularis; folia alterna stipulata ant cirrosa, ultimis opposita exstipulata.

a) II el i a the a e: irregulares, stipulatac, ecirrosac. (Zygoph. rep.) Mclianthus T.L. Chitonia Moz. Sess.?

1) Cardiospermeae: cirrosae. Conspect. 5235-5239.

c) Acereae: oppositifoliae exstipulatae ecirrosae samara diptera. Negundo Mnch. Acer $L$.

i) Sapindeac: typicae triloculares grandihilatac.

a) II ippocastaneac: oppositifoliae ligitatae (Zygophylleas ficre irregulari revocantes). 5202. 5201. et Calothyrsus et Macrothyrsus spach.

b) Do dlon a eeac: alternifoliae, capsulace (plurimis membranaceae) loculis 2-pleiospermis.

Conspect. 5223-5282 et Cossignia Commers. Diplopeltis Endl. Ia gonia A. St. Hil. (52"25. Phacocarpus .rart.)

c) Cupanieae: alternifoliac, capsulac loculis 1-spermis.

Toulicia Anbl. Schmidelia $L$, Irina $\mathrm{Bl}$. Prostea Camb. Lepisanthas Bl. Sapindus L. Erioglossum Bl. Monlinsia Camb. Cupdria Plum. (Tina K. S. Ratonia DeC:) Blighia Kön. (Akeesia Tuss. Bonania Raf.) Stalmania Lam. Dimereza Lab. Talisia Aubl. Ipphelium L. (Euphoria Comm. Bimocarpus Lour. Scytalia (iart.) Thouinia Poit. (Thyana IIamilt.) hypelate $R, B r$. Melicocca $T_{\text {. }}$ (Schlcichera $W_{\text {. }}$ - Matayba Aubl. (Ephielis Schreb.) Aphania Bl.? Alectryon Giitn.?

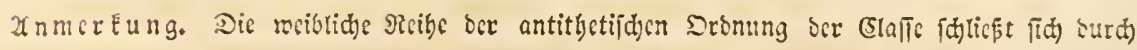

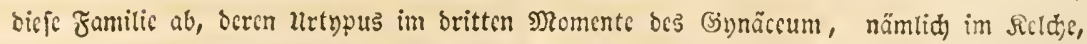
grgeben ift, turch) ifn roirb bic Röfung gefunbert: "gynacceum absolvitur". Durdi

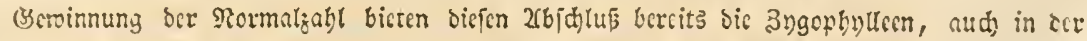

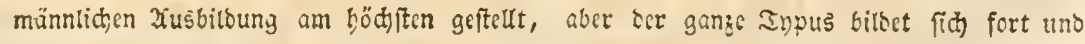

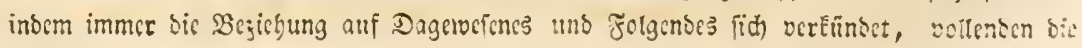




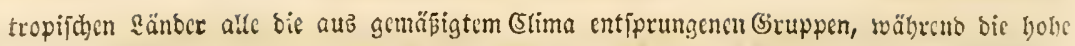

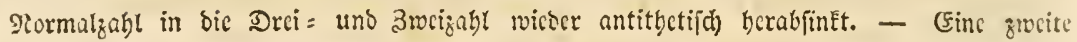

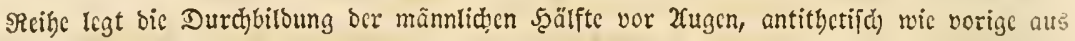

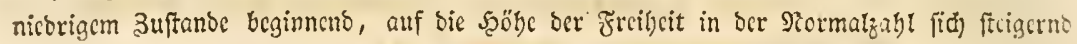
unt in ben gebunbenen abctphif

12t. Familic. Maluengewärific: Malvaceae.

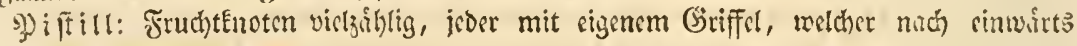

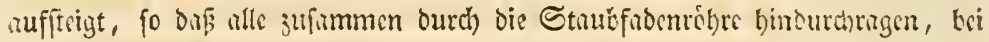
Den vollendeten Formen gelungt oer FruchtEnoten uno Griffsl jur (Finhsit (Kietuieae), bat Domn $5(3-10)$ Narben oder in Fogro,ia mur cine Ecuten=

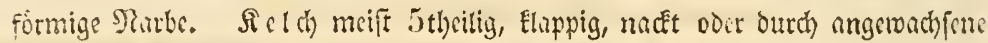

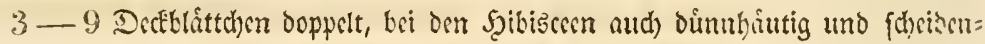
artig (Abchnosclus, Hymenocalyx etc.), wotei bis Scitle mehr feld)artig cr=

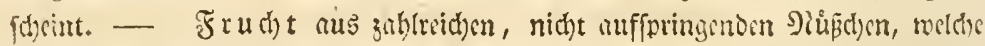
1) um cinen mittelftínbigen Eurjen Sammentriger berum quirlformig gejtelit

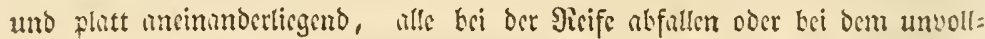

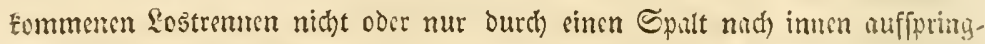

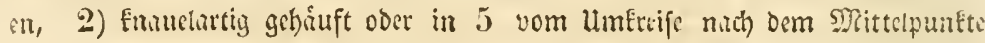

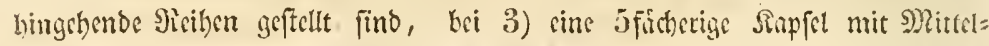

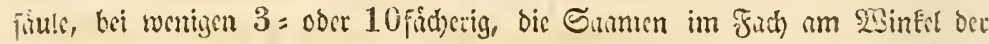

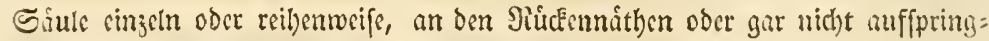

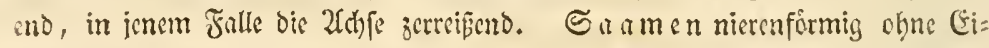

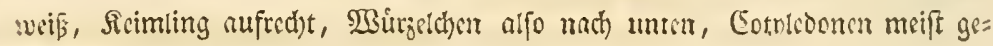
foltet. Gramenfduate bei sinigen wollerngeno (Gossypinum).

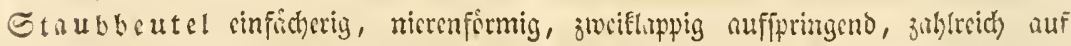

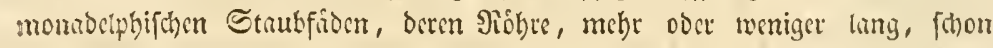
yon ber Mitte aus bis nuch ober mit 2fntheren befsçt ift. SBci Astrajnea umb Hymenocalys bic Strubbutel 2focherig, bei erfferet auch beftimmtziflig. -

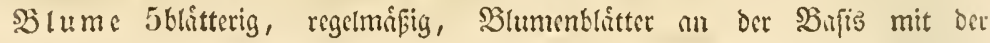

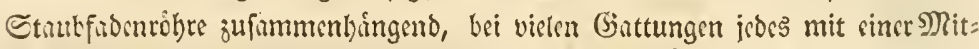
telterte, in ocr Sinofpe und nach dem Serblitgen jufummengsorsht, zufum= merthangend abfallend.

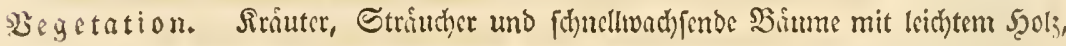

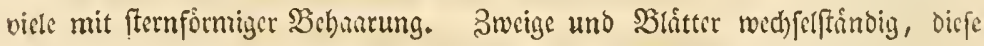
meiff gefticlt und handformig getbeilt oder sefig, lappig und urigetbcilt, bam abcr

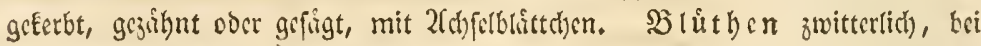

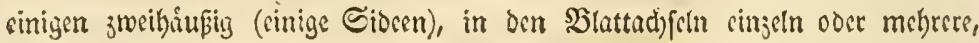
aud) traubig.

(Sruppen:

1) II a I reac: earpidia verticillata secedentia.

a) Lava tereae: antherac indefinitue, calyx bracteolatus.

a) genuin a e: germina et stigmata subulata 00 , carpidia sicca. Malva $L$. Lavatera $L$, Althaca $L$. Modiola Mnch. Splacralcea St. Hit. 
G) Pavonicae: germina subquina definita, stigmata doplicata capitellatil, carpidia sicca.

Urena L. Pavonia Cav. Thorntonia Rchb. Lopirdia Mluit. I.ebretonia scherk. Gucthea N.v.F.

$\gamma)$ II alvavisceae: carpidia 5 baccata subconnata.

Arhania Su.

b) Astrapaeariac: antherae definitae aliae 1-locilares steriles, aliat biloculares fertiles, germen subcoalitum 5. loculare.

Astrapaea Lindl.

c) Sideac: calyx ebracteolatus, corolla,

a) Sideae genuin ale: germina indefinita 1-sperma secedentia.

Silla L. Gayia Kuth. Periptera DeC.

B) II al achreac: germina 5-definita, stigniata duplicata, petalis obliqua. (Pavonieac repetitae.)

Malachra $L$.

y) Sidaria e: germina in capsulam coalescentia nec omnino secellentia.

«a) Anodea e: carpidia indefinita 1-sperma.

Nuttalia Bart. Anoda Cav. Cristaria (av. ")

B3) Bastardieae: capsula 5-loculari-loculicida valvis melis septiferis.

Bastardia $K$.

yø) Abutileae: capsulae vesicariae carpidiis subtrispermib.

Abutilon Dill.

2) Mal opea e: carpilia glomerato-agrgregata.

a) Malope a genuinae: carpidia inordinata, calyx tribracteolaters.

Malope $L$.

b) Palavieae: carpidia inordinata, calys ebracteolatus.

Palavia Cav.

c) li it a i b e li e a e: carpidia radiatim5-acervulata, calyx plurilırarteolatus. liitaibelia $\mathrm{W}$.

i) Hibiscea e: capsula perfecta, loculi plurimis pleiosperni.

a) Lagunaeeae: calyx ebracteolatus.

Lagunea Cav. Ingenhousia Moc. Sess.

b) Hymenocalyceae: calyx spathaceus bracteolatus, antherae hiloculares.

Hymenocalyx Zenk,

c) lietmiea e: calyx bracteolatus, antherae uniloculares.

๔) genuin a e: capsula 5-locularis, calyx regularis.

Senra Cav. Hibiscus L. Mbelmosshus Medik. Kiosteletzkya Presl. Lagunaria DeC. Polychlacna Don. Gossypiun $L$.

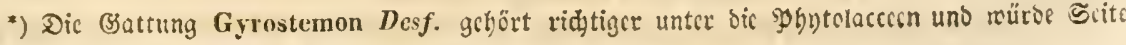
230. aนَ Plyy tolacca folgen müfịen. 
B) F ug os i a a : capsula 3-4-locularis, stigma trifidum aut claratum! Fugosia Juss. Redontea Vent.

y) Thespesieac: capsula imperfecte aut perfecte 10 locularis. Thespesia Corr. Parita Scop. (Paritium A. St. Ilil.) D'ecaschista Whigt. et Arn.

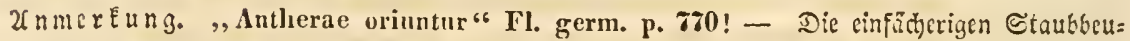

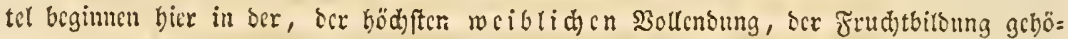
rigen Ela cigene 2fntitycfe ber Familic vollenect fie burdy Astrapaea unb Hymenocalyx, uns äthn=

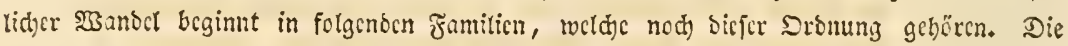

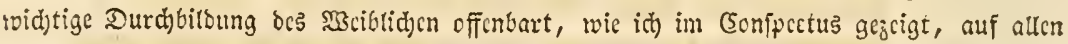

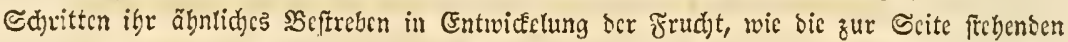
Ranunfulacecn beweijern, was unter bon Syftematiéern unferer zcit für bie Ralvnecen enstid ber talentwolle Meisner wicber erfannt hat, Spicr ift bic, bom ulrweibliden cnt=

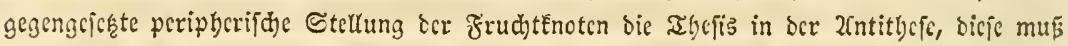

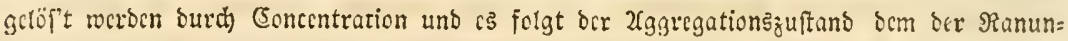
Eetn und 2fnemonecn analog, weld)cr auch) an jeincm Sorte bie methrfäd)erige Sapjel, jo wic

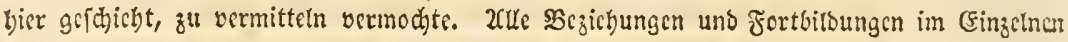
Siliften ziemlich llar vorliegen.

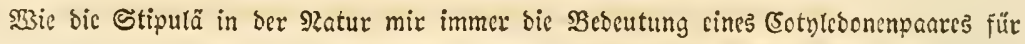

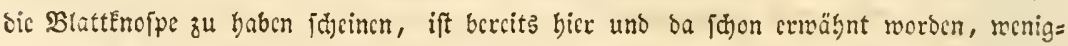
ftcns zeigt fich bicfe Eretärung immer ticfer begrïndet, als andere mir beEanntc. Daß

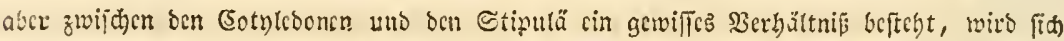

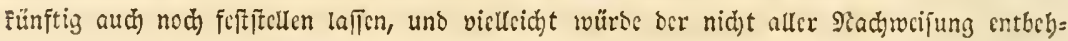

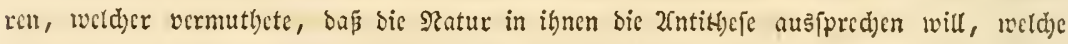

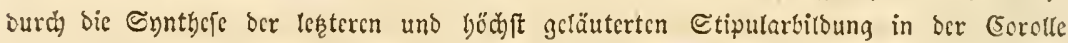

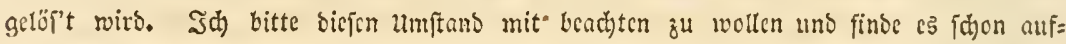
fallent, Daß bei cotyledonibus convolutis an mehrerern Stclfen cine aestivatio corollae convoluta, bei ben SRagnoliacecn nit ftcifen (Soty)tcoonen und Netalen stipulae convolutae borkemunen, bic syctalcn bod) atts bem gerollten 3uftande fich entfalten.

125. Familie. Etorchichnabelgetmächic: Geraniaceac.

P̧iftill: 1) Frudtenoten cinfaum(id), ju 5, kci 'Tropaeolum uno Flockea ju

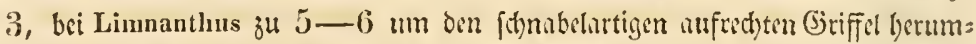

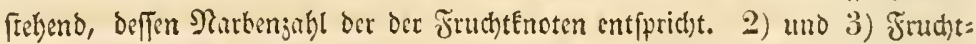

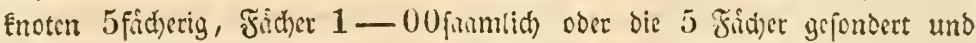
jedes mit befonderem (sirifel (IIeritiera), meift bie Girffé in cincu verwad)= fen. Seld) 5blitterig oder 5theilig, in ber Inofpe bie iuperen Iheilftucke aufliegend, bei cinigen bas oberfte an ber $\mathfrak{B a f i s}$ in eimen angerwad)fenen (Pelargonium) oder freien Sporn (Misgallana, Tropaenlum) tibergebeno. \$si 2) und 3) ber Reld) Elappig, dic 2(bjd)nitte entmeder flad) (Hermannieac,

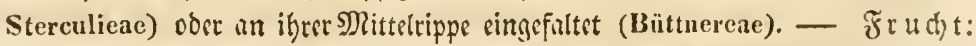
bei 1) bie frudtfficher 1 fanmig, von unten fid) aktofeno, igre şille (jedes

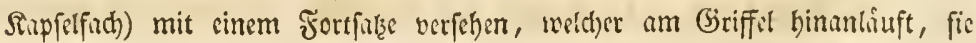
lofen fich zuerft unten ab uno werben won ienem Fortfaze, weldyer fith auch vou 
unten nad) oken bebt, in sinem Bogen emporgeboben, fpringen unch innen nuf und ftetten oen Sanmen aub, bei einigen (Krodimu unb sinigen Pelarg.) brehen fich jene Fortfốge ftrickartig um oen Griffel zufummen. Ganmen

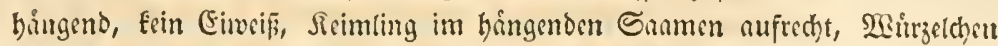

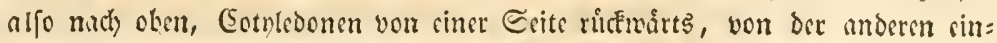

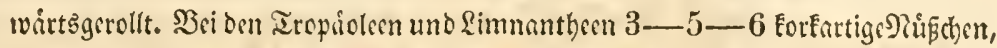

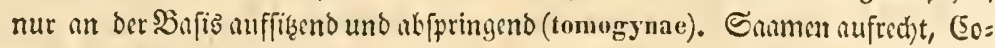

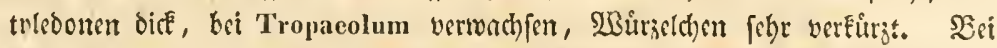
Mugallana cine oreifligelige, cinfaumige Shuß. 2) 5 fíd)erige אapfel, a. an

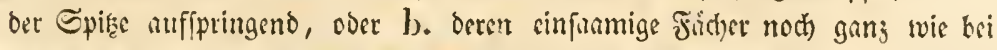
(ieranium von unten fidh ablopeno; c. Fíd)er gefondert, 1-00fanmig,

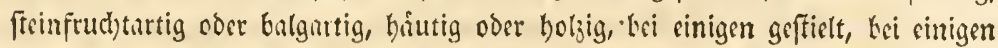

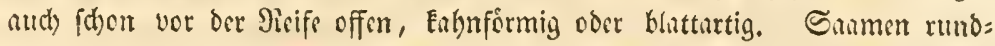

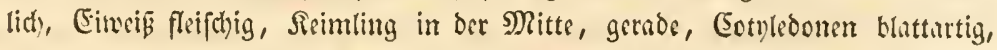

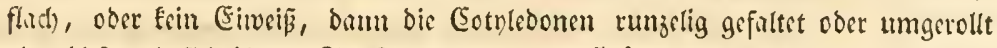

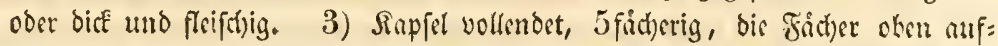

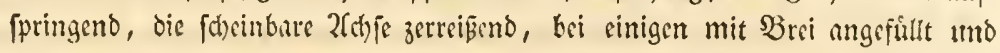
nicht auffipringento (Theobroma); Sanmen reihenweife, bei den erfen nur

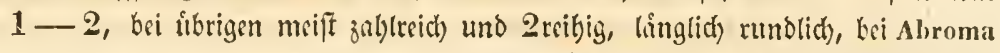

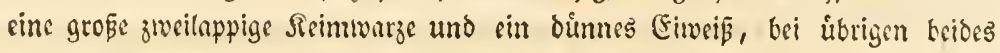
nid)t mehr vorbanden.

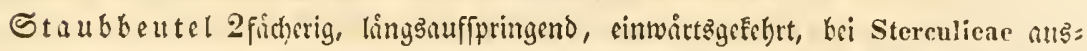

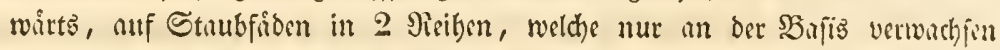
fino (Geranieae gemninae, Magallana, Hernandieae, Büttnereae) ober gamz frei (Tropaeolum, Limnantlieae, Mhynchotheca), ober potnadelphifó, (Mon-

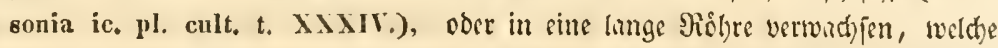

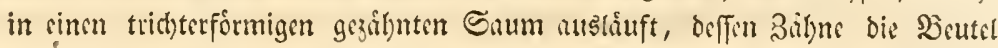
tragen (Sterculieae geniinae). Die \$cutel fohten kei Lrodium ber inneren Etrubfadenreibe, bei Geranium find fie vorbanden und find bie fpatter auf: fpringenden. SBei Tropieolum fpringen bie 4 zuerft auf, wolthe ben 4 un=

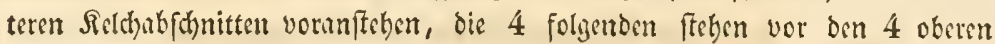

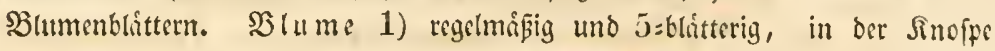
meift zufummengeoreht, mit ber erften Etaubfadenrsilye und mit dem Sistde

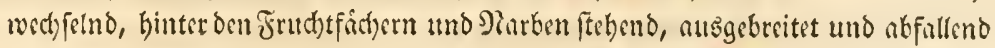
bei Erodium uno Geranium, bei P'elargonium unregelmífig 5 blâtterig umb ab: falleno; Ginnelfento, unregetmificia 5blitterig und geftreift bsi Tropaeoleae, regelmispig 3: und 5blitterig uno gefercift bei Limnantheae. Bai 2) uno 3)

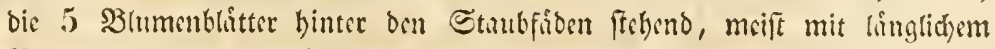

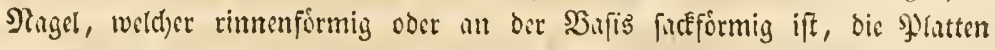

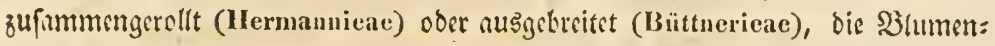
blitter fehr flein (Lasiopetaleae), oder ginzlid) feglend (Rhynchotheca, Sterculieae geminae).

Begetation. 1) Siråuter und Etriaud)er mit mehr oder minder fuftigem, fno: 
tigen Etengel, Şläter meift wechfelnd, bei gabeliftigem Stengel auch grgen:

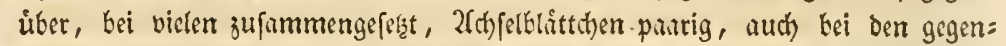

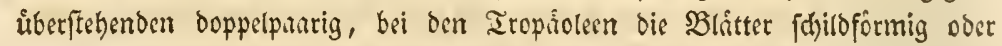
gefingert, bei ben \&imnantheen gefiedert, wie bei vorigen oh ne 2(d) ferblittchen. 2) uno 3) Straucher uno zum Theil großße Báume, Blâtter meift ungetheilt, bei wenigen handformig, befonders berzformig ober linglid, bei mebreren ges

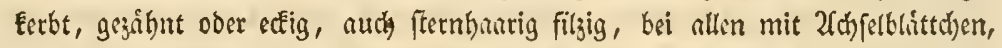
biefe bei sterculieae genuinae meift bald abfallent. SB tuthen zwitterlid, bei

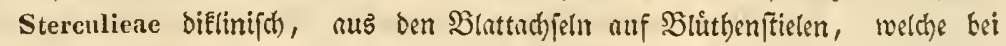
Tropaeoleae und Limnantheae cinblithbig find, bei Geranium sanguineum

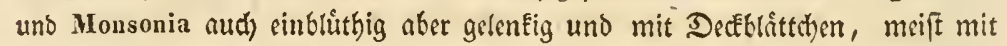

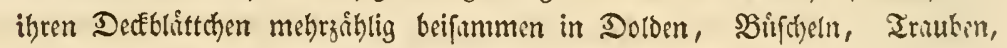

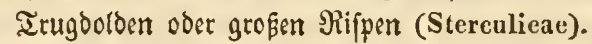

(S) ruppen:

1) Geranie ae: calyx imbricatns, carpilia monosperma 5, aliis $3-6$ aut in Magallana, in 1-spermum contracta. Albumen uullum, semina in a. pendula, cotyledones convolutae aut planae, in b. et e. crassae, Tropaeolo connatac.

a) genuinae: filamenta petalis aequinumera, duplicata aut triplicata, monalelpha aut polyadelpha, folia stipulata.

a) Erodiea e segulares stam, alternis ant singulis fertilibus.

Erodium l'Herit. Geraniun $L$.

B) Pelargonieac s. irregulares staminibus partim sterilibus:

Hoarea Sucet. Dimacria Linll. Campylia Sweet. Myrrhidium DeC. Jenkinsonia Sweet. Chorisma Lindl. Pelargiun DeC. Cynosbata DeC. Peristera $D_{c} C$. Otidia Lindl. Polyactium DeC. Isopetalum Sicect.

y) IIonsonieae: polyalelphae.

Monsonia L. fil. Sarcocaulon $D e C$.

b) Tropacoleac: stamina petalis irregularibus uareescentibus incougrua, folia exstipulata. (Petala superiora calycogena.)

Magallana Cav. 'Tropacolum $L$.

c) Limuntheae: stmuina petalis regularibus marcescentibus duplicata, folia exstipulata.

Flockea $W$. Limnanthus $R, B r{ }^{*}$ )

*) SRidjt lange nachber, ats id auf $\mathfrak{G}$. 145. bei Floerkea vorläufig auf Limnantheae vcr=

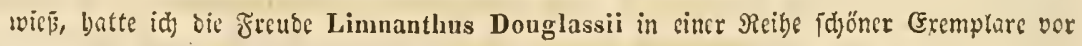

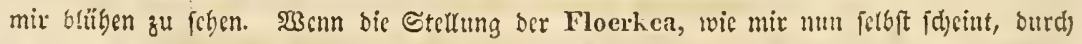

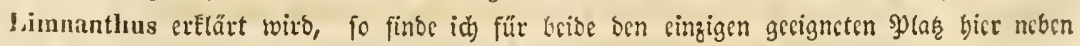

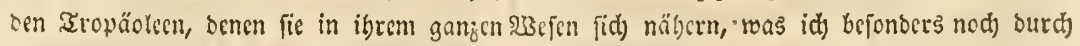

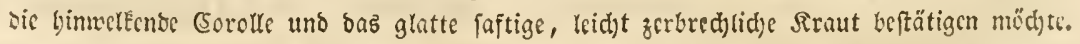
Die (Sotylebonen fint unverwadjien, bie Shlume bereitet bier bentlid) idjon Oxalis vor, bic oritte Familie vor'äntïg vortünoend. 
2) Stereuliariac: calyx valratus, partitionibus planis, carpidia pleinsperma atit capsula perfecta. Albumen carnosum olciferm et emlnyo erectus, rectus aut albumen nullum et cotyledones curvatae, convolutac contortuplicatac aut plano-convexae et crassae.

a) Il ermannieae: Jermaphroditie, corolla contorta, stamina 5-1080 ima basi monadelpha.

c) Maluernieae: stamina 5. petalis anteposita, capsula 5-locularis. Mahernia $L$. IIcrmania $L$,

B) Waltherieae: stanina 5. petalis anteposita, capsula demum 1locularis 1-sperma.

Waltheria $L$. Altheria $A, P . T h$.

r) Dombe yea e: stamina $10-15-30-40$ plurimis partim stetilia, capsula 5-incularis.

Pentapetes L. Assonia Cav. Dombeya Cav. Mellania Forsk. Pterospermum Schreb. Trochetia DeC. Ruizia Cav. - Hugonia L.? quae calyce imbricato differt. -

b) Rhy ch ot heceac: corolla nulla, capsula, albumen carnosum, embryo inversus!

Rhynchotheca Rz. Pav.

c) Sterculieae: diclines, stamina in tubum connata, antheris in ejus

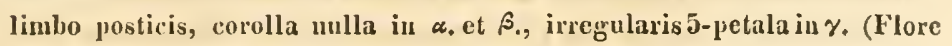
et fructu Zanthoxyleis parallelae).

œ) Triplaceae: carpidia drupacea indehiscentia aut membranacea globosa vel ventricosa dehiscentia.

Heritiera Ait, - Pterygota Schott. Endl. Biasolettia Prsl.*) Chiclaca Prsl, Triphaca Lour.

B) Stercul i a c genuinae: carpidia follicularia iam ante maturitatem quibusdam aperta:

ace) So ut hwelli eae: carpidia subsessilia.

Sterculia $L$, Sonthwellia Salisb. Trichosiphum Schott. Endl.

Cola Schott, Eindl. Cavallium Schott. Findl.

$\beta \beta)$ Il il degardicae: carpiclia stipitata alata,

Hildegardia Schott. Endl.

ry) Bl'icliychit oneae: carpidia stipitata aptera, ante uaturitatem aperta, nembranacea :

Erythropsis Lindl. Firmiana Marsigl. Scaphimm Schott, Endl. - carpidia clausa subconnexa: Brachychiton Scholt, Endl. Poccilodermis Scholt. Futl.

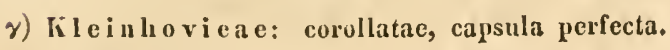

Kleinhovia $L$.

*) 2rittir älb Biasolettia koch. (ucrgl. S. 219.), baher idj) biefe aljo Freyera nenne, bem

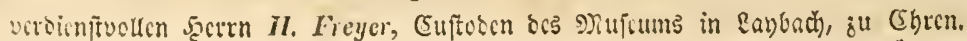


3) Büttnereae: calyx ralvatus partitionihus induplicatis, petala staninilus postposita patula, stamina basi monadelpha, pluribus sterilia fertilibus alterna. Capsula perfecta.

a) genuinae: petala conspicua, eapsulae loculi 1 -2-spermi. stamina 5 fertilia, sterilia nulla: Melochia $L$. Rirdleia $V_{\text {ent. }}$ stamina alia sterilia: Ayenia $L$, Büttneria Loeffl. Rulingia $R, B r$.

b) Lasiopetaleae: petala nulla aut minima squamuliformia. Kerandrenia Gay. 'Thomasia Gay. Guichenotia Gay. Lasiopetalum Sm. Seringia Gay. (Gaya Spr.) Macarthuria Hïg.

c) The obrome a e: petala conspicua, eapsulae loculi 00-spermi.

Commersonia Forst. Guazuma Plum. Glossestemon Desf. Abroma L. fil. Theobroma $L$.

Zf nmerEung. Der Iypus der Geraniaceen, stamina absolvuntur "Fl. germ, p. 785.,

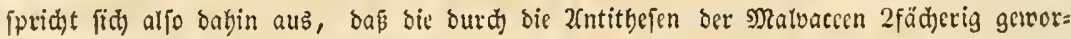

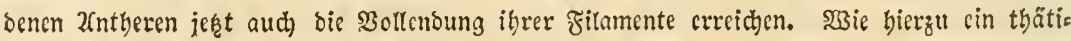

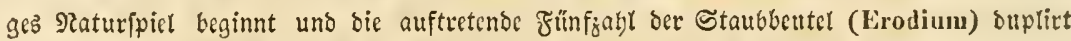
(Geranium), burch Bertümmernng wieber unterbrod)en (Pelargonium), burd) Becorci.

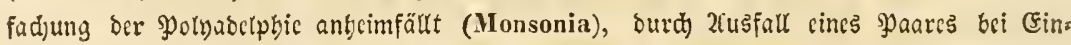
tritt bes analogen Radjbarverbättniffes ber Sapindaceen, wieber atts dem Inpus ber 3weci=

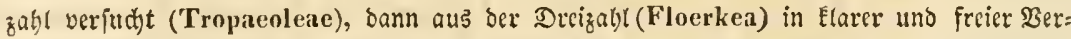
boppelung geroonnen wird (Limnanthus, in befien $\mathfrak{B}$ (hithen id) nod) offter 6 ats 5 Frud)t=

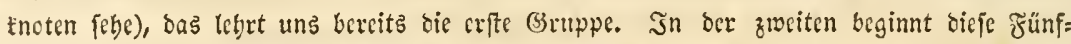
zabl von neuem uno madyt ähntiche Fortfdyritte burch bie Dombeyacen, bis ricber bie

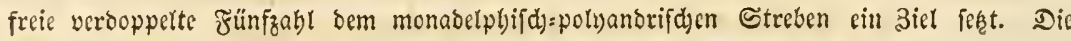

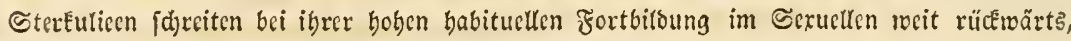
bie Staubfabenröhre ber Nharvecn viederhotend; jie ziehen fid), a!s hödffter (Segenfus der ganzen Reibe, nod) weiter zurtide ats bic Malveen, und fins, wie fdjon Rhynchotheca, obne Eorolle, bis ifnen Kleinlovia bie Eorolle wieber getwinnt. Dic şüttureen gleidjen ałfe bagcrociene Berbättniffe aus, ftellen bie Brunbzablen feft, für alle Gphären oer

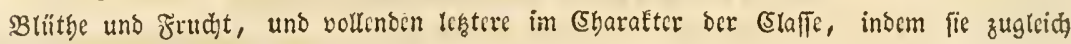
bas in ber 2entitbeje wieder auftatdyenoe Eiveis, in ihnen ganz überwunden bat. So bit=

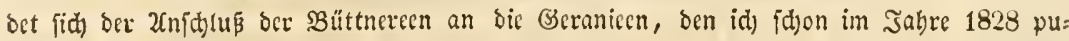
blicitte und zu meiner Freube bann aud von Röper, in friner an ticfen SRaturblicken fo reidien $\widetilde{d}$ rift: ", de floribus et affinitatibus Balsaminearum" 1830. wicoer ge= ahnet jothe.

126. Familie. Saucrflecgewänfie: Oxalideae.

Spiftill: Fruchtenoten 5 Eantig und 5 fâcherig, Biriffel 5 oder einer mit 5 , bei Caesarea nur 3 Narben, biefe bei einigen fopfformig, bei Caryocir $4-5$ (Sirffel, bei Adansonia 9-10ppaltige, fternformig ausgebreitete Narbe.")

*) SBei Cavanillesia Rz, Pav., (Pourretia $W$.) iđreiben einige nad) $R z$. uno Pavon. ,stigma capitatum", sie neucre 2Cb6iloung von Bonpland pl. aequill. t. 133. zcigt mir in: ceffen ein stigma multifilum. 
Seld) 5 blitterig oder glocenformig, 5paltig oder theilig, bei sinigen $3=$ und

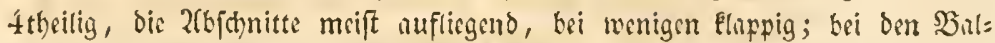
fumineen 4 blifterig beginnend, 2 quecrgeffellte suberfe Jield)blitter beçelute= ifhnlich, 2 verticul geftelte corollinif(t), diss untere gefpornt, bas ohere mit bem Beftreten in 2 fith ju theilen. $F r u c t: 5$ fitherige fiapfel oder Becre in 1) mit Mittelfiule beginneno, bei 2) und 3) fich roieder auflofend, bei Ungeria

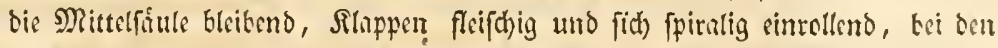

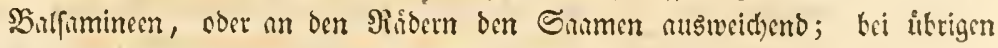
biutig ober ledernetig, bei ben leseteren auch wicber fleifhig uno verwarbfon,

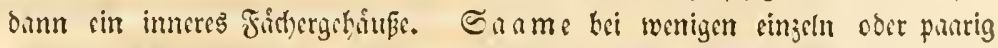
in ben Fichern, meift 1 = oder 2rihig, bei 1) a. Einmenbaut binn, Ecin

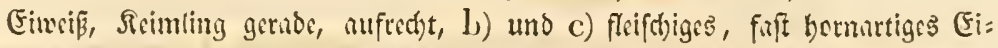

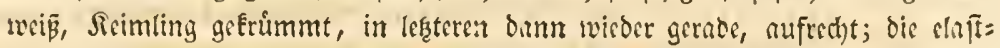
if (i) fid) atrollenden Slappen ber Balfamineen ffreuen bis Samen aus, bei Oxaleae genuinae ift dic Sanmenhaut biff, jieht fich vom reifen San=

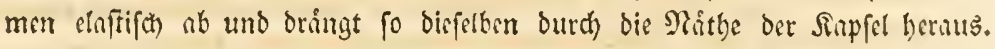

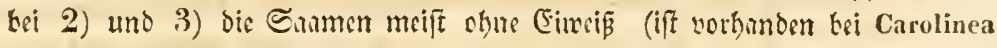
uno Cheirostemon), (Sotyldoonen incift runzelig gefaltet ober gerollt ober we: nigftens getogen (Allansonia), bri Cheirostemon flud), bei Rhizoboleae flcin,

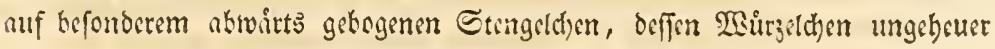
grosi ift.

Staubbeutel 2ficherig, lings aufpringend, cimwirtgefefhrt bei ben Balfami= neen, bei biefen folon in ber jungen Jinofpe aufpringend; fermer bei ben Dras

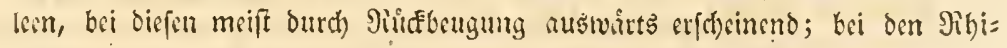

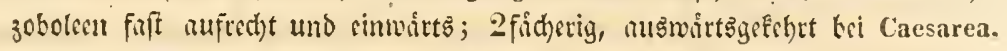
sinficherig focinend und nur mit unocutlicher Suceridscidewand hei ben 5ृe=

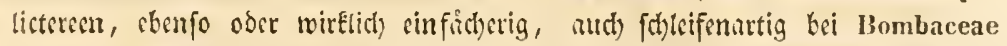

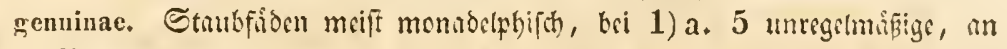
oer Bafiz fpigig, nud nur locter auffikend, unch oben etwas zufammenbingeno,

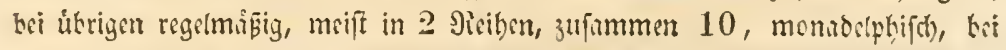
Oxalis die frrien (Enden nuth) mit einem ober bem anteren Subne, bei anderen

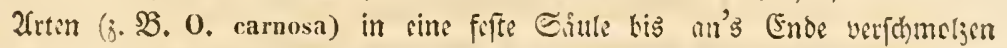

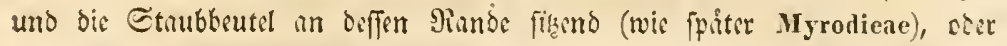

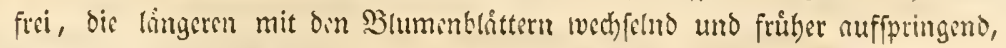
die 5 Eưrjeren vor din Ṡluminblittem, bri Averrlioa Caranbola biefe, vor son Slumenblîttern, unfuchtbar. Bei Caesarea ued)feln 5 Driffen mit den Blumentlittern und frehen binn noch binter ben lingeren Stubfifden. -

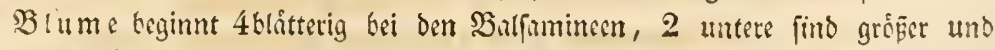
baben lingere Slifgel (hort, bot. t. 101. Fig, e.), 2 obere (oufelbft Fig. cc.) fino Elciner und baken einen Furzeren Nangel, jwifchen biefen feblt bus fünfte. Bsi allen übrigen find 5 Şlumenblatter, enttweder alle regelmásig ober un=

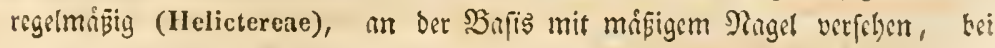




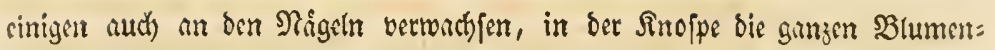
blatter zufammengerollt.

Segetation: 1) Situter mit faftigem Stengel, Slitter cinfind, uno gezấnt, zet= frreut (Impatiens, Balsamina), ober gegenuiber (Balsan, setac, trilob. etc. Caesarea), oder zufammengefert, $2=3=4=53$ åblig, oder mit blattirtigem

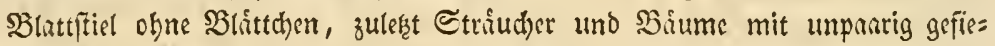
betten Blittern, 2(6)jelblattchen parrig. 2) Strifud)er mit ganzen, meift

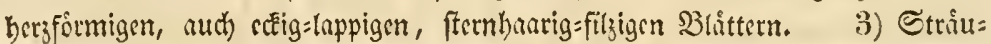

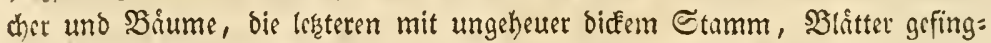

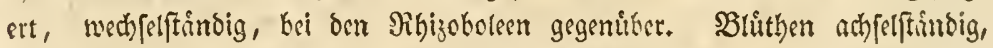
Sticle sin: oder mebrbluthig, gelenfig mit Dectblittchen.

Bruppen:

1) Oxalea e: caly $4-5$ sepalus, antherae $5-10$ biloculares, stigmata 5 , in antithesi 3 .

a) Bals am ineae: calcaratac. (Cal, 4 scpalus, Cor. 4 pet. stam, 5., 3. petalis alterna et 2 superiora sepalo snmmo antepositis; - proxima Hydrocera 5 sep. 5 pet. - antherae biloculares colnerentes; caps. 5valvis $\mathbf{5}$-locularis locnlis $\mathbf{1 - 5}$ spermis, semina exalbuminosa, in Inpatiente singula, invicem superimposita unile columella flexusa, in Balsamina serialia, in Hydrocera rerticillatim posita.)

Impatiens L. Balsamina Riv. Hydrocera Bl.

b) Caesarellae: regulares calyce valvato, stamina libera 10 . antherae extrorsae, glandulae 5. post stamina longiora petalis alterna, pistillum 3 -fidum 3-loculare. - Folia simplicia opposita.

Caesarea Camb.

c) Oxaleac genuinae: regulares ealyce imbricato, antherae retrorsae.

c) Acetoselleae: 10 andro-mouadelphae rectembryae. Oxalis $L$.

B) Ledocarpeae: libere 10-andrae, curvembryae. (Caesareau repetentia).

Ledocarpon Desf.

भ) Carambolea e: liberae, ct subliberae baccatae, rectembryae.

Biophytum DeC. Arerrhoa L. Bilimbia (Rhecd.) lichb.

2) Helictereae: calyx campannatus et corolla 5 petala irregulares, staminum tulus elongatus, antherae oblongac, longitudinaliter subbiloculares incumbenti-adnatae. Stipulatac. (Sterculieas repetentes).

a) Isoreae: tubus stamineus germinis stipitem raginans, filamentis apicen versus liberis germen circunstantibus, carpidia in stipite connexa 2-00-sperma intus dehiscentia, columella nulla,

Helicteres L. Isora Rheed. Methoricum Schott. Endl. Alicteres Neck. Orthothecium Schotl. Endl.

b) Ungerieac: columella centralis persistens! antherae in tubi 5 fidi limbo ternariae.

Ungeria Schott. Endl. 
c) Iyrodieac: tubus stamineus germinis stipitem vaginans aut germen sessile superans, antherne in limbi margine sessiles.

\&) Reevesicae: capsula stipitata loculicile 5-valvis, semina loculix gemina alata.

Reevesia Lindl.

B) Quararibeae: drupa aut capsula bilocularis disperma sessilis. Plaginnthus Forst, Quararibea Aubl. Myrodia Sw.

y) Matisicae: drupa magna 5 locularis putaminibus 1-spermis. Matisia K. H.B.

3) Bombaceae: calyx campanulatus, corolla regnlaris ant nulla.

a) Rhizoboleae: stamina polyadelpha, petala carnosa; antherae biloculares suberectae; styli $4-5$ ? Radicula maxima! Folia opposili digitata. (Oxaleas schizostyles repetentes).

Caryocar L. (P'ekea Aubl. Saonari Aubl. Rhizobolus Gärt.) - Opliclus Lour?

b) Cheirostenoneae: petala nulla, symnema (filamentorum connatorum fascis) apice digitato-5-fidun laciniis dorso antheriferís. (Helictereas revocaus!)

Cheirostemou $\mathrm{Hl}$. Bpl.

c) Bomb a e e a e genuinae: petala corollina, antherae 1 - vel 2-luculares (Typorum familiae summa et finis!).

a) Eriodendreae: capsula 2-5 locularis, semina lana involuta.

«a) uniseriali-monadelphae:

Bomly cospermum Presl.? - Ochroma Sw. Erione Schott. Findt, Eriodendron DeC. Gossampinns Hamilt. Caupylanthera Sichott. Endl. Eriotheea Schott, Endl. Montezmuna Moc. Sessé,

$\beta \beta)$ dupli- et multipli-seriali-monadelphae :

Chorisia Kuntk. Salmasia Scholl. Endl.

ry) polyadelphac:

Bombax $L$.

3) Po u r r t i ea e: eapsula demum 1-Incul, 1-sperma, (flos fugacissinus!)

Pourretia $W$. (Cavanillesia $R z$. Pav.)

y) Adansonieac: capsula 5-10-12-locularis, senina nuda aut in pulpa nillulantia.

Carolinea $L$. fil. Durio L. Adausonia $L$.

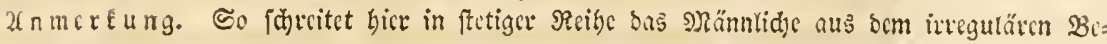
ginnen ber Balfamineen vorwärts unb witb in Caesarea unb in ben äêten Sxalcen vell=

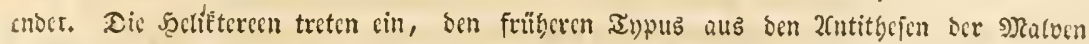

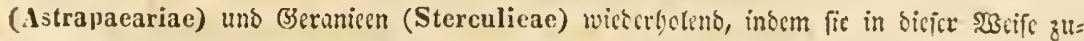

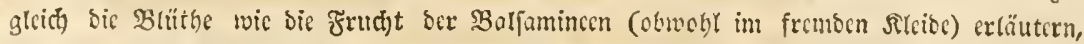
Dic cigenthümridje Staubfabenverbindung, wie fie bci Oxalis fid) mannigfaltig geftaltct,

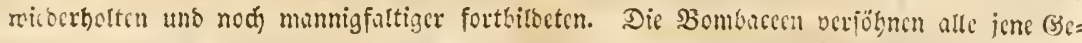

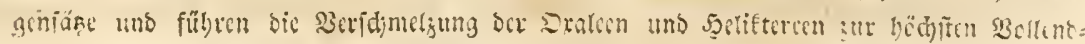




\section{Etielblüthige.}

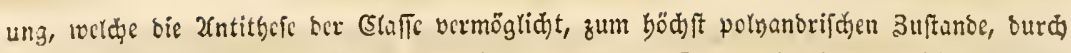

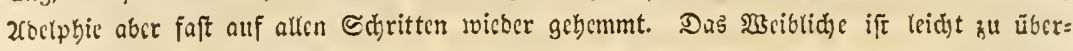

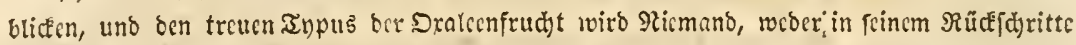
zutur Baljamine, nod) in feincr Sallenoung, in oer Adansonia digitata, verfennen.

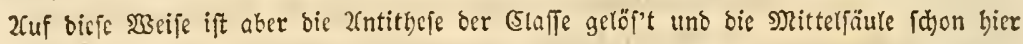

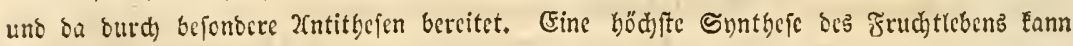
barum in ocr britten Dronung ocr (Elaffe in Einleit beginnen uno von bicfer Einbeit nur

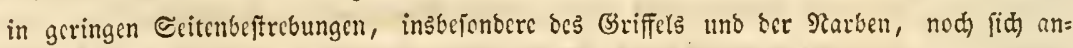
fangs entfernenb, bie bödfte Conecntration erlangen, in weldyer wir bas normale reiblide Gtteben, inuner auf ber Babn feincr Platur wandelno, etÉcunen.

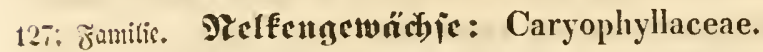

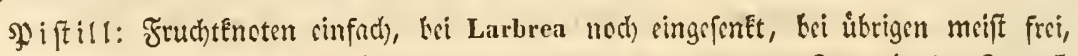
Earmentriger mittelftindig, oben in Sertindung mit ben $2-4$ corr $3-5$ (Griffeln, meld)e loings innerfeits ftigmatifd) und nach aupen umgetogen find, bei 2) und 3) auch Eeulen = uno Eopfformig. Seld 1) 56litterig oder 5: theilig, bei menigen 4 blitterig ober 4 theilig, bei Diantheae mebr oder weniger

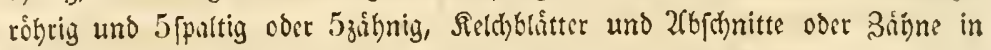

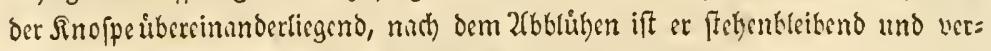
trocknend. 2) uno 3) 5theilig, 2(bfí)nitte meift ftumpf, anksu [bei 3)] meift mit grosem Drüfenpante befeft, in oer Sinofpe Elappig, nad) bem 2lkblithen meiff jethenblikend. - $\mathfrak{r u d}_{\mathrm{t}}$ : 1) Sappel bei einigen (Silene) auf einsm Stift (gynophorum) emporgehoben, bei oan meiften ribend, 1fictherig, bei si= nigen bod), (owie bei 2) uno 3) 3 fâcherig, bei wenigen in ber $\mathfrak{B a j i s ~} 4$ fód)erig, bei Cueubalus kerrenurtig, Sanmentríger nad) ber Sefrudhtung oben abgerif= fen, ouber alz freie Mittelfâtule, meift vielfaumig, bei anderen $3-2-1$ = faumig, bei Arenaria Ponae pl. erit, 260. fand ich 3 mufthelformige gefranjte Darfilarfduppen, in benen die Sanmen tiegen: Fig. E. F., $23 a n$ rippenloz, an oer Epite in 5-10 3ithnen auffpringend oder 2-4= bei andern 3-5= Elappig. Sarmen meifít nicrenformig, geformalt oder glatt, aud) planconver

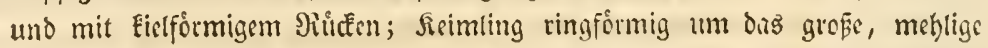

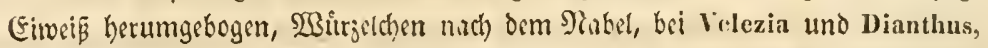
fowic bei Erythroxyleae, beren Saume bingend uno nach Berţimmerung von 2 Frichern nur cinjeln ift, findet fich orr firimling getabe. Bei 3) cine 3:

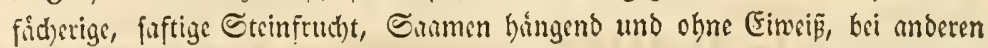

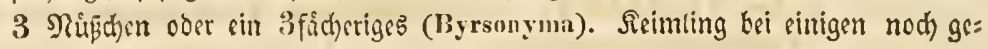
Erimmt, bei anderen gerube, Eotnledonen faft klatturtig.

Staubbeutel: 2focherig, aufliegend bei cinigen uno faft allen 2) uno 3) nuf= rect)t, lingbauffpringent, meift 10 , bei wenigen $3-5$, nuch $4-8$, auf

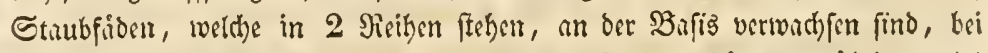
Larbrea und nod ein prat anderen aud bem Selthe angaingend, ůkrigens bei

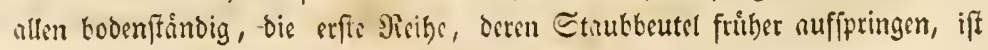




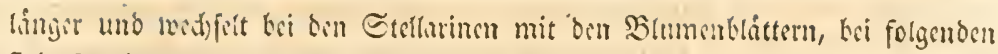

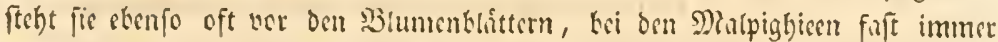

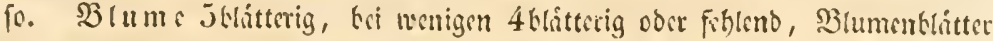

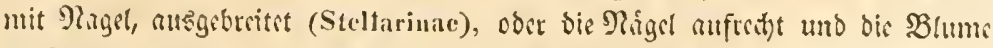
glocemartig (Lcucolunimu, Gypsophila, Tunica), ober bie \$ुhtte flud) ans= gitreitit (Velezia, Diauthus, Silene, Cuculalus, Lychnideac), biefe giml ober gefpalten ober grzihnt und geftumft (Dianthns, Malpighieac), bri einigin am

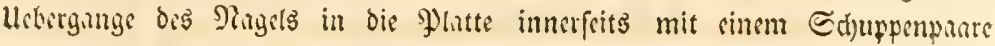

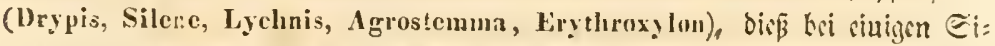

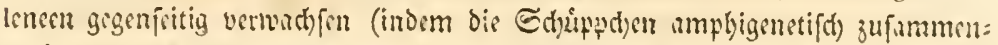

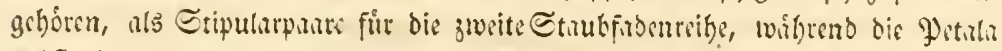

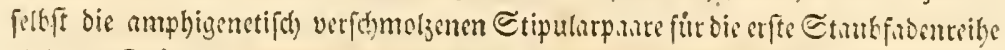
fino). Drifonpolfter feblt, nur bei sinigen Etellatinen finden fids) Sorifen: puare meben ber erften Etrubfabenreibe, withe fich bei bon Eiseneen, beren Fortbiloung 2flls emporbcht, jur coronula bilben und ats Etipularpante ber jweiten SRibe nad) amphigenctifder Berfdymelzung hinftreben.

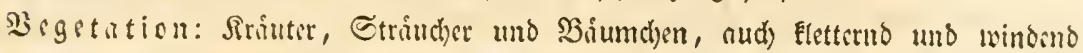
(Cucubalus, Banisteria, Triopteris); Blatter gegemiber, fticllog uno mit

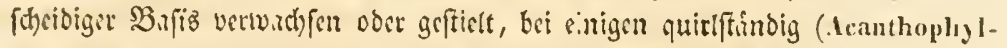

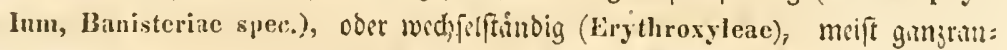

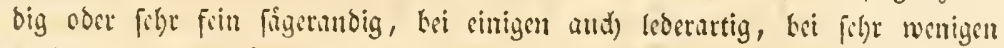
luthtig oder hamoformig (Banisteria), meift fahl soer einfach bebaut, bri Malpighia ureus, macrophylla ete. unterfeits glisartig jerbrectlidfe, ifher

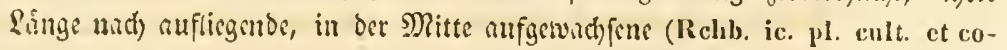

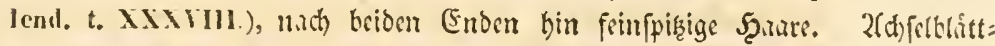

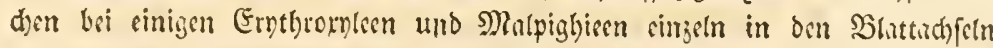

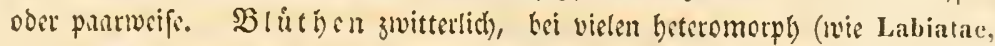

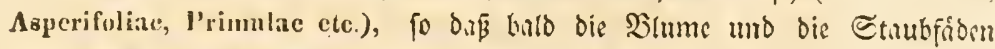

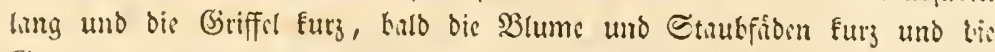
Griffel lung erfot)einen; Felch bei einigen mit partigen Deckfd)uppen (Di:nthus),

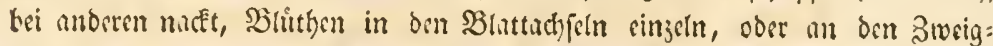
fpirsen sinzelu, bei anderen uno Malpighieen audi) boldig, traubig uno rifpig, bie

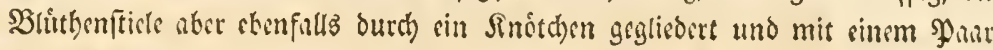
Dedeblittchen verfeten.

(Brtipen:

1) Caryopliylleac: calyx imbricato $4--5$ sepalus aut $4-5$ fidus (Antherac incumbentes).

a) Stellarinae: capsula dentibus aut valvulis dehiscens, calyx 4-5 sepalus.

a) Sa gin e a e: $2-4$ gynae.

ж) digynae staminibus binariis 4-8,

Buffonia Sauvag. Mochringia $L$. Leucostemma Benth.

$\beta \beta)$ digynae staminibus quinariis $5-10$. 
Gouflein Rob. Cast. cf. Rckb. pl. erit. 585. Pichoglottis Fisch. C. A, Mey. Brachystemura D. Don. Odontostemna Benth.

yy) tetragynae, staminihis $\div-8$.

Sagina L. Mönchia Fish. Lanarchia Rchb. - Staminibus 10:

Alsinella Benth. (Rchb. pl. crit, ic, 875.) Spergulastrum Vickx.

B) Arenarinae: 3-gryae.

ux) stamina (binaria) 8:

Siebera Schrud.

$\beta \beta$ ) stamina (ternaria) 3 :

Holosteum $L$.

yy) stamina quinaria, nempe 5:

Merkia Fisch, Triplateia Barll, - ant 10: Cherleria llall. Strl-

lacia L, Larbrea St. Hil. Sabulina Rchb. Alsinanthuo Desv. Honckenya Ehrhe, Arenaria L. Plinthine lichb. Eremogone Fisch. et C. A. Mey. Schiedea Cham. Dolophragma Fenzl.

y) Cerastieae: 5-gynae, singulae 10-anirae Sagiurits et Arenarinas repetentes:

Spergella Rchb, Malachium Fries. Corastium l. (at Leucodonium Jichb.)

b) Drypeae: capsula opereulitu-ciremuscissa! petala squamulis geminis coronata.

Drypis Ger.

c) Diantheae: capsula dentibus aut valvulis dehistens, calyx tubu. losus 5-fidus aut 5-dentatus. (Cucubalus est baccifer, calyx nomullis profunde 5-fidus).

a) ge nu inae 2-gynae,

«) pentantherae:

- Banffya Baumg.

$\beta \beta)$ deeantherae curvembryae:

Gypsophila L, Heterochroa Bunge. Acanthophyllum C. A. Mey. Saponaria $L$. Tunica Scop.

yr) decantherae rectembryate:

Velezia $L$. Dianthus $L$.

6) Sileneac: 3-gynae.

Silene 2. (Corone IIffgg. Conoimorphis Oth. Atocion Oth. Heliosperma Rchb, Silenc Oth. Pumilio R. Viscago Hall.) (ucubalus $L$.

y) Lychnideae: 5-gynac.

Melanilyum Clns. Lychnis $L$, Coceyganthe Rchl. Viscaria Mit. Githago Desf, Agrostemma $L_{\text {* }}$

2) Rrythroxyle ae: calyx valvatus, fructus monosprernus, semina albuminosa, petala coronulata. Folia (aliis oppositi) altema stipulata

Erythroxylon $L$. Sethia $K . I I, B$. 


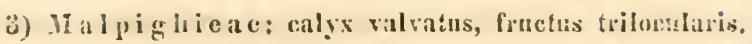

a) Banisterieac: trigynae samariferae.

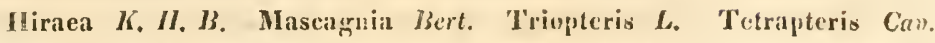
Banisteria $L$. Ifeteropteris $K$. II. B. P'eixuta A. Juss, Vargasia Bert. ete.

1) Hiptageac: submonogynat, tristignaticate fructu sicco.

Hiptage Gï̈rt. 'Tristellaria $A, P$ Th. Tliryallis $L$. Gaudichaudia K. I. B. Camarea A. St. Hit. Aspiearpa Nich. Fimbriaria St. Hil.

c) Malpighieac genuinae: trigynae drupaceae.

Caucanthus Forsk. Galphimia Cav, Bunchosia Juss. Byrsonima hich. Malpighia $L$. Pteranilra $A$. Juss.

अumer mirs, wic unjer Galium zur Cinchona, unfere Sasifraga zur Weinuannia, Cunonia

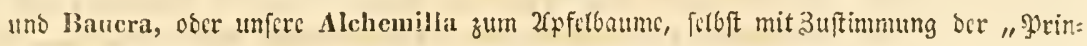

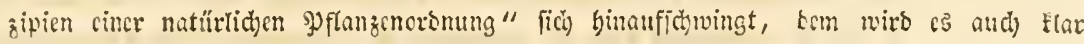

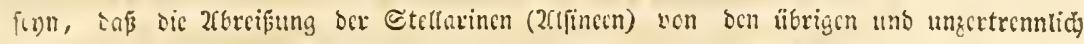

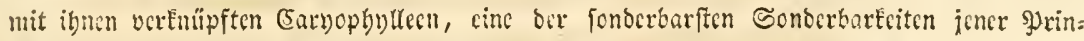

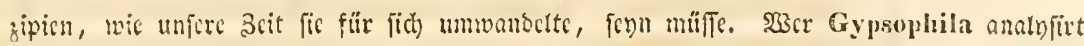

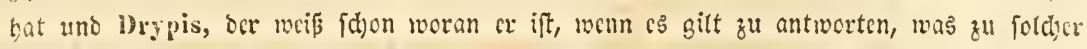

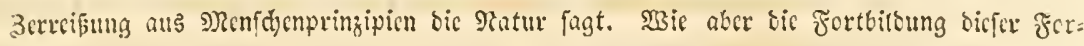

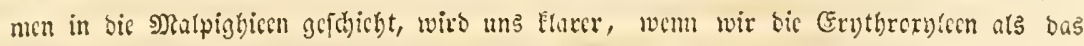

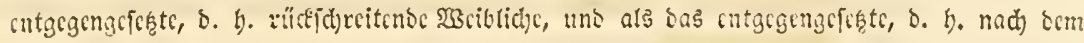

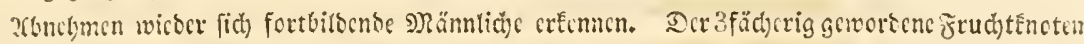

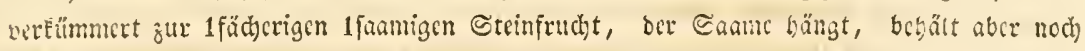

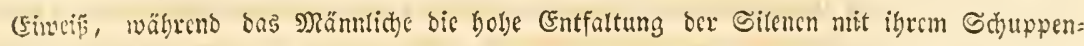

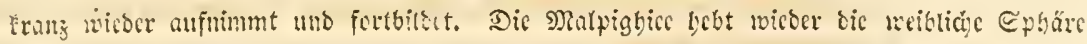

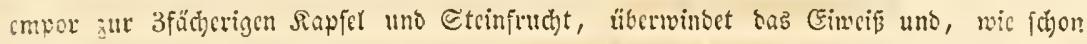
Velezia uno Diantlus getban, bic Sitümmung ocs Conamen unb Stintings, uno bic faftige

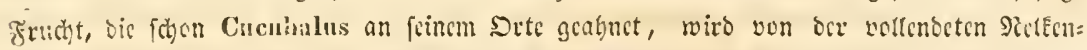

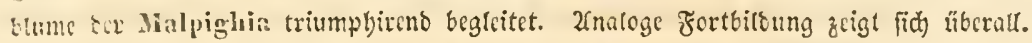

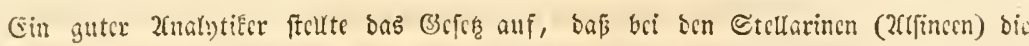

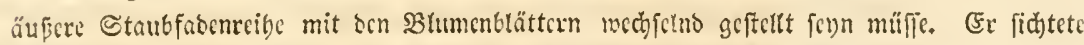

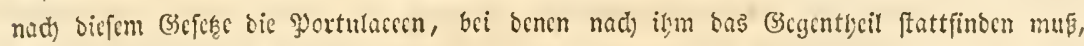

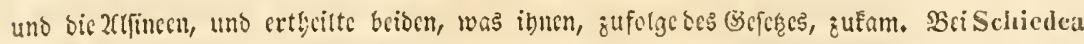

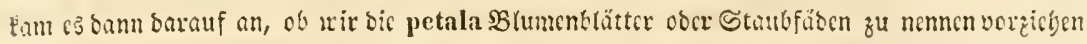
wollten (vergl. Endl. Atacta uno Wien, Aunal.). Folgen roir nach), fo finden uir bas

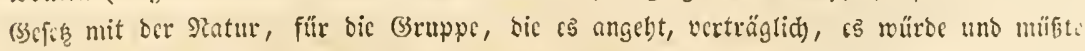

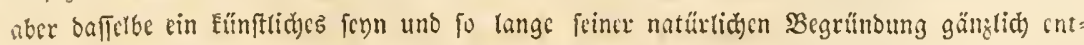
behren, ałs wir nidht in Stande wären, zu beweifen, baß in ber unmittelbaren fortbirs:

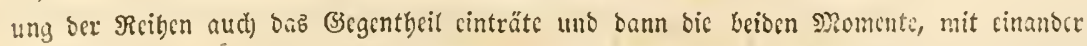

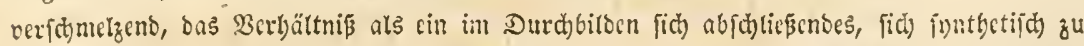
volfenden vermöd)te. Uno fo ift fo yut in Sagrina uns Buffonia, wic ber ocr Cinchona in Galium ober ser ocs 2fpfot= baumes in Alchemillin; aber cine "Pflangcnorsnung Alsineae", getrent ven ciner

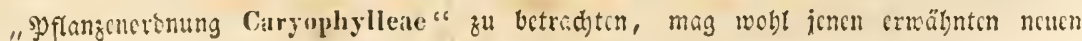


"Yringipicu" entipredben, nur nidt cine Conjequeng mit Jussien's und DeCandolle's

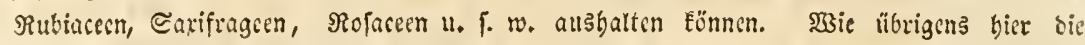
Battungen ibre Begrünoung vorzüglich in Ser Entwicketung bes weiblichen \$pringips fin:

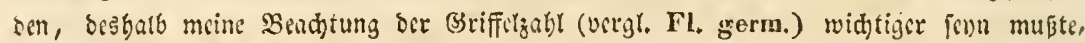
als ctiva bie Beactung ber Drilien, bie fo menig abfdyncioeno find, als bic corona, weldie

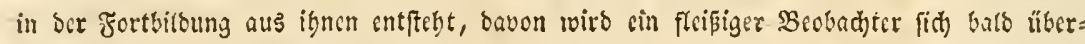
sculgen. -

128. Fanilic. Shergetwäd): Theaceae.

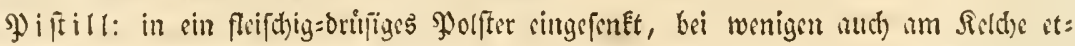

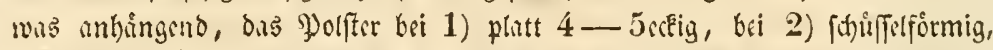
bei 3) fehtend, Frudtffroten $3-4-5$ fircherig, Findber $1=$ bis mebrfium=

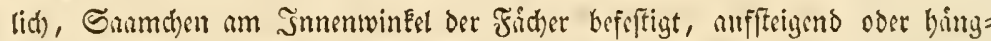

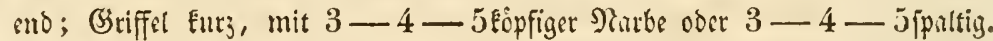

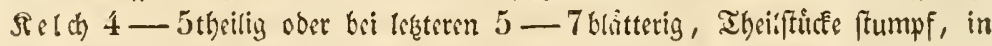
Der Snofpe jiegeturtig, nath oem 2fbthiben ftebentsibend. - Frudit

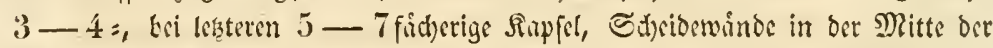

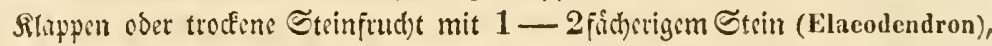

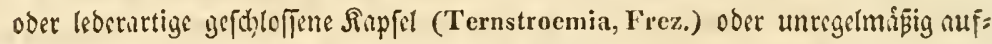
fpringend (Ternstroemiae spec.) ober regermī̄̈ig fact)tbeilig (Celastreae, Laplacea, Kielmeyera, Mahmea, Bonnetia), bei einigen bic Fsicher flugelfrucht: artig zufammengrond (Ptelilium, Hippocratea), aud) bectenurtig (Tralliana, Authodon, Salacia, Johnia), Sumentriger leiftenurtig von ber Mittel=

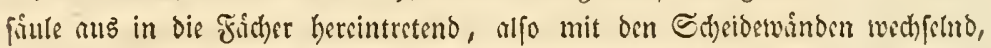
bei Cochlospermum 1 fintherig, Sanmdyen an 2 Sriten ber unvollemmenen Sd)eiderwande, ober bei einigen anfangs wandftandig, ben \&eiften ber Mittel=

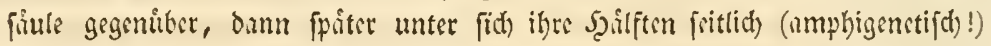

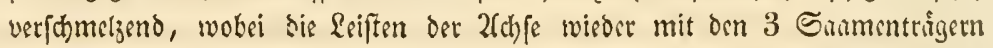

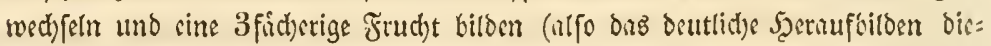
fer bochften 2fntithefe aus dem wanofarmigen 3uftumbe ber erfen und nicbrig:

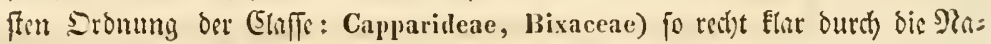

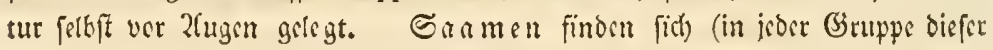

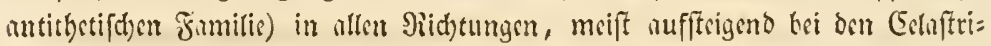

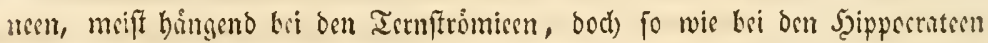
in bcioen Giruppen aud das (Bigentlyeil, j. B. bci Bonnetia unfrect)t. Einige Gittungen biken eincn 2frillus (Evonymus, Celastrus, Cochlospermum), ober

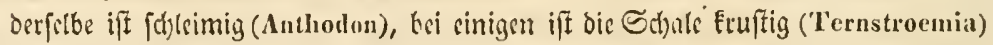
bei nndern unten, oben ober feitlid) gefligelt (Hippocratea, Laplacea, Kielmeyera); bei einigen if nod) sin fleifhiges (Fimeiz (Celastrineac, Cochlosper-

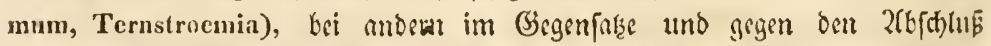
hin, ift is verzegrt (Hippocrateae, Laplacea, Kielmeyera, Thea, Camellii).

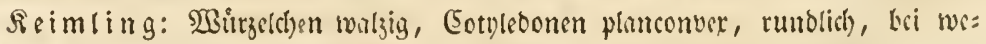
nigen sine borizontale Eanmenlage, quer uno fuft nisrenformig (Thea) und

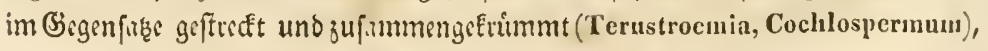


bit andern fatwindet die Srrummung (Freziera) und kei nllen hibrigen ift bit

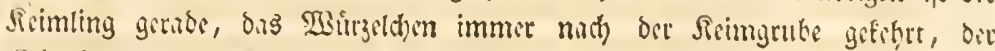
ficimling alfo im Earmen aufredt)t.

Staubbeutel: 1) $4-5$ auf bem झुolfer oder an beffen Siande, 2fäct)erig,

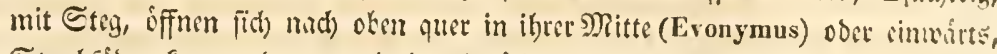
Etaubfidoen furz und unten breit, einfictberig bei Ilippocratea und Anthoton,

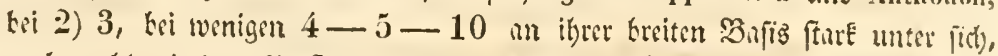

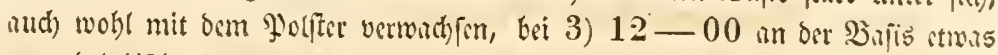
monidelphifd oder poibroclphifh ober frei, 2Beutel bei sinigen geftedft und ju=

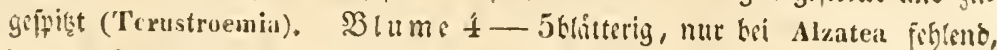

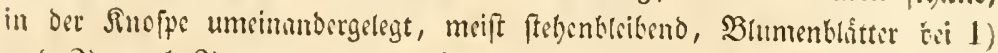
und 2), anch) 3) Frezicra etc. linglid) phatt und bifflid), mit krciter Birfis,

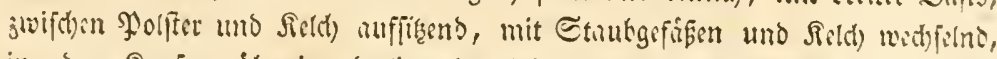
in ort Snope ikereinmortiegend, bri einigen von 3) $5-9-12$, breiter (Ventenatia), auch) muffelartig ('Tliea), jonft ebenjo, fogut kei cinigon an ber Bajis verwicbjon (Ternstroemia, Eurya, Gordonia).

Sigetation: Etrututber und Biame, 3meige und Blitter bei, 1) und 2) jum Theil gegenuber, hei menizen quilffindig (Alzatea), Jiveiglein aud trebl $4=$ Erantig (Evonymus), bei 1) und 2) aud) Elettetno (Celastrus, Tralliana. Ilip-

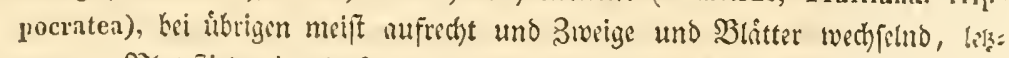

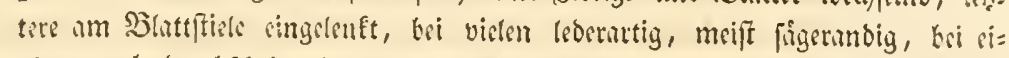
niger aud) burdbidsinend punEtirt, bei Cochlospermun bandformig gefpalten.

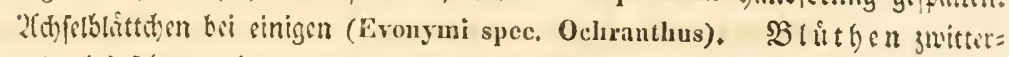

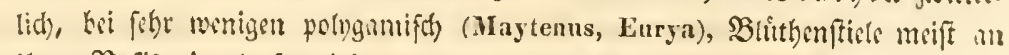

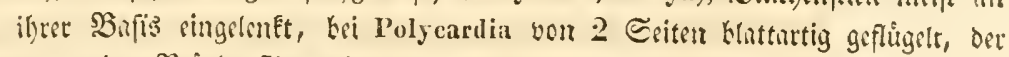

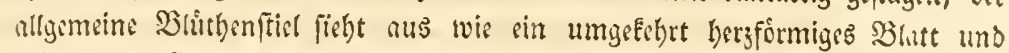

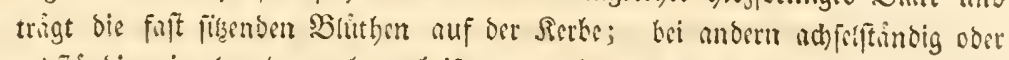

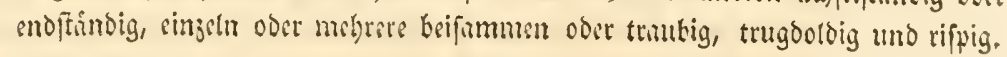
Bruppen :

1) Celastreac: ad pulvinar glandulosum, germen circumdans $\not-5-6-$ andrac.

a) Evony meac: corollatae circa pulvinar depressum.

Evonymus 'T', L. Celastrus L. Maytenus Feuill. Polycardia Juss. Elacodendron Jeq. (Rubentia Commers, Sclirebera Rtz. Nerija R.rb. huc ex p. 223.) Ptelidium A.P.T\%. (Seringia $\mathrm{spr}$ ) Tralliana Lour.

b) Alz a tea e : apetalae.

Mzatea $R z$. Puv.

c) Ochrant he a e: corollatae cirea pulvinar cyathiforme.

Ochrantle Lindl.

2) Hip pocratea e: monalelplo $3-5-10$ andrac cxalbminosae.

a) gen 1 in a e: 3-antlerae fructu capsulari.

Hippocratca I. Raddisia Leand,

b) Salaciea e: 3-antherae baccatae.

Inthodun $R=$, Pal. Salacia L. Jolnia Roxb. 


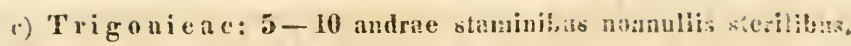

Trigonia Aubl. Lacepredea $K, I, h$.

3) Ternstroemicae: 12-00-andme ant mon-pols-aleiphite.

a) genuinae: hermaphroditae calyce bibracteatu.

Ternstroemia :lut, (Taonabo Aubl.) Cleyera Thab. Freziera sin. Letsomia Rz. Pav. Gecria Bl. Siuravia $\mathbb{H}$. (Sauranja DeC. et Ayiatelia DeC. Palava Rz. Pav,) Stenartir Cav. Mlalachodendron Car.

b) Reinwartteae: polygamo-dioicae.

Eurya Thub. Reinwardta Bl. (Vanalpbena Leschen. Siapha Murunh. Marumia Rinu. Biumia Sp.)

c) Cancilieae: hermaphoritae calyce ehrateato.

Coehlospermun K. H. B. (Misimilisnea et Wittelsbachia Mart.) Architaea Mart. Bonnetia Mart, Fsaburea Aubl. (Bonnetia Schreb.) Marila $S w$. Caraipa Aubl. Thea i. Gordonia Ellis. Kielmegera Nat. Schima Reine. Laplacea li. II B. (Haemo:huris Salisb. jam savign. zoolog. Wiekstrüinia Sirked. Lindleya X. v. T..) Camellia $L_{\text {. }}$ Ventenatia P. 13 .

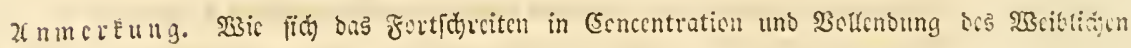

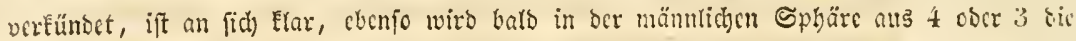

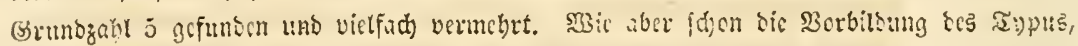

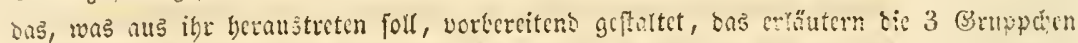

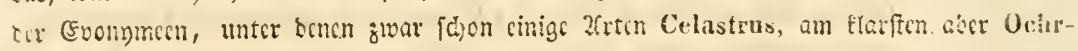
anthe zur Icenfirönice (id binbiltoen.

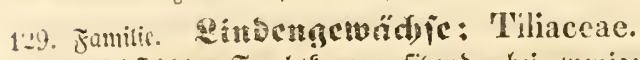

sp iftill: Frud)tEnoten fięend, bei twenigen auf sinem Stift (gynophorun, d:cfo

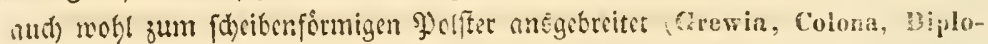
phractum, Triumfelta), 3-5fótictig, kut mentigen 2= oocr mebrfiftherig.

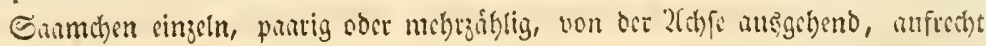

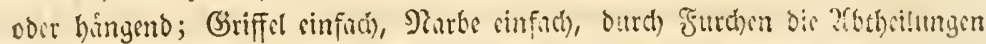
der Fibher angebeutet, bei andert gefpalten (Corchorus). Sield) 4-5:

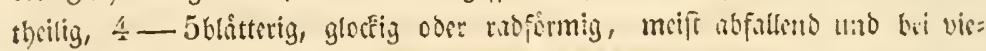

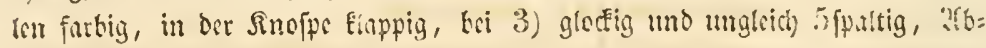

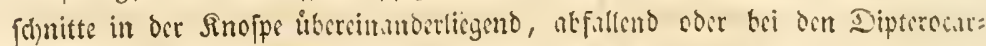

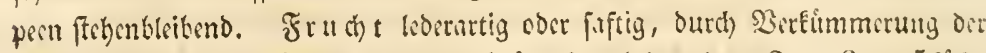

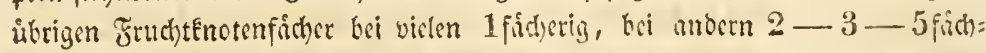
erig, F̧ad)er 1-2-metrifamig, auch Steinfrudt, (Elacocarpeac), birfi

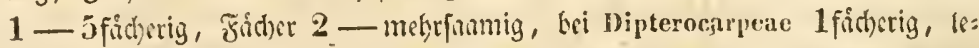

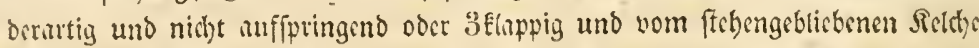

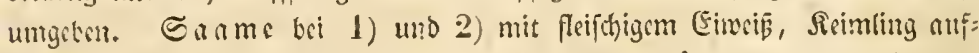

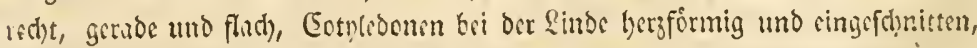

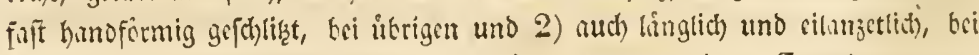

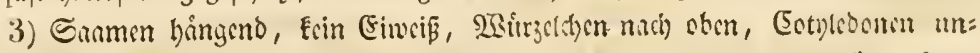
gleich und foficf aufinanderligento (Shorea, Vateria), aud runjelig gefaltet (1)ryobalanops). 
Etaubbeutel 2fädrig, bei 1) aufliegeno, furg und und innen anffptingent, boi 2) und 3) geftreft und angetwad)fen, tri 2) an ber Epike mit 2 Schorn, bri 3) bafelbft mit Lingefpalten aufpringent. Ctrubfioon meift mnteftimmt: siblig und zablrcich), frit oder bei wenizen unter 3) strons zufummenlyingent, Lei 2) brei= bis viermal fo visle ats 93 tumentlitter, nlfo 15-20, kri 3)

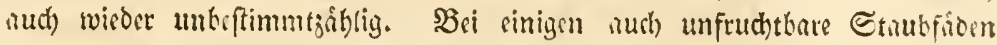

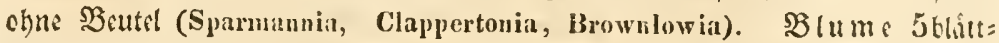

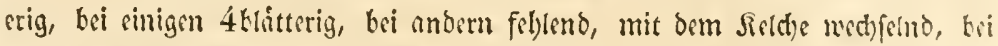
sinigen an ber Baffis mit siner Drife oder Echupps, nut bei Elateosarpeate um sin

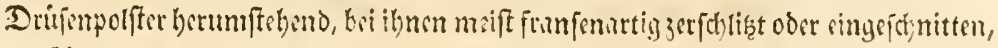
bei 2fnwefenbeit eines Frtichtenctentrigers an deffen Bapis singefugt, in Der Sincipe umeinandergetrgt bis jum Siporebten (Diplerocarpese), boum aud) un Dir Bafis zufunmenthingeno.

Sigetution: twenige Srrituter (Antichorus, Corehorus ete.), meift Etriudger uno

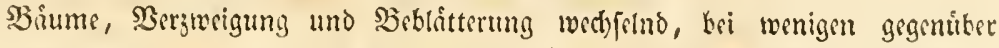
(Friesin, Dicera serrata. Aristotelia), Bllitter bei vielen herzfơrmig ober pi= formig und figerundig, bri sinigen Corchorns bie unterften Srigegihnte in lange Borften auggebeno, 2laferblittchen parrig, jung abfullend; bei Tilia bie

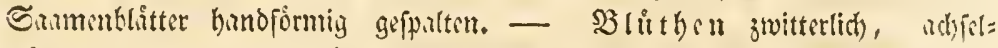
ftindig singeln oder mehrziffig, in gefticlten Dolden (Sparmannia) oder Irug= botben mit gropen Berflutt (Tilia), ober geEniuclt (Trinnfetta), trubiz

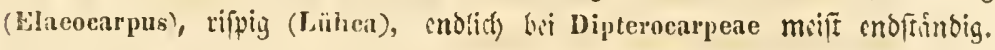
(3̈ruppen:

1) Tiliariae: stamina 00 . antherae introrsum longitulinaliter dehiscentes; semina alhuminosa. Pancis pulvinar e gynophoro.

a) genuinae holostyles pentapetalae:

Tilia L. Lindnera Richb. Grewia Juss. Colona Cav. (Columhia P.) Diplophractum Desf. Huntingia L. Apeiba Aubl. Alemria Voc. Sess. Iühen $W$. Esperal $W$. Porpa $3 /$, Berria Rxb. Brownlowia H.rb.

b) Corehorcae, sehizostyles.

Corchorus L. (Chorenrus [s. Coretioides DeC.] et Coreta P. Br.) Ceratocoreta DeC. Ganjil Rniph. Guazumoiles DeC.)

c) Sparmannieae, holostyles apetalae et 4-peialae ant irregulariter5petalac et circa stamina fertilia filameintosae. - Apetalae:

Alatia Rz. Pae. Foreolaria DeC. Ablania Aubl. ('Trichocarpus Schreb.) Sloanea Plum. (Gyuostona DeC. Hyriochaeta De C

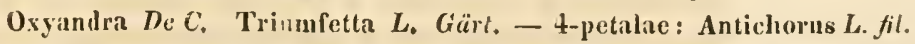
Helioearpus $L$. Triunfettaria $R$. (Hartramia Gärt, Lam, non Hedg.) - filamentosae: Clappertonia Mcisn. (Honckenya $W_{\text {, nou Ehrh.) }}$ Sparmannia Thinb.

2) Elacocarpeac: stamina petalis utplurimum fiubriatis ant incisis tripla aut quadrupla, antherac poris apice rlehiscentes, semina albuminosa. Pulvinar carnosum. (Aristoteliae antitheticae petala integra). 
a) genuinae holostyles 5-petalae.

Elacocarpus L. (Imehneria Scop. Ganitrus Giat.) Villea Mut. Mollia Mart. Tricuspidlaria Rz. Pav. Accratium DeC, Monocera Jacq.

b) Aristotelieate schizostyli-tristiguaticae holopetaliae. Aristotelia lllerit.

c) Fricsicae holostyles 4-pctalne.

Friesia DcC, Dicera Forst, Acronotia $D$ t.

3) Dipterocarpeac: stamina definita suhusolyadelpha aut libera, antherae alnatae subulatae, apieis rima duplici dehisecutes, petala integra, semina magna subsolitaria exalbuminosa. Pulvinar 0.

a) synetalac.

Hopea Roxb.

b) libere 5-petalae, antherae muticae.

Dipteroearpus Giärt.

c) lihcre 5-petalac, antherarum connectivo in mucronem deciduun producto.

Vatica L. (Dryobalanops Gïrl. et Pterygium Corr. Shorea Banks.) Seidlia Kostel. (Vateria lane. Rxb.) Vateria $\mathbf{L}$.

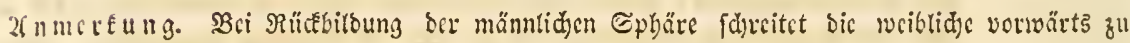

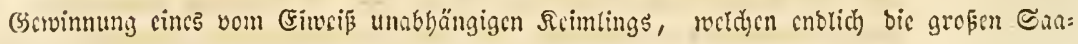
men ber Dipterecarpecn gewinnen. Der neue Familimtupus beginut mit ber norsifdyen

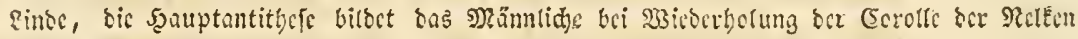

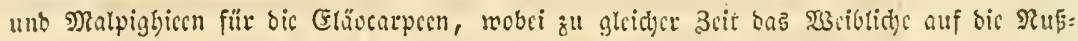

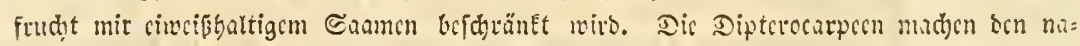

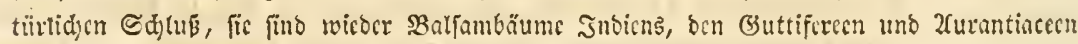

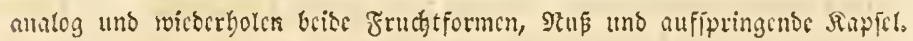

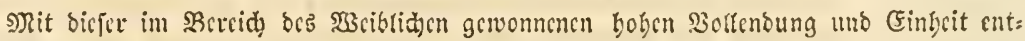

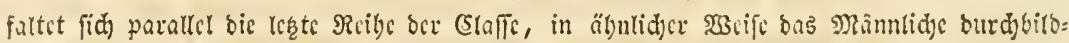

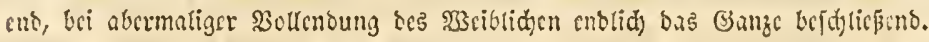

130. řmitic. \$̧artbengewäd)je: Hypericineae.

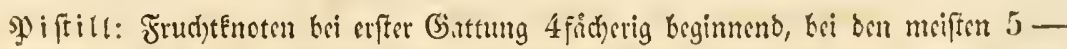

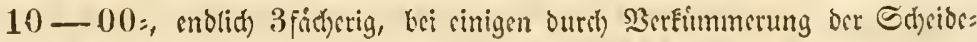

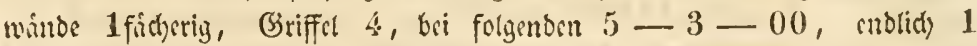
(Limum hologynum, monogynum, Hyperiea monogyna). Sharben Eopf:

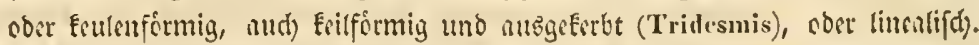

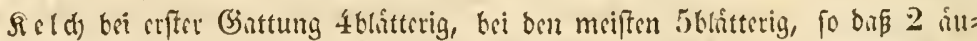

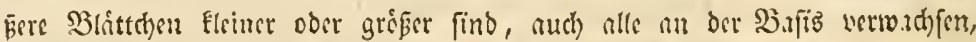

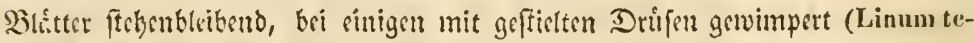

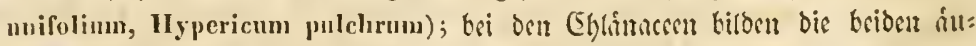

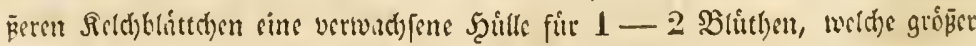

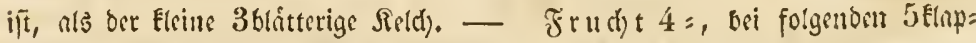

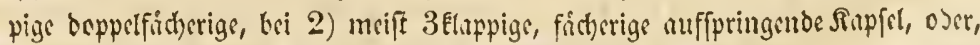

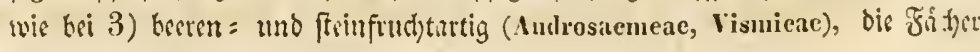


pringin ganj ab unb lojin bic Mittelfảhle auf (Linear, Drosanthene, Elodeinae, Tridesmeae), ober die Mittelfiule ift vollendet uno bleibeno (Androsaemeae, Brathydeae, Chlenaceae). - $\subseteq$ a a $m$ e $n$ hingend (Lineae, Haronga, Clienaceae), borizontal (Hypericeac), oder aufferigend (P'sorospermum), in ocn Finchern einzstn(Lineae, Eliaea, llaronga, Chlenac.),

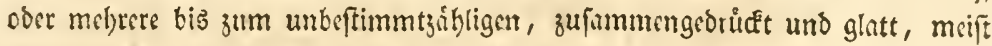
puntitrt odir fcin waiglich) (Linene, Vismieae, Chlenacene), obcr linglid', audi) gefrimmt (Itypericeae), nuch geflugglt ('Tridesmeae), (Firrcis bei Linum catharticum nach St. Hilaire, not) vorbanden, bunn und fleifhig, bei Chlenaceae chenfulls flcifchig odar bornartig (A.P. Th). Sirimling óthaltig, grin (Lineac, Chlenaceae), mit platten vom rundtithen, Eursen uno frumpfen

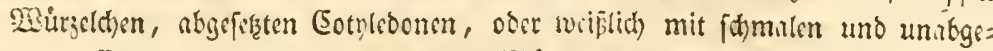

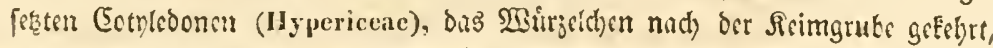
bi Psorospermum geftummt, Fei utrigen gernds.

Etaubbeutel 2ficherig, lings nach innen auffpringend, nufliegend, nufrect odir

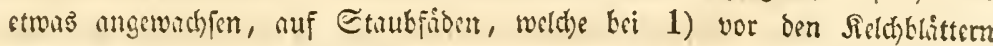

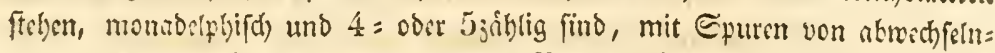
Den kettellofen Fitoen, weld)e alfo ben Blumenblittern vornfteben, bai 2 ,

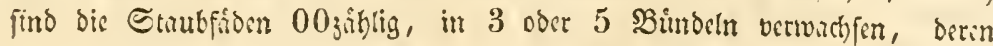
Ningel (synnema) in lef̧terem Falle ben SSlumenblittern voranftelen, im $\mathrm{fr}=$

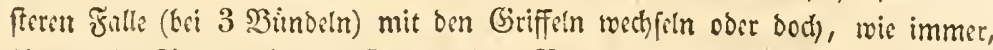
binter bic Echsibeninde geftellt find. Bei Llodea keftimmt triadelphifd),

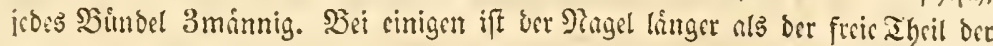

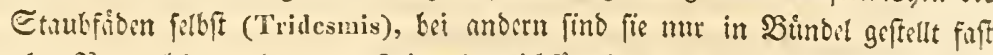

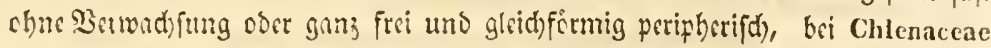

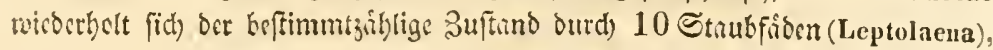
und geht aus dicfem in Den monadeiphifot)=003ibligen utber, wo dann bei

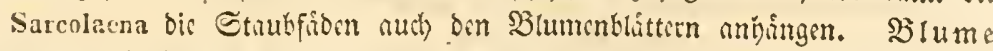
Eiginnt 4blitterig (liadiola, Ascypum), keifolgenden J̈blitterig, bei ber leşten Gisttung (Sarcoiaena) 6blatterig, in ber Sinofpe und nad bem 21bblihen ju= fammengerollt, bit einigen butch bie Ningel strons zufummentingeno (Linum campanulatum, Chlenaceae), von regelmápigem ltmtip ober bei ans Dern făief keiffơmig (Lini sp. et IIypericene), bci cinigen abfallend (Lineae,

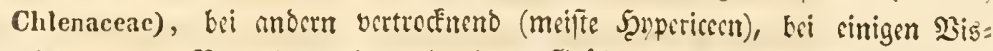

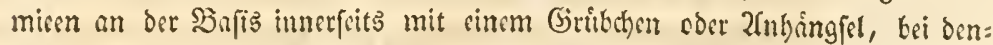
fetben auch bypognnifoc Drufar odir Gduppen, mit ben Nígeln (synnemata) Der Stimbrabenbundel abwethfind.

Sigetation. Sinuter und Stritud)r, mit gegenikerftehender (Radiola, Lini spec. Hgpericene) obcr quitlattiger (Linum quadrifolium, Hyper. Coris, Bratlys) ober med)felnoer (Lini spec. Chlenaceac) Bergmeigung uno Scblatterung,

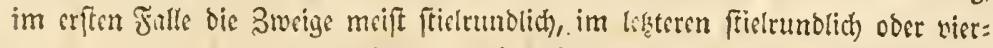
Eantig und bei einigen mit fifttigen Jlingstrindern (Linum aethiopicum, Hyprrician quadrangulum, tetrapterum), Slatter meift fikend, kei anderen ge= 
fitelt (Linmm trigynum, aethiopicum, Iyper, spec. Vismieae, Chlenaceae), ganz unb grnjantndig, bei einigen feingefingt, bei ben meifen nerbig (Lineae, Iypericene, Chlenaceae), ath firoernervig (Limum trigynum, Androsaemun, Sorcolnena), bei vielen mit durd) jheinenden PुunÊten ober Delbebåltern (Linum marginatum, Hypericum perforatum), bei Linum aethiopicum bie

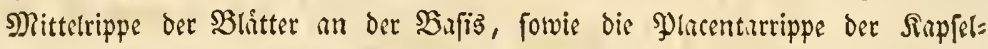

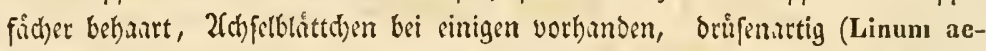
thiopicum etc.) abfallend bei Chlenaceae; amd) die Stengel und ?weige, Feldte, Blumenblatter und Stuubfiben bei einigen mit fowntsen Dilbebiltern in Form von ŞunEten ober Streifhen (vittulae Spach.). Die Silchblatter tri sinigen mit geftielten Drifen gerwimpert (z. 23. Linum tenuifolium, gallicum, corymbulosum, IIyper. pulchr. nummul. Elod. barbatum efe.).

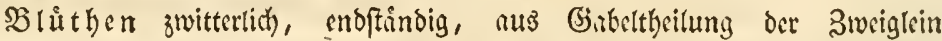
(Radiola, Ilypericeae, Cillenaceae) trugooldig ober aus einfitigen Irinten trugboldig (Lini et Hyperici species), bei wenigen fult cinjeln (Linum trigynum, Hyper. Ascyron), bei cinigen bie Silutbenfficle zufammengebridft und flügctimbig (Linum aethiopicum, Hyperici spec.), auth) getenkig (Iyper. articulatum s. Eliaea, Chlenacene). (J) bemerke nuth in biefer Familie den beteromorphen 3uftand, baß bald Blume und Staubfíden uno bald wieder bie Giriffel linget find.

(3ruppen:

1) Lin e a : monalelphae $4-5$ andrae, filamentorum rudimentis sterilibus fertilibus alternis; semina loculis singula ant loculis divisis gemina.

a) Radiol e a e: quaternariae:

Radiola Dill.

b) Lineae genuinae: quinariae exstipulatae.

a) stigmata capitellata, antherae erectae:

Cathartolinum Rchb.

ß) stigmata incrassato-clavata (petalis concolora), antherac sub anthesi horizontaliter incumbentes:

Linum $L$.

भ) stigmata imposita antheriformia flava, antherae sub anthesi horizontaliter incumbentes:

Adenolinum Rchb.

c) Macroline a : quinariae stipulatae,

œ) stigmata capitellata:

Linopsis Rchb.

B) stigmata oblongata, horizontaliter incumbentia :

Xantholinum Rchb.

y) stigmata imposita reniformia, filamenta fertilia utrinque unidentata, (practer sterile) rulimentum filanentis alternum: Nacrolinum Rchb.")

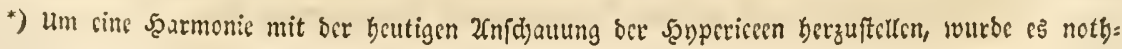


2) II yericeac: polyalelphae aut polyandrac, poly-microspermac. (Oligosperma et Lincas repetentia genera sulsunt: Drosanthc, Eremocarpus, Eliaea, Ancistrolobus, Haronga).

Laneretia $D_{e} C$. teste cl. Spach. Frankeniaceis pertinet, Eucryphia cum Carpodonto Lab. juncta Terustrocuieis omnino magis affincs.

a) Ascyrcac: quaternariae: sep. 4. pet. 4. stam. 00. sulmonadelpha styli 2-4. capsula 1-locularis.

Ascyron $L$.

b) II y pericea genuinae: petala 5. sccuriformi-iuacquilatera convolnta aut involuta, (basi inappendicnlata, stamiua tri- pent- hex- oct-adelpha, submoualelpha aut libera, squamae alternac nullac. Caps. septicida $3-5-6-8$-locularis aut baccans, radicula tercs recta).

4) Drosantheac: capsula tricocca, coccis 1-3-spermis, demum cum placenta deciduis.

Eremocarpus Spach. Drosanthe Spach.

B) Andros a emcac: cepsula triloculari-septicida, placenta centrali valvisque persistentibus.

Hypericum L. (IIolosepalum, Milleporum, Adenosepalım, Drosocarpium, Coridium, Crossophyllum, Olympia Spach.) Webbia Spach. Campylopelwa Rchb. (Campylopus Spach. non P.B. nec

menoig, aud) bie viclgeftaltige Battung Linum auf natürlic)en Sufammenhang ocr näber

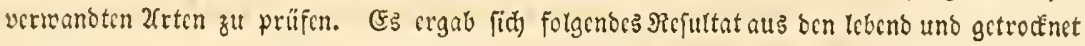
vorlicgenoen 2erten, hri benen bic brigeregten 2(utoren für bic Epccies als Linum gebören.

Catluartolinum pratense ( $L_{*}$ cath. $L_{0}$ ), virginianum $L_{0}$ - fol. alterna aut sparsa fl, flavo: gallicum $L$. (aureum $W^{r}$. $K_{\text {. }}$ ) corymbulosum Rchb. Fl. germ. alternum Lam. bicolor Desf. sardoum Mïll. - 1. subcoeruleo: agreste Brot. tenuifolium $L$. suffiruticosum $L$. salsolö̈des $D e C$.

Linum usitatissimum L. (asiaticum Mart.) humile Mill. hologynum Rchb. Fl. grerm. diffusum Schrad. (pallens Hort.) Rchb, hort. t. 128, inacquale Prsl. strictum $L$. narbonense $L$, lacre Scop. Sieberi $1 h c h b$, (hirsut. Sicb, e Canea).

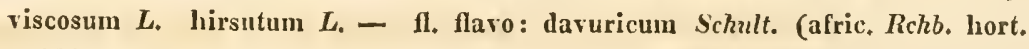
t. 46.). -

A d e nolinum austriacum $L$, marginatum Poir. (syn. in Fl, germ.) angustifolium Iruds. pallescens Led. perenne $L_{0}$ alpicola Rchb, (c Carinthia.) alpinum $L$. sibiricun $D e C$. pyrenaeum $D e C$. (narbon, Lap.) nerrosum $\boldsymbol{W} . K$.

Linopsis - huc sp. africanae - L. acthiopica (Thnb.) quadrifolia $\left(L_{.}\right)-$ fol. superiora alterna: africana (L.) maritima $(L$.$) - sp. americana : corymbosa$ (1öpp.).

Xantholinum nodiflorum $L$. campanulatum $L$, tauricum $W$. capitatum Kit. flarum $L_{\text {s }}$ arborcum $L$.

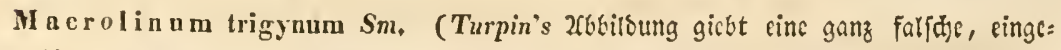
bittete 2(nalyfe, bie id mit ocr SRatur gar nid)t üsereinftimmen febc). 
Brid.) Psorophytum Spach. Androsaemum All. Eremanthe Spach. Campylosporus Spach. Norysca Spach. Roscyna Spach.

y) Brathydeae: petala post anthesin involuta, stamina submonadelpha aut libera.

Myriandra Spach. Brathydium Spach. Brathys Mut. Isophyllum Spach.

c) Vismieac: petala aequilatera, pluribus basi foveolata aut appendiculata, stamina tri-polyadelpha, synnemata squamulis alterna.

a) Elodeinae: stamiua triadelpha, semina teretia aptera, embryo rectus:

Elodea Adans. (Triadenium Rafin. Martia Spr.) Elodes Spach. Triadenia Spach.

B) Tridesmeae: stamina triadel pha, semina alata,

Eliaea Camb. Tridesmis Spach. Ancistrolobus Spach. CratoxyIon Blume.

y) Vismieae genuinae: stam. pentadel pha, squamulae synnematibus alternae (drupa aut bacca, sem. definita aut indefuita pendula, horizontalia aut aulscendentia).

๙a) drupa subearnosa, pyrenae 5, 1-2-spermae, semina cylindrica laevia pendula, esubryo reetus, radicula cotyledones aequans: Harouga A.P.Th.

$\beta \beta$ ) bacea polysperma, semina cylindrica lıorizontalia, embryo rectus:

Vismia l'and.

yr) bacca oligosperma, semina sub e re c ta, radicula recurva cotyledonibus brevior.

Psorosperinum spach.

3) Cli le n a e a : 10-90 andro-monalelphae, oligo-nacro-spermae, pedunculi artieulati.

a) stamina 00, capsula 3-locularis loculis 2-spermis:

Sarculacua A. P. Th.

b) stamiua definita 10. capsula demum 1-locularis 1-sperma.

Leptolaena A.P. Th.

c) stamina 010, capsulil 3-locularis loculis 00-spermis:

petala 5: Schizulacua A.P.Th. - pet, 6: Rhodolaena A.P.Th.

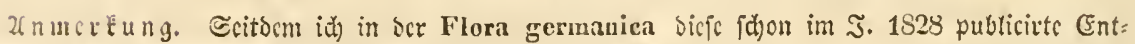

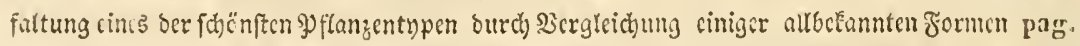

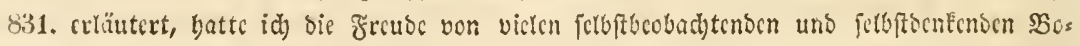

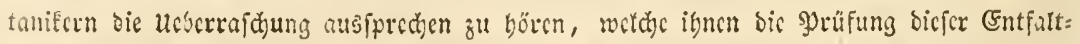

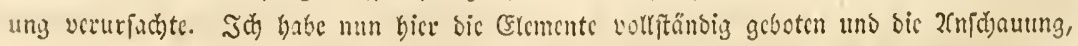

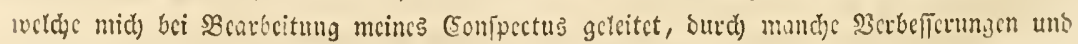

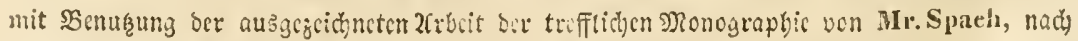

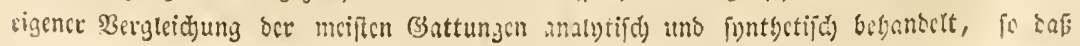


Jibermann mit bicfer Expefition leidht bie sahn ber Ratur zu verfolgen vermag. 2Bic

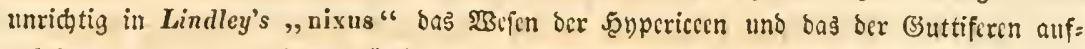

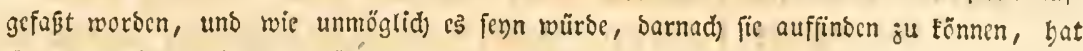
Spach bercits genügeno crläutert.

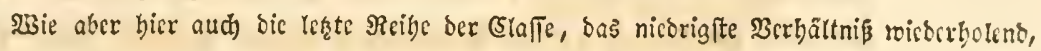
aus ber 3weizagl beginnt, unb zlwar nidht alkein bie Rabiolecn, fonbern aud sie 2(scyreer,

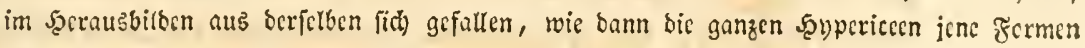

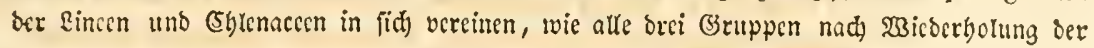
nicberen Sorbilbung fich) fortbitsen und alle nur erfïntiche Berbättniffe imnig verid)metzen,

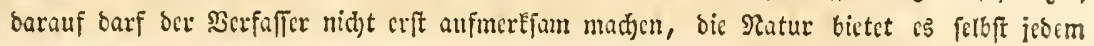
ofinem 2cuge.

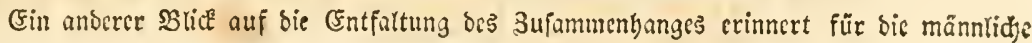

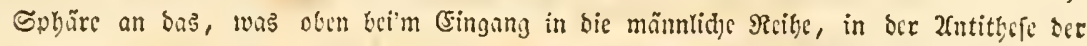

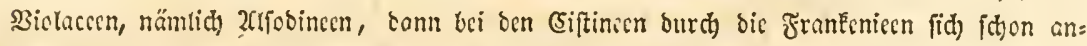
gebeutct, in ber gweiten Sronung burd bie Geranieae genuinae, Dombercen uns SBütt=

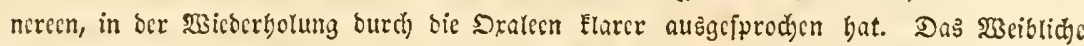

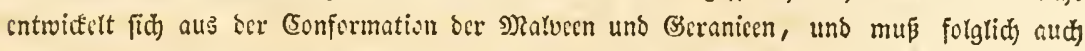

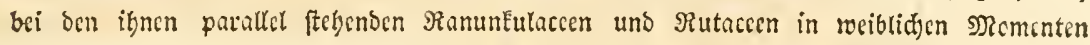

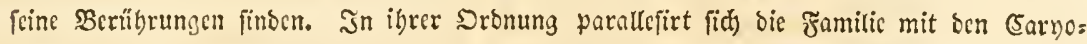

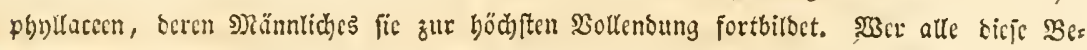

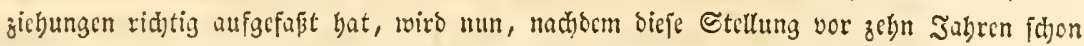
als in ber scatur ocgrüntet vorlag, die abermalige 3crreißung ber Familic, bic weite Ents fernung oer fimen von ben Şypericen, die Unterfteckung jence unter bie Beranicen, ober

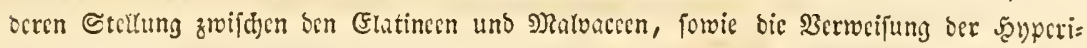

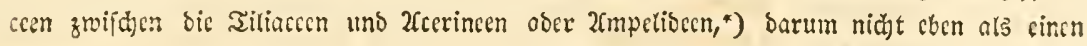

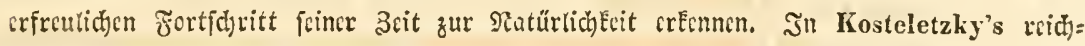

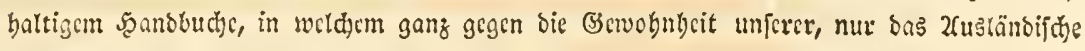

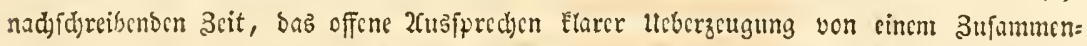

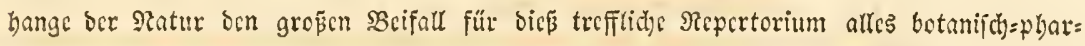

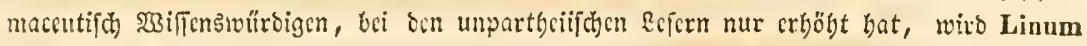
zivar an bic Sratibeen angcichtoffen, body abet S. 1911. Sic innere ticfere Utbarzengung

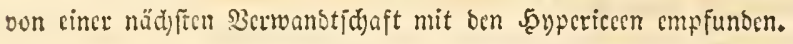

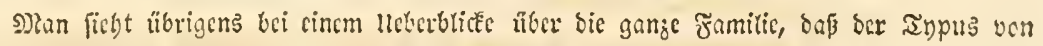

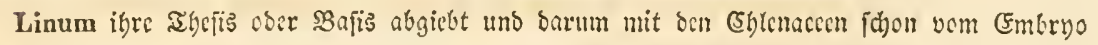

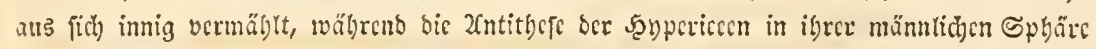
weitcr aus ben (Srenzen beraustritt, als bic analogen Earyopbyuleen in ifrer weiblichen

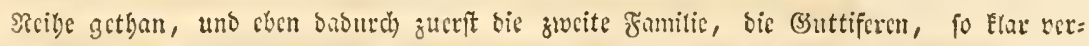

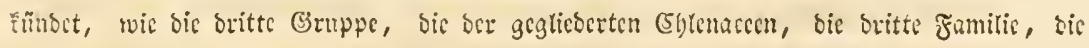
scr 2Curantiacen, zucrit andeutct.

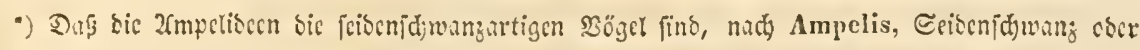

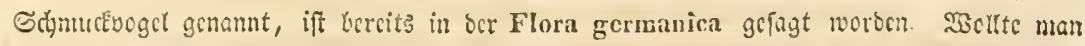

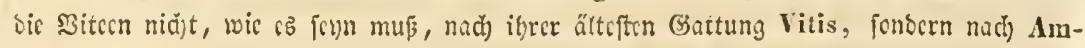
pelopsis bencunen, fo muf̧ten fic 2imperepfibecn genannt werben. 


\section{Familie. Guttngetwöthje: Guttiferae,}

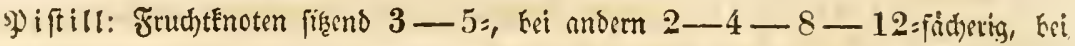

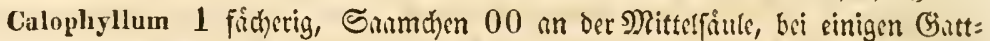
ungen unter 2) und 3) in ben Fishern cinzeln ober wenige: Griffel febr

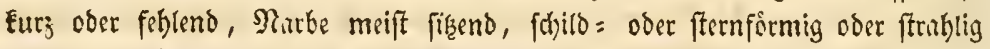
gefurcht. Field $2-4-6-7$ blitterig oder theilig, leocrartig ziegel= förmig, meift fartig uno fetenenbleibeno, bei Strombosia cin fochildartig (wie bie

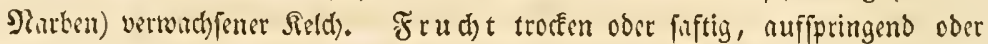

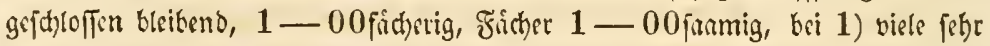

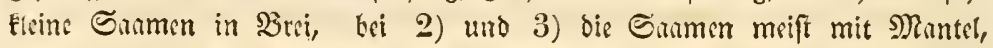

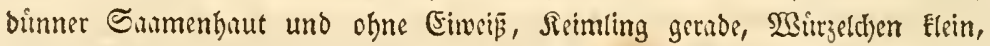

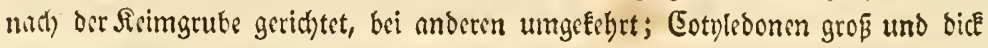
und vertwad) fen.

Staubbeutel 2ficharig, innerfeits lingsinffipringend, angemadfon, nur bei liavetieae an ber Eprige auffpringeno, Etunbfáden 00, nur kei wenigen 5

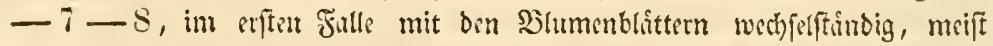

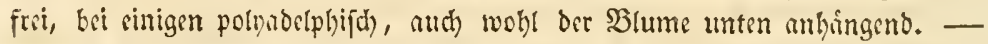

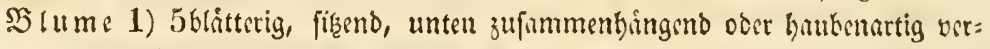
twrtb)en, abfallend, leberartig, in ber Sinofpe umgetegt, bei Maregravia in

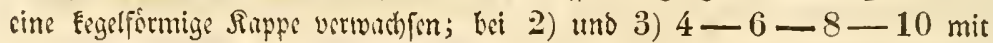

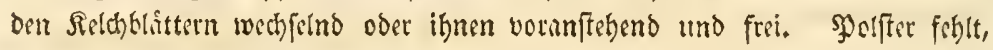
cublid) nut Kai Chrysopia 5lappig.

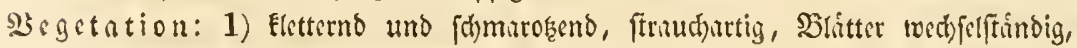

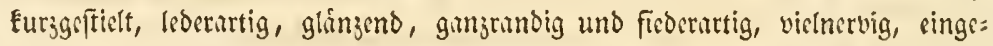

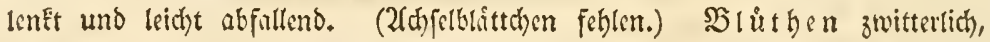
bei wernigen biflinifd), polngrmifd) oder biôcifd) (Clusia, Haretia, Mammen),

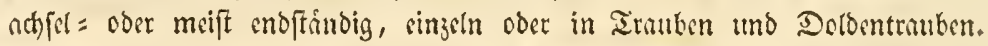
("Epi " ift nimlid) nicht immer spica, fondern Margrav. spiciflora bu, cben fo eine Dolbentrutuke mit langeftielten SBtuttben und ebenforvenig eime

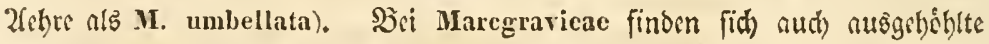
uto Enrppenformige $\mathfrak{B r u c t e r t}$.

Gruppen:

1) Marcgravieac: fructus coriaceus, semina 00 minima in pulpa uidulantia.

a) Strombosicae: calyce peltato.

Strombosia Blume.

b) Maregravieac genuinae : calyce partito, corolla calsptrata.

Maregravia L. Antholoma Lab.

c) IR uyschieac: calyx partitus bibractcolatus, cor. libere 5-petala.

Norantea Aubl. Ruyschia Jacq.

2) Clusiariae: plurimae diclines, capsula dehisecnse

a) Haretieac: antherae apice dehiscentes.

Haretia K.H.B. Renggeria Meisn. (Schwciggeria Mart, non al.) Arrudea Camb. 
b) II a rilea e: antherae longitudinaliter dehiscentes, connectiro producto. Iarila $S w$. Anisosticte Bartl.

c) Clusi a e: untherae longitudinaliter deliscentes, connectiro truncato.

«) Toromiteae: capsula $3-4-5$-locularis, connectirnm apiculatum.

Verticillaria Rz. Pav. Tovomita Aubl. (Marialvea Mart. Micranthera Choys. Ochrocarpus A.P.Th.)

B) Mesuea e: capsula 1-locularis bivalvis.

Mesua $L$.

r) Clusie a e gen uinae: capsula 5-10 locularis 5-10 valvis, Quapoya Aubl. Clnsia $L$.

3) Garcinicae s. baccatae.

a) Symplioniea e: bacca 00-locularis.

«) Chrysopi eac calyx 5-partitus, stamina 00 monalelpha. Moronobea Aubl. Symphonia L. fil. Chrysopia A.P.TH.

B) Gynotrocheae: cal. 4-partitus, stamina 8, definita. Gynotroches $B l$.

y) Plat onie ae: cal. 5-partitus stam, 00, pentadelpha, Platonia Mart.

b) Cal ophylle a e: bacca 1-locularis, stamina cohaerentia. Canella $L$. Calophyllum $L$. Kayea $W$ all. Apoterium $B l$.

c) Ma nostanca e: baca 00-locularis, stamina libera.

a) Rhe ediea e: stigma infundibulare. Rlıeedia $L$.

ß) M amme ariae: stigma stellatum, calyx 2-sepalus decilluus. Mamivea $L$.

भ) Cambogieae: stigma stellatum, calyx $4-5-6$ sepalus aut partitus.

Garcinia L. Stalagmites Murr. Pentadesma Don.

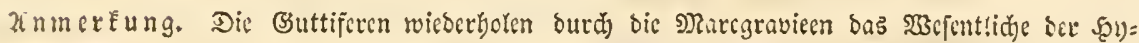
pericen, entfalten iber typifden Formen unter ben Clufieen und bereiten als Garcinien bie Sęsperibeen vor. Darum erjdeint bier in erferen noch cimmal ber zicgelartige Field)

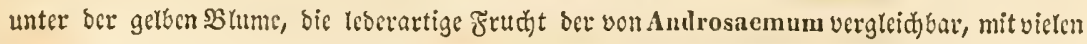

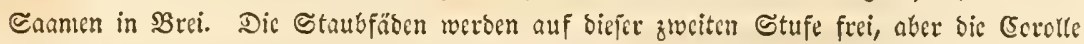

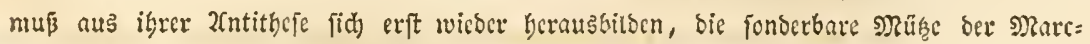
gravia und oer 2(ntholoma ift gleidjam nur bas Etement cincr Sorolk. Die Etufice fagt

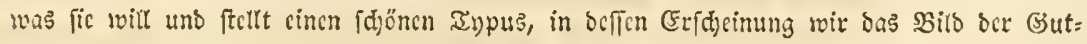

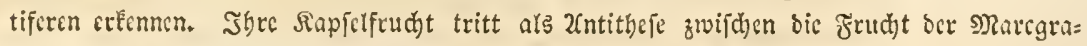

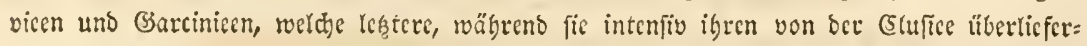

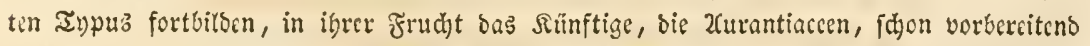

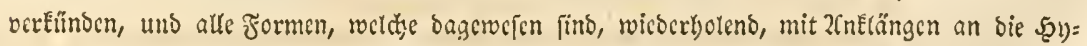

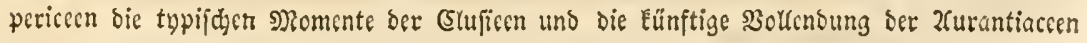
veridgmetzen. 
132. ชamilie. Drangengetwätjp: Hesperideae.

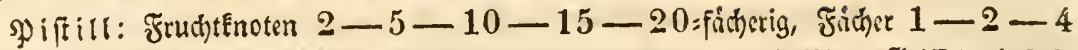
- 00:fanmlict), bis vielzáhligen (Swictenieae) zweirrihig; Griffel sinfach,

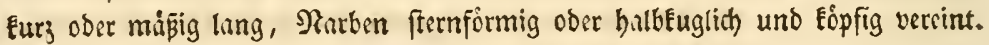
Selth noch $5=$ ober bei andern $4=$ ober 3 theilig, bei ben lesteren vollenbet

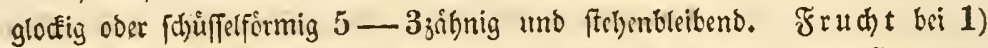
a. b. cine Sapfel und Eteinfudyt ober Beere, bie אTrppen ber erfferen mit Edreibewsinden in ber Mitte, $1-2$ (bei wenigen Gattungen 4) Sinmen

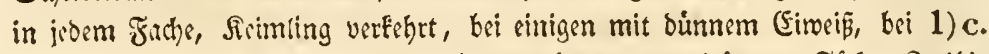

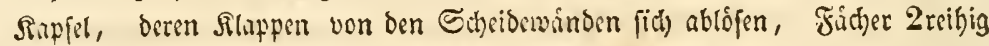
00ศanmig, flad), unterwiatts (Cedrela) oder oberwánts (Chlorosylon, Swie-

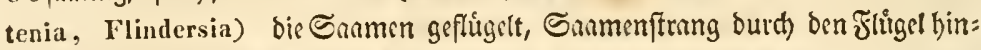

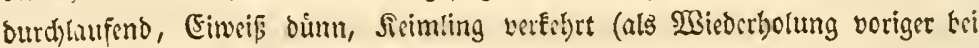
Cedrela), ooer quer uno obme Eimsis (Flintersia), oder aufrect) (Swietenia),

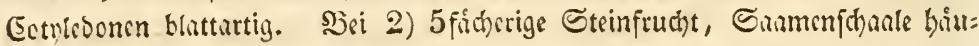

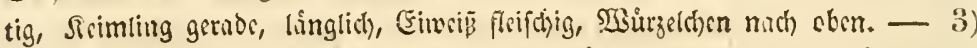

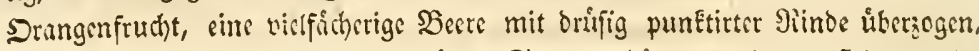

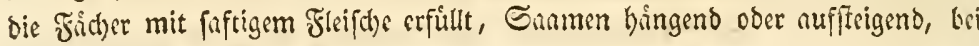
cinigen mit mehreren Seimlinisn verfahen, Sisimling gernde, aufred)t, Eotnle=

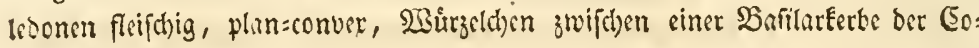
trleoonen singrfentt.

Staubbeutel aufredt, 2ficherig, lingsanuffpringeno, tri 1) mift $8-10$, tri wonigen 5-6, auf einem aus ben Etmubfión ginzlich verwathfenen (5n:

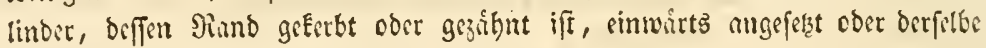
nuch) oten in Etrubfíbon zertheilt. 2) $10-20-00$ Strubbentsl, $\delta_{c}=$ rin fleifthiger Steg fid fitwer bic Beutel binaus in cine Epize fortfeget. 3) $8-10-20-60$, bei wenigen 5 (Limoniaesp.), auf Etnubfîben,

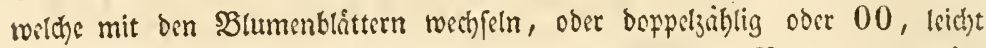

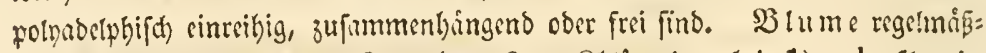
ig, 5 blátterig, bei wenigen $3-4-6-8$ blottterig, bei 1) a. b. Elsppig,

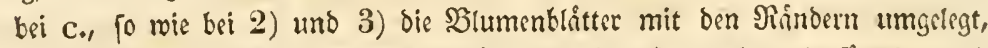

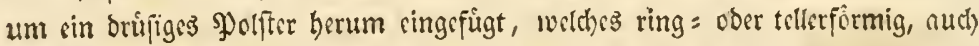

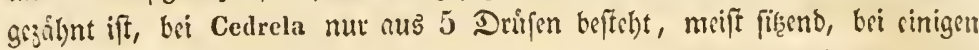
ctivas zufammenthangend, bei Swietenia uns Soymida nuz cinem Eurzen Irnget entipringend.

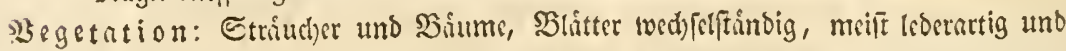
glimzeno, bei 1) a.b. zufammengeforet ober einfudh, bei c. unfautig ober pan=

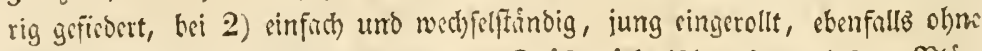

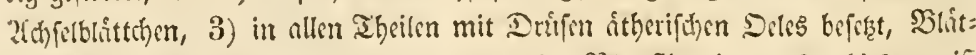

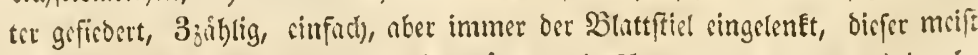

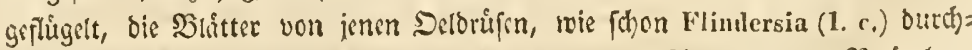

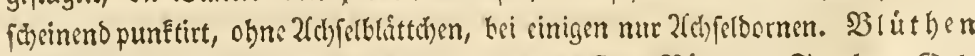

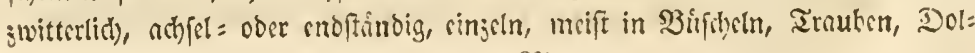
Dintrauben, Trugbolden (Hunirieac), Siffurn. 
(s)ruppen:

1) Mlicac: corolla valrata (Swietenicis subcontorta), filamenta in cylindrum connata, antheris $8-10-12$ margini introrsis.

a) grenuinae: grerminis loculi bi- (paucis 1-paucissimis 4-6) -ovulati, albumen tenue, ralicula porrecta, cotyledones foliaceac. (Folia sintplicia aut composita).

Quivisia Juss. Calodryum Desv. Neregamia $W_{g} g h t$. Arn. Turraea

1. Melia I. Azadarichta A. Juss. - stam. superne distincta : Mal lea A. Juss. Cipadessa Bl.

b) Trichilicae: germinis loculi $1-2-o v u l a t i$, albumen 0 . radicula inter cotyledones crassas inflexa.

a) $\mathrm{X}$ ylocarpea e: cylindrus filamentorum abbreviatus antheras 8 10) (quibustam 5-(i) subincluleus.

Sylocarpus Коен. Carapa Aubl. Goniochiton Bl. Synoun A.Juss. Lansium Bl. Nemedra A. Juss. Aphanamixis Bl. Aglaia Lour. Milnea R.xb.

B) Trieliticae genuinae: cylinirus filamentorum elongato-tubulosus aut conicus, antheris $8-10$ (quilusdam $5-6-i$ ) in fauce zerens.

ж\%) Guarecae antherae (i- 10 inclusae.

Guarea L. Sandoricum Cav. Bidyunocheton Bi. Cabralea A. Juss. Epicharis Bl. Hartighsea A. Juss. Visoxylon Bl. Chisocheton $B l$.

$\beta \beta$ ) Gerumea stamina basi tantum coalita petalis aequinumera i. e. 5 .

Geruma Forsk.

gr) II ey ne a e: antherae petalis duplicatae exsertae.

Heynea Rxb. Trichilia L. Mosehoxylon A. Juss. Ekebergia Sparrm. Walsura Rxb. - Odontanulra kinth.

y) Calpandriea e: 00-andro-monalclphae.

Calpandria $\mathrm{Bl}$. Stemmatosiphon Poll.

c) Swietenieae: corolla subcoutorta, filamenta in cylindrum coalita aut sublibera ovula loculis 4-00, biserialia imbricata, capsulae ralrulis a septis dehiscentibus, semina plana aut alata, cotyledones foliaceae.

๔) genu in a e s. quinariae, antherae in filamentorum cylindro. (Yetala breve unguiculata. cmbryo Swieteniae erectus).

Swietenia L. Suynida A.Juss. Chickrassia A. Juss.

$\beta)$ Ki a yeac s. quaternariae.

Khaya A. Juss.

भ) Cedreleae 8. quinariae staminibus distinctis. (Cltimae iau folia punctata, embryo exalbuminosus transversus, Cedrclae inversus). Chloroxylon Dec. Cedrela L. Oxleya Ilook. Flindersia R. Br. 
2) Humirieac: filamenta 10-20-00 monadelpha, connectivo producto; drupa 5-locularis loculis $1-2$ spermis, scminibus albuminosis.

Ilumiria Aubl. Sacoglottis Mart. Helleria N.v. L.

3) Anrantieae: stamina subcohacentia aut libera, baeca plurimis aethereo-oleoso cortice obducta succosa.

a) Limonieae: loculorum orub solitaria paucis gemina.

a) At a l in ti e a e 8-10 andro-monadelphae.

Atalantia Corr. - ovula 2. superimposita: Lavanga Hamilt. Feronia Corr.

B) Triphasiea e 6-andrae flore ternario.

Triphasia Lour.

y) Bergereae 8-10 andrae (Limoniae sp. etiam5-andrae) germine stipitato. .

Bergera Koen. Sclerostylis $B l$. Glycosmis Corr. Limonia $L$.

b) Clausen ea e: 8-10 andrae (i. e. libere diplostemoneae), germinis loculis hiovulatis, ovulis superimpositis.

Murraya Koen. Cookia Som. Clausena Burm. Micromelum Bl.

c) Citrea : libere aut subfasciculatim $20-60$ andrae, bacca 00-iocularis locnlis 09 spermis.

Aegle Corr. Citrus $L$.

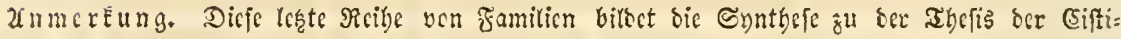

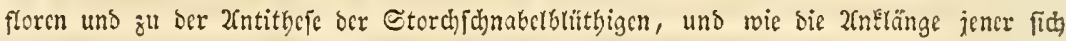

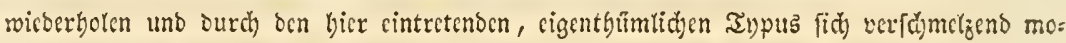
Dificirten, ift bereits crwähnt worben.

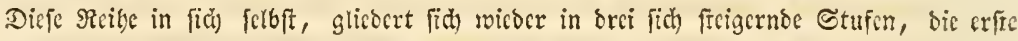

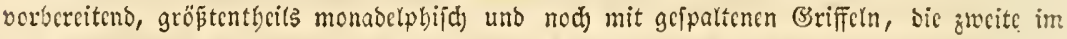

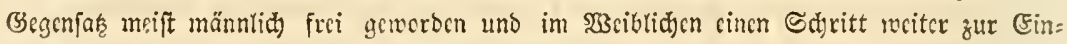
beit gelangt, mit unterorüctem Sriffel unb fternförmig, wie sic 3eiger eincr Uha fid aus=

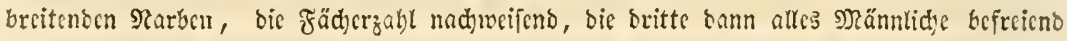

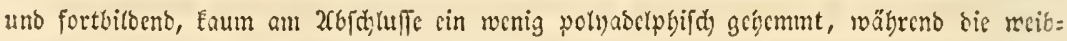

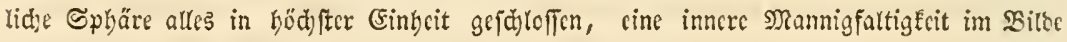
Der Sitget verborgen.

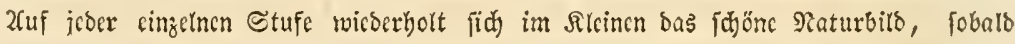
wir barauf, worauf wir in jeber Stufe eben follen, unjer zenge zu tenfen verftechen.

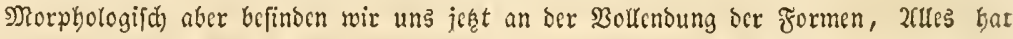
iid) in feiner Sphäre ausgebildet und gegliebert und bas jajönfte (5benmaaß aller centrifă) uns excentrifh geftellten aheile ift wirf(id) erreidjt.

Edjon bas $\mathfrak{B}$ latt, weldycs wir in Fciner ticfiten Entftelyung bei ben Egloroplynten,

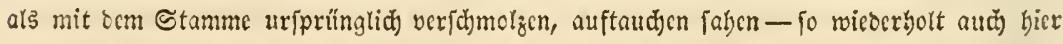
in ber böchften (Elaffe nod) Pachynema bie blattartigen fladjen Etämmodyen und 3rocige,

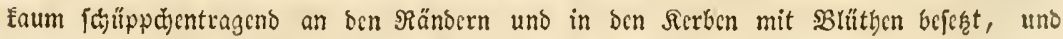

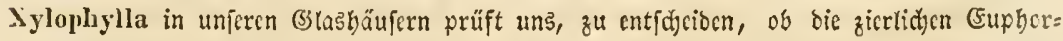

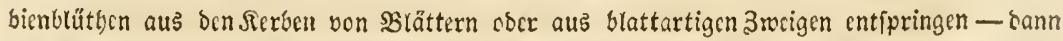
frei aus Sinoten fdycisig berauswachicn, enditid fid glieberno, labet uns bier sin, frine 


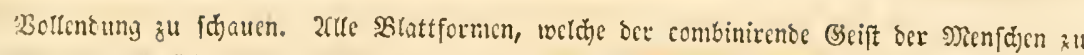

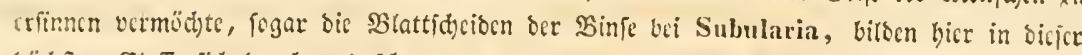

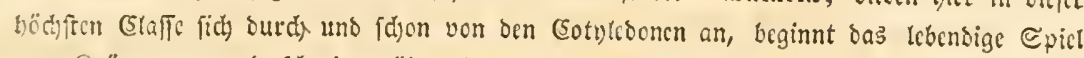

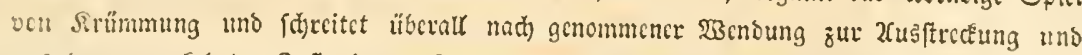

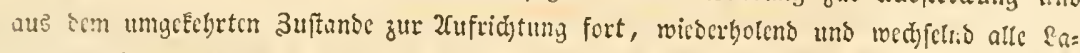

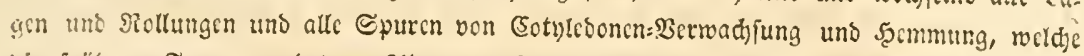

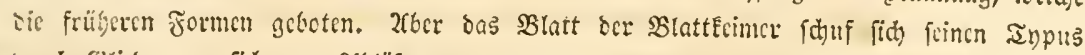

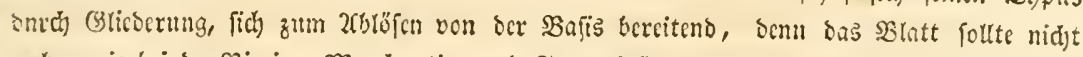

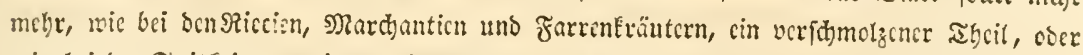

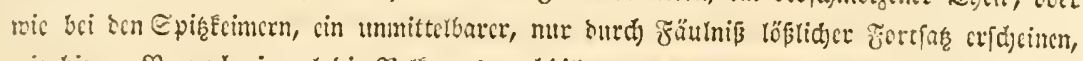

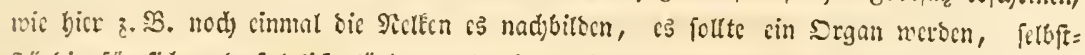
ftünsig fïr fich) uno folglid) tösbar uns eigentgümlic) felbft fid geftaltons. Dic (slicbers

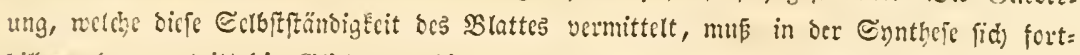
biloen, barum tritt bic (Blicocrung bicr - nad) antitbctifder 2fnoeutung bicfes Berónät= niliç in Qunssia u. 2 . - burd) bie ferennirenoen sinecn für bic unteren Slätter, wäh=

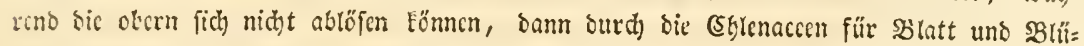

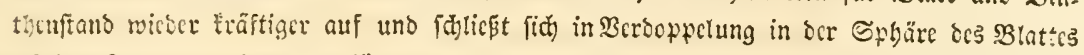

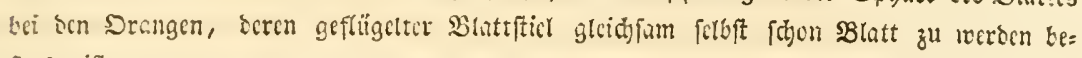
forcot ifr.

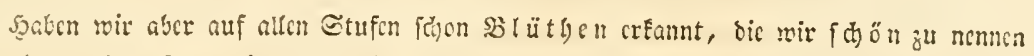

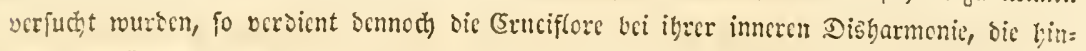

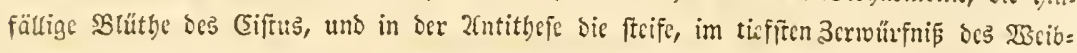

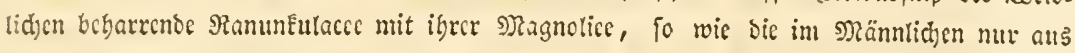

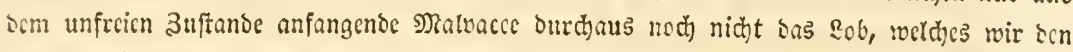

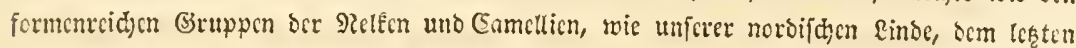
blittyenouftenden Baume unferes Sommers, nebft feinem tropifdjen Sefolge, fo gern zu

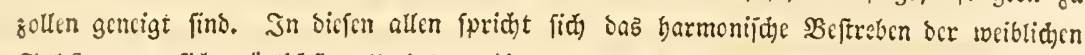

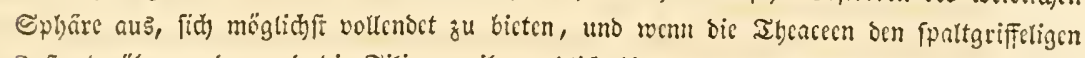

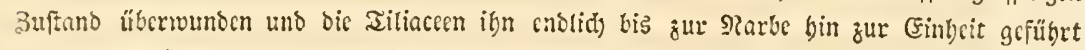
babcn, fo ftört uns mur nod) bic Distarmonic in ber 2(usbitsung ber Saumen, icr sinte

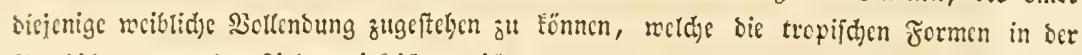

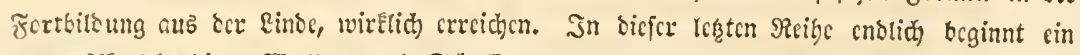

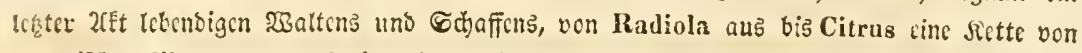

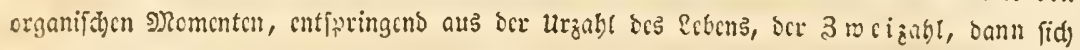

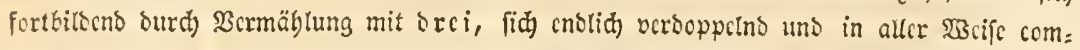

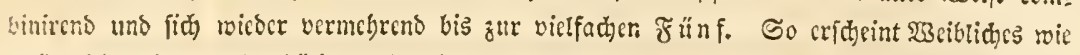

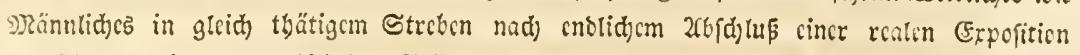
Des Jocalce rincs vegetabilijđ)en Gicfraltens und Eebens.

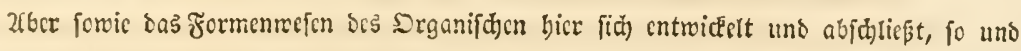

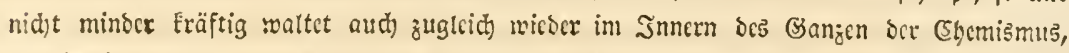

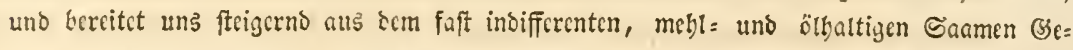

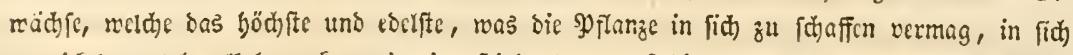

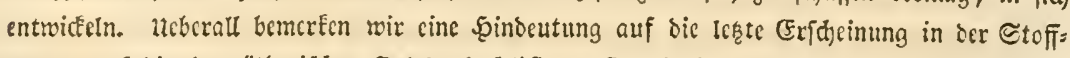

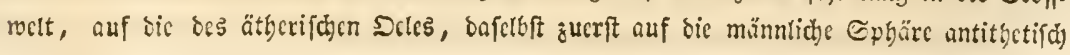




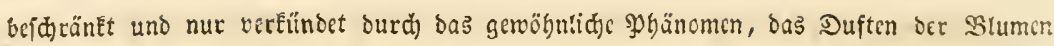

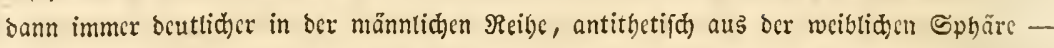

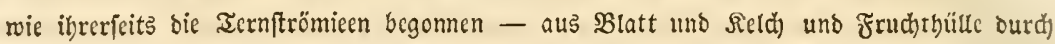

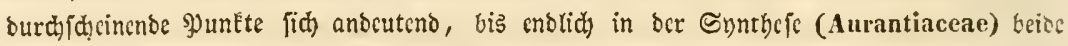

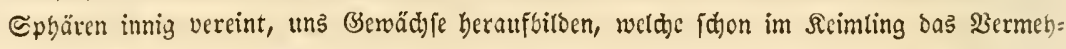
ren unb Schaffen aus fich fetbft burch cin Drängen nad) Sermebrung in fich felbft flat

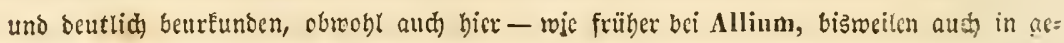
genwärtiger (Staाাe bei Polyembryum uno Evouymus, bicfe (5mbryonenvermehrung fichon

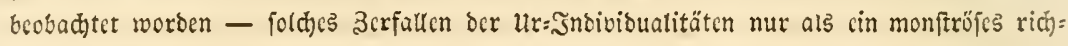
tig bezciơnet weroen oürfte, böher bod) bedungen und, entgegengefect bem 3erfallen bes গু

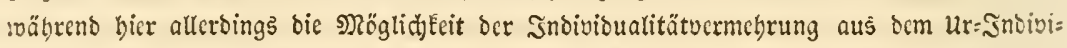

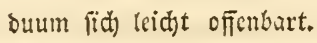

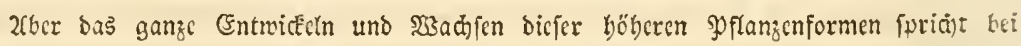

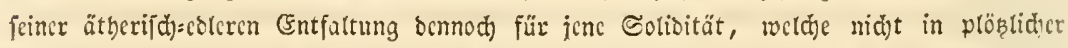

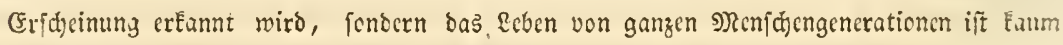

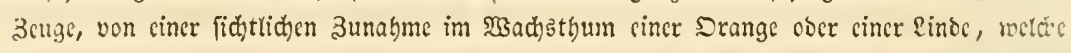

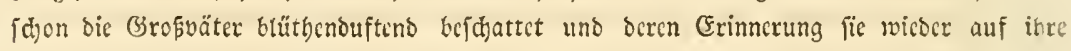

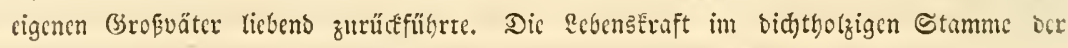

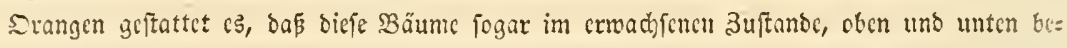

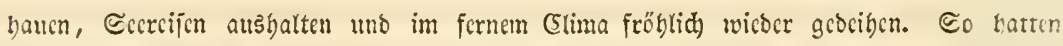

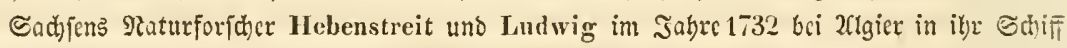

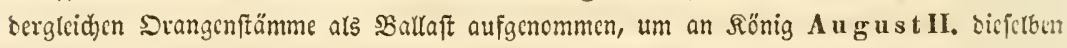

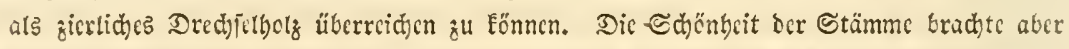

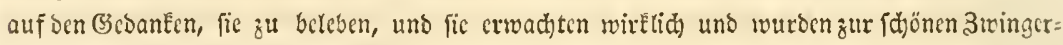

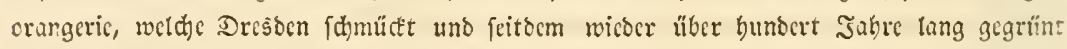
uns geblüht bat, ofne merftiçe 3nnahme bes Durchmefiers ber Etämme. So fünoen mir

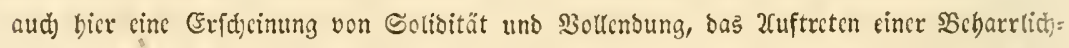

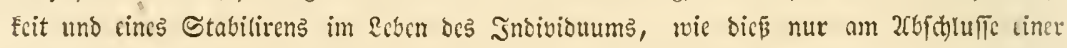

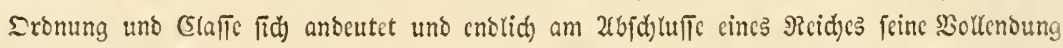
żu erreidjen vermag.

Nan rilltmt uns bic 26oogatopflaumc (Persea gratissima), bic Mrangofrutbt (Manfera indica), bic Mrombinpflaume (Spondias Mombin), dic Ehryfobalane (Chrysobalanus Icaco etc.), ben Srciapfe! (Achras sapota, Grias canliflora), dic Gujauc (Psilimu

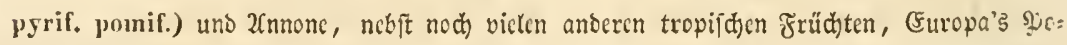

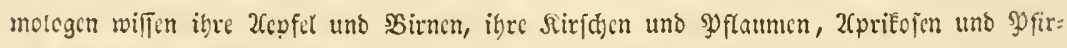

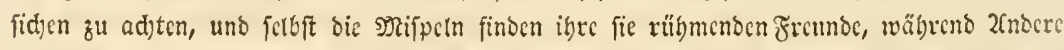

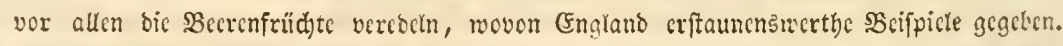
Zuber bic rabre Bollenbung ber Srganifation fpridft fid nod nidt ba aus, wo bie colle

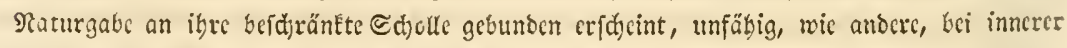

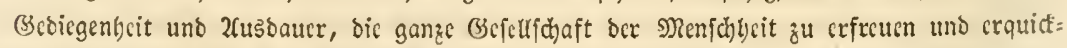

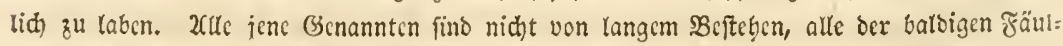

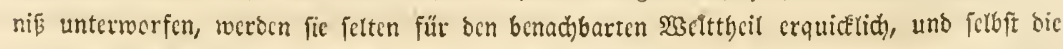

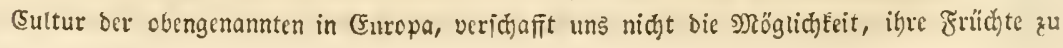




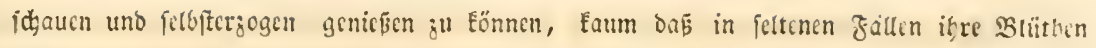

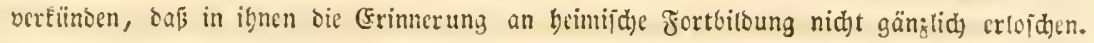

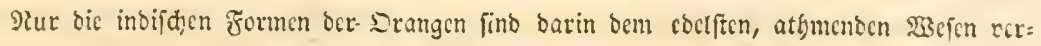
glcidbar, Daß fic cine Ergichungsfähigleit weit von ibrer (Scburtsftätte berahren, foldhe Entfernung in Ergebung crtragen und bcimifa) weroen, bas fremoe Bolf wicocr blühend

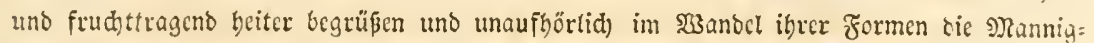

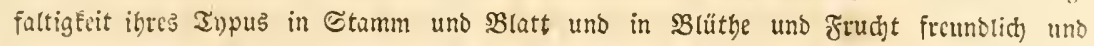
wie beimifd entfatten. Soldye 2(nichauung unb Empfindung errvecft aber bic Ernote our

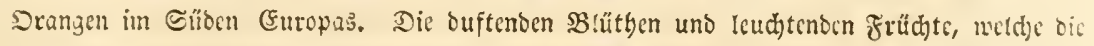

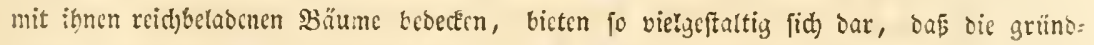

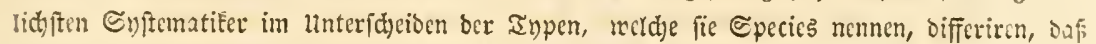

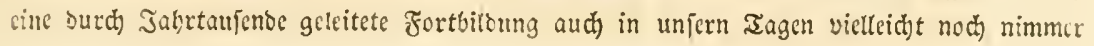

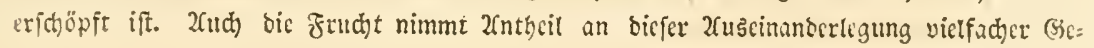
falten, fogar in normal geroorbenen Manfitrojitäten, bald rücefdyreitend in igrer 9Retamor=

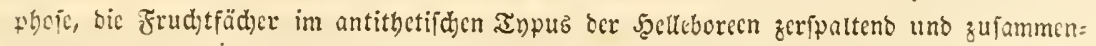

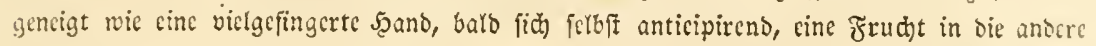

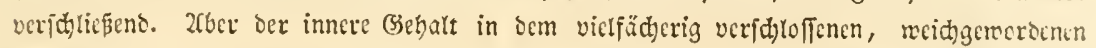

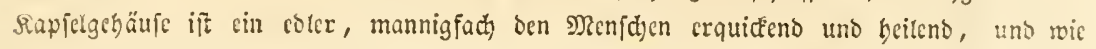
Foton in Dftintion bie Nangoftane beticet ift uno bie Frudts ber Feronia elephantun und bic Marmełcira ber bunbertjäbrigen 2egle, alte aber nur ifrem cigenen Baterlanbe

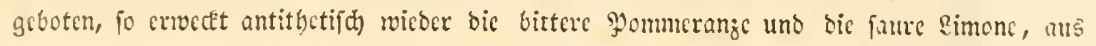

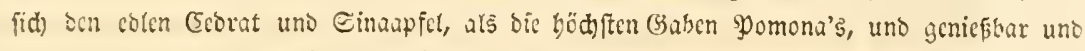
surd) Eabung belebeno, für alle Bölece ber (Frobe.

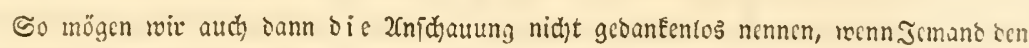

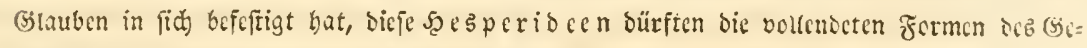
mäd) srcidbs, mit allem Redte genannt werben.

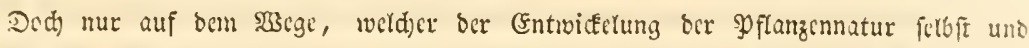
ourchgreifeno forgt, ift übcrhaupt cine Röjung ber Frage, ja ferbft bic Etcllung ier

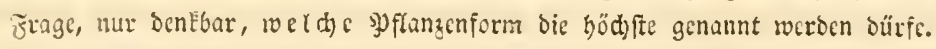

und bat uns bic satur cinen ariabneifonen Faden vertichen, wie wir glauben sürfen,

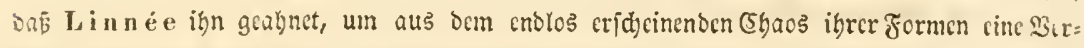

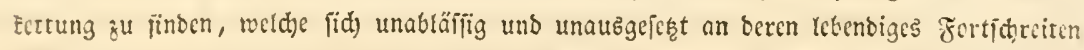

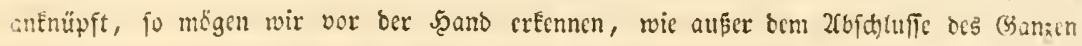

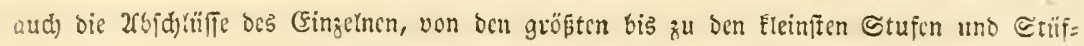
d)en, in ifree 2ert, zunelymeno uno fecigerno fid in fich felber geftalten.

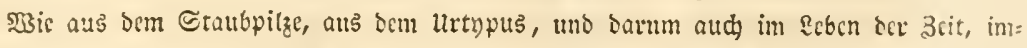

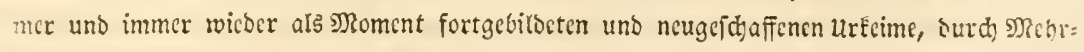

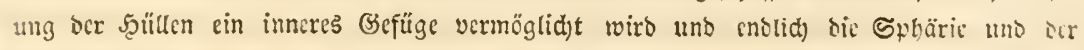

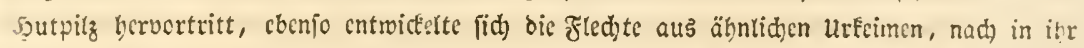

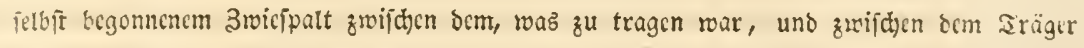

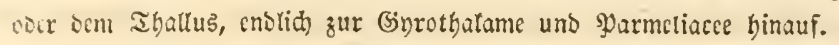

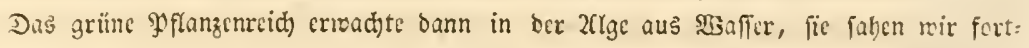
bitcen, immer mütterlich nur in berrtiche Formen geftaltet, bis bann, żum (Etemente ser Ruft

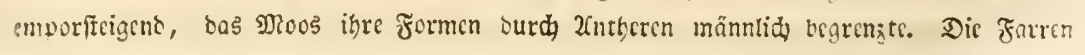




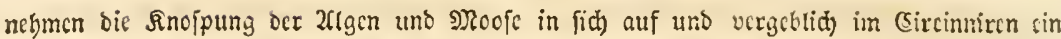

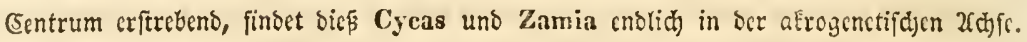

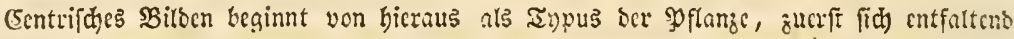

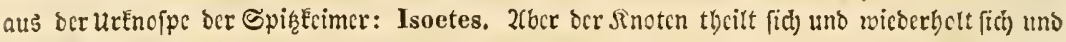

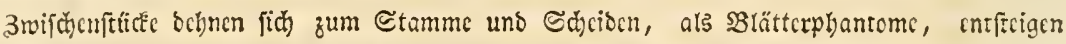

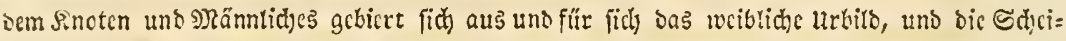

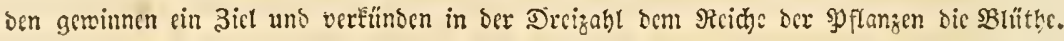

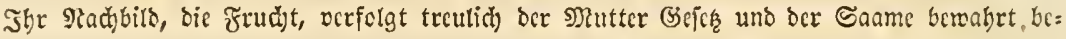

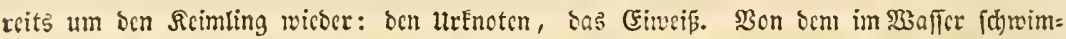

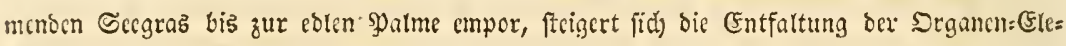

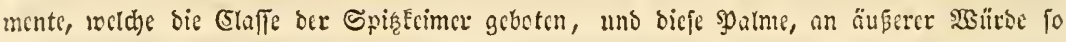

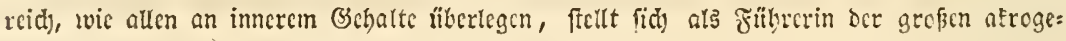

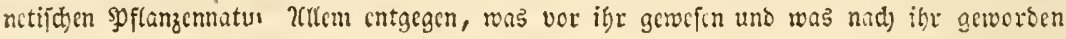
(2intithefe).

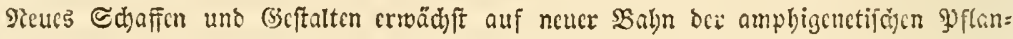
zennatur, nadbocm bas şattgcbilbe in ben şlattfeimern fich freigenacht (folia decidua) uns fid felbft einen (Segenfas burd) fich geftellt bat (cotyledones oppositae). Und was früher aus Sinoten und unmittelbar anbängenden Scheisen geboren, bas febch wir von

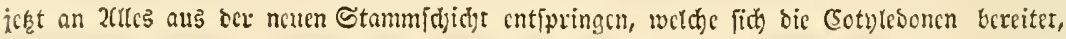

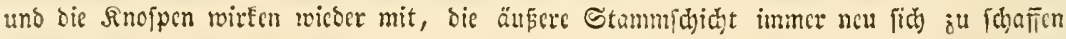
(ङnnthcie).

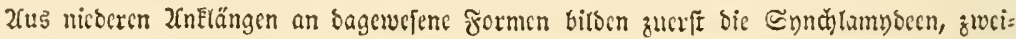
felbtumig nod, im einfacton Blattleben fich fort bis zum cstcn forbccr = unb 3immetbaum.

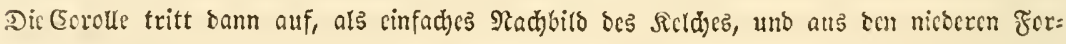

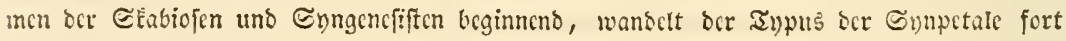
bis in bic viclgeftaltigen Formen ber Sthodoracecn und Eapotacen. (IIlffï, vgl. E. 102.).

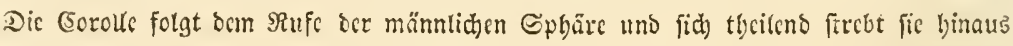

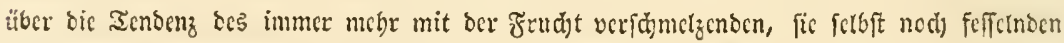

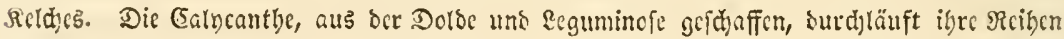

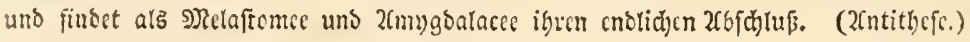

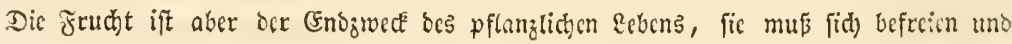

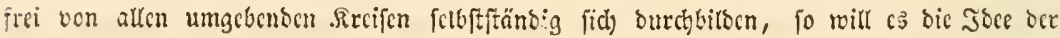

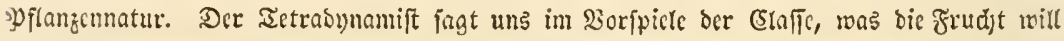

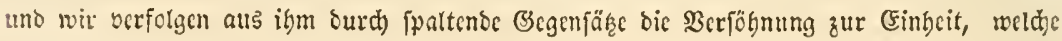
dic Srange gewonnen. (Sinthefe.)

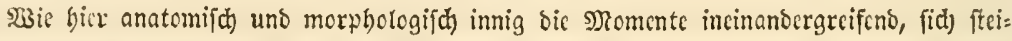
gern, fo ftcigert fich auch phyfiologif das Fortichreiten ber Dauer von cinjährigen Sirät:= tern und ভtauben zu Sträuchern und Säumen, fo fteigert fich bann ber Berbalt an ๔tof:

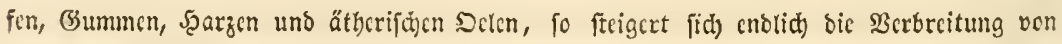
ber nörolichen Eros bis bin unter ben tropifăen Şimmel.

DenEen wir jef̧t cimmal, bieß 2lites fei 3ufall in unferem Syftem, glauben wir nid)t

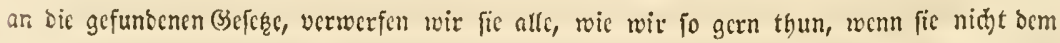

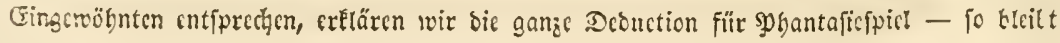


Etielblüthige.

Sronung: Cäulenfrüdytige.

Srangengervädj] uns bennod - - cin Refultat, alls ciner objectioen sefdauung bet ङ山öpfungentnommen - rie manuns - nod feines geboten. -

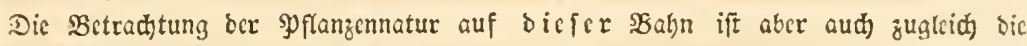

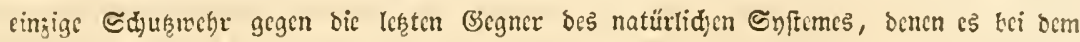

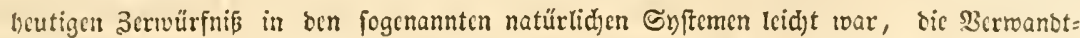

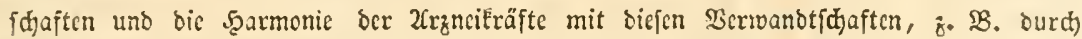
Euphorbia unb Carcarilla, wic burd) fo mandje anocre Bcifpicle zrocifecthaft machen zu

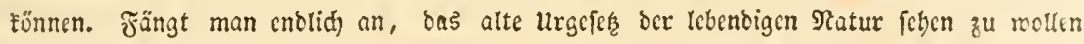

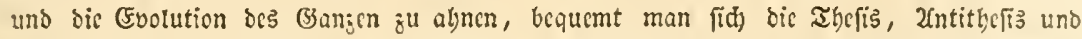

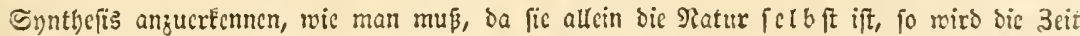

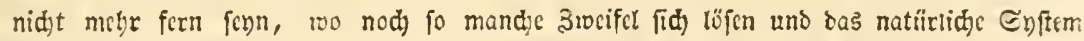
burin feinen sriumpf feicu virb, dof cs cin mirelid natürtides roiro! - 


\section{Crflärung tuis Bitte.}

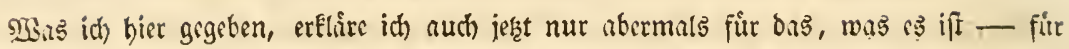

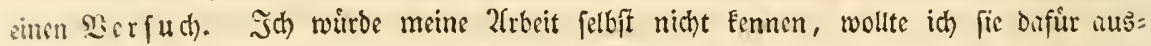

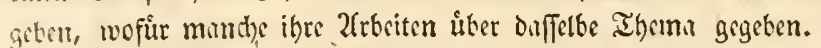

Jch babe mich nur beftebt, zu zsigen, dus in ber von mir feit fiebenjebn Jahren

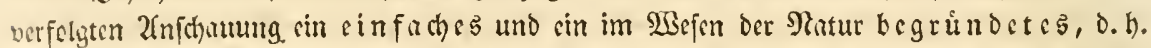
to wic fie ferkft ift, ein lebendigfortfdreitenoes Prinzip befolgt iff, und dicfe $\mathfrak{B}_{e}=$ folgung mag man Eunftig, wo fie es thid)t fenn follte, confequent madjen, bas wiro Nie= mano dirnebarer erfenenen, als ich felleft.

Jch hake mich vorläufig bethadigt

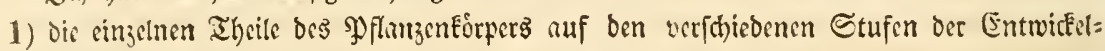
ung des Pflunzenreiches, als refentlich) biefelben wieder ju finden.

2) Dicfe Theile nach Marbgabe ifere Erfd)cinung als Materielles uno Retendiges, ifrer

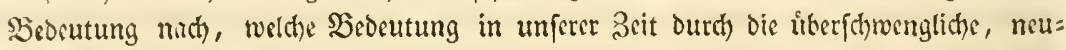

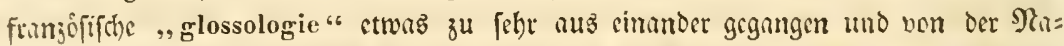
turwerftindlichéeit akgeleitet worden, wieber frei zu machen, D. h. mit ihren wahren, sinfather Samen: Epore, Sanme, Snoppe, STurzel, Stumm, Statt, Schcioe,

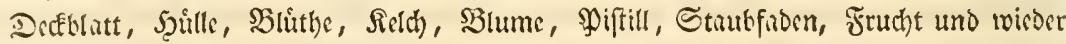
Sanme, u. T. tw. zu bejcichnen.

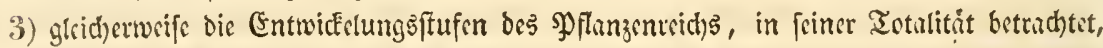

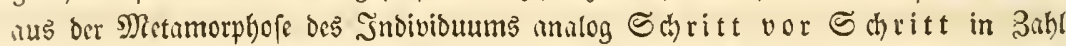

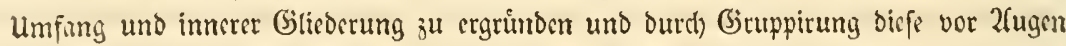
jul legen, endich mit den allerverffindichften, immer von oen be Fannteften und siteften Battungen bergenommenen Samen, bic fo entftundemen Girtwpen zu be= jeidjuen.

4) Durdi) bie von mir im Saghe 1820 erfonnte, Dann in ifrer Eridgeinung immer tiefer verfolgte und rafflos und mit immer gimftiger vermebrten Şülfsmitteln fortge=

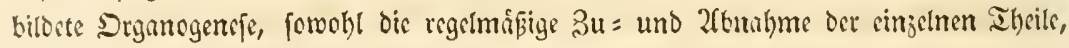

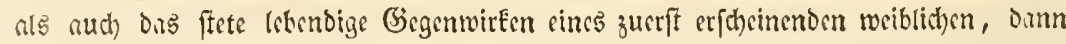

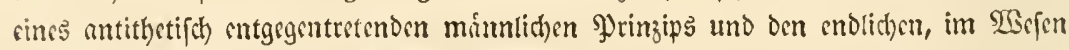

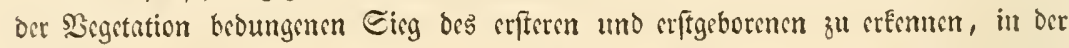

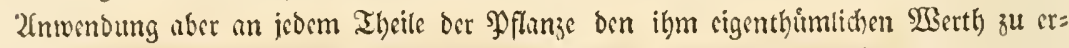

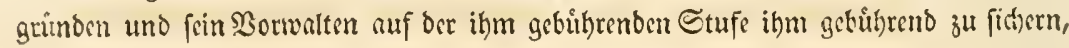
mit cinem Sorte: die 2lllgegenwart der Metamorphofe in oer ganjen गु) flanzennatur vor bas objectio zu febengemobnte 2 uge zu fubren.

5) Der vou mir gemachten uno, fovisl mir befannt ift, juerfit von mir atsgefprodhenen

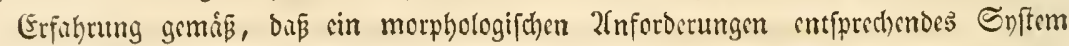
nid)t gedad)t werben Fonne, obne jugleid) im anatomifasen uno phyfiologifacen (Ent= wickelungsgange zu harmoniren, in oer (Erfd)cinung feiner Giruppen uno Sindividuen

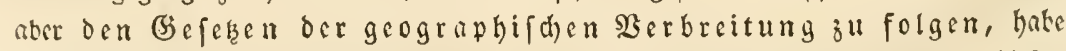
ich bei immer weiterer Prifung midh bemilht, meine 2(noronung aus allen biefen

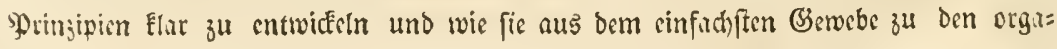


nenrcid)ffen, combinirteften formen fid) weiter geftiltet, fo aud) aus ber norbliden

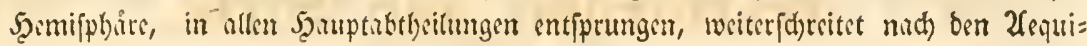
noctialregionen in: bie 3onen ber Tropen.

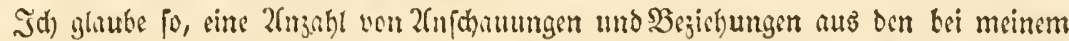

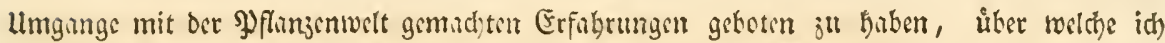

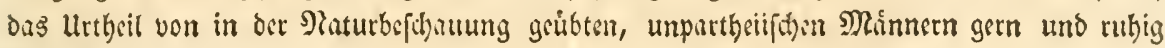
ertwirte.

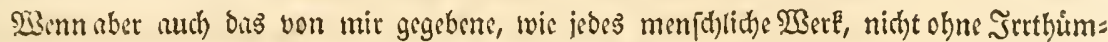

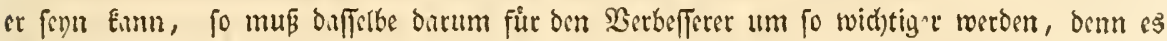
ift eben bariu unfer Sntereffe und jugleid die wahre Sbedeutung bes S rganifden unt פat utlichen am tiefifen begriubet, wenn twir einen Gegenftund yor uns baben, in wel=

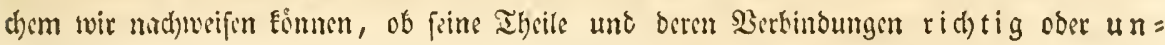

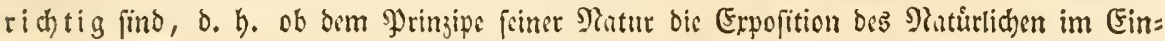
zcluct: wicber entfpridt ober nid) t. Und id balte bus cben fur bie wahre, lebendige

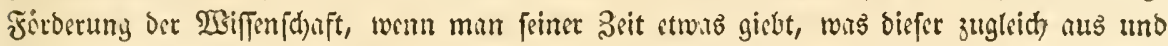

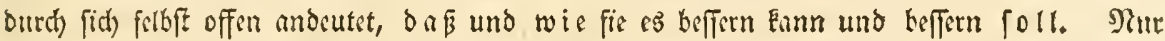
gete man nichts, tons man aus ber Natur mad)t, fur ftubil aus, fonft fabilifitt man fid) felbft. 230 mir baker aus der Natur nacbgewiefen wirb, baí id feblte, boffe id), wie if immer gethan, ber erfte zat fern, welder dem Befferen folgt.

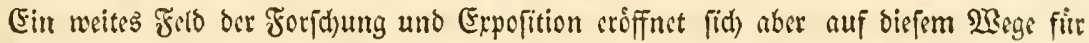

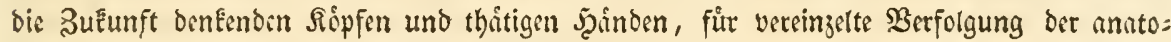

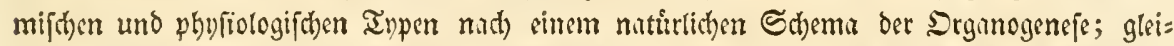
b)en Sroff fimbst die Thatitigerit in ber Entwicfelung nller Finzelbeiten ber materiell=organi=

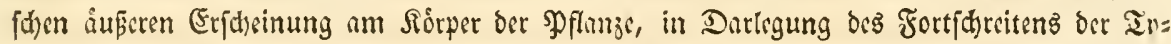

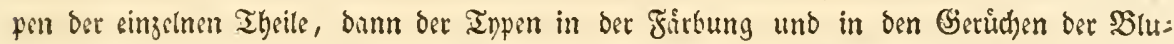

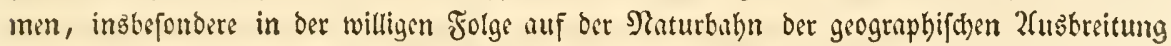

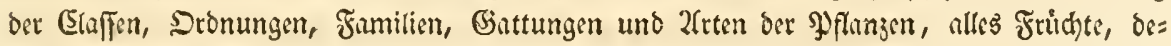

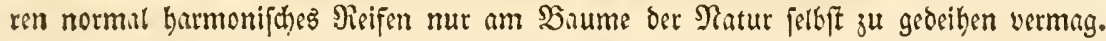

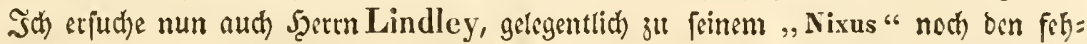
lenden $\mathcal{E}_{\text {an }}$ on und bie fehlende Drganogene pe geben zu wollen, denn mur canon und

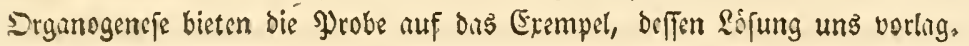

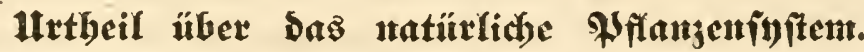

„Cette méthode offre encore un interêt d'un genre particulier, en montrant plusicurs caractères tellement associès qu'ils ne peuvent exister l'un sans l'autre, et is resoudre ce qu'on peut nommer - des problèmes en botanique."

„Plus les ressemblances sont nombreuses, plus les familles et par suite le caractère général, est plus chargè."

A. L. de Jussicu. 


\section{Driter \$achball Der Satitheie im Opponenten mit Reigung jux @intbeje.}

1, STlir find

1) D.rourd), wo.ls wir hier gelefan brtben, eitrigermaken,

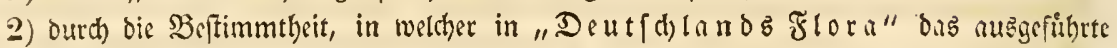
Beifpiel der Drganogenefe der Eruciferen uno ßefedeen und beren Etelluny im Softeme bebundelt worden - noch mebr

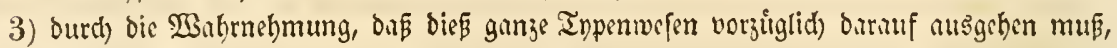
umfere nun cinmal angenommenen uno wenigftens burd) 2fagewefhnung fur naturtid gebultenen Syfteme zu prufen und wabrfdeinlict) bod in ihren ifolitten Theilen als

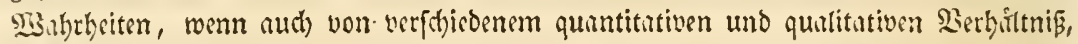
erfernen zu loffen - am allermeiften

überzengt worbern, bar an bieferm Typentwefen etwas ift.

grair hitben uns barum zu ber Bemuihung entfhloffen, ben Gegenftand nummehr be=

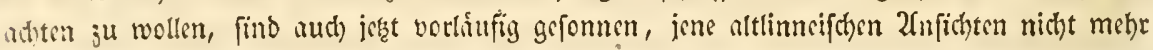
zu verbunnen uno bie vom Berfaner im Jabre 1828 ausgefprobenen Worte: "brei

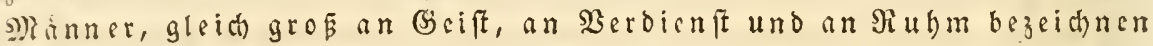
jene Epodien, oeren Frad) te wir jegt zu arnoten beginnen," nidst zu wider= legen, fonbarn zu unferen sigenen Ueberzangung mat)en zu wollen. 2tberbings leuchtet uns

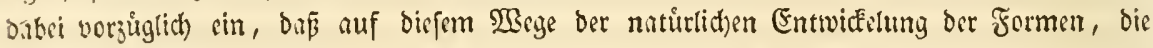
v. Şumboldifhe Entdectung ber grographifden Berfiltuife, in innige Şarmonic mit bem Syftem treten und sines in bem andern sinft feime Begrundung unb fein Zitel finben burfte.

\section{Echlufingort an Den Dppouenteut.}

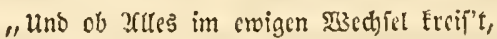

FE bebarret im 2Bebjal cin rubiger Esift!"

Du fishft, lieber Sefer, wie es ber Pflumanfpecies, die wir Syftem numnten, am

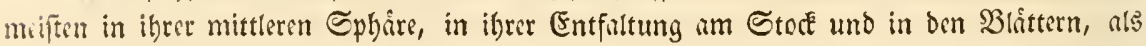

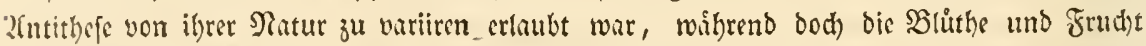
den Iypus dor Gattung — wenn Du nur willft, als etwas פ̧ofitives - weit mehr

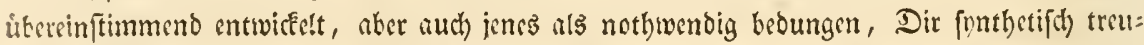
(id) Zfles berwabt und nur bewa

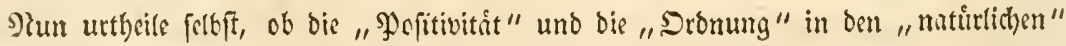

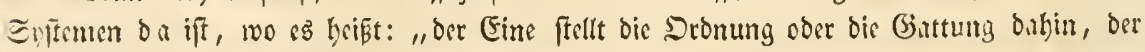
Unbere borthin, wir warten aber feit lange vergeblich nod) auf ben britten, weldher uns fas

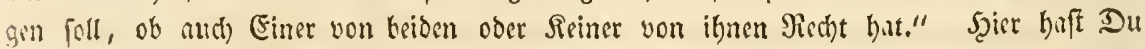

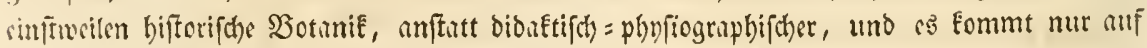

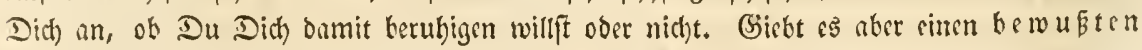

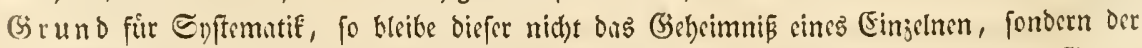

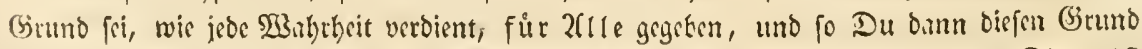

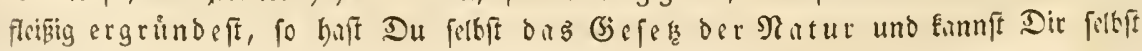




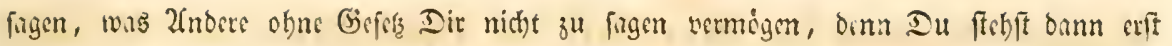

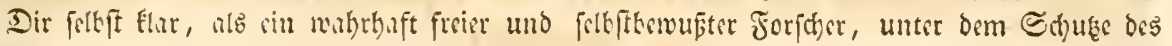

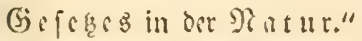

D.13 ift num unjere Pofitivitit, welde Esine Etabilitit und bod and frine

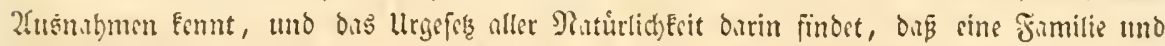

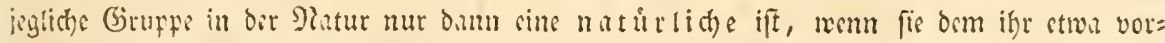

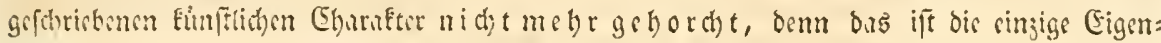

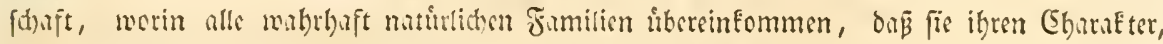

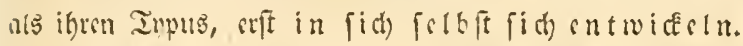

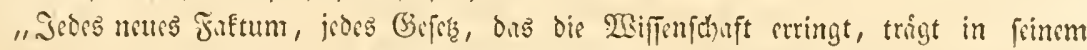

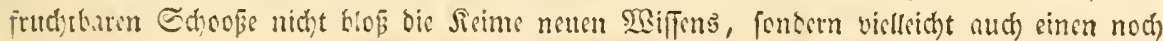

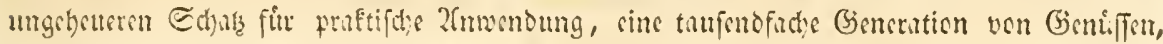

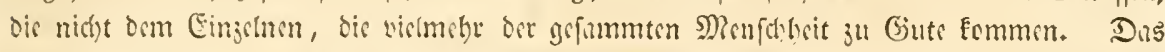

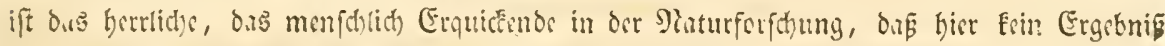

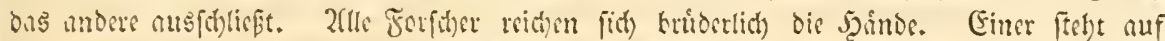

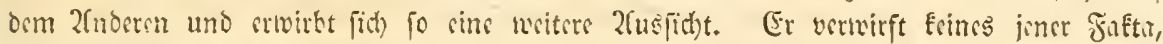

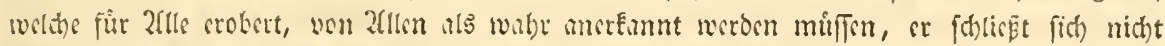

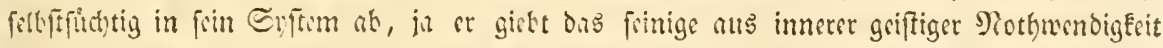

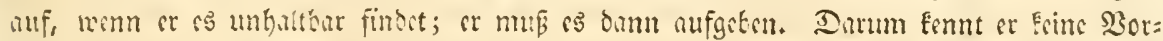

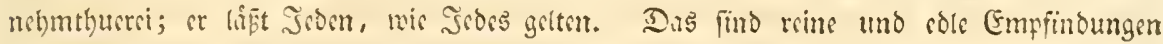

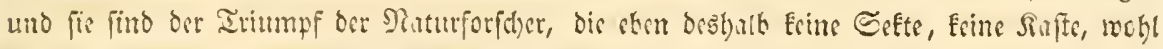

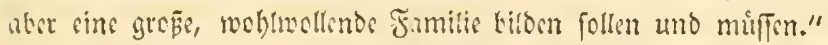

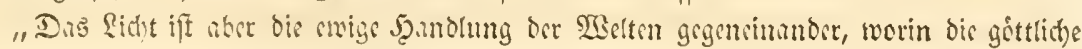

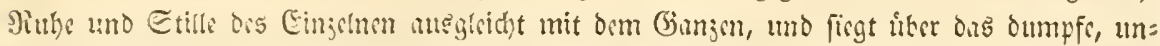

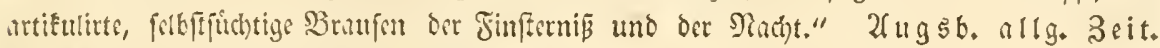
1836. o. 19. 21pr. $176-177$.

Gegen bie Mittebenden bat darum sken ber Menfen bie Pflicht, mit ifnen jull leben

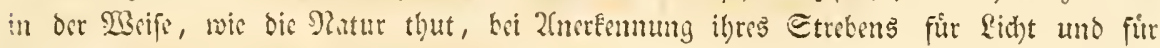

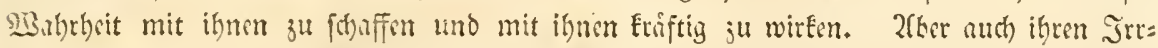

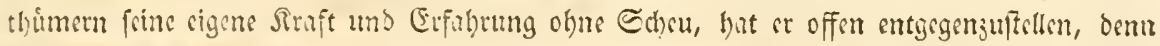

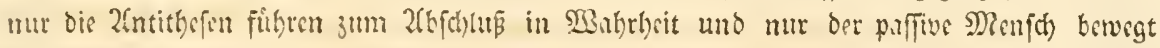

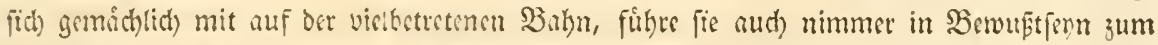

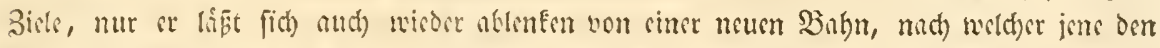

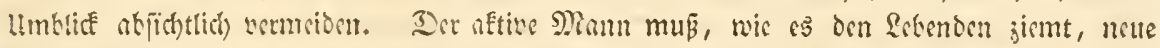

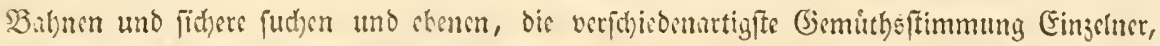

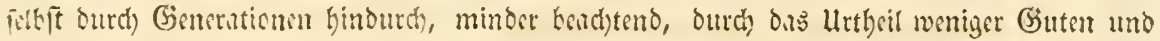

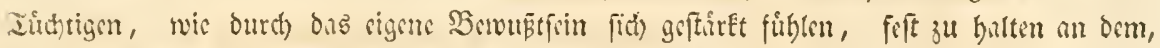

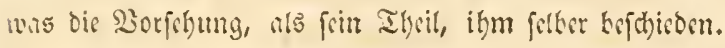

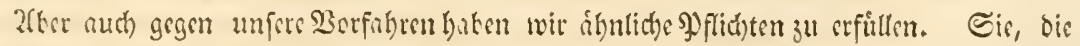

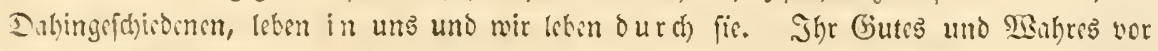

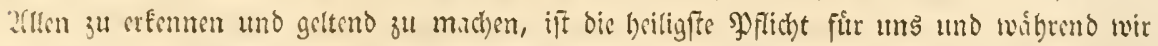

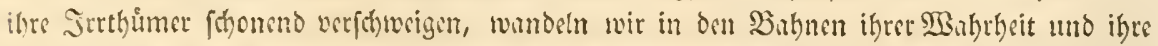

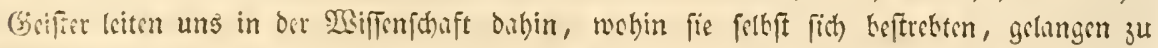

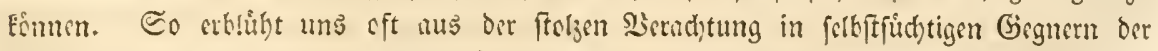

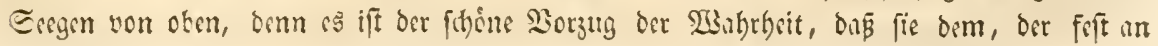




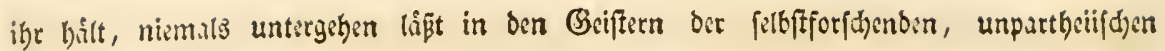
Denfer.

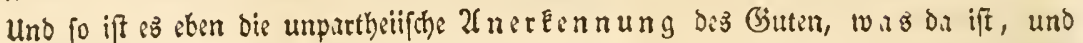

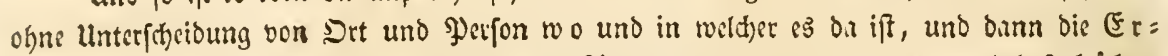

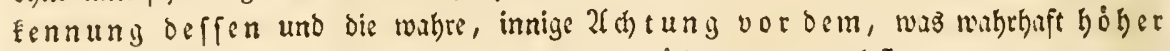
ift, nis wir felbit, wilche uns Elar midben funn, ủber uns felbft.

\$O $\mathrm{m}$ Ceyn zum Ceyn gebt alles Reben üfer,

Beftultung reift zur umgeftaltung nur,

uno bie Erfdjcinung đdjoct vorüber.

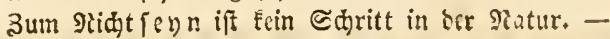

शुs ir fino nidht, um zu fern,

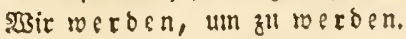

actoge. 


\title{
Mic $\mathfrak{s}$ i it $\mathfrak{r}$
}

\author{
ber

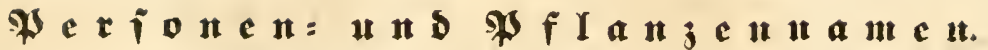

NB. Zule gyflamgengattungem, reche blos nad) ihrer Nummer aus bem Conspectus aufgeführt worben, find hier weggeblieben.

A

Abasicarpon. Andrz. S. 260 Actinotus. Lab.

Ahatia. Rz. Pav. 2Jt? -303 Adimia. Wall.

Abelmoselius. Medik, 287 A d anson.

Abietinae.

Ablania. Aubl.

Abutileae.

Abutilun. Dill.

Acalyphis. $L$.

Acalyplieae.

Acanthariae.

Acantheae.

- crenninae.

Acanthunychia. De C. 235 Ademosua. $\mathrm{R}$. Br.

Acantlopilyllum. C: $A, M$. 248 Adhadota. IIerm.

Acanthoprasium.

Acantluus. $\boldsymbol{L}$.

Acer. $L$.

Acerantluss. Morr.

Aceratium. DeC:

Acerene.

Acetosa. $P$.

Acetoselleac.

Achania. Siv.

S. 221 Aglaia. Lour.

9:0 S. 313

Achetaria. Cham. Schlecht. 195 Argialitis. R. J3r.

Achras. $L$.

Achyrantlieae.

Achyrospermum. Bnth.

Acidoton. S $w$.

Acinos.

Acinotum. De $C_{\text {* }}$

Acnualenia. Bartl.

Acmenis. DeC.

Aconitum. $L$.

Aconogonumu. Meisn.

Acorineas

Acrocarpica.

Acrocephalus. Bnth.

Acroglochin. Scherd.

Acronodia. $B l$.

Acroschistae.

Acrotreina. Jack.

Actiea. L.

Actaeariae.

Actinospora. Ture.

168 Adansonia. $L$.

$30:$ Adansonieae.

287 Adelia. 1.

287 Ailenacanthus. N, $v, E$.

281 Adenibulra. $\| r$.

281 Adruogramma. Rchb.

190 Adeuolinum. Rchb.

190 Adenophora. Fisch.

190 Adenosepalum. Spach.

188 Adiantheae.

190 Aillumia. Biafin.

285 Adonideae.

$2(j)$ Adonis, L.

304 Adoxa. $L$.

28.5 Arastaci. DeC.

o:ji Adrastaeariae.

2!)t Alriana. Gaudich.

28 Aechmanthera. N, v. F,

215 Aegiceras. $L$

2:3!) A egricereae.

188 Aegle. Corr.

281 Negochloa. Benth.

189 Aegoporlium. $L$.

efio Acollanthus. Mart.

282 A Aeschynantluus. Jeeq.

5is Actleilema. R. Br.

27 Aethionema. R. Br.

\%otiops.

15:3 Acthusi. $L$.

18.20 Alopecura.

4. 3̈6 A grimonia. ' $T . L$.

2!5. Agrimonieae.

2155 A grosteae.

281 A grostemna. $L$.

140 A grostideae.

28: $A$ h I qu is t.

-3iti Aigopricen. L. fil.

g06i Ailanthus. Dsf.

18 (i) A ton.

308 Ajuga. I.

190 Aizoideat.

190 - genuinac.

139 Aizoideen.

2fit Aizoon. $I$.

ำช Akeesia. Tuss,

276 Alingieae.

201 Alangium. Lam.

278 Alchemilla. $L$.

¿5 Alch emilleae.

281 Alchornea. Sw.

190) Aldrovanda. Monti.

203 Alectryon. Gïrt.

-14 Aleurites. Forst.

214 Alegria. Moc. Sess.

314 Alcpilla. La Roch.

194 Alfieri, I ittorio.

219 Alicteres. Nech.

189 Alismacenc

1!) Alismeac.

I90 Allionieae.

259 Aluseidea. St. Ilit.

18!) Alonsoa. Cav .

169 A gardh. 11.16.44.45.53.92 A l s chinger.

ous Agrassizia. Chav.

304 Agssizia. Spach.

26il A gasyllis. Spr.

278 gathelepis. Chois.

278 Agatlooles. Don.

278 Agathosiua, $\|$.

¿to 4 gaveae.

.) Alsinantlus. Osv。

198 Alsinastrum. ' $T$ '

$\because 4 i$ Alsine. $L$.

20 Allsinclla. Benth.

196 Alsodeia. A, I.'Th.

211 Alsodineae.

280 - genuinae.

191 Alstroemerieae.

253

눈

141

넝

$14 i$

11

281

282

189

237

239

237

240

285

247

248

242

242

281

$2 \% 1$

ำ

281

303

201

41

9) 4

143

144

174

20.

19!)

188

11

298 
Althaea. $L$.

Altheria. A.P. Th. Alysseae.

- genuinae.

Alyssum. $L$.

Alzatea. Rz. Pav.

Alzateae.

Amanoa. Aubl.

Awaratus. Buth,

Amarantheae.

- genuinae.

Amarantlus. $L$.

Amarenus. Piesl.

Amarylleae.

Amaryllineae.

Ambleia.

Aublygnoneae.

Aublygonum. Meisn.

Ambrarial. Cr.

Ambrosiene.

Aubrosineae.

Amentaceae.

Ametliysten. L.

Amethystoides.

Ammi. $L$.

Amulineae.

- gemuinae.

- seselinene.

- teretiusculae.

v. $A$ m m o n.

Ammyrsine. Prsh.

Amoneae.

Amonia. Nestl.

Amoria. Presl.

Ampelopsis. Mchx.

Amperea. A.Juss.

Amplibolocarpae.

Amphicenianthae.

- labiatifl.

- ligulifl.

- tubulifi.

Amplifgrnanthae.

- Jahiatiflorae.

- lignlifiorae.

- tubuliflorae.

Amphirrhoge. Rchb.

Amphirrhogeae.

Amphirrhox. Spr.

Amphischistae.

Amphiseopia. N.v. E.

Amygdalaceac.

Ariygralaceen.

Amyridenc.

Antimena. A.Juss.

Aubaseac.

Analonsis. I.

Anacampseros. Sims.

Anacarilieac.

Anagrallideae.

Anagallidium. Griscb.

Anagallis. L.

Ananasiease.

Anarrhinmm. Dsf.

Anastaltial. L.

Antastaticeac.

Anuvininu. Rheed.

Anaxagron. St. Jit.

Ancistrolobus. Spach.
S. 286|Anchietes St. Hil.

29I Anchonium. $D e C$.

25!) Ancistrocarpus. $K, H . B$.

25!) Anda. Piso.

254) Andrachne. L.

301 Andreateacea.

301 Andreacaceen

282 Andreoskia. DeC.

188 Andrographideae.

23:) Andrographis. Wall.

23!9 Andromeda. $L$.

209 Andromedeae.

226 Andropogoneat.

151 Androsiceite.

151 Androsaemeae.

188 Androsaemum.

236i A n d rzeiow s ky.

236i Andizeiowskya. Rchb.

180 . Anemoue. $L$.

18: Anemoneae.

142 - genninae.

181 Anesorriza. Cham.

18!) Anethum. T. L.

18!) Angelica. $L$.

219 Angelicene.

219 Angrlonit. K. II. B.

21!) Angincarpicae.

2200 Angolamia. S'cop.

20(1) Ingophora Cav.

21 Angophorcae.

2nti Anisactis. DeC.

158 Anisochilus, Wall.

243 Anisoduntium. Rchb.

$22(i)$ Anisodus. $L k$.

221 Anisonueles. H. Br.

$28 \mathrm{I}$ Inisosciadino. DeC.

13!) Anisosticte. Barll.

182 Anoda. Cav.

182. Anolene.

15:2 Anisum. Ad

18. Anogra. Spach.

18\% Anoilit. L.

182 Anonariac.

182 Anoneac.

182 - grenninae.

26i9 Anopterns. Labill.

269) Anredicra. Juss.

2 (6) Anthrisens. I?.

¿5!) Intichorus. I. $f$

194 Antidrosma. L.

25t Amirhinatiae.

2ost Antiprhilatitr.

20ㄴㄹ Antirthimu. L.

Es1 Antherereis. Lavó.

23:) Inthum roteac.

2:) Anthurleistit. Afz.

$2: 37$ Anthurlendron. Rehb.

224 Antloulon. Rz. l'av.

2014 Anthoionia. Lnb.

211 Anthosperineac.

onf. Anthosperimum. I.

15i Anthustromma. A. Juss.

198 sintlos llidiate.

258 Anthillis. It.

253 Anychia. Uchx.

ôs Apatelivt. DeC.

zoss Apeilua Aubl.

¿Ut A Jianes. $L$.
S. 2(i!) A phania. Bl.

25:) Aphanochilus. 188

S. 285

23!) A phanomyxis. Bl. 313

281 I phanostemma. St. Hil. 278

28:2 Aphragmus. Audrz. 260

137 A pin. 'T. $L$. 21!

137 fpolophyllum. A, Juss. $\quad 282$

$2(i 0)$ A pocyneae. 211

190 Aporlotes. 183

199 A postasieac. 155

20ni A poterinn. Bl. 311

206i Aquifolineene. 214

$141 j$ Aquilearia. $L . \quad 278$

204 Arabidene. 260

317 - genuinae.

308 Arabidopsis. 'DeC.

18. 75) Arabis. $L$ 2(i)

2 (i) Arabisa. Rchb. 2filt

276 A riceae. $1+2$

276 Aralia. $L . \quad 2$. 21

27 A A raliacene. 、 221

200 Araucariae. 168

20 Arbuteas. 206

200 Arluntus. $L$. $20 t^{\circ}$

20 Archangelica. Ifoffm. 200

198 Archemora, De C.

190 Arritiea, Mart. 302

247 Arctopus. L

253 Aretostapliylos. Adans. 20(i

253 Arlisia. Sw. 204

220 Arulisicale.

189) Areeariae. 158

188 Aremonia. Neck. . 243

291 Arenaria. L. 24)8

188 Arenarinente. - 298

21! Argemone. T. L. 2tit

310 Argemoneac. 26it

288 Areytliamneac. 281

287 Argthannia. P. Brw. 281

219 Aristolochia.

$2 \pm$ Aristolochiaceat. 178

278 Aristolohicie. 173

288 Aristotelia. l'Jforit. 3104

278 Aristoteliene.

278 Armeria. W. $20 \%$

230 Armericie. 2013

240 Armlenchtergewächse. 101

zly Armoracia. G. M. Fl. W'ett. 2(i)

ä03 Armoracienc.

281 Ancbia. Forsk. 1952

198 Amolilia. Iil. 2:3

198 Aruot. W'ollier.

$1 ! 8$ Aroidene. $14 \%$

1!3!) A romalendrou. $B l$.

136 A $\mathrm{r}$ olll it ri it. 18!)

211 Aronew ächse. 130

207 Arracinclat. Brencr. 21!

301 Arrudear. Camb. 310

310 Artinbutrys. $\mathrm{H}, \mathrm{Br}$. 288

180 Aricdia. L. 2. 2030

180 Arilionarias. 16 (id

281 Arthropliyllan, Bl. 20,

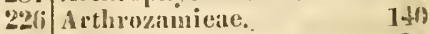

"2oti Artucarpeat. $17 \%$

epos Artoncenia. Dev. 1!y!

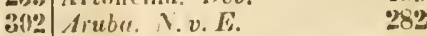

303 Arnulinaceas. $1+6$

2421 sarineate. 
A scherson. Asclepiadeae.

Aselepiateen.

Aselepienc.

Asera. Schott.

Astyreae.

Aserron. $L$.

Asimina. Ad.

Asperiloliaceae. Schizos:arpicae.

Idiocarpicae. Capsulares.

Asperugu. $L$.

Asphodeleac.

Aspicarpa. Rich.

Assonia. Cav.

Astartea. $D_{\mathrm{e}} C$.

Astephaneae.

Astericintha. N.v. E.

Asteriscinm. Cham.

Astericarpeae.

Asterocalpus. Neek.

Astilbe. Hamilt.

Astoma. Dec.

Astomaea. Rchb.

Astragaleae.

Astrantia. ' $\boldsymbol{T}$. $L$.

Astrapaen. Lindl.

Astrapaeariae.

Astrephia. Difr.

Astrodon.

Astrotrichia. DeC.

Astydamia. $\mathrm{DeC}$.

Asystasia. $B l$.

Atalantia. Corr.

Atalantieae.

Atamisquea. Miers.

Athamanta. $L$.

Athenaen. Sichreb.

Atherospermeat.

Atocion. Oth.

Atragene. $L$.

A traphaxis. $L$.

A trema. $D e C$.

A triplex. $L$.

Atriplicineae.

Atropa. $L$

Atropeae.

Aubletia. Rich.

Aubrietia. ddans.

Aucuba. 'Thb.

Andibertia. Bnth.

Aurantieae.

Aurinia. C. A. M.

Autommarchia. Colla.

Avenilcene.

Averrhoil. $L$.

Avicularia. Meisn.

Axonoteclitum. Fuzl.

Axyris. $L$.

Ayenia. $L$.

Aylmeria. Mart.

Azadarichta. 1.Juss.

Azalea. $L$

Azorella. Lam.

Ba:azia.

Baea. Commers.

Buekea. $L$
S. 11. 106|Baekecao

207 Baeobotrys. Forst.

208 Balgkernflechten.

go8 Balanophoreae.

$27 t$ Billarlia. Cambess,

307 Ballota. L.

208 Balsamina. Riv.

278 Balsamineae.

1!)' Bambuseae.

192 Banara. Aubl.

193 Banisteria. $I$.

193 Banisterieae.

193 Banflya. Baumg.

154 Barleria. $L$.

2!y!) Barlerieae.

$2 ! 11$ Barosina. $\boldsymbol{W}$.

253 Barraldeia. A. P. Th.

208 Barraultia. Spr.

190 Barreria. Scop.

221 Barringtonieae,

2(j) B a r tling.

26il Birtlingia. Brngn.

230 Bartonia, Sims.

218 Bartramia, Gärt, Lam.

218 Basella. $L$.

226 Raselleae.

221 Bassia. Koen.

287 Bastardia. $K$.

287 Bastardieae.

178 Batrachium, DeC.

188 B a u er.

221 Bauera. Andr.

2:0 Bituereae.

190 B a n h in.

314 Baumannia. Spach.

314 Beitsonia, Roxb.

207 Beaufortia. R. Br.

220 Begonia. $\boldsymbol{L}$.

273 Begonieae.

174 Beilsch inidt.

298 Belangera. Camb.

298 B ell.

23(i Beloperone. N. v. F.

218 B en th a m.

238 Berbereae.

238 - genuinae.

201 Berheridene

201 Berberis. L.

28" Bergenia. Mneh.

25!) Ber"era. Koen.

22I IBergereae.

18! Berrial. L.

sి14 Beringeria.

250 Ber n a r d. Juss.

28: Bernh al di. 11. 32. 46.67.

14 (i) Bernardia. Houst.

2!) 4 Bernhardieae.

Eib(j) Berria. Rxb.

23(j) Bertervil. De C.

2ub Bertolone.

2y2

203 B e s s e r.

31: Bessera. Spr.

gori Beta. $L$.

201 B e t e ke.

18? Betokea. $D_{e} C$.

198 Betonica.

Detulealc.
S. 253 Beurreria. $J c q . \quad$ S. 193

$2(0+$ Beyrichia. Cham. Schlecht. 19y

1 ï3 B i a so I e t t o.

163 Biasolcttia, Koch.

$231 i$ 291. An

188 Biasolettia. Prsl. 29]

294 Bifora. Hoffm. . 208

2!)+ Bignoniariac. 198

146i Bignonieae, 198

27t Billardiera. Sm. 270

gg!) Billardiereae 280

2!)!! Billotia. Colla.

29)8 Bilimbia. (Rheed.) Rehb. 294

190 Bineetaria. Forsk. 215

190 biophytum, DeC. 294

282 $B$ is ch of f. $\quad 11.56 .81$

282 Biscutella. $L$. 25!

282 Biscutelleat.

214 Bistella. Desf.

251 Bistorta. $T$.

11. 15. 16 Bivonnea, Fl.Mex. 235

25.3 Bivonaea, Rafin. 281

23ㄹ Bixa. $L$. 274

303 Bixaceae.

23!) Bixaceen.

289) Bixene. 274

215 Bixinene. 27\%?

287 Bladhia. Thnb. 204

287 Blairia. $L$. 206

27tj Blandovieae. .

20 Hasicae. 137

230 Blattmoose

230) Blechum. Juss. 1:40

28 Blephilia. Rafin. 188

246 Bliurhia. Kön. 285

272 Blitanthus. Rchb. 238

25: Bliteae.

2ü(j) Blitum. L.

236 Blochmannia. Weig. 2310

18 B I uff.

230 B I u m e. 11. 143. 149. 155, 164.

20174

190 Blumenbachia. Schrad, 232

12. 2(i2) Blumenbachieae. $23 \%$

205 Blımia. Spr. 30 ?

$2\left(j_{0}\right)$ Bneagea. St. Hil. 278

2 (i5 Bocageae.

$26.5)$ Boceonia. L.

230 Bocenieae. 263

31413 o eber. 18

314 v. Boen inghasen. 11

248 Boeninghausenia. Rchb. 282

188 Buerhavieae. 184

4 Bojer.

G8 Buisduvalia. Spach. 246i

Esl Bolax. Commers. 201

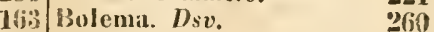

203 Bombincene. 245

25!) - erenuinae. 295

3i(i) Bombax. $L$. 295

219 Bumbycospermum. Prsl. 295

18. 3l Bougard. 18

2(i) Bonjeinia. lichb.

23!) Honnayal. Lnk. 199)

11 Bonnetia. Mart. 302

178 Bonnetia. Schreb. 302

188 B $0 \mathrm{nl}$ I and.

1720 Bontia. $l$. $\quad 196$ 


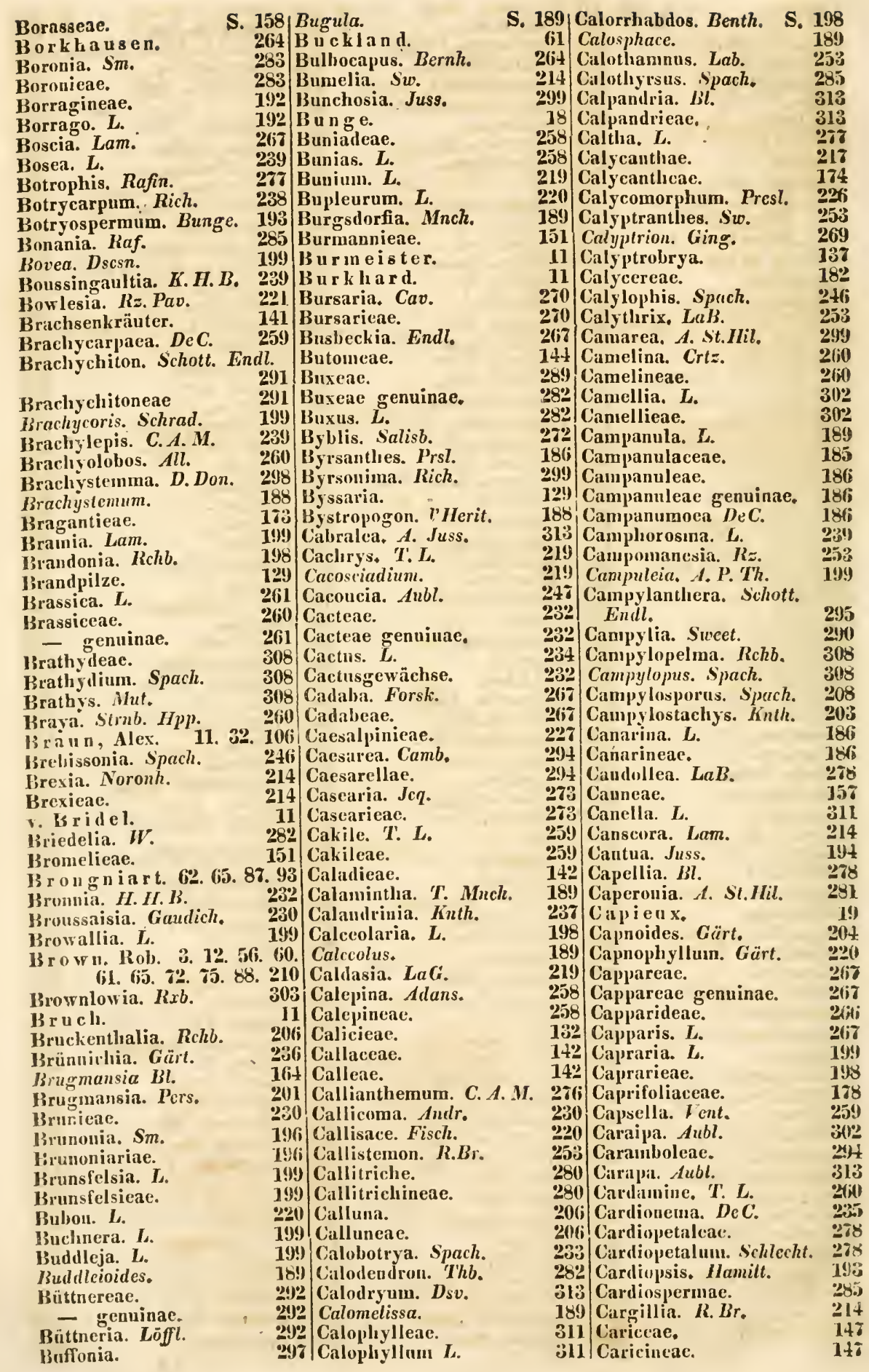


Cansiera.

Carisseac.

Carmelia, DeC.

Caroides. DeC.

Carolinea. L. fil

Carota.

Carrichtera. DeC.

Carum. L.

Ciarus.

Caryocar. $L$.

(aryophyllaceas.

raryophylleae.

Caryophyllus. T. $L$.

Casaleis. st.Hil.

Cassiaccae.

Cassiacten.

Cassini.

Cassipourea.

Castela. 'Turp.

Casteleae.

Casnarina.

Catepha. Leschen.

Catluartolinunı. Rchb.

Catin ra. Aubl.

Catovtachys.

Caturns. $L$.

Cavallium. Schott. Findl.

Cavanilla. Lam.

Caranillesia. Rz.Pav.

Caucalinae.

Cancalis. L.

Caucanthus, Forsh.

Canlerpeae.

Caulinia. $I T$.

Canloplyylleae.

Cauloplıyllum. Mchx.

Ceanotheae.

Cedrela. $L$.

Cedrela. Lour.

Cedreleae.

Celastreae.

Celastrus, $L$.

Celsia. L.

Celtideae.

Centanrella. Michx.

Centaurinae.

Centinorlia. J. Bauh.

Centrantliera $\boldsymbol{R} . \boldsymbol{B r}$.

Centranthus.

Centropogon. Presl.

Centunculus. I.

Cephäelideac.

Cephäelis.

Cephalantheac.

Ceplaalantluus.

Cephalohyptis.

Ceploalostigma, $D_{c} C$

Cephalotus.

Ceramanthe, Rchb.

Ceramiaceae.

Ceramieae.

Ceranthera. P. $B$.

Ceranthercae.

Ceranthus Schreb.

Cerasene.

Cerastieac.

Cerastium, $L$.
S. 170|Ceratiola. Rich.

211 Ceratocarpus. I.

220 Ceratocaulon. Brnh.

219 Ceratocephitleac.

$2 ! 5$ Ceratocephalus. Mnch.

2?0 Ceratocoretit. DeC.

2 (ii) Ceratonieas.

21! Ceratopetalum. Sm.

7. 21.82. 283 Ceratophylleac.

2!)5 - genuinae.

295 Ceratostemina. Juss.

g!ir Ceratoyylon. Blume.

253 Cerocarpus. K. H. B.

$27($ Cercodia, Murr.

207 Cereaster. DeC.

ב-70reus, Juss.

84. 88. 2(it Cervaria. Kiv.

17!) Cesal pini.

¿8: Cestrum. $L$.

$28:$ Chaerophyllum, $L$.

170 Chuetocrater. Rz. Pav.

201 Chaetonychia $D_{e} C$.

306 Chailletieac.

25.3 Chamaedrys.

188. Chamaelaucieae.

281 Chanaelaucium. Dsf.

29! Chamaeledeae.

215. Chama enerium, $T$.

29:- Chamaepitys.

21!) Chamaesilleritis.

219) Cinamira. 'Thb.

2(y) Ch a mi s so.

136i Chnmissonia. $L k$.

16 ? Chara. $L$

20.5. Characene.

(6) Chasmonia. Prsl.

20) Cheimntheas.

2220 Cheiranthus, $L$.

313 Cheiropsis, De C.

23. Cheirostemon, $\mathrm{Hb}, \mathrm{Bpl}$

313 Cheirostemoneae.

301 Chelidonieae.

s01 Chelidoninm. C.Bauh.L.

19!) Chelone, $L$.

172 Chenopoleae.

211 - genninae.

18: Chenopodium. L,

2ü6 Sherleria. Hall.

198 Chiazospermum, Brnh.

17 Chishaea. Prsl.

186 Chichrassia. A. Juss,

$20 \div$ Chilodia, $R, B r$.

180 Chimonantheae.

180 Chimophila. Prsh. Ratl.

181) Chionantluss. $L$.

180 Chironia. L.

18?) Chironieae.

18 i Chisocheton. $B l$.

2:30 Chitunia, Moz,Sess,

198 Chlenacenc.

136 Chlora. $L$.

136 Chlorantheae.

2(i) Cloridleae.

269 Chlorophytá.

213 Chloroxylon, $D_{\mathrm{C}} C$.

254 Chorcorus. $D_{e} C$.

298 Chorilaena. Endl.

gyslChorisia. Kunth.
S. 282 Chorisına. Lindl.

238 Clurisura $D$ C.

201 Choulant.

276 Cluristiania, Prsl. 27 t

276 Chrysangia. Lk. 186

30.3 Chryseis, Lindl. 264

227 Chrysema. 213

230 Chrysitricheac. 147

162 Clirysobalanease. 254

162. Chrysohotrya. Spach. 233

206 Chrysopia. A. P. Th. 311

30s Chrysopiene. 311

2t' Chrysophylleae. 214

24 Chrysopliyllun. L. 214

234 Chrysusplenieae. 230

234 Chrysosplenium. L. 230

230 Cianitis. Reinu. 230

1 Cicendia. Adans. 211

201 Cichoriaceac. 182

21.) Cicntil, $L$. 219

272 Cipadessa. Bl. 313

235 Cimicifuga $\boldsymbol{L}$. 277

224 Coptis, Salisb. $\quad 277$

189 Cinchona. 180

253 Cinchoneat. 180

253 Circaea. $L$. $\quad 246$

206 Circaeene. 246

246 Cissene. 221

189 Cissus. L. 221

1 (t) Cisteae. 272

260 - genuinae. $\quad 272$

11. '262 Cistineae. $2 \%$

$2-66$ Cistus. T. L. 272

162 Cistusgewächse. $\quad 270$

161 Citrene $\quad 314$

188 Citrus, L. 314

260 Cladoniaceae. 132

260 Cladostepheac. 136

277 Claoxylon. A. Juss. $\quad 281$

295 Clappertonia. Meisn. 303

295 Clarisia. Abat. 240

264 Clarkia. Prsh. $\quad 240$

264 Clansena. Burm. $\quad 314$

198 Clanseneae. 314

238 Claytonia. L. 237

235 Clematideae. $\quad 276$

238 Clematis. L. $L$

298 Cleome. $L$. 267

264 Cleomeac. 266

291 - genuinae. 267

313 Cleomella. DeC. 267

190 Cleonia. $L . \quad 189$

174 Clermontia. Gaudich。 186

206 Clethra. L. 206

213 Cleyera. Thnb. 302

211 Cliffortia. $\boldsymbol{L}$. 243

210 Cliffortieae. 243

313 Clintonia. Dougl. 186

285 Clintonieae. 186

308 Clusia. L. 311

211 Clusiariae. $\quad 310$

167 Clusieae.

146 - genuinae. 311

135 Cluytia. 282

313 Cluytieae. 281

282 Clypeola. L. .. 259

298 Clypeoleae. $\quad 259$ 
Cnemidostachys, Mart. S. 281 Coprosmeae.

Cneoreae. 282 Corchoreae.

Cneorum. $L$.

Cnidium. Cuss.

Cnidoscolus. Pohl.

Cobaea. Cav.

Cocoinae.

Coccoloba $L$.

Coccolobene.

Coceyganthe. Rchb.

Cochilearia. $L$.

Cochlidiosperma, Rchb.

Cochlospermun, $K, H, B$.

Codia. Forst.

Codiaeum. $R m p h$.

Codon. $L$.

Codonopsis. $D_{c} C$.

Coelanthum. Fnzl.

Coelocline, $A, D e C$.

Coffeariae.

Coffeineae.

Coffea.

Cola. Schott, Endl.

Colbertia. Salisb.

Colchiceae.

Colebrookia, $R x b$.

Coleoilles.

Coleophyta.

Coleus. Lour.

Colla.

Colladonia. DeC.

Collemacene.

Collinsia. Nutt.

Collinsonia $L$.

Collomia. Nutt.

Colona. Cav.

Colquhounia. Wall.

Columbia. P.

Columelleas.

Colmmellia. Rz. Pav.

Combe.

Combreteae.

- genuinae.

Combretum, Löffl.

Commelinaceae.

Commelineae.

Commia. Lour.

Compositae.

Conceveibum, Rich.

Condylocarpus. Hoffm.

Confervaceae.

Conferreae.

Coniferae.

Coniocarpicae.

Coniocybeae.

Conioselinum. Fisch.

Conium. $L$.

Connareae.

Conolıoria. Aubl.

Conoliorieac.

Conoimorpha Oth.

Conopodium $K$.

Contortae.

Convallarieae.

Convolvulaceae.

Convolvuleae.

Cookia, Sonn.

Coprosma.

220 Corda.

158 Cordiene.

236 Cordiera.

156 Coriariene.

236 Corideae.

150 Coris. $L$.

291 Corneac.

152 Coronariae.

189 Coronillene,

198 Cortusa $L$.

190 Cortusene.

194 Corydaleae.

148 toca.

209 Croton. L.

153 Crotoneae.
28'2 Corchorus, $L$.

$19+$ Cordia. L. 193 Cryptocarpus. K. H. B. 239

236 Cordylocarpus. $D_{s f}$.

298 Corema. Don.

260 Coreosma. Spach.

198 Coreta. P. Br.

302 Coretioitlcs. DeC.

230 Coriandreae.

281 Coriandrum. I.

194 Coriaria. Nissol.

27s Coridium. Spach.

180 Corispermum. $\boldsymbol{L}$.

180 Corniculatae.

278 Cornus, T. L.

188 Corone. IIffgg.

141 Coronopus. 'T.

189 Correa de Serra.

253 Correa. Sm.

219 Corrigiola. $L$.

133 Cortia. DeC.

303 Cory dalis. Vent.

188 Corymbiene.

303 Corynandra. Schrad, Rchb

213 Corynandreae.

213 Corynostyleae.

5 Coryuostylis, Mart.

247 Coryphieae.

247 Corypliariae.

247 Cosimanthus Nolte, nach $\mathbf{E u}$ -

148 Cossignia Commers.

281 Courtoisia. Rchb.

182 Contoubea. Aubl.

281 Crambe, 'T'. $L$.

220 Craniotome. Rchb.

135 Crantzia. Nutt.

135 Crataeva. $L$.

168 Cratericarpun. Spaeh.

131 Craw fortia. Wall.

132 Cremolobus. $D_{e} C$.

220 Crescentia, $L$.

2 i9 Cristaria. Cav.

224 Crithmum, T.L.

269 Crossandra. Sulisb.

269 Crossophyllum. Spach.

298 Crossostigma. Spach.

219 Crossostylis, Forst.

194 Crotonopsis. Mchx.

194 Crowea. Sm.

314 Crozophora. Ncck,

180/Crozophoreae.
193 Cryptophragminm. N.v.E. 190

180 Cryptostegia. R.Br. 208

259 Cryptostegieae. 208

28:2 Cryptotaenia. DeC. 209

233 Cucubalus. L. 298

303 Cncurbitacene. 181

303 Cucurbiteae. 184

218 Cunila, L. 188

283 Cunonia. $\boldsymbol{L}_{\text {. }} 230$

283 Cunoniaceae. 230

204 Cupania. Plum. 285

308 Cupanieae. 25 .

204 Cupressinae, 165

239 Curatella. $\boldsymbol{L}_{*}$.

228 Cuscuta. L. 239

221 Cuscuteae. 239

221 Cusparieae. 282

153 Cussonia. Thnb. 221

298 Cuvier, 26

226 Cyanea. Gaudich. $186^{\circ}$

202 Cyanocephalus. 189

50 Cyathoideae. 139

283 Cybianthus, Mart. 204

237 Cycarleacene. 139

220 Cycadeae. 139

204 Cyclamen, $L$. 204

204 Cyclantheae. 158

264 Cyelolepis, Moq. Tand. 238

$26+$ Cyclospermum, LaG, 219

18 "2 Cyclostegia. 188

267 Cylactis. Rafin. 243

267 Cymaria. Benth. 189

269 Cyminosma. Giört. 282

269 Cymopteris, Rafin. 220

158 Cynancheae. 208

158 - genuinac. 208

\begin{tabular}{ll} 
Cynareae, & \\
193 & 182 \\
\hline &
\end{tabular}

285 Cynocrambeae. 243

194 Cynoglosseae. 192

21 Cynoglossum, L. 193

259 Cynomorieae. 164

188 Cynosbata. DeC. 290

220 Cynosciadium, $D_{e} C$. 220

267 Cypereae. 147

246 Cypergräser 147

211 Cyperineac. 147

249 Cyperoidene, 147

199 Cypripeditae. 155

287 Cypselea. 'Turp. 237

220 Cyrta. 189

190 Cyrtanilra Forst. 199

308 Cyrtandreac. 199

246 Cysticapnos, Boerh. $26 \pm$

253 Cytineae, 164

281 Cytineen. $16 t$

281 Cytinus. $16 t$

281

282 Dacrydium. 166

281 Dactylaena. Schrad. 267

281 Dactylicapnos. Wall, 264
218 Cuminuin. C. Bauh, L. 219 
Dactylophyllum.

Dalbergicae.

Daleclınıpia. Plum.

Dantia. A. P.' Th.

Daplineae.

Darwinieae,

Dasyanthera. Prsl.

Dasyloma. $D e C$.

Dasyuema, Schott.

Datisca. $L$.

Datisceac.

Datura. $L$.

Datureae.

Dancineae.

Daucus. $L$.

Davilla, I and.

Deca isne. $\quad$ Y3 Dimetopia. DeC. Dimocarpus. Lour 45. 50.53.6\%.63. 72.7\%.84. Dionaea. Ellis. 85. 67. 30.91. 140.145. 180. Winscoreae. 155.196.218.219.272 Winscorinea.

1) e Candolle, $1 . \quad 43$ biosma. $L$.

Decasthista. Whigh. et Diosmear. Arn. 298 - genuinase

Defforgia. Lam. 233 Diospyreae.

Dejanira. Cham. Schlecht, 211 loiospyros, L.

Deilosma. Andrz. 260 Hiotis. Schreb.

Delima. $L$.

Delimeac.

Delissea. Goudich.

Delissenae.

Delphinieac.

Delphinimm. $\boldsymbol{L}$.

Dendrouscen, Benth.

Denhamia, Meisn.

Dentaria. T.L.

Dentellarinae.

277 Diphylleia, Mchx.

277 Diploliena, $R \cdot B r$.

186 Diplopeltis. Fndl.

186 Diplophraetum. $D s f$.

27i l)iplotacteac.

27 Diplotaxis, DeC.

$26+$ Diposis. DeC.

267 Dipuriliunı. Burt.

260 Dipsaceac.

203 Dipteracanthus.

Desfon tia ines. 9. 50.287 Dipterocarpeae.

Desfontainia. Rz. Pav. 201 Dipterocarpus. Gürt.

Desrousscaux. FiDiptercome. Fisch, et

Detarieas.

Detharding.

Dentzia. 'Thab.

Deverra. De $C$.

Diamorplı. Nutt. 228 Mey.

11 Dirca.

230 Jisandra. $L$.

220 Dischillum. Ging.

230 Dischimia. Chois.

Diamoste, Cham.Schlecht. 199 Discocapnos. Cham.

Diantlieae. - gelluinae.

Dianthus, $L$.

Diapensia. $L$.

Diascia. $L k$,

Dicera. Forst.

Diceranilra. Bnth.

Diceratium, DeC.

Diccras, Pers.

Diclooglotis, Fisch, C.A. Mey.

Dichroma. Cav.

Dicliptera. Juss,

Dicliptercae.

Diconangia. Mitch.

Dictannns, $L$.

Dietyantha, Rafin.

Didesmus. Dsv,

Didiscus $D_{e} C$.

Didymandra. $\boldsymbol{~} \boldsymbol{}$.

Didymocalpus, Jack.

Didy moche ton. Bl.
298 Uiscopleura. DeC.

298 Disoxylon. Bl.

206 Ditaxis. I ahl.

198 Ditoca. Bnks et Gärtn.

304 Dolnowskya. Prsl.

189 Dodartia. $L$.

260 Dudecatheon. $\boldsymbol{L}$

199 l) o e be 1.

Doslonaceac.

298 l)oering.

198 Doldengew ïchse.

190 Doliocarpeae.

190 Doliocarpus. Rol.

233 Dolophrama. Fensl.

282 Jombeya. Cav.

173 Jombeyeac.

2590011.

221 Donatia. Forst.

172 Dondia. Spr.

199 Dontostemon. C. $\boldsymbol{A}$. $M$

313 Dopatrium. Hamilt.
298 Distelkarden.
S. 264 Dorema. Don. S. 220

264 Dorstenieac. 172

11.94 Drabis. $L$.

201 Urabeae. 259

233 Dracacneae. 157

11 Dracuneulinae. $1+2$

198 Urehblïthler. 209

198 Drosinthe. Spaek. 307

283 Wrosantheae. 307

1 Drosera, $L$. 271

278 Drosereac. 271

277 Drosophyllum, Lh. 271

278 Mrosocarpium. Spach. 308

190 Drummondia. DeC. 230

290 Drusa. DeC. 221

285 Irymaria, $\boldsymbol{W}$. 236

220 Drymis. Forst. 278

$2 \Varangle 5$ Diymosphace. 189

272 Dryobalanops. Gärt. $30 \pm$

153 Drypetes. I'ah!. 28?

152 Drypeae. $295^{\circ}$

282 Drypis. Ger. 298

282 1) u p on cl.

282 Dugnetia. St.Hil. 278

$21+$ Dulichieae. 147

214 Dunalia. K.H.B. 201

238 U u ri o. L. 295

265 Dw y g oub s k y.

202 Dyschoriste. N.v.F. 190

285 Dysophylla, Blume. 188

303 Ebenaceae. 214

260 Ebermaiera. N.v.E. 190

260 Echieac. 19:

221 Echiopsis. Rchb. 19:-

283 Echinacanthus. 190

17i Echinocactus. Salm.Dyer. 233

190 Echinocarpus. Bl. 27t

$30+$ Echinopeac. 182

304 Echinophora. T. L. 219

Echinospermum. Su. 193

180 Echinosphace. 169

170 Echinosphaera. Sieb. 281

198/ Ficliteae. 211

269 [F ckermann.

196 E l r en berg. 11.21.36. 65.

264 . 11 .

219 Ehrenbergia. 285

313 Ehretia. L. 193

177 Eiben. 165

281 Eichwa I ते.

235 Einomeia. 173

186 Eis engrein. 11. 16.40.41

199 Ekebergia. Sparim. 313

204 Eklon. 11

11 Elaeagneae. 169

285 Elaeocarpeae. 303

11 - grenainae. 304

217 Elacocarpus. $L$. $30 t$

27 Elaeococca. Commers. 281

277 Elaeorleudron. $J c q$. 301

298 Elaeoselinum, Koch. 219

291 Elatine. L. 248

291 Elatineae. 245

11 Eleplantopeae. 182

230 Eliaea, Camb. 308

221 Ellisia, $L . \quad 193$

260 Ellisiene. 193

199 Elunigera, Rchb. 198 
Elodea. Adans.

Elodeinae.

Elodes. Spach.

EI 8 hol t 7 .

Elsholtzia $W$.

Flvasia.

Elrasieae.

Elythracanthus. N. $v \cdot F$.

Elytraria. Iahl.

Elitrarieae.

Einbelia. Burm.

Emloryouteris. Gärt.

Enex. Neck.

Eimpedoclea. St.Hil.

Fimperloclea.

Empetreie.

- genuinae.

Eimpetrum. $L$.

Enartinrocarpus. Lab.

Encyauthus. Lour.

End I i che r. 11.24.56.60.6 69.89 .90 .

Hndodeca. Rafin.

Endopogon. N.v.F.

Enemion. Rafin.

Lingelinan n.

Entophyllocarpica.

Epacrea.e.

Epacrideae.

Ephedra.

Epliedreae.

Fiphielis. Schreb.

Epicharis. $13 l$.

Epidendreate.

Epigaen. $L$.

Epifobieate.

Epilobiam. $L$.

Epimediene.

Epimedium. L.

Epipacteac.

Tipiphyllum. Herm.

Equisetaceae.

Equisetum.

Erantliewsin. R. Rr.

Erantlis. Salisb.

Eremantice. Spach.

Eremocarpus. spach.

Eremogone. Fisch. C. A.M.

Eremophila. R. Br.

Eremostacliys. Bnth.

Erianthera. N.v.E.

Erica.

Ericacene.

Ericariac.

Ericeae.

Erigenia. Nutt.

Lriuns. $L$.

Erioeauleae.

Eriodentreae.

Eriodeudron. DeC.

Erioglossum. Bl.

Eriogoneat.

Eriogynia. IIook.

Erione. Schott, Endl.

Eriope. Benth.

Eriophyton. Bnth.
S. 308| Friosphaeria.

308 Eriostachys.

308 Eriostemon. Sm. .

264 Eriosynaphe. DeC.

188 Eriotheca. Schoti. Endl.

283 Eriothymus. Bnth.

283 Friotis. Dec. et Lib.

190 Erubatos. DeC.

190 Erodiene.

190 Eródinu.

$20 \frac{4}{4}$ Erophila. DeC.

215 Erpetion. DeC. Sweet.

236 Ernea. 'T'.

27 Erucistrum. Jahb.

188 Eryngimm. $T$. $L$.

282 Erysinea.

282 Lrysimnm. $L$.

282 Erythriles. Renealm. P.

259 Erythraeariae.

206 Erythrochiton. $N$. et $M$.

S. 189| Exostemma.

108 Eysen hard t.

S. 180

282

220 Fabaceae. 226

295 Faluricia. Gärtn. 253

je() Eallemalgen. 135

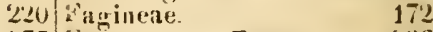

277 a croverum. P. 236

290 Fagraea. Thab. 211

290 Falcaria. Riv. 219

259 Farsetia. 'Turr. 259

$269)$ iaserpilze. 130

200 ferlia. Mnch. $17 x$

260) Fee.

2 2 Fentzl. 11. 75

260 Fentzlia. Benth. $19 \frac{4}{2}$

260 Feronia. Corr. 314

$21 \mathrm{i}$ ferrarieae. 1511

'210 Ferula. ' $T$. $L$. $2: 0$

$282 \mid$ Ferulago. $K . \quad 220$

291 Ferriss a c.

27.3 Festucaceae. $1+6$

273 Festuceae. 146

298 Ficaria. Dill. 276

290 licarieae. $2 \% 6$

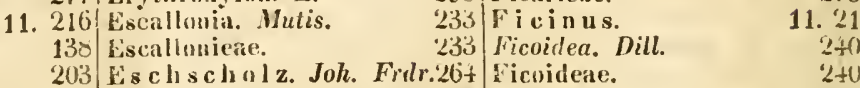

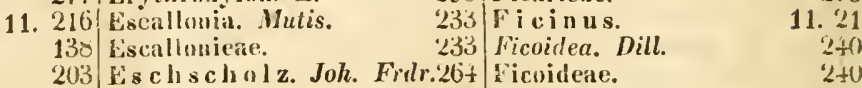

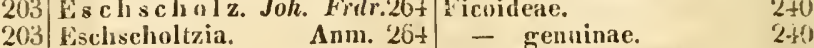

166 Eschweiler. 11.117 Fimbriaria. St. Hil. 299

166 Escoliedia. Rz. Pav. 19S Fimbristyleae. 147

285 Esminchia. Rchb. 295 $\mathrm{F}$ ingerhut. 11

313 Esperi. " ${ }^{\prime} . \quad 303$ Firuiana. Marsigl. 291

155 Fubasis. Salisb. 221 Fischer. 18. 236

206 Lucapnos. Brnh. 264 F itzinger. 30

246 Euchatis. Bartl. 282 Flacourtia. Commers, l'Ierit.

246 Eucharidium, Fisch, C. A.M. 267

2630 246 Flacourtianeae. $26 \%$

\begin{tabular}{l|ll|ll}
265 & Enchysia. Prsl. & 186 & Flacourtieae. & 207 \\
155 & Enclidicae. & 158 & Flummula. Rùpp. & 277
\end{tabular}

234 Luclidium. R. Br. 250 Flechten. 131

165 Eudema. H.B. 260 Fle is cher. 11

165 Ludesmia. 253 Fliudersia. $\mathrm{K} . \mathrm{Br}$. 313

190 Engenia. Mich. 253 Floerkea. $\boldsymbol{H}^{\text {. }} 290$

277 Lugenicase. 253 Florideae. 135

308 Eugilia. 194 Flügrea. $\boldsymbol{W}$. 282

307 Eunomia. DeC. 259 Foeniculum. Alans. 220

235 Forestiera. Poir. 170

283 Forgesia. Commers. 233

301 Forsythia. Iahl. 213

301 Fotliergilla. L. fil. 242

281 Fothergilleat. 2\%2

280 Foveoliaria. DeC. 303

280 Fonquiera. $K .71, \boldsymbol{l}, 232$

285 Fouquicreac. 232

302 Fragosil. Rz. Pav. 221

188 Franciscea. Pohl. 199

180 Franguliacene. 223

189 Frankenia. I. 272

193 Frankenicae. 272

260 Frankoa. Cav. 230

248 Frasera. Walt. 211

211 Fraxinus. $L$. 213

210 Fresenius. 11

192 Freyera. Rchb. 291. Aniu.

219 Freylinia. Colla. $\quad 199$

167 Ereziera. Sw. 302 
Fries, 10.11: 15. 16. j1. 53.| Gendarussa, Rumph:

Fricsia. $D c C$.

Friesiear.

Fitzelle.

Frostia.

Inchsia. $\boldsymbol{L}$.

Fucineac.

Fucoi'eac.

Fuernrolir.

Fugosia. Juss.

Fingosicar.

Fuireneac.

Fumaria. $L$

Funaricae.

- genuinac.

Ga er tner.

Gaertnera. Itam.

Gaissenia. Rafin.

Galacineac.

Galax. L

Galbanum.

Galearia. Presl.

Galeneac.

Galcobdolon.

Galenpsis. $L$.

Galerienlaria.

Gallertaleren.

Gallertfleciten.

Galopina.

Galphimia. Cav.

Galvesia. Juss.

Gaujiz. $R m p h$.

Ganitns. (ï̈)t.

Ganzblumiere.

Garcia. Rohr.

Garcinia. L.

Garcinieae.

Gardenia.

Garleniene.

Gardognia, Rz. Puv.

Garidella. $L$.

Gasterothalami.

Gastonia Commers.

Gandin.

G a ud i c ha a d.

Gaudichandia K. II. B.

Gaulthiera. Kalm.

Gaura. L.

Ganridium. Spach.

Gil $w$ le $r$.

(i a y.

Groya. Gaud.

Girya. Kuth.

Giaya. sipr.

Gaylussacia. $K, J I, B$.

Gaylussaciene.

Gajopliytum. A. Juss.

Gialipera. Alubl.

Giurdneria. Hull.

Gelilera. Fisch. C. A. M.

Geeria. $\boldsymbol{B l}$.

Geliler.

Geliörutfrüchtige

Geisblattgewärhse.

Gelenkfruchtilgen.

felonium. Rxb. 7j. 85. 117 Gencts llis. De C.

304 Geniospormm. Hall.

304 Geniostoma. Forst.

11. 62. 12t Genisteac.

164 - genuinae.

$2+6$ Gentiana. $\boldsymbol{L}$.

136 Gentianeae.

136 - genninac.

11. $28.9+$ Geusalycene. 288 Geocluorda Cham 18 (j) Grammeionium. Rchb.

cht. Granmostiadium. $D_{c} C, 219$

$1+i$ Geofroyeac.

'26t Ger' un il $r$.

26 Gernniaceac.

$26 t$ Geranieac.

$1: 29$ - geuninac.

1. 32. 41.82 Geranimu $L$.

211 Gerarlia. $L$.

277 Germancu.

230 Germua. Forsk.

230 Germmeae.

220 Gesnereae.

226 Gilia. Cav.

226 Gilicae.

18s Gilibertia. Rz. Pav.

186 Gilliesia.

189 Gisckia. L.

135 Gisekiene.

133 Gialıago. Def.

180. Gladiniene

299 Glancicae.

198 Glaucium. $T$.

303 Glanx. $L$

$30+$ Gleclion. Spr.

17. Alcichenieac.

281 Glinus. I.

311 Globularia. $L$.

311 Glubulariaceae.

180 Globuliariaceen.

180 Giobularicae.

189 Glocekler.

27 frlossarilien. Mart.

13:. Glossoma. Schreb.

221 Glossostylis. Cham, et Schlecht. Guttiferae.

lei Glossula.

235) Blycineae.

206 Crlycosmis. Corr.

$2+6$ Gnaphaliene.

$2+6$ Godetia. Spach.

Fli Getlic. 5. 7.

S. 19) Gomaniacear.

25: Grofficill. Rob. Cast.

S. 222

18!i Grithors ky.

298

11. $81 \mathrm{i}$

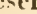

g(i) Grifia. Rclub.

220 Gir. Goluka. (IIacr.) 2I!

211 Grahamia. Gill. Ilook. 237

210 Gramilieae. 14.5

210. Grammatothcea. Presl. 186

198 Granateac.

208 Granadilleac. 208

21. 7() Graphithalami, 133

288 Graptoplyyllum, N.v, E. 190

$2 y 0$ Gratiola. $L$.

2!30 Gratiolene. 199

290 Grewia. Juss,

1!) Griesselich.

184 Grielum.

31: G i is eb a ch. 11. 210. 212

313 Griselinia, Forst. 281

$199 \mathrm{Groh}$ h. .

134 Gronovia. $L$.

194 Gronoviene.

por Grossularia $T$

151 Grossularicac.

239) Grïnflauzen. 13.5

239 Guajaceae. 285

298 Guarea. $\boldsymbol{L}$. 313

15) Guareac. 313

$20+$ Guatteria, RE. Puv. 278

$2(i t)$ Guattericae. 278

$20+$ Guazumoides. $D c C$. 303

18!) Guettaria. 180

1:3) Guctardeae. 180

23i Guichenotia. Gay. 292

195 Guidonia. Plum, 278

195 G u illemin. 93

195 Guilleminia, $K . M . B . \quad 233$

195 Guilleminia, Neck, 221

18.5 Guthuik. 11

z(i?) Gunnerene. 170

Gol Gutagewäclise.

196 Gymnanthus. Sw.

183 Gymnogrammeac. 13:)

¿206 Gymnaudia. P'all. 1998

31 Gymnanthera, $R, B r . \quad 308$

18:- Gymneiu. 18!)

2t6 Gymmocarpum. Forsk. 2005

80. 30 43 45. 28 gymnocylix.

20 Goetliea J, v, E. 287 Gymanlropsis. De C

28;G Geppert. 11. 55 Gijnostoma. DcC.

29:- Gol d ha e h.

206 Goldhachia. DeC.

206 Goldbachiene.

246 Guldenssia. N. $v$ F.

283 Gomphia. Schreb.

210 Gompliostegia. Bnth.

281 Goniocarpus. Koen.

302 Goniochiton. $\boldsymbol{B l}$.

20 Gonolobeae.

228 Goudenicae.

178 rordonia. Ellis.

130 Gossampinus. Hamilt.

281 Gossypium. L.
18 Gyotrocheae. 311

25!) Gynotroches. Bl. 311

190 Gymnostachym. N.v. F. 198

283 Gyrostemou. Dsf. 28\%. Anin.

18!) Gyrothalami. 133

$2+4$

318 II a berle.

11

186 II accquetia. Neck. 21

30\% IInemadoreate. 151

2!)5 Hacmocharis. Sulisb.

2or Haruca. I ent.
259 Gypsophila. $L, \quad 0298$

205 IIalmelia. A. DeC. 278 
Halenia. Brkb.

Iralimolobos. 'Tseh.

Ialimolobos. 'Tseh. 260 Hemiphragma. Wallr. v. II all.

II aller.

IIalleria. $\boldsymbol{L}_{\text {. }}$

Halimocnemis. C. A.M.

Halocnemum. M. $B$.

IIalogeton. C. A. M.

Halorageae.

IIalorageen.

Haloragis. Forst.

IIamadryas. Commers.

IIamelia.

Iamamelideac

II a m p e.

Haplanthus.

Haplophyllum. A. Jass.

H a r k a rt.

Harmalum. $T$.

Haronga. A.P.Th.

Harris on.

Histumannia. Spach.

Hartheugewäclise.

Hartigisea. A. Juss.

llasselquistia. $\boldsymbol{L}$.

Havetia. K. II. L.

Haveticae.

II a y e.

Hebenstreitia. Chois.

llecaterium. $K \approx$.

IItera. $\boldsymbol{L}$.

llederaceae.

Hedeoma. Pers.

H e d wig, Joh.

Il ed wig, Roman.

Hedyotideac

Hedyotis.

Hedysareae.

liegetseliweiler.

Heidengewächse.

Heinroth.

Helia. Mart.

Heliantheae.

Heliantlemum. $\boldsymbol{T}$.

Helictereac.

II licteres.

Helicteroides. DeC.

Heliocarpus. $L$.

Heliophila. $L$.

Heliosperma. Rchb.

Heliotropiceae.

Heliotropium. $\boldsymbol{L}$.

Helleborastra.

Helleborene.

- genuinae.

Helleborns. $I$.

Helleria. $N, v . F$.

Heloscialium. Ḱoch.

IIelosiene.

Hemerocalleae.

Hemerocallideae.

Hemiadelphis. N.v. E.

Hemiandra. $\mathrm{R}, \mathrm{Br}$.

Iemichorista. N. v. F

Hemidesmeae.

Hemidesinus. R. Br.

Hemigenia. IR. Mr.
S. 211 Hemimeris. $\boldsymbol{L}$.

Hemisphace.

II II mistemma. Juss,

72. Hemistemoneac.

199 Hemistoma.

239) IIendecandra. Eschsch

238 Henkelia. Spr.

¿39) Hen schel.

244 Hensehelia. Prsl.

244 Hepatica. Dill.

244 IIeraclemu. $L$.

276 Herbiel.

180 $1 \mathrm{e} \mathrm{er}$ er.

176 IItritiera.

zu 11 Ilemannia. I

190 Hermannieac.

2S: Ilermas, $L$

11 llermesiu. Bpl.

282 Hermupoa. Löffl.

(6) Ilerniarieae.

246 Herpestes. Giartn.

304 IIerschellia. Boud.

313 Ilesiodlea. Mnch.

200 II esperidere.

310 II esperinae.

310 Hesperis, $L$.

11. 89. 117 Ilesperothymus.

19(i) Ileteranthesia.

281 Iletrranthia. N. v. F,

20I Heterochroa, Bunge.

22I Ileterocylix.

189 Heteromelissa.

ni) IIeteromorpla. Cham. Schle

1!)

180 lleteropteris. H. K. B.

180 Heteroptycha. DeC.

226 Heterosphace.

11 II enclicra. $L$,

20.5 I enchereae.

20 Hexacentris. N.v. F,

211 Hexalobus. A. DeC

182 Hexipteril. Hook.

272 Ileynea. Rxb.

294 Ileynecie.

$29 t$ II ey n h old.

232 Hilbertia. Andr.

303 Ilibbertiene.

260 - genuinac.

298 Ilibiscene.

193 Hibiseus. $L$.

278 Hildegardia. Schott Endl.

277 IIIlegarlieac.

278 Hillousc.

277 I ind en bur g.

3It Hippocastaneac.

219 Ilippocraten. $L$

$16+$ Hippocrateae.

154 - genuinae

154 Hippomane. $L$.

190 Hippomaneac.

190 Hippuridene.

190 Hippuris. $L$.

208 Hiptage. Gürt.

208 Iliptageae.

18!) II imeil. K, II I3.
308 IIerniaria. $T \cdot L$.

193 II i l a i re, Geoffr. St.
S. 198 Hisingerı. Hcll.

S. 281

199 Iladnikia. $K_{\text {. }}$ - 21

277 Hoaren. Sweet.

277 H of $\mathrm{fmann}$.

188 v. Hof $\mathrm{m}$ an nse gge. 11.77

281 Hohenackeria. Fisch, et C.d..V.

199

(i5 Hoitzia. Juss. 19t

278 H o 11 . 11. 89

286 Holmskioldia. Retz. 190

220 Hololacline. Ehrnb. 240

11 Holosepalum. Spach. 308

5 Holosteum, $L$,

291 Holostiguna. Spach. 248

291 Homalanthus, Juss, 281

291 Iromalinene. 257

219) IIomalophylleae. 18;

281 Homoianthac. 18:

267 labiatill. 18를

235 ligulifl. 18'2

235 tubulifl. 182

199 Honekenya. Fihh. gus

201 Honckcnya. IF. 303

189 $1100 \mathrm{k}$ e r. 12. 54, 62. 82. 141

312 Hopea. $\mathbf{R} x$. $20+$

2(i0) H ор е. 10. 11. 31. !)

260 Horminum. L. 18!)

189 Horminum. ' $I$ '. 1843

18!) Hornem ann.

199 Hornemannia. $W$. 199

298 Horns u e h. 11. 52

189 H 0 rn $n$ n $\mathrm{r}$.

189 IIorsfieldia. $B l$. 2021

18!

180

299) Huanaca. Cav. . 221

219 Hudsonia. $L$.

230 II u e buer.

2029) Miigeli(t. Benth. 191

1!)1 IÏ̈gelia. $\tilde{A} c \hat{b} b$,

278 Hugonia. L.

259 Hugueninia. Rchb. 2fio

313 Hultenia. Dunort. 24:

313 v. H u mbold t. 11. 53. 9l

11 Humiria, Aubl. 31

278 Hunnemannia. Sweet. 264

277 Hunteria. Roxb. 21

278 Hura $L$. 231

287 Intlinge. $1: 0$

287 Hutchinsia. R. Br. עอ!!

29) Iyaenancluc. Lamb. 282

291 Hyala. l'Herit.

291 Hyalostemma. Wall,

91 II yantheae.

20 If banthus. Jacq. 2(;)

285 Hydnoearpus. Giüt. 267

301 Hydnoreac.

301 Hylrangea. $L$. 2331

301 Hydranceac.

281 Hydrastis. $L$. ע77

281 Hydrocera. $B l$. 29.

244 Hydrochareac. 144

$24 t$ Hydrocharidene. 144

29)! IIydroeotyle. T. L.

2!jy Hydrocotyleac.

29!) II ydrolea. I. 
Ifyilrolceac.

Hydrnphylleae.

IIydrophyllım. $\boldsymbol{L}$.

Hygropluila. N.v. E.

IIy grophileae.

llymenanthera. B $3 k s$.

llymenanthereao.

Hymenini.

Ilymenocal yeeap.

Ilymenucalyx. Zenk.

11 ynemocrater. Fisch. et $C$. 1. . Mey.

Hymenolaena. $D_{\varepsilon} C$.

Ilymenoplyylleae.

1 ymenopliysa. C. A. M.

Ilymenosphace.

Ilyoscyameae.

Ilyoseyamus. $L$.

Hypecoincac.

Hypeenum, $L$.

liypelate. R, Br.

Mypenia. Buth.

llypericincae.

Ilypericeae.

- genuinae.

Hypericum. $L$.

Ilypertelis. $\boldsymbol{F} . M_{c y}$.

IIypoëstes. Sol.

IIypoxideac.

liypscla. Piesh.

Ilyptis. Icq.

llyssopus. L.

Jaborosa. Juss.

Jacea. Commers.

v. Jacquin.

Jacquinia. $L$.

Jaç̨uinieae.

Jaeger.

Jambolilera. $\boldsymbol{L}$.

Jamlosa. Hmph.

Janipha. TK.II. B,

Jasione. L.

Jasmineae.

Jasminum, T. $L$,

Jatroplia. $\boldsymbol{L}$.

Iberidene.

Iberis, $L$.

Jeffer'sonia. Bart.

Jenkinsonia. Sweet.

Jiraseckia. Schm。

Jlicineae.

lllecebreae.

Illecebrum. $\boldsymbol{L}$.

Illicieac.

- genuinae.

Illicium. $L$.

Imbricaria.. Commers.

Impaticus. $L$.

Imperatoria. $\boldsymbol{L}$.

Ingenhousia. Moc. Sess.

Inocarpus. Forst.

Joannesia. Felloz.

Johnia. Rxb.

Jolirenia. $D_{\subset} C$.

Jnnidieae.

Jonidium. Vent.
S. $194 \mid$ Jenopsilium. $D_{c} C$.

193 Josephinia. Vent.

193 Jussinia. Commers.

190 Jovellana. Cav.

1901 Ipon:opsis.

(6i) Irilcáe.

$2(i)$ lrina. Blo

130 Irlbachia. Vart.

287 loucana. Anbl.

287 Isanthera. N.v.E.

satideac.

21!) Isatis. $L$.

139 Isertia.

250) 3- Isuardia. L.

18! Isoeteac.

201 Isoctes.

201 Isopetalum. Swect.

Efit Isophyllum. Spach.

2 (it) Isuplexis. Lindl.

285 Isopyrease.

18!? Isopyrum, $L$.

304 Isora. Rheed.

305 Isoreac.

308 Isotoma. $R, B r$

307 Itea. $I$.

2ast Inglanieac.

190 Juncaceae.

151 Juncaria. Clus.

186i. Junceae.

189) Juncus, $L$.

189) $\mathrm{J} u \mathrm{ng}$.

Jungermanniaceae.

201 Jungermanniaceen.

2(i) Jungemanniaeae.

11.31 Jus $i$ un 4 (日.

10. Kre ys i g.

S. 259 Khayeae.

S. 313

198 Kibera. Adans. 260

2.53 lielweyera, .llart.

1!3 Ki eser. 43. 117

$19+$ riiggrelaria. $L$.

14!) liiggelariene. $261 \%$

285 lifischleger.

211 Kirschlegeria. Spack. 24t;

273 Ii irtland.

1!9) Kitatil,clia. $W$.

188 Ii itaibelicae.

258 li t tel.

30)

287

11.64 .186

258 Ikittelia. Rchb. 111.01. $186^{\circ}$

180 Kílaprothia. K.II.B.

$2+6$ lileinhovia. $L$. 291

189) Ḱleinhovieac.

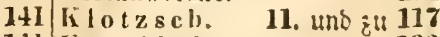

$1+1$ Kinauclllechten. 13:

290 línciflia. spach. 240

308 Kinoufferhten. " 132

198 liunvitonia. Salisb. 20̈ti

278 Knowltonieac.

277 k o c h. 11.33.35.38.80, 65, 81.

294 85. 88

2!)4 Kochia, Rth. 2:3!

186 liochicae.

233 Koelbing.

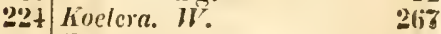

151 Koenigia. $L$.

235. Kolbea.

152. Kinlbenschosser. ' $16 \%$

752 Koniga. R.Br.

1 Kosteletzky. 11.211

136i Kosteletzkya. Presl. $28 \mathrm{~g}$

138 Kramericae.

1330 Krapfia. $D \in C$.

204 123.180.104.25i Kronlilicn.

204 Jussieu, Adr. 68 Krubera. Hoffm. 220)

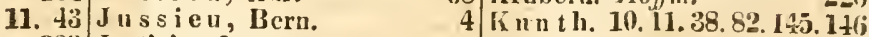

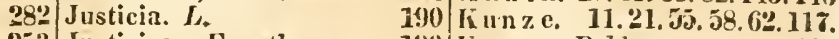

253 Justicieae Eranthemeae 190 Kunzea. Rch̉b. S.

281 - Gendarussae. 190 Kürbisgewächse. $19 \pm$

186 - Rnelliariae. 190 K ït $\mathrm{z}$ ing.

213 Jussicua. Houst. 281 Kundmannia. Scop. 2010

214 Jussicua. $L$.

281 Jussieucae.

259) Ixieac.

25!)

265.) Kützchenblüthler.

290 Kallstroemia. Scop.

207 Kalmia. L.

214 Kamettia. Kastls.

235 Ka.nt.

235 Kapperngewächsc.

258 Kasthofer.

278 liaulf uss.

278 Kayca. Wall.

215 Keithia. Benth.

294 Kelchblithige.

220 Kelchflechten.

287 K er.

215 Kezaudrenia. Gay.

281 Kiernalgen.

301 Kierneri. Med.

200 Ketmieae.

2(i9) - genuinae.

E(69) Khnaga. 1. Juss.

$2 \pm 6$ Labiatae.

187

150 La eh mann.

Lacepedea, $K . H, B$,

181 Lacideae.

285 Lacistemeac:

206 Laetia. $L$. 27

211 L a gasea.

5 Lagerstroemieae.

266 Laget ta.

11 Lagochilus. Bnth, 188

11. 21.58 Lagoecia. T, 218

311 Lagopsis. Buth. 188

189) Lagotis. Gürtn. 195

218 Lagunaceae.

132 Lagnuaria. $D_{i} C$.

7. 89. Lagunea. Cav. 283

$2 ! 9^{2}$ Lahayct. R, S. 235

13(j L a m a k.

2 tio Lamarkea. Gaudich. 353

287 L a m b e r t.

287 Laminarineae.

313 Lamiopsis. I88

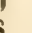

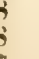


Lamiotypus.

Lannimu. $L$.

Lansiun. Bl.

Lancretia. $D_{e} C$.

Lipathum. ' $T$.

Laplacea. K.II.B.

Lurtirea. St. Ilil.

L i r tez.

Latrvenblüthler.

La s c l.

Lil serpitium. T.L.

Lisionthera. P. $\boldsymbol{l}$.

Lasiucorys. Bnth.

lasinuetaleae.

Lasiopetaluu. $S m$.

Laureac.

laturintace.

Lau rineen.

Laviulula, $L$.

Lavatera. L.

Lavatereae.

- genuinae.

Lavauxia. Spech.

Jarradia. I elloz.

Lilvradiene.

1.cbretouia. Schrk.

isecanocurpus. N. I. E.

Leclica. L.

Lecheeae.

lecirlineae.

Lecokia. De C.

Lesythideac.

lierleate.

Ledebour.

Led!:u.

L.ciocirpeae.

L(d)carpun, Diff.

l.eeit. I.

Leluman.

Lehmannia. Spr.

l.ciololia. Rchb.

l.cioschizucarpicae.

Lenineae.

Lerineac.

lestibulariae.

hemuis, Rz. l'al.

lemitis. linth.

Lemice. IJ chr.

Leonuriss. $L$.

devechinia. $1 /$.

Lepilarathis, 17 .

Irviticite.

leppidium. $\boldsymbol{L}$.

Lepisanthis, Bl.

levtacanthus. N.v.F.

Lcptalium. DeC.

Leptarrhena R.Br.

Leptociulis. Nutt.

Leptolacena. A. P.'Th.

leptosipliom. Benth.

leptopyrum, likb.

Leptospermeac.

Leptuspermum, Forst.

l.eptostitchya. N.v.F.

Leptrina. Rafin.

Lepropelulon. Ell.

Lepuropetalon. Nitt.

l.eria. DeC.
S. 18 ? Tetsomia. Rz. Pav.

188 Leucas. $R, j, j$.

313 Jencocarpus, Don.

317 Lcucocarpus. Wich.

2:(i Leucodonium. Lichb.

3̈!!2 Lencolaena. R.BBr.

2 29) Lcucosceptrum. \$m.

2!: Leneostemma,

1 19? Levisticum. J. Bauh. et $K$.

11. 32 Lewisia. Pursle.

220) Libnuotis. Crtz.

$21+$ Liboschitz.

18 Lichenes.

2!)? Lichtenstrinia. Cham.

29? Liglitfootia, l'Iferit.

17fi Liunsticum. T,L.

17.) Liuustrun. $L$.

17.5 Lilac. ' $T$ '.

180. Liliengrïser.

2ofi limmantlieae.

$2 \times 6$ Limnantlicmeac.

286i Limnanthemum. Gm.

$2+6$ Limnanthus. R.Br.

go Limopliila, $K B$ r.

270 Limonia. $L$

26 i Limniene.

239 Limosella. $L$

272 Linantlus, Benth.

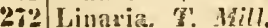

13:2 Liulackeria. Prsl.

2I!) Lindle y, Vowort. 12-18. Ludwigia. $L$

25144.50 .330 .65 .80 .91 .143 .145 , Lïhea. $\mathrm{W}$.

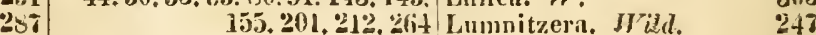

18 Lindenbergia, Lehm. $19 ! 9$ Lunaria. $L$.

207 Limlengewiillise. $\quad 302$ Lunularinae.

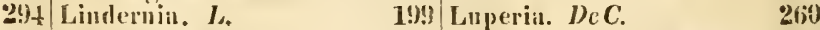

29) Lindleya. Finth. 273 Lnoirister. Presl. 2026

21t Livalleyu. N. v, F. $\quad 30$ Lupinus. $L$.

11 Listurera. Relib. 303 Lapnlinuria. -

201 Lincae. 20l6 Lupulinac. 17?

2 (i) - genuinae. 306ilurilie.

188 Link. 11.88.50.76. 116 Lutcolu, $T$.

136) Linkia. P. 20I Luxemburria. St.llil. 2\{j9

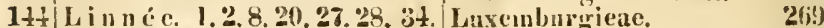

198 37. 49. 5\%. Jii.6il. (18. 70. 7\%.73. Lum!a, Dsf. 15\%

$20476.57 .6 !), 86.83 .84 .92 .123$. Lychnilcae. 293

188 272 Lychnis. L. 2!8

26 Linociera. $\$ m$. 213 Lycinpsis. Sp. $2+6$

188 Linopsis. .Rchb. 3Cx́ lycium. L.

189) Linmu. L, 306 Lyeopertacei.

199. Lippenblïthler. $\quad$ 187 Iytopersicum. L. 20

25!) Lirindendreite. 288 Lyeopodiatene.

250!) Liriodendion L. 278 Lyenpus. $L$. 188

285. lisianthus. P.Mr. 2I1 Lyonia. Nutt. 20ij

190 Lisionotus, Don. 199 Lysimachicac. 204

260 Lithnearpea. Bl. 172 Lysipoma. Kuth. 1810

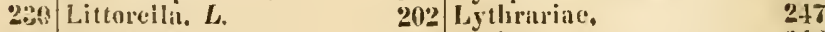

21!) Luasia. Ailans. 23" Lytlireic. 2t8

318 Loitsateric. 231

$19+$ Lolitsitcen.

zot Loascile.

2.53 Lubclia. L.

25i. Cohcliariac.

190 Lobelicie.

237 Lobocarpus. Whigt, Arn.

20 ( Iobularia. Dsv.

230) Lochneria. Scop.

1o: Luefflingiz. Io.
231

281

2⿺辶

2!) 2

159

12

2(i.)

172

II)

1eii 
Macrolineae.

Macrolinum. $R$ chb.

Macromelissa.

Macropodium. R.Br.

Macrostylis. Bartl.

Macrotliyrsus. Spach.

Macrotys. Rafin.

Maerua. Forsk.

Magellana. Cav.

M a guol.

Magmia. A. St.JIt.

Magydaris. Loch.

ilaliernia.

Mahcrnieae.

Mahonia. Nutt.

Maluri'lit. Aubl.

Majoraua. 'T.

Maks imowits ch.

Maláchimu. Fries.

Malachodendron. Cav.

Malaclira. L.

Malachreie.

Malaxideae.

Malcolmia. R.Jir.

MIalesherbia. $R z, P a v$.

Maiesherbieae.

Mallechten.

Mallea. A.Juss.

Hallogonum. Fuzl.

Malope. $L$.

Malopeae, - genuinae.

Malpighia. $L$

Halpighieac.

- senuinac.

Malva. $L$.

Malvaceac.

Malvarisceae.

Malveac.

Malrengewächse.

Mammea. $\boldsymbol{L}$.

Hammeariac.

Mammillaria. Haw.

MI in $\mathrm{n}$.

Mancanilla. Plum.

Maudragurat. ' $T$ '.

Mamdingreac.

Manglilia. Juss.

Magnolia. L.

Magnoliariac.

Magnolieac.

- genuinae.

Manglietia. Bl.

Maugustaneac.

Maninot. Adaus.

Mamulea. $l$.

Míapar. A.Juss,

Maprounea. Aubl.

Miralia, $A, P{ }^{\prime} T \%$,

Maratlireac.

Marattiaceise.

Marceravia. $L$.

Marcgravieae.

- genuinae.

Marchantiaceac.

Mlarelıantiaceen.

Marchanticate.

Marialva. Mart.
S. 8nz Varianthus, Ilüg. S. 270/Melicrtus. Forst.

S. 267

807 Marila. Sw. (302?) - 311 Melissi. $L$.

18!) Mariluae.

2(i) HI a r y u a $\boldsymbol{r}^{\mathrm{t}, \mathrm{Clam} .}$

28: Marrubieae.

285) Marrubium. L.

2тz. II a rscliall v, Bieber-

2 (i) stein.

ges0 Marsilitrene.

MIarsiliaveen,

285.) Marsypiantles, Mart.

21!) $r .11$ is 1 t e 115 .

2!)1 Martia. Spr.

311 Velissear.

11 Melissoiles.

188 Melistaurum. Forst. 273

Melittear. 189

18 Melittis. L. 189

138 Melocactus, C. Bauh, 233

138 Melochia. L. 292

189 Heniocus. Dsv. 259

11 Henispermeac. 146

308. Menouvillea. DeC, 259

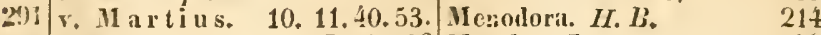

2(i.) 57.59.106 Mentha. L.

ü!: Martynia. Horst. 198 Mentheae. 188

188 Uartynicac. 198 II entzelia. Plum. 232

18 Marumia, Keinu. 302 Mentzelieae. 232

2utd Mascinnia. Bert. 299 Henyantheae. 210

302. Maschilocarpicae. $\quad 138$ Menyanthes. L. 210

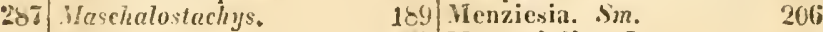

285 Vastichina. 109 Hercurialis. L. 281

$15 j$ Mastixia, $B l$. 221 Veriandra. Buth, 189

Z60 Mataplea. Aubl. 15ó Merimea. Cambess, 243

ans Matisia. K.II.B. 295 Hextens. 11.18.38

208 Matisieac. 295 Mesembryanther:eac. 240

151 Matourea. Aubl. 199/Mesembryantleum, Dill. 240

313 Inatthewsia. Hook. Arn, 260 Messerschmidtia. L. 193

20f Matthiola. R. Br. 260 Mesona. Jil. 109

285 Mathioleac. 260 Hesosphaeria. 189

295. Iattia. Schult. 19:3 Mesua. L.

28 Manchartia. Neck.? 219 Mesweac. 311

2!)!) II a uke. 11 Methoniceac.

2!y!) 31mmandia. Jeq. 195 Methoricum. Schott. Endl. 291

gut)!) II a n tz. $\quad$ II Metrosideros. Gïrt. 253

Esti Vaximilianea. Mart. 302 Metzleria. Mrsl. 106

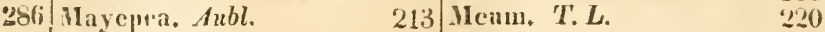

$286 \mid$ II a h hef. 11 . II eyen. 11.50 .165

286 Mastems. Feuill. 301 M y er, C. A. 11.18.236

286. Mazus. Lour. 199 M e yer, $\mathbf{L}_{*}$

311 Meconopsis. Viguier. 264 Me yer, W. 11.51.11T

311 Vecrburgia. Mnch. 235 Meyeria. N.v. E. 191

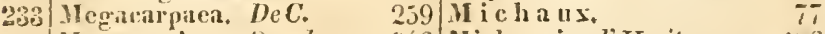

11 Megraterim. Spach. 246 Michanxia. l'Iterit. 156

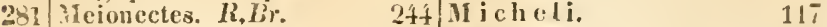

201 II is ner. 11.22.262.288 Nichelia. L. 278

201 ileviera. DeC. 156 Micrantheae. 251

214 Slerkia. Fisch. 298 Micranthemum. Prsl. 226

2- Melalenca. L. 253 Micranthera. Choys. 311

278 Melalenceac. 253 Micranthes. Jlaw. 230

278 Mclampodicae. 152 Micranthenm. Desf. 281

278 Melampyreae. 197 .llicrocarpaca. R.Lr. 199

278 Helampyrmm, T.L. 197. Hicrocorys, H.Br. 189

311 Helandryum. Clus. 298 Microdon. Chois. 196

281 . Helanim. DeC. 269 Micromelum, Bl. $31+$

199 . Melanopsillium. Colla. 253 . Micromeria. Bnth. 169

281 Melanoselinum, Hoffm. 220 Micropleura. LaG+ 221

281 Melantheae. 152 Microsphace. 159

221 Melastomeac. $2+8$ Hierostachys, A. Juss, 281

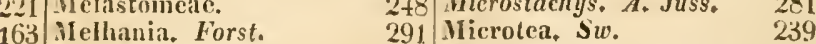

139 Melia, L. 313 Miersia. 15

$\begin{array}{lll}139 & \text { Melia, L. } & 157 \\ 310 & \text { Melieae. } & 313 \text { Miliusa, Lesch. }\end{array}$

$\begin{array}{lllll}310 & \text { grenninae. } & 313 & \text { Milleporum. Spuch. } & 30 \text { J } \\ 310 & \text { Mcliantheac. } & 255 & \text { Millingtonia. Roxb. } & 265\end{array}$

137 Mclianthus. T. L. 285 Millinglonicae, 265

137 Melicocca, $L$. 28 J Milnea. Rxb. 313

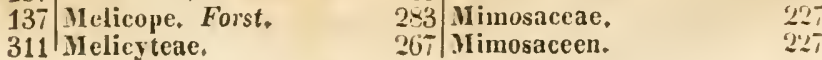




\begin{tabular}{|c|c|c|c|c|c|}
\hline Mimoseae. & $228 \mid$ & linsia. Camb. & 285 & Negundo, Mnch. & .285 \\
\hline Mimulus. $L$. & 199 & Mozinna. Ort. & 281 & Nilitris. Gärt. & 253 \\
\hline Mimusopeae. & 215 & Mucedinei. & 130 & Nelkengewä̀chse. & 295 \\
\hline Mimusops. $L$. & 215 & orini. & 130 & Nelsonia. R. Br. & 190 \\
\hline Minthidium. & 189 & Miitzenmoose. & 137 & Nelumbeac. & 144 \\
\hline lostachys, & 188 & neae. & 221 & Nemedra. A. Juss. & 313 \\
\hline $\begin{array}{l}\text { Minuartia. Loeffl. } \\
\text { Mirlo e l. } \\
\text { 8.50.51.1 }\end{array}$ & 235 & um. $P$. & 221 & Nemesia. Ient. & 198 \\
\hline $\begin{array}{l}\text { M irls e } 1.8 .50 .51 .1 \\
\text { Misandra. Commers. }\end{array}$ & 170 & gria. $L$. & 303 & Nemia. Berg. & 199 \\
\hline $\begin{array}{l}\text { dra. Commers. } \\
\text { llus. Presl. }\end{array}$ & 172 & ria. Dsv. & 258 & Nemopluila. Barton. & 193 \\
\hline $\begin{array}{l}\text { llus. Presl. } \\
\text { a. T, L. }\end{array}$ & 226 & Koen. & 314 & ilene. & \\
\hline $\begin{array}{l}\text { Mitella. T, } L, \\
\text { Mitelleae. }\end{array}$ & 230 & uleae. & $\begin{array}{l}272 \\
159\end{array}$ & $\begin{array}{l}\text { Nep } \\
\text { Nep }\end{array}$ & $t$ \\
\hline $\begin{array}{l}\text { Mitelleac. } \\
\text { Mitrasacme. Lab. }\end{array}$ & $\begin{array}{l}230 \\
210\end{array}$ & hia. Dumort. & 186 & riae. & 188 \\
\hline aemeac. & 210 & & 40 & & \\
\hline 1. Forst. & 235 & $\mathbf{M}$ & 182 & elinn. $L$. & 285 \\
\hline ra. Juss. & 215 & n. T. L. & 258 & gamia. Wght. Arn. & \\
\hline pilze. 1 & 129 & e C. & 253 & he. & 211 \\
\hline a. Cham.Schlecht. 1 & 199 & eae. & 196 & Nerija. Rxb. & 301 \\
\hline Mnch. & 286 & m. & 196 & Neslia, Dsv. & 260 \\
\hline gia. & 297 & 1. Prsl. & 186 & gewächse. & 172 \\
\hline Ehrh. & 298 & is. $L_{*}$ & 192 & inthus. N.v.E. & 0 \\
\hline & 213 & L. & 276 & B. Juss. & 0 \\
\hline ium. Juss, & 214 & ra. Spach. & 308 & N & 0 \\
\hline Mo h $1 . \quad 11,40.6$ & .79. & eae. & 170 & obeae. & \\
\hline & 124 & en. & 170 & a. Gürt. & 1 \\
\hline & 83 & ia. $D_{s v}$ & 240 & a. $L$. & 1 \\
\hline räclıse. & 262 & & 170 & ne. & \\
\hline art. & $30+$ & ta. $D e C$ & 303 & a. DeC. & \\
\hline$V$ & 235 & ylleae. & 244 & sergia, Rs.Pav. & \\
\hline neas. & $23 j$ & yllum. I raill. & 244 & $\begin{array}{l}\text { lir. T.L. } \\
\text { llastrum. Mnch. }\end{array}$ & $\begin{array}{l}217 \\
277\end{array}$ \\
\hline $\begin{array}{l}\text { gemlintae. } \\
\text { ugo. } L \text {. }\end{array}$ & $\begin{array}{l}233 \\
236\end{array}$ & Siw. & $\begin{array}{l}113 \\
295\end{array}$ & $\begin{array}{l}\text { Nigellastrum. Mnch. } \\
\text { Nigelleae. }\end{array}$ & 277 \\
\hline permum. Koch. & 219 & & 295 & $A g$. & \\
\hline la. $L$ & 188 & im. $\mathrm{DeC}$ & 290 & a. L. & \\
\hline$L$ & 180 & un. Schott. & 253 & ene. & 0 \\
\hline la, Bnth. & 174 & . Scop, & 219 & änter. & $t$ \\
\hline & 188 & $L$. & $20 \div$ & . Rchb. & 9 \\
\hline Aubl. & 282 & 2 & $20+1$ & ia. $K, H . B$. & 69 \\
\hline 1. Mich. & 199 & 1 & 252 & $L$. & 0 \\
\hline 2. J $\quad \in \mathbb{C} q$ & 304 & & 252 & ae. & 0 \\
\hline us. Fisch. & 190 & Myrteae. & 253 & 3 & 11 \\
\hline Dun. & 278 & genuinae. & 253 & 31 & 21 \\
\hline s. Schrad. & 239 & 9.11 & 253 & uech. & 305 \\
\hline - Salisb. & 186 & Myrtilleae. & 206 & $6 \mathrm{~N}$ & 135 \\
\hline s, Brgn. Fndl, & 281 & & & - I ent. & 213 \\
\hline i $L_{\text {. }}$ & 206 & zen. & 245 & ace. & 189 \\
\hline eae. & 206 & itten. & 200 & as. $R \cdot B r$ & 260 \\
\hline L.fil. & 290 & Iflechten. & 132 & lete. Bnth. & 188 \\
\hline e. & 290 & s. $L$ & 162 & . Bart. & 287 \\
\hline a. $\mathrm{Moc}$ & 295 & . L. & 194 & 4 & 201 \\
\hline Mick.L. & 237 & 1a. 'Thnb+ & 265 & 51 & 4 \\
\hline & 237 & ia. $D e C$. & 277 & 72 & \\
\hline & 172 & & 151 & L. & $?$ \\
\hline e $C_{\text {. }}$ & 258 & hwertel. & 151 & a. $D_{0}$ & \\
\hline R.Br. & 199 & & 151 & 11. I ent. & 20 \\
\hline ia. $D e C$. & 261 & 11 & $18:$ & ancene. & 1 \\
\hline d. & 178 & 8 Nasturtieae. & 260 & aeeae. & \\
\hline a. Lam. & $\begin{array}{l}178 \\
283\end{array}$ & $\begin{array}{c}8 \\
3 \\
3 \\
(3) \%\end{array}$ & & Dysseate. & 16 \\
\hline Lam. & $\begin{array}{l}280 \\
283\end{array}$ & & 20 & yärt. & \\
\hline e. & 76 & 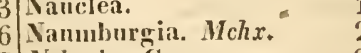 & 201 & Selereb. & \\
\hline . Plum. & 267 & Gm. & 235 & 55 Ochneas. & \\
\hline & 267 & 7 Nectonxia. K.II.B. & 201 & - genuin & \\
\hline$\vdots$ & 311 & ] Nees v. Fsen b & & Ochradeneac. & \\
\hline & 22 & Ch. G. 7.8.12. & 3 & Ochradenus. Delile. & \\
\hline Muscho: & 189 & 117.1 & 175 & 70 Ochr & \\
\hline in. $A$. & 313 & $3 \mathrm{Ne}$ & & Och & \\
\hline Iosta. & 10 & 1.5 & & Ochir & \\
\hline
\end{tabular}


Ochroma. Sw.

Ochthudium. DeC.

Orymoideac.

Ocimmun. $L$.

Oriontandra. Finth.

Odoutarhena. C. A. M.

Oijontostemma, Benth.

Oenantlic, $L$.

Oenothera. $L$.

Oenothereae.

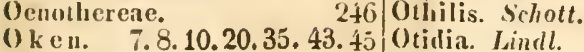

Olacincae.

Olea. 'T. $L$.

Olcincae.

Olericeac.

Olisia.

Oliviera, Fent.

Olympia. Spach.

Omalauthus. A.Juss.

Onbrophytum. Popp.

Omphiluvarpus, $\ell^{3}, B$.

Omplaslodes. $T$.

Omplialea. $L$.

Onagra.

Onagraceac.

Onagreac.

Onobrycheae.

Oocephalus.

Opntows ky.

Opercularia.

Operinlarineae.

Ophelia. Don.

Ophelus. Lour.?

Oplinglosseae.

Ophirease.

Oplurydeae.

Opitz.

Opopanax. $K$.

Opuntia. ' $T$.

Opuntieac.

Orangengewächse.

Orchideae.

- genuinae.

Orclideen.

Ureas. Cham.Schlecht.

Oreoselinum. Clus. T.M. $B$.

Origancae.

Origanmus. $L$.

Orlaya. Iloffm.

Ornus. 1 .

Orobanclieae.

Orohium, Rchb.

Orontiene.

Oropliea. $B l$.

Ortega.

Ortega. I

Ortegini. Iiiffl.

Ortholcucas.

Orthopodium.

Orthosiphon. Bnth.

Orthosporum. C.A.M.

Orthostemon, $B r$.

Orthotheciun. Schott. Endl

Ortmann.

Orvala.

Oryehmophragmos.Bunge.

Orygia. Forsk.
S. 295 Oryzeac.

258 Oscillatarineae.

189 Osmorrliza. Rafin.

189 Osmundaceae.

313 Osmundaceen.

259 Osmundere.

298 Ostericum. II $/ f m$.

220 Osterluzeien.

246 Osyrideac.

214 Otnstegia. Benth.

213 Ottoa. K.H.B.

213 Ondneia. R.Br.

238 Onrisia. Comm.

188 Oxalene.

219 ... genuinae.

305 Oxalideat.

281 Oxalis. L.

161 Oxleya. Hook.

215 On yamlra. DeC.

193 Oxyenecus, Pers.

281 Oxyria. IIill.

246

245 Pachylophis. Spach.

$2+6$ Pacliyuma, $R$. Br.

226 Pachyplesrum. Led.

189 Pachysandra. Hchx.

1 I Paederota. $L$.

180 Paconia. $L$.

180 Paconicae.

211 P'agamea, Aubl.

295 Palizecycaleat.

13!) Palaeuphyta.

16i Palava, K̇.Pav.

15.5 Palavia. Cav.

11. 3+ Palavieae.

220 Palimbia. Ress.

234 Palisot Beauvois.

23: I alias.

31? Palmae.

$154-$ genuinae.

155. Palmenfarrn.

154 Palmstruckia. Rtz.

260 Panax. $L$.

Pandincae.

220 Panderia. Fisc

188 Pangim, $B l$.

188 l'auiceae.

220 l'anopice. Noronh.

213 Hanzeria. Much.

19:) Papaver. T. L.

200 Papaveraceae.

18: Papavercae.

288 - genuinae.

эร Papayaceac.

23.) Papilionaceac.

235. I'aralea. Aubl.

188 Paranesns. Presl.

189 I'aratropia. $B l$.

18!) Parilleae.

23!) Parilia. Denst.

11 Parilium. Gärt.

.294 l'arita. Scop.

st Paritium. St. Hil.

188 Parkericae.

261 Parmentier.

206 Parmeliaceae.
S. 146| Parnassia. $L$ S, 211

135) Jaronychia, Juss, 235

219) Paronychicae. 235

139) Iaropsieae. 208

139) l'urrya. $\boldsymbol{R}, \boldsymbol{B}$ r. 260

13!) Inssilloreac. 208

200 l'astinaca. 'T. $L$. 220

173 l'atagonula. $L$. 193

167 ['atrisia. K. II. B. 273

278 Patrisiene. 273

2!) Paullinieae, 285

188/I'avonia. Cav. 287

220 I'aronieac. $28 \mathrm{~T}$

260 I'ectinaria. 189

198 I'edalium. L. 198

293 I'edicellia. Lour. 283

29:3 l'edicularinae. 197

2!!2 I'edilanthus. Neck. 281

2!)4 Pegranum, $\boldsymbol{L}$ 282

sిIs Peixota. A. Juss. 299

$30:$ l'ekea. Aubl. 295

206j Pelargium. DeC. 290

236 Pelargonieae. 290

l'eliosantheae. 142

246 Peltaria. L. 259

278 Peltodon. Pohl. 189

220 Pencueariae. 167

282 I'entarrypta. Lehm. 219

198 l'entadesma. Don. 311

27\% Pentaloba. Lour. 269

278 I'entilobeae. 269

211 I'entapetes. $\boldsymbol{L}$. 291

139 Pentaphalanx. 253

140) I'enthoreat. 230

302 l'enthorum. $L$. $\quad 230$

287 l'entstemon. l'Ilerit. 190

287 Peplidium. Delil. 199

220) Peranemaceae. 139

8! Perlicium. 182

18 Pereskia. Plum. $23 \pm$

158 Pereskiene. 234

158 Percrularinae. 208

13!) Perilleridia. Rchb. 219

19!) Perilta. L. $18 \gamma$

2.I I'erilomia. K. H.B. 189

14!) l'eriploca. $L$. 280

23!) l'eriplocene. 208

268 - genuinae. 208

14(i) Periptera. DeC. 2-i

281 I'eristera. DeC. 2960

188 Peristrophe. N.v.E. 190

2(j) Peritumat. DeC.

$26 \%$ Peritumene. 2660

2 (it) Peritropia. $2: 26$

264 Pernettia. Gaudich. 206

184 l'ersicaria. $T$. 236

225) l'ersicariene. 236

215 l'ersinatac. 197

206 ['e sion. 77. 117

201 l'ersomieac. 169

153) I'etagnia. Guss. 21

22:3 l'etalidium. N. v. E, 190

214 l'elermann. 11. 120

288 l'etit-'Thonars. 9.50

288 Petiveriae. L. 239

139 Petirria.

169 I'etrusillis. R. Br. 259

133 l'etromarula. DeC. 
Petroselinum. Hoffm、 S. 219|Pistolochia.

Petunia. Juss.

Pencedaneac.

Peucedanum. $L$.

Peumene.

Pfeifenflechten.

$P f e$ iffer.

Phacelia. Juss.

Pliacocapnos, Brnh.

Phacocarpus. Mart.

Phaeostoma. Spach.

Phalarideae.

Pharnaceum. $L$.

Phaseoleae.

Phebalium. Vent.

Phellandrium. $T \cdot L$.

Pliladelpheae.

Philadelphns. $\boldsymbol{L}$.

Phillyrea. T, L.

Philomedia. Noronh.

P'hilotheca. Rolg.

I'hilydrum.

Phleboanthe, Fisch.

Phlebophyllum, N.v. F.

P'hlogacanthus, N,v, E

Phlogineae.

Phlomis. $L$.

Phlomiteae.

Phlomitis, $\boldsymbol{R}$.

I'hlox. $L$.

Phocuiceae.

Pholidia. R, Br.

Phylacanthus, $N$, et . h.

Phyllantheae.

'hyllis.

Jhyliocladas.

['liyllndoce, Sulisb.

liyllostegia. Benth.

physalideac.

Physalis, $L$.

Jhysiphora. Soland.

I'hysocalyx, Pohi.

Plysospermun. Cuss.

Jliysostegia. Benth.

J'hysosternon. Mart.

Phyteumi. $L$.

Phytolacea. $L$.

Pliy tolacceac.

Picrorrhiza. Royle.

l'ieuer.

Pigea. $D_{c} C$.

Pileantlius. La $B$.

f'ilocarpus, Vahl.

J'ilse.

Pimpinella. L.

Pinaria. DeC.

Pinguicula, $L$

Piparea. Aubl.

Pipareanae.

Pipereac.

Pipcrella.

Jivipca. Aubl.

Piriqueta. Aubl.

Pisonicac.

Pistacieae.

Pistia.

Pistiaceae.

Pistiaceen.
201 Pistoloclicac.

220 'ittosporeac.

220 - genuinae.

17t Pittosporeae. Banks.

13:- Pitumba. Aubl.

11 Plagianthus,

193 Plagiobotrye.

264 Plagiostoma.

285 Plagiotis.

246 Plananthere.

146 Plintageac.

236 PIantagineae.

226 Plantagio. $L$.

282 Platancae.

220 I' latner.

230 Platonia. Mart.

230 P'latonieae.

213 Platunieae.

283 Plattycapnos. DeC. Brnh

282 P'latycodon. DeC.

14 Platyspermae.

189 Platyspermum. Hoffm.

190 Platyspermm. Jlook.

190 Platystemon. Bouth.

194 Platystemonere.

188 P'atystigna. Benth.

188 Plectranthns, l'Ierit.

188 P'lectophytum. K, $I, B$,

$19+$ Ilectritis, Lindl.

158 Plcthiosphace.

196 Pleurandra. LaB.

198 Pleurogyne. Fschch.

281 Pleurospernum. Hoffm.

180 l'linthine. Rchb.

166 Plocama. Ait.

206 Pluknetia. Plum.

189 Plumbagene.

201 I'lumbagineae.

201 PIumbarineen.

269 PInmbago. $T$.

198 Porlocarpene.

219 I'odocarpus.

189 Porlogyne. Hffes.

267 Podopliyllmu. $L$.

186 Podopteris. $\mathbf{l l}$. $\boldsymbol{l}$.

239 Podostenoneare.

'30 Poecilolermis. 198

11 Poepig.

269 Pogogyuc. Bnth.

253 P'ogonitis. Rachb.

282 Pogrostemon. Dsf.

129 Polanisia. Rufin.

219 Polembryun, A.Juss.

260 I'olcmonieate

198 I'olemonium. $L$.

269 I'olia, Lour,?

269 L'oliantleae.

173 Polium.

189 Pollichia, Solend.

199 I'olyactinus. DeC.

232 P'olyalthea. $B l$.

274 Polycardia, Juss,

224 Polyearpaea. Lam.

143 Polycarpeae.

143 Iolyearpon. Löffl

143 l'olycenia. Chois.
S. 173/Poiychlaena. Don.

173 Polyonenum. $L$.

270 Polydesmit

270 Polygalacene.

270 Polygalaccen.

273 Polygaleae.

192 Polyoument 250

188 - 236

169) Polyrouella. 236

163 P. Mchx 236

"202 Pyldene. 136

202 Dest. 221

202 Pulypodiene. - 130

202 Polytareniat. Dec. 220

172 Pomacene.

20 Pomax. 180

311 Pombalia. Iand, $26 !$

311 Pombalieac. 269

190 "Nongatieac. 156

264 l'ontedcreae. 15 t

186 Poranthera. Rudge.

219 Poraquiba. Aubl. 214

220 P'orcelia. Rz. Pud. 2-8

260 H'orocirpac. 261

$26+1$ lorpa. Bl.

$26+$ Portulaca. $L$. 303

"264 P'orislaciscenc. .

189 Portulacaccen. 235

221 L'ortulataria. Jact. 237

178 Portulaceae. $\quad 236$

189 - genuinae. 237

277 Portulacellac. 237

'2ll Posidunia. Cout. et Foen, 152

219 Potalia. Aubl. 211

29o Potameac. 14

180 Potamogetoneas.

281 Polamophila. Silerls.

202 Potentilleae.

201 - genuinae. 21

201 Poterieas.

203 Poirretia. II.

166 Pourvetieac. 290

166 l'ozoa. Lat.

267 Lraeformativi. 12!?

265 Prangos. Lindl. 219

236 Prasieae. 15!

162 - genuinae. $18 \%$

91 Prasium, L. 18\%

10. 11. 16t Prepusa Mart. 106

189 I'rescot.

$2.6 j$ l'ieslaen. 18

188 Peslate. Mart. 193

$10-188^{\circ}$

267 Presl. 11. 55.94

282 Iretrea. Gay. 195

194 Prérost.

$19 \pm$ Prieurea. $D_{C} C$. 246

235 Primulacene. 20 i

15 t Primulacen. 204

189 Primuleac. 204

235 Prismatocarpus, l'Herit. 146

290 Prockia. I'. Bron.

278 I'rockieac.

301 Promaya. Ifïg. 270

235 Proserpinaca. $L$.

235 Prostantlıera. Lab. 190

235 Prostantlicreac. 189

196 P'rostea. Camb. 205 
Prosthesia, Bl.

Proteaceae.

Proteaceen.

Proteene.

Prunella. $I_{2}$

Prunelleae.

Pseudauisomcles.

Pseudomelissa.

Pseudothymbra.

Psirliear.

Psidium. L.

Psilonema. C. A. M.

P'soroplytum. Spach.

l'sorospermmm. Spach.

P'syehine, Dsf.

P'sychineae.

Psychotria.

P'syeliotrieac.

Psychrophita. $D_{c} \mathrm{C}$.

Psyllirae.

Psyllium. $T$.

P'telidium, A.P.Th,

Pterandra. A.Juss.

P'trroidene.

J'teronenrum. $\boldsymbol{D}_{\mathrm{c}} \boldsymbol{C}$.

P'teroselinum. $R \mathrm{chb}$.

Pterosperisum. Schreb.

Pterospora, Nutt.

Pterostegia. Fisch. C.A.M.

P'terostigma. Buth.

Pterygium. Corr.

Pterygota. Scholt. Findl.

Ptilotrichum. C. A. M.

Ptychotis. $K$.

Pugroninm. Gärt.

P'nlsatilla. $T$.

P'nlsatilleae.

Pulverarieae.

Pumilio. R.

Juniea. T. $L$.

P'urkinje.

Purkinjea. Prsl.

P'utoria. $\boldsymbol{P}$.

Putorieac.

Pycnanthemum. Mchx.

Pycnobotrys.

Pycnosphace.

Pyenostacliys, Hook.

Pycnothymus.

Pyramidium.

Pyrola. L.

Pyroleac.

Pyxidanthera. Mchx.

Quadrella, $D_{c} C$.

Quapoya. Aubl.

(juarariben. Aubl.

Quararibeae.

Quassieae.

Qucria. Gürt.

(Queria. Loeffl.

Quinquineac.

Quintinia. DcC.

Quisqualis. Rumph.

Quivisia. Juss.

Rabenlorst.

Radiola, Dill.
S. 269) Radioleae.

16i9) It a di i 8 .

1(i) Rafl'esia.

16!) Rafllesiene.

169 Ramondia. Rich.

169 Ranunculaceac.

18!) Lanuneulariae.

189) Ranun:ulene.

18! - genuinae.

253 Ranunculus. $L$

353) Ranunkelgewäclıse.

25!) Kaphincae.

208 Raphanistreac.

3̈(8) Raphanistrum. $T$.

25) Raplianus. $L$.

25!) Raphislospora. N.v. F.

180 Rapistrum. Boerh.

180 liapuntium. ' $T$.

27 Ka p a il. 10. 18. 28. 56.

20 '2

202 Ratonia. DeC.

301 Rautengewächse.

299) lRauwolfieae.

138 li a y.

2(i0) Razumovia. Spr.

200 12 eammuria. $L$,

29) Ii eammurieae.

206 Relris. Spach.

236 Rebonillarieae.

199 Rechia. Moc, Sesst.

$30+$ liecchieae.

gy1 Redontea. Ient.

25!) Rodowskia. Cham.

219 Reevesia. Lindl.

¿58 Reevesieae.

zog lik e i che l.

27(j) Rehmanuia. Libosch.

131 lic inwardt.

298 Reinwardta. $B l$.

247 lienggeria. Meisn.

62. 124 Requienia. $D_{e} C$

$20+$ Reseda. T. $L$.

181) Reserleae.

180 Kesedella. Rchb.

188 Lestiaceae.

18!) li e un.

189 Rhabdia. Mart.

189 Thabdocaulon.

188 R hamnene.

18!) R hamneen.

206 Rleedia. L。

206 Rheedieae.

206 Rheum, L.

Rliuncantlıus. $\boldsymbol{N}, v, E$.

267 Rhinintlieae.

311 Rhinanthera, $B l$.

295 Rhipsalideae.

295 Rhipsalis. Gärt.

283 Rhizoholeae.

235 Rhizobolus.

285 Rhizobotrya. Tsch.

180 Rhizophoreac.

233 Rholochiton. Zucc.

247 Rhodotendreae.

313 Khododendron. $L$.

Rhodolnena, A, P. Th.

11 Kliodomeleae.

306 Rliodora. $L$.
S. 306 Rliodoreac.

11. 11 89) 3 liorlotliamnus, Rchb.

1 (it Rliyuclotlicea.

1 it Rhyoliotheceac. 2!)1

19!) Kilies. $T$. 2233

255 Ricciene. 130

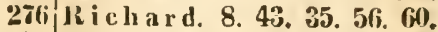

276 61, 65. 21, 72. 86. 88. 8!).

$27(i)$ 166. 168, 170

276 Richardia. 142

275 Richeria. Iahl. 282

25!) Kiclıter. 3. 11. 69. 81

259 licineae. 281

25!) - genuinae. 281

25!) Ricinocarpus, $D_{s f}$. 281

19) Ricinus, $L$, 281

259 Ricotic. L.

186 IRiedleia. I'ent.

293

117 Rindera. Pall. 139

2o!) Rinorea. Anbl.

211 Kitchiea. $\mathrm{R}, \mathrm{Br}$. 267

1. 2. 49 Ritgen.

I!) Kivina. $L$.

240 Rivineae. 239

$240 \mathrm{R}$ i vinus.

233 Robertsonia. Haw.

135 Robsonia. Berland.

278 Rochelia, Rchb. 193

27\% Rogeria. Gay. 198

288 Roëlla. L. 186

25!) Ro emer. 11

2!) v. Roemer. 58

39.5) Roenseria. Medik. 2(it

11 Roper. 4.11.93. 29?

199 Inoeperia. Spr. $\mathbf{2 8 1}$

11 Rolirer. 11

302 Rolirkolben. 149

310 Rollandia. Gaudich. 186

206 Rollinia. St. Hil. 258

2(j1 Rondeletia. 180

2(j) Rorelleae. 201

201 Roridula. $L$. 플

148 Roriduleac.

11 Rosa. ' $T$. L.

193 Rosncene.

$18 !)$ Rosaceen. 241

2.2. lioseae. 243

202 - genuinae. 243

sิll kosenumeller.

311 Roxyna. Spach. 308

236 Rosinarims. 'T.L.

190 li os smï s s ler.

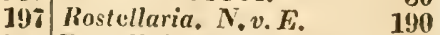

26 Rostellularia. Rchb. 190

23s Ros t kovius.

2ue R o th. 11. 34, 85

295 Rottlera. $R x b$. 11. 281

295 Rottlcra, $V$. 19!)

2ti0 Rommen. Poit. 2017

179) lRoussea. $\$ \mathrm{Sm}$. 214

198 Roxburglia. 153

207 r. Royen. $\quad$ 2. 4 !)

208 Royeni. $L$. 214

308 Koylea. Wall. 188

136 Rubentia. Commers. כิ01

207/Rubeae. 243 
Rubiaceae.

Rubiaccen.

Rubus. $L$.

Rud o I p li i. J. C.:L.

Ruellia. $\boldsymbol{L}$

Ruellieae.

Ruizia. Cav.

Rulingia. R. Br.

Rumex, $L$.

Rumia. Hoffm.

Rumiceae.

Rungia. N.v. $E$.

li precht.

Rusceae.

Russelia. Jcq.

Ruta. $L$.

Rutacene.

Rutariae.

Ruteae.

- genuinae.

Rutlie.

Ruyschia. Jacq.

Ruyschieae.

Ryanaea. DeC.

Ryania. Vahl.

Sabaea. R. Br.

Sabaeariae.

Sabalinae.

Sabbatia. Adans

Sabulina. Rchb.

Saccliarinae.

Sacoglottis. Mart.

Sagina. L.

Sagineae.

Sagoineac.

Sagonea. Aubl.

Salacia. $L$

Salacieae.

Salicariea e.

Saliceae.

Salicorneae.

Salicornia, $\boldsymbol{L}$.

S a $l$ is bury.

Salishuria.

Salmasia. Schott. Findl

Salm-Reyferscheid D y k, Fürst Joseph.

Salpiglossis. Rz. Pav.

Salsola. $L$.

Salsoleae.

- genuinae.

Salvia. T. L.

Salviariae.

Salviene.

Salviniaceae.

Salviniaccen.

Salviniene.

Samara.

Sambuceae.

Saubucus.

Samoleate.

Samolus. $L$

Samyla. Löfll.

Samydeac.

- genuinae:

Sandoricum. Cav.

Sanguinaria. Dill. L.
S. 179|Sanguisorbeae.

179 - genuinae.

243 Sanicoria. $D e C$.

11. 53 Sanicula. T, L.

190 Saniculeae.

190 Santalaceae.

291 Santalaceen.

2 2)2 Santalene.

236 - genuinae.

219 Saouari. Aubl.

236 Sapindaceae.

190 Sapindaceen.

11 Sapindeae.

153 Sapindus. $L$.

199 Sapinm. Jacq.

282 Saponaria. $L$.

279 Sapotaceac.

28\% Sapotaceen.

$28^{\circ}$ Sapoteae.

28. - genuinae.

11 Saracenia. $L$.

310 Saracenicae.

310 Saracha. Rz. Pav.

273 Sarcocapnos. DeC.

273 Sarcocaulon. DeC.

Sarcococca. Lindl.

210 Sarcocolla. Rchb.

210 Sarcolaena. A. P. Th.

158 Sarcophyteac.

211 Sarcopliytum.

298 Sircustyles. Prsl.

146 Sargasseae.

314 Samentaceae.

$2 y 8$ Satureja. $L$.

2!) Savignya. $D e C$.

158 Sanravia. $W$.

194 Saurauja. DeC.

301 Sancrkleegrewächse.

301 Savia. $W$.

248 S a uter.

17․ Sauvagesia. Jcq.

238 Sauragesieae.

238 Saxifraga. $L$.

78 Saxifragariac.

166 Saxifrageate.

295 - genuinae.

Scabioseae.

11 Scabrita. Iahl.

201 Scacvoleae.

239) Scilligeria. $D e C$.

239) Scandicineae.

239 Scandix. $L$.

188 Seaphinm. Schott. Endl.

188 S cili it be I.

188 Schachtelhalme.

138 Schan rinia, $C, M$ et

138

138 S ch a ner.

214 Sehefficldia. Forst.

178 Scheibenllcchten.

178 Sclıcidenptianzen.

204 S c lue 11 ing.

204 S chelver.

273 Sehepperia. Neck.

273 Sclienchzeria. $L$.

273 S chiede.

313 Schiedea. Cham.

2 (i5) $\mathrm{S}$ li iller.
S. 242 Schima, Reinw. - S. 302

242 Schimmelpilze. 130

221 S c hi in p er.

22: Schirerekia. Andrz. 259

221 Schizanthus. $R$ z. Pav, 201

166 Selizocarya, Spach. $2+6$

166i Schizola ena, A.P. Th. 308

167 Schkuhr. 82

167 Schlauchalgen. 135

$2 ! 5$ Schlawchlinge. $\quad 130$

284 v. S c hlechtendal. $\quad 11$

284 Schleichera. W. 285

285 Schmetterlingsblüthige. 225

285 Schmidelia. L. 285

281 S c l m i d t, E. L. W. $\quad 11$

298 S c h mid t, G. L.E. $\quad 11$

213 S ch m id t, Z.A.F. 11

213 S chmidt, Wilib 80. 84

214 Schoberia, C. A. M, et Led. 23!)

214 Schoberiene. 23 !

272 S chönheit. 11

272 Schoeneae. 147

201 S chot t. $\quad 11.164 .235$

$2\left(i 4\right.$ Schouwia. $D_{e} C$. 25!

290 S clirader. 11.20 . 37

282 Schrebera. Rtz. 301

16ir Schubert, C.

308 v. S c hubert, G. H.

164 Schubertia. Bl. 221

$16 t$ S chuebler. 11

230 Schüblera, $M$. 210

$131 i$ Schïsselflechten.

152 Schufia. Spach. 246

188 Schultesia. Mart. 211

260) S chultz. 11. 5 ?

302 S cllult z, C.J. 11

302 Schnlzia. Spr. 219

292 Schw a e grichen. 11. 20

28. Schweiggeria. Mart. 310

11 Schweigreria. Spr. 26!

270 Schweinitzia. Fill, 206

2(j) Schwertel. 149

230 S ch y chows k y

229 Seiadopliyllum. P. Browne. 221

22!) Scilleae. 154

230 Suirpene, 148

178 Scirpinate. 147

214 Scitamineae. 156

18ti Sclerantlicae, . 235

219 Scleranthus. $L$. 235

219 Selerineac. 147

219 Sclerochliena. R. Br.

291 Sillerosciadium. $K$. 20

11 Selerostylis, Bl. 314

165 Sclerothrix. Prsl. 230

Led. Scoparia. $L$. 207

23!) Siopirimae. 204

11. 87 Scopolina. Schult. 201

204 Scordium. 18!

130. Scorodonia. 189

141 Scrolularia. L. 198

5 Scrofulareae. 1918

43 Serofularinae. 197

267 Scutellaria. L. 189

152 Scutellarieae.

32 Scybalium, Schott. Endl. 164

298 Scynopsole. Endl. 164

5 Scytaliu. Gärt. 
Sebastiania. Spr. Securinega. Juss. Sedene.

Segregatae.

Segnieria. Lour.

Seideln.

Seillia. Hostl.

Selagiuelleas.

Selenia. Nutt.

Selinum, $L$.

Senacia. Commers.

Senebiera. Poir.

Senra. Cav.

Seringia. Gay.

Serpyllum.

Seringe.

Seringia. Spr.

Serpicula. L.

Sersalisia. R.Br.

Sesameac.

Sesamoilles, $T$.

Sesaumun.

Sescli. L.

Sessea. Rz. Pav.

Sesuvieae.

Sesnvium. $L$.

Sethia, K. H. B.

Seymeria, Pursh.

Shorea. Banks.

Siagronarrhen.

Siapha. Noronh.

Sibthorpia. $L$.

Sitla. $L$.

Sidariae.

Sideae.

- genuinae.

Sideritis, $L$.

Sideroxylon. L.

siebera. Schrad.

v. S i e b o ld.

Silaus. Bess.

Silene. $L$.

Siler. Scop.

Silerineae.

Simsenlilien.

Sinapeae.

Sinapis. $L$.

Siphidia.

Siphocampyla. Cham.

Siphoniae. Rich.

Siphonostegia, Bnth.

Siphulea.

Sisarum. Riv.

Sison. LaG.

Sisymbreac.

- genuinae.

Sisymbrimn, $\boldsymbol{L}$

Sium. L.

Skinuera. Forst.

Slevortia. Rchb.

Sloanea, Plum.

Smelowskia. C. A. M.

Smilateae.

Smilacineae.

S m ith.

Smyrineae.

Smyrnium. L

Sobelewstia. M. B.
S. 281/Solaná.

28. Solanaceae.

230 Solandra. .Sw

182 Solaneac.

28!) Solanum, L.

16i) Soldanella. L.

304 Solea. spr.

16 Solenopsis. Prsl.

25!) Solenespermae.

220 Solenostemon.

270 Solenantluss. Led.

25!) Sollya, Lindl.

287 Sonneratia. $L$.

29) Sophoreac.

189) Sorintlius. Led.

87. 89. 185 S oret.

301 Sontlıwellia. Salisb.

244 Sontliwellieae.

214 Soymida. A. Juss.

$198 \mathrm{~S}$ jia ch.

?(i) Spallanzania. Pollin.

1!)8 Spananthe. Jcq.

220 Sparganieae.

198 Suarmannia. Thnb.

237 Spirmannieae.

23\% Spathularia. St. Hil.

298 Specularia, DeC.

198 s pen er.

304 Spergella, Rchb.

189 Spergula. $L$.

302 Spergulastrum, Mch $x$.

198 Speranleae.

287 S permacocene.

287 Sphacele. Bnih.

287 Splacralcea. St. Hil.

287 Sphacriacei.

188 Sphaerophoreac.

214 Sphaerostigma, $D e C$.

2!) Spliaerotheca, Cham.

11. 30 Spliagnaceae.

220 Spliagmum.

298 Sphallerucarpus. Bess.

220 Sphenoclea. Gärt.

220 Sphondylum, Hoffm.

151 Sípicaria.

?60 Spinacia, T. $L$.

260 Spiraecae.

173 Spirantliera. $N$, et $M$.

186 Spondieae.

281 S prengel.

191 S pritieim.

132 Stachydeae.

219 Stachymacris.

219 Stachyobolrys.

$2(0)$ Stachiotypus.

260 Stacliys, $L$.

2(i0) Stacklonsia. Sm.

219) Stachhonsieae.

276 Stalmauia. Lam.

211 Stalagmites. Murr.

303. Sanleya. Nutt.

260 Stapelicae.

183 Staphylea. $L$.

153 Staulryleeae.

12. 71) S t a rke, J, Chr.

219 Statice. $L$.

219) Staticeae.

258 Staubflechten.
S. 201 Staubfruchtflechten. - S. 132

$200)$ S teinlieil. 76

201 Stellaria. $L$. 298

201 Stellarineae. 297

201 Stellatae. 179

204 Stemmatosiphon. Pohl. 313

$2(i)$ Stemotia, $L$. 199

181 S Stenocliilus. R. Br. $\quad 196$

21!) Stenogyne. Benth. 189

189 Stenopetalum. R. Br. $\quad \mathbf{1 6 0}$

192 Stenosiplion. Spach. 246

270 Stenosiphonium. N, v,E. 190

253 S t e pli an. 18

22(j) Stephania. $W$. 26iz

20 Sterculia. $L_{*} \quad \mathbf{2 9 1}$

5 Sterculiariae.

291 Sterculicie. 291

2y) 1 - genuinae. 291

313 Sterigmostemon. $M . B$. 25 !

84 Sterigma. $D_{c} C$. 25 !

243 v. S ternberg, Graf

201 Kaspar.

149) Ste ind el.

303 Steudelia. Prsl 11.90

303 Steudelieae. 236

2(j) S t e ven.

186 Stevenia. Adans. 260

11.35 Stewartia. Cav. 302

298 Stielbliithige. 256

236 Stigmarota. Lour. . 2 (i7

2ys Stilago. $L$.

236 Stilbe. Berg. : $\quad 203$

180 Stilbeae. 203

188 Stillingia. Garden. $\quad \mathbf{2 8 1}$

286 Stipulicida. Mchx. 235

130 Stockesia. 189

132 Stomatechium. Lehm. 192

246 Storclischnabelgewächse. 288

204 Stramonium. Gärt. 201

137 Stratioteae.

137 Streptanthus. Nutt. $\mathbf{2 6 0}$

21!) Sireptocarpus. Lindl. 199

186 Streptopodium.

220 Streulinge.

189 Strobilantlus. $B l . \quad 190$

238. Strombosia. Bl. 310

243 Strombosieae.

283 S t r v e.

22t Strychncae.

11. 20, 79 $\mathrm{S}$ iा $\mathrm{r}$ m. 82

5 Stylidiariae. 186

188 Stylitieae. 186i

184 Stýlocerus, A. Juss, 281

189 Stylophorum. Nutt. 264

188 Styphelieae. Nutt. 203

188 Styraceac.

282. Styracilluae. - 170

$28 \%$ Suacda. Pall.

285 Subularia. $L . \quad 260$

311 S u icow.

260 Succowia. Med. 260

208 Sumachineae.

28.5 suter.

28.) Sutera. Rih. 199

43 Swarzieac.

203 Swertia. L. 211

203 Swerticae. . . 211

131 Swietenia. $L$. 313 
Swietenieae.

- genninae.

Symplionia. L, fil.

Symphonieae.

Symphyandra. $D_{e} C$.

Symphysia. Prsl.

Symphysicae.

Symphytum. Is

Sympieza. Schlecht.

Symplocos. $\boldsymbol{L}$.

Synactinia. Rchb.

Synandra. Nutt.

Synantliereae.

- genuinae.

Syncarpicae.

Synchlamydeae.

Synclistae.

- nucamentaceae.

- articulatae.

- continuae.

Syndesmon. IIfgg.

Syngenesisten.

Synoum. A. Juss.

Synpetalae.

Syuzyganthera. Rz.Pav.

Syrenia. Andrz.

Syringa. $L$.

Syzyginm. Gäri.

Szorits.

Szubert.

Tabacina. $R$.

Tabacum.

Taccaceac.

Tacceae.

Tichia. Aubl.

Tachybota. Aubl.

Talauma. Juss.

Talaumeae.

Talineae.

Talinum. Ad.

Talisia. Aubl.

Tamarisceae.

Tamariscineae.

Tamarix. T.L.

Taugalgen.

Taonabo. Aubl.

Taphrosperumum. C.A.M.

Targioneae.

Tasmaunia. $R . B r$.

Tasmannieac.

T'auscl.

Tauscheria. Fisch.

Taxineae.

Taxeac.

Taxus.

Teedia. Rud.

'I'eesdalia. $R, B$

'Telephicae.

Telephium. T. $L$.

Telf a ir.

Tellerflechten.

Tellima. R. Br.

Teloxis. Moq. Tand.

Temus. Mol.

Tenore.

Terebintliaceac.

Terebinthaceen.
S. 313 Terebinthinae.

313 Terminalieae.

311 Ternstroemia. Mut.

311 Ternstroenieac.

186 - genuinae.

20 i Tetracellium. Turczan.

206i Tetracera, $\boldsymbol{L}$ 。

1920 Tetraceratium, $D_{e} C$.

206 Tetradenia. Benth.

215 Tetradiclis. Stev.

206 Tetrailynamae.

189 Tetragonia. I.

180 Tetragonieae.

180 Tetranieles. $\mathcal{R} . \mathrm{Br}$.

182 Tetrapoma. Tiurczan.

161 Tetrapteris. Cav.

258 Tetrapterygium. Fisch, C 258 A. $M$.

258 Tetrataenium.

25!) Teucrieae.

$2 \bar{\gamma}($ Tencriopsis.

180 'Tencris.

313 Tencrium. T. $L$.

18. Thalaunantliae.

17: Thalictrene.

$2(0)$ 'Thalictrum. $\boldsymbol{L}$.

213 Thapsia. 'T. $L$.

253 Thapsieae.

18 Thea. $L$.

11.18 Theaceae.

Thecacoris. A. Juss.

201 Theegewäclıse.

201 Thelyuronum. L.

142 Theophast.

142 Theophyrasta. $L$.

211 Thesieac.

269) Thespesia. Corr.

278 Thespesieae.

278 Thibaudia. Rz. Pav.

237 Thlaspi. Dill. L.

237 Thlaspideae.

285 - biscutatae.

240 - naviculares.

240 'Thomasia. Gay.

240 Thorntonia. Rchb.

13̈6 Thoninia. Poit.

302 Thryallis. $\boldsymbol{L}$.

260 Thunbergia. $L$

136 Thunbergiene.

278 'Thyana. Han:ilt.

278 'Thylachiun. Lour.

11 Thymbra. $L$.

258 Thymeleaceae.

16 .5 Tlymus. $L$.

166 Thysanocarpus. Jlook.

166 Thysselinum.Dod,Riv.Sp

1999 Tiarella. $\boldsymbol{L}$.

259 Tiarislium. Lehm.

23ii Ticorea, Aubl.

287 Tiedemannia. $D e C$.

(i) Tilia. L.

133 Tiliaceac.

230 Tiliariae.

238 - geuninae.

278 Tillandsiene.

31 Tina. R.S.

223 Tiniaria. Meisn.

203 Titlymaleae.
S. 224/Tittmannia. Rchb.

247 Tordylineae.

302 Tortylium. T.

302 Torenia. I

302 Torfmonre.

e(i) Toricellia. De C

27 Torilis. Alens. Spr.

260 Tovaria. J?z. Pav.

188 Toulicia. Aubl.

218 Tovomita. Aubl.

256 Tovomiteac.

240 T o n r e f or t.

240 Tournefortia. $L$.

244 Tournefortiea.

$2(i)$ Toxicodeadron. $T / 2 b$

2y9 Tozzia. L

Torzieae.

256 Trachelium. $L$.

20 Tracliymene. $R d g$.

189 Trachypleurum, Fichb.

189 Trachyschizocaryicae.

189 Trachysciatium. DeC.

189 'Trachytella. $D_{\mathrm{c}} \mathrm{C}$.

256 Teragia. Plum.

$27(j$ Tragium. Spr. 211

2รil Tragopyum. M. B.

220 'Fragoriganum. 188

200 Tragoselinum. T. ㄴ.

302 Tralliana. Lour. 301

300 Trapa. $L$. $2+6$

28\% 'Trapea. $24 \%$

300 Traubenfarrn.

$2+30$. Tratretter. 18

36 Trautvetteria. Fisch, et $C$.

204

167 Tremandreae.

287

288 Trepociurus

288 Treviranus. 11.35.51, 11!

206 'Triadenin

\%5) Triadenium. Rafin. 308

259 Triadica

25y 'Trianthema. L.

25!) Tribulea.

292 Tribulus. T. $L$.

287 Tricera. Schreb. $28 \%$

285 Trichilia. $L$. 313

oyy) Trichiliea

191 - genuinae.

190 Trichlis: Hall. .

285 Trichocarpus, Schrcb.

267 Trichodesma. R. Br. 19\%2

189 Trichogonium. DeC. 220

1(i) Triclosiphum. Schott.

189 Findl. 291

258 Trichospermum. $B l$. 27t

220 Trichosphaeria. 184

230 Trichosporum. Don. (non

193 Fries.) 19y

28* Triclostemma. $L$. 189

220 Tricuspislaria. $R$ z.Pav. 304

303 Trillesineae. 318

302 Tridesuis. Spach. 308

303 Trifolieac. 220

303 - genuinac.

151 Trifolium. $L$. 24;

236.) Triglochineae. 144

280 Trigonia. Aubl. 302 
Trigonieae.

Trigynaea. Schlecht.

Trimeris. Prsl.

Trinia Hoffm.

Trinius.

Triopteris. $L$.

Triphaca. Lour.

Triphaceae.

Triphasia. Lour.

Triplıasicae.

Triplysaria. Fisch et Mey.

Triplaris. $\boldsymbol{L}$.

Triplateia. Bertl.

Triplostegia. Wall.

Tripterocuecus. Endl.

Tristania. $\boldsymbol{R} . \boldsymbol{B r}$.

Tristellaria. A.P.Th.

Triticeac.

Triumfetta. L. Gärtn.

Trimufettaria, $\boldsymbol{R}$.

Trixis, Mitch. Gürtn.

Truchetia. De C.

Trochiseanthes. $K$.

Trullieac.

Trollius. $L$.

Tropacoleae.

Tropaeolum. $L$.

Iubercularii.

Tulbaghia.

Tulbaghieac.

'Iullia.

Tuna. Dill,

Tunica. Scop.

Tupistreae.

Turaria. Mol.

Turczinninow.

Turgenia. Hoffm.

Turnera. Plum.

Turnercae.

Turp in.

Turpinia. Fent.

Turraea. $L$.

Turrita.

Turritina.

Turritis. $\boldsymbol{L}$.

Tylonimm. Presl.

Typhaceac.

Typheae.

Vaccinieae.

Vaccinium. L.

Valulia, Thb.

Vahlieae.

Vaillant.

Valeriana. $L$.

Valerianeac.

Valerianclla. $T$.

Valeriandleae.

Vallea. Mut.

Ianalphena. Leschen.

Vancouveria. Morr.

Vandeae.

Vandellia. L.

Vanilleae.

Vargasia. Bert.

Varronia. $L$.

Vateria. $L$.

Vatica. $L$.
S, 302 Vaucheriene.

278 Veilchengewïchse.

186 Tulaen. DeC.

21!) I clarum. Plin.

18 Velezia. $L$.

$29 y$ Vella. $L$.

291 Velleac.

291 Vellosieae.

314 Venana. Lam.

314 Ventenatia. $P$. B.

198 Veratrene.

236 Verbaseariac.

298 Verbascea.

178 Verlascum. $L$.

282 Verluena. $L$

253 Verbencac.

299) Veronica. $\boldsymbol{L}$.

$14(i$ Veronicene.

31:3 Verticillaria. Rz. Pav.

$30:$ Verticordia. $D$ c $C$

244 Vesicaria. Lam.

$2 ! 1$ v. I es t.

2:0 Vestia. $W$.

277 Viburnum. $L$.

278 Vicieac.

200 Vicatia. De C.

290 Viemülitige.

12!) Villarsia. I ent.

154 Vinceae.

$15+$ Viola. $L$.

$188^{\prime}$ Violaceae.

234 | Violeac.

298 - genuinae.

142 Viorna. Pers.

215 Virgnlaria. Rz.Pav.

18 Iiscago. Hall.

219 Viscaria, Riv.

232 Viticella. Dill.

232 Vitis. $L$.

152 Viviania, Colla.

285 Vismia. Iand.

313 Vismieae.

260 - genuinae.

260 Vismea. $L$.

260 Ileckia, Rafin.

186 Ullucus. Laz.

148 Ulmeae.

189 Ulospermum. Lk.

206 Ulvaceae.

206 Ulveae.

230 Umbellaria.

230 Unbelliferae.

1.37. 65 - genuinac.

\begin{tabular}{l|l}
178 & Unger. \\
178 & Ungeria. Schott. Endl.
\end{tabular}

178 Ungerieae.

178 Unona. $L$.

304 Vochysieae.

302 Vo ig t.

265 Totonita, Aubl.

155 Voyra. Aubl.

199 Uredinei.

155 Urena, $L$.

29y) v. Vriese.

193 Urpilze.

304 Urticaceae.

304 Urticeac.
S. 136 Usneaceae.

268 Usteria. Cav.

S. 133

(j0 Utricularia. $L$

298 Uvaria. L. 278

2 (jo Uvariene.

2 (i0) Uredalia. R.Br. 195

151

214 Walılenbergia. Schrad. 186

302 W a it $\%$.

152 Walkera, Schreb. 283

198 IV a I I i cli.

199 W a ll rotli. $\quad 11.51,117$

199 Wallrothia. Spr.

190 Walsura. Rxb. 313

190 Waltheria.

198 IValtherieae. ' 291

198 Warzenpilze. 129

311 Wasserliesche. 143

253 Wasserriemen. 141

25!) IV at $\mathrm{s}$ o 11.

11 Webbia. Spach. 308

201 Wedelfarrn. 138

178. Weidriche. 247

226 We i in man n. 11

219 Weiumannia. $L$. 230

256 Weinmannicae. $\mathbf{2 3 0}$

210 IV elw itschia. Rchb. 194

211 Wenderoth. 11. 33

268 W'endtia. Hoffm.

2(j8) Westringia. Sm. 18!

2 (jo Uickstroemia. Schirad. 302

2(69) W i e g m a n n, 11.32.36.94

278 W i eland.

198 Wigandiı, K.H.B. 194

298 IV ilbrandt.

298 Willdenow. 31. 78

277 Willemetia, C. A.M. 239

221 IV il son. 141

253 Wimmer. 11.86

308 Windengewãchse.

308 wink ler. zu 11

308 Wintereae. 278

215 Wittelsbachia. Mart, 302

189) Wormia, Rottb. 278

237. Anm. Wulfenia. Jacq. 198

172 Wydleria. DeC.

220 Wylia. Hoffm. 219

135

136

189) Xanthieae, 182

217 Tanthiophaea. 189

218 Xantholinum. Rehb. $\quad 307$

177

2y) Xanthosia. Rudg.

294 Xeroteac. 152

278 Xyladenius. Hamilt. 274

248 Xylocarpeac. 313

7.43 Xylocarpu\%. Koen. 313

221 Xylopia. P. Brwn. 278

211 Xylopleurum, Spach. $2+6$

129 Xyrideac. 148

287

11. 36 Zahlhrucknera. Rchb. 230

129) Zamiaceac. 139

172 Zamieac.

172 Zannichcllieae. 144 
Zanthoxyleae.

Zapfenbäume.

Zapfenfarrn.

Zaukenlilien.

Zausehneria. Prsl.

Zenker.

Zieria. Forst.
S. $282 \mid$ Zictersia.

168 Zilla. Forsk.

139 Zimmermann.

152 Zippelia. Rchb

246 Zizia. $K$.

11.22, 51,53.117 Ziziphora. $L$.

283 Zoegea.
S. $188 \mid$ Zosimia. Hoffm.

258 Zostera.

11 Zostereae.

164 Zweifelblumige.

219 Zygnemene.

188 Zygophylleac.

182 - genuinae.
S. 220

14:

141

161

135

28.5

285 


